\title{
Interventions for preventing obesity in children (Review)
}

Brown T, Moore THM, Hooper L, Gao Y, Zayegh A, ljaz S, Elwenspoek M, Foxen SC, Magee L, O'Malley C, Waters E, Summerbell CD

Brown T, Moore THM, Hooper L, Gao Y, Zayegh A, ljaz S, Elwenspoek M, Foxen SC, Magee L, O'Malley C, Waters E, Summerbell CD. Interventions for preventing obesity in children.

Cochrane Database of Systematic Reviews 2019, Issue 7. Art. No.: CD001871.

DOI: 10.1002/14651858.CD001871.pub4.

www.cochranelibrary.com 
TABLE OF CONTENTS

ABSTRACT

PLAIN LANGUAGE SUMMARY

SUMMARY OF FINDINGS

BACKGROUND

OBJECTIVES

METHODS

RESULTS

Figure 1.

Figure 2.

Figure 3.

Figure 4.

Figure 5.

Figure 6.

DISCUSSION

AUTHORS' CONCLUSIONS

ACKNOWLEDGEMENTS

REFERENCES

CHARACTERISTICS OF STUDIES

DATA AND ANALYSES

Analysis 1.1. Comparison 1 Dietary interventions versus control: age 0-5 years, Outcome 1 zBMI.

Analysis 2.1. Comparison 2 Physical activity interventions versus control: age 0-5 years, Outcome 1 zBMI. Physical activity vs control - setting.

Analysis 2.2. Comparison 2 Physical activity interventions versus control: age 0-5 years, Outcome 2 BMI. Physical activity vs control - setting.

Analysis 3.1. Comparison 3 Diet and physical activity interventions versus control: age 0-5 years, Outcome 1 zBMI. Diet and physical activity vs control - setting.

Analysis 3.2. Comparison 3 Diet and physical activity interventions versus control: age 0-5 years, Outcome 2 zBMI. Diet and physical activity vs control - duration.

Analysis 3.3. Comparison 3 Diet and physical activity interventions versus control: age 0-5 years, Outcome 3 BMI. Diet and physical activity vs control - setting.

Analysis 3.4. Comparison 3 Diet and physical activity interventions versus control: age 0-5 years, Outcome 4 BMI. Diet and physical activity vs control - duration.

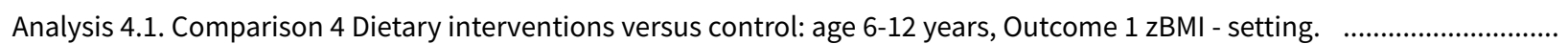

Analysis 4.2. Comparison 4 Dietary interventions versus control: age 6-12 years, Outcome 2 BMI - setting.

Analysis 5.1. Comparison 5 Physical activity interventions versus control: age 6-12, Outcome 1 zBMI. Physical activity vs control - setting.

Analysis 5.2. Comparison 5 Physical activity interventions versus control: age 6-12, Outcome 2 zBMI. Physical activity vs control - duration.

Analysis 5.3. Comparison 5 Physical activity interventions versus control: age 6-12, Outcome 3 BMI. Physical activity vs control - setting.

Analysis 5.4. Comparison 5 Physical activity interventions versus control: age 6-12, Outcome 4 BMI. Physical activity vs control - duration.

Analysis 6.1. Comparison 6 Diet and physical activity interventions vs control: age 6-12 years, Outcome 1 zBMI. Diet and physical activity vs control - setting.

Analysis 6.2. Comparison 6 Diet and physical activity interventions vs control: age 6-12 years, Outcome 2 zBMI. Diet and physical activity vs control - duration.

Analysis 6.3. Comparison 6 Diet and physical activity interventions vs control: age 6-12 years, Outcome 3 BMI. Diet and physical activity vs control - setting.

Analysis 6.4. Comparison 6 Diet and physical activity interventions vs control: age 6-12 years, Outcome 4 BMI. Diet and physical activity vs control - duration.

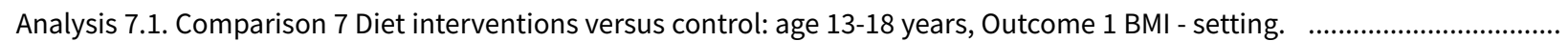

Analysis 8.1. Comparison 8 Physical activity interventions versus control: age 13-18 years, Outcome 1 zBMI - setting. ..............

Analysis 8.2. Comparison 8 Physical activity interventions versus control: age 13-18 years, Outcome 2 zBMI - duration. .......... 
Analysis 8.3. Comparison 8 Physical activity interventions versus control: age $13-18$ years, Outcome 3 BMI - setting. ............... 530 Analysis 8.4. Comparison 8 Physical activity interventions versus control: age 13-18 years, Outcome 4 BMI - duration. ............ 531 Analysis 9.1. Comparison 9 Diet and physical activity interventions versus control: age 13-18 years, Outcome 1 zBMI - setting. .. 532 Analysis 9.2. Comparison 9 Diet and physical activity interventions versus control: age 13-18 years, Outcome 2 zBMI - duration. 533 Analysis 9.3. Comparison 9 Diet and physical activity interventions versus control: age 13-18 years, Outcome 3 BMI - setting. .. 533 Analysis 9.4. Comparison 9 Diet and physical activity interventions versus control: age 13-18 years, Outcome 4 BMI - duration. . 534 Analysis 10.1. Comparison 10 Dietary interventions versus physical activity interventions: age 6-12 years, Outcome 1 zBMI. ... 535 Analysis 10.2. Comparison 10 Dietary interventions versus physical activity interventions: age 6-12 years, Outcome 2 BMI. .... 535 Analysis 11.1. Comparison 11 Diet and physical activity versus physical activity interventions: age 6-12 years, Outcome 1 zBMI. 536 Analysis 11.2. Comparison 11 Diet and physical activity versus physical activity interventions: age 6-12 years, Outcome 2 BMI. . 536 Analysis 12.1. Comparison 12 Dietary interventions versus diet and physical activity interventions: age 6-12 years, Outcome $1 \quad 536$ zBMI.

Analysis 12.2. Comparison 12 Dietary interventions versus diet and physical activity interventions: age 6-12 years, Outcome 2 BMI.

ADDITIONAL TABLES

APPENDICES

WHAT'S NEW

HISTORY 
[Intervention Review]

\section{Interventions for preventing obesity in children}

Tamara Brown 1,2a, Theresa HM Moore ${ }^{3,4 b}$, Lee Hooper ${ }^{5}$, Yang Gao6, Amir Zayegh7 ${ }^{7}$, Sharea ljaz 3,4 , Martha Elwenspoek ${ }^{3,4}$, Sophie C Foxen ${ }^{8}$, Lucia Magee 9 , Claire O'Malley1,2c, Elizabeth Waters 10 , Carolyn D Summerbell1,2

1Department of Sport and Exercise Sciences, Durham University, Durham, UK. 2Fuse, the NIHR Centre for Translational Research in Public Health, Durham, UK. ${ }^{3}$ Population Health Sciences, Bristol Medical School, University of Bristol, Bristol, UK. ${ }^{4}$ NIHR CLAHRC West at University Hospitals Bristol NHS Foundation Trust, Bristol, UK. ${ }^{5}$ Norwich Medical School, University of East Anglia, Norwich, UK. ${ }^{6}$ Department of Sport and Physical Education, Hong Kong Baptist University, Kowloon, Hong Kong. ${ }^{7}$ General Medicine, The Royal Children's Hospital, Melbourne, Australia. 8Defence Medical Services, Royal Air Force High Wycombe, Naphill, UK. 9 Medical Department, Royal United Hospital, Bath, UK. 10University of Melbourne, Parkville, Australia

a Tamara Brown and Theresa Moore contributed equally to this work and are considered joint first authors. Tamara Brown now works at Teesside University, Middlesbrough, UK. $b$ Tamara Brown and Theresa Moore contributed equally to this work and are considered joint first authors.. CThis author now works at Teesside University, Middlesbrough, UK

Contact: Theresa HM Moore, Population Health Sciences, Bristol Medical School, University of Bristol, Canynge Hall, Bristol, BS8 2PS, UK. theresa.moore@bris.ac.uk, theresa.moore@bristol.ac.uk.

Editorial group: Cochrane Public Health Group.

Publication status and date: New search for studies and content updated (conclusions changed), published in Issue 7, 2019.

Citation: Brown T, Moore THM, Hooper L, Gao Y, Zayegh A, ljaz S, Elwenspoek M, Foxen SC, Magee L, O'Malley C, Waters E, Summerbell CD. Interventions for preventing obesity in children. Cochrane Database of Systematic Reviews 2019, Issue 7. Art. No.: CD001871. DOI: 10.1002/14651858.CD001871.pub4.

Copyright @ 2019 The Cochrane Collaboration. Published by John Wiley \& Sons, Ltd.

\section{A B S T R A C T}

\section{Background}

Prevention of childhood obesity is an international public health priority given the significant impact of obesity on acute and chronic diseases, general health, development and well-being. The international evidence base for strategies to prevent obesity is very large and is accumulating rapidly. This is an update of a previous review.

\section{Objectives}

To determine the effectiveness of a range of interventions that include diet or physical activity components, or both, designed to prevent obesity in children.

\section{Search methods}

We searched CENTRAL, MEDLINE, Embase, PsychINFO and CINAHL in June 2015. We re-ran the search from June 2015 to January 2018 and included a search of trial registers.

\section{Selection criteria}

Randomised controlled trials (RCTs) of diet or physical activity interventions, or combined diet and physical activity interventions, for preventing overweight or obesity in children (0-17 years) that reported outcomes at a minimum of 12 weeks from baseline.

\section{Data collection and analysis}

Two authors independently extracted data, assessed risk-of-bias and evaluated overall certainty of the evidence using GRADE. We extracted data on adiposity outcomes, sociodemographic characteristics, adverse events, intervention process and costs. We meta-analysed data as guided by the Cochrane Handbook for Systematic Reviews of Interventions and presented separate meta-analyses by age group for child 0 to 5 years, 6 to 12 years, and 13 to 18 years for zBMI and BMI. 


\section{Main results}

We included 153 RCTs, mostly from the USA or Europe. Thirteen studies were based in upper-middle-income countries (UMIC: Brazil, Ecuador, Lebanon, Mexico, Thailand, Turkey, US-Mexico border), and one was based in a lower middle-income country (LMIC: Egypt). The majority (85) targeted children aged 6 to 12 years.

Children aged 0-5 years: There is moderate-certainty evidence from 16 RCTs $(n=6261)$ that diet combined with physical activity interventions, compared with control, reduced BMI (mean difference (MD) $-0.07 \mathrm{~kg} / \mathrm{m}^{2}, 95 \%$ confidence interval (Cl) -0.14 to -0.01 ), and had a similar effect ( $11 \mathrm{RCTs}, \mathrm{n}=5536$ ) on zBMI (MD $-0.11,95 \% \mathrm{Cl}-0.21$ to 0.01 ). Neither diet (moderate-certainty evidence) nor physical activity interventions alone (high-certainty evidence) compared with control reduced BMI (physical activity alone: $\mathrm{MD}-0.22 \mathrm{~kg} / \mathrm{m}^{2}, 95 \%$ $\mathrm{Cl}-0.44$ to 0.01 ) or zBMI (diet alone: $\mathrm{MD}-0.14,95 \% \mathrm{Cl}-0.32$ to 0.04 ; physical activity alone: $\mathrm{MD} 0.01,95 \% \mathrm{Cl}-0.10$ to 0.13 ) in children aged $0-5$ years.

Children aged 6 to 12 years: There is moderate-certainty evidence from 14 RCTs $(n=16,410)$ that physical activity interventions, compared with control, reduced BMI (MD $-0.10 \mathrm{~kg} / \mathrm{m}^{2}, 95 \% \mathrm{Cl}-0.14$ to -0.05$)$. However, there is moderate-certainty evidence that they had little or no effect on $\mathrm{zBMI}(\mathrm{MD}-0.02,95 \% \mathrm{Cl}-0.06$ to 0.02$)$. There is low-certainty evidence from $20 \mathrm{RCTs}(\mathrm{n}=24,043)$ that diet combined with physical activity interventions, compared with control, reduced $z B M I(M D-0.05 \mathrm{~kg} / \mathrm{m} 2,95 \% \mathrm{Cl}-0.10$ to -0.01$)$. There is high-certainty evidence that diet interventions, compared with control, had little impact on zBMI (MD $-0.03,95 \% \mathrm{Cl}-0.06$ to 0.01$)$ or $\mathrm{BMI}(-0.02 \mathrm{~kg} / \mathrm{m} 2,95 \% \mathrm{Cl}-0.11$ to 0.06$)$.

Children aged 13 to 18 years: There is very low-certainty evidence that physical activity interventions, compared with control reduced BMI (MD $-1.53 \mathrm{~kg} / \mathrm{m} 2,95 \% \mathrm{Cl}-2.67$ to $-0.39 ; 4 \mathrm{RCTs} ; \mathrm{n}=720)$; and low-certainty evidence for a reduction in $\mathrm{zBMI}(\mathrm{MD}-0.2,95 \% \mathrm{Cl}-0.3$ to $-0.1 ; 1$ RCT; $n=100)$. There is low-certainty evidence from eight RCTs $(n=16,583)$ that diet combined with physical activity interventions, compared with control, had no effect on BMI (MD $-0.02 \mathrm{~kg} / \mathrm{m} 2,95 \% \mathrm{Cl}-0.10$ to 0.05 ); or $\mathrm{ZBMI}$ (MD 0.01, $95 \% \mathrm{Cl}-0.05$ to $0.07 ; 6 \mathrm{RCTs} ; \mathrm{n}=$ 16,543). Evidence from two RCTs (low-certainty evidence; $n=294$ ) found no effect of diet interventions on BMI.

Direct comparisons of interventions: Two RCTs reported data directly comparing diet with either physical activity or diet combined with physical activity interventions for children aged 6 to 12 years and reported no differences.

Heterogeneity was apparent in the results from all three age groups, which could not be entirely explained by setting or duration of the interventions. Where reported, interventions did not appear to result in adverse effects (16 RCTs) or increase health inequalities (gender: 30 RCTs; socioeconomic status: 18 RCTs), although relatively few studies examined these factors.

Re-running the searches in January 2018 identified 315 records with potential relevance to this review, which will be synthesised in the next update.

\section{Authors' conclusions}

Interventions that include diet combined with physical activity interventions can reduce the risk of obesity (zBMI and BMI) in young children aged 0 to 5 years. There is weaker evidence from a single study that dietary interventions may be beneficial.

However, interventions that focus only on physical activity do not appear to be effective in children of this age. In contrast, interventions that only focus on physical activity can reduce the risk of obesity (BMI) in children aged 6 to 12 years, and adolescents aged 13 to 18 years. In these age groups, there is no evidence that interventions that only focus on diet are effective, and some evidence that diet combined with physical activity interventions may be effective. Importantly, this updated review also suggests that interventions to prevent childhood obesity do not appear to result in adverse effects or health inequalities.

The review will not be updated in its current form. To manage the growth in RCTs of child obesity prevention interventions, in future, this review will be split into three separate reviews based on child age.

\section{PLAIN LANGUAGE SUMMARY}

\section{Do diet and physical activity strategies help prevent obesity in children (aged 0 to 18 years)?}

\section{Background}

More children are becoming overweight and obese worldwide. Being overweight as a child can cause health problems, and children may be affected psychologically and in their social life. Overweight children are likely also to be overweight as adults and continue to experience poor physical and mental health.

\section{Searching for studies}

We searched many scientific databases to find studies that looked at ways of preventing obesity in children. We included studies aimed at all ages of children. We only included studies if the methods they were using were aimed at changing children's diet, or their level of physical 
activity, or both. We looked only for the studies that contained the best information to answer this question, 'randomised controlled trials' or RCTs.

\section{What we found}

We found 153 RCTs. The studies were based mainly in high-income countries such as the USA and European countries although $12 \%$ were in middle-income countries (Brazil, Ecuador, Egypt, Lebanon, Mexico, Thailand and Turkey). Just over half the RCTs (56\%) tried out strategies to change diet or activity levels in children aged 6 to 12 years, a quarter were for children aged 0 to 5 years and a fifth (20\%) were for teenagers aged 13 to 18 . The strategies were used in different settings such as home, preschool or school and most were targeted towards trying to change individual behaviour.

\section{Did they work?}

One widely accepted way of assessing if a child is overweight is to calculate a score based on their height and how much they weigh, and relating this to the weight and height of many children their age in their country. This is called the zBMI score. We found 61 RCTs involving over 60,000 children, that had reported zBMI scores. Children aged 0 to 5, and children aged 6 to 12 who were helped with a strategy to change their diet or activity levels reduced their zBMI score by 0.07 and 0.04 units respectively compared to children who were not given a strategy. This means these children were able to reduce their weight. This change in zBMI, when provided to many children across a whole population, is useful for governments in trying to tackle the problems of obesity in children. Strategies to change diet or physical activity, or both, given to adolescents and young adults aged 13 to 18 years, did not successfully reduce zBMI.

We looked to see if the strategies were likely to work fairly for all children, for example girls and boys, children from wealthy or less wealthy backgrounds, children from different racial backgrounds. Not many RCTs reported this, but in those that did, there was no indication that the strategies increased inequalities. However we could not find enough RCTs with this information to help us answer this question. We also looked to see if children were harmed by any of the strategies, for example by having injuries, losing too much weight or developing damaging views about themselves and their weight. Not many RCTs reported this, but in those that did, none reported any harms from children who had been given strategies to change their diet or physical activity.

We looked at how well the RCTs were done to see if they might be biased. We decided to downgrade some information based on these assessments. The quality of the evidence was 'moderate' for children aged 0 to 5 for zBMI, 'low' for children aged 6 to 12 and moderate for adolescents (13 to 18$)$.

\section{Our conclusions}

Strategies for changing diet or activity levels, or both, of children in order to help prevent them becoming overweight or obese are effective in making modest reductions in zBMI score in children aged 0 to 5 years and in children aged 6 to 12 years. This can be useful to parents and children concerned about children becoming overweight. It can also be useful for governments, trying to tackle a growing trend of children who are becoming obese or overweight. We found less evidence for adolescents and young people aged 13 to 18 , and the strategies given to them did not reduce their zBMI score. 


\section{SUMMARY OF FINDINGS}

\section{Summary of findings for the main comparison. Dietary interventions compared to control for preventing obesity in children aged 0 to 5 years}

Dietary interventions compared to control for preventing obesity in children aged 0 to 5 years

Patient or population: children aged 0-5 years

Setting: healthcare setting

Intervention: dietary interventions

Comparison: control

\begin{tabular}{|c|c|c|c|c|c|}
\hline \multirow[t]{2}{*}{ Outcomes } & \multicolumn{2}{|c|}{ Anticipated absolute effects ${ }^{*}(95 \% \mathrm{Cl})$} & \multirow{2}{*}{$\begin{array}{l}\text { № of participants } \\
\text { (studies) }\end{array}$} & \multirow{2}{*}{$\begin{array}{l}\text { Certainty of the } \\
\text { evidence } \\
\text { (GRADE) }\end{array}$} & \multirow[t]{2}{*}{ Comments } \\
\hline & Risk with control & Risk with dietary interventions & & & \\
\hline
\end{tabular}

${ }^{*}$ The risk in the intervention group (and its $95 \%$ confidence interval) is based on the assumed risk in the comparison group and the relative effect of the intervention (and its $95 \% \mathrm{Cl}$ ).

Cl: confidence interval; MD: mean difference; RCT: randomised controlled trial; zBMI: body-mass index z score

\section{GRADE Working Group grades of evidence}

High certainty: we are very confident that the true effect lies close to that of the estimate of the effect.

Moderate certainty: we are moderately confident in the effect estimate: the true effect is likely to be close to the estimate of the effect, but there is a possibility that it is substantially different.

Low certainty: our confidence in the effect estimate is limited: the true effect may be substantially different from the estimate of the effect.

Very low certainty: we have very little confidence in the effect estimate: the true effect is likely to be substantially different from the estimate of effect.

1Risk of bias: there is only one study and it has one domain (incomplete outcome data) rated as high risk of bias, with $22 \%$ of participants dropping out of the study.

Summary of findings 2. Physical activity interventions compared to control for preventing obesity in children aged 0 to 5 years

\section{Physical activity interventions compared to control for preventing obesity in children aged 0 to 5 years}

Patient or population: children aged 0-5 years

Setting: childcare/preschool or healthcare setting

Intervention: physical activity interventions

Comparison: control 


\begin{tabular}{|c|c|c|c|c|c|}
\hline \multirow[t]{2}{*}{ Outcomes } & \multicolumn{2}{|c|}{ Anticipated absolute effects ${ }^{\star}(95 \% \mathrm{Cl})$} & \multirow{2}{*}{$\begin{array}{l}\text { № of participants } \\
\text { (studies) }\end{array}$} & \multirow{2}{*}{$\begin{array}{l}\text { Certainty of the } \\
\text { evidence } \\
\text { (GRADE) }\end{array}$} & \multirow[t]{2}{*}{ Comments } \\
\hline & Risk with control & $\begin{array}{l}\text { Risk with physical activity interven- } \\
\text { tions }\end{array}$ & & & \\
\hline $\begin{array}{l}\text { Body-mass index } \\
\text { (BMI) }\end{array}$ & $\begin{array}{l}\text { The mean BMI ranged from } \\
15.94 \text { to } 16.4 \mathrm{~kg} / \mathrm{m}^{2}\end{array}$ & $\begin{array}{l}\text { MD } 0.22 \mathrm{~kg} / \mathrm{m}^{2} \text { lower } \\
\text { ( } 0.44 \text { lower to } 0.01 \text { higher) }\end{array}$ & $\begin{array}{l}2233 \\
\text { (5 RCTs) }\end{array}$ & $\begin{array}{l}\oplus \oplus \oplus \oplus \\
\text { High }\end{array}$ & $\begin{array}{l}\text { Physical activity interventions } \\
\text { likely do not reduce BMI }\end{array}$ \\
\hline $\begin{array}{l}\text { Body-mass index z } \\
\text { score (zBMI) }\end{array}$ & $\begin{array}{l}\text { The mean zBMI ranged from } \\
-0.15 \text { to }-0.22\end{array}$ & $\begin{array}{l}\text { MD } 0.01 \text { higher } \\
\text { ( } 0.10 \text { lower to } 0.13 \text { higher) }\end{array}$ & $\begin{array}{l}1053 \\
\text { (4 RCTs) }\end{array}$ & $\begin{array}{l}\oplus \oplus \oplus \oplus \\
\text { High }\end{array}$ & $\begin{array}{l}\text { Physical activity interventions } \\
\text { likely do not reduce zBMI }\end{array}$ \\
\hline
\end{tabular}

${ }^{\star}$ The risk in the intervention group (and its $95 \%$ confidence interval) is based on the assumed risk in the comparison group and the relative effect of the intervention (and its $95 \% \mathrm{Cl})$.

BMI: body-mass index; CI: confidence interval; MD: mean difference; RCT: randomised controlled trial; zBMI: body-mass index z score

\section{GRADE Working Group grades of evidence}

High certainty: we are very confident that the true effect lies close to that of the estimate of the effect.

Moderate certainty: we are moderately confident in the effect estimate: the true effect is likely to be close to the estimate of the effect, but there is a possibility that it is substantially different.

Low certainty: our confidence in the effect estimate is limited: the true effect may be substantially different from the estimate of the effect.

Very low certainty: we have very little confidence in the effect estimate: the true effect is likely to be substantially different from the estimate of effect.

\section{Summary of findings 3. Diet and physical activity interventions combined compared to control for preventing obesity in children aged 0 to 5 years}

\section{Diet and physical activity interventions combined compared to control for preventing obesity in children age 0-5 years}

Patient or population: children aged 0-5 years

Setting: childcare/preschool, health system, wider community or home

Intervention: combined diet and physical activity interventions

Comparison: control

\begin{tabular}{|c|c|c|c|c|c|}
\hline \multirow[t]{2}{*}{ Outcomes } & \multicolumn{2}{|c|}{ Anticipated absolute effects ${ }^{\star}(95 \% \mathrm{Cl})$} & \multirow{2}{*}{$\begin{array}{l}\text { № of participants } \\
\text { (studies) }\end{array}$} & \multirow{2}{*}{$\begin{array}{l}\text { Certainty of the } \\
\text { evidence } \\
\text { (GRADE) }\end{array}$} & \multirow[t]{2}{*}{ Comments } \\
\hline & Risk with control & $\begin{array}{l}\text { Risk with diet and physical ac- } \\
\text { tivity interventions }\end{array}$ & & & \\
\hline $\begin{array}{l}\text { Body-mass index z } \\
\text { score (zBMI) }\end{array}$ & $\begin{array}{l}\text { The mean zBMI ranged } \\
\text { from } 0.15 \text { to } 0.98\end{array}$ & $\begin{array}{l}\text { MD } 0.07 \text { lower ( } 0.14 \text { lower to } 0.01 \\
\text { lower) }\end{array}$ & $\begin{array}{l}6261 \\
(16 \text { RCTs) }\end{array}$ & $\begin{array}{l}\oplus \oplus \oplus \ominus \\
\text { Moderate } 1\end{array}$ & $\begin{array}{l}\text { Diet and physical activity interven- } \\
\text { tions potentially slightly reduce zBMI }\end{array}$ \\
\hline
\end{tabular}




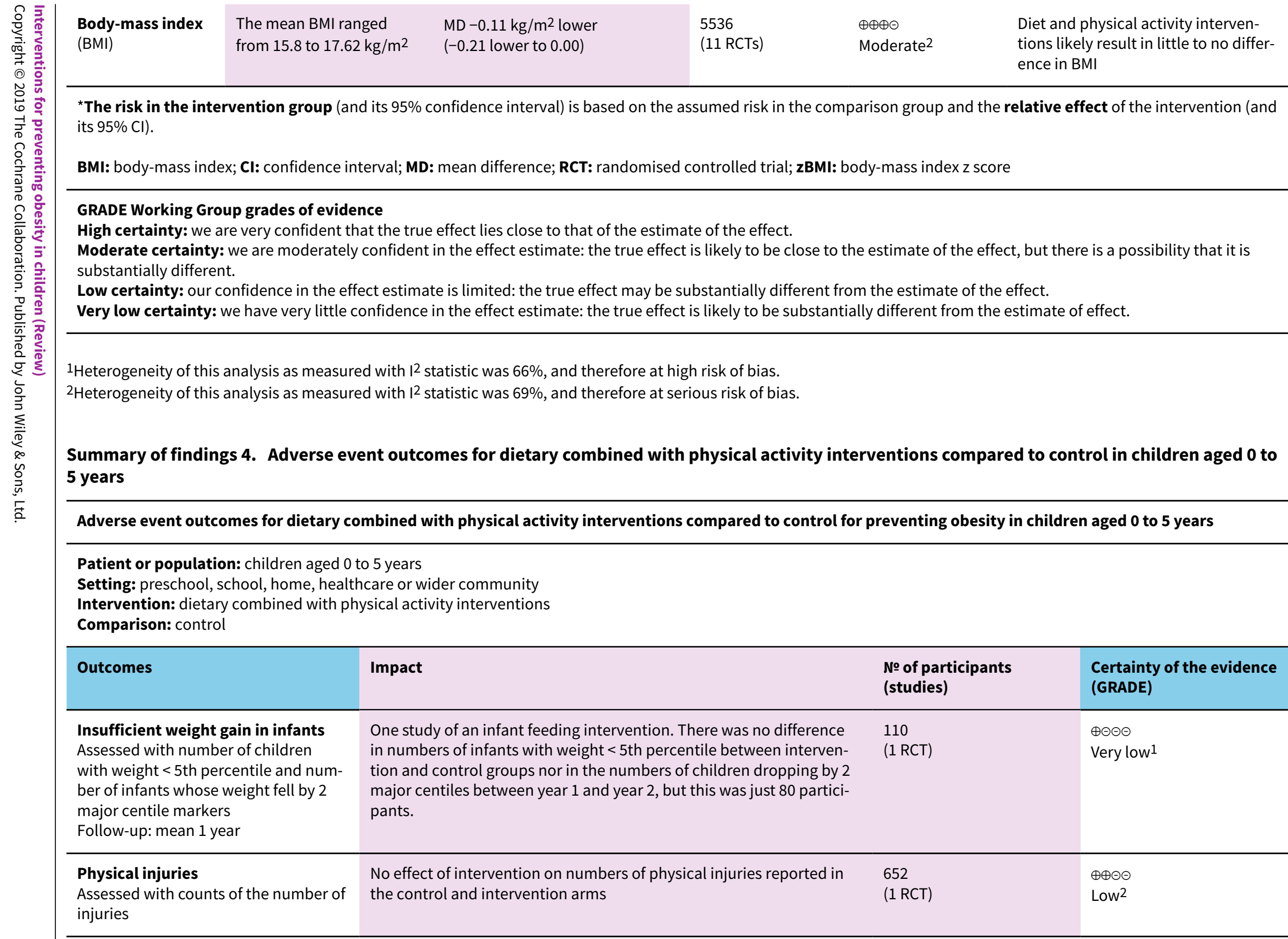




\begin{tabular}{|c|c|c|c|c|c|c|c|}
\hline \multicolumn{2}{|l|}{ Adverse events } & \multicolumn{3}{|c|}{ No 'adverse events' reported } & \multicolumn{2}{|c|}{$\begin{array}{l}983 \\
\text { (2 RCTs) }\end{array}$} & $\begin{array}{l}\oplus \oplus \ominus \ominus \\
\text { Low }^{3}\end{array}$ \\
\hline \multicolumn{2}{|c|}{$\begin{array}{l}\text { Infections } \\
\text { Assessed with parental questionnaire } \\
\text { Follow-up: range } 2 \text { months to } 4 \\
\text { months }\end{array}$} & \multicolumn{3}{|c|}{$\begin{array}{l}\text { No effect of intervention on numbers of reported infections. These da- } \\
\text { ta are very uncertain. A single study of just } 41 \text { participants found simi- } \\
\text { lar numbers of (parent-reported) infections in children in the interven- } \\
\text { tion and control groups. }\end{array}$} & \multicolumn{2}{|c|}{$\begin{array}{l}709 \\
(1 \mathrm{RCT})\end{array}$} & $\begin{array}{l}\oplus \oplus \ominus \ominus \\
\text { Low }^{2}\end{array}$ \\
\hline \multicolumn{2}{|c|}{$\begin{array}{l}\text { Accidents } \\
\text { Assessed with parental questionnaire } \\
\text { Follow-up: range } 2 \text { months to } 4 \\
\text { months }\end{array}$} & \multicolumn{3}{|c|}{$\begin{array}{l}\text { No effect on number of accidents. These data are very uncertain. A } \\
\text { single study of just } 41 \text { participants found similar numbers of (par- } \\
\text { ent-reported) accidents in children in the intervention and control } \\
\text { groups. }\end{array}$} & \multicolumn{2}{|c|}{$\begin{array}{l}42 \\
(1 \mathrm{RCT})\end{array}$} & $\begin{array}{l}\oplus \ominus \odot \odot \\
\text { Very low } 4\end{array}$ \\
\hline \multicolumn{8}{|c|}{$\mathbf{R C T}$ : randomised controlled trial } \\
\hline \multicolumn{8}{|c|}{$\begin{array}{l}\text { GRADE Working Group grades of evidence } \\
\text { High certainty: we are very confident that the true effect lies close to that of the estimate of the effect. } \\
\text { Moderate certainty: we are moderately confident in the effect estimate: the true effect is likely to be close to the estimate of the effect, but there is a possibility that it is } \\
\text { substantially different. } \\
\text { Low certainty: our confidence in the effect estimate is limited: the true effect may be substantially different from the estimate of the effect. } \\
\text { Very low certainty: we have very little confidence in the effect estimate: the true effect is likely to be substantially different from the estimate of effect. }\end{array}$} \\
\hline \multirow{2}{*}{\multicolumn{8}{|c|}{$\begin{array}{l}\text { 1Downgraded three times. Twice for imprecision, as evidence based on just one study with only } 110 \text { participants. Downloaded once for risk of } \\
\text { at high risk of bias and two unclear from a total of six items. } \\
\text { 2Downgraded twice for imprecision because this outcome was reported in one of } 26 \text { studies. } \\
\text { 3Downgraded three times for imprecision as this outcome was measured in only one of } 26 \text { studies and only } 42 \text { participants. } \\
\text { Summary of findings 5. Dietary interventions compared to control for preventing obesity in children aged } 6 \text { to } 12 \text { years }\end{array}$}} \\
\hline & & & & & & & \\
\hline \multicolumn{8}{|c|}{ Dietary interventions compared to control for preventing obesity in children aged 6 to 12 years } \\
\hline \multicolumn{8}{|c|}{$\begin{array}{l}\text { Patient or population: children aged } 6-12 \text { years } \\
\text { Setting: school or wider community } \\
\text { Intervention: dietary interventions } \\
\text { Comparison: control }\end{array}$} \\
\hline \multirow[t]{2}{*}{ Outcomes } & \multicolumn{3}{|c|}{ Anticipated absolute effects ${ }^{*}(95 \% \mathrm{Cl})$} & \multirow{2}{*}{\multicolumn{2}{|c|}{$\begin{array}{l}\text { № of participants } \\
\text { (studies) }\end{array}$}} & \multirow{2}{*}{$\begin{array}{l}\text { Certainty of the } \\
\text { evidence } \\
\text { (GRADE) }\end{array}$} & \multirow[t]{2}{*}{ Comments } \\
\hline & Risk with contr & & Risk with dietary interventions & & & & \\
\hline $\begin{array}{l}\text { Body-mass index z } \\
\text { score (zBMI) }\end{array}$ & $\begin{array}{l}\text { The mean zBMI } \\
0.09 \text { to } 0.41\end{array}$ & nged from & MD 0.03 lower ( 0.06 lower to 0.01 higher) & $\begin{array}{l}7231 \\
\text { (9 RCTs) }\end{array}$ & & $\begin{array}{l}\oplus \oplus \oplus \oplus \\
\text { High }\end{array}$ & $\begin{array}{l}\text { Dietary interventions } \\
\text { alone do not reduce zBMI }\end{array}$ \\
\hline
\end{tabular}




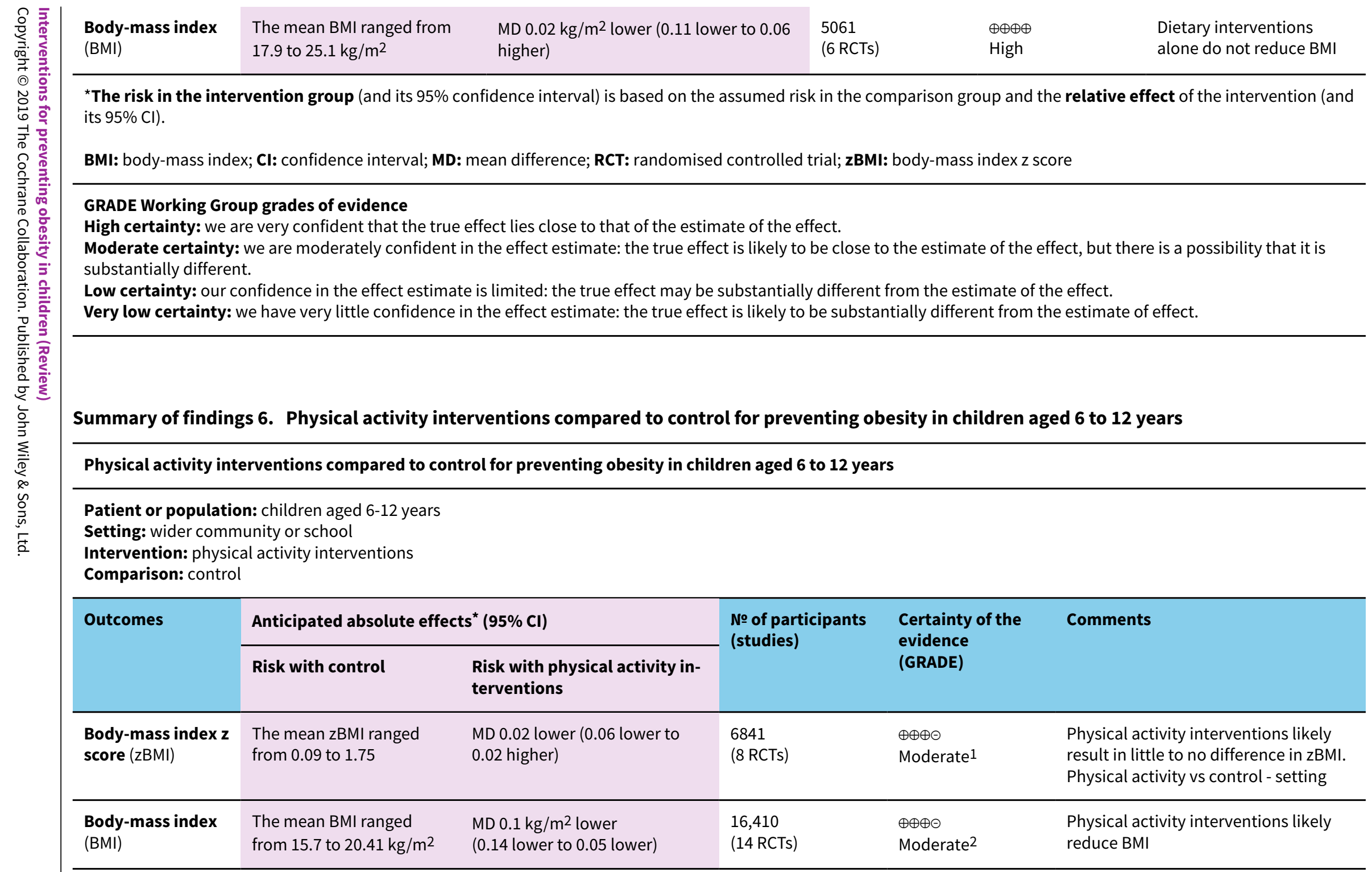

${ }^{\star}$ The risk in the intervention group (and its $95 \%$ confidence interval) is based on the assumed risk in the comparison group and the relative effect of the intervention (and its $95 \% \mathrm{Cl})$.

BMI: body-mass index; Cl: confidence interval; MD: mean difference; RCT: randomised controlled trial; zBMI: body-mass index z score 
High certainty: we are very confident that the true effect lies close to that of the estimate of the effect.

Moderate certainty: we are moderately confident in the effect estimate: the true effect is likely to be close to the estimate of the effect, but there is a possibility that it is

substantially different.

Low certainty: our confidence in the effect estimate is limited: the true effect may be substantially different from the estimate of the effect.

Very low certainty: we have very little confidence in the effect estimate: the true effect is likely to be substantially different from the estimate of effect.

${ }^{1}$ Four of seven studies have at least one domain judged to be high risk of bias. In addition removal of these studies substantially changes the effect of having an intervention, from no effect to there being a positive effect of the intervention.

${ }^{2}$ Removal of six studies, rated high risk of bias, increased the effect size and narrowed the confidence interval.

\section{Summary of findings 7 . Adverse event outcomes for physical activity interventions compared to no intervention in children aged 6 to 12 years}

\section{Adverse event outcomes for physical activity interventions compared to control for preventing obesity in children aged 6 to 12 years}

Patient or population: children aged 6-12 years

Setting: preschool, school, home, healthcare or wider community

Intervention: physical activity

Comparison: control

\begin{tabular}{|c|c|c|c|}
\hline Outcomes & Impact & $\begin{array}{l}\text { № of participants } \\
\text { (studies) }\end{array}$ & $\begin{array}{l}\text { Certainty of the evidence } \\
\text { (GRADE) }\end{array}$ \\
\hline Physical injuries & $\begin{array}{l}\text { No effect on numbers of children with physical injuries in the control } \\
\text { and intervention arms }\end{array}$ & $\begin{array}{l}912 \\
(1 \mathrm{RCT})\end{array}$ & $\begin{array}{l}\oplus \oplus \ominus \ominus \\
\text { Low } 1\end{array}$ \\
\hline $\begin{array}{l}\text { Underweight } \\
\text { Assessed with counts of children as- } \\
\text { sessed as underweight }\end{array}$ & $\begin{array}{l}\text { No effect on number (proportion) of children designated as under- } \\
\text { weight }\end{array}$ & $\begin{array}{l}5266 \\
\text { (3 RCTs) }\end{array}$ & $\begin{array}{l}\oplus \oplus \oplus \oplus \\
\text { High } 1\end{array}$ \\
\hline $\begin{array}{l}\text { Depression } \\
\text { Assessed with child's depression inven- } \\
\text { tory }\end{array}$ & $\begin{array}{l}\text { Depression was reduced in children in the intervention group (MD } \\
-0.21,95 \% \mathrm{Cl}-0.42 \text { to }-0.001 \text { ) } \\
\text { Baseline depression score of the control group was } 2.09 \text { (SD 2.74) }\end{array}$ & $\begin{array}{l}225 \\
(1 \mathrm{RCT})\end{array}$ & $\begin{array}{l}\oplus \oplus \ominus \ominus \\
\text { Low }^{2}\end{array}$ \\
\hline $\begin{array}{l}\text { Body satisfaction } \\
\text { Assessed with Silhouettes scale, Self- } \\
\text { perceived body shape scale and the } \\
\text { Body Dissatisfaction scale }\end{array}$ & $\begin{array}{l}\text { No effect of intervention on reported body satisfaction at the end of } \\
\text { the intervention }\end{array}$ & $\begin{array}{l}225 \\
(1 \mathrm{RCT})\end{array}$ & $\begin{array}{l}\oplus \oplus \ominus \ominus \\
\text { Low }^{2}\end{array}$ \\
\hline
\end{tabular}

Cl: confidence interval; MD: mean difference; $\mathbf{R C T}$ : randomised controlled trial 
GRADE Working Group grades of evidence

High certainty: we are very confident that the true effect lies close to that of the estimate of the effect.

Moderate certainty: we are moderately confident in the effect estimate: the true effect is likely to be close to the estimate of the effect, but there is a possibility that it is substantially different.

Low certainty: our confidence in the effect estimate is limited: the true effect may be substantially different from the estimate of the effect.

Very low certainty: we have very little confidence in the effect estimate: the true effect is likely to be substantially different from the estimate of effect.

1Downgraded for risk of bias because this study has one domain at high risk of bias. Downgraded for imprecision because only one of 22 studies reported this outcome.

2Downgraded for risk of bias as one domain of the bias tool was at high risk of bias. Downgraded for imprecision as the study included only 225 participants.

Summary of findings 8. Diet and physical activity interventions combined compared to control for preventing obesity in children aged 6 to 12 years Diet and physical activity interventions combined compared to control for preventing obesity in children aged 6 to 12 years

Patient or population: children aged 6-12 years

Setting: home, wider community or school

Intervention: diet and physical activity interventions

Comparison: control

\begin{tabular}{|c|c|c|c|c|c|}
\hline \multirow[t]{2}{*}{ Outcomes } & \multicolumn{2}{|c|}{ Anticipated absolute effects ${ }^{\star}(95 \% \mathrm{Cl})$} & \multirow{2}{*}{$\begin{array}{l}\text { № of participants } \\
\text { (studies) }\end{array}$} & \multirow{2}{*}{$\begin{array}{l}\text { Certainty of the } \\
\text { evidence } \\
\text { (GRADE) }\end{array}$} & \multirow[t]{2}{*}{ Comments } \\
\hline & Risk with control & $\begin{array}{l}\text { Risk with diet and physical ac- } \\
\text { tivity interventions }\end{array}$ & & & \\
\hline $\begin{array}{l}\text { Body-mass index z } \\
\text { score (zBMI) }\end{array}$ & $\begin{array}{l}\text { The mean zBMI ranged } \\
\text { from } 0.05 \text { to } 0.9\end{array}$ & $\begin{array}{l}\text { MD } 0.05 \text { lower ( } 0.10 \text { lower to } 0.01 \\
\text { lower) }\end{array}$ & $\begin{array}{l}24,043 \\
(20 \mathrm{RCTs})\end{array}$ & $\begin{array}{l}\oplus \oplus \ominus \ominus \\
\text { Low }^{1}\end{array}$ & $\begin{array}{l}\text { Diet and physical activity interven- } \\
\text { tions combined may reduce zBMI } \\
\text { slightly }\end{array}$ \\
\hline $\begin{array}{l}\text { Body-mass index } \\
\text { (BMI) }\end{array}$ & $\begin{array}{l}\text { The mean BMI ranged } \\
\text { from } 17.57 \text { to } 24.8 \mathrm{~kg} / \mathrm{m}^{2}\end{array}$ & $\begin{array}{l}\text { MD } 0.05 \mathrm{~kg} / \mathrm{m}^{2} \text { lower ( } 0.11 \text { lower } \\
\text { to } 0.01 \text { higher) }\end{array}$ & $\begin{array}{l}19,498 \\
(25 \mathrm{RCTs})\end{array}$ & $\begin{array}{l}\oplus \oplus \ominus \ominus \\
\text { Low }^{2}\end{array}$ & $\begin{array}{l}\text { Diet and physical activity interven- } \\
\text { tions combined may result in little to } \\
\text { no difference in BMI }\end{array}$ \\
\hline
\end{tabular}

${ }^{*}$ The risk in the intervention group (and its 95\% confidence interval) is based on the assumed risk in the comparison group and the relative effect of the intervention (and its $95 \% \mathrm{Cl})$.

BMI: body-mass index; CI: confidence interval; MD: mean difference; RCT: randomised controlled trial; zBMI: body-mass index z score

\section{GRADE Working Group grades of evidence}

High certainty: we are very confident that the true effect lies close to that of the estimate of the effect.

Moderate certainty: we are moderately confident in the effect estimate: the true effect is likely to be close to the estimate of the effect, but there is a possibility that it is

substantially different.

Low certainty: our confidence in the effect estimate is limited: the true effect may be substantially different from the estimate of the effect. 
1 Heterogeneity was very high with an 12 statistic of $87 \%$.

2If studies at high risk of bias are removed, the effect of the intervention is increased from being consistent with having no effect, to indicating that the intervention reduced body-mass index in comparison to the control.

Summary of findings 9. Adverse event outcomes for dietary combined with physical activity interventions compared to no intervention or usual care for preventing obesity in children aged 6 to 12 years

Adverse event outcomes for dietary combined with physical activity interventions compared to control for preventing obesity in children aged 6 to 12 years

Patient or population: children aged 6 to 12 years

Setting: school or wider community

Intervention: combined dietary and physical activity interventions

Comparison: control

\begin{tabular}{|c|c|c|c|}
\hline Outcomes & Impact & $\begin{array}{l}\text { № of participants } \\
\text { (studies) }\end{array}$ & $\begin{array}{l}\text { Certainty of the evidence } \\
\text { (GRADE) }\end{array}$ \\
\hline $\begin{array}{l}\text { Underweight } \\
\text { Assessed with counts of children as- } \\
\text { sessed as underweight }\end{array}$ & $\begin{array}{l}\text { No effect on number (proportion) of children designated as under- } \\
\text { weight }\end{array}$ & $\begin{array}{l}784 \\
(2 \mathrm{RCTs})\end{array}$ & $\begin{array}{l}\oplus \oplus \oplus \ominus \\
\text { Moderate } 1\end{array}$ \\
\hline $\begin{array}{l}\text { Depression } \\
\text { Assessed with Child's Depression In- } \\
\text { ventory }\end{array}$ & $\begin{array}{l}\text { Depression was reduced in children in the intervention group (MD } \\
-0.21,95 \% \mathrm{Cl}-0.42 \text { to }-0.001 \text { ) } \\
\text { Baseline depression score of the control group was } 2.09 \text { (SD 2.74) }\end{array}$ & $\begin{array}{l}225 \\
(1 \mathrm{RCT})\end{array}$ & $\begin{array}{l}\oplus \oplus \odot \ominus \\
\text { Low }^{2}\end{array}$ \\
\hline $\begin{array}{l}\text { Increased weight concern } \\
\text { Assessed with scales for weight con- } \\
\text { cern }\end{array}$ & No effect of the intervention on concern about weight & $\begin{array}{l}285 \\
(2 \mathrm{RCTs})\end{array}$ & $\begin{array}{l}\oplus \oplus \oplus \oplus \\
\text { High }\end{array}$ \\
\hline $\begin{array}{l}\text { Body satisfaction } \\
\text { Assessed with Silhouettes scale, Self- } \\
\text { perceived Body Shape scale and the } \\
\text { Body Dissatisfaction scale }\end{array}$ & $\begin{array}{l}\text { No effect of intervention (diet and physical activity) on reported body } \\
\text { satisfaction at the end of the intervention }\end{array}$ & $\begin{array}{l}1128 \\
(3 \mathrm{RCTs})\end{array}$ & $\begin{array}{l}\oplus \oplus \oplus \oplus \\
\text { High }\end{array}$ \\
\hline Visits to a healthcare provider & $\begin{array}{l}\text { Visits to a healthcare provider were similar in the intervention and } \\
\text { control groups; } N=1 \text { in intervention and } N=2 \text { in control }\end{array}$ & $\begin{array}{l}60 \\
(1 \mathrm{RCT})\end{array}$ & $\begin{array}{l}\oplus \oplus \oplus \ominus \\
\text { Low }^{3}\end{array}$ \\
\hline $\begin{array}{l}\text { Adverse events related to taking of } \\
\text { blood samples }\end{array}$ & $\begin{array}{l}<3 \% \text {, similar numbers in the intervention (1.6\%) and control }(1.7 \%) \\
\text { groups (RD } 0.00,95 \% \mathrm{Cl}-0.01 \text { to } 0.01 \text { ) }\end{array}$ & $\begin{array}{l}4603 \\
(1 \mathrm{RCT})\end{array}$ & $\begin{array}{l}\oplus \oplus \oplus \ominus \\
\text { Moderate } 4\end{array}$ \\
\hline
\end{tabular}




\begin{tabular}{|c|c|c|c|c|}
\hline 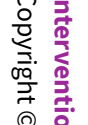 & $\begin{array}{l}\text { Underweight } \\
\text { Assessed with waist circumference of } \\
\text { children }<10 \text { th centile }\end{array}$ & $\begin{array}{l}\text { Waist circumference of children }<10 \text { th centile for weight did not differ } \\
\text { between the intervention and control group }(P=0.373)\end{array}$ & $\begin{array}{l}724 \\
(1 \mathrm{RCT})\end{array}$ & $\begin{array}{l}\oplus \oplus \oplus \ominus \\
\text { Moderate }\end{array}$ \\
\hline 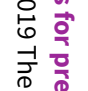 & Injuries & $\begin{array}{l}\text { Similar numbers of children were reported with injuries in the inter- } \\
\text { vention }(11 \%, \mathrm{~N}=2) \text { and control }(4.7 \%, \mathrm{~N}=1) \text { groups }\end{array}$ & $\begin{array}{l}60 \\
(1 \mathrm{RCT})\end{array}$ & $\begin{array}{l}\oplus \oplus \oplus \ominus \\
\text { Low }^{3}\end{array}$ \\
\hline
\end{tabular}

${ }^{*}$ The risk in the intervention group (and its $95 \%$ confidence interval) is based on the assumed risk in the comparison group and the relative effect of the intervention (and its $95 \% \mathrm{Cl}$ ).

Cl: confidence interval; MD: mean difference; RCT: randomised controlled trial; RD: risk difference

\section{GRADE Working Group grades of evidence}

High certainty: we are very confident that the true effect lies close to that of the estimate of the effect.

Moderate certainty: we are moderately confident in the effect estimate: the true effect is likely to be close to the estimate of the effect, but there is a possibility that it is

substantially different.

Low certainty: our confidence in the effect estimate is limited: the true effect may be substantially different from the estimate of the effect.

Very low certainty: we have very little confidence in the effect estimate: the true effect is likely to be substantially different from the estimate of effect.

1Downgraded for risk of bias because one of the studies had an outcome rated as high risk of bias.

2Downgraded for risk of bias as one domain of the bias tool was at high risk of bias. Downgraded for imprecision as the study included only 225 participants.

${ }^{3}$ Downgraded twice for imprecision, only 60 participants, and only three events.

${ }^{4}$ Downgraded once for imprecision as there were very few events.

\section{Summary of findings 10. Diet interventions compared to control for preventing obesity in children aged 13 to 18 years}

Diet interventions compared to control for preventing obesity in children aged 13 to 18 years

Patient or population: children aged $13-18$ years

Setting: home or school

Intervention: diet interventions

Comparison: control

\begin{tabular}{|c|c|c|c|c|c|}
\hline \multirow[t]{2}{*}{ Outcomes } & \multicolumn{2}{|c|}{ Anticipated absolute effects ${ }^{\star}(95 \% \mathrm{Cl})$} & \multirow{2}{*}{$\begin{array}{l}\text { № of participants } \\
\text { (studies) }\end{array}$} & \multirow{2}{*}{$\begin{array}{l}\text { Certainty of the } \\
\text { evidence } \\
\text { (GRADE) }\end{array}$} & \multirow[t]{2}{*}{ Comments } \\
\hline & Risk with control & Risk with diet interventions & & & \\
\hline $\begin{array}{l}\text { Body-mass index } \\
\text { (BMI) }\end{array}$ & $\begin{array}{l}\text { The mean BMI was } 24.8 \\
\mathrm{~kg} / \mathrm{m}^{2}\end{array}$ & $\begin{array}{l}\text { MD } 0.13 \mathrm{~kg} / \mathrm{m}^{2} \text { lower ( } 0.50 \text { lower to } 0.23 \\
\text { higher) }\end{array}$ & $\begin{array}{l}294 \\
(2 \mathrm{RCTs})\end{array}$ & $\begin{array}{l}\oplus \oplus \ominus \ominus \\
\text { LoW }^{1,2}\end{array}$ & $\begin{array}{l}\text { Diet interventions may result } \\
\text { in little to no difference in BMI }\end{array}$ \\
\hline
\end{tabular}

${ }^{\star}$ The risk in the intervention group (and its $95 \%$ confidence interval) is based on the assumed risk in the comparison group and the relative effect of the intervention (and its $95 \% \mathrm{Cl}$ ). 


\section{GRADE Working Group grades of evidence}

High certainty: we are very confident that the true effect lies close to that of the estimate of the effect.

Moderate certainty: we are moderately confident in the effect estimate: the true effect is likely to be close to the estimate of the effect, but there is a possibility that it is substantially different.

Low certainty: our confidence in the effect estimate is limited: the true effect may be substantially different from the estimate of the effect.

Very low certainty: we have very little confidence in the effect estimate: the true effect is likely to be substantially different from the estimate of effect.

${ }^{1}$ There are two studies and one has two domains at high risk of bias.

2There are two studies with 294 participants in total.

\section{Summary of findings 11. Physical activity interventions compared to control for preventing obesity in children aged 13 to 18 years}

Physical activity interventions compared to control for preventing obesity in children aged 13 to 18 years

\section{Patient or population: children aged $13-18$ years}

Setting: school

Intervention: physical activity interventions

Comparison: control

\begin{tabular}{|c|c|c|c|c|c|}
\hline \multirow[t]{2}{*}{ Outcomes } & \multicolumn{2}{|c|}{ Anticipated absolute effects* $(95 \% \mathrm{Cl})$} & \multirow{2}{*}{$\begin{array}{l}\text { № of participants } \\
\text { (studies) }\end{array}$} & \multirow{2}{*}{$\begin{array}{l}\text { Certainty of the } \\
\text { evidence } \\
\text { (GRADE) }\end{array}$} & \multirow[t]{2}{*}{ Comments } \\
\hline & Risk with control & $\begin{array}{l}\text { Risk with physical activity in- } \\
\text { terventions }\end{array}$ & & & \\
\hline $\begin{array}{l}\text { Body-mass index z } \\
\text { score (zBMI) }\end{array}$ & $\begin{array}{l}\text { The mean zBMI was } 0.21 \\
\text { to } 0.81\end{array}$ & $\begin{array}{l}\text { MD } 0.2 \text { lower ( } 0.3 \text { lower to } 0.1 \\
\text { lower) }\end{array}$ & $\begin{array}{l}100 \\
(1 \mathrm{RCT})\end{array}$ & $\begin{array}{l}\oplus \oplus \ominus \ominus \\
\text { Low }^{1,2}\end{array}$ & $\begin{array}{l}\text { The evidence suggests physical activi- } \\
\text { ty interventions reduce zBMI }\end{array}$ \\
\hline $\begin{array}{l}\text { Body-mass index } \\
\text { (BMI) }\end{array}$ & $\begin{array}{l}\text { The mean BMI was } 20.4 \\
\text { to } 26.65 \mathrm{~kg} / \mathrm{m}^{2}\end{array}$ & $\begin{array}{l}\text { MD } 1.53 \mathrm{~kg} / \mathrm{m}^{2} \text { lower } \\
\text { (2.67 lower to } 0.39 \text { lower) }\end{array}$ & $\begin{array}{l}720 \\
(4 \mathrm{RCTs})\end{array}$ & $\begin{array}{l}\oplus \ominus \odot \ominus \\
\text { Very low } 3,4\end{array}$ & $\begin{array}{l}\text { The evidence is very uncertain about } \\
\text { the effect of physical activity interven- } \\
\text { tions on BMI }\end{array}$ \\
\hline
\end{tabular}

${ }^{\star}$ The risk in the intervention group (and its 95\% confidence interval) is based on the assumed risk in the comparison group and the relative effect of the intervention (and its $95 \% \mathrm{Cl})$.

BMI: body-mass index; CI: confidence interval; MD: mean difference; RCT: randomised controlled trial; zBMI: body-mass index z score

\section{GRADE Working Group grades of evidence}

High certainty: we are very confident that the true effect lies close to that of the estimate of the effect.

Moderate certainty: we are moderately confident in the effect estimate: the true effect is likely to be close to the estimate of the effect, but there is a possibility that it is 
Low certainty: our confidence in the effect estimate is limited: the true effect may be substantially different from the estimate of the effect.

Very low certainty: we have very little confidence in the effect estimate: the true effect is likely to be substantially different from the estimate of effect.

1One study with only 100 participants.

2Evidence from one study, which we rated at high risk of bias for blinding of participants.

3 When we removed the data from studies with at least one domain at high risk of bias, the treatment effect reduces to show no difference between intervention and control.

${ }^{4}$ Heterogeneity is very high ( $93 \%$ value for 12 stastic). Also, one study has values that show an extremely positive effect of the intervention. When we removed this study of 80 participants, the positive effect of the intervention is removed.

\section{Summary of findings 12. Adverse events outcomes for physical activity interventions compared to control in children aged 13 to 18 years}

\section{Adverse event outcomes for physical activity interventions compared to control for preventing obesity in children age 13 to 18 years}

Patient or population: children aged 13-18 years

Intervention: physical activity

Comparison: control (no intervention or usual care)

\begin{tabular}{|c|c|c|c|}
\hline Outcomes & Impact & $\begin{array}{l}\text { № of participants } \\
\text { (studies) }\end{array}$ & $\begin{array}{l}\text { Certainty of the evidence } \\
\text { (GRADE) }\end{array}$ \\
\hline $\begin{array}{l}\text { Body satisfaction } \\
\text { Assessed with Silhouettes scale, Self-perceived } \\
\text { Body Shape and Body Dissatisfaction scale }\end{array}$ & $\begin{array}{l}\text { No effect of intervention on reported body satisfaction at } \\
\text { the end of the intervention }\end{array}$ & $\begin{array}{l}190 \\
(1 \mathrm{RCT})\end{array}$ & $\begin{array}{l}\oplus \oplus \ominus \ominus \\
\text { Low }^{1,2}\end{array}$ \\
\hline $\begin{array}{l}\text { Unhealthy weight gain } \\
\text { Assessed with counts of children with unhealthy } \\
\text { weight gain }\end{array}$ & No effect of intervention on unhealthy gains in weight & $\begin{array}{l}546 \\
(2 \mathrm{RCTs})\end{array}$ & $\begin{array}{l}\oplus \oplus \oplus \ominus \\
\text { Moderate } 3\end{array}$ \\
\hline $\begin{array}{l}\text { Self-acceptance/self-worth } \\
\text { Assessed with Harter self-worth scale }\end{array}$ & $\begin{array}{l}\text { One study }(\mathrm{N}=190) \text { reported no effect of intervention on } \\
\text { self-acceptance. A second CRt of the same intervention } \\
\text { reported improved self-worth in those children who re- } \\
\text { ceived the intervention }\end{array}$ & $\begin{array}{l}546 \\
(2 \mathrm{RCTs})\end{array}$ & $\begin{array}{l}\oplus \oplus \oplus \ominus \\
\text { Moderate } 3\end{array}$ \\
\hline $\begin{array}{l}\text { Binge eating } \\
\text { Assessed with percent of episodes of binge eating } \\
\text { in the past month }\end{array}$ & No effect of intervention on binge eating & $\begin{array}{l}556 \\
(2 \mathrm{RCTs})\end{array}$ & $\begin{array}{l}\oplus \oplus \oplus \ominus \\
\text { Moderate } 3\end{array}$ \\
\hline
\end{tabular}

RCT: randomised controlled trial

\section{GRADE Working Group grades of evidence}

High certainty: we are very confident that the true effect lies close to that of the estimate of the effect.

Moderate certainty: we are moderately confident in the effect estimate: the true effect is likely to be close to the estimate of the effect, but there is a possibility that it is 
Low certainty: our confidence in the effect estimate is limited: the true effect may be substantially different from the estimate of the effect.

1Downgraded as this study has two domains at high risk of bias.

2Downgraded for imprecision as study had only 190 participants.

${ }^{3}$ Downgraded for risk of bias, as both studies had at least one domain at high risk of bias.

\section{Summary of findings 13. Diet and physical activity interventions combined compared to control for preventing obesity in children aged 13 to 18} years

\section{Diet and physical activity interventions combined compared to control for preventing obesity in children aged 13 to 18 years}

Patient or population: children aged $13-18$ years

Setting: home or school

Intervention: diet and physical activity interventions

Comparison: control

\begin{tabular}{|c|c|c|c|c|c|}
\hline \multirow[t]{2}{*}{ Outcomes } & \multicolumn{2}{|c|}{ Anticipated absolute effects* $(95 \% \mathrm{Cl})$} & \multirow{2}{*}{$\begin{array}{l}\text { № of participants } \\
\text { (studies) }\end{array}$} & \multirow{2}{*}{$\begin{array}{l}\text { Certainty of the } \\
\text { evidence } \\
\text { (GRADE) }\end{array}$} & \multirow[t]{2}{*}{ Comments } \\
\hline & Risk with control & $\begin{array}{l}\text { Risk with diet and physical } \\
\text { activity interventions com- } \\
\text { bined }\end{array}$ & & & \\
\hline $\begin{array}{l}\text { Body-mass index z } \\
\text { score (zBMI) }\end{array}$ & $\begin{array}{l}\text { The mean zBMI ranged } \\
\text { from } 0.21 \text { to } 0.81\end{array}$ & $\begin{array}{l}\text { MD } 0.01 \text { higher ( } 0.05 \text { lower to } \\
0.07 \text { higher) }\end{array}$ & $\begin{array}{l}16,543 \\
\text { (6 RCTs) }\end{array}$ & $\begin{array}{l}\oplus \oplus \ominus \ominus \\
\text { Low }^{1}\end{array}$ & $\begin{array}{l}\text { Combined diet and physical activity in- } \\
\text { terventions may result in little to no dif- } \\
\text { ference in zBMI }\end{array}$ \\
\hline $\begin{array}{l}\text { Body-mass index } \\
\text { (BMI) }\end{array}$ & $\begin{array}{l}\text { The mean BMI ranged } \\
\text { from } 18.99 \text { to } 24.57 \mathrm{~kg} / \\
\mathrm{m}^{2}\end{array}$ & $\begin{array}{l}\text { MD } 0.02 \mathrm{~kg} / \mathrm{m}^{2} \text { lower ( } 0.1 \text { lower } \\
\text { to } 0.05 \text { higher) }\end{array}$ & $\begin{array}{l}16,583 \\
\text { (8 RCTs) }\end{array}$ & $\begin{array}{l}\oplus \oplus \ominus \ominus \\
\operatorname{Low}^{2,3}\end{array}$ & $\begin{array}{l}\text { Combined diet and physical activity in- } \\
\text { terventions may result in little to no dif- } \\
\text { ference in BMI }\end{array}$ \\
\hline
\end{tabular}

${ }^{\star}$ The risk in the intervention group (and its 95\% confidence interval) is based on the assumed risk in the comparison group and the relative effect of the intervention (and its $95 \% \mathrm{Cl})$.

BMI: body-mass index; Cl: confidence interval; MD: mean difference; RCT: randomised controlled trial; zBMI: body-mass index z score

\section{GRADE Working Group grades of evidence}

High certainty: we are very confident that the true effect lies close to that of the estimate of the effect.

Moderate certainty: we are moderately confident in the effect estimate: the true effect is likely to be close to the estimate of the effect, but there is a possibility that it is substantially different.

Low certainty: our confidence in the effect estimate is limited: the true effect may be substantially different from the estimate of the effect.

Very low certainty: we have very little confidence in the effect estimate: the true effect is likely to be substantially different from the estimate of effect. 
${ }^{1}$ Heterogeneity is very high, measured at $92 \%$ with $\mathrm{I}^{2}$ statistic

$250 \%$ of the studies in this meta-analysis are at high risk of bias.

${ }^{3}$ Heterogeneity is high, measured at $58 \%$ with $\mathrm{I}^{2}$ statistic.

Summary of findings 14. Adverse event outcomes for dietary combined with physical activity interventions compared to control for preventing obesity in children aged 13 to 18 years

Adverse events outcomes for dietary combined with physical activity interventions compared to control for preventing obesity in children aged13 to 18 years

Patient or population: children aged 13-18 years

Setting: school

Intervention: diet and physical activity

Comparison: control (no intervention or usual care)

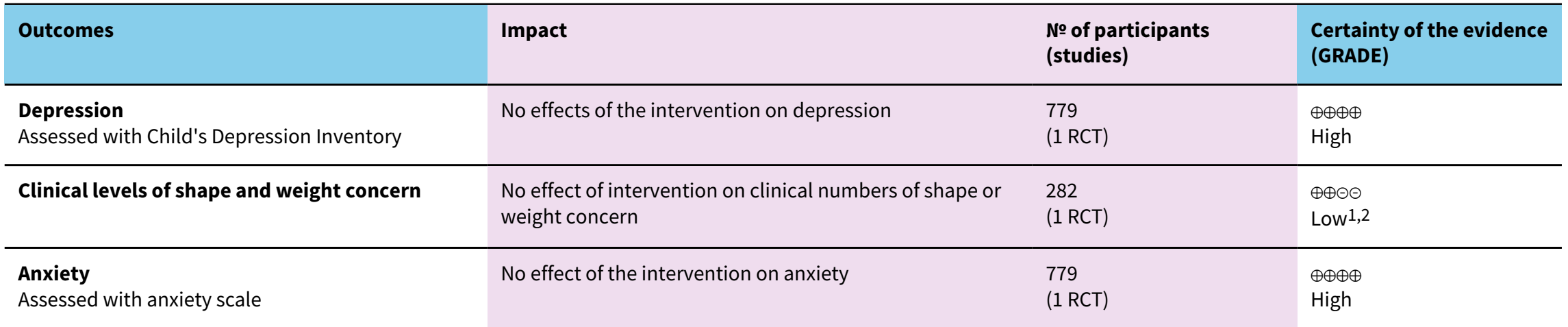

RCT: randomised controlled trial

GRADE Working Group grades of evidence

High certainty: we are very confident that the true effect lies close to that of the estimate of the effect.

Moderate certainty: we are moderately confident in the effect estimate: the true effect is likely to be close to the estimate of the effect, but there is a possibility that it is

substantially different.

Low certainty: our confidence in the effect estimate is limited: the true effect may be substantially different from the estimate of the effect.

Very low certainty: we have very little confidence in the effect estimate: the true effect is likely to be substantially different from the estimate of effect.

1Downgraded for risk of bias because these data appear to be from a post hoc subgroup analysis.

2Downgraded for imprecision as the number of participants was small.

Summary of findings 15. Dietary interventions compared to physical activity interventions for preventing obesity in children aged 6 to 12 years

Dietary interventions compared to physical activity interventions for preventing obesity in children aged 6 to 12 years 
Patient or population: children aged 6-12 years

Setting: school

Intervention: dietary interventions

Comparison: physical activity interventions

\begin{tabular}{|c|c|c|c|c|c|}
\hline \multirow[t]{2}{*}{ Outcomes } & \multicolumn{2}{|c|}{ Anticipated absolute effects ${ }^{*}(95 \% \mathrm{Cl})$} & \multirow{2}{*}{$\begin{array}{l}\text { № of participants } \\
\text { (studies) }\end{array}$} & \multirow{2}{*}{$\begin{array}{l}\text { Certainty of the } \\
\text { evidence } \\
\text { (GRADE) }\end{array}$} & \multirow[t]{2}{*}{ Comments } \\
\hline & $\begin{array}{l}\text { Risk with physical } \\
\text { activity interven- } \\
\text { tions }\end{array}$ & $\begin{array}{l}\text { Risk with dietary in- } \\
\text { tervention }\end{array}$ & & & \\
\hline $\begin{array}{l}\text { Body-mass index } \\
\text { (BMI) }\end{array}$ & $\begin{array}{l}\text { The mean } \mathrm{BMI} \\
\text { ranged from } 17.4 \text { to } \\
18.8 \mathrm{~kg} / \mathrm{m}^{2}\end{array}$ & $\begin{array}{l}\text { MD } 0.03 \mathrm{~kg} / \mathrm{m}^{2} \text { lower } \\
\text { ( } 0.25 \text { lower to } 0.2 \text { high- } \\
\text { er) }\end{array}$ & $\begin{array}{l}4917 \\
\text { (2 RCTs) }\end{array}$ & $\begin{array}{l}\oplus \oplus \oplus \oplus \\
\text { High }\end{array}$ & $\begin{array}{l}\text { Dietary interventions result in little to no difference } \\
\text { in BMI compared to physical activity interventions } \\
\text { when delivered in schools to children aged 6-12 } \\
\text { years }\end{array}$ \\
\hline $\begin{array}{l}\text { Body-mass index z } \\
\text { score (zBMI) }\end{array}$ & $\begin{array}{l}\text { The mean zBMI was } \\
0.2\end{array}$ & $\begin{array}{l}\text { MD } 0.11 \text { lower } \\
(0.62 \text { lower to } 0.4 \\
\text { higher) }\end{array}$ & $\begin{array}{l}1205 \\
(1 \mathrm{RCT})\end{array}$ & $\begin{array}{l}\oplus \oplus \oplus \oplus \\
\text { High }\end{array}$ & $\begin{array}{l}\text { 'Dietary interventions' results in little to no differ- } \\
\text { ence in zBMI compared to physical activity inter- } \\
\text { ventions when delivered in schools to children aged } \\
6-12 \text { years }\end{array}$ \\
\hline
\end{tabular}

${ }^{\star}$ The risk in the intervention group (and its $95 \%$ confidence interval) is based on the assumed risk in the comparison group and the relative effect of the intervention (and its $95 \% \mathrm{Cl})$.

BMI: body-mass index; Cl: confidence interval; MD: mean difference; RCT: randomised controlled trial; zBMI: body-mass index z score

\section{GRADE Working Group grades of evidence}

High certainty: we are very confident that the true effect lies close to that of the estimate of the effect.

Moderate certainty: we are moderately confident in the effect estimate: the true effect is likely to be close to the estimate of the effect, but there is a possibility that it is substantially different.

Low certainty: our confidence in the effect estimate is limited: the true effect may be substantially different from the estimate of the effect.

Very low certainty: we have very little confidence in the effect estimate: the true effect is likely to be substantially different from the estimate of effect.

Summary of findings 16. Diet and physical activity interventions combined compared to physical activity interventions alone for preventing obesity in children aged 6 to 12 years

\section{Diet and physical activity interventions combined compared to physical activity interventions alone for preventing obesity in children aged 6 to 12 years}

Patient or population: children aged 6-12 years

Setting: school

Intervention: combined diet and physical activity interventions 
Comparison: physical activity interventions alone

\begin{tabular}{|c|c|c|c|c|c|}
\hline \multirow[t]{2}{*}{ Outcomes } & \multicolumn{2}{|c|}{ Anticipated absolute effects* $(95 \% \mathrm{Cl})$} & \multirow{2}{*}{$\begin{array}{l}\text { № of participants } \\
\text { (studies) }\end{array}$} & \multirow{2}{*}{$\begin{array}{l}\text { Certainty of the } \\
\text { evidence } \\
\text { (GRADE) }\end{array}$} & \multirow[t]{2}{*}{ Comments } \\
\hline & $\begin{array}{l}\text { Risk with physical } \\
\text { activity interven- } \\
\text { tions }\end{array}$ & $\begin{array}{l}\text { Risk with diet and } \\
\text { physical activity inter- } \\
\text { ventions combined }\end{array}$ & & & \\
\hline $\begin{array}{l}\text { Body-mass index } \\
\text { (BMI) }\end{array}$ & $\begin{array}{l}\text { The mean BMI was } \\
17.7 \mathrm{~kg} / \mathrm{m}^{2}\end{array}$ & $\begin{array}{l}\text { MD } 0.04 \mathrm{~kg} / \mathrm{m}^{2} \text { lower } \\
\text { (1.05 lower to } 0.97 \text { high- } \\
\text { er) }\end{array}$ & $\begin{array}{l}3946 \\
(1 \mathrm{RCT})\end{array}$ & $\begin{array}{l}\oplus \oplus \oplus \oplus \\
\text { High }\end{array}$ & $\begin{array}{l}\text { Combined dietary and physical activity interven- } \\
\text { tions result in little to no difference in BMI compared } \\
\text { to physical activity interventions when delivered in } \\
\text { schools to children aged } 6-12 \text { years }\end{array}$ \\
\hline $\begin{array}{l}\text { Body-mass index z } \\
\text { score (zBMI) }\end{array}$ & $\begin{array}{l}\text { The mean zBMI was } \\
0.15\end{array}$ & $\begin{array}{l}\text { MD } 0.16 \text { lower ( } 0.57 \text { low- } \\
\text { er to } 0.25 \text { higher) }\end{array}$ & $\begin{array}{l}3946 \\
(1 \mathrm{RCT})\end{array}$ & $\begin{array}{l}\oplus \oplus \oplus \oplus \\
\text { High }\end{array}$ & $\begin{array}{l}\text { Combined dietary and physical activity intrventions } \\
\text { result in little to no difference in zBMI compared to } \\
\text { physical activity interventions when delivered in } \\
\text { schools to children aged 6-12 years }\end{array}$ \\
\hline
\end{tabular}

*The risk in the intervention group (and its $95 \%$ confidence interval) is based on the assumed risk in the comparison group and the relative effect of the intervention (and its $95 \% \mathrm{Cl})$.

BMI: body-mass index; CI: confidence interval; MD: mean difference; RCT: randomised controlled trial; zBMI: body-mass index z score

\section{GRADE Working Group grades of evidence}

High certainty: we are very confident that the true effect lies close to that of the estimate of the effect.

Moderate certainty: we are moderately confident in the effect estimate: the true effect is likely to be close to the estimate of the effect, but there is a possibility that it is substantially different.

Low certainty: our confidence in the effect estimate is limited: the true effect may be substantially different from the estimate of the effect.

Very low certainty: we have very little confidence in the effect estimate: the true effect is likely to be substantially different from the estimate of effect.

Summary of findings 17. Dietary interventions alone compared to diet and physical activity interventions combined for preventing obesity in children aged 6 to 12 years

Dietary interventions alone compared to diet and physical activity interventions combined for preventing obesity in children aged 6 to 12 years

Patient or population: children aged 6-12 years

Setting: school

Intervention: dietary interventions alone

Comparison: combined diet and physical activity interventions

Anticipated absolute effects ${ }^{\star}(95 \% \mathrm{Cl})$

Outcomes

\section{№ of participants}

(studies)

\section{Certainty of the}

evidence
Comments

ran




\begin{tabular}{|c|c|c|c|c|c|c|}
\hline & $\begin{array}{l}\text { Risk with diet and } \\
\text { physical activity in- } \\
\text { terventions com- } \\
\text { bined }\end{array}$ & $\begin{array}{l}\text { Risk with dietary in- } \\
\text { tervention }\end{array}$ & \multicolumn{2}{|r|}{ (GRADE) } & \\
\hline 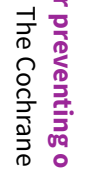 & $\begin{array}{l}\text { Body-mass index } \\
\text { (BMI) }\end{array}$ & $\begin{array}{l}\text { The mean BMI was } \\
17.4 \mathrm{~kg} / \mathrm{m}^{2}\end{array}$ & $\begin{array}{l}\text { MD } 0.28 \mathrm{~kg} / \mathrm{m}^{2} \text { low- } \\
\text { er (1.67 lower to } 1.11 \\
\text { higher) }\end{array}$ & $\begin{array}{l}3971 \\
(1 \mathrm{RCT})\end{array}$ & $\begin{array}{l}\oplus \oplus \oplus \oplus \\
\text { High }\end{array}$ & $\begin{array}{l}\text { Dietary interventions alone result in little to no dif- } \\
\text { ference in BMI compared to diet and physical activi- } \\
\text { ty interventions combined when delivered in schools } \\
\text { to children aged } 6-12 \text { years }\end{array}$ \\
\hline 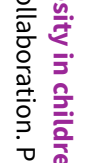 & $\begin{array}{l}\text { Body-mass index z } \\
\text { score (zBMI) }\end{array}$ & $\begin{array}{l}\text { The mean zBMI was } \\
0.2\end{array}$ & $\begin{array}{l}\text { MD } 0.05 \text { higher ( } 0.38 \\
\text { lower to } 0.48 \text { higher) }\end{array}$ & $\begin{array}{l}3971 \\
(1 \mathrm{RCT})\end{array}$ & $\begin{array}{l}\oplus \oplus \oplus \oplus \\
\text { High }\end{array}$ & $\begin{array}{l}\text { Dietary interventions alone result in little to no dif- } \\
\text { ference in zBMI compared to diet and physical activi- } \\
\text { ty interventions combined when delivered in schools } \\
\text { to children aged } 6-12 \text { years }\end{array}$ \\
\hline
\end{tabular}

${ }^{*}$ The risk in the intervention group (and its $95 \%$ confidence interval) is based on the assumed risk in the comparison group and the relative effect of the intervention (and its $95 \% \mathrm{Cl}$ ).

BMI: body-mass index; CI: confidence interval; MD: mean difference; RCT: randomised controlled trial; zBMI: body-mass index z score

\section{GRADE Working Group grades of evidence}

High certainty: we are very confident that the true effect lies close to that of the estimate of the effect.

Moderate certainty: we are moderately confident in the effect estimate: the true effect is likely to be close to the estimate of the effect, but there is a possibility that it is substantially different.

Low certainty: our confidence in the effect estimate is limited: the true effect may be substantially different from the estimate of the effect.

Very low certainty: we have very little confidence in the effect estimate: the true effect is likely to be substantially different from the estimate of effect. 


\section{B A C K G R O U N D}

Obesity prevention is an international public health priority (WHO 2016), and there is growing evidence of the impact of overweight and obesity on short- and long-term functioning, health and wellbeing (Reilly 2011). In a wide range of countries (including more recently, middle- and low-income countries), high and increasing rates of overweight and obesity have been reported over the last 30 to 40 years (WHO 2016).

The global evidence suggests that the prevalence of overweight and obesity in children started to rise at the end of the 1980's GBD Obesity Collaboration 2014. By 2010, 43 million children under five years of age were overweight or obese, with approximately 35 million of these children living in low- and middle-income countries (de Onis 2010). Internationally, childhood obesity rates continue to rise in some countries (e.g. Mexico, India, China, Canada), although there is evidence of a slowing of this increase or a plateauing in some age groups in some countries (WHO 2016). The World Health Organization (WHO) Commission on Ending Childhood Obesity (WHO 2016), found that childhood obesity, including obesity in preschool children and adolescents, is reaching alarming proportions in many countries and poses an urgent and serious challenge. The Sustainable Development Goals, set by the United Nations in 2015, also identify prevention and control of non-communicable diseases, including obesity, as core priorities (United Nations).

Once childhood obesity is established, it is difficult to reverse through interventions (Al-Khudairy 2017; Mead 2017), and tracks through to adulthood (Singh 2008; Whitaker 1997), strengthening the case for primary prevention. Adult obesity is associated with increased risk of heart disease, stroke, metabolic syndrome, type 2 diabetes and some cancers (Bhaskaran 2014; Yatsuya 2010). Children who are obese have poorer psychological well-being and elevated levels of a number of cardiometabolic risk factors (Kipping 2008a). Obesity co-morbidities including high blood pressure, high blood cholesterol and insulin insensitivity are being observed at an increasingly early age. Childhood obesity may cause musculoskeletal problems, obstructive sleep apnoea, asthma and a number of psychological issues (NHS England 2014). Childhood obesity is associated with type 2 diabetes and heart disease in adulthood and middle-age mortality (Public Health England 2015). Treating obesity is very expensive and, in the UK, it was estimated (in 2014) that the NHS spends GBP 5.1 billion per year on obesity related illnesses (Dobbs 2014).

Primary preventive efforts are likely to have optimal effects if started in early childhood with parental involvement (Summerbell 2012). From birth to starting primary school is a crucial time point for obesity prevention interventions, when diet and activity behaviour are being established between parent and child. Lifestyle modification interventions to improve dietary quality, increase physical activity levels and reduce sedentary behaviours, often using behaviour-changing techniques and involving parents or carers, or both, are the mainstay for interventions in preschoolaged children. By intervening at such an early age, it may be possible to prevent obesity levels continuing to rise for future generations and is crucial to reducing health inequalities (Marmot 2010). As highlighted by the Commission (WHO 2016), adolescence may be a critical time for excess weight gain, in that this age group normally have more freedom in food and beverage choices made outside the home compared with younger children. This, alongside the fact that physical activity usually declines during adolescence, particularly in girls, offers both opportunities and barriers for those developing interventions.

Obesity prevalence is also inextricably linked to the degree of relative social inequality, with greater social inequality associated with a higher risk of obesity in most high-income countries (even in infants and young children (Ballon 2018)), but in most lowand middle-income countries the reverse relationship is observed (Monteiro 2004). It is therefore critical that in preventing obesity we are also reducing the associated gap in health inequalities, ensuring that interventions do not inadvertently have more favourable outcomes in those with a more socio-economically advantaged position in society. The available knowledge base on which to develop a platform of obesity prevention action and base decisions about appropriate public health interventions to reduce the risk of obesity across the whole population, or targeted towards those at greatest risk, still remains limited (Gortmaker 2011; HillierBrown 2014).

The WHO Commission (WHO 2016), states that progress in tackling childhood obesity has been slow and inconsistent, and obesity prevention and treatment requires a whole-of-government approach in which policies across all sectors systematically take health into account, avoid harmful health impacts, and thus improve population health and health equity. Indeed, it is now acknowledged that tackling obesity requires a systems approach and policy initiatives across government departments that are joined-up (Rutter 2017). However, as Knai and colleagues have noted in relation to Chapter 2 of the Childhood Obesity Plan for England, it suffers from continued reliance on self-regulation at an individual level (Knai 2018). The WHO Commission (WHO 2016), suggests that upstream interventions providing guidance and training to caregivers working in child-care settings and institutions on appropriate advice on diet, physical activity and sleep for preschool children may be particularly important. The WHO Commission (WHO 2016), also suggests that upstream interventions may be particularly important for adolescents, for example, targeting the marketing of unhealthy foods such as sugarsweetened beverages; tackling the obesogenic environment, such as take-away food outlets.

The aim of this review was to update the evidence base for children given the exponential growth of studies in this field over the last five to 10 years, and thus ensure that the review remains current and policy and practice-relevant, with particular regard for health equity. We have updated this Cochrane Review to include data reported in randomised controlled trials (RCTs) published up to and including 2015. In this update, we present data by age group, from 0 to 5 years, 6 to 12 years; and 13 to 18 years. We also provide a list of RCTs published between 2016 and 2018, which we deem, from the information reported in the abstract, as likely to meet the inclusion criteria of this review.

Going forward, we will split the review into three reviews based on child age: from 0 to 5 years; 6 to 12 years, and 13 to 18 years. It is reasonable to believe that different interventions might work differently in children of different ages. For example, meaningful parent engagement may be a key factor for the effectiveness of interventions for preschool children, but this may not be the case for adolescents; adolescents may find online interventions easy to use, and attractive and engaging, because of their cognitive ability 
and affinity for social media, but these types of interventions might not work well for younger children.

\section{Description of the condition}

Overweight and obesity are terms used to describe an excess of adiposity (or fatness) above the ideal for good health. Current expert opinion supports the use of body-mass index (BMI) cut-off points to determine weight status (as healthy weight, overweight or obese) for children and adolescents and several standard BMI cutoffs have been developed (Cole 2000; Cole 2007; de Onis 2004; de Onis 2007). Despite this, there is no consistent application of this methodology by experts and a variety of percentile-based methods are also used, which can make it difficult to compare RCTs that have used different measures and weight outcomes.

Overweight and obesity in childhood are known to have significant impact on both physical and psychosocial health (reviewed in Lobstein 2004). Indeed, many of the cardiovascular consequences that characterise adult-onset obesity are preceded by abnormalities that begin in childhood. Hyperlipidaemia, hypertension, abnormal glucose tolerance (Freedman 1999), and type 2 diabetes (Arslanian 2002), occur with increased frequency in obese children and adolescents and children. In addition, obesity in childhood and adolescence are known to be independent risk factors for adult obesity (Must 1992; Must 1999; Power 1997; Singh 2008; Whitaker 1997), underpinning the importance of obesity prevention efforts.

\section{Modifiable determinants of childhood obesity}

Obesity results from a sustained positive energy imbalance and a variety of genetic, behavioural, cultural, environmental and economic factors have been implicated in its development (reviewed in Lobstein 2004). The interplay of these factors is complex and has been the focus of considerable research, however, the burden of obesity is not experienced uniformly across a population, with the highest levels of the condition experienced by those most disadvantaged. In high-income countries there is a significant trend observed between obesity and lower socioeconomic status, while in some developing countries the contrary is found, with children from relatively affluent families more vulnerable to obesity.

\section{Description of the intervention}

This review involves assessing educational, behavioural and health promotion interventions. We use the terms 'intervention' and 'programme' interchangeably throughout this review. The Ottawa Charter defines four action areas for health promotion: 1) actions to develop personal skills, which are actions targeted at individual skills, behaviours, or knowledge and beliefs; 2) actions to strengthen community actions, which are actions targeted at communities and include environmental and settings-based approaches to health promotion; 3) actions to reorientate health services, which are actions within the health sector and relate to the delivery of services; and 4) actions to build healthy public policy and create supportive environments, which are intersectoral in nature and relate to creating physical, social and policy environments that promote health WHO 1986.

\section{Why it is important to do this review}

Governments internationally are being urged to take action to prevent childhood obesity and to address the underlying determinants of the condition. To provide decision makers with high-quality research evidence to inform their planning and resource allocation, this review aims to provide an update of the evidence from RCTs designed to compare the effect of interventions to prevent childhood obesity with the effect of receiving an alternative intervention or no intervention. We aimed to update the previous review (Waters 2011), which concluded that many diet and exercise interventions to prevent obesity in children appeared ineffective in preventing weight gain, but could be effective in promoting a healthy diet and increased levels of physical activity. The previous review also urged reconsideration of the appropriateness of study durations, designs and intervention intensity as well as making recommendations in relation to comprehensive reporting of RCTs. Overall however, although there was insufficient evidence to determine that any one particular programme could prevent obesity in children, the evidence suggested that comprehensive strategies to increase the healthiness of children's diets and their physical activity levels, coupled with psycho-social support and environmental change were most promising. We incorporated research evidence that has been published since that time and is also consistent with emerging issues in relation to evidence reviews and synthesis (Higgins 2011a). We also noted the important work around implementation of policies and interventions to prevent obesity in children (Wolfenden 2016a). In addition, to meet the growing demand from public health and health promotion practitioners and decision makers, we have attempted to include information related not only to the impact of interventions on preventing obesity, but also information related to how outcomes were achieved, how interventions were implemented, the context in which they were implemented (Wang 2006), and the extent to which they work equitably (Tugwell 2010). This new aspect of the review was partly guided by the Systematic Reviews of Health Promotion and Public Health Interventions (Armstrong 2007), more recommendations for complex reviews and useful evidence for decision makers (Waters 2011), and informed by expert opinion.

\section{O B J E C T IVES}

The main objective of the review was to determine the effectiveness of a range of interventions that include diet or physical activity components, or both, designed to prevent obesity in children, by updating the 2011 version of the review (Waters 2011). Specific objectives include:

- evaluation of the effect of dietary educational interventions versus control on changes in $\mathrm{zBMI}$ score, BMI and adverse events among children under 18 years;

- evaluation of the effect of physical activity interventions versus control on changes in zBMI score, BMI and adverse events among children under 18 years;

- evaluation of combined effects of dietary educational interventions and physical activity interventions versus control on changes in zBMI score and BMI among children under 18 years

- evaluation of the effect of dietary educational interventions versus physical activity interventions on changes in zBMI score, $\mathrm{BMI}$ and adverse events among children under 18 years. 
Secondary aims were to examine the characteristics of the programmes and strategies to answer the question, 'what works for whom, why and at what cost?'. Secondary objectives include the evaluation of sociodemographic characteristics, process indicators (such as intensity, duration, setting and delivery of intervention) and contextual factors that might contribute to the outcome of the interventions. Specific objectives include:

- evaluation of sociodemographic characteristics of participants (socioeconomic status, gender, age, ethnicity, geographical location, etc.);

- evaluation of particular process indicators (i.e. those that describe why and how a particular intervention has worked).

\section{METHODS}

\section{Criteria for considering studies for this review}

\section{Types of studies}

We included data from RCTs that were designed or had an underlying intention to prevent obesity. We included RCTs that had an active intervention period of any duration, provided that the studies reported follow-up outcome data at a minimum of 12 weeks from baseline. We included RCTs in which individuals or groups of individuals were randomised, however, for those with group randomisation we only included cluster-RCTs with six or more groups. We categorised RCTs primarily according to the target age group ( 0 to 5 years, 6 to 12 years, and 13 to 18 years). We excluded RCTs published before 1990. The global evidence suggests that the prevalence of overweight and obesity in children, including preschool children, started to rise at the end of the 1980s (de Onis 2010; GBD Obesity Collaboration 2014). Given the lag time between the conception, funding, and the completion of RCTs, we considered a 1990 publication date as a pragmatic and reasonable starting point for the literature in the area.

\section{Types of participants}

We included RCTs of children with a mean age of less than 18 years at baseline. We included RCTs where children were part of a family group receiving the intervention if outcome evaluation could be extracted separately for the children. In order to reflect a public health approach that recognises the prevalence of a range of weight within the general population of children we included RCTs where the participants included children who were overweight or obese. We included RCTs that restricted eligibility according to weight if the eligibility was not restricted to children with obesity. We also included RCTs where children were 'at risk' for obesity, for example their parent(s) was/were overweight, or the children had low levels of physical activity. RCTs that only enrolled children who were obese at baseline we considered to be focused toward treatment rather than prevention and we therefore excluded them. We excluded RCTs of interventions designed to prevent obesity in pregnant women and RCTs designed for children with a critical illness or severe co-morbidities.

\section{Types of interventions}

\section{Strategies}

We included educational, health promotion, psychological, family, behavioural therapy, counselling, management strategies.

\section{Interventions included}

We included various types of diet or physical activity interventions, or both. We included RCTs of interventions that included diet and nutrition, or exercise and physical activity, or both; interventions may also have included other elements such as lifestyle change (e.g. changes to sedentary behaviour or sleep) and social support. We included complementary feeding RCTs, which aimed to promote a healthy weight in babies and toddlers. We also included interventions that aimed to increase motor skills in young children, where the rationale for these interventions was based on the evidence that greater motor skills in young children lead to higher levels of physical activity as the child grows older. We excluded RCTs where the rationale of the intervention was other than preventing obesity.

\section{Setting}

We included interventions in any setting. These included interventions within the wider community (including faith-based settings), school and out-of-school-hours care, home, healthcare, and childcare or preschool/nursery/kindergarten.

\section{Types of comparison}

We included RCTs that compared diet or physical activity interventions, or both, with a non-intervention control group who received no treatment or usual care, or another active intervention (i.e. head-to-head comparisons).

\section{Intervention personnel}

There was no restriction on who delivered the interventions, for example, researchers, primary care physicians (general practitioners), nutrition/diet professionals, teachers, physical activity professionals, health promotion agencies, health departments, faith leaders or others.

\section{Indicators of theory and process}

We collected data on indicators of intervention process and evaluation, health promotion theory underpinning intervention design, modes of strategies, and attrition rates from these studies. We compared where possible, whether the effect of the intervention varied according to these factors. We included this information in descriptive analyses and used it to guide the interpretation of findings and recommendations.

\section{Interventions excluded}

We excluded RCTs of interventions designed specifically for the treatment of childhood obesity and RCTs designed to treat eating disorders such as anorexia and bulimia nervosa. We excluded any drug or surgery interventions, as these are treatment interventions. We excluded RCTs that were exclusively focused on breast or bottle feeding; for example, RCTs that solely evaluated the effect of various protein levels in infant formulas. We also excluded RCTs that focused solely on strength and fitness training (not aimed at obesity prevention).

\section{Types of outcome measures}

To be included, studies had to report one or more of the following primary review outcomes, presenting a baseline and a postintervention measurement. We focused on reporting the results for 
the anthropometric outcomes (primary outcomes) and listing other outcomes.

\section{Primary outcomes}

- zBMI score/BMI

- Prevalence of overweight and obesity

- Weight and height

- Ponderal index

- Per cent fat content

- Skin-fold thickness

\section{Summary of findings}

We present 'Summary of findings' tables in which we report zBMI score, $\mathrm{BMI}$ and adverse events for the three age groups of children ( 0 to 5 years, 6 to 12 years and 13 to 18 years), and three intervention types (diet, physical activity, diet and physical activity combined).

\section{Search methods for identification of studies}

\section{Electronic searches}

We searched the following databases for this update and for previous versions of this review. We did not exclude studies based on language.

For the 2015 update (in this review we included and synthesised data from all studies identified)

- Cochrane Central Register of Controlled Trials (CENTRAL; 2010, Issue 1 to 2016 Issue 6) in the Cochrane Library

- MEDLINE (Ovid) January 2010 to June 2015

- Embase (Ovid) January 2010 to June 2015

- Cumulative Index to Nursing and Allied Health Literature (CINAHL) (Ovid) March 2010 to June 2015

- PsycINFO (Ovid) 2010 to June 2015

For the 2018 update (see Characteristics of studies awaiting classification for studies identified as potentially relevant from screening titles and abstracts)

- Cochrane Central Register of Controlled Trials (CENTRAL; 2015, Issue 6 to 2018, Issue 1), in the Cochrane Library

- MEDLINE (Ovid) June 2015 to January 2018

- Embase (Ovid) June 2015 to January 2018

- Cumulative Index to Nursing and Allied Health Literature (CINAHL) (Ovid) June 2015 to January 2018

- PsycINFO (Ovid) June 2015 to January 2018

Complete search strategies and search dates for each database can be found in the Appendices.

- Update 2018 (Appendix 1). Potentially relevant studies stored in Studies awaiting classification

- Update 2015 (Appendix 2). All study data assessed for inclusion and synthesised

- Update 2010 (Appendix 3). All study data assessed for inclusion and synthesised

- Update 2005 (Appendix 4). All study data assessed for inclusion and synthesised

\section{Searching other resources}

For the 2018 update on 22 January 2018 we searched ClinicalTrials.gov with the filter 'Applied Filters: Child (birth-17)' . We also searched the WHO International Clinical Trials Registry Platform, search portal (ICTRP), using the filter for studies in children. In addition, we scanned the reference lists of key systematic reviews and references of included studies.

\section{Data collection and analysis}

\section{Selection of studies}

For the 2015 update, one review author (TB) performed title and abstract screening, and another review author (CS) checked a random subsample (10\%). For the 2018 update, two review authors (TB and ME) independently assessed all titles and abstracts in duplicate using RAYYAN software (Rayyan-QCRI 2016). For titles and abstracts that potentially met the inclusion criteria, we obtained the full text of the article for further evaluation. Two review authors (from TB, CO and ME), independently assessed the fulltext reports of studies against a list of criteria for inclusion. We resolved differences in opinion or uncertainty through a process of discussion. Occasionally we brought in a third review author (CS, TM).

\section{Data extraction and management}

We developed a data extraction form, based on the Effective Public Health Practice Project Quality Assessment Tool for quantitative studies (Thomas 2003), with additional data extraction items specifically related to implementation. For studies identified between 2010 and 2015 we extracted information relevant to equity using the PROGRESS (Place, Race, Occupation, Gender, Religion, Education, Socio-economic status (SES), Social status) checklist (Ueffing 2009). And to facilitate full understanding of interventions we also incorporated items from the TIDieR checklist and guide (Hoffman 2014). We also extracted information relevant to assessing risk of bias, source and involvement of funders, data on indicators of intervention process and evaluation, health promotion theory underpinning intervention design, modes of strategies, and attrition rates. Two review authors (CO, TB) independently extracted data from included papers into the data extraction form for each study.

This review sought to identify studies that had reported on sociodemographic characteristics known to be important from an equity perspective using the PROGRESS checklist (Ueffing 2009).

We attempted to capture factors that we could use to assess implementability of the interventions. These included: programme reach (i.e. was the intervention available to all those to whom it would be relevant?); programme acceptability (was the intervention acceptable to the target population?); and programme integrity (was the programme implemented as planned?). A comprehensive process evaluation allowed us to monitor variability in context and delivery, and to identify barriers and facilitators to implementation.

\section{Assessment of risk of bias in included studies}

We assessed the risk of bias of included RCTs using the 'Risk of bias' tool (Higgins 2017). At least two review authors assessed each study as being at 'high', 'low' or 'unclear' risk of bias for each item. Review authors were not blinded with respect to study authors, 
institution or journal. We used discussion and consensus to resolve any disagreements.

We incorporated performance and detection bias under the item 'blinding' in the 'Risk of bias' tool. We assessed this to be at low risk for RCTs that reported blinding of outcome assessors, and high risk for RCTs reporting that outcome assessors were not blinded.

We assessed RCTs as low risk for attrition bias if an adequate description of participant flow through the study was provided, the proportion of missing outcome data was relatively balanced between groups and the reasons for missing outcome data were provided and we considered them unlikely to bias the results. We assessed RCTs 'high' risk for attrition if attrition was $30 \%$ or greater at final follow-up.

For cluster-randomised trials we made an additional assessment listed as 'other bias' based on the advice for dealing with clusterRCTs (Higgins 2011a). For 'timing of recruitment of clusters', we rated RCTs at 'high' risk of bias if the studies had recruited the clusters after randomisation and at 'low' risk of bias if recruitment occurred before randomisation.

For selective outcome reporting we searched for both trial registrations and protocols. Where we were unable to find a trial registration or protocol, we recorded 'selective outcome reporting as unclear. If all relevant primary outcomes reported in the study report or protocol were reported in the results of the paper, we marked these as low risk of bias. If relevant primary outcomes reported in the study report or protocol were not reported (in the results paper) we recorded these as high risk of bias. Where studies reported an outcome in the results paper that they had not prespecified in the protocol or trials register, we reported this as high risk of bias. For RCTs where we could not locate a protocol or trial registration document, we recorded risk of bias as unclear. See Table 8.5 and Section 8.1.3 of the Cochrane Handbook for Systematic Reviews of Interventions (Higgins 2011b).

\section{Measures of treatment effect}

For this update we focused on reporting the results for the anthropometric outcomes and listed other outcomes. We conducted meta-analyses to investigate the impact of included interventions on zBMI scores and BMI. We did not undertake a meta-analysis of the effects of the interventions on prevalence of overweight or obesity. Most of the RCTs did not report prevalence and used highly variable methods for the classification of overweight and obesity. Different methods of classification of weight status in children produce very different prevalence estimates, and so limit comparisons between RCTs.

\section{Unit of analysis issues}

We assessed each cluster-RCT to see if the analysis had accounted for clustering. For any studies that had not adjusted for clustering we created an approximate analysis of the cluster-RCT by inflating the standard errors (SE) See section 16.3.6 of the Cochrane Handbook for Systematic Reviews of Interventions (Higgins 2011a). This method requires the intra-cluster correlation coefficient (ICC), an estimate of the variability within and between clusters, for the RCT. Where a study does not report this, it is possible to use an external estimate of ICC. We selected external estimates of 0.02 and 0.04 by looking at the ICCs reported in other clusterRCTs, discounting extremes and looking at the published literature
(Ukoumunne 1999). We ran sensitivity analyses using 1) no adjustment, 2) adjustment for clustering assuming ICC of 0.02 , and 3) adjustment for clustering assuming ICC of 0.04 . We did this for both BMI and zBMI. All values of unadjusted SE and approximate adjusted SE plus data required to calculate them are listed in Appendix 5.

\section{Studies with multiple treatment groups}

For RCTs with more than one intervention group we considered 1 ) if all the intervention groups were relevant to the review, and 2) if all the intervention groups were relevant for a specific meta-analysis. In situations where only one intervention group was relevant to the meta-analysis, we would treat it as a two-armed RCT. For RCTs with more than two arms of relevance to the same meta-analysis and with one control arm, we included data from both treatment arms. To avoid double counting of participants we halved the number of participants in the control arm. For factorial RCTs we included all the arms of the trials as if they were distinct trials. See Cochrane Handbook of Systematic Reviews of Interventions Section 16.5.4 and 16.5.6 (Higgins 2011a).

\section{Dealing with missing data}

We noted missing data on the data extraction form and took them into account when judging the risk of bias of each study. We excluded RCTs for which insufficient data were available from quantitative analyses (e.g. in study reports, and when missing data could not be obtained). We did not impute any missing data.

\section{Assessment of heterogeneity}

We used $\mathrm{I}^{2}$ statistic to assess heterogeneity (Higgins 2003) using suggested assessments of heterogeneity such that 12 of $0 \%$ to $40 \%$ : might not be important; $30 \%$ to $60 \%$ : may represent moderate heterogeneity; $50 \%$ to $90 \%$ : may represent substantial heterogeneity; $75 \%$ to $100 \%$ : considerable heterogeneity. We decided to pool data whatever the value of $\mathrm{I}^{2}$ statistic indicated in the meta-analysis and to explore heterogeneity by running subgroup analyses using different variables, for example, setting, duration of intervention, type of intervention to see if variability could be explained. For our 'Summary of findings' table, and given the varied nature of intervention types, setting, and characteristics of baseline populations, we chose to downgrade evidence once for RCTs with greater than $60 \%$ value for $1^{2}$ statistic and to downgrade evidence twice for RCTs with greater than $85 \%$ value of $\mathrm{I}^{2}$ statistic. For the main analyses we will not use the $\mathrm{Chi}^{2}$ or $\mathrm{I}^{2}$ statistics to assess differences between the subgroups for BMI or zBMI. We consider the age groups to be distinct populations, and therefore assessment of differences between the three age groups is not appropriate for the purposes of this review (Deeks 2017).

\section{Assessment of reporting biases}

We assessed reporting bias and other small study effects following methods set out in Chapter 10 of the Cochrane Handbook for Systematic Reviews of Interventions Higgins 2011d. For those metaanalyses with more than 10 studies we prepared funnel plots using Stata version 15 (Stata 2019), and tested for asymmetry with Egger tests (Egger 1997a), using the commands 'metabias' and 'metafunnel' Harbord 2009. 


\section{Data synthesis}

We analysed zBMI scores and BMI data using the generic inverse variance method with a random-effects model (Deeks 2017). The order of preference for data was prespecified. In preference we took difference in means between intervention and control that were reported for the end of the intervention and had been adjusted for clustering or baseline variation, or both. However, if only unadjusted data were available we used those. If difference in mean data were unavailable we used change scores: the change in outcome from baseline to follow-up (Cochrane Handbook for Systematic Reviews of Interventions, chapter 9.4.5.2; Deeks 2017). If standard deviation (SD) was not reported we derived it, where possible, from $95 \%$ confidence intervals, $P$ values or SE, using the calculator provided in Review Manager 5 (RevMan 5 (Review Manager 2014)), and equations provided in Chapter 9 of the Cochrane Handbook for Systematic Reviews of Interventions (Deeks 2017). We did not use data from RCTs where the difference in means between the two arms at baseline was more than the change in mean in either arm (suggesting that the baseline measure would dominate the outcome data) unless the study presented the change (and variance of that change) for each arm, or had adjusted for the baseline difference.

For RCTs that reported more than one intervention arm, we presented the data for each intervention arm compared with the control arm, with the number of participants in the control arm halved to ensure no double counting.

We have presented only outcome data reported immediately post-intervention. We did not analyse data for subsequent postintervention follow-up.

We have presented analyses stratified by age group with three categories: 0 to 5 years, 6 to 12 years, and 13 to 18 years. This was based on what would be meaningful for decision makers. These age categories correspond to stages of child development and childhood settings. We believe the populations, children aged 0 to 5 years, children aged 6 to 12 years and young people aged 13 to 18 years, to be too different developmentally to be considered as a single sample. Interventions that are likely to work on a 3 or 4 year old, are unlikely to work in adolescents, and vice versa. We present the effects of BMI and zBMI for each of the three age groups as the main analyses in this review.

For cluster-RCTs that had not adjusted for clustering we approximated analysis for clustering using ICC $=0.04$, based upon methods described in the Cochrane Handbook for Systematic Reviews of Interventions (Higgins 2011a), and on sensitivity analyses of the value of ICC to use for the approximation: 1 ) no clustering or ICC $=0,2$ ) ICC of 0.02 , and 3) ICC of 0.04 . This is described in more detail in section Unit of analysis issues, and in Sensitivity analysis. See Appendix 5 for lists of unadjusted and approximately adjusted SE.

\section{Subgroup analysis and investigation of heterogeneity}

We explored heterogeneity in the nine primary analyses:

- age 0 to 5 years: dietary interventions, physical activity interventions, and combined dietary and physical interventions; zBMI and BMI;
- age 6 to 12 years: dietary intervention, physical activity interventions, and combined dietary and physical interventions; zBMI and BMI;

- age 13 to 18 years: dietary intervention, physical activity interventions, and combined dietary and physical interventions; zBMI and BMI.

by two subgroup analyses, 1) main setting of the intervention (childcare/preschool, school, health service, wider community, home), and 2) duration of active intervention period ( $\leq 12$ months, $>12$ months).

\section{GRADE and 'Summary of findings' table}

We created 'Summary of findings' tables to summarise the size and certainty of effects of the interventions. This was based on the five GRADE considerations (risk of bias, consistency of effect, imprecision, indirectness and publication bias). We used GRADEpro software (GRADEpro GDT 2015), and followed methods described in the Cochrane Handbook for Systematic Reviews of Interventions: Section 8.5 (Higgins 2017), and Chapter 12 (Schünemann 2017).

To determine the consistency of effects for each comparison we looked at the $\mathrm{I}^{2}$ statistic value. For comparisons where the metaanalysis had an $1^{2}$ statistic value above $60 \%$ we determined these to be at 'serious' inconsistency. If the $\mathrm{I}^{2}$ statistic was above $85 \%$ we considered this to be 'very serious' inconsistency. We assessed the risk of bias across all the RCTs contributing to the pooled effect. We assessed the effect of risk of bias by comparing the overall treatment effect from all studies with a sensitivity analysis in which we excluded all studies with at least one domain at high risk of bias. If the estimates from the overall versus the sensitivity analysis were in opposite directions, we downgraded the estimate twice for risk of bias rating it as 'very serious'. If the treatment effects from the overall analysis and the sensitivity analysis were largely congruent then we did not downgrade.

\section{Sensitivity analysis}

Fifteen cluster-RCTs had not accounted for clustering in their analysis (Annesi 2013; Bonis 2014; Cao 2015; Farias 2015; Herscovici 2013; Klein 2010; Lazaar 2007; Llargues 2012; Melnyk 2013; Natale 2014; Robbins 2006; Sallis 1993; Sevinc 2011; Spiegel 2006; Thivel 2011). Three of these studies did not contribute data to any metaanalyses (Farias 2015; Sallis 1993; Sevinc 2011). We approximated adjustment for clustering using the method described in the Cochrane Handbook for Systematic Reviews of Interventions (Higgins 2011a). We selected a range of ICC coefficients (no adjustment, ICC $=0.02$ and ICC $=0.04$ ). We ran meta analyses using unadjusted SE and SE adjusted for ICC $=0.02$ and ICC $=0.04$ for both BMI and $\mathrm{ZBMI}$. Using sensitivity analysis, we observed that the pooled effect sizes for each meta-analysis was changed very little by the choice of value for ICC (see Appendix 5). In order to be conservative in our selection of ICC we chose an ICC of 0.04 and have presented pooled metaanalyses in which the SE of RCTs that had not taken account of clustering have been approximately adjusted using an ICC of 0.04 . 


\section{RESULTS}

\section{Description of studies}

\section{Results of the search}

This is the fourth update of this review, the search dates for which were 1999, 2002, 2005, 2010, and 2015. The 2010 to 2015 search retrieved 18,106 unique new records. We read 279 of these records in full and added 108 new RCTs. In total, since 1999, searches for this review have retrieved 46,107 unique records, and we have included 153 RCTs (210 papers). See Figure 1 for the PRISMA flow chart (Moher 2009). There are 62 RCTs $(n=88,383)$ contributing data to meta-analysis of zBMIs and $72 \mathrm{RCTs}(\mathrm{n}=77,286)$ contributing data to meta-analysis of BMI. Note, these figures do not add up to 153 (to reflect number of included studies) because some studies report both zBMI and BMI whilst other studies report neither. Twenty-four RCTs reported both BMI and zBMI scores. The records retrieved from searching and the RCTs identified since 1999 appear to be increasing exponentially (see Figure 2). We ran the searches for a fifth update (search date January 2018) and have listed papers with potential for inclusion identified from this search in 'Studies awaiting classification'. However, we have not yet synthesised data from these studies in this review.

\section{Figure 1. Flow of records}

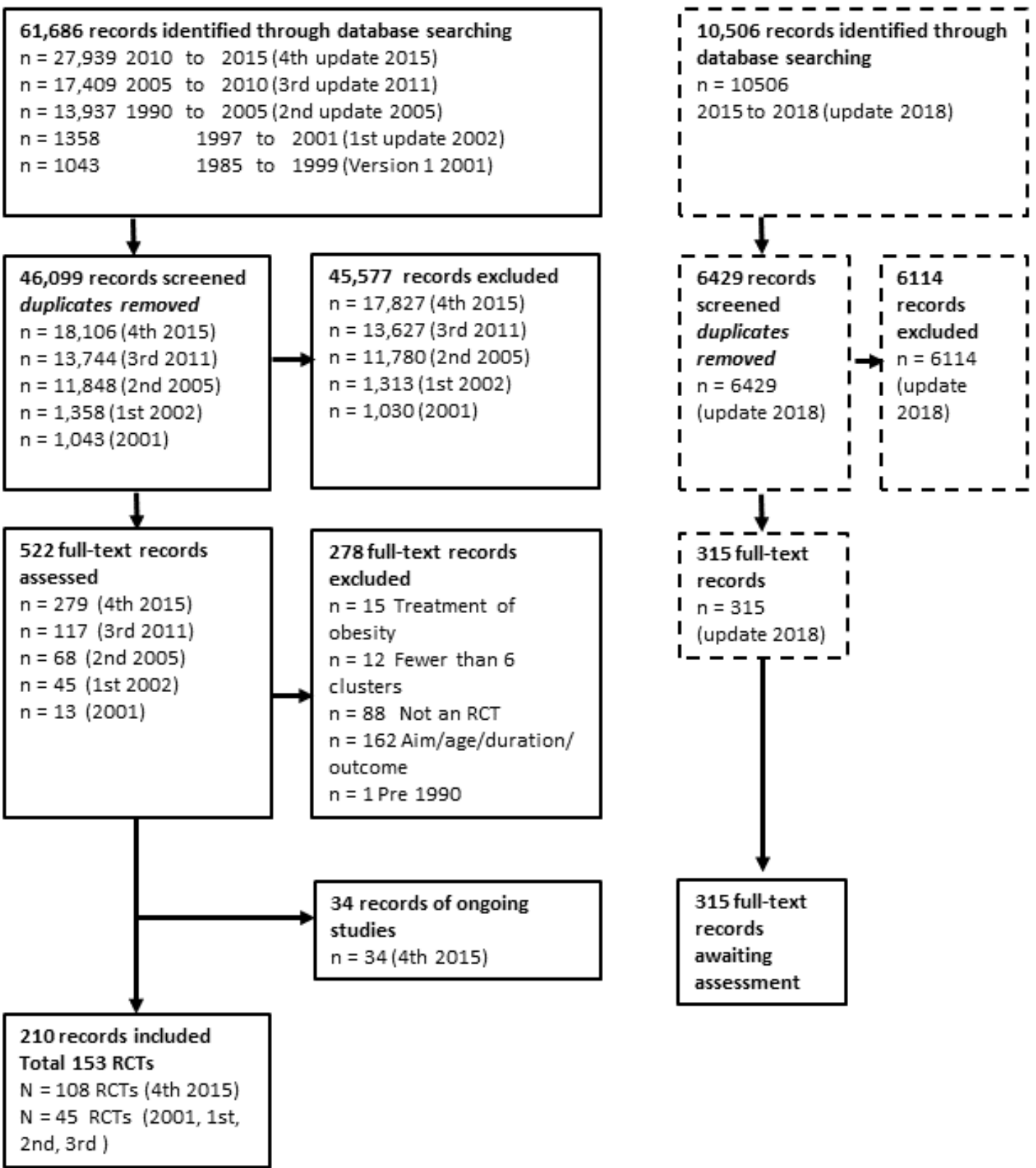


Figure 2. Increase in number of records retrieved and studies included in this systematic review from 2001 until 2017

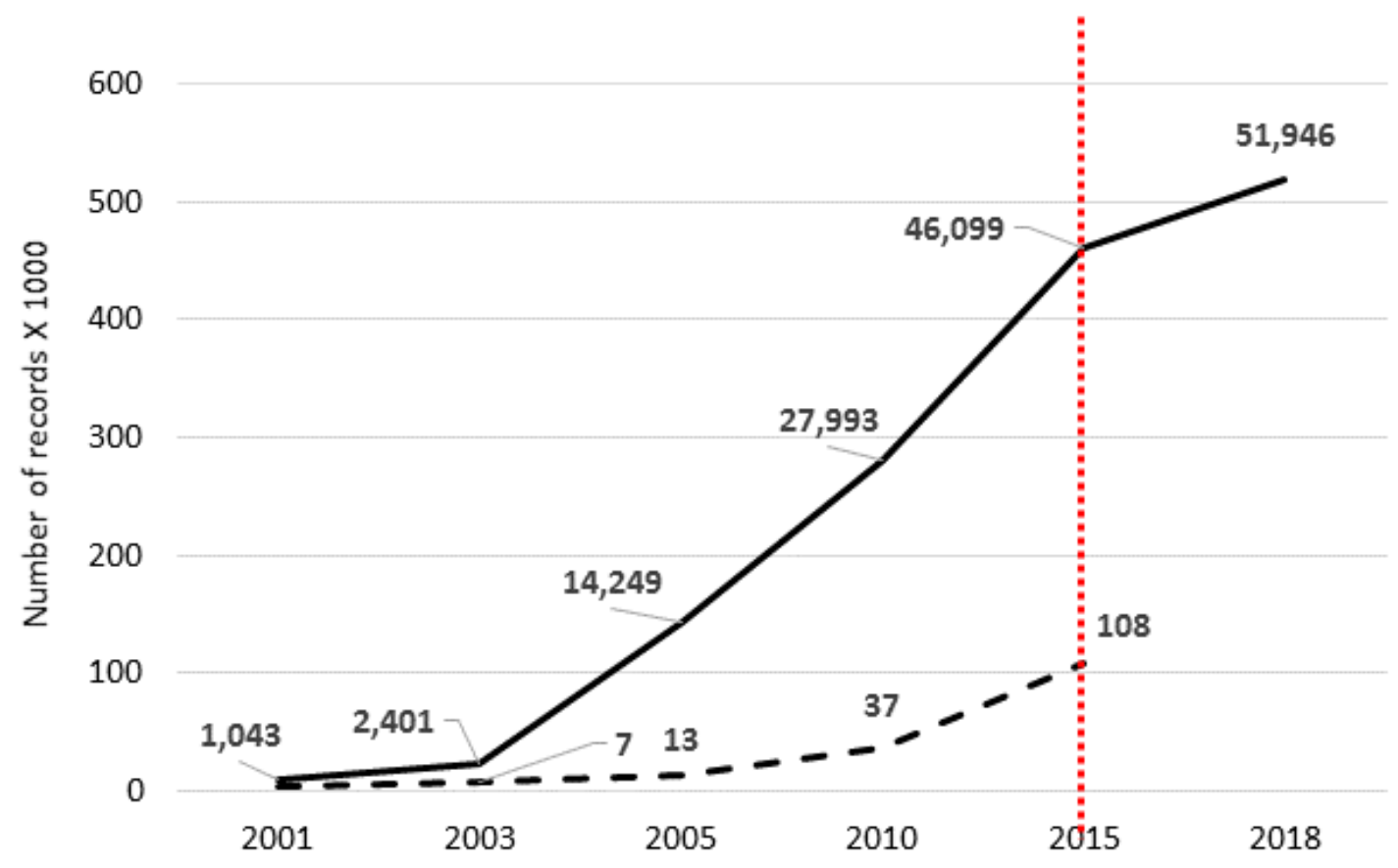

Number of records

- - Number of included studies

Change in inclusion criteria 2001 to 2010 RCTs and CCTs, Post 2015 RCTs only

\section{Included studies}

We included 153 RCTs in this review. We have listed details of each in the Characteristics of included studies table and Figure 3 , and have summarised additional material relating to the theory underpinning the intervention, setting, age, country, and intervention period in Table 1, Table 2 and Table 3. Information about type of comparator is listed in Table 4 and information related to funding source is summarised in Table 5. We have listed studies reporting adverse events in Table 6, Table 7 and Table 8. We have summarised included studies reporting zBMI or BMI, and therefore included in the meta-analyses, in Table 9, and we have listed them in more detail in Table 10, Table 11, Table 12, Table 13, Table 14 and Table 15. 
Figure 3. Distribution of studies by location, age of children and type of intervention. * Total number of locations is 154 and not 153 (number of studies) as one study, Lana 2014, was located in both Spain and Mexico. Papadaki 2010 was located in 7 countries across Europe.
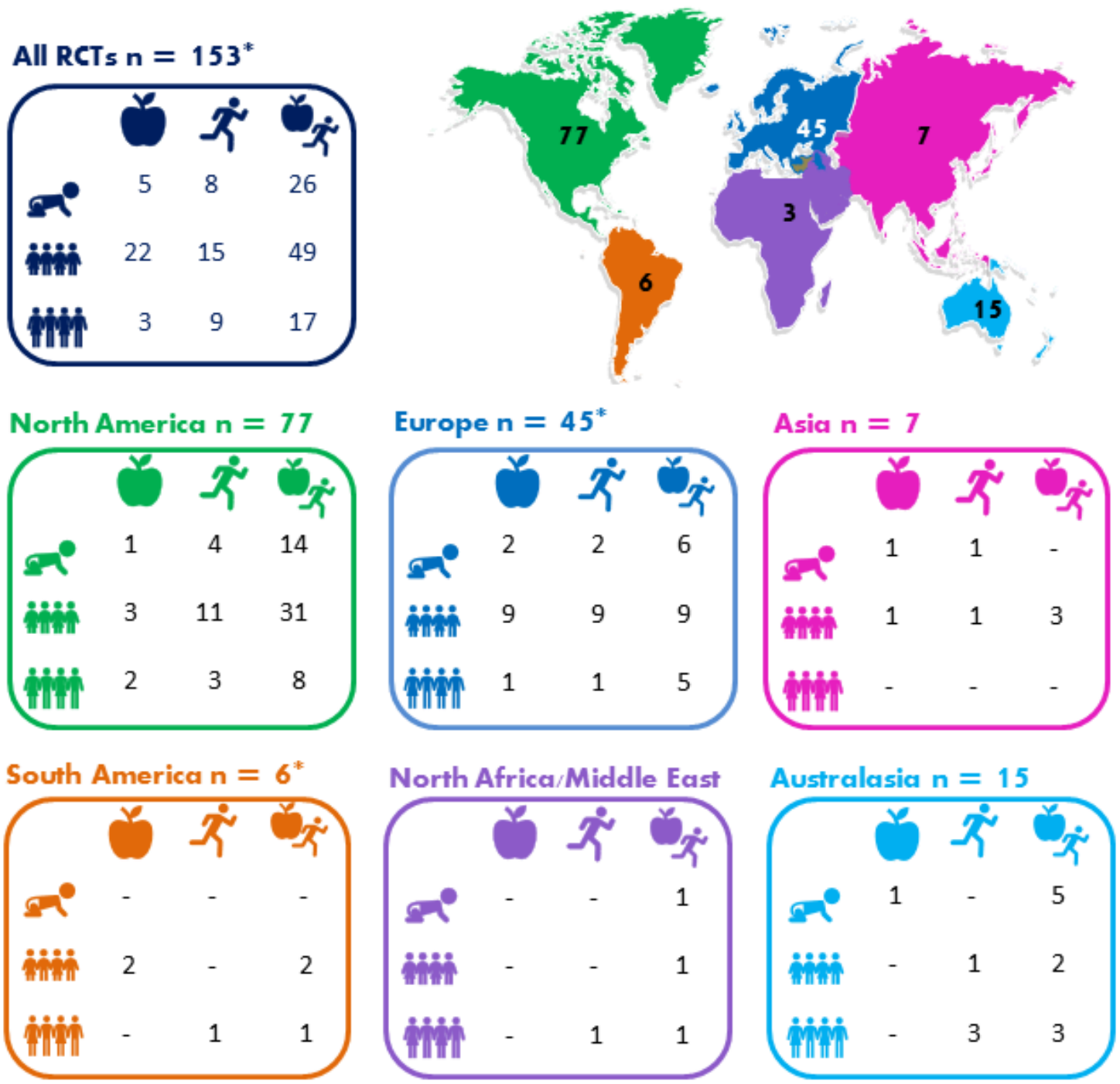
Participants
Age 0-5
Intervention types
袖 Age 6-12
îî Age 13-18

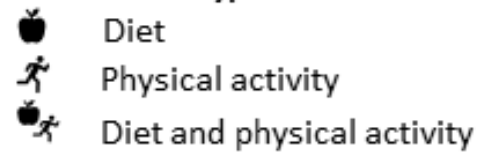




\section{Study design}

We included 108 cluster-RCTs and 45 RCTs in this review ( $n=210$ references).

\section{Participants}

Most RCTs were conducted in North America ( $n=77,50 \%$ ), with most of these in the USA ( $n=69 ; 45 \%)$; the remainder were conducted in Europe ( $n=45,29 \%)$, Australasia ( $n=15,10 \%)$, Asia $(n=7,5 \%)$, South America $(n=6,4 \%)$; and the Middle East and North Africa ( $n=3,2 \%$ ) (Figure 3). Based on the World Bank classification of countries by income, most RCTs were conducted in high-income countries $(n=139 ; 91 \%)$ with $13(8 \%)$ in upper-middleincome countries, and one (1\%) in a lower-middle-income country (Appendix 6). We categorised settings as 'school' including primary, middle and secondary schools ( $n=91,59 \%$ ), 'community' ( $n=$ $23,15 \%)$, 'health care' ( $n=6,4 \%)$, 'childcare' including nurseries; child-care centres; kindergartens and preschools $(n=22,14 \%)$ and 'home' ( $\mathrm{n}=11,7 \%)$. Twenty-two (14\%) RCTs included more than one setting, for example school-based RCTs with homework or parental involvement were also classed as 'home-based'. For the purpose of meta-analyses, we placed RCTs into subgroups according to the main setting, that is, the setting where most of the intervention was carried out. Of the 153 included RCTs, 39 (25\%) targeted children aged 0 to 5 years, $85(56 \%)$ targeted children aged 6 to 12 years, one included children aged 0 to 5 and 6 to 12 , and 29 (19\%) RCTs targeted children aged 13 to 18 years (Figure 3 ).

\section{Interventions}

Ninety-three $(61 \%)$ RCTs included a combination of diet and physical activity intervention. Thirty-nine (21\%) RCTs compared physical activity with control and 21 (14\%) RCTs compared dietonly with control (Figure 3). Ninety-one (59\%) RCTs reported some form of theoretical underpinning, the most common being Social Cognitive Theory (Table 1, Table 2, Table 3). Thirty five (23\%) RCTs took measures to address potential inequalities in the development of the intervention or the design of the RCT; 15 in the 0 to 5 age group, 17 in the 6 to 12 age group, and four in the 13 to 18 age group. One hundred and sixteen RCTs (76\%) were interventions that were implemented for 12 months or less, 25 (16\%) for one to two years, and $12(8 \%)$ were implemented for more than two years.

There were 15 (10\%) RCTs that had more than one intervention group; 12 of these types of RCTs evaluated various components such as targeting or including parents (Beech 2003; Haerens 2006), different strategies (Bonsergent 2013; Wilksch 2015; Williamson 2012), or settings (Crespo 2012), online only versus online plus text messaging (Lana 2014), different diets/nutrition advice (Epstein 2001; Paineau 2008; Papadaki 2010; Paul 2011), and different types/ intensities of physical activity (Salmon 2008). We did not analyse the effects of these various components as they were outside the scope of the review. We did, however, include all the comparison groups (where data allowed) in the meta-analyses compared to control.

Three (2\%) RCTs (Meng 2013; Sevinc 2011; Warren 2003), directly evaluated dietary interventions versus physical activity interventions and were head-to-head comparisons that fulfilled our inclusion criteria (see Objectives). Unfortunately, only one of these RCTs (Meng 2013), reported data suitable for inclusion in metaanalyses, so we did not undertake meta-analysis of head-to-head comparisons of diet and physical activity but described the results narratively.

We have given additional details about the interventions for each study in the Characteristics of included studies tables.

\section{Settings}

In terms of settings, the included studies were conducted in a range of difference places: childcare $(n=22)$; healthcare $(n=6)$; home $(n$ $=11)$; school $(n=90)$; and the wider community $(n=24)$. In children aged 0 to 5 years: childcare $(n=22)$; healthcare $(n=5)$; home $(n=6)$; school $(n=2)$; and the wider community $(n=4)$. In children aged 6 to 12 years: home $(n=3)$; school $(n=64)$; and the wider community $(n=18)$. In children aged 13-18 years: healthcare $(n=1)$; home $(n=$ $2)$; school ( $n=24)$; and the wider community $(n=2)$.

We looked at the change in the profile of settings for interventions to prevent childhood obesity before 2011 (earlier) compared with 2011 to 2015 (later), given the call for more upstream interventions over the last 10 to 15 years. Overall, we did not see any clear trend for a shift towards more upstream interventions over time. In children aged 0 to 5 years, settings in earlier studies included childcare $(n=7)$ and home $(n=2)$; later studies included childcare $(n=15)$, healthcare $(n=5)$, home $(n=4)$, school $(n=2)$ and the wider community $(n=4)$. In children aged 6 to 12 years, settings in earlier studies included home $(n=2)$, school $(n=29)$ and the wider community $(n=12)$; later studies included home $(n=1)$, school ( $=35)$ and the wider community $(n=6)$. In children aged 13 to 18 years, settings in earlier studies included healthcare $(n=1)$, home $(n=1)$, school $(n=10)$ and the wider community $(n=1)$; later studies included home $(n=1)$, school $(n=14)$ and the wider community ( $=1$ ).

\section{Comparisons}

The type of control comparison groups varied across the 153 RCTs (Table 4), the vast majority of RCTs included 'no intervention', 'usual care' or 'waiting list' comparisons. We considered these three to be essentially similar because usual care in a prevention intervention is no intervention. There were also RCTs that included relatively more active control comparisons (not expected to affect outcomes of interest) such as school-readiness programmes, selfesteem programmes, an alcohol and drug programme, health and safety programmes, general health programmes and self-help programmes. In many cases, particularly in school-based RCTs, it was not always clear whether the intervention was instead of, or as well as, the usual care condition (i.e. standard diet and physical activity curriculum); for this reason we included these types of RCTs (i.e. those with a concomitant intervention component) along with those RCTs that included no-intervention comparisons, usual-care comparisons and waiting-list comparisons. These variations in the type of control comparison groups should be borne in mind when considering the results of the meta-analyses.

\section{Outcomes}

Details of all outcomes reported in RCTs can be found in Characteristics of included studies. The most common measures of adiposity reported were zBMI and BMI. Sixteen RCTs reported adverse events. 


\section{Funding sources}

\section{Funding sources 0 to 5 years}

The majority of RCTs declared non-industry funding in their publications, that is, not-for-profit charitable organisations and government institutes $(n=28 ; 72 \%)$. See Table 5 . No RCTs were funded wholly by industry. Five RCTs (13\%) (Daniels 2012; De Vries 2015; Paul 2011; Puder 2011; Roth 2015), described mixed funding from both industry and not-for-profit organisations, of which three included sponsorship from baby food manufacturers (Daniels 2012; Paul 2011; Puder 2011). Another two declared that both research and writing of the trial reports had been done independently from the funders: Puder 2011 received industry funding from two organisations that make infant nutrition, Wyeth Nutrition (https://www.wyethnutrition.com/), and Nestlé (www.nestlefoundation.org/e/research.html), and Roth 2015 was partially funded by a grant from a health insurance organisation, Barmer Ersatzkasse (www.barmer.de/en). Both RCTs had industry funding mediated through not-for-profit foundations, a grant from the Wyeth foundation, and an "unrestricted educational grant from Nestlé" (Puder 2011). Three RCTs that received some industry sponsorship did not report if the research and writing were independent of funding. Sponsorship for De Vries 2015 derived from a telecommunications firm, Hutchison-Whampoa (www.ckh.com.hk), Daniels 2012 from an infant food manufacturer HJ Heinz (www.heinzbaby.co.uk/), and the third, Paul 2011, was given infant food for the research by Gerber, a subsidiary of Nestlé (medical.gerber.com/).

\section{Funding sources 6 to 12 years}

The majority of RCTs declared non-industry funding in their publications (69; 81\%). See Table 5. One study reported being funded by industry (Damsgaard 2014). This funding came from food sponsors, who provided foods for the study (Danæg A/S, Naturmælk, Lantmännen A/S, Skærtoft Mølle A/S, Kartoffelpartnerskabet, AkzoNobel Danmark, Gloria Mundi, and Rose Poultry $\mathrm{A} / \mathrm{S}$ ), and a charitable trust from a bank (Nordea Foundation). Sponsorship was independent of the research and writing. Seven RCTs described mixed funding from both industry and not-for-profit organisations, of which two reported that both research and writing of the trial reports had been done independently from the funders. James 2004 had sponsorship from the pharmaceutical industry: Glaxo Smith Klein (www.gsk.com/en$\mathrm{gb} /$ ); Aventis (www.sanofi.com/en/); and Pfizer (www.pfizer.com/). Paineau 2008 received funding from CEDUS (www.sucre-info.com/ le-cedus/), the professional organisation for the sugar beet sector in France. Five RCTs did not report if research or writing were independent of funding: Grydeland 2014 (chocolate manufacture); Kain 2014 (food processing company); Muckelbauer 2010 (association of the German water and gas industries); Papadaki 2010 (food provided by numerous sponsors including Coca-Cola, Kelloggs and Unilever); Rodearmel 2006 (W.K. Kellogs Institute for Food and Nutrition Research).

\section{Funding sources 13 to 18 years}

The majority of RCTs declared non-industry funding in their publications (26; 90\%). See Table 5. Two RCTs stated they received no funding at all for their research (Shin 2015; Weeks 2012). Two RCTs received funding from both non-industry and industry sources. Bonsergent 2013 received industry funding from The Wyeth foundation (owned by Nestlé), and research and writing were independent of this funding. Patrick 2006 reported that three study authors were co-owners and received income from The Centre for Health Interventions, San Diego, California, which was developing products related to the trial.

\section{Theoretical basis of interventions}

Forty-nine per cent (19/39) of RCTs of children aged 0 to 5 years, $56 \%(48 / 85)$ of RCTs of children aged 6 to 12 years, and $70 \%(21 / 30)$ of RCTs of children aged 13 to 18 years reported a theoretical basis informing the study design. In total, we identified 35 different theories (Table 1; Table 2; Table 3; Appendix 7).

Included RCTs used three theories (precaution adaption process model, socioecological model, and theory of planned behaviour) in interventions given to children of 0 to 5 , and 13 to 18 years. We found the health belief model in interventions given to 0 to 5 year olds, and 6 to 12 year olds; the social learning theory in RCTs given to children aged 6 to 12 , and 13 to 18 years. All three age groups received interventions based on self-determination theory and social cognitive theory (Table 1; Table 2; Table 3; Appendix 7).

There were 11 theories underpinning interventions for 0 to 5 year olds, of which four were unique (anticipatory guidance; attachment theory; exposure theory; theories of information processing).

There were 14 theories underpinning interventions of children aged 6 to 12, of which 10 were unique (family systems theory; sociocultural theory; ecological and developmental systems theories; environmental change theory; group socialisation theory; investigation, vision, action and change methodology; health promotion model; behavioural choice theory; theory of reasoned action, constructivism; and youth development and resiliencybased approaches). Of the 85 RCTs of children aged 6 to 12 , the most predominant theory used was social cognitive theory.

There were 12 theories underpinning interventions for 13 to 18 year olds, seven theories were unique (skills model; informationmotivation behavioural control theory; implementation intentions; attitude, social influence and self-efficacy (ACE model); socioecological model, self-determination theory; and theory of interactive technology). Of the 29 RCTs of children aged 13 to 18 years the most predominant theory used was social cognitive theory.

\section{Implementation factors}

\section{Economic information}

All RCTs reported details of personnel who delivered the intervention (Characteristics of included studies). Only one study out of all 153 RCTS included a formal economic evaluation (Llargues 2012). This was for the AVall programme for 6 to 12 year olds (Llargues 2011; Mora 2015). Six of 39 RCTs for children aged 0 to 5 years reported on intervention costs (Bonvin 2013; Campbell 2013; Klein 2010; Natale 2014; Reilly 2006; Rush 2012). Seven of 85 RCTs for children aged 6 to 12 years reported intervention costs (Brandstetter 2012; Coleman 2005; Hendy 2011; Kipping 2008; Martinez-Vizcaino 2014; Rush 2012; Vizcaino 2008). Two of 30 RCTs for children aged 13 to 18 years, reported on direct intervention costs (Christiansen 2013; Ebbeling 2006). 


\section{Strategies to address disadvantage/diversity 0 to 5 years}

Fifteen RCTs adopted a range of methods to ensure diversity or to moderate the effects of disadvantage. Seven RCTs included either cultural training for staff delivering interventions (Fitzgibbon 2011; Harvey-Berino 2003), or had modified, tailored or specifically designed interventions for specific cultural settings (Fitzgibbon 2006; Natale 2014; Puder 2011; Slusser 2012; Story 2012). Two RCTs specifically set out to address diversity by selecting specific communities (Fitzgibbon 2005; Fitzgibbon 2006), and seven adopted recruitment strategies aimed at increasing diversity (Annesi 2013; Bellows 2013a; Haines 2013; Nemet 2011; Ostbye 2012; Skouteris 2016; Wen 2012). Two RCTs described methods they used to overcome environmental barriers to participation related to inequality (Fitzgibbon 2005; Fitzgibbon 2011).

\section{Strategies to address disadvantage/diversity 6 to 12 years}

Seventeen RCTs adopted strategies to address disadvantage/ diversity. Methods to address issues of diversity and inequity included involving participant groups in the design and delivery of the intervention (Baranowski 2003; Beech 2003; Robinson 2003; Story 2003), specifically tailoring the interventions to be culturally relevant (Brown 2013; Caballero 2003; Coleman 2005; De Heer 2011; Gutin 2008; Habib-Mourad 2014; Robbins 2006; Robinson 2003; Robinson 2010; Stolley 1997; Story 2003), consideration of language (Spiegel 2006), and specifically addressing the intervention for populations at risk of inequity (Habib-Mourad 2014; HaireJoshu 2010; Levy 2012; Madsen 2013). In addition to the RCTs that reported intervention strategies to address disadvantage/ diversity, 15 RCTs reported on recruitment strategies to address disadvantage/diversity.

\section{Strategies to address disadvantage/diversity 13 to 18 years}

Of the 30 RCTs targeted towards the 13 to 18 years age group, one study reported incorporating intervention strategies (Shin 2015), and three RCTs reported on recruitment strategies to address disadvantage/diversity (Lubans 2011; Singh 2009; Smith 2014).

\section{Other aspects of implementation from process evaluations}

It is worth noting that many of the included RCTs across all age groups reported one or more elements of process evaluation, including dose, exposure, attendance, adherence, intervention fidelity, feasibility of intervention, child satisfaction or acceptability, reach, and retention.

Donnelly 2009 reported intensity of lesson delivery. This RCT also investigated the effect of teacher participation in classroom physical activity. They found that teacher participation in the activity appeared to positively influence student activity levels in the study.

Child or teacher (or intervention deliverer) satisfaction with the intervention was a relatively common factor to measure in the studies we included in this review. In previous versions of this Cochrane Review, we highlighted the important link between how much the child and teacher enjoy the intervention (and, particularly for younger children, whether they consider it to be 'fun'), and recruitment, adherence and retention.

Many of the process evaluations raised practical issues relating to the intervention, which were barriers or facilitators of implementation. For example, Kipping 2008 reported that teachers found it difficult to adhere to the intervention requirements as intervention lessons were difficult to accommodate into the school timetable. Robbins 2006 similarly identified important barriers to increasing physical activity in some girls, with lack of suitable places, resources and social support for physical activity limiting compliance with the intervention programme. Robinson 2003 explored barriers to attendance and found transportation to be an important factor. Coleman 2005 published implementationrelated information in a separate paper (Heath 2002), and provided recommendations to practitioners covering some of the contextual factors to consider when adapting the programme to their own context.

Habib-Mourad 2014 reported on implementation, dose and context. Failure to succeed in modifying the school's food environment was due to lobbying and lack of support of some of the school authorities. The study was based in Lebanon which is a politically unstable context, with security threats and social unrest.

\section{Studies awaiting classification}

Two RCTs require translation and are awaiting classification; these RCTs are listed in Characteristics of studies awaiting classification(Lichtenstein 2011; Walther 2011). RCTs identified that were ongoing at the time of the 2015 search have been listed under Characteristics of studies awaiting classification. We ran an update search from May 2015 to January 2018 to identify all potential RCTs for this review. This search identified 6342 unique records and we identified 315 papers to read in full (Figure 1). We have added these records to the category 'Studies awaiting classification'. Because we have not yet assessed these records for inclusion to the review, the table entries for these records are empty. Ongoing RCTs and those awaiting classification will be incorporated into future updates of this review.

\section{Excluded studies}

Studies excluded at full-text stage are listed in Characteristics of excluded studies.

\section{Risk of bias in included studies}

The Characteristics of included studies reports the risk of bias results for the 153 included RCTs. We present a 'Risk of bias' graph (Figure 4) with review authors' judgements about each 'Risk of bias' item presented as percentages across all included RCTs. We present a 'Risk of bias' summary (Figure 5), with review authors' judgements about each 'Risk of bias' item for each included study. When a study included insufficient information in the relevant papers to allow us to make a judgement for a particular domain, we gave RCTs a rating of unclear. 
Figure 4. 'Risk of bias' graph: review authors' judgements about each 'Risk of bias' item presented as percentages across all included studies

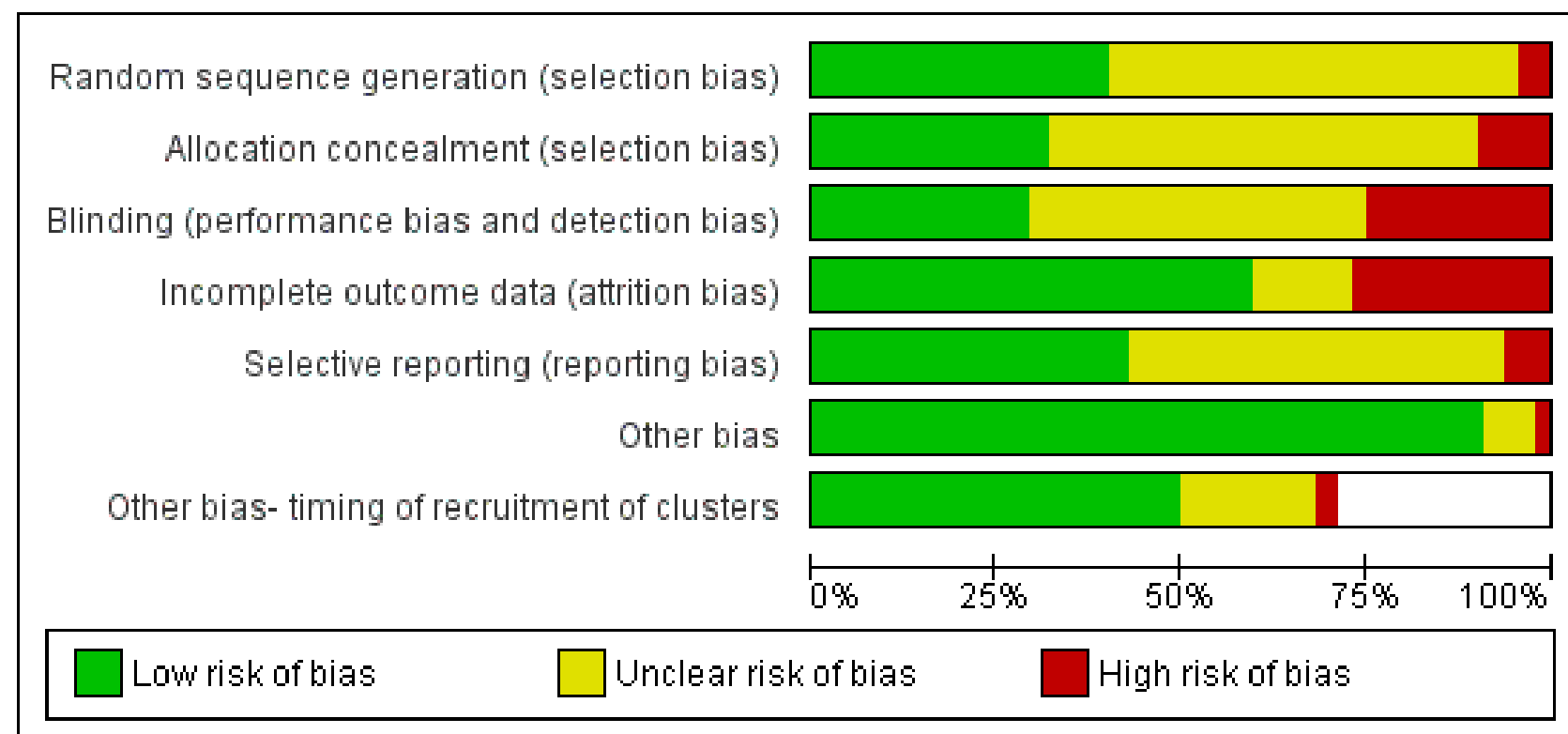


Figure 5. 'Risk of bias' summary: review authors' judgements about each 'Risk of bias' item for each included study

\begin{tabular}{|c|c|c|c|c|c|c|c|}
\hline & 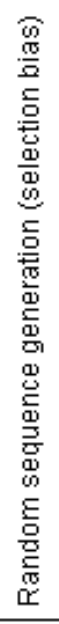 & 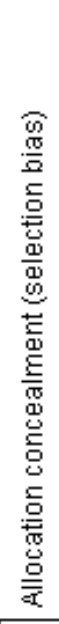 & 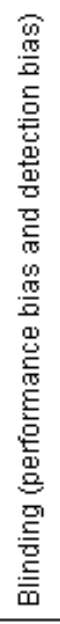 & 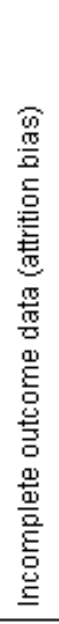 & 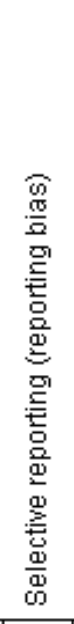 & 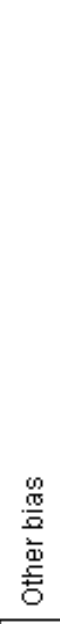 & 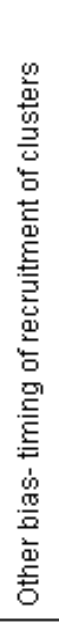 \\
\hline Alkon 2014 & $?$ & + & + & $\odot$ & $\odot$ & + & $?$ \\
\hline Amaro 2006 & $?$ & ? & $?$ & $\odot$ & $?$ & + & $?$ \\
\hline Andrade 2014 & + & + & $\odot$ & + & $?$ & + & $\odot$ \\
\hline Annesi 2013 & + & $?$ & $?$ & $\odot$ & $?$ & $?$ & $?$ \\
\hline Baranowski 2003 & + & + & $?$ & + & $?$ & + & \\
\hline Baranowski 2011 & $?$ & $?$ & $\oplus$ & $\oplus$ & $?$ & $?$ & \\
\hline Barkin 2012 & + & + & $\odot$ & $\oplus$ & + & + & \\
\hline Beech 2003 & $?$ & $?$ & $?$ & 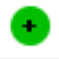 & $?$ & + & \\
\hline Bellows 2013a & $?$ & $?$ & $\odot$ & + & $\odot$ & + & $?$ \\
\hline Birken 2012 & + & + & $\odot$ & $\odot$ & $\odot$ & $?$ & \\
\hline Black 2010 & $?$ & $?$ & + & + & + & + & \\
\hline Bohnert 2013 & $\odot$ & - & $\odot$ & $\odot$ & $?$ & + & \\
\hline Bonis 2014 & $?$ & + & $?$ & + & $?$ & + & + \\
\hline Bonsergent 2013 & $\oplus$ & + & $?$ & $\odot$ & + & + & + \\
\hline Bonuck 2014 & + & + & $\odot$ & 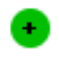 & $?$ & $?$ & \\
\hline Bonvin 2013 & $?$ & + & + & $?$ & $\odot$ & $\oplus$ & $?$ \\
\hline Brandstetter 2012 & $?$ & + & + & + & $\Theta$ & + & 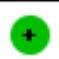 \\
\hline Branscum 2013 & $?$ & $?$ & $?$ & $?$ & $?$ & + & + \\
\hline Brown 2013 & $?$ & $?$ & $\odot$ & $\odot$ & $?$ & $?$ & \\
\hline Caballero 2003 & + & $?$ & + & + & $?$ & + & + \\
\hline Campbell 2013 & 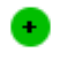 & + & $\odot$ & + & + & $?$ & + \\
\hline Cao 2015 & $?$ & $?$ & $?$ & $\Theta$ & $?$ & + & + \\
\hline
\end{tabular}


Figure 5. (Continued)

\begin{tabular}{|c|c|c|c|c|c|c|c|}
\hline Cao 2015 & $?$ & $?$ & $?$ & $\odot$ & $?$ & + & + \\
\hline Chen 2010 & + & $?$ & - & $?$ & $?$ & + & \\
\hline Chen 2011 & + & $\odot$ & $?$ & $\odot$ & $?$ & + & \\
\hline Christiansen 2013 & $?$ & $?$ & $?$ & + & + & + & + \\
\hline Coleman 2005 & $\odot$ & $?$ & - & + & $\odot$ & + & + \\
\hline Coleman 2012 & $?$ & $\odot$ & $\odot$ & + & + & + & + \\
\hline Crespo 2012 & $?$ & $\odot$ & + & $\odot$ & $?$ & + & + \\
\hline Cunha 2013 & $?$ & + & - & $?$ & + & + & $?$ \\
\hline Damsgaard 2014 & + & $\odot$ & - & $?$ & + & $?$ & - \\
\hline Daniels 2012 & $?$ & + & + & 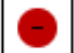 & + & + & \\
\hline De Bock 2012 & $?$ & $?$ & + & 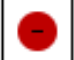 & $?$ & + & + \\
\hline De Coen 2012 & 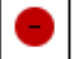 & $\odot$ & $?$ & $?$ & + & + & $?$ \\
\hline De Heer 2011 & $?$ & $?$ & $?$ & + & $?$ & $?$ & + \\
\hline Dennison 2004 & $?$ & + & $\odot$ & $\odot$ & $?$ & + & + \\
\hline De Ruyter 2012 & + & + & + & + & + & + & \\
\hline De Vries 2015 & $\Theta$ & $\Theta$ & + & + & + & + & $?$ \\
\hline Dewar 2013 & $?$ & + & $\odot$ & $\odot$ & + & + & + \\
\hline Donnelly 2009 & $?$ & $?$ & + & $\odot$ & + & + & ? \\
\hline Ebbeling 2006 & + & + & $?$ & $\odot$ & $?$ & + & \\
\hline El Ansarai 2010 & $?$ & $?$ & - & $?$ & $?$ & + & \\
\hline Elder 2014 & $?$ & $?$ & $?$ & $\odot$ & $?$ & + & + \\
\hline Epstein 2001 & $?$ & $?$ & $?$ & + & $?$ & + & \\
\hline Ezendam 2012 & + & + & $?$ & $\theta$ & + & + & 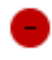 \\
\hline Fairclough 2013 & + & $\odot$ & - & $\theta$ & + & $\odot$ & $?$ \\
\hline Farias 2015 & $?$ & $\odot$ & $?$ & $\odot$ & - & + & $?$ \\
\hline Feng 2004 & $?$ & $?$ & $?$ & + & $?$ & + & + \\
\hline Fitggibbon 2005 & $?$ & $?$ & + & $\oplus$ & + & + & + \\
\hline Fitggibbon 2006 & $?$ & + & $?$ & + & + & $\odot$ & + \\
\hline Fitzgibbon 2011 & $?$ & $?$ & $?$ & + & $?$ & $\odot$ & + \\
\hline Foster 2008 & $?$ & $?$ & $?$ & + & $?$ & + & + \\
\hline French 2011 & $?$ & $?$ & $\odot$ & $\odot$ & $?$ & $\odot$ & + \\
\hline
\end{tabular}


Figure 5. (Continued)

\begin{tabular}{|c|c|c|c|c|c|c|c|}
\hline French 2011 & $?$ & $?$ & $\odot$ & $\odot$ & $?$ & $\odot$ & + \\
\hline Fulkerson 2010 & $?$ & $?$ & $\odot$ & $\odot$ & $?$ & $\oplus$ & \\
\hline Gentile 2009 & $?$ & $?$ & $?$ & $?$ & + & $\oplus$ & + \\
\hline Gortmaker 1999a & $\odot$ & 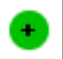 & + & $\odot$ & $?$ & $\oplus$ & $\odot$ \\
\hline Grydeland 2014 & $?$ & ? & $\Theta$ & $\odot$ & $\odot$ & ? & $\oplus$ \\
\hline Gutin 2008 & + & $\odot$ & $\odot$ & $\odot$ & + & $\oplus$ & $?$ \\
\hline Habib-Mourad 2014 & + & $\odot$ & $?$ & + & $?$ & $\oplus$ & + \\
\hline Haerens 2006 & $?$ & $?$ & $?$ & $\odot$ & $?$ & + & $?$ \\
\hline Haines 2013 & $\odot$ & $\odot$ & $?$ & $\odot$ & + & + & \\
\hline Haire-Joshu 2010 & + & $?$ & $?$ & $\odot$ & $?$ & $\oplus$ & $?$ \\
\hline $\operatorname{Han} 2006$ & $?$ & $?$ & $?$ & $\odot$ & $?$ & + & $?$ \\
\hline Harvey-Berino 2003 & $?$ & $?$ & + & $\odot$ & $?$ & 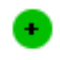 & \\
\hline HEALTHY Study Gp 2010 & $\odot$ & + & $?$ & + & + & $?$ & $?$ \\
\hline Hendy 2011 & $?$ & $?$ & + & $?$ & - & $?$ & \\
\hline Herscovici 2013 & $?$ & $?$ & $?$ & + & $?$ & + & 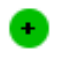 \\
\hline Howe 2011 & $\odot$ & $?$ & $?$ & $?$ & $?$ & $\oplus$ & \\
\hline James 2004 & $\odot$ & $\odot$ & $?$ & $\odot$ & $?$ & $\oplus$ & $\oplus$ \\
\hline Jansen 2011 & + & $?$ & $\odot$ & $\odot$ & + & $\oplus$ & + \\
\hline Johnston 2013 & $\odot$ & $?$ & $\odot$ & $\odot$ & $?$ & + & + \\
\hline Kain 2014 & $?$ & $?$ & $?$ & $?$ & $?$ & $\oplus$ & + \\
\hline Keller 2009 & $?$ & $?$ & $?$ & $\odot$ & $?$ & + & \\
\hline Khan 2014 & + & $?$ & + & $?$ & + & $\oplus$ & \\
\hline Kipping 2008 & $?$ & + & + & $\odot$ & $?$ & + & + \\
\hline Kipping 2014 & + & + & + & + & + & $\oplus$ & + \\
\hline Klein 2010 & $?$ & $?$ & $?$ & $\odot$ & $?$ & + & $?$ \\
\hline Klesges 2010 & $?$ & $?$ & 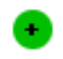 & $?$ & + & + & \\
\hline Kriemler 2010 & $\odot$ & + & $?$ & $\odot$ & + & $\oplus$ & $\oplus$ \\
\hline Lana 2014 & + & + & $\odot$ & $\odot$ & + & $\oplus$ & \\
\hline Lazaar 2007 & + & + & $?$ & $\odot$ & $?$ & + & $?$ \\
\hline Lew 2012 & $?$ & $?$ & $?$ & + & $?$ & + & + \\
\hline Li $2010 a$ & $?$ & $?$ & 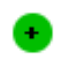 & $\odot$ & $?$ & $\oplus$ & + \\
\hline
\end{tabular}


Figure 5. (Continued)

\begin{tabular}{|c|c|c|c|c|c|c|c|}
\hline Li 2010a & $?$ & $?$ & $\odot$ & $\odot$ & $?$ & $\odot$ & + \\
\hline Llargues 2012 & $?$ & $?$ & $?$ & $\odot$ & $\odot$ & $\odot$ & $?$ \\
\hline Lubans 2011 & $?$ & $?$ & $\odot$ & $\odot$ & $\odot$ & $\bullet$ & $\odot$ \\
\hline Macias-Cervantes 2009 & + & $?$ & + & $\odot$ & $?$ & $\odot$ & \\
\hline Madsen 2013 & $?$ & $?$ & $\odot$ & $\odot$ & + & $\odot$ & $\odot$ \\
\hline Magnusson 2012 & $?$ & $?$ & 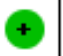 & $\odot$ & $?$ & $\oplus$ & + \\
\hline Marcus 2009 & $?$ & $?$ & $?$ & $\odot$ & $\odot$ & $\oplus$ & + \\
\hline Martinez-Vizcaino 2014 & $\odot$ & + & $\odot$ & $\odot$ & $\odot$ & $\odot$ & + \\
\hline Mauriello 2010 & $?$ & $?$ & $\odot$ & $\odot$ & $\odot$ & $\odot$ & + \\
\hline Melnyk 2013 & $?$ & $?$ & + & $\odot$ & $\odot$ & + & + \\
\hline Meng 2013 & + & $?$ & $?$ & $\odot$ & $\odot$ & + & $?$ \\
\hline Mihas 2010 & + & $?$ & $\odot$ & $\odot$ & $?$ & $\odot$ & + \\
\hline Morgan 2011 & + & + & - & $\odot$ & $?$ & $\odot$ & \\
\hline Mo-suwan 1998 & $?$ & $?$ & $?$ & $\odot$ & + & + & + \\
\hline Muckelbauer 2010 & $?$ & $\odot$ & $\odot$ & $\odot$ & $\oplus$ & + & + \\
\hline Natale 2014 & $?$ & $?$ & $?$ & $\odot$ & $?$ & 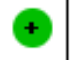 & $?$ \\
\hline Nemet 2011 & + & $?$ & + & $?$ & $\odot$ & $\odot$ & $?$ \\
\hline Neumark-Sxtainer 2003 & $?$ & $\odot$ & $?$ & $\odot$ & + & + & $\theta$ \\
\hline Neumark-Sztainer 2010 & $?$ & $?$ & $?$ & $\odot$ & $\odot$ & + & + \\
\hline Nollen 2014 & $?$ & $?$ & $?$ & $\odot$ & ? & $\odot$ & \\
\hline Nyberg 2015 & $?$ & $?$ & $?$ & $\odot$ & $\odot$ & $\odot$ & $\odot$ \\
\hline Ostbye 2012 & + & $?$ & $?$ & $\odot$ & + & $\odot$ & \\
\hline Paineau 2008 & + & $\odot$ & 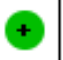 & $\odot$ & + & + & + \\
\hline Papadaki 2010 & + & $?$ & - & $\odot$ & $\odot$ & 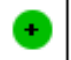 & \\
\hline Pate 2005 & $?$ & $?$ & $?$ & $?$ & $?$ & + & + \\
\hline Patrick 2006 & $?$ & $?$ & $?$ & $\odot$ & $?$ & + & \\
\hline Paul 2011 & $?$ & $?$ & $\odot$ & $\odot$ & $\odot$ & + & \\
\hline Peralta 2009 & + & $?$ & + & $\odot$ & $?$ & + & \\
\hline Puder 2011 & + & + & + & $\odot$ & $\oplus$ & $\oplus$ & + \\
\hline Reed 2008 & $?$ & $\odot$ & $?$ & $\odot$ & $\odot$ & $\oplus$ & + \\
\hline Reilly 2006 & $\odot$ & $\odot$ & $\oplus$ & + & + & $\oplus$ & + \\
\hline
\end{tabular}


Figure 5. (Continued)

\begin{tabular}{|c|c|c|c|c|c|c|c|}
\hline \multirow[b]{2}{*}{ Reilly 2006} & & & & & & & \\
\hline & + & + & + & + & $\odot$ & $\odot$ & $\odot$ \\
\hline Robbins 2006 & $\odot$ & $\odot$ & $?$ & $\odot$ & $?$ & $\odot$ & $\odot$ \\
\hline Robinson 2003 & + & $\odot$ & $\odot$ & $\odot$ & $\odot$ & $\odot$ & \\
\hline Robinson 2010 & $\odot$ & $?$ & $\oplus$ & $\odot$ & $\odot$ & $\odot$ & $\odot$ \\
\hline Rodearmel 2006 & $?$ & $?$ & $?$ & $\odot$ & $?$ & $\odot$ & $\odot$ \\
\hline Rosario 2012 & $\odot$ & + & $\odot$ & $\odot$ & + & $\odot$ & + \\
\hline Rosenkranz 2010 & + & $?$ & + & + & $\odot$ & $\odot$ & + \\
\hline Roth 2015 & + & + & $\odot$ & $\odot$ & $?$ & $\odot$ & $\odot$ \\
\hline Rush 2012 & $?$ & $?$ & $\oplus$ & $\odot$ & $\odot$ & $\odot$ & $?$ \\
\hline Safdie 2013 & $?$ & $?$ & $?$ & $\odot$ & $?$ & $\odot$ & $\odot$ \\
\hline Sahota 2001 & + & + & $\odot$ & $\odot$ & $?$ & $\odot$ & + \\
\hline Sallis 1993 & $\odot$ & $?$ & $?$ & $\odot$ & $?$ & $\odot$ & $?$ \\
\hline Salmon 2008 & + & + & + & $?$ & $?$ & $\odot$ & + \\
\hline Santos 2014 & $\odot$ & + & + & $\odot$ & $\odot$ & $\odot$ & + \\
\hline Sevinc 2011 & $?$ & $?$ & $?$ & $\odot$ & $?$ & $\odot$ & + \\
\hline Shin 2015 & $?$ & $?$ & $?$ & $\odot$ & $?$ & $\odot$ & \\
\hline Sichieri 2009 & $?$ & + & $?$ & $\odot$ & $\odot$ & + & $\odot$ \\
\hline Siegrist 2013 & $?$ & $?$ & + & 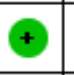 & 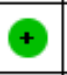 & $\odot$ & + \\
\hline Simon 2008 & $\odot$ & + & $?$ & $\odot$ & $\odot$ & $\odot$ & $\odot$ \\
\hline Singh 2009 & + & + & $\odot$ & $\odot$ & + & $\odot$ & $\odot$ \\
\hline Skouteris 2016 & + & + & + & $?$ & $\odot$ & $\odot$ & \\
\hline Slusser 2012 & + & + & + & $\odot$ & $?$ & $\odot$ & \\
\hline Smith 2014 & + & + & $\Theta$ & $\odot$ & $\odot$ & $\odot$ & $\oplus$ \\
\hline Spiegel 2006 & $?$ & $?$ & $?$ & $?$ & $?$ & $\odot$ & $?$ \\
\hline Stolley 1997 & $?$ & $?$ & $?$ & $\odot$ & $?$ & + & \\
\hline Story 2003 & + & $?$ & $?$ & 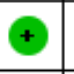 & $\odot$ & $\odot$ & \\
\hline Story 2012 & $?$ & $?$ & $?$ & + & $\odot$ & $\odot$ & $?$ \\
\hline Telford 2012 & $?$ & $?$ & $?$ & $?$ & + & $\odot$ & $?$ \\
\hline Thivel 2011 & $?$ & $?$ & $?$ & $?$ & $?$ & + & + \\
\hline Velez 2010 & $?$ & $?$ & $?$ & $\Theta$ & $?$ & + & \\
\hline Verbestel 2014 & $?$ & $\odot$ & - & $\odot$ & $?$ & $\odot$ & $\oplus$ \\
\hline
\end{tabular}


Figure 5. (Continued)

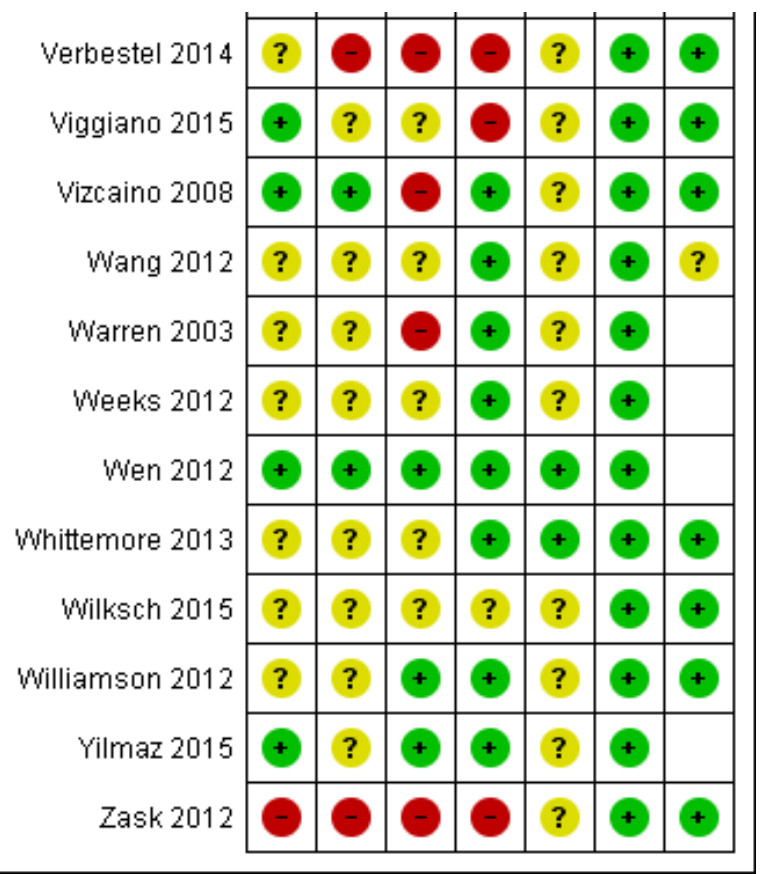

\section{Allocation}

In RCTs included in the meta-analyses, we rated relatively few RCTs as 'high' risk of bias. Often, study reports did not clearly specify sequence generation and allocation concealment; half of the RCTs (55/110) were at 'low' risk of bias for generation of random sequence, with nearly half, $47 \%$ (52/110) without enough information to allow us to make a judgement. There were similar proportions for allocation concealment, 38\% (42/110) at 'low' risk of bias and $52 \%(57 / 110)$ at 'unclear'. For those RCTs that were not included in the meta-analyses, 10\% (4/42) were at high risk of bias for random sequence generation and the proportion with insufficient information on which to make a judgement $32 / 42$ (76\%) was much higher than for RCTs that were included in the metaanalysis; 74\% (31/42) of RCTs did not report enough information for allocation concealment.

\section{Blinding}

We rated a quarter (27/110) of RCTs included in the meta-analyses as 'high' risk of bias. With $44 \%$ providing insufficient information to judge bias and $30 \%(34 / 110)$ rated as 'low' risk of bias. For RCTs not included in the meta-analyses the proportions were similar, with a higher proportion reporting insufficient information to judge bias $(50 \%(21 / 42)$. It is feasible to obscure how interventions were allocated from the outcome assessors; however it is not possible to conceal allocation of interventions from the participants themselves. Especially in RCTs with individual randomisation. Therefore, a 'high' risk of bias judgement is to be expected for this item.

\section{Incomplete outcome data}

We rated $26 \%(29 / 110)$ of RCTs, included in the meta-analyses, as high for attrition bias. In most cases this was because more than $30 \%$ of participants were lost to follow-up and analyses did not account for attrition. Other reasons included: unbalanced completion rates in study groups; not providing reasons for missing data; not providing missing data by study group; and differences in characteristics related to study outcomes between completers and non-completers. We rated $62 \%(68 / 110)$ of RCTs as low risk of bias from missing data. We based our decisions on the provision of an adequate description of participant flow through the study and with missing outcome data relatively balanced between groups and judged to be unlikely to be related to the outcomes of interest.

We assessed similar proportions of RCTs, not included in the metaanalyses, as high risk of bias $(29 \%, 12 / 42)$ but there were fewer at low risk of bias $(52 \%, 22 / 42)$.

We rated relatively few RCTs, included in the meta-analysis, $12 \%$ $(13 / 110)$ as unclear for attrition bias, mainly because they did not adequately report participant flow. Of RCTs, not included in the meta-analyses, we assessed a greater proportion (19\%; 8/43) as unclear risk of bias.

\section{Selective reporting}

We rated $51 \%(56 / 110)$ of RCTs included in the meta-analyses as 'low' risk of bias whereas a much lower proportion of $21 \%$ (9/42) were 'low' risk of bias for RCTs that were not included in the meta-analyses. Only four RCTs included in the meta-analyses were recorded as high risk of bias whereas $14 \%(6 / 42)$ of those not included in the meta-analyses were high risk of bias. The reasons that studies, in the meta-analyses, acquired a grade of 'High' risk of bias included: failure to report outcomes of BMI or zBMI despite these outcomes being listed, a priori, in trial registers/ protocols or reporting of BMI or ZBMI when these outcomes had not been prespecified in trials registers or protocols. There were many RCTs, $64 \%$ of those in the meta-analyses and $45 \%$ for those not included in the meta-analyses, that had no prespecified record, either protocol or trial registration report, of the planned clinical trial. 


\section{Other potential sources of bias}

We categorised 'other' bias as risk of study contamination and the majority, 90\% (99/110) of RCTs in the meta-analyses, were low risk of bias. We rated three RCTs (3\%) as 'high' risk and eight (7\%) as 'unclear' risk. The proportions of RCTs assessed as low (93\%), high $(0 \%)$ or unclear $(7 \%)$ risk were very similar for RCTs without data in the meta-analyses, and those judged to be at 'high' risk were at risk of contamination.

\section{Timing of recruitment of clusters}

This assessment related only to cluster-RCTs. We judged RCTs as high risk of bias if they had recruited the clusters after randomisation. The majority of RCTs, both those included in the meta-analyses (69\%) and those not included in the analyses (74\%), were at low risk of bias. Approximately a third of RCTs did not have enough information to allow us to make a judgement: $26 \%(21 / 81)$ of RCTs in the meta-analyses, and $26 \%$ (7/27) of RCTs not in the meta-analyses. Six per cent (5/81) of RCTs in the meta-analyses had recruited participants after randomisation and were at 'high' risk of bias. No RCTs not in the meta-analyses had recruited participants after randomisation.

\section{Publication bias, or small study effect}

None of the meta-analyses with more than 10 studies had evidence of funnel plot asymmetry as tested using the Egger test (Egger 1997a). P values ranged from 0.304 to 0.958 . This indicates we could find no evidence of small study effects or publication bias. See Figure 6.

Figure 6. Funnel plots of all comparisons with more than $\mathbf{1 0}$ studies. A Funnel plot of comparison 3. Diet and physical activity interventions versus control in children aged 0-5 years. Outcome: zBMI. No evidence of asymmetry (Egger test $P=\mathbf{0 . 9 5 8}$ ). B Funnel plot of comparison 3. Diet and physical activity interventions versus control in children aged 0-5 years. Outcome: BMI. No evidence of asymmetry (Egger test $P=0.529)$. C Funnel plot of comparison 5. Physical activity interventions versus control in children aged 6-12. Outcome: BMI. No evidence of asymmetry (Egger test $P=0.763$ ). D Funnel plot of comparison 6. Physical activity interventions versus control in children aged 6-12. Outcome: zBMI. No evidence of asymmetry (Egger test $P=0.304$ ). E Funnel plot of comparison 
6. Physical activity interventions versus control in children aged 6-12. Outcome: BMI. No evidence of asymmetry (Egger test $\mathrm{P}=\mathbf{0 . 7 6 8}$ ).

A

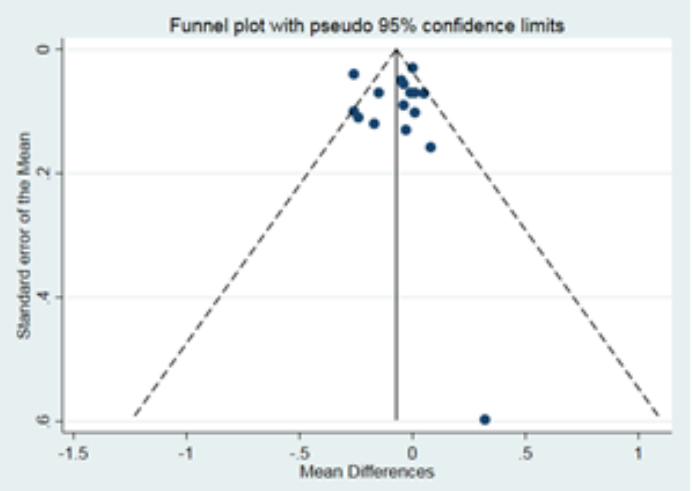

$\mathrm{C}$

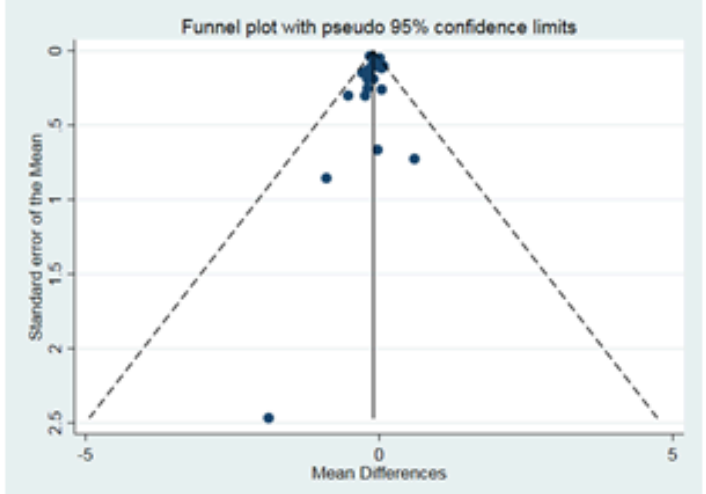

$E$

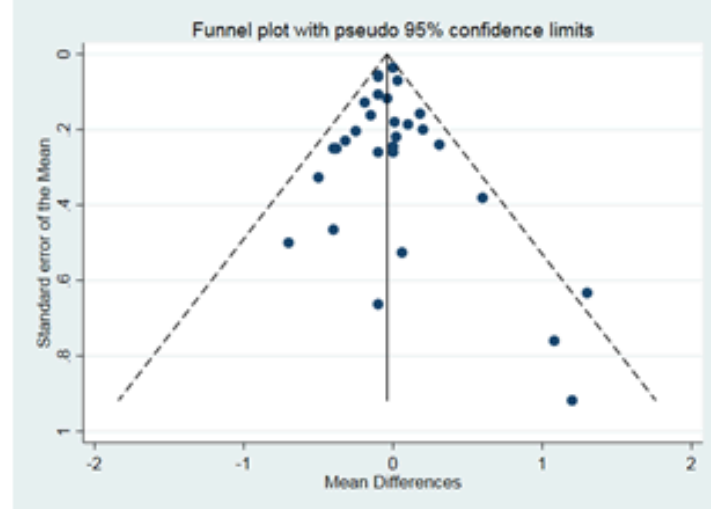

B

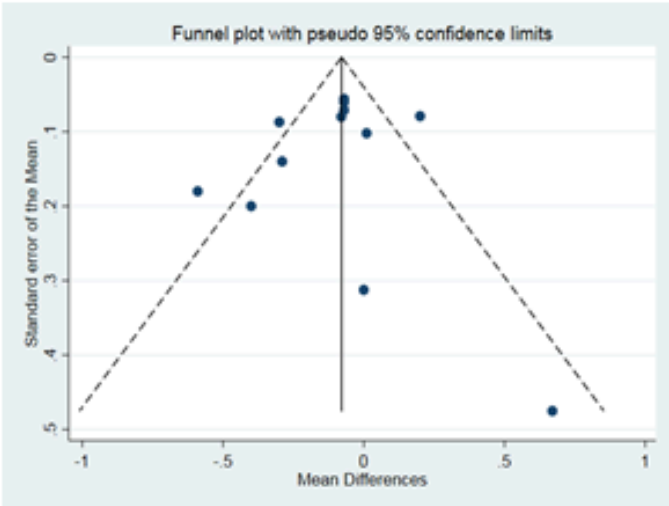

D

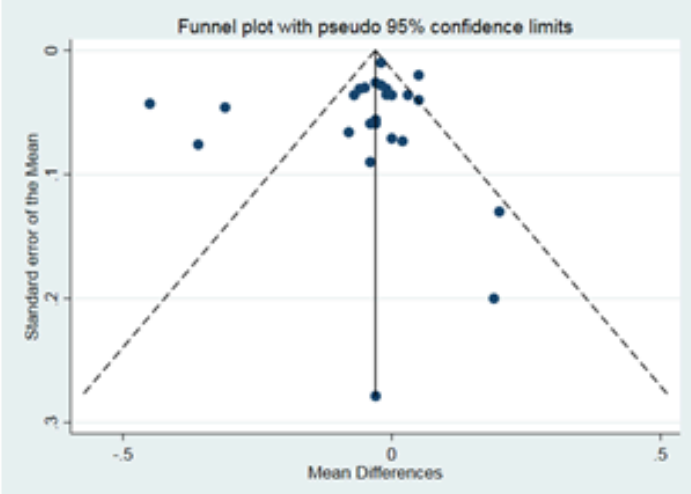


Figure 6. (Continued)

\section{Effects of interventions}

See: Summary of findings for the main comparison Dietary interventions compared to control for preventing obesity in children aged 0 to 5 years; Summary of findings 2 Physical activity interventions compared to control for preventing obesity in children aged 0 to 5 years; Summary of findings 3 Diet and physical activity interventions combined compared to control for preventing obesity in children aged 0 to 5 years; Summary of findings 4 Adverse event outcomes for dietary combined with physical activity interventions compared to control in children aged 0 to 5 years; Summary of findings 5 Dietary interventions compared to control for preventing obesity in children aged 6 to 12 years; Summary of findings 6 Physical activity interventions compared to control for preventing obesity in children aged 6 to 12 years; Summary of findings 7 Adverse event outcomes for physical activity interventions compared to no intervention in children aged 6 to 12 years; Summary of findings 8 Diet and physical activity interventions combined compared to control for preventing obesity in children aged 6 to 12 years; Summary of findings 9 Adverse event outcomes for dietary combined with physical activity interventions compared to no intervention or usual care for preventing obesity in children aged 6 to 12 years; Summary of findings $\mathbf{1 0}$ Diet interventions compared to control for preventing obesity in children aged 13 to 18 years; Summary of findings 11 Physical activity interventions compared to control for preventing obesity in children aged 13 to 18 years; Summary of findings 12 Adverse events outcomes for physical activity interventions compared to control in children aged 13 to 18 years; Summary of findings 13 Diet and physical activity interventions combined compared to control for preventing obesity in children aged 13 to 18 years; Summary of findings 14 Adverse event outcomes for dietary combined with physical activity interventions compared to control for preventing obesity in children aged 13 to 18 years; Summary of findings 15 Dietary interventions compared to physical activity interventions for preventing obesity in children aged 6 to 12 years; Summary of findings 16 Diet and physical activity interventions combined compared to physical activity interventions alone for preventing obesity in children aged 6 to 12 years; Summary of findings $\mathbf{1 7}$ Dietary interventions alone compared to diet and physical activity interventions combined for preventing obesity in children aged 6 to 12 years

\section{Summary of outcomes}

\section{ZBMI}

Fifty-eight RCTs reported zBMI, 20 in the age group 0 to 5, 31 in the age group 6 to 12, and seven in the age group 13 to 18 years. We have given a full breakdown of RCTs reporting zBMI grouped by intervention type, and age group in Table 9 and Table 10.

\section{BMI}

Seventy-two RCTs reported BMI, 16 in the age group 0 to $5 ; 43$ in the age group 6 to 12; and 13 in the age group 13 to 18 years. We have given a full breakdown of RCTs reporting BMI grouped by intervention type and age group in Table 9 and Table 11.

\section{Adverse events}

Sixteen RCTs reported adverse events, four in the 0 to 5 age group (Table 6), eight in the 6 to 12 age group (Table 7), and four in the 13 to 18 age group (Table 8 ).

\section{Comparison 1: age 0 to 5 years, dietary interventions versus control}

ZBMI

Moderate-certainty evidence from one RCT (520 participants) indicated that dietary interventions versus control for preventing obesity did not reduce zBMI scores in children aged 0 to 5 years. The mean difference in zBMI was -0.14 (95\% confidence interval (CI) -0.32 to 0.04$)$. See Analysis 1.1 and Summary of findings for the main comparison.

\section{BMI}

No studies reported BMI.

\section{Adverse events}

No studies reported adverse events.

\section{Comparison 2: age 0 to 5 years, physical activity interventions versus control}

\section{$z B M I$}

High-certainty evidence from four RCTs (1053 participants) indicated that physical activity interventions versus control for preventing obesity did not reduce $\mathrm{zBMI}$ in children aged 0 to 5 years. The mean difference in zBMI was 0.01 ( $95 \% \mathrm{Cl}-0.10$ to 0.13$)$. See Analysis 2.1 and Summary of findings 2 . We found no differences in subgroup by setting.

\section{BMI}

High-certainty evidence from five RCTs (2233 participants) indicated that physical activity interventions versus control for preventing obesity did not reduce BMI in children aged 0 to 5 years. The mean difference in BMI was $-0.22 \mathrm{~kg} / \mathrm{m}^{2}\left(95 \% \mathrm{Cl}-0.44 \mathrm{~kg} / \mathrm{m}^{2}\right.$ to $\left.0.01 \mathrm{~kg} / \mathrm{m}^{2}\right)$. See Analysis 2.2 and Summary of findings 2. We found no differences in subgroup by setting. 


\section{Adverse events}

No studies reported adverse events.

\section{Comparison 3: age 0 to 5 years, diet and physical activity interventions versus control}

\section{$z B M I$}

Moderate-certainty evidence from 16 RCTs (6261 participants) indicated that combined diet and physical activity interventions versus control for preventing obesity lead to a small reduction of zBMI in children aged 0 to 5 years. The mean difference in zBMI was $-0.07(95 \% \mathrm{Cl}-0.14$ to -0.01$)$. See Analysis 3.1 and Summary of findings 3 . We found no differences in subgroup by setting or duration of intervention.

\section{BMI}

Moderate-certainty evidence from 11 RCTs (5536 participants) indicated that combined diet and physical activity interventions versus control for preventing obesity reduce BMI in children aged 0 to 5 years. The mean difference in BMI was $-0.11 \mathrm{~kg} / \mathrm{m}^{2}(95 \%$ $\mathrm{Cl}-0.21 \mathrm{~kg} / \mathrm{m}^{2}$ to $0.00 \mathrm{~kg} / \mathrm{m}^{2}$ ). See Analysis 3.3 and Summary of findings 3 . We found no differences in subgroup by duration of intervention.

Subgroup analyses of settings revealed that there were differences in effect of interventions based upon setting in which they were delivered $\left(\mathrm{Chi}^{2}=12.31, \mathrm{df}=2(\mathrm{P}=0.002), \mathrm{I}^{2}=83.8 \%\right)$. Evidence from two RCTs delivered at home (778 participants) indicated that diet and physical activity interventions reduced BMI (mean difference (MD) $-0.33 \mathrm{~kg} / \mathrm{m}^{2}, 95 \% \mathrm{Cl}-0.55 \mathrm{~kg} / \mathrm{m}^{2}$ to $-0.10 \mathrm{~kg} / \mathrm{m}^{2}$ ) and one RCT of 75 participants set in the wider community, found a large reduction in $\mathrm{BMI}\left(\mathrm{MD}-0.59 \mathrm{~kg} / \mathrm{m}^{2}, 95 \% \mathrm{Cl}-0.94 \mathrm{~kg} / \mathrm{m}^{2}\right.$ to $-0.24 \mathrm{~kg} /$ $\mathrm{m}^{2}$ ) but this RCT was at high risk of bias for blinding and with just 75 participants was also imprecise. Data from eight RCTs of diet and physical activity interventions delivered in a childcare or preschool setting showed no evidence of effect on BMI (MD $-0.05 \mathrm{~kg} / \mathrm{m}^{2}, 95 \%$ $\mathrm{Cl}-0.14 \mathrm{~kg} / \mathrm{m}^{2}$ to $0.05 \mathrm{~kg} / \mathrm{m}^{2}$ ). See Analysis 3.3 and Summary of findings 3 .

\section{Adverse events}

Four RCTs reported five types of adverse event; infection, injury, accident, sufficiency of weight gain in infants and a catch-all of 'adverse events'. See Table 6 and Summary of findings 4 . In assessing the safety of the 'Soothe/Sleep' and introduction of solids' interventions on weight status in terms of sufficiency of weight gain, Paul 2011 reported that they had detected no significant differences among treatment groups for insufficient weight gain. Fitzgibbon 2006 reported there were no adverse events during the study although they provided no data or information on what measures they used. Puder 2011 reported that there were no injuries or other adverse events during physical activity sessions in the intervention classes. Roth 2015 reported that the physical activity intervention did not lead to a significant difference between the intervention and control group in rates of accidents and infections.

None of the RCTs reported that the interventions led to more adverse events than the control. There is no evidence that diet and physical activity interventions adversely affect any of these outcomes. However, for the outcomes of insufficient weight gain and infections we have little certainty of the evidence because it is drawn from few participants, a single RCT or RCTs at high risk of bias.

\section{Comparison 4: age 6 to 12 years, dietary interventions versus control}

$z B M I$

High-certainty evidence from nine RCTs (7231 participants) indicated that dietary interventions versus control for preventing obesity do not affect zBMI in children aged 0 to 5 years (MD -0.03 , $95 \% \mathrm{Cl}-0.06$ to 0.01 ). See Analysis 4.1 and Summary of findings 5 . We found no differences in subgroup by setting.

\section{BMI}

High-certainty evidence from six RCTs (5061 participants) indicated that dietary interventions versus control for preventing obesity do not affect BMI in children aged 0 to 5 years (MD $-0.02 \mathrm{~kg} / \mathrm{m}^{2}, 95 \%$ $\mathrm{Cl}-0.11 \mathrm{~kg} / \mathrm{m}^{2}$ to $0.06 \mathrm{~kg} / \mathrm{m}^{2}$ ). See Analysis 4.2 and Summary of findings 5 . We found no differences in subgroup by setting.

\section{Adverse events}

No studies reported adverse events.

\section{Comparison 5: age 6 to 12 years, physical activity interventions versus control}

\section{$z B M I$}

Moderate-certainty evidence from eight RCTs (6841 participants) indicated that physical activity interventions versus control for preventing obesity do not affect $\mathrm{zBMI}$ in children aged 6 to 12 years (MD $-0.02,95 \% \mathrm{Cl}-0.06$ to 0.02 ). See Analysis 5.1 and Summary of findings 6 . We found no differences in subgroup by setting.

\section{BMI}

Moderate-certainty evidence from 14 RCTs (16,410 participants) indicated that physical activity interventions versus control for preventing obesity reduce BMI in children aged 6 to 12 years (MD $-0.10 \mathrm{~kg} / \mathrm{m}^{2}, 95 \% \mathrm{Cl}-0.14 \mathrm{~kg} / \mathrm{m}^{2}$ to $-0.05 \mathrm{~kg} / \mathrm{m}^{2}$ ). See Analysis 5.3 and Summary of findings 6 . We found no differences in subgroup by setting.

\section{Adverse events}

One RCT (Li 2010a), reported that children who received physical activity interventions versus control did not have any additional physical injuries compared to those who were assigned to the control group. However, we are a little uncertain of the evidence as it is drawn from a single RCT with one domain at high risk of bias. Three RCTs reported that their physical activity interventions did not cause underweight (high-certainty evidence). A culturally tailored after-school dance and screen-time-reduction intervention (Robinson 2010), for low-income, preadolescent African-American girls significantly reduced depressive symptoms, and there was no evidence for increased weight concerns or body dissatisfaction. However, we have little confidence in the evidence because it is drawn from few participants. See Table 7 and Summary of findings 7. 
Comparison 6: age 6 to 12 years, diet and physical activity interventions versus control

\section{ZBMI}

Low-certainty evidence from 20 RCTs (24,043 participants) indicated that combined diet and physical activity interventions versus control for preventing obesity reduce $\mathrm{zBMI}$ in children aged 6 to 12 years (MD $-0.05,95 \% \mathrm{Cl}-0.10$ to -0.01 ). See Analysis 6.1 and Summary of findings 8 . We found no differences in subgroup by setting.

\section{BMI}

Low-certainty evidence from 25 RCTs (19,498 participants) indicated that combined diet and physical activity interventions versus control for preventing obesity did not reduce $\mathrm{BMI}$ in children aged 6 to 12 years (MD $-0.05 \mathrm{~kg} / \mathrm{m}^{2}, 95 \% \mathrm{Cl}-0.11 \mathrm{~kg} / \mathrm{m}^{2}$ to 0.01 $\mathrm{kg} / \mathrm{m}^{2}$ ). See Analysis 6.3 , and Summary of findings 8 . We found no differences in subgroup by setting.

\section{Adverse events}

Five of the 52 studies targeting children aged 6 to 12 years assessed adverse or unintended consequences of the interventions. The studies used a variety of measures to assess adverse effects, including prevalence of underweight, unhealthy eating practices, teasing, stigmatisation, body image perceptions, satisfaction and self-worth. The majority of studies did not report any adverse outcomes. One study (Beech 2003), reported similar numbers of visits to a healthcare provider in the intervention and control groups, but this evidence is very uncertain as the study was small and the number of events low. One study (HEALTHY Study Gp 2010), reported similar numbers of adverse events related to collection of blood samples in the intervention and control groups. This evidence is uncertain as, although the study was large (4603 participants), there were few events.

Two studies reported that the proportion of children underweight was similar among children who received the intervention and those who had the control (Foster 2008; HEALTHY Study Gp 2010; moderate-certainty evidence). There is moderate-certainty evidence from a third study, Siegrist 2013, who measured waist circumference of children below the 10th centile for weight, and several underweight children in both intervention and control groups showed a decrease in waist circumference. There were no significant differences between the intervention and control groups however. This suggests that these reductions were not related to the intervention. The study authors reported that this finding may indicate that normal and underweight children are attempting to lose weight independent of and during participation in lifestylechange interventions and they found no evidence that this was affected by the intervention.

Two studies reported high-certainty evidence that concern about weight among the participants was similar between those children who received the intervention compared to those who did not (Beech 2003; Robinson 2010).

High-certainty evidence from three studies reported no differences between children in the intervention groups and those in the control groups in the measure of body satisfaction (Beech 2003; Foster 2008; Robinson 2010). One RCT (Beech 2003), reported that children who received physical activity interventions did not have any additional physical injuries compared to those who were assigned to the control group. However, we are uncertain of the evidence as it is drawn from a single RCT with only 60 participants. See Table 7 and Summary of findings 9.

\section{Comparison 7: age 13 to 18 years, dietary interventions versus control}

ZBMI

No studies reported zBMI.

BMI

Low-certainty evidence from two RCTs (294 participants) indicated that dietary interventions versus control for preventing obesity do not affect BMI in children aged 0 to 5 years (MD $-0.13 \mathrm{~kg} / \mathrm{m}^{2}, 95 \%$ $\mathrm{Cl}-0.50 \mathrm{~kg} / \mathrm{m}^{2}$ to $0.23 \mathrm{~kg} / \mathrm{m}^{2}$ ). See Analysis 7.1 and Summary of findings 10 . We found no differences in subgroup by setting.

\section{Adverse events}

No studies reported adverse events.

\section{Comparison 8: age 13 to 18 years, physical activity interventions versus control}

\section{$z B M I$}

Low-certainty evidence from one RCT (100 participants) set in school indicated that physical activity interventions versus control for preventing obesity reduce zBMI score in children aged 13 to 18 years (MD $-0.20,95 \% \mathrm{Cl}-0.30$ to -0.10 ). See Analysis 8.1. and Summary of findings 11 .

\section{BMI}

Very low-certainty evidence from four RCTs (720 participants) indicated that physical activity interventions versus control for preventing obesity reduce BMI in children aged 13 to 18 years (MD $-1.53 \mathrm{~kg} / \mathrm{m}^{2}, 95 \% \mathrm{Cl}-2.67 \mathrm{~kg} / \mathrm{m}^{2}$ to $-0.39 \mathrm{~kg} / \mathrm{m}^{2}$ ). See Analysis 8.3 and Summary of findings 11 . We found no differences in subgroup by setting.

\section{Adverse events}

Two RCTs (Neumark-Sztainer 2003; Neumark-Sztainer 2010), reported four types of adverse event: unhealthy weight control behaviour, body satisfaction, unhealthy weight gain, selfacceptance and binge eating (Table 8 Summary of findings 12). None reported that the interventions led to more adverse events than the control. However, for the outcome of body satisfaction we have little confidence in the evidence because it is drawn from one RCT of 190 participants (low-certainty evidence). One RCT (Neumark-Sztainer 2010), reported that unhealthy weight control behaviour in girls was improved as part of an evaluation of the impact of the New Moves school-based intervention aimed at preventing weight-related problems in adolescent girls. See Table 8 and Summary of findings 12 .

\section{Comparison 9: age 13 to 18 years, diet and physical activity interventions versus control}

\section{zBMI}

Low-certainty evidence from six RCTs (16,543 participants) indicated that combined dietary and physical activity interventions versus control for preventing obesity do not affect $\mathrm{zBMI}$ score in 
children aged 13 to 18 years (MD 0.01, 95\% Cl -0.05 to 0.07 ). See Analysis 9.1 and Summary of findings 13 . We found no differences in subgroup by setting.

\section{BMI}

Low-certainty evidence from eight RCTs (16,583 participants) indicated that combined dietary and physical activity interventions versus control for preventing obesity do not affect $\mathrm{BMI}$ in children aged 13 to 18 years (MD $-0.02 \mathrm{~kg} / \mathrm{m}^{2}, 95 \% \mathrm{Cl}-0.10 \mathrm{~kg} / \mathrm{m}^{2}$ to $0.05 \mathrm{~kg} /$ $\left.\mathrm{m}^{2}\right)$. See Analysis 9.3. and Summary of findings 14. All studies were in one setting, school.

\section{Adverse events}

Two RCTs (Melnyk 2013; Wilksch 2015), reported three types of adverse event: depression; anxiety; and clinical levels of shape and weight concern (Table 8; Summary of findings 14). None reported that the interventions led to more adverse events than the control. However, for the outcome of clinical levels of shape and weight concern we have little confidence in the evidence because it is drawn from one RCT of 282 participants (lowcertainty evidence). Wilksch 2015 reported on the efficacy of a five-week obesity-prevention programme (Life Smart) and two eating disorder-prevention programmes (Media Smart and HELPP) against each other and a no-intervention control condition. 'Media Smart' was the only programme to show benefit on disordered eating. Melnyk 2013 reported on the efficacy of a 15-week COPE (Creating Opportunities for Personal Empowerment) programme, versus an attention control programme (Healthy Teens), on the healthy lifestyle behaviours, psychosocial outcomes, social skills, and academic performance of a culturally diverse sample of high school adolescents. Teens in the COPE group with extremely elevated depression scores at pre-intervention had significantly lower depression scores than the Healthy Teens group $(P=0.02)$. See Table 8 and Summary of findings 14.

Comparison 10: age 0 to 5 years, dietary interventions versus physical activity interventions

\section{zBMI}

No studies reported zBMI.

\section{BMI}

No studies reported BMI.

Comparison 11: age 6 to 12 years, dietary interventions versus physical activity interventions

\section{ZBMI}

High-certainty evidence from one RCT (1205 participants) indicated that dietary interventions have a similar effect to physical activity interventions on zBMI in children aged 6 to 12 years (MD - $0.11,95 \%$ $\mathrm{Cl}-0.62$ to 0.4 ). See Analysis 10.1 and Summary of findings 15 .

\section{BMI}

High-certainty evidence from two RCTs (4917 participants) indicated that dietary interventions have a similar effect to physical activity interventions on $\mathrm{BMI}$ in children aged 6 to 12 years (MD $-0.03 \mathrm{~kg} / \mathrm{m}^{2}, 95 \% \mathrm{Cl}-0.25 \mathrm{~kg} / \mathrm{m}^{2}$ to $0.20 \mathrm{~kg} / \mathrm{m}^{2}$ ). See Analysis 10.2 and Summary of findings 15 .
Comparison 12: age 13 to 18 years, dietary interventions versus physical activity interventions

$z B M I$

No studies reported zBMI.

BMI

No studies reported BMI.

Comparison 13: age 0 to 5 years, diet and physical activity interventions combined versus physical activity interventions

zBMI

No studies reported zBMI.

BMI

No studies reported BMI.

Comparison 14: age 6 to 12 years, diet and physical activity interventions combined versus physical activity interventions

ZBMI

High-certainty evidence from one RCT (3946 participants) indicated that combined diet and physical activity interventions have a similar effect to physical activity interventions on zBMI in children aged 6 to 12 years (MD $-0.16,95 \% \mathrm{Cl}-0.57$ to 0.25 ). See Analysis 11.1 and Summary of findings 16.

\section{BMI}

High-certainty evidence from one RCT (3946 participants) indicated that combined diet and physical activity interventions have a similar effect to physical activity interventions on $\mathrm{BMI}$ in children aged 6 to 12 years (MD $-0.04 \mathrm{~kg} / \mathrm{m}^{2}, 95 \% \mathrm{Cl}-1.05 \mathrm{~kg} / \mathrm{m}^{2}$ to $0.97 \mathrm{~kg} /$ $\mathrm{m}^{2}$ ). See Analysis 11.2 and Summary of findings 16 .

Comparison 15: age 13 to 18 years, diet and physical activity interventions combined versus physical activity interventions

ZBMI

No studies reported zBMI.

\section{BMI}

No studies reported BMI.

Comparison 16: age 0 to 5 years, diet and physical activity interventions combined versus dietary interventions

$z B M I$

No studies reported zBMI.

\section{BMI}

No studies reported BMI.

Comparison 17: age 6 to 12 years, diet and physical activity interventions combined versus dietary interventions

$z B M I$

High-certainty evidence from one RCT (3971 participants) indicated that combined diet and physical interventions have a similar effect to dietary interventions on zBMI in children aged 6 to 12 years 
(MD $0.05,95 \% \mathrm{Cl}-0.38$ to 0.48 ). See Analysis 12.1 and Summary of findings 17.

\section{BMI}

High-certainty evidence from one RCT (3971 participants) indicated that combined diet and physical interventions have a similar effect to dietary interventions on BMI in children aged 6 to 12 years (MD $-0.28 \mathrm{~kg} / \mathrm{m}^{2}, 95 \% \mathrm{Cl}-1.67 \mathrm{~kg} / \mathrm{m}^{2}$ to $1.11 \mathrm{~kg} / \mathrm{m}^{2}$ ). See Analysis 12.2 and Summary of findings 17.

\section{Comparison 18: age 13 to 18 years, diet and physical activity interventions combined versus dietary interventions}

\section{$z B M I$}

No studies reported zBMI.

\section{BMI}

No studies reported BMI.

\section{Heterogeneity}

\section{Age 0 to 5 years}

There was only one study for the comparison of dietary interventions versus control, outcome BMI (Analysis 1.1), so an assessment of heterogeneity was not applicable. Heterogeneity measured using the $1^{2}$ statistic was $0 \%$ for the meta-analysis of zBMI of studies assessing physical activity versus control (Analysis 2.1). For Analysis 2.2, physical activity versus control, heterogeneity for the outcome BMI was $54 \%$, and it was not reduced by the introduction of subgroups of setting or duration. We found moderate heterogeneity for the comparison diet and physical activity versus control, outcome zBMI, of $\mathrm{I}^{2}=66 \%$ (Analysis 3.1), which was reduced in the subgroups 'childcare/preschool' to $16 \%$, and 'wider community' to $0 \%$, but increased to substantial levels in the subgroup 'home' to $I^{2}=86 \%$. This subgroup had just three studies with divergent intervention effects. We found moderate heterogeneity for the comparison diet and physical activity versus control for the outcome BMI (Analysis 3.3), of $12=69 \%$, which was reduced only for the subgroup 'home' to $0 \%$ but remained moderate for the subgroup 'childcare/preschool', which included most of the studies $(12=63 \%)$. Subgrouping by duration of intervention did not reduce heterogeneity for any comparison.

\section{Age 6 to 12 years}

We found no heterogeneity for the comparison of dietary interventions versus control, outcome BMI (Analysis 4.2), and moderate heterogeneity of $\mathrm{I}^{2}=42 \%$ for the outcome $\mathrm{zBMI}$ (Analysis 4.1). All the RCTs in these comparisons were 12 months or less in duration and subgroups were not applicable. For Analysis 5.1, physical activity versus control, outcome zBMI, heterogeneity was moderate $(12=33 \%)$ and it was not reduced by the introduction of subgroups of setting or duration. There was very low heterogeneity for Analysis 5.3, physical activity versus control, outcome BMI, of $\mathrm{I}^{2}$ $=5 \%$.

We found substantial heterogeneity for the comparison, diet and physical activity versus control, outcome zBMI (Analysis 6.1), of I2 $=87 \%$. The heterogeneity was reduced in subgroup 'school' Just exceeding moderate levels $1^{2}=77 \%$; however, for the subgroup 'wider community', heterogeneity increased to substantial levels, 12
$=94 \%$. There was very low heterogeneity for Analysis 6.3, diet and physical activity versus control, outcome BMI, of $\mathrm{I}^{2}=17 \%$.

\section{Age 13 to 18 years}

We found no heterogeneity for the comparison of dietary interventions versus control, outcome BMI $(12=0 \%)$ (Analysis 7.1). There was only one study for the comparison of physical activity interventions versus control, outcome BMI (Analysis 8.1), and an assessment of heterogeneity was not applicable. For Analysis 8.3, physical activity versus control, outcome BMI, there were only four studies and heterogeneity was substantial, $12=93 \%$. All four RCTs were in the 'school' setting subgroup with a duration of 12 months or less.

For Analysis 9.1, dietary and physical activity interventions combined versus control, outcome zBMI, there was substantial heterogeneity of the meta-analysis of six RCTs $1^{2}=92 \%$. All bar one of the studies was set in 'school', and subgrouping did not reduce heterogeneity measured by the $1^{2}$ statistic value. However, subgrouping by duration (Analysis 9.2) reduced heterogeneity, those with interventions of 12 months or less had an 12 of $60 \%$ and for studies with a duration of more than 12 months, $1^{2}=57 \%$.

For Analysis 9.3, dietary and physical activity interventions combined versus control, outcome BMI, there were only eight RCTs and heterogeneity was moderate at $\mathrm{I}^{2}=58 \%$. All were set in schools. Subgrouping by duration reduced heterogeneity in the studies that were less than 12 months $\left(I^{2}=18 \%\right)$ with studies of greater duration having higher heterogeneity, $(12=75 \%)$.

\section{Equity and disadvantage}

This review sought to identify studies that had reported on characteristics known to be important from an equity and disadvantage perspective. For this process, we utilised the PROGRESS (Place, Race, Occupation, Gender, Religion, Education, Socio-economic status (SES), Social status) framework (Ueffing 2009). Where reported, interventions did not appear to increase health inequalities. We recorded where outcomes were analysed by any of the eight PROGRESS categories. For gender (the $G$ in PROGRESS), 30 studies reported outcomes analysed by gender; seven studies in the 0 to 5 age group, 14 studies in the 6 to 12 age group, and nine studies in the 13 to 18 age group.

\section{Subgroup analyses by gender in children aged 0 to 5 years}

Seven of the 39 RCTs analysed the effects of the intervention by gender. One RCT indicated that their intervention had a greater effect in girls compared to boys for reducing BMI (Mo-suwan 1998), and another for reducing skinfold thickness (De Vries 2015). In contrast, one study reported that BMI reduction in the intervention group occurred only in the boys (Klein 2010). Four RCTs reported no difference in the effect of the intervention, compared to control, on adiposity measures between boys and girls (Crespo 2012; Keller 2009; Nemet 2011; Story 2012).

\section{Subgroup analyses by gender in children aged 6 to 12 years}

Four of the 85 RCTs analysed the effects of the intervention by gender. Some of those RCTs that did not were interventions that only targeted boys or girls. Of the RCTs that did not undertake analysis by gender, 9 RCTs reported post hoc, subgroup analyses on gender and measures of adiposity, and reported no effect of the 
intervention compared to control on: zBMI (Elder 2014; Herscovic 2013; Johnston 2013; Khan 2014); BMI (Elder 2014; Herscovici 2013; Johnston 2013; Llargues 2012; Martinez-Vizcaino 2014; Sevinc 2011); per cent body fat changes or weight gain in white girls only (Telford 2012).

Two RCTs indicated that, after the intervention, girls were less likely to be obese than boys (Cao 2015; Levy 2012), and three RCTs indicated that zBMI, BMI or per cent body fat were reduced, compared to control, in girls but not in boys (Grydeland 2014, Li 2010a; Williamson 2012). In contrast, two RCTs indicated that outcomes for boys were improved compared with those for girls for zBMI (Kain 2014), and per cent body fat (Williamson 2012). Martinez-Vizcaino 2014 analysed several secondary adiposityrelated outcomes and found that some improved more in girls (skinfold thickness, per cent body fat) while others improved more in boys (waist circumference).

\section{Subgroup analyses of gender in children age 13 to 18 years}

Nine of the 29 RCTs for children aged 13 to 18 analysed results by gender. Five RCTs found no effect of intervention compared to control, by gender, on zBMI or BMI (Ebbeling 2006; Patrick 2006; Viggiano 2015; Weeks 2012; Wilksch 2015). Four RCTs assessed the effect of intervention by gender on secondary measures such as per cent body fat and skinfold thickness, and reported some differences in these measures between genders but no differences on zBMI or BMI (Black 2010; El Ansarai 2010; Haerens 2006; Singh 2009).

\section{Subgroup analysis by socio-economic status, migrant status, ethnicity and rural/urban setting in children aged 0 to 5 years}

Five of the 39 RCTs reported on the effect of the intervention by socio-economic status (SES). Two RCTs reported that interventions had greater effects in children from families with better educational levels or SES (Puder 2011; Rush 2012), one study reported that the intervention reduced $\mathrm{BBMI}$ more in children from a lower SES background compared to those from high-SES (De Coen 2012). Two RCTs found no difference in the effect of the intervention by parental education level on zBMI (Campbell 2013), or BMI (Bonvin 2013).

Of the five RCTs that reported on the effect of interventions by SES, three of these studies also reported on other PROGRESS categories. Two RCTs reported the effect of the intervention by migrant status and found no difference on BMI (Bonvin 2013; Puder 2011). One study (Rush 2012), reported that children of Maori ethnicity had a slightly (but not significantly) greater increase in BMI and per cent body fat compared with children from European origin. This intervention also reported a more favourable, but not statistically significant, effect of the intervention in children attending rural schools compared with urban schools, and in children attending schools in less deprived areas compared with schools in areas of deprivation.

\section{Subgroup analysis by socio-economic status, migrant status, ethnicity and rural/urban setting in children aged 6 to 12 years}

Six of the 85 RCTs reported on the effect of the intervention by SES. Two RCTs reported no interaction between SES and BMI (De Heer 2011; Simon 2008). Two RCTs in high-income countries reported that higher parental SES related to more favourable outcomes: reduced child waist circumference, per cent body fat (Elder 2014), BMI and waist to hip ratio (Grydeland 2014). Two RCTs in upper-middle-income countries (Mexico and Turkey) reported that higher parental SES was related to less favourable outcomes; the probability of moving from overweight to obese (Levy 2012), and increase in BMI (Sevinc 2011).

Five of the 85 RCTs reported on the effect of the intervention by ethnicity. Two RCTs found no interaction of intervention effect with ethnicity (Johnston 2013; Rush 2012); two RCTs reported that the intervention was more effective for African American participants (Foster 2008; Gortmaker 1999a), and one RCT reported that the intervention worked better at preventing weight gain (zBMI) in white girls (Williamson 2012).

\section{Subgroup analysis by socio-economic status, migrant status, ethnicity and rural/urban setting in children aged 13 to 18 years}

Two of the 29 RCTs reported that they had conducted analyses to assess the effect of the intervention by ethnicity, and did not find any significant difference (Pate 2005; Singh 2009).

\section{DISCUSSION}

\section{Summary of main results}

This review includes 153 RCTs of programmes aimed at preventing obesity in children aged from 0 to 18 years. There were $39(25 \%)$ RCTs targeting children aged 0 to 5 years, 85 (56\%) RCTs targeted children aged 6 to 12 years, and 29 (19\%) RCTs targeted children aged 13 to 18 years. One study recruited children aged five years and 10 years. The duration of 116 interventions was 12 months or less, 25 interventions lasted between one and two years, and 12 interventions were implemented for more than two years. Ninety three $(61 \%)$ RCTs included a combination of diet and physical activity interventions. Thirty nine (21\%) RCTs compared physical activity with control and 21 (14\%) RCTs compared diet-only with control. The studies delivered the interventions mostly at school ( $n=91 ; 59 \%)$, in community settings $(n=24 ; 15 \%)$, at child-care centres or preschools $(n=22 ; 14 \%)$, and a minority at home $(n=11$; $7 \%)$ or health centres $(n=6 ; 4 \%)$. Twenty-two $(14 \%)$ RCTs included more than one setting. These interventions were all targeted at the individual or interpersonal level of the Socioecological Model (SEM) (Stokols 1992), or both. We looked at the change in the profile of settings for interventions to prevent childhood obesity before 2011 compared with 2011 to 2015 , given the call for more upstream interventions over the last 10 to 15 years. We identified only 11 studies that we categorised as being set in the wider environment (not in a childcare, school, home, or healthcare setting). Of note, we did not identify any RCTs that were conducted in a faith-based setting.

This systematic review of RCTs for preventing obesity in children found that there was some evidence that diet and physical activity interventions combined could reduce measures of adiposity in children aged 0 to 5 years. For children aged 6 to 12 years, physical activity interventions reduced measures of adiposity compared to control. A combination of diet and physical activity interventions might reduce adiposity, but we are very uncertain about this. For children aged 13 to 18 years, physical activity interventions might reduce adiposity, but we are very uncertain about this. The effects observed in this review should be viewed with some caution in light of the findings from a recent review by McCrabb 2019, who conducted a systematic review to assess the difference between the efficacy of obesity interventions when assessed in a RCT, 
compared with the effectiveness of that intervention when scaledup and implemented in a real world setting. Across all measures of weight status, the effects reported in scaled-up interventions were typically $75 \%$ or less of the effects reported in the efficacy trials (McCrabb 2019).

\section{Children aged 0 to 5 years}

This systematic review of RCTs for preventing obesity in children aged 0 to 5 years found evidence of which we can be moderately certain, that combination dietary and physical activity interventions compared to control reduce $\mathrm{zBMI}$ and $\mathrm{BMI}$ in children aged 0 to 5 years. However, the reduction is very small. Examination of the effects of dietary combined with physical activity interventions on BMI shows that the effect of interventions differed between settings, so that there appears to be no effect of combined diet and physical interventions on BMI set in childcare/ preschool ( $\mathrm{n}=8 \mathrm{RCTs}$ ) but interventions delivered at home or the wider community reduced BMI. However, when we removed one study reporting a very large reduction in BMI, the overall effect was reduced. There was moderate-certainty evidence that diet interventions alone compared to control, and high-certainty evidence that physical activity interventions alone compared to control, did not reduce either BMI or zBMI.

\section{Children aged 6 to 12 years}

Physical activity interventions compared to control reduced BMI in children aged 6 to 12 years, and we are moderately certain of this effect, however we found no reduction in zBMI. Dietary combined with physical activity interventions compared to control reduced zBMI in children aged 6 to 12 years (low-certainty evidence). We found evidence, in which we are very confident, that dietary interventions did not reduce either BMI or zBMI in children aged 6 to 12 years.

\section{Children aged 13 to 18 years}

We found that physical activity interventions delivered on their own, compared to control, might or might not reduce BMI (very low-certainty evidence), and might reduce zBMI (low-certainty evidence) in children aged 13 to 18 years. Dietary interventions alone and dietary interventions combined with physical activity interventions have no effect on either BMI or ZBMI, but we have limited confidence in this evidence.

There was considerable variability in RCTs as measured using the $\mathrm{I}^{2}$ statistic and many meta-analyses were characterised by moderate or low values for heterogeneity. Subgroup analysis by duration of intervention reduced heterogeneity in only one comparison: Combined dietary and physical activity versus control for children aged 13 to 18 years, where substantial heterogeneity was reduced to moderate for the outcome BMI. Examining heterogeneity using the subgroup setting did not consistently reduce heterogeneity, in some subgroups heterogeneity increased.

Characterising a clinically relevant effect size in adiposity for children is not straightforward. There are few relevant publications that discuss this, and most have been run in a population of children who are obese. In a sample of obese children (mean age 10.7 years, range 4 to 15 years; mean zBMI 2.5, range 2.0 to 4.0 ), weight loss was associated with an improvement in the atherogenic profile and in insulin resistance, but only if the zBMI decreased by at least 0.5 units over a one-year period (Reinehr 2004). In another sample of children with obesity, Ford 2010 used a reduction in zBMI of 0.25 . The WAVES obesity prevention study applied a reduction in $\mathrm{zBMI}$ of 0.25 with which to calculate power in order to detect any clinically significant differences in zBMI between intervention and control groups (Adab 2015b). Another obesity prevention study used a reduction of 0.125 in zBMI (Williamson 2008). Therefore, the reduction in zBMI observed in this review is approximately half that of the most conservative estimate. The clinical significance of this reduction on a population level (including children of all weights) is uncertain. It could correspond to a small but clinically important shift in population BMI if sustained over several years; however, most of the evidence relates to interventions of 12 months or less and only a minority of RCTs reported post-intervention follow-up, which makes it difficult for us to have confidence that the outcomes of often short-term interventions are sustained over the longer term. Because BMI of children will vary with their growth trajectory, we do not have an example of a clinically meaningful difference in BMI.

Only three RCTs, in children aged 6 to 12 years, compared one type of active intervention with another. We found no evidence that any of the three types of intervention (diet, physical activity or combined diet and physical activity) were more effective than each other. However, it is worth highlighting that descriptions of most interventions (where reported in enough detail) included some element of advice on diet or physical activity, regardless of whether the intervention was categorised as a diet or physical activity intervention.

There is huge variety in the types of approaches used in the interventions, even within the categories of 'diet' and 'physical activity' which limits our ability to compare interventions across RCTs. In addition, the components of interventions are usually evaluated as a whole, rather than in isolation. This makes it difficult to draw firm assumptions about the effectiveness of individual intervention components. It might be the case that it is the components of the interventions acting in synergy rather than individual components that lead to intervention success. What we can say (if we focus on beneficial effects that occur for both zBMI and $\mathrm{BMI}$ ), is that diet or physical activity interventions, or both, to prevent obesity, are effective in reducing zBMI and BMI in children aged up to 12 years. And for adolescents and young people aged 13 to 18 years, diet or physical activity interventions alone are not effective in reducing $\mathrm{zBMI}$ and $\mathrm{BMI}$.

Evidence from newly identified RCTs from low- and middle-income countries for this updated review is an important contribution, in terms of context and external validity, particularly for policy-makers in those countries. This updated review also confirms, importantly, that interventions to prevent childhood obesity do not appear to result in adverse effects or health inequalities, but we noted that the analysis of outcomes by PROGRESS factors (including SES) was rarely conducted and continues to be a stubborn problem. Those responsible for policy and practice need to know which interventions are not only feasible, effective, and affordable, but also address inequalities.

Only fifteen studies (9.8\%) reported costs, and just one study reported a full economic evaluation. Most studies with costs were published after 2011 


\section{Overall completeness and applicability of evidence}

This update included 13 studies from upper-middle-income countries (Andrade 2014; Crespo 2012; Cunha 2013; Farias 2015; Habib-Mourad 2014; Lana 2014; Levy 2012; Macias-Cervantes 2009; Mo-suwan 1998; Safdie 2013; Sevinc 2011; Sichieri 2009; Yilmaz 2015), and one from a lower-middle-income country (El Ansarai 2010). Information from these studies makes an important contribution, in terms of context and external validity, to the existing evidence base for policy-makers.

The type and intensity of the interventions varied considerably, and it is perhaps too simplified to categorise interventions by type 'diet' or 'physical activity' or a combination of both. For example, within the category 'physical activity' interventions, the intensity of the activity could vary considerably, from education about the value of physical activity to daily physical activity sessions of specific intensity. Physical activity interventions could also include reducing sedentary behaviour, which could be 'screen time'. Diet interventions could focus on water or sugar-sweetened beverages. This update includes interventions delivered online, or via mobile/text, and 'exergaming'; some interventions include other lifestyle components that are known determinants of energy-balance-related behaviours, such as routines for sleep and mealtimes, parenting styles and feeding behaviours. We suggest future categorisations need to be more sophisticated and take into account factors that might influence the ability of participants to engage with interventions.

Most interventions reviewed for this update focused on the individual (personal) level of the SEM (Stokols 1992), rather than upstream (environment, policy) levels, because of the nature of our inclusion criteria (RCTs). We looked at the change in the profile of settings for interventions to prevent childhood obesity before 2011 compared with 2011 to 2015 , given the call for more upstream interventions over the last 10 to 15 years. We identified only 11 studies that we categorised as being set in the wider environment (not in a childcare, school, home, or healthcare setting). Of note, we did not identify any RCTs that were conducted in a faithbased setting. Given the importance placed on health and wellbeing within many faiths, particularly for Muslims, we noted that none of the interventions we reviewed were based in a religious setting. A recent scoping review exposes the extent to which health promotion, including interventions to prevent obesity in children, occurs in Islamic religious settings (Rai 2019). Overall, we did not see any clear trend for a shift towards more upstream interventions between these two time periods. We recommend the findings from high-quality reviews of community-based and policy interventions to tackle childhood obesity (Wolfenden 2016a), alongside those from this review.

The methods of implementation are less varied, with the interventions delivered by staff, teachers, academics, investigators, or via electronic media, or a combination of these methods. To provide useful evidence to decision makers, and those wishing to replicate effective interventions, we have attempted to provide a synthesis of a variety of implementation factors reported in the studies. We believe this information is required to move beyond simply the question of what works in obesity prevention, to the other important questions of how it worked, will it work in another context or under different conditions, and is it feasible or appropriate for others to implement.
Assessment of publication biases and small-study effects using the funnel plots revealed no apparent funnel plot asymmetry that might indicate a sample of studies free from publication bias. However, we know that $28 \%$ of studies in this review do not contribute data to any meta-analysis. In addition, update searches of this review have identified potentially many more RCTs with data to add (Studies awaiting classification).

\section{Quality of the evidence}

We did not include data from $43(28 \%)$ included studies in any meta-analyses due to inadequate reporting of data summarising the effects of interventions. We were unable to make a judgement about risk of bias for 379 of 1021 (37\%) 'Risk of bias' items assessed in RCTs. For studies in the meta-analysis we were unable to make a judgement about risk of bias for 250 of 742 (33\%) 'Risk of bias' items. This figure is higher, by nearly half, in those studies that did not contribute data to the meta-analyses (129 of 279, $46 \%$ ). Approximately half of judgements (range $45 \%$ to $52 \%$ ) for random sequence generation, allocation concealment, blinding and selective outcome reporting were unclear for RCTs included in the meta-analysis. For RCTs that did not contribute to the metaanalysis the number of unclear items for these domains was much higher (range $50 \%$ to $76 \%$ ). We are aware that a judgement of a 'Risk of bias' item of 'unclear' could indicate either no bias at all, or high risk of bias. Certainty of evidence of effects (using GRADE) was downgraded to 'moderate' or 'low', depending on the level of heterogeneity, and the effect of removing studies rated at 'high' risk of bias, from the analysis. Heterogeneity was not adequately explained by subgroup analyses.

\section{Potential biases in the review process}

We made several changes to the planned methods as set out in the protocol. This was partly because the protocol methods are now very dated. For example, published updates of the Cochrane methods for assessment of risk of bias have been revised twice since this review was first published (Higgins 2011c; Higgins 2016). All changes are set out in the section Differences between protocol and review. We made other changes because the rate of publication of new, relevant, studies on this topic appears to be increasing exponentially. This has outstripped the resources we had in which to complete the update. We restricted analysis of RCTs to the outcomes zBMI and BMI. We are aware of the issue of outcome reporting bias (Dwan 2010; Kirkham 2010). Because we are looking at healthy populations of children, and our interventions of interest could be aimed at healthcare issues other than preventing obesity, many RCTs might report a wide variety of outcomes not relevant to this review. This coupled with the exponential increase in research in this area (Figure 2), means that it was not feasible to include all RCTs that might potentially have reported all adiposity outcomes. In addition it is important to not include outcomes that might overwhelm readers or are trivial to decision makers, and this review already has 32 meta-analyses (McKenzie 2016). Approaches to systematic reviews of public health prevention topics have included restriction of selection of studies or analyses by outcome for these reasons (McKenzie 2016; Verbeek 2017). In future this Cochrane Review will be split into smaller reviews each focusing on specific age groups/ development stages of children. In these reviews we will reassess the review question, inclusion criteria, objectives, methods and outcomes. 


\section{Agreements and disagreements with other studies or reviews}

Other comprehensive reviews on this topic have found similar results, in that there is a modest effect or no effect of interventions, that target individual change, to prevent obesity in children. Of course, one can always find the rare study that shows that an intervention is effective, but the evidence base taken together suggests that the effect of these interventions is, at best, modest. The WHO Commission on Ending Childhood obesity (WHO 2016), suggests that part of the failure of interventions that target individual behaviour change, such as those included in this Cochrane Review, is due to the fact that they target individual behaviour change. The WHO Commission suggests that upstream interventions may be particularly important, and more effort is required in this area. Example interventions for adolescents, including tackling the marketing of unhealthy foods such as sugarsweetened beverages, and the obesogenic environment such as take-away food outlets. For preschool children, providing guidance and training to caregivers working in child-care settings and institutions on diet, physical activity, and sleep may be particularly important. It is now acknowledged that tackling obesity requires a systems approach, and policy initiatives across government departments should be joined up (Rutter 2016; Rutter 2017). Incorporating evidence from interventions at a policy level into a traditional Cochrane Review of RCTs is challenging, and the research community need to help and support policy-makers and stakeholders in bringing the totality of the evidence base together in a balanced and accessible format.

\section{AUTHORS' CONCLUSIONS}

\section{Implications for practice}

This review update provides policy-makers with a more robust evidence base because it is restricted to randomised controlled trials (RCTs), and it includes three times as many studies as the 2011 version (Waters 2011). The body of evidence in this review demonstrates that a range of diet combined with physical activity interventions can have a modest beneficial effect on obesity in children aged 0 to 5 years. The body of evidence in this review also demonstrates that a range of physical activity interventions can have a modest beneficial effect on obesity in children aged 6 to 18 years. The clinical significance, at a population level, of these small, statistically significant benefits over the short term is difficult to assess and, at best, minor. However, we know that the diet and physical activity behaviours that are adopted in childhood track throughout life. The potential cumulative effect of small but sustainable changes towards a healthier diet and a more physically active lifestyle could, at least in theory, reap long-term benefits for the promotion of healthy weight for individuals, communities and populations. It is important to note that a healthy diet and a physically active lifestyle have many health benefits beyond the promotion of a healthy weight.

A very important finding from this update is that interventions to prevent childhood obesity do not appear to cause any harms or adverse events, including eating disorders or weight concern. Also, there is no evidence that interventions to prevent childhood obesity increase inequalities. Only a few studies assessed the costs and cost effectiveness of interventions included in this review.
Evidence from newly identified studies from middle-income countries is an important contribution to this update, in terms of context and external validity, particularly for policy-makers in those countries. We found some evidence that cultural factors that impact on implementation may vary between countries.

\section{Implications for policy}

The interventions included in this update mainly focused on changing individual (personal) behaviours and were conducted in childcare centres, schools, homes and healthcare centres. About $15 \%$ of the interventions were conducted in the wider community, mainly local public community or recreation centres. If we are serious about tackling childhood obesity, this will require the implementation of these wider community-level interventions, together with upstream environmental and policy interventions. Taking a systems approach to tackling childhood obesity does not mean that we only focus on upstream or downstream interventions, but that we intervene at parts of the system where we believe will have the greatest impact. Policy makers also need to keep a watchful eye on progress of interventions over time, because systems have a habit of successfully adapting to such challenges.

\section{Implications for research}

This review includes potentially relevant RCT evidence that is not yet synthesised into the review. The rationale for this was that the evidence on this topic is accruing at the rate of 2000 to 4000 records per year, or approximately 200 potentially relevant, full-text papers to assess per year, which has important resource implications in terms of review preparation. Added to this we feel the current scope and design of this review is too broad to identify subtle differences in what works for whom in which setting. By publishing the synthesis of the 2015 search we present the most up-to-date, synthesised evidence. We will now divide this review into three smaller reviews by age group of children/young people ( 0 to 5,6 to 12 and 13 to 18 years). We will draft new protocols for these reviews, in which we can assess and revise all methods. For example, assessment of risk of bias going forward could use the new Cochrane 'Risk of bias' tool (ROB2), which is domainbased and is focused on the bias relating to specific extracted outcome data. This new tool uses signalling questions and helps review authors come to more definitive decisions about the bias. The search might be investigated to ensure that all potentially relevant studies are captured, and it might be possible to reduce sensitivity to avoid identifying literature of no relevance to the review. Future reviews on this topic require a more nuanced categorisation of interventions, setting and participant types. We suggest that further categorisation of diet and physical activity interventions by type (including dose) may help to identify more effective intervention components.

We do not anticipate the effect sizes we found in this review for the 6 to 12-year-old age group to change significantly with the addition of more interventions that target individual-level energybalance-related behaviours. However, we do recommend that further research in the early years and adolescence is conducted, and that research should include a wider range of community settings (including faith-based settings).

We suggest that interventions and strategies to prevent obesity in children should include follow-up over several years, and we understand that funding issues for such follow-up work can be 
problematic. We suggest that research on long-term follow-up of existing studies that have been completed, would provide important information on the sustainability of behaviour change and impact on weight. We understand the barriers to conducting this type of work, such as ethical approval and data protection issues. We also understand the perceived higher prestige attached to primary research compared with secondary or follow-up research. We urge funding bodies and journal editors to place a higher value on this type of research activity.

We also suggest that a better understanding of process and implementation, using evaluation methods by which one can better compare the results of one study with the next (and summarise the information for reviews such as this), would be extremely useful. This type of activity is critical for the successful translation of interventions from one context to another, and across different countries.

We also urge researchers to not only collect information at baseline on gender and other PROGRESS (Place, Race, Occupation, Gender, Religion, Education, Socio-economic status (SES), Social status) factors, including SES, but also to analyse the effect of the intervention by these factors. We understand the reluctance of researchers to perform multiple, post-hoc analyses of this type however these are necessary if we are to provide confidence for practice and policy that the interventions we deem effective do not increase inequalities.

We urge researchers and funding bodies in all countries to support research on childhood obesity in low- and middleincome countries, and better understand the experiences of nutrition transition and rapid weight gain. In the context of some countries, this research should aim to address the double burden of malnutrition. We applaud the work of the Global Challenge Research Fund (GCRF), UK, and similar funding streams.

Finally, we support the research recommendations set out by the WHO Commission on Ending Childhood Obesity (WHO 2017).

\section{ACKNOWLEDGEMENTS}

This work is dedicated to Elizabeth Waters, who sadly died in 2015. Liz was instrumental in creating the Cochrane Public Health Group. She and Carolyn Summerbell co-led this Cochrane systematic review, Interventions for Preventing Obesity in Children, from its inception to 2015.

The review authors would like to thank: All organisations who helped to fund this review (see Sources of support); the Review Advisory Group for their guidance: Colin Bell (Associate Professor, School of Medicine, Deakin University, Australia) and Lisa Gibbs (Associate Professor, Melbourne School of Population and Global Health, University of Melbourne, Australia); Tim Armstrong and Temo Waqanivalu from the World Health Organization for their support and peer review; Liz Bickerdike and Toby Lasserson and other team members from the Cochrane Editorial Unit, Cochrane Central Executive, for help with data checking and providing detailed constructive feedback; Rebecca Armstrong (previously the joint Co-ordinating Editor), Daniel Francis (Editor), Luke Wolfenden (Coordinating Editor) and Jodie Doyle (Managing Editor) of Cochrane Public Health; Anonymous copy editor from The Cochrane copy edit team for astonishing, and much valued, attention to detail; Julian Higgins (CLAHRC West) and Jelena Savovic (CLAHRC West) for expertise and wisdom; Lauren Scott (CLAHRC West) for advice on analysis of cluster-RCTs; James Nobles for advice on implementation; Geraldine Cutler, Anna Ferguson and Zoe Trinder-Widess (Patient and Public Involvement Panel CLAHRC West) for shaping of the plain language summary and Alison Richards (CLAHRC West) for some of the literature searches.

The views expressed in this article are those of the author(s) and not necessarily those of the NHS, the NIHR, or the Department of Health and Social Care (all UK), or the Cochrane Collaboration or World Health Organization. 


\section{R E F E R E N C E S}

\section{References to studies included in this review}

Alkon 2014 \{published data only\}

Alkon A, Crowley AA, Benjamin Neelon SE, Hill S, Pan Y, Nguyen V, et al. Nutrition and physical activity randomized control trial in child care centers improves knowledge, policies, and children's body mass index. BMC Public Health 2014;14:215. [DOI: doi:10.1186/1471-2458-14-215]

\section{Amaro 2006 \{published data only\}}

Amaro S, Viggiano A, Di Costanzo A, Madeo I, Viggiano A, Baccari ME, et al. Kalèdo, a new educational board-game, gives nutritional rudiments and encourages healthy eating in children: a pilot cluster randomized trial. European Journal of Pediatrics 2006;165(9):630-5.

\section{Andrade 2014 \{published data only\}}

Andrade S, Lachat C, Ochoa-Aviles A, Verstraeten R, Huybregts L, Roberfroid D, et al. A school-based intervention improves physical fitness in Ecuadorian adolescents: a clusterrandomized controlled trial. International Journal of Behavioral Nutrition and Physical Activity 2014;11:153.

\section{Annesi 2013 \{published data only\}}

* Annesi JJ, Smith AE, Tennant GA. Effects of a cognitivebehaviorally based physical activity treatment for 4- and 5-yearold children attending US preschools. International Journal of Behavioral Medicine 2013;20(4):562-6.

Annesi JJ, Smith AE, Tennant GA. Reducing high BMI in African American preschoolers: effects of a behavior-based physical activity intervention on caloric expenditure. Southern Medical Journal 2013;106(8):456-9.

\section{Baranowski 2003 \{published data only\}}

Baranowski T, Baranowski JC, Cullen KW, Thompson DI, Nicklas T, Zakeri IE, et al. The fun, food, and fitness project (FFFP): the Baylor GEMS pilot study. Ethnicity and Disease 2003;13 Suppl 1:S30-9.

\section{Baranowski 2011 \{published data only\}}

Baranowski T, Baranowski J, Thompson D, Buday R, Jago R, Griffith MJ, et al. Video game play, child diet, and physical activity behavior change: a randomized clinical trial. American Journal of Preventive Medicine 2011;40(1):33-8.

\section{Barkin 2012 \{published data only\}}

Barkin SL, Gesell SB, Po'e EK, Escarfuller J, Tempesti T. Culturally tailored, family-centered, behavioral obesity intervention for Latino-American preschool-aged children. Pediatrics 2012;130(3):445-56.

\section{Beech 2003 \{published data only\}}

Beech BM, Klesges RC, Kumanyika SK, Murray DM, Klesges L, McClanahan B, et al. Child- and parent-targeted interventions: the Memphis GEMS pilot study. Ethnicity and Disease 2003;13 Suppl 1:S40-53.
Bellows 2013a \{published data only\}

Bellows LL, Davies PL, Anderson J, Kennedy C. Effectiveness of a physical activity intervention for Head Start preschoolers: a randomized intervention study. American Journal of Occupational Therapy 2013;67(1):28-36.

Birken 2012 \{published data only\}

Birken CS. Office-based randomized controlled trial to reduce screen time in preschool children. Pediatrics 2012;130(6):1110-5.

\section{Black 2010 \{published data only\}}

Black MM, Hager ER, Le K, Anliker J, Arteaga SS, Diclemente C, et al. Challenge! Health promotion/obesity prevention mentorship model among urban, black adolescents. Pediatrics 2010;126(2):280-8.

\section{Bohnert 2013 \{published data only\}}

Bohnert AM, Ward AK. Making a difference: evaluating the Girls in the Game (GIG) after-school program. Journal of Early Adolescence 2013;33(1):5-16.

Bonis 2014 \{published data only\} Bonis M, Loftin M, Ward D, Tseng TS, Clesi A, Sothern M. Improving physical activity in daycare interventions. Childhood Obesity 2014;10(4):334-41.

\section{Bonsergent 2013 \{published data only\}}

Bonsergent E, Agrinier N, Thilly N, Tessier S, Legrand K, Lecomte E, et al. PRALIMAP Trial Group. Overweight and obesity prevention for adolescents: a cluster randomized controlled trial in a school setting. American Journal of Preventive Medicine 2013;44(1):30-9.

\section{Bonuck 2014 \{published data only\}}

Bonuck K, Avraham SB, Lo Y, Kahn R, Hyden C. Bottle-weaning intervention and toddler overweight. Journal of Pediatrics 2014;164(2):306-12.

\section{Bonvin 2013 \{published data only\}}

Bonvin A, Barral J, Kakebeeke TH, Kriemler S, Longchamp A, Schindler $C$, et al. Effect of a governmentally-led physical activity program on motor skills in young children attending child care centers: a cluster randomized controlled trial. International Journal of Behavioral Nutrition and Physical Activity 2013;10:90. [DOI: 10.1186/1479-5868-10-90]

\section{Brandstetter 2012 \{published data only\}}

Brandstetter S, Klenk J, Berg S, Galm C, Fritz M, Peter R, et al. Overweight prevention implemented by primary school teachers: a randomised controlled trial. Obesity Facts 2012;5(1):1-11.

Branscum 2013 \{published data only\}

Branscum P, Sharma M, Wang LL, Wilson B. A true challenge for any superhero: an evaluation of a comic book obesity prevention program. Family \& Community Health 2013;36(1):63-76 
Brown 2013 \{published data only\}

Brown B, Noonan C, Harris KJ, Parker M, Gaskill S, Ricci C, et al. Developing and piloting the Journey to Native Youth Health program in Northern Plains Indian communities. Diabetes Educator 2013;39(1):109-18.

\section{Caballero 2003 \{published data only\}}

* Caballero B, Clay T, Davis SM, Ethelbah B, Rock BH, Lohman T, et al. Pathways: a school-based, randomized controlled trial for the prevention of obesity in American Indian schoolchildren. American Journal of Clinical Nutrition 2003;78(5):1030-8.

Davis S, Gomez Y, Lambert L, Skipper B. Primary prevention of obesity in American Indian Children. Annals of the New York Academy of Sciences 1993;699:167-80.

Stone EJ, Norman JE, Davis SM, Stewart D, Clay TE, Caballero B, et al. Design, implementation, and quality control in the Pathways American-Indian multicenter trial. Preventive Medicine 2003;37:S13-S23.

\section{Campbell 2013 \{published data only\}}

Cameron AJ, Ball K, Hesketh KD, McNaughton SA, Salmon J, Crawford DA, et al. Variation in outcomes of the Melbourne Infant, Feeding, Activity and Nutrition Trial (InFANT) program according to maternal education and age. Preventive Medicine 2014;58:58-63.

* Campbell KJ, Lioret S, McNaughton SA, Crawford DA, Salmon. A parent-focused intervention to reduce infant obesity risk behaviors: a randomized trial. Pediatrics 2013;131(4):652-60.

Cao 2015 \{published data only\}

* Cao Z-J, Wang SM, Chen Y. A randomized trial of multiple interventions for childhood obesity in China. American Journal of Preventive Medicine 2015;48(5):552-60.

Cao ZJ, Wang SM, Zheng WJ, Guo JN, Qu SX. Evaluation on the effectiveness of intervention comprehensive program on child obesity using generalized estimating equation. Chinese Journal of Epidemiology 2014;35(7):773-8.

\section{Chen 2010 \{published data only\}}

* Chen JL, Weiss S, Heyman MB, Lustig RH. Efficacy of a childcentred and family-based program in promoting healthy weight and healthy behaviors in Chinese American children: a randomized controlled study. Journal of Public Health 2010;32(2):219-29.

Chen JL, Weiss SJ, Heyman MB, Cooper B, Lustig RH. The Active Balance childhood program for improving coping and quality of life in Chinese American children. Nursing Research 2010;59(4):270-9.

\section{Chen 2011 \{published data only\}}

Chen JL, Weiss S, Heyman MB, Cooper B, Lustig RH. The efficacy of the web-based childhood obesity prevention program in Chinese American adolescents (Web ABC study). Journal of Adolescent Health 2011;49(2):148-54.
Christiansen 2013 \{published data only\}

Christiansen LB, Toftager M, Boyle E, Kristensen PL, Troelsen J. Effect of a school environment intervention on adolescent adiposity and physical fitness. Scandinavian Journal of Medicine \& Science in Sports 2013;23(6):e381-9.

\section{Coleman 2005 \{published data only\}}

* Coleman KJ, Tiller CL, Sanchez J, Heath EM, Sy O, Milliken G, et al. Prevention of the epidemic increase in child risk of overweight in low-income schools. Archives of Pedatrics and Adolescent Medicine 2005;159:217-24.

Heath EM, Coleman KJ. Adoption and institutionalization of the Child and Adolescent Trial for Cardiovascular Health (CATCH) in El Paso, Texas. Health Promotion Practice 2003;4:159-64.

Heath EM, Coleman KJ. Evaluation of the institutionalization of the Coordinated Approach to Child Health (CATCH) in a US/ Mexico border community. Health Education and Behavior 2002;29:444-600.

\section{Coleman 2012 \{published data only\}}

Coleman KJ, Shordon M, Caparosa SL, Pomichowski ME, Dzewaltowski DA. The healthy options for nutrition environments in schools (Healthy ONES) group randomized trial: using implementation models to change nutrition policy and environments in low income schools. International Journal of Behavioral Nutrition and Physical Activity 2012;9:80.

\section{Crespo 2012 \{published data only\}}

Crespo NC, Elder JP, Ayala GX, Slymen DJ, Campbell NR, Sallis JF, et al. Results of a multi-level intervention to prevent and control childhood obesity among Latino children: the Aventuras Para Ninos Study. Annals of Behavioral Medicine 2012;43(1):84-100.

\section{Cunha 2013 \{published data only\}}

Cunha DB, de Souza BS, Pereira RA, Sichieri R. Effectiveness of a randomized school-based intervention involving families and teachers to prevent excessive weight gain among adolescents in Brazil. PLOS ONE 2013;8(2):e57498.

\section{Damsgaard 2014 \{published data only\}}

Damsgaard CT, Dalskov SM, Laursen RP, Ritz C, Hjorth MF, Lauritzen L, et al. Provision of healthy school meals does not affect the metabolic syndrome score in 8-11-year-old children, but reduces cardiometabolic risk markers despite increasing waist circumference. British Journal of Nutrition 2014;112(11):1826-36.

\section{Daniels 2012 \{published data only\}}

Daniels LA, Mallan KM, Battistutta D, Nicholson JM, Perry R, Magarey A. Evaluation of an intervention to promote protective infant feeding practices to prevent childhood obesity: outcomes of the NOURISH RCT at 14 months of age and 6 months post the first of two intervention modules. International Journal of Obesity 2012;36(10):1292-8.

Daniels LA, Mallan KM, Nicholson JM, Battistutta DLA, Mallan KM, Nicholson JM, et al. Outcomes of an early feeding practices intervention to prevent childhood obesity. Pediatrics 2013;132(1):e109-18. 
De Bock 2012 \{published data only\}

De Bock F, Breitenstein L, Fischer J. Positive impact of a preschool-based nutritional intervention on children's fruit and vegetable intake: results of a cluster-randomized trial. Public Health Nutrition 2012;15(3):466-75.

\section{De Coen 2012 \{published data only\}}

De Coen V, De Bourdeaudhuij I, Vereecken C, Verbestel V, Haerens L, Huybrechts I, et al. Effects of a 2-year healthy eating and physical activity intervention for 3-6-year-olds in communities of high and low socio-economic status: the POP (Prevention of Overweight among Pre-school and school children) project. Public Health Nutrition 2012;15(9):1737-45.

\section{De Heer 2011 \{published data only\}}

De Heer HD, Koehly L, Pederson R, Morera O. Effectiveness and spillover of an after-school health promotion program for Hispanic elementary school children. American Journal of Public Health 2011;101(10):1907-13.

\section{Dennison 2004 \{published data only\}}

Dennison BA, Russo TJ, Burdick PA, Jenkins PL. An intervention to reduce television viewing by preschool children. Archives of Pediatrics \& Adolescent Medicine 2004;158(2):170-6.

\section{De Ruyter 2012 \{published data only\}}

De Ruyter JC, Olthof MR, Seidell JC, Katan MB. A trial of sugarfree or sugar-sweetened beverages and body weight in children. New England Journal of Medicine 2012;367(15):1397-1406.

De Vries 2015 \{published data only\}

De Vries AG, Huiting HG, Van den Heuvel ER, L'Abée C, Corpeleijn E, Stolk RP. An activity stimulation programme during a child's first year reduces some indicators of adiposity at the age of two-and-a-half. Acta Paediatrica 2015;104(4):414-21.

\section{Dewar 2013 \{published data only\}}

Dewar DL, Morgan PJ, Plotnikoff RC, Okely AD, Batterham M, Lubans DR. Exploring changes in physical activity, sedentary behaviors and hypothesized mediators in the NEAT girls group randomized controlled trial. Journal of Science and Medicine in Sport 2014;17(1):39-46.

* Dewar DL, Morgan PJ, Plotnikoff RC, Okely AD, Collins CE, Batterham $\mathrm{M}$, et al. The nutrition and enjoyable activity for teen girls study: a cluster randomized controlled trial. American Journal of Preventive Medicine 2013;45(3):313-7.

Lubans DR, Morgan PJ, Okely AD, Dewar D, Collins CE, Batterham $\mathrm{M}$, et al. Preventing obesity among adolescent girls: one-year outcomes of the nutrition and enjoyable activity for teen girls (NEAT Girls) cluster randomized controlled trial. Archives of Pediatrics and Adolescent Medicine 2012;166(9):821-7.

\section{Donnelly 2009 \{published data only\}}

* Donnelly JE, Greene JL, Gibson CA, Smith BK, Washburn RA, Sullivan DK, et al. Physical Activity Across the Curriculum (PAAC): a randomized controlled trial to promote physical activity and diminish overweight and obesity in elementary school children. Preventive Medicine 2009;49(4):336-41. [1096-0260: (Electronic)]

Gibson CA, Smith BK, Dubose KD, Greene JL, Bailey BW, Williams SL, et al. Physical activity across the curriculum: year one process evaluation results. International Journal of Behavioral Nutrition and Physical Activity 2008;5:36. [DOI: 10.1186/1479-5868-5-36]

\section{Ebbeling 2006 \{published data only\}}

Ebbeling CB, Feldman HA, Osganion SK, Chomitz VR, Ellenbogen SJ, Ludwig DS. Effects of decreasing sugarsweetened beverage consumption on body weight in adolescents: a randomized, controlled pilot study. Pediatrics 2006;117:673-80.

\section{El Ansarai 2010 \{published data only\}}

El Ansari W, El Ashker S, Moseley L. Associations between physical activity and health parameters in adolescent pupils in Egypt. International Journal of Environmental Research and Public Health 2010;7(4):1649-69.

\section{Elder 2014 \{published data only\}}

Elder JP, Crespo NC, Corder K, Ayala GX, Slymen DJ, Lopez NV, et al. Childhood obesity prevention and control in city recreation centres and family homes: the MOVE/me Muevo Project. Pediatric Obesity 2014;9(3):218-31.

\section{Epstein 2001 \{published and unpublished data\}}

Epstein LH, Gordy CC, Raynor HA, Beddome M, Kilanowski CK, Paluch R. Increasing fruit and vegetable intake and decreasing fat and sugar intake in families at risk for childhood obesity. Obesity Research 2001;9(3):171-8.

Ezendam 2012 \{published data only\}

* Ezendam NP, Brug J, Oenema A. Evaluation of the web-based computer-tailored FATaintPHAT intervention to promote energy balance among adolescents: results from a school cluster randomized trial. Archives of Pediatrics \& Adolescent Medicine 2012;166(3):248-55.

Ezendam NP, Noordegraaf VS, Kroeze W, Brug J, Oenema A. Process evaluation of FATaintPHAT, a computer-tailored intervention to prevent excessive weight gain among Dutch adolescents. Health Promotion International 2013;28(1):26-35.

\section{Fairclough 2013 \{published data only\}}

Fairclough SJ, Hackett AF, Davies IG, Gobbi R, Mackintosh KA, Warburton GL, et al. Promoting healthy weight in primary school children through physical activity and nutrition education: a pragmatic evaluation of the CHANGE! randomised intervention study. BMC Public Health 2013;13:626.

\section{Farias 2015 \{published data only\}}

Farias ES, Gonçalves EM, Morcillo AM, Guerra-Júnior G, Amancio OM. Effects of programmed physical activity on body composition in post-pubertal schoolchildren. Jornal de Pediatria 2015;91(2):122-9. 
Feng 2004 \{published data only\}

Feng BS, Zhang HM, Zhong QY. The early intervention research of health education on the simple obesity of low ages children. Maternal and Child Health Care China 2004;19(10):23-5.

\section{Fitzgibbon 2005 \{published data only\}}

Fitzgibbon ML, Stolley MR, Dyer AR, VanHorn L, KauferChristoffel K. A community-based obesity prevention program for minority children: rationale and study design for Hip-Hop to Health Jr. Preventive Medicine 2002;34:289-97.

* Fitzgibbon ML, Stolley MR, Schiffer L, Van Horn L, KauferChristoffel K, Dyer A. Two-year follow-up results for HipHop to Health Jr.: a randomized controlled trial for overweight prevention in preschool minority children. Journal of Pediatrics 2005;May:618-25.

Stolley MR, Fitzgibbon ML, Dyer A, Van Horn L, KauferChristoffel K, Schiffer L. Hip-Hop to Health Jr., an obesity prevention program for minority preschool children: baseline characteristics of participants. Preventive Medicine 2003;36:320-9

\section{Fitzgibbon 2006 \{published data only\}}

Fitzgibbon ML, Stolley MR, Schiffer L, Van Horn L, KauferChristoffel K, Dyer A. Hip-Hop to Health Jr. for Latino preschool children. Obesity 2006;14(9):1616-25.

\section{Fitzgibbon 2011 \{published data only\}}

* Fitzgibbon ML, Stolley MR, Schiffer LA, Braunschweig CL, Gomez SL, Van Horn, et al. Hip-Hop to Health Jr. Obesity prevention effectiveness trial: postintervention results. Obesity 2011;19(5):994-1003.

Kong A, Buscemi J, Stolley MR, Schiffer LA, Kim Y, Braunschweig CL, et al. Hip-Hop to Health Jr. Randomized effectiveness trial 1-year follow-up results. American Journal of Preventive Medicine 2016;50(2):136-44. [DOI: http:// dx.doi.org/10.1016/j.amepre.2015.07.008]

\section{Foster 2008 \{published data only\}}

Foster GD, Sherman S, Borradaile KE, Grundy KM, Vander Veur SS, Nachmani J, et al. A policy-based school intervention to prevent overweight and obesity. Pediatrics 2008;121(4):e794-802. [1098-4275: (Electronic)]

Rappaport EB, Daskalakis C, Sendecki JA. Using routinely collected growth data to assess a school-based obesity prevention strategy. International Journal of Obesity 2013;37(1):79-85.

\section{French 2011 \{published data only\}}

French SA, Gerlach AF, Mitchell NR, Hannan PJ, Welsh EM. Household obesity prevention: Take Action--a grouprandomized trial. Obesity 2011;19(10):2082-8.

\section{Fulkerson 2010 \{published data only\}}

Fulkerson JA, Rydell S, Kubik MY, Lytle L, Boutelle K, Story M, et al. Healthy Home Offerings via the Mealtime Environment (HOME): feasibility, acceptability, and outcomes of a pilot study. Obesity 2010;18 Suppl 1:S69-74.

\section{Gentile 2009 \{published data only\}}

Gentile DA, Welk G, Eisenmann JC, Reimer RA, Walsh DA, Russell DW, et al. Evaluation of a multiple ecological level child obesity prevention program: Switch what you Do, View, and Chew. BMC Medicine 2009;7:49. [1741-7015: (Electronic)]

\section{Gortmaker 1999a \{published data only\}}

Gortmaker SL, Peterson K, Wiecha J, Sobal AM, Dixit S, Fox MK, et al. A school-based, interdisciplinary curriculum in grades 6 and 7 reduced obesity in girls. Evidence Based Nursing 2000;3:13.

Gortmaker SL, Peterson K, Wiecha J, Sobal AM, Dixit S, Fox MK, et al. Reducing obesity via a school-based interdisciplinary intervention among youth. Archives of Pediatrics and Adolescent Medicine 1999;153(4):409-18.

Wang LY, Yang Q, Lowry R, Wechsler H. Economic analysis of a school-based obesity prevention program. Obesity Research 2003;11(11):1313-24.

\section{Grydeland 2014 \{published data only\}}

Bjelland M, Hausken SE, Bergh IH, Grydeland M, Klepp K-I, Andersen LF, et al. Changes in adolescents' and parents' intakes of sugar-sweetened beverages, fruit and vegetables after 20 months: results from the HEIA study - a comprehensive, multicomponent school-based randomized trial. Food and Nutrition Research 2015;59:25932.

* Grydeland M, Bjelland M, Anderssen SA, Klepp K-I, Bergh IH, Andersen LF, et al. Effects of a 20-month cluster randomised controlled school-based intervention trial on BMI of schoolaged boys and girls: the HEIA study. British Journal of Sports Medicine 2014;48(9):768-73.

\section{Gutin 2008 \{published data only\}}

* Gutin B, Yin Z, Johnson M, Barbeau P. Preliminary findings of the effect of a 3-year after-school physical activity intervention on fitness and body fat: the Medical College of Georgia Fitkid Project. International Journal of Pediatric Obesity 2008;3 Suppl 1:3-9. [1747-7174: (Electronic)]

Wang LY, Gutin B, Barbeau P, Moore JB, Hanes J, Johnson MH, et al. Cost-effectiveness of a school-based obesity prevention program. Journal of School Health 2008;78:619-24.

Yin Z, Gutin B, Johnson MH, Hanes J Jr, Moore JB, Cavnar M, et al. An environmental approach to obesity prevention in children: Medical College of Georgia FitKid Project year 1 results. Obesity Research 2005;13:2153-61.

Yin Z, Hanes J Jr, Moore JB, Humbles P, Barbeau P, Gutin B. An after-school physical activity program for obesity prevention in children: The Medical College of Georgia FitKid Project. Evaluation and the Health Professions 2005;28:67-89.

Yin Z, Moore JB, Johnson MH, Barbeau P, Cavnar M, Thornburg J, et al. The Medical College of Georgia FitKid Project: the relations between program attendance and changes in outcomes in year 1. International Journal of Obesity 2005;29:S40-S45. 
Yin Z, Moore JB, Johnson MH, Vernon MM, Gutin B. The impact of a 3-year after-school obesity prevention program in elementary school children. Childhood Obesity 2012;8(1):60-70.

\section{Habib-Mourad 2014 \{published data only\}}

Habib-Mourad C. An intervention to promote healthy eating and physical activity in Lebanese school children: Health-E-PALS, a pilot cluster randomised controlled trial. Obesity Facts. 2014.

\section{Haerens 2006 \{published data only\}}

* Haerens L, Deforche B, Maes L, Cardon G, Stevens V, De Bourdeaudhuij I. Evaluation of a 2-year physical activity and healthy eating intervention in middle school children. Health Education Research 2006;21(6):911-21.

Haerens L, Deforche B, Maes L, Stevens V, Cardon G, De Bourdeaudhuij I. Body mass effects of a physical activity and healthy food intervention in middle schools. Obesity 2006;14(5):847-54. [0268-1153: (Print)]

\section{Haines 2013 \{published data only\}}

Haines J, McDonald J, O'Brien A, Sherry B, Bottino C. Healthy Habits, Happy Homes: randomized trial to improve household routines for obesity prevention among preschool-aged children. JAMA Pediatrics 2013;167(11):1072-9.

\section{Haire-Joshu 2010 \{published data only\}}

Haire-Joshu D, Nanney MS, Elliott M, Davey C, Caito N, Loman D, et al. The use of mentoring programs to improve energy balance behaviors in high-risk children. Obesity 2010;18 Suppl 1:S75-83.

\section{Han 2006 \{published data only\}}

Han X, Ling P, Chen Y. The outcome evaluation of the elementary students about 3-year in system-intervention of nutrition dinner in Yangpu District, Shanghai. Health Education and Health Promotion 2006;1:21-4.

\section{Harvey-Berino 2003 \{published data only\}}

Harvey-Berino J, Rourke J. Obesity prevention in preschool Native-American children: a pilot study using home visiting. Obesity Research 2003;11:606-11.

\section{HEALTHY Study Gp 2010 \{published data only\}}

* HEALTHY Study Group. A school-based intervention for diabetes risk reduction. New England Journal of Medicine 2010;363(5):443-53.

Hall WJ. School factors as barriers to and facilitators of a preventive intervention for pediatric type 2 diabetes. Translational Behavioral Medicine 2014;4(2):131-40.

Marcus MD, Foster GD, El Ghormli L. Shifts in BMI category and associated cardiometabolic risk: prospective results from HEALTHY study. Pediatrics 2012;129(4):e983-91.

Volpe SL, Hall WJ, Steckler A, Schneider M, Thompson D, Mobley C, et al. Process evaluation results from the HEALTHY nutrition intervention to modify the total school food environment. Health Education Research 2013;28(6):970-8.
Hendy 2011 \{published data only\}

Hendy HM, Williams KE, Camise TS. Kid's Choice Program improves weight management behaviors and weight status in school children. Appetite 2011;56(2):484-94.

\section{Herscovici 2013 \{published data only\}}

Herscovici CR, Kovalskys I, De Gregorio MJ. Gender differences and a school-based obesity prevention program in Argentina: a randomized trial. Revista Panamericana de Salud Publica [Pan American Journal of Public Health] 2013;34(2):75-82.

\section{Howe 2011 \{published data only\}}

Howe CA, Harris RA, Gutin B. A 10-month physical activity intervention improves body composition in young black boys. Journal of Obesity 2011;2011:358581.

\section{James 2004 \{published data only\}}

* James J, Thomas P, Cavan D, Kerr D. Preventing childhood obesity by reducing consumption of carbonated drinks: cluster randomised controlled trial. BMJ 2004;328(7450):22.

James J, Thomas P, Kerr D. Preventing childhood obesity: two year follow-up results from the Christchurch obesity prevention programme in schools (CHOPPS). BMJ 2007;335(7623):762. [1468-5833: (Electronic)]

\section{Jansen 2011 \{published data only\}}

Jansen W, Borsboom G, Meima A, Zwanenburg EJ-V, Mackenbach J. Effectiveness of a primary school-based intervention to reduce overweight. International Journal of Pediatric Obesity 2011;6(2-2):e70-7.

\section{Johnston 2013 \{published data only\}}

Johnston CA, Moreno JP, El-Mubasher A, Gallagher M, Tyler C, Woehler D. Impact of a school-based pediatric obesity prevention program facilitated by health professionals. Journal of School Health 2013;83(3):171-81.

Kain 2014 \{published data only\}

Kain J, Concha F, Moreno L, Leyton B. School-based obesity prevention intervention in Chilean children: effective in controlling, but not reducing obesity. Journal of Obesity 2014;2014:618293.

\section{Keller 2009 \{published data only\}}

Keller A, Klossek A, Gausche R, Hoepffner W, Kiess W, Keller E. Prevention for obesity in children [Gezielte primäre Adipositasprävention bei Kindern]. Deutsche Medizinische Wochenschrift 2009;134:13-8.

Khan 2014 \{published data only\}

Khan NA, Raine LB, Drollette ES, Scudder MR, Pontifex MB, Castelli DM, et al. Impact of the FITKids physical activity intervention on adiposity in prepubertal children. Pediatrics 2014;133(4):e875-83.

\section{Kipping 2008 \{published data only\}}

Kipping RR, Payne C, Lawlor DA. Randomised controlled trial adapting US school obesity prevention to England. Archives of Disease in Chidhood 2008;93(6):469-73. [1468-2044: (Electronic)] 
Kipping 2014 \{published data only\}

Kipping RR, Howe LD, Jago R, Campbell R, Wells S, Chittleborough CR, et al. Effect of intervention aimed at increasing physical activity, reducing sedentary behaviour, and increasing fruit and vegetable consumption in children: Active for Life Year 5 (AFLY5) school based cluster randomised controlled trial. BMJ 2014;348:g3256.

\section{Klein 2010 \{published data only\}}

Klein D, De Toia D, Weber S, Wessely N, Koch B, Dordel S, et al. Effects of a low threshold health promotion intervention on the BMI in pre-school children under consideration of parental participation. e-SPEN, the European e-Journal of Clinical Nutrition and Metabolism 2010;5(3):e125-31.

\section{Klesges 2010 \{published data only\}}

Klesges RC, Obarzanek E, Kumanyika S. The Memphis girls' health enrichment multi-site studies (GEMS): an evaluation of the efficacy of a 2-year obesity prevention program in African American girls. Archives of Pediatrics \& Adolescent Medicine 2010;164(11):1007-14.

\section{Kriemler 2010 \{published data only\}}

Hartmann T, Zahner L, Puhse U, Puder J. Effects of a schoolbased physical activity program on physical and psychosocial quality of life in elementary school children: a clusterrandomized trial. Pediatric Exercise Science 2010;22(4):511-22.

* Kriemler S, Zahner L, Schindler C, Meyer U, Hartmann T, Hebestreit $\mathrm{H}$, et al. Effect of school based physical activity programme (KISS) on fitness and adiposity in primary schoolchildren: cluster randomised controlled trial. BMJ (Clinical Research Ed.) 2010;340:c785.

Meyer U, Schindler C, Zahner L, Ernst D, Hebestreit H, Van Mechelen W, et al. Long-term effect of a school-based physical activity program (KISS) on fitness and adiposity in children: a cluster-randomized controlled trial. PLOS ONE 2014;9(2):e87929.

\section{Lana 2014 \{published data only\}}

Lana A, Faya-Ornia G, Lopez ML. Impact of a web-based intervention supplemented with text messages to improve cancer prevention behaviors among adolescents: results from a randomized controlled trial. Preventive Medicine 2014;59:54-9.

\section{Lazaar 2007 \{published data only\}}

Lazaar N, Aucouturier J, Ratel S, Rance M, Meyer M, Duché P. Effect of physical activity intervention on body composition in young children: influence of body mass index status and gender. Acta Paediatrica 2007;96(9):1315-20.

\section{Levy 2012 \{published and unpublished data\}}

Levy TS, Morales Ruán C, Amaya Castellanos C, Salazar Coronel A, Jiménez Aguilar A, Méndez Gómez Humarán I. Effectiveness of a diet and physical activity promotion strategy on the prevention of obesity in Mexican school children. BMC Public Health 2012;12:152. [DOI: 10.1186/1471-2458-12-152]

\section{Li 2010a $\{$ published data only\}}

Li YP, Hu XQ, Schouten EG, Liu AL, Du SM, Li LZ, et al. Report on childhood obesity in China (8): effects and sustainability of physical activity intervention on body composition of Chinese youth. Biomedical and Environmental Sciences 2010;23(3):180-7.

\section{Llargues 2012 \{published data only\}}

Llargues E, Franco R, Recasens A, Nadal A, Vila M, Perez MJ, et al. Assessment of a school-based intervention in eating habits and physical activity in school children: the AVall study. Journal of Epidemiology and Community Health 2011;65(10):896-901.

* Llargues E, Recasens A, Franco R, Nadal A, Vila M, Perez MJ, et al. Medium-term evaluation of an educational intervention on dietary and physical exercise habits in schoolchildren: the Avall 2 study. Endocrinologia y Nutricion 2012;59(5):288-95.

Mora T, Llargues E, Recasens A. Does health education affect BMI? Evidence from a school-based randomised-control trial. Economics and Human Biology 2015;17(C):190-201.

\section{Lubans 2011 \{published data only\}}

Lubans DR, Morgan PJ, Aguiar EJ, Callister R. Randomized controlled trial of the Physical Activity Leaders (PALs) program for adolescent boys from disadvantaged secondary schools. Preventive Medicine 2011;52(3-4):239-46.

\section{Macias-Cervantes 2009 \{published data only\}}

Macias-Cervantes MH, Malacara JM, Garay-Sevilla ME, DiazCisneros FJ. Effect of recreational physical activity on insulin levels in Mexican/Hispanic children. The European Journal of Pediatrics 2009;168(10):1195-202. [1432-1076: (Electronic)]

\section{Madsen 2013 \{published data only\}}

Madsen K, Thompson H, Adkins A, Crawford Y. Schoolcommunity partnerships: a cluster-randomized trial of an afterschool soccer program. JAMA Pediatrics 2013;167(4):321-6.

\section{Magnusson 2012 \{published data only\}}

Hrafnkelsson H, Magnusson KT, Thorsdottir I, Johannsson E, Sigurdsson E. Result of school-based intervention on cardiovascular risk factors. Scandinavian Journal of Primary Health Care 2014;32(4):149-55.

* Magnusson KT, Hrafnkelsson H, Sigurgeirsson I, Johannsson E, Sveinsson T. Limited effects of a 2-year schoolbased physical activity intervention on body composition and cardiorespiratory fitness in 7-year-old children. Health Education Research 2012;27(3):484-94.

\section{Marcus 2009 \{published data only\}}

Marcus C, Nyberg G, Nordenfelt A, Karpmyr M, Kowalski J, Ekelund U. A 4-year, cluster-randomized, controlled childhood obesity prevention study: STOPP. International Journal of Obesity 2009; Vol. 33, issue 4:408-17. [03070565]

\section{Martinez-Vizcaino 2014 \{published data only\}}

Martinez-Vizcaino V, Sanchez-Lopez M, Notario-Pacheco B, Salcedo-Aguilar F, Solera-Martínez M, Franquelo-Morales $\mathrm{P}$, et al. Gender differences on effectiveness of a school-based physical activity intervention for reducing cardiometabolic risk: a cluster 
randomized trial. International Journal of Behavioral Nutrition and Physical Activity 2014;11:154.

Mauriello 2010 \{published data only\}

Mauriello LM, Ciavatta MM, Paiva AL, Sherman KJ, Castle PH, Johnson JL, et al. Results of a multi-media multiple behavior obesity prevention program for adolescents. Preventive Medicine 2010;51(6):451-6.

\section{Melnyk 2013 \{published data only\}}

Melnyk BM, Jacobson D, Kelly S, Belyea M, Shaibi G, Small L, et al. Promoting healthy lifestyles in high school adolescents: a randomized controlled trial. American Journal of Preventive Medicine 2013;45(4):407-15.

\section{Meng 2013 \{published data only\}}

Meng L, Xu H, Liu A, Van Raaij J, Bemelmans W, Hu X, et al. The costs and cost-effectiveness of a school-based comprehensive intervention study on childhood obesity in China. PLOS ONE 2013;8(10):e77971.

\section{Mihas 2010 \{published data only\}}

Mihas C, Mariolis A, Manios Y, Naska A, Arapaki A, MariolisSapsakos $T$, et al. Evaluation of a nutrition intervention in adolescents of an urban area in Greece: short- and longterm effects of the VYRONAS study. Public Health Nutrition 2010;13(5):712-9.

\section{Morgan 2011 \{published data only\}}

Morgan PJ, Lubans DR, Callister R, Okely AD, Burrows TL, Fletcher R, et al. The 'Healthy Dads, Healthy Kids' randomized controlled trial: efficacy of a healthy lifestyle program for overweight fathers and their children. International Journal of Obesity 2011;35(3):436-47.

\section{Mo-suwan 1998 \{published and unpublished data\}}

Mo-Suwan L. Increasing obesity in school children in a transitional society and the effect of the weight control program. Southeast Asian Journal of Tropical Medicine and Public Health 1993;24(3):590-94.

* Mo-Suwan L, Pongprapai S, Junjana C, Peutpaiboon A. Effects of a controlled trial of a school-based exercise program on the obesity indexes of preschool children. American Journal of Clinical Nutrition 1998;68:1006-111.

\section{Muckelbauer 2010 \{published data only\}}

Muckelbauer R, Libuda L, Clausen K, Toschke A. Immigrational background affects the effectiveness of a school-based overweight prevention program promoting water consumption. Obesity 2010;18(3):528-34.

\section{Natale 2014 \{published data only\}}

Natale RA, Lopez-Mitnik G, Uhlhorn SB, Asfour L, Messiah SE. Effect of a child care center-based obesity prevention program on body mass index and nutrition practices among preschoolaged children. Health Promotion Practice 2014;15(5):695-705.
Nemet 2011 \{published data only\}

* Nemet D, Geva D, Eliakim A. Health promotion intervention in low socioeconomic kindergarten children. Journal of Pediatrics 2011;158(5):796-801.

Nemet D, Geva D, Pantanowitz M, Igbaria N, Meckel Y, Eliakim A. Health promotion intervention in Arab-Israeli kindergarten children. Journal of Pediatric Endocrinology 2011;24(11-12):1001-7.

Nemet D, Geva D, Pantanowitz M, Igbaria N, Meckel Y, Eliakim A. Long term effects of a health promotion intervention in low socioeconomic Arab- Israeli kindergartens. BMC Pediatrics 2013;13:45.

\section{Neumark-Sztainer 2003 \{published data only\}}

Friend S, Flattum CF, Simpson D, Nederhoff DM, NeumarkSztainer D. The researchers have left the building: what contributes to sustaining school-based interventions following the conclusion of formal research support?. Journal of School Health 2014;84(5):326-33.

* Neumark-Sztainer D, Story M, Hannan PJ, Rex J. New Moves: a school-based obesity prevention program for adolescent girls. Preventive Medicine 2003;37(1):41-51.

\section{Neumark-Sztainer 2010 \{published data only\}}

Neumark-Sztainer DR, Friend SE, Flattum CF, Hannan PJ, Story MT, Bauer KW, et al. New moves-preventing weightrelated problems in adolescent girls a group-randomized study. American Journal of Preventive Medicine 2010;39(5):421-32.

\section{Nollen 2014 \{published data only\}}

Nollen NL, Mayo MS, Carlson SE, Rapoff MA, Goggin KJ, Ellerbeck EF. Mobile technology for obesity prevention: a randomized pilot study in racial- and ethnic-minority girls. American Journal of Preventive Medicine 2014;46(4):404-8.

\section{Nyberg 2015 \{published data only\}}

Nyberg G, Sundblom E, Norman A, Bohman B, Hagberg J, Elinder LS. Effectiveness of a universal parental support programme to promote healthy dietary habits and physical activity and to prevent overweight and obesity in 6-year-old children: the healthy school start study, a cluster-randomised controlled trial. PLOS ONE 2015;10(2):e0116876.

\section{Ostbye 2012 \{published data only\}}

Ostbye T, Krause KM, Stroo M, Lovelady CA, Evenson KR, Peterson BL, et al. Parent-focused change to prevent obesity in preschoolers: results from the KAN-DO study. Preventive Medicine 2012;55(3):188-95.

\section{Paineau 2008 \{published data only\}}

Paineau DL, Beaufils F, Boulier A, Cassuto DA, Chwalow J, Combris $\mathrm{P}$, et al. Family dietary coaching to improve nutritional intakes and body weight control: a randomized controlled trial. Archives of Pediatrics \& Adolescent Medicine. 2008/01/09 2008; Vol. 162, issue 1:34-43. [1538-3628: (Electronic)]

Papadaki 2010 \{published data only\}

Damsgaard CT. Higher protein diets consumed ad libitum improve cardiovascular risk markers in children of overweight 
parents from eight European countries [Erratum appears in J Nutr. 2014 Dec;144(12):2094]. Journal of Nutrition 2013;143(6):810-7.

* Papadaki A, Linardakis M, Larsen T. The effect of protein and glycemic index on children's body composition: the DiOGenes randomized study. Pediatrics 2010;126(5):e1143-52.

\section{Pate 2005 \{published data only\}}

Pate RR, Ward DS, Saunders RP, Felton G, Dishman RK, Dowda M. Promotion of physical activity among high-school girls: a randomized controlled trial. American Journal of Public Health 2005;95(9):1582-7.

\section{Patrick 2006 \{published data only\}}

Patrick K, Calfas KJ, Norman GJ, Zabinski MF, Sallis JF, Rupp J, et al. Randomized controlled trial of a primary care and homebased intervention for physical activity and nutrition behaviors. Archives of Pediatrics and Adolescent Medicine 2006;160:128-36.

\section{Paul 2011 \{published data only\}}

Paul IM, Savage JS, Anzman SL, Beiler JS, Marini ME, Stokes JL, et al. Preventing obesity during infancy: a pilot study. Obesity 2011;19(2):353-61.

\section{Peralta 2009 \{published data only\}}

Peralta LR, Jones RA, Okely AD. Promoting healthy lifestyles among adolescent boys: the Fitness Improvement and Lifestyle Awareness Program RCT. Preventive Medicine 2009;48(6):537-42. [1096-0260: (Electronic)]

\section{Puder 2011 \{published data only\}}

Burgi F, Niederer I, Schindler C, Bodenmann P, MarquesVidal P, Kriemler S, et al. Effect of a lifestyle intervention on adiposity and fitness in socially disadvantaged subgroups of preschoolers: a cluster-randomized trial (Ballabeina). Preventive Medicine 2012;54(5):335-40.

Niederer I, Bürgi F, Ebenegger V, Marques-Vidal P, Schindler C, Nydegger A, et al. Effects of a lifestyle intervention on adiposity and fitness in overweight or low fit preschoolers (Ballabeina). Obesity 2013;21(3):E287-93.

* Puder JJ, Marques-Vidal P, Schindler C, Zahner L, Niederer I, Bürgi $F$, et al. Effect of multidimensional lifestyle intervention on fitness and adiposity in predominantly migrant preschool children (Ballabeina): cluster randomised controlled trial. BMJ 2011;343:d6195.

\section{Reed 2008 \{published data only\}}

Naylor P, Macdonald H, Reed K, McKay HA. Action Schools BC: a socio-ecological approach to modifying disease risk factors in elementary school children. Preventing Chronic Disease 2006;3(2):A6017-A6019.

Naylor P, Macdonald HM, Zebedee JA, Reed KE, McKay HA. Lessons learned from Action Schools BC: an active schools model to promote physical activity in elementary schools. Journal of Science and Medicine in Sport 2006;9(5):413-9.

* Reed KE, Warburton DE, Macdonald HM, Naylor PJ, McKay HA. Action Schools! BC: a school-based physical activity intervention designed to decrease cardiovascular disease risk factors in children. Preventive Medicine 2008;46(6):525-31. [0091-7435: (Print)]

\section{Reilly 2006 \{published data only\}}

Reilly JJ, Kelly L, Montgomery C, Williamson A, Fisher A, McColl JH, et al. Physical activity to prevent obesity in young children: cluster randomised controlled trial. BMJ 2006;333(7577):1041.

Robbins 2006 \{published data only\}

Robbins LB, Gretebeck KA, Kazanis AS, Pender NJ. Girls on the move program to increase physical activity participation. Nursing Research 2006;55(3):206-16.

\section{Robinson 2003 \{published data only\}}

Robinson TN, Killen JD, Kraemer HC, Wilson DM, Matheson DM, Haskell WL, et al. Dance and reducing television viewing to prevent weight gain in African-American girls: the Stanford GEMS pilot study. Ethnicity and Disease 2003;13 Suppl 1:S65-77.

Robinson 2010 \{published data only\}

Robinson TN, Matheson DM, Kraemer HC, Wilson DM, Obarzanek E, Thompson NS, et al. A randomized controlled trial of culturally tailored dance and reducing screen time to prevent weight gain in low-income African American girls: Stanford GEMS. Archives of Pediatrics \& Adolescent Medicine 2010;164(11):995-1004.

\section{Rodearmel 2006 \{published data only\}}

Rodearmel SJ, Wyatt HR, Barry MJ, Dong F, Pan D, Israel RG, et al. A family-based approach to preventing excessive weight gain. Obesity 2006;14(8):1393-401.

\section{Rosario 2012 \{published data only\}}

Rosario R, Araujo A, Oliveira B, Padrao P, Lopes O, Teixeira V, et al. Impact of an intervention through teachers to prevent consumption of low nutrition, energy-dense foods and beverages: a randomized trial. Preventive Medicine 2013;57(1):20-5.

* Rosario R, Oliveira B, Araujo A, Lopes O, Padrao P, Moreira A, et al. The impact of an intervention taught by trained teachers on childhood overweight. International Journal of Environmental Research and Public Health 2012;9(4):1355-67.

\section{Rosenkranz 2010 \{published data only\}}

Rosenkranz RR, Behrens TK, Dzewaltowski DA. A grouprandomized controlled trial for health promotion in Girl Scouts: healthier troops in a SNAP (Scouting Nutrition \& Activity Program). BMC Public Health 2010;10:81.

Roth 2015 \{published data only\}

Roth K, Kriemler S, Lehmacher W, Ruf KC, Graf C, Hebestreit H. Effects of a physical activity intervention in preschool children. Medicine and Science in Sports and Exercise 2015;47(12):254251 .

\section{Rush 2012 \{published data only\}}

Rush E, Reed P, McLennan S, Coppinger T, Simmons D, Graham D. A school-based obesity control programme: Project 
Energize. Two-year outcomes. British Journal of Nutrition 2012;107(4):581-7.

\section{Safdie 2013 \{published data only\}}

Salfdie M, Jennings-Aburto N, Lévesque L, Janssen I, Campirano-Núñez F, López-Olmedo N, et al. Impact of a schoolbased intervention program on obesity risk factors in Mexican children. Salud Publica de Mexico 2013;55(Suppl 3):S374-S387.

\section{Sahota 2001 \{published data only\}}

* Sahota P, Rudolf MC, Dixey R, Hill AJ, Barth JH, Cade J. Evaluation of implementation and effect of primary school based intervention to reduce risk factors for obesity. BMJ 2001;323:1027-9.

Sahota P, Rudolf MC, Dixey R, Hill AJ, Barth JH, Cade J. Randomised controlled trial of primary school based intervention to reduce risk factors for obesity. BMJ 2001;323:1029-32.

\section{Sallis 1993 \{published data only\}}

Sallis JF, McKenzie TL, Alcaraz JE, Kolody B, Hovell MF, Nader PR. Project SPARK. Effects of physical education on adiposity in children. Annals of the New York Academy of Sciences 1993;699:127-36.

\section{Salmon 2008 \{published data only\}}

Salmon J, Ball K, Crawford D, Booth M, Telford A, Hume C, et al. Reducing sedentary behaviour and increasing physical activity among 10-year-old children: overview and process evaluation of the 'Switch-Play' intervention. Health Promotion International 2005;20(1):7-17.

* Salmon J, Ball K, Hume C, Booth M, Crawford D. Outcomes of a group-randomized trial to prevent excess weight gain, reduce screen behaviours and promote physical activity in 10year-old children: switch-play. International Journal of Obesity 2008;32(4):601-12. [1476-5497: (Electronic)]

\section{Santos 2014 \{published data only\}}

Santos RG, Durksen A, Rabbanni R, Chanoine JP, Lamboo Miln A, Mayer T, et al. Effectiveness of peer-based healthy living lesson plans on anthropometric measures and physical activity in elementary school students: a cluster randomized trial. [Erratum appears in JAMA Pediatr. 2015 Jan;169(1):96]. JAMA Pediatrics 2014;168(4):330-7.

\section{Sevinc 2011 \{published data only\}}

Sevinc O, Bozkurt AI, Gundogdu M, Bas Aslan U, Agbuga B, Aslan $S$, et al. Evaluation of the effectiveness of an intervention program on preventing childhood obesity in Denizli, Turkey. Turkish Journal of Medical Sciences 2011;41(6):1097-105.

\section{Shin 2015 \{published data only\}}

Shin A, Surkan PJ, Coutinho AJ, Suratkar SR, Campbell RK, Rowan M, et al. Impact of Baltimore healthy eating zones: an environmental intervention to improve diet among African American youth. Health Education \& Behavior 2015;42 (1 Suppl):97S-105S.
Sichieri 2009 \{published data only\}

Sichieri R, Paula Trotte A, de Souza RA, Veiga GV. School randomised trial on prevention of excessive weight gain by discouraging students from drinking sodas. Public Health Nutrition 2009;12(2):197-202. [1368-9800: (Print)]

Siegrist 2013 \{published data only\}

Siegrist M, Lammel C, Haller B, Christle J, Halle M. Effects of a physical education program on physical activity, fitness, and health in children: the JuvenTUM project. Scandinavian Journal of Medicine \& Science in Sports 2013;23(3):323-30.

Simon 2008 \{published data only\}

Simon C, Kellou N, Dugas J, Platat C, Copin N, Schweitzer B, et al. A socio-ecological approach promoting physical activity and limiting sedentary behavior in adolescence showed weight benefits maintained 2.5 years after intervention cessation. International Journal of Obesity 2014;38(7):936-43.

* Simon C, Schweitzer B, Oujaa M, Wagner A, Arveiler D, Triby E, et al. Successful overweight prevention in adolescents by increasing physical activity: a 4-year randomized controlled intervention. International Journal of Obesity 2008;32(10):1489-98. [1476-5497: (Electronic)]

Simon C, Wagner A, DiVita C, Rauscher E, Klein-Platat C, Arveiler $D$, et al. Intervention centred on adolescents' physical activity and sedentary behaviour (ICAPS): concept and 6-month results. International Journal of Obesity 2004;28:S96-S103.

Simon C, Wagner A, Platat C, Arveiler D, Schweitzer B, SchliengerJL, et al. ICAPS: a multilevel program to improve physical activity in adolescents. Diabetes Metab 2006; 2006;32:41-49.

\section{Singh 2009 \{published data only\}}

* Singh AS, Chin A Paw MJ, Brug J, Van Mechelen W. Dutch obesity intervention in teenagers: effectiveness of a schoolbased program on body composition and behavior. Archives of Pediatrics \& Adolescent Medicine 2009;163(4):309-17. [1538-3628: (Electronic)]

Singh AS, Chin A Paw MJ, Brug J, Van Mechelen W. Shortterm effects of school-based weight gain prevention among adolescents. Archives of Pediatrics \& Adolescent Medicine 2007;161:565-71.

Singh AS, Paw MJMCA, Kremers SPJ, Visscher TLS, Brug J, Van Mechelen W. Study Protocol: Design of the Dutch Obesity Intervention in Teenagers (NRG-DOiT): systematic development, implementation and evaluation of a school-based intervention aimed at the prevention of excessive weight gain in adolescents. BMC Public Health 2006;6:304.

\section{Skouteris 2016 \{published data only\}}

Skouteris H, Hill B, McCabe M, Swinburn B, Busija L. A parentbased intervention to promote healthy eating and active behaviours in pre-school children: evaluation of the MEND 2-4 randomized controlled trial. Pediatric Obesity 2016;11(1):4-10. 
Slusser 2012 \{published data only\}

Slusser W, Frankel F, Robison K, Fischer H, Cumberland W. Pediatric overweight prevention through a parent training program for 2-4 year old Latino children. Childhood Obesity 2012;8(1):52-9.

\section{Smith 2014 \{published data only\}}

Smith JJ, Morgan PJ, Plotnikoff RC, Dally KA, Salmon J, Okely $A D$, et al. Smart-phone obesity prevention trial for adolescent boys in low-income communities: the ATLAS RCT. Pediatrics 2014;134(3):e723-31.

Spiegel 2006 \{published data only\}

Spiegel SA, Foulk D. Reducing overweight through a multidisciplinary school-based intervention. Obesity 2006;14(1):88-96.

\section{Stolley 1997 \{published data only\}}

Stolley MR, Fitzgibbon ML. Effects of an obesity prevention program on the eating behaviour of African American mothers and daughters. Health Education and Behaviour 1997;24(2):152-64.

\section{Story 2003 \{published data only\}}

Rochon J, Klesges RC, Story M, Robinson TN, Baranowski T, Obarzanek E, et al. Common design elements of the girls health enrichment multi-site studies (GEMS). Ethnicity and Disease 2003;13(Suppl 1):S6-S14.

Story M, Sherwood NE, Himes JH, Davis M, Jacobs DR Jr, Cartwright $Y$, et al. An after-school obesity prevention program for African-American girls: the Minnesota GEMS pilot study. Ethnicity and Disease 2003;13(Suppl 1):S54-64.

Story M, Sherwood NE, Obarzanek E, Beech BM, Baranowski JC, Thompson NS, et al. Recruitment of African-American preadolescent girls into an obesity prevention trial: the GEMS pilot studies. Ethnicity and Disease 2003;13(Suppl 1):S78-S87.

\section{Story 2012 \{published data only\}}

Story M, Hannan O, Fulkerson JA, Rock BH, Smyth M, Arcan C, et al. Bright Start: description and main outcomes from a group-randomized obesity prevention trial in American Indian children. Obesity 2012;20(11):2241-9.

Telford 2012 \{published data only\}

Telford RD, Cunningham RB, Fitzgerald R, Olive LS, Prosser L, Jiang $\mathrm{X}$, et al. Physical education, obesity, and academic achievement: a 2-year longitudinal investigation of Australian elementary school children. American Journal of Public Health 2012;102(2):368-74.

\section{Thivel 2011 \{published data only\}}

Thivel D, Isacco L, Lazaar N, Aucouturier J, Ratel S, Dore E, et al. Effect of a 6-month school-based physical activity program on body composition and physical fitness in lean and obese schoolchildren. European Journal of Pediatrics 2011;170(11):1435-43.
Velez 2010 \{published data only\}

Velez A, Golem DL, Arent SM. The impact of a 12-week resistance training program on strength, body composition, and selfconcept of Hispanic adolescents. Journal of Strength \& Conditioning Research 2010;24(4):1065-73.

Verbestel 2014 \{published data only\} Verbestel V, De Coen V, Van Winckel M, Huybrechts I, Maes L, De Bourdeaudhuij I. Prevention of overweight in children younger than 2 years old: a pilot cluster-randomized controlled trial. Public Health Nutrition 2014;17(6):1384-92.

Viggiano 2015 \{published data only\}

Viggiano A, Viggiano E, Di Costanzo A, Viggiano A, Andreozzi E, Romano V, et al. Kaledo, a board game for nutrition education of children and adolescents at school: cluster randomized controlled trial of healthy lifestyle promotion. European Journal of Pediatrics 2015;174(2):217-28.

\section{Vizcaino 2008 \{published data only\}}

Vizcaíno VM, Aguilar FS, Gutiérrez RF, Martínez MS, López MS, Martínez SS, et al. Assessment of an after-school physical activity program to prevent obesity among 9- to 10-year-old children: a cluster randomized trial. International Journal of Obesity 2008;32:12-22.

\section{Wang 2012 \{published data only\}}

Wang Y, Yuan Y, Xu GF. Evaluating the effect of school-based children obesity prevention and control. Chinese Health Service Management 2012;4:317-9.

\section{Warren 2003 \{published data only\}}

Warren JM, Henry CJ, Lightowler HJ, Bradshaw SM, Perwaiz S. Evaluation of a pilot school programme aimed at the prevention of obesity in children. Health Promotion International 2003;18(4):287-96.

\section{Weeks 2012 \{published data only\}}

Weeks BK, Beck BR. Twice-weekly, in-school jumping improves lean mass, particularly in adolescent boys. Pediatric Obesity 2012;7(3):196-204.

\section{Wen 2012 \{published data only\}}

Hayes A, Lung T, Wen LM, Baur L, Rissel C, Howard K. Economic evaluation of "healthy beginnings" an early childhood intervention to prevent obesity. Obesity 2014;22(7):1709-15.

* Wen LM, Baur LA, Simpson JM, Rissel C, Wardle K, Flood VM. Effectiveness of home based early intervention on children's BMI at age 2: randomised controlled trial. [Erratum appears in BMJ. 2013;346:f1650]. BMJ 2012;344:e3732.

\section{Whittemore 2013 \{published data only\}}

Whittemore R, Jeon S, Grey M. An internet obesity prevention program for adolescents. Journal of Adolescent Health 2013;52(4):439-47.

\section{Wilksch 2015 \{published data only\}}

Wilksch SM, Paxton SJ, Byrne SM, Austin SB, McLean SA, Thompson KM, et al. Prevention across the spectrum: a randomized controlled trial of three programs to reduce risk 
factors for both eating disorders and obesity. Psychological Medicine 2015;45(9):1811-23.

Williamson 2012 \{published data only\}

Newton RL, Thomson JL, Rau KK, Ragusa SA, Sample AD, Singleton NN, et al. Psychometric characteristics of process evaluation measures for a rural school-based childhood obesity prevention study: Louisiana Health. American Journal of Health Promotion 2011;25(6):417-21.

* Williamson DA, Champagne CM, Harsha DW, Han H, Martin CK, Newton RL Jr, et al. Effect of an environmental school-based obesity prevention program on changes in body fat and body weight: a randomized trial. Obesity 2012;20(8):1653-61.

\section{Yilmaz 2015 \{published data only\}}

Yilmaz G, Demirli CN, Karacan CD. An intervention to preschool children for reducing screen time: a randomized controlled trial. Child: care, health and development 2015;41(3):443-9.

\section{Zask 2012 \{published data only\}}

Zask A, Adams JK, Brooks LO, Hughes DF. Tooty Fruity Vegie: an obesity prevention intervention evaluation in Australian preschools. Health Promotion Journal of Australia 2012;23(1):10-5.

Zask A, Barnett LM, Rose L, Brooks LO, Molyneux M, Hughes D, et al. Three year follow-up of an early childhood intervention: is movement skill sustained?. International Journal of Behavioral Nutrition and Physical Activity 2012;9:127.

\section{References to studies excluded from this review}

\section{Adab 2014 \{published data only\}}

Adab P, Pallan MJ, Cade J, Ekelund U, Barrett T, Daley A, et al. Preventing childhood obesity, phase II feasibility study focusing on South Asians: BEACHeS. BMJ Open 2014;4(4):e004579.

\section{Alexander 2014 \{published data only\}}

Alexander AG, Grant WL, Pedrino KJ, Lyons PE. A prospective multifactorial intervention on subpopulations of predominately Hispanic children at high risk for obesity. Obesity 2014;22(1):249-53.

\section{Almas 2013 \{published data only\}}

Almas A, Muhammad I, Tazeen J. School-based physical activity programme in preadolescent girls (9-11 years): a feasibility trial in Karachi, Pakistan. Archives of Disease in Childhood 2013;98(7):515-9.

\section{Al-Nakeeb 2007 \{published data only\}}

Al-Nakeeb Y, Duncan MJ, Lyons M, Woodfield L. Body fatness and physical activity levels of young children. Annals of Human Biology 2007;34(1):1-12. [0301-4460: (Print)]

\section{Alves 2008 \{published data only\}}

Alves JG, Galé CR, Souza E, Batty GD. Effect of physical exercise on bodyweight in overweight children: a randomized controlled trial in a Brazilian slum. Cad Saude Publica 2008;24(Suppl 2):S353-9.

\section{Annesi 2015 \{published data only\}}

Annesi JJ, Howton A, Johnson PH, Porter KJ. Pilot testing a cognitive-behavioral protocol on psychosocial predictors of exercise, nutrition, weight, and body satisfaction changes in a college-level health-related fitness course. Journal of American College Health 2015;63(4):268-78.

\section{Anzman-Frasca 2013 \{published data only\}}

Anzman-Frasca S, Liu S, Gates KM, Paul IM, Rovine MJ, Birch LL. Infants' transitions out of a fussing/crying state are modifiable and are related to weight status. Infancy 2013;18(5):662-86.

\section{Ara 2006 \{published data only\}}

Ara I, Vicente-Rodriguez G, Perez-Gomez J, Jimenez-Ramirez J, Serrano-Sanchez JA, Dorado C, et al. Influence of extracurricular sport activities on body composition and physical fitness in boys: a 3-year longitudinal study. International Journal of Obesity 2006;30(7):1062-71. [0307-0565: (Print)]

\section{Arbeit 1992 \{published data only\}}

Arbeit ML, Johnson CC, Mott DS, Harsha DW, Nicklas TA, Webber LS, et al. The Heart Smart cardiovascular school health promotion: behavior correlates of risk factor change. Preventive Medicine 1992;21(1):18-32.

\section{Ask 2006 \{published data only\}}

Ask AS, Hernes S, Aarek I, Johannessen G, Haugen M. Changes in dietary pattern in 15 year old adolescents following a 4 month dietary intervention with school breakfast--a pilot study. Nutrition Journal 2006;5:33.

\section{Ask 2010 \{published data only\}}

Ask AS, Hernes S, Aarek I, Vik F, Brodahl C, Haugen M. Serving of free school lunch to secondary-school pupils - a pilot study with health implications. Public Health Nutrition 2010;13(2):238-44.

\section{Bacardi-Gascon 2012 \{published data only\}}

Bacardi-Gascon M, Pérez-Morales ME, Jiménez-Cruz A. A six month randomized school intervention and an 18-month follow-up intervention to prevent childhood obesity in Mexican elementary schools. Nutricion Hospitalaria 2012;27(3):755-62.

Balas-Nakash 2010 \{published data only\}

Balas-Nakash M, Benitez-Arciniega A, Perichart-Perera O, Valdes-Ramos R, Vadillo-Ortega F. The effect of exercise on cardiovascular risk markers in Mexican school-aged children: comparison between two structured group routines. Salud Publica de Mexico 2010;52(5):398-405.

\section{Baranowski 2012 \{published data only\}}

Baranowski T, Abdelsamad D, Baranowski J, O'Connor TM, Thompson D, Barnett A, et al. Impact of an active video game on healthy children's physical activity. Pediatrics 2012;129(3):e636-42.

\section{Beard 2012 \{published data only\}}

Beard J. The effects of traditional and progressive physical education courses on adolescents' body compositions and student satisfaction. Dissertation Abstracts International Section A: Humanities and Social Sciences 2012;73(4-A):173. 


\section{Bellows 2013b \{published data only\}}

Bellows LL, Johnson SL, Davies PL, Anderson J, Gavin WJ, Boles RE. The Colorado LEAP study: rationale and design of a study to assess the short term longitudinal effectiveness of a preschool nutrition and physical activity program. BMC Public Health 2013;13:1146.

\section{Bergh 2012a \{published data only\}}

Bergh IH, Van Stralen MM, Grydeland M, Bjelland M, Lien N, Andersen LF, et al. Exploring mediators of accelerometer assessed physical activity in young adolescents in the Health In Adolescents Study - a group randomized controlled trial. BMC Public Health 2012;12:814.

\section{Bergh 2012b \{published data only\}}

Bergh IH, Bjelland M, Grydeland M, Lien N, Andersen LF, Klepp KI, et al. Mid-way and post-intervention effects on potential determinants of physical activity and sedentary behavior, results of the HEIA study - a multi-component schoolbased randomized trial. International Journal of Behavioral Nutrition and Physical Activity 2012;9:63.

\section{Berry 2007 \{published data only\}}

Berry D, Savoye M, Melkus G, Grey M. An intervention for multiethnic obese parents and overweight children. Applied Nursing Research 2007;20(2):63-71.

\section{Berry 2011 \{published data only\}}

Berry D, Colindres M, Sanchez-Lugo L, Sanchez M, Neal M, Smith-Miller C. Adapting, feasibility testing, and pilot testing a weight management intervention for recently immigrated Spanish-speaking women and their 2- to 4-year-old children. Hispanic Health Care International 2011;9(4):186-93.

\section{Berry 2014 \{published data only\}}

Berry DC, McMurray RG, Skelly AH, Neal M, Hall EG, Aimyong N, et al. The family partners for health study: A cluster randomized controlled trial for child and parent weight management. Nutrition and Diabetes 2014;4:e101.

\section{Bilic-Kirin 2013 \{published data only\}}

Bilic-Kirin V, Burazin J, Buljan V, Milicic V, Gmajnic R. Influence of health education regarding correct diet on anthropometric indexes in children. Collegium Antropologicum 2013;37(4):1089-94

\section{Birch 2012 \{published data only\}}

Birch LL, Anzman-Frasca S, Paul IM. Starting early: obesity prevention during infancy. Nestle Nutrition Institute Workshop Series 2012;73:81-94.

\section{Bjelland 2015 \{published data only\}}

Bjelland M, Hausken SE, Bergh IH, Grydeland M, Klepp K-I, Andersen LF, et al. Changes in adolescents' and parents' intakes of sugar-sweetened beverages, fruit and vegetables after 20 months: results from the HEIA study - a comprehensive, multicomponent school-based randomized trial. Food and Nutrition Research 2015;59:25932.

\section{Bollela 1999a \{published data only\}}

Bollella MC, Boccia LA, Nicklas TA, Lefkowitz KB, Pittman BP, Zang EA, et al. Assessing dietary intake in preschool children: The Healthy Start Project - New York. Nutrition Research 1999;19(1):37-48

Bollela 1999b \{published data only\}

Bollella MC, Spark A, Boccia LA, Nicklas TA, Pittman BP, Williams CL. Nutrient intake of Head Start children: home vs. school. Journal of the American College of Nutrition 1999;18(2):108-14.

\section{Borys 2000 \{published data only\}}

Borys J-M, Lafay L. Nutritional education of children to influence the dietary habits of all the family [L'information nutritionelle des enfants pour modifier les habitudes alimentaires de toute la famille]. Revue Medicale de la Suisse Romande 2000;120:207-9.

\section{Briganti 2014 \{published data only\}}

Briganti S. The Italian E.A.T. Project: effectiveness of a multicomponent school-based health promotion study on measures of fatness and behavior in teenagers. Eating and Weight Disorders 2014;Conference:444-5.

Bruss 2010 \{published data only\}

Bruss MB, Michael TJ, Morris JR, Applegate B, Dannison L, Quitugua JA, et al. Childhood obesity prevention: an intervention targeting primary caregivers of school children. Obesity 2010;18(1):99-107.

\section{Buchan 2010 \{published data only\}}

Buchan DS, Ollis S, Thomas NE, Baker JS. The influence of a high intensity physical activity intervention on a selection of health related outcomes: an ecological approach. BMC Public Health 2010;10:8.

\section{Burguera 2011 \{published data only\}}

Burguera B, Colom A, Pinero E, Yanez A, Caimari M, Tur J, et al. ACTYBOSS: activity, behavioral therapy in young subjects--afterschool intervention pilot project on obesity prevention. Obesity Facts 2011;4(5):400-6.

\section{Burke 1998 \{published data only\}}

Burke V, Milligan RA, Thompson C, Taggart AC, Dunbar DL, Spencer MJ, et al. A controlled trial of health promotion programs in 11-year-olds using physical activity "enrichment" for higher risk children. Journal of Pediatrics 1998;132(5):840-8.

\section{Buscemi 2015 \{published data only\}}

Buscemi J, Blumstein L, Kong A, Stolley MR, Schiffer L, Odoms-Young A, et al. Retaining traditionally hard to reach participants: lessons learned from three childhood obesity studies. Contemporary Clinical Trials 2015;42:98-104.

\section{Cairella 1998 \{published data only\}}

Cairella G, Romagnoli F, Cantarelli P, Valentini P, Tarsitani G. School oriented intervention on dietary education: results of phase 1. International Journal of Obesity 1998;22:S254 (Abstract). 


\section{Cameron 2014 \{published data only\}}

Cameron SL, Gray AR, Taylor RW, Lawrence JA, Galland BC, Hanna MB, et al. Excessive growth from 6 to 24 months of age: results from the prevention of overweight in infancy (POI) randomised controlled trial. Archives of Disease in Childhood 2014; Conference:var.pagings.

\section{Campbell 2014 \{published data only\}}

Campbell F, Conti G, Heckmann JJ, Moon SH, Pinto R, Pungello $E$, et al. Early childhood investments substantially boost adult health. Science 2014;343(6178):1478-85.

\section{Carrel 2005 \{published data only\}}

Carrel AL, Clark RR, Peterson S, Eickhoff J, Allen DB. Schoolbased fitness changes are lost during the summer vacation. Archives of Pediatrics and Adolescent Medicine 2007;161:561-4.

* Carrel AL, Clark RR, Peterson SE, Nemeth BA, Sullivan J, Allen DB. Improvement of fitness, body composition, and insulin sensitivity in overweight children in a school-based exercise program. Archives of Pediatrics and Adolescent Medicine 2005;159:963-8.

\section{Casazza 2006 \{published data only\}}

Casazza K, Ciccazzo M. The method of delivery of nutrition and physical activity information may play a role in eliciting behavior changes in adolescents. Eating Behaviours 2007;8:73-82.

\section{Centis 2012 \{published data only\}}

Centis E, Marzocchi R, Di Luzio R, Moscatiello S, Salardi S, Villanova N, et al. GC Croce School of Health Promotion. A controlled, class-based multicomponent intervention to promote healthy lifestyle and to reduce the burden of childhood obesity. Pediatric Obesity 2012;7(6):436-45.

\section{Chomitz 2003 \{published data only\}}

Chomitz VR, Collins J, Kim J, Kramer E, McGowan R. Promoting healthy weight among elementary school children via a health report card approach. Archives of Pediatrics \& Adolescent Medicine 2003;157(8):765-72.

\section{Copeland 2010 \{published data only\}}

Copeland AL. A school-based alcohol, tobacco, and drug prevention program for children: the wise mind study. Cognitive Therapy and Research 2010;34(6):522-32.

\section{Cordova 2012 \{published data only\}}

Cordova A, Gerardo V, Antoni S, Jose R-M. Physical activity and cardiovascular risk factors in Spanish children aged 11-13 years. Revista Espanola de Cardiologia 2012;65(7):620-6.

\section{Cruz 2014 \{published data only\}}

Cruz TH, Davis SM, FitzGerald CA, Canaca GF, Keane PC. Engagement, recruitment, and retention in a trans-community, randomized controlled trial for the prevention of obesity in rural American Indian and Hispanic children. Journal of Primary Prevention 2014;35(3):135-49.

\section{Cullen 1996 \{published data only\}}

Cullen KJ, Cullen AM. Long-term follow-up of the Busselton six-year controlled trial of prevention of children's behaviour disorders. Journal of Pediatrics 1996;129(1):136-9.

D'Agostino 1999 \{published data only\}

D'Agostino CD, D'Andrea T, Talbot -Nix S, Williams CL. Increasing nutrition knowledge in preschool children: the Healthy Start Project, Year 1. Journal of Health Education 1999;30(4):217-21.

Daley 2006 \{published data only\}

Daley AJ, Copeland RJ, Wright NP, Roalfe A, Wales JK. Exercise therapy as a treatment for psychopathologic conditions in obese and morbidly obese adolescents: a randomized, controlled trial. Pediatrics 2006; Vol. 118, issue 5:2126-34.

Daniels 2014 \{published data only\}

Daniels LA, Mallan KM, Battistutta, Nicholson JM, Meedeniya JE, Bayer JK, et al. Child eating behavior outcomes of an early feeding intervention to reduce risk indicators for child obesity: the NOURISH RCT. Obesity 2014;22(5):E104-11.

Danielzik 2005 \{published data only\}

Danielzik S, Pust S, Landsberg B, Müller MJ. First lessons from the Kiel Obesity Prevention Study (KOPS). International Journal of Obesity 2005;29(S2):S78-83.

da Silva 2013 \{published data only\}

da Silva LS, Fisberg M, de Souza Pires MM, Nassar SM, Sottovia CB. The effectiveness of a physical activity and nutrition education program in the prevention of overweight in schoolchildren in Criciuma, Brazil. European Journal of Clinical Nutrition 2013;67(11):1200-4

Davis 2011 \{published data only\}

Davis JN, Ventura EE, Cook LT, Gyllenhammer LE, Gatto NM, Davis JN. LA Sprouts: a gardening, nutrition, and cooking intervention for Latino youth improves diet and reduces obesity. Journal of the American Dietetic Association 2011;111(8):1224-30.

\section{DeBar 2011 \{published data only\}}

DeBar LL. Student public commitment in a school-based diabetes prevention project: impact on physical health and health behavior. BMC Public Health 2011;11:711.

\section{De Bock 2010 \{published data only\}}

De Bock F, Fischer JE, Hoffmann K, Renz-Polster H. A participatory parent-focused intervention promoting physical activity in preschools: design of a cluster-randomized trial. [Erratum appears in BMC Public Health. 2012;12:355]. BMC Public Health 2010;10:49.

Della 2013 \{published data only\} Della Santa AP. Study of the effectiveness of an educational model to prevent and control childhood obesity. Annals of Nutrition and Metabolism 2013;Conference:var.pagings.

\section{De Ruyter 2013 \{published data only\}}

De Ruyter JC, Katan MB, Kuijper LD, Liem DG, Olthof MR. The effect of sugar-free versus sugar-sweetened beverages on 
satiety, liking and wanting: an 18 month randomized doubleblind trial in children. PLOS ONE 2013;8(10):e78039.

\section{de Silva-Sanigorski 2010a \{published data only\}}

de Silva-Sanigorski AM, Bell AC, Kremer P, Nichols M, Crellin M, Smith M, et al. Reducing obesity in early childhood: results from Romp \& Chomp, an Australian community-wide intervention program. American Journal of Clinical Nutrition 2010;91(4):831-40.

\section{de Silva-Sanigorski 2010b \{published data only\}}

de Silva-Sanigorski AM, Bolton K, Haby M, Kremer P, Gibbs L, Waters $\mathrm{E}$, et al. Scaling up community-based obesity prevention in Australia: background and evaluation design of the Health Promoting Communities: Being Active Eating Well initiative. BMC Public Health 2010;10:65.

\section{de Silva-Sanigorski 2010c \{published data only\}}

de Silva-Sanigorski A, Prosser L, Carpenter L, Honisett S, Gibbs L, Moodie M, et al. Evaluation of the childhood obesity prevention program Kids--'Go for your life'. BMC Public Health 2010;10:288.

\section{de Silva-Sanigorski 2012 \{published data only\}}

de Silva-Sanigorski AM, Bell AC, Kremer P, Park J, Demajo L, Smith M, et al. Process and impact evaluation of the Romp \& Chomp obesity prevention intervention in early childhood settings: lessons learned from implementation in preschools and long day care settings. Childhood Obesity 2012;8(3):205-15.

\section{Dixon 2000 \{published data only\}}

Dixon LB, Tershakovec AM, McKenzie J, Shannon B. Diet quality of young children who received nutrition education promoting lower dietary fat. Public Health Nutrition 2000;3(4):411-6.

\section{Donnelly 1996 \{published data only\}}

Donnelly JE, Jacobsen DJ, Whatley JE, Hill JO, Swift LL, Cherrington $\mathrm{A}$, et al. Nutrition and physical activity program to attenuate obesity and promote physical and metabolic fitness in elementary school children. Obesity Research 1996;4(3):229-43.

\section{Donnelly 2011 \{published data only\}}

Donnelly JE. Classroom-based physical activity, cognition, and academic achievement. Preventive medicine 2011;52(Suppl):S36-42.

\section{Donnelly 2013 \{published data only\}}

Donnelly JE, Greene JL, Gibson CA, Sullivan DK, Hansen DM, Hillman $\mathrm{CH}$, et al. Physical activity and academic achievement across the curriculum (A + PAAC): rationale and design of a 3year, cluster-randomized trial. BMC Public Health 2013;13:307.

\section{Dzewaltowski 2010 \{published data only\}}

Dzewaltowski DA. HOP'N after-school project: an obesity prevention randomized controlled trial. International Journal of Behavioral Nutrition and Physical Activity 2010;7:90.

\section{Economos 2007 \{published data only\}}

Economos CD, Hyatt RR, Goldberg JP, Must A, Naumova EN, Collins JJ, et al. A community intervention reduces $\mathrm{BMI}$ z-score in children: Shape Up Somerville first year results. Obesity 2007;15(5):1325-36.

\section{Economos 2013 \{published data only\}}

Economos CD, Hyatt RR, Must A, Goldberg JP, Kuder J, Naumova EN, et al. Shape Up Somerville two-year results: a community-based environmental change intervention sustains weight reduction in children. Preventive medicine 2013;57(4):322-7.

\section{Ehlert 2010 \{published data only\}}

Ehlert JJ. SNAAKS: student nutrition attitudes, action, knowledge, and skills: a nutrition program tailored to the needs of predominantly black and Hispanic college freshmen. Dissertation Abstracts International: Section B: The Sciences and Engineering 2010;71(3-B):234.

Elinder 2012 \{published data only\}

Elinder LS, Heinemans N, Hagberg J, Quetel A-K, Hagstromer M. A participatory and capacity-building approach to healthy eating and physical activity- SCIP-school: a 2-year controlled trial. International Journal of Behavioral Nutrition and Physical Activity 2012;9:145.

\section{Eskicioglu 2014 \{published data only\}}

Eskicioglu P, Halas J, Senechal M, Wood L, McKay E, Villeneuve $S$, et al. Peer mentoring for type 2 diabetes prevention in first nations children. Pediatrics 2014;133(6):e1624-31.

\section{Evans 2012 \{published data only\}}

Evans EM, Schmidt MD, Mojtahedi MC, Guest DD, Das BM, Mailey EL. Peer led behavioral intervention and changes in weight status in female college freshman. FASEB journal 2012;26(1 Suppl):257.3.

\section{Fitzgibbon 2013 \{published data only\}}

Fitzgibbon ML, Stolley MR, Schiffer L, Kong A, Braunschweig CL, Gomez-Perez SL, et al. Family-based hip-hop to health: outcome results. Obesity 2013;21(2):274-83.

\section{Flattum 2011 \{published data only\}}

Flattum C, Friend Sa, Story M, Neumark-Sztainer D. Evaluation of an individualized counseling approach as part of a multicomponent school-based program to prevent weightrelated problems among adolescent girls. Journal of the American Dietetic Association 2011;111(8):1218-23.

Flodmark 1993 \{published data only\}

Flodmark CE, Ohlsson T, Ryden O, Sveger T. Prevention of progression to severe obesity in a group of obese school children treated with family therapy. Pediatrics 1993;91(5):880-4.

\section{Florea 2005 \{published data only\}}

Florea IM. The Goodbodies Program: physical activity and motor skill performance influences on fitness, and body composition in overweight children. University of South Carolina. United States -- South Carolina: University of South Carolina, 2005; Vol. AAT 3181946:by Florea, Ioana Madalina,. 
Flores 1995 \{published data only\}

Flores R. Dance for health: improving fitness in African American and Hispanic adolescents. Public Health Reports 1995;110(2):189-93.

\section{Fonseca 2007 \{published data only\}}

Fonseca H, Matos MG. Obesity, overweight and lifestyle/ psychosocial associated factors: a comparative study in a four year time. Journal of Adolescent Health 2007; Vol. 40, issue 2 Suppl 1:S35-6.

\section{Fotu 2011 \{published data only\}}

Fotu KF. Outcome results for the Ma'alahi Youth Project, a Tongan community-based obesity prevention programme for adolescents. Obesity Reviews 2011;12 Suppl 2:41-50.

Francis 2010 \{published data only\}

Francis M, Nichols SS, Dalrymple N. The effects of a schoolbased intervention programme on dietary intakes and physical activity among primary-school children in Trinidad and Tobago. Public Health Nutrition 2010;13(5):738-47.

\section{French 2012 \{published data only\}}

French GM, Nicholson L, Skybo T, Klein EG, Schwirian PM, Murray-Johnson L, et al. An evaluation of mother-centered anticipatory guidance to reduce obesogenic infant feeding behaviors. Pediatrics 2012;130(3):e507-17.

\section{Frenn 2013 \{published data only\}}

Frenn M, Pruszynski JE, Felzer H, Zhang J. Authoritative feeding behaviors to reduce child BMI through online interventions. Journal for Specialists in Pediatric Nursing 2013;18(1):65-77.

\section{Gabriele 2010 \{published data only\}}

Gabriele JM, Stewart TM, Sample GJ. Development of an internet-based obesity prevention program for children. Journal of Diabetes Science and Technology 2010;4(3):723-32.

\section{Gao 2013 \{published data only\}}

Gao Z, Hannan P, Xiang P, Stodden DF, Valdez VE. Video game-based exercise, Latino children's physical health, and academic achievement. American Journal of Preventive Medicine 2013;44(3 Suppl 3):S240-S246.

\section{Gately 2005 \{published data only\}}

Gately PJ, Cooke CB, Barth JH, Bewick BM, Radley D, Hill AJ. Children's residential weight-loss programs can work: a prospective cohort study of short-term outcomes for overweight and obese children. Pediatrics 2005;116(1):73-7. [1098-4275: (Electronic)]

\section{Gatti 2015 \{published data only\}}

Gatti C, Suhas E, Côté S, Anassour Laouan-Sidi E, Dewailly É, Lucas M. Obesity and metabolic parameters in adolescents: a school-based intervention program in French Polynesia. Journal of Adolescent Health 2015;56(2):174-80.

\section{Gatto 2015 \{published data only\}}

Gatto NM, Martinez LC, Spruijt-Metz D, Davis JN. LA Sprouts randomized controlled nutrition and gardening program reduces obesity and metabolic risk in Latino youth. Obesity 2015;23(6):1244-51.

\section{Gesell 2012 \{published data only\}}

Gesell SB. Understanding the social networks that form within the context of an obesity prevention intervention. Journal of Obesity 7498;2012:749832.

\section{Goldfield 2006 \{published data only\}}

Goldfield GS, Mallory R, Parker T, Cunningham T, Legg C, Lumb A, et al. Effects of open-loop feedback on physical activity and television viewing in overweight and obese children: a randomized, controlled trial. Pediatrics 2006;118(1):e157-66.

Goldfield 2007 \{published data only\}

Goldfield GS, Mallory R, Parker T, Cunningham T, Legg C, Lumb A, et al. Effects of modifying physical activity and sedentary behavior on psychosocial adjustment in overweight/obese children. Journal of Pediatric Psychology 2007;32(7):783-93.

\section{Graf 2011 \{published data only\}}

Graf C, Dordel S. The CHILT I project (Children's Health Interventional Trial). A multicomponent intervention to prevent physical inactivity and overweight in primary schools. Bundesgesundheitsblatt, Gesundheitsforschung, Gesundheitsschutz 2011;54(3):313-21.

\section{Greening 2011 \{published data only\}}

Greening L, Harrell KT, Low AK, Fielder CE. Efficacy of a schoolbased childhood obesity intervention program in a rural southern community: TEAM Mississippi Project. Obesity 2011;19(6):1213-9.

\section{Hakanen 2010 \{published data only\}}

Hakanen M, Lagström H, Pahkala K, Sillanmäki L, Saarinen M, Niinikoski $\mathrm{H}$, et al. Dietary and lifestyle counselling reduces the clustering of overweight-related cardiometabolic risk factors in adolescents. Acta Paediatrica 2010;99(6):888-95.

\section{Hardy 2010 \{published data only\}}

Hardy LL, King L, Kelly B, Farrell L, Howlett S. Munch and Move: evaluation of a preschool healthy eating and movement skill program. International Journal of Behavioral Nutrition and Physical Activity 2010;7:80.

\section{Harrell 1998 \{published data only\}}

Harrell JS, Gansky SA, McMurray RG, Bangdiwala SI, Frauman AC, Bradley CB. School-based interventions improve heart health in children with multiple cardiovascular disease risk factors. Pediatrics 1998;102 (2 Pt 1):371-80.

\section{Harrell 1999 \{published data only\}}

Harrell JS, McMurray RG, Gansky SA, Bangdiwala SI, Bradley CB. A public health vs a risk-based intervention to improve cardiovascular health in elementary school children: the Cardiovascular Health in Children Study. American Journal of Public Health 1999;89(10):1529-35. 


\section{Hartmann 2010 \{published data only\}}

Hartmann T, Zahner L, Pühse U, Schneider S, Puder JJ, Kriemler S. Physical activity, bodyweight, health and fear of negative evaluation in primary school children. Scandinavian Journal of Medicine \& Science in Sports 2010;20(1):e27-34.

Hatzis 2010 \{published data only\}

Hatzis CM, Papandreou C, Kafatos AG. School health education programs in Crete: evaluation of behavioural and health indices a decade after initiation. Preventive Medicine 2010;51(3-4):262-7.

\section{Hauner 2012 \{published data only\}}

Hauner H, Much D, Vollhardt C, Brunner S, Schmid D, Sedlmeier E-M, et al. Effect of reducing the n-6:n-3 longchain PUFA ratio during pregnancy and lactation on infant adipose tissue growth within the first year of life: an openlabel randomized controlled trial. American Journal of Clinical Nutrition 2012;95(2):383-94.

\section{Hawthorne 2011 \{published data only\}}

Hawthorne A, Shaibi G, Gance-Cleveland B, McFall S. Grand Canyon Trekkers: school-based lunchtime walking program. Journal of School Nursing 2011;27(1):43-50.

\section{He 2004 \{published data only\}}

He Y-F, Wang W-Y, Fu P, Sun Y, Yu S-Y, Chen R, et al. Effects of a comprehensive intervention programme on simple obesity of children in kindergarten. Chinese Journal of Pediatrics 2004;42(5):1-6.

\section{Hernandez 2014 \{published data only\}}

Hernandez AE, Marcus M, Hirst K, Faith MS, Goldberg L, Treviño RP. Impact of implementation and conduct of the HEALTHY primary prevention trial on student performance. American Journal of Health Promotion 2014;29(1):55-8.

\section{Herrick 2012 \{published data only\}}

Herrick H, Thompson H, Kinder J, Madsen KR. Use of SPARK to promote after-school physical activity. Journal of School Health 2012;82(10):457-61.

\section{Hoelscher 2010 \{published data only\}}

Hoelscher DM, Springer AE, Ranjit N, Perry CL, Evans AE, Stigler M, et al. Reductions in child obesity among disadvantaged school children with community involvement: the Travis County CATCH Trial. Obesity 2010;18 Suppl 1:S36-44.

\section{Hoffman 2011 \{published data only\}}

Hoffman JA, Thompson DR, Franko DL, Power TJ, Leff SS, Stallings VA. Decaying behavioral effects in a randomized, multiyear fruit and vegetable intake intervention. Preventive Medicine 2011;52(5):370-5.

\section{Hollar 2010 \{published data only\}}

Hollar D, Messiah SE, Lopez-Mitnik G. Effect of a two-year obesity prevention intervention on percentile changes in body mass index and academic performance in low-income elementary school children. American Journal of Public Health 2010;100(4):646-53.

\section{Hollar 2010a \{published data only\}}

Hollar D, Messiah SE, Lopez-Mitnik G. Healthier options for public schoolchildren program improves weight and blood pressure in 6- to 13-year-olds. Journal of the American Dietetic Association 2010;110(2):261-7.

Hollar 2010b \{published data only\}

Hollar D, Lombardo M, Lopez-Mitnik G, Hollar TL, Almon M, Agatston AS, et al. Effective multi-level, multi-sector, schoolbased obesity prevention programming improves weight, blood pressure, and academic performance, especially among lowincome, minority children. Journal of Health Care for the Poor and Underserved 2010;21(2 Suppl):93-108.

Hollywood 2013 \{published data only\} Hollywood E, Comiskey C, Begley T, Snel A, O'Sullivan K, Quirke $\mathrm{M}$, et al. Measuring and modelling body mass index among a cohort of urban children living with disadvantage. Journal of Advanced Nursing 2013;69(4):851-61.

Hopper 1996 \{published data only\}

Hopper CA, Gruber MB, Munoz KD, MacConnie SE. School-based cardiovascular exercise and nutrition programs with parent participation. Journal of Health Education 1996;27(5):S32-S39.

\section{Horodynski 2004 \{published data only\}}

Horodynski MA, Hoerr S, Coleman G. Nutrition education aimed at toddlers: a pilot program for rural low-income families. Family and Community 2004;27(4):103-13.

\section{Howard 1996 \{published data only\}}

Howard JK, Bindler RM, Synoground G, Van Gemet FC. A cardiovascular risk reduction program for the classroom. Journal of School Nursing 1996;12(4):4-11.

\section{Huang 2012 \{published data only\}}

Huang SY, Hogg J, Zandieh S, Bostwick SB. A ballroom dance classroom program promotes moderate to vigorous physical activity in elementary school children. American Journal of Health Promotion 2012;26(3):160-5.

\section{Huberty 2011 \{published data only\}}

Huberty JL, Beets MW, Beighle A, Welk G. Environmental modifications to increase physical activity during recess: preliminary findings from ready for recess. Journal of Physical Activity \& Health 2011;8 Suppl 2:S249-56.

\section{Ildiko 2007 \{published data only\}}

Ildikó V, Zsófia M, János M, Andreas P, Dóra NE, András P, et al. Activity-related changes of body fat and motor performance in obese seven-year-old boys. Journal of Physiological Anthropology 2007;26(3):333-7.

\section{Jago 2006 \{published data only\}}

Jago R, Jonker ML, Missaghian M, Baranowski T. Effect of 4 weeks of Pilates on the body composition of young girls. Preventive Medicine 2006;42(3):177-80.

\section{Jago 2011 \{published data only\}}

Jago R, McMurray RG, Drews KL, Moe EL, Murray T, Pham TH, et al. HEALTHY intervention: fitness, physical activity, and 
metabolic syndrome results. Medicine and Science in Sports and Exercise 2011;43(8):1513-22.

\section{Jensen 2013a \{published data only\}}

Jensen BW, Nielsen BM, Husby I, Bugge A, El-Naaman B, Andersen LB, et al. Association between sweet drink intake and adiposity in Danish children participating in a long-term intervention study. Pediatric Obesity 2013;8(4):259-70.

\section{Jensen 2013b \{published data only\}}

Jensen BW, Nichols M, Allender S, de Silva-Sanigorski A, Millar L, Kremer P, Lacy K, Swinburn B. Inconsistent associations between sweet drink intake and 2-year change in BMI among Victorian children and adolescents. Pediatric Obesity 2013;8(4):271-83.

\section{Jiang 2007 \{published data only\}}

Jiang J, Xia X, Greiner T, Wu G, Lian G, Rosenqvist U. The effects of a 3-year obesity intervention in schoolchildren in Beijing. Child Care Health Development 2007;33(5):641-6.

\section{Johnson 2012 \{published data only\}}

Johnson BA, Kremer PJ, Swinburn BA, de Silva-Sanigorski AM, Johnson BA. Multilevel analysis of the Be Active Eat Well intervention: environmental and behavioural influences on reductions in child obesity risk. International Journal of Obesity 2012;36(7):901-7.

\section{Johnston 2012 \{published data only\}}

Johnston JD, Massey AP, Marker-Hoffman RL. Using an alternate reality game to increase physical activity and decrease obesity risk of college students. Journal of Diabetes Science and Technology 2012;6(4):828-38.

\section{Jurg 2006 \{published data only\}}

Jurg ME, Kremers SP, Candel MJ, Van der Wal MF, De Meij JS. A controlled trial of a school-based environmental intervention to improve physical activity in Dutch children: JUMP-in, kids in motion. Health Promotion International 2006;21(4):320-30.

\section{Karanja 2010 \{published data only\}}

Karanja N, Lutz T, Ritenbaugh C, Maupome G, Jones J, Becker T, et al. The TOTS community intervention to prevent overweight in American Indian toddlers beginning at birth: a feasibility and efficacy study. Journal of Community Health 2010;35(6):667-75.

\section{Karanja 2012 \{published data only\}}

Karanja N, Aickin M, Lutz T, Mist S, Jobe J. A community-based intervention to prevent obesity beginning at birth among American Indian children: study design and rationale for the PTOTS study. Journal of Primary Prevention 2012;33(4):161-74.

\section{Karp 2014 \{published data only\}}

Karp SM, Barry KM, Gesell SB, Po'e EK, Dietrich MS, Barkin SL. Parental feeding patterns and child weight status for Latino preschoolers. Obesity Research \& Clinical Practice 2014;8(1):e88-97.

\section{Katz 2010 \{published data only\}}

Katz DL, Cushman D, Reynolds J, Njike V, Treu JA, Walker J, et al. Putting physical activity where it fits in the school day: preliminary results of the $\mathrm{ABC}$ (Activity Bursts in the Classroom) for fitness program. Preventing Chronic Disease 2010;7(4):A82.

Katz 2011 \{published data only\}

Katz DL. Teaching healthful food choices to elementary school students and their parents: the nutrition detectives program. Journal of School Health 2011;81(1):21-8.

\section{Kesztyus 2013 \{published data only\}}

Kesztyus D, Schreiber A, Wirt T, Wiedom M, Dreyhaupt J, Brandstetter S, et al. Economic evaluation of URMEL-ICE, a school-based overweight prevention programme comprising metabolism, exercise and lifestyle intervention in children. European Journal of Health Economics 2013;14(2):185-95.

\section{Kilanowski 2010 \{published data only\}}

Kilanowski JF. Nutrition \& physical education in the summer migrant classroom (MEP). Clinical and Translational Science 2010; Conference(var.pagings):S30-1.

Kim 2014 \{published data only\}

Kim N, Seo D-C, King M. Long-term predictors of blood pressure among adolescents during an 18-month school-based obesity prevention intervention. Journal of Adolescent Health 2014;55(4):521-7.

\section{King 2014 \{published data only\}}

King MH, Lederer AM, Sovinski D, Knoblock HM, Meade RK, Seo DC, et al. Implementation and evaluation of the HEROES initiative: a tri-state coordinated school health program to reduce childhood obesity. Health Promotion Practice 2014;15(3):395-405.

\section{Klakk 2013 \{published data only\}}

Klakk H, Chinapaw M, Heidemann M, Andersen LB, Wedderkopp N. Effect of four additional physical education lessons on body composition in children aged 8-13 years-a prospective study during two school years. BMC Pediatrics 2013;13:170.

\section{Klish 2012 \{published data only\}}

Klish WJ, Karavias KE, White KS, Balch AJ, Fraley JK, Mikhail C, et al. Multicomponent school-initiated obesity intervention in a high-risk, Hispanic elementary school. Journal of Pediatric Gastroenterology and Nutrition 2012;54(1):113-6.

Koblinsky 1992 \{published data only\} Koblinsky SA, Guthrie JF, Lynch L. Evaluation of a nutrition education program for Head Start parents. Society for Nutrition Education 1992;24:No 1

\section{Kremer 2011 \{published data only\}}

Kremer P, Waqa G, Vanualailai N, Schultz JT, Roberts G, Moodie M, et al. Reducing unhealthy weight gain in Fijian adolescents: results of the Healthy Youth Healthy Communities study. Obesity Reviews 2011;12 Suppl 2:29-40.

Krombholz 2012 \{published data only\}

Krombholz H. The impact of a 20-month physical activity intervention in child care centers on motor performance and 
weight in overweight and healthy-weight preschool children. Perceptual and Motor Skills 2012;115(3):919-32.

\section{LaBresh 2014 \{published data only\}}

LaBresh KA, Ariza AJ, Lazorick S, Furberg RD, Whetstone L, Hobbs $C$, et al. Adoption of cardiovascular risk reduction guidelines: a cluster-randomized trial. Pediatrics 2014;134(3):e732-8.

\section{Lachausse 2012 \{published data only\}}

Lachausse RG. My student body: effects of an internet-based prevention program to decrease obesity among college students. Journal of American College Health 2012;60(4):324-30.

\section{Lagstrom 1997 \{published data only\}}

Lagstrom H, Jokienen E, Seppanen R, Ronnemaa T, Viikari J, Valimaki I, et al. Nutrient intakes by young children in a prospective randomized trial of a low-saturated fat, lowcholesterol diet. Archives of Pediatric and Adolescence Medicine 1997;151:181-8.

\section{Lakes 2013 \{published data only\}}

Lakes KD, Bryars T, Sirisinahal S, Salim N, Arastoo S, Emmerson N, et al. The Healthy for Life Taekwondo pilot study: a preliminary evaluation of effects on executive function and BMI, feasibility, and acceptability. Mental Health and Physical Activity 2013;6(3):181-8.

\section{Lambourne 2013 \{published data only\}}

Lambourne K, Washburn RA, Lee J, Betts JL, Thomas DT, Smith BK, et al. A 6-month trial of resistance training with milk supplementation in adolescents: effects on body composition. International Journal of Sport Nutrition and Exercise Metabolism 2013;23(4):344-56.

\section{Lazorick 2014 \{published data only\}}

Lazorick S, Crawford Y, Gilbird A, Fang X, Burr V, Moore V, et al. Long-term obesity prevention and the motivating adolescents with technology to choose health program. Childhood Obesity 2014;10(1):25-33.

\section{LeMaster 2011 \{published data only\}}

LeMaster J, Matisziw T, McElroy J, Nilon C, Sayers S, Stanis S. Playgrounds without borders: methods for a playground environmental intervention among U.S. schoolchildren. Internet Journal of Epidemiology 2011;10(1):No pagination.

\section{Li 2010b \{published data only\}}

Li Y, Hu X, Zhang Q, Liu A, Fang H, Hao L, et al. The nutritionbased comprehensive intervention study on childhood obesity in China (NISCOC): a randomised cluster controlled trial. BMC Public Health 2010;10:229.

\section{Lin 2012 \{published data only\}}

Lin ST, Xu ZY, Wang JJ, Li BH, Pei ZC, Wang HJ, et al. Physical activities and dietary intervention on metabolic syndrome in children. Chung-Hua Liu Hsing Ping Hsueh Tsa Chih Chinese Journal of Epidemiology 2012;33(2):135-9.
Lionis 1991 \{published data only\}

Lionis C, Kafatos A, Vlachonikolis J, Vakaki M, Tzortzi M, Petraki A. The effects of a health education intervention program among Cretan adolescents. Preventive Medicine 1991;20(6):685-99.

\section{Llaurado 2014 \{published data only\}}

Llaurado E, Tarro L, Moriña D, Queral R, Giralt M, Solà R. EdAl-2 (Educacio en Alimentacio) programme: reproducibility of a cluster randomised, interventional, primary school-based study to induce healthier lifestyle activities in children. BMJ Open 2014;4(11):e005496.

\section{Lloyd 2012 \{published data only\}}

Lloyd JJ, Wyatt KM, Creanor S. Behavioural and weight status outcomes from an exploratory trial of the Healthy Lifestyles Programme (HeLP): a novel school-based obesity prevention programme. BMJ Open 2012;2(3):e000390.

\section{Louzada 2012 \{published data only\}}

Louzada ML, Campagnolo PD, Rauber F, Vitolo MR. Longterm effectiveness of maternal dietary counseling in a lowincome population: a randomized field trial. Pediatrics 2012;129(6):e1477-84.

\section{Lubans 2012b \{published data only\}}

Lubans DR, Morgan PJ, Collins CE, Okely AD, Burrows R. Mediators of weight loss in the 'Healthy Dads, Healthy Kids' pilot study for overweight fathers. International Journal of Behavioral Nutrition \& Physical Activity 2012;9:45.

\section{Luepker 1996 \{published data only\}}

Luepker RV, Perry CL, McKinlay SM, Nader PR, Parcel GS, Stone EJ, et al. Outcomes of a field trial to improve children's dietary patterns and physical activity: the child and adolescent trial for cardiovascular health (CATCH). JAMA 1999;275(10):768-76.

\section{Lumeng 2015 \{published data only\}}

Lumeng JC. Changes in body mass index associated with Head Start participation. Pediatrics 2015;135(2):e449-56.

Lynch 2012 \{published data only\}

Lynch WC, Martin J, Eldridge G, Bailey SJ, Benke C, Paul L. Childhood obesity prevention in rural settings: background, rationale, and study design of '4-Health,' a parent-only intervention. BMC Public Health 2012;12:255.

Lytle 2006 \{published data only\}

Lytle LA, Kubik MY, Perry C, Story M, Birnbaum AS, Murray DM. Influencing healthful food choices in school and home environments: results from the TEENS study. Preventative Medicine 2006;43(1):8-13.

\section{Maggiulli 2015 \{published data only\}}

Maggiulli LR. A quasi-experimental study of a health and wellness group intervention for adolescents in residential treatment facilities (RTFs) and examination of risk factors as treatment success predictors. Dissertation Abstracts International Section A: Humanities and Social Sciences 2015;75(11-AE):191. 
Mailey 2012 \{published data only\}

Mailey EL. Unobserved mental health profiles are associated with weight and physical activity change in female college freshmen: a latent profile analysis. Mental Health and Physical Activity 2012;5(1):76-84.

\section{Manger 2012 \{published data only\}}

Manger WM, Manger LS, Minno AM, Killmeyer M, Holzman RS, Schullinger JN, et al. Obesity prevention in young schoolchildren: results of a pilot study. Journal of School Health 2012;82(10):462-8.

\section{Manios 1998 \{published data only\}}

Manios Y, Kafatos A, Mamalakis G. The effects of a health education intervention initiated at first grade over a 3 year period: physical activity and fitness indices. Health Education Research 1998;13(4):593-606.

\section{Manios 1999 \{published data only\}}

Manios Y, Moschandreas J, Hatzis C, Kafatos A. Evaluation of a health and nutrition education program in primary school children of Crete over a three-year period. Preventive Medicine 1999;28:149-59.

\section{Martinez-Andrade 2014 \{published data only\}}

Martinez-Andrade GO, Cespedes EM, Rifas-Shiman SL, RomeroQuechol G, González-Unzaga MA, Benítez-Trejo MA, et al. Feasibility and impact of Creciendo Sanos, a clinic-based pilot intervention to prevent obesity among preschool children in Mexico City. BMC Pediatrics 2014;14:77.

\section{Matvienko 2010 \{published data only\}}

Matvienko O, Ahrabi-Fard I. The effects of a 4-week afterschool program on motor skills and fitness of kindergarten and first-grade students. American Journal of Health Promotion 2010;24(5):299-303.

\section{McAuley 2010 \{published data only\}}

McAuley KA, Taylor RW, Farmer VL, Hansen P, Williams SM, Booker CS, et al. Economic evaluation of a community-based obesity prevention program in children: the APPLE project. Obesity 2010;18(1):131-6.

\section{McCallum 2007 \{published data only\}}

McCallum Z, Wake M, Gerner B, Baur L A, Gibbons K, Gold L, et al. Outcome data from the LEAP (Live, Eat and Play) trial: a randomized controlled trial of a primary care intervention for childhood overweight/mild obesity. International Journal of Obesity 2007;31:630-6.

McCallum Z, Wake M, Gerner B, Harris C, Gibbons K, Gunn J, et al. Can Australian general practitioners tackle childhood overweight/obesity? Methods and processes from the LEAP (Live, Eat and Play) randomized controlled trial. Journal of Paediatrics and Child Health 2005;41:488-94.

\section{McGarvey 2004 \{published data only\}}

McGarvey E, Keller A, Forrester M, Williams R, Seward D, Suttle DE. Feasibility and benefits of a parent-focused preschool child obesity intervention. American Journal of Public Health 2004;94(9):1490.

\section{McMurray 2002 \{published data only\}}

McMurray RG, Harrell JS, Bangdiwala SI, Bradley CB, Deng S, Levine A. A school-based intervention can reduce body fat and blood pressure in young adolescents. Journal of Adolescent Health 2002;31(2):125-32.

\section{Melnyk 2007 \{published data only\}}

Melnyk BM, Small L, Morrison-Beedy D, Strasser A, Spath L, Kreipe R, et al. The COPE Healthy Lifestyles TEEN program: feasibility, preliminary efficacy, \& lessons learned from an after school group intervention with overweight adolescents. Journal of Pediatric Health Care 2007;21(5):315-22. [0891-5245: (Print)]

Millar 2011 \{published data only\}

Millar L, Kremer P, de Silva-Sanigorski A, McCabe MP, Mavoa H, Moodie M, et al. Reduction in overweight and obesity from a 3year community-based intervention in Australia: the 'It's Your Move!' project. Obesity Reviews 2011;12 Suppl 2:20-8.

\section{Mistry 2011 \{published data only\}}

Mistry AM. Differences in food intake, nutrition knowledge and fitness assessment measurements in high school students who have completed the nutricise 4 life program and students who have not. FASEB journal 2011;Conference(April):var.pagings.

Moodie 2010a \{published data only\}

Moodie ML, Carter RC, Swinburn BA, Haby MM. The costeffectiveness of Australia's Active After-School Communities program. Obesity 2010;18(8):1585-92.

\section{Moodie 2010b \{published data only\}}

Moodie M, Richardson J, Rankin B, Lezzi A, Sinha K. Predicting time trade-off health state valuations of adolescents in four Pacific countries using the Assessment of Quality-of-Life (AQoL-6D) instrument. Value in Health 2010;13(8):1014-27.

Moodie 2013 \{published data only\}

Moodie ML, Herbert JK, de Silva-Sanigorski AM, Mavoa HM, Keating CL, Carter RC, et al. The cost-effectiveness of a successful community-based obesity prevention program: the be active eat well program. Obesity 2013;21(10):2072-80.

\section{Morgan 2012 \{published data only\}}

Morgan PJ, Saunders KL, Lubans DR. Improving physical selfperception in adolescent boys from disadvantaged schools: psychological outcomes from the Physical Activity Leaders randomized controlled trial. Pediatric Obesity 2012;7(3):e27-32.

\section{Muckelbauer 2011 ppublished data only\}}

Muckelbauer R, Libuda L, Clausen K, Kersting M. Approaches for the prevention of overweight through modified beverage consumption in the elementary school setting. The "trinkfit" study. Bundesgesundheitsblatt, Gesundheitsforschung, Gesundheitsschutz 2011;54(3):339-48.

\section{Mustila 2012a \{published data only\}}

Mustila T, Raitanen J, Keskinen P, Saari A, Luoto R. Lifestyle counseling during pregnancy and offspring weight development until four years of age: follow-up study of a controlled trial. Journal of Negative Results in Biomedicine 2012;11:11. 
Mustila 2012b \{published data only\}

Mustila T, Keskinen P, Luoto R. Behavioral counseling to prevent childhood obesity - study protocol of a pragmatic trial in maternity and child health care. BMC Pediatrics 2012;12:93.

\section{Mustila 2012c \{published data only\}}

Mustila T, Raitanen J, Keskinen P, Saari A, Luoto R. Lifestyle counselling targeting infant's mother during the child's first year and offspring weight development until 4 years of age: a followup study of a cluster RCT. BMJ Open 2012;2(1):e000624.

\section{Mustila 2013 \{published data only\}}

Mustila T, Raitanen J, Keskinen P, Saari A, Luoto R. Pragmatic controlled trial to prevent childhood obesity in maternity and child health care clinics: pregnancy and infant weight outcomes (the VACOPP Study). BMC Pediatrics 2013;13:80.

\section{Navarro 2013 \{published data only\}}

Navarro JI, Sigulem DM, Ferraro AA, Polanco JJ, Barros AJ. The double task of preventing malnutrition and overweight: a quasi-experimental community-based trial. BMC Public Health 2013;13:212

\section{Nichols 2014 \{published data only\}}

Nichols SD. Sustainability of a curriculum-based intervention on dietary behaviours and physical activity among primary school children in Trinidad and Tobago. West Indian Medical Journal 2014;63(1):68-77.

\section{Niinikoski 1997 \{published data only\}}

Niinikoski H, Viikari V, Ronnemaa T, Helenius H, Jokinen E, Lapinleimu H, et al. Regulation of growth of 7- to 36- monthold children by energy and fat intake in the prospective, randomized STRIP baby trial. Pediatrics 1997;100(5):810-6.

\section{Nogueira 2014 \{published data only\}}

Nogueira RC. An in-school exercise intervention to enhance bone and reduce fat in girls: The CAPO Kids trial. Bone 2014;68:92-9.

\section{Obarzanek 1997 \{published data only\}}

Obarzanek E, Hunsberger SA, Van Horn L, Hartmuller VV, Barton BA, Stevens VJ, et al. Safety of a fat-reduced diet: The Dietary Intervention Study in Children (DISC). Pediatrics 1997;100(1):51-9.

\section{Oehrig 2001 \{published data only\}}

Oehrig E, Geiss HC, Haas G-M, Schwandt P. The prevention education program (PEP) Nuremberg: design and baseline data of a family oriented intervention study. International Journal of Obesity 2001;25(Suppl 1):S89-S92.

\section{Padilla 2012 \{published data only\}}

Padilla-Raygoza N, Diaz-Guerrero R, Ruiz-Paloalto L. Intervention in lifestyle as management of obesity in school age children in Celaya. Obesity Facts 2012;Conference:var.pagings.

\section{Pettman 2014 \{published data only\}}

Pettman T, Magarey A, Mastersson N, Wilson A, Dollman J. Improving weight status in childhood: results from the Eat Well
Be Active community programs. International Journal of Public Health 2014;59(1):43-50.

Plachta-Danielzik 2011 \{published data only\}

Plachta-Danielzik S, Landsberg S, Lange D, Seiberl J, Müller MJ. Eight-year follow-up of school-based intervention on childhood overweight - The Kiel obesity prevention study. Obesity Facts 2011;4(1):35-43.

Pope 2013 \{published data only\}

Pope L, Harvey-Berino J. Burn and earn: a randomized controlled trial incentivizing exercise during fall semester for college first-year students. Preventive Medicine 2013;56(3-4):197-201.

\section{Pratt 2013 \{published data only\}}

Pratt CA, Boyington J, Esposito L, Pemberton VL, Bonds D, Kelley M, et al. Childhood Obesity Prevention and Treatment Research (COPTR): interventions addressing multiple influences in childhood and adolescent obesity. Contemporary Clinical Trials 2013;36(2):406-13.

\section{Prins 2012 \{published data only\}}

Prins RG, Brug J, Van Empelen P, Oenema A. Effectiveness of YouRAction, an intervention to promote adolescent physical activity using personal and environmental feedback: a cluster RCT. PLOS ONE 2012;7(3):e32682.

\section{Puma 2013 \{published data only\}}

Puma J, Romaniello C, Crane L, Scarbro S, Belansky E, Marshall J. Long-term student outcomes of the Integrated Nutrition and Physical Activity Program. Journal of Nutrition Education and Behavior 2013;45(6):635-42.

\section{Quarles 2011 \{published data only\}}

Quarles J. Walking school bus and its impact on BMI. Dissertation Abstracts International: Section B: The Sciences and Engineering 2011;72(10-B):352.

\section{Rask-Nissila 2000 \{published data only\}}

Rask-Nissila L, Jokinen E, Terho P, Tammi A, Lapinleimu H, Ronnemaa T, et al. Neurological development of 5 year old children receiving a low-saturated fat, low-cholesterol diet since infancy: a randomized controlled trail. JAMA 2000;284(8):993-1000.

\section{Rawlins 2013 \{published data only\}}

Rawlins E, Baker G, Maynard M, Harding S. Perceptions of healthy eating and physical activity in an ethnically diverse sample of young children and their parents: the DEAL prevention of obesity study. Journal of Human Nutrition and Dietetics 2013;26(2):132-44.

Reed 2013 \{published data only\}

Reed JA, Maslow AL, Long S, Hughey M. Examining the impact of 45 minutes of daily physical education on cognitive ability, fitness performance, and body composition of African American youth. Journal of Physical Activity \& Health 2013;10(2):185-97. 
Reinehr 2007 \{published data only\}

Reinehr T, Temmesfeld M, Kersting M, de Sousa G, Toschke AM. Four-year follow-up of children and adolescents participating in an obesity intervention program. International Journal of Obesity 2007; Vol. 31, issue 7:1074-7. [0307-0565: (Print)]

\section{Resaland 2014 \{published data only\}}

Resaland G. Key results and implications from the Sogndal school intervention study-a prospective controlled intervention study of two-year school-based 60 minutes daily physical activity on cardiovascular disease risk factors. Obesity Facts 2014; Conference:var.pagings.

\section{Resnicow 2005 \{published data only\}}

Resnicow K, Taylor R, Baskin M, McCarty F. Results of Go Girls: a weight control program for overweight African-American adolescent females. Obesity Research 2005;13(10):1739-48.

\section{Richmond 2013 \{published data only\}}

Richmond SA. Sport injury and obesity prevention in junior high school students. Dissertation Abstracts International: Section B: The Sciences and Engineering 2013;74(1-B):No pagination.

\section{Robinson 1999 \{published data only\}}

Robinson TN. Can a school-based intervention to reduce television use decrease adiposity in children in grades 3 and 4 . Western Journal of Medicine 2000;173(1):40.

* Robinson TN. Reducing children's television viewing to prevent obesity: a randomised controlled trial. JAMA 1999;282(16):1561-7.

\section{Ronsley 2013 \{published data only\}}

Ronsley R, Lee AS, Kuzeljevic B, Panagiotopoulos C. Healthy Buddies reduces body mass index z-score and waist circumference in Aboriginal children living in remote coastal communities. Journal of School Health 2013;83(9):605-13.

\section{Roofe 2010 \{published data only\}}

Roofe NL. The impact of nutrition and health education intervention on kindergarten students' nutrition and exercise knowledge. Dissertation Abstracts International: Section B: The Sciences and Engineering 2010;71(9-B):11481.

\section{Roofe 2011 \{published data only\}}

Roofe NL. Improving families' nutrition knowledge through service learning. Journal of Allied Health 2011;40(4):194-8.

\section{Rueda 2013 \{published data only\}}

Rueda JM, Khosla P. Impact of breakfasts (with or without eggs) on body weight regulation and blood lipids in university students over a 14-week semester. Nutrients 2013;5(12):5097-113.

\section{Rush 2014 \{published data only\}}

Rush E, McLennan S, Obolonkin V, Vandal AC, Hamlin M, Simmons D, et al. Project Energize: whole-region primary school nutrition and physical activity programme; evaluation of body size and fitness 5 years after the randomised controlled trial. British Journal of Nutrition 2014;111(2):363-71.

\section{Rush 2014a \{published data only\}}

Rush E, Obolonkin V, McLennan S, Graham D, Harris J. Lifetime cost effectiveness of a through-school nutrition and physical programme: Project Energize. Obesity Research \& Clinical Practice 2014;8(2):e115-22.

\section{Sadowsky 1999 \{published data only\}}

Sadowsky HS, Sawdon JM, Scheiner ME, Sticklin AM. Eight week moderate intensity exercise intervention elicits body composition change in adolescents. Cardiopulmonary Physical Therapy Journal 1999;10(2):38-44.

\section{Sanders 2014 \{published data only\}}

Sanders LM, Perrin EM, Yin HS, Bronaugh A, Rothman RL. "Greenlight study": a controlled trial of low-literacy, early childhood obesity prevention. Pediatrics 2014;133(6):e1724-37.

Schuna 2013 \{published data only\}

Schuna JM Jr, Lauersdorf RL, Behrens TK, Liguori G, Liebert ML. An objective assessment of children's physical activity during the Keep It Moving! after-school program. Journal of School Health 2013;83(2):105-11.

\section{Sherwood 2013a \{published data only\}}

Sherwood NE, Levy RL, Langer SL, Senso MM, Crain AL, Hayes MG, et al. Healthy Homes/Healthy Kids: a randomized trial of a pediatric primary care-based obesity prevention intervention for at-risk 5-10 year olds. Contemporary Clinical Trials 2013;36(1):228-43.

\section{Shofan 2011 \{published data only\}}

Shofan Y, Kedar O, Branski D, Berry E, Wilschanski M. A schoolbased program of physical activity may prevent obesity. European Journal of Clinical Nutrition 2011;65(6):768-70.

\section{Sigmund 2012 \{published data only\}}

Sigmund E, El Ansari W, Sigmundova D. Does school-based physical activity decrease overweight and obesity in children aged 6-9 years? A two-year non-randomized longitudinal intervention study in the Czech Republic. BMC Public Health 2012;12:570.

\section{Sigmund 2013 \{published data only\}}

Sigmund E, Sigmundova D. Longitudinal 2-year follow-up on the effect of a non-randomised school-based physical activity intervention on reducing overweight and obesity of Czech children aged 10-12 years. International Journal of Environmental Research and Public Health 2013;10(8):3667-83.

\section{Simonetti 1986 \{published data only\}}

Simonetti D'Arca A, Tarsitani G, Cairella M, Siani V, De Filippis S, Mancinelli S, et al. Prevention of obesity in elementary and nursery school children. Public Health 1986;100:166-73.

\section{Singhal 2010 \{published data only\}}

Singhal N, Misra A, Shah P, Gulati S. Effects of controlled schoolbased multi-component model of nutrition and lifestyle interventions on behavior modification, anthropometry and metabolic risk profile of urban Asian Indian adolescents in North India. European Journal of Clinical Nutrition 2010;64:364-73. 


\section{Singhal 2011 \{published data only\}}

Singhal N, Misra A, Shah P, Gulati S, Bhatt S, Sharma S, et al. Impact of intensive school-based nutrition education and lifestyle interventions on insulin resistance, beta-cell function, disposition index, and subclinical inflammation among Asian Indian adolescents: a controlled intervention study. Metabolic Syndrome \& Related Disorders 2011;9(2):143-50.

\section{Slusser 2013 \{published data only\}}

Slusser WM, Sharif MZ, Erausquin JT, Kinsler JJ, Collin D, Prelip ML. Improving overweight among at-risk minority youth: results of a pilot intervention in after-school programs. Journal of Health Care for the Poor and Underserved 2013;24(2 Suppl):12-24.

\section{Smith 2012 \{published data only\}}

Smith NR, Clark C, Fahy AE, Tharmaratnam V, Lewis DJ, Thompson C, et al. The Olympic Regeneration in East London (ORiEL) study: protocol for a prospective controlled quasiexperiment to evaluate the impact of urban regeneration on young people and their families. BMJ Open 2012;2(4):e001840.

\section{Spark 1998 \{published data only\}}

Spark A, Pfau J, Nicklas TA, Williams CL. Reducing fat in preschool meals: description of the foodservice intervention component of Healthy Start. Journal of Nutrition Education 1998;30(3):170.

\section{Spencer 2012 \{published data only\}}

Spencer RA. The role of peer mentors in enhancing school-based physical activity: the Heart Healthy Kids (H2K) program phase 2. Canadian Journal of Cardiology 2012; Conference(Suppl 181):var.pagings.

\section{Spieker 2015 \{published data only\}}

Spieker EA, Sbrocco T, Theim KR, Maurer D, Johnson D, Bryant E, et al. Preventing Obesity in the Military Community (POMC): the development of a clinical trials research network. International Journal of Environmental Research and Public Health 2015;12(2):1174-95.

\section{Springer 2013 \{published data only\}}

Springer AE, Kelder SH, Byrd-Williams CE, Pasch KE, Ranjit N, Delk JE, et al. Promoting energy-balance behaviors among ethnically diverse adolescents: overview and baseline findings of The Central Texas CATCH Middle School Project. Health Education \& Behavior 2013;40(5):559-70.

\section{Stenevi-Lundgren 2009 \{published data only\}}

Stenevi-Lundgren S, Daly RM, Linden C, Gardsell P, Karlsson MK. Effects of a daily school based physical activity intervention program on muscle development in prepubertal girls. European Journal of Applied Physiology 2009;105(4):533-41. [1439-6327: (Electronic)]

\section{Stephens 1998 \{published data only\}}

Stephens MB, Wentz SW. Supplemental fitness activities and fitness in urban elementary school classrooms. Family Medicine 1998;30(3):220-3.

\section{Stewart 1995 \{published data only\}}

Stewart KJ, Lipis PH, Seemans CM, McFarland, Weinhofer JJ, Brown CS. Heart Healthy Knowledge, food patterns, fatness and cardiac risk factors in children receiving nutrition education. Journal of Health Education 1995;26(6):381-90.

Stice 2015 \{published data only\}

Stice E, Yokum S, Burger K, Rohde P, Shaw H, Gau JM. A pilot randomized trial of a cognitive reappraisal obesity prevention program. Physiology and Behavior 2015;138:124-32.

\section{Stock 2007 \{published data only\}}

Stock S, Miranda C, Evans S, Plessis S, Ridley J, Yeh S, et al. Healthy Buddies: a novel, peer-led health promotion program for the prevention of obesity and eating disorders in children in elementary school. Pediatrics 2007;120(4):e1059-e1068.

Strauss 2011 \{published data only\}

Strauss A, Herbert B, Mitschek C, Duvinage K, Koletzko B. TigerKids: successful health promotion in preschool settings. Bundesgesundheitsblatt - Gesundheitsforschung Gesundheitsschutz 2011;54(3):322-9.

Swinburn 2011 \{published data only\}

Swinburn BA, Millar L, Utter J, Kremer P, Moodie M, Mavoa H, et al. The Pacific Obesity Prevention in Communities project: project overview and methods. Obesity Reviews 2011;12 Suppl 2:3-11

\section{Talvia 2004 \{published data only\}}

Talvia S, Lagstrom H, Rasanen M, Salminen M, Rasanen L, Salo P, et al. A randomized intervention since infancy to reduce intake of saturated fat. Archives of Pediatrics \& Adolescent Medicine 2004;158(1):41.

\section{Tamir 1990 \{published data only\}}

Tamir D, Feurstein A, Brunner S, Halfon S, Reshef A, Palti H. Primary prevention of cardiovascular diseases in childhood: changes in serum total cholesterol, high density lipoprotein, and body mass index after 2 years of intervention in Jerusalem schoolchildren age 7-9 years. Preventive Medicine 1990;19:22-30

\section{Tarro 2014a \{published data only\}}

Tarro L, Llaurado E, Albaladejo R, Morina D, Arija V, Sola R, et al. A primary-school-based study to reduce the prevalence of childhood obesity--the EdAl (Educacio en Alimentacio) study: a randomized controlled trial. Trials 2014;15:58.

\section{Tarro 2014b \{published data only\}}

Tarro L, Llaurado E. Follow-up of a healthy lifestyle education program (the Educacio en Alimentacio Study): 2 years after cessation of intervention. Journal of Adolescent Health 2014;55(6):782-9.

\section{Taveras 2011 \{published data only\}}

Taveras EM, Blackburn K, Gillman MW, Haines J, McDonald J, Price $S$, et al. First steps for mommy and me: a pilot intervention to improve nutrition and physical activity behaviors of postpartum mothers and their infants. Maternal and Child Health Journal 2011;15(8):1217-27. 
Taylor 2005 \{published data only\}

Taylor MJ, Mazzone M, Wrotniak BH. Outcome of an exercise and educational intervention for children who are overweight. Pediatric Physical Therapy 2005; Vol. 17, issue 3:180-8. [0898-5669: (Print)]

Telford 2013a \{published data only\}

Telford RD, Cunningham RB, Waring P, Telford RM, Olive LS, Abhayaratna WP. Physical education and blood lipid concentrations in children: the LOOK randomized cluster trial. PLOS ONE 2013;8(10):e76124.

Telford 2013b \{published data only\}

Telford RD. Physical education can improve insulin resistance: the LOOK randomized cluster trial. Medicine and Science in Sports and Exercise 2013;45(10):1956-64.

Tershakovec 1998 \{published data only\}

Tershakovec AM, Jawad AF, Stallings VA, Zemel BS, McKenzie JM, Stolley PD, et al. Growth of hypercholesterolemic children completing physician-initiated low-fat dietary intervention. The Journal of Pediatrics 1998;133(1):28-34.

Toftager 2014 \{published data only\}

Toftager M, Christiansen LB, Ersbøll AK, Kristensen PL, Due P, Troelsen J. Intervention effects on adolescent physical activity in the multicomponent SPACE study: a cluster randomized controlled trial. PLOS ONE 2014;9(6):e99369.

Toruner 2010 \{published data only\}

Toruner EK, Savaser S. A controlled evaluation of a schoolbased obesity prevention in Turkish school children. Journal of School Nursing 2010;26(6):473-82.

\section{Toruner 2015 \{published data only\}}

Toruner EK. Efficacy of a school-based healthy life program in Turkey. Children's Health Care 2015;44(1):69-86.

\section{Treuth 2007 \{published data only\}}

Treuth MS, Catellier DJ, Schmitz KH, Pate RR, Elder JP, McMurray RG, et al. Weekend and weekday patterns of physical activity in overweight and normal-weight adolescent girls. Obesity 2007;15(7):1782-8.

\section{Trudeau 2000 \{published data only\}}

Trudeau F, Espindola R, Laurencelle L, Dulac F, Rajic M, Shephard RJ. Follow-up of participants in the Trois-Rivieres growth and development study: examining their health-related fitness and risk factors as adults. American Journal of Human Biology 2000;12:207-13.

* Trudeau F, Shephard RJ, Arsenault F, Laurencelle L. Changes in adiposity and body mass index from late childhood to adult life in the Trois-Rivieres study. American Journal of Human Biology 2001;13(3):349-55.

\section{Tucker 2011 \{published data only\}}

Tucker S, Lanningham-Foster L, Murphy J, Olsen G, Orth K, Voss J, et al. A school based community partnership for promoting healthy habits for life. Journal of Community Health 2011;36(3):414-22.
Utter 2011 \{published data only\}

Utter J, Scragg R, Robinson E, Warbrick J, Faeamani G, Foroughian S, et al. Evaluation of the Living 4 Life project: a youth-led, school-based obesity prevention study. Obesity Reviews 2011;12 Suppl 2:51-60.

\section{Vandongen 1995 \{published data only\}}

Vandongen R, Jenner DA, Thompson C, Taggart AC, Spickett EE, Burke $V$, et al. A controlled evaluation of a fitness and nutrition intervention program on cardiovascular health in 10- to 12-yearold children. Preventive Medicine 1995;24(1):9-22.

\section{Van Ryzin 2013 \{published data only\}}

Van Ryzin MJ, Nowicka P. Direct and indirect effects of a familybased intervention in early adolescence on parent-youth relationship quality, late adolescent health, and early adult obesity. Journal of Family Psychology 2013;27(1):106-16.

\section{Wake 2011 \{published data only\}}

Wake M, Price A, Clifford S, Ukoumunne O. Does an intervention that improves infant sleep also improve overweight at age 6 ? Follow-up of a randomised trial. Archives of Disease in Childhood 2011;96(6):526-32.

\section{Wallen 2010 \{published data only\}}

Wallen EF. A school-intervention with increased physical activity and healthy food choices puts a halt to obesity in youth with intellectual disability. Obesity Reviews 2010;Conference:var.pagings.

\section{Wang 2011 \{published data only\}}

Wang LY, Nichols LP, Austin SB. The economic effect of Planet Health on preventing bulimia nervosa. Archives of Pediatrics \& Adolescent Medicine 2011;165(8):756-62.

Weber 2014 \{published data only\}

Weber M, Grote V, Closa-Monasterolo R, Escribano J, Langhendries J-P, Dain E, et al. European Childhood Obesity Trial Study. Lower protein content in infant formula reduces BMI and obesity risk at school age: follow-up of a randomized trial. American Journal of Clinical Nutrition 2014;99(5):1041-51.

\section{Willi 2012 \{published data only\}}

Willi SM, Hirst K, Jago R, Buse J, Kaufman F, El Ghormli L, et al. HEALTHY Study Group. Cardiovascular risk factors in multiethnic middle school students: the HEALTHY primary prevention trial. Pediatric Obesity 2012;7(3):230-9.

Williams 1998 \{published data only\}

* Williams CL, Spark A, Strobino BA, Bollella MC, D'Agostio CA, Brotanek J, et al. Cardiovascular risk reduction in a preschool population: The Healthy Start Project. Preventive Cardiology 1998;2:45-55.

Williams CL, Squillace MM, Bollella MC, Brotanek J, Campanaro L, D'Agostino C, et al. Healthy Start: a comprehensive health education program for preschool children. Preventive Medicine 1998;27:216-23.

Williams CL, Strobino BA, Bollella M, Brotanek J. Cardiovascular risk reduction in preschool children: The "Healthy Start" 
Project. Journal of the American College of Nutrition 2004;23(2):117-23.

Williams 2011 \{published data only\}

Williams AD, Warrington V. Get Fit Kids: a feasibility study of a pedometer-based walking program. Bariatric Nursing \& Surgical Patient Care 2011;6(3):139-43.

\section{Williamson 2006 \{published data only\}}

Williamson DA, Walden HM, White MA, York-Crowe E, Newton RL Jr, Alfonso A, et al. Two-year internet-based randomized controlled trial for weight loss in African-American girls. Obesity 2006;14(7):1231-43.

\section{Williamson 2007 \{published data only\}}

Williamson DA, Copeland AL, Anton SD, Champagne C, Han H, Lewis L, et al. Wise Mind project: a school-based environmental approach for preventing weight gain in children. Obesity 2007;15(4):906-17.

\section{Wilson 2010 \{published data only\}}

Wilson AM, Magarey AM, Dollman J, Jones M, Mastersson N. The challenges of quantitative evaluation of a multi-setting, multi-strategy community-based childhood obesity prevention programme: lessons learnt from the Eat Well Be Active community programs in South Australia. Public Health Nutrition 2010;13(8):1262-70.

\section{Winter 2011 \{published data only\}}

Winter S, Sass DA. Healthy \& Ready to Learn: examining the efficacy of an early approach to obesity prevention and school readiness. Journal of Research in Childhood Education 2011;25(3):304-25.

\section{Wright 2012 \{published data only\}}

Wright K, Norris K, Newman GJ, Suro Z. Improving healthy dietary behaviors, nutrition knowledge, and self-efficacy among underserved school children with parent and community involvement. Childhood Obesity 2012;8(4):347-56.

\section{Wright 2014a \{published data only\}}

Wright DR, Taveras EM, Gillman MW, Horan CM, Hohman KH, Gortmaker SL, et al. The cost of a primary care-based childhood obesity prevention intervention. BMC Health Services Research 2014;14:44.

\section{Wright 2014b \{published data only\}}

Wright K, Suro Z. Using community--academic partnerships and a comprehensive school-based program to decrease health disparities in activity in school-aged children. Journal of Prevention \& Intervention in the Community 2014;42(2):125-39.

\section{Yang 2014 \{published data only\}}

Yang H, Xiao F, Yin D, Li R, Xin Q, Zheng X, et al. Impact analysis on the health management programs among community-based 0-36-month-olds on their growth and development. Chung-Hua Liu Hsing Ping Hsueh Tsa Chih Chinese Journal of Epidemiology 2014;35(11):1244-8.

\section{Yildirim 2013 \{published data only\}}

Yildirim M, Singh AS, te Velde S. Mediators of longitudinal changes in measures of adiposity in teenagers using parallel process latent growth modeling. Obesity 2013;21(11):2387-95.

\section{Yin 2012 \{published data only\}}

Yin Z, Parra-Medina D, Cordova A, He M, Trummer V, Sosa E, et al. Miranos! Look at us, we are healthy! An environmental approach to early childhood obesity prevention. Childhood Obesity 2012;8(5):429-39.

\section{Zanirati 2011 \{published data only\}}

Zanirati VF, Vasconcelos de PD, Pires BL, Souza LA, dos Santos LC. Impact of nutrition education workshops in the nutritional profile of children inserted in the integrated school program. Revista de Atencao Primaria a Saude 2011;14(4):408-16.

\section{Zhou 2014 \{published data only\}}

Zhou Z, Ren H, Yin Z, Wang L, Wang K. A policy-driven multifaceted approach for early childhood physical fitness promotion: impacts on body composition and physical fitness in young Chinese children. BMC Pediatrics 2014;14:118.

\section{References to studies awaiting assessment}

\section{Adamo 2013 \{published data only\}}

Adamo KB, Grattan K, Harvey A, Naylor PJ, Temple V, Wilson S, et al. Does a physical activity daycare intervention impact body composition and gross motor skills? A pilot randomized control trial. Canadian Journal of Diabetes 2013;37:S262-3.

\section{Adamo 2014 \{published data only\}}

Adamo KB, Barrowman N, Naylor PJ, Yaya S, Harvey A, Grattan KP, et al. Activity Begins in Childhood (ABC) - inspiring healthy active behaviour in preschoolers: study protocol for a cluster randomized controlled trial. Trials 2014;15:305.

\section{Adamo 2017 \{published data only\}}

Adamo KB, Wasenius NS, Grattan KP, Harvey AL, Naylor PJ, Barrowman NJ, et al. Effects of a preschool intervention on physical activity and body composition. Journal of Pediatrics 2017;188:42-49.e2.

\section{Allender 2016 \{published data only\}}

Allender S, Millar L, Hovmand P, Bell C, Moodie M, Carter R, et al. Whole of systems trial of prevention strategies for childhood obesity: WHO STOPS Childhood Obesity. International Journal of Environmental Research and Public Health 2016;13(11):16.

\section{Alvarez-Bueno 2017 \{published data only\}}

Alvarez-Bueno C, Cavero-Redondo I, Garrido-Miguel M, NotarioPacheco B, Martinez-Andres M, Sanchez-Lopez M. Effectiveness of the MOVI-KIDS physical activity intervention improving children's academic achievement. Obesity Facts 2017;10:232.

\section{Anderson 2014 \{published data only\}}

Anderson LM, Symoniak ED, Epstein LH. A randomized pilot trial of an integrated school-worksite weight control program. Health Psychology 2014;33(11):1421-5. 
Anderson 2016 \{published data only\}

Anderson EL, Howe LD, Kipping RR, Campbell R, Jago R, Noble SM, et al. Long-term effects of the Active for Life Year 5 (AFLY5) school-based cluster-randomised controlled trial. BMJ Open 2016;6(11):e010957.

\section{Andrade 2016 \{published data only\}}

Andrade S, Lachat C, Cardon G, Ochoa-Aviles A, Verstraeten R, Van Camp J, et al. Two years of school-based intervention program could improve the physical fitness among Ecuadorian adolescents at health risk: subgroups analysis from a clusterrandomized trial. BMC Pediatrics 2016;16:51.

\section{Annesi 2017 \{published data only\}}

Annesi JJ, Walsh SM, Greenwood BL, Mareno N, UnruhRewkowski JL. Effects of the Youth Fit 4 Life physical activity/ nutrition protocol on body mass index, fitness and targeted social cognitive theory variables in 9- to 12 -year-olds during after-school care. Journal of Paediatrics and Child Health 2017;53(4):365-73.

\section{Arlinghaus 2017 \{published data only\}}

Arlinghaus KR, Moreno JP, Reesor L, Hernandez DC, Johnston CA. Companeros: high school students mentor middle school students to address obesity among Hispanic adolescents. Preventing Chronic Disease 2017;14:E92.

\section{Armstrong 2015 \{published data only\}}

Armstrong B, Lim CS, Janicke DM. Park density impacts weight change in a behavioral intervention for overweight rural youth. Behavioral Medicine 2015;41(3):123-30.

\section{Atkinson 2015 \{published data only\}}

Atkinson MJ, Wade TD. Mindfulness-based prevention for eating disorders: a school-based cluster randomized controlled study. International Journal of Eating Disorders 2015;48(7):1024-37.

\section{Bacopoulou 2015 \{published data only\}}

Bacopoulou F, Efthymiou V, Papaefthymiou M, Landis G, Palaiologos G, Kaklea M, et al. Metabolic syndrome in Greek adolescents and the effect of 6-month educational/behavioural school interventions. Hormone Research in Paediatrics 2015;84:424.

\section{Barbosa 2015 \{published data only\}}

Barbosa Filho VC, Lopes Ada S, Lima AB, de Souza EA, Gubert Fdo A, Silva KS, et al. Fortaleca sua Saude" Working Group. Rationale and methods of a cluster-randomized controlled trial to promote active and healthy lifestyles among Brazilian students: the "Fortaleca sua Saude" program. BMC Public Health 2015;15:1212.

\section{Barbosa 2017 \{published data only\}}

Barbosa Filho VC, da Silva KS, Mota J Vieira NF, Gubert FD, Lopes AD. "For whom was it effective?" Moderators of the effect of a school-based intervention on potential physical activity determinants among Brazilian students. Preventive Medicine 2017;97:80-5.

\section{Barnes 2015 \{published data only\}}

Barnes AT, Plotnikoff RC, Collins CE, Morgan PJ. Feasibility and preliminary efficacy of the MADE4Life Program: a pilot randomized controlled trial. Journal of Physical Activity \& Health 2015;12(10):1378-93.

\section{Beck 2017 \{published data only\}}

Beck AL, Fernandez A, Rojina J, Cabana M. Randomized controlled trial of a clinic-based intervention to promote healthy beverage consumption among Latino children. Clinical Pediatrics 2017;56(9):838-44.

\section{Beets 2014 \{published data only\}}

Beets MW, Glenn Weaver R, Turner-McGrievy G, Huberty J, Ward DS, Freedman DA, et al. Making healthy eating and physical activity policy practice: the design and overview of a group randomized controlled trial in afterschool programs. Contemporary Clinical Trials 2014;38(2):291-303.

\section{Belanger 2016 \{published data only\}}

Belanger M, Humbert L, Vatanparast $\mathrm{H}$, Ward S, Muhajarine N, Chow AF, Eet al. A multilevel intervention to increase physical activity and improve healthy eating and physical literacy among young children (ages 3-5) attending early childcare centres: the Healthy Start-Depart Sante cluster randomised controlled trial study protocol. BMC Public Health 2016;16:313.

\section{Benden 2014 \{published data only\}}

Benden ME, Zhao H, Jeffrey CE, Wendel ML, Blake JJ. The evaluation of the impact of a stand-biased desk on energy expenditure and physical activity for elementary school students. International Journal of Environmental Research and Public Health 2014;11(9):9361-75.

\section{Bergh 2014 \{published data only\}}

Bergh IH, Van Stralen MM, Bjelland M, Grydeland M, Lien N, Klepp KI, et al. Post-intervention effects on screen behaviours and mediating effect of parental regulation: the HEalth In Adolescents study--a multi-component school-based randomized controlled trial. BMC Public Health 2014;14:200.

\section{Bips \{published data only\}}

Leibniz-Institut Prävention und Epidemiologie BIPS. Evaluation of the health effects of „JolinchenKids - Fit and healthy in daycare", a program for health promotion targeting 3-6 year-old children, at 68 daycare facilities in Germany. http:// www.drks.de/DRKS00011065.

\section{Birnbaum 2017 \{published data only\}}

Birnbaum J, Geyer C, Kirchberg F, Manios Y, Koletzko B, ToyBoxstudy Group. Effects of a kindergarten-based, family-involved intervention on motor performance ability in 3- to 6-yearold children: the ToyBox-study. Journal of Sports Sciences 2017;35(4):377-84.

\section{Bogart 2016 \{published data only\}}

Bogart LM, Elliott MN, Cowgill BO, Klein DJ, Hawes-Dawson J, Uyeda K, et al. Two-year BMI outcomes from a school-based intervention for nutrition and exercise: a randomized trial. Pediatrics 2016;137(5):e20152493. 
Bonuck 2016 \{published data only\}

Bonuck KA, Blank A, True-Felt B, Chervin R. Promoting sleep health among families of young children in head start: protocol for a social-ecological approach. Preventing Chronic Disease 2016;13:E121.

\section{Braun 2016 \{published data only\}}

Braun M Cook C Mukherjea R Liska D A. A randomized, controlled, parallel study to assess the effects of soy protein on body composition and general health parameters in healthy children. FASEB Journal. Conference: Experimental Biology. 2016.

\section{Brophy-Herb 2017 \{published data only\}}

Brophy-Herb H E Horodynski M Contreras D Kerver J Kaciroti N Stein M Lee H J Motz B Hebert S Prine E Gardiner C Van Egeren L A Lumeng J C. Effectiveness of differing levels of support for family meals on obesity prevention among head start preschoolers: the simply dinner study. BMC Public Health 2017;17(1):184.

\section{Bryant 2017 \{published data only\}}

Bryant M Burton W Cundill B Farrin A J Nixon J Stevens J Roberts K Foy R Rutter H Hartley S Tubeuf S Collinson M Brown J. Effectiveness of an implementation optimisation intervention aimed at increasing parent engagement in HENRY, a childhood obesity prevention programme - the Optimising Family Engagement in HENRY (OFTEN) trial: study protocol for a randomised controlled trial. Trials [Electronic Resource] 2017;18(1):40.

\section{Buhler 2015 \{published data only\}}

Buhler A. [Recruitment of Families at Risk for Obesity: The Kindergarten Experience]. Gesundheitswesen 2015;77(Suppl 1):S25-6.

\section{Burgermaster 2017 \{published data only\}}

Burgermaster Marissa Koroly Jenna Contento Isobel Koch Pamela Gray Heewon L. A Mixed-Methods Comparison of Classroom Context During Food, Health \& Choices, a Childhood Obesity Prevention Intervention. Journal of School Health 2017;87(11):811-22.

\section{Burke 2017 \{published data only\}}

Burke N L Shomaker L B Brady S Reynolds J C Young J F Wilfley D E Sbrocco T Stephens M Olsen C H Yanovski J A Tanofsky-Kraff M. Impact of age and race on outcomes of a program to prevent excess weight gain and disordered eating in adolescent girls. Nutrients 2017;9(9):947.

\section{Buscemi 2014 \{published data only\}}

Buscemi J Odoms-Young A Stolley M L Blumstein L Schiffer L Berbaum M L McCaffrey J Montoya A M Braunschweig C Fitzgibbon M L. Adaptation and dissemination of an evidence-based obesity prevention intervention: design of a comparative effectiveness trial. Contemporary Clinical Trials 2014;38(2):355-60.

\section{Bustos 2016 \{published data only\}}

Bustos N Olivares S Leyton B Cano M Albala C. Impact of a school-based intervention on nutritional education and physical activity in primary public schools in Chile (KIND) programme study protocol: cluster randomised controlled trial. BMC Public Health 2016;16(1):1217.

\section{Byrd-Bredbenner 2015 \{published data only\}}

Byrd-Bredbenner Carol Worobey J Martin-Biggers J Hongu N Hernandez G. HomeStyles: Shaping Home Environments and Lifestyle Practices to Prevent Childhood Obesity: A Randomized Controlled Trial. Journal of Nutrition Education \& Behavior 2015;47(4):S99.

\section{Byrd-Bredbenner 2017 \{published data only\}}

Byrd-Bredbenner C Martin-Biggers J Koenings M Quick V Hongu N Worobey J. HomeStyles, A Web-Based Childhood Obesity Prevention Program for Families With Preschool Children: Protocol for a Randomized Controlled Trial. JMIR Research Protocols 2017;6(4):e73.

Byrd-Bredbenner 2017a \{published data only\}

Byrd-Bredbenner C Quick V Martin-Biggers J. Homestyles: A randomized controlled trial. Annals of Nutrition and Metabolism 2017;71(Supplement 2):1235.

\section{Byrd-Bredbenner 2018 \{published data only\}}

Byrd-Bredbenner C Martin-Biggers J Povis G A Worobey J Hongu $\mathrm{N}$ Quick V. Promoting healthy home environments and lifestyles in families with preschool children: HomeStyles, a randomized controlled trial. Contemporary Clinical Trials 2018;64:139-51.

\section{Byrne 2016 \{published data only\}}

Byrne R Magarey A Daniels L. Maternal perception of weight status in first-born Australian toddlers aged 12-16 months - the NOURISH and SAIDI cohorts. Child: Care, Health \& Development 2016;42(3):375-81.

\section{Caballero 1998 \{published data only\}}

Caballero B Davis S Davis C E Ethelbah B Evans M Lohman T Stephenson L Story M White J. Pathways: A schoolbased program for the primary prevention of obesity in American Indian children. Journal of Nutritional Biochemistry 1998;9(9):535-43

\section{Camacho-Cardenosa 2016 \{published data only\}}

Camacho-Cardenosa A Brazo-Sayavera J Camacho-Cardenosa M Marcos-Serrano M Timon R Olcina G. Effects of High Intensity Interval Training on Fat Mass Parameters in Adolescents. Revista Espanola de Salud Publica 2016;90:e1-9.

\section{Campbell 2016 \{published data only\}}

Campbell K J Hesketh K D McNaughton S A Ball K McCallum Z Lynch J Crawford D A. The extended Infant Feeding, Activity and Nutrition Trial (InFANT Extend) Program: a cluster-randomized controlled trial of an early intervention to prevent childhood obesity. BMC Public Health 2016;16:166.

\section{Cao 2014 \{published data only\}}

Cao Z Wang S Zheng W Guo J Qu S. Evaluation on the effectiveness of intervention comprehensive program on child obesity, using Generalized Estimating Equation. [Chinese]. Zhonghua liu xing bing xue za zhi = Zhonghua liuxingbingxue zazhi 2014;35(7):773-8. 


\section{Carlin 2015 \{published data only\}}

Carlin A Murphy M H Gallagher A M. The WISH study: The effect of peer-led Walking in ScHools on school-time physical activity. Proceedings of the Nutrition Society. Conference: Irish Section Meeting Nutrition at Key Life Stages: New Findings, New Approaches. Ireland.. 2015; Vol. 74, issue OCE4.

\section{Carraway 2015 \{published data only\}}

Carraway Marissa Errickson. Project mentor+: Mentorled exercise with cognitive-behavioral therapy to improve perceived competence, reduce social anxiety, and increase physical activity in overweight adolescents. Greenville: East Carolina University, 2015:99.

\section{Cavero-Redondo 2017 \{published data only\}}

Cavero-Redondo I Alvarez-Bueno C Garrido-Miguel M MartinezVizcaino V Diez-Fernandez A Fernandez-Infante A. Physical activity intervention program (movi-kids study) on preventing obesity in preschoolers. Obesity Facts 2017;10:246.

\section{Cecchetto 2017 \{published data only\}}

Cecchetto F H Pena D B Pellanda L C. Playful Interventions Increase Knowledge about Healthy Habits and Cardiovascular Risk Factors in Children: The CARDIOKIDS Randomized Study. Arquivos Brasileiros de Cardiologia 2017;109(3):199-206.

\section{Chamberland 2017 \{published data only\}}

Chamberland K Sanchez M Panahi S Provencher V Gagnon J Drapeau V. The impact of an innovative web-based school nutrition intervention to increase fruits and vegetables and milk and alternatives in adolescents: a clustered randomized trial. International Journal of Behavioral Nutrition \& Physical Activity 2017;14(1):140.

\section{Chen 2015 \{published data only\}}

Chen Y Ma L Ma Y Wang H Luo J Zhang X Luo C Wang H Zhao H Pan D Zhu Y Cai L Zou Z Yang W Ma J Jing J. A national school-based health lifestyles interventions among Chinese children and adolescents against obesity: rationale, design and methodology of a randomized controlled trial in China. BMC Public Health 2015;15:210.

\section{Chen 2017 \{published data only\}}

Chen E Miller G E Yu T Brody G H. Unsupportive parenting moderates the effects of family psychosocial intervention on metabolic syndrome in African American youth. International Journal of Obesity 2017;06:06.

\section{Cloutier 2015 \{published data only\}}

Cloutier M M Wiley J Wang Z Grant A Gorin A A. The Early Childhood Obesity Prevention Program (ECHO): an ecologicallybased intervention delivered by home visitors for newborns and their mothers. BMC Public Health 2015;15:584.

\section{Cocca 2016 \{published data only\}}

Cocca A Lomas R Enriquez M Cocca M Ceballos O. Effects of a school-based intervention program on metabolic syndrome parameters in school-aged youth. Obesity Reviews 2016;17:131-2.

\section{Costa 2017 \{published data only\}}

Costa C S Campagnolo P D Lumey L H Vitolo M R. Effect of maternal dietary counselling during the 1st year of life on glucose profile and insulin resistance at the age of 8 years: a randomised field trial. British Journal of Nutrition 2017;117(1):134-41.

\section{Creanor 2016 \{published data only\}}

Creanor S Lloyd J Hillsdon M Dean S Green C Taylor R S Ryan E Wyatt K He L P Trial Management Group. Detailed statistical analysis plan for a cluster randomised controlled trial of the Healthy Lifestyles Programme (HeLP), a novel school-based intervention to prevent obesity in school children. Trials [Electronic Resource] 2016;17(1):599.

\section{Cunningham-Sabo 2016 \{published data only\}}

Cunningham-Sabo L Lohse B Smith S Browning R Strutz E Nigg C Balgopal M Kelly K Ruder E. Fuel for Fun: a clusterrandomized controlled study of cooking skills, eating behaviors, and physical activity of 4 th graders and their families. BMC Public Health 2016;16:444.

\section{Daniels 2014a \{published data only\}}

Daniels Lynne Allison Mallan Kimberley Margaret Battistutta Diana Nicholson Jan Maree Meedeniya Josephine Emma Bayer Jordana Kim Magarey Anthea. Child eating behavior outcomes of an early feeding intervention to reduce risk indicators for child obesity: The NORISH RCT. Obesity 2014;22(5):E104-11.

\section{Daniels 2015 \{published data only\}}

Daniels L A Mallan K M Nicholson J M Thorpe K Nambiar S Mauch C E Magarey A. An Early Feeding Practices Intervention for Obesity Prevention. Pediatrics 2015;136(1):e40-9.

\section{Daniels 2015a \{published data only\}}

Daniels L Heath A L Williams S M Cameron S L Fleming E A Taylor B J Wheeler B J Gibson R S Taylor R W. Baby-Led Introduction to SolidS (BLISS) study: a randomised controlled trial of a baby-led approach to complementary feeding. BMC Pediatrics 2015;15:179.

\section{Davis 2016 \{published data only\}}

Davis A M Stough C O Black W R Dean K Sampilo M Simpson $S$ Landrum Y. Outcomes of a weight management program conjointly addressing parent and child health. Children's Health Care 2016;45(2):227-40.

\section{Davis 2016a \{published data only\}}

Davis S M Myers O B Cruz T H Morshed A B Canaca G F Keane $P$ C O'Donald E R. CHILE: Outcomes of a group randomized controlled trial of an intervention to prevent obesity in preschool Hispanic and American Indian children. Preventive Medicine 2016;89:162-8.

\section{de Greeff 2016 \{published data only\}}

de Greeff J W Hartman E Mullender-Wijnsma M J Bosker R J Doolaard S Visscher C. Effect of Physically Active Academic Lessons on Body Mass Index and Physical Fitness in Primary School Children. Journal of School Health 2016;86(5):346-52. 


\section{Delisle 2015 \{published data only\}}

Delisle C Sandin S Forsum E Henriksson H Trolle-Lagerros Y Larsson C Maddison R Ortega F B Ruiz J R Silfvernagel K Timpka $\mathrm{T}$ Lof M. A web- and mobile phone-based intervention to prevent obesity in 4-year-olds (MINISTOP): a population-based randomized controlled trial. BMC Public Health 2015;15:95.

\section{de Moraes 2017 \{published data only\}}

de Moraes M M Mediano M F F de Souza R A G Moura A S da Veiga $G$ V Sichieri R. Discouraging soft drink consumption reduces blood glucose and cholesterol of Brazilian elementary students: Secondary analysis of a randomized controlled trial. Preventive Medicine 2017;100:223-8.

\section{de Villiers 2016 \{published data only\}}

de Villiers A Steyn N P Draper C E Hill J Gwebushe N Lambert E V Lombard C. Primary School Children's Nutrition Knowledge, Self-Efficacy, and Behavior, after a Three-Year Healthy Lifestyle Intervention (HealthKick). Ethnicity \& Disease 2016;26(2):171-80.

Dordevic 2015 \{published data only\}

Dordevic A L Bonham M P Ware R S Brennan L Truby H. Study protocol: evaluation of 'JenMe', a commercially-delivered weight management program for adolescents: a randomised controlled trial. BMC Public Health 2015;15:563.

\section{Doring 2016 \{published data only\}}

Doring N Ghaderi A Bohman B Heitmann B Larsson C Berglind $D$ Hansson $L$ Sundblom E Magnusson M Blennow M Tynelius $P$ Forsberg L Rasmussen F. Primary prevention of childhood obesity within child health services: The PRIMROSE cluster-RCT. Obesity Facts 2016;9:35.

\section{Doring 2016a \{published data only\}}

Doring N Ghaderi A Bohman B Heitmann B L Larsson C Berglind D Hansson L Sundblom E Magnusson M Blennow M Tynelius P Forsberg L Rasmussen F. Motivational Interviewing to Prevent Childhood Obesity: A Cluster RCT. Pediatrics 2016;137(5):e20153104

\section{Drummy 2016 \{published data only\}}

Drummy C Murtagh E M McKee D P Breslin G Davison G W Murphy $\mathrm{MH}$. The effect of a classroom activity break on physical activity levels and adiposity in primary school children. Journal of Paediatrics and Child Health. 2016.

\section{Dundee Family Health Study \{published data only\}}

ISRCTN13385965 Trial registration number. Dundee Family Health Study. WHO ISRCTN ISRCTN13385965 2017.

\section{Dunker 2017 \{unpublished data only\}}

Dunker K L L Claudino A M. Preventing weight-related problems among adolescent girls: A cluster randomized trial comparing the Brazilian 'New Moves' program versus observation. Obesity Research and Clinical Practice. (In Press) 2017.

\section{Dunton 2015 \{published data only\}}

Dunton G Ebin V J Efrat M W Efrat R Lane C J Plunkett S. The Use of Refundable Tax Credits to Increase Low-Income Children's After-School Physical Activity Level. Journal of Physical Activity \& Health 2015;12(6):840-53.
Eat Healthy for Families \{published data only\}

NCT03394326 Trial registration number. Eat Healthy for Families. https://ClinicalTrials.gov/show/NCT03394326 2017:NCT03394326.

\section{Edwardson 2015 \{published data only\}}

Edwardson C L Harrington D M Yates T Bodicoat D H Khunti K Gorely T Sherar L B Edwards R T Wright C Harrington K Davies M J. A cluster randomised controlled trial to investigate the effectiveness and cost effectiveness of the 'Girls Active' intervention: a study protocol. BMC Public Health 2015;15:526.

Effectiveness of the run-a-mile intervention \{published data only\}

School of Public Health Birmingham University. Effectiveness of the run-a-mile intervention. WHO ISRCTN http://isrctn.com/ ISRCTN12698269 2016:ISRCTN12698269.

\section{Eldridge 2016 \{published data only\}}

Eldridge G Paul L Bailey S J Ashe C B Martz J Lynch W. Effects of parent-only childhood obesity prevention programs on BMIz and body image in rural preteens. Body Image 2016;16:143-53.

Eline 2016 \{published data only\}

Eline V L'Hoir M Van Grieken A Hein R Magda B B. Effectiveness of a primary prevention program of overweight in 0-3 year old children, the BBOFT+ study; a cluster randomized trial. Journal of Pediatric Gastroenterology and Nutrition 2016;62:725.

Eneli 2015 \{published data only\}

Eneli I U Tylka T L Hummel J Watowicz R P Perez S A Kaciroti $\mathrm{N}$ Lumeng J C. Rationale and design of the Feeding Dynamic Intervention (FDI) study for self-regulation of energy intake in preschoolers. Contemporary Clinical Trials 2015;41:325-34.

\section{Eno 2017 \{published data only\}}

Eno Persson J Bohman B Tynelius P Rasmussen F Ghaderi A. Prevention of Childhood Obesity in Child Health Services: Follow-Up of the PRIMROSE Trial. Childhood Obesity 2017;12:12.

\section{Esquivel 2015 \{published data only\}}

Esquivel M Li F Novotny R. Wellness policy and the head start environment: the moderating role of head start teachers in the children's healthy living program in Hawai'i. FASEB journal. 2015; Vol. 29, issue 1 Meeting Abstracts.

\section{Evans 2016 \{published data only\}}

Evans A Ranjit N Hoelscher D Jovanovic C Lopez M McIntosh A Ory M Whittlesey L McKyer L Kirk A Smith C Walton C Heredia $\mathrm{N}$ I Warren J. Impact of school-based vegetable garden and physical activity coordinated health interventions on weight status and weight-related behaviors of ethnically diverse, lowincome students: Study design and baseline data of the Texas, Grow! Eat! Go! (TGEG) cluster-randomized controlled trial. BMC Public Health 2016;16:973.

Families Preventing Diabetes Together \{published data only\} NCT02721602 Trial registration number. Families Preventing Diabetes Together. https://ClinicalTrials.gov/show/ NCT02721602. https://ClinicalTrials.gov/show/NCT02721602, 2016:NCT02721602. 


\section{Fangupo 2015 \{published data only\}}

Fangupo Louise J Heath Anne-Louise M Williams Sheila M Somerville Megan R Lawrence Julie A Gray Andrew R Taylor Barry J Mills Virginia C Watson Emily O Galland Barbara C Sayers Rachel M Hanna Maha B Taylor Rachael W. Impact of an earlylife intervention on the nutrition behaviors of 2-y-old children: a randomized controlled trial. American Journal of Clinical Nutrition 2015;102(3):704-12.

\section{Farmer 2017 \{published data only\}}

Farmer V L Williams S M Mann J I Schofield G McPhee J C Taylor $\mathrm{RW}$. The effect of increasing risk and challenge in the school playground on physical activity and weight in children: a cluster randomised controlled trial (PLAY). International Journal of Obesity 2017;41(5):793-800.

\section{Folkvord 2016 \{published data only\}}

Folkvord F Anschutz D J Buijzen M. The association between BMI development among young children and (un)healthy food choices in response to food advertisements: a longitudinal study. International Journal of Behavioral Nutrition \& Physical Activity 2016;13:16.

\section{Friedrich 2015 \{published data only\}}

Friedrich R R Caetano L C Schiffner M D Wagner M B Schuch I. Design, randomization and methodology of the TriAtiva Program to reduce obesity in school children in Southern Brazil. BMC Public Health 2015;15:363.

\section{Fulkerson 2014 \{published data only\}}

Fulkerson J A Neumark-Sztainer D Story M Gurvich O Kubik M Y Garwick A Dudovitz B. The Healthy Home Offerings via the Mealtime Environment (HOME) Plus study: design and methods. Contemporary Clinical Trials 2014;38(1):59-68.

Fulkerson 2015 \{published data only\} Fulkerson J A Friend S Flattum C Horning M Draxten M NeumarkSztainer D Gurvich O Story M Garwick A Kubik M Y. Promoting healthful family meals to prevent obesity: HOME Plus, a randomized controlled trial. International Journal of Behavioral Nutrition \& Physical Activity 2015;12:154.

Fulkerson 2017 \{published data only\}

Fulkerson J A Friend S Horning M Flattum C Draxten M NeumarkSztainer D Gurvich O Garwick A Story M Kubik M Y. Family Home Food Environment and Nutrition-Related Parent and Child Personal and Behavioral Outcomes of the Healthy Home Offerings via the Mealtime Environment (HOME) Plus Program: A Randomized Controlled Trial. Journal of the Academy of Nutrition \& Dietetics 2017;01:01.

\section{Galland 2016 \{published data only\}}

Galland B Taylor B Gray A Heath A Lawrence J Sayers R Cameron S Hanna M Dale K Coppell K Taylor R. Early life prevention of obesity by targeting sleep, or food and activity: A randomized controlled trial. Sleep 2016;39:A339-40.

\section{Gallotta 2015 \{published data only\}}

Gallotta M C Emerenziani G P lazzoni S Meucci M Baldari C Guidetti L. Impacts of coordinative training on normal weight and overweight/obese children's attentional performance. Frontiers in Human Neuroscience 2015;9:577.

Gallotta 2016 \{published data only\}

Gallotta M C Iazzoni S Emerenziani G P Meucci M Migliaccio S Guidetti L Baldari C. Effects of combined physical education and nutritional programs on schoolchildren's healthy habits. PeerJ 2016;2016(4):e1880.

Gao 2017 \{published data only\}

Gao Z Pope Z Lee J E Stodden D Roncesvalles N Pasco D Huang C C Feng D. Impact of exergaming on young children's school day energy expenditure and moderate-to-vigorous physical activity levels. Journal of Sport and Health Science 2017;6(1):11-6.

\section{Gholami 2015 \{published data only\}}

Gholami M Wiedemann A Knoll N Schwarzer R. Mothers improve their daughters' vegetable intake: a randomized controlled trial. Psychology Health \& Medicine 2015;20(1):1-7.

Goldfield 2016 \{published data only\}

Goldfield G S Harvey A L J Grattan K P Temple V Naylor P J Alberga A S Ferraro Z M Wilson S Cameron J D Barrowman $\mathrm{N}$ Adamo K B. Effects of Child Care Intervention on Physical Activity and Body Composition. American Journal of Preventive Medicine 2016;51(2):225-31.

\section{Gorin 2014 \{published data only\}}

Gorin A A Wiley J Ohannessian C M Hernandez D Grant A Cloutier M M. Steps to Growing Up Healthy: a pediatric primary care based obesity prevention program for young children. $B M C$ Public Health 2014;14:72.

\section{Gortmaker 1999b \{published data only\}}

Gortmaker SL, Cheung LW, Peterson KE, Chomitz G, Cradle JH, Dart $\mathrm{H}$, et al. Impact of a school-based interdisciplinary intervention on diet and physical activity among urban primary school children. Archives of Pediatrics and Adolescent Medicine 1999;153:975-83.

\section{Greve 2015 \{published data only\}}

Greve J Heinesen E. Evaluating the impact of a school-based health intervention using a randomized field experiment. Economics \& Human Biology 2015;18:41-56.

Grillich 2016 \{published data only\}

Grillich L Kien C Takuya Y Weber M Gartlehner G. Effectiveness evaluation of a health promotion programme in primary schools: a cluster randomised controlled trial. BMC Public Health 2016;16:679.

\section{Gross 2016 \{published data only\}}

Gross R S Mendelsohn A L Gross M B Scheinmann R Messito M J. Randomized Controlled Trial of a Primary Care-Based Child Obesity Prevention Intervention on Infant Feeding Practices. Journal of Pediatrics 2016;174:171-177.e2. 
Gruber 2015 \{published data only\}

Gruber R Somerville G. Preliminary results from a multicomponent obesity prevention school based program. Sleep. 2015;38:A392-a393.

\section{Grydeland 2013 \{published data only\}}

Grydeland M, Bergh IH, Bjelland M, Lien N, Andersen LF, Ommundsen $\mathrm{Y}$, et al. Intervention effects on physical activity: the HEIA study - a cluster randomized controlled trial. International Journal of Behavioral Nutrition and Physical Activity 2013;10:17.

\section{Gunawardena 2016 \{published data only\}}

Gunawardena N Kurotani K Indrawansa S Nonaka D Mizoue T Samarasinghe D. School-based intervention to enable school children to act as change agents on weight, physical activity and diet of their mothers: a cluster randomized controlled trial. International Journal of Behavioral Nutrition \& Physical Activity 2016;13:45.

\section{Ha 2014 \{published data only\}}

Ha A S Lonsdale C Ng J Y Lubans D R. A school-based rope skipping intervention for adolescents in Hong Kong: protocol of a matched-pair cluster randomized controlled trial. BMC Public Health 2014;14:535.

\section{Habib-Mourad 2014a \{published data only\}}

Habib-Mourad C Ghandour L A Moore H J Nabhani-Zeidan M Adetayo K Hwalla N Summerbell C. Promoting healthy eating and physical activity among school children: findings from Health-E-PALS, the first pilot intervention from Lebanon. BMC Public Health 2014;14:940.

\section{Haines 2016 \{published data only\}}

Haines J Rifas-Shiman S L Gross D McDonald J Kleinman K Gillman M W. Randomized trial of a prevention intervention that embeds weight-related messages within a general parenting program. Obesity 2016;24(1):191-9.

\section{Haire-Joshu 2015 \{published data only\}}

Haire-Joshu D L Schwarz C D Peskoe S B Budd E L Brownson $\mathrm{R} C$ Joshu $C \mathrm{E}$. A group randomized controlled trial integrating obesity prevention and control for postpartum adolescents in a home visiting program. International Journal of Behavioral Nutrition \& Physical Activity 2015;12(1):88.

\section{Hammersley 2017 \{published data only\}}

Hammersley M L Jones R A Okely A D. Time2bHealthy - An online childhood obesity prevention program for preschoolaged children: A randomised controlled trial protocol. Contemporary Clinical Trials 2017;61:73-80.

\section{Handel 2017 \{published data only\}}

Handel M N Larsen S C Rohde J F Stougaard M Olsen N $J$ Heitmann B L. Effects of the Healthy Start randomized intervention trial on physical activity among normal weight preschool children predisposed to overweight and obesity. PLOS ONE [Electronic Resource] 2017;12(10):e0185266.

\section{Hankonen 2016 \{published data only\}}

Hankonen N Heino M T Araujo-Soares V Sniehotta F F Sund R Vasankari T Absetz P Borodulin K Uutela A Lintunen T Haukkala A. 'Let's Move It' - a school-based multilevel intervention to increase physical activity and reduce sedentary behaviour among older adolescents in vocational secondary schools: a study protocol for a cluster-randomised trial. BMC Public Health 2016;16:451.

\section{Hannon 2015 \{published data only\}}

Hannon T S Carroll A E Palmer K N Saha C Childers W K Marrero $D$ G. Rationale and design of a comparative effectiveness trial to prevent type 2 diabetes in mothers and children: the ENCOURAGE healthy families study. Contemporary Clinical Trials 2015;40:105-11.

\section{Harder-Lauridsen 2014 \{published data only\}}

Harder-Lauridsen N M Birk N M Ried-Larsen M Juul A Andersen L $B$ Pedersen B K Krogh-Madsen R. A randomized controlled trial on a multicomponent intervention for overweight school-aged children - Copenhagen, Denmark. BMC Pediatrics 2014;14:273.

Healthy Caregivers-Healthy Children (HC2) Phase II \{published data only\}

NCT00119132 Trial registration number. Healthy CaregiversHealthy Children (HC2) Phase II. https://ClinicalTrials.gov/show/ NCT02697565 2015:NCT02697565.

\section{Hejazi 2017 \{published data only\}}

Hejazi S Peyman N Esmaily H. Effect of educational intervention based on Self-Efficacy on preventive behaviors of overweight and obesity among secondary-school female students in Mashhad. [Persian]. Iranian Journal of Endocrinology and Metabolism 2017;19(4):261-9.

\section{Helle 2017 \{published data only\}}

Helle C Hillesund E R Omholt M L Overby N C. Early food for future health: a randomized controlled trial evaluating the effect of an eHealth intervention aiming to promote healthy food habits from early childhood. BMC Public Health 2017;17(1):729.

\section{Helping pre-school children to avoid obesity \{published data only\}}

ISRCTN22620137 Trial registration number. Helping pre-school children to avoid obesity ISRCTN22620137. WHO ISRCTN 2016:ISRCTN22620137.

\section{Hingle 2015 \{published data only\}}

Hingle M D Turner T Kutob R Merchant N Roe D J Stump C Going $S$ B. The EPIC Kids Study: a randomized family-focused YMCAbased intervention to prevent type 2 diabetes in at-risk youth. BMC Public Health 2015;15:1253.

\section{Hjorth 2016 \{published data only\}}

Hjorth M F Sjodin A Dalskov S M Damsgaard C T Michaelsen K F Biltoft-Jensen A Andersen R Ritz C Chaput J P Astrup A. Sleep duration modifies effects of free ad libitum school meals on adiposity and blood pressure. Applied Physiology, Nutrition, \& Metabolism = Physiologie Appliquee, Nutrition et Metabolisme 2016;41(1):33-40. 
Hohman 2016 \{published data only\}

Hohman EE, Savage JS, Paul IM, Birch LL. INSIGHT study parenting intervention to prevent childhood obesity improves patterns of dietary exposures in infants. FASEB Journal. Conference: Experimental Biology. 2016; Vol. 30.

\section{Hohman 2017 \{published data only\}}

Hohman EE, Paul IM, Birch LL, Savage JS. INSIGHT responsive parenting intervention is associated with healthier patterns of dietary exposures in infants. Obesity 2017;25(1):185-91.

\section{Hohman 2018 \{published data only\}}

Hohman E E Savage J S Birch L L Beiler J S Paul I M. Pacifier Use and Early Life Weight Outcomes in the Intervention Nurses Start Infants Growing on Healthy Trajectories Study. Childhood Obesity 2018;14(1):58-66.

\section{Hollar 2015 \{published data only\}}

Hollar D, Heitz C, Zhou W. More young children in an obesity prevention intervention in ms and la head start centers improve/maintain BMI percentile and waist circumference compared to nonparticipants. Circulation. 2015; Vol. 131.

\section{Hollis 2015 \{published data only\}}

Hollis J L Sutherland R Campbell L Morgan P Lubans D Nathan $\mathrm{N}$ Wolfenden L Okely T Davies L Gilham K Cohen K Wiggers J. A socio-ecologically framed, school-based physical activity intervention has beneficial effects on obesity outcomes in adolescents from low SES communities: the PA4E1 RCT. Obesity Facts 2015;8:110.

\section{Hollis 2016 \{published data only\}}

Hollis J L Sutherland R Campbell L Morgan P J Lubans D R Nathan N Wolfenden L Okely A D Davies L Williams A Cohen K E Oldmeadow C Gillham K Wiggers J. Effects of a 'schoolbased' physical activity intervention on adiposity in adolescents from economically disadvantaged communities: secondary outcomes of the 'Physical Activity 4 Everyone' RCT. International Journal of Obesity 2016;40(10):1486-93.

\section{Horodynski 2015 \{published data only\}}

Horodynski M A Silk K Hsieh G Hoffman A Robson M. Tools for teen moms to reduce infant obesity: a randomized clinical trial. BMC Public Health 2015;15:22.

\section{Horton 2013 \{published data only\}}

Horton L A Parada H Slymen D J Arredondo E Ibarra L Ayala G X. Targeting children's dietary behaviors in a family intervention: 'Entre familia: reflejos de salud'. Salud Publica de Mexico 2013;55(Suppl 3):397-405.

\section{Hrafnkelsson 2014 \{published data only\}}

Hrafnkelsson H Magnusson K T Thorsdottir I Johannsson E Sigurdsson E L. Result of school-based intervention on cardiovascular risk factors. Scandinavian journal of primary health care 2014;32(4):149-55.

\section{Huang 2015 \{published data only\}}

Huang T Larsen K T Jepsen J R Moller N C Thorsen A K Mortensen $\mathrm{E} L$ Andersen $L \mathrm{~B}$. Effects of an obesity intervention program on cognitive function in children: A randomized controlled trial. Obesity 2015;23(10):2101-8.

Huang 2015a \{published data only\}

Huang T Larsen K T Moller N C Ried-Larsen M Frandsen U Andersen $L$ B. Effects of a multi-component camp-based intervention on inflammatory markers and adipokines in children: A randomized controlled trial. Preventive Medicine 2015;81:367-72.

\section{Hughes 2016 \{published data only\}}

Hughes S O Power T G Beck A Betz D Calodich S Goodell L S Hill L G Hill R Jaramillo J A Johnson S L Lanigan J Lawrence A Martinez A D Nesbitt M Overath I Parker L Ullrich-French S. Strategies for Effective Eating Development-SEEDS: Design of an Obesity Prevention Program to Promote Healthy Food Preferences and Eating Self-Regulation in Children From LowIncome Families. Journal of Nutrition Education \& Behavior 2016;48(6):405-418.e1.

\section{Hull 2016 \{published data only\}}

Hull P C Buchowski M Canedo J R Beech B M Du L Koyama T Zoorob R. Childhood obesity prevention cluster randomized trial for Hispanic families: outcomes of the healthy families study. Pediatric Obesity 2016;24:24.

\section{Iaia 2017 \{published data only\}}

Iaia M Pasini M Burnazzi A Vitali P Allara E Farneti M. An educational intervention to promote healthy lifestyles in preschool children: a cluster-RCT. International Journal of Obesity 2017;41(4):582-90.

\section{Jaakkola 2015 \{published data only\}}

Jaakkola J M Pahkala K Viitala M Ronnemaa T Viikari J Niinikoski H Lagstrom H Jula A Simell O Raitakari O. Association of Adiponectin with Adolescent Cardiovascular Health in a Dietary Intervention Study. Journal of Pediatrics 2015;167(2):353-360.e1

\section{Janeiro \{published data only\}} NCT03128775. Trial registration number. Nudging Students Towards Healthy Diet and Physical Activity to Prevent Obesity. Clinical trials.gov NCT0312877 Rio de Janeiro State University Oswaldo Cruz Foundation.

\section{Jones 2015 \{published data only\}}

Jones R A Kelly J Cliff D P Batterham M Okely A D. Acceptability and Potential Efficacy of Single-Sex After-School Activity Programs for Overweight and At-Risk Children: The Wollongong SPORT RCT. Pediatric Exercise Science 2015;27(4):535-45.

\section{Katan 2016 \{published data only\}}

Katan M B De Ruyter J C Kuijper L D J Chow C C Hall K D Olthof $M$ R. Impact of masked replacement of sugar-sweetened with sugar-free beverages on body weight increases with initial bmi: Secondary analysis of data from an 18 month double-blind trial in children. PLOS ONE 2016;11(7):e0159771.

Kaufman-Shriqui 2016 \{published data only\}

Kaufman-Shriqui V Fraser D Friger M Geva D Bilenko N Vardi H Elhadad N Mor K Feine Z Shahar D R. Effect of a School-Based 
Intervention on Nutritional Knowledge and Habits of LowSocioeconomic School Children in Israel: A Cluster-Randomized Controlled Trial. Nutrients 2016;8(4):234.

Kennedy 2018 \{published data only\}

Kennedy S G Smith J J Morgan P J Peralta L R Hilland T A Eather N Lonsdale C Okely A D Plotnikoff R C Salmon J O Dewar D L Estabrooks P A Pollock E Finn T L Lubans D R. Implementing Resistance Training in Secondary Schools: A Cluster Randomized Controlled Trial. Medicine \& Science in Sports \& Exercise 2018;50(1):62-72.

\section{Kesztyus 2017 \{published data only\}}

Kesztyus D Lauer R Kesztyus T Kilian R Steinacker J M Join the Healthy Boat" Study Group. Costs and effects of a state-wide health promotion program in primary schools in Germany - the Baden-Wurttemberg Study: A cluster-randomized, controlled trial. PLoS ONE [Electronic Resource] 2017;12(2):e0172332.

\section{Kharofa 2015 \{published data only\}}

Kharofa R Y Copeland K A Sucharew H Meurer J R. Randomized controlled trial of a Wellness Action Plan to promote healthy diet and activity in pediatric primary care. Preventive Medicine Reports 2015;2:899-905.

\section{Kipping 2016 \{published data only\}}

Kipping R Jago R Metcalfe C White J Papadaki A Campbell R Hollingworth W Ward D Wells S Brockman R Nicholson A Moore L. NAP SACC UK: protocol for a feasibility cluster randomised controlled trial in nurseries and at home to increase physical activity and healthy eating in children aged 2-4 years. BMJ Open 2016;6(4):e010622.

\section{Knowlden 2015 \{published data only\}}

Knowlden A P Sharma M Cottrell R R Wilson B R Johnson M L. Impact evaluation of Enabling Mothers to Prevent Pediatric Obesity through Web-Based Education and Reciprocal Determinism (EMPOWER) Randomized Control Trial. Health Education \& Behavior 2015;42(2):171-84.

\section{Knowlden 2016 \{published data only\}}

Knowlden A Sharma M. One-Year Efficacy Testing of Enabling Mothers to Prevent Pediatric Obesity Through Web-Based Education and Reciprocal Determinism (EMPOWER) Randomized Control Trial. Health Education \& Behavior 2016;43(1):94-106.

\section{Knowlden 2017 \{published data only\}}

Knowlden A P Conrad E. Two-Year Outcomes of the Enabling Mothers to Prevent Pediatric Obesity Through Web-Based Education and Reciprocal Determinism (EMPOWER) Randomized Control Trial. Health Education \& Behavior 2017;45(2):262-76.

\section{Kobel 2014 \{published data only\}}

Kobel S Wirt T Schreiber A Kesztyus D Kettner S Erkelenz N Wartha O Steinacker J M. Intervention effects of a school-based health promotion programme on obesity related behavioural outcomes. Journal of Obesity 2014;2014:476230.
Kobel 2017 \{published data only\}

Kobel S Lammle C Wartha O Kesztyus D Wirt T Steinacker J M. Effects of a Randomised Controlled School-Based Health Promotion Intervention on Obesity Related Behavioural Outcomes of Children with Migration Background. Journal of Immigrant \& Minority Health 2017;19(2):254-62.

Kobel 2017a \{published data only\}

Kobel S Wartha O Wirt T Dreyhaupt J Lammle C Friedemann E M Kelso A Kutzner C Hermeling L Steinacker J M. Design, Implementation, and Study Protocol of a Kindergarten-Based Health Promotion Intervention. BioMed Research International 2017;2017:4347675.

Kocken 2016 \{published data only\}

Kocken P L Scholten A M Westhoff E De Kok B P Taal E M Goldbohm R A. Effects of a Theory-Based Education Program to Prevent Overweightness in Primary School Children. Nutrients 2016;8(1):04.

\section{Kong 2016 \{published data only\}}

Kong A Buscemi J Stolley M R Schiffer L A Kim Y Braunschweig C L Gomez-Perez S L Blumstein L B Van Horn L Dyer A R Fitzgibbon M L. Hip-Hop to Health Jr. Randomized Effectiveness Trial: 1Year Follow-up Results. American Journal of Preventive Medicine 2016;50(2):136-44.

\section{Kovalskys 2016 \{published data only\}}

Kovalskys I Rausch Herscovici C Indart P Zonis Luciana Z L Anez E Orellana L. Maintaining levels of physical activity (PA) among 9- 11 y old children is a challenge. Evidence from SALTEN, a school-based obesity prevention intervention in Argentina. Obesity Reviews 2016;17:145.

\section{Kovalskys 2017 \{published data only\}}

Kovalskys I Indart Rougier P Luciana L Rauch Herscovici C De Gregorio M J. Mini salten: A case study on learnings from Argentina on outcomes and challenges. Annals of Nutrition and Metabolism 2017;71(Supplement 2):96-7.

\section{Kovalskys 2017a \{published data only\}}

Kovalskys I Rausch Herscovici C Indart Rougier P De Gregorio M J Zonis L Orellana L. Study Protocol of MINI SALTEN: a technology-based multi-component intervention in the school environment targeting healthy habits of first grade children and their parents. BMC Public Health 2017;17(1):401.

Kuni 2015 \{published data only\}

Kuni B Ruhling N E Hegar U Roth C Schmitt H. Ball games and nutrition counseling improve postural control in overweight children. BMC Pediatrics 2015;15:205.

\section{La 2016 \{published data only\}}

La Torre G Mannocci A Saulle R Sinopoli A D'Egidio V Sestili C Manfuso R Masala D. [GiochiAMO! The protocol of a school based intervention for the promotion of physical activity and nutrition among children]. Clinica Terapeutica 2016;167(5):152-5. 
LaChausse 2017 \{published data only\}

LaChausse Robert G. A clustered randomized controlled trial to determine impacts of the Harvest of the Month program. Health Education Research 2017;32(5):375-83.

\section{Lakshman 2015 \{published data only\}}

Lakshman R Whittle F Hardeman W Suhrcke M Wilson E Griffin S Ong K K. Effectiveness of a behavioural intervention to prevent excessive weight gain during infancy (The Baby Milk Trial): study protocol for a randomised controlled trial. Trials [Electronic Resource] 2015;16:442.

\section{Lappe 2017 \{published data only\}}

Lappe J M McMahon D J Laughlin A Hanson C Desmangles J C Begley M Schwartz M. The effect of increasing dairy calcium intake of adolescent girls on changes in body fat and weight. American Journal of Clinical Nutrition 2017;105(5):1046-53.

\section{Larsen 2015 \{published data only\}}

Larsen A L Robertson T Dunton G. RE-AIM analysis of a randomized school-based nutrition intervention among fourth-grade classrooms in California. Translational Behavioral Medicine 2015;5(3):315-26.

Lau 2015 \{published data only\}

Lau W C Zhang S Maddison R. The effect of a school-based active video game intervention on children's aerobic fitness, physical activity level, and exercise related psychological variables: A preliminary RCT trial. Obesity Facts 2015;8:145.

Leme 2015 \{published data only\}

Leme A C Philippi S T. The "Healthy Habits, Healthy Girls" randomized controlled trial for girls: study design, protocol, and baseline results. Cadernos de Saude Publica 2015;31(7):1381-94.

\section{Leme 2016 \{published data only\}}

Leme A C Lubans D R Guerra P H Dewar D Toassa E C Philippi $S$ T. Preventing obesity among Brazilian adolescent girls: Sixmonth outcomes of the Healthy Habits, Healthy Girls-Brazil school-based randomized controlled trial. Preventive Medicine 2016;86:77-83

\section{Lent 2014 \{published data only\}}

Lent M R Vander Veur S S McCoy T A Wojtanowski A C Sandoval B Sherman S Komaroff E Foster G D. A randomized controlled study of a healthy corner store initiative on the purchases of urban, low-income youth. Obesity 2014;22(12):2494-500.

\section{Lerner-Geva 2015 \{published data only\}}

Lerner-Geva L Bar-Zvi E Levitan G Boyko V Reichman B PinhasHamiel O. An intervention for improving the lifestyle habits of kindergarten children in Israel: a cluster-randomised controlled trial investigation. Public Health Nutrition 2015;18(9):1537-44.

\section{Li 2017 \{published data only\}}

Li B Liu W J Adab P Pallan M Hemming K Frew E Lin R Martin J Liu W Cheng K K. Cluster-randomised controlled trial to assess the effectiveness and cost-effectiveness of an obesity prevention programme for Chinese primary school-aged children: the CHIRPY DRAGON study protocol. BMJ Open 2017;7(11):e018415.

\section{Liberato 2016 \{published data only\}}

Liberato S C Brimblecombe J K Moodie M Nutton G Sayers S Ball $\mathrm{K}$. Assessing the impact of a multi-component intervention to improve dietary intake of indigenous Australian children and their families living in remote communities. Obesity Reviews 2016;17:159.

\section{Lichtenstein 2011 \{published data only\}}

Lichtenstein STeufel. Prevention of obesity in primary school: A school-based prevention program reduces the risk for obesity in school children. Monatsschrift fur Kinderheilkunde 2011;159(8):751-7.

\section{Llargués 2017 \{published data only\}}

Llargués Esteve Recasens Ma Assumpta Manresa JosepMaria Jensen Bjarne Bruun Franco Rosa Nadal Anna Vila Maria Recasens Isabel Pérez M José Castell Conxa. Four-year outcomes of an educational intervention in healthy habits in schoolchildren: the Avall 3 Trial. European Journal of Public Health 2017;27(1):42-7.

\section{Lloyd 2014 \{published data only\}}

Lloyd A B Lubans D R Plotnikoff R C Morgan P J. Impact of the 'Healthy Dads, Healthy Kids' lifestyle programme on the activity- and diet-related parenting practices of fathers and mothers. Pediatric Obesity 2014;9(6):e149-55.

\section{Lloyd 2015 \{published data only\}}

Lloyd Jenny Wyatt Katrina. Uptake, retention and engagement of children participating in the cluster randomised controlled trial of the Healthy Lifestyles Programme (HeLP). Education \& Health 2015;33(4):88-95.

\section{Lloyd 2017 \{published data only\}}

Lloyd J Creanor S Price L Abraham C Dean S Green C Hillsdon M Pearson V Taylor R S Tomlinson R Logan S Hurst A Ryan E Daurge W Wyatt $\mathrm{K}$. Trial baseline characteristics of a cluster randomised controlled trial of a school-located obesity prevention programme; the Healthy Lifestyles Programme (HeLP) trial. BMC Public Health 2017;17(1):291.

\section{Lloyd 2017a \{published data only\}}

Lloyd J McHugh C Minton J Eke H Wyatt K. The impact of active stakeholder involvement on recruitment, retention and engagement of schools, children and their families in the cluster randomised controlled trial of the Healthy Lifestyles Programme (HeLP): a school-based intervention to prevent obesity. Trials [Electronic Resource] 2017;18(1):378.

\section{Lloyd 2018 \{published data only\}}

Lloyd J Creanor S Logan S Green C Dean S G Hillsdon M Abraham C Tomlinson R Pearson V Taylor R S Ryan E Price L Streeter A Wyatt K. Effectiveness of the Healthy Lifestyles Programme (HeLP) to prevent obesity in UK primary-school children: a cluster randomised controlled trial. The Lancet Child and Adolescent Health 2018;2(1):35-45.

\section{Lopes 2016 \{published data only\}}

Lopes W A Leite N da Silva L R Brunelli D T Gaspari A F Radominski R B Chacon-Mikahil M P Cavaglieri C R. Effects of 12 weeks of combined training without caloric restriction on 
inflammatory markers in overweight girls. Journal of Sports Sciences 2016;34(20):1902-12.

Lubans 2010 \{published data only\}

Lubans DR Morgan PJ Dewar D. The Nutrition and Enjoyable Activity for Teen Girls (NEAT girls) randomized controlled trial for adolescent girls from disadvantaged secondary schools: rationale, study protocol, and baseline results. BMC Public Health 2010;10:652.

\section{Lubans 2012a \{published data only\}}

Lubans DR, Morgan PJ, Callister R. Potential moderators and mediators of intervention effects in an obesity prevention program for adolescent boys from disadvantaged schools. Journal of Science and Medicine in Sport 2012;15(6):519-25.

Lubans 2016 \{published data only\}

Lubans D Smith JJ Peralta LR Plotnikoff RC Oakley AD Salmonm J Eather N Dewar Dl, et al. A school-based intervention incorporating smartphone technology to improve healthrelated fitness among adolescents: rationale and study protocol for the NEAT and ATLAS 2.0 cluster randomised controlled trial and dissemination study. BMJ Open 2016;6:e010448.

\section{Lubans 2016a \{published data only\}}

Lubans D R Smith J J Morgan P J Beauchamp M R Miller A Lonsdale C Parker P Dally K. Mediators of Psychological Well-being in Adolescent Boys. Journal of Adolescent Health 2016;58(2):230-6.

\section{Lubans 2016b \{published data only\}}

Lubans D R Smith J J Plotnikoff R C Dally K A Okely A D Salmon $J$ Morgan P J. Assessing the sustained impact of a school-based obesity prevention program for adolescent boys: the ATLAS cluster randomized controlled trial. International Journal of Behavioral Nutrition \& Physical Activity 2016;13:92.

\section{Lumeng 2017 \{published data only\}}

Lumeng J C Miller A L Horodynski M A Brophy-Herb H E Contreras D Lee H Sturza J Kaciroti N Peterson K E. Improving Self-Regulation for Obesity Prevention in Head Start: A Randomized Controlled Trial. Pediatrics 2017;139(5):e20162047.

\section{Luszczynska 2016 \{published data only\}}

Luszczynska A Horodyska K Zarychta K Liszewska N Knoll N Scholz U. Planning and self-efficacy interventions encouraging replacing energy-dense foods intake with fruit and vegetable: A longitudinal experimental study. Psychology \& Health 2016;31(1):40-64.

\section{Lynch 2016 \{published data only\}}

Lynch B A Gentile N Maxson J Quigg S Swenson L Kaufman T. Elementary School-Based Obesity Intervention Using an Educational Curriculum. Journal of Primary Care \& Community Health 2016;7(4):265-71.

\section{Madsen 2015 \{published data only\}}

Madsen K Linchey J Gerstein D Ross M Myers E Brown K Crawford P. Energy Balance 4 Kids with Play: Results from a Two-Year Cluster-Randomized Trial. Childhood Obesity 2015;11(4):375-83.

\section{Madsen 2017 \{published data only\}}

Madsen K A Linchey J Ritchie L Thompson H R. The Fit Study: Design and rationale for a cluster randomized trial of schoolbased BMI screening and reporting. Contemporary Clinical Trials 2017;58:40-6.

\section{Magarey 2016 \{published data only\}}

Magarey A Mauch C Mallan K Perry R Elovaris R Meedeniya J Byrne R Daniels L. Child dietary and eating behavior outcomes up to 3.5 years after an early feeding intervention: The NOURISH RCT. Obesity 2016;24(7):1537-45.

\section{Maha 2016 \{published data only\}}

Maha Nubani Huseini M Donchin M. School-based intervention program to promote healthy eating \& physical activity among palestinian mothers. European Journal of Preventive Cardiology 2016;23:S62.

\section{Martinez-Vizcaino 2015 \{published data only\}}

Martinez-Vizcaino V Mota J Solera-Martinez M Notario-Pacheco B Arias-Palencia N Garcia-Prieto J C Gonzalez-Garcia A AlvarezBueno C Sanchez-Lopez M Movi-Kids group. Rationale and methods of a randomised cross-over cluster trial to assess the effectiveness of MOVI-KIDS on preventing obesity in preschoolers. BMC Public Health 2015;15:176.

\section{McEachan 2016 \{published data only\}}

McEachan R R Santorelli G Bryant M Sahota P Farrar D Small N Akhtar S Sargent J Barber S E Taylor N Richardson G Farrin A J Bhopal R S Bingham D D Ahern S M Wright J Bi B childhood obesity scientific group. The HAPPY (Healthy and Active Parenting Programmme for early Years) feasibility randomised control trial: acceptability and feasibility of an intervention to reduce infant obesity. BMC Public Health 2016;16:211.

\section{McManus 2015 \{published data only\}}

McManus R M Donovan L Miller D Mottola M Giroux I RosasArellano P. Families defeating diabetes (FDD): A Canadian intervention for family-centered diabetes prevention following gestational diabetes (GDM): Initial results. Diabetes 2015;64:A632.

\section{Melnyk 2015 \{published data only\}}

Melnyk B M Jacobson D Kelly S A Belyea M J Shaibi G Q Small L O'Haver J A Marsiglia F F. Twelve-Month Effects of the COPE Healthy Lifestyles TEEN Program on Overweight and Depressive Symptoms in High School Adolescents. Journal of School Health 2015;85(12):861-70.

\section{Messiah 2017 \{published data only\}}

Messiah S E Lebron C Moise R Sunil Mathew M Sardinas K Chang C Palenzuela J Walsh J Shelnutt K P Spector R Altare F Natale R. Healthy caregivers-healthy children ( $\mathrm{HC2}$ ) phase 2: Integrating culturally sensitive childhood obesity prevention strategies into childcare center policies. Contemporary Clinical Trials 2017;53:60-7.

\section{Minossi 2015 \{published data only\}}

Minossi V Pellanda L C. The "Happy Heart" educational program for changes in health habits in children and their 
families: protocol for a randomized clinical trial. BMC Pediatrics 2015;15:19.

\section{Mohammed 2015 \{published data only\}}

Mohammed Nawi A Che Jamaludin F I. Effect of Internet-based Intervention on Obesity among Adolescents in Kuala Lumpur: A School-based Cluster Randomised Trial. The Malaysian Journal of Medical Science 2015;22(4):47-56.

\section{Moir 2016 \{published data only\}}

Moir C Meredith-Jones K Taylor B J Gray A Heath A M Dale K Galland B Lawrence J Sayers R M Taylor R W. Early Intervention to Encourage Physical Activity in Infants and Toddlers: A Randomized Controlled Trial. Medicine \& Science in Sports \& Exercise 2016;48(12):2446-53.

\section{Moore 2016 \{published data only\}}

Moore Justin B Brinkley Jason Morris Sara F Oniffrey Theresa M Kolbe Mary Bea. Effectiveness of Community-Based Minigrants to Increase Physical Activity and Decrease Sedentary Time in Youth. Journal of Public Health Management \& Practice 2016;22(4):370-8.

\section{Mora 2015a \{published data only\}}

Mora T Llargues E Recasens A. Does health education affect BMI? Evidence from a school-based randomised-control trial. Economics \& Human Biology 2015;17:190-201.

\section{More jumping and better eating at home and school \{published} data only\}

ISRCTN58093412 Trial registration number. More jumping and better eating at home and school [MINI SALTEN: study assessing the efficacy of a virtual intervention targeted to parents and aimed at preventing obesity in 6 year old children of public schools of Buenos Aires]. WHO ISRCTN http://isrctn.com/ ISRCTN58093412 2016:ISRCTN58093412.

\section{Morgan 2014 \{published data only\}}

Morgan P J Collins C E Plotnikoff R C Callister R Burrows T Fletcher R Okely A D Young M D Miller A Lloyd A B Cook A T Cruickshank J Saunders K L Lubans D R. The 'Healthy Dads, Healthy Kids' community randomized controlled trial: a community-based healthy lifestyle program for fathers and their children. Preventive Medicine 2014;61:90-9.

Muller 2016 \{published data only\}

Muller U M Walther C Adams V Mende M Adam J Fikenzer K Machalica K C Erbs S Linke A Schuler G. Long term impact of one daily unit of physical exercise at school on cardiovascular risk factors in school children. European Journal of Preventive Cardiology 2016;23(13):1444-52.

\section{Muzaffar 2014 \{published data only\}}

Muzaffar H Castelli D M Scherer J Chapman-Novakofski K. The impact of web-based HOT (Healthy Outcomes for Teens) Project on risk for type 2 diabetes: a randomized controlled trial. Diabetes Technology \& Therapeutics 2014;16(12):846-52.

\section{Muzaffar 2016 \{published data only\}}

Muzaffar H, Nickols-Richardson SM. PAWS (Peer-education About Weight Steadiness) club: Rationale and design for a randomized controlled study. FASEB Journal. Conference: Experimental Biology. 2016; Vol. 30.

Nanney 2016 \{published data only\}

Nanney M S Shanafelt A Wang Q Leduc R Dodds E Hearst M Kubik M Y Grannon K Harnack L. Project BreakFAST: Rationale, design, and recruitment and enrollment methods of a randomized controlled trial to evaluate an intervention to improve school breakfast program participation in rural high schools. Contemporary Clinical Trials Communications 2016;3:12-22.

Natale 2016 \{published data only\}

Natale R N Messiah S E Chang C Sardinas K Fitzgibbons J Peraza S. Healthy caregivers-healthy children: A primary prevention program with preschool children. Cardiology (Switzerland) 2016;134:71.

Natale 2017 \{published data only\}

Natale R A Messiah S E Asfour L S Uhlhorn S B Englebert N E Arheart K L. Obesity Prevention Program in Childcare Centers: Two-Year Follow-Up. American Journal of Health Promotion 2017;31(6):502-10.

\section{Natale 2017a \{published data only\}}

Natale Ruby R Camejo Stephanie T Asfour Lila Uhlhorn Susan B Delamater Alan Messiah Sarah E. Promoting healthy weight among children with developmental delays. Journal of Early Intervention 2017;39(1):51-65.

\section{Nezami 2016 \{published data only\}}

Nezami B T Lytle L A Tate D F. A randomized trial to reduce sugar-sweetened beverage and juice intake in preschool-aged children: description of the Smart Moms intervention trial. $B M C$ Public Health 2016;16(1):837.

\section{Nezami 2017 \{published data only\}}

Nezami BT. Healthy weight behaviors and weight change in parents and preschool-aged children. Chapel Hill: The University of North Carolina at Chapel Hill, 2017:201.

\section{Nezami 2017a \{published data only\}}

Nezami B T Ward D S Lytle L A Ennett S T Tate D F. A mHealth randomized controlled trial to reduce sugar-sweetened beverage intake in preschool-aged children. Pediatric Obesity 2017;08:08.

\section{Nogueira 2014a \{published data only\}}

Nogueira R C Weeks B K Beck B R. An in-school exercise intervention to enhance bone and reduce fat in girls: the CAPO Kids trial. Bone 2014;68:92-9.

\section{Nogueira 2015 \{published data only\}}

Nogueira R C Weeks B K Beck B R. Targeting bone and fat with novel exercise for peripubertal boys: the CAPO kids trial. Pediatric exercise science 2015;27(1):128-39.

Nogueira 2017 \{published data only\}

Nogueira R C Weeks B K Beck B. One-Year Follow-up of the CAPO Kids Trial: Are Physical Benefits Maintained?. Pediatric Exercise Science 2017;29(4):486-95. 
Novotny 2015 \{published data only\}

Novotny R Nigg C R Li F Wilkens L R. Pacific kids DASH for health (PacDASH) randomized, controlled trial with DASH eating plan plus physical activity improves fruit and vegetable intake and diastolic blood pressure in children. Childhood Obesity 2015;11(2):177-86.

\section{Novotny 2017 \{published data only\}}

Novotny R Wilkens L R Nigg C R Braun K Butel J Areta A Coleman P Belyeu-Camacho T Greenberg J Bersamin A Guerrero R L Barber L R Fialkowski M K De La Cruz-Talbert E. Effectiveness of the Children's Healthy Living $(\mathrm{CHL})$ multilevel multicomponent community intervention program in 5 US affiliated pacific jurisdictions. FASEB Journal. Conference: Experimental Biology. 2017; Vol. 31, issue 1 Supplement 1.

\section{Nudging, Healthy Diet and Physical Activity \{published data only\}}

NCT03136016 Trial registration number. Nudging, Healthy Diet and Physical Activity. Design of a School Randomized Trial for Nudging Students Towards Healthy Diet and Physical Activity. https://clinicaltrials.gov/show/NCT03136016 WHO ClinicalTrials.gov 2017:NCT03136016.

\section{NUTRICIA \{published data only\}}

NUTRICIA Universiteit Leiden Wageningen University Danone Research. Baby's First Bites: Promoting Vegetable Intake in Infants and Toddlers. https://ClinicalTrials.gov/show/ NCT03348176.

\section{Nyberg 2016 \{published data only\}}

Nyberg G Norman A Sundblom E Zeebari Z Elinder L S. Effectiveness of a universal parental support programme to promote health behaviours and prevent overweight and obesity in 6-year-old children in disadvantaged areas, the Healthy School Start Study II, a cluster-randomised controlled trial. International Journal of Behavioral Nutrition \& Physical Activity 2016;13:4.

\section{Nystrom 2017 \{published data only\}}

Nystrom C D Sandin S Henriksson P Henriksson H TrolleLagerros Y Larsson C Maddison R Ortega F B Pomeroy J Ruiz J R Silfvernagel K Timpka T Lof M. Mobile-based intervention intended to stop obesity in preschool-aged children: the MINISTOP randomized controlled trial. American Journal of Clinical Nutrition 2017;105(6):1327-35.

\section{Ochoa 2017 \{published data only\}}

Ochoa A Ochoa Aviles A Andrade Tenesaca D S Verstraeten R Huybregts L Lachat C Ramirez Jimbo P L Andrade Munoz D J Donoso Moscoso S P. Effect of the school-based health promotion intervention activital on dietary intake and waist circumference: A cluster randomized controlled trial. Annals of Nutrition and Metabolism 2017;71(Supplement 2):1272-3.

\section{Ochoa-Aviles 2017 \{published data only\}}

Ochoa-Aviles A Verstraeten R Huybregts L Andrade S Van Camp J Donoso S Ramirez P L Lachat C Maes L Kolsteren P. A school-based intervention improved dietary intake outcomes and reduced waist circumference in adolescents: a cluster randomized controlled trial. Nutrition Journal 2017;16(1):79.
Olsen 2017 \{published data only\}

Olsen N J Angquist L Mortensen E L Heitmann B L. Primary prevention of excessive fat gain among 2-6 year olds. Results from the "healthy start" randomized intervention. Annals of Nutrition and Metabolism 2017;71(Supplement 2):735-6.

Omorou 2015 \{published data only\}

Omorou A Y Langlois J Lecomte E Vuillemin A Briancon S. Erratum: Adolescents' Physical Activity and Sedentary Behavior: A Pathway in Reducing Overweight and Obesity. The PRALIMAP 2-Year Cluster Randomized Controlled Trial (Journal of physical activity \& health). Journal of physical activity \& health 2015;12(5):628-35.

\section{Omorou 2015a \{published data only\}}

Omorou A Y Langlois J Lecomte E Vuillemin A Briancon S Pralimap Trial Group. Adolescents' Physical Activity and Sedentary Behavior: A Pathway in Reducing Overweight and Obesity. The PRALIMAP 2-Year Cluster Randomized Controlled Trial. Journal of Physical Activity \& Health 2015;12(5):628-35.

\section{Pamplona 2015 \{published data only\}}

Pamplona Cunha H Caetano R Oliveira M V Rosini N Oppermann Moura S A Machado M J Silva E L. Effectiveness of lifestyle intervention in reducing cardiometabolic risk factors in students with dyslipidemia or abdominal obesity. Clinical Chemistry and Laboratory Medicine 2015;53:S310.

\section{Parkinson 2015 \{published data only\}}

Parkinson K N Jones A R Tovee M J Ells L J Pearce M S AraujoSoares $V$ Adamson A J. A cluster randomised trial testing an intervention to improve parents' recognition of their child's weight status: study protocol. BMC Public Health 2015;15:549.

\section{Paul 2016 \{published data only\}}

Paul IM, Savage JS, Anzman-Frasca S, Marini ME, Mindell JA, Birch LL. INSIGHT Responsive Parenting Intervention and Infant Sleep. Pediatrics 2016;138(1):e20160762.

\section{Pellanda 2015 \{published data only\}}

Pellanda L C Minossi V Borges R F Hattge Jr S P. The happy heart educational program randomized clinical trial: Benefits for children with cardiovascular risk factors and their caregivers are sustained after a year of follow-up. European Heart Journal 2015;36:114.

\section{Penalvo 2013 \{published data only\}}

Penalvo J L Santos-Beneit G Sotos-Prieto M Martinez R Rodriguez C Franco M Lopez-Romero P Pocock S Redondo J Fuster V. A cluster randomized trial to evaluate the efficacy of a school-based behavioral intervention for health promotion among children aged 3 to 5. BMC Public Health 2013;13:656.

\section{Penalvo 2015 \{published data only\}}

Penalvo J L Santos-Beneit G Sotos-Prieto M Bodega P Oliva B Orrit X Rodriguez C Fernandez-Alvira J M Redondo J Vedanthan R Bansilal S Gomez E Fuster V. The SI! Program for Cardiovascular Health Promotion in Early Childhood A ClusterRandomized Trial. Journal of the American College of Cardiology 2015;66(14):1525-34. 
Pinket 2016 \{published data only\}

Pinket A S Van Lippevelde W De Bourdeaudhuij I Deforche B Cardon G Androutsos O Koletzko B Moreno L A Socha P lotova V Manios Y De Craemer M ToyBox-Study Group. Effect and Process Evaluation of a Cluster Randomized Control Trial on Water Intake and Beverage Consumption in Preschoolers from Six European Countries: The ToyBox-Study. PLOS ONE [Electronic Resource] 2016;11(4):e0152928.

\section{Price 2015 \{published data only\}}

Price S Ferisin S Sharifi M Steinberg D Bennett G Wolin K Y Horan C Koziol R Marshall R Taveras E M. Development and Implementation of an Interactive Text Messaging Campaign to Support Behavior Change in a Childhood Obesity Randomized Controlled Trial. Journal of Health Communication 2015;20(7):843-50.

\section{Prina 2014 \{published data only\}}

Prina S Royer H. The importance of parental knowledge: evidence from weight report cards in Mexico. Journal of Health Economics 2014;37:232-47.

\section{Quintiliani 2014 \{published data only\}}

Quintiliani L M DeBiasse M A Branco J M Bhosrekar S G Rorie J A Bowen D J. Enhancing physical and social environments to reduce obesity among public housing residents: rationale, trial design, and baseline data for the Healthy Families study. Contemporary Clinical Trials 2014;39(2):201-10.

\section{Raat 2013 \{published data only\}}

Raat H Struijk M K Remmers T Vlasblom E van Grieken A Broeren S M te Velde S J Beltman M Boere-Boonekamp M M L'Hoir M $P$. Primary prevention of overweight in preschool children, the BeeBOFT study (breastfeeding, breakfast daily, outside playing, few sweet drinks, less TV viewing): design of a cluster randomized controlled trial. BMC Public Health 2013;13:974.

\section{Raine 2017 \{published data only\}}

Raine L B Khan N A Drollette E S Pontifex M B Kramer A F Hillman C H. Obesity, Visceral Adipose Tissue, and Cognitive Function in Childhood. Journal of Pediatrics 2017;187:134-140.e3.

\section{Rangan 2017 \{published data only\}}

Rangan A Zheng M Olsen N J Rohde J F Heitmann B L. Dietary intake, weight gain and sleep patterns in young children predisposed to overweight. Annals of Nutrition and Metabolism 2017;71(Supplement 2):538-9.

\section{Razani 2016 \{published data only\}}

Razani N Kohn M A Wells N M Thompson D Hamilton Flores H Rutherford G W. Design and evaluation of a park prescription program for stress reduction and health promotion in lowincome families: The Stay Healthy in Nature Everyday (SHINE) study protocol. Contemporary Clinical Trials 2016;51:8-14.

\section{Redfern 2016 \{published data only\}}

Redfern J Enright G Raadsma S Allman-Farinelli M InnesHughes C Khanal S Lukeis S Rissel C Gyani A. Effectiveness of a behavioral incentive scheme linked to goal achievement: study protocol for a randomized controlled trial.[Erratum appears in Trials. 2016;17(1):126 Note: Khanal, Santash [Corrected to Khanal, Santosh]; PMID: 26957296]. Trials [Electronic Resource] 2016;17:33.

Rerksuppaphol 2017 \{published data only\}

Rerksuppaphol L Rerksuppaphol S. Internet Based Obesity Prevention Program for Thai School Children- A Randomized Control Trial. Journal of Clinical and Diagnostic Research JCDR 2017;11(3):SC07-11.

Reyes-Morales 2016 \{published data only\}

Reyes-Morales H Gonzalez-Unzaga M A Jimenez-Aguilar A Uribe-Carvajal R. Effect of an intervention based on childcare centers to reduce risk behaviors for obesity in preschool children. [Spanish]. Boletin Medico del Hospital Infantil de Mexico 2016;73(2):75-83.

\section{Richmond 2016 \{published data only\}}

Richmond S A Kang J Doyle-Baker P K Nettel-Aguirre A Emery C A. A School-Based Injury Prevention Program to Reduce Sport Injury Risk and Improve Healthy Outcomes in Youth: A Pilot Cluster-Randomized Controlled Trial. Clinical Journal of Sport Medicine 2016;26(4):291-8.

\section{Rosario 2015 \{published data only\}}

Rosario R Araujo A Padro P Lopes O Pereira B Moreira P. Characteristics of a successful program to decrease BMI and LNED intake in school children. Obesity Facts 2015;8:128.

Rosario 2017 \{published data only\}

Rosario R Araujo A Padrao P Lopes O Moreira A Pereira B Moreira P. Prevention of childhood obesity: Lessons learned from a school-based intervention program. Obesity Facts 2017;10:52.

\section{Roth 2010 \{published data only\}}

Roth K, Mauer S, Obinger M, Ruf K. Prevention through Activity in Kindergarten Trial (PAKT): a cluster randomised controlled trial to assess the effects of an activity intervention in preschool children. BMC Public Health 2010;10:410.

\section{Ruiter 2015 \{published data only\}}

Ruiter E L Fransen G A Molleman G R van der Velden K Engels R $C$. The effectiveness of a web-based Dutch parenting program to prevent overweight in children 9-13 years of age: study protocol for a two-armed cluster randomized controlled trial. BMC Public Health 2015;15:148.

Rush 2016 \{published data only\}

Rush E Cairncross C Williams M H Tseng M Coppinger T McLennan S Latimer K. Project Energize: intervention development and 10 years of progress in preventing childhood obesity. BMC Research Notes 2016;9:44.

Salazar 2014 \{published data only\}

Salazar G Vasquez F Concha F Rodriguez Mdel P Berlanga Mdel R Rojas J Munoz A Andrade M. Pilot nutrition and physical activity intervention for preschool children attending daycare centres (JUNJI): primary and secondary outcomes. Nutricion hospitalaria 2014;29(5):1004-12. 
Sanchez-Lopez 2015 \{published data only\}

Sanchez-Lopez M Pardo-Guijarro M J Del Campo D G Silva P Martinez-Andres M Gulias-Gonzalez R Diez-Fernandez A Franquelo-Morales $P$ Martinez-Vizcaino V Movi-Kids group. Physical activity intervention (Movi-Kids) on improving academic achievement and adiposity in preschoolers with or without attention deficit hyperactivity disorder: study protocol for a randomized controlled trial. Trials [Electronic Resource] 2015;16:456.

\section{Santos 2012 \{published data only\}}

Santos R Durksen A Chanoine J P Miln A L Mayer T McGavock J. Healthy buddies $\odot$ manitoba: A clustered randomized controlled trial of peer-based healthy living lessons plans on body weight and physical activity in early years students. Canadian Journal of Diabetes 2012;1:S20.

\section{Santos 2015 \{published data only\}}

Santos I S Matijasevich A Assuncao M C Valle N C Horta B L Goncalves H D Gigante D P Martines J C Pelto G Victora C G. Promotion of Weight Gain in Early Childhood Does Not Increase Metabolic Risk in Adolescents: A 15-Year Follow-Up of a Cluster-Randomized Controlled Trial. Journal of Nutrition 2015;145(12):2749-55

\section{Santos 2016 \{published data only\}}

Santos I S Bassani D G Matijasevich A Halal C S Del-Ponte B da Cruz S H Anselmi L Albernaz E Fernandes M Tovo-Rodrigues $L$ Silveira M F Hallal P C. Infant sleep hygiene counseling (sleep trial): protocol of a randomized controlled trial. BMC Psychiatry 2016;16(1):307.

\section{Santos-Beneit 2015 \{published data only\}}

Santos-Beneit G Sotos-Prieto M Pocock S Redondo J Fuster $\mathrm{V}$ Penalvo J L. Association between anthropometry and high blood pressure in a representative sample of preschoolers in madrid. Revista Espanola de Cardiologia 2015;68(6):477-84.

\section{Savage 2016 \{published data only\}}

Savage JS, Birch LL, Marini M, Anzman-Frasca S, Paul IM. Effect of the INSIGHT Responsive Parenting Intervention on Rapid Infant Weight Gain and Overweight Status at Age 1 Year: A Randomized Clinical Trial. JAMA Pediatrics 2016;170(8):742-9.

\section{Sayers 2017 \{published data only\}}

Sayers R Galland B Cameron S Gray A Heath A Lawrence J Newlands A Taylor B Taylor R. Does early parental education prevent infant sleep problems?. Journal of Sleep Research 2017;26(Supplement 1):9.

\section{Schoffman 2017 \{published data only\}}

Schoffman DE. Enhancing Parent-child communication and promoting physical activity and healthy eating through mobile technology: A randomized trial. Columbia: University of South Carolina, 2017:182.

\section{Schuh 2017 \{published data only\}}

Schuh D S Goulart M R Barbiero S M Sica C D Borges R Moraes D W Pellanda L C. Healthy School, Happy School: Design and Protocol for a Randomized Clinical Trial Designed to Prevent
Weight Gain in Children. Arquivos Brasileiros de Cardiologia 2017;108(6):501-7.

\section{Schwartz 2015 \{published data only\}}

Schwartz R Vigo A de Oliveira L D Justo Giugliani E R. The Effect of a Pro-Breastfeeding and Healthy Complementary Feeding Intervention Targeting Adolescent Mothers and Grandmothers on Growth and Prevalence of Overweight of Preschool Children. PLOS ONE [Electronic Resource] 2015;10(7):e0131884.

Seguin 2017 \{published data only\}

Seguin R A Morgan E H Hanson K L Ammerman A S Jilcott Pitts S B Kolodinsky J Sitaker M Becot F A Connor L M Garner J A McGuirt J T. Farm Fresh Foods for Healthy Kids (F3HK): An innovative community supported agriculture intervention to prevent childhood obesity in low-income families and strengthen local agricultural economies. BMC Public Health 2017;17(1):306.

\section{Seward 2016 \{published data only\}}

Seward K Wolfenden L Finch M Wiggers J Wyse R Jones J Gillham K Yoong S L. Multistrategy childcare-based intervention to improve compliance with nutrition guidelines versus usual care in long day care services: a study protocol for a randomised controlled trial. BMJ Open 2016;6(6):e010786.

Sgambato 2016 \{published data only\}

Sgambato M R Cunha D B Henriques V T Estima C C Souza B S Pereira R A Yokoo E M Paravidino V B Sichieri R. PAAPPAS community trial protocol: a randomized study of obesity prevention for adolescents combining school with household intervention. BMC Public Health 2016;16(1):809.

\section{Shah 2016 \{published data only\}}

Shah N B Fenick A M Rosenthal M S. A Healthy Weight for Toddlers? Two-Year Follow-up of a Randomized Controlled Trial of Group Well-Child Care. Clinical Pediatrics 2016;55(14):1354-7.

\section{Sherwood 2015 \{published data only\}}

Sherwood N E JaKa M M Crain A L Martinson B C Hayes M G Anderson J D. Pediatric Primary Care-Based Obesity Prevention for Parents of Preschool Children: A Pilot Study. Childhood Obesity 2015;11(6):674-82.

\section{Simons 2014 \{published data only\}}

Simons M Chinapaw M J van de Bovenkamp M de Boer M R Seidell J C Brug J de Vet E. Active video games as a tool to prevent excessive weight gain in adolescents: rationale, design and methods of a randomized controlled trial. BMC Public Health 2014;14:275.

\section{Simons 2015 \{published data only\}}

Simons M Brug J Chinapaw M J de Boer M Seidell J de Vet E. Replacing Non-Active Video Gaming by Active Video Gaming to Prevent Excessive Weight Gain in Adolescents. PLOS ONE [Electronic Resource] 2015;10(7):e0126023.

\section{Skouteris 2010 \{published data only\}}

Skouteris H, McCabe M, Swinburn B, Hill B. Healthy eating and obesity prevention for preschoolers: a randomised controlled trial. BMC Public Health 2010;10:220. 
Skouteris 2014 \{published data only\}

Skouteris H Edwards S Rutherford L Cutter-MacKenzie A Huang T O'Connor A. Promoting healthy eating, active play and sustainability consciousness in early childhood curricula, addressing the Ben10TM problem: a randomised control trial. BMC Public Health 2014;14:548.

\section{Smedegaard 2016 \{published data only\}}

Smedegaard S Christiansen L B Lund-Cramer P Bredahl T Skovgaard T. Improving the well-being of children and youths: a randomized multicomponent, school-based, physical activity intervention. BMC Public Health 2016;16(1):1127.

\section{Smith 2015 \{published data only\}}

Smith J D Montano Z Dishion T J Shaw D S Wilson M N. Preventing weight gain and obesity: indirect effects of the family check-up in early childhood. [Erratum appears in Prev Sci. 2015 Apr;16(3):420; PMID: 25740012]. Prevention Science 2015;16(3):408-19.

\section{Smith 2017 \{published data only\}}

Smith J J Morgan P J Lonsdale C Dally K Plotnikoff R C Lubans $D$ R. Mediators of change in screen-time in a school-based intervention for adolescent boys: findings from the ATLAS cluster randomized controlled trial. Journal of Behavioral Medicine 2017;40(3):423-33.

\section{Smith 2018 \{published data only\}}

Smith $\mathrm{E}$. The effects of access and education on preschool children's fruit and vegetable intake. Columbus: The Ohio State University, 2018:214.

\section{Sobko 2016 \{published data only\}}

Sobko T Tse M Kaplan M. A randomized controlled trial for families with preschool children - promoting healthy eating and active playtime by connecting to nature. BMC Public Health 2016;16:505.

\section{Spieker 2015a \{published data only\}}

Spieker E A Sbrocco T Theim K R Maurer D Johnson D Bryant E Bakalar J L Schvey N A Ress R Seehusen D Klein D A Stice E Yanovski J A Chan L Gentry S Ellsworth C Hill J W Tanofsky-Kraff M Stephens M B. Preventing Obesity in the Military Community (POMC): the development of a clinical trials research network. International Journal of Environmental Research \& Public Health [Electronic Resource] 2015;12(2):1174-95.

\section{Steenbock 2017 \{published data only\}}

Steenbock B Zeeb H Rach S Pohlabeln H Pischke C R. Design and methods for a cluster-controlled trial conducted at sixtyeight daycare facilities evaluating the impact of "JolinchenKids - Fit and Healthy in Daycare", a program for health promotion in 3- to 6-year-old children. [Erratum appears in BMC Public Health. 2017 Sep 22;17 (1):736; PMID: 28938882]. BMC Public Health 2017;18(1):6.

\section{Stettler 2015 \{published data only\}}

Stettler N Wrotniak B H Hill D L Kumanyika S K Xanthopoulos M S Nihtianova S Shults J Leff S S Pinto A Berkowitz R I Faith M S. Prevention of excess weight gain in paediatric primary care: beverages only or multiple lifestyle factors. The Smart
Step Study, a cluster-randomized clinical trial. Pediatric Obesity 2015;10(4):267-74.

\section{Suchert 2015 \{published data only\}}

Suchert V Isensee B Sargent J Weisser B Hanewinkel R lauft Study Group. Prospective effects of pedometer use and class competitions on physical activity in youth: A cluster-randomized controlled trial. Preventive Medicine 2015;81:399-404.

\section{Sun 2017 \{published data only\}}

Sun A, Cheng J, Bui Q, Liang Y, Ng T, Chen JL. Home-Based and Technology-Centered Childhood Obesity Prevention for Chinese Mothers With Preschool-Aged Children. Journal of Transcultural Nursing 2017;28(6):616-24.

\section{Sutherland 2016 \{published data only\}}

Sutherland R Reeves P Campbell E Lubans D R Morgan P J Nathan N Wolfenden L Okely A D Gillham K Davies L Wiggers J. Cost effectiveness of a multi-component school-based physical activity intervention targeting adolescents: the 'Physical Activity 4 Everyone' cluster randomized trial. International Journal of Behavioral Nutrition \& Physical Activity 2016;13:94.

\section{Tang 2016 \{published data only\}}

Tang M, Griese KE, Krebs NF. Dietary intakes of formula-fed infants consuming a meat-or dairy-based complementary diet: A semi-controlled feeding trial. FASEB Journal. Conference: Experimental Biology. 2016.

\section{Tarro 2017 \{published data only\}}

Tarro L Aceves-Martins M Papell-Garcia I Arola L Giralt M Llaurado E Sola R. A youth-led, social marketing intervention run by adolescents to encourage healthy lifestyles among younger school peers (EYTO-kids project): A protocol for pilot cluster randomized controlled trial (Spain). International Journal of Environmental Research and Public Health 2017;14(8):923.

\section{Taveras 2012 \{published data only\}}

Taveras EM. Healthy Habits, Happy Homes: methods and baseline data of a randomized controlled trial to improve household routines for obesity prevention. Preventive Medicine 2012;55(5):418-26.

\section{Taylor 2013 \{published data only\}}

Taylor NJ. Using intervention mapping to develop a culturally appropriate intervention to prevent childhood obesity: The HAPPY (Healthy and Active Parenting Programme for Early Years) study. International Journal of Behavioral Nutrition and Physical Activity 2013;10:142.

\section{Taylor 2016 \{published data only\}}

Taylor R W Heath A L Galland B C Cameron S L Lawrence J A Gray A R Tannock G W Lawley B Healey D Sayers R M Hanna M Meredith-Jones K Hatch B Taylor B J. Three-year follow-up of a randomised controlled trial to reduce excessive weight gain in the first two years of life: protocol for the POI follow-up study. BMC Public Health 2016;16(1):771. 
Taylor 2017 \{published data only\}

Taylor B Taylor R Gray A Galland B Heath A Lawrence J Hanna M Hatch $B$. The prevention of obesity in infancy by targeting sleep or food and activity: RCT outcomes at 5 years. Obesity Facts 2017;10:23.

\section{Taylor 2017a \{published data only\}}

Taylor R W Williams S M Fangupo L J Wheeler B J Taylor B J Daniels L Fleming E A McArthur J Morison B Erickson L W Davies R S Bacchus S Cameron S L Heath A M. Effect of a BabyLed Approach to Complementary Feeding on Infant Growth and Overweight: A Randomized Clinical Trial. JAMA Pediatrics 2017;171(9):838-46.

\section{Thayer 2017 \{published data only\}}

Thayer LM. Food explorers: Family edition a theory-based text message and social media pilot intervention for families to address child diet. Chapel Hill: The University of North Carolina at Chapel Hill, 2017:209.

\section{The Healthy School Start Plus Intervention Study \{published data only\}}

NCT03390725 Trial registration number. The Healthy School Start Plus Intervention Study. https://ClinicalTrials.gov/show/ NCT03390725. https://ClinicalTrials.gov/show/NCT03390725, 2017:NCT03390725.

\section{Thompson 2013 \{published data only\}}

Thompson D Mahabir R Bhatt R Boutte C Cantu D Vazquez I Callender C Cullen K Baranowski T Liu Y Walker C Buday R. Butterfly Girls; promoting healthy diet and physical activity to young African American girls online: rationale and design. BMC Public Health 2013;13:709.

\section{Tirlea 2016 \{published data only\}}

Tirlea L Truby H Haines T P. Pragmatic, Randomized Controlled Trials of the Girls on the Go! Program to Improve Self-Esteem in Girls. American Journal of Health Promotion 2016;30(4):231-41.

\section{Tomayko 2016 \{published data only\}}

Tomayko E J Prince R J Cronin K A Adams A K. The Healthy Children, Strong Families intervention promotes improvements in nutrition, activity and body weight in American Indian families with young children. Public Health Nutrition 2016;19(15):2850-9.

\section{Tomayko 2017 \{published data only\}}

Tomayko E J Prince R J Cronin K A Parker T Kim K Grant V M Sheche J N Adams A K. Healthy Children, Strong Families 2: A randomized controlled trial of a healthy lifestyle intervention for American Indian families designed using community-based approaches. Clinical Trials 2017;14(2):152-61.

\section{Toscano 2017 \{published data only\}}

Toscano C V A Carvalho H M Ferreira J P. Exercise Effects for Children With Autism Spectrum Disorder: Metabolic Health, Autistic Traits, and Quality of Life. Perceptual \& Motor Skills 2017;125(1):126-146.

\section{van Grieken 2017 \{published data only\}}

van Grieken A Vlasblom E Wang L Beltman M Boere-Boonekamp M M L'Hoir M P Raat H. Personalized Web-Based Advice in Combination With Well-Child Visits to Prevent Overweight in Young Children: Cluster Randomized Controlled Trial. Journal of Medical Internet Research 2017;19(7):e268.

\section{van Nassau 2014 \{published data only\}}

van Nassau F Singh A S Cerin E Salmon J van Mechelen W Brug $J$ Chinapaw M J. The Dutch Obesity Intervention in Teenagers (DOiT) cluster controlled implementation trial: intervention effects and mediators and moderators of adiposity and energy balance-related behaviours. International Journal of Behavioral Nutrition \& Physical Activity 2014;11:158.

\section{Vilchis-Gil 2016 \{published data only\}}

Vilchis-Gil J Klunder-Klunder M Duque X Flores-Huerta S. Decreased Body Mass Index in Schoolchildren After Yearlong Information Sessions With Parents Reinforced With Web and Mobile Phone Resources: Community Trial. Journal of Medical Internet Research 2016;18(6):e174.

\section{Wade 2017 \{published data only\}}

Wade Tracey D Wilksch Simon M Paxton Susan J Byrne Susan M Austin S. Do universal media literacy programs have an effect on weight and shape concern by influencing media internalization?. International Journal of Eating Disorders 2017;50(7):731-8.

\section{Wagner 2017 \{published data only\}}

Wagner K A Braun E Armah S M Horan D Smith L G Pike J Tu W Hamilton M T Delp E J Campbell W W Boushey C J Hannon T S Gletsu-Miller N. Dietary Intervention for Glucose Tolerance In Teens (DIG IT): Protocol of a randomized controlled trial using health coaching to prevent youth-onset type 2 diabetes. Contemporary Clinical Trials 2017;53:171-7.

\section{Walther 2011 \{published data only\}}

Walther C, Walther C. [Effects of daily physical exercise at school on cardiovascular risk--results of a 2-year cluster-randomized study]. [German]. Deutsche Medizinische Wochenschrift 2011;136(46):2348-54

\section{Walton 2015 \{published data only\}}

Walton K Filion A J Darlington G Morrongiello B Haines J. Parents and tots together: Adaptation of a family-based obesity prevention intervention to the Canadian context. Canadian Journal of Diabetes 2015;39:S72-3.

Walton 2016 \{published data only\}

Walton K Filion A J Gross D Morrongiello B Darlington G Randall Simpson J Hou S Haines J. Parents and Tots Together: Pilot randomized controlled trial of a family-based obesity prevention intervention in Canada. Canadian Journal of Public Health. Revue Canadienne de Sante Publique 2016;106(8):e555-62.

\section{Wang 2017 \{published data only\}}

Wang Z Xu F Ye Q Tse L A Xue H Tan Z Leslie E Owen N Wang Y. Childhood obesity prevention through a community-based cluster randomized controlled physical activity intervention 
among schools in China: The health legacy project of the 2nd world summer youth olympic games (YOG-obesity study). International Journal of Obesity 2017;05:05.

\section{Wasser 2015 \{published data only\}}

Wasser H Bentley M. Mothers and others: designing a randomized trial to prevent obesity among infants and toddlers. FASEB journal Meeting Abstracts. 2015; Vol. 29, issue 1 Meeting Abstracts.

\section{Waters 2017 \{published data only\}}

Waters E Gibbs L Tadic M Ukoumunne O C Magarey A Okely A D de Silva A Armit C Green J O'Connor T Johnson B Swinburn B Carpenter L Moore G Littlecott H Gold L. Cluster randomised trial of a school-community child health promotion and obesity prevention intervention: findings from the evaluation of fun ' $n$ healthy in Moreland!.[Erratum appears in BMC Public Health. 2017 Sep 22;17 (1):736; PMID: 28938882]. BMC Public Health 2017;18(1):92.

\section{Welk 2015 \{published data only\}}

Welk G J Chen S Nam Y H Weber T E. A formative evaluation of the SWITCH obesity prevention program: print versus online programming. BMC Obesity 2015;2:20.

\section{Wen 2015 \{published data only\}}

Wen L M Baur L A Simpson J M Xu H Hayes A J Hardy L L Williams M Rissel C. Sustainability of Effects of an Early Childhood Obesity Prevention Trial Over Time: A Further 3Year Follow-up of the Healthy Beginnings Trial. JAMA Pediatrics 2015;169(6):543-51.

\section{Wendel 2016 \{published data only\}}

Wendel M L Benden M E Zhao H Jeffrey C. Stand-Biased Versus Seated Classrooms and Childhood Obesity: A Randomized Experiment in Texas. American Journal of Public Health 2016;106(10):1849-54.

\section{Wessel 2015 \{published data only\}}

Wessel J O'Kelly-Phillips E Palmer K Saha C Hannon T Carroll A Marrero D G. Comparative effectiveness study of the diabetes prevention program in families: preliminary results. Circulation. 2015; Vol. 131.

\section{Wessel 2015a \{published data only\}}

Wessel J Phillips E Palmer K Saha C Hannon T S Carroll A Marrero D G. Comparative effectiveness trial of the diabetes prevention program in families. Diabetes 2015;64:A633.

\section{Wieland 2016 \{published data only\}}

Wieland M L Weis J A Hanza M M Meiers S J Patten C A Clark M M Sloan J A Novotny P J Njeru J W Abbenyi A Levine J A Goodson M Porraz Capetillo M G Osman A Hared A Nigon J A Sia I G. Healthy immigrant families: Participatory development and baseline characteristics of a community-based physical activity and nutrition intervention. Contemporary Clinical Trials 2016;47:22-31.

\section{Wilken 2013 \{published data only\}}

Wilken L R Novotny R Fialkowski M K Boushey C J Nigg C Paulino Y Leon Guerrero R Bersamin A Vargo D Kim J Deenik
J. Children's Healthy Living ( $\mathrm{CHL}$ ) Program for remote underserved minority populations in the Pacific region: rationale and design of a community randomized trial to prevent early childhood obesity. BMC Public Health 2013;13:944.

\section{Wilksch 2017 \{published data only\}}

Wilksch S M Paxton S J Byrne S M Austin S B O'Shea A Wade T $D$. Outcomes of three universal eating disorder risk reduction programs by participants with higher and lower baseline shape and weight concern. International Journal of Eating Disorders 2017;50(1):66-75.

\section{Wolfenden 2016b \{published data only\}}

Wolfenden L Wiggers J Morgan P Razak L A Jones J Finch M Sutherland R Lecathelinais C Gillham K Yoong S L. A randomised controlled trial of multiple periods of outdoor free-play to increase moderate-to-vigorous physical activity among 3 to 6 year old children attending childcare: study protocol. BMC Public Health 2016;16:926.

\section{Wright 2016 \{published data only\}}

Wright C M Duquesnay P J Anzman-Frasca S Chomitz V R Chui K Economos C D Langevin E G Nelson M E Sacheck J M. Study protocol: the Fueling Learning through Exercise (FLEX) study - a randomized controlled trial of the impact of school-based physical activity programs on children's physical activity, cognitive function, and academic achievement. BMC Public Health 2016;16(1):1078.

\section{Xin 2016 \{published data only\}}

Xin H Qing Y Zhiyong W Huafeng Y Shengxiang Q Xupeng C Fei X. Effectiveness of lifestyle intervention to prevent obesity among primary school students in Nanjing, China. Obesity Reviews 2016;17:123-4.

\section{Xu 2015 \{published data only\}}

Xu F Ware R S Leslie E Tse L A Wang Z Li J Wang Y. Effectiveness of a Randomized Controlled Lifestyle Intervention to Prevent Obesity among Chinese Primary School Students: CLICK-Obesity Study. PLOS ONE [Electronic Resource] 2015;10(10):e0141421.

\section{Xu 2016 \{published data only\}}

Xu F Wang Z Ye Q Ah Tse L Xue H Tan Z Wang Y. Policy-oriented, school-based physical activity intervention to prevent childhood obesity in China (the health legacy project of the second Summer Youth Olympic Games): A cluster randomised trial. The Lancet Diabetes and Endocrinology 2016;3:S9.

\section{Xu 2017 \{published data only\}}

Xu H Li Y Zhang Q Hu X L Liu A Du S Li T Guo H Li Y Xu G Liu W Ma J Ma G. Comprehensive school-based intervention to control overweight and obesity in China: a cluster randomized controlled trial. Asia Pacific Journal of Clinical Nutrition 2017;26(6):1139-51.

\section{Yoshinaga 2016 \{published data only\}}

Yoshinaga M Ogata H Aoki M Ito Y Hamajima T Miyazaki A Tokuda M Lin L Horigome H Takahashi H Nagashima M. Efficacy of walking as a lifestyle modification approach for childhood 
obesity. A randomized controlled trial. European Heart Journal 2016;37(Supplement 1):248.

\section{Zafiropulos 2015 \{published data only\}}

Zafiropulos V Chatzi V Dimitropoulakis P Markaki A Fthenakis Z G Thalassinos N Fragkiadakis G A. Preliminary results of a dietary intervention among primary school children. Obesity Facts 2015;8:133.

\section{Zota 2016 \{published data only\}}

Zota D Dalma A Petralias A Lykou A Kastorini C M Yannakoulia M Karnaki P Belogianni K Veloudaki A Riza E Malik R Linos A. Promotion of healthy nutrition among students participating in a school food aid program: a randomized trial. International Journal of Public Health 2016;61(5):583-92.

\section{References to ongoing studies}

\section{Adab 2015b \{published data only\}}

Adab P, Pallan MJ, Lancashire ER, Hemming K, Frew E, Griffin T, et al. A cluster-randomised controlled trial to assess the effectiveness and cost-effectiveness of a childhood obesity prevention programme delivered through schools, targeting 6-7 year old children: the WAVES study protocol. BMC Public Health 2015;15:488.

\section{Adams 2012 \{published data only\}}

Adams AK, LaRowe TL, Cronin KA, Prince RJ, Wubben DP, Parker, et al. The Healthy Children, Strong Families intervention: design and community participation. Journal of Primary Prevention 2012;33(4):175-85.

\section{Barlow 2008 \{unpublished data only\}}

Barlow J. Empowering mothers to prevent obesity at weaning. tcru.ioe.ac.uk/nsf/Default.aspx?tabid=336.

\section{Bundy 2011 \{published data only\}}

Bundy AC. The Sydney playground project: popping the bubblewrap--unleashing the power of play: a cluster randomized controlled trial of a primary school playgroundbased intervention aiming to increase children's physical activity and social skills. BMC Public Health 2011;11:680.

\section{Draper 2010 \{published data only\}}

Draper CE, de Villiers A, Lambert E. HealthKick: a nutrition and physical activity intervention for primary schools in low-income settings. BMC Public Health 2010;10:398.

\section{Dreyhaupt 2012 \{published data only\}}

Dreyhaupt J, Koch B, Wirt T, Schreiber A, Brandstetter S, Kesztyus D, et al. Evaluation of a health promotion program in children: study protocol and design of the cluster-randomized Baden-Wurttemberg primary school study. BMC Public Health 2012;12:157. [DRKS-ID: DRKS00000494]

\section{Flattum 2015 \{published data only\}}

Flattum C, Draxten M, Horning M, Fulkerson J. HOME Plus: program design and implementation of a family-focused, community-based intervention to promote the frequency and healthfulness of family meals, reduce children's sedentary behavior, and prevent obesity. International Journal of Behavioral Nutrition and Physical Activity 2015;12:53.

Glazebrook 2011 \{published data only\}

Glazebrook C. Evaluating the effectiveness of a schools-based programme to promote exercise self-efficacy in children and young people with risk factors for obesity: steps to active kids (STAK). BMC Public Health 2011;11:830.

Hesketh 2013 \{published data only\}

Hesketh KD. The Melbourne Infant Feeding, Activity and Nutrition Trial (InFANT) program follow-up. Contemporary Clinical Trials 2013;34(1):145-51.

\section{Horodynski 2011a \{published data only\}}

Horodynski MA, Baker S, Coleman. The Healthy Toddlers Trial Protocol: an intervention to reduce risk factors for childhood obesity in economically and educationally disadvantaged populations. BMC Public Health 2011;11:581.

\section{Horodynski 2011b \{published data only\}}

Horodynski MA. Healthy babies through infant-centered feeding protocol: an intervention targeting early childhood obesity in vulnerable populations. BMC Public Health 2011;11:868.

Miller 2012 \{published data only\}

Miller AL, Horodynski MA, Herb HE, Peterson KE, Contreras D, Kaciroti N, et al. Enhancing self-regulation as a strategy for obesity prevention in Head Start preschoolers: the growing healthy study. BMC Public Health 2012;12:1040.

\section{Murphy 2013 \{published data only\}}

Murphy M, Porter J, Yusuf H, Ntouva A, Newton T, Kolliakou A, et al. Considerations and lessons learned from designing a motivational interviewing obesity intervention for young people attending dental practices: a study protocol paper. Contemporary Clinical Trials 2013;36(1):126-34.

\section{Myers 2014 \{published data only\}}

Myers EF, Gerstein DE, Foster J, Ross M, Brown K, Kennedy E, et al. Energy balance for kids with play: design and implementation of a multi-component school-based obesity prevention program. Childhood Obesity 2014;10(3):251-9.

Olsen 2012 \{published data only\}

Olsen NJ, Buch-Andersen T, Handel MN, Ostergaard LM, Pedersen J, Seeger C, et al. The Healthy Start project: a randomized, controlled intervention to prevent overweight among normal weight, preschool children at high risk of future overweight. BMC Public Health 2012;12:590.

\section{Ostbye 2015 \{published data only\}}

Ostbye T, Mann CM, Vaughn AE, Namenek Brouwer RJ, Benjamin Neelon SE, Hales D, et al. The keys to healthy family child care homes intervention: study design and rationale. Contemporary Clinical Trials 2015;40:81-9.

Paul 2014 \{published data only\}

Paul IM, Williams JS, Anzman-Frasca S, Beiler JS, Makova KD, Marini ME, et al. The Intervention Nurses Start Infants Growing 
on Healthy Trajectories (INSIGHT) study. BMC Pediatrics 2014;14:184.

\section{Piek 2010 \{published data only\}}

Piek JP. Rationale, design and methods for a randomised and controlled trial to evaluate "Animal Fun" - a program designed to enhance physical and mental health in young children. $B M C$ Pediatrics 2010;10:78.

\section{Po'e 2013 \{published data only\}}

Po'e EK, Heerman WJ, Mistry RS, Barkin SL. Growing Right Onto Wellness (GROW): a family-centered, community-based obesity prevention randomized controlled trial for preschool childparent pairs. Contemporary Clinical Trials 2013;36(2):436-49.

\section{Reifsnider 2013 \{published data only\}}

Reifsnider E, McCormick DP, Cullen KW, Szalacha L, Moramarco MW, Diaz A, et al. A randomized controlled trial to prevent childhood obesity through early childhood feeding and parenting guidance: rationale and design of study. BMC Public Health 2013;13:880.

\section{Sanchez-Gomez 2012 \{published data only\}}

Sánchez-Gómez LM, Fernández-Luque MJ, Ruiz-Díaz L, SánchezAlcalde R, Sierra-García B, Mayayo-Vicente S, et al. A clusterrandomised clinical trial comparing two cardiovascular health education strategies in a child population: the Savinghearts project. BMC Public Health 2012;12:1024.

\section{Sherwood 2013 \{published data only\}}

Sherwood NE, French SA, Veblen-Mortenson S, Crain AL, Berge J, Kunin-Batson A, et al. NET-Works: linking families, communities and primary care to prevent obesity in preschoolage children. Contemporary Clinical Trials 2013;36(2):544-54.

\section{Siegrist 2011 \{published data only\}}

Siegrist M, Hanssen H, Lammel C, Haller B, Halle M. A cluster randomised school-based lifestyle intervention programme for the prevention of childhood obesity and related early cardiovascular disease (JuvenTUM 3). BMC Public Health 2011;11:258

\section{Slawson 2015 \{published data only\}}

Slawson DL, Dalton WT, Dula TM, Southerland J, Wang L, Littleton MA, et al. College students as facilitators in reducing adolescent obesity disparity in Southern Appalachia: Team Up for Healthy Living. Contemporary Clinical Trials 2015;43:39-52.

\section{Sobko 2011 \{published data only\}}

Sobko T, Svensson Vi, Ek A, Ekstedt M, Karlsson H, Johansson E, et al. A randomised controlled trial for overweight and obese parents to prevent childhood obesity--Early STOPP (STockholm Obesity Prevention Program). BMC Public Health 2011;11:336.

\section{Taylor 2011 \{published data only\}}

Taylor BJ, Heath AL, Galland BC, Gray AR, Lawrence JA, Sayers RM, et al. Prevention of Overweight in Infancy (POI.nz) study: a randomised controlled trial of sleep, food and activity interventions for preventing overweight from birth. BMC Public Health 2011;11:942.

\section{Tovar 2013 \{published data only\}}

Tovar A, Hennessy E, Must A, Hughes SO, Gute DM, Sliwa S, et al. Feeding styles and evening family meals among recent immigrants. International Journal of Behavioral Nutrition and Physical Activity 2013;10:84.

Walters 2012 \{published data only\}

Walters KL. Project hli?dx(w)/Healthy Hearts Across Generations: development and evaluation design of a tribally based cardiovascular disease prevention intervention for American Indian families. Journal of Primary Prevention 2012;33(4):197-207.

\section{Ward 2011 \{published data only\}}

Ward DS, Vaughn AE, Bangdiwala KI, Campbell M, Jones DJ, Panter AT, et al. Integrating a family-focused approach into child obesity prevention: rationale and design for the My Parenting SOS study randomized control trial. BMC Public Health 2011;11:431.

\section{Waters 2007 \{published and unpublished data\}}

Waters E. Fun 'n healthy in Moreland! A 5-year schoolcommunity-based health promotion and obesity prevention study for primary school children. www.mchs.org.au/ 2007.

\section{Wen 2012a \{published data only\}}

Wen LM, Baur LA, Rissel, Flood V, Simpson JM, Hayes A, et al. Healthy Beginnings Trial Phase 2 study: follow-up and cost-effectiveness analysis. Contemporary Clinical Trials 2012;33(2):396-401.

\section{Wyatt 2013 \{published data only\}}

Wyatt KM, Lloyd JJ, Abraham C, Creanor S, Dean S, Densham E, et al. The Healthy Lifestyles Programme (HeLP), a novel schoolbased intervention to prevent obesity in school children: study protocol for a randomised controlled trial. Trials 2013;14:95.

\section{Xu 2012 \{published data only\}}

Xu F, Ware R, Tse LA, Wang Z, Hong X, Song A, et al. A schoolbased comprehensive lifestyle intervention among Chinese kids against obesity (CLICK-Obesity): rationale, design and methodology of a randomized controlled trial in Nanjing city, China. BMC Public Health 2012;12:316.

\section{Zoorob 2013 \{published data only\}}

Zoorob R, Buchowski MS, Beech B. Healthy families study: design of a childhood obesity prevention trial for Hispanic families. Contemporary Clinical Trials 2013;35(2):108-21.

\section{Additional references}

\section{Al-Khudairy 2017}

Al-Khudairy L, Loveman E, Colquitt JL, Mead E, Johnson RE, Fraser $\mathrm{H}$, et al. Diet, physical activity and behavioural interventions for the treatment of overweight or obese adolescents aged 12 to 17 years. Cochrane Database of Systematic Reviews 2017, Issue 6. [DOI: 10.1002/14651858.CD012691; PUBMED: 28639320] 


\section{Armstrong 2007}

Armstrong $\mathrm{R}$, Waters $\mathrm{E}$ on behalf of the Guidelines for Systematic Reviews in Health Promotion and Public Health Taskforce. Health Promotion and Public Health Systematic Review Handbook. Version 2. Victoria: Cochrane Health Promotion and Public Health Field, 2007.

\section{Ballon 2018}

Ballon M, Botton J, Charles MA, Carles S, de Lauzon-Guillain B, Forhan A, et al. on behalf of the EDEN Mother-Child Cohort Study Group. Socioeconomic inequalities in weight, height and body mass index from birth to 5 years. International Journal of Obesity 2018;42:1671-9.

\section{Bhaskaran 2014}

Bhaskaran K, Douglas I, Forbes H, dos-Santos-Silva I, Leon DA, Smeeth L. Body-mass index and risk of 22 specific cancers: a population-based cohort study of 5.24 million UK adults. Lancet 2014;384(9945):755-65. [PUBMED: 25129328]

\section{Cole 2000}

Cole TJ, Bellizzi MC, Flegal KM, Dietz WH. Establishing a standard definition for child overweight and obesity worldwide: international survey. BMJ 2000;320:1240-53.

\section{Cole 2007}

Cole TJ, Flegal KM, Nicholls D, Jackson AA. Body mass index cut offs to define thinness in children and adolescents: international survey. BMJ 2007; Vol. 335, issue 7612:194.

\section{de Onis 2004}

de Onis M, Garza C, Victora CG, Onyango AW, Frongillo EA, Martines J. The WHO Multicentre Growth Reference Study: planning, study design, and methodology. Food and Nutrition Bulletin 2004; Vol. 25, issue 1 Suppl:S15-26.

\section{de Onis 2007}

de Onis M, Garza C, Onyango AW, Borghi E. Comparison of the WHO child growth standards and the CDC 2000 growth charts. Journal of Nutrition 2007; Vol. 137, issue 1:144-8.

\section{de Onis 2010}

de Onis M, Blossner M, Borghi E. Global prevalence and trends of overweight and obesity among preschool children. American Journal of Clinical Nutrition 2010;92(5):1257-64. [PUBMED: 20861173]

\section{Deeks 2017}

Deeks JJ, Higgins JP, Altman DG (editors), on behalf of the Cochrane Statistical Methods Group. Chapter 9: Analysing data and undertaking meta-analyses. In: Higgins JPT, Churchill R, Chandler J, Cumpston MS (editors), Cochrane Handbook for Systematic Reviews of Interventions version 5.2.0 (updated June 2017), Cochrane, 2017. Available from www.training.cochrane.org/handbook.

\section{Dobbs 2014}

Dobbs R, Sawers C, Thompson F, Manyika J, Woetzel J, Child P, et al. Overcoming Obesity: An Initial Economic Analysis. Shanghai: McKinsey Global Institute, 2014.

\section{Dwan 2010}

Dwan K, Gamble C, Kolamunnage-Dona R, Mohammed S, Powell C, Williamson PR. Assessing the potential for outcome reporting bias in a review: a tutorial. Trials 2010; Vol. 11, issue 52. [DOI: 10.1186/1745-6215-11-52]

\section{Egger 1997a}

Egger M, Davey Smith G, Schneider M, Minder C. Bias in meta-analysis detected by a simple, graphical test. $B M J$ 1997;315:629-34.

\section{Egger 1997b}

Egger G, Swinburn B. An "ecological” approach to the obesity pandemic. BMJ 1997;315:477-480.

\section{Ford 2010}

Ford AL, Hunt LP, Cooper A, Shield JP. What reduction in BMI SDS is required in obese adolescents to improve body composition and cardiometabolic health?. Archives of Disease in Childhood 2010;95(4):256-61.

\section{Freedman 1999}

Freedman DS, Dietz WH, Srinivasan SR, Berenson GS. The relation of overweight to cardiovascular risk factors among children and adolescents: the Bogalusa Heart Study. Pediatrics 1999;103(6 Pt 1):1175-82.

\section{GBD Obesity Collaboration 2014}

The GBD 2013 Obesity Collaboration. Global, regional and national prevalence of overweight and obesity in children and adults 1980-2013: a systematic analysis. Lancet 2014;384(9945):766-81. [DOI: 10.1016/S0140-6736(14)60460-8.]

\section{GRADEpro GDT 2015 [Computer program]}

McMaster university (developed by Evidence Prime). GRADEpro GDT. Version Date Accessed: 20 April 2019. Hamilton (ON): McMaster university (developed by Evidence Prime), 2015.

\section{Harbord 2009}

Harbord RM, Harris RJ, Sterne JA. Updated tests for small-study effects in meta-analyses. Stata Journal 2009;9(2):197-210.

\section{Heath 2002}

Heath EM, Coleman KJ. Evaluation of the institutionalization of the Coordinated Approach to Child Health (CATCH) in a US/ Mexico border community. Health Education and Behavior 2002;29:444-60.

\section{Higgins 2003}

Higgins JP, Thompson SG, Deeks JJ, Altman DG. Measuring inconsistency in meta-analyses. BMJ 2003;327:557-60.

\section{Higgins 2011a}

Higgins JP, Deeks JJ, Altman DG (editors). Chapter 16: Special topics in statistics. In: Higgins JPT, Green S editor(s). Cochrane Handbook for Systematic Reviews of Interventions Version 5.1.0. The Cochrane Collaboration, 2011.

\section{Higgins 2011b}

Higgins JPT, Atman D, Sterne JAC, on behalf of the Cochrane Statistical Methods Group and the Cochrane Bias Methods 
Group. Chapter 8: Assessing risk of bias in included studies. In: Higgins JPT, Green S editor(s). Cochrane Handbook for Systematic Reviews of Interventions. Version 5.1.0 [updated March 2011]. The Cochrane Collaboration, 2011.

\section{Higgins 2011C}

Higgins JP, Green S (editors). Cochrane Handbook for Systematic Reviews of Interventions Version 5.1.0 [updated March 2011]. The Cochrane Collaboration, 2011. www.handbook.cochrane.org.

\section{Higgins 2011d}

Sterne JAC, Egger M, Moher D. Chapter 10: Addressing reporting bias. In: Higgins JPT, Green S editor(s). Cochrane Handbook for Systematic Reviews of Interventions Version 5.1.0. The Cochrane Collaboration, 2011.

\section{Higgins 2016}

Higgins JP, Sterne JA, Savović J, Page MJ, Hróbjartsson A, Boutron I, et al. A revised tool for assessing risk of bias in randomized trials. In: Chandler J, McKenzie J, Boutron I, Welch V (editors). Cochrane Methods. Cochrane Database of Systematic Reviews 2016, Issue 10 (Suppl 1). sites.google.com/ site/riskofbiastool/welcome/rob-2-0-tool?authuser=0. [DOI: 10.1002/14651858.CD201601; https://sites.google.com/site/ riskofbiastool/welcome/rob-2-0-tool?authuser=0]

\section{Higgins 2017}

Higgins JP, Altman DG, Sterne JA (editors). Chapter 8: Assessing risk of bias in included studies. In: Higgins JPT, Churchill R, Chandler J, Cumpston MS (editors), Cochrane Handbook for Systematic Reviews of Interventions version 5.2.0 (updated June 2017), Cochrane. Available from www.training.cochrane.org/handbook 2017.

\section{Hillier-Brown 2014}

Hillier-Brown FC, Bambra CL, Cairns-Nagi JM, Kasim A, Moore HJ, Summerbell CD. A systematic review of the effectiveness of individual, community and societal level interventions at reducing socioeconomic inequalities in obesity amongst children. BMC Public Health 2014;14:830.

\section{Hoffman 2014}

Hoffman T, Glasziou P, Boutron I, Milne R, Perera R, Moher D, et al. Better reporting of interventions: template for intervention description and replication (TIDieR) checklist and guide. BMJ 2014;348:g1687.

\section{Kain 2004}

Kain J, Uauy R, Albala, Vio F, Cerda R, Leyton B. Schoolbased obesity prevention in Chilean primary school children: methodology and evaluation of a controlled study. International Journal of Obesity 2004;28(4):483-93.

\section{Kipping 2008a}

Kipping R, Jago R, Lawlor D. Obesity in children. Part 1: Epidemiology, measurement, risk factors, and screening. BMJ 2008;337:a1824.

\section{Kirkham 2010}

Kirkham JJ, Dwan KM, Altman DG, Gamble C, Dodd S, Smyth R, et al. The impact of outcome reporting bias in randomised controlled trials on a cohort of systematic reviews. BMJ 2010; Vol. 340:c365. [DOI: 10.1136/bmj.c365]

\section{Knai 2018}

Knai C, Lobstein T, Petticrew M, Rutter H, Savona N. England's childhood obesity action plan II. BMJ 2018;362:bmj.k3098. [DOI: 10.1136/bmj.k3098]

\section{Lefebvre 2011}

Lefebvre C, Manheimer E, Glanville J. Chapter 6: Searching for studies. In: Higgins JPT, Green S (editors). Cochrane Handbook for Systematic Reviews of Interventions Version 5.1.0 (updated March 2011). The Cochrane Collaboration, 2011. Available from www.handbook.cochrane.org.

\section{Llargues 2011}

Llargues E, Franco R, Recasens A, Nadal A, Vila M, Pérez MJ, et al. Assessment of a school-based intervention in eating habits and physical activity in school children: the AVall study. Journal of Epidemiology and Community Health 2011;65(10):896-901.

\section{Lobstein 2004}

Lobstein T, Bauer L, Uauy R. Obesity in children and young people: a crisis in public health. Obesity Reviews 2004;5 Suppl 1:1-104.

\section{Marmot 2010}

Marmot M, Allen J, Goldblatt P, Boyce T, McNeish D, Grady M, et al. Fair Society, Healthy Lives (The Marmot Review). London: Institute of Health Equity, 2010.

\section{McCrabb 2019}

McCrabb S, Lane C, Hall A, Milat A, Bauman A, Sutherland R, et al. Scaling-up evidence-based obesity interventions: a systematic review assessing intervention adaptations and effectiveness and quantifying the scale-up penalty. Obesity Reviews 2019;1:1-19. [DOI: 10.1111/obr.12845]

\section{McKenzie 2016}

McKenzie JE, Brennan SE, Ryan RE, Thompson HJ, Johnstone RV, Thomas J. Chapter 3: Defining the Criteria for including studies and how they will be grouped for synthesis. Draft version (January 2019) for inclusion in:. Higgins JPT, Thomas J, Chandler J, Cumpston M, Li T, Page MJ, Welch EV (editors) Cochrane Handbook for Systematic Reviews of Interventions. London: Cochrane. 2016. [DOI: 10.1002/14651858.CD201601; https://sites.google.com/site/ riskofbiastool/welcome/rob-2-0-tool?authuser=0]

\section{Mead 2017}

Mead E, Brown T, Rees K, Azevedo LB, Whittaker V, Jones D, et al. Diet, physical activity and behavioural interventions for the treatment of overweight or obese children from the age of 6 to 11 years. Cochrane Database of Systematic Reviews 2017, Issue 6. [DOI: 10.1002/14651858.CD012651; PUBMED: 28639319] 


\section{Moher 2009}

Moher D, Liberati A, Tetzlaff J, Altman DG, the PRISMA Group (2009). Preferred reporting items for systematic reviews and meta-analyses: the PRISMA Statement. PLoS Medicine 2009;6(7):e1000097. [DOI: 10.1371/journal.pmed1000097]

\section{Monteiro 2004}

Monteiro CA, Conde WL, Lu B, Popkin BM. Obesity and inequities in health in the developing world. International Journal of Obesity and Related Metabolic Disorders 2004;28(9):1181-6.

\section{Mora 2015}

Mora T, Llargués E, Recasens A. Does health education affect BMI? Evidence from a school-based randomised-control trial. Economics and Human Biology 2015;17(C):190-201.

\section{Must 1992}

Must A, Jacques PF, Dallal GE, Bajema CJ, Dietz WH. Long-term morbidity and mortality of overweight adolescents. A followup of the Harvard Growth Study of 1922 to 1935. New England Journal of Medicine 1992;327(19):1350-5.

\section{Must 1999}

Must A, Strauss RS. Risks and consequences of childhood and adolescent obesity. International Journal of Obesity and Related Metabolic Disorders 1999;23 Suppl 2:S2-11.

\section{NHS England 2014}

NHS England. Health Survey for England 2014. The Joint Health Surveys Unit of NatCen Social Research and the Research Department of Epidemiology and Public Health at University College London 2014.

\section{Pangrazi 2003}

Pangrazi RP, Beighle A, Vehige T, Vack C. Impact of Promoting Lifestyle Activity for Youth (PLAY) on children's physical activity. Journal of School Health 2003;73(8):317-21.

\section{Power 1997}

Power C, Lake JK, Cole TJ. Measurement and long-term health risks of child and adolescent fatness. International Journal of Obesity and Related Metabolic Disorders 1997;21(7):507-26.

\section{Public Health England 2015}

Public Health England. Childhood Obesity: Applying all our Health. Public Health England. London 2015.

\section{Rai 2019}

Rai KK, Dogra S, Barber S, Adab P, Summerbell C, on behalf of the 'Childhood Obesity Prevention in Islamic Religious Settings' Programme Management Group'. A scoping review and systematic mapping of health promotion interventions associated with obesity in Islamic religious settings in the UK. Obesity Reviews Accepted April 2019.

\section{Rayyan-QCRI 2016}

Ouzzani M, Hammady H, Fedorowicz Z, Elmagarmid A. Rayyan - a web and mobile app for systematic reviews. Systematic Reviews 2016;5:210. [DOI: 10.1186/s13643-016-0384-4]

\section{Reilly 2011}

Reilly JJ, Kelly J. Long-term impact of overweight and obesity in childhood and adolescence on morbidity and premature mortality in adulthood: systematic review. International Journal of Obesity 2011;35(7):891-8.

\section{Reinehr 2004}

Reinehr T, Andler W. Changes in the atherogenic risk factor profile according to degree of weight loss. Archives of Disease in Childhood 2004;89:419-22.

\section{Review Manager 2014 [Computer program]}

Nordic Cochrane Centre, The Cochrane Collaboration. Review Manager 5 (RevMan 5). Version 5.3. Copenhagen: Nordic Cochrane Centre, The Cochrane Collaboration, 2014.

\section{Rutter 2016}

Rutter H, Glonti K. Towards a new model of evidence for public health. Lancet 2016;388:S7. [DOI: 10.1016/ S0140-6736(16)32243-7]

\section{Rutter 2017}

Rutter H, Savona N, Glonti K, Bibby J, Cummins S, Finegood DT, et al. The need for a complex systems model of evidence for public health. Lancet 2017;390(10112):2602-4.

\section{Schünemann 2017}

Schünemann HJ, Oxman AD, Vist GE, Higgins JP, Deeks JJ, Glasziou P, et al. on behalf of the Cochrane Applicability and Recommendations Methods Group. Chapter 12: Interpreting results and drawing conclusions. In: Higgins JPT, Churchill R, Chandler J, Cumpston MS (editors), Cochrane Handbook for Systematic Reviews of Interventions version 5.2.0 (updated June 2017). Cochrane, 2017. Available from www.training.cochrane.org/handbook.

\section{Singh 2008}

Singh AS, Mulder C, Twisk JW, Van Mechelen W, Chinapaw MJ. Tracking of childhood overweight into adulthood: a systematic review of the literature. Obesity Reviews 2008;9(5):474-88.

\section{Stata 2019 [Computer program]}

StataCorp. Stata Statistical Software:. Version Release 15. College Station, TX: StataCorp LLC, 2017.

\section{Stokols 1992}

Stokols D. Establishing and maintaining healthy environments. Toward a social ecology of health promotion. American Psychologist 1992;47(1):6-22.

\section{Stunkard 1983}

Stunkard A, Sorenson T, Schulsinger F. Use of the Danish Adoption Register for the study of obesity and thinness.. In: Kety S, Rowland L, Sidman R, Matthysse S editor(s). The Genetics of Neurological and Psychiatric Disorders. New York: Raven Press, 1983:115-120.

\section{Summerbell 2012}

Summerbell CD, Moore HJ, Vogele C, Kreichauf S, Wildgruber A, Manios $\mathrm{Y}$, et al. Evidence-based recommendations for the development of obesity prevention programs targeted at 
preschool children. Obesity Reviews 2012;13 Suppl 1:129-32. [PUBMED: 22309071]

\section{Thomas 2003}

Thomas BH, Ciliska D, Dobbins M, Micucci S. National Collaborating Centre for Methods and Tools. Quality assessment tool for quantitative studies.. Effective Public Health Practice Project. Hamilton, Canada: McMaster University, 2003, issue Retrieved from http://www.nccmt.ca/resources/ search/14.

\section{Ueffing 2009}

Ueffing E, Tugwell P, Welch V, Petticrew M, Kristjansson E, for the Cochrane Health Equity Field. C1, C2 Equity Checklist for Systematic Review Authors. Version 2009-05-28. Accessed at www.equity.cochrane.org/files/equitychecklist.pdf 2009.

\section{Ukoumunne 1999}

Ukoumunne OC, Gulliford MC, Chinn S, Sterne JA, Burney PG. Methods for evaluating area-wide and organisation-based interventions in health and health care: a systematic review. Health Technology Assessment 1999;3(5):iii-92. [PUBMED: 10982317]

\section{United Nations}

United Nations. Sustainable development goals. Goal 3: ensure healthy lives and promote well-being for all at all ages. https:// www.who.int/sdg/targets/en/ Accessed 31 December 2018.

\section{Verbeek 2017}

Verbeek J, ljaz S, Mischke C. Inclusion criteria for outcomes of studies not clearly reported in Cochrane systematic reviews. Journal of Clinical Epidemiology 2017;87:98-106. [PUBMED: 28478083]

\section{Wang 2006}

Wang S, Moss JR, Hiller JE. Applicability and transferability of interventions in evidence-based public health. Health Promotion International 2006;21(1):76-83.

\section{Whitaker 1997}

Whitaker RC, Wright JA, Pepe MS, Seidel KD, Dietz WH. Predicting obesity in young adulthood from childhood and parental obesity. New England Journal of Medicine 1997;337(13):869-73.

\section{WHO 1986}

World Health Organization. The Ottawa Charter for Health Promotion. https://www.who.int/healthpromotion/ conferences/previous/ottawa/en/. Geneva: First International Conference on Health Promotion, Ottawa, 21 November, 1986.

\section{WHO 2016}

World Health Organization. Consideration of the evidence on childhood obesity for the Commission on Ending Childhood Obesity: report of the ad hoc working group on science and evidence for ending childhood obesity. World Health Organization, Geneva, Switzerland 2016.

\section{WHO 2017}

World Health Organization. Report of the Commission on Ending Childhood Obesity. Implementation plan: executive summary. apps.who.int/iris/bitstream/handle/10665/259349/ WHO-NMH-PND-ECHO-17.1-eng.pdf? sequence=1\&isAllowed=y 2017. [(WHO/NMH/PND/ECHO/17.1). Licence: CC BY-NC-SA 3.0 IGO]

\section{Williamson 2008}

Williamson DA, Champagne CM, Harsha D, Han H, Martin CK, Newton R Jr. Design and methods for a childhood obesity prevention program in rural schools. Contemporary Clinical Trials 2008;29(5):783-95.

\section{Wolfenden 2016a}

Wolfenden L, Jones J, Williams CM, Finch M, Wyse RJ, Kingsland $M$, et al. Strategies to improve the implementation of healthy eating, physical activity and obesity prevention policies, practices or programmes within childcare services. Cochrane Database of Systematic Reviews 2016, Issue 10. [DOI: 10.1002/14651858.CD011779.pub2]

\section{Yatsuya 2010}

Yatsuya H, Folsom AR, Yamagishi K, North KE, Brancati FL, Stevens J. Race- and sex-specific associations of obesity measures with ischemic stroke incidence in the Atherosclerosis Risk in Communities (ARIC) study. Stroke 2010;41(3):417-25. [PUBMED: 20093637]

\section{References to other published versions of this review Campbell 2001}

Campbell K, Waters E, O'Meara S, Summerbell C. Interventions for preventing obesity in childhood. A systematic review. Obesity Reviews 2001;2(3):149-57.

\section{Campbell K 2002}

Campbell K, Waters E, O'Meara S, Kelly S, Summerbell C. Interventions for preventing obesity in children. Cochrane Database of Systematic Reviews 2002, Issue 2. [DOI: 10.1002/14651858.CD001871]

\section{Summerbell 2005}

Summerbell C, Waters E, Edmunds L, Kelly S, Brown T, Campbell K. Interventions for preventing obesity in children. Cochrane Database of Systematic Reviews 2005, Issue 3. [DOI: 10.1002/14651858.CD001871.pub2]

\section{Waters 2011}

Waters E, de Silva-Sanigorski A, Burford BJ, Brown T, Campbell KJ, Gao Y, et al. Interventions for preventing obesity in children. Cochrane Database of Systematic Reviews 2011, Issue 12. [DOI: 10.1002/14651858.CD001871.pub3]

* Indicates the major publication for the study 
CHARACTERISTICS OF STUDIES

Characteristics of included studies [ordered by study ID]

Alkon 2014

Methods

Study name: Nutrition and physical activity self-assessment for child care (NAP SACC)

Study design: cluster-RCT

Intervention period: 7 months

Follow-up period (post-intervention): nil

Differences in baseline characteristics: reported

Reliable outcomes: reported

Protection against contamination: reported

Unit of allocation: childcare centre

Unit of analysis: individual (controlling for clustering effect)

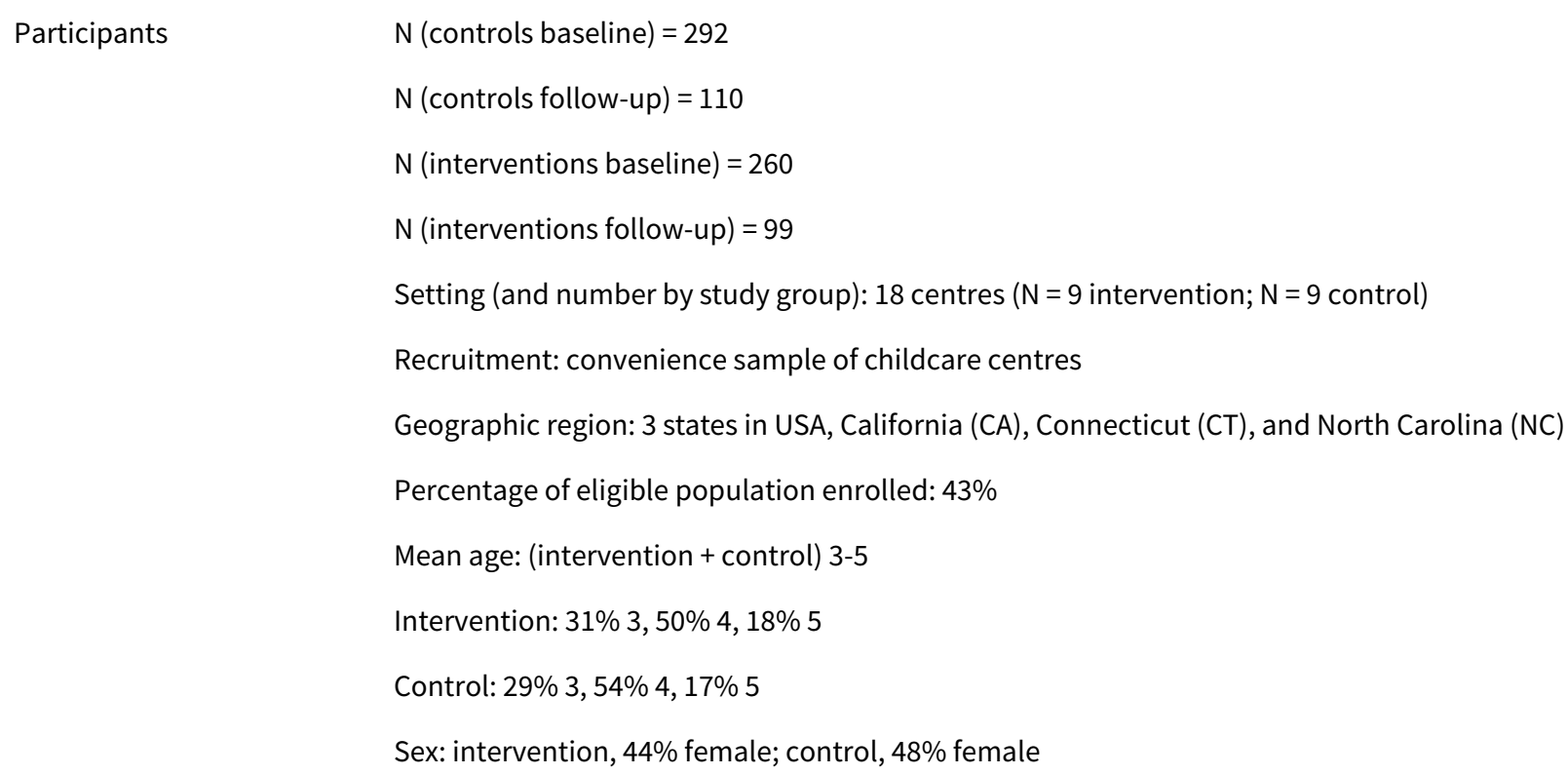
trition and PA environments in childcare settings by improving the nutritional quality of food and beverages, the amount and quality of PA, staff-child interactions, and centre nutrition and PA policies and practices.

Trained nurse childcare health consultants facilitated 5, one-hour workshops

for child care providers and other staff (e.g. cooks, administrators) at each of the intervention centres on the following:

- childhood obesity

- healthy eating for young children

- PA for young children

- personal health and wellness

- working with families to promote healthy behaviours. 
Alkon 2014 (Continued)

7 of the intervention centres also received the parent workshop, "Raising Healthy Kids." The CCHCs worked with the centre directors to write or update the centre's nutrition and PA policies. They also provided at least monthly on-site consultations and additional phone or email consultations and distributed posters and information sheets on nutrition and PAs. The posters were displayed in the childcare centres, and the information sheets were given to the childcare providers and parents. Examples of some common issues addressed during the consultation visits were the type of milk served, healthy snacks, and ideas for structured PA.

The nurse CCHCs conducted a mean (SD) of 11 (3) on-site visits and 8 (6) off-site consultations per centre over the 7-month intervention, in addition to the provider and parent workshops.

Dietary and PA intervention vs control

Outcome measures
- Primary outcome: zBMI, \% overweight, \% obese
- Secondary outcomes: provider and parent knowledge survey, nutrition and PA policies, nutrition and
PA practices
Process evaluation: reported (fidelity)

Implementation-related factors
Theoretical basis: NR

Resources for intervention implementation: reported

Who delivered the intervention: reported

PROGRESS categories assessed at baseline: child: gender; parent: race/ethnicity, education, occupation, SES

PROGRESS categories analysed at outcome: NR

Outcomes relating to harms/unintended effects: NR

Intervention included strategies to address diversity or disadvantage: NR

Economic evaluation: NR

Notes NCT01921842

Funding: grant \#R40 MC 08727 through the U.S. Department of Health and Human Services, Health Resources and Services Administration, Maternal and Child Health Research Program

Each of the centres received USD 500 for its participation in the study. The intervention centre directors were asked to purchase equipment or supplies to support PA. The programme has been used by a number of states and incorporated into the US public health campaign Let's Move.

\section{Risk of bias}

\begin{tabular}{lll}
\hline Bias & Authors' judgement & Support for judgement \\
\hline $\begin{array}{l}\text { Random sequence genera- } \\
\text { tion (selection bias) }\end{array}$ & Unclear risk & $\begin{array}{l}\text { Randomisation at childcare centre } \\
\text { Quote: "The centers were matched on size and the proportion of children eli- } \\
\text { gible for income subsidies and then randomly assigned to the NAP SACC inter- } \\
\text { vention or control group." }\end{array}$ \\
\hline $\begin{array}{l}\text { Allocation concealment } \\
\text { (selection bias) }\end{array}$ & Low risk & $\begin{array}{l}\text { Randomisation done at childcare centre level } \\
\text { Quote: "The centers were matched on size and the proportion of children eli- } \\
\text { gible for income subsidies and then randomly assigned to the NAP SACC inter- } \\
\text { vention or control group." }\end{array}$
\end{tabular}


Alkon 2014 (Continued)

Blinding (performance bias and detection bias)

All outcomes

Low risk Research assistant blinded to group assignment completed the centre's written policy assessments, centre-level observational

measures, and child-level height and weight measurements

Incomplete outcome data High risk

(attrition bias)

Although the pre-intervention heights and weights included children randomly selected in each site and data were missing at random, there was an imbalance. The total at the pre-intervention period, 268 of the $552(49 \%)$ children enrolled in the study, was limited by availability of resources. There were more children (336) with post-intervention heights and weights, but only children with matched data were included in the centre-level analyses (209)

\begin{tabular}{lll}
\hline $\begin{array}{l}\text { Selective reporting (re- } \\
\text { porting bias) }\end{array}$ & Low risk & Trial registration document checked. All outcomes reported \\
\hline Other bias & Low risk & No other threats to validity noted \\
\hline $\begin{array}{l}\text { Other bias- timing of re- } \\
\text { cruitment of clusters }\end{array}$ & Unclear risk & $\begin{array}{l}1 \text { control centre, which withdrew when it was unable to complete the required } \\
\text { number of study questionnaires, was replaced with a matched centre prior to } \\
\text { intervention }\end{array}$ \\
\hline
\end{tabular}

Amaro 2006

\begin{tabular}{|c|c|}
\hline \multirow[t]{8}{*}{ Methods } & Study design: cluster-RCT \\
\hline & Intervention period: 24 weeks \\
\hline & Follow-up period (post-intervention): nil \\
\hline & Differences in baseline characteristics: reported \\
\hline & Reliable outcomes: reported \\
\hline & Protection against contamination: NR \\
\hline & Unit of allocation: classrooms \\
\hline & Unit of analysis: child (controlling for clustering effect of classroom) \\
\hline \multirow[t]{10}{*}{ Participants } & $\mathrm{N}($ controls baseline $)=103$ \\
\hline & $\mathrm{N}$ (controls follow-up) $=88$ \\
\hline & $\mathrm{N}$ (interventions baseline) $=188$ \\
\hline & $\mathrm{N}$ (interventions follow-up) $=153$ \\
\hline & Setting: schools ( $N=3$; intervention: 10 classrooms, control: 6 classrooms) \\
\hline & Recruitment: middle school students in Naples \\
\hline & Geographic region: Italy \\
\hline & Percentage of eligible population enrolled: $95 \%$ \\
\hline & Mean age: intervention, $12.3 \pm 0.8$; control, $12.5 \pm 0.7$ \\
\hline & Sex: male and female \\
\hline Interventions & Board game Kaledo to increase nutrition knowledge \\
\hline
\end{tabular}


Amaro 2006 (Continued)

- 1 play session/week lasting 15-30 min with 2 players on each team

- Players match difference between the total energy intake given by the nutrition cards and the total energy expenditure given by the activity cards

- At the end of the game the player with the least difference between energy intake and expenditure is the winner

Dietary intervention vs control

\begin{tabular}{ll}
\hline Outcomes & Height, weight \\
& PA \\
& - Nutrition knowledge \\
& Dietary intake \\
Process evaluation: NR
\end{tabular}

Implementation-related factors
Theoretical basis: NR

Resources for intervention implementation (e.g. funding needed or staff hours required): reported Who delivered the intervention: reported

PROGRESS categories assessed at baseline: reported (race)

PROGRESS categories analysed at outcome: NR

Outcomes relating to harms/unintended effects: NR

Intervention included strategies to address diversity or disadvantage: NR

Economic evaluation: NR

Notes

Funding: study has been made possible by contributions from the Italian Association Amici di Raoul Follereau (AIFO), Commune of Naples and from the 2nd University of Naples

\section{Risk of bias}

\begin{tabular}{lll}
\hline Bias & Authors' judgement & Support for judgement \\
\hline $\begin{array}{l}\text { Random sequence genera- } \\
\text { tion (selection bias) }\end{array}$ & Unclear risk & Quote: "Classrooms were randomly assigned to the conditions." \\
\hline $\begin{array}{l}\text { Allocation concealment } \\
\text { (selection bias) }\end{array}$ & Unclear risk & NR \\
\hline $\begin{array}{l}\text { Blinding (performance } \\
\text { bias and detection bias) } \\
\text { All outcomes }\end{array}$ & Unclear risk & NR \\
\hline $\begin{array}{l}\text { Incomplete outcome data } \\
\text { (attrition bias) }\end{array}$ & High risk & 2 clusters lost from intervention and 1 lost from control group \\
$\begin{array}{l}\text { All outcomes } \\
\text { Selective reporting (re- } \\
\text { porting bias) }\end{array}$ & Unclear risk & Neither protocol nor trial registration documents were available. \\
\hline \begin{tabular}{l} 
Other bias \\
\hline $\begin{array}{l}\text { Other bias- timing of re- } \\
\text { cruitment of clusters }\end{array}$
\end{tabular} & Unclear risk & No other threats to validity \\
\hline
\end{tabular}


Study name: ACTIVITAL

Study design: cluster-RCT

Intervention period: 28 months

Follow-up period (post-intervention): nil

Differences in baseline characteristics: reported

Reliable outcomes: reported

Protection against contamination: reported

Unit of allocation: school

Unit of analysis: individual with clustering by school

$$
\begin{aligned}
& N(\text { controls baseline })=740 \\
& N(\text { controls follow-up })=521 \text { (for BMI) } \\
& N \text { (interventions baseline })=700 \\
& N(\text { interventions follow-up })=539 \text { (for BMI) }
\end{aligned}
$$

Setting (and number by study group): 20 schools ( $N=10$ intervention; $N=10$ control)

Recruitment: all 8th and 9th graders from 20 schools in urban Cuenca were invited to participate.

Geographic region: Cuenca, Ecuador

Percentage of eligible population enrolled: $71 \%$ (20/28 paired schools)

Mean age: intervention $12.9 \pm 0.8$; control $12.9 \pm 0.8$

Sex: intervention, $66.4 \%$ female; control, $59.3 \%$ female

Analysed the effects of a school-based health promotion intervention on physical fitness and explored if the effect varied with school characteristics

- Individual-based strategies

- Book 1 (curriculum 90 min every 2 weeks): to create awareness of importance of adequate PA throughout adolescence, to increase knowledge and enhance decision-making skills. Thought textbooks and pedagogic materials for teachers and students

- Book 2 (curriculum 90 min every 2 weeks): to encourage the adolescents to be physically active for at least 60 min per day and to spend maximum 2 hour per day on sedentary activities.

- Environment-based strategies

- Parental workshops: in total six workshops were performed. Informative leaflets supporting the content of the workshop were distributed to each participant during the workshops. Two workshops focused on decreasing sedentary time and increasing PA ( $1^{\text {st }}$ year) and dealing with barriers for PA (2nd year). Parents attendance was mandatory through a letter signed by each school principal Each leaflet included theoretical information, advises and benefits on the particular topic of the workshops

- Social event: 1 hour interactive session with young athletes was given. Athletes shared their personal sport experiences and gave advice on active lifestyles and PA.

- Walking trail and posters: Using line markings, a walking trail was drawn on the school's playground. The length of the trail was the perimeter of playground, so adolescents could use it.. 3 posters suspended on the school walls adjacent to the trail, with phrases like: "Do you like to talk? "Walk and Talk" 
Andrade 2014 (Continued)

- Posters for classroom and food tuck shop: five different posters with key messages on PA and pictures of young athletes to encourage students to be active and eat healthy

Dietary and PA intervention vs control
Implementation-related factors

\section{Outcome measures}

- Primary outcome: physical fitness (EUROFIT battery), screen time (questionnaires) and PA (accelerometers)

- Secondary outcomes: zBMI, overweight prevalence

Process evaluation: reported (attendance)

Theoretical basis: SCT, IMB model, Control theory, TTM and TPB

Resources for intervention implementation: reported

Who delivered the intervention: reported

PROGRESS categories assessed at baseline: reported (gender)

PROGRESS categories analysed at outcome: gender (data NR)

Outcomes relating to harms/unintended effects: NR

Intervention included strategies to address diversity or disadvantage: NR

Economic evaluation: NR

$\begin{array}{ll}\text { Notes } & \text { NCT01004367 } \\ & \text { Funding: this work was supported by generous financial support from VLIR-UOS and Nutrition 3rd } \\ \text { World and conducted within the cooperation between the Cuenca University (Ecuador) and the Ghent } \\ \text { University (Belgium) } \\ \text { Mestizo ethnicity - no further details reported }\end{array}$

\section{Risk of bias}

\begin{tabular}{|c|c|c|}
\hline Bias & Authors' judgement & Support for judgement \\
\hline \multirow{2}{*}{$\begin{array}{l}\text { Random sequence genera- } \\
\text { tion (selection bias) }\end{array}$} & Low risk & Random number generation \\
\hline & & $\begin{array}{l}\text { Quote: "We randomly selected } 10 \text { pairs in Stata (version } 12.0 \text {, Stata Corpora- } \\
\text { tion, Texas, USA) using a random number generation with random allocation } \\
\text { of the intervention within each pair." }\end{array}$ \\
\hline $\begin{array}{l}\text { Allocation concealment } \\
\text { (selection bias) }\end{array}$ & Low risk & Randomisation at school level \\
\hline $\begin{array}{l}\text { Blinding (performance } \\
\text { bias and detection bias) } \\
\text { All outcomes }\end{array}$ & High risk & $\begin{array}{l}\text { Blinded staff measured outcomes but study authors acknowledge that they } \\
\text { cannot rule out that they could have observed elements of the interventions } \\
\text { such as posters/walking trail in intervention schools }\end{array}$ \\
\hline \multirow{2}{*}{$\begin{array}{l}\text { Incomplete outcome data } \\
\text { (attrition bias) } \\
\text { All outcomes }\end{array}$} & Low risk & $\begin{array}{l}\text { There was } 26 \% \text { dropout, unbalanced, missing data analysis showed no major } \\
\text { differences }\end{array}$ \\
\hline & & $\begin{array}{l}\text { Quote: "An intention-to-treat analysis was performed to assess the interven- } \\
\text { tion effect using mixed linear regression models with the pair-matching as ran- } \\
\text { dom effect." }\end{array}$ \\
\hline
\end{tabular}


Andrade 2014 (Continued)

Selective reporting (re- Unclear risk $\quad$ Trial registration found. The trial registration mentions "Anthropometry" as a porting bias) secondary outcome but fails to specify what specific outcome will be reported e.g. BMI or zBMI. BMI is reported in the study report but may have been selected.

\begin{tabular}{|c|c|c|}
\hline Other bias & Low risk & No other threats to validity \\
\hline \multirow[t]{2}{*}{$\begin{array}{l}\text { Other bias- timing of re- } \\
\text { cruitment of clusters }\end{array}$} & High risk & $\begin{array}{l}\text { Clusters were selected before randomisation but it seems student recruit- } \\
\text { ment/exclusion happened after clusters were assigned }\end{array}$ \\
\hline & & $\begin{array}{l}\text { Quote: "In each school, two 8th grades and two 9th grades were randomly se- } \\
\text { lected and all students in those grades were invited to participate in the study" }\end{array}$ \\
\hline
\end{tabular}

Annesi 2013

\begin{tabular}{ll}
\hline Methods & Study name: Start for life \\
& Study design: cluster-RCT \\
& Intervention period: 9 months \\
& Follow-up period (post-intervention): nil \\
& Differences in baseline characteristics: reported \\
& Reliable outcomes: reported \\
& Protection against contamination: NR \\
& Unit of allocation: class \\
& Unit of analysis: individual
\end{tabular}

Participants Very confusing reporting of participant numbers.

Abstract (Annessi 2013_2610) reports baseline data for intervention group $N=716$, control group $N=$ 1699 table 1); then 690 vs 464 (intervention vs control respectively, table 2) over 9 months

$\mathrm{N}$ (controls baseline $)=464$

$N($ controls follow-up $)=464$

$\mathrm{N}$ (interventions baseline $)=690$

$N$ (interventions follow-up) $=690$

Setting: YMCA-affiliated preschools: in the abstract (Annesi 2013_2610) it says that 18 treatment and 8 control classes were included, but in the text under methods it says 60 treatment and 38 control classes were included; 9 treatment and 8 control classes reported in Annesi 2013_3075

Recruitment: randomly selected from YMCA-affiliated preschools

Geographic region: south-eastern USA

Percentage of eligible population enrolled: NR

Mean age: intervention + control: $4.4 \pm 0.5$ "no difference in age between groups"

Sex: intervention + control: $47.6 \%$ female "no difference in sex between groups" 
Annesi 2013 (Continued)

Interventions 30-min/day preschool-based intervention (Start for life), with a foundation in SCT that emphasises the use of self-regulation skills and feelings of mastery (self-efficacy), was administered for 9 months to 4 and 5-year-old African American children. Preschool teachers in the Start for life treatment group received additional 4-h training where the administration of PAs supported by cognitive-behavioural methods was taught. They also retained a binder of daily lesson plans.

Note: in the control condition of usual care, the 30 min reserved for structured PA was under the control of the classroom teachers. It varied widely from class to class, generally consisting of a variety of gross motor activities and use of playground equipment (e.g. sliding boards, tricycles). Activities and movements ranged in intensity from light to vigorous and were highly variable.

PA vs control

Process outcomes: NR

Implementation-related factors
Theoretical basis: SCT and Self-efficacy theory

Resources for intervention implementation: NR

Who delivered the intervention: reported

PROGRESS categories assessed at baseline: gender, race/ethnicity

PROGRESS categories analysed at outcome: NR

Outcomes relating to harms/unintended effects: NR

Intervention included strategies to address diversity or disadvantage: a large sample size of mostly minority children was used to maximise generalisability to underserved subgroups that have a notably high prevalence of overweight and obesity in the USA

Economic evaluation: NR

Notes $\quad$ Funding: NR

Confirmation by email correspondence with study author, "there was random assignment throughout" and these 2 references are linked to the same study:

- Annesi JJ, Smith AE, Tennant GA., Annesi JJ. Effects of a cognitive-behaviourally based PA treatment for 4- and 5-year-old children attending US preschools. Int J Behav Med 2013 Dec;20(4):562-6. Ref ID: 2610

- Annesi JJ, Smith AE, Tennant GA., Annesi JJ. Reducing high BMI in African American preschoolers: effects of a behaviour-based PA intervention on caloric expenditure. South Med J 2013 Aug;106(8):456-9. Ref ID: 3075

In the abstract (Annesi 2013_2610) it says that 18 treatment and 8 control classes were included, but in the text under methods it says 60 treatment and 38 control classes were included; 9 treatment and 8 control classes reported in Annesi 2013_3075. Therefore data extracted for the larger sample from Annesi_2013_2610

African American children primarily, the socioeconomic strata were all lower to lower-middle classes.

\section{Risk of bias}

Bias Authors' judgement Support for judgement


Annesi 2013 (Continued)

Random sequence genera- Low risk tion (selection bias)
YMCA-managed after-school care sites in the southeastern USA were randomly assigned to either the experimental 'Start for life treatment or the comparison treatment via computer-generated random numbers. Study author confirmed "there was random assignment throughout"

\begin{tabular}{lll}
\hline $\begin{array}{l}\text { Allocation concealment } \\
\text { (selection bias) }\end{array}$ & Unclear risk & NR \\
\hline $\begin{array}{l}\text { Blinding (performance } \\
\text { bias and detection bias) }\end{array}$ & Unclear risk & NR \\
All outcomes & &
\end{tabular}

\begin{tabular}{|c|c|c|}
\hline $\begin{array}{l}\text { Incomplete outcome data } \\
\text { (attrition bias) } \\
\text { All outcomes }\end{array}$ & High risk & $\begin{array}{l}\text { Flow of children through the study impossible to determine. in the abstract } \\
\text { (Annesi 2013_2610) it says that } 18 \text { treatment and } 8 \text { control classes were in- } \\
\text { cluded, but in the text under methods it says } 60 \text { treatment and } 38 \text { control } \\
\text { classes were included; } 9 \text { treatment and } 8 \text { control classes reported in An- } \\
\text { nesi 2013_3075. Therefore we extracted data for the larger sample from An- } \\
\text { nesi_2013_2610 }\end{array}$ \\
\hline $\begin{array}{l}\text { Selective reporting (re- } \\
\text { porting bias) }\end{array}$ & Unclear risk & Protocol/trial registration documents were unavailable \\
\hline Other bias & Unclear risk & Contamination NR \\
\hline $\begin{array}{l}\text { Other bias- timing of re- } \\
\text { cruitment of clusters }\end{array}$ & Unclear risk & No information \\
\hline
\end{tabular}

\section{Baranowski 2003}

\begin{tabular}{|c|c|}
\hline \multirow[t]{2}{*}{ Methods } & $\begin{array}{l}\text { Study design: RCT } \\
\text { Follow-up: } 12 \text { weeks } \\
\text { Differences in baseline characteristics: reported } \\
\text { Reliable outcomes: yes for anthropometry and accelerometry } \\
\text { Protection against contamination: NR, but set in } 2 \text { camps } \\
\text { Unit of allocation: child } \\
\text { Unit of analysis: child }\end{array}$ \\
\hline & All analyses were performed according to ITT principles \\
\hline \multirow[t]{4}{*}{ Participants } & $\begin{array}{l}N \text { (controls baseline) }=16 \\
N(\text { controls follow-up) }=14 \\
N \text { (interventions baseline })=19 \\
N \text { (interventions follow-up) }=17\end{array}$ \\
\hline & $\begin{array}{l}\text { Recruitment: all consenting 8-year old, African American girls = 50th percentile for age and gender BMI, } \\
\text { with a parent willing to be involved. Set in Texas, USA }\end{array}$ \\
\hline & Proportion of eligibles participating: not stated, but children needed access to internet \\
\hline & $\begin{array}{l}\text { Mean age: intervention, } 8.3 \text { (SD 0.3); control: } 8.4 \text { (SD 0.3) years } \\
\text { Sex: girls only }\end{array}$ \\
\hline Interventions & $\begin{array}{l}\text { Set in summer camps and homes, the intervention was delivered by trained personnel in camp and re- } \\
\text { searchers via a website. The intervention was designed to prevent obesity and aimed to increase fruit, } \\
\text { vegetable and water consumption, and enhance PA. Intervention continued via a website with weekly } \\
\text { visits. The pilot also evaluated the feasibility of a larger trial. } \\
\text { Controls received usual camp activities and asked to visit control website once a month. }\end{array}$ \\
\hline
\end{tabular}


Baranowski 2003 (Continued)

(Combined effects of dietary interventions and PA interventions vs control)

\begin{tabular}{|c|c|}
\hline Outcomes & $\begin{array}{l}\text { - } \text { BMI } \\
\text { - } \text { Paist circumference } \\
\text { - } \text { DEXA) for \% body fat } \\
\text { - } \mathrm{PA:CSA} \text { accelerometer } \\
\text { - } \text { Modified SAPAC } \\
\text { - } \text { GEMS Activity Questionnaire (GAQ) computerised } \\
\text { - } \text { Dietary intake measured by two } 24-h \text { recalls using NDS-R } \\
\text { - Monitoring website usage } \\
\text { Process evaluation: reported }\end{array}$ \\
\hline $\begin{array}{l}\text { Implementation-related } \\
\text { factors }\end{array}$ & $\begin{array}{l}\text { Theoretical basis: SCT and Family Systems theory } \\
\text { Resources for intervention implementation (e.g. funding needed or staff hours required): NR } \\
\text { Who delivered the intervention: reported } \\
\text { PROGRESS categories assessed at baseline: reported (race, education, SES) } \\
\text { PROGRESS categories analysed at outcome: NR } \\
\text { Outcomes relating to harms/unintended effects: NR } \\
\text { Intervention included strategies to address diversity or disadvantage: reported } \\
\text { Economic evaluation: NR }\end{array}$ \\
\hline Notes & $\begin{array}{l}\text { Funding: this research was largely funded by a grant from the National Heart Lung and Blood Insti- } \\
\text { tute, U01 HL-65160. This work is also a publication of the United States Department of Agriculture } \\
\text { (USDA/ARS) Children's Nutrition Research Center, Department of Pediatrics, Baylor College of Medi- } \\
\text { cine, Houston, Texas, and was funded, in part, by federal funds from the USDA/ARS under Cooperative } \\
\text { Agreement No. 58-6250-6001 }\end{array}$ \\
\hline
\end{tabular}

\section{Risk of bias}

Bias Authors' judgement Support for judgement

$\begin{array}{ll}\begin{array}{l}\text { Random sequence genera- } \\ \text { tion (selection bias) }\end{array} & \text { Low risk }\end{array} \quad \begin{aligned} & \text { Quote: "Random assignment was conducted in an urn randomisation proce- } \\ & \text { dure, through telephone contact to the coordinating centre..." }\end{aligned}$

\begin{tabular}{ll}
\hline $\begin{array}{l}\text { Allocation concealment } \\
\text { (selection bias) }\end{array}$ & Low risk \\
& $\begin{array}{l}\text { Quote: "Random assignment was conducted in an urn randomisation proce- } \\
\text { dure, through telephone contact to the coordinating centre..." Statistically } \\
\text { significant differences between groups in BMl at baseline but very few peo- } \\
\text { ple in study, so this is in-line with possibility of baseline imbalance through } \\
\text { 'chance'. }\end{array}$ \\
\hline
\end{tabular}

\begin{tabular}{|c|c|c|}
\hline $\begin{array}{l}\text { Blinding (performance } \\
\text { bias and detection bias) } \\
\text { All outcomes }\end{array}$ & Unclear risk & NR \\
\hline $\begin{array}{l}\text { Incomplete outcome data } \\
\text { (attrition bias) } \\
\text { All outcomes }\end{array}$ & Low risk & $\begin{array}{l}\text { No participants reported as lost. } \\
\text { Quote: "Data were analyzed according to "Intention- to-Treat" (ITT) princi- } \\
\text { ples." }\end{array}$ \\
\hline
\end{tabular}


Baranowski 2003 (Continued)
Selective reporting (re-
Unclear risk
Protocol/trial registration documents were unavailable porting bias)

Other bias

Low risk

Baranowski 2011

\begin{tabular}{ll}
\hline Methods & Study design: RCT \\
Intervention period: 3 months \\
Follow-up period (post-intervention): 2 months \\
Differences in baseline characteristics: reported \\
Reliable outcomes: reported \\
Protection against contamination: NR \\
Unit of allocation: individual \\
Unit of analysis: individual
\end{tabular}

Participants

$\mathrm{N}$ (control baseline $)=50$

$\mathrm{N}$ (control follow-up) $=40$

$\mathrm{N}($ intervention baseline $)=103$

$\mathrm{N}$ (intervention follow-up) $=93$

Setting (and number by study group): home-based (laboratory-based assessment)

Recruitment: included children between 50 percentile and 95 percentile BMI; children were recruited primarily with advertisements on a radio station whose listening audience included parents of children in the targeted age groups from ethnic minority communities (African-American, Hispanic)

Geographic region: Houston, Texas, USA

Percentage of eligible population enrolled: $68 \%$

Mean age: $10-12,42.5 \%=10$ years; $32.7 \%=11$ years $; 24.8 \%=12$ years

Sex: intervention $43.7 \%$ female; control: $44.0 \%$ female

Interventions

Evaluate outcome from playing "Escape from Diab" (Diab) and “Nanoswarm:

Invasion from Inner Space" (Nano) video games on children's diet, PA and adiposity.

"Escape from Diab" and "Nanoswarm: Invasion from Inner Space" (hereinafter called Diab and Nano) were video games designed to lower risks of type 2 diabetes and obesity by changing youth diet and PA behaviours.

Each game had 9 sessions and a minimum of approximately 40 min of game-play per session. This totaled approximately $6 \mathrm{~h}$ of new game-play per game. A session-by session description of each of the components in Diab is in the game overview grid. Each session had a knowledge minigame designed to provide practical knowledge related to change goals. Energy balance was divided into 18 sequential learning activities such that each ensuing learning session was predicated on mastering that material, which built on material in the previous session. Goal setting included action and coping (anticipatory problem solving) implementation intentions; a behavioural inoculation component involving a motivational message with a reasons statement linking the selected behaviour change to a personally select- 
ed value; and a goal-behaviour menu tailored to usual dietary or PA behaviours. A similar structure was used for Nano.

Children were allowed to take as long as desired in completing all sessions, but completing all sessions was required in the intervention group. Project staff called participants within 3 days of an expected session not played. The time from baseline to post was the time needed to play both games, which was roughly 3 months, but varied by participant.

The control group received a knowledge enhancing internet experience presented in 2 parts (one for Diab, one for Nano). Each part included a booklet with two discs: 1 disc connecting to 8 sessions of game-based websites (each related to diet, PA and obesity), with questions on the disc to be answered after each session (with immediate feedback); and the 2nd containing a knowledge-based nutrition game (Part 1: "Good Food and Play Make a Balance Day" and Part 2: "Dish It Up") that was played with the 8 session websites.

Diet and PA combination intervention vs control

\begin{tabular}{ll}
\hline Outcomes & Outcome measures \\
- Primary outcome: fruit and vegetable intake, PA, BMI, zBMI, triceps, waist circumference \\
- Secondary outcomes: primary/secondary NR
\end{tabular}

Process evaluation: reported (enjoyment of the game)

Implementation-related factors
Theoretical basis: SCT, Self-determination and Persuasion theories

Resources for intervention implementation: reported

Who delivered the intervention: reported

PROGRESS categories assessed at baseline: gender, race/ethnicity; parent: education (the sample had more 10-year-olds, men/boys, white people, and parents with a college degree or higher)

PROGRESS categories analysed at outcome: NR

Outcomes relating to harms/unintended effects: NR

Intervention included strategies to address diversity or disadvantage: NR

Economic evaluation: NR

\section{Notes}

Funding: this research was primarily funded by a grant from the National Institute of Diabetes and Digestive and Kidney Diseases (5 U44 DK66724-01). This work is also a publication of the U.S. Department of Agriculture (USDA/ARS) Children's Nutrition Research Center, Department of Pediatrics, Baylor College of Medicine, Houston,Texas, and had been funded in part with federal funds from the USDA/ARS under Cooperative Agreement No.58-6250-6001. Sample size was set by the funding agency. there was $80 \%$ power to detect a small-to-moderate overall effect (Cohen's $d=0.25$.

Children were required to have BMI percentile between 50 percentile and 95 percentile at baseline.

Reply from study author re duration of intervention: "The time from baseline to post was the time needed to play both games, which was roughly three months, but varied by participant. We called in a control participant to equal the times between pre and post in both groups."

Graduated incentives were provided for child participation in data collection: USD 25 for baseline assessment; USD 30 for between-game assessments; USD 35 for immediate postgame assessment; and USD 40 for 2-month follow-up.

Treatment group participants were loaned 24-inch iMac computers with the games and Microsoft Windows XP operating system preinstalled, but had no applications other than the video game interventions. Intervention co-ordinators monitored child use of the games by organising and reviewing email messages each time a child completed a session, answering call-in questions, guiding repair of minor hardware or software malfunctions, and arranging for speedy repair of larger malfunctions. 
Baranowski 2011 (Continued)

Post-game questionnaires with children and interviews with parents revealed that most children $(80 \%-$ 90\%) enjoyed playing both Diab and Nano.

\section{Risk of bias}

\begin{tabular}{|c|c|c|}
\hline Bias & Authors' judgement & Support for judgement \\
\hline $\begin{array}{l}\text { Random sequence genera- } \\
\text { tion (selection bias) }\end{array}$ & Unclear risk & Randomisation, no further details \\
\hline $\begin{array}{l}\text { Allocation concealment } \\
\text { (selection bias) }\end{array}$ & Unclear risk & Randomisation, no further details \\
\hline $\begin{array}{l}\text { Blinding (performance } \\
\text { bias and detection bias) } \\
\text { All outcomes }\end{array}$ & Low risk & $\begin{array}{l}\text { For anthropometric assessments and 24-h dietary recalls, data collectors were } \\
\text { blinded to group assignment }\end{array}$ \\
\hline $\begin{array}{l}\text { Incomplete outcome data } \\
\text { (attrition bias) } \\
\text { All outcomes }\end{array}$ & Low risk & $\begin{array}{l}10 \% \text { attrition in intervention group at final follow-up and } 20 \% \text { in control } \\
\text { There were no significant differences in any demographic variables between } \\
\text { those retained or eliminated from the sample. } \\
\text { There were no differences in demographics or anthropometrics between par- } \\
\text { ticipants with or without missing data. Only } 7.5 \% \text { of all the data were missing } \\
\text { across all } 4 \text { time periods. Little's Chi } 2 \text { test of all variables indicated data were } \\
\text { missing completely at random (Chi } 2=549.25, \mathrm{df}=547, \mathrm{P}=0.465) \text {. Analyses } \\
\text { were performed with and without imputed data and the results were similar. }\end{array}$ \\
\hline $\begin{array}{l}\text { Selective reporting (re- } \\
\text { porting bias) }\end{array}$ & Unclear risk & Protocol/trial registration documents were unavailable. \\
\hline Other bias & Unclear risk & $\begin{array}{l}\text { Despite randomisation there were differences in mean levels of fruit and veg- } \\
\text { etables, nonfat vegetables, total energy, MVPA, counts/min, BMI percentile } \\
\text { and zBMI, by group at baseline. Analyses adjusted for the baseline measure, } \\
\text { demographic characteristics, social desirability of response, and duration of } \\
\text { game play. Despite random assignment to conditions, initial differences in key } \\
\text { measures may have impaired the ability to detect changes. }\end{array}$ \\
\hline
\end{tabular}

\section{Barkin 2012}

$\begin{array}{ll}\text { Methods } & \text { Study name: Salud con la familia } \\ & \text { Study design: RCT } \\ \text { Intervention period: } 12 \text { weeks } \\ \text { Follow-up period (post-intervention): nil } \\ \text { Differences in baseline characteristics: reported } \\ \text { Reliable outcomes: reported } \\ \text { Protection against contamination: NR } \\ \text { Unit of allocation: parent-child dyads } \\ \text { Unit of analysis: individual }\end{array}$

Participants N(controls baseline $)=52$


Barkin 2012 (Continued)

$$
\begin{aligned}
& N \text { (controls follow-up) }=40 \\
& N \text { (interventions baseline) }=54 \\
& N \text { (interventions follow-up) }=35 \\
& \text { Setting (and number by study group): } 1 \text { community recreation centre }
\end{aligned}
$$

Recruitment: a bilingual research assistant approached individuals in the waiting areas of co-operating community agencies (e.g. social service agencies, paediatric clinics, community centres), also advertised via multiple mechanisms: flyers at community organisations and businesses; Spanish language radio

Geographic region: urban neighbourhood, Tennessee, USA

Percentage of eligible population enrolled: $40 \%$

Mean age: intervention $4.2 \pm 0.9$; control $4.1 \pm 0.9$

Sex: intervention, $45.7 \%$ female; control, $55 \%$ female Latino-American preschool-aged children.

12 weekly, 90-min group skills-building sessions for parents and children designed to improve nutritional family habits, increase weekly PA, and decrease media use (sedentary activity), conducted in Spanish by trained facilitator and set in the community centre. Participants were randomly assigned to small social groups at each session (6-8 parent-child dyads), and assigned small group activities (engaging both parents and children as the focus of the intervention) and specific group roles. The content was based on a best-practice culturally tailored programme for Latino-American families developed by the National Latino Children's Institute.

Control group received a brief school readiness programme ( 3 times for 60 min each session during the 12 weeks) conducted in the same community centre, designed to improve school readiness in preschool-aged children through increased parental verbal engagement (e.g. daily reading, playing word games, how to talk to children). The programme was based on the Dialogic Reading Model-C.A.R. (Comment and Wait, Ask Questions and Wait, and Respond by Adding More), an empirically tested curriculum that teaches parents to read picture books with their children.

Dietary and PA intervention vs control

$\begin{array}{ll}\text { Outcomes } & \text { Outcome measures } \\ \text { - Primary outcome: BMI } \\ \text { - Secondary outcomes: none } \\ \text { Process evaluation: reported (fidelity) }\end{array}$

Implementation-related factors
Theoretical basis: SCT and TTM of Change Resources for intervention implementation: reported Who delivered the intervention: reported

PROGRESS categories assessed at baseline: child: gender, race/ethnicity (country of origin); parent: race/ethnicity (country of origin, acculturation), education

PROGRESS categories analysed at outcome: NR

Outcomes relating to harms/unintended effects: NR

Intervention included strategies to address diversity or disadvantage: NR

Economic evaluation: NR 
Barkin 2012 (Continued)

Funding: supported by a Project Diabetes Implementation grant from the State of Tennessee (GR-09-25517-00) awarded to Dr Barkin and funds awarded to Dr Barkin from the Vanderbilt Clinical and Translational Science Award (National Center for Research Resources/NIH) (1 UL1 RR024975). Dr Gesell was supported by the American Heart Association Clinical Research grant Program (09CRP2230246). None of the funders contributed to the design and conduct of the study; collection, management, analysis, or interpretation of the data; or preparation, review, or approval of the manuscript.

$42 \%$ of participating preschool-aged children were overweight or obese at baseline.

Both transportation to and from study sessions and on-site child care services (for siblings) were provided free of charge to all study participants. Participants received small incentives after each wave of data collection (e.g. cutting board, kitchen timer, gift card to local supermarket), a total value of USD 60 per parent-child dyad over the study period.

\section{Risk of bias}

\begin{tabular}{lll}
\hline Bias & Authors' judgement & Support for judgement \\
\hline $\begin{array}{l}\text { Random sequence genera- } \\
\text { tion (selection bias) }\end{array}$ & Low risk & Computer-generated randomisation \\
\hline $\begin{array}{l}\text { Allocation concealment } \\
\text { (selection bias) }\end{array}$ & Low risk & $\begin{array}{l}\text { A biostatistician not otherwise involved in the study, generated the randomi- } \\
\text { sation list, and condition assignments were placed in non-transparent en- } \\
\text { velopes, which were sealed and numbered consecutively }\end{array}$ \\
\hline $\begin{array}{l}\text { Blinding (performance } \\
\text { bias and detection bias) }\end{array}$ & High risk & $\begin{array}{l}\text { Neither research staff nor participants were blinded to other participants' con- } \\
\text { dition allocation. }\end{array}$ \\
\hline $\begin{array}{l}\text { Incomplete outcome data } \\
\text { (attrition bias) }\end{array}$ & Low risk & $\begin{array}{l}\text { Attrition rate from initial exposure to 3-month follow-up was lower in the con- } \\
\text { trol group (15\%) than in the intervention group (36\%), (6 weeks between base- } \\
\text { line data collection and first intervention and control sessions) but the groups } \\
\text { of dyads who completed the intervention and control conditions did not sig- } \\
\text { nificantly differ on demographic characteristics or anthropometric measure- } \\
\text { ments at baseline. }\end{array}$ \\
\hline $\begin{array}{l}\text { Selective reporting (re- } \\
\text { porting bias) }\end{array}$ & Low risk & $\begin{array}{l}\text { Trial register found. BMI mentioned as a primary outcome in the trial registra- } \\
\text { tion document. }\end{array}$ \\
\hline $\begin{array}{l}\text { Other bias } \\
\text { L }\end{array}$ & Low risk & \begin{tabular}{l} 
No further bias identified \\
\hline
\end{tabular}
\end{tabular}

Beech 2003

\begin{tabular}{ll}
\hline Methods & Study design: RCT \\
& Intervention period: 12 weeks \\
& Follow-up period (post-intervention): nil \\
Differences in baseline characteristics: reported & Reliable outcomes: reported \\
Protection against contamination: NR \\
Unit of allocation: child \\
Unit of analysis: child
\end{tabular}

Participants

Pre-adolescent African-American girls

$\mathrm{N}$ (controls baseline) 18 
Beech 2003 (Continued)

$$
\begin{aligned}
& N(\text { controls follow-up })=18 \\
& N(\text { interventions baseline })=\text { child programme } 21+21=42 \\
& N(\text { interventions follow-up) =parent programme } 21+21=42 \\
& \text { Setting: unclear if at houses or at university centres }
\end{aligned}
$$

Recruitment: girls and their families were recruited through public service announcements on several local African-American radio stations, participation of GEMS investigators in live radio talk shows, and flyers distributed at local elementary schools.

Geographic region: Memphis, USA

Percentage of eligible population enrolled:

Mean age overall: $8.9(0.8)$; range 8 -10 years; intervention age: child-targeted group 8.7 (0.8); parent-targeted group 9.1 (0.7); control: $8.9(0.8)$

Sex: girls only

Ethnicity: African-American only

Interventions

Intervention: the active interventions involved highly interactive weekly group sessions for 12 weeks with either girls (child-targeted programme) or parents/caregivers (parent-targeted programme). Content focused on knowledge and behaviour-change skills to promote healthy eating and increased PA. Control: the comparison intervention focused on global self-esteem. The participants attended 3 monthly, 90-min sessions over the 12-week pilot study

\begin{tabular}{ll}
\hline Outcomes & BMI \\
- Waist circumference \\
- Physical maturation \\
- DEXA for \% body fat \\
- Blood samples for insulin \\
$\circ$ accelerometer CSA \\
$\circ$ modified SAPAC \\
$\circ$ GEMS Activity Questionnaire (GAQ) computerised \\
- Dietary intake measured by two 24-h recalls using NDS-R \\
Psychological variables: \\
$\circ$ body image using modified (Stunkard 1983) body silhouettes \\
$\circ$ weight control behaviours using McKnight Risk Factor Survey \\
$\circ$ parental food preparation practices \\
$\circ$ Self-Perception Profile for Children \\
$\circ$ Healthy Growth Study for physical activity expectations \\
$\circ$ a self-efficacy measure
\end{tabular}

Process evaluation: reported

Implementation-related

factors

Notes Funding: NR

\section{Risk of bias}


Beech 2003 (Continued)

Random sequence genera- Unclear risk Authors refer to randomisation but do not specify a procedure. tion (selection bias)

\begin{tabular}{|c|c|c|}
\hline $\begin{array}{l}\text { Allocation concealment } \\
\text { (selection bias) }\end{array}$ & Unclear risk & NR \\
\hline \multirow{2}{*}{$\begin{array}{l}\text { Blinding (performance } \\
\text { bias and detection bias) } \\
\text { All outcomes }\end{array}$} & \multirow[t]{2}{*}{ Unclear risk } & NR \\
\hline & & $\begin{array}{l}\text { Quote: "interview sessions were held in conjunction with the post-test assess- } \\
\text { ment sessions and were conducted by a study investigator who was not in- } \\
\text { volved in the direct delivery of the interventions." }\end{array}$ \\
\hline \multirow{2}{*}{$\begin{array}{l}\text { Incomplete outcome data } \\
\text { (attrition bias) } \\
\text { All outcomes }\end{array}$} & \multirow[t]{2}{*}{ Low risk } & No missing outcome data \\
\hline & & $\begin{array}{l}\text { Quote: "Complete data were collected at follow up for } 100 \% \text { of the study popu- } \\
\text { lation" }\end{array}$ \\
\hline
\end{tabular}

\begin{tabular}{lll}
\hline $\begin{array}{l}\text { Selective reporting (re- } \\
\text { porting bias) }\end{array}$ & Unclear risk & Protocol/trial registration document were unavailable \\
\hline Other bias & Low risk & No further bias identified \\
\hline
\end{tabular}

\section{Bellows 2013a}

\begin{tabular}{|c|c|}
\hline \multirow[t]{8}{*}{ Methods } & Study design: cluster-RCT \\
\hline & Intervention period: 18 months \\
\hline & Follow-up period (post-intervention): nil \\
\hline & Differences in baseline characteristics: reported \\
\hline & Reliable outcomes (apart from steps): reported \\
\hline & Protection against contamination: NR \\
\hline & Unit of allocation: school \\
\hline & Unit of analysis: individual \\
\hline \multirow[t]{10}{*}{ Participants } & $\mathrm{N}$ (controls baseline $)=131$ \\
\hline & $N$ (controls follow-up) = 103 \\
\hline & $\mathrm{N}$ (interventions baseline) $=132$ \\
\hline & $\mathrm{N}$ (interventions follow-up) = 98 \\
\hline & Setting: 8 community Head Start centres ( 4 intervention, 4 control) \\
\hline & Recruitment: NR \\
\hline & Geographic region: USA, no further details \\
\hline & Percentage of eligible population enrolled: unclear \\
\hline & Mean age (months): intervention plus: 53.0 months \pm 6.8 ; control: $51.5 \pm 6.6$ months \\
\hline & Sex: intervention + control: $45 \%$ female \\
\hline Interventions & The Food Friends: Get Movin' With Mighty Moves programme. \\
\hline
\end{tabular}


Bellows 2013a (Continued)

The Mighty Moves intervention lasted 18 weeks and was conducted in the classroom 4 days/week for 15-20 min each day, for a total of 72 lessons. Lessons comprised multiple activities (143 total activities) and were led by the classroom teacher. Each week's activities focused on a skill or group of skills from $1 / 3$ gross motor skill categories: stability (trunk strength), locomotor (running, hopping, skipping), or manipulation (ball skills). Early in each week, children were introduced to a motor skill, and movement concepts were added as the week progressed. Later in the programme, skill patterns were incorporated into activities.

$\begin{array}{ll}\text { Outcomes } & \text { Outcome measures } \\ \text { - Gross motor skill performance } \\ \text { - PA } \\ \text { Unclear which were primary and secondary outcomes } \\ \text { Process outcomes: reported (fidelity) }\end{array}$

Implementation-related factors
Theoretical basis: NR

Resources: NR

Who delivered the intervention: reported

PROGRESS categories assessed at baseline: gender, race/ethnicity

PROGRESS categories analysed at outcome: NR

Outcomes relating to harms/unintended effects: NR

Intervention included strategies to address diversity or disadvantage: all participants were considered to have low SES because of their enrolment in Head Start.

Economic evaluation: NR

NCT01937481
Funding: this project is supported by Agriculture and Food Research Initiative Competitive Grant no.
2010-85215-20648 from the USDA National Institute of Food and Agriculture. Additional support for this
research was funded by a career development award from the NIH (K23DK087826) awarded to REB.

\section{Risk of bias}

\begin{tabular}{|c|c|c|}
\hline Bias & Authors' judgement & Support for judgement \\
\hline $\begin{array}{l}\text { Random sequence genera- } \\
\text { tion (selection bias) }\end{array}$ & Unclear risk & Randomly assigned, no further details \\
\hline $\begin{array}{l}\text { Allocation concealment } \\
\text { (selection bias) }\end{array}$ & Unclear risk & NR \\
\hline $\begin{array}{l}\text { Blinding (performance } \\
\text { bias and detection bias) } \\
\text { All outcomes }\end{array}$ & High risk & Blinding of data collection was not possible \\
\hline $\begin{array}{l}\text { Incomplete outcome data } \\
\text { (attrition bias) } \\
\text { All outcomes }\end{array}$ & Low risk & $76 \%$ retention. Loss and reasons balanced between groups \\
\hline $\begin{array}{l}\text { Selective reporting (re- } \\
\text { porting bias) }\end{array}$ & Low risk & Protocol/trial registration document were unavailable \\
\hline
\end{tabular}


Bellows 2013a (Continued)

\begin{tabular}{lll} 
Other bias & Low risk & No other threats to validity \\
\hline $\begin{array}{l}\text { Other bias- timing of re- } \\
\text { cruitment of clusters }\end{array}$ & Unclear risk & NR
\end{tabular}

Birken 2012

\begin{tabular}{ll}
\hline Methods & Study design: RCT \\
Intervention period: 10 min (brief intervention) \\
Follow-up period (post-intervention): 1 year \\
Differences in baseline characteristics: reported \\
Reliable outcomes: reported \\
Protection against contamination: study authors report potential for contamination \\
Unit of allocation: individual \\
Unit of analysis: individual
\end{tabular}
Participants N (controls baseline $)=79$
$\mathrm{N}$ (controls follow-up) $=68$
$\mathrm{N}$ (interventions baseline $)=81$
$N$ (interventions follow-up) $=64$
Setting (and number by study group): 1 community-based, primary care paediatric group practice, with 3 physicians
Recruitment: at child's 3-year health maintenance visit
Geographic region: Toronto, Canada

Percentage of eligible population enrolled: $91 \%$ (53\% assessed for eligibility of those due for health visit)

Mean age: intervention $3.12 \pm 0.19$; control $3.08 \pm 0.12$

Sex: intervention, $44 \%$ female; control, $49 \%$ female screen time, meals in front of the TV, and BMI

Parents in the intervention group received a 10-min behavioural counselling intervention by trained study personnel directly after the health maintenance visit, which included information on the health impact of screen time in children and provided strategies to decrease screen time. These strategies included suggestions such as removing the TV from the child's bedroom, encouraging meals to be eaten without the TV on, and budgeting of the child's screen time.

Families were encouraged to try a 1- week TV turn off, in which children were encouraged to spend time without the TV and were provided with a calendar and stickers to reward the children for days without the TV. Contingency planning for time spent not watching TV was promoted.

Activities for the child, during this session, included providing a story to parents about TV viewing (The Berenstain Bears and Too Much TV) and creating a list of non TV-related activities. The intervention group also received a Canadian Pediatric Society handout titled 'Promoting Good Television Habits' 
Birken 2012 (Continued)

Parents of children in both the intervention and control groups received standardised counselling from trained study personnel on safe media use, which included information on TV rating systems, internet safety, and limiting exposure to violent programming. They both received a previously published Canadian Pediatric Society parent handout titled "Managing Media in the Home."

PA intervention vs control

$\begin{array}{ll}\text { Outcomes } & \text { Outcome measures } \\ \text { - Primary outcome: screen time } \\ \text { - Secondary outcomes: zBMI, number of meals with TV, TV in bedroom } \\ \text { Process evaluation: NR }\end{array}$

Implementation-related factors
Theoretical basis: concepts of goal setting, positive reinforcement, monitoring, and cognitive restructuring

Resources for intervention implementation: NR

Who delivered the intervention: reported

PROGRESS categories assessed at baseline: child: gender; parent: education, occupation, race/ethnicity (country of origin)

PROGRESS categories analysed at outcome: NR

Outcomes relating to harms/unintended effects: NR

Intervention included strategies to address diversity or disadvantage: NR

Economic evaluation: NR

\section{NCT00959309}

Funding: supported in part by a Paediatric Consultants Research Grant, Hospital for Sick Children, Toronto. The Paediatric Outcomes Research Team is supported by a grant from the Hospital for Sick Children Foundation. The funding organisations were not involved in any of the following: design and conduct of the study; collection, management, analysis, and interpretation of the data; and preparation, review, or approval of the manuscript.

The intervention group had a clinically significantly higher zBMI at baseline,

compared with the control group $(0.66 \pm 1.18$ vs $0.30 \pm 0.83)$ adjusted in analysis

Study authors estimate cost of implementing this intervention to all children: if implemented as an additional counselling service at the primary care visit, this intervention would be a significant cost. For example, if we calculate direct costs for physician counselling for all children in Ontario attending a primary care practice

and use an existing fee code for smoking cessation counselling in

Ontario, the cost would be > CAD 2 million annually.

\section{Risk of bias}

\section{Bias}

Random sequence genera- Low risk tion (selection bias)

\section{Authors' judgement Support for judgement}

Computer-generated randomisation

Sequentially numbered, opaque, identical, sealed envelopes (selection bias) 
Birken 2012 (Continued)
Blinding (performance
Low risk
Assessors were blinded
bias and detection bias)
All outcomes

Incomplete outcome data Low risk $\quad 79 \%$ and $86 \%$ follow-up in the intervention and control groups, respectively
(attrition bias)

All outcomes

$\begin{aligned} & \text { Selective reporting (re- } \\ & \text { porting bias) }\end{aligned}$
Low risk Trial registration document checked. All outcomes reported

\begin{tabular}{ll}
\hline Other bias $\quad$ Unclear risk $\quad$ Contamination possible \\
\hline
\end{tabular}

Black 2010

\begin{tabular}{|c|c|}
\hline \multirow[t]{8}{*}{ Methods } & Study design: RCT \\
\hline & Intervention period: 12 weeks \\
\hline & Follow-up period (post-intervention): 21 months \\
\hline & Differences in baseline characteristics: reported \\
\hline & Reliable outcomes: reported \\
\hline & Protection against contamination: NR \\
\hline & Unit of allocation: individual \\
\hline & Unit of analysis: individual \\
\hline \multirow[t]{12}{*}{ Participants } & $\mathrm{N}($ controls baseline $)=114$ \\
\hline & $N$ (controls follow-up) = 93 (1st follow-up); 90 final \\
\hline & $\mathrm{N}$ (interventions baseline) $=121$ \\
\hline & $\mathrm{N}$ (interventions follow-up) = 91 (1st follow-up); 89 final \\
\hline & Setting (and number by study group): home- and community-based \\
\hline & Recruitment: 2 samples: \\
\hline & $\begin{array}{l}\text { - } \mathrm{N}=84 \text { participated in a longitudinal investigation of growth and development (17.9\% experienced } \\
\text { growth faltering by age } 2 \text { years, but by age } 6 \text { years growth had recovered) }\end{array}$ \\
\hline & - $\mathrm{N}=151$ recruited from middle schools \\
\hline & $\begin{array}{l}\text { Geographic region: resident in low-income communities surrounding a mid-Atlantic urban, university } \\
\text { medical centre }\end{array}$ \\
\hline & Percentage of eligible population enrolled: NR \\
\hline & Mean age: intervention + control: $13.3(11-16)$ \\
\hline & Sex: intervention + control: $49 \%$ female \\
\hline
\end{tabular}

Interventions

To evaluate a 12-session, home- and community-based health promotion/obesity prevention programme. 
Black 2010 (Continued)

A manualised 12-session (12-week) intervention based on SCT, developed with a board of African American adolescents, and a rap music video promoting healthy eating and PA. Principles of mentorship (role modelling and support), participatory learning, and goal-setting were central to the intervention.

Participants were paired with race- and gender-matched college-enrolled (age 19-25 years) mentor. Mentoring took place in both the home and the community (mentors accompanied the adolescents to neighbourhood convenience stores and playgrounds to promote healthy dietary choices and PA).

In addition to setting dietary and PA goals, tracking and evaluating progress and revising goals as necessary, intervention adolescents made and tasted healthy snacks and engaged in PA.

Diet and PA combined intervention vs control

Outcome measures
- Primary outcome: zBMI, \% overweight or obese, \% body fat, fat mass, fat-free mass
- Secondary outcomes: PA, dietary intake
Process evaluation: reported (fidelity)

Implementation-related factors
Theoretical basis: SCT and MI

Resources for intervention implementation: NR

Who delivered the intervention: reported

PROGRESS categories assessed at baseline: child: gender, race/ethnicity; parent: education, SES, social status

PROGRESS categories analysed at outcome: child: gender

Outcomes relating to harms/unintended effects: NR

Intervention included strategies to address diversity or disadvantage: NR

Economic evaluation: NR

\section{Notes}

Funding: this research was supported by grant R40MC00241 from the Maternal and Child Health Research Program, US Department of Health and Human Services to Maureen Black, Ph.D., and the University of Maryland General Clinical Research Center grant M01 RR16500, General Clinical Research Centers Program, National Center for Research Resources (NCRR), NIH

Mentors received approximately $40 \mathrm{~h}$ of training, including $\mathrm{MI}$ and had weekly supervision during the intervention

\section{Risk of bias}

\begin{tabular}{lll}
\hline Bias & Authors' judgement & Support for judgement \\
\hline $\begin{array}{l}\text { Random sequence genera- } \\
\text { tion (selection bias) }\end{array}$ & Unclear risk & $\begin{array}{l}\text { Randomly stratified by growth history, weight status, gender and age, no fur- } \\
\text { ther details }\end{array}$ \\
\hline $\begin{array}{l}\text { Allocation concealment } \\
\text { (selection bias) }\end{array}$ & Unclear risk & NR \\
\hline $\begin{array}{l}\text { Blinding (performance } \\
\text { bias and detection bias) } \\
\text { All outcomes }\end{array}$ & Low risk & $\begin{array}{l}\text { Research assistants were unaware of participants' intervention status or base- } \\
\text { line data re collection of anthropometric measures }\end{array}$ \\
\hline
\end{tabular}

Incomplete outcome data Low risk $\quad$ Loss is overall $<30 \%$ and ITT analyses were conducted
(attrition bias)


Black 2010 (Continued)

Selective reporting (re- Low risk Trial registration document checked. All outcomes reported porting bias)

Other bias $\quad$ Low risk No additional threats to validity

Bohnert 2013

\begin{tabular}{|c|c|}
\hline \multirow[t]{8}{*}{ Methods } & Study design: RCT \\
\hline & Intervention period: 30 weeks \\
\hline & Follow-up period (post-intervention): nil \\
\hline & Differences in baseline characteristics: reported \\
\hline & Reliable outcomes: reported \\
\hline & Protection against contamination: NR \\
\hline & Unit of allocation: individual \\
\hline & Unit of analysis: individual \\
\hline \multirow[t]{10}{*}{ Participants } & $N($ controls baseline $)=24$ \\
\hline & $N($ controls follow-up) $=37$ \\
\hline & $\mathrm{N}$ (interventions baseline) $=52$ \\
\hline & $\mathrm{N}$ (interventions follow-up) = 96 \\
\hline & Setting (and number by study group): elementary schools ( $N=52$ intervention girls, $N=24$ control girls) \\
\hline & Recruitment: brief announcements at 5 urban elementary (public) schools ( $3 \mathrm{rd}, 4$ th and 5 th grade girls) \\
\hline & Geographic region: underserved, urban, low-income communities in Chicago, USA \\
\hline & Percentage of eligible population enrolled: $100 \%$ \\
\hline & Mean age: intervention: $9.02 \pm 0.93$; control: $9.38 \pm 1.13$ \\
\hline & Sex: $100 \%$ female \\
\hline \multirow[t]{2}{*}{ Interventions } & $\begin{array}{l}\text { To examine the effectiveness of 'Girls in the Game' after-school programmes (GIG ASPs) in promoting } \\
\text { social-emotional development and reducing BMI and obesogenic behaviours among a group of urban, } \\
\text { low-income, African American and Latina girls. The GIG After-school programme is a 30-week curricu- } \\
\text { lum that includes } 10 \text { three-week modules. Each session is led by trained GIG coaches, is approximate- } \\
\text { ly } 90 \text { min in length. } 50 \% \text { covers physical instruction and energetic activity through traditional and non- } \\
\text { traditional sports and fitness activities, and } 50 \% \text { addresses age-appropriate health education, nutrition } \\
\text { education, and leadership and life skills topics. A healthy snack or meal was provided at each session. A } \\
\text { small prize was provided to the "girl of the day". }\end{array}$ \\
\hline & $\begin{array}{l}\text { Curriculum is evidence-based and utilises SAFE (sequenced, active, focused, explicit) practices. Specif- } \\
\text { ically, each lesson follows a structured plan and builds upon previous lessons to achieve their objec- } \\
\text { tive (i.e. sequenced). GIG also utilises engaging and interactive methods to help girls achieve skills, and } \\
\text { girls are encouraged to come up with solutions (i.e. active). Finally, GIG programme leaders devote a } \\
\text { set amount of time each week (e.g. } 45 \mathrm{~min} / \mathrm{session} \text { ) to teaching these skills (i.e. focused), and girls have } \\
\text { a clear understanding (i.e. provided with "topic of the day") about what they are expected to learn (i.e. } \\
\text { explicit). }\end{array}$ \\
\hline
\end{tabular}


Bohnert 2013 (Continued)

Diet and PA combined intervention vs control

Outcomes Outcome measures

- Primary outcome: zBMI, nutrition, PA, body image/weight perception, self-report social-emotional development

- Secondary outcomes: NR

Process evaluation: reported (attendance, programme quality, implementation, and engagement)

Implementation-related factors
Theoretical basis: SCT and Sociocultural theory

Resources for intervention implementation: NR

Who delivered the intervention: reported

PROGRESS categories assessed at baseline: child: race/ethnicity; school: SES (low-income)

PROGRESS categories analysed at outcome: NR

Outcomes relating to harms/unintended effects: NR

Intervention included strategies to address diversity or disadvantage: NR

Economic evaluation: NR

Notes

Funding: this trial was funded by a grant from the Chicago Consortium to Lower Obesity in Chicago Children (CLOCC:AU 508485)

GIG staff and study personnel collected data on attendance, programme quality, curriculum implementation, and participant engagement from programme girls only.

Across all sites, on average, girls who participated in the programme throughout the year attended $73.6 \%$ of GIG sessions. Ratings of programme quality were high at all programme sites particularly for safe environment $(M=4.78, S D=0.23)$, supportive environment $(M=3.84, S D=0.24)$, and interaction ( $M$ $=3.93, \mathrm{SD}=0.36)$ domains, which were all above normative score distributions in validity studies. The 4th domain, engagement, was relatively lower $(M=2.64, S D=0.28)$, but still at the higher end of the distribution for Youth Programme Quality Assessment Scales. Implementation data suggest that curriculum was implemented very well across the 5 school sites $(M=1.85, S D=0.12)$ and participant engagement was high $(M=1.81, S D=0.16)$ on average.

\section{Risk of bias}

Bias Authors' judgement Support for judgement

Random sequence genera- High risk Random numbers table but girls were not assigned to the control group if tion (selection bias) spaces in the programme were still available (i.e. filling programme slots took priority over balancing sample sizes between GIG and control groups)

\begin{tabular}{lll}
$\begin{array}{l}\text { Allocation concealment } \\
\text { (selection bias) }\end{array}$ & High risk & See above \\
\hline $\begin{array}{l}\text { Blinding (performance } \\
\text { bias and detection bias) } \\
\text { All outcomes }\end{array}$ & High risk & $\begin{array}{l}\text { Blinding of outcome assessors NR but GIG staff were involved in collecting } \\
\text { questionnaire data }\end{array}$ \\
\hline
\end{tabular}

Incomplete outcome data High risk
(attrition bias)

(attrition bias)

All outcomes 
Bohnert 2013 (Continued)
Selective reporting (re-
Unclear risk
Protocol/trial registration documents were unavailable. porting bias)

Other bias

Low risk

No additional threats to validity

Bonis 2014

Study name: Nutrition and physical activity self-assessment for child care (NAP SACC)
Study design: cluster-RCT
Intervention period: 6 months
Follow-up period (post-intervention): nil
Differences in baseline characteristics: reported
Reliable outcomes: reported
Protection against contamination: NR
Unit of allocation: childcare facility
Unit of analysis: individual

Participants (controls baseline) $=123$
$\mathrm{~N}$ (controls follow-up) $=99$
$\mathrm{~N}$ (interventions baseline) $=128$
$\mathrm{~N}$ (interventions follow-up) $=110$
Setting (and number by study group): childcare facilities ( $\mathrm{N}=13$ intervention facilities, $\mathrm{N}=13$ control fa-
cilities)
Recruitment: letters from the Louisiana State Department of Public Health were mailed to licensed
childcare facilities, which stated that participation and completion of the NAP SACC project could be
substituted for participation in a mandatory annual state safety seminar to maintain their state licen-
sure. The first 30 facilities that responded positively were included in the study
Geographic region: licensed childcare facilities in Louisiana, USA
Percentage of eligible population enrolled: $98 \%$
Mean age: intervention: $3.81 \pm 0.75$; control: $3.9 \pm 0.85$
Sex: intervention, $52 \%$ female; control: $52 \%$ female

Interventions

To determine whether the NAP SACC programme would improve PA levels in randomly selected licensed Louisiana daycare centres.

4 dietitians with PA training experience were contracted to become NAP SACC certified and who then trained the childcare providers over $4 \times 1$-h workshops and provided monthly visits to assist with implementation of the guidelines.

- The NAP SACC consultants delivered to the staff of each treatment facility 4 workshops that demonstrated the importance of PA and nutrition. The workshop topics included overweight, nutrition, PA, and growing healthy kids.

- The consultants maintained regular contact with the treatment facility staff and provided support in addressing any barriers that would prevent achievement of their specific facility improvement plan. 
Bonis 2014 (Continued)

- They also distributed educational information to the parent/guardians that focused on PA and nutrition recommendations at home.

- Each treatment facility director completed the NAP SACC self-assessment tool that assessed their centre on 14 key areas in PA and nutrition, with response options ranging from "minimal" to "best practice." Based on the responses, the facility director with guidance from the NAP SACC consultant chose 3-4 areas for improvement and prepared a unique facility improvement plan.

The control centres were given access to the NAP SACC programme after completion of the project.

Diet and PA combined intervention vs control

\begin{tabular}{ll}
\hline Outcomes & Outcome measures \\
- Primary outcome: PA \\
Process evaluation: reported (implementation) \\
\hline $\begin{array}{l}\text { Implementation-related } \\
\text { factors }\end{array}$ \\
Resources for intervention implementation: reported \\
Who delivered the intervention: reported \\
PROGRESS categories assessed at baseline: gender \\
PROGRESS categories analysed at outcome: NR \\
Outcomes relating to harms/unintended effects: NR \\
Intervention included strategies to address diversity or disadvantage: NR \\
Economic evaluation: NR \\
$\begin{array}{l}\text { Funding: the study was funded by the Office of Public Health-Maternal and Child Health Department of } \\
\text { Louisiana (New Orleans, LA) } \\
\text { Training of staff and implementation carried out as part of the intervention }\end{array}$ \\
\hline Notes
\end{tabular}

\section{Risk of bias}

\begin{tabular}{lll}
\hline Bias & Authors' judgement & Support for judgement \\
\hline $\begin{array}{l}\text { Random sequence genera- } \\
\text { tion (selection bias) }\end{array}$ & Unclear risk & Randomly selected child care facilities; no further details \\
\hline $\begin{array}{l}\text { Allocation concealment } \\
\text { (selection bias) }\end{array}$ & Low risk & $\begin{array}{l}\text { The facilities were randomly designated to either the treatment or control } \\
\text { group by a team member using simple randomisation without knowledge of } \\
\text { the facilities' names, demographics, or location. Cluster randomisation }\end{array}$ \\
\hline $\begin{array}{l}\text { Blinding (performance } \\
\text { bias and detection bias) }\end{array}$ & Unclear risk & NR \\
\hline $\begin{array}{l}\text { All outcomes } \\
\begin{array}{l}\text { Incomplete outcome data } \\
\text { All outcomes }\end{array}\end{array}$ & Low risk & $17 \%$ attrition, balanced between groups \\
\hline $\begin{array}{l}\text { Selective reporting (re- } \\
\text { porting bias) }\end{array}$ & Unclear risk & Protocol/trial registration documents were unavailable. \\
\hline
\end{tabular}


Bonis 2014 (Continued)

Other bias Low risk No additional threats to validity

Other bias- timing of re- Low risk Figure shows recruitment happened prior to randomisation

cruitment of clusters

Bonsergent 2013

\begin{tabular}{|c|c|}
\hline \multirow[t]{9}{*}{ Methods } & Study name: PRomotion de l'ALIMentation et de \\
\hline & Study design: cluster-RCT ( $2 \times 2 \times 2$ factorial) \\
\hline & Intervention period: 2 years \\
\hline & Follow-up period (post-intervention): nil \\
\hline & Differences in baseline characteristics: reported \\
\hline & Reliable outcomes: reported \\
\hline & Protection against contamination: NR \\
\hline & Unit of allocation: school \\
\hline & Unit of analysis: school \\
\hline
\end{tabular}

\begin{tabular}{|c|c|}
\hline Participants & $\begin{array}{l}\text { Baseline: } \\
\text { - } \text { education = 3424; no education }=2947 \\
\text { - } \text { environmental = } 3150 ; \text { no environmental = } 3221 \\
\text { - } \text { screening= } 3191 ; \text { no screening = } 3180 \\
\text { Follow-up: } \\
\text { - education = 1949; no education }=1589 \\
\text { - } \text { environmental = } 1728 ; \text { no environmental = } 1810 \\
\text { - } \text { screening = 1687; no screening = } 1851\end{array}$ \\
\hline
\end{tabular}

Setting (and number by study group): 24 public secondary schools (8 groups, 3 schools in each group)

Recruitment: all adolescents entering the selected high schools in Grade 10 in 2006 or 2007 (according to the school) and in Grade 11 in 2007 or 2008

Geographic region: Lorraine, Northeast France

Percentage of eligible population enrolled: $84 \%$

Mean age: intervention + control: $15.8 \pm 0.7$

Sex: intervention + control: $52.9 \%$ female

Interventions

To evaluate the impact of 3 strategies ("education," "environment," "screening and care") aimed at preventing overweight and obesity in adolescents in a high school setting. The prevention strategies were education (development of nutritional knowledge and skills); environment (creation of a favourable environment by improving availability of "healthy" dietary items and PA); and screening and care (detection of overweight/obesity and, if necessary, adapted care management).

Each study group $(\mathrm{A}-\mathrm{H})$ received all, some or none of the 3 strategies below:

Educational strategy: 
Bonsergent 2013 (Continued)

- First high school year (grade 10):

- $5 \mathrm{~h}$ of lectures on nutritional needs

- $2 \mathrm{~h}$ and personal work for groups on nutritional rhythms or environment

- organisation of a 1-day or half-a-day PRALIMAP party

- 2nd high school year (grade 11):

- $6 \mathrm{~h}$ of lectures on nutritional environment

- $2 \mathrm{~h}$ and personal work for collective groups on influence of eco-citizenship, nutritional security measures and prices of food and drink and PA

- organisation of a 1-day or half-a-day PRALIMAP party

Diet and PA combined intervention vs control

\begin{tabular}{ll}
\hline Outcomes & Outcome measures \\
& - Primary outcome: BMI \\
& Process evaluation: reported (implementation) \\
\hline $\begin{array}{l}\text { Implementation-related } \\
\text { factors }\end{array}$ & Theoretical basis: NR \\
& Resources for intervention implementation: reported \\
& Who delivered the intervention: reported \\
& PROGRESS categories assessed at baseline: child: gender; parent: occupation, social class, SES (family \\
& income) \\
& PROGRESS categories analysed at outcome: NR \\
& Outcomes relating to harms/unintended effects: NR \\
Intervention included strategies to address diversity or disadvantage: NR \\
Economic evaluation: NR
\end{tabular}

Notes NCT00814554

Funding: the PRALIMAP trial was funded by grants from public and private sectors. Special acknowledgements are addressed to ARH Lorraine, Conseil Régional de Lorraine, DRASS de Lorraine, GRSP de Lorraine, Fondation Coeurs et Artères, Fondation Wyeth, Ministère de l'enseignement supérieur et de la recherche, Inca, IRESP, Régime local d'assurance maladie d'Alsace Lorraine and Urcam de Lorraine. All trial steps, design, data collection, analysis, write-ups, and reports are and will be performed independently of any funding or sponsoring agency.

Staff resources: public health professionals of Nancy University (for screening and care strategy), health education professionals external to the high schools (PRALIMAP monitors), and supported and supervised high school professionals (the teachers) in the implementation of strategies. The teachers conducted the education strategy (no mention of training).

The process evaluation showed that, of 11 planned hours of dietary and PA lectures, the 12 "education schools" performed 4.8+/-0.8 hours on average (range 3-6); menu offerings were considerably improved over the 2-year period of intervention in the 12 environment schools, with more fruits and vegetables and fewer sugary drinks and snacks. However, this trend also was noted, to a lesser extent, in the 12 "no-environment schools," probably because of the French nutritional policy which followed since 2001. Adapted care management (ie: the screening strategy), comprising 7 group sessions, was implemented in full in 8 high schools, partially implemented in 1, and not implemented at all in 3.

\section{Risk of bias}


Bonsergent 2013 (Continued)

Random sequence genera- Low risk tion (selection bias)
Although NR, this is a $2 \times 2 \times 2$ factorial cluster RCT that has been stratified by department and type of education. It would have been highly unusual for a trial of such complexity to be organised by means other than computer-based randomisation and selection processes.

\begin{tabular}{|c|c|c|}
\hline $\begin{array}{l}\text { Allocation concealment } \\
\text { (selection bias) }\end{array}$ & Low risk & $\begin{array}{l}\text { Although NR, this is a } 2 \times 2 \times 2 \text { factorial cluster RCT that has been stratified by } \\
\text { department and type of education. It would have been highly unusual for a tri- } \\
\text { al of such complexity to be organised by means other than computer-based } \\
\text { randomisation and selection processes. }\end{array}$ \\
\hline
\end{tabular}

\begin{tabular}{|c|c|}
\hline $\begin{array}{l}\text { Blinding (performance } \\
\text { bias and detection bias) } \\
\text { All outcomes }\end{array}$ & Unclear risk \\
\hline
\end{tabular}

\begin{tabular}{lll}
\hline $\begin{array}{l}\text { Incomplete outcome data } \\
\text { (attrition bias) } \\
\text { All outcomes }\end{array}$ & High risk & $\begin{array}{l}52 \%-58 \% \text { attrition, significant differences between completers and non-com- } \\
\text { pleters }\end{array}$ \\
\hline $\begin{array}{l}\text { Selective reporting (re- } \\
\text { porting bias) }\end{array}$ & Low risk & Protocol/trial registration document seen. All outcomes reported \\
\hline Other bias & Low risk & $\begin{array}{l}\text { The study authors report no important interaction between the effects of the } \\
\text { different interventions. "No interaction was detected among the three strate- } \\
\text { gies (education, environment, screening)" }\end{array}$
\end{tabular}

Other bias- timing of re- Low risk CONSORT Figure shows enrolment happened before allocation

cruitment of clusters

\section{Bonuck 2014}

\begin{tabular}{|c|c|}
\hline \multirow[t]{8}{*}{ Methods } & Study design: RCT \\
\hline & Intervention period: 12.1 months, range $10.6-14.4$ months \\
\hline & Follow-up period (post-intervention): nil \\
\hline & Differences in baseline characteristics: reported \\
\hline & Reliable outcomes: reported \\
\hline & Protection against contamination: reported \\
\hline & Unit of allocation: parent-child dyads \\
\hline & Unit of analysis: parent-child dyads \\
\hline \multirow[t]{7}{*}{ Participants } & $\mathrm{N}($ controls baseline $)=150$ \\
\hline & $\mathrm{N}$ (controls follow-up) $=130$ \\
\hline & $\mathrm{N}$ (interventions baseline) $=149$ \\
\hline & $N$ (interventions follow-up) $=121$ \\
\hline & $\begin{array}{l}\text { Setting (and number by study group): community: } 2 \times \text { WICs ( } N=78 \text { intervention, } N=78 \text { control in } 1 \text { site; } \\
N=71 \text { intervention, } N=72 \text { control in other site) }\end{array}$ \\
\hline & Recruitment: participants recruited at children's one-year-old visits \\
\hline & Geographic region: Bronx, New York, USA \\
\hline
\end{tabular}


Bonuck 2014 (Continued)

Percentage of eligible population enrolled: $100 \%$

Mean age: intervention + control: 12.6 months (range 10-15.5 months)

Sex: intervention + control: $52 \%$ female

Interventions

To evaluate 3 research questions

- Does a WIC-based counselling intervention reduce (milk) bottle use?;

- Does this intervention reduce energy intake from bottles?

- Does this intervention reduce the risk of a child being > 85th percentile weight-for-length?

WIC nutritionists delivered the educational intervention counselling guided by a flip-chart, which was developed with input from the WIC sites' staff and clients. The team provided guidance in how to use the flip-chart, but no formal training was given. WIC nutrition staff remained constant throughout the intervention period. The flip-chart presents messages about healthy weight, dental caries, and iron deficiency anemia effects from bottle-weaning. It recommends that parents gradually replace bottles with cups. Though no transitional cup type is specified, in a supplemental " $Q$ \& A" section for nutritionists' reference, there is a recommendation to use a lidded cup filled only halfway if a parent expresses concerns about spillage. At baseline, the intervention group also received a pamphlet to share with family members and a lidded, 2-handled 6-ounce sippy cup with a hard spout and no internal "leak proof" valve. Follow-up diet and anthropometric assessments were scheduled concurrent with quarterly required visits to WIC for nutritional counselling and check disbursement, through the next 12 months.

Diet intervention (bottle use) vs control

Outcome measures
- Primary outcome: weight-for-length z-score $>$ 85th percentile, bottle use, energy intake
- Secondary outcomes: NR
Process evaluation: NR

Implementation-related factors
Theoretical basis: NR

Resources for intervention implementation: reported (downloadable)

Who delivered the intervention: reported

PROGRESS categories assessed at baseline: child: gender, race/ethnicity

PROGRESS categories analysed at outcome: NR

Outcomes relating to harms/unintended effects: NR

Intervention included strategies to address diversity or disadvantage: NR

Economic evaluation: NR

Notes NCT00756626.

Funding: funded by the US Department of Agriculture, National Institute of Food and Agriculture (2007-04556 to K.B.)

All participants low-income. Intervention delivered as part of routine care in an existing service. Culturally tailored-Spanish and English resources.

\section{Risk of bias}

\section{Bias}

\section{Authors' judgement Support for judgement}


Bonuck 2014 (Continued)

$\begin{array}{ll}\begin{array}{l}\text { Random sequence genera- } \\ \text { tion (selection bias) }\end{array} & \text { Low risk } \\ \end{array}$

\begin{tabular}{lll}
\hline $\begin{array}{l}\text { Allocation concealment } \\
\text { (selection bias) }\end{array}$ & Low risk & See above \\
\hline
\end{tabular}

\begin{tabular}{lll}
\hline $\begin{array}{l}\text { Blinding (performance } \\
\text { bias and detection bias) } \\
\text { All outcomes }\end{array}$ & High risk & No masking \\
\hline $\begin{array}{l}\text { Incomplete outcome data } \\
\begin{array}{l}\text { (attrition bias) } \\
\text { All outcomes }\end{array}\end{array}$ & Low risk & Loss for BMI was $<20 \%$ and balanced \\
\hline
\end{tabular}

\begin{tabular}{lll}
\hline $\begin{array}{l}\text { Selective reporting (re- } \\
\text { porting bias) }\end{array}$ & Unclear risk & Protocol/trial registration document seen. All outcomes reported \\
\hline Other bias & Unclear risk & Contamination was possible \\
\hline
\end{tabular}

Bonvin 2013

\begin{tabular}{|c|c|}
\hline \multirow[t]{9}{*}{ Methods } & Study name: Youp'la Bouge \\
\hline & Study design: cluster-RCT \\
\hline & Intervention period: 9 months \\
\hline & Follow-up period (post-intervention): nil \\
\hline & Differences in baseline characteristics: reported \\
\hline & Reliable outcomes: reported \\
\hline & Protection against contamination: NR \\
\hline & Unit of allocation: childcare centres \\
\hline & Unit of analysis: individual accounting for clustering \\
\hline \multirow[t]{10}{*}{ Participants } & $\mathrm{N}($ controls baseline $)=315$ \\
\hline & $N($ controls follow-up) $=308$ \\
\hline & $\mathrm{N}$ (interventions baseline) $=313$ \\
\hline & $\mathrm{N}$ (interventions follow-up) $=280$ \\
\hline & Setting (and number by study group): public childcare centres ( $N=136$, average $23-28$ children in each) \\
\hline & $\begin{array}{l}\text { Recruitment: a 3rd of the public childcare centres were randomly selected and invited by mail to partic- } \\
\text { ipate }\end{array}$ \\
\hline & Geographic region: 3 cantons (geographic government area) in the French-speaking part of Switzerland \\
\hline & Percentage of eligible population enrolled: $46 \%$ \\
\hline & Mean age: intervention: $3.3 \pm 0.6$; intervention + control: $3.4 \pm 0.6$ \\
\hline & Sex: intervention: $49 \%$ female; control: $51 \%$ female \\
\hline
\end{tabular}


Bonvin 2013 (Continued)

Interventions

To study a PA programme in preschools to see if it improves their motor skills and benefits their health - including looking for effects on BMI

- Behavioural strategies to improve child: parent and educator knowledge about PA benefits and to increase pleasure, self-efficacy and skills and to integrate PA into the daily life of the childcare centre

- PA intervention that included non-prescriptive: training and support of the educators; rearrangement of the childcare built environment; encouragement of parental involvement; recommendation of daily PA

- USD 1500 for the rearrangement of the environment and specific recommendations on providing an indoor movement space

- Childcare centres were encouraged to involve parents and invite them to an information session. Parents received flyers containing information about the intervention

- $5 \mathrm{x}$ workshops for childcare educators delivered by the co-ordinator, by sport scientists specialised in PA and health and by physicians. They covered

- movement and motor development;

- moving - a pleasure and a need;

- practical aspect of PA;

- health promotion in childcare centres;

- implementation of the project

- Meetings between trained educators and study co-ordinator for exchanging ideas every 2 months during the intervention

- Co-ordinator available to educators if they had questions or concerns during the intervention

The control group did not receive any intervention and continued their regular programme (corresponding to a waiting list for a future participation).

NOTE: no precise mandatory demands were made regarding the daily PA time or the use of a structured PA curriculum.

PA intervention (motor skills) vs control

Outcome measures
- Primary outcome: motor skills
- Secondary outcomes: PA, quality of life (PedsQL ${ }^{\mathrm{TM}}$ Score), BMI
Process evaluation: reported

Implementation-related factors

Theoretical basis: SEM (Egger 1997b)

Resources for intervention implementation: reported

Who delivered the intervention: reported

PROGRESS categories assessed at baseline: child: gender; parent: race/ethnicity (parent born outside Switzerland), education

PROGRESS categories analysed at outcome: parent: race/ethnicity (parent born outside Switzerland), education (data not shown)

Outcomes relating to harms/unintended effects: NR

Intervention included strategies to address diversity or disadvantage: NR

Economic evaluation: NR

Notes NCT00967460

Funding: geographical governmental institutions in France conducted the intervention, No report of who funded the evaluation and publication. 
Bonvin 2013 (Continued)

Resources: each childcare center received a budget of USD 1500 for the rearrangement of their environment (equipment and space). Resources included Co-ordinator x 1, specialised trainers, flyers and documentation for parent sessions.

Process: process evaluation indicated that all intervention centres provided at least 1 , and 5 centres $(17 \%) \geq 2$ educators for training. These educators attended all workshops. The educators were either strongly $(50 \%)$ or moderately $(50 \%)$ motivated. The management was either strongly $(70 \%)$ or moderately (30\%) involved. All intervention centres rearranged their indoor environment and purchased PA indoor equipment ( $69 \%$ of it portable/mobile), while $28 \%$ also purchased outdoor equipment (only mobile); $69 \%$ of the centres provided free access to a movement space and $72 \%$ organised an information session with parents (i.e. parental involvement).

Implementation: childcare centres and parents were highly satisfied with the programme, which allowed its further widespread implementation over the following years outside of a study setting. The study also allowed the study authors to identify the predictors that improve the effectiveness of the implementation. Based on the study findings, the programme adapted its content and created a label that requires Youp'là Bouge childcare centres to comply with the following requirements: 1) $90 \mathrm{~min} /$ day of PA (10 min of which structured PA); 2) at least one trained educator per childcare center; 3) a written PA policy to integrate the different intervention components; 4) wherever possible, free access to an indoor movement space and the purchase of specifically mobile equipment; 5) at least one parental information session/year.

\section{Risk of bias}

\begin{tabular}{|c|c|c|}
\hline Bias & Authors' judgement & Support for judgement \\
\hline $\begin{array}{l}\text { Random sequence genera- } \\
\text { tion (selection bias) }\end{array}$ & Unclear risk & Randomisation stated, no further details \\
\hline $\begin{array}{l}\text { Allocation concealment } \\
\text { (selection bias) }\end{array}$ & Low risk & $\begin{array}{l}\text { Recruitment, selection and a blinded randomisation of the childcare centres } \\
\text { were performed by a governmental co-ordinator not involved in the assess- } \\
\text { ment of the programme. }\end{array}$ \\
\hline $\begin{array}{l}\text { Blinding (performance } \\
\text { bias and detection bias) } \\
\text { All outcomes }\end{array}$ & Low risk & Trained researchers blinded to group allocation provided the assessments \\
\hline $\begin{array}{l}\text { Incomplete outcome data } \\
\text { (attrition bias) } \\
\text { All outcomes }\end{array}$ & Unclear risk & $\begin{array}{l}\text { Study was powered to account for attrition, however analyses focuses only on } \\
\text { those children who were present on the test day, study flow is complex and } \\
\text { varies between outcomes }\end{array}$ \\
\hline $\begin{array}{l}\text { Selective reporting (re- } \\
\text { porting bias) }\end{array}$ & Low risk & Protocol/trial registration document seen. All outcomes reported. \\
\hline Other bias & Low risk & $46 \%$ of eligible population enrolled, unclear if this representative \\
\hline $\begin{array}{l}\text { Other bias- timing of re- } \\
\text { cruitment of clusters }\end{array}$ & Unclear risk & $\begin{array}{l}\text { It seems that no new people joined after randomisation (figure } 3 \text { ) but base- } \\
\text { lines were done after randomisation and not all (within a cluster) had their } \\
\text { baseline data collected and some without baseline measures were measured } \\
\text { at final follow-up. }\end{array}$ \\
\hline
\end{tabular}

Brandstetter 2012

$\begin{array}{ll}\text { Methods } & \text { Study design: cluster-RCT } \\ & \text { Intervention period: } 10 \text { months (school year) } \\ & \text { Follow-up period (post-intervention): } 2 \text { months (varied) }\end{array}$


Brandstetter 2012 (Continued)

Differences in baseline characteristics: reported

Reliable outcomes: reported

Protection against contamination: reported

Unit of allocation: class

Unit of analysis: individual accounting for clustering

\begin{tabular}{|c|c|}
\hline \multirow[t]{4}{*}{ Participants } & $\mathrm{N}($ controls baseline $)=579$ \\
\hline & $\mathrm{N}$ (controls follow-up) $=495$ \\
\hline & $\mathrm{N}$ (interventions baseline) $=540$ \\
\hline & $\mathrm{N}$ (interventions follow-up) $=450$ \\
\hline
\end{tabular}

Setting (and number by study group): 32 primary schools, $16=$ intervention $(N=450,16=$ control $(N=$ 495); hospital-based setting for outcome measurements

Recruitment: all principals of elementary schools within the Ulm region in Southern Germany were informed in writing about the study and were asked to invite first-grade teachers to participate

Geographic region: Ulm, Southern Germany

78\% Geographic region: Ulm, Southern Germany

Mean age: intervention: $7.61 \pm 0.42$; control: $7.53 \pm 0.42$

Sex: intervention: $44.9 \%$ female; control: $47.9 \%$ female

To describe the effects of URMEL-ICE for overweight prevention on children's BMI and other measures of fat mass

Intervention to educate grade 2 students re PA, TV time, SSB consumption. Intervention was integrated into 2 nd grade curriculum, implemented by existing classroom teachers. Intervention consisted of 29 teaching lessons (lasting 30-60 min), 2 exercise blocks per day (5-7 min each) and 6 family homework lessons that required students to work with parents/family to complete. Intervention lasted for 1 year.

Intervention was developed with experienced teachers to ensure anchoring in existing curriculum

SCT provided the methodological framework, emphasised action alternatives and easily accomplishable goals. Modified teaching to promote more PA in class time and provided suggestions for involving parents. Teachers were provided with $4 \times 2.5$-h training sessions.

Diet and PA combined intervention vs control

Outcome measures
- Primary outcome: BMI
- Secondary outcomes: waist circumference, skinfolds (triceps, subscapular)
Process evaluation: reported (implementation)

Implementation-related factors
Theoretical basis: SCT

Resources for intervention implementation: reported

Who delivered the intervention: reported

PROGRESS categories assessed at baseline: child: gender, race/ethnicity; parent: education PROGRESS categories analysed at outcome: NR 
Brandstetter 2012 (Continued)

Outcomes relating to harms/unintended effects: NR

Intervention included strategies to address diversity or disadvantage: NR

Economic evaluation: NR (cost reported)

Notes $\quad$ Funding: this study was funded by the Baden-Württemberg Stiftung (Stuttgart, Germany).

It was implemented during regular class time by the classroom teacher within the existing curriculum (mainly social studies) in order to ensure programme implementation without additional personnel or materials in everyday teaching.

Costs/resources: teachers took part in 4 training sessions ( $2.5 \mathrm{~h}$ each). Teachers and schools had no direct costs to cover (for materials or for additional teaching time). However, in terms of indirect costs for the schools, the intervention required 29 regular teaching units mainly in social sciences during 1 school year (that corresponds to the weekly working time of teachers) and additionally $10 \mathrm{~h}$ of training sessions. From the perspective of the intervention provider costs were limited to personnel costs of the teacher training sessions and material costs of the intervention materials (one folder per teacher).

Follow-up measurements in our study took place after a 6-week summer break.

\section{Risk of bias}

\begin{tabular}{|c|c|c|}
\hline Bias & Authors' judgement & Support for judgement \\
\hline $\begin{array}{l}\text { Random sequence genera- } \\
\text { tion (selection bias) }\end{array}$ & Unclear risk & Stratified randomisation, no further details \\
\hline $\begin{array}{l}\text { Allocation concealment } \\
\text { (selection bias) }\end{array}$ & Low risk & Allocation procedure performed blinded \\
\hline $\begin{array}{l}\text { Blinding (performance } \\
\text { bias and detection bias) } \\
\text { All outcomes }\end{array}$ & Low risk & Participants were measured in a separate setting (hospital \\
\hline $\begin{array}{l}\text { Incomplete outcome data } \\
\text { (attrition bias) } \\
\text { All outcomes }\end{array}$ & Low risk & $83 \%$ and $85 \%$ retention in intervention and control groups respectively \\
\hline $\begin{array}{l}\text { Selective reporting (re- } \\
\text { porting bias) }\end{array}$ & High risk & $\begin{array}{l}\text { Protocol/trial registration documents were unavailable. BMI was reported. zB- } \\
\text { MI was reported for baseline, but not at follow-up }\end{array}$ \\
\hline Other bias & Low risk & $\begin{array}{l}\text { Intervention and control group differed in the time lag between the } 2 \text { points } \\
\text { of measurements. In addition, time periods for investigating the children were } \\
\text { rather long: } 6 \text { months at baseline and } 4 \text { months at follow-up. Data adjusted for } \\
\text { time lag effects. }\end{array}$ \\
\hline
\end{tabular}

Other bias- timing of re- Low risk Figure shows recruitment happened prior to randomisation cruitment of clusters

\section{Branscum 2013}

$\begin{array}{ll}\text { Methods } & \text { Study design: cluster-RCT } \\ \text { Intervention period: } 4 \text { weeks } \\ \text { Follow-up period (post-intervention): } 8 \text { weeks } \\ \text { Differences in baseline characteristics: reported }\end{array}$


Branscum 2013 (Continued)

Reliable outcomes: reported

Protection against contamination: reported

Unit of allocation: after school care groups

Unit of analysis: after school care groups

\begin{tabular}{|c|c|}
\hline \multirow[t]{10}{*}{ Participants } & $\mathrm{N}($ controls baseline $)=43$ \\
\hline & $N($ controls follow-up) $=43$ \\
\hline & $\mathrm{N}$ (interventions baseline) $=37$ \\
\hline & $\mathrm{N}$ (interventions follow-up) $=37$ \\
\hline & $\begin{array}{l}\text { Setting (and number by study group): } 12 \text { Mid-Western Young Men's } \mathrm{C} \\
\text { programmes ( } \mathrm{N}=6 \text { in each intervention group) }\end{array}$ \\
\hline & Recruitment: programme facilitator approached parents at pick-up \\
\hline & Geographic region: Ohio, USA \\
\hline & Percentage of eligible population enrolled: NR \\
\hline & Mean age: intervention + control: 8-11 \\
\hline & Sex: intervention: $53 \%$ female; control: $43 \%$ female \\
\hline
\end{tabular}

Interventions

To pilot test the 'comics for health' intervention, a new comic-book programme designed to help children learn and engage in behaviours associated with the prevention of obesity.

Programmes were randomised to either a theory-based or a knowledge-based version of the intervention.

$4 \times 30$-min lessons provided to each group, intervention lasted 4 weeks. The pedagogical techniques used to mediate changes differed for the 2 groups. In the theory-based intervention group the following constructs were operationalised: self-efficacy, self-control. Activities included taking small achievable steps for learning and mastering new skills, and participating in role plays to practice new skills and behaviours in pretend setting with either peer or parent. The knowledge-based group techniques were based on only building knowledge regarding healthy eating and PA.

Lesson 1: engaging in no more than $2 \mathrm{~h}$ of screen time/day

Lesson 2: consuming water and sugar-free drinks instead of SSBS

Lesson 3: participating in at least $60 \mathrm{~min}$ of PA/day

Lesson 4: consuming 5 servings of fruits and vegetables/day

Both interventions culminated with the children creating an original comic book or strip. Activities for making the comic were identical for both programmes, in which children were taught basic concepts of storytelling and character development. However, children in the theory-based intervention were asked to develop their comic stories on the health issues covered during the intervention, whereas children in the knowledge-based intervention were not asked to incorporate the health messages.

Theory-based dietary and PA intervention vs knowledge-based dietary and PA intervention

Outcomes

Outcome measures

- Primary outcome: BMI percentile, dietary intake (fruit and vegetable consumption, SSB consumption), PA and screen-time engagement

- Secondary outcomes: constructs of SCT (self-efficacy, self-control, and expectations), process evaluation 
Branscum 2013 (Continued)

Process evaluation: reported (fidelity, dose, reach, context)

Implementation-related factors
Theoretical basis: SCT

Resources for intervention implementation: NR

Who delivered the intervention: reported

PROGRESS categories assessed at baseline: child: gender, race/ethnicity

PROGRESS categories analysed at outcome: NR

Outcomes relating to harms/unintended effects: NR

Intervention included strategies to address diversity or disadvantage: NR

Economic evaluation: NR
Funding: this work was supported by the United Health HEROES grant provided by Youth Service America and an internal faculty-mentoring grant, provided by the College of Education, Criminal Justice, and Human Services at the University of Cincinnati.

Separate paper on process evaluation. Implementation: most lessons recording $100 \%$ tasks completed, lessons implemented in both intended order and length. After-school staff members reported that the programme was well received by children. $70.4 \%$ children attended each lesson on the initial day of delivery. Sources of contamination identified.

\section{Risk of bias}

\begin{tabular}{lll}
\hline Bias & Authors' judgement & Support for judgement \\
\hline $\begin{array}{l}\text { Random sequence genera- } \\
\text { tion (selection bias) }\end{array}$ & Unclear risk & NR. \\
& Quote: "This study used a group randomized controlled design" \\
\hline $\begin{array}{l}\text { Allocation concealment } \\
\text { (selection bias) }\end{array}$ & Unclear risk & NR \\
\hline $\begin{array}{l}\text { Blinding (performance } \\
\text { bias and detection bias) } \\
\text { All outcomes }\end{array}$ & Unclear risk & $\begin{array}{l}\text { After-school staff members were initially blinded from knowing which pro- } \\
\text { gramme their site received }\end{array}$ \\
\hline
\end{tabular}

Incomplete outcome data Unclear risk $\quad$ Only reports number of children assessed, no details of study flow
(attrition bias)

All outcomes

\begin{tabular}{lll}
\hline $\begin{array}{l}\text { Selective reporting (re- } \\
\text { porting bias) }\end{array}$ & Unclear risk & Protocol/trial registration documents were unavailable. \\
\hline Other bias & Low risk & $\begin{array}{l}\text { Sources of contamination identified but study authors report similar risk to } \\
\text { both groups of outside contamination }\end{array}$ \\
\hline $\begin{array}{l}\text { Other bias- timing of re- } \\
\text { cruitment of clusters }\end{array}$ & Low risk & No figure; text suggests recruitment happened prior to randomisation \\
\hline
\end{tabular}

\section{Brown 2013}

Methods Study design: RCT


Brown 2013 (Continued)

Intervention period: 12 weeks

Follow-up period (post-intervention): nil

Differences in baseline characteristics: reported (data not shown)

Reliable outcomes: reported

Protection against contamination: NR

Unit of allocation: individual

Unit of analysis: individual

\section{Participants}

$\mathrm{N}($ controls baseline $)=38$

$\mathrm{N}$ (controls follow-up) $=32$

$\mathrm{N}$ (interventions baseline $)=38$

$\mathrm{N}$ (interventions follow-up) $=31$

Setting (and number by study group): classrooms, community and fitness centres in 2 American Indian reservations, 8 groups ( $N=4$ intervention groups and $N=4$ control groups, average 8 youths per group)

Recruitment: potentially eligible youths from school rosters were blocked by site and grade and randomly ordered within blocks for recruitment.

Geographic region: 2 American Indian reservations in North-Central and Southwestern Montana

Percentage of eligible population enrolled: $82 \%$

Mean age: intervention + control: $11.4 \pm 1.1$

Sex: intervention + control: $50 \%$ female

The purpose of this study was to develop a lifestyle change programme for Native American youth by modifying the Diabetes Prevention Program (DPP) and assess implementation indicators and shortterm behavioural and physiological outcomes of the intervention among a small pilot sample.

'Journey DPP' was an intervention that modified the original Diabetes Prevention Program for Native American Youth. 9-sessions, each session implemented every 1.5 weeks, lasting 12 weeks

Modifying the original DPP (through community-based participatory research) for Native American youth included adding cultural components, addressing youth's knowledge of and access to healthy food, including hands-on interactive learning activities and using a group format to deliver the intervention. Group sessions were held after school in classrooms and community and fitness centres. Sessions were led by tribally enrolled community members (called lifestyle educators) from each of the 2 participating reservations. Cultural aspects were incorporated throughout the programme and included emphasis on traditional activities (such as berry picking, horseback riding, dancing, hunting, hiking, and camping), use of storytelling and native language to convey information, and participation of elders

Control group was a health-orientated comparison that addressed risks for alcohol and drug use.

Participants in both conditions received USD 150 worth of incentives (e.g. pedometers, balls, jump ropes, athletic shoes) and a certificate of completion. Participants' parents or guardians also received a USD 25 voucher redeemable from local grocery stores

Diet and PA combined intervention vs control

\section{Outcomes}

Outcome measures

- Primary outcome: dietary intake, nutrition knowledge, PA, PA score, screen time, BMI $\left(\mathrm{kg} / \mathrm{m}^{2}\right)$, BMI percentile (\%), zBMI (NR whether outcomes primary or secondary) 
Brown 2013 (Continued)

\section{- Secondary outcomes:}

Process evaluation: reported (recruitment, retention, completion, implementation, satisfaction)

Implementation-related factors
Theoretical basis: TTM-Stages of Change and SCT

Resources for intervention implementation: NR

Who delivered the intervention: reported

PROGRESS categories assessed at baseline: child: gender; all children were Native American

PROGRESS categories analysed at outcome: NR

Outcomes relating to harms/unintended effects: NR

Intervention included strategies to address diversity or disadvantage: culturally tailored

Economic evaluation: NR
Funding: NR. The paper states, "Beginning in 2004, the University of Montana and both reservation communities formed a collaborative partnership to reduce diabetes risk factors in Native American youth. Subsequently, the partnership wrote the federal NIH grant application and established a code of research ethics for the study.

Interviews conducted at the end of the study suggested that the lifestyle educators had high confidence in their ability to implement the program's behavioural and educational strategies of goal setting and problem solving. Educators reported difficulty in keeping some participants interested in the sessions and suggested having more interactive learning activities in the program. Educators expressed interest in having more information and activities that included the participants' families."

\section{Risk of bias}

\begin{tabular}{lll}
\hline Bias & Authors' judgement & Support for judgement \\
\hline $\begin{array}{l}\text { Random sequence genera- } \\
\text { tion (selection bias) }\end{array}$ & Unclear risk & Blocked by site and grade and randomly ordered within blocks for recruitment \\
\hline $\begin{array}{l}\text { Allocation concealment } \\
\text { (selection bias) }\end{array}$ & Unclear risk & NR \\
\hline $\begin{array}{l}\text { Blinding (performance } \\
\text { bias and detection bias) } \\
\text { All outcomes }\end{array}$ & High risk & $\begin{array}{l}\text { Data were collected by trained tribal and university research staff. Neither staff } \\
\text { nor participants were blinded to condition assignment. Also tribal partners } \\
\text { wanted to implement an alcohol and drug prevention curriculum for the com- } \\
\text { parison condition, given intervention was not blinded this may have intro- } \\
\text { duced performance/detection bias }\end{array}$ \\
\hline
\end{tabular}

Incomplete outcome data Low risk Low attrition ( $84 \%$ completed) and balanced between groups
(attrition bias)

All outcomes

$\begin{array}{lll}\begin{array}{l}\text { Selective reporting (re- } \\ \text { porting bias) }\end{array} & \text { Unclear risk } & \begin{array}{l}\text { Protocol/trial registration documents were unavailable. Risk could not be as- } \\ \text { sessed. }\end{array}\end{array}$

Other bias Unclear risk Insufficient details reported to assess risk of contamination

Methods Study design: cluster-RCT


Caballero 2003 (Continued)

Intervention period: 3 years

Follow-up (post-intervention): nil

Differences in baseline characteristics: reported

Reliable outcomes: yes

Protection against contamination: adequately addressed

Unit of allocation: school

Unit of analysis: child

Unit of analysis errors addressed. Primary analysis applied the ITT principle and missing data at fol-

low-up was imputed based on a prediction equation developed using control school data and Rubin's multiple imputation method.

Participants
N (controls baseline) $=835$
N (interventions baseline) $=879$
N (interventions follow-up) $=727$
N of schools: 41
Recruitment: all consenting American Indian students in grades 3-5 (8 to 11 years) from schools in Ari-
zona, New Mexico, South Dakota, USA
Proportion of eligibles participating: not stated, but schools had to provide: $>15$ 3rd graders; $90 \%$
American Indian; retention of 3-5 grades over 70\% in past 3 years; school meals prepared on site; facili-
ties for PA programme; approval of study by school, community and tribal authorities
Mean age: 7.6 (SD 0.6) years
Sex: both sexes included but no figures given
School-based multi-component trial utilising school curriculum and existing staff resources trained by
licensed SPARK (Sports, Play and active Recreation for Kids, see Sallis 1993 ) instructors and Pathways
personnel who also acted as mentors. The intervention aimed to attenuate obesity and reduce per-
centage body fat.
4 components included improved PA, food service, classroom curriculum and family involvement pro-
gramme.
Control programme NR, presumably usual curriculum
Combined effects of dietary interventions and PA interventions vs control

Outcomes

- BMI

- TSF and subscapular skinfold

- Bioelectrical impedance

- PA:

- TriTrac R3D accelerometer

- checklist standardised from pilot work used as a 24-h recall questionnaire

- Knowledge attitudes and beliefs:

- self-report questionnaires developed in pilot

- Dietary intake measured by modified 24-h recall

- Observations of school meals

- Analysis of school menus for energy, protein, carbohydrate, fat, sodium and fibre using the NDS-R

Process evaluation: reported

Implementation-related factors
Theoretical basis: Social Learning theory and principles of American Indian culture and practice Resources for intervention implementation (e.g. funding needed or staff hours required): reported Who delivered the intervention: reported

PROGRESS categories assessed at baseline: reported (gender)

PROGRESS categories analysed at outcome: reported (gender) 
Caballero 2003 (Continued)

Outcomes relating to harms/unintended effects: NR

Intervention included strategies to address diversity or disadvantage: reported

Economic evaluation: NR

Notes

Funding: supported by National Heart, Lung, and Blood Institute grants U01- HL-50869, -50867, -50905,

-50885 , and -50907

\section{Risk of bias}

\begin{tabular}{|c|c|c|}
\hline Bias & Authors' judgement & Support for judgement \\
\hline $\begin{array}{l}\text { Random sequence genera- } \\
\text { tion (selection bias) }\end{array}$ & Low risk & $\begin{array}{l}\text { Schools were assigned to intervention and control groups by a process of } \\
\text { stratified randomisation }\end{array}$ \\
\hline $\begin{array}{l}\text { Allocation concealment } \\
\text { (selection bias) }\end{array}$ & Unclear risk & NR \\
\hline $\begin{array}{l}\text { Blinding (performance } \\
\text { bias and detection bias) } \\
\text { All outcomes }\end{array}$ & Low risk & $\begin{array}{l}\text { Quote: "To avoid operator bias, measurement teams were not involved in de- } \\
\text { livering the intervention. Training, certification and cross-validation of mea- } \\
\text { surement staff were done centrally or regionally, supervised by the Measure- } \\
\text { ment Committee." }\end{array}$ \\
\hline $\begin{array}{l}\text { Incomplete outcome data } \\
\text { (attrition bias) } \\
\text { All outcomes }\end{array}$ & Low risk & Missing data balanced across groups and imputation method given \\
\hline $\begin{array}{l}\text { Selective reporting (re- } \\
\text { porting bias) }\end{array}$ & Unclear risk & Protocol/trial register not found. \\
\hline Other bias & Low risk & No other threats to validity \\
\hline $\begin{array}{l}\text { Other bias- timing of re- } \\
\text { cruitment of clusters }\end{array}$ & Low risk & $\begin{array}{l}\text { Likely no recruitment after randomisation (figure 1) } \\
\text { Quote: "Children were enrolled in the study, and baseline measurements were } \\
\text { made at the end of the } 2 \text { nd grade.... After the baseline measurements were } \\
\text { made, upper and lower \%BF strata were defined for schools at each site, and } \\
\text { random allocation was determined for each stratum." }\end{array}$ \\
\hline
\end{tabular}

\section{Campbell 2013}

\section{Methods}

Study name: Melbourne infant, feeding, activity and nutrition trial (InFANT) program

Study design: cluster-RCT

Intervention period: 15 months

Follow-up period (post-intervention): nil

Differences in baseline characteristics: reported

Reliable outcomes: reported

Protection against contamination: NR

Unit of allocation: first-time parents' groups

Unit of analysis: individual accounting for clustering 
Campbell 2013 (Continued)

\begin{tabular}{|c|c|}
\hline \multirow[t]{3}{*}{ Participants } & $N($ controls baseline $)=271$ \\
\hline & $\mathrm{N}$ (controls follow-up) $=239$ \\
\hline & $\mathrm{N}$ (interventions baseline) $=271$ \\
\hline
\end{tabular}

Setting (and number by study group): 62 parent-group clusters from 28 eligible local government areas (intervention N = 31 parents' groups and 271 children; control N = 31 parents' groups and 271 children)

Recruitment: 14 LGAs were randomly selected from the 28 eligible LGAs located within a $60-\mathrm{km}$ radius of the research centre. 50\% of eligible first-time parents' groups (rounded to next even number) within each LGA were randomly selected (62/103 groups) and approached by research staff for recruitment during 1 of the standard nurse-facilitated group sessions.

Geographic region: Melbourne, Australia

Percentage of eligible population enrolled: $86 \%$

Mean age: intervention: $3.9 \pm 1.6$ (months); control: $3.9 \pm 1.6$ (months)

Sex: intervention: $48.3 \%$ female; control: $46.5 \%$ female BMI

Parents were offered six 2-h dietitian-delivered quarterly sessions over 15 months focusing on parental knowledge, skills, and social support around infant feeding, diet, PA, and TV viewing. Control group parents received 6 newsletters on non obesity-focused themes; all parents received usual care from child health nurses.

Diet and PA combined intervention vs control

Outcomes Outcome measures

- Primary outcome: dietary intake, PA, TV viewing

- Secondary outcomes: zBMI

Process evaluation: reported (perceived group session usefulness and relevance; fidelity)

Implementation-related factors
Theoretical basis: SCT

Resources for intervention implementation: reported

Who delivered the intervention: reported

PROGRESS categories assessed at baseline: child: gender; maternal education

PROGRESS categories analysed at outcome: maternal education (secondary reference for Campbell 2013 examines moderating effect of zBMI by maternal education)

Outcomes relating to harms/unintended effects: NR

Intervention included strategies to address diversity or disadvantage: NR

Economic evaluation: reported (costs of resources)

\section{Notes}

\section{ISRCTN81847050}

Funding: supported by the National Health and Medical Research Council (grant 425801). Additional funds were supplied by the Heart Foundation Victoria and Deakin University. 
The total estimated cost of delivering the programme, based on the costs of the intervention adjusted for the fact that a trial setting sees an artificially small number of families included relative to the workforce employed, was approximately AUD 500 per family.

\section{Risk of bias}

\begin{tabular}{|c|c|c|}
\hline Bias & Authors' judgement & Support for judgement \\
\hline $\begin{array}{l}\text { Random sequence genera- } \\
\text { tion (selection bias) }\end{array}$ & Low risk & $\begin{array}{l}\text { Randomisation (stratified by LGA) was conducted by an independent statisti- } \\
\text { cian; balanced ( } 1: 1 \text { ) randomisation; randomly ordered list of LGAs }\end{array}$ \\
\hline $\begin{array}{l}\text { Allocation concealment } \\
\text { (selection bias) }\end{array}$ & Low risk & $\begin{array}{l}\text { Randomisation of first-time parents' groups (clusters) occurred after recruit- } \\
\text { ment to avoid selection bias. Randomisation (stratified by LGA) was conducted } \\
\text { by an independent statistician. }\end{array}$ \\
\hline $\begin{array}{l}\text { Blinding (performance } \\
\text { bias and detection bias) } \\
\text { All outcomes }\end{array}$ & High risk & $\begin{array}{l}\text { Staff measuring height and weight were not blinded to intervention status be- } \\
\text { cause they also delivered the intervention. All dietary recalls, data entry, and } \\
\text { analyses were conducted with staff blinded to participant's group allocation. }\end{array}$ \\
\hline $\begin{array}{l}\text { Incomplete outcome data } \\
\text { (attrition bias) } \\
\text { All outcomes }\end{array}$ & Low risk & $\begin{array}{l}\text { Low attrition ( } 88 \% \text { completed) and balanced between groups. In addition, par- } \\
\text { ticipating parents excluded from mid-intervention analyses ( } 5 \text { months from } \\
\text { baseline) due to missing data and loss to follow-up were more likely at base- } \\
\text { line to have low levels of maternal education ( } 57.5 \% \text { vs } 36.1 \% \text { ). Kept at low } \\
\text { risk- because we are not using data from mid-intervention analysis. }\end{array}$ \\
\hline $\begin{array}{l}\text { Selective reporting (re- } \\
\text { porting bias) }\end{array}$ & Low risk & Protocol seen. All outcomes reported \\
\hline Other bias & Unclear risk & Insufficient details reported to assess risk of contamination \\
\hline $\begin{array}{l}\text { Other bias- timing of re- } \\
\text { cruitment of clusters }\end{array}$ & Low risk & $\begin{array}{l}\text { Randomisation of first-time parents' groups (clusters) occurred after recruit- } \\
\text { ment to avoid selection bias. }\end{array}$ \\
\hline
\end{tabular}

Cao 2015

$\begin{array}{ll}\text { Methods } & \text { Study name: Family-Individual-School (FIS) } \\ & \text { Study design: cluster-RCT } \\ \text { Intervention period: } 34 \text { months (10 months, } 22 \text { months, } 34 \text { months) } \\ \text { Follow-up period (post-intervention): nil } \\ \text { Differences in baseline characteristics: reported } \\ \text { Reliable outcomes: reported } \\ \text { Protection against contamination: reported } \\ \text { Unit of allocation: school } \\ \text { Unit of analysis: individual accounting for clustering } \\ \text { N (controls baseline) }=1158 \\ \text { N (controls follow-up) }=828\end{array}$


Cao 2015 (Continued)

$$
\begin{aligned}
& \mathrm{N}(\text { interventions baseline })=1287 \\
& \mathrm{~N}(\text { interventions follow-up })=985
\end{aligned}
$$

Setting (and number by study group): 14 primary schools ( $N=1287$ intervention children and 7 schools, $\mathrm{N}=1159$ control children and 7 schools)

Recruitment: all 26 primary schools in a district of the city were divided into 3 groups according to average obesity prevalence; according to the economic level of the communities in which the schools were located and the condition of school sports fields and canteens, $4 / 7$ schools with high obesity prevalence were selected; $6 / 12$ schools with middle obesity prevalence and $4 / 6$ with low obesity prevalence were selected

Geographic region: Shanghai, China

Percentage of eligible population enrolled: $100 \%$

Mean age: intervention: $7.01 \pm 0.44$; control: $6.81 \pm 0.24$

Sex: intervention: $45.2 \%$ female; control: $47.4 \%$ female

To evaluate the effectiveness of an intervention targeted at school, family and the individual level to prevent childhood obesity

- School components:

- health education

- 6-h health education course per semester

- obesity-related health information dissemination through school publicity platform such as blackboard newspaper, morning meeting and class meeting and brochures.

- theme class meetings or seminars about childhood obesity provided by health teacher

- dietary intervention

- teachers' control of eating speed for students during lunch and advice on eating less junk foods.

- reducing fat content at canteens

- making more fruits and vegetables available

- exercises intervention

- 20-m music shuttle run 2-3 times/week

- ensure the participation rate of regular school PE and extracurricular activities

- >1-h PA time each school day. Featured sports activities such as rope skipping and football

- Family components:

- health education

- parent-school meeting every semester

- distribution of brochures on childhood obesity prevention and intervention

- parents' participation of obesity prevention lectures

- dietary intervention

- information to parents about balanced diet principles and methods

- instructions to parents about healthy eating habits of children

- exercises intervention

- a strip of skipping rope provided to each student and appropriate level of PA at home supervised and monitored by parents.

- parents' completion of "Students' Extracurricular PA Registration Form" during summer and winter vacations, including frequency, duration, intensity, and other information of PA

Control group received no intervention

Diet and PA combined intervention vs control

- Primary outcome: prevalence of obesity/overweight, zBMI

- Secondary outcomes: NR 
Cao 2015 (Continued)

Process evaluation: NR

Implementation-related factors
Theoretical basis: NR

Resources for intervention implementation: reported

Who delivered the intervention: reported

PROGRESS categories assessed at baseline: child: gender

PROGRESS categories analysed at outcome: child: gender, age (for overweight prevalence only)

Outcomes relating to harms/unintended effects: NR

Intervention included strategies to address diversity or disadvantage: NR

Economic evaluation: NR

Notes $\quad$ Funding: Shanghai Municipal Health Bureau: Award Number 12GWZX0301

Study authors reported that successful completion of intervention activities required administrative measures and expert resources as well as financial support.

\section{Risk of bias}

Bias Authors' judgement Support for judgement

Random sequence genera- Unclear risk Schools allocated to intervention or control in matched pairs, based on obesition (selection bias) ty level of the school; divided randomly by sortation

\begin{tabular}{lll}
\hline $\begin{array}{l}\text { Allocation concealment } \\
\text { (selection bias) }\end{array}$ & Unclear risk & \\
\hline $\begin{array}{l}\text { Blinding (performance } \\
\text { bias and detection bias) } \\
\text { All outcomes }\end{array}$ & Unclear risk & NR \\
\hline
\end{tabular}

\begin{tabular}{lll}
\hline $\begin{array}{l}\text { Incomplete outcome data } \\
\text { (attrition bias) } \\
\text { All outcomes }\end{array}$ & High risk & $\begin{array}{l}\text { 29\%-31\% attrition rate and completer analysis only. No information on peo- } \\
\text { ple who dropped out or reasons why. Numbers missing similar from each in- } \\
\text { tervention group. }\end{array}$ \\
\hline $\begin{array}{l}\text { Selective reporting (re- } \\
\text { porting bias) }\end{array}$ & Unclear risk & Protocol/trial registration documents were unavailable. \\
\hline $\begin{array}{l}\text { Other bias } \\
\begin{array}{l}\text { Other bias- timing of re- } \\
\text { cruitment of clusters }\end{array}\end{array}$ & Low risk & No other apparent threats to validity \\
\hline
\end{tabular}

Chen 2010

Study name: The active balance childhood program
Study design: RCT
Intervention period: 8 weeks
Follow-up period (post-intervention): 4 months in control and intervention group, and 6 months in in-
tervention group (control group was a waiting list group, and received the intervention during the last 2


Chen 2010 (Continued)

months of follow-up in the intervention group). Therefore, assume the only follow-up that can be used to compare groups is 4 months post-intervention ( $\mathrm{T} 2$ in intervention group; $\mathrm{T} 3$ in control group).

Differences in baseline characteristics: reported

Reliable outcomes (apart from steps): reported

Protection against contamination: NR (and risk likely to be high)

Unit of allocation: child + parent (family)

Unit of analysis: child

The study authors do not report that analyses were performed according to ITT principles.

Participants

8-10-year-old Chinese American children who were normal weight or overweight and their parents were eligible for enrolment if they met the following criteria:

- the adult and child self-identify ethnicity as Chinese or of Chinese origin

- they reside in the same household

- a dyad of 1 adult and 1 child was the minimum necessary for a household to participate.

$\mathrm{N}($ controls baseline $)=32$

$\mathrm{N}$ (controls 2 months $)=$ unclear

$\mathrm{N}$ (controls 6 months after baseline $)=$ unclear

$\mathrm{N}$ (intervention baseline) $=35$

$\mathrm{N}$ (intervention 2 months and end intervention) = unclear

$\mathrm{N}$ (intervention 6 months after baseline and 4 months after end intervention) = unclear

Of the 67 children who were included at baseline, "Fifty-seven children and their families (85\%) completed baseline and follow-up measures; $94 \%$ of children in the intervention group and $75 \%$ of children in the control group completed baseline and follow-up measures".

NOTE: see Fig (flow chart for protocol) below. $\mathrm{T}=$ baseline for both groups, $\mathrm{T} 1=2$ months after baseline (and end of intervention in intervention group) in both groups. T2 in intervention group and T3 in control group $=6$ month follow-up

Setting: 'study site'. Unclear but probably research centre

Recruitment: participants were recruited from Chinese language programmes in the San Francisco Bay area.

Geographic region: San Francisco, California, USA

Percentage of eligible population enrolled: unclear

Mean age: (intervention + control) 8.97 (SD 0.89); intervention plus: $9.14 \pm 0.85$; control: $8.78 \pm 0.91$

Sex: 29 of 67 children were girls (43.2\%). "Approximately $54 \%$ of children in the intervention group and $59 \%$ of children in the control group were boys"

Interventions

The study authors developed an individual tailored child-centred and family-focused behavioural programme (Active Balance Childhood ( $A B C$ ) study) that focused on promoting healthy weight management and healthy lifestyles (adequate dietary intake and improved PA) in Chinese-American children, ages $8-10$, and their families. The features of the intervention are described clearly and in detail in this paper. Importantly, the intervention was certainly family-focused and the parents were fully engaged and involved with the intervention. 
Chen 2010 (Continued)

Implementation of the intervention was NR, but study authors concluded that the intervention was feasible.

Outcomes

Outcome measures anthropometry, blood pressure, measures of dietary intake, PA, knowledge and self-efficacy regarding PA, and diet at baseline and 2, 6 and 8 months after baseline assessment

- Primary outcome: BMI

Note: methods of analysis were interesting (see below)

Implementation-related factors

\section{Theoretical basis: SCT}

Resources for intervention implementation (e.g. funding needed or staff hours required): NR

Who delivered the intervention: assume research team

PROGRESS categories assessed at baseline: NR

PROGRESS categories analysed at outcome: NR

Outcomes relating to harms/unintended effects: NR

Intervention included strategies to address diversity or disadvantage: reported (for Chinese community)

Economic evaluation: NR

Notes

Funding: this publication was made possible by grant number KL2 RR024130 to J.L.C. from the National Center for Research Resources, a component of the NIH and NIH Roadmap for Medical Research, Chinese Community Health Care Association community grants and in part by NIH grant DK060617 to M.B.H.

\section{Risk of bias}

\begin{tabular}{lll}
\hline Bias & Authors' judgement & Support for judgement \\
\hline $\begin{array}{l}\text { Random sequence genera- } \\
\text { tion (selection bias) }\end{array}$ & Low risk & $\begin{array}{l}\text { Children and parents were randomly assigned to the intervention group or the } \\
\text { waiting list control group by a computer-generated random number assign- } \\
\text { ment }\end{array}$ \\
\hline $\begin{array}{l}\text { Allocation concealment } \\
\text { (selection bias) }\end{array}$ & Unclear risk & NR \\
\hline $\begin{array}{l}\text { Blinding (performance } \\
\text { bias and detection bias) } \\
\text { All outcomes }\end{array}$ & High risk & No mention that outcome assessors were blind to allocation \\
\hline $\begin{array}{l}\text { Incomplete outcome data } \\
\text { (attrition bias) }\end{array}$ & Unclear risk & $\begin{array}{l}\text { 85\% followed up in total but more loss in (waiting list) control than interven- } \\
\text { tion; reasons NR; ITT NR }\end{array}$ \\
$\begin{array}{l}\text { Qutcomes } \\
\text { Quote: "Fifty-seven children and their families (85\%) completed baseline and } \\
\text { follow-up measures; } 94 \% \text { of children in the intervention group and 75\% of chil- } \\
\text { dren in the control group completed baseline and follow-up measures." }\end{array}$ \\
$\begin{array}{l}\text { Selective reporting (re- } \\
\text { porting bias) }\end{array}$ & Unclear risk & \begin{tabular}{l} 
Protocol/trial registration documents were unavailable. \\
\hline \begin{tabular}{l} 
Other bias \\
\hline
\end{tabular}
\end{tabular} \\
\hline
\end{tabular}


Chen 2011

\section{Methods}

Study name: Web ABC study

Study design: RCT

Intervention period: 8 weeks

Follow-up period (post-intervention): 6 months

Differences in baseline characteristics: reported

Reliable outcomes: reported

Protection against contamination: NR

Unit of allocation: individual

Unit of analysis: individual

$$
\begin{aligned}
& N(\text { controls baseline })=27 \\
& N(\text { controls follow-up })=24 \\
& N(\text { interventions baseline })=27 \\
& N(\text { interventions follow-up })=26
\end{aligned}
$$

Setting (and number by study group): 54 participants ( $N=27$ intervention; $N=27$ control) from community centres

Recruitment: convenience sample of 12-15-year-old participants who accessed community programmes

Geographic region: San Francisco, USA

Percentage of eligible population enrolled: $86 \%$

Mean age: (intervention + control:12.52 (3.15)

Sex: intervention, $41 \%$ female; control, $52 \%$ female
Interventions
Aim: to examine the efficacy of the Web $A B C$ programme in promoting healthy lifestyles and healthy weight in Chinese-American adolescents.

Intervention was designed to be individually tailored to the behavioural stage of the adolescent. For instance, if the adolescent was in the 'Preparation' stage in PA area, he/she would receive information on ways of being active and various types of fun activities he/she could do.

Both adolescent and parental sessions/lessons lasted $15 \mathrm{~min}$ each. Content/themes of the 8-week adolescent programme included the following

- Week 1: understanding how the body works and how to recognise and cope with feelings

- Week 2: apply adequate problem-solving techniques and develop healthy coping skills

- Week 3: use various relaxation techniques and develop healthy coping skills

- Week 4: nutrition 101: understanding food and health

- Week 5: nutrition 102: make smart food choices

- Week 6: understanding the importance of an adequate activity level

- Week 7: being cool and active: various fun activities for youth and families

- Week 8: being yourself and using fun ways to improve your health and maintain a healthy lifestyle

There were 3 internet sessions for parents designed to coach parents in the skills needed to help their adolescents improve their progress toward healthy lifestyles and healthy weights. 
Chen 2011 (Continued)

Participants could log on to the programme and complete sessions/lessons from home, the library, or the community centre. Completed online therefore no need for a facilitator.

Control group details: participants in the control group also logged on to the website using a preassigned username and password. Every week for 8 weeks, adolescents received general health information that was not tailored, adapted from the American Academy of Pediatrics, the CDC, and the American Heart Association, related to nutrition, dental care, safety, common dermatology care, and risk-taking behaviours using similar format as the intervention group (text, graphics, comics, and voice-over). Parents also received three internet sessions related to general information on the topics taught in the control group. Information was presented in English to the adolescents and in English and Chinese to the parents. Each lessons lasted for about 15 min.

Diet and PA combined intervention vs control

Outcome measures
- Primary: BMI
- Secondary: waist-to-hip ratio, blood pressure, PA, food intake, diet and PA knowledge, diet and PA
self-efficacy
Process evaluation: NR (NB log-on rate)

Implementation-related factors
Theoretical basis: TTM-Stages of Change and SCT

Resources for intervention implementation: NR

Who delivered the intervention: reported - internet-based

PROGRESS categories assessed at baseline: child: gender; parent: race/ethnicity (acculturation) education, occupation, SES (family income)

PROGRESS categories analysed at outcome: NR

Outcomes relating to harms/unintended effects: NR

Intervention included strategies to address diversity or disadvantage: NR

Economic evaluation: NR
Funding: this publication was made possible by grant number KL2 RR024130 to J.L.C. from the National Center for Research Resources, a component of the NIH and NIH Road map for medical research, Hellman research grant, and in part by NIH grant DK060617 to M.B.H.

No details provided relating to costs of intervention and resources but authors reported it is relatively low cost intervention because it is internet-based

\section{Risk of bias}

\begin{tabular}{lll}
\hline Bias & Authors' judgement & Support for judgement \\
\hline $\begin{array}{l}\text { Random sequence genera- } \\
\text { tion (selection bias) }\end{array}$ & Low risk & Computer- generated random assignment \\
\hline $\begin{array}{l}\text { Allocation concealment } \\
\text { (selection bias) }\end{array}$ & High risk & $\begin{array}{l}\text { Convenience sampling used prior to randomisation; site co-ordinators helped } \\
\text { to identify eligible participants, introducing possibility of bias }\end{array}$ \\
\hline $\begin{array}{l}\text { Blinding (performance } \\
\text { bias and detection bias) } \\
\text { All outcomes }\end{array}$ & Unclear risk & NR \\
\hline
\end{tabular}


Chen 2011 (Continued)

\begin{tabular}{|c|c|c|}
\hline $\begin{array}{l}\text { Incomplete outcome data } \\
\text { (attrition bias) } \\
\text { All outcomes }\end{array}$ & Low risk & $\begin{array}{l}\text { Total loss }<10 \% ; 11 \% \text { from control and } 3 \% \text { from intervention. No significant } \\
\text { differences were found in baseline variables between adolescents who provid- } \\
\text { ed follow-up data and adolescents who were lost to follow-up }\end{array}$ \\
\hline
\end{tabular}

Selective reporting (re- Unclear risk Protocol/trial registration documents were unavailable.
porting bias)

\begin{tabular}{|c|c|c|}
\hline Other bias & Low risk & No other apparent threats to validity \\
\hline
\end{tabular}

Christiansen 2013

$\begin{array}{ll}\text { Methods } & \text { Study design: cluster-RCT } \\ \text { Intervention period: } 2 \text { years } \\ \text { Follow-up period (post-intervention): nil } \\ \text { Differences in baseline characteristics: reported } \\ \text { Reliable outcomes: reported } \\ \text { Protection against contamination: NR } \\ \text { Unit of allocation: school } \\ \text { Unit of analysis: individual accounting for clustering }\end{array}$

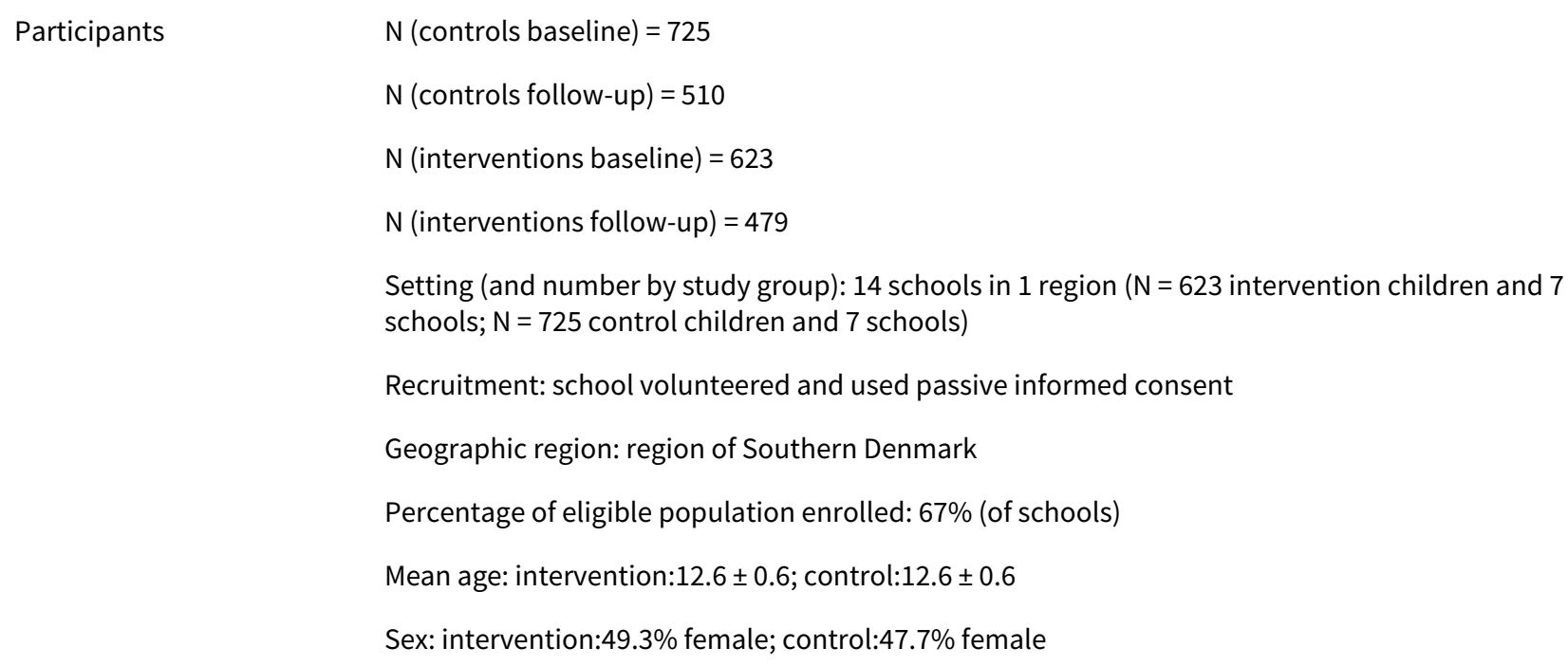

To evaluate the effect of an intervention targeting the physical and organisational school environment for noncurricular PA on adiposity, aerobic fitness, and musculoskeletal strength in Danish adolescents

Intervention was designed to change the organisational and physical environment of the school comprising 11 components, of which intervention schools were obliged and supported to implement as many components as possible, but full implementation was not required.

Physical environment changes included the following intervention components:

- upgrading the existing school outdoor area for PA including unfixed equipment

- developing and building specially designed playgrounds for adolescents (Playspots)

- improving safety for active transport to/from school 
Christiansen 2013 (Continued)

Organisational environment changes included the following:

- formulate and implement school PA policy

- school theme week once a year focusing on learning about and doing PA during school lessons

- teachers educated as "kickstarters" who facilitate and motivate PA during recess

- establish school's play patrol: older students were trained to initiate play and games for younger children during school's recess

- mandatory outdoor recess and/or free access to gym/sports hall

- school's traffic patrol: older students helped younger children cross the streets near the school

- students educated and trained in safe cycling

- establishing an after-school fitness programme

PE classes were not subject to intervention, but remained at the usual practice of 1.5 to $2 \mathrm{~h} /$ week at all schools (including control)

PA vs control

Outcomes Outcome measures

- Primary outcome: waist circumference, shuttle run, handgrip

- Secondary outcomes: NR

Process evaluation: reported (implementation)

Implementation-related Theoretical basis: Social Ecological framework

factors

Resources for intervention implementation: reported

Who delivered the intervention: reported

PROGRESS categories assessed at baseline: child: gender; parent: race/ethnicity, SES (household income)

PROGRESS categories analysed at outcome: NR

Outcomes relating to harms/unintended effects: NR

Intervention included strategies to address diversity or disadvantage: NR

Economic evaluation: reported

Funding: the SPACE study is a part of the Center for Intervention Research in Health Promotion and Disease Prevention. The SPACE-study is funded by TrygFonden.

All intervention schools upgraded their outdoor areas (10 000-20 000 $)$ and established Playspots (EUR 65,000-250,000). They also implemented PA policy, kickstarters, mandatory outdoor recess, and school theme week. The school's play patrol, school's traffic patrol, and cyclist education were already implemented if feasible at most schools, and did not directly change apart from being included in the school's PA policy. The improvement of cycling infrastructure was partly met in 2 schools, but lack of financial support made it impossible to implement in the remaining 5 schools. The organisation of the after-school fitness programme was implemented in 2 local areas, but lack of voluntary instructors made the component impossible in the other 5 areas. Interviews with school leaders after intervention revealed that all schools planned to continue the organisational components of the interventions, but with minor adjustments especially to the mandatory outdoor recess

\section{Risk of bias}

Bias Authors' judgement Support for judgement


Christiansen 2013 (Continued)

Random sequence genera- Unclear risk Schools matched in pairs and randomised one by one by drawing school tion (selection bias) names from a bag

\begin{tabular}{lll}
\hline $\begin{array}{l}\text { Allocation concealment } \\
\text { (selection bias) }\end{array}$ & Unclear risk & NR \\
\hline $\begin{array}{l}\text { Blinding (performance } \\
\text { bias and detection bias) }\end{array}$ & Unclear risk & NR \\
All outcomes & & \\
\hline
\end{tabular}

Incomplete outcome data Low risk

(attrition bias)

All outcomes

\begin{abstract}
$<30 \%$ loss to follow-up, and comparisons of those lost from intervention to those lost from control showed no difference. In total, those lost to follow-up were significantly older, lower SES (household income $<50 \%$ of the median income), larger waist circumference and shorter shuttle run. Outcomes were adjusted for age, sex and corresponding baseline value. Even though relatively more comparison group students were lost to follow-up ( $29.7 \%$ vs $23.1 \%)$, there were no significant differences between lost to follow-up students by intervention on the outcome, demographic, or active behaviour measures (data not shown).
\end{abstract}

\begin{tabular}{lll}
\hline $\begin{array}{l}\text { Selective reporting (re- } \\
\text { porting bias) }\end{array}$ & Low risk & Protocol seen. All pre-specified outcomes from protocol paper were reported \\
\hline Other bias & Low risk & No other apparent threats to validity \\
\hline $\begin{array}{l}\text { Other bias- timing of re- } \\
\text { cruitment of clusters }\end{array}$ & Low risk & Figure shows recruitment happened prior to randomisation \\
\hline
\end{tabular}

Coleman 2005

\begin{tabular}{ll}
\hline Methods & Study design: cluster-RCT \\
Intervention period: 4 years \\
Follow-up period (post-intervention): nil \\
Differences in baseline characteristics: reported \\
Reliable outcomes: reported \\
Protection against contamination: NR \\
Unit of allocation: school \\
Unit of analysis: school \\
All analyses were performed according to ITT principles \\
N (controls baseline) $=473$ \\
N (interventions baseline) $=423$ \\
N (interventions follow-up) $=744$ \\
Setting (and number by study group): 8 schools ( $\mathrm{N}=4$ intervention; $\mathrm{N}=4$ control) \\
Recruitment: intervention schools chosen randomly from schools that had applied to participate in the \\
programme in 1999. Control schools matched by district and geographic location. All children in 3rd \\
grade invited to participate
\end{tabular}


Percentage of eligible population enrolled: $94 \%$

Mean age: intervention: $8.3 \pm 0.5$ years (boys), $8.2 \pm 0.45$ years (girls); control: $8.3 \pm 0.5$ years (boys), $8.3 \pm$ 0.5 years (girls)

Sex: intervention: $47 \%$ female; control: $47 \%$ female

Interventions

Intervention schools: received money (USD 3500 in 1st year, USD 2500 in 2nd year, USD 1500 for 3rd year and USD 1000 for 4th year) for purchasing equipment and paying substitutes so that PE teachers and food service staff could attend training, and for promotion of $\mathrm{CATCH}$ programme at each school. Classroom materials were also subsidised (CATCH PE guidebook, PE activity box for grades 3 through 5, curriculum material for grades 3 through 5 and the EATSMART manual).

Control schools: did not receive any of the El Paso CATCH programme materials and did not attend any training for the programme. Received USD 1000 at the start of each school year to encourage participation

Also received some data i.e. at start of 4 th grade, the 3 rd grade summary results were provided to both intervention and control schools

Combined effects of dietary interventions and PA interventions vs control

- Risk of overweight or overweight
- Anthropometry (height, weight, waist to hip ratio, BMI)
- Aerobic fitness
- PE outcomes (time spent in moderate PA (goal $\geq 50 \%)$, time spent in vigorous PA (goal $\geq 20 \%)$ )
- Cafeteria outcomes (fat in school lunches $\geq 30 \%$ ), sodium in school lunches (goal $=600-1000 \mathrm{mg})$ )
Process evaluation: reported

Implementation-related factors
Theoretical basis: NR

Resources for intervention implementation (e.g. funding needed or staff hours required): reported Who delivered the intervention: reported

PROGRESS categories assessed at baseline: reported (race, gender, SES)

PROGRESS categories analysed at outcome: reported (gender)

Outcomes relating to harms/unintended effects: NR

Intervention included strategies to address diversity or disadvantage: reported

Economic evaluation: NR

Notes

Funding: this work was funded by the Patient Care and Outcomes Research Grant program from the American Heart Association, Dallas, Tex (9970182N)

\section{Risk of bias}

Bias Authors' judgement Support for judgement

Random sequence genera- High risk tion (selection bias)

"Participant schools were chosen randomly from those schools that had completed an application to participate" in CATCH programme. Not clear how this was done. Control schools matched and assigned, probably not using randomly generated sequence. Study authors describe design as quasi-experimental

\begin{tabular}{|c|c|c|}
\hline $\begin{array}{l}\text { Allocation concealment } \\
\text { (selection bias) }\end{array}$ & Unclear risk & $\begin{array}{l}\text { Allocation may have been concealed but it is not clear. There was cluster allo- } \\
\text { cation. Control schools were first matched to these schools primarily by dis- }\end{array}$ \\
\hline
\end{tabular}




\begin{tabular}{lll}
\hline $\begin{array}{l}\text { Blinding (performance } \\
\text { bias and detection bias) } \\
\text { All outcomes }\end{array}$ & High risk & Blinding probably not carried out for participants or outcome assessors \\
\hline $\begin{array}{l}\text { Incomplete outcome data } \\
\text { (attrition bias) } \\
\text { All outcomes }\end{array}$ & Low risk & ITT analysis conducted \\
\hline $\begin{array}{l}\text { Selective reporting (re- } \\
\text { porting bias) }\end{array}$ & High risk & $\begin{array}{l}\text { Protocol/trial registration documents were unavailable. Incomplete reporting } \\
\text { of outcome data. No anthropometry data at endpoint (study authors state no } \\
\text { effect but no data provided) }\end{array}$ \\
\hline $\begin{array}{l}\text { Other bias } \\
\begin{array}{l}\text { Other bias- timing of re- } \\
\text { cruitment of clusters }\end{array}\end{array}$ Low risk & No other threats to validity \\
\hline
\end{tabular}

Coleman 2012

\begin{tabular}{|c|c|}
\hline \multirow[t]{8}{*}{ Methods } & Study design: cluster-RCT \\
\hline & Intervention period: 2 years (and 1 baseline year) \\
\hline & Follow-up period (post-intervention): nil \\
\hline & Differences in baseline characteristics: reported \\
\hline & Reliable outcomes: reported \\
\hline & Protection against contamination: NR \\
\hline & Unit of allocation: school \\
\hline & Unit of analysis: individual accounting for clustering \\
\hline \multirow[t]{10}{*}{ Participants } & $\mathrm{N}($ controls baseline $)=300$ \\
\hline & $\mathrm{N}$ (controls follow-up) $=216$ \\
\hline & N (interventions baseline) $=279$ \\
\hline & $\mathrm{N}$ (interventions follow-up) = 208 \\
\hline & $\begin{array}{l}\text { Setting (and number by study group): } 6 \text { elementary and } 2 \text { middle schools ( } N=3 \text { elementary and } 1 \text { mid- } \\
\text { dle schools, } N=3 \text { elementary and } 1 \text { middle schools) }\end{array}$ \\
\hline & Recruitment: all schools agreed to participate \\
\hline & Geographic region: low-income school district, South Carolina, USA \\
\hline & Percentage of eligible population enrolled: $69 \%$ in elementary schools; $63 \%$ in middle schools \\
\hline & Mean age: intervention + control: $8.9 \pm 1.6$ \\
\hline & Sex: intervention + control: $57 \%$ female \\
\hline Interventions & $\begin{array}{l}\text { The 'Healthy Options for Nutrition Environments in Schools' (Healthy ONES) study is an evidence-based } \\
\text { public health (EBPH) randomised group trial that adapted the Institute for Healthcare Improvement's }\end{array}$ \\
\hline
\end{tabular}


(IHI) rapid improvement process model to implement school nutrition policy and environmental change.

The multilevel intervention was implemented with the following 4 steps

- Step 1: recruit stakeholders (advisory board and monitoring teams in intervention schools)

- Step 2: gauge organisational readiness/conduct environmental audit (during baseline year)

- Step 3: engage stakeholders to create strategy for change (at the end of the baseline year)

- Step 4: intervention implementation via PDSA (plan, do, study, act) learning cycles

Intervention goals were to:

- eliminate unhealthy foods and beverages on campus

- develop nutrition services as the main source on campus for healthful eating

- promote school-staff modelling of healthful eating

Providers were advisory board, change team, research team and teachers

Diet vs control

Outcome measures
- Primary outcome: zBMI, percentage overweight/obesity
- Secondary outcomes: outside food and beverage items
Process evaluation: reported (the intervention focuses on process of implementation)

Implementation-related

Theoretical basis: Ecological and Developmental Systems Theories and BEM

factors

Resources for intervention implementation: reported

Who delivered the intervention: reported

PROGRESS categories assessed at baseline: child: gender, race/ethnicity

PROGRESS categories analysed at outcome: NR

Outcomes relating to harms/unintended effects: NR

Intervention included strategies to address diversity or disadvantage: targeted low-income school district

Economic evaluation: NR

Notes

Funding: funding for this study was provided by the United States Department of Agriculture (USDA) National Research Initiative (NRI) award \#2007-5521505323 / (2007-55215-18241).

Participants: $43 \%$ were overweight or obese and $25 \%$ were obese with an average $\mathrm{zBMI}$ of $0.77 \pm 1.06$.

Healthy ONES provided a process for implementing environment and policy change with existing staff and required substitution rather than addition of activities; relatively low cost

\section{Risk of bias}

\begin{tabular}{lll}
\hline Bias & Authors' judgement & Support for judgement \\
\hline $\begin{array}{l}\text { Random sequence genera- } \\
\text { tion (selection bias) }\end{array}$ & Unclear risk & $\begin{array}{l}\text { Elementary schools matched by location and size and all school randomised, } \\
\text { no other details }\end{array}$ \\
\hline $\begin{array}{l}\text { Allocation concealment } \\
\text { (selection bias) }\end{array}$ & High risk & $\begin{array}{l}\text { The assignment of schools was done by the first study author. Interven- } \\
\text { tion-group children had significantly higher zBMls at baseline than con- }\end{array}$ \\
\hline
\end{tabular}


trol-group children. Children who had measures for all time points had significantly higher zBMIs and rates of overweight or obesity at baseline when compared to children who did not have measures for all time points.

\begin{tabular}{|c|c|c|}
\hline $\begin{array}{l}\text { Blinding (performance } \\
\text { bias and detection bias) } \\
\text { All outcomes }\end{array}$ & High risk & $\begin{array}{l}\text { Both the intervention and measurement of outcomes were conducted by the } \\
\text { same people who were not blinded to condition. }\end{array}$ \\
\hline
\end{tabular}

\begin{tabular}{lll}
\hline $\begin{array}{l}\text { Incomplete outcome data } \\
\text { (attrition bias) } \\
\text { All outcomes }\end{array}$ & Low risk & 27\% attrition, balanced. ITT done \\
\hline $\begin{array}{l}\text { Selective reporting (re- } \\
\text { porting bias) }\end{array}$ & Low risk & $\begin{array}{l}\text { Protocol/trial registration documents were unavailable. zBMI data and \% over- } \\
\text { weight/obese only reported in text despite these being the primary outcome } \\
\text { measures (non-significant). }\end{array}$ \\
\hline $\begin{array}{ll}\text { Other bias } & \text { Low risk }\end{array}$ & Figure shows recruitment happened prior to randomisation \\
\hline $\begin{array}{l}\text { Other bias- timing of re- } \\
\text { cruitment of clusters }\end{array}$ & Low risk & \\
\hline
\end{tabular}

\title{
Crespo 2012
}

Methods

Study design: cluster-RCT

Intervention period: 1 year

Follow-up period (post-intervention): 2 years

Differences in baseline characteristics: reported

Reliable outcomes: reported (for weight)

Protection against contamination: NR

Unit of allocation: parent-child dyads

Unit of analysis: individual accounting for clustering

\section{Participants}

\author{
$\mathrm{N}($ controls baseline $)=227$ \\ $\mathrm{N}$ (controls follow-up) $=134$ \\ Family + community N (interventions baseline) $=165$ \\ Family + community $\mathrm{N}$ (interventions follow-up) $=83$ \\ Family only $\mathrm{N}$ (interventions baseline) $=198$ \\ Family only N (interventions follow-up) $=96$ \\ Community only $\mathrm{N}$ (interventions baseline) $=218$ \\ Community only $\mathrm{N}$ (interventions follow-up) $=128$ \\ Setting (and number by study group): 13 elementary schools ( $N=3$ schools in each group, 808 dyads) \\ Recruitment: parents were recruited directly on school grounds, during school presentations, and \\ through fliers sent home with students \\ Geographic region: South Bay region of San Diego County, adjacent to US-Mexico Border
}


Mean age: intervention + control: $5.9 \pm 0.9$

Sex: intervention + control: $50 \%$ female

Interventions

To evaluate the impact of a multi-level promotora-based (Community Health Advisor) intervention to promote healthy eating and PA and prevent excess weight gain among Latino children

- Family-only

- promotoras discussed with participants ways to overcome barriers to healthy eating and PA, ways to prepare healthy meals in the home, benefits of promoting healthy eating and PA in their children (e.g. behavioural benefits), ways to set appropriate goals for the family and monitor healthy eating in the home, and modelling healthy eating.

- 1 home visit/month for 7 months (over 1 school year)

- Community-only

- School playgrounds (improvements) and salad bars (implementation and improvement); community parks (improvements); restaurant health child menus,

- Posters, newsletters, frequent produce buyer cards in grocery stores.

- 3 years

- Family + community

- Combined modifying home (parenting) and community (school, park, and food retail) environments - see above

- Measurement-only control

Diet and PA combined intervention vs control

Outcomes Outcome measures

- Primary outcome: zBMI scores, BMI percentile, percentage overweight ( $\geq 85$ th, 95 th percentile) percentage obesity ( $\geq 95$ th percentile weight for age)

- Secondary outcomes: dietary intake, physical activity, sports participation, TV viewing

Process evaluation: reported (implementation)

Implementation-related factors
Theoretical basis: SCT, HBM resources for intervention implementation: reported

Who delivered the intervention: reported

PROGRESS categories assessed at baseline: child: gender, race/ethnicity; parent: race/ethnicity, education

PROGRESS categories analysed at outcome: child, gender

Outcomes relating to harms/unintended effects: NR

Intervention included strategies to address diversity or disadvantage: culturally tailored, i.e. bilingual and bicultural evaluation assistants

Economic evaluation: NR

Notes

Funding: the Aventuras para Niños study was funded by the National Heart, Lung and Blood Institute (5R01HL073776). Additional support was provided to Dr. Elder and Dr. Ayala by the CDC (5U48DP000036), to Dr. Ayala by the American Cancer Society (RSGPB 113653), to Dr. Arredondo by the American Cancer Society (PFT-04-156-01), and to Dr. Crespo by the National Institute of Diabetes and Digestive and Kidney Diseases (F31DK079345) and the National Heart, Lung and Blood Institute (T32HL079891).

Intervention groups differed in length and intensity

\section{Risk of bias}


Crespo 2012 (Continued)

\begin{tabular}{|c|c|c|}
\hline Bias & Authors' judgement & Support for judgement \\
\hline $\begin{array}{l}\text { Random sequence genera- } \\
\text { tion (selection bias) }\end{array}$ & Unclear risk & $2 \times 2$ factorial design, randomised design, no further details \\
\hline $\begin{array}{l}\text { Allocation concealment } \\
\text { (selection bias) }\end{array}$ & High risk & NR \\
\hline $\begin{array}{l}\text { Blinding (performance } \\
\text { bias and detection bias) } \\
\text { All outcomes }\end{array}$ & Low risk & $\begin{array}{l}\text { Measurement staff were blinded to participants' study condition. Behavioural } \\
\text { measures were self-report }\end{array}$ \\
\hline $\begin{array}{l}\text { Incomplete outcome data } \\
\text { (attrition bias) } \\
\text { All outcomes }\end{array}$ & High risk & $\begin{array}{l}\text { 41\%-52\% attrition impacted on power to detect effects, although dropout sta- } \\
\text { tus was not significant in the analyses models. ITT done. } \\
\text { Quote: "All available data were utilized. Thus, although a participant may have } \\
\text { data missing at M2, M3, or M4, data available at non-missing time points were } \\
\text { still included in the analysis." }\end{array}$ \\
\hline $\begin{array}{l}\text { Selective reporting (re- } \\
\text { porting bias) }\end{array}$ & Unclear risk & Protocol/trial registration documents were unavailable. \\
\hline Other bias & Low risk & No other potential threats to validity \\
\hline $\begin{array}{l}\text { Other bias- timing of re- } \\
\text { cruitment of clusters }\end{array}$ & Low risk & Figure shows recruitment happened prior to randomisation. \\
\hline
\end{tabular}

Cunha 2013

\begin{tabular}{|c|c|}
\hline \multirow[t]{8}{*}{ Methods } & Study design: cluster-RCT \\
\hline & Intervention period: 9 months \\
\hline & Follow-up period (post-intervention): nil \\
\hline & Differences in baseline characteristics: reported \\
\hline & Reliable outcomes: reported (for BMI) \\
\hline & Protection against contamination: NR \\
\hline & Unit of allocation: class \\
\hline & Unit of analysis: individual accounting for clustering \\
\hline \multirow[t]{7}{*}{ Participants } & $\mathrm{N}($ controls baseline $)=281$ \\
\hline & $\mathrm{N}$ (controls follow-up) $=282$ \\
\hline & $N$ (interventions baseline) $=293$ \\
\hline & $\mathrm{N}$ (interventions follow-up) $=277$ \\
\hline & Setting (and number by study group): 20 classes in 20 schools \\
\hline & $\begin{array}{l}\text { ( } N=20 \text { classes, } 1 \text { class in each school, } N=10 \text { intervention classes and } 293 \text { participants and } N=10 \text { con- } \\
\text { trol classes and } 281 \text { participants) }\end{array}$ \\
\hline & Recruitment: selected 20 schools from 35, no further details \\
\hline
\end{tabular}


Cunha 2013 (Continued)

Geographic region: municipality of Duque de Caxias, Rio de Janeiro, Brazil

Percentage of eligible population enrolled: $98 \%$

Mean age: intervention: $11.2 \pm 1.3$; control: $11.2 \pm 1.3$

Sex: intervention: $47.7 \%$ female; control: $48.6 \%$ female
To evaluate the effectiveness of an intervention involving families and teachers to prevent excessive weight gain among adolescents in Brazil

Students attended 9 nutritional education sessions (1/month for 9 months) during the 2010 academic year provided by external trained nutritionists.

Encouraging students to change their eating habits and food consumption via trained nutritionists giving monthly 1-h sessions in the classrooms on the following themes:

- healthy eating

- native Brazilian eating habits

- excessive sugar in processed food

- marriage of the rice and beans

- the beauty of fruits

- super water: a super-hero

- cookies

- mini-market

- food advertisements

Each session included:

- activities, related to the subject, to be conducted at the school

- folders explaining the intervention programme and suggesting the participation of the family, such as reducing purchase of sodas and increasing the purchase of fruit, to be sent home

- strategies for reinforcement of themes by the teachers, using exercises prepared for this purpose, such as specific popular histories or maths games

- a set of messages sent to families in the form of illustrated booklets and recipes.

Parents/guardians and teachers received information on the same subjects.

The control group received a 1-hour section of orientation on general health and advice on eating, at the end of the study

Diet intervention vs control

- Primary outcome: BMI

- Secondary outcomes: body fat, percentage overweight/obese, dietary intake

Process evaluation: reported (compliance)

Implementation-related factors
Theoretical basis: TTM

Resources for intervention implementation: reported

Who delivered the intervention: reported

PROGRESS categories assessed at baseline: child: race/ethnicity

PROGRESS categories analysed at outcome: NR

Outcomes relating to harms/unintended effects: NR 
Cunha 2013 (Continued)

Intervention included strategies to address diversity or disadvantage: NR (area selected is one of the poorest in Brazil)

Economic evaluation: NR

Notes

\section{NCT01046474}

Funding: this work was supported by Foundation of Support of Research of the State of Rio de Janeiro - FAPERJ (E261029422008); National Counsel of Technological and Scientific Development - CNPQ (474288/2009-9); Pan American Health and Education Foundation - PAHEF. The funders had no role in study design, data collection and analysis, decision to publish, or preparation of the manuscript. $14 \%$ of final sample were participants who entered the study after random allocation; schools selected that were in low violence areas.

\section{Risk of bias}

\begin{tabular}{|c|c|c|}
\hline Bias & Authors' judgement & Support for judgement \\
\hline $\begin{array}{l}\text { Random sequence genera- } \\
\text { tion (selection bias) }\end{array}$ & Unclear risk & $\begin{array}{l}\text { Each pair in the ranking sequence was randomly drafted with } 1 \text { class being as- } \\
\text { signed to the experimental group and } 1 \text { to the control group. Randomisation } \\
\text { process was conducted by the investigators. }\end{array}$ \\
\hline $\begin{array}{l}\text { Allocation concealment } \\
\text { (selection bias) }\end{array}$ & Low risk & Opaque envelopes \\
\hline $\begin{array}{l}\text { Blinding (performance } \\
\text { bias and detection bias) } \\
\text { All outcomes }\end{array}$ & High risk & NR \\
\hline $\begin{array}{l}\text { Incomplete outcome data } \\
\text { (attrition bias) } \\
\text { All outcomes }\end{array}$ & Unclear risk & $\begin{array}{l}\text { Very low attrition }(<5 \%) \text { however } 14 \% \text { of final sample were participants who } \\
\text { entered the study after random allocation }\end{array}$ \\
\hline $\begin{array}{l}\text { Selective reporting (re- } \\
\text { porting bias) }\end{array}$ & Low risk & Protocol/trial registration document seen. All outcomes reported \\
\hline Other bias & Low risk & \\
\hline $\begin{array}{l}\text { Other bias- timing of re- } \\
\text { cruitment of clusters }\end{array}$ & Unclear risk & $\begin{array}{l}\text { Figure shows } 14 \% \text { of final sample were participants who entered the study af- } \\
\text { ter random allocation }\end{array}$ \\
\hline
\end{tabular}

\section{Damsgaard 2014}

\section{Methods}

Study name: The optimal well-being, development and health for Danish children through a healthy new Nordic diet (OPUS) school meal study

Study design: cluster-RCT - cross-over

Intervention period: 3 months

Follow-up period (post-intervention): 3

Differences in baseline characteristics: reported

Reliable outcomes: reported

Protection against contamination: reported

Unit of allocation: school 
Damsgaard 2014 (Continued)

Unit of analysis: individual accounting for clustering

\begin{tabular}{|c|c|}
\hline \multirow[t]{8}{*}{ Participants } & $\mathrm{N}$ (intervention + controls baseline $)=823$ \\
\hline & $\mathrm{N}$ (intervention + controls follow-up) $=613-733$ \\
\hline & $\begin{array}{l}\text { Setting (and number by study group): } 46 \text { classes in } 9 \text { schools ( } 4-8 \text { classes pe } \\
\text { class in each school, } N=10 \text { intervention classes and } 293 \text { participants and } N \\
\text { participants) }\end{array}$ \\
\hline & Recruitment: schools were recruited by telephone and email \\
\hline & Geographic region: eastern part of Denmark (Zealand and Lolland-Falster) \\
\hline & Percentage of eligible population enrolled: $32 \%$ schools; $82 \%$ participants \\
\hline & Mean age: intervention + control: $10.0 \pm 0.6$ \\
\hline & Sex: intervention + control: $48 \%$ female \\
\hline
\end{tabular}

Interventions

To assess the impact of introducing a nutritionally balanced full school meal programme (new Nordic diet - NND) on the overall cardiometabolic profile

Children aged 8-11 years received freshly prepared school lunch and snacks or usual packed lunch from home (control) each for 3 months. 3-month cross-over trial ( 3 months intervention then 3 months control and vice versa)

During the 3-month NND period, the children were served a mid-morning snack, an ad libitum hot lunch meal and an afternoon snack, and twice a week dessert was served, consisting either of fresh fruit or of a fruit-based snack. The lunch meals and snacks were designed according to the NND guidelines, which are based on seasonal, local Nordic ingredients. The intention was that the NND should contain less meat and more berries, cabbage, root vegetables, legumes, potatoes, wild plants, whole grains, nuts, fish and seaweed than the average Danish diet. The school meals were designed to cover $40 \%-45 \%$ of the daily energy requirement of an 11 -year-old boy.

School lunches were served buffet style, and neither total energy intake nor the intakes of specific food groups were strictly controlled. However, children were encouraged to taste everything and to keep a reasonable plate distribution where vegetables and potatoes/grains constituted most of the plate. The NND school meals were free of charge, children cooked, tasted and served the food, and the $15 \mathrm{~min}$ usually set aside for lunch were increased to 20-25 $\mathrm{min}$. The menus for OPUS school meal study were developed by chefs with feedback from nutrition scientists from the Division of Nutrition, The Technical University of Denmark. The meals were produced locally at each school by trained chefs and kitchen personnel hired for the study.

Control group usually had a home-packed lunch, typically consisting of cold open-faced rye bread sandwiches with meat topping and some fresh fruits, which were consumed during the usual lunch break.

Diet intervention vs control

Outcomes Outcome measures

- Primary outcome: METs score

- Secondary outcomes: cardiometabolic markers (blood pressure, arterial pressure, heart rate, cholesterol, plasma triglycerols, HOMA-IR), and body composition (waist circumference, zBMI, fat mass, fatfree mass, android:total fat mass), dietary intakes

Process evaluation: NR

Implementation-related factors
Theoretical basis: NR

Resources for intervention implementation: reported that cost of programme and sample of amount of food waste was measured but results NR 
Who delivered the intervention: reported

PROGRESS categories assessed at baseline: child: race/ethnicity; parent: education

PROGRESS categories analysed at outcome: NR

Outcomes relating to harms/unintended effects: NR

Intervention included strategies to address diversity or disadvantage: NR

Economic evaluation: NR

\section{Notes}

Funding: Nordea Foundation grant no. 02-2010-0389; Danæg A/S, Naturmælk, Lantmännen A/S, Skærtoft Mølle A/S, Kartoffelpartnerskabet, AkzoNobel Danmark, Gloria Mundi and Rose Poultry A/S provided foods in kind for the study. The Nordea Foundation and the food sponsors had no role in the design and analysis of the study or in the writing of this article. Nordea foundation are a grant-awarding trust from a bank.

Cross-over trial

\section{Risk of bias}

\begin{tabular}{lll}
\hline Bias & Authors' judgement & Support for judgement \\
\hline $\begin{array}{l}\text { Random sequence genera- } \\
\text { tion (selection bias) }\end{array}$ & Low risk & $\begin{array}{l}\text { Quote: "The nine schools were randomly assigned to the order in which the } \\
\text { classes would receive NND or control by use of R statistical software (www.r- } \\
\text { project.org). Randomisation was performed in clusters corresponding to year } \\
\text { group, so that all 3rd-grade classes at a particular school received the NND and } \\
\text { control in the same order, and the 4th-grade classes at that school received } \\
\text { the NND and control in the opposite order." }\end{array}$ \\
&
\end{tabular}

Allocation concealment High risk (selection bias)
Quote: "Randomisation was done by a statistician not involved in data collection or analysis and, for logistical reasons, before the children were invited to participate in the study."

Outcomes adjusted for sex and baseline values but non-completers less likely to be of high educational background and more likely to be immigrants/descendants and not clear if there were statistically significant differences between groups that were not adjusted for in the analyses - baseline characteristic presented for total study population only; also number of participants per group per outcome were reported

\begin{tabular}{|c|c|c|}
\hline $\begin{array}{l}\text { Blinding (performance } \\
\text { bias and detection bias) } \\
\text { All outcomes }\end{array}$ & High risk & Not blinded \\
\hline $\begin{array}{l}\text { Incomplete outcome data } \\
\text { (attrition bias) } \\
\text { All outcomes }\end{array}$ & Unclear risk & $\begin{array}{l}\text { Group-wise loss }(\mathrm{N}) \text { and reasons NR. Dropout is } 30 \% \text { if we take it from clus- } \\
\text { ter-randomisation to the final measurement but clusters were not lost. ITT was } \\
\text { done with imputations and these tested in sensitivity analyses. }\end{array}$ \\
\hline $\begin{array}{l}\text { Selective reporting (re- } \\
\text { porting bias) }\end{array}$ & Low risk & $\begin{array}{l}\text { Protocol and registry data compared with results paper. All outcomes report- } \\
\text { ed }\end{array}$ \\
\hline Other bias & Unclear risk & $\begin{array}{l}\text { Outcomes adjusted for sex and baseline values but non-completers less like- } \\
\text { ly to be of high educational background and more likely to be immigrants/de- } \\
\text { scendants and not clear if there were statistically significant differences be- } \\
\text { tween groups that were not adjusted for in the analyses - baseline characteris- } \\
\text { tic presented for total study population only; also number of participants per } \\
\text { group per outcome were reported }\end{array}$ \\
\hline
\end{tabular}


Damsgaard 2014 (Continued)

Other bias- timing of re- High risk cruitment of clusters
Quote: "Randomisation was done by a statistician not involved in data collection or analysis and, for logistical reasons, before the children were invited to participate in the study"

Daniels 2012

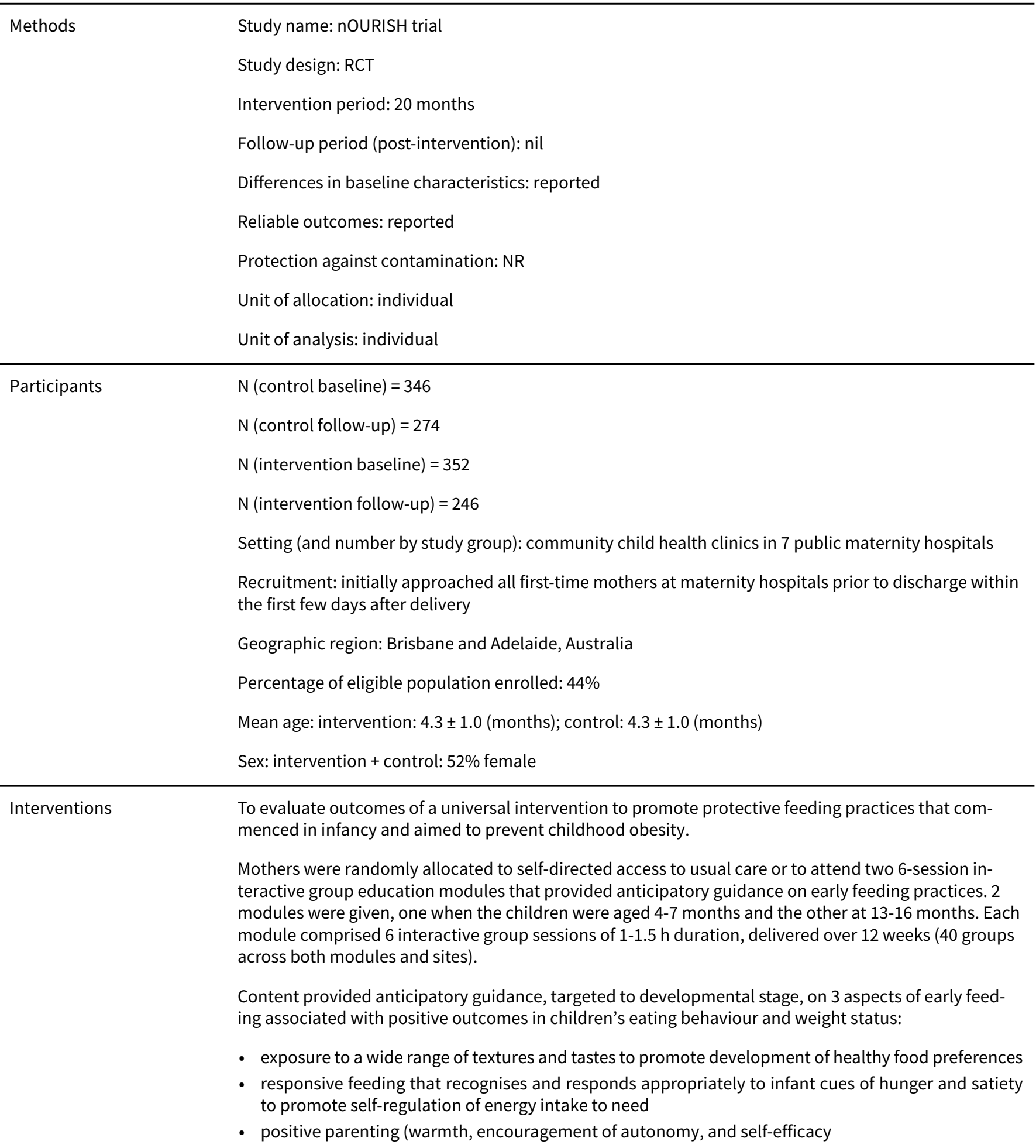


Daniels 2012 (Continued)

+ written material was given summarising every session.

Sessions were co-facilitated by a dietitian $(N=13)$ and a psychologist $(N=13)$

NOTE: content as presented to mothers focused on healthy eating patterns and growth, rather than obesity prevention. Mothers participating in the 2 nd intervention module were offered onsite child care provided by adjunct care providers.

The control group had standard access to universal community child health services

Diet intervention vs control

Outcome measures
- Primary outcome: maternal feeding practices and child-feeding strategies
- Secondary outcomes: weight, weight z score, length/height, length/height z score, BMI, zBMI
Process evaluation: reported (attendance)

Implementation-related factors
Theoretical basis: Attachment theory, Anticipatory Guidance and a Social Cognitive approach

Resources for intervention implementation: reported

Who delivered the intervention: reported

PROGRESS categories assessed at baseline: parent: race/ethnicity, education, SES

PROGRESS categories analysed at outcome: NR

Outcomes relating to harms/unintended effects: NR

Intervention included strategies to address diversity or disadvantage: NR

Economic evaluation: NR

Notes

Funding: nOURISH was funded from 2008-2010 by the Australian National Health and Medical Research Council (grant 426704). Additional funding was provided by HJ Heinz (postdoctoral fellowship, Dr Mallan), Meat \& Livestock Australia, Department of Health South Australia, Food Standards Australia New Zealand, Queensland University of Technology, and National Health and Medical Research Council Career Development Award (390136, Dr Nicholson).

Attendance At $>2$ sessions for module 1 was $N=229$ (65\%) and module 2 was $N=130$ (45\% of those retained at module commencement).

Study ongoing and details of results when infant aged 3.5 and 5 years also to be reported.

A separate paper (Daniels 2012) reports outcomes at 6 months post baseline, i.e. after the first of 2 intervention modules

\section{Risk of bias}

\begin{tabular}{lll}
\hline Bias & Authors' judgement & Support for judgement \\
\hline $\begin{array}{l}\text { Random sequence genera- } \\
\text { tion (selection bias) }\end{array}$ & Unclear risk & $\begin{array}{l}\text { Randomly allocated according to a permuted-blocks randomisation sched- } \\
\text { ule generated by the Institute's Research Methods Group, which includes this } \\
\text { study's statistician, none of whom were involved in data collection or interven- } \\
\text { tion delivery. }\end{array}$ \\
& $\begin{array}{l}\text { Block sizes generated based on location of assessment clinic therefore possi- } \\
\text { ble element of selection bias. }\end{array}$
\end{tabular}


Daniels 2012 (Continued)

$\begin{array}{ll}\begin{array}{l}\text { Allocation concealment } \quad \text { Low risk } \\ \text { (selection bias) }\end{array} & \begin{array}{l}\text { Randomly allocated according to a permuted-blocks randomisation sched- } \\ \text { ule generated by the Institute's Research Methods Group, which includes this } \\ \text { study's statistician, none of whom were involved in data collection or interven- } \\ \text { tion delivery. }\end{array} \\ & \begin{array}{l}\text { Block sizes generated based on location of assessment clinic therefore possi- } \\ \text { ble element of selection bias. }\end{array}\end{array}$

Blinding (performance Low risk Outcome assessors blinded (participants not blinded)
bias and detection bias)
All outcomes

\begin{tabular}{lll}
\hline $\begin{array}{l}\text { Incomplete outcome data } \\
\text { (attrition bias) } \\
\text { All outcomes }\end{array}$ & High risk & $\begin{array}{l}\text { Total attrition was 22\%. Withdrawal was higher among younger and less-edu- } \\
\text { cated mothers and in the intervention group than in the control group. }\end{array}$ \\
\hline $\begin{array}{l}\text { Selective reporting (re- } \\
\text { porting bias) }\end{array}$ & Low risk & Protocol seen; all outcomes specified in methods were reported in results \\
\hline Other bias & Low risk & No additional threats to validity \\
\hline
\end{tabular}

\section{De Bock 2012}

$\begin{array}{ll}\text { Methods } & \text { Study design: cluster-RCT } \\ \text { Intervention period: } 6 \text { months } \\ \text { Follow-up period (post-intervention): nil } \\ \text { Differences in baseline characteristics: reported } \\ \text { Reliable outcomes: reported } \\ \text { Protection against contamination: NR } \\ \text { Unit of allocation: individual } \\ \text { Unit of analysis: individual }\end{array}$

Participants
$\begin{aligned} & N \text { (control baseline })=183 \\ & N(\text { control follow-up })=N R(N=202 \text { intervention + control at follow-up }) \\ & N(\text { intervention baseline })=194 \\ & N(\text { intervention follow-up })=N R\end{aligned}$

Setting (and number by study group): 18 preschools (10 preschools, $N=194$ children in intervention group; 8 preschools, $\mathrm{N}=183$ children in control group)

Recruitment: had applied to participate in the nutritional intervention module of a state-sponsored health promotion programme 'Komm mit in das gesunde Boot'

Geographic region: 3 areas of Baden-Württemberg in South West Germany

Percentage of eligible population enrolled: $78 \%$ of preschools, $80 \%$ participants

Mean age: intervention + control: $4.26 \pm 0.78$

Sex: intervention + control: $46.8 \%$ female 
De Bock 2012 (Continued)

Interventions
To assess the effects of a preschool-based nutritional intervention on both behavioural outcomes, like children's fruit, vegetable and water consumption, and anthropometric measures.

6-month intervention administered once weekly by a nutrition expert consisting of joint meal preparation and activities for children and parents such as tasting and preparing nutritious, fresh foods.

Fifteen 2-hour sessions once weekly over a period of 6 months. 10 modules only targeted children, another 5 parents and children, or parents exclusively, involving parents by targeting them alone (discussions on parents' modelling role and nutritional needs of children) or together with their children. Intervention activities consisted of familiarising with different food types and preparation methods as well as cooking and eating meals together in groups of children, teachers and parents. One session additionally focused on healthy drinking behaviours.

Models for healthy eating within the intervention include:

- use of nutrition experts

- play acting with 'pirate dolls' used as props enjoying fruit and vegetables

- active parental involvement

- involvement of other preschool peers

Waiting list control

Dlet intervention vs control

- Primary outcome: fruit and vegetable intake, ?PA

- Secondary outcomes: BMI, skinfold thickness, waist-to-height ratio, consumption of water and sugared drinks

Process evaluation: reported (fidelity)

Implementation-related factors
Theoretical basis: Social Learning theory and Exposure theory

Resources for intervention implementation: NR

Who delivered the intervention: reported

PROGRESS categories assessed at baseline: child: gender, race/ethnicity; parent: education

PROGRESS categories analysed at outcome: NR

Outcomes relating to harms/unintended effects: NR

Intervention included strategies to address diversity or disadvantage: NR

Economic evaluation: NR

Notes

Funding: this work was supported by a grant from the Baden-Württemberg Stiftung. F.D.B. is supported by the European Social Fund and by the Ministry of Science, Research and the Arts Baden-Württemberg.

This paper focuses on the nutritional intervention element but protocol reports that PA is a primary outcome.

On average, 23.1 (SD 12.1) children participated regularly in the lessons; 16.5 (SD 9.5) parents present at the parents'-only and parent and children's sessions. Reports that sustainability measurements not available from all participating preschools.

\section{Risk of bias}


De Bock 2012 (Continued)

Random sequence genera- Unclear risk Stratified the recruited preschools before randomisation to balance aggregate tion (selection bias) preschool social background and immigrant proportion

$\begin{aligned} & \text { Allocation concealment } \quad \text { Unclear risk } \\ & \text { (selection bias) }\end{aligned}$

(selection bias)

Blinding (performance Low risk Study personnel were blinded to group assignment
bias and detection bias)
All outcomes

Incomplete outcome data High risk $\quad 58 \%$ of the children provided both pre and post-intervention measurements
(attrition bias)

All outcomes

Selective reporting (re- Unclear risk Protocol/trial registration documents were unavailable porting bias)

\begin{tabular}{lll}
\hline Other bias & Low risk & No additional threats to validity \\
\hline $\begin{array}{l}\text { Other bias- timing of re- } \\
\text { cruitment of clusters }\end{array}$ & Low risk & Figure shows recruitment happened prior to randomisation \\
& $\begin{array}{l}\text { Quote: "we stratified the recruited pre-schools before randomization to bal- } \\
\text { ance aggregate pre-school social background and immigrant proportion" }\end{array}$ \\
\hline
\end{tabular}

De Coen 2012

\begin{tabular}{|c|c|}
\hline \multirow[t]{9}{*}{ Methods } & Study name: Prevention of overweight among pre-school and school children (POP) \\
\hline & Study design: cluster-RCT \\
\hline & Intervention period: 20 months \\
\hline & Follow-up period (post-intervention): nil \\
\hline & Differences in baseline characteristics: reported (data not shown) \\
\hline & Reliable outcomes: reported (for BMI) \\
\hline & Protection against contamination: NR \\
\hline & Unit of allocation: community \\
\hline & Unit of analysis: ?individual accounting for nesting within schools; also community level \\
\hline \multirow[t]{7}{*}{ Participants } & $\mathrm{N}$ (control baseline $)=557$ \\
\hline & $\mathrm{N}$ (control follow-up) $=442$ \\
\hline & $\mathrm{N}$ (intervention baseline) $=1032$ \\
\hline & $\mathrm{N}$ (intervention follow-up) $=670$ \\
\hline & $\begin{array}{l}\text { Setting (and number by study group): } 31 \text { pre/schools in } 6 \text { communities (local authority town or munici- } \\
\text { pality). } \mathrm{N}=3 \text { communities in each group including low-, medium- and high-SES. }\end{array}$ \\
\hline & $\begin{array}{l}\text { Recruitment: all pre-primary and primary schools in the } 6 \text { communities were invited to participate (vol- } \\
\text { untary) }\end{array}$ \\
\hline & Geographic region: Flanders, Belgium \\
\hline
\end{tabular}


De Coen 2012 (Continued)

Percentage of eligible population enrolled: $63 \%$ of schools, $49 \%$ participants

Mean age: intervention: $4.86 \pm 1.25$; control: $5.04 \pm 1.29$

Sex: intervention: $47.1 \%$ female; control: $54.7 \%$ female
To examine the effects of a 2-year multi-component intervention in local communities with different socio-economic characteristics on the prevention of overweight among 3-6-year-old children

Intervention focused on:

- increasing daily consumption of water and decreasing soft drinks consumption

- increasing daily milk consumption

- increasing daily consumption of vegetables and fruit

- decreasing daily consumption of sweets and savoury snacks

- increasing daily PA

- decreasing screen-time behaviour involved

Involved community, parents, regional Health Boards. School was the most important setting for the implementation of the intervention. All intervention schools were requested to implement 5 Healthy Weeks per intervention year ( 1 for each cluster of topics) with a minimum $1 \mathrm{~h}$ of classroom time dedicated to the topic together with extracurricular activities.

7 modules:

- the organisation of the POP project at school level

- the organisation of classroom activities (Healthy Weeks), including suggested dose and content

- development of an active playground

- implementation of health-related PE

- environmental and policy changes to increase the availability of water at school (e.g. water fountains)

- environmental and policy changes to increase to availability of vegetables and fruits at school

- educational strategies for parents on all topics

No details reported of control

Diet and PA combination intervention vs control

\section{Outcomes}

\section{Outcome measures}

- Primary outcome: zBMI

- Secondary outcomes: dietary intake, PA, screen time

Process evaluation: reported (implementation)

Implementation-related factors
Theoretical basis: SEM

Resources for intervention implementation: reported

Who delivered the intervention: reported

PROGRESS categories assessed at baseline: child: gender; parent: education

PROGRESS categories analysed at outcome: SES (maternal education)

Outcomes relating to harms/unintended effects: NR

Intervention included strategies to address diversity or disadvantage: NR

Economic evaluation: NR

Notes

Funding: the study was commissioned, financed and steered by the Ministry of the Flemish Community (Department of Economics, Science and Innovation; Department of Welfare, Public Health and Family) 
De Coen 2012 (Continued)

Teachers received EUR 250 from the research project to buy materials or finance environmental changes. Regional Health Boards received EUR 500 for their input in the project.

All schools implemented the requested classroom hour. Regarding the snack and playground policy, it was clear that the requested adjustments asked for more time investment and at the time of observation, most schools had not yet met the standard.

\section{Risk of bias}

\begin{tabular}{lll}
\hline Bias & Authors' judgement & Support for judgement \\
\hline $\begin{array}{l}\text { Random sequence genera- } \\
\text { tion (selection bias) }\end{array}$ & High risk & $\begin{array}{l}\text { From each pair of matched communities the researchers allocated 1 randomly } \\
\text { to the intervention condition. }\end{array}$ \\
\hline $\begin{array}{l}\text { Allocation concealment } \\
\text { (selection bias) }\end{array}$ & High risk & $\begin{array}{l}\text { From each pair of matched communities the researchers allocated 1 randomly } \\
\text { to the intervention condition. }\end{array}$ \\
\hline $\begin{array}{l}\text { Blinding (performance } \\
\text { bias and detection bias) }\end{array}$ & Unclear risk & $\begin{array}{l}\text { NR for researchers; schools were aware of the fact that they were in an inter- } \\
\text { vll outcomes } \\
\text { community. }\end{array}$ \\
\hline $\begin{array}{l}\text { Incomplete outcome data } \\
\begin{array}{l}\text { (attrition bias) } \\
\text { All outcomes }\end{array}\end{array}$ & Unclear risk & $\begin{array}{l}\text { 21\% dropout for intervention vs 35\% dropout for intervention group, across all } \\
\text { SES communities. Across the conditions, participants with a low SES dropped } \\
\text { out significantly more at the follow-up. }\end{array}$ \\
\hline $\begin{array}{l}\text { Selective reporting (re- } \\
\text { porting bias) }\end{array}$ & Low risk & $\begin{array}{l}\text { Protocol not sought; all outcomes specified in methods were reported in re- } \\
\text { sults }\end{array}$ \\
\hline $\begin{array}{l}\text { Other bias } \\
\text { Other bias- timing of re- } \\
\text { cruitment of clusters }\end{array}$ & Unclear risk & No additional threats to validity \\
\hline
\end{tabular}

\section{De Heer 2011}

$\begin{array}{ll}\text { Methods } & \text { Study design: cluster-RCT } \\ \text { Intervention period: } 12 \text { weeks } \\ \text { Follow-up period (post-intervention): nil } \\ \text { Differences in baseline characteristics: reported } \\ \text { Reliable outcomes: reported } \\ \text { Protection against contamination: NR } \\ \text { Unit of allocation: classroom } \\ \text { Unit of analysis: individual accounting for clustering }\end{array}$

$\begin{array}{ll}\text { Participants } & \text { N (controls baseline })=354 \\ & N(\text { controls follow-up })=326 \\ & \text { N (interventions basic baseline })=292 \\ & \text { N (interventions basic follow-up })=242\end{array}$


Recruitment: students were recruited by making announcements and passing out consent forms during PE classes

Geographic region: El Paso, Texas, USA

Percentage of eligible population enrolled: $53 \%$

Mean age: intervention: $9.24 \pm 0.87$; control: $9.10 \pm 1.08$

Sex: intervention, $45.9 \%$ female; control, $44.6 \%$ female

Interventions

The intervention was a 12-week culturally tailored after-school programme meeting twice a week. The after-school programme ran twice weekly for 12 weeks, for a total of 24 sessions at each school. Each session took place in the schoolyard or in the multipurpose room and comprised a 20-to 30-min health education component followed by 45-60 $\mathrm{min}$ of PA.

The researchers hired bilingual community health workers through the human resources department of the University of Texas at El Paso to teach the health education curriculum. To teach the PA component of the programme, senior-level student teachers from the University of Texas at EI Paso PE Teacher Education programme were recruited through announcements in several upper-level courses required for the PE teaching certification.

Diet and PA vs control

\begin{tabular}{|c|c|}
\hline \multirow[t]{3}{*}{ Outcomes } & $\begin{array}{l}\text { Outcome measures } \\
\text { - Primary outcome: age- and gender-adjusted BMI percentile, BMI, aerobic capacity, dietary intentions } \\
\text { and knowledge }\end{array}$ \\
\hline & Primary/secondary not specified \\
\hline & Process outcome: reported (dose) \\
\hline
\end{tabular}

Implementation-related Theoretical basis: ecological principles, SCT

factors

Resources: NR, but study authors state 'resources were limited'

Who delivered the intervention: reported

PROGRESS categories assessed at baseline: gender

PROGRESS categories analysed at outcome: gender, SES

Outcomes relating to harms/unintended effects: NR

Intervention included strategies to address diversity or disadvantage: selected a bilingual health education curriculum, 'Bienestar' (well-being), that is culturally targeted to Mexican Americans

Economic evaluation: NR

Notes

Funding: this project was supported by pilot research grants from the Center for Border Health Research through the Paso del Norte Health Foundation and by the NIH Hispanic Health Disparities Research Center (grant P20MD002287-01).

Population was predominately Hispanic. Demographic variables such as age, gender, and self-reported ethnicity were collected at baseline. However, many students were apparently not aware of their ethnicity because more than half marked don't know or other. Consequently, the study authors decided not to include self-reported ethnicity in any of the analyses.

Intervention exposure predicted lower $\mathrm{BMI}(\mathrm{P}=0.045)$, higher aerobic capacity $(\mathrm{P}=0.012)$, and greater intentions to eat healthily $(P=0.046)$ for the classroom at follow-up. Intervention effectiveness increased with increasing proportions of intervention participants in a classroom. 
De Heer 2011 (Continued)

Risk of bias

\begin{tabular}{|c|c|c|}
\hline Bias & Authors' judgement & Support for judgement \\
\hline $\begin{array}{l}\text { Random sequence genera- } \\
\text { tion (selection bias) }\end{array}$ & Unclear risk & Randomisation, no further details \\
\hline $\begin{array}{l}\text { Allocation concealment } \\
\text { (selection bias) }\end{array}$ & Unclear risk & NR \\
\hline $\begin{array}{l}\text { Blinding (performance } \\
\text { bias and detection bias) } \\
\text { All outcomes }\end{array}$ & Unclear risk & NR \\
\hline $\begin{array}{l}\text { Incomplete outcome data } \\
\text { (attrition bias) } \\
\text { All outcomes }\end{array}$ & Low risk & $\begin{array}{l}17 \% \text { attrition in intervention group and } 8 \% \text { in control, in bivariate analyses, we } \\
\text { detected no significant baseline differences in demographic characteristics or } \\
\text { any of the dependent variables between dropouts and those who completed } \\
\text { both baseline and follow-up measurements. }\end{array}$ \\
\hline $\begin{array}{l}\text { Selective reporting (re- } \\
\text { porting bias) }\end{array}$ & Unclear risk & Protocol/trial registration documents were unavailable. \\
\hline Other bias & Unclear risk & $\begin{array}{l}\text { Intervention classrooms also contained a spill-over group }(\mathrm{N}=251) \text { that did } \\
\text { not join the after-school programme but that completed measurements and } \\
\text { surveys. This spill-over group was analysed separately. }\end{array}$ \\
\hline
\end{tabular}

Other bias- timing of re- Low risk Figure shows recruitment happened prior to randomisation.

cruitment of clusters

De Ruyter 2012

Methods Study name: Double-blind, randomized intervention study in kids (DRINK)

Study design: RCT

Intervention period: 18 months

Follow-up period (post-intervention): nil

Differences in baseline characteristics: reported

Reliable outcomes: reported

Protection against contamination: reported

Unit of allocation: individual

Unit of analysis: individual

Participants $\quad \begin{aligned} & N \text { (control baseline) }=322 \\ & N \text { (control follow-up) }=252 \\ & N \text { (intervention baseline) }=319 \\ & N \text { (intervention follow-up) }=225 \\ & \text { Setting (and number by study group): } 8 \text { elementary schools }\end{aligned}$


De Ruyter 2012 (Continued)

Recruitment: no details

Geographic region: Zaanstreek, Purmerend and Haarlem - 3 suburbs in an urbanised area $16-33 \mathrm{~km}$ from Amsterdam

Percentage of eligible population enrolled: $95 \%$

Mean age: intervention: $8.2 \pm 1.8$; control: $8.2 \pm 1.8$

Sex: intervention: $46 \%$ female; control: $47 \%$ female

Interventions

To examine the effect on weight gain of masked replacement of SSBs with noncaloric, artificially sweetened beverages.

Intervention participants received $250 \mathrm{~mL}$ (8 oz) per day of a sugar-free, artificially sweetened beverage (sugar-free group) and control participants received a similar sugar-containing beverage that provided $104 \mathrm{kcal}$ (sugar group). Beverages were distributed through schools. Participating children received a box at school each week labelled with their name and containing 8 cans, 1 for each day of the week plus 1 extra to be used as a spare in case a can was misplaced.

Diet (beverage) intervention vs control

Outcomes

Outcome measures

- Primary outcome: zBMI

- Secondary outcomes: waist-to-height ratio, the sum of the 4 skinfold-thickness measurements, fat mass (electrical impedance), weight, height, height $z$ score, waist circumference

Process evaluation: reported (adherence)

Implementation-related Theoretical basis: NR

factors

Resources for intervention implementation: reported

Who delivered the intervention: reported

PROGRESS categories assessed at baseline: child: gender, race/ethnicity; parent: education

PROGRESS categories analysed at outcome: NR

Outcomes relating to harms/unintended effects: reported

Intervention included strategies to address diversity or disadvantage: NR

Economic evaluation: NR

Notes $\quad$ NCT00893529

Funding: supported by grants from the Netherlands Organization for Health Research and Development (120520010), the Netherlands Heart Foundation (2008B096), and the Royal Netherlands Academy of Arts and Sciences (ISK/741/PAH)

It is customary for children in Dutch elementary schools to consume a beverage brought from home in class during a morning break around 10 am under supervision of the teacher.

Developed custom drinks for this study to ensure that the sugar-free and sugar-containing drinks tasted and looked essentially the same.

At 18 months, $26 \%$ of the children had stopped consuming the beverages.

\section{Risk of bias}


De Ruyter 2012 (Continued)

Random sequence genera- Low risk tion (selection bias)
Quote: "An Excel visual basic macro program randomly assigned children to sugar-sweetened or sugar-free beverages within each school so that mean age, gender and initial BMI were equal between treatments "

A 2nd macro stratified children

Allocation concealment Low risk Independent statistician not otherwise involved in study

(selection bias)

\begin{tabular}{|c|c|c|}
\hline $\begin{array}{l}\text { Blinding (performance } \\
\text { bias and detection bias) } \\
\text { All outcomes }\end{array}$ & Low risk & $\begin{array}{l}\text { Double-blinded. Blinding of participants was tested, and correct responses } \\
\text { were higher than chance but this is one of very few studies in the area which } \\
\text { participants are blinded }\end{array}$ \\
\hline
\end{tabular}

Incomplete outcome data Low risk (attrition bias)

Analyses in which missing values were imputed suggested that results for the All outcomes full cohort would have been similar to those for the children who completed the study. $29 \%$ dropout in intervention group and $22 \%$ in the control group. ITT analyses conducted on $100 \%$ of participants and also completer analyses.

\begin{tabular}{lll}
\hline $\begin{array}{l}\text { Selective reporting (re- } \\
\text { porting bias) }\end{array}$ & Low risk & Protocol seen; all outcomes specified in methods were reported in results \\
\hline Other bias & Low risk & No other apparent threats to validity \\
\hline
\end{tabular}

\section{De Vries 2015}

\begin{tabular}{|c|c|}
\hline \multirow[t]{9}{*}{ Methods } & Study name: Groningen expert centre for kids with obesity (GECKO) \\
\hline & Study design: cluster-RCT \\
\hline & Intervention period: 11 months \\
\hline & Follow-up period (post-intervention): 18 months \\
\hline & Differences in baseline characteristics: reported \\
\hline & Reliable outcomes: reported \\
\hline & Protection against contamination: reported \\
\hline & Unit of allocation: nurse \\
\hline & Unit of analysis: individual accounting for clustering \\
\hline \multirow[t]{9}{*}{ Participants } & $\mathrm{N}$ (control baseline) $=65$ \\
\hline & $N($ control follow-up) $=54$ \\
\hline & $\mathrm{N}$ (intervention baseline) $=96$ \\
\hline & $\mathrm{N}$ (intervention follow-up) $=89$ \\
\hline & Setting (and number by study group): Well Baby Clinics; intervention: 7 nurses \\
\hline & ( $N=96$ children); control: 6 nurses $(N=65$ children) \\
\hline & $\begin{array}{l}\text { Recruitment: parents were informed about the current study during the 3rd trimester of pregnancy by } \\
\text { the general practitioner, midwife or gynaecologist or at their 1st visit to the Well Baby Clinic. }\end{array}$ \\
\hline & Geographic region: Drenthe, one of the northern provinces of the Netherlands \\
\hline & Percentage of eligible population enrolled: $70 \%$ \\
\hline
\end{tabular}


De Vries 2015 (Continued)

Mean age: intervention + control: 2 weeks

Sex: intervention: $40 \%$ female; control: $57 \%$ female
To evaluate the effect of early stimulation of PA on growth, body composition, motor activity and motor development in toddlers.

The intervention group received recommendations from a nurse during a home visit 2 weeks after birth and during regular visits at the Well Baby Clinic at 2, 4, 8 and 11 months of age. After every consultation, parents received a printed copy of the recommendations. 5 visits by participants and parents, the 1 st a home visit at 2 weeks old, and the rest to the Well Baby Clinic at 2, 4, 8 and 11 months of age. Follow-up visit at age 2.5 years took place either at clinic or at home.

Before each intervention visit (5 in total), the intervention nurses received special training from child physiotherapists on how to implement the stimulation programme.

The focus at 2 weeks was to engage symmetric handling and encourage use of coloured toys and sounds. The focus at 2 months was to encourage variation in the infant's position and location of play, and the focus at 4 months was to expand on this. At 8 months, the recommendations were to encourage the infant to crawl and thereby enlarge his playing area. Then at 11 months, parents were instructed to encourage their infant to walk without support.

Parents in the control group received standard care without activity

recommendations

PA intervention vs control

- Primary outcome: BMI, sum of skinfolds

- Secondary outcomes: \% overweight, weight, height, waist circumference, hip circumference, skinfolds (triceps, biceps, subscapular, supra-iliacal), \% body fat, motor skills (Bayley score), PA

Process evaluation: NR

Implementation-related factors
Theoretical basis: NR

Resources for intervention implementation: NR

Who delivered the intervention: reported

PROGRESS categories assessed at baseline: child: gender; parent: education, SES (income)

PROGRESS categories analysed at outcome: gender

Outcomes relating to harms/unintended effects: NR

Intervention included strategies to address diversity or disadvantage: NR

Economic evaluation: NR

Funding: this research was funded by an unrestricted grant from Hutchison Whampoa Ltd. and the University of Groningen

GECKO also included a birth cohort study; only birthweight was reported at baseline no other anthropometric outcomes were reported at baseline (aged 2 weeks)

\section{Risk of bias}

\section{Bias}

\section{Authors' judgement Support for judgement}


De Vries 2015 (Continued)

Random sequence genera- High risk Randomisation was carried out manually by a GECKO researcher, who drew tion (selection bias) pieces of paper from a bag. No further details of allocation. This method is highly susceptible to subversion or alteration.

\begin{tabular}{|c|c|c|}
\hline $\begin{array}{l}\text { Allocation concealment } \\
\text { (selection bias) }\end{array}$ & High risk & $\begin{array}{l}\text { Randomisation was carried out manually by a GECKO researcher, who drew } \\
\text { pieces of paper from a bag. No further details of allocation. This method is } \\
\text { highly susceptible to subversion or alteration. }\end{array}$ \\
\hline
\end{tabular}

$\begin{array}{ll}\begin{array}{l}\text { Blinding (performance } \\ \text { bias and detection bias) }\end{array} & \text { Low risk }\end{array}$

All outcomes

\begin{tabular}{lll}
\hline $\begin{array}{l}\text { Incomplete outcome data } \\
\text { (attrition bias) } \\
\text { All outcomes }\end{array}$ & Low risk & $\begin{array}{l}\text { Low attrition rate (17\% intervention, 7\% control), study reports that dropout } \\
\text { did not differ between the intervention ( } \mathrm{N}=7) \text { and control groups ( } \mathrm{N}=11, \mathrm{P}= \\
0.06)\end{array}$ \\
\hline $\begin{array}{l}\text { Selective reporting (re- } \\
\text { porting bias) }\end{array}$ & Low risk & Protocol/trial registration document seen. All outcomes reported \\
\hline $\begin{array}{l}\text { Other bias } \\
\text { Other bias- timing of re- }\end{array}$ & Low risk & No further threats to validity \\
\hline cruitment of clusters & & $\begin{array}{l}\text { NR; it is likely that nurses were randomised first and newborns assigned to } \\
\text { them over time later }\end{array}$ \\
\hline
\end{tabular}

\section{Dennison 2004}

\begin{tabular}{ll}
\hline Methods & Study design: cluster-RCT \\
Intervention period: 12 weeks \\
\\
Follow-up (Post-intervention): nil \\
Differences in baseline characteristics: NR \\
Reliable outcomes: reported \\
Protection against contamination: reported \\
Unit of allocation: nursery \\
Unit of analysis: unclear
\end{tabular}

\begin{tabular}{ll}
\hline Participants & (controls baseline) $=83$ \\
$\mathrm{~N}$ (controls follow-up) $=73$ \\
$\mathrm{~N}$ (interventions baseline) $=93$ \\
$\mathrm{~N}$ (interventions follow-up) $=90$ \\
Setting: school (8 intervention and 8 control) \\
Geographic region: New York State, USA \\
Proportion of eligibles participating: not stated \\
Mean age: 4.0 years \\
Sex: both sexes included but no figures given
\end{tabular}

Interventions

Preschool- and daycare centre-based intervention delivered by one early childhood teacher and a music teacher. This was part of larger 'Brocodile the Crocodile' health promotion programme, which lasted for 39 weeks for $1 \mathrm{~h}$ each week including 32 sessions on healthy eating. 7 educational sessions assessed intervention to encourage reduction of TV viewing for both parents and children. Controls received materials and activities about health and safety.

PA interventions vs control

Outcomes $\quad$ BMI


Dennison 2004 (Continued)

- Triceps skinfolds

- Parental estimates of child's sedentary activity in previous week in hours, and to estimate number of hours usually spent in these activities for each weekend day and each week day

Alternate activities as a result of reduced TV viewing were not stated/measured

Process evaluation: NR

Implementation-related factors
Theortetical basis: NR

Resources for intervention implementation (e.g. funding needed or staff hours required): reported Who delivered the intervention: reported

PROGRESS categories assessed at baseline: reported (race, occupation)

PROGRESS categories analysed at outcome: NR

Outcomes relating to harms/unintended effects: NR

Intervention included strategies to address diversity or disadvantage: NR

Economic evaluation: NR

Notes

Funding: this study was supported in part by grant 1-R01-HL65144 from the NIH, National Heart, Lung, and Blood Institute, Bethesda

\section{Risk of bias}

\section{Bias Authors' judgement Support for judgement}

Random sequence genera- Unclear risk tion (selection bias)
The generation of the randomisation sequence was not described. The study authors do state that "Randomisation performed in random permutations of the numbers 1 and 2..." But do not say how the permutations were generated.

\section{Allocation concealment Low risk \\ (selection bias)}

Blinding (performance $\quad$ High risk $\quad$ Not blinded
bias and detection bias)
All outcomes

\begin{tabular}{ll}
\hline $\begin{array}{l}\text { Incomplete outcome data } \\
\text { (attrition bias) }\end{array}$ & Low risk \\
All outcomes & ing data.
\end{tabular}

\begin{tabular}{lll}
\hline $\begin{array}{l}\text { Selective reporting (re- } \\
\text { porting bias) }\end{array}$ & Unclear risk & Protocol/trial registration documents were unavailable. \\
\hline Other bias & Low risk & No other apparent threats to validity \\
\hline $\begin{array}{l}\text { Other bias- timing of re- } \\
\text { cruitment of clusters }\end{array}$ & Low risk & $\begin{array}{l}\text { Figure } 1 \text { indicates recruitment happened prior to randomisation. Centres } \\
\text { agreed to participate, then randomisation was performed at the centre level } \\
\text { on all centres at the start of the study }\end{array}$ \\
\hline
\end{tabular}


Dewar 2013 (Continued)

\author{
Study design: cluster-RCT \\ Intervention period: 12 months \\ Follow-up period (post-intervention): 12 months \\ Differences in baseline characteristics: reported \\ Reliable outcomes: reported \\ Protection against contamination: NR \\ Unit of allocation: school \\ Unit of analysis: individual accounting for clustering
}

\begin{tabular}{|c|c|}
\hline \multirow[t]{10}{*}{ Participants } & $\mathrm{N}$ (control baseline) $=179$ \\
\hline & $\mathrm{N}$ (control follow-up) = 97 \\
\hline & $\mathrm{N}$ (intervention baseline) $=178$ \\
\hline & $\mathrm{N}$ (intervention follow-up) $=77$ \\
\hline & $\begin{array}{l}\text { Setting (and number by study group): } 12 \text { secondary schools in low-income communities (178 girls in } 6 \\
\text { intervention schools and } 179 \text { girls in } 6 \text { control schools) }\end{array}$ \\
\hline & Recruitment: NR in this paper \\
\hline & Geographic region: New South Wales, Australia \\
\hline & Percentage of eligible population enrolled: $67 \%$ schools \\
\hline & Mean age: intervention: $13.20 \pm 0.45$; control: $13.15 \pm 0.44$ \\
\hline & Sex: intervention + control: $100 \%$ female. \\
\hline
\end{tabular}

NEAT Girls combined a range of strategies to promote lifestyle (e.g. walking to school) and lifetime PA (e.g. RT), improve dietary intake, and reduce sedentary behaviours

Intervention components included enhanced school sport sessions, lunchtime PA sessions, nutrition workshops, interactive educational seminars, pedometers for self-monitoring, student handbooks, parent newsletters, and text messages to reinforce and encourage targeted health behaviours.

Control group was provided with equipment packs and a condensed

version of the intervention following the completion of 24-month assessments.

Diet and PA combination intervention vs control

Outcome measures
- Primary outcome: BMI
- Secondary outcomes: zBMI; \% body fat (bioelectrical impedance analysis); PA (accelerometers); di-
etary intake; and recreational screen-time (self-report), self-esteem
Process evaluation: reported (attendance, fidelity)

$\begin{array}{ll}\begin{array}{l}\text { Implementation-related } \\ \text { factors }\end{array} & \text { Theoretical basis: SCT } \\ & \text { Resources for intervention implementation: reported } \\ & \text { Who delivered the intervention: NR }\end{array}$


Dewar 2013 (Continued)

PROGRESS categories assessed at baseline: child: race/ethnicity, gender, SES

PROGRESS categories analysed at outcome: NR

Outcomes relating to harms/unintended effects: NR

Intervention included strategies to address diversity or disadvantage: NR

Economic evaluation: NR

\section{Notes}

\section{ACTRN1261000033004}

Funding: this research project is funded by an Australian Research Council Discovery Project Grant (DP1092646). This sponsor had no involvement in the design or implementation of this study, in analyses of data, or in the drafting of this paper.

Process: a total of 148 girls received the intervention (83.1\%). Students'

mean (SD) attendance at school sport sessions was $60.6 \%$ (26.0\%). On average, girls attended $65.0 \%$ $(25.1 \%)$ of the nutrition workshops, $24.6 \%(28.1 \%)$ of the optional lunch-time sessions, and completed $8.8 \%(25.7 \%)$ of the home PA and nutrition challenges.

Intervention delivery fidelity was found to be $74.0 \%$. All 4 of the parental newsletters were sent to valid addresses for $74.5 \%$ of girls in the intervention group. A total of 58 text messages were sent to $91 \%$ of girls in the intervention group. Overall, girls were satisfied with the programme (mean (SD), 3.52 (1.24); rating scale, $1=$ strongly disagree to $5=$ strongly agree). The enhanced school sport sessions $(41.7 \%)$ and the nutrition workshops (38.7\%) were the 2 intervention components enjoyed most by girls.

Resources: the intervention was focused on promoting lifetime PAs, reducing sedentary behaviours, and encouraging low-cost healthy eating, and it was delivered during 4 school terms (i.e. 12 months) at no additional financial cost to the school or students. All intervention schools were provided with a standard equipment pack (value = USD 1300), which consisted of a range of equipment (e.g. elastic tubing RT devices, fitness balls, and yoga and Pilates resources) designed to support the promotion of lifetime PAs.

\section{Risk of bias}

\begin{tabular}{lll}
\hline Bias & Authors' judgement & Support for judgement \\
\hline $\begin{array}{l}\text { Random sequence genera- } \\
\text { tion (selection bias) }\end{array}$ & Unclear risk & Not described \\
\hline $\begin{array}{l}\text { Allocation concealment } \\
\text { (selection bias) }\end{array}$ & Low risk & $\begin{array}{l}\text { An independent researcher randomised each pair of schools to either the NEAT } \\
\text { Girls intervention or control groups. 12 schools were matched (ie, } 6 \text { pairs of } \\
\text { schools) based on their geographic location, size, and demographics. }\end{array}$ \\
\hline $\begin{array}{l}\text { Blinding (performance } \\
\text { bias and detection bias) }\end{array}$ & High risk & $\begin{array}{l}\text { Data collection was conducted by trained research assistants blinded to group } \\
\text { allocation at baseline only }\end{array}$ \\
\hline $\begin{array}{l}\text { Incomplete outcome data } \\
\text { (attrition bias) }\end{array}$ & High risk & $\begin{array}{l}114 \text { (64.0\%) and 123 (68.7\%) girls were retained in the intervention and control } \\
\text { groups; because of participant attrition, the analyses were underpowered to } \\
\text { detect small changes in BMl }\end{array}$ \\
\hline $\begin{array}{l}\text { Selective reporting (re- } \\
\text { porting bias) }\end{array}$ & Low risk & \begin{tabular}{l} 
Protocol accessed. All outcomes specified in protocol were reported in results. \\
\hline $\begin{array}{l}\text { Other bias } \\
\text { Other bias- timing of re- } \\
\text { cruitment of clusters }\end{array}$
\end{tabular} \\
\hline \hline
\end{tabular}




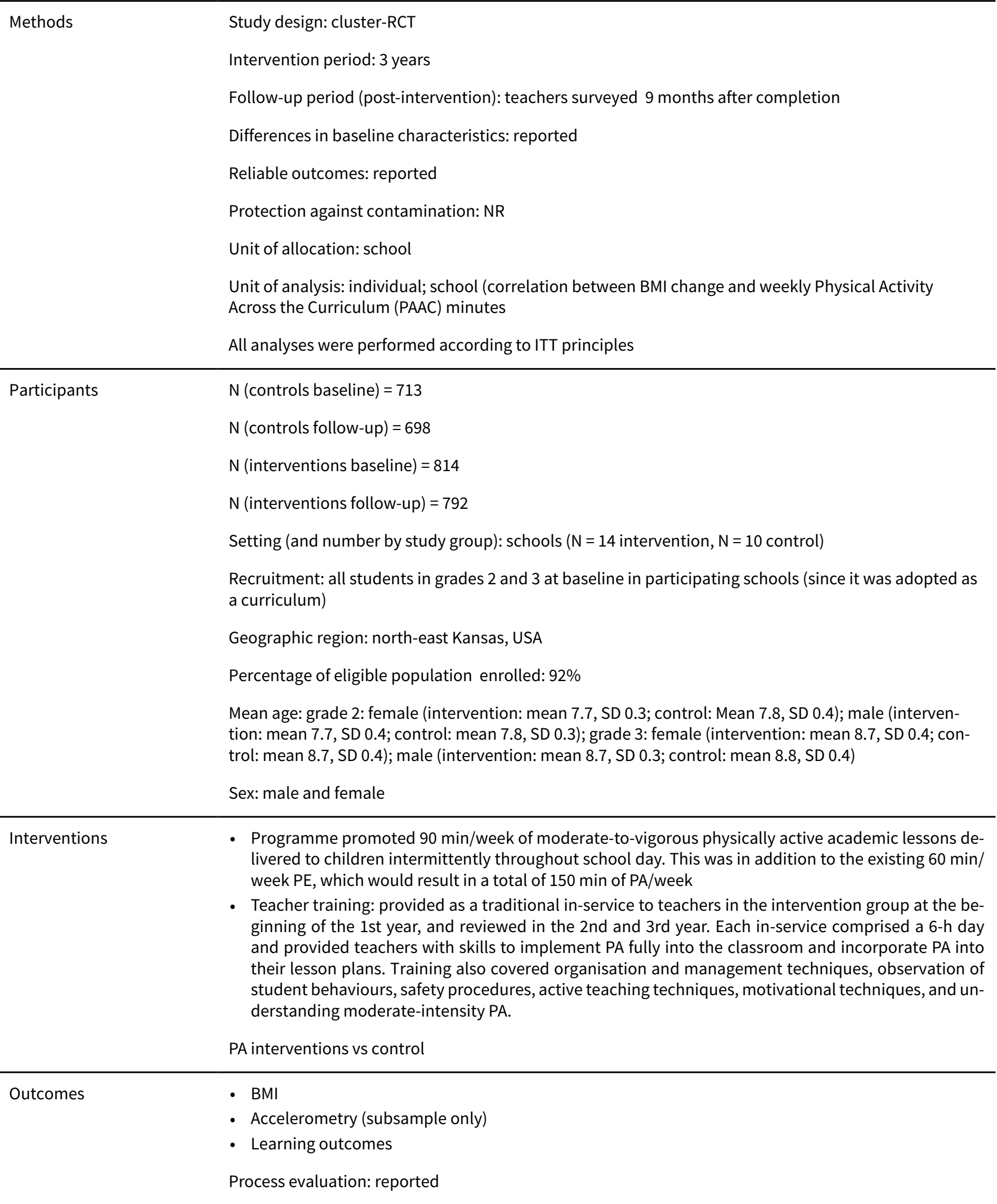


Donnelly 2009 (Continued)

Implementation-related factors
Theoretical basis: NR

Resources for intervention implementation (e.g. funding needed or staff hours required): reported Who delivered the intervention: reported

PROGRESS categories assessed at baseline: reported (race, gender, SES)

PROGRESS categories analysed at outcome: reported (gender)

Outcomes relating to harms/unintended effects: NR

Intervention included strategies to address diversity or disadvantage: NR

Economic evaluation: NR

Notes

Funding: this work was supported by grant NIH NIDDK R01 061489 from the National Institute of Diabetes and Digestive and Kidney Disease, Bethesda, MD. The authors would like to thank the International Life Sciences Institute for Health Promotion for educational materials.

\section{Risk of bias}

\begin{tabular}{|c|c|c|}
\hline Bias & Authors' judgement & Support for judgement \\
\hline $\begin{array}{l}\text { Random sequence genera- } \\
\text { tion (selection bias) }\end{array}$ & Unclear risk & $\begin{array}{l}\text { NR. } 24 \text { schools were randomly assigned to treatment or control stratified by } \\
\text { district and size. }\end{array}$ \\
\hline $\begin{array}{l}\text { Allocation concealment } \\
\text { (selection bias) }\end{array}$ & Unclear risk & NR \\
\hline $\begin{array}{l}\text { Blinding (performance } \\
\text { bias and detection bias) } \\
\text { All outcomes }\end{array}$ & Low risk & $\begin{array}{l}\text { Research assistants blinded to condition for measurement of primary and sec- } \\
\text { ondary outcomes and data entry. Research assistants who conducted class- } \\
\text { room visitations not blinded }\end{array}$ \\
\hline $\begin{array}{l}\text { Incomplete outcome data } \\
\text { (attrition bias) } \\
\text { All outcomes }\end{array}$ & High risk & $\begin{array}{l}2 \text { schools ( } 8 \% \text { ) discontinued participation; } 1 \text { due to closing of the school and } 1 \\
\text { refused randomisation to control. } 2.5 \% \text { of participants lost to follow-up }\end{array}$ \\
\hline $\begin{array}{l}\text { Selective reporting (re- } \\
\text { porting bias) }\end{array}$ & Low risk & Protocol found. All outcomes listed in the protocol were reported in results. \\
\hline Other bias & Low risk & No additional threats to validity \\
\hline $\begin{array}{l}\text { Other bias- timing of re- } \\
\text { cruitment of clusters }\end{array}$ & Unclear risk & NR \\
\hline
\end{tabular}

Ebbeling 2006

\begin{tabular}{ll} 
Methods & Study design: RCT \\
& Intervention period: 25 weeks \\
& Follow-up (post-intervention): nil \\
& Differences in baseline characteristics: reported \\
& Reliable outcomes: reported \\
& Protection against contamination: reported \\
& Unit of allocation: child \\
& Unit of analysis: child \\
\hline Participants & $\mathrm{N}$ (controls baseline) $=50$ \\
\hline
\end{tabular}

Interventions for preventing obesity in children (Review) 
Ebbeling 2006 (Continued)

$\mathrm{N}$ (controls follow-up) $=50$

$\mathrm{N}$ (interventions baseline) $=53$

$\mathrm{N}$ (interventions follow-up) $=53$

Setting (and number by study group): home (intervention $N=53$; control $N=50$ )

Recruitment: local high school provided mailing lists. Adolescents aged $13-18$ years who reported consuming at least 1 serving per day of SSB and lived predominately in 1 household were eligible.

Geographic region: USA

Percentage of eligible population enrolled: $77 \%$

Mean age: intervention: $16.0 \pm 1.1$ years; control: $15.8 \pm 1.1$ years

Sex: intervention: $55 \%$ female; control: $54 \%$ female

Interventions

Intervention

- Weekly home deliveries of noncaloric beverages for 25 weeks: the target number of individual beverage servings (i.e. $360 \mathrm{~mL}$ or $12 \mathrm{fl}$ oz per referent serving) delivered to each home was based on household size: 4 servings/day for the participant and 2 servings/day for each additional member of the household. Beverage preferences selected from a wide variety of options (e.g. bottled water and 'diet' beverages including soft drinks, iced teas, lemonades, and punches). A regional supermarket delivery service filled the orders and delivered the beverages, with research staff co-ordinating and monitoring the process

- Monthly telephone calls to reinforce instructions, provide education and counselling, etc

- Refrigerator magnets with messages under the theme of "Think Before You Drink" and an additional message cautioned subjects to beware of misleading beverage labels and advertisements

Control

- Participants in control group asked to continue their usual beverage consumption habits throughout the 25-week intervention period

- Received weekly home deliveries of noncaloric beverages for 4 weeks after completion of follow-up measurements, as a benefit for having participated in the study

Dietary interventions vs control

$\begin{array}{ll}\text { Outcomes } & \text { BMI } \\ \text { - Energy intake from SSBs } \\ \text { - Noncaloric beverage intake }(\mathrm{mL}) \\ \text { - PA (MET) } \\ \text { - TV viewing }(\mathrm{h}) \\ \text { Process evaluation: reported }\end{array}$

Implementation-related factors
Theoretical basis: NR

Resources for intervention implementation (e.g. funding needed or staff hours required): reported Who delivered the intervention: reported

PROGRESS categories assessed at baseline: reported (race, gender, SES)

PROGRESS categories analysed at outcome: reported (gender)

Outcomes relating to harms/unintended effects: NR

Intervention included strategies to address diversity or disadvantage: NR

Economic evaluation: NR 
Ebbeling 2006 (Continued)

Notes
Funding: this study was supported by grants R01 DK63554 and K01 DK62237 from the National Institute of Diabetes and Digestive Kidney Diseases, the Charles H. Hood Foundation, and grant M01 RR02172 awarded by the NIH to support the General Clinical Research Center at Children's Hospital Boston

Estimated that the cost involved in delivering their intervention was approximately 35 USD per student over 25 weeks.

\section{Risk of bias}

\begin{tabular}{lll}
\hline Bias & Authors' judgement & Support for judgement \\
\hline $\begin{array}{l}\text { Random sequence genera- } \\
\text { tion (selection bias) }\end{array}$ & Low risk & $\begin{array}{l}\text { Eligible participants were entered sequentially onto a list of random group as- } \\
\text { signments prepared in advance by the study statistician, stratified by gender } \\
\text { and BMI. Sequence of random assignments was permutated within stratum in } \\
\text { blocks of 2, 4 and } 6\end{array}$ \\
\hline $\begin{array}{l}\text { Allocation concealment } \\
\text { (selection bias) }\end{array}$ & Low risk & $\begin{array}{l}\text { To avoid any bias in the enrolment procedure, personnel conducting recruit- } \\
\text { ment were masked to the sequence }\end{array}$ \\
\hline $\begin{array}{l}\text { Blinding (performance } \\
\text { bias and detection bias) }\end{array}$ & Unclear risk & $\begin{array}{l}\text { Interviewer for dietary and PA recall interviews was masked to group assign- } \\
\text { ment. Not clear whether people conducting BMI measures (primary endpoint) } \\
\text { were masked to group assignment. Participants not masked }\end{array}$ \\
\hline $\begin{array}{l}\text { Incomplete outcome data } \\
\text { (attrition bias) } \\
\text { All outcomes }\end{array}$ & Low risk & All participants completed study \\
\hline $\begin{array}{l}\text { Selective reporting (re- } \\
\text { porting bias) }\end{array}$ & Unclear risk & Protocol/trial registration documents were unavailable. \\
\hline \begin{tabular}{l} 
Other bias \\
\hline
\end{tabular} & Low risk & No additional threats to validity \\
\hline
\end{tabular}

\section{El Ansarai 2010}

Study design: RCT (paper reports it is a cross-sectional study but it isn't as the same 160 participants
were measured at baseline and 3-month follow-up)
Intervention period: 3 months
Follow-up period (post-intervention): nil
Differences in baseline characteristics: reported
Reliable outcomes: reported
Protection against contamination: NR
Unit of allocation: individual
Unit of analysis: individual
N (control baseline) $=80$
N (control follow-up) $=80$
N (intervention baseline) $=80$
N (intervention follow-up) $=80$


Setting (and number by study group): 1 secondary school

Recruitment: a little minority of schools in Mansoura city have both indoor and outdoor sport facilities and sport equipment, which were needed for the study. 1 secondary school in Mansoura city was selected due to the availability of both indoor and outdoor sport facilities and sport kits at the school.

Geographic region: Mansoura city, Nile Delta, Lower Egypt

Percentage of eligible population enrolled: $44 \%$ agreed to participate, based on the completed PA readiness questionnaires, 20 pupils were excluded because of reported medical condition(s). A further 20 girls were randomly selected and put in 'reserve' because there were 20 more girls than boys

Mean age: intervention: $15.7 \pm 1.8$ years

Sex: intervention + control: $56 \%$ female

Interventions

To assess the relationships between a PA programme and health parameters in adolescent school pupils in Egypt

The PA intervention programme comprised an 'after-school' $1 \mathrm{~h}$ of moderate exercise 3 times/week for 3 months. Both the controls and the intervention pupils attended the 'normal' exercise schedule provided by the school; in addition, the intervention group attended after-school PA programme from about 2-3 o'clock in the afternoon.

PA intervention vs control

\begin{tabular}{ll}
\hline Outcomes & Outcome measures \\
- Primary outcome: cholesterol, blood pressure, heart rate \\
- Secondary outcomes: weight, BMI, body fat \\
Process evaluation: NR
\end{tabular}

Implementation-related

Theoretical basis: NR

factors

Resources for intervention implementation: reported

Who delivered the intervention: reported

PROGRESS categories assessed at baseline: gender

PROGRESS categories analysed at outcome: gender

Outcomes relating to harms/unintended effects: NR

Intervention included strategies to address diversity or disadvantage: NR

Economic evaluation: NR

Notes

Funding: NR

Have contacted study authors to confirm this is an RCT

\section{Risk of bias}

\section{Bias}

Authors' judgement Support for judgement

Random sequence genera- Unclear risk tion (selection bias)

Groups randomly allocated, no further details

$\begin{array}{lll}\text { Allocation concealment } & \text { Unclear risk } & \text { NR } \\ \text { (selection bias) }\end{array}$


El Ansarai 2010 (Continued)
Blinding (performance
High risk
Participants not blinded
bias and detection bias)

All outcomes

Incomplete outcome data $\quad$ Unclear risk
$\begin{aligned} & \text { (attrition bias) } \\ & \text { All outcomes }\end{aligned}$

\begin{tabular}{lll}
\hline $\begin{array}{l}\text { Selective reporting (re- } \\
\text { porting bias) }\end{array}$ & Unclear risk & Protocol/trial registration documents were unavailable \\
\hline Other bias & Low risk & No additional threats to validity \\
\hline
\end{tabular}

Elder 2014

\begin{tabular}{|c|c|}
\hline \multirow[t]{8}{*}{ Methods } & Study design: cluster-RCT \\
\hline & Intervention period: 24 months \\
\hline & Follow-up period (post-intervention): nil \\
\hline & Differences in baseline characteristics: reported \\
\hline & Reliable outcomes: reported \\
\hline & Protection against contamination: NR \\
\hline & Unit of allocation: recreation centres \\
\hline & Unit of analysis: families accounting for clustering \\
\hline \multirow[t]{10}{*}{ Participants } & $\mathrm{N}$ (control baseline) $=270$ \\
\hline & $N$ (control follow-up) $=256$ \\
\hline & $\mathrm{N}$ (intervention baseline) $=271$ \\
\hline & $\mathrm{N}$ (intervention follow-up) $=238$ \\
\hline & $\begin{array}{l}\text { Setting (and number by study group): community: } 30 \text { recreation centres; intervention group } N=15 \\
\text { recreation centres and } 271 \text { families and control group } N=15 \text { recreation centres and } 270 \text { families }\end{array}$ \\
\hline & $\begin{array}{l}\text { Recruitment: targeted phone calls; } 8600 \text { telephone numbers were obtained from a market research } \\
\text { company. In addition, } 1000 \text { families were contacted at public locations, such as libraries, schools, com- } \\
\text { munity events (street fairs, special gatherings) and the } 30 \text { participating recreation centres }\end{array}$ \\
\hline & Geographic region: San Diego County, USA \\
\hline & Percentage of eligible population enrolled: $47 \%$ families screened \\
\hline & Mean age: intervention + control: $6.6 \pm 0.7$ \\
\hline & Sex: intervention + control: $54.9 \%$ female \\
\hline \multirow[t]{4}{*}{ Interventions } & To promote healthy eating and PA among 5- to 8-year-old children \\
\hline & The targeted nutrition behaviours addressed by the family health coaches included: \\
\hline & $\begin{array}{l}\text { - increase consumption of vegetables and fruits through modifications in meal and snack purchasing } \\
\text { and preparation }\end{array}$ \\
\hline & - decrease consumption of SSBs through changes in food purchasing and limit setting \\
\hline
\end{tabular}


Elder 2014 (Continued)

- increase healthy portions by modifying food consumption behaviours

- reduce eating out and when eating out, select healthy options

- increase availability and accessibility of healthy foods and beverages in the home

- reduce screen time and avoid eating in front of the TV

- increase the number of meals eaten together as a family

The targeted PA behaviours included:

- increase the amount of MVPA to $60 \mathrm{~min} /$ day on most days of the week

- increase availability and accessibility of PA opportunities in the home and community

- increase the variety of fun, and developmentally appropriate and culturally appropriate PA opportunities

Interventions:

- Telephone survey about the family's recreation centre use (10 min; prior to introductory workshop) once;

- Introductory group workshop at the recreation centre ( $1.5 \mathrm{~h}$; month 1 of intervention) once;

- Home visit (1 h; within the first 6 months of intervention) once;

- Mailed tip sheets (approximately monthly during intervention) 8 times;

- Phone consultations on tip sheet (10 min; twice per tip sheet) 18 times;

- Group workshops at the recreation centre ( $1.5 \mathrm{~h}$; quarterly during intervention) three times.

Providers:

- 2 full-time family health coaches, a full-time recreation specialist, a half-time recreation assistant and a full-time intervention coordinator.

- Control: at the 1-year measurements, interactive booths were set up at the recreation centre for families to receive take-home information and giveaways on non-obesity-related topics. Children participated in crafts and science experiments. Families received information on dental care, fire safety, environmental awareness and video

Diet and PA combination intervention vs control

Outcomes Outcome measures

- Primary outcome: BMI, BMI percentile, zBMI, waist circumference, $\%$ body fat

- Secondary outcomes: PA and sedentary time, dietary intake

Process evaluation: reported (fidelity)

Implementation-related factors
Theoretical basis: NR

Resources for intervention implementation: reported

Who delivered the intervention: reported

PROGRESS categories assessed at baseline: child: gender, race/ethnicity; parent: gender, race/ethnicity (acculturation), education, occupation, SES (income), marital status

PROGRESS categories analysed at outcome: child: gender; parent: acculturation

Outcomes relating to harms/unintended effects: NR

Intervention included strategies to address diversity or disadvantage: NR

Economic evaluation: NR

Funding: this study was supported by the NIH grant NIDDK R01DK072994. NCC was supported by grants T32HL079891 and F31KD079345. KC was supported by the Medical Research Council Epidemiology Unit (Unit Programme number U106179474) and the Centre for Diet and Activity Research (CEDAR), a UKCRC 
Elder 2014 (Continued)

Public Health Research: Centre of Excellence. Funding from the British Heart Foundation, Economic and Social Research Council, Medical Research Council, the National Institute for Health Research, and the Wellcome Trust, under the auspices of the UK Clinical Research Collaboration, is gratefully acknowledged.

Context: recreation centres were affected by a municipal, then a statewide economic downturn resulting in increased responsibilities of recreational staff, and decreased staffing and reduced hours and programmes due to downsizing of municipal government. The overall dose was limited.

\section{Risk of bias}

\begin{tabular}{|c|c|c|}
\hline Bias & Authors' judgement & Support for judgement \\
\hline $\begin{array}{l}\text { Random sequence genera- } \\
\text { tion (selection bias) }\end{array}$ & Unclear risk & Randomised, no further details \\
\hline $\begin{array}{l}\text { Allocation concealment } \\
\text { (selection bias) }\end{array}$ & Unclear risk & NR \\
\hline $\begin{array}{l}\text { Blinding (performance } \\
\text { bias and detection bias) } \\
\text { All outcomes }\end{array}$ & Unclear risk & NR \\
\hline $\begin{array}{l}\text { Incomplete outcome data } \\
\text { (attrition bias) } \\
\text { All outcomes }\end{array}$ & Low risk & $\begin{array}{l}\text { Low attrition: } 5 \% \text { control and } 12 \% \text { intervention groups lost to follow-up, base- } \\
\text { line values adjusted for in follow-up analyses }\end{array}$ \\
\hline $\begin{array}{l}\text { Selective reporting (re- } \\
\text { porting bias) }\end{array}$ & Unclear risk & Protocol/trial registration documents were unavailable \\
\hline Other bias & Low risk & No additional threats to validity \\
\hline $\begin{array}{l}\text { Other bias- timing of re- } \\
\text { cruitment of clusters }\end{array}$ & Low risk & Figure shows recruitment happened prior to randomisation \\
\hline
\end{tabular}

Epstein 2001

$\begin{array}{ll}\text { Methods } & \text { Study design: RCT } \\ \text { Intervention period: } 1 \text { year } \\ \text { Follow-up (post-intervention): nil } \\ \text { Differences in baseline characteristics: reported } \\ \text { Reliable outcomes: yes } \\ \text { Protection against contamination: not clear } \\ \text { Unit of allocation: family } \\ \text { Unit of analysis: child } \\ \text { For percentage of overweight (height and weight measured but NR) } \\ \text { N (controls baseline) } 13 \text { (low fat/sugar) } \\ \text { N (controls follow-up) }=13 \\ \text { N (interventions baseline) }=13 \text { (fruit and vegetables) } \\ \text { N (interventions follow-up) }=13 \\ \text { 2 interventions, } 13 \text { children in each intervention group. } 30 \text { started but only } 26 \text { children provided base- } \\ \text { line data } \\ \text { Geographic region: New York State, USA } \\ \text { Proportion of eligibles participating: not stated } \\ \text { Mean age: } 8.8 \text { (1.8) (low fat/sugar); } 8.6 \text { (1.9) (fruit/vegetables) } \\ \text { Sex: both sexes included (boys/girls 6/7 (low fat/sugar); } 3 / 10 \text { (fruit/vegetables)) }\end{array}$


Epstein 2001 (Continued)

Interventions
- Families with obese parents and non-obese children were randomised to groups in which parents were provided with a comprehensive behavioural weight-control programme and were encouraged to increase fruit and vegetable intake.

- Comparison groups were encouraged to decrease intake of high fat/high sugar foods

Dietary interventions vs control

\begin{tabular}{ll}
\hline Outcomes & Percentage of overweight \\
- Servings/day of fruits and vegetables \\
- Servings/day of high fat/high sugar foods \\
Process evaluation: NR
\end{tabular}

Implementation-related factors
Theoretical basis: NR

Resources for intervention implementation (e.g. funding needed or staff hours required): reported Who delivered the intervention: reported

PROGRESS categories assessed at baseline: reported (gender)

PROGRESS categories analysed at outcome: reported (gender)

Outcomes relating to harms/unintended effects: NR

Intervention included strategies to address diversity or disadvantage: NR

Economic evaluation: NR

Notes

Funding: this study was funded in part by NIH Grant HD34284 (to L.H.E.)

\section{Risk of bias}

\begin{tabular}{lll}
\hline Bias & Authors' judgement & Support for judgement \\
\hline $\begin{array}{l}\text { Random sequence genera- } \\
\text { tion (selection bias) }\end{array}$ & Unclear risk & $\begin{array}{l}\text { Sequence generation NR. Families (parent-child dyads) who met entrance cri- } \\
\text { teria were randomly assigned to 1/2 groups; no further details }\end{array}$ \\
\hline $\begin{array}{l}\text { Allocation concealment } \\
\text { (selection bias) }\end{array}$ & Unclear risk & NR \\
\hline $\begin{array}{l}\text { Blinding (performance } \\
\text { bias and detection bias) } \\
\begin{array}{l}\text { All outcomes } \\
\text { Incomplete outcome data }\end{array}\end{array}$ & Unclear risk & Low risk \\
$\begin{array}{l}\text { (attrition bias) } \\
\text { All outcomes }\end{array}$ & A total of 15 families began in each of the 2 groups. Complete 1-year data were \\
\hline $\begin{array}{l}\text { Selective reporting (re- } \\
\text { porting bias) }\end{array}$ & Unclear risk & Protocol/trial registration documents were unavailable \\
\hline \begin{tabular}{l} 
Other bias \\
\hline
\end{tabular} & Low risk & No additional threats to validity. \\
\hline
\end{tabular}

\section{Ezendam 2012}

Methods Study name: FATaintPHAT (VETisnietVET in Dutch)


Ezendam 2012 (Continued)

Study design: cluster-RCT

Intervention period: 10 weeks

Follow-up period (post-intervention): 21.5 months

Differences in baseline characteristics: reported

Reliable outcomes: reported

Protection against contamination: NR

Unit of allocation: school

Unit of analysis: individual accounting for clustering

Participants
$N$ (control baseline) $=398$
$N$ (control follow-up) $=340$
$N$ (intervention baseline) $=485$
Setting (and number by study group): 20 secondary schools, $\mathrm{N}=11$ intervention schools and 485 partic-
ipants, $\mathrm{N}=9$ control schools and 398 participants
Recruitment: targeted phone calls; 8600 telephone numbers were obtained from a market research
company. In addition, 1000 families were contacted at public locations, such as libraries, schools, com-
munity events (street fairs, special gatherings) and the 30 participating recreation centres

Geographic region: Rotterdam, Netherlands

Percentage of eligible population enrolled: $33 \%$ schools, $59 \%$ participants

Mean age: intervention: $12.7 \pm 0.7$; control: $12.6 \pm 0.6$

Sex: intervention: $41.1 \%$ female; control: $50.3 \%$ female

Interventions

To evaluate the short- and long-term results of a Web-based computer-tailored intervention aiming to increase PA, decrease sedentary behaviour, and promote healthy eating to contribute to the prevention of excessive weight gain among adolescents

Internet-delivered intervention - 8 modules addressing weight management and energy balance-related behaviours.

- Each module consisted of information about the behaviour-health link, an assessment of behaviour and determinants, individually tailored feedback on behaviour and determinants, and an option to formulate an implementation intention to prompt specific goal setting and action planning.

- The feedback provided included several elements: behavioural feedback (comparing the student's behaviour with guidelines for that behaviour (normative feedback) and with behaviour of peers (comparative feedback)), prompts for intention formation, decisional balance information to change attitudes, prompts for barrier identification, instructions on how to perform and/or change a behaviour to improve self-efficacy, and suggestions on how to organise social support

- The intervention was accessible through the internet. The teachers were asked to allocate $15 \mathrm{~min}$ for each of 8 lessons over 10 weeks to work with the programme according to a teacher manual.

The control school implemented the regular curriculum.

Diet and PA combination intervention vs control

Outcomes

Outcome measures

- Primary outcome: BMI, \% overweight, waist circumference 
Process evaluation: reported - separate publication

Implementation-related factors
Theoretical basis: TPB, Precaution Adoption Process Model, Implementation intentions

Resources for intervention implementation: reported

Who delivered the intervention: reported

PROGRESS categories assessed at baseline: child: gender, race/ethnicity, education (pre-university vs vocational schools)

PROGRESS categories analysed at outcome: NR

Outcomes relating to harms/unintended effects: NR

Intervention included strategies to address diversity or disadvantage: NR

Economic evaluation: NR
Notes

\section{ISRCTN15743786}

Funding: this study was funded by grant 62200020 from ZonMw, the Netherlands Organization for Health Care Research and Development.

More schools in the intervention group were vocational schools

Process evaluation (see Ezendam 2012): $81 \%$ was exposed to all intervention modules and $73 \%$ reported to have put the advice into practise. Half and one-3rd of the students appreciated the tailored advice positively and neutrally, respectively.

Students attending vocational training appreciated FATaintPHAT better than students attending university preparation education. No associations were found between behavioural outcomes with appreciation and use.

\section{Risk of bias}

\section{Bias \\ Authors' judgement Support for judgement}

Random sequence genera- Low risk Schools were randomised after stratification according to educational level tion (selection bias) (vocational or pre-university training) using a random-number generator

$\begin{array}{ll}\begin{array}{l}\text { Allocation concealment } \\ \text { (selection bias) }\end{array} & \text { Methods NR } \\ & \text { Quote: "Students in the intervention group were more likely to participate } \\ & \\ & \\ & \text { tervention." }\end{array}$

Blinding (performance Unclear risk NR

bias and detection bias)

All outcomes

\section{Incomplete outcome data High risk}

(attrition bias)

All outcomes
3 schools withdrew after randomisation and before baseline characteristics were recorded. In the intervention group, $15 \%$ of the students were lost to follow-up and in the control group, $12 \%$ were lost. Paper reports loss to follow-up did not differ according to study condition, educational level, ethnicity, or sex.

Schools were stratified according to educational level (vocational for students attending vocational training; pre-university for students preparing for bachelor degree education) and randomly assigned to either the intervention (11 
Ezendam 2012 (Continued)

schools) or control group (12 schools of which 3 schools dropped out after randomisation, although allocation was concealed).

\begin{tabular}{lll}
\hline $\begin{array}{l}\text { Selective reporting (re- } \\
\text { porting bias) }\end{array}$ & Low risk & Protocol seen; all outcomes specified in methods were reported in results \\
\hline Other bias & Low risk & No additional threats to validity \\
\hline $\begin{array}{l}\text { Other bias- timing of re- } \\
\text { cruitment of clusters }\end{array}$ & High risk & $\begin{array}{l}\text { Recruitment of schools occurred before randomisation but participants were } \\
\text { recruited after randomisation. }\end{array}$ \\
\hline
\end{tabular}

\section{Fairclough 2013}

\section{Methods}

Study name: CHANGE! (Children's health, activity and nutrition: get educated!)

Study design: cluster-RCT

Intervention period: 20 weeks

Follow-up period (post-intervention): 10 weeks

Differences in baseline characteristics: reported

Reliable outcomes: reported

Protection against contamination: reported

Unit of allocation: school

Unit of analysis: individual accounting for clustering

\begin{tabular}{|c|c|}
\hline \multirow[t]{10}{*}{ Participants } & $\mathrm{N}$ (control baseline) $=152$ \\
\hline & $\mathrm{N}$ (control follow-up) = 117 \\
\hline & $\mathrm{N}$ (intervention baseline) $=166$ \\
\hline & $\mathrm{N}$ (intervention follow-up) $=89$ \\
\hline & Setting (and number by study group): 12 primary schools, 6 intervention schools and 6 control schools \\
\hline & $\begin{array}{l}\text { Recruitment: schools were randomly selected (1 high- and } 1 \text { low-SES school within each Neighbour- } \\
\text { hood Management Area) }\end{array}$ \\
\hline & Geographic region: Wigan, UK \\
\hline & Percentage of eligible population enrolled: $100 \%$ schools, $76 \%$ participants \\
\hline & Mean age: intervention: $10.6 \pm 0.3$; control: $10.7 \pm 0.3$ \\
\hline & Sex: intervention: NR; control: NR \\
\hline \multirow[t]{3}{*}{ Interventions } & To assess the effectiveness of the CHANGE! intervention on measures of body size, PA and food intake \\
\hline & $\begin{array}{l}\text { - Year } 6 \text { class teachers from the intervention schools received } 4 \mathrm{~h} \text { of training in the delivery of the cur- } \\
\text { riculum resource, and so were fully familiarised with the curriculum prior to implementation. }\end{array}$ \\
\hline & $\begin{array}{l}\text { - The CHANGE! curriculum consisted of } 20 \text { weekly lesson plans, worksheets, homework tasks, lesson } \\
\text { resources, and a CD-ROM. The lessons were of 60-min duration and provided an opportunity for chil- } \\
\text { dren to discuss, explore, and understand the meaning and practicalities of PA and nutrition as key } \\
\text { elements of healthy lifestyles. }\end{array}$ \\
\hline
\end{tabular}


- The core message of the PA and sedentary behaviour components was "move more, sit less" with no specific prescription given as to what forms of PA the children should do

- The nutrition components focused on topics such as, energy balance, macronutrients, and eating behaviours.

- The homework tasks supplemented the classroom work and targeted family involvement in food and PA related tasks. Curriculum was adapted from existing resources.

Classes in the control schools received normal instruction and did not teach a specific unit of PSHE focused on healthy eating and PA

Diet and PA combination intervention vs control

\begin{tabular}{ll}
\hline Outcomes & Outcome measures \\
& - Primary outcome: BMI, zBMI, waist circumference \\
- Secondary outcomes: PA, sedentary time \\
Process evaluation: NR
\end{tabular}

Implementation-related factors

Theoretical basis: SCT

Resources for intervention implementation: reported

Who delivered the intervention: reported

PROGRESS categories assessed at baseline: gender, SES (IMD score)

PROGRESS categories analysed at outcome: gender, SES

Outcomes relating to harms/unintended effects: NR

Intervention included strategies to address diversity or disadvantage: NR

Economic evaluation: NR

\section{ISRCTN03863885}

Funding: Liverpool John Moores University (UK)

Intervention was integrated within the existing curriculum and delivery by class teachers was a sustainable approach, that was undertaken at minimal financial cost.

\section{Risk of bias}

\begin{tabular}{lll}
\hline Bias & Authors' judgement & Support for judgement \\
\hline $\begin{array}{ll}\text { Random sequence genera- } \\
\text { tion (selection bias) }\end{array}$ & Low risk & $\begin{array}{l}\text { Quote: "Schools were stratified to ensure an equal distribution of high and low } \\
\text { SES schools, which were randomly allocated to an Intervention ( } \mathrm{n}=6 \mathrm{schools}) \\
\text { or Comparison condition ( } \mathrm{n}=6 \text { schools) using a random number generator } \\
\text { (SPSS Inc., Chicago, IL)." }\end{array}$ \\
\hline
\end{tabular}

\begin{tabular}{|c|c|c|}
\hline $\begin{array}{l}\text { Allocation concealment } \\
\text { (selection bias) }\end{array}$ & High risk & $\begin{array}{l}\text { Randomisation of schools was not blinded and was conducted by the research } \\
\text { team }\end{array}$ \\
\hline
\end{tabular}

\begin{tabular}{ll}
\hline Blinding (performance & High risk \\
bias and detection bias) & \\
All outcomes & \\
\hline
\end{tabular}


Fairclough 2013 (Continued)

Selective reporting (re- Low risk Protocol/trial registration document seen. All outcomes reported porting bias)

\begin{tabular}{lll}
\hline Other bias & Low risk & No additional threats to validity \\
\hline $\begin{array}{l}\text { Other bias- timing of re- } \\
\text { cruitment of clusters }\end{array}$ & Unclear risk & Figure shows clusters recruited prior to randomisation only \\
\hline
\end{tabular}

\section{Farias 2015}

\begin{tabular}{ll}
\hline Methods & Study design: cluster-RCT \\
Intervention period: 1 school year, no further details \\
Follow-up period (post-intervention): nil \\
Differences in baseline characteristics: reported \\
Reliable outcomes: reported \\
Protection against contamination: NR \\
Unit of allocation: class \\
Unit of analysis: individual
\end{tabular}

Participants

$$
\begin{aligned}
& N(\text { control baseline })=284 \\
& N(\text { control follow-up })=195 \\
& N(\text { intervention baseline })=283 \\
& N(\text { intervention follow-up })=191
\end{aligned}
$$

Setting (and number by study group): 1 secondary school, 5 classes in intervention group and 5 classes in control group

Recruitment: NR

Geographic region: Colé-gio Meta, Rio Branco, AC, Brazil

Percentage of eligible population enrolled: NR

Mean age: intervention: $15.9 \pm 0.8$; control: $16.0 \pm 0.8$

Sex: intervention: $43.1 \%$ female; control: $50.7 \%$ female

- Both groups had 2 PE classes weekly, lasting 60 min each. Each class had 83 PE classes totaling 415 annual classes.

- Intervention group underwent programmed PA with heart rate monitoring, consisting of 3 parts: aerobic activity (exercises for flexibility, muscular strength, jumping rope, walking, alternating running, continuous jumping, recreational games), lasting $30 \mathrm{~min}$; sports games (volleyball, soccer, handball), lasting $20 \mathrm{~min}$ and stretching lasting $10 \mathrm{~min}$.

- Control group performed the usual PA at school, such as reception and games through exercise, callisthenics, learning the fundamentals of sports, and sports activities.

PA combination intervention vs control. 
Farias 2015 (Continued)

Outcome measures
- Primary outcome: weight, height, MBI, zBMI, overweight/obesity prevalence, waist circumference,
sum of skinfolds; \% body fat; lean mass, fat mass
- Secondary outcomes: NR
Process evaluation: NR

Implementation-related

Theoretical basis: NR

factors

Resources for intervention implementation: NR

Who delivered the intervention: NR

PROGRESS categories assessed at baseline: gender, SES

PROGRESS categories analysed at outcome: NR

Outcomes relating to harms/unintended effects: NR

\begin{tabular}{ll}
\hline Notes & Funding: CNPq (Conselho Nacional de Desenvolvimento Científico eTecnológico) --- process n. \\
& $475959 / 2010-8$
\end{tabular}

\section{Risk of bias}

\begin{tabular}{lll}
\hline Bias & Authors' judgement & Support for judgement \\
\hline $\begin{array}{l}\text { Random sequence genera- } \\
\text { tion (selection bias) }\end{array}$ & Unclear risk & NR \\
\hline $\begin{array}{l}\text { Allocation concealment } \\
\text { (selection bias) }\end{array}$ & High risk & $\begin{array}{l}\text { NR. Baseline differences but only baseline outcome value adjusted for in } \\
\text { analyses }\end{array}$ \\
\hline $\begin{array}{l}\text { Blinding (performance } \\
\text { bias and detection bias) }\end{array}$ & Unclear risk & NR \\
\begin{tabular}{ll} 
All outcomes \\
\hline $\begin{array}{l}\text { Incomplete outcome data } \\
\text { (attrition bias) }\end{array}$
\end{tabular} & High risk & $31 \%$-33\% dropout - no reasons provided, completer analysis \\
$\begin{array}{l}\text { All outcomes } \\
\text { Selective reporting (re- } \\
\text { porting bias) }\end{array}$ & High risk & $\begin{array}{l}\text { Protocol/trial registration documents were unavailable. Weight, BMI and zBMI } \\
\text { post-intervention data NR although measured }\end{array}$ \\
\hline \begin{tabular}{l} 
Other bias \\
\hline $\begin{array}{l}\text { Other bias- timing of re- } \\
\text { cruitment of clusters }\end{array}$
\end{tabular} & Unclear risk & NR \\
\hline
\end{tabular}

Feng 2004

$\begin{array}{ll}\text { Methods } & \text { Study design: cluster-RCT } \\ \text { Intervention period: } 3 \text { years } \\ \text { Follow-up period (post-intervention): nil } \\ \text { Differences in baseline characteristics: reported }\end{array}$


Feng 2004 (Continued)

Reliable outcomes: reported

Protection against contamination: NR

Unit of allocation: kindergarten classes

Unit of analysis: individual

Participants
N (control baseline) $=1118$
N (intervention baseline) $=1120$
N (intervention follow-up) $=1086$
Setting (and number by study group): 21 kindergartens
Recruitment: NR
Geographic region: Huangshi City, Hubei Province, China
Percentage of eligible population enrolled: NR
Mean age: intervention: $3.12 \pm 0.83 ;$ control: $3.10 \pm 0.90$
Sex: Ratio of males to females in intervention gropup 1.09 in control group 1.023

Interventions

To summarise and appraise the validity and feasibility of the effect of the early intervention on children of simple obesity

- Health workshops on how to deal with simple obesity were delivered to kindergarten teachers and all parents in intervention group every year. No details were reported.

- One-by-one face-to-face consultations to obese children and their parents about how to prevent obesity and how to correct relevant unhealthy behaviours.

Diet and physical intervention vs control (no concrete PA or nutrition intervention offered in this study

Outcome measures
- Primary outcome: prevalence of overweight/obesity, incidence of obesity
- Secondary outcomes: NR
Process evaluation: NR

Implementation-related

Theoretical basis: NR

factors

Resources for intervention implementation: NR

Who delivered the intervention: NR

PROGRESS categories assessed at baseline: gender

PROGRESS categories analysed at outcome: NR

Outcomes relating to harms/unintended effects: NR

Intervention included strategies to address diversity or disadvantage: NR

Economic evaluation: NR

Notes

Funding: NR

Review author (G Yang) data extracted this study as it is published in Chinese (English abstract) 
Feng 2004 (Continued)

Risk of bias

\begin{tabular}{lll}
\hline Bias & Authors' judgement & Support for judgement \\
\hline $\begin{array}{l}\text { Random sequence genera- } \\
\text { tion (selection bias) }\end{array}$ & Unclear risk & Divided into 2 groups at random \\
\hline $\begin{array}{l}\text { Allocation concealment } \\
\text { (selection bias) }\end{array}$ & Unclear risk & NR \\
\hline $\begin{array}{l}\text { Blinding (performance } \\
\text { bias and detection bias) } \\
\text { All outcomes }\end{array}$ & Unclear risk & NR \\
\hline $\begin{array}{l}\text { Incomplete outcome data } \\
\text { (attrition bias) } \\
\text { All outcomes }\end{array}$ & Low risk & 1086 out of 1120 (97.0\%) in intervention group and 1074 out of 1118 (96.1\%) \\
\hline $\begin{array}{l}\text { Selective reporting (re- } \\
\text { porting bias) }\end{array}$ & Unclear risk & Protocol/trial registration document were unavailable \\
\hline \begin{tabular}{l} 
Other bias \\
\hline $\begin{array}{l}\text { Other bias- timing of re- } \\
\text { cruitment of clusters }\end{array}$
\end{tabular} & Low risk & No additional threats to validity \\
\hline
\end{tabular}

Fitzgibbon 2005

Methods
Study design: cluster-RCT
Intervention period: 14 weeks
Follow-up period (post-intervention): 2 years
Differences in baseline characteristics: reported
Reliable outcomes: reported
Protection against contamination: NR
Unit of allocation: preschool
Unit of analysis: individual
To assess possible bias in results because of children leaving school or missing anthropometric data at
a specific follow-up, 2 additional analyses were conducted in which study authors imputed BMI 1 and 2
years post-intervention from prior (baseline, post-intervention, or Year 1 ) or subsequent (Year 2 ) values
of BMI.

\footnotetext{
Participants

$\mathrm{N}($ controls baseline $)=212$

$\mathrm{N}$ (controls follow-up) = post-intervention $(\mathrm{N}=183) ; 1$-year follow-up $(\mathrm{N}=146) ; 2$-year follow-up $(\mathrm{N}=$ 154)

$\mathrm{N}$ (interventions baseline $)=197$

$\mathrm{N}$ (interventions follow-up) = post-intervention ( $N=179)$; 1-year follow-up ( $N=143) ;$ 2-year follow-up ( $N$ =146)
} 
Fitzgibbon 2005 (Continued)

Setting (and number by study group): preschools (intervention $N=6$; control $N=6$ )

Recruitment: 12 Head Start sites administered through the Archdiocese of Chicago and that served primarily African-American children were recruited to participate. All children at these sites were eligible to participate.

Geographic region: Chicago, USA

Percentage of eligible population enrolled: NR

Mean age: intervention, $48.6 \pm 7.6$ months; control, $50.8 \pm 6.4$ months

Sex: intervention, $49.7 \%$ female; control, $50.5 \%$ female

Child intervention:

- 14 weeks ( 3 times/week) of a diet/PA intervention delivered by trained early childhood educators

- Each session included:

- 20 min nutrition activity reflecting the food pyramid

- 20 min aerobic activity based on overall moderate/vigorous movement

Parent intervention:

- Received weekly newsletters that mirrored the children's curriculum

- Accompanying homework assignments $(N=12)$ designed to be an interactive activity between children and parents. Parents received a small monetary incentive for completing and returning homework.

Control intervention:

- 14-week (once a week) curriculum that taught general health concepts such as seat belt safety, immunisation and dental health.

- Parents received weekly newsletters that mirrored the curriculum, but no homework assignments

Combined effects of dietary interventions and PA interventions vs control

Outcomes

- Primary: change in BMI from baseline to Year 1 post-intervention and Year 2 post-intervention

- Secondary:

- dietary intake

- PA

$\circ$ TV viewing

Process evaluation: reported

Implementation-related factors
Theoretical basis: reported (SCT as the primary framework, and concepts from Self-determination theory)

Resources for intervention implementation (e.g. funding needed or staff hours required): reported Who delivered the intervention: reported

PROGRESS categories assessed at baseline: reported (gender, race, education)

PROGRESS categories analysed at outcome: NR

Outcomes relating to harms/unintended effects: reported

Intervention included strategies to address diversity or disadvantage: reported

Economic evaluation: NR 
Fitzgibbon 2005 (Continued)

Intervention design reported in secondary reference for Fitzgibbon 2005 (Fitzgibbon et al Preventive Medicine 2002;34:289-97).

This study is linked with results reported for another 12 preschools servicing Latino communities in Fitzgibbon 2006.

\section{Risk of bias}

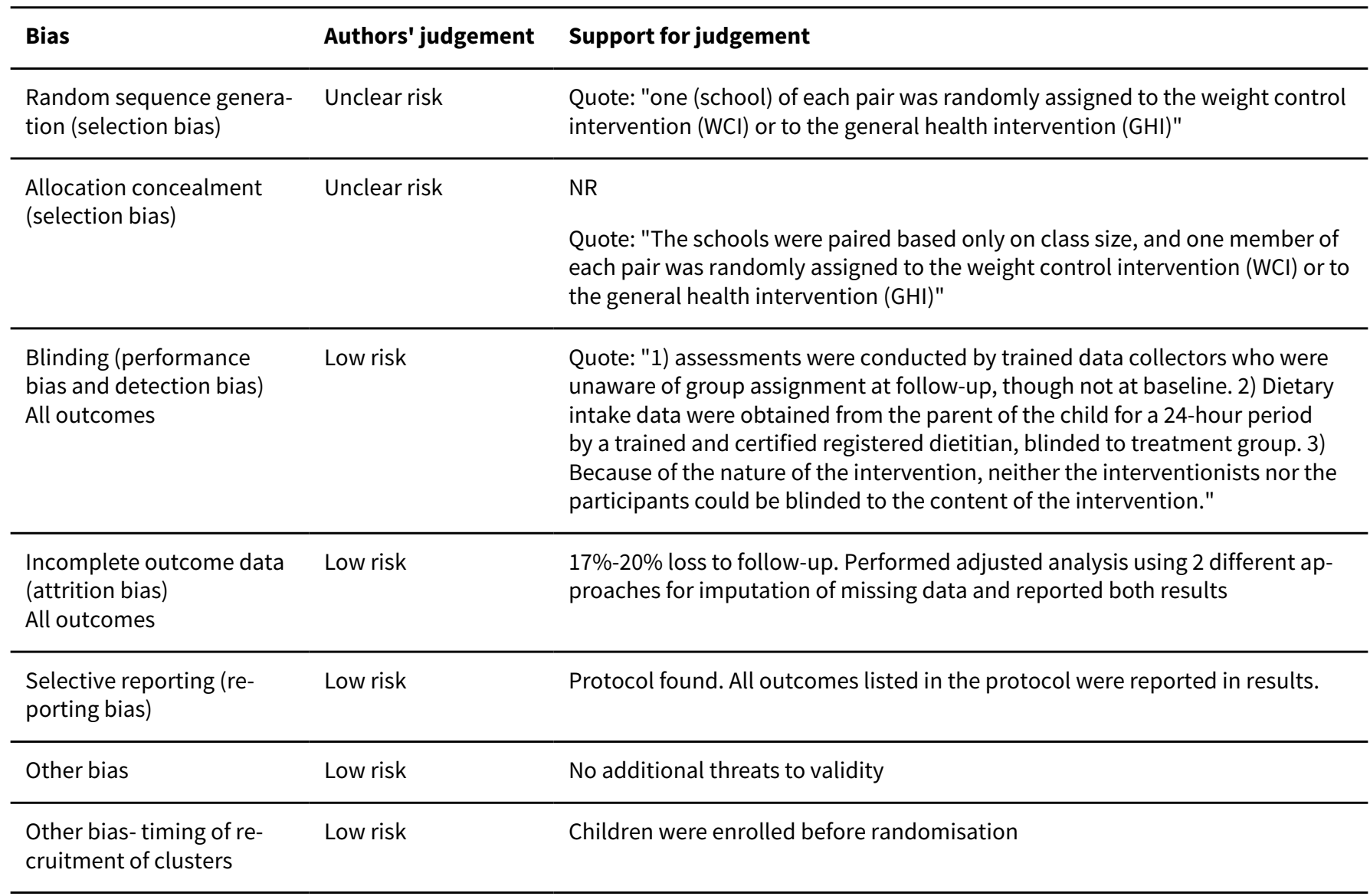

\section{Fitzgibbon 2006}

\section{Methods}

Study design: cluster-RCT

Intervention period: 14 weeks

Follow-up period (post-intervention): 2 years

Differences in baseline characteristics: reported

Reliable outcomes: reported

Protection against contamination: NR

Unit of allocation: preschool

Unit of analysis: individual

All analyses were performed according to ITT principles

Participants N(controls baseline $)=199$


Fitzgibbon 2006 (Continued)

$\mathrm{N}$ (controls follow-up) = post-intervention $(\mathrm{N}=193)$; 1-year follow-up $(\mathrm{N}=165) ;$ 2-year follow-up $(\mathrm{N}=$ 165)

$N$ (interventions baseline $)=202$

$\mathrm{N}$ (interventions follow-up) = post-intervention ( $N=196)$; 1-year follow-up ( $N=178) ; 2$-year follow-up ( $N$ $=176)$

Setting (and number by study group): preschools (intervention $\mathrm{N}=6$; control $\mathrm{N}=6$ )

Recruitment: 12 Head Start sites administered through the Archdiocese of Chicago and that served primarily Latino children were recruited to participate. All children at these sites were eligible to participate.

Geographic region: Chicago, USA

Percentage of eligible population enrolled: NR

Mean age: intervention: $50.8 \pm 7.3$ months; control: $51.0 \pm 7.0$ months

Sex: intervention: $47.5 \%$ female; control: $51.3 \%$ female

Interventions

Child intervention:

- 14 weeks (3 times/week) of a diet/PA intervention delivered by trained early childhood educators.

- Each session included:

- 20 min nutrition activity reflecting the food pyramid

- 20 min aerobic activity based on overall moderate/vigorous movement

- Curriculum was linguistically and culturally appropriate and delivered in both Spanish and English

Parent intervention:

- Received weekly newsletters that mirrored the children's curriculum

- Accompanying homework assignments $(N=12)$ designed to be an interactive activity between children and parents. Parents received a small monetary incentive for completing and returning homework.

Control intervention:

- 14 week (once a week) curriculum that taught general health concepts such as seat belt safety, immunisation and dental health

- Parents received weekly newsletters that mirrored the curriculum, but no homework assignments

Combined effects of dietary interventions and PA interventions vs control

Outcomes

- Primary: change in BMI from baseline to Year 1 post-intervention and Year 2 post-intervention

- Secondary:

- dietary intake

- PA

- TV viewing

Process evaluation: reported

Implementation-related factors
Theoretical basis: SCT

Resources for intervention implementation (e.g. funding needed or staff hours required): reported Who delivered the intervention: reported

PROGRESS categories assessed at baseline: reported (gender, race, education)

PROGRESS categories analysed at outcome: NR 
Fitzgibbon 2006 (Continued)

Outcomes relating to harms/unintended effects: reported

Intervention included strategies to address diversity or disadvantage: reported

Economic evaluation: NR

Funding: supported by a grant from the National Heart, Lung, and Blood Institute (Grant HL58871)
Intervention design reported in secondary reference for Fitzgibbon 2005 (Fitzgibbon et al Preventive
Medicine 2002;34:289-97).
This study is linked with results reported for another 12 preschools primarily servicing African-Ameri-
can children in Fitzgibbon 2005.

\section{Risk of bias}

\begin{tabular}{|c|c|c|}
\hline Bias & Authors' judgement & Support for judgement \\
\hline $\begin{array}{l}\text { Random sequence genera- } \\
\text { tion (selection bias) }\end{array}$ & Unclear risk & $\begin{array}{l}12 \text { Head Start sites that were administered through the Archdiocese of Chicago } \\
\text { and that served primarily Latino children were recruited to participate. The } 12 \\
\text { schools were then randomly assigned to the intervention group or the control } \\
\text { group. }\end{array}$ \\
\hline $\begin{array}{l}\text { Allocation concealment } \\
\text { (selection bias) }\end{array}$ & Low risk & NR, but clusters likely assigned simultaneously \\
\hline $\begin{array}{l}\text { Blinding (performance } \\
\text { bias and detection bias) } \\
\text { All outcomes }\end{array}$ & Unclear risk & NR \\
\hline $\begin{array}{l}\text { Incomplete outcome data } \\
\text { (attrition bias) } \\
\text { All outcomes }\end{array}$ & Low risk & $\begin{array}{l}\text { Participant flow provided with numbers missing similar between intervention } \\
\text { and control groups }\end{array}$ \\
\hline $\begin{array}{l}\text { Selective reporting (re- } \\
\text { porting bias) }\end{array}$ & Low risk & Protocol found. All outcomes listed in the protocol were reported in results. \\
\hline Other bias & Low risk & No additional threats to validity \\
\hline $\begin{array}{l}\text { Other bias- timing of re- } \\
\text { cruitment of clusters }\end{array}$ & Low risk & $\begin{array}{l}12 \text { Head Start sites that were administered through the Archdiocese of Chicago } \\
\text { and that served primarily Latino children were recruited to participate. The } 12 \\
\text { schools were then randomly assigned to the intervention group or the control } \\
\text { group. }\end{array}$ \\
\hline
\end{tabular}

Fitzgibbon 2011

$\begin{array}{ll}\text { Methods } & \text { Study design: RCT } \\ & \text { Intervention period: } 14 \text { weeks } \\ & \text { Follow-up period (post-intervention): } 12 \text { months } \\ & \text { Differences in baseline characteristics: reported } \\ & \text { Reliable outcomes: reported } \\ & \text { Protection against contamination: NR } \\ & \text { Unit of allocation: school }\end{array}$


Fitzgibbon 2011 (Continued)

Unit of analysis: individual accounting for class and school

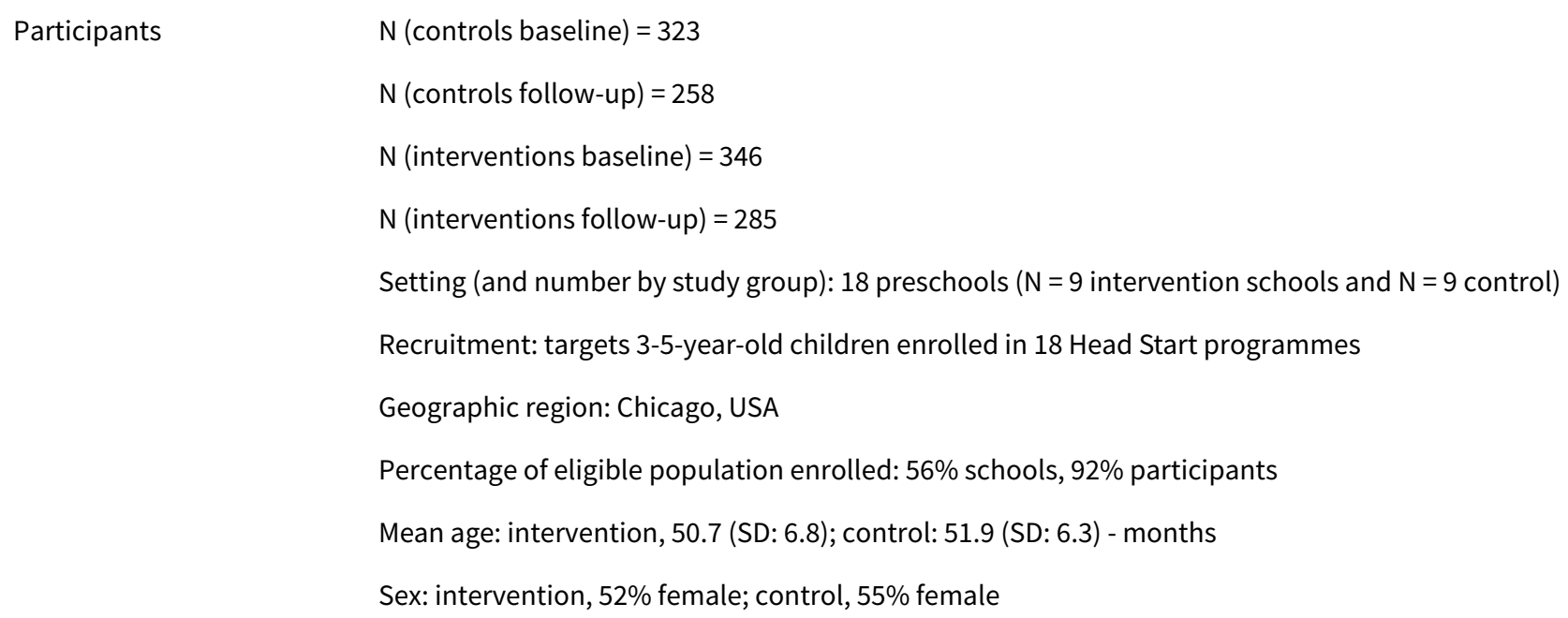
tion delivered by teachers that builds on results of Hip hop to health junior. Adapted curriculum so that teachers were asked to teach 2 weekly sessions, with the option of including a 3rd session if they chose.

Each week focused on a particular theme with a specific objective. Each session included a 20-min lesson related to healthy eating and exercise, as well as a 20- minute PA component. Lessons featured the colourful 'Pyramid Puppets' that represent the 7 food groups of the food pyramid. In addition, the intervention incorporated songs and raps that were included on a CD for teachers to play for their students. The CD also included 2 fully scripted exercise routines.

Parents also received a weekly newsletter that paralleled the children's curriculum in content and included a homework assignment. Parents received USD 5 for each of the homework assignments that they completed and returned. Each parent also received the same $C D$ that the teacher used in the classroom so that the nutrition concepts and importance of PA could be reinforced in the home.

Description of control: 14 weeks long and taught once a week. The children learned a variety of health concepts, including car seat and seat belt safety, immunisations, dental health, and the procedures for calling 911. Parents received a weekly newsletter that mirrored the weekly theme of the school-based curriculum but were not asked to complete homework assignments.

Diet and PA combination intervention vs control

- Primary outcome: BMI and zBMI

- Secondary outcomes: dietary intake, PA, screen time

Process evaluation: NR

Implementation-related factors
Theoretical basis: SCT, Self-Determination theory

Resources for intervention implementation: reported

Who delivered the intervention: reported

PROGRESS categories assessed at baseline: gender, race/ethnicity; parent: gender, education, SES (income), occupation, social status (marital)

PROGRESS categories analysed at outcome: NR

Outcomes relating to harms/unintended effects: NR 
Fitzgibbon 2011 (Continued)

Intervention included strategies to address diversity or disadvantage: no, but study targeted at low-income, black minority children. Also cultural modifications such as addressing environmental considerations (social support, unsafe neighbourhoods, economic restrictions, conflicting responsibilities)

Economic evaluation: NR

Notes Funding: the Hip-hop to health obesity prevention effectiveness trial was supported by the National Heart, Lung and Blood Institute (HL081645)

Teacher training: for intervention and control groups the initial training sessions were $3 \mathrm{~h}$. Following the 1st formal session, the intervention co-ordinator conducted 3 in-school training sessions for the intervention teachers and 1 in-school session for the control teachers.

Resources for sessions: any paperwork/booklets (not described), puppets and CDs used in lessons, weekly newsletter for parents, CD for parents (same as used by teacher in classroom to convey nutrition concepts and importance of PA)

\section{Risk of bias}

\begin{tabular}{lll}
\hline Bias & Authors' judgement & Support for judgement \\
\hline $\begin{array}{l}\text { Random sequence genera- } \\
\text { tion (selection bias) }\end{array}$ & Unclear risk & Randomisation, no further details \\
\hline $\begin{array}{l}\text { Allocation concealment } \\
\text { (selection bias) }\end{array}$ & Unclear risk & NR \\
\hline $\begin{array}{l}\text { Blinding (performance } \\
\text { bias and detection bias) } \\
\text { All outcomes }\end{array}$ & Unclear risk & $\begin{array}{l}\text { Both parents and interviewers were aware of group assignments. No further } \\
\text { details }\end{array}$ \\
\hline $\begin{array}{l}\text { Incomplete outcome data } \\
\text { (attrition bias) } \\
\text { All outcomes }\end{array}$ & Low risk & Low attrition. 18\% to 20\% at 1 year for BMI at 14 weeks \\
\hline $\begin{array}{l}\text { Selective reporting (re- } \\
\text { porting bias) }\end{array}$ & Unclear risk & Protocol/trial registration document were unavailable \\
\hline $\begin{array}{l}\text { Other bias } \\
\begin{array}{l}\text { Other bias- timing of re- } \\
\text { cruitment of clusters }\end{array}\end{array}$ & Low risk & No other potential threats to validity \\
\hline
\end{tabular}

\section{Foster 2008}

\section{Methods}

Study design: cluster-RCT

Intervention period: 2 years

Follow-up period (post-intervention): nil

Differences in baseline characteristics: reported

Reliable outcomes: reported (anthropometry, dietary intake, PA and sedentary behaviour)

Protection against contamination: all schools were under the direction of the district's Food Service Division, which agreed to make the necessary changes in intervention schools, while making no changes to the control schools. 
Foster 2008 (Continued)

Unit of allocation: school

Unit of analysis: individual

Study authors imputed missing data using the multiple imputation procedure with the Markov chain Monte Carlo algorithm as well as the LOCF method for comparison

\section{Participants}

$$
\begin{aligned}
& N(\text { controls baseline })=600 \\
& N(\text { controls follow-up })=365 \\
& N(\text { interventions baseline })=749 \\
& N(\text { interventions follow-up })=479
\end{aligned}
$$

Setting (and number by study group): schools ( $N=5$ intervention, $N=5$ control)

Recruitment: within schools, written parental consent and child assent required

Geographic region: Philadelphia, USA

Percentage of eligible population enrolled: school level: $83 \%$. Across participating schools, consent rate was $70 \% \pm 15 \%$

Mean age: intervention, $11.13 \pm 1$ years; control, $11.2 \pm 1$ years

Sex: intervention, $52 \%$ female; control: $55 \%$ female

School self assessment

- Assessed environments using the CDC School Health Index

- School formed a Nutrition Advisory Group to guide assessment

- Schools subsequently developed an action plan for change with a variety of strategies, e.g. limiting use of food as reward/punishment, fundraising etc

Nutrition education

- $50 \mathrm{~h}$ of food and nutrition education/student/school year based on National Center for Education Statistics guidelines

- Integrated into classroom subjects; integrative and interdisciplinary

Nutrition policy

- All food sold and served in the schools was changed to meet the nutritional standards based on dietary guidelines for Americans

Social marketing

- Several techniques: raffle tickets; slogan and character development

Family/parent outreach

- Home and school association meetings, report card nights, parent education meetings, weekly nutrition workshops. Parent challenges re PA and healthy eating.

- Schools encouraged parents to send healthy foods and discouraged unhealthy foods

Staff training

- All school staff offered 10 hours/year of training in nutrition education to receive curricula and supporting materials e.g. Planet Health and Know your body, and curriculum lesson packets etc

Combined effects of dietary interventions and PA interventions vs control 
Foster 2008 (Continued)

- Incidence of overweight and obesity
- Prevalence and remission of overweight and obesity
- Dietary intake and PA
- Sedentary behaviours
- Potential adverse effects
Process evaluation: NR

Implementation-related factors
Theoretical basis: settings-based approach; CDC Guidelines to Promote Lifelong Healthy Eating and PA Resources for intervention implementation (e.g. funding needed or staff hours required): NR

Who delivered the intervention: reported

PROGRESS categories assessed at baseline: reported (race, gender, SES)

PROGRESS categories analysed at outcome: reported (race, gender)

Outcomes relating to harms/unintended effects: reported

Intervention included strategies to address diversity or disadvantage: NR

Economic evaluation: NR
Funding: this study was supported by grants from the CDC (R06/CCR321534-01) and the US Department of Agriculture/Food and Nutrition Service through the Pennsylvania Nutrition Education Program as part of Food Stamp Nutrition Education

\section{Risk of bias}

\begin{tabular}{lll}
\hline Bias & Authors' judgement & Support for judgement \\
\hline $\begin{array}{ll}\text { Random sequence genera- } \\
\text { tion (selection bias) }\end{array}$ & Unclear risk & NR \\
& & $\begin{array}{l}\text { Quote: "the schools were randomly assigned as intervention or control } \\
\text { schools." }\end{array}$ \\
\hline
\end{tabular}

Allocation concealment Unclear risk NR

(selection bias)

Quote: "Schools within each cluster were approached to participate in a predetermined, random order. When 2 schools in each cluster agreed to participate, the schools were randomly assigned as intervention or control schools."

\begin{tabular}{lll}
\hline $\begin{array}{l}\text { Blinding (performance } \\
\text { bias and detection bias) } \\
\text { All outcomes }\end{array}$ & Unclear risk & $\begin{array}{l}\text { Heights and weights were measured annually on a digital scale and wall- } \\
\text { mounted stadiometer by a trained research team with a standardised proto- } \\
\text { col. The team was not blinded to treatment condition. }\end{array}$ \\
\hline $\begin{array}{l}\text { Incomplete outcome data } \\
\text { (attrition bias) }\end{array}$ & Low risk & $\begin{array}{l}\text { Clusters not lost and individual dropout NR but they did imputations and sen- } \\
\text { sitivity analysis. }\end{array}$ \\
& $\begin{array}{l}\text { Quote: "To account for attrition at the student level, we imputed } \\
\text { missing data at year } 2 \text { using the multiple imputation (MI) procedure with the } \\
\text { Markov chain Monte Carlo algorithm.....In addition, to assess the consistency } \\
\text { of our findings, data were analysed using the more conventional baseline car- } \\
\text { ried forward and last observation carried forward methods." }\end{array}$
\end{tabular}

\begin{tabular}{lll}
\hline $\begin{array}{l}\text { Selective reporting (re- } \\
\text { porting bias) }\end{array}$ & Unclear risk & Protocol/trial registration document were unavailable \\
\hline Other bias & Low risk & No additional threats to validity. \\
\hline
\end{tabular}


Foster 2008 (Continued)

Other bias- timing of recruitment of clusters
Low risk Recruitment happened before randomisation.

Quote: "Schools within each cluster were approached to participate in a predetermined, random order. When 2 schools in each cluster agreed to participate, the schools were randomly assigned as intervention or control schools."

French 2011

\begin{tabular}{|c|c|}
\hline \multirow[t]{9}{*}{ Methods } & Study name: Take action \\
\hline & Study design: cluster-RCT \\
\hline & Intervention period: 1 year \\
\hline & Follow-up period (post-intervention): nil \\
\hline & Differences in baseline characteristics: NR \\
\hline & Reliable outcomes: yes \\
\hline & Protection against contamination: NR \\
\hline & Unit of allocation: household \\
\hline & Unit of analysis: household and individual accounting for cluster \\
\hline \multirow[t]{10}{*}{ Participants } & $\mathrm{N}$ (controls baseline) $=45$ households \\
\hline & $\mathrm{N}$ (controls follow-up) $=44$ households \\
\hline & $\mathrm{N}$ (interventions baseline) $=45$ households \\
\hline & $\mathrm{N}$ (interventions follow-up) $=43$ households \\
\hline & $\begin{array}{l}\text { Setting (and number by study group): } 90 \text { households, } 158 \text { adults, } 75 \text { adolescents aged } 12-17 \text { years, } 84 \\
\text { children aged } 5-11 \text { years, and } 23 \text { children }<5 \text { years. This publication reports outcomes only for the ado- } \\
\text { lescents (and adults). }\end{array}$ \\
\hline & $\begin{array}{l}\text { Recruitment: community (libraries, work sites, schools, daycare centres, health clinics, religious institu- } \\
\text { tions, parks, health clinics, grocery stores etc. }\end{array}$ \\
\hline & Geographic region: Minnesota, USA \\
\hline & Percentage of eligible population enrolled: $31 \%$ (randomised households) \\
\hline & Mean age: adolescents range $12-17$ years \\
\hline & Sex: NR \\
\hline
\end{tabular}

The intervention included both household environment and individual - level behavioural components. The household environment intervention included:

- placement of TV time-limiting devices on all household TV sets;

- provision of guidelines about household food availability;

- provision of a home scale for daily self-weighing (adults only)

The individual behavioural intervention component promoted specific individual behaviour changes related to weight control that were consistent with the 
French 2011 (Continued)

household-level intervention.

The intervention was delivered using $6 \mathrm{x}$ monthly (first 6 months) face-to-face group meetings (at the University), telephone calls, and monthly newsletters. Control households received no intervention.

Diet and PA combination intervention vs control

\begin{tabular}{|c|c|}
\hline \multirow[t]{2}{*}{ Outcomes } & $\begin{array}{l}\text { Outcome measures } \\
\text { - Primary outcome: household mean zBMI (but reports adolescent zBMI) } \\
\text { - Secondary outcomes: eating behaviours, dietary intake, PA, TV viewing, dollars per person spent eat- } \\
\text { ing out }\end{array}$ \\
\hline & Process evaluation: reported (participation) \\
\hline
\end{tabular}

Implementation-related factors
Theoretical basis: NR

Resources for intervention implementation: reported

Who delivered the intervention: reported

PROGRESS categories assessed at baseline: household: gender, race/ethnicity, social status (marital), SES (income), education

PROGRESS categories analysed at outcome: NR

Outcomes relating to harms/unintended effects: NR

Intervention included strategies to address diversity or disadvantage: NR

Economic evaluation: NR

Notes

Funding: this study was supported by grant \#1U54CA116849 and \#R21CA137240 from the NIH/National Cancer Institute.

Only cost mentioned was for the USD 25 gift card for local grocery store for those households who attended the group sessions. Various resources (i.e. scales, goal sheets, telephone call time/cost, incentives such as sports balls, had weights gift cards etc).

Intervention participation. Over $73 \%$ of the 45 intervention households attended at least $4 / 6$ face-toface group sessions and completed $\geq 50 \%$ of the home activities. About $20 \%$ of households had perfect attendance and home activity completion rates. Within-household attendance, or the average percent of eligible household members who attended each session, was 59\%. Two-3rds (68\%) of households had $\geq 50 \%$ household members attending sessions, and one- $3 \mathrm{rd}$ of households had $\geq 75 \%$ household members attending sessions. TV-limiting devices were placed in 93\% of intervention households. The average duration the devices were kept attached to the TVs was 10.6 months. Monitors were programmed to a weekly mean of $29.8 \mathrm{~h}$ (range 11-70), a $44 \%$ reduction from baseline ( $52.8 \mathrm{~h}$ weekly). $28 / 42$ households kept the TV monitors on the TV after the end of the study. Session evaluations were administered during the last face-to-face group session. 83\% of the intervention participants rated overall sessions as satisfactory or very satisfactory (on a 5-point scale).

\section{Risk of bias}

\begin{tabular}{lll}
\hline Bias & Authors' judgement & Support for judgement \\
\hline $\begin{array}{l}\text { Random sequence genera- } \\
\text { tion (selection bias) }\end{array}$ & Unclear risk & $\begin{array}{l}\text { Households were randomised following the completion of the 4th week of re- } \\
\text { ceipt annotation }\end{array}$ \\
\hline $\begin{array}{l}\text { Allocation concealment } \\
\text { (selection bias) }\end{array}$ & Unclear risk & NR \\
\hline
\end{tabular}


French 2011 (Continued)

Blinding (performance High risk Households randomised to the control group were informed of their group asbias and detection bias) signment, unlikely that the research staff taking measurements were blinded.

All outcomes

$\begin{array}{ll}\begin{array}{l}\text { Incomplete outcome data } \\ \text { (attrition bias) }\end{array} & \text { Low risk } \\ \text { All outcomes } & \text { NR }\end{array}$

\begin{tabular}{lll}
\hline $\begin{array}{l}\text { Selective reporting (re- } \\
\text { porting bias) }\end{array}$ & Unclear risk & Protocol/trial registration document were unavailable \\
\hline Other bias & High risk & $\begin{array}{l}\text { Of contamination (household is the focus of the intervention, not individual } \\
\text { adolescent) }\end{array}$ \\
\hline $\begin{array}{l}\text { Other bias- timing of re- } \\
\text { cruitment of clusters }\end{array}$ & Low risk & Recruitment happened prior to randomisation. \\
\hline
\end{tabular}

\section{Fulkerson 2010}

Methods

Study name: Healthy home offerings via the mealtime environment (HOME): feasibility, acceptability, and outcomes of a pilot study

Trial design: Pilot RCT

Intervention period: 3 months

Follow-up period (post-intervention): 6 months

Differences in baseline characteristics: Yes (none of the baseline demographic or weight related characteristics differed significantly by condition)

Reliable outcomes: reported (BMI percentiles/BMI z scores) only in text not tabulated (p6) - no baseline data for these reported as not primary aim/outcome as pilot study

Protection against contamination: Not reported

Unit of allocation: Families

Unit of analysis: individual

\begin{tabular}{|c|c|}
\hline \multirow[t]{10}{*}{ Participants } & $\mathrm{N}$ (controls baseline) $=22$ \\
\hline & $\mathrm{N}$ (controls follow-up) $=22$ \\
\hline & $\mathrm{N}$ (interventions baseline) $=22$ \\
\hline & $\mathrm{N}$ (interventions follow-up) $=22$ \\
\hline & $\begin{array}{l}\text { Setting (and number by study group): } 44 \text { families (parent and child dyads) in community centres or } \\
\text { churches, } \mathrm{N}=22 \text { intervention, } \mathrm{N}=22 \text { control families }\end{array}$ \\
\hline & Recruitment: from 2 elementary schools via flyers, school newsletters and small group presentations \\
\hline & Geographic region: Minneapolis, USA \\
\hline & Percentage of eligible population enrolled: $90 \%$ \\
\hline & Mean age: range 8-10 years (intervention + control) \\
\hline & Sex: $52 \%$ female (intervention + control) \\
\hline
\end{tabular}


Fulkerson 2010 (Continued)

Interventions
To develop, implement, and test the feasibility and acceptability of the HOME program.

Intervention comprised a childhood obesity prevention intervention aimed at increasing the quality of foods in the home and at family meals. Each session included a healthy snack, separate parent and child group time, family meal preparation, interactive nutrition education activities, a group meal, homework assignment, take-home materials, and session evaluations. Activities were hands-on and interactive. Parent group time enabled parents to learn from each other in regards to dealing with picky eaters, meal planning, etc.

Child group time included taste-testing, along with learning meal planning and cooking skills. The intervention components at each session focused on a specific topic (e.g. increasing fruits and vegetables).

The intervention programme was implemented by the study authors and trained students.

Sessions were held at rented space in a church and community centre (with kitchen and dining facilities) within close proximity to participants' homes in the early evening (18:00-19:30). Families participated in five 90-min intervention sessions in a multiple family-group format (3-8 families at one time). All family members (other adults and siblings) were encouraged to attend the programme. Babysitting was available for children (<8 years). Each session was offered to families twice at each location within a 2-week period to allow for scheduling flexibility.

Families randomised to the control condition participated in home assessments only and were sent written intervention materials at the end of the study.

Dietary intervention vs control

- Primary outcome: process measures: feasibility, satisfaction, intervention dose (attendance and homework completion) and fidelity (implementation)

- Secondary outcomes: BMI percentiles and zBMI, family dinner frequency, parental self-efficacy, child food preparation skills, home food availability, nutrition quality of foods served at family meals, dietary assessment

Process evaluation: reported

Implementation-related factors
Theoretical basis: SCT

Resources for intervention implementation: reported

Who delivered the intervention: reported

PROGRESS categories assessed at baseline: child: gender, race/ethnicity; parent: gender, race/ethnicity, education, occupation

PROGRESS categories analysed at outcome: NR

Outcomes relating to harms/unintended effects: NR

Intervention included strategies to address diversity or disadvantage: NR

Economic evaluation: NR

Notes

Funding: this study was funded by the NIH (NIDDK R21 DK72997). The funders played no role in the design, implementation or write-up of the study.

\section{Risk of bias}




\section{Fulkerson 2010 (Continued)}

Random sequence genera- Unclear risk Randomised, no further details tion (selection bias)

Allocation concealment $\quad$ Unclear risk
(selection bias)

Blinding (performance High risk NR, unlikely to be blinded outcome measures as collected in home
bias and detection bias)
All outcomes

\begin{tabular}{lll}
\hline $\begin{array}{l}\text { Incomplete outcome data } \\
\text { (attrition bias) } \\
\text { All outcomes }\end{array}$ & Low risk & \\
\hline $\begin{array}{l}\text { Selective reporting (re- } \\
\text { porting bias) }\end{array}$ & Unclear risk & Protocol/trial registration document were unavailable \\
\hline Other bias & Low risk & No additional threats to validity \\
\hline
\end{tabular}

Gentile 2009

$\begin{array}{ll}\text { Methods } & \text { Study design: cluster-RCT } \\ \text { Intervention period: } 8 \text { months (1 academic year) } \\ \text { Follow-up period (post-intervention): } 6 \text { months } \\ \text { Differences in baseline characteristics: reported } \\ \text { Reliable outcomes: reported } \\ \text { Protection against contamination: reported } \\ \text { Unit of allocation: school } \\ \text { Unit of analysis: individual (with adjustment for school) }\end{array}$

$\begin{array}{ll}\text { Participants } & N \text { (controls baseline) }=653 \\ N \text { (controls follow-up) }=619 \text { (post-intervention), } 587 \text { (follow-up) } \\ N \text { (interventions baseline) }=670 \\ N \text { (interventions follow-up) }=582 \text { (post-intervention), } 529 \text { (follow-up) } \\ \text { Setting: school (intervention } N=5 \text {, control } N=5) \\ \text { Recruitment: students in 3rd-5th grade from } 10 \text { schools in two States } \\ \text { Geographic region: USA } \\ \text { Percentage of eligible population enrolled: 63\% } \\ \text { Mean age: intervention: 9.6 (0.9) years; control: 9.6 (0.9) years } \\ \text { Sex: both male and female } \\ \text { - The Switch programme promoted healthy active lifestyles by encouraging students to "Switch what } \\ \text { you do, chew, and view". The specific 'do' 'view', and 'chew' goals were to be active for } \geq 60 \text { min/day, }\end{array}$


Gentile 2009 (Continued)

to limit total screen time to $\leq 2 \mathrm{~h} /$ day, and to eat $\geq 5$ fruits/vegetables/day. The intervention utilised overlapping behavioural and environmental strategies employed at multiple ecological levels.

- Social marketing: the community component was designed to promote awareness of the importance of healthy lifestyles and the prevention of childhood obesity in the targeted communities, and included paid advertising, (e.g. billboards) and unpaid media emphasising the key messages.

- Curriculum: the school curriculum component was designed to reinforce the Switch messages and facilitate the family component of the intervention. Teachers were provided with materials and ways to integrate key concepts into their existing curricula.

- Family: the family component was designed to provide parents (and children) with materials and resources via monthly resource packs sent home to facilitate the adoption of the healthy target behaviours by the family.

Combined effects of dietary interventions and PA interventions vs control

Outcomes - Height and weight, screen time, fruit and vegetable intake, PA (steps)

Process evaluation: NR

Implementation-related factors

\section{Theoretical basis: SEM}

Resources for intervention implementation (e.g. funding needed or staff hours required): NR

Who delivered the intervention: reported

PROGRESS categories assessed at baseline: reported (race, gender)

PROGRESS categories analysed at outcome: reported (gender)

Outcomes relating to harms/unintended effects: NR

Intervention included strategies to address diversity or disadvantage: NR

Economic evaluation: NR

\section{NCT00685555}

Funding: in Lakeville, Minnesota, Switch was sponsored by Medica Foundation, the Healthy and Active America Foundation, and Fairview Health Services. In Cedar Rapids, lowa Switch was sponsored by Cargill, Inc. and the Healthy and Active America Foundation. The Switch program is a programme of the National Institute on Media and the Family, a non-profit organisation. Several of the study authors were employed by the Institute to create the programme or to conduct the research, or consulted with the Institute on the design or analysis.

\section{Risk of bias}

\begin{tabular}{lll}
\hline Bias & Authors' judgement & Support for judgement \\
\hline $\begin{array}{l}\text { Random sequence genera- } \\
\text { tion (selection bias) }\end{array}$ & Unclear risk & $\begin{array}{l}\text { Quote: "Schools were matched within district by enrollment and percent free/ } \\
\text { reduced-cost lunch and then randomly assigned to the experimental (three in } \\
\text { Cedar Rapids and two in Lakeville) or control (three in Cedar Rapids and two in } \\
\text { Lakeville) condition." }\end{array}$ \\
\hline $\begin{array}{l}\text { Allocation concealment } \\
\text { (selection bias) }\end{array}$ & Unclear risk \\
\hline $\begin{array}{l}\text { Blinding (performance } \\
\text { bias and detection bias) } \\
\text { All outcomes }\end{array}$ & Unclear risk & NR \\
\hline
\end{tabular}


Gentile 2009 (Continued)

Incomplete outcome data Unclear risk Cluster NR lost; individual numbers don't match between CONSORT figure and (attrition bias) baseline data table 1 . based on figure 1 , the loss is $21 \%$ in intervention and All outcomes $10 \%$ in control.

Selective reporting (re- Low risk Protocol seen; all outcomes specified in methods were reported in results
porting bias)

\begin{tabular}{lll}
\hline Other bias & Low risk & No additional threats to validity \\
\hline $\begin{array}{l}\text { Other bias- timing of re- } \\
\text { cruitment of clusters }\end{array}$ & Low risk & Figure suggests recruitment happened prior to randomisation. \\
\hline
\end{tabular}

\section{Gortmaker 1999a}

\begin{tabular}{|c|c|}
\hline Methods & $\begin{array}{l}\text { Study design: cluster-RCT } \\
\text { Follow-up: over } 2 \text { school years (18 months) } \\
\text { Differences in baseline characteristics: reported } \\
\text { Reliable outcomes: self-report outcome measures were developed or modified from existing mea- } \\
\text { sures. If not designed for youth sample the measures were validated for use in this sample. } \\
\text { Protection against contamination: not clear } \\
\text { Unit of allocation: school } \\
\text { Unit of analysis: child } \\
\text { Unit of analysis errors addressed } \\
\text { All analyses were performed according to ITT principles. Also used indicator variables with mean sub- } \\
\text { stitution to control for missing behavioural data and re-estimated regressions that excluded observa- } \\
\text { tions with missing data for sensitivity analyses. }\end{array}$ \\
\hline Participants & $\begin{array}{l}N \text { (intervention follow-up) }=641 \\
N \text { (control follow-up) }=654 \\
\text { Outcome data collected for: } 82 \% \text { of baseline N enrolled: }(81 \% \text { intervention and } 82 \% \text { control) } \\
65 \% \text { of eligible population }=1560 \\
\text { N participants: } 1295 \\
\text { N of schools: } 10 \\
\text { Setting: school } \\
\text { Geographic region: Massachusetts, USA } \\
\text { Age: mean age } 11.7 \text { years } \\
\text { Sex: } 48 \% \text { girls }\end{array}$ \\
\hline Interventions & $\begin{array}{l}\text { School-based interdisciplinary intervention utilising the school curriculum and existing school teachers } \\
\text { to promote } 4 \text { major subjects and PE. Sessions focused on decreasing TV viewing, decreasing consump- } \\
\text { tion of high-fat foods, increasing fruit and vegetable consumption and increasing MVPA } \\
\text { Control programme NR, presumably usual school curriculum } \\
\text { Combined effects of dietary interventions and PA interventions vs control }\end{array}$ \\
\hline Outcomes & $\begin{array}{l}\text { - } \text { BMI } \\
\text { - } \text { Friceps skinfold } \\
\text { - } 11 \text {-item TV and video measure } \\
\text { - MVPA (measured by Youth Activity Questionnaire) } \\
\text { - Dietary intake (measured by Food Frequency Questionnaire) including } \\
\circ \% \text { energy from fat and saturated fat } \\
\circ \text { fruit and vegetable intake } \\
\circ \text { total energy intake }\end{array}$ \\
\hline
\end{tabular}


Gortmaker 1999a (Continued)

Process evaluation: reported

Implementation-related factors
Theoretical basis: Behavioural Choice and SCT

Resources for intervention implementation (e.g. funding needed or staff hours required): reported Who delivered the intervention: reported

PROGRESS categories assessed at baseline: reported (race, gender)

PROGRESS categories analysed at outcome: reported (race, gender)

Outcomes relating to harms/unintended effects: reported

Intervention included strategies to address diversity or disadvantage: NR

Economic evaluation: NR

\section{Notes}

Funding: supported in part by grant HD-30780 from the National Institutes of Child Health and Human Developement, Bethesda, Md and Prevention Research Centre Grant U48/CCU115807 from the CDC, Atlanta, Ga.

\section{Risk of bias}

\begin{tabular}{|c|c|c|}
\hline Bias & Authors' judgement & Support for judgement \\
\hline $\begin{array}{l}\text { Random sequence genera- } \\
\text { tion (selection bias) }\end{array}$ & Low risk & Quote: "... were randomly assigned (using a random number table)..." \\
\hline $\begin{array}{l}\text { Allocation concealment } \\
\text { (selection bias) }\end{array}$ & Low risk & $\begin{array}{l}\text { Randomisation was conducted at school level and all were randomised at start } \\
\text { of study. Student intervention status was assigned based on school enrolment. }\end{array}$ \\
\hline $\begin{array}{l}\text { Blinding (performance } \\
\text { bias and detection bias) } \\
\text { All outcomes }\end{array}$ & Low risk & Outcome assessors were blinded \\
\hline $\begin{array}{l}\text { Incomplete outcome data } \\
\text { (attrition bias) } \\
\text { All outcomes }\end{array}$ & Low risk & $\begin{array}{l}\text { Missing data is } 50 \% \text {; balanced across groups and reasons for missing data giv- } \\
\text { en. Analysis done with both imputed missing data (mean substitution) and } \\
\text { without these data and results were similar (data NR) }\end{array}$ \\
\hline $\begin{array}{l}\text { Selective reporting (re- } \\
\text { porting bias) }\end{array}$ & Unclear risk & Protocol/trial registration documents were unavailable. \\
\hline Other bias & Low risk & No additional threats to validity \\
\hline $\begin{array}{l}\text { Other bias- timing of re- } \\
\text { cruitment of clusters }\end{array}$ & Low risk & No CONSORT figure; text indicates recruitment done prior to randomisation \\
\hline
\end{tabular}

\section{Grydeland 2014}

$\begin{array}{ll}\text { Methods } & \text { Study name: Health in adolescents (HEIA) } \\ \text { Study design: cluster-RCT } \\ \text { Intervention period: } 20 \text { months } \\ \text { Follow-up period (post-intervention): nil } \\ \text { Differences in baseline characteristics: reported }\end{array}$


Grydeland 2014 (Continued)

Reliable outcomes: reported

Protection against contamination: reported

Unit of allocation: school

Unit of analysis: individual

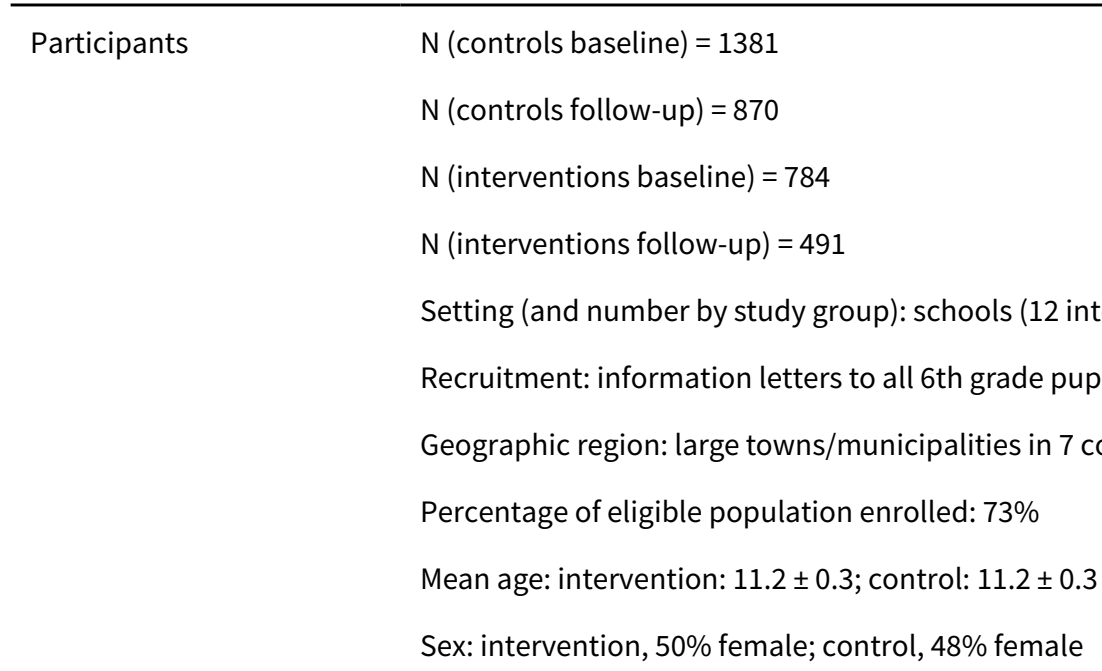

Interventions

To investigate effects of a multi-component school-based intervention programme targeting PA, sedentary and dietary behaviours on anthropometric outcomes

The multilevel intervention included collaboration with school principals and teachers, school-health services and parent committees.

Multiple intervention efforts were orchestrated to promote a healthy diet and to increase awareness of healthy choices, to increase participants' PA during school hours and leisure time, and to reduce screen-time.

In summary, the components of the intervention included:

- Classroom activities led by teachers

- lessons once a month with student booklets

- a fruit and veg break once a week to eat fruit

- posters for the classroom

- PA session once per week

- provision of sports equipment to each class

- active commuting campaigns (18 weeks total)

- computer-tailored individual modules

- Home/parents: monthly fact sheets and brochures on healthy eating and PA recommendations

- Training sessions for teachers and meetings yearly at the school

Diet and PA combined intervention vs control

Outcomes Outcome measures

- Primary outcome: BMI, zBMI, waist to hip ratio, waist circumference

- Secondary outcomes: sedentary behaviours, PA, consumption of fruit, vegetables, sugar-sweetened soft drinks and fruit drinks

Process evaluation: reported 
Resources for intervention implementation: NR

Who delivered the intervention: reported

PROGRESS categories assessed at baseline: child: gender; parent: education

PROGRESS categories analysed at outcome: child: gender; parent: education

Outcomes relating to harms/unintended effects: NR

Intervention included strategies to address diversity or disadvantage: NR

Economic evaluation: NR

Notes

Funding: the study Health in adolescents (HEIA) was funded by the Norwegian Research Council (grant number 175323/V50) with supplementary funds

from the Throne Holst Nutrition Research Foundation, University of Oslo and also from the Norwegian School of Sport Sciences.

Student booklets for classroom activities, posters for the classroom, sports equipment given to each class, monthly fact sheets and brochures to parents, and training material for teachers.

As only $2 \%$ of the variance in BMI and waist circumference was explained by group, they did not adjust for clustering in the analysis. Interaction effects by gender, pubertal status and parental educational level were tested in separate analyses as a 2nd step using 2-way ANCOVA/logistic regressions with the interaction terms as covariates.

\section{Risk of bias}

\begin{tabular}{|c|c|c|}
\hline Bias & Authors' judgement & Support for judgement \\
\hline $\begin{array}{l}\text { Random sequence genera- } \\
\text { tion (selection bias) }\end{array}$ & Unclear risk & Randomly assigned by 'blind draw' \\
\hline $\begin{array}{l}\text { Allocation concealment } \\
\text { (selection bias) }\end{array}$ & Unclear risk & NR \\
\hline $\begin{array}{l}\text { Blinding (performance } \\
\text { bias and detection bias) } \\
\text { All outcomes }\end{array}$ & High risk & Investigators and participants not blinded \\
\hline $\begin{array}{l}\text { Incomplete outcome data } \\
\text { (attrition bias) } \\
\text { All outcomes }\end{array}$ & Low risk & $4 \%$ attrition, equal across groups \\
\hline $\begin{array}{l}\text { Selective reporting (re- } \\
\text { porting bias) }\end{array}$ & Low risk & $\begin{array}{l}\text { Protocol seen; all relevant outcomes were reported. Protocol paper described } \\
\text { a thorough economic evaluation; however this was NR in the full paper and no } \\
\text { reference to a different economic evaluation paper }\end{array}$ \\
\hline Other bias & Unclear risk & Contamination possible \\
\hline $\begin{array}{l}\text { Other bias- timing of re- } \\
\text { cruitment of clusters }\end{array}$ & Low risk & Recriutment happened prior to randomisation \\
\hline
\end{tabular}

Gutin 2008

$\begin{array}{ll}\text { Methods } & \text { Study design: cluster-RCT } \\ \text { Intervention period: } 3 \text { years }\end{array}$


Gutin 2008 (Continued)

Follow-up period (post-intervention): nil

Differences in baseline characteristics: reported

Reliable outcomes: reported

Protection against contamination: NR

Unit of allocation: school

Unit of analysis: individual

All analyses were performed according to ITT principles

\section{Participants}

$\mathrm{N}$ (controls baseline $)=289$

$\mathrm{N}$ (controls 1 year follow-up) $=265$ (for analysis, $\mathrm{N}=265$ )

$\mathrm{N}$ (controls 3 year follow-up) $=168($ for analysis, $\mathrm{N}=168)$

$\mathrm{N}$ (interventions baseline) $=312$

$\mathrm{N}$ (interventions 1 year follow-up) $=260$ (for analysis, $\mathrm{N}=182$ )

$\mathrm{N}$ (interventions 3 year follow-up) $=148$ (for analysis, $N=42$ )

Setting (and number by study group): school ( $N=9$ intervention; $N=9$ control)

Recruitment: all consenting students in participating schools who would be beginning 3rd grade at the start of the intervention.

Geographic region: Augusta/Richmond County, Georgia, USA

Percentage of eligible population enrolled: $52 \%$

Mean age: $8.5 \pm 0.6$ years

Sex: $52 \%$ female

Interventions

2-hour after-school intervention sessions were offered 5 days/week on school days for 3 school years, however students did not have to attend every day to continue in the programme. The programme included:

- 40 min of academic enrichment activities, during which healthy snacks were provided (healthy snacks could be construed as a modest dietary intervention) followed by:

- 80 min of MVPA, which were a variety of activities designed to improve sport skills, aerobic fitness, strength, and flexibility, and 40 min were devoted to vigorous PA. The activities were designed to be mastery-oriented rather than competitive.

Control group received regular health screenings and diet/PA information.

PA interventions vs control

Outcomes

- \% body fat, bone density, fat mass, fat-free soft tissue, BMI, waist circumference, CV fitness, CV risk factors (total cholesterol, HDL cholesterol, resting blood pressure), self-reported free-living PA, PA enjoyment, motivation for PA, perceived competence, goal orientation

For reported outcomes at 1 year and 3 years, participants who stayed in the same schools for the intervention period and who returned for all measurements were included. Of these, control participants were compared with intervention participants who had an adequate exposure to the intervention, as indicated by $? 40 \%$ attendance at the after-school sessions ( $\mathrm{N}$ for analysis reported above).

Process evaluation: reported 
Gutin 2008 (Continued)

Implementation-related factors
Theoretical basis: environmental change

Resources for intervention implementation (e.g. funding needed or staff hours required): reported Who delivered the intervention: reported

PROGRESS categories assessed at baseline: reported (race, gender, education, SES)

PROGRESS categories analysed at outcome: NR

Outcomes relating to harms/unintended effects: NR

Intervention included strategies to address diversity or disadvantage: reported

Economic evaluation: NR

Notes

Funding: this project was funded by the NIH (RO1DK93361)

Data extracted from 4 publications (see secondary references for Gutin 2008):

Yin et al. Eval Health Prof 2005;28:67 (intervention rationale, design, process and implementation factors)

Yin et al. Obes Res 2005;13:2153 (1 year outcomes)

Yin et al. Int J Obes 2005;29:S40 (1 year outcomes: post-hoc analysis of dose response relationship between outcomes and level of programme attendance)

Gutin et al. Int J Ped Obes 2008 (3 year outcomes)

\section{Risk of bias}

\begin{tabular}{|c|c|c|}
\hline Bias & Authors' judgement & Support for judgement \\
\hline $\begin{array}{l}\text { Random sequence genera- } \\
\text { tion (selection bias) }\end{array}$ & Low risk & Sequence generated using random number table \\
\hline $\begin{array}{l}\text { Allocation concealment } \\
\text { (selection bias) }\end{array}$ & High risk & $\begin{array}{l}\text { Performed recruitment over } 2 \text { periods. During the } 2 \text { nd recruitment period, par- } \\
\text { ents/students were informed of intervention assignment of school. Found no } \\
\text { interaction effect of time of consent on primary outcome variables. }\end{array}$ \\
\hline $\begin{array}{l}\text { Blinding (performance } \\
\text { bias and detection bias) } \\
\text { All outcomes }\end{array}$ & High risk & Participants and schools were not blind \\
\hline $\begin{array}{l}\text { Incomplete outcome data } \\
\text { (attrition bias) } \\
\text { All outcomes }\end{array}$ & High risk & $\begin{array}{l}\text { Dropout at } 3 \text { years NR; } 1 \text {-year individual loss was overall } 15 \% \text { with } 20 \% \text { loss in } \\
\text { intervention and } 10 \% \text { loss in control; analysis was not ITT. }\end{array}$ \\
\hline $\begin{array}{l}\text { Selective reporting (re- } \\
\text { porting bias) }\end{array}$ & Low risk & Protocol seen; all outcomes from the protocol are in papers \\
\hline Other bias & Low risk & No additional threats to validity \\
\hline $\begin{array}{l}\text { Other bias- timing of re- } \\
\text { cruitment of clusters }\end{array}$ & Unclear risk & No information on the timing of recruitment in relation to randomisation \\
\hline
\end{tabular}


Habib-Mourad 2014

\section{Methods}

Study name: Health-E-PALS

Study design: cluster-RCT

Intervention period: 12 weeks

Follow-up period (post-intervention): nil

Differences in baseline characteristics: reported

Reliable outcomes: reported

Protection against contamination: NR

Unit of allocation: school

Unit of analysis: individual accounting for cluster

\title{
Participants
}

\author{
$\mathrm{N}($ control baseline $)=181$ \\ $\mathrm{N}$ (control follow-up) $=175$ \\ $\mathrm{N}$ (intervention baseline) $=193$ \\ $\mathrm{N}$ (intervention follow-up) $=188$
}

Setting (and number by study group): 4 private and 4 public schools ( 2 each in each group)

Recruitment: schools were purposively selected to include socioeconomically and religiously diverse catchment areas.

Geographic region: Beirut, Lebanon

Percentage of eligible population enrolled: all students in Grades 4 and 5 (aged 9-11 years) were invited to take part

Mean age: intervention: $10.3 \pm 0.9$; control: $10.1 \pm 1.0$

Sex: intervention: $43 \%$ female; control: $57 \%$ female

To evaluate a pilot multi-component school intervention that is culturally appropriate to promote healthy eating and PA among children aged 9-11 years.

The intervention specifically targeted obesity-related behaviours in 9-11 year olds including: increasing consumption of fruits and vegetables, favouring healthy over high-energy-dense snacks and drinks, increasing the habit of having breakfast daily, increasing MVPA, and decreasing overall sedentary behaviour. 45-minute classroom sessions were delivered each week for 12 weeks. Classroom sessions were delivered mainly by the 1st study author, a specialist in community nutrition, with the support of 1 research assistant who is also a nutritionist.

Several co-ordinated components as follows:

- 12 culturally appropriate classroom sessions using fun and interactive activities were delivered once a week for 3 consecutive months. The activities were incorporated into the school curriculum.

- At the end of the intervention, the teachers received extensive 2-day training with the complete educational kit and teachers' manual, to be able to implement the sessions later on.

- A family programme consisting of meetings, health fairs as well as information packets was sent home along with some food samples and recipes 
Habib-Mourad 2014 (Continued)

- A 'Health-E-PALS' educational Kit with the following

- classroom posters (10)

- take-home pamphlets (12 for each student)

- food diary booklet (1 for each student)

- PA booklet (1 for each student)

- set of 60 food cards

- board game: treasure game

- traffic lights signs

- food counter box (1 for each student)

- pedometers ( 1 for each student)

A food service intervention targeting the school shops and the lunch boxes sent by the family (recommendations concerning the healthy list of snacks and drinks that should be available to children in the shop were provided to shop administrators. Posters encouraging healthy food choices were posted at the points of sales whenever possible).

Students in the control schools received their usual curriculum during the intervention period.

Diet and PA combination intervention vs control

Outcome measures
- Primary outcome: dietary habits, PA, screen time, knowledge, self-efficacy, BMI, waist circumference
- Secondary outcomes: NR
Process evaluation: reported (implementation, dose, context)

Implementation-related factors

Theoretical basis: SCT

Resources for intervention implementation: reported

Who delivered the intervention: NR

PROGRESS categories assessed at baseline: gender

PROGRESS categories analysed at outcome: NR

Outcomes relating to harms/unintended effects: NR

Intervention included strategies to address diversity or disadvantage: adapted to the culture of Lebanese and Arab populations

Economic evaluation: NR

Notes Funding: this research was funded by an Eastern Mediterranean Regional Office Special Grant for Research in Priority Areas of Public Health (EMRO/WHO)

Failure to succeed in modifying the school's food environment due to lobbying and lack of support of some of the school authorities. Lebanon is a politically unstable context, with security threats and social unrests.

\section{Risk of bias}

\begin{tabular}{lll}
\hline Bias & Authors' judgement & Support for judgement \\
\hline $\begin{array}{l}\text { Random sequence genera- } \\
\text { tion (selection bias) }\end{array}$ & Low risk & $\begin{array}{l}\text { Coin toss used to randomise schools. } \\
\text { Quote: "Then, within each matched pair, one school was randomly assigned } \\
\text { (by the toss of a coin) to receive the intervention, and the other school served } \\
\text { as the control. Ultimately, four schools received the intervention (2 private and } \\
2 \text { public) and four others were control schools." }\end{array}$ \\
\hline
\end{tabular}


Habib-Mourad 2014 (Continued)

$\begin{aligned} & \text { Allocation concealment } \\ & \text { (selection bias) }\end{aligned}$ High risk, but assume 'High risk' as coin tossing is easily subverted.

Blinding (performance $\quad$ Unclear risk
bias and detection bias)
All outcomes

Incomplete outcome data Low risk Very low attrition (3\%) balanced in each group
(attrition bias)
All outcomes

\begin{tabular}{lll}
\hline $\begin{array}{l}\text { Selective reporting (re- } \\
\text { porting bias) }\end{array}$ & Unclear risk & Protocol/trial registration document were unavailable \\
\hline Other bias & Low risk & No additional threats to validity \\
\hline $\begin{array}{l}\text { Other bias- timing of re- } \\
\text { cruitment of clusters }\end{array}$ & Low risk & Figure suggests that recruitment happened prior to randomisation. \\
\hline
\end{tabular}

Haerens 2006

\begin{tabular}{|c|c|}
\hline \multirow[t]{8}{*}{ Methods } & Study design: cluster-RCT \\
\hline & Intervention period: 2 school years \\
\hline & Follow-up period (post-intervention): nil \\
\hline & Differences in baseline characteristics: reported \\
\hline & Reliable outcomes: reported \\
\hline & Protection against contamination: NR \\
\hline & Unit of allocation: school \\
\hline & Unit of analysis: individual \\
\hline \multirow[t]{9}{*}{ Participants } & $\mathrm{N}$ (baseline) = 2840 (not available by condition) \\
\hline & $N($ controls follow-up) = 1452 \\
\hline & $\mathrm{N}$ (interventions follow-up) $=554$ \\
\hline & $\begin{array}{l}\text { Setting: schools (intervention: } 10 \text { ( } 5 \text { standard intervention, } 5 \text { standard intervention + parent support), } \\
\text { control: 5) }\end{array}$ \\
\hline & $\begin{array}{l}\text { Recruitment: students in } 7 \text { th and 8th grades from schools with technical and vocational education in } \\
\text { West-Flanders }\end{array}$ \\
\hline & Geographic region: Belgium \\
\hline & Percentage of eligible population enrolled: $95 \%$ \\
\hline & Mean age: $13.1(0.8)$ years (no breakdown by condition) \\
\hline & Sex: both male and female \\
\hline \multirow[t]{2}{*}{ Interventions } & 2 intervention groups: \\
\hline & - standard intervention \\
\hline
\end{tabular}


Haerens 2006 (Continued)

- standard intervention + parent involvement

Standard intervention

- School work group

- Received background information and guidelines on how to address intervention topics

- Intervention manual and educational materials

- planning and review meetings every 3 months (1-h)

- schools promoted students being physically active during breaks, at noon or during after-school hours

- resources and sports equipment made available for students

- child physical fitness test

- computer-tailored intervention advice for PA and reducing fat intake

- School promotions, social marketing and educational strategies that focused on 3 behavioural changes

- increasing fruit consumption to at least 2 pieces a day

- reducing soft drink consumption and increasing water consumption to $1.5 \mathrm{~L} /$ day

- reducing fat intake

Parent involvement

- Social marketing and educational materials via school papers and newsletters

- CD with the adult computer-tailored intervention for fat intake and PA

- Encouraged to discuss intervention with children and create supportive home environment for behaviour change

Combined effects of dietary interventions and PA interventions vs control

\begin{tabular}{ll}
\hline Outcomes & zBMI \\
& - PA (questionnaire and accelerometry for a subset of students) \\
& Diet (fat intake, fruit, water and soft drinks; questionnaire) \\
Process evaluation: reported
\end{tabular}

Implementation-related factors

\section{Theoretical basis: reported (TPB, TTM)}

Resources for intervention implementation (e.g. funding needed or staff hours required): reported

Who delivered the intervention: reported

PROGRESS categories assessed at baseline: reported (gender, SES)

PROGRESS categories analysed at outcome: reported (gender)

Outcomes relating to harms/unintended effects: NR

Intervention included strategies to address diversity or disadvantage: NR

Economic evaluation: NR
Funding: this study was supported by the Policy Research Centre Sport, PA and Health funded by the Flemish Government

\section{Risk of bias}

\begin{tabular}{lll}
\hline Bias & Authors' judgement & Support for judgement \\
\hline $\begin{array}{l}\text { Random sequence genera- } \\
\text { tion (selection bias) }\end{array}$ & Unclear risk & $\begin{array}{l}\text { Quote: "The } 15 \text { schools were randomly assigned to the intervention or control } \\
\text { conditions" }\end{array}$ \\
\hline
\end{tabular}


Haerens 2006 (Continued)

\begin{tabular}{lll}
$\begin{array}{l}\text { Allocation concealment } \\
\text { (selection bias) }\end{array}$ & Unclear risk & \\
\hline $\begin{array}{l}\text { Blinding (performance } \\
\text { bias and detection bias) }\end{array}$ & Unclear risk & NR \\
All outcomes & &
\end{tabular}

\begin{tabular}{lll}
\hline $\begin{array}{l}\text { Incomplete outcome data } \\
\text { (attrition bias) }\end{array}$ & High risk & 25\% overall dropout, NR by group \\
$\begin{array}{ll}\text { All outcomes } \\
\text { Quote: "Pupils not participating at follow-up were significantly older and con- } \\
\text { sumed significantly more soft drinks than pupils participating at follow-up." }\end{array}$ & Protocol/trial registration document were unavailable \\
$\begin{array}{l}\text { Selective reporting (re- } \\
\text { porting bias) }\end{array}$ & Unclear risk & No additional threats to validity \\
\hline $\begin{array}{l}\text { Other bias } \\
\text { Other bias- timing of re- }\end{array}$ & Unclear risk & NR \\
\hline cruitment of clusters & &
\end{tabular}

Haines 2013

\begin{tabular}{|c|c|}
\hline \multirow[t]{9}{*}{ Methods } & Study name: Healthy habits, happy homes \\
\hline & Study design: RCT \\
\hline & Intervention period: 6 months \\
\hline & Follow-up period (post-intervention): nil \\
\hline & Differences in baseline characteristics: reported \\
\hline & Reliable outcomes: reported \\
\hline & Protection against contamination: NR \\
\hline & Unit of allocation: parent-child dyads \\
\hline & Unit of analysis: individual \\
\hline \multirow[t]{9}{*}{ Participants } & $\mathrm{N}$ (control baseline $)=59$ \\
\hline & $\mathrm{N}$ (control follow-up) $=56$ \\
\hline & $\mathrm{N}$ (intervention baseline $)=62$ \\
\hline & $\mathrm{N}$ (intervention follow-up) $=55$ \\
\hline & Setting (and number by study group): home-based \\
\hline & $\begin{array}{l}\text { Recruitment: families were identified from patient records at } 4 \mathrm{CHCs} \text { that served primarily low-income, } \\
\text { and racial/ethnic minority families. Mailed out potential participants a letter introducing them to the } \\
\text { study, inviting them to take part and an opt-out telephone number should the family choose not to par- } \\
\text { ticipate. }\end{array}$ \\
\hline & Geographic region: Boston, USA \\
\hline & Percentage of eligible population enrolled: $24 \%$ of those contacted \\
\hline & Mean age: intervention: $4.1 \pm 1.1$; control: $4.0 \pm 1.1$ \\
\hline
\end{tabular}


Haines 2013 (Continued)

Sex: ilntervention: $43.6 \%$ female; control: $51.8 \%$ female
To examine the effectiveness of a home-based intervention to improve household routines known to be associated with childhood obesity among a sample of low-income, racial/ethnic minority families.

The Healthy habits, happy homes intervention is a home-based intervention that uses individually tailored counselling by health educators to encourage behaviour change. The intervention was informed by findings from focus groups with $74 \mathrm{racial} / \mathrm{ethnic}$ minority parents of young children. Major components of the intervention included:

- motivational coaching by a health educator during 4 home visits and 4 health coaching telephone calls

- mailed educational materials and incentives

- weekly text messages on adoption of household routines

4 bilingual educators were trained to do the MI during the home visits and coaching calls. Each home visit included:

- a check-in to review progress and setbacks to behaviour change

- discussion of behaviour-change goals and collaborative goal setting

- a concrete activity or tool the parent could use to support behaviour change.

The monthly coaching calls were designed to assess participants' progression making changes, provide support for challenges that arose, and reinforce study messages. The intervention focused on promotion of 4 household behaviours: eating meals together as a family, obtaining adequate sleep, limiting TV time, and removing the TV from the child's bedroom. In addition to the coaching, home visits and calls, parents received text messages twice weekly for 16 weeks and then weekly for the last 8 weeks of the programme.

Control: families randomised to the control condition received 4 monthly mailed packages that included educational materials on reaching developmental milestones during early childhood and low-cost incentives (e.g. coloring books).

Diet and PA intervention vs control
Outcomes

Outcome measures

- Primary outcome: frequency of family meals, child sleep duration, child weekday and weekend day TV viewing, presence of a TV in the room

- Secondary outcomes: BMI

Process evaluation: reported (attendance, satisfaction)

Implementation-related factors

Theoretical basis: NR
Resources for intervention implementation: reported

Who delivered the intervention: reported

PROGRESS categories assessed at baseline: child: gender, race/ethnicity; parent: education, SES (household income), marital status

PROGRESS categories analysed at outcome: NR

Outcomes relating to harms/unintended effects: NR

Intervention included strategies to address diversity or disadvantage: targeted low-income, and racial/ ethnic minority families

Economic evaluation: NR 
Haines 2013 (Continued)

Funding: this work was supported by the CDC and the National Center for Chronic Disease Prevention and Health Promotion (Prevention Research Centers grant 1U48DP00194)

Role of the Sponsors: the sponsors had no role in the design and conduct of the study; collection, management, analysis, and interpretation of the data; and preparation, review, or approval of the manuscript; and decision to submit the manuscript for publication

Participants received USD 40 for completing the baseline visit and USD 50 for completing the 6-month follow-up visit.

Among the 62 families randomised to intervention, 48 (77\%) completed all 4 home visits. Fewer families completed the phone calls; 23 (37\%) completed all 4 phone calls.

Parents' satisfaction was assessed using a survey to rate how satisfied they were with the programme components and how helpful each component was in guiding their approach to their child's behaviours. Among the 55 intervention families who completed the process survey at follow-up, $89 \%$ reported being "satisfied" or "very satisfied" with the programme as a whole; $98 \%$ were "satisfied" or "very satisfied" with the counselling received during home visits; and $98 \%$ were "satisfied" or "very satisfied" with the counselling received during coaching calls. Nearly all parents $(98 \%)$ reported they would recommend the programme to friends and family.

\section{Risk of bias}

\section{Bias \\ Authors' judgement Support for judgement}

Random sequence genera- Low risk tion (selection bias)

Stratum was recruitment site blocked by child sex; condition was assigned by blocks of 4 in each strata. Our statistical programmer used a computerized routine to randomly assign the stratified blocks to the intervention and control condition.

\begin{tabular}{|c|c|c|}
\hline $\begin{array}{l}\text { Allocation concealment } \\
\text { (selection bias) }\end{array}$ & Low risk & $\begin{array}{l}\text { Assignments were implemented through sealed, sequentially numbered indi- } \\
\text { vidual envelopes that the research assistant opened following the completion } \\
\text { of baseline assessments. }\end{array}$ \\
\hline
\end{tabular}
of baseline assessments.

Blinding (performance $\quad$ Unclear risk NR
bias and detection bias)
All outcomes

Incomplete outcome data Low risk

(attrition bias)

Overall 92\% completed follow-up; $8 \%$ individual attrition (from total families

All outcomes enrolled) with both groups being balanced

\begin{tabular}{lll}
\hline $\begin{array}{l}\text { Selective reporting (re- } \\
\text { porting bias) }\end{array}$ & Low risk & $\begin{array}{l}\text { Protocol seen; all outcomes specified in methods have been reported in re- } \\
\text { sults }\end{array}$ \\
\hline Other bias & Low risk & No other threats to validity \\
\hline
\end{tabular}

Haire-Joshu 2010

Study name: Partners of all ages reading about diet and exercise (PARADE)
Study design: cluster-RCT
Intervention period: 4 months
Follow-up period (post-intervention): mean time elapsed between pretest and post-test was 5.7
months (SD 2.6) with a minimum of 2.1 months and maximum of 16.2 months
Differences in baseline characteristics: reported


Haire-Joshu 2010 (Continued)

Reliable outcomes: reported

Protection against contamination: NR

Unit of allocation: sites

Unit of analysis: individual accounting for clustering

\begin{tabular}{ll}
\hline Participants & N (control baseline $)=364,74$ sites \\
& $N$ (control follow-up $)=155,43$ sites \\
& N (intervention baseline $)=418,45$ sites \\
& N (intervention follow-up) $=296,69$ sites
\end{tabular}

Setting (and number by study group): sites (74 intervention and 45 control); visits occurred in various community settings including libraries, community centres, after-school areas, or outside of the classroom setting

Recruitment: recruited from OASIS Intergenerational Reading

Program (OASIS) and Big Brothers, Big Sisters Inc. (BBBS)

Geographic region: primarily urban and suburban, St. Louis, Missouri, USA

Percentage of eligible population enrolled: NR

Mean age: intervention: $8.3 \pm 1.4$; control: $8.7 \pm 1.7$

Sex: intervention: $48 \%$ female; control: $32 \%$ female as an element of mentoring programmes for high-needs children.

The intervention was delivered over a 4-month period by trained mentors. The curriculum of the intervention was designed to focus on content to enhance knowledge of dietary and activity guidelines, identify common and accessible activities, and low cost and accessible fruits and vegetables. Each module was packaged to contain all programme materials including individual visit lesson plan, a storybook, and a parent action newsletter (described in further detail below). The intervention was developed using a community-based participatory approach and included identifying core content through a series of developmental meetings with mentoring programme staff, structured interviews and pilot testing with children, parents and mentors. PARADE mentors delivered 8 lesson plans, 8 child-focused computer-tailored storybooks, and 8 parent action support newsletters addressing positive diet and activity behaviour patterns.

Training of mentors: mentors were adults active in the participating organisations, who volunteered to be a mentor to a child. PARADE training was $2 \mathrm{~h}$ and included a review of all materials, lesson plan objectives, and tailoring of storybooks. Training sessions were conducted as a normal part of ongoing mentor training; 201 mentors completed training.

Control: control children received the standard tutoring programme, which consisted of routine 1-h visits with the child. Intervention families received the standard tutoring program plus PARADE

Diet and PA intervention vs control

\begin{tabular}{|c|c|}
\hline \multirow[t]{3}{*}{ Outcomes } & Outcome measures \\
\hline & $\begin{array}{l}\text { - Primary outcome: } \\
\text { - child nutrition and PA knowledge, daily caloric intake, percent of calories consumed from fat, daily } \\
\text { servings of fruits and vegetables, percent of time spent in PA, zBMI }\end{array}$ \\
\hline & $\begin{array}{l}\text { - parental daily caloric intake, percent of calories consumed from fat, daily servings of fruits and } \\
\text { vegetables, and min walked per week }\end{array}$ \\
\hline
\end{tabular}


Haire-Joshu 2010 (Continued)

- Secondary outcomes: child reported attempt to challenge self to eat 5 fruits or vegetables a day or to be active for at least $1 \mathrm{~h}$ each day

Process evaluation: reported (attendance)

Implementation-related factors

Theoretical basis: Ecological Model, SCT

Resources for intervention implementation: reported

Who delivered the intervention: reported

PROGRESS categories assessed at baseline: child, gender, race/ethnicity; parent: race/ethnicity, education, income, employment, marital status

PROGRESS categories analysed at outcome: NR

Outcomes relating to harms/unintended effects: NR

Intervention included strategies to address diversity or disadvantage: targeted underserved and 'high needs' children already in mentoring programmes, which are used to reach children at risk for poor educational outcomes.

Economic evaluation: NR

Notes

Funding: provided by National Institute of Nursing Research (R01NR05079) and the American Cancer Society (TURPG 0028601)

$56 \%$ of children in the analysis group had received all 8 sessions and $82 \%$ had received at least 6 sessions. Evaluation of PARADE by the parent revealed that $84 \%$ read the tailored storybooks with their child, and $88 \%$ reported that their child liked the books as much or more than other books.

Parents were given a USD 15 gift card for completing the pretest and post-test survey PARADE needed to fit within the delivery structure of the ongoing mentoring programmes.

\section{Risk of bias}

\begin{tabular}{|c|c|c|}
\hline Bias & Authors' judgement & Support for judgement \\
\hline $\begin{array}{l}\text { Random sequence genera- } \\
\text { tion (selection bias) }\end{array}$ & Low risk & Randomly assigned sites, computer-generated randomisation scheme \\
\hline $\begin{array}{l}\text { Allocation concealment } \\
\text { (selection bias) }\end{array}$ & Unclear risk & NR \\
\hline $\begin{array}{l}\text { Blinding (performance } \\
\text { bias and detection bias) } \\
\text { All outcomes }\end{array}$ & Unclear risk & NR \\
\hline $\begin{array}{l}\text { Incomplete outcome data } \\
\text { (attrition bias) } \\
\text { All outcomes }\end{array}$ & High risk & $\begin{array}{l}29 \% \text { attrition in intervention and } 57 \% \text { attrition in control group sites. Also the } \\
\text { mean time elapsed between pretest and post-test was } 5.7 \text { months (SD 2.6) with } \\
\text { a minimum of } 2.1 \text { months and maximum of } 16.2 \text { months }\end{array}$ \\
\hline $\begin{array}{l}\text { Selective reporting (re- } \\
\text { porting bias) }\end{array}$ & Unclear risk & Protocol/trial registration documents were unavailable \\
\hline Other bias & Low risk & No additional threats to validity \\
\hline $\begin{array}{l}\text { Other bias- timing of re- } \\
\text { cruitment of clusters }\end{array}$ & Unclear risk & No CONSORT figure, NR in text \\
\hline
\end{tabular}




\begin{tabular}{ll}
\hline Methods & Study design: cluster-RCT \\
& Intervention period: 3 years \\
& Follow-up period (post-intervention): nil \\
& Differences in baseline characteristics: reported \\
& Reliable outcomes: reported \\
& Protection against contamination: NR \\
& Unit of allocation: schools \\
& Unit of analysis: individual
\end{tabular}

Participants

$\mathrm{N}$ (control baseline $)=1400$

$\mathrm{N}($ control follow-up $)=1342$

$\mathrm{N}($ intervention baseline $)=1400$

$\mathrm{N}$ (intervention follow-up) $=1328$

Setting (and number by study group): 10 elementary schools (5 in each group)

Recruitment: cluster random sampling of schools: 2 schools from each area

Students were selected from Grades 1-4. 70 students in each grade in each school were selected. The participants were chosen according to their student ID, but study authors did not report on "how"

Geographic region: south, north, east, west and middle parts of Yangpu district, Shanghai, China

Percentage of eligible population enrolled: NR

Mean age: NR (grades 1-4; aged 6-10 years)

Sex: intervention group: $47 \%$ female; control: $48 \%$ female

Interventions

To evaluate the intervention outcomes among elementary students in Yangpu district after a 3-year nutrition intervention, to set up a comprehensive intervention system on elementary students' nutritious lunch with health promotion strategies.

"Precede-proceed" model:

- provide healthy lunch to students in the intervention group

- set up regulations for lunch in the intervention schools and lunch providers

- improve canteen's environment

- appoint nutritionists in the lunch providers to supervise and monitor lunch provision, as well as act as a 'bridge' among school, family, and community

- train the nutritionists in lunch providers and relevant teachers in the schools

- deliver newspapers (about nutritional knowledge) to students and teachers

- improve the environment near the schools (to set up a healthy food-friendly environment)

- a variety of education means adopted to residents near the schools (including blackboard, broadcast, cooking training course, leaflets)

- Supervisions of local community health centres and local centres for disease control to the schools and lunch providers

Diet intervention vs control 
Han 2006 (Continued)

- Primary outcome: prevalence of overweight/obesity, knowledge/attitude and practice, physical health index, anemia prevalence rate

- Secondary outcomes: primary/secondary not specified

Process evaluation: NR

Implementation-related factors
Theoretical basis: NR

Resources for intervention implementation: NR

Who delivered the intervention: reported

PROGRESS categories assessed at baseline: NR

PROGRESS categories analysed at outcome: NR

Outcomes relating to harms/unintended effects: NR

Intervention included strategies to address diversity or disadvantage: NR

Economic evaluation: NR

Review author (G Yang) data extracted this study as it is published in Chinese (English abstract)

\section{Risk of bias}

\section{Bias}

Authors' judgement Support for judgement

Random sequence genera- Unclear risk Randomised

tion (selection bias)

Allocation concealment Unclear risk NR

(selection bias)

Blinding (performance
bias and detection bias)

\begin{tabular}{lll}
\hline $\begin{array}{l}\text { Incomplete outcome data } \\
\text { (attrition bias) } \\
\text { All outcomes }\end{array}$ & Low risk & $\begin{array}{l}\text { Low attrition 1328/1400 (94.9\%) in intervention group and 1342/1400 (96.1\%) } \\
\text { completed the intervention }\end{array}$ \\
\hline $\begin{array}{l}\text { Selective reporting (re- } \\
\text { porting bias) }\end{array}$ & Unclear risk & Protocol/trial registration documents were unavailable \\
\hline $\begin{array}{l}\text { Other bias } \\
\begin{array}{l}\text { Other bias- timing of re- } \\
\text { cruitment of clusters }\end{array}\end{array}$ & Unclear risk & NR \\
\hline
\end{tabular}

\section{Harvey-Berino 2003}

$\begin{array}{ll}\text { Methods } & \text { Study design: RCT } \\ \text { Intervention period: } 16 \text { weeks } \\ \text { Follow-up (post-intervention): nil } \\ \text { Differences in baseline characteristics: reported }\end{array}$


Harvey-Berino 2003 (Continued)

Reliable outcomes: reported

Protection against contamination: NR

Unit of allocation: child

Unit of analysis: child

\begin{tabular}{|c|c|}
\hline \multirow[t]{5}{*}{ Participants } & $\begin{array}{l}N \text { (controls baseline })=20 \\
N(\text { controls follow-up) }=17 \\
N \text { (intervention baseline })=20 \\
N \text { (intervention follow-up) }=20\end{array}$ \\
\hline & $\begin{array}{l}\text { Recruitment: children aged } 9 \text { months- } 3 \text { years, child was walking, mother BMI > 25, mother agreed to } \\
\text { keep all appointments. Set in Northern New York State, USA, Quebec and Ontario, Canada }\end{array}$ \\
\hline & Proportion of eligibles participating: not stated \\
\hline & Mean age: 21 months (no SD reported) \\
\hline & Sex: both sexes included; $54 \%$ boys \\
\hline \multirow[t]{2}{*}{ Interventions } & $\begin{array}{l}\text { Home visiting programme delivered by an indigenous peer educator who was extensively trained. The } \\
\text { intervention was an adaptation of the Active Parenting Curriculum where } 11 \text { parenting topics were cov- } \\
\text { ered in } 16 \text { weeks. The focus for the treatment group was exclusively on how to improve parenting skills } \\
\text { to develop appropriate eating and exercise behaviours to prevent obesity. } \\
\text { Controls received the usual parenting support programme }\end{array}$ \\
\hline & Combined effects of dietary interventions and PA interventions vs control \\
\hline \multirow[t]{10}{*}{ Outcomes } & - Maternal BMI \\
\hline & - $\mathrm{N}$ classified $>$ 85th and 95th weight for height $\mathrm{z}$ centile scores \\
\hline & $\begin{array}{l}\text { - Diet: 3-day food records analysed for total calorie and fat intake using Nutritionist IV computer pro- } \\
\text { gramme }\end{array}$ \\
\hline & - PA: Tritrac R3D accelerometer (mother and child) \\
\hline & - Psychological variables: \\
\hline & - Outcomes expectations \\
\hline & - Self-efficacy \\
\hline & - Intentions \\
\hline & - Child Feeding Questionnaire \\
\hline & Process evaluation: NR \\
\hline
\end{tabular}

Implementation-related

Theoretical basis: NR

factors

Resources for intervention implementation (e.g. funding needed or staff hours required): reported

Who delivered the intervention: reported

PROGRESS categories assessed at baseline: reported (race, occupation, gender, education)

PROGRESS categories analysed at outcome: NR

Outcomes relating to harms/unintended effects: NR

Intervention included strategies to address diversity or disadvantage: reported

Economic evaluation: NR

\section{Risk of bias}


Harvey-Berino 2003 (Continued)

Random sequence genera- Unclear risk Quote: "Subjects were randomly assigned to one of two treatment groups" tion (selection bias)

\begin{tabular}{lll}
\hline $\begin{array}{l}\text { Allocation concealment } \\
\text { (selection bias) }\end{array}$ & Unclear risk
\end{tabular}

Blinding (performance Low risk Outcome assessors were blinded

bias and detection bias)

All outcomes

\begin{tabular}{ll}
\hline $\begin{array}{l}\text { Incomplete outcome data } \\
\text { (attrition bias) }\end{array}$ & Low risk \\
$\begin{array}{ll}\text { All outcomes } & \text { Quote: "Two mother/child pairs were lost to follow-up at the 16-week assess- } \\
\text { ment. An additional case had incomplete follow-up data. Therefore, complete } \\
\text { data were available for 93\% of the sample" }\end{array}$ \\
\hline
\end{tabular}

\begin{tabular}{lll}
\hline $\begin{array}{l}\text { Selective reporting (re- } \\
\text { porting bias) }\end{array}$ & Unclear risk & Protocol/trial registration documents were unavailable \\
\hline Other bias & Low risk & No additional threats to validity \\
\hline
\end{tabular}

$\begin{array}{ll}\text { Methods } & \text { Study design: cluster-RCT } \\ \text { Intervention period: } 3 \text { years } \\ \text { Follow-up period (post-intervention): nil } \\ \text { Differences in baseline characteristics: reported } \\ \text { Reliable outcomes: reported } \\ \text { Protection against contamination: NR } \\ \text { Unit of allocation: school } \\ \text { Unit of analysis: school }\end{array}$

\section{Participants}

$$
\begin{aligned}
& N(\text { control baseline })=3191 \\
& N(\text { control follow-up })=2296 \\
& N(\text { intervention baseline })=3222 \\
& N(\text { intervention follow-up })=2307
\end{aligned}
$$

Setting (and number by study group): 42 middle schools at 7 field sites ( 21 in each group)

Recruitment: each field site was responsible for the recruitment of 6 schools; eligibility was based on ability to enrol a sufficient number of predominately minority and lower-SES students. Study staff met with district superintendents and school principals to verify the eligibility of schools, and to ascertain how appropriate the school would be for conducting the trial. Sixth grade students were recruited employing a variety of techniques

Geographic region: USA

Percentage of eligible population enrolled: the rate of parental consent and child assent was 58.9\%; $57.6 \%$ of students agreed to health screening at baseline 
HEALTHY Study Gp 2010 (Continued)

Mean age: intervention: $11.3 \pm 0.5$; control: $11.3 \pm 0.6$

Sex: intervention: $52.6 \%$ female; control: $52.9 \%$ female

Interventions To evaluate the effects of a 3-year, multi-component, school-based programme on risk factors for type 2 diabetes

The intervention consisted of 4 integrated components: nutrition, PA, behavioural knowledge and skills, and communications and social marketing.

The nutrition component targeted the quantity and nutritional quality of foods and beverages that were served throughout the school environment (cafeteria, vending machines, a la carte options, snack bars, school stores, fundraisers, and classroom celebrations). The physical-education component was designed to increase the amount of time students spent in MVPA, defined as activity sufficient to raise the heart rate to $\geq 130$ beats per minute. Behavioural knowledge and skills were communicated with the use of a classroom-based programme, FLASH (Fun Learning Activities for Student Health), which targeted self-awareness, knowledge, behavioural skills (e.g. self-monitoring and goal setting), and peer involvement for behavioural change. Communication strategies and social marketing integrated and supported the intervention.

The intervention was delivered over five semesters (Spring 2007, Fall 2007, Spring 2008, Fall 2008, Spring 2009). Study interventionists; research dietitians; PA co-ordinators

Diet and PA combination intervention vs control

Outcomes Outcome measures

- Primary outcome: $\mathrm{BMI} \geq 85$ th percentile

- Secondary outcomes: BMI $\geq 95$ th percentile, zBMI, waist circumference $\geq 90$ th percentile, waist circumference, fasting insulin, fasting glucose, shifts in BMI categories (see secondary references for HEALTHY Study Gp 2010 (Marcus et al 2012))

Process evaluation: reported (implementation: see secondary references for HEALTHY Study Gp 2010 (Volpe et al. 2013; barriers and facilitators: Hall et al 2014))

Implementation-related factors

\section{Theoretical basis: NR}

Resources for intervention implementation: reported

Who delivered the intervention: reported

PROGRESS categories assessed at baseline: gender, race/ethnicity; parent: education

PROGRESS categories analysed at outcome: NR

Outcomes relating to harms/unintended effects: reported

Intervention included strategies to address diversity or disadvantage: NR but black and Hispanic children of lower SES were oversampled

Economic evaluation: NR
NCT00458029

Funding: supported by grants (U01-DK61230, U01-DK61249, U01-DK61231, and U01-DK61223) from the National Institute of Diabetes and Digestive and Kidney Diseases of the NIH to the Studies to Treat or Prevent Pediatric Type 2 Diabetes (STOPP-T2D) collaborative group, with additional support from the American Diabetes Association.

The intervention was facilitated by staff and funds provided by the study. Such an efficacy study cannot assess the feasibility, effectiveness, or sustainability of an intervention programme outside a study setting. Overall, the observed fidelity of implementing nutrition strategies improved from baseline to the end of the study. By the last semester, all but 2 nutrition process evaluation goals were met. The most 
HEALTHY Study Gp 2010 (Continued)

challenging goal to implement was serving high fibre foods, including grain-based foods and legumes. The easiest goals to implement were lowering the fat content of foods offered and offering healthier beverages. The most challenging barriers experienced by research dietitians and food service staff were costs, availability of foods and student acceptance. Forming strong relationships between the research dietitians and food service staff was identified as a key strategy to meet HEALTHY nutrition goals. Barriers included teacher frustration that intervention activities detracted from tested subjects, student resistance and misbehaviour, classroom-management problems, communication-equipment problems, lack of teacher/staff engagement, high cost and limited availability of nutritious products, inadequate facility space, and large class sizes. Facilitators included teacher/staff engagement, effective classroom management, student engagement, schools with direct control over food service, support from school leaders, and adequate facilities and equipment. Schools received annual compensation for participation that could be used at the discretion of the school administration for programme enhancement. Schools assigned to intervention received USD 2000 in year 1, USD 3000 in year 2 and USD 4000 in year 3, and those assigned to control USD 2000 in year 1, USD 4000 in year 2 and USD 6000 in year 3 . The control school amounts became higher because the intervention schools received additional compensation in terms of PE equipment and food service costs. The amounts escalated each year as a retention strategy.

\section{Risk of bias}

\begin{tabular}{lll}
\hline Bias & Authors' judgement & Support for judgement \\
\hline $\begin{array}{l}\text { Random sequence genera- } \\
\text { tion (selection bias) }\end{array}$ & Low risk & $\begin{array}{l}\text { The co-ordinating centre developed a stratified randomisation scheme. The } \\
\text { stratification factors were field centre and } 6 \text { th grade size to assign comparable } \\
\text { within cluster (school) sample sizes across treatment arms at each field centre. }\end{array}$ \\
\hline $\begin{array}{l}\text { Allocation concealment } \\
\text { (selection bias) }\end{array}$ & Low risk & $\begin{array}{l}\text { The co-ordinating centre developed a stratified randomisation scheme. The } \\
\text { stratification factors were field centre and 6th grade size to assign comparable } \\
\text { within cluster (school) sample sizes across treatment arms at each field centre. }\end{array}$ \\
\hline $\begin{array}{l}\text { Blinding (performance } \\
\text { bias and detection bias) } \\
\text { All outcomes }\end{array}$ & Unclear risk & $\begin{array}{l}\text { Study staff and key school administrative personnel were informed of the ran- } \\
\text { domisation assignment early on. Students and their parents blinded but only } \\
\text { during recruitment and health screening stages. To minimise staff bias, study } \\
\text { staff who delivered the intervention appeared in the intervention schools on- } \\
\text { ly and were separate from study staff who administered data collection proce- } \\
\text { dures in both intervention and control schools. }\end{array}$ \\
\hline
\end{tabular}

Incomplete outcome data Low risk Student attrition was identical (27.5\%) in the intervention and control schools. (attrition bias)

All outcomes

Selective reporting (re- Low risk Protocol seen; all outcomes specified in methods were reported in results
porting bias)

Other bias Unclear risk

\begin{abstract}
Contamination: there was a minimal amount of cross-activity between schools at the middle school level, but HEALTHY-branded items were distributed at both intervention and control schools as part of retention and incentives so that the study logo was a familiar sight. Perhaps the greatest potential for cross-over occurred where a single food service corporation served both intervention and control schools and wanted to take advantages of efficiencies by placing only one order. The study staff administering the nutrition intervention component actively monitored school orders and purchases, and formed alliances at the district and corporate food service levels to restrict the intervention to only the 3 assigned schools.
\end{abstract}

Other bias- timing of re- $\quad$ Unclear risk
cruitment of clusters


Hendy 2011

\begin{tabular}{|c|c|}
\hline \multirow[t]{9}{*}{ Methods } & Study design: RCT \\
\hline & Intervention period: 3 months \\
\hline & Follow-up period (post-intervention): 6 months \\
\hline & Differences in baseline characteristics: reported \\
\hline & Reliable outcomes: reported \\
\hline & Protection against contamination: $\mathrm{NR}$ \\
\hline & Unit of allocation: individual \\
\hline & Unit of analysis: individual \\
\hline & Analyses were not performed according to ITT principles \\
\hline \multirow[t]{11}{*}{ Participants } & $\begin{array}{l}\text { Note: of } 382,341 \text { provided data, of which } 11 \text { were underweight and removed from analysis, leaving } 330 \\
\text { at baseline }\end{array}$ \\
\hline & $\mathrm{N}$ (controls baseline $)=$ unclear \\
\hline & $\mathrm{N}$ (controls follow-up) $=$ unclear \\
\hline & $\mathrm{N}$ (interventions baseline) = unclear \\
\hline & $\mathrm{N}$ (interventions follow-up) = unclear \\
\hline & Setting: 1 elementary/primary school \\
\hline & Recruitment: unclear \\
\hline & Geographic region: small town in eastern Pennsylvania, USA \\
\hline & Percentage of eligible population enrolled: unclear \\
\hline & Mean age: unclear (1st to 4 th graders) \\
\hline & Sex: intervention+ control: $\mathrm{N}=382,211$ boys and 171 girls \\
\hline
\end{tabular}
- fruit and vegetables and SSBs, and steps/day. Small teams of parent volunteers delivered the KCP

The KCP group (called the "LIONS") received stars punched into their nametags for each of three "Good Health Behaviors" that included eating $1 / 8$ cup fruit and vegetables ("the size of a ping pong ball") 1st during their meal (FVFIRST), choosing a low-fat and low sugar healthy drink (HDRINK), and having 5000 exercise steps recorded on their pedometers (EXERCISE).

The control group (called the "TIGERS") received stars punched into their nametags for each of three "Good Citizenship Behaviors" that included talking quietly during meals, keeping their meal area clean, and respecting others by not touching them or their things.

Diet and PA vs control

\begin{tabular}{l} 
Outcome measures \\
- Primary outcome: BMI percentile, fruit and vegetable intake, SSB intake, PA \\
Primary/secondary outcome measures not specified \\
Process measure: NR \\
\hline
\end{tabular}


Hendy 2011 (Continued)

Implementation-related factors
Theoretical basis: SCT, Self-determination theory, Group Socialization theory

Resources for intervention implementation: reported

Who delivered the intervention: reported

PROGRESS categories assessed at baseline: NR

PROGRESS categories analysed at outcome: NR

Outcomes relating to harms/unintended effects: NR

Intervention included strategies to address diversity or disadvantage: NR

Economic evaluation: estimations of the dollar costs per child per month of KCP application were provided, with suggestions for additional cost reductions

The study authors reported the change in BMI\% from baseline to end of the intervention for:

- the group of children who were overweight in the control group

- the group of children who were normal weight in the control group

- the group of children who were overweight in the intervention group

- the group of children who were normal in the intervention group

Funding source: Grants from Penn State University

\section{Risk of bias}

\begin{tabular}{|c|c|c|}
\hline Bias & Authors' judgement & Support for judgement \\
\hline \multirow{2}{*}{$\begin{array}{l}\text { Random sequence genera- } \\
\text { tion (selection bias) }\end{array}$} & Unclear risk & Randomly allocated, no further details \\
\hline & & Quote: "children were randomly assigned to one of the two study groups" \\
\hline $\begin{array}{l}\text { Allocation concealment } \\
\text { (selection bias) }\end{array}$ & Unclear risk & NR \\
\hline $\begin{array}{l}\text { Blinding (performance } \\
\text { bias and detection bias) } \\
\text { All outcomes }\end{array}$ & Low risk & Nurse who measured height and weight in children was blind to allocation \\
\hline $\begin{array}{l}\text { Incomplete outcome data } \\
\text { (attrition bias) } \\
\text { All outcomes }\end{array}$ & Unclear risk & $\begin{array}{l}\text { For BMI of } 200 \text { average weight children, } 186(93 \%) \text { had data at } 6 \text { months. Flow } \\
\text { of study participants through treatment and control groups unclear }\end{array}$ \\
\hline $\begin{array}{l}\text { Selective reporting (re- } \\
\text { porting bias) }\end{array}$ & High risk & $\begin{array}{l}\text { Protocol/trial registration documents were unavailable. From the text of the } \\
\text { RCT: } \mathrm{zBMI} \text { calculated but NR, focus is on BMI percentile; primary/secondary } \\
\text { outcomes not specified; post hoc subgroup analyses presented for BMI for av- } \\
\text { erage weight children and overweight children }\end{array}$ \\
\hline Other bias & Unclear risk & Contamination not discussed \\
\hline
\end{tabular}

\section{Herscovici 2013}

\section{Methods}

$$
\text { Study design: cluster-RCT }
$$

Intervention period: 6 months

Follow-up period (post-intervention): nil 
Herscovici 2013 (Continued)

Differences in baseline characteristics: reported

Reliable outcomes: reported

Protection against contamination: NR

Unit of allocation: schools

Unit of analysis: individual

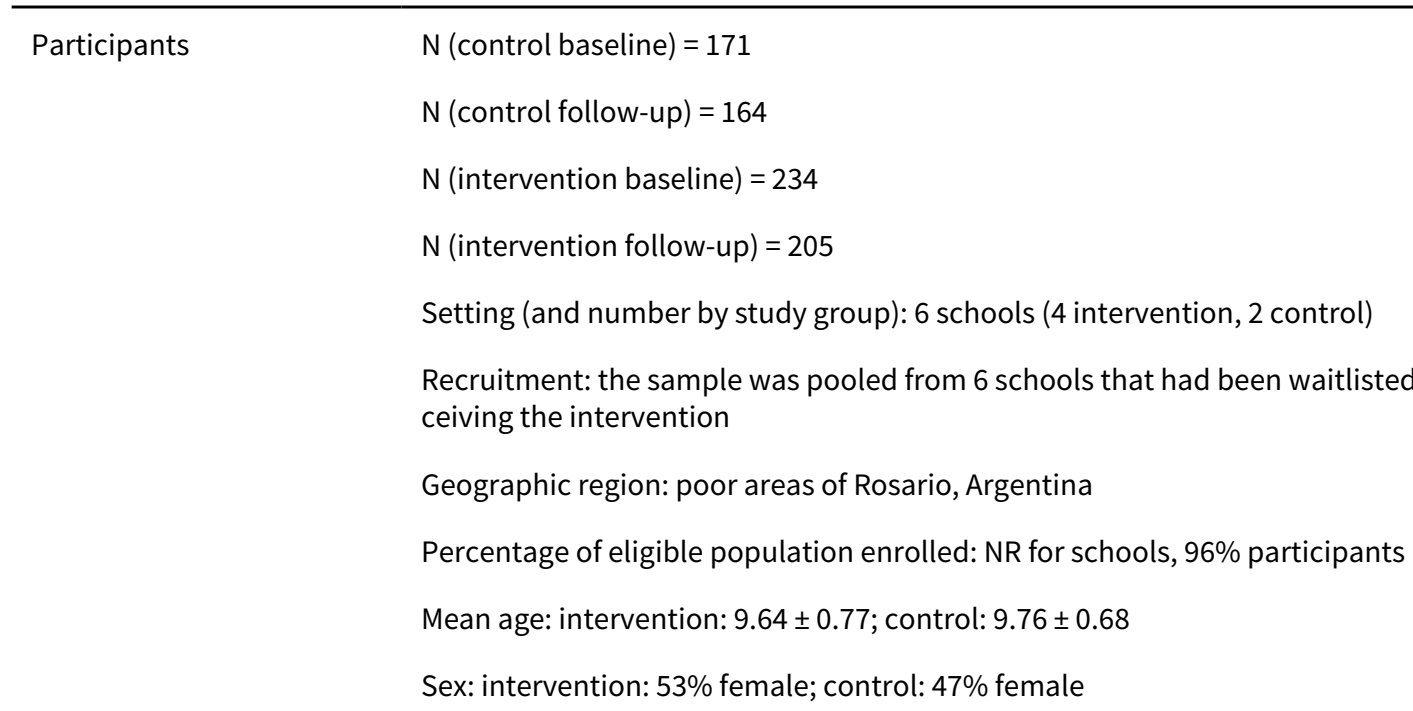

Interventions

To evaluate changes in BMI and food intake among children at schools that received the Healthy Snack Bar intervention

For the intervention arm, the participating grades took part in 4 workshops: 3 for the children (Healthy eating, Body in motion, and Healthy body); and one for their parents/ caregivers. Workshops lasted 40 min, were conducted monthly by an interdisciplinary team, and had an interactive modality.

The intervention consisted of 5 parts: the 4 workshops, plus modifications to the school cafeteria menu.

- Workshop 1: Healthy eating. The 1st workshop aimed to help children identify healthy foods, understand why healthy foods improve health, and contemplate the disadvantages of including competitive options in their diet (e.g. pros and cons of fat and sodium consumption). This workshop specifically encouraged the intake of 5 healthy food items targeted by the programme: orange juice (100\% orange, no sugar added), whole fruits, low-sugar cereal, skim milk, and vegetables (fresh, canned, or cooked).

- Workshop 2: Body in motion. The 2nd workshop aimed to get children motivated about PA, and to understand the health-related benefits of regular exercise.

- Workshop 3: Healthy body. The 3rd workshop sought to help children establish the connection between good eating habits, regular PA, and a healthy body. An additional goal was to enable children to identify a healthy menu based on nutritional components.

- Workshop 4: parent/caregiver. The 4th workshop aimed to provide dietary education to the children's parents/caregivers and emphasised the importance of PA.

- School Snack Bar. At the start of the study, the school snack bar options were modified to include 3 of the aforementioned 5 healthy food items stimulated by the programme (orange juice, fruit, and lowsugar cereal).

Control: no details

Diet and PA intervention vs control 
Herscovici 2013 (Continued)

- Primary outcome: zBMI, children's intake of healthy and unhealthy foods

- Secondary outcomes: NR

Process evaluation: reported (attendance)

Implementation-related factors
Theoretical basis: NR

Resources for intervention implementation: reported

Who delivered the intervention: reported

PROGRESS categories assessed at baseline: gender

PROGRESS categories analysed at outcome: gender

Outcomes relating to harms/unintended effects: NR

Intervention included strategies to address diversity or disadvantage: targeted at poor areas

Economic evaluation: NR

Notes

Funding: this work was supported by the International Life Sciences Institute (ILSI) Research Foundation (Washington, D.C., USA, and ILSI Argentina, Buenos Aires, Argentina).

Parents' and/or caregivers' attendance was 53\% and was not considered exclusion criteria

\section{Risk of bias}

\begin{tabular}{lll}
\hline Bias & Authors' judgement & Support for judgement \\
\hline $\begin{array}{l}\text { Random sequence genera- } \\
\text { tion (selection bias) }\end{array}$ & Unclear risk & Simple randomisation after schools matched by socioeconomic status \\
\hline $\begin{array}{l}\text { Allocation concealment } \\
\text { (selection bias) }\end{array}$ & Unclear risk & $\begin{array}{l}\text { NR. Overall, boys were more overweight and obese than girls (31\% vs } 24.3 \%), \\
\text { and for the former, a statistically significant difference was found in their zB- } \\
\text { Mls, with boys in the control group being slightly heavier than boys in the in- } \\
\text { tervention group. Controlled for gender in analyses }\end{array}$
\end{tabular}

Blinding (performance
bias and detection bias)

\begin{tabular}{lll}
\hline $\begin{array}{l}\text { Incomplete outcome data } \\
\text { (attrition bias) } \\
\text { All outcomes }\end{array}$ & Low risk & Low attrition balanced between groups \\
\hline $\begin{array}{l}\text { Selective reporting (re- } \\
\text { porting bias) }\end{array}$ & Unclear risk & Protocol/trial registration documents were unavailable \\
\hline Other bias & Low risk & No additional threats to validity \\
\hline $\begin{array}{l}\text { Other bias- timing of re- } \\
\text { cruitment of clusters }\end{array}$ & Low risk & Figure shows enrolment happened prior to randomisation \\
\hline
\end{tabular}

Howe 2011

$\begin{array}{ll}\text { Methods } & \text { Study design: RCT } \\ & \text { Intervention period: } 10 \text { months }\end{array}$


Follow-up period (post-intervention): nil

Differences in baseline characteristics: NR for whole intervention group

Intervention group was divided into attenders (ATT) and non-attenders (NATT), participating in $\geq 60 \%$ or $<60 \%$ of the intervention, respectively.

Reliable outcomes: reported

Protection against contamination: reported

Unit of allocation: individual

Unit of analysis: individual

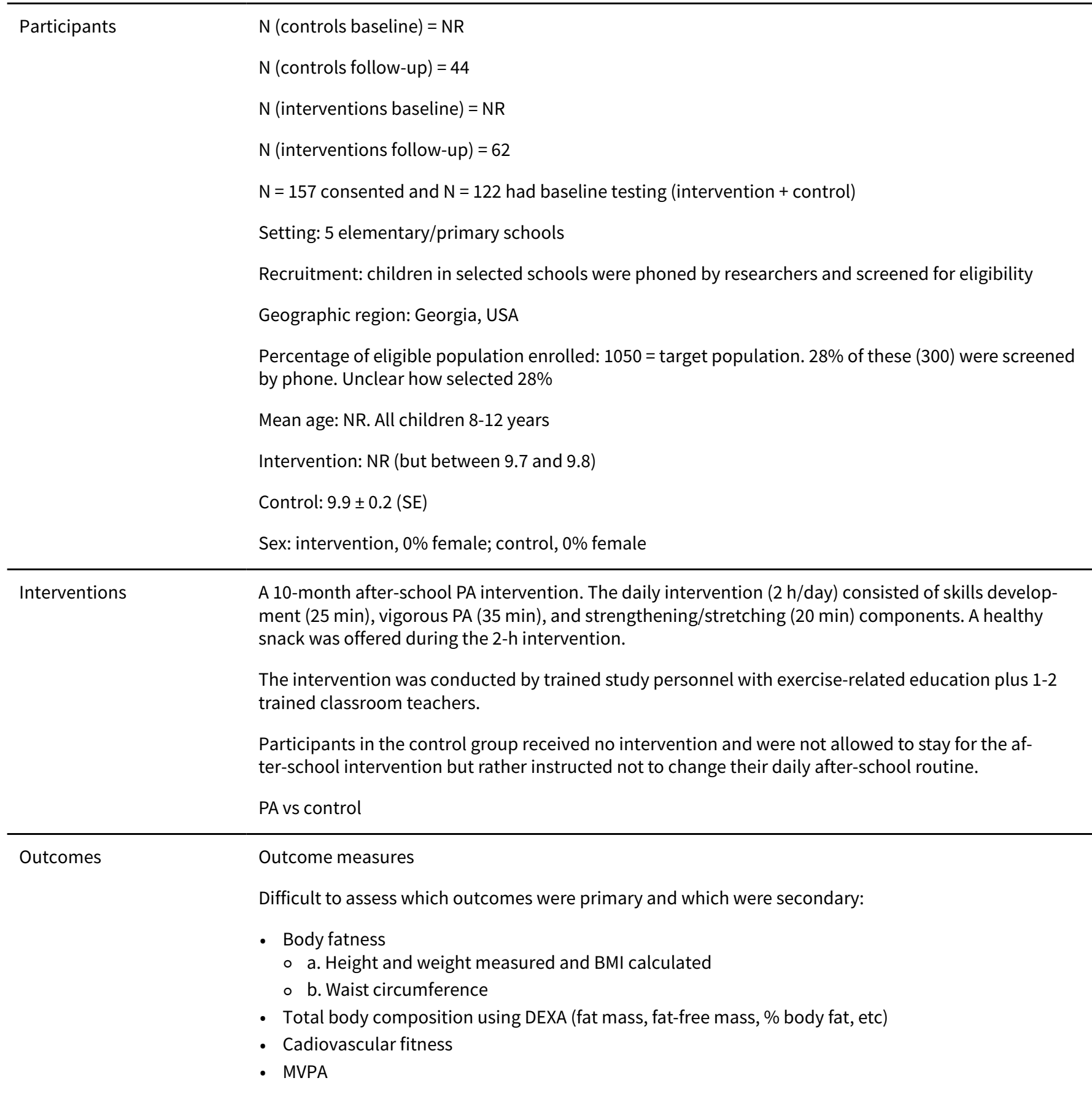


Howe 2011 (Continued)

Process: NR

Implementation-related factors
Theoretical basis: NR

Resources for intervention implementation: NR

Who delivered the intervention: reported

PROGRESS categories assessed at baseline: NR

PROGRESS categories analysed at outcome: NR

Outcomes relating to harms/unintended effects: NR

Intervention included strategies to address diversity or disadvantage: NR

Economic evaluation: NR

Notes All children were African American boys

\section{Risk of bias}

\begin{tabular}{lll}
\hline Bias & Authors' judgement & Support for judgement \\
\hline $\begin{array}{l}\text { Random sequence genera- } \\
\text { tion (selection bias) }\end{array}$ & High risk & No mention of method of randomisation \\
& $\begin{array}{l}\text { Quote: "participants was randomized into either the intervention group ( } \mathrm{n}=62) \\
\text { or the control group ( } \mathrm{n}=44) \text { with a ratio of three to two, respectively. In the in- } \\
\text { stance of siblings, the } 1 \text { st to be tested was randomized and the remaining sib- } \\
\text { ling(s) was/were placed in the same group." }\end{array}$
\end{tabular}

\begin{tabular}{|c|c|c|}
\hline $\begin{array}{l}\text { Allocation concealment } \\
\text { (selection bias) }\end{array}$ & Unclear risk & NR \\
\hline $\begin{array}{l}\text { Blinding (performance } \\
\text { bias and detection bias) } \\
\text { All outcomes }\end{array}$ & Unclear risk & NR \\
\hline $\begin{array}{l}\text { Incomplete outcome data } \\
\text { (attrition bias) } \\
\text { All outcomes }\end{array}$ & Unclear risk & $\begin{array}{l}\text { Insufficient information, } N=157 \text { consented and } N=122 \text { had baseline testing } \\
\text { (intervention + control), } N=106 \text { randomised }\end{array}$ \\
\hline $\begin{array}{l}\text { Selective reporting (re- } \\
\text { porting bias) }\end{array}$ & Unclear risk & Protocol/trial registration documents were unavailable \\
\hline Other bias & Low risk & None identified \\
\hline
\end{tabular}

\section{James 2004}

\section{Methods}

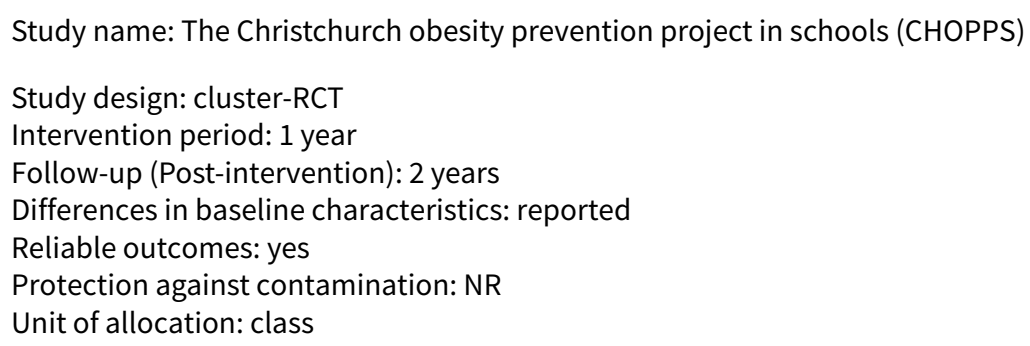


James 2004 (Continued)

Unit of analysis: class

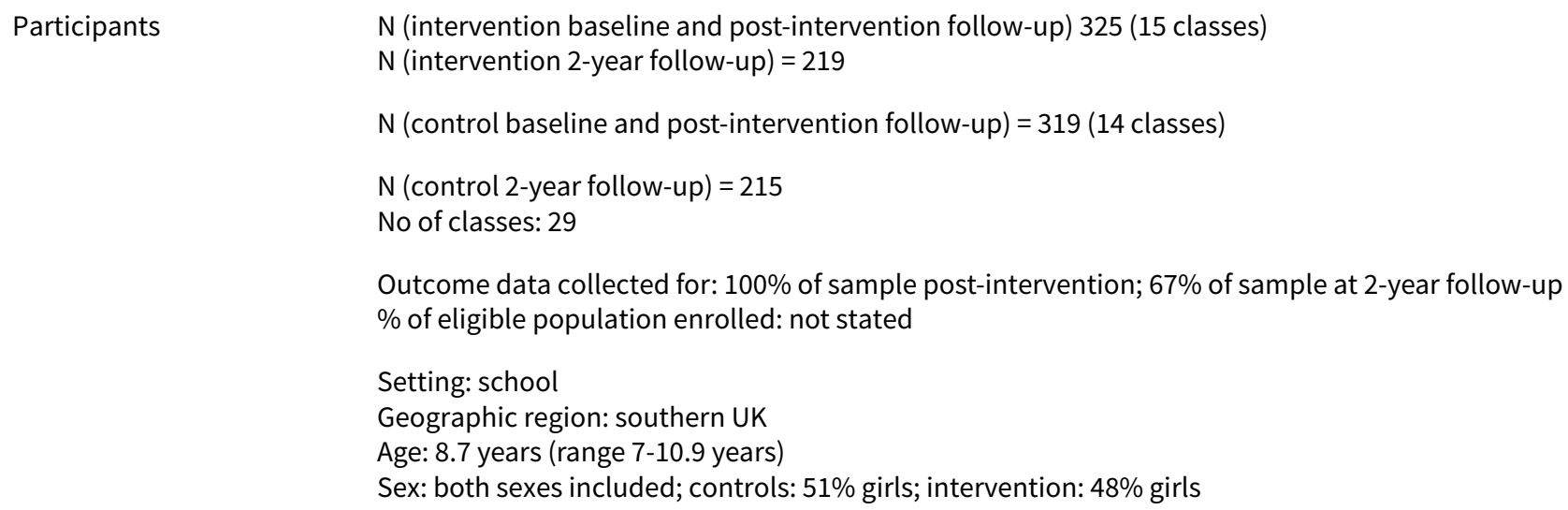

Interventions

School-based educational intervention aiming to prevent obesity by reducing consumption of carbonated drinks, delivered by the study author and supported by existing staff. 3 sessions, $1 /$ term, promoted drinking water and a reduction of carbonated drinks

Control programme NR, presumably usual school curriculum

Dietary intervention vs controls

\begin{tabular}{ll}
\hline Outcomes & BMI \\
- & Proportion of children overweight or obese (based on converting BMI values to centile values and \\
measuring the proportion above the 91st centile) \\
- Carbonated drink consumption and water consumption using a drinks diary \\
Process evaluation: NR
\end{tabular}

Implementation-related factors

Theoretical basis: NR

Resources for intervention implementation (e.g. funding needed or staff hours required): NR

Who delivered the intervention: reported

PROGRESS categories assessed at baseline: reported (gender)

PROGRESS categories analysed at outcome: reported (gender)

Outcomes relating to harms/unintended effects: NR

Intervention included strategies to address diversity or disadvantage: NR

Economic evaluation: NR

Notes

Funding: this project was funded from unrestricted educational grants from GlaxoSmithKline, Aventis, and Pfizer and from internal resources within Bournemouth Diabetes and Endocrine Centre. The external funding bodies had no input into protocol development, data collection, or analysis or interpretation. 2 of the study authors had one child each in one of the schools, NR whether intervention or control.

\section{Risk of bias}

\begin{tabular}{lll}
\hline Bias & Authors' judgement & Support for judgement \\
\hline $\begin{array}{l}\text { Random sequence genera- } \\
\text { tion (selection bias) }\end{array}$ & Low risk & $\begin{array}{l}\text { Quote: "clusters were randomised according to a random number table, with } \\
\text { blinding to schools or classes" }\end{array}$ \\
\hline
\end{tabular}


James 2004 (Continued)

$\begin{array}{ll}\begin{array}{l}\text { Allocation concealment } \\ \text { (selection bias) }\end{array} & \text { Quote: "clusters were randomised according to a random number table, with } \\ \text { blinding to schools or classes" }\end{array}$

blinding to schools or classes"

Blinding (performance Unclear risk Cannot be determined

bias and detection bias)

All outcomes

\begin{tabular}{|c|c|c|}
\hline $\begin{array}{l}\text { Incomplete outcome data } \\
\text { (attrition bias) }\end{array}$ & Low risk & $\begin{array}{l}\text { No clusters lost; individual loss was low: } 10 \% \text { in intervention and } 13 \% \text { in con- } \\
\text { trol group and reasons match }\end{array}$ \\
\hline
\end{tabular}

All outcomes

Selective reporting (re- Unclear risk $\quad$ Protocol/trial registration documents were unavailable
porting bias)

\begin{tabular}{lll}
\hline Other bias & Low risk & No additional threats to validity \\
\hline $\begin{array}{l}\text { Other bias- timing of re- } \\
\text { cruitment of clusters }\end{array}$ & Low risk & Figure indicates participants were recruited prior to randomisation \\
\hline
\end{tabular}

Jansen 2011

Study name: 'Lekker Fit!' (Enjoy being fit!)
Study design: cluster-RCT
Intervention period: 8 months
Follow-up period (post-intervention): nil
Differences in baseline characteristics: reported
Reliable outcomes: reported
Protection against contamination: NR
Unit of allocation: school
Unit of analysis: individual accounting for clustering

\begin{tabular}{|c|c|}
\hline \multirow[t]{10}{*}{ Participants } & $\mathrm{N}$ (control baseline) = 1499 \\
\hline & $\mathrm{N}$ (control follow-up) = 1168 \\
\hline & $\mathrm{N}$ (intervention baseline) $=1271$ \\
\hline & $\mathrm{N}$ (intervention follow-up) = 1048 \\
\hline & $\begin{array}{l}\text { Setting (and number by study group): } 20 \text { schools in total, } 2622 \text { children (10 ( } N=1382) \text { control, } 10(N= \\
1240) \text { intervention) }\end{array}$ \\
\hline & Recruitment: all primary schools in inner-city areas of Rotterdam were free to apply for participation \\
\hline & Geographic region: Rotterdam, Netherlands \\
\hline & Percentage of eligible population enrolled: $74 \%$ schools \\
\hline & Mean age: Grade 3-5: intervention, 7.7 (1.0), control: 7.8 (1.0) Grade 6-8: 10.8 (1.0), control: 10.8 (1.0) \\
\hline & $\begin{array}{l}\text { Sex: Grade 3-5: intervention, } 50.5 \% \text { female; control, } 51 \% \text { female. Grade } 6-8 \text { : intervention, } 52.8 \% \text { female; } \\
\text { control, } 49 \%\end{array}$ \\
\hline
\end{tabular}


Jansen 2011 (Continued)

Interventions
To reduce overweight and inactivity in children by addressing both behavioural and environmental determinants.

Multicomponent intervention delivered by teachers and integrated into curriculum. Main components of the intervention were the implementation of 3 PE sessions a week by a professional PE teacher, additional sport and play activities outside school hours and an educational programme.

A 2 nd component of the intervention was the organisation of additional sport and play activities outside school hours that can be attended on a voluntary basis.

A 3rd component is classroom education with 3 main lessons on healthy nutrition, active living and healthy lifestyle choices adapted for each grade. The lessons were provided by the classroom teacher, and comprised a homework assignment, a theoretical part and a practical part, during which knowledge was applied in activities. Each lesson finishes with joint goal setting.

A 4th component was the administration of the Eurofit test, comprising measurements of height, weight and 9 different fitness tests, at the beginning and the end of the school year.

Other components were a health-promotion gathering at the beginning of the school year for parents and the involvement of local sport clubs. Local sport clubs were involved in providing some of the PE classes and PA activities outside school hours

Control schools continued with their usual curriculum. The usual curriculum of primary schools in the Netherlands consists of two PE sessions a week by the classroom teacher or a PE teacher, dependent on the school's policy.

Diet and PA intervention vs control

Outcome measures
- Primary outcome: BMI, \% overweight, waist circumference and fitness
- Secondary outcomes: NR
Process evaluation: NR

Implementation-related

Theoretical basis: TPB, Ecological Model

factors

Resources for intervention implementation: reported

Who delivered the intervention: reported

PROGRESS categories assessed at baseline: gender, race/ethnicity

PROGRESS categories analysed at outcome: NR

Outcomes relating to harms/unintended effects: NR

Intervention included strategies to address diversity or disadvantage: participants recruited from deprived inner city areas

Economic evaluation: NR

\section{Risk of bias}

\begin{tabular}{lll}
\hline Bias & Authors' judgement & Support for judgement \\
\hline $\begin{array}{l}\text { Random sequence genera- } \\
\text { tion (selection bias) }\end{array}$ & Low risk & $\begin{array}{l}\text { Randomisation took place within each pair with the toss of a coin by an officer } \\
\text { of the municipal education service in the presence of 1st study author. }\end{array}$
\end{tabular}


Jansen 2011 (Continued)

\begin{tabular}{|c|c|c|}
\hline $\begin{array}{l}\text { Allocation concealment } \\
\text { (selection bias) }\end{array}$ & Unclear risk & NR \\
\hline $\begin{array}{l}\text { Blinding (performance } \\
\text { bias and detection bias) } \\
\text { All outcomes }\end{array}$ & High risk & $\begin{array}{l}\text { Data collection staff (as well as the pupils and teachers) would certainly be } \\
\text { aware of which person or school was in which study condition. }\end{array}$ \\
\hline $\begin{array}{l}\text { Incomplete outcome data } \\
\text { (attrition bias) } \\
\text { All outcomes }\end{array}$ & High risk & $\begin{array}{l}82 \% \text { (in intervention) vs } 78 \% \text { (in control) retention for BMI at follow-up, } 3 \\
\text { matched pairs of schools were lost after randomisation: "2 started with inter- } \\
\text { vention components before study" also lost prior to baseline data collection; } \\
\text { due to organisational problems in data collection follow-up measures on waist } \\
\text { circumference were lacking for the pupils in the highest grade of another inter- } \\
\text { vention school. Imputation used for missing data }\end{array}$ \\
\hline
\end{tabular}

Selective reporting (re- Low risk Protocol/trial registration document seen. All outcomes reported
porting bias)

\begin{tabular}{lll}
\hline Other bias & Low risk & No additional threats to validity \\
\hline $\begin{array}{l}\text { Other bias- timing of re- } \\
\text { cruitment of clusters }\end{array}$ & Low risk & Figure 1 suggests recruitment happened prior to randomisation \\
\hline
\end{tabular}

Johnston 2013

Study design: cluster-RCT
Intervention period: 2 years
Follow-up period (post-intervention): nil
Differences in baseline characteristics: reported
Reliable outcomes: reported
Protection against contamination: NR
Unit of allocation: school
Unit of analysis: individual accounting for clustering

Participants
$N($ control baseline $)=326$
$N($ control follow-up $)=237$
$N($ intervention baseline $)=509$
$N($ intervention follow-up $)=392$

Setting (and number by study group): 7 elementary schools in a large suburban independent school district (4 intervention schools and 3 control schools)

Recruitment: schools were contacted via 2 phone calls, an email sent from the research staff to appropriate school personnel, and an email sent by the school district notifying the schools' personnel

Geographic region: southwest of Houston, TX, USA

Percentage of eligible population enrolled: $17 \%$ schools, $89 \%$ participants

Mean age: $7-9$; intervention: $7.8 \pm 0.4$; control: $7.7 \pm 0.4$ 
Johnston 2013 (Continued)

Sex: intervention: $38.2 \%$ female, $\mathrm{N}=186$ overweight/obese; $46.7 \%$ female, $\mathrm{N}=300$ normal weight; control: $45.9 \% \%$ female, $\mathrm{N}=135$ overweight/obese; $54.2 \%$ female, $\mathrm{N}=177$ normal weight

Interventions

The goal of the intervention was to slow the rate of weight gain in children through training staff to promote more healthful behaviours in their students.

Integrated health and PE into existing school core curriculum using MI to address resistance to change. The intervention "professional-facilitated intervention" ( PFI ) employed trained health professionals who assisted teachers in creating and implementing lesson plans incorporating healthy messages. Health professionals worked with school administration, cafeteria staff and elective teachers to create a healthier school environment. Teachers assisted by trained health professionals, $20 \mathrm{~h}$ of didactic training, $40 \mathrm{~h}$ of in vivo training, and $40 \mathrm{~h}$ of supervised practice. Weekly supervision with 2 clinical psychologists and a registered dietician for $60 \mathrm{~min}$. All school staff involved.

Control: self-help condition where schools received the same curriculum materials and were encouraged to incorporate healthy messages into their existing curricula. No additional training or support was provided.

Diet and PA combination intervention vs control

Outcome measures
- Primary outcome: zBMI
- Secondary outcomes: academic end-of-year grades
Process evaluation: reported: fidelity (intervention group only)

Implementation-related factors

\section{Theoretical basis: NR; MI strategies}

Resources for intervention implementation: reported

Who delivered the intervention: reported

PROGRESS categories assessed at baseline: gender, race/ethnicity

PROGRESS categories analysed at outcome: gender, race/ethnicity

Outcomes relating to harms/unintended effects: NR

Intervention included strategies to address diversity or disadvantage: NR, from diverse ethnic backgrounds (Asian $=25.3 \%$, black $=23.3 \%$, Hispanic $=23.1 \%$, white $=28.3 \%$ )

Economic evaluation: NR

Notes Funding: NR

Results are split by weight status, overall sample NR

Teachers and other school staff from schools assigned to the PFI condition attended $93 \%$ of meetings with the health professionals over 2 years. Only 3 teachers out of 20 in the PFI condition did not meet the standard of 5 teaching moments per week, 1 integrated lesson weekly, 1 activity every 2 weeks, and 1 school-wide activity per semester.

Study author provided change in weight, BMI and zBMI for the Asian overweight/obese subgroup by treatment condition

\section{Risk of bias}

Bias Authors' judgement Support for judgement

Random sequence genera- Low risk Randomised using a random number generator
tion (selection bias)


Johnston 2013 (Continued)

Allocation concealment $\quad$ Unclear risk $\quad$ NR
(selection bias)

\begin{tabular}{|c|c|c|}
\hline $\begin{array}{l}\text { Blinding (performance } \\
\text { bias and detection bias) } \\
\text { All outcomes }\end{array}$ & High risk & $\begin{array}{l}\text { Blinding of participants and study staff to the condition that they were in was } \\
\text { not possible. }\end{array}$ \\
\hline
\end{tabular}

\begin{tabular}{lll}
\hline $\begin{array}{l}\text { Incomplete outcome data } \\
\text { (attrition bias) } \\
\text { All outcomes }\end{array}$ & Low risk & \\
\hline $\begin{array}{l}\text { Selective reporting (re- } \\
\text { porting bias) }\end{array}$ & Unclear risk & Protocol/trial registration documents were unavailable \\
\hline Other bias & Low risk & No additional threats to validity \\
\hline $\begin{array}{l}\text { Other bias- timing of re- } \\
\text { cruitment of clusters }\end{array}$ & Low risk & Figure 1 suggests recruitment happened prior to randomisation \\
\hline
\end{tabular}

Kain 2014

$\begin{array}{ll}\text { Methods } & \text { Study design: cluster-RCT } \\ & \text { Intervention period: } 12 \text { months } \\ & \text { Follow-up period (post-intervention): nil } \\ & \text { Differences in baseline characteristics: NR } \\ & \text { Reliable outcomes: reported } \\ & \text { Protection against contamination: NR } \\ & \text { Unit of allocation: school } \\ & \text { Unit of analysis: individual }\end{array}$

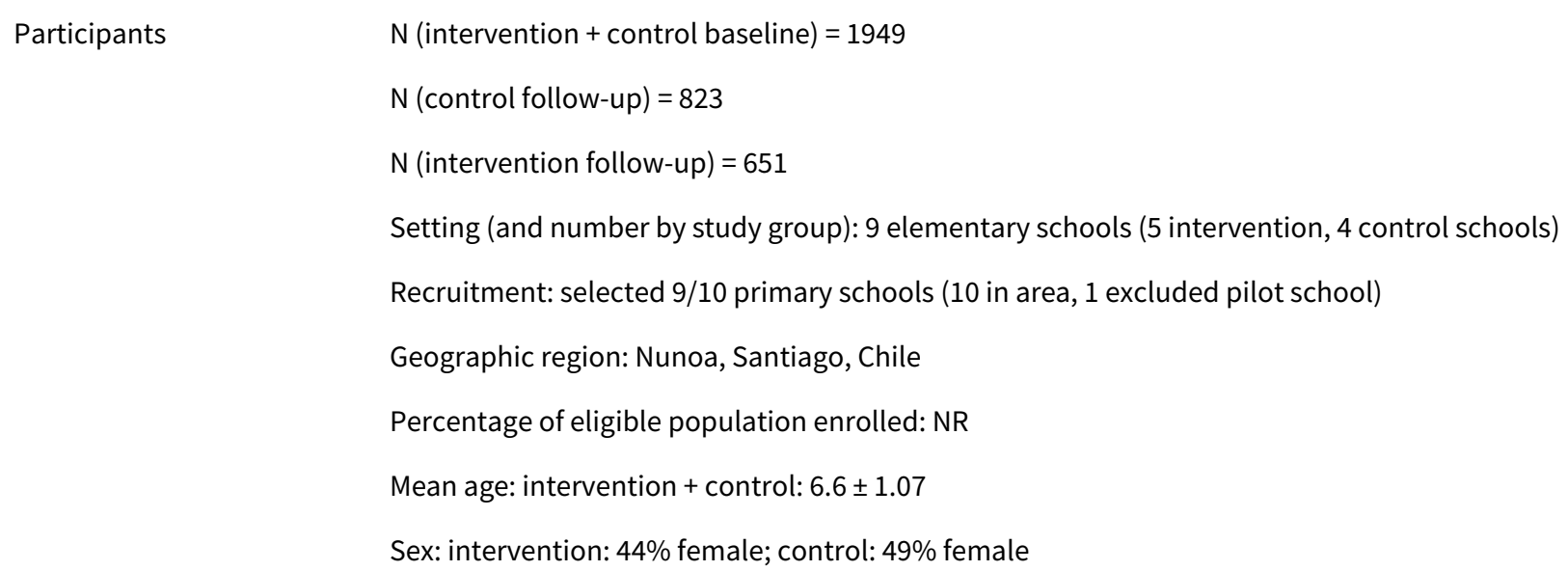


Kain 2014 (Continued)

- Training in 5 intervention schools

- 2 nutritionists trained teachers from kindergarten-3rd grade on the correct application of the contents of a special booklet that included 8 sessions of 90 min each on healthy eating for the children (6 h of training) $N=38$ teachers

- Teachers of PE classes from 1st-3rd grade were trained $(6 \mathrm{~h})$ by a specialist on the use of a book, containing a leaflet for each class which included drawings of different exercises recommended to increase MVPA ( $\mathrm{N}=12$ teachers)

- Training of kiosk owners (4 h) using a book which showed how to gradually offer $80 \%$ of healthy foods ( $N=8$ owners)

- Parents

- During 1 regular school meeting, the study nutritionist briefly explained in every class the objectives of the programme and specifically the types and combination of snacks considered to be 'healthy'

Period 2: March-November 2012

- Training teachers of children 1 st-3rd grade

- At the beginning of the school year we repeated the training process for newly hired teachers (3 teachers for healthy eating and 1 for PE (6 hrs each)

- Parents of children 1 st-3rd grade

- Twice during the year in each class, the study nutritionist briefly interacted with parents (15 min)

- PA

- In 2011 there were 2 weekly PE classes, 1 lasting 90 min and the other $45 \mathrm{~min}$. In 2012, the duration of the 2 nd class increased to $90 \mathrm{~min}$. Control schools followed the regular curriculum.

Diet and PA combination intervention vs control

\section{Outcomes}

Outcome measures

- Primary outcome: zBMI, obesity prevalence

- Secondary outcomes: healthy eating knowledge, types of food brought to school

Process evaluation: reported: implementation

Implementation-related factors
Theoretical basis: NR

Resources for intervention implementation: reported

Who delivered the intervention: reported

PROGRESS categories assessed at baseline: gender

PROGRESS categories analysed at outcome: gender

Outcomes relating to harms/unintended effects: NR

Intervention included strategies to address diversity or disadvantage: NR

Economic evaluation: NR

\section{Notes}

Funding: this study was supported by the Chilean Ministry of Education, Chile Deportes (Government Sports Promotion Agency) and an unrestricted grant from Corpora Tresmontes.

This multicomponent intervention included a set of activities related to healthy eating and PA as part of a wider programme. It is important to point out that specifically these activities (and not others) were implemented because school principals and teachers only accepted the implementation and evaluation of the ones we report here. The only curricular initiative consisted in extending PE class time, while the others included training classroom teachers to deliver contents on healthy eating and PE teachers to improve the quality of their classes. \% class time in MVPA declined (24.5-16.2) while remaining unchanged (24.8-23.7\%) in classes conducted by untrained and trained teachers, respectively. 
Kain 2014 (Continued)

We were not able to implement two activities that were programmed: greater parental involvement and the transformation of the school kiosk into one that offers $80 \%$ of healthy foods.

\section{Risk of bias}

\begin{tabular}{|c|c|c|}
\hline Bias & Authors' judgement & Support for judgement \\
\hline $\begin{array}{l}\text { Random sequence genera- } \\
\text { tion (selection bias) }\end{array}$ & Unclear risk & Randomisation stratified by SES, no further details \\
\hline $\begin{array}{l}\text { Allocation concealment } \\
\text { (selection bias) }\end{array}$ & Unclear risk & NR \\
\hline $\begin{array}{l}\text { Blinding (performance } \\
\text { bias and detection bias) } \\
\text { All outcomes }\end{array}$ & Unclear risk & NR \\
\hline $\begin{array}{l}\text { Incomplete outcome data } \\
\text { (attrition bias) } \\
\text { All outcomes }\end{array}$ & Unclear risk & Insufficient reporting of attrition/exclusions; $76 \%$ retention apparently \\
\hline $\begin{array}{l}\text { Selective reporting (re- } \\
\text { porting bias) }\end{array}$ & Unclear risk & $\begin{array}{l}\text { Protocol/trial registration documents were unavailable. RCT report presents } \\
\text { outcomes by individual schools and by boys/girls but not by overall interven- } \\
\text { tion vs control }\end{array}$ \\
\hline Other bias & Low risk & No additional threats to validity \\
\hline $\begin{array}{l}\text { Other bias- timing of re- } \\
\text { cruitment of clusters }\end{array}$ & Low risk & $\begin{array}{l}\text { No CONSORT Figure; text indicates recruitment happened prior to randomisa- } \\
\text { tion }\end{array}$ \\
\hline
\end{tabular}

Keller 2009

$\begin{array}{ll}\text { Methods } & \text { Study design: RCT } \\ \text { Intervention period: } 12 \text { months } & \text { Follow-up period (post-intervention): nil } \\ & \text { Differences in baseline characteristics: N/A } \\ & \text { Reliable outcomes: N/A } \\ & \text { Protection against contamination: N/A } \\ & \text { Unit of allocation: individual } \\ & \text { Unit of analysis: individual } \\ & \text { N (controls baseline) }=185 \\ & N \text { (controls follow-up) }=134 \\ & N \text { (interventions baseline) }=59 \\ & N \text { (interventions follow-up) }=49 \\ \text { Participants } & \text { Setting: home }\end{array}$


Keller 2009 (Continued)

Recruitment: the network CrescNet collected data (participant height and weight) from $>300,000$ children and 365 were selected at risk of obesity (age 4-7 years) to participate

Geographic region: Germany

Percentage of eligible population enrolled: $33 \%$

Mean age: intervention, $5.9 \pm 1.4$; control: $5.6 \pm 1.2$

Sex: both male and female

Interventions

- The paediatrician carried out a low-threshold intervention that consisted of an age-adapted nutrition and exercise programme to inspire the awareness of the adequate nourishment and motion

- 3-monthly measurement of height and weight by paediatrician and consultation about aims to change lifestyle (diet and exercise) and progress to targets based on results of questionnaire (PA) and food diaries

- 3 food diaries over period of 12 months, each for 5 days including 1 weekend. Dietician passed recommendations for dietary change (based on food diaries) to paediatrician for consultation with family and child

Combined effects of dietary interventions and PA interventions vs control

$\begin{array}{ll}\text { Outcomes } & \text { Height, weight } \\ & \text { Process evaluation: N/A } \\ & \text { Theoretical basis: NR } \\ \text { Implementation-related } & \text { Resources for intervention implementation (e.g. funding needed or staff hour } \\ \text { factors } & \text { Who delivered the intervention: reported } \\ & \text { PROGRESS categories assessed at baseline: reported (gender) } \\ & \text { PROGRESS categories analysed at outcome: reported (gender) } \\ & \text { Outcomes relating to harms/unintended effects: NR } \\ \text { Intervention included strategies to address diversity or disadvantage: NR } \\ \text { Economic evaluation: NR }\end{array}$

Notes

Funding: NR. The study author declaration states the study authors received no financial incentive, but stops short of saying a) who funded the research and b) that data and analysis were separated from any financial backers.

Quote: "The authors declare that they have no financial ties with a company whose product plays an important role in the article (or with a companies that distribute a competitor product (Die autoren erklaren, dass sie keine finanziellen Verbindungen mit einer Firmer haben, deren Produkt in dem Artikel eine wichtige Rolle spielt (oder mit einer Firma, die ein Konzkurrenzprodukt vertreibt)"

\section{Risk of bias}

\section{Bias}

Authors' judgement Support for judgement

Random sequence genera- Unclear risk Randomisation; no further details

tion (selection bias)

\begin{tabular}{l}
$\begin{array}{l}\text { Allocation concealment } \quad \text { Unclear risk } \\
\text { (selection bias) }\end{array}$ \\
\hline
\end{tabular}


Keller 2009 (Continued)
Blinding (performance
Unclear risk
Cannot translate
bias and detection bias)
All outcomes

\begin{tabular}{lll}
\hline $\begin{array}{l}\text { Incomplete outcome data } \\
\text { (attrition bias) } \\
\text { All outcomes }\end{array}$ & High risk & $\begin{array}{l}<30 \% \text { retained in intervention group while 72\% retained in control group after } \\
\text { randomisation to study completion at one year }\end{array}$ \\
\hline $\begin{array}{l}\text { Selective reporting (re- } \\
\text { porting bias) }\end{array}$ & Unclear risk & Protocol/trial registration documents were unavailable \\
\hline Other bias & Low risk & No further threats to validity
\end{tabular}

Khan 2014

Study name: FITKids (Fitness improves thinking in kids)
Study design: RCT
Intervention period: 9 -months
Follow-up period (post-intervention): nil
Differences in baseline characteristics: reported
Reliable outcomes: reported
Protection against contamination: NR
Unit of allocation: individual
Unit of analysis: individual

\begin{tabular}{|c|c|}
\hline \multirow[t]{10}{*}{ Participants } & $\mathrm{N}$ (control baseline $)=1100$ \\
\hline & $\mathrm{N}$ (control follow-up) $=90$ \\
\hline & $\mathrm{N}$ (intervention baseline) $=110$ \\
\hline & $\mathrm{N}$ (intervention follow-up) = 103 \\
\hline & Setting (and number by study group): after school \\
\hline & Recruitment: NR \\
\hline & Geographic region: Illinois, USA \\
\hline & Percentage of eligible population enrolled: $78 \%$ \\
\hline & Mean age: intervention: $8.8 \pm 0.5$; control: $8.8 \pm 0.6$ \\
\hline & Sex: intervention: $49 \%$ female; control: $45 \%$ female \\
\hline \multirow[t]{2}{*}{ Interventions } & $\begin{array}{l}\text { To investigate the effect of a 9-month PA intervention on cardiorespiratory fitness and adiposity among } \\
\text { prepubertal children. (Main aim of study was cognitive health) }\end{array}$ \\
\hline & $\begin{array}{l}\text { The intervention group received a 2-h intervention ( } 5 \text { days/week for } 9 \text { months) based on the 'Child } \\
\text { and adolescent trial for cardiovascular health (CATCH)' curriculum. This is an evidence-based PA pro- } \\
\text { gramme that provides MVPA in a non-competitive environment. The sessions consisted of } 70 \text { min of in- } \\
\text { termittent MVPA. }\end{array}$ \\
\hline
\end{tabular}


Khan 2014 (Continued)

Each session began with 20-25 min at PA stations focused on a health-related fitness component (e.g. cardiorespiratory fitness, muscular strength). After the fitness activities, a healthful snack was provided during the 15-min educational component (topics included goal setting, self-management, and self-efficacy).

After the educational component, participants engaged in 50-55 min of organisational games or sportoriented activities (e.g. dribbling a basketball). The sessions concluded with a 15-min cool-down period. A target heart zone for each child was established as 55\%-80\% of the child's maximum heart rate, and time below, time in, and time above the target heart zone was recorded. Trained research staff members encouraged participants to maintain their heart rate within the target zone throughout the session, with the exception of the time spent in the educational component.

Wait list control

PA intervention vs control

Outcome measures
- Primary outcome: event-related brain potentials, task performance, academic achievement (NR here)
- Secondary outcomes: BMI, zBMI, fat-free mass index, fat mass index, \% fat mass, \% central fat mass,
estimated visceral adipose tissue area, cardiorespiratory fitness (V02 max percentile)
- Also: magnetic resonance imaging, functional magnetic resonance imaging, eye tracking, virtual re-
ality, diet and brain function (NR here)

Process evaluation: reported (attendance)

Implementation-related factors
Theoretical basis: NR

Resources for intervention implementation: reported

Who delivered the intervention: reported

PROGRESS categories assessed at baseline: gender, race/ethnicity, SES

PROGRESS categories analysed at outcome: gender

Outcomes relating to harms/unintended effects: NR

Intervention included strategies to address diversity or disadvantage: NR

Economic evaluation: NR

Funding: all phases of this study were supported by NIH grant HD055352. Funded by the NIH

A USD 100 incentive was provided at pretest and follow-up. No monetary incentive was provided for participation in the after-school intervention, which was provided at no cost.

Actual setting is unclear, presume schools/community setting, participants visited the University laboratory for measurement.

Fidelity: attendance for the 150 -day programme ranged from $37 \%-99 \%$, with $85 \%$ of the participants attending $>70 \%$ of the intervention sessions.

\section{Risk of bias}

\begin{tabular}{lll}
\hline Bias & Authors' judgement & Support for judgement \\
\hline $\begin{array}{l}\text { Random sequence genera- } \\
\text { tion (selection bias) }\end{array}$ & Low risk & $\begin{array}{l}\text { Pairs of participants were matched for demographics and fitness, and a coin } \\
\text { was flipped to determine group assignment }\end{array}$ \\
\hline
\end{tabular}


Khan 2014 (Continued)

$\begin{array}{ll}\begin{array}{l}\text { Allocation concealment } \\ \text { (selection bias) }\end{array} & \begin{array}{l}\text { Randomisation was performed by an independent researcher who was not in- } \\ \text { volved in the data collection. No description of allocation }\end{array}\end{array}$

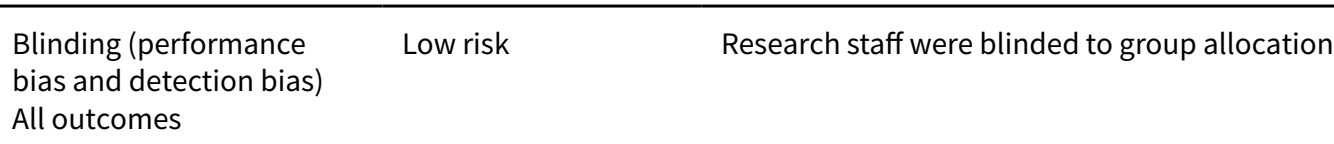

\begin{tabular}{|c|c|c|}
\hline $\begin{array}{l}\text { Incomplete outcome data } \\
\text { (attrition bias) } \\
\text { All outcomes }\end{array}$ & Unclear risk & $\begin{array}{l}\text { Low attrition ( } 12 \% \text {, across groups) and missing data at follow-up were imput- } \\
\text { ed with values observed at baseline. However, the participants who were lost } \\
\text { to follow-up had a significantly higher zBMI (MD } 0.60 ; \mathrm{Cl} 0.15 \text { to } 0.20 \text { ) compared } \\
\text { with trial completers. }\end{array}$ \\
\hline $\begin{array}{l}\text { Selective reporting (re- } \\
\text { porting bias) }\end{array}$ & Low risk & Protocol/trial registration document seen. All outcomes reported \\
\hline Other bias & Low risk & No additional threats to validity \\
\hline
\end{tabular}

\section{Kipping 2008}

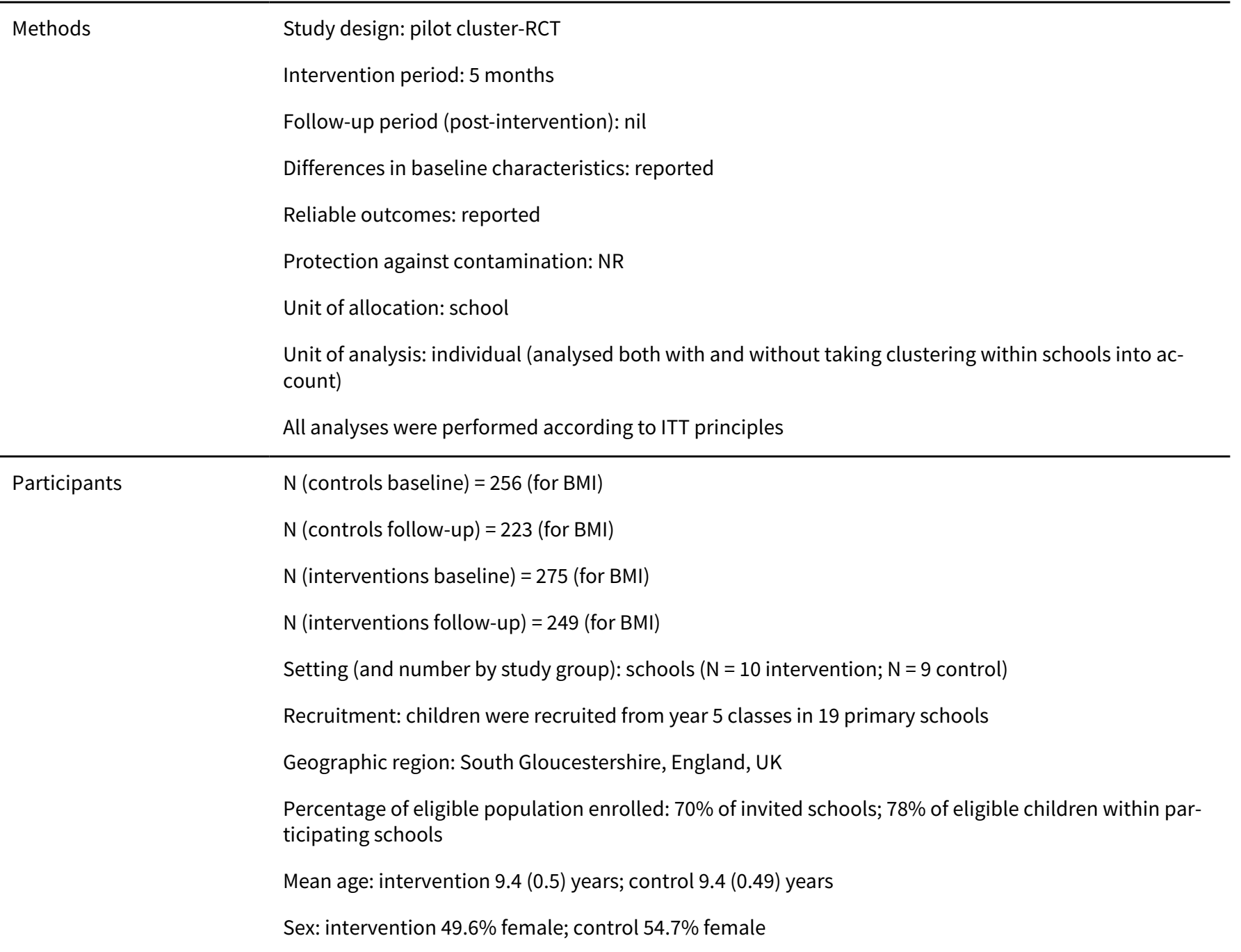


Kipping 2008 (Continued)

- 16 lessons on healthy eating, increasing PA and reducing TV viewing

- Changes from original programme included shortening the lesson plans, change US phrasing or references and change pyramid structure of food groups to the balance of good health. The pilot also did not include 2 staff meetings.

- 2 teachers provided a training session for 10 teachers who would be delivering the sessions.

- Materials provided to the schools, including lesson plans for 9 PA lessons, 6 nutrition lessons and 1 screen viewing session

Combined effects of dietary interventions and PA interventions vs control

- Primary outcome: reduction in time spent doing screen-based activities
- Other outcomes:
$\circ$ BMI
$\circ$ Obesity
$\circ$ Walks/cycles to and from school also included since there was a difference between groups at base-
line
Numbers included in final analysis
$\circ$ Intervention: BMI $75 \%$, screen questionnaire $48 \%$ and activity questionnaire $51 \%$
$\circ$ Control: BMI $64 \%$, screen questionnaire $47 \%$ and activity questionnaire $61 \%$

Process evaluation: reported

Implementation-related factors

\section{Theoretical basis: SCT and Behavioural Choice theory}

Resources for intervention implementation (e.g. funding needed or staff hours required): reported

Who delivered the intervention: reported

PROGRESS categories assessed at baseline: reported (gender)

PROGRESS categories analysed at outcome: reported (gender)

Outcomes relating to harms/unintended effects: NR

Intervention included strategies to address diversity or disadvantage: NR

Economic evaluation: NR (however cost of intervention materials was included)

Notes $\quad$ Funding: received from the Department of Health via the South West Public Health Group, South
Gloucestershire Council, and DAL was funded by a

Department of Health Career Scientist Award, which also funded data entry

\section{Risk of bias}

Bias Authors' judgement Support for judgement

$\begin{array}{ll}\text { Random sequence genera- } & \text { Unclear risk } \\ \text { tion (selection bias) } & 27 \text { schools in South Gloucestershire were invited to take part in the study and } \\ \end{array}$
tion (selection bias) 19 agreed to be in the study; "cluster randomised",no further details

\begin{tabular}{ll}
\hline $\begin{array}{l}\text { Allocation concealment } \\
\text { (selection bias) }\end{array}$ & $\begin{array}{l}\text { Allocation was at the school level and all schools allocated at the start of the } \\
\text { study, after schools were invited to participate and notified that they would be } \\
\text { allocated to either intervention or control groups. }\end{array}$ \\
$\begin{array}{l}\text { Quote: "Random allocation to intervention or control school was concealed } \\
\text { and done by one of the authors (DAL)" }\end{array}$ \\
\hline
\end{tabular}

Blinding (performance $\quad$ Low risk Outcome assessors and analysts were blinded
bias and detection bias)

All outcomes 
Kipping 2008 (Continued)

\begin{tabular}{|c|c|c|}
\hline $\begin{array}{l}\text { Incomplete outcome data } \\
\text { (attrition bias) } \\
\text { All outcomes }\end{array}$ & High risk & $\begin{array}{l}25 \% \text { individual loss in intervention compared to } 36 \% \text { in control; Reason was } \\
\text { mostly incomplete completion of personal identifiers on self-report question- } \\
\text { naires. Missing ones were not included in analysis }\end{array}$ \\
\hline
\end{tabular}

\begin{tabular}{lll}
\hline $\begin{array}{l}\text { Selective reporting (re- } \\
\text { porting bias) }\end{array}$ & Unclear risk & Protocol or trial register not found \\
\hline Other bias & Low risk & No other additional threats to validity \\
\hline $\begin{array}{l}\text { Other bias- timing of re- } \\
\text { cruitment of clusters }\end{array}$ & Low risk & Figure indicates recruitment happened prior to randomisation \\
\hline
\end{tabular}

\section{Kipping 2014}

Study name: Active for life year 5 (AFLY5)
Study design: cluster-RCT
Intervention period: 6-7 months (2/3 school terms)
Follow-up period (post-intervention): 5-6 months
Differences in baseline characteristics: reported
Reliable outcomes: reported
Protection against contamination: NR
Unit of allocation: schools
Unit of analysis: individual, accounting for clustering

Participants (control baseline) $=1157$
$N$ (control follow-up) $=N R$
$N$ (intervention baseline) $=1064$
N (intervention follow-up) = NR
Setting (and number by study group): 60 schools (30 schools in each group)
Recruitment: all state primary and junior schools with children in years 4-6 (age 8-11 years) in the areas
covered by Bristol City Council (93 schools) and North Somerset Council (55 schools) were invited to
participate
Geographic region: Bristol and North Somerset, England, UK
Percentage of eligible population enrolled: $41 \%$ schools, 99\% participants
Mean age: intervention: 9.5 \pm 0.3; control: 9.5 \pm 0.3
Sex: intervention: $49 \%$ female; control: $52 \%$ female
To investigate the effectiveness of a school-based intervention to increase PA, reduce sedentary behav-
iour, and increase fruit and vegetable consumption in children
- Training for year 5 classroom teachers and learning support assistants (provided by a nutritionist and
a PE specialist)
Provision of 16 lesson plans and teaching materials


Kipping 2014 (Continued)

- Provision of 10 parent-child interactive homework activities

- Provision of written information; written information for parents

Diet and PA combination intervention vs control

Outcome measures
- Primary outcome: MVPA, sedentary activity, fruit and vegetable consumption
- Secondary outcomes: screen viewing, snack consumption, high-fat food consumption, high-energy
drink consumption, BMI, waist circumference, general overweight/obesity, central overweight/obe-
sity

Process evaluation: NR

Implementation-related factors
Theoretical basis: SCT

Resources for intervention implementation: reported

Who delivered the intervention: reported

PROGRESS categories assessed at baseline: gender, SES (school deprivation score)

PROGRESS categories analysed at outcome: NR

Outcomes relating to harms/unintended effects: NR

Intervention included strategies to address diversity or disadvantage: NR

Economic evaluation: NR
Notes

\section{ISRCTN50133740}

Funding: the AFLY5 RCT is funded by the UK National Institute for Health Research (NIHR) Public Health Research Programme (09/3005/04), which also paid the salary of SW. DAL and LDH work in a unit that receives funds from the UK Medical Research Council (MRC) (MC_UU_12013/5). RRK and RC work in Centre for the Development and Evaluation of Complex Interventions for Public Health Improvement (DECIPHer), which receives funding from the British Heart Foundation, Cancer Research UK, the Economic and Social Research Council (RES-590-28-0005), the MRC, the Welsh Assembly Government, and the Wellcome Trust WT087640MA), under the auspices of the UK Clinical Research Collaboration. LDH is supported by a UK MRC population health scientist fellowship (G1002375). None of the funders had involvement in the Trial Steering Committee, data analysis, data interpretation, data collection, or writing of the paper.

The process evaluation in the pilot study found that the teachers thought the intervention should be extended to include parents if it was to be maximally effective.

Training and all materials provided. schools were financially compensated for the cost of replacement teachers while their staff attended training.

\section{Risk of bias}

\begin{tabular}{lll}
\hline Bias & Authors' judgement & Support for judgement \\
\hline $\begin{array}{l}\text { Random sequence genera- } \\
\text { tion (selection bias) }\end{array}$ & Low risk & $\begin{array}{l}\text { Grouped schools into six mutually exclusive strata by these two characteristics } \\
\text { and randomly }\end{array}$ \\
& $\begin{array}{l}\text { allocated them to control or intervention within these strata. One author who } \\
\text { was unaware of any characteristics of the schools did the randomisation (iden- } \\
\text { tification numbers were used to relate schools to the two stratifying variables, } \\
\text { and had no knowledge of which schools these numbers linked to). }\end{array}$ \\
\hline
\end{tabular}


Kipping 2014 (Continued)

\begin{tabular}{|c|c|c|}
\hline $\begin{array}{l}\text { Allocation concealment } \\
\text { (selection bias) }\end{array}$ & Low risk & $\begin{array}{l}\text { Randomisation was concealed by using the Bristol Randomised Trials Collabo- } \\
\text { ration's automated (remote) system. }\end{array}$ \\
\hline
\end{tabular}

\begin{tabular}{|c|c|c|}
\hline $\begin{array}{l}\text { Blinding (performance } \\
\text { bias and detection bias) }\end{array}$ & Low risk & $\begin{array}{l}\text { The fieldworkers who collected data from the children were all blinded to } \\
\text { school allocation }\end{array}$ \\
\hline
\end{tabular}

All outcomes

\begin{tabular}{|c|c|c|}
\hline $\begin{array}{l}\text { Incomplete outcome data } \\
\text { (attrition bias) } \\
\text { All outcomes }\end{array}$ & Low risk & $82-83 \%$ follow-up for weight \\
\hline $\begin{array}{l}\text { Selective reporting (re- } \\
\text { porting bias) }\end{array}$ & Low risk & $\begin{array}{l}\text { Protocol seen; All outcomes specified in methods have been reported in re- } \\
\text { sults }\end{array}$ \\
\hline Other bias & Low risk & No additional threats to validity \\
\hline $\begin{array}{l}\text { Other bias- timing of re- } \\
\text { cruitment of clusters }\end{array}$ & Low risk & Recruitment happened prior to randomisation \\
\hline
\end{tabular}

Klein 2010

\begin{tabular}{|c|c|}
\hline \multirow[t]{9}{*}{ Methods } & Study name: Kindergarten mobil (KiMo)-project \\
\hline & Study design: cluster-RCT \\
\hline & Intervention period: 5 months (intervention) vs 6 months (control) \\
\hline & Follow-up period (post-intervention): nil \\
\hline & Differences in baseline characteristics: reported \\
\hline & Reliable outcomes: reported \\
\hline & Protection against contamination: NR \\
\hline & Unit of allocation: kindergartens \\
\hline & Unit of analysis: individual \\
\hline \multirow[t]{10}{*}{ Participants } & $\mathrm{N}$ (control baseline $)=\mathrm{NR}$ \\
\hline & $\mathrm{N}$ (control follow-up) = 361 \\
\hline & $\mathrm{N}$ (intervention baseline) $=\mathrm{NR}$ \\
\hline & $\mathrm{N}$ (intervention follow-up) $=678$ \\
\hline & Setting (and number by study group): 27 kindergartens ( 16 intervention, 11 control) \\
\hline & Recruitment: NR \\
\hline & Geographic region: different districts of Cologne, Germany \\
\hline & Percentage of eligible population enrolled: NR \\
\hline & Mean age: intervention + control: $4.7 \pm 1.0$ \\
\hline & Sex: intervention + control: $46 \%$ female \\
\hline
\end{tabular}


Klein 2010 (Continued)

Interventions
To evaluate the effects of a low-threshold health-promotion intervention on anthropometry and motor abilities of preschool children in 16 intervention vs 11 control kindergartens

An information meeting for parents and educators was arranged after baseline testing in each of the 16 intervention kindergartens (duration: $90-120 \mathrm{~min}$ ). The theoretical model used as the basis of the intervention was a combination between the TPB and of the precaution adoption process model. Major aims were to enhance the parents' and educators' awareness of a healthy lifestyle and to impact parental skills and competencies concerning nutrition, PA and stress management. Contents included the importance of PA for children, the consequences of physical inactivity, basics of healthy nutrition and self-management.

The overall and individual results of the motor tests were presented. Performance for each test item was classified according to age and gender performance and presented in the form of medals. A gold medal represented the classification "mega super" and "very very super", a silver medal "very super" and "super", and the bronze medal "a bit super". This classification was chosen instead of school grades in order to achieve a high degree of participation and not to frustrate the children and parents. In addition, they received a fitness pass with the test results including raw data and classification as well as body height and weight and they presented key guidelines. Individual questions were clarified and advisory service was offered. Finally an oral feedback was obtained concerning the content of the information meeting. On average, $60 \%$ of the children were represented by at least 1 parent $(\mathrm{N}=466)$. For those parents who did not attend, the fitness passes were handed over to the head of the kindergarten. The head of the kindergarten and additionally 1 educator of each group were present at the information meeting.

Diet and PA intervention vs control (health education)
Implementation-related factors
Outcome measures

- Primary outcome: BMI, motor abilities

- Secondary outcomes: primary/secondary NR

Process evaluation: reported (attendance at information evening)

Theoretical basis: TPB, Precaution Adoption Process

Resources for intervention implementation: reported

Who delivered the intervention: reported

PROGRESS categories assessed at baseline: gender

PROGRESS categories analysed at outcome: gender

Outcomes relating to harms/unintended effects: NR

Intervention included strategies to address diversity or disadvantage: NR

Economic evaluation: reports direct costs

Notes Funding: NR. About EUR 1000 per kindergarten.

At the information meeting on average $60 \%$ of the children were represented by at least 1 parent

\section{Risk of bias}

Bias Authors' judgement Support for judgement

Random sequence genera- Unclear risk Kindergartens randomised, no further details tion (selection bias) 
Klein 2010 (Continued)

\begin{tabular}{lll}
$\begin{array}{l}\text { Allocation concealment } \\
\text { (selection bias) }\end{array}$ & Unclear risk & NR \\
\hline $\begin{array}{l}\text { Blinding (performance } \\
\text { bias and detection bias) }\end{array}$ & Unclear risk & NR \\
All outcomes & &
\end{tabular}

\begin{tabular}{ll}
\hline $\begin{array}{l}\text { Incomplete outcome data } \\
\text { (attrition bias) }\end{array}$ & High risk \\
All outcomes & $\begin{array}{l}\text { Only reports outcomes for participants with baseline and follow-up data; in- } \\
\text { tervention children were significantly older and taller than those of the control } \\
\text { and had a lower BMI but this was adjusted for in the analyses }\end{array}$ \\
\hline
\end{tabular}

\begin{tabular}{lll}
\hline $\begin{array}{l}\text { Selective reporting (re- } \\
\text { porting bias) }\end{array}$ & Unclear risk & Protocol or trial register not found \\
\hline Other bias & Low risk & No additional threats to validity \\
\hline $\begin{array}{l}\text { Other bias- timing of re- } \\
\text { cruitment of clusters }\end{array}$ & Unclear risk & NR \\
\hline
\end{tabular}

Klesges 2010

Methods

Study name: Memphis girls health enrichment multi-site studies (GEMS)

Study design: RCT

Intervention period: 24 months

Follow-up period (post-intervention): nil

Differences in baseline characteristics: reported

Reliable outcomes: reported

Protection against contamination: reported

Unit of allocation: individual

Unit of analysis: individual

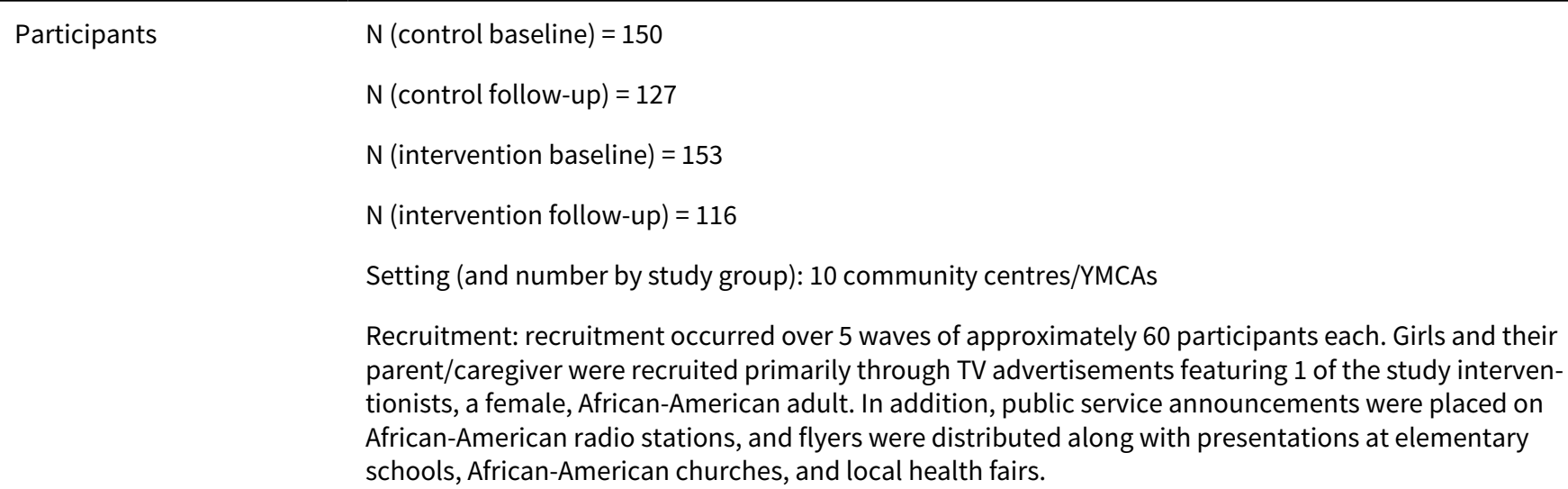

Geographic region: Memphis, Tennessee, USA

Percentage of eligible population enrolled: $90 \%$ of screened

Mean age: intervention: $9.3 \pm 0.9$; control: $9.3 \pm 0.9$ 
The obesity prevention intervention provided practical experience with nutrition and PA. Girls and caregivers participated in the obesity prevention intervention through a combination of separate and joint sessions. Girls developed behavioural goals to eat a nutritionally balanced diet, reduce consumption of SSBs and high-fat high caloric foods, increase intake of water, vegetables and fruits, increase MVPA and decrease sedentary behaviour. Behavioural strategies included skill-building, self-monitoring, feedback and positive reinforcement, goal-setting, problem-solving and social support. Caregivers were encouraged to make changes in the home food environment such as increasing the availability of healthy foods.

Both intervention groups had the same number and frequency of sessions. Meetings occurred weekly for 14 weeks and then monthly for 20 months (34 sessions over 2 years). Sessions lasted approximately $90 \mathrm{~min}$. During the 2 nd year, both interventions transitioned to monthly field trips within the community. Interventionists were African-American women with experience teaching and working with children. There were separate interventionists for the obesity prevention and alternative intervention groups, and they were trained only for their assigned intervention.

The alternative intervention targeted the girls only and was designed to provide meaningful benefits with the goal of improving self-esteem and social efficacy. There was no focus on changing behaviours at home or activities related to diet, PA or body weight.

Diet and PA combination intervention vs alternative intervention

Outcome measures
Outcomes
- $\begin{aligned} & \text { Primary outcome: BMI } \\ & \text { etary intake, PA }\end{aligned}$

Process evaluation: reported (fidelity)

\author{
Implementation-related Theoretical basis: NR \\ factors \\ Resources for intervention implementation: NR \\ Who delivered the intervention: NR \\ PROGRESS categories assessed at baseline: NR \\ PROGRESS categories analysed at outcome: NR \\ Outcomes relating to harms/unintended effects: NR \\ Intervention included strategies to address diversity or disadvantage: targeted African-American girls \\ Economic evaluation: NR
}

Funding: research was supported by co-operative agreements HL62662 and HL62663 from the National Heart, Lung, and Blood Institute, NIH.

Included children with $\mathrm{BMI} \geq 25$ th age-sex specific percentile or have at least 1 parent with $\mathrm{BMI} \geq 25 \mathrm{~kg} /$ $\mathrm{m} 2$. Girls were excluded if they had BMI $>35 \mathrm{~kg} / \mathrm{m} 2$.

Of the $10 \%$ randomly videotaped sessions, the Project Director determined whether the objectives were consistently implemented. The range of sessions judged acceptable was $92 \%-100 \%$ (reflects ratings based on 1 = strongly agree or $2=$ agree). Session attendance over the 2 years averaged 27.8 (SD $=8.05)$ for the obesity prevention intervention and $27.9(S D=8.10)$ for the alternative intervention, in- 
cluding make-up sessions, which comprised about $50 \%$ of all attendance $(P=0.94)$. The pilot for this study is Beech 2003

\section{Risk of bias}

\begin{tabular}{|c|c|c|}
\hline Bias & Authors' judgement & Support for judgement \\
\hline \multirow[t]{2}{*}{$\begin{array}{l}\text { Random sequence genera- } \\
\text { tion (selection bias) }\end{array}$} & Unclear risk & $\begin{array}{l}\text { Randomisation was stratified by recruitment wave, and within wave, by com- } \\
\text { munity centre }\end{array}$ \\
\hline & & $\begin{array}{l}\text { In the } 1 \text { st } 2 \text { centres, randomisation was done in } 2 \text { mirror-image blocks of } 15 \text {. } \\
\text { Recruitment fell short at both centres, so in the remaining waves, randomisa- } \\
\text { tion was done in independent blocks of } 5 \text { at each centre. }\end{array}$ \\
\hline $\begin{array}{l}\text { Allocation concealment } \\
\text { (selection bias) }\end{array}$ & Unclear risk & NR \\
\hline $\begin{array}{l}\text { Blinding (performance } \\
\text { bias and detection bias) } \\
\text { All outcomes }\end{array}$ & Low risk & $\begin{array}{l}\text { Although neither participants nor intervention staff were blinded to treatment } \\
\text { assignment, intervention staff did not perform any measurements after ran- } \\
\text { domisation, and measurement staff were blinded to treatment assignment. }\end{array}$ \\
\hline $\begin{array}{l}\text { Incomplete outcome data } \\
\text { (attrition bias) } \\
\text { All outcomes }\end{array}$ & Unclear risk & Retention was $76 \%$ intervention, $85 \%$ control \\
\hline $\begin{array}{l}\text { Selective reporting (re- } \\
\text { porting bias) }\end{array}$ & Low risk & Protocol/trial registration document seen. All outcomes reported \\
\hline Other bias & Low risk & Intervention and control sessions were conducted on different days \\
\hline
\end{tabular}

\section{Kriemler 2010}

\begin{tabular}{ll}
\hline Methods & Study name: KISS \\
Study design: cluster-RCT \\
Intervention period: 9 months \\
Follow-up period (post-intervention): 3 years \\
Differences in baseline characteristics: reported \\
Reliable outcomes: reported \\
Protection against contamination: reported \\
Unit of allocation: school \\
Unit of analysis: individual accounting for clustering (class and school) \\
N (control baseline) $=205$ \\
N (control follow-up) $=100$ \\
N (intervention baseline) $=297$ \\
N (intervention follow-up) $=189$ \\
Setting (and number by study group): 28 classes from 15 elementary schools (16 classes from 9 schools \\
in intervention group and 12 classes from 6 schools in control group)
\end{tabular}


Recruitment: 15 schools were randomly selected from 95 schools; then 15 schools were randomly assigned into intervention

Geographic region: 2/26 provinces of Switzerland (Aargau and Baselland)

Percentage of eligible population enrolled: $15 \%$ schools

Mean age: intervention: $6.9 \pm 0.3$ 1st graders; control: $6.9 \pm 0.31$ st graders; intervention: $11.0 \pm 0.5$ - 5th graders; control: $11.3 \pm 0.65$ th graders

Sex: intervention: $52 \%$ female; control: $50 \%$ female

Interventions

To assess the effectiveness of a school-based PA programme during 1 school year on physical and psychological health in young schoolchildren

Multi-component PA programme of 9 months including daily PE (i.e. 2 additional lessons/week on top of 3 regular lessons), short PA breaks during academic lessons, and daily PA homework.

Children in both groups had 3 PE lessons/week, which are compulsory by law. The intervention group had 2 additional PE lessons on the remaining school days. A team of expert PE teachers prepared all 5 $\mathrm{PE}$ lessons for the children in the intervention group. All intervention classes received the same curriculum. The 3 compulsory weekly PE lessons ( $45 \mathrm{~min}$ each) were given by the usual classroom teachers according to the specified curriculum, whereas the 2 additional weekly lessons (45 min each) were taught mostly outdoors by PE teachers.

In addition, 3-5 short activity breaks (2-5 min each) during academic lessons, comprising motor skill tasks such as jumping or balancing on 1 leg, power games, or co-ordinative tasks, were introduced every day. The children received daily PA homework of about 10 min' duration prepared by the PE teachers. This included aerobic, strength, or motor skill tasks such as brushing their teeth while standing on 1 leg, hopping up and down the stairs, rope jumping, or comparable activities.

PA combination intervention vs control

\section{Outcomes}

Outcome measures

- Primary outcome: sum of 4 skinfolds, aerobic fitness, PA, quality of life

- Secondary outcomes: BMI, CV risk score (comprising all components of the metabolic syndrome including waist circumference)

Process evaluation: NR

Implementation-related factors
Theoretical basis: SEM

Resources for intervention implementation: reported

Who delivered the intervention: reported

PROGRESS categories assessed at baseline: child: gender; parent: race/ethnicity, education

PROGRESS categories analysed at outcome: NR

Outcomes relating to harms/unintended effects: NR

Intervention included strategies to address diversity or disadvantage: NR

Economic evaluation: NR

\section{ISRCTN15360785}

Funding: this study was funded by the Swiss Federal Office of Sports (grant number SWI05-013), the Swiss National Science Foundation (grant number PMPDB-114401), and the Diabetes Foundation of the Region of Basel. The funding sources had no role in the design and conduct of the study or in the collection, management, analysis, and interpretation of the data. 
The level of adherence to the intervention outside school (PA homework) was insufficient, which is a limitation of this study.

\section{Risk of bias}

\begin{tabular}{|c|c|c|}
\hline Bias & Authors' judgement & Support for judgement \\
\hline $\begin{array}{l}\text { Random sequence genera- } \\
\text { tion (selection bias) }\end{array}$ & Low risk & $\begin{array}{l}\text { Randomly selected and assigned in a } 4: 3 \text { ratio after stratification for grade; se- } \\
\text { lected } 28 / 190 \text { consenting classes on the basis of a computer-generated ran- } \\
\text { dom number table that was in the hands of a person not involved in the study }\end{array}$ \\
\hline $\begin{array}{l}\text { Allocation concealment } \\
\text { (selection bias) }\end{array}$ & Low risk & $\begin{array}{l}\text { Randomly selected and assigned in a } 4: 3 \text { ratio after stratification for grade; se- } \\
\text { lected } 28 / 190 \text { consenting classes on the basis of a computer-generated ran- } \\
\text { dom number table that was in the hands of a person not involved in the study }\end{array}$ \\
\hline $\begin{array}{l}\text { Blinding (performance } \\
\text { bias and detection bias) } \\
\text { All outcomes }\end{array}$ & Unclear risk & $\begin{array}{l}\text { Assessors responsible for the measurements were blinded to the group alloca- } \\
\text { tion for all measurements except skinfold and waist circumference measures }\end{array}$ \\
\hline $\begin{array}{l}\text { Incomplete outcome data } \\
\text { (attrition bias) } \\
\text { All outcomes }\end{array}$ & High risk & $\begin{array}{l}36 \% \text { vs } 51 \% \text { (intervention vs control) attrition at } 3 \text { years post intervention. } \\
\text { Quote: "More obese children and those with a migrant background dropped } \\
\text { out tried to account for this possible bias by adding a propensity score to our } \\
\text { model (to adjust for differential participation) showing that our results re- } \\
\text { mained the same despite adjustment for participation differences. This is es- } \\
\text { pecially true for zBMI, for which we had a participation bias in favour of initial- } \\
\text { ly leaner children being more prevalent in the intervention than in the control } \\
\text { group" }\end{array}$ \\
\hline $\begin{array}{l}\text { Selective reporting (re- } \\
\text { porting bias) }\end{array}$ & Low risk & All pre-specified outcomes have been reported \\
\hline Other bias & Low risk & No additional threats to validity \\
\hline $\begin{array}{l}\text { Other bias- timing of re- } \\
\text { cruitment of clusters }\end{array}$ & Low risk & Figure shows recruitment happened prior to randomisation \\
\hline
\end{tabular}

Lana 2014

\begin{tabular}{ll}
\hline Methods & Study design: RCT \\
Intervention period: 9 months \\
Follow-up period (post-intervention): nil \\
Differences in baseline characteristics: reported \\
Reliable outcomes: NR; self-reported BMI \\
Protection against contamination: NR \\
Unit of allocation: individual \\
Unit of analysis: individual \\
\hline
\end{tabular}

Participants N(control baseline $)=284$


Lana 2014 (Continued)

\author{
$\mathrm{N}$ (control follow-up) $=316$ \\ $\mathrm{N}$ (intervention baseline $)=283$ \\ $\mathrm{N}$ (intervention follow-up) $=177$ group 1 \\ $\mathrm{N}$ (intervention follow-up) $=244$ group 2 \\ Setting (and number by study group): online (attending school)
}

Recruitment: the programme was supported by the educational authorities of Spain and Mexico and diffused among secondary education schools in both countries. Programme information was sent by email to all teachers. Links and banners were placed on the main educational portals. Participation was voluntary, but most interested teachers encouraged their students to participate.

Geographic region: Spain and Mexico

Percentage of eligible population enrolled: $51.9 \%$

Mean age: intervention: 12 years (26.6\%), 13 years (38.5\%), 14 years $(25.7 \%), \geq 15$ years $(9.2 \%)$; control: 12 years $(20.5 \%), 13$ years $(42.7 \%), 14$ years $(27.4 \%), \geq 15$ years $(9.4 \%)$

Sex: intervention: $55.4 \%$ female; control: $54.2 \%$ female

Interventions

To assess the impact of a web-based intervention supplemented with text messages to reduce cancer risk linked with smoking, unhealthy diet, alcohol consumption, obesity, sedentary lifestyle and sun exposure

1 control group and 2 experimental groups, which received exclusively the online intervention (experimental group 1) or the intervention supplemented with encouraging weekly text messages (experimental group 2).

Mechanism: how to prevent and treat main cancer-risk behaviours using the theoretical framework of the 'Attitude, social influence and self-efficacy (ASE) model

- emphasising advantages of following the recommendations and disadvantages of risk behaviours

- creating a healthy online social environment and

- strengthening the skills to avoid risk behaviours.

The section with the highest educational capacity contained problems or challenges that students had to solve. They were related both with subjects of their curriculum (e.g. math, literature or science) and with the risk-behaviour prevention. The website also provided other services, such as expert dietetic advice after analysing common homemade recipes and 24 -h food recalls, peer-starred educational videos, forums and chat lines to discuss cancer-related topics, documents and web links with selected information and online educational games.

Text messages were sent weekly to those who provided mobile numbers to encourage compliance with healthy behaviours. For instance, a text message focused on a healthy diet was the following: 'Don't be fooled! The best way to be pretty on the outside is by being pretty on the inside. Fruits and vegetables are your best makeup'.

Diet and PA combination intervention vs control

Outcomes Outcome measures

- Primary outcome: 6 cancer-risk behaviours: smoking, unhealthy diet, alcohol consumption. \% overweight/obesity, sedentary lifestyle and sun exposure

Process evaluation: NR

Implementation-related factors
Theoretical basis: ASE, TTM

Resources for intervention implementation: reported 
Lana 2014 (Continued)

Who delivered the intervention: reported

PROGRESS categories assessed at baseline: child: gender; parent: education, SES (income)

PROGRESS categories analysed at outcome: NR

Outcomes relating to harms/unintended effects: NR

Intervention included strategies to address diversity or disadvantage: NR

Economic evaluation: NR

Funding: this study was funded by the Spanish Ministry of Health. The financial backer had no role in the study design or in the collection, analysis and interpretation of data. Both the writing of the manuscript and the decision to submit it for publication belong to the study authors, who acted independently of the financial backer. All contributors had access to data

\section{Risk of bias}

\begin{tabular}{lll}
\hline Bias & Authors' judgement & Support for judgement \\
\hline $\begin{array}{l}\text { Random sequence genera- } \\
\text { tion (selection bias) }\end{array}$ & Low risk & $\begin{array}{l}\text { Randomisation using a computer program } \\
\text { Quote: "Participants were randomly assigned to either the control group (CG) } \\
\text { or experimental group (EG) using a computer program." }\end{array}$ \\
\hline $\begin{array}{l}\text { Allocation concealment } \\
\text { (selection bias) }\end{array}$ & Low risk & $\begin{array}{l}\text { Allocation was computer assigned. (From Protocol). Significant differences at } \\
\text { baseline and follow-up for demographic characteristics between groups }\end{array}$ \\
\hline $\begin{array}{l}\text { Blinding (performance } \\
\text { bias and detection bias) }\end{array}$ & Low risk & Presumed low as all self-reported, and computer-based \\
\hline $\begin{array}{l}\text { Incomplete outcome data } \\
\text { (attrition bias) } \\
\text { All outcomes }\end{array}$ & High risk & High dropout; 63\% across groups, which varied across the 3 groups \\
\hline $\begin{array}{l}\text { Selective reporting (re- } \\
\text { porting bias) }\end{array}$ & Low risk & Protocol seen; all outcomes specified in methods were reported in results \\
\hline \begin{tabular}{l} 
Other bias \\
\hline
\end{tabular} & Low risk & No additional threats to validity \\
\hline
\end{tabular}

Lazaar 2007

$\begin{array}{ll}\text { Methods } & \text { Study design: cluster-RCT } \\ \text { Intervention period: } 6 \text { months } \\ \text { Follow-up period (post-intervention): nil } \\ \text { Differences in baseline characteristics: reported } \\ \text { Reliable outcomes: reported } \\ \text { Protection against contamination: NR } \\ \text { Unit of allocation: school }\end{array}$


Lazaar 2007 (Continued)

Unit of analysis: individual

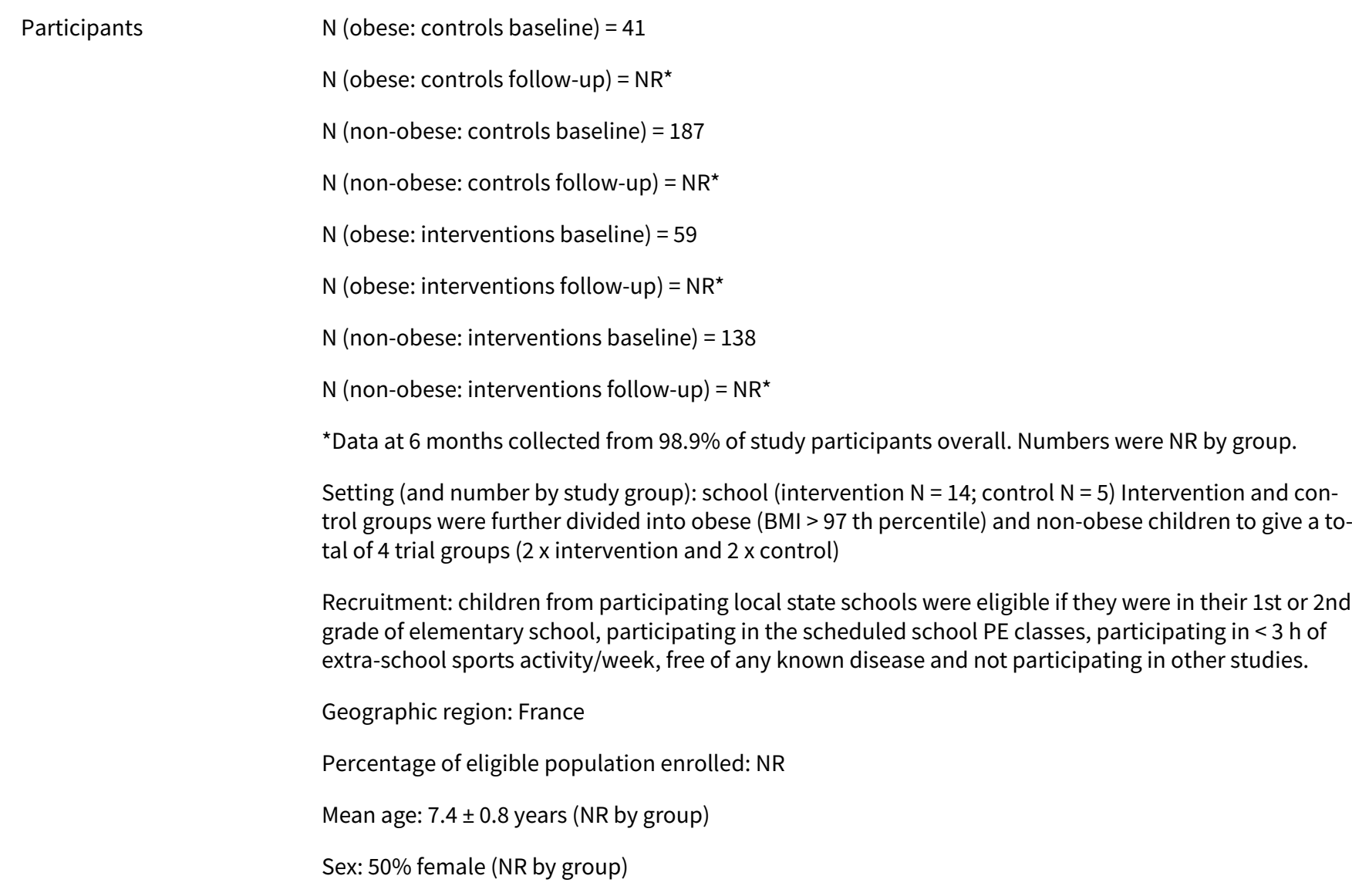

Interventions

Control: all children took part in scheduled school PE classes:

- Two 1-h sessions each week held within the school timetable

- Aimed at providing children with a rational basis for their activity programmes and for exercise in general

- Various combinations of 5-min exercises: exercises on co-ordination, exercises devoted to posture and balance, relaxation techniques, rhythm and music, exercises devoted to creative movement, games relating to group participation etc.

- Activities increased in intensity and duration throughout the study

Intervention: children in the intervention group were required to follow an additional PA programme:

- Two 1-h sessions each week held after class

- Objective: a playful physical practice and 45 min of dynamic exercise within the hour

- Exercise programme designed to enhance the joy of movement, body awareness and team spirit

- Based on traditional games aimed at minimising children's inactivity

- During a session, 2 children were randomly selected to monitor their energy expenditure and estimate the average intensity of the sessions and quantify the total duration of PA

PA interventions vs control

Outcomes

- Primary: obesity status 
Lazaar 2007 (Continued)

$$
\begin{aligned}
& \text { - Secondary: } \\
& \circ \text { BMI } \\
& \circ \text { zBMI } \\
& \circ \text { waist circumference } \\
& \circ \text { skinfold thickness } \\
& \circ \text { fat-free mass }
\end{aligned}
$$

Process evaluation: reported

Implementation-related

factors
Theoretical basis: NR

Resources for intervention implementation (e.g. funding needed or staff hours required): NR

Who delivered the intervention: reported

PROGRESS categories assessed at baseline: reported (gender)

PROGRESS categories analysed at outcome: reported (gender)

Outcomes relating to harms/unintended effects: NR

Intervention included strategies to address diversity or disadvantage: NR

Economic evaluation: NR

Notes

\begin{tabular}{|c|c|c|}
\hline Bias & Authors' judgement & Support for judgement \\
\hline $\begin{array}{l}\text { Random sequence genera- } \\
\text { tion (selection bias) }\end{array}$ & Low risk & A draw was carried out to choose intervention schools \\
\hline $\begin{array}{l}\text { Allocation concealment } \\
\text { (selection bias) }\end{array}$ & Low risk & $\begin{array}{l}\text { All eligible children from within schools were automatically assigned to groups } \\
\text { based according to school assignment and based on their individual BMI }\end{array}$ \\
\hline $\begin{array}{l}\text { Blinding (performance } \\
\text { bias and detection bias) } \\
\text { All outcomes }\end{array}$ & Unclear risk & NR \\
\hline $\begin{array}{l}\text { Incomplete outcome data } \\
\text { (attrition bias) } \\
\text { All outcomes }\end{array}$ & High risk & $\begin{array}{l}\text { Only report total }(\mathrm{N}=425) \text {, and group numbers }(138 ; 59 ; 187 ; 41) \text { once in text, } \\
\text { so unclear if these were analysed or randomised numbers. supplementary da- } \\
\text { ta in tables also have no numbers. }\end{array}$ \\
\hline $\begin{array}{l}\text { Selective reporting (re- } \\
\text { porting bias) }\end{array}$ & Unclear risk & $\begin{array}{l}\text { No protocol available; no tables visible - link does not work; text indicates that } \\
\text { post hoc analyses were conducted but these are not listed in the methods. } \\
\text { Outcomes for zBMI are presented only for post hoc subgroup analyses (gender, } \\
\text { baseline obesity). Main group differences are not presented }\end{array}$ \\
\hline Other bias & Low risk & No additional threats to validity \\
\hline $\begin{array}{l}\text { Other bias- timing of re- } \\
\text { cruitment of clusters }\end{array}$ & Unclear risk & NR: no consort figure in the paper or refereed to as supplementary file \\
\hline
\end{tabular}

Funding: this study was supported by grants from French National Plan for Nutrition and health (PNNS), the Comité Régional Exécutif des Actions de Santé d'Auvergne (CREAS), the Caisse Régionale d'Assurance Maladie d'Auvergne (CRAMA), the Appert Institutes, the town of Clermont-Ferrand and schools' governing bodies of Clermont-Ferrand

\section{Risk of bias}


Levy 2012

$\begin{array}{ll}\text { Methods } & \text { Study name: Nutrition on the go } \\ \text { Study design: 2-stage cluster-RCT } \\ \text { Intervention period: } 6 \text { months } \\ \text { Follow-up period (post-intervention): nil } \\ \text { Differences in baseline characteristics: reported } \\ \text { Reliable outcomes: reported } \\ \text { Protection against contamination: NR } \\ \text { Unit of allocation: school } \\ \text { Unit of analysis: individual accounting for cluster }\end{array}$

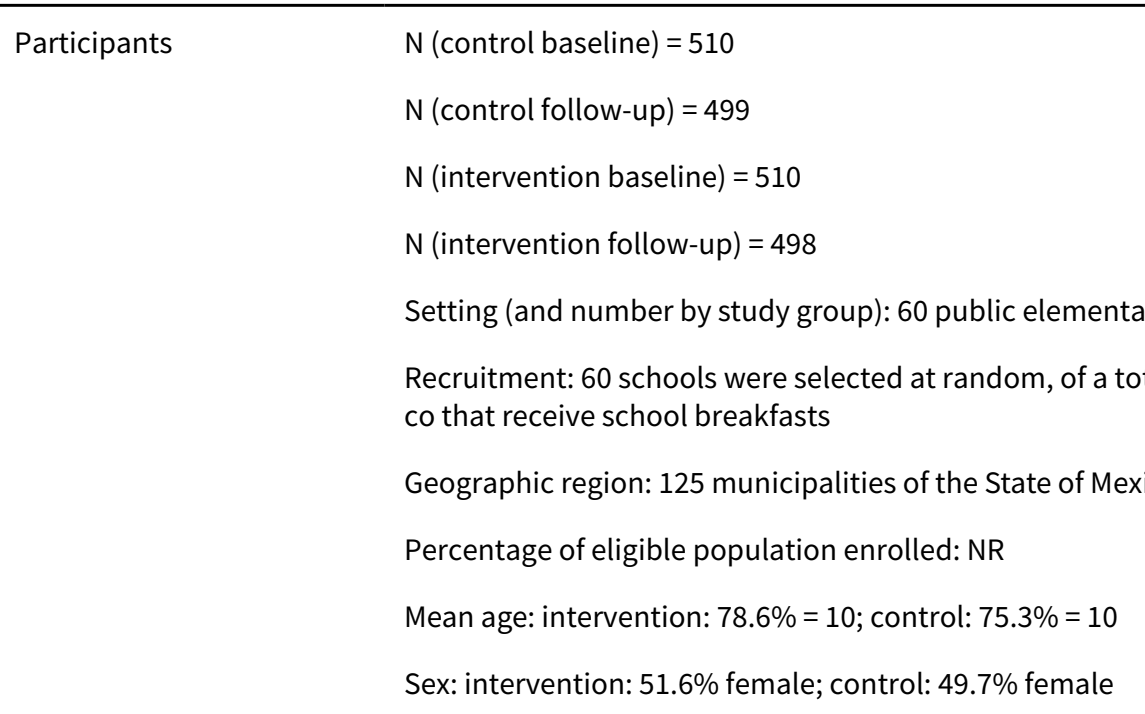

Interventions

To evaluate the effectiveness of a diet and PA strategy among school-aged children in the State of Mexico-known as 'Nutrition on the go' to maintain BMI, as a basis for establishing public health policy

The strategy mentions 4 components as listed below. However, does not give details about how components 1-3 are carried out. This paper appears to concentrate on component 4, 'Healthy break', further details of which are provided.

"The strategy "Nutrition on the Go" consisted of 4 components:

- a gradual decrease of the energy content of school breakfasts by reducing the fat content in milk, not increasing carbohydrates, decreasing the sugar content of the cereals provided and including fruit.

- the gradual regulation of food offered within the school, through the technical council of the State of Mexico.

- gradual adherence to the PA programme, according to the requirements of the Ministry of Public Education

- implementation of an educational campaign, called 'Healthy break' for healthy eating and PA. The objectives of this programme are to promote consuming 1 fruit and 1 vegetable, drinking pure water and performing PA (organised games and callisthenics) during break."

The components of the intervention are described as follows: (labelled here a to $j$ as described in the paper). 
"(a) Nutrition and PA workshops. These were divided into 6 sessions which included participatory recreational activities for children to gain knowledge and skills to properly select healthy foods and promoting PA.

(b) Puppet Theatre, based on the theory of peer learning. The 5th grade students participating in the study presented a puppet show to students from 1st to third grades after they studied the script and rehearsed for the performance.

(c) Two day workshop in each school for elementary school teachers. "The workshops sought to convey to teachers the importance of healthy eating and PA through dynamic and playful activities to promote participation.

(d) A session was held for store personnel to convey information about healthy eating, make suggestions about types of food to sell in schools and recommend the daily sale of vegetables, fruit and pure water. The importance of the responsibility of the cooperative (the food store inside the school) for preserving the health of the school community was addressed.

(e) School PA systems were used to promote consumption of water. Water bottles were delivered to children and teachers to encourage consumption.

(f) Physical activation. Organized activities involving motion were conducted twice per week. Activities per-formed each day before the start of classes included warm-ups, activation and relaxation. Recommendations to support physical activation were provided through the school guide and a CD with music for established activities. Weekly activation sessions gradually increased from 2 to 5 days.

(g) Broadcasting of audio spots on the schools' PA systems. Spots were broadcast 3 times per week during the break. The central messages were aimed at promoting the consumption of fruits, vegetables and pure water during break and to promote PA in children, with an average length of 1 min and 15 seconds per spot.

(h) Organized games during break (once per week). Active and safe participation of teachers and children was promoted during break

(i) Placement of banners at the entrance of the school. In order to highlight the campaign in the school community, a banner was hung that read, "This school pro- motes healthy breaks.

(j) Calendars with healthy recipes for school lunches were provided to parents.

Diet and PA combination intervention vs control"

\section{Outcome measures}

- Primary outcome: \% overweight, $\%$ obese, BMI

- Secondary outcomes: dietary intake, PA, knowledge, self-efficacy

Process evaluation: NR

Theoretical basis: NR

Resources for intervention implementation: reported

Who delivered the intervention: NR

PROGRESS categories assessed at baseline: gender, SES

PROGRESS categories analysed at outcome: gender, SES

Outcomes relating to harms/unintended effects: NR

Intervention included strategies to address diversity or disadvantage: NR; intervention targeted children receiving school breakfasts

Economic evaluation: NR 
Levy 2012 (Continued)

Notes
Funding: "this study was supported by: state system for the comprehensive development of the family, State of Mexico (DIFEM)

Materials were validated including a pilot study and an efficacy study prior to this RCT.

Subjects were beneficiaries of a school breakfast program in both federal and state educational systems with morning and evening shifts."

\section{Risk of bias}

\begin{tabular}{lll}
\hline Bias & Authors' judgement & Support for judgement \\
\hline $\begin{array}{l}\text { Random sequence genera- } \\
\text { tion (selection bias) }\end{array}$ & Unclear risk & $\begin{array}{l}\text { Randomly assigned schools then randomly selected participants within the } \\
\text { schools, no further details }\end{array}$ \\
\hline $\begin{array}{l}\text { Allocation concealment } \\
\text { (selection bias) }\end{array}$ & Unclear risk & $\begin{array}{l}\text { Randomly assigned schools then randomly selected participants within the } \\
\text { schools, no further details }\end{array}$ \\
\hline $\begin{array}{l}\text { Blinding (performance } \\
\text { bias and detection bias) } \\
\text { All outcomes }\end{array}$ & Unclear risk & $\begin{array}{l}\text { Quote: "A blind cluster-randomized field trial was conducted with fifth grade } \\
\text { school children. No indication who was blind" }\end{array}$ \\
\hline $\begin{array}{l}\text { Incomplete outcome data } \\
\text { (attrition bias) } \\
\text { All outcomes }\end{array}$ & Low risk & Loss to follow-up was 3.2\% and was evenly distributed by treatment group \\
\hline $\begin{array}{l}\text { Selective reporting (re- } \\
\text { porting bias) }\end{array}$ & Unclear risk & Protocol mentioned, but we were unable to find it \\
\hline $\begin{array}{l}\text { Other bias } \\
\begin{array}{l}\text { Other bias- timing of re- } \\
\text { cruitment of clusters }\end{array}\end{array}$ & Low risk & Figure 1 indicates that recruitment happened prior to randomisation \\
\hline
\end{tabular}

Li 2010a

$\begin{array}{ll}\text { Methods } & \text { Study name: The happy } 10 \text { program } \\ \text { Study design: cluster-RCT } \\ \text { Intervention period: } 12 \text { months } \\ \text { Follow-up period (post-intervention): } 12 \text { months } \\ \text { Differences in baseline characteristics: reported } \\ \text { Reliable outcomes: reported } \\ \text { Protection against contamination: NR } \\ \text { Unit of allocation: schools } \\ \text { Unit of analysis: individual accounting for clustering } \\ \text { N (control baseline) }=2371 \\ \text { N (control follow-up) }=2092 \\ \text { N (intervention baseline) }=2329\end{array}$


Li 2010a (Continued)

$\mathrm{N}$ (intervention follow-up) $=2028$

Setting (and number by study group): 20 schools (10 schools in each group)

Recruitment: randomly selected 2 districts, Dong Cheng and Chong Wen, from the eight districts in urban Beijing. 10 primary schools from each district were randomly chosen

Geographic region: Dong Cheng and Chong Wen districts, Beijing, China

Percentage of eligible population enrolled: $26 \%$ of schools were randomly selected, $96 \%$ participants

Mean age: intervention + control: $9.3 \pm 0.7$

Sex: intervention + control: $48 \%$ female

Interventions

The objective is to determine whether a large-scale PA intervention could affect body composition in primary school students in Beijing, China

The Happy 10 program was based on the principle of TAKE10!® (take10.net/). It consisted of 2 daily 10 min PA sessions conducted in the break between classes. The programme provided a variety of safe, moderate, age-, and space-appropriate exercises. Teaching materials included activity cards, video demonstrations, tracking posters, and stickers. Each activity card introduced 1 exercise and explained how to perform it. The videos showed students from the pilot study performing the activities. Teachers could either demonstrate the activity or show it on a video. The tracking poster and stickers were used to illustrate the progress of each class.

There were several activity models directly from TAKE 10! Program, such as "invisible jump rope"; "copy cat"; "all about you"; "stories on the move!"; "stories in space". Clear introductions were colourfully printed in the activity card. Students, teachers and parents were encouraged to develop new activity models, as were the programme staff. Many new programmes, much more than that directly from TAKE 10!, were developed, such as "story in zoo"; "story in farm"; "who is wearing yellow today"; "time like a colt"; "happy and health"; "little frog".

The 10-min sessions consisted of 4 parts:

- the teacher or student selected the cards to determine the activities

- several children were chosen to model the exercises in the front of the classroom and the other students followed along (1-3 activities were performed at each session)

- a cool-down period took place after the activities

- the students were taught a health message. If they chose the "invisible jump rope", each student pretended to have an invisible jump rope and began to jump. Teacher called out numbers from 1-10 starting with 1 . Everyone jumped as they counted up to that number. Starting at 20 and counting backwards, while students did the invisible jump rope backwards. The students jumped more and more quickly as the teacher was increasing the counting speed. Some teachers also combined math calculating into the activities.

The average caloric expenditure for both 10-min sessions ranged from 60-70 kcal/ school day, which translated to $43-50 \mathrm{kcal} /$ day, as measured by PA sensors. The average MET rate/session ranged from 4.8 to $7.3 \mathrm{kcal} / \mathrm{kg} /$ hour. All activities were of moderate to vigorous intensity.

PA intervention vs control

Outcomes Outcome measures

- Primary outcome: BMI

- Secondary outcomes: weight, height, zBMIs, fat-free mass, fat mass, \% body fat, weight status (underweight, normal weight, overweight, obese)

Process evaluation: reported (attendance)

Implementation-related Theoretical basis: NR

factors

Resources for intervention implementation: reported 
Who delivered the intervention: NR

PROGRESS categories assessed at baseline: gender, SES

PROGRESS categories analysed at outcome: gender, SES

Outcomes relating to harms/unintended effects: NR

Intervention included strategies to address diversity or disadvantage: NR; intervention targeted children receiving school breakfasts

Economic evaluation: NR

Notes ChicTR-TRC-00000053

Funding: this research was supported by Nutricia Research Foundation. This is the pilot to Meng 2013

\title{
Risk of bias
}

\begin{tabular}{|c|c|c|}
\hline Bias & Authors' judgement & Support for judgement \\
\hline $\begin{array}{l}\text { Random sequence genera- } \\
\text { tion (selection bias) }\end{array}$ & Unclear risk & Randomisation, no further details \\
\hline $\begin{array}{l}\text { Allocation concealment } \\
\text { (selection bias) }\end{array}$ & Unclear risk & Randomisation, no further details \\
\hline $\begin{array}{l}\text { Blinding (performance } \\
\text { bias and detection bias) } \\
\text { All outcomes }\end{array}$ & Low risk & $\begin{array}{l}\text { The research staff who conducted the measurement were blinded to the inter- } \\
\text { vention assignment. }\end{array}$ \\
\hline $\begin{array}{l}\text { Incomplete outcome data } \\
\text { (attrition bias) } \\
\text { All outcomes }\end{array}$ & Low risk & $12.3 \%$ attrition, balanced \\
\hline $\begin{array}{l}\text { Selective reporting (re- } \\
\text { porting bias) }\end{array}$ & Unclear risk & $\begin{array}{l}\text { Trial registration number did not link to a usable record in the Chinese trial } \\
\text { registry. No protocol found. }\end{array}$ \\
\hline Other bias & Low risk & No additional threats to validity \\
\hline $\begin{array}{l}\text { Other bias- timing of re- } \\
\text { cruitment of clusters }\end{array}$ & Low risk & Figure shows recruitment happened prior to randomisation \\
\hline
\end{tabular}

\section{Llargues 2012}

\section{Methods}

\author{
Study name: AVall and AVall2 \\ Study design: cluster-RCT \\ Intervention period: 2 years \\ Follow-up period (post-intervention): 4 years \\ Differences in baseline characteristics: reported \\ Reliable outcomes: reported \\ Protection against contamination: NR \\ Unit of allocation: school
}




\begin{tabular}{|c|c|}
\hline \multirow[t]{10}{*}{ Participants } & $\mathrm{N}($ control baseline $)=\mathrm{NR}(\mathrm{N}=704$ intervention + control $)$ \\
\hline & $\mathrm{N}$ (control follow-up) $=237$ \\
\hline & $\mathrm{N}$ (intervention baseline) $=\mathrm{NR}$ \\
\hline & $\mathrm{N}$ (intervention follow-up) $=272$ \\
\hline & $\begin{array}{l}\text { Setting (and number by study group): } 16 \text { primary schools ( } 10 \text { put } \\
\text { schools in each group }\end{array}$ \\
\hline & Recruitment: all schools in the town of Granollers were recruited \\
\hline & Geographic region: Granollers, Spain \\
\hline & Percentage of eligible population enrolled: $84.9 \%$ participants \\
\hline & Mean age: intervention + control: $6.03 \pm 0.3$ \\
\hline & Sex: intervention: $45.3 \%$ female; control: $45.6 \%$ female \\
\hline
\end{tabular}

Interventions

The aim of this study was to evaluate the effect of an intervention that modified food and PA habits on the progression of BMI in a population of school children using Research, Vision, Action and Change (IVAC) methodology

"The educational methodology IVAC,17 based on the principle that the school children are actors able to operate over their environment, was used. The children investigate and reflect on how the environment determines their health and lifestyle, while the teacher assists them in developing skills to change these conditions. This educational method allows the inclusion of activities related to healthy food habits and PA in any subject of the curriculum."

"Over the 2-year period, six meetings with the research team, the teachers and the educators took place in order to monitor the activities accomplished and to plan subsequent actions. Every classroom used $3 \mathrm{~h}$ a week to develop activities related to health food habits and/or PA. This time was part of regular classes- math, science, language, knowledge of the environment- developing posters, food tables, games, crafts, cooking workshops and promotion of games in the playground."

Diet and PA combination intervention vs control

$\begin{array}{ll}\text { Outcomes } & \text { Outcome measures } \\ \text { - Primary outcome: BMI, weight status } \\ \text { - Secondary outcomes: eating habits, PA } \\ \text { Process evaluation: NR }\end{array}$

Implementation-related factors

Theoretical basis: Investigation, Vision, Action and Change (IVAC) Methodology

Resources for intervention implementation: reported

Who delivered the intervention: reported

PROGRESS categories assessed at baseline: gender, race/ethnicity

PROGRESS categories analysed at outcome: gender, parental education and race/ethnicity

Outcomes relating to harms/unintended effects: NR

Intervention included strategies to address diversity or disadvantage: NR

Economic evaluation: reported (Mora 2015 - direct medical costs) 
Llargues 2012 (Continued)

Notes

\section{NCT01156805}

Funding: study was supported by the Department of Education and Health of the Catalonian Government and the principals of all the schools concerned. Full costs of implementing the AVall project are reported in secondary reference for Llargues 2012, Mora et al 2015 - average cost per treated child was EUR 245.8; an annual cost of 41 s per treated child.

4 years after the intervention, the average BMI was reduced by $1.13 \mathrm{~kg} / \mathrm{m} 2$ and implies $1.6 \mathrm{~kg}$ for treated children with average height. Thus, we compute the ratio of net intervention costs and net intervention effects: EUR 41/1.13 kg/m2 or EUR 25.6/kg

\section{Risk of bias}

Bias Authors' judgement Support for judgement

Random sequence genera- Unclear risk tion (selection bias)

Allocation concealment Unclear risk
(selection bias)
The 16 schools were grouped into strata, depending on whether they were public or not, and they had the same number of classes of 1st primary course. Each school in the groups was randomly assigned, no further details

The 16 schools were grouped into strata, depending on whether they were public or not, and they had the same number of classes of 1st primary course. Each school in the groups was randomly assigned, no further details. Significant baseline imbalances between groups, but analysis tested whether any variable, mainly those that were unbalanced at baseline, including baseline $\mathrm{BMI}$, changed the effect seen in the intervention.

Blinding (performance
bias and detection bias)

\begin{tabular}{|c|c|c|}
\hline $\begin{array}{l}\text { Incomplete outcome data } \\
\text { (attrition bias) } \\
\text { All outcomes }\end{array}$ & Low risk & $\begin{array}{l}\text { Complete data on anthropometric variables were obtained in } 509 \text { of the } 704 \\
\text { children }(72.3 \%), 237(78.8 \%) \text { in the control group and } 272(72.7 \%) \text { in the in- } \\
\text { tervention group at } 4 \text { year follow-up. At } 6 \text { year follow-up anthropometric mea- } \\
\text { surements were no longer available for an additional } 83 \text { schoolchildren. The } \\
\text { average BMI values for those who dropped out of the panel in the } 2 \text { nd and } 3 \text { rd } \\
\text { waves }(2010 \text { to } 2012) \text { proved not to be statistically significant, which provides } \\
\text { us with internal validity for the randomised assignment ( } 16.67 \text { vs. } 16.63 \text {, differ- } \\
\text { ence P value }=0.44) \text {. Thus, the treatment effect should not be confounded by } \\
\text { the presence of selection bias. }\end{array}$ \\
\hline
\end{tabular}

\begin{tabular}{lll}
\hline $\begin{array}{l}\text { Selective reporting (re- } \\
\text { porting bias) }\end{array}$ & Low risk & Protocol/trial registration document seen. All outcomes reported \\
\hline Other bias & Low risk & No additional threats to validity \\
\hline $\begin{array}{l}\text { Other bias- timing of re- } \\
\text { cruitment of clusters }\end{array}$ & Unclear risk & NR \\
\hline
\end{tabular}

Lubans 2011

$\begin{array}{ll}\text { Methods } & \text { Study name: Physical activity leaders (PALs) program } \\ & \text { Study design: cluster-RCT } \\ \text { Intervention period: } 6 \text { months Recruited June to December } 2009 \\ \text { Follow-up period (post-intervention): nil }\end{array}$


Lubans 2011 (Continued)

Differences in baseline characteristics: reported

Reliable outcomes: reported

Protection against contamination: NR

Unit of allocation: school

Unit of analysis: individual accounting for cluster

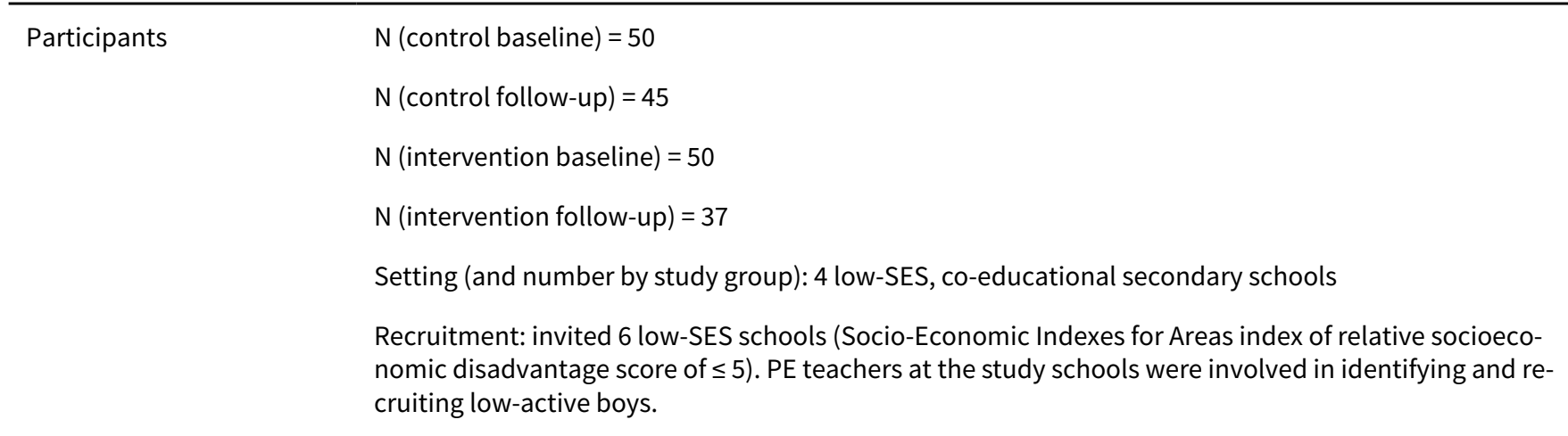

Geographic region: Hunter Region, New South Wales (NSW), Australia

Percentage of eligible population enrolled: $67 \%$ schools

Mean age: intervention: $14.4 \pm 0.7$; control: $14.2 \pm 0.4$

Sex: intervention: $0 \%$ female; control: $0 \%$ female

The PALs program was a multi-component school-based intervention and included school sport sessions, interactive seminars, lunch-time activities, PA and nutrition handbooks, leadership sessions and pedometers for self-monitoring. The programme was developed in reference to Bandura's SCT (2004) and the intervention components, behaviour change strategies and targeted constructs are listed and described in Table 1. The intervention was focused on the promotion of lifestyle (i.e. activities that are performed as part of everyday life, such as walking to school and using the stairs) and lifetime activities (i.e. activities that may be easily carried into adulthood because they generally need one or two people, for example, RT). The PA sessions focused on the use of elastic tubing RT devices, known as Gymsticks ${ }^{\text {TM }}$ (Gymstick International, Lahti, Finland). Participants completed self- and teacher-directed fitness sessions. A flexible intervention delivery model was utilised to allow teachers to adapt the programme to the needs of their students. Teachers were encouraged to set up fitness circuits to maximise participation, but students were also given basic RT programmes to promote exercise independence. The RT programs included 2 sets of $8-12$ repetitions for 10 exercises. The RT programmes were focused on all the major muscle groups and included a variety of exercises, which changed over the intervention period.

A unique aspect of this study was that it encouraged participants to become PA leaders in their schools and at home and provided accreditation to formalise their achievements. Participants who satisfied the accreditation criteria were presented with leadership certificates at school assemblies. The criteria for accreditation were, (i) attendance at $\geq 6$ school sport sessions, (ii) attendance at $\geq 5$ lunch-time sessions, (iii) attendance at $\geq 5$ PA leadership sessions and (iv) submission of the PA and nutrition handbook. The PALs program was delivered at the wait-list control group schools at the completion of the 6month study.

PA intervention vs control

Outcome measures
- Primary outcome: weight, , BMI, zBMI, overweight/obesity prevalence


Lubans 2011 (Continued)

- Secondary outcomes: \% body fat, waist circumference, upper body muscular endurance, abdominal strength, lower body strength, PA, dietary intake (fruit, vegetables, sugary drinks, water)

Process evaluation: reported (recruitment, retention, attendance, satisfaction)

Implementation-related factors

\section{Theoretical basis: SCT}

Resources for intervention implementation: reported

Who delivered the intervention: reported

PROGRESS categories assessed at baseline: NR

PROGRESS categories analysed at outcome: NR

Outcomes relating to harms/unintended effects: reported

Intervention included strategies to address diversity or disadvantage: NR, but teachers selected low-active boys and disadvantaged schools targeted

Economic evaluation: NR

Notes

\section{ACTRN12610000330044}

Funding: this study is funded by grant DP1092646 from the Australian Research Council.

Intervention delivered over 2 school terms at no cost to the school or students (in Australian secondary schools, extra-curricular/co-curricular school sport programmes are often delivered off campus and may involve weekly fees). RT is rarely offered in Australian schools. On average, participants in the intervention group attended $7 / 10$ school sport sessions, $6 / 8$ lunch-time sessions, $4 / 6$ physical

activity leadership sessions and 29/50 participants submitted their completed PA and nutrition handbooks. Approximately 50\% (23 participants) of the intervention group satisfied the requirements for PALs accreditation. Overall, participants were satisfied with the programme $(4.0 \pm 0.9)$.

\section{Risk of bias}

\begin{tabular}{lll}
\hline Bias & Authors' judgement & Support for judgement \\
\hline $\begin{array}{l}\text { Random sequence genera- } \\
\text { tion (selection bias) }\end{array}$ & Unclear risk & Randomised, no further details \\
& $\begin{array}{l}\text { Quote: "Following baseline assessments, the 12 schools were matched (ie, } 6 \\
\text { pairs of schools) based on their geographic location, size, and demographics. } \\
\text { An independent researcher then randomized each pair to either the NEAT Girls } \\
\text { intervention or control groups." }\end{array}$ \\
\hline
\end{tabular}

Allocation concealment $\quad$ Unclear risk $\quad$ Not described
(selection bias)

\begin{tabular}{|c|c|c|}
\hline $\begin{array}{l}\text { Blinding (performance } \\
\text { bias and detection bias) } \\
\text { All outcomes }\end{array}$ & High risk & $\begin{array}{l}\text { Research assistants and participants were not blinded to treatment allocation } \\
\text { at 3- and 6-month assessments }\end{array}$ \\
\hline $\begin{array}{l}\text { Incomplete outcome data } \\
\text { (attrition bias) } \\
\text { All outcomes }\end{array}$ & Low risk & $82 \%$ retention rate at final follow-up; ITT done \\
\hline $\begin{array}{l}\text { Selective reporting (re- } \\
\text { porting bias) }\end{array}$ & Low risk & Protocol seen; all outcomes specified in protocol have been reported \\
\hline Other bias & Low risk & No other threats to validity \\
\hline
\end{tabular}


Lubans 2011 (Continued)

Other bias- timing of re- Low risk Clusters recruited prior to randomisation cruitment of clusters

\begin{tabular}{|c|c|}
\hline \multirow[t]{8}{*}{ Methods } & Study design: RCT \\
\hline & Intervention period: 12 weeks \\
\hline & Follow-up period (post-intervention): nil \\
\hline & Differences in baseline characteristics: reported \\
\hline & Reliable outcomes: reported \\
\hline & Protection against contamination: NR \\
\hline & Unit of allocation: individual \\
\hline & Unit of analysis: individual \\
\hline \multirow[t]{10}{*}{ Participants } & $\mathrm{N}$ (controls baseline $)=38$ \\
\hline & $N($ controls follow-up) $=30$ \\
\hline & $\mathrm{N}$ (interventions baseline) $=38$ \\
\hline & $\mathrm{N}$ (interventions follow-up) $=32$ \\
\hline & Setting: community \\
\hline & $\begin{array}{l}\text { Recruitment: children aged 6-9 years attending public schools in } 4 \text { neighbourhoods in León, Guanajua- } \\
\text { to, Mexico }\end{array}$ \\
\hline & Geographic region: Mexico \\
\hline & Percentage of eligible population enrolled: NR \\
\hline & Median age: intervention: 8 (6.1-9.1); control: 7.5 (6.9-8.4) \\
\hline & Sex: both male and female \\
\hline
\end{tabular}

Interventions

Intervention children were instructed to modify their PA to obtain an increase of at least 2500 steps/day over the baseline level. To attain this, 2 strategies were used:

- to increase incidental PA (i.e. walk to school, to accompany their parents at shopping and to help in the domestic work at home

- involvement in recreational activities 3 times/week in a Municipal Sport Center (60 min sessions of age-appropriate recreational activities)

PA interventions vs control

Outcomes

- Anthropometric measurements: height, weight, waist circumference, triceps skinfold

- Laboratory measurements: glucose, triglycerides, cholesterol, HDL-C, LDL-C, HOMA-IR

- Basal PA (steps/day, by pedometer)

- CV fitness (VO2 max): by treadmill

- Food intake

Process evaluation: reported 
Macias-Cervantes 2009 (Continued)

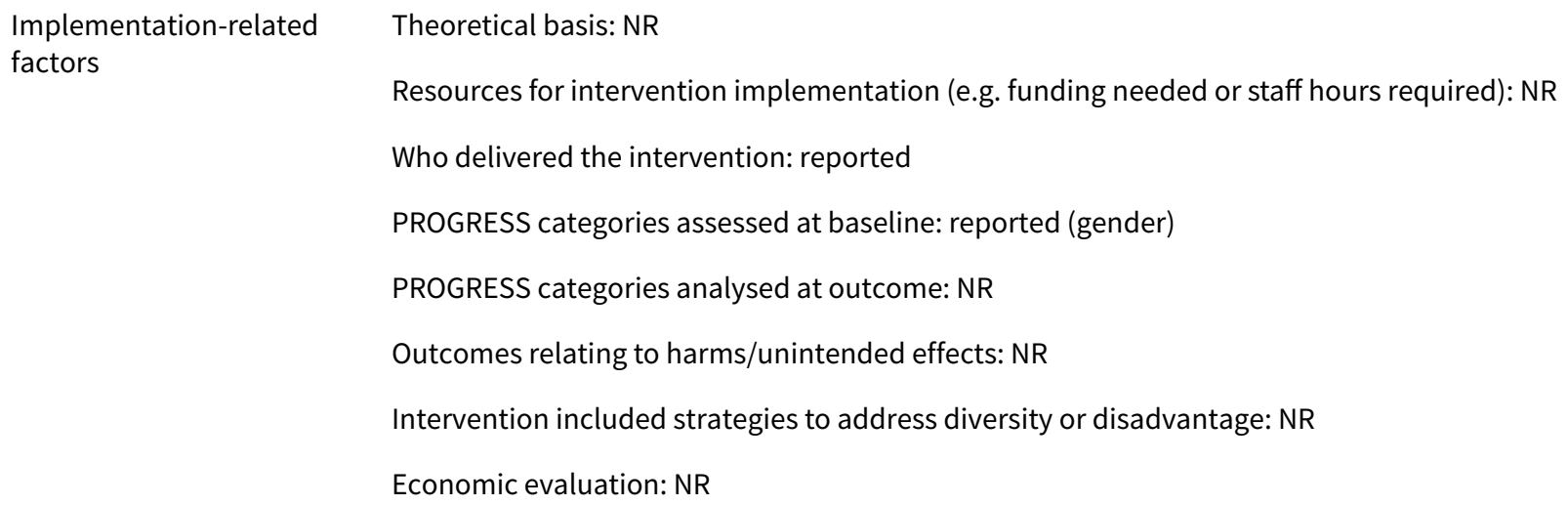

\section{Risk of bias}

\begin{tabular}{|c|c|c|}
\hline Bias & Authors' judgement & Support for judgement \\
\hline $\begin{array}{l}\text { Random sequence genera- } \\
\text { tion (selection bias) }\end{array}$ & Low risk & Quote: "Randomization was carried out with a lottery" \\
\hline $\begin{array}{l}\text { Allocation concealment } \\
\text { (selection bias) }\end{array}$ & Unclear risk & NR \\
\hline $\begin{array}{l}\text { Blinding (performance } \\
\text { bias and detection bias) } \\
\text { All outcomes }\end{array}$ & Low risk & Not blinded but unlikely to influence results \\
\hline $\begin{array}{l}\text { Incomplete outcome data } \\
\text { (attrition bias) } \\
\text { All outcomes }\end{array}$ & Low risk & $\begin{array}{l}81 \% \text { retention of participants. Similar numbers of dropouts between groups } \\
\text { and reasons for withdrawal recorded }\end{array}$ \\
\hline $\begin{array}{l}\text { Selective reporting (re- } \\
\text { porting bias) }\end{array}$ & Unclear risk & Protocol and trial register report sought but not found \\
\hline Other bias & Low risk & $\begin{array}{l}\text { Baseline imbalance reported, but does not affect BMI or skinfold thickness. } \\
\text { Children in experimental group were taller with larger waist circumference }(\mathrm{P}< \\
\text { 0.04). But BMI and skinfold thickness were similar }\end{array}$ \\
\hline
\end{tabular}

\section{Madsen 2013}

\section{Methods}

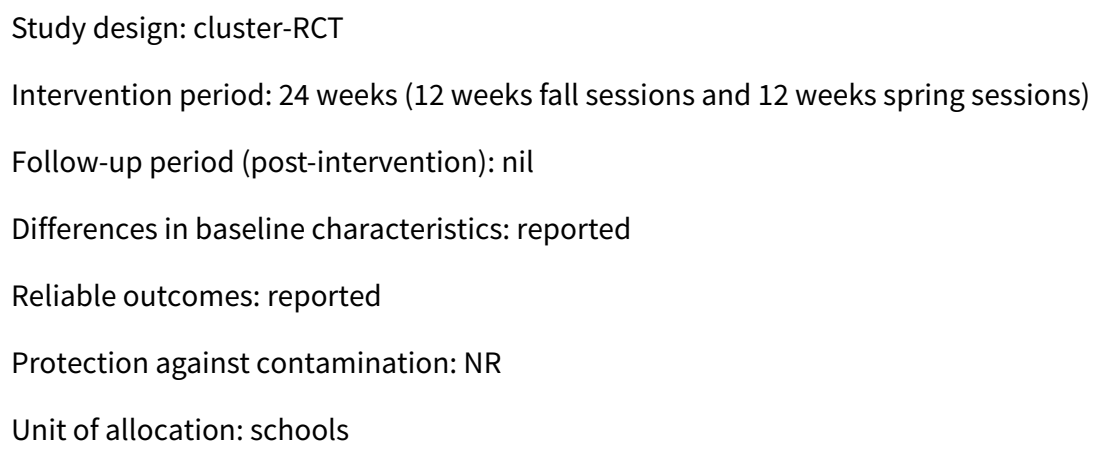




\begin{tabular}{|c|c|}
\hline \multirow[t]{10}{*}{ Participants } & $\mathrm{N}($ controls baseline $)=74$ \\
\hline & $N($ controls follow-up $)=71$ \\
\hline & $\mathrm{N}$ (interventions baseline) $=82$ \\
\hline & $\mathrm{N}$ (interventions follow-up) $=79$ \\
\hline & $\begin{array}{l}\text { Setting (and number by study group): after-school soccer } \\
\text { tion and } 3 \text { control) }\end{array}$ \\
\hline & Recruitment: 60 schools that had not offered SCORES in $t$ \\
\hline & Geographic region: San Francisco, USA \\
\hline & Percentage of eligible population enrolled: $12 \%$ schools, \\
\hline & Mean age: intervention: $9.8 \pm 0.6$; control: $9.8 \pm 0.7$ \\
\hline & Sex: intervention, $38 \%$ female; control, $42 \%$ female \\
\hline
\end{tabular}

Interventions

To evaluate the impact of a community-based, after-school soccer and youth development programme, 'America SCORES', on students' PA, weight status, and fitness

America SCORES is an after-school soccer programme. Primary aim is building competencies and skills that will support student's overall development, including teamwork; leadership and academic commitment. It is offered to youth who would otherwise have limited access to extracurricular activities.

As part of the revised programme, students were involved in the following

- students spend 2-3 days/week in soccer drills or games for a minimum of $1 \mathrm{~h}$ of soccer on 2 afternoons each week

- 1 hour of creative writing on 2 afternoons each week during each 12 -week session (fall and spring).

Who delivered: was initially delivered by trained SCORES soccer and writing coaches, however due to schools having fewer resources and funds to pay contract staff, SCORES adapted the programme and moved to the train-the-trainer model, whereby SCORES trained the district's after-school staff to operate the programme.

Training: under the train-the-trainer model, the 3 SCORES schools received the SCORES curriculum, 6 $\mathrm{h}$ of training in the fall before the programme began, and an additional 6-hour training in the spring. Training included lesson planning and execution, student soccer and poetry skill development, and behaviour management. SCORES provided coaches with soccer coaching manuals featuring $>100$ soccer practice activities and games and a writing programme curriculum with examples and activities. Additionally, SCORES staff visited each of the intervention school sites multiple times during the course of the study to provide technical assistance.

PA intervention vs control

- Primary outcome: MVPA

- Secondary outcomes: zBMI, cardiorespiratory fitness

Process evaluation: reported (attendance)

Implementation-related factors
Theoretical basis: NR

Resources for intervention implementation: reported

Who delivered the intervention: reported 
PROGRESS categories analysed at outcome: NR

Outcomes relating to harms/unintended effects: NR

Intervention included strategies to address diversity or disadvantage: offered to youth who would otherwise have limited access to extracurricular activities; curriculum refined based on 20 years' experience of working in low-income, disadvantaged schools

Economic evaluation: NR

Notes $\quad$ Funding: this work was by the following grants: NIH/NICHDK23HD054470 and American Heart Association $0865005 \mathrm{~F}$

Participants ( $n=156)$ were diverse (42\% Latino, 32\% Asian, and $12 \%$ African American) and $76(49 \%)$ had a BMI at or above the 85 th percentile.

Note: one intervention school did not receive the SCORES intervention as the new principal withdrew prior to the start of the study.

Historically, schools pay up to half of the cost of operating the SCORES program, while SCORES has raised the balance through

grants and private donations.

While the initial plan was to study the traditional SCORES model previously described, owing to significant budget cuts in the district in 2009-2010, schools had fewer resources to contract staff from outside agencies, such as SCORES, to deliver their programs. SCORES responded by moving to a train-the trainer model in which SCORES trained the district's after-school staff to operate the program.

While staff almost met the goal of offering 12 weeks of SCORES programming in the fall (mean, 11.3 weeks), in the spring, only 7 weeks of SCORES were offered on average (this was driven by low compliance at 1 school). Based on average attendance rates, students in intervention schools were exposed to an average of 1.4 hours of soccer each week.

\section{Risk of bias}

\begin{tabular}{|c|c|c|}
\hline Bias & Authors' judgement & Support for judgement \\
\hline $\begin{array}{l}\text { Random sequence genera- } \\
\text { tion (selection bias) }\end{array}$ & Unclear risk & Randomly allocated, no further details \\
\hline $\begin{array}{l}\text { Allocation concealment } \\
\text { (selection bias) }\end{array}$ & Unclear risk & NR \\
\hline $\begin{array}{l}\text { Blinding (performance } \\
\text { bias and detection bias) } \\
\text { All outcomes }\end{array}$ & High risk & Neither schools nor researchers were blinded to assignment \\
\hline $\begin{array}{l}\text { Incomplete outcome data } \\
\text { (attrition bias) } \\
\text { All outcomes }\end{array}$ & Low risk & $96 \%$ retention \\
\hline $\begin{array}{l}\text { Selective reporting (re- } \\
\text { porting bias) }\end{array}$ & Low risk & Protocol/trial registration document seen. All outcomes reported \\
\hline Other bias & Low risk & No additional threats to validity \\
\hline $\begin{array}{l}\text { Other bias- timing of re- } \\
\text { cruitment of clusters }\end{array}$ & Low risk & Figure shows recruitment happened prior to randomisation \\
\hline
\end{tabular}




\author{
Study design: cluster-RCT \\ Intervention period: 2 years \\ Follow-up period (post-intervention): nil \\ Differences in baseline characteristics: reported \\ Reliable outcomes: reported \\ Protection against contamination: NR \\ Unit of allocation: school \\ Unit of analysis: individual, accounting for clustering
}

Participants
$\begin{aligned} & \mathrm{N} \text { (control baseline) }=170 \\ & \mathrm{~N} \text { (control follow-up) }=81 \\ & \mathrm{~N} \text { (intervention baseline) }=151 \\ & \text { Setting (and number by study group): } 6 \text { elementary schools } \\ & \text { Recruitment: } 6 \text { elementary schools were randomly selected, all children attending } 2 \text { nd grade in these } \\ & \text { schools were invited }\end{aligned}$

Geographic region: Reykjavik, Iceland

Percentage of eligible population enrolled: $83 \%$ participants

Mean age: intervention: $7.3 \pm 0.3 \mathrm{~N}=128$; control: $7.4 \pm 0.3, \mathrm{~N}=138$

Sex: intervention: $51 \%$ female, $N=128$; control: $60 \%$ female, $N=138$

Interventions

To report the effectiveness of a 2-year school-based intervention on fitness and nutrition and the status of an array of common CV disease risk factors among these 7-year-old schoolchildren

- Teachers' involvement

- The teachers received training at bimonthly workshop meetings and through informal on-site meetings, both to provide teachers with expert information on PA and nutrition and to give them opportunities for dialogue with their colleagues regarding the evolvement of the intervention.

- Teachers' PA log books were the basis for estimating the time children spent doing PA under a teacher's supervision at school.

- $P A$

- PA intervention was progressive in nature, starting with approximately $30 \mathrm{~min} /$ day at the start of the study and increasing to approximately $60 \mathrm{~min} /$ day in the latter intervention year, where teachers who implemented the intervention used various strategies to better integrate PA into the daily routine at school.

- Nutrition

- The main focus of the dietary intervention was on increasing fruit and vegetable intake, with both educational material and homework assignments. Food-based dietary guidelines on fish, fish liver oil and milk intake were also in focus, and parents, teachers, and school food service staff were involved in the intervention.

Diet and PA combination intervention vs control 
- Primary outcome: BMI, waist circumference, lean mass, whole body \% fat, sum of 5 skinfolds, cardiorespiratory fitness

- Secondary outcomes: max heart beats/min, blood pressure, cholesterol, triglycerides, fasting glucose, $\mathrm{HbA} 1 \mathrm{C} \%$, insulin, dietary intake (see Hrafnkelsson 2014, secondary reference for Magnusson 2012)

Process evaluation: NR

Implementation-related factors
Theoretical basis: NR

Resources for intervention implementation: reported

Who delivered the intervention: reported

PROGRESS categories assessed at baseline: child; gender; parent: education, SES (family income)

PROGRESS categories analysed at outcome: NR

Outcomes relating to harms/unintended effects: NR

Intervention included strategies to address diversity or disadvantage: NR

Economic evaluation: NR

Notes

Funding: the study was primarily funded by the Icelandic Centre for Research (RANNIS), but also supported by the city of Reykjavik, the Ministry of Education, Science and Culture and BRIM Seafood.

At baseline the intervention school children had on average 0.43 lower zBMIs than the children in the control schools ( $95 \% \mathrm{Cl}-0.94$ to 0.08 ), adjusted for school clustering.

Gifts were given to all children in the intervention and control schools in the form of backpacks in the fall of 2007 and athletic T-shirts at the end of the intervention.

The goal of $60 \mathrm{~min}$ of PA/day was not achieved at the end of the study; high teacher turnover possibly affected decrease in PA.

When the study ended, Iceland was in a state of financial crisis.

\section{Risk of bias}

\begin{tabular}{lll}
\hline Bias & Authors' judgement & Support for judgement \\
\hline $\begin{array}{l}\text { Random sequence genera- } \\
\text { tion (selection bias) }\end{array}$ & Unclear risk & $\begin{array}{l}\text { Schools paired on number of students and social background and randomly } \\
\text { assigned, no further details }\end{array}$ \\
\hline $\begin{array}{l}\text { Allocation concealment } \\
\text { (selection bias) }\end{array}$ & Unclear risk & $\begin{array}{l}\text { Schools paired on number of students and social background and randomly } \\
\text { assigned, no further details }\end{array}$ \\
\hline $\begin{array}{l}\text { Blinding (performance } \\
\text { bias and detection bias) } \\
\text { All outcomes }\end{array}$ & Low risk & $\begin{array}{l}\text { Researchers did not know which group was the intervention group, no further } \\
\text { details }\end{array}$ \\
\hline $\begin{array}{l}\text { Incomplete outcome data } \\
\text { (attrition bias) } \\
\text { All outcomes }\end{array}$ & High risk & $\begin{array}{l}\text { High attrition, 46\% in control and 30\% in intervention (of those measured at } \\
\text { baseline) for \% body fat }\end{array}$ \\
\hline $\begin{array}{l}\text { Selective reporting (re- } \\
\text { porting bias) }\end{array}$ & Unclear risk & Protocol or trial register not found \\
\hline \begin{tabular}{l} 
Other bias \\
\hline
\end{tabular} & Low risk & No additional threats to validity \\
\hline
\end{tabular}


Magnusson 2012 (Continued)

Other bias- timing of re- Low risk Figure indicates that recruitment happened prior to randomisation cruitment of clusters

\begin{tabular}{|c|c|}
\hline \multirow[t]{8}{*}{ Methods } & Study design: cluster-RCT \\
\hline & Intervention period: 4 years \\
\hline & Follow-up period (post-intervention): nil \\
\hline & Differences in baseline characteristics: reported \\
\hline & Reliable outcomes: reported (anthropometry and accelerometry) \\
\hline & Protection against contamination: NR \\
\hline & Unit of allocation: school \\
\hline & $\begin{array}{l}\text { Unit of analysis: child. Primary analysis used observed cases, but sensitivity analyses were carried out } \\
\text { using FAS population (evaluated with replacement for missing data by LOCF) }\end{array}$ \\
\hline \multirow[t]{10}{*}{ Participants } & $\mathrm{N}$ (controls baseline) $=1465$ \\
\hline & $\mathrm{N}$ (controls follow-up) $=1300$ \\
\hline & $\mathrm{N}$ (interventions baseline) $=1670$ \\
\hline & $\mathrm{N}$ (interventions follow-up) = 1538 \\
\hline & Setting: schools ( $N=5$ intervention, $N=5$ control) \\
\hline & Recruitment: all consenting students in selected schools up to 4 th school year \\
\hline & Geographic region: Sweden \\
\hline & Percentage of eligible population enrolled: $90 \%-100 \%$ \\
\hline & Mean age: control: 7.5 (1.3) years; intervention: 7.4 (1.3) years \\
\hline & Sex: both sexes included \\
\hline \multirow[t]{7}{*}{ Interventions } & $\begin{array}{l}\text { - Intervention was designed to change the school environment to promote healthy eating and PA during } \\
\text { school and in after-school care. }\end{array}$ \\
\hline & $\begin{array}{l}\text { - Daily PA ( } 30 \text { min per child) was integrated into regular school curriculum and facilitated by classroom } \\
\text { teachers }\end{array}$ \\
\hline & $\begin{array}{l}\text { - Classroom teachers encouraged healthy eating, eating less sweetened foods, and to choose healthy } \\
\text { items for school lunch and afternoon snack (provided by schools) }\end{array}$ \\
\hline & $\begin{array}{l}\text { - School changes in items provided to increase health (lower sugar, more fibre, lower fat etc), eliminate } \\
\text { unhealthy celebration foods and restrict foods for excursions and sports days }\end{array}$ \\
\hline & - Awareness raising activities included STOPP newsletter to parents and schools twice a year \\
\hline & - School nurses were also trained in obesity-related problems \\
\hline & Combined effects of dietary interventions and PA interventions vs control \\
\hline
\end{tabular}

Outcomes

- Prevalence overweight/obese

- PA, accelerometer

- Eating habits 
Marcus 2009 (Continued)

Process evaluation: reported

Implementation-related factors

Theoretical basis: NR

Resources for intervention implementation (e.g. funding needed or staff hours required): NR

Who delivered the intervention: reported

PROGRESS categories assessed at baseline: reported (place, race, occupation, gender, education, social status)

PROGRESS categories analysed at outcome: reported (gender, education)

Outcomes relating to harms/unintended effects: reported

Intervention included strategies to address diversity or disadvantage: NR

Economic evaluation: NR

\section{Notes}

Funding: the study was supported by grants from Stockholm County Council, Swedish Council for working life and social research, Swedish Research Council, Freemason's in Stockholm Foundation for Children's Welfare and Signhild Engkvist Foundation.

\section{Risk of bias}

\begin{tabular}{|c|c|c|}
\hline Bias & Authors' judgement & Support for judgement \\
\hline $\begin{array}{l}\text { Random sequence genera- } \\
\text { tion (selection bias) }\end{array}$ & Unclear risk & $\begin{array}{l}\text { Quote: "Five of the selected schools were thereafter randomized to interven- } \\
\text { tion and five schools to control." }\end{array}$ \\
\hline $\begin{array}{l}\text { Allocation concealment } \\
\text { (selection bias) }\end{array}$ & Unclear risk & NR \\
\hline $\begin{array}{l}\text { Blinding (performance } \\
\text { bias and detection bias) } \\
\text { All outcomes }\end{array}$ & Unclear risk & NR \\
\hline \multirow{2}{*}{$\begin{array}{l}\text { Incomplete outcome data } \\
\text { (attrition bias) } \\
\text { All outcomes }\end{array}$} & Low risk & $\begin{array}{l}\text { Figure } 1 \text { indicates all randomised (FAS) included in a sensitivity analysis - data } \\
\text { not presented, these results were not different. }\end{array}$ \\
\hline & & $\begin{array}{l}\text { Quote: "The primary analysis was carried out using the observed cases popu- } \\
\text { lation, and a sensitivity analysis was performed using the FAS population. The } \\
\text { FAS population was evaluated with replacement for missing data by the last } \\
\text { observation carried forward approach, i.e. where only one measurement was } \\
\text { observed, and the estimated change in BMIsds was set to } 0 . "\end{array}$ \\
\hline $\begin{array}{l}\text { Selective reporting (re- } \\
\text { porting bias) }\end{array}$ & Low risk & Protocol/trial registration document seen. All outcomes reported \\
\hline Other bias & Low risk & No additional threats to validity \\
\hline $\begin{array}{l}\text { Other bias- timing of re- } \\
\text { cruitment of clusters }\end{array}$ & Low risk & Figure shows recruitment happened prior to randomisation \\
\hline
\end{tabular}

Martinez-Vizcaino 2014

$\begin{array}{ll}\text { Methods } & \text { Study design: cluster-RCT } \\ & \text { Intervention period: } 9 \text { months }\end{array}$


Martinez-Vizcaino 2014 (Continued)

Follow-up period (post-intervention): nil

Differences in baseline characteristics: reported

Reliable outcomes: reported

Protection against contamination: reported

Unit of allocation: schools

Unit of analysis: individual accounting for cluster

\begin{tabular}{|c|c|}
\hline \multirow[t]{10}{*}{ Participants } & $\mathrm{N}($ control baseline $)=823$ \\
\hline & $\mathrm{N}$ (control follow-up) $=492$ \\
\hline & $\mathrm{N}$ (intervention baseline $)=769$ \\
\hline & $\mathrm{N}$ (intervention follow-up) $=420$ \\
\hline & Setting (and number by study group): 20 schools ( 10 in each group, mostly rural) \\
\hline & Recruitment: NR \\
\hline & Geographic region: Cuenca, Spain \\
\hline & $\begin{array}{l}\text { Percentage of eligible population enrolled: } 100 \% \text { schools, } 63.6 \% \text { of intervention and } 70.6 \% \text { of control } \\
\text { children had consent and baseline variables measured (randomised schools prior to consent) }\end{array}$ \\
\hline & $\begin{array}{l}\text { Mean age: intervention: } 9.4 \pm 0.7 \text { (girls); intervention: } 9.4 \pm 0.7 \text { (boys); control: } 9.5 \pm 0.7 \text { (girls); control: } \\
9.5 \pm 0.7 \text { (boys) }\end{array}$ \\
\hline & Sex: intervention: $55 \%$ female; control: $49 \%$ female \\
\hline \multirow[t]{2}{*}{ Interventions } & $\begin{array}{l}\text { To assess the impact of a standardised PA programme on adiposity and cardiometabolic risk factors in } \\
\text { schoolchildren }\end{array}$ \\
\hline & $\begin{array}{l}\text { MOVI-2 consisted of an extracurricular play-based and non-competitive PA programme. MOVI- } 2 \text { includ- } \\
\text { ed basic sports games, traditional games, and other outdoor activities such as cycling or gymkhanas } \\
\text { (www.movidavida.org/). The programme included two 90-minute PA sessions during the weekdays in } \\
\text { the evening from } 4-5.30 \text { pm and one } 150 \text {-min session on Saturday morning each week. In the weekday } \\
\text { sessions there was a break of } 5 \text { min and in the Saturday session there were } 2 \text { breaks of } 5 \text { min where chil- } \\
\text { dren could drink water. All activities were implemented by monitors with technical qualifications in PA } \\
\text { and sports, PE teachers, or PA science graduates, specifically engaged and adequately trained for the } \\
\text { programme. }\end{array}$ \\
\hline
\end{tabular}

All activities were performed indoors in the school's gymnasium and require materials habitual in most European primary school gymnasium (soft rubber balls, road signal cones, flag waist bands, plastic gymnastic loops). Games were classified into 2 big categories: a) endurance games in which the main PA was running (i.e. chasing, sprinting, dribbling, hopping, and such) and b) resistance games in which there were also locomotion involving opposition from a partner (lifting, pushing, wrestling, hauling, and such). Each game session lasted approximately $90 \mathrm{~min}$ and included 9 games of $5.5 \pm 1.4 \mathrm{~min}$ of duration interspersed by periods of $4 \pm 1$ min for recovery and organisation.

PA intervention vs control

Outcomes Outcome measures

- Primary outcome: BMI, waist circumference, prevalence overweight/obesity, TSF thickness; \% body fat; fat-free mass, blood pressure

- Secondary outcomes: NR

Process evaluation: reported (PA intensity and energy expenditure, satisfaction/compliance, cost) 
Martinez-Vizcaino 2014 (Continued)

Implementation-related Theoretical basis: SEM
factors

Resources for intervention implementation: reported

Who delivered the intervention: reported

PROGRESS categories assessed at baseline: gender, race/ethnicity (born abroad); parent: education, occupation

PROGRESS categories analysed at outcome: gender (parental employment status also used as poten-

tial confounder)

Outcomes relating to harms/unintended effects: reported

Intervention included strategies to address diversity or disadvantage: NR

Economic evaluation: reported

Notes NCT01277224

Funding: this study was funded by the Ministry of Education and Science-Junta de Comunidades de

Castilla-La Mancha (PII1109-0259-9898 and POII10-0208-5325), and Ministry of Health (FIS PI081297).

Additional funding was obtained from the Research Network on Preventative Activities and Health Promotion (Ref. - RD06/0018/0038).

The cost of our intervention was EUR 28/month/child (wholly subsidised by research grant)

\section{Risk of bias}

\begin{tabular}{lll}
\hline Bias & Authors' judgement & Support for judgement \\
\hline $\begin{array}{l}\text { Random sequence genera- } \\
\text { tion (selection bias) }\end{array}$ & Low risk & A computer-generated procedure was used for randomisation \\
\hline $\begin{array}{l}\text { Allocation concealment } \\
\text { (selection bias) }\end{array}$ & Low risk & Opaque envelopes used for allocation \\
\hline $\begin{array}{l}\text { Blinding (performance } \\
\text { bias and detection bias) } \\
\text { All outcomes }\end{array}$ & High risk & $\begin{array}{l}\text { Blinding of the school allocation was done for the laboratory determina- } \\
\text { tions but not for other outcome variables, because they were measured in the } \\
\text { school setting - anthropometric and blood pressure determinations were not } \\
\text { blinded to intervention allocation }\end{array}$ \\
\hline
\end{tabular}

\begin{tabular}{lll}
\hline $\begin{array}{l}\text { Incomplete outcome data } \\
\text { (attrition bias) } \\
\text { All outcomes }\end{array}$ & Low risk & \\
\hline $\begin{array}{l}\text { Selective reporting (re- } \\
\text { porting bias) }\end{array}$ & Low risk & Protocol seen; all outcomes specified in methods were reported in results \\
\hline Other bias & Low risk & $\begin{array}{l}\text { In towns with } \geq 2 \text { schools, only } 1 \text { was chosen at random to avoid contamina- } \\
\text { tion of the intervention }\end{array}$
\end{tabular}

\begin{tabular}{l}
$\begin{array}{l}\text { Other bias- timing of re- Low risk } \\
\text { cruitment of clusters }\end{array}$ \\
\hline
\end{tabular}

Mauriello 2010

Methods Study design: cluster-RCT


Mauriello 2010 (Continued)

Intervention period: 2 months

Follow-up period (post-intervention): 10 months

Differences in baseline characteristics: reported

Reliable outcomes: NR

Protection against contamination: NR

Unit of allocation: schools

Unit of analysis: individual accounting for clustering

\section{Participants}

$$
\begin{aligned}
& N(\text { control baseline })=672 \\
& N(\text { control follow-up })=457 \\
& N(\text { intervention baseline })=1128 \\
& N(\text { intervention follow-up })=725
\end{aligned}
$$

Setting (and number by study group): 8 high schools in 4 states in USA

Recruitment: school administrators invited students from various classes to participate. Some schools over-recruited students due to the ease of incorporating the research into their schedules, making it easier to retain students in the research in subsequent semesters

Geographic region: Rhode Island, Massachusetts, New York, Tennessee, USA

Percentage of eligible population enrolled: $97 \%$ participants

Mean age: NR: (14-17 years)

Sex: intervention: $51.9 \%$ female; control: $50.8 \%$ female intervention for adolescents.

Multi-media intervention for PA, fruit and vegetable consumption, and limited TV viewing. Interactive technology to provide individually tailored messages to high school students. Health in Motion addresses recommended guidelines for 3 target energy-balance behaviours related to obesity risk:

- PA (at least 60 min on at least 5 days per week)

- fruit and vegetable consumption (at least 5 servings of fruits and vegetables each day)

- limited TV viewing (2 hours or less of TV each day).

Individualised tailoring is based on the theoretical constructs (stage of change, decisional balance, selfefficacy, and processes of change) of the TTM of Behavior Change.

The treatment group received 3 intervention sessions (baseline, 1 month, and 2 months), in addition to 6- and 12-month follow-up assessments. The control group completed assessments at baseline, 2, 6, and 12 months. All sessions were administered via computers in school computer laboratories.

Control: no treatment

Diet and PA intervention vs control (health education)

Outcomes Outcome measures

- Primary outcome, PA, fruit and vegetable consumption, TV viewing

- Secondary outcomes: movement to action or maintenance stages $(A / M)$ among those in a pre-action stage at baseline for each behaviour: PA, fruit and vegetable consumption, TV viewing, risk reduction, 
Mauriello 2010 (Continued)

co-variation of behaviour change, stability in action and maintenance stages, weight status, movement to overweight using BMI (self-report)

Process evaluation: reported (dose)

Implementation-related factors
Theoretical basis: TTM of Behaviour Change

Resources for intervention implementation: reported

Who delivered the intervention: reported

PROGRESS categories assessed at baseline: gender, race/ethnicity

PROGRESS categories analysed at outcome: NR

Outcomes relating to harms/unintended effects: NR

Intervention included strategies to address diversity or disadvantage: NR

Economic evaluation: NR
Funding: funding for this research was provided by the National Heart, Lung, and Blood Institute (Grant \# R43 HL074482).

Most treatment participants (90.2\%) received at least 3 intervention sessions. Due to a programming error discovered in the 1 st week of the trial, some treatment group participants $(21.5 \%)$ received an extra dose of the intervention. Overall, the average number of intervention sessions was 3.09.

\section{Risk of bias}

\begin{tabular}{lll}
\hline Bias & Authors' judgement & Support for judgement \\
\hline $\begin{array}{l}\text { Random sequence genera- } \\
\text { tion (selection bias) }\end{array}$ & Unclear risk & $\begin{array}{l}\text { Schools were stratified based on race/ethnicity, geographic location, and per- } \\
\text { centage of students receiving reduced priced lunches and then randomly as- } \\
\text { signed }\end{array}$ \\
\hline $\begin{array}{l}\text { Allocation concealment } \\
\text { (selection bias) }\end{array}$ & Unclear risk & NR \\
\hline $\begin{array}{l}\text { Blinding (performance } \\
\text { bias and detection bias) } \\
\text { All outcomes }\end{array}$ & High risk & Quote: "Research assistants who were not blind to the group assignment" \\
\hline $\begin{array}{l}\text { Incomplete outcome data } \\
\text { (attrition bias) } \\
\text { All outcomes }\end{array}$ & High risk & $\begin{array}{l}\text { 34\%-36\% attrition; multiple imputation for missing data done and complete } \\
\text { datasets analysed }\end{array}$ \\
\hline $\begin{array}{l}\text { Selective reporting (re- } \\
\text { porting bias) }\end{array}$ & Low risk & $\begin{array}{l}\text { Trial registration found. All outcomes listed in the trial registration document } \\
\text { were reported in results. }\end{array}$ \\
\hline $\begin{array}{l}\text { Other bias } \\
\begin{array}{l}\text { Other bias- timing of re- } \\
\text { cruitment of clusters }\end{array}\end{array}$ Low risk & No additional threats to validity \\
\hline
\end{tabular}

Melnyk 2013

Methods

Study name: COPE (Creating opportunities for personal empowerment) 
Melnyk 2013 (Continued)

Study design: cluster-RCT

Intervention period: 15 weeks

Follow-up period (post-intervention): 6 months

Differences in baseline characteristics: reported

Reliable outcomes: reported

Protection against contamination: reported

Unit of allocation: school

Unit of analysis: individual not accounting for clustering

\section{Participants}

$$
\begin{aligned}
& N(\text { control baseline })=433 \\
& N(\text { control follow-up })=341 \\
& N(\text { intervention baseline })=374 \\
& N(\text { intervention follow-up })=286
\end{aligned}
$$

Setting (and number by study group): 11 high schools in 2 school districts

Recruitment: research team members introduced the study to all students in each participating health class and sent consent/assent packets home with those teens who expressed interest in study participation

Geographic region: southwest USA

Percentage of eligible population enrolled: $52 \%$ participants

Mean age: intervention: $14.75 \pm 0.76$; control: $14.74 \pm 0.70$

Sex: intervention: $49.2 \%$ female; control: $54.5 \%$ female ercise, Nutrition) programme (referred to here as COPE), vs an attention control programme (Healthy teens), on the healthy lifestyle behaviours, BMI, psychosocial outcomes, social skills, and academic performance of high school adolescents aged 14-16 years

Intervention: the COPE programme is a manualised 15-session educational and cognitive-behavioural skills-building programme guided by cognitive theory, with PA as a component of each session. The COPE intervention was pilot-tested 3 times with white, Hispanic, and African-American adolescents as a group intervention in high school settings. Each session of COPE contains 15-20 min of PA (e.g. walking, dancing, kick-boxing movements), not intended as an exercise training programme, but rather to build beliefs in the teens that they can engage in and sustain some level of PA on a regular basis. Teens received a COPE manual with homework activities for each of the 15 sessions that reinforced the content and skills in the programme. A parent newsletter describing the content of the COPE programme also was sent home with the teens 4 times during the course of the 15-week programme, and the teens were instructed to review each newsletter with their parent(s) as part of their homework assignments.

Control: the Healthy Teens programme was designed as a 15-week attention control programme to control for the time the health teachers in the COPE group spent delivering the experimental content to their students. Health teachers received a full-day training workshop on the Healthy teens content. The content was manualised and focused on safety and common health topics/issues for teens, such as road safety, dental care, infectious diseases, immunisations, and skin care.

Control teens also received a manual with homework assignments each week that focused on the topics being covered in class and were asked to review with his or her parent a newsletter that was sent home with the teens 4 times during the programme. The control programme was administered in a for- 
Melnyk 2013 (Continued)

mat like that of the COPE intervention and included the same number and length of sessions as the experimental programme, but there was no overlap of content between the two programmes.

Diet and PA intervention vs control

\begin{tabular}{ll}
\hline Outcomes & Outcome measures \\
& - Primary outcome: BMI, PA \\
- Secondary outcomes: depressive and anxiety symptoms, social skills, substance use, and academic \\
performance \\
Process evaluation: reported (fidelity) \\
\hline Implementation-related $\quad$ Theoretical basis: Cognitive theory \\
Resources for intervention implementation: reported \\
Who delivered the intervention: reported \\
PROGRESS categories assessed at baseline: gender, race/ethnicity \\
PROGRESS categories analysed at outcome: NR \\
Outcomes relating to harms/unintended effects: depressive and anxiety symptoms \\
Intervention included strategies to address diversity or disadvantage: NR \\
Economic evaluation: NR
\end{tabular}

Notes

NCT01704768

Funding: this study was funded by the NIH/ National Institute of Nursing Research 1R01NR012171.

The study team observed incidents of decreased fidelity to the intervention that occurred at least once, in approximately half of the classrooms.

\section{Risk of bias}

\begin{tabular}{lll}
\hline Bias & Authors' judgement & Support for judgement \\
\hline $\begin{array}{l}\text { Random sequence genera- } \\
\text { tion (selection bias) }\end{array}$ & Unclear risk & Random assignment, no further details \\
\hline $\begin{array}{l}\text { Allocation concealment } \\
\text { (selection bias) }\end{array}$ & Unclear risk & Random assignment, no further details \\
\hline $\begin{array}{l}\text { Blinding (performance } \\
\text { bias and detection bias) } \\
\text { All outcomes }\end{array}$ & Low risk & States blinded, but not who \\
\hline $\begin{array}{l}\text { Incomplete outcome data } \\
\text { (attrition bias) } \\
\text { All outcomes }\end{array}$ & Low risk & $\begin{array}{l}\text { Retention was 76\% in intervention group at 6 months and 78\% in control at } 6 \\
\text { months; analyses were performed using all available data (i.e. ITT), including } \\
\text { participants who subsequently dropped out of the study. }\end{array}$ \\
\hline $\begin{array}{l}\text { Selective reporting (re- } \\
\text { porting bias) }\end{array}$ & Low risk & Protocol/trial registration document seen. All outcomes reported \\
\hline $\begin{array}{l}\text { Other bias } \\
\begin{array}{l}\text { Other bias- timing of re- } \\
\text { cruitment of clusters }\end{array}\end{array}$ & Low risk & No additional threats to validity \\
\hline
\end{tabular}


Study design: cluster-RCT

Intervention period: 2 semesters during 1 academic year, the actual implemented duration is 8.9 months because it was interrupted by the 2 regular holidays ( 1 month summer holiday and 2 months winter holiday)

Follow-up period (post-intervention): nil

Differences in baseline characteristics: reported

Reliable outcomes: reported

Protection against contamination: NR

Unit of allocation: class

Unit of analysis: individual accounting for cluster

Participants

$\mathrm{N}$ (all control baseline $)=4500$

$N$ (control Beijing follow-up $)=460$

$\mathrm{N}$ (control other cities follow up $)=3280$

$\mathrm{N}$ (all intervention baseline $)=5250$

$\mathrm{N}$ (nutrition intervention Beijing follow-up) $=615$

$N$ (PA intervention Beijing follow-up $)=590$

$\mathrm{N}$ (nutrition + PA intervention other cities follow-up) $=3356$

Setting (and number by study group): primary schools

Recruitment: 2-step cluster sampling was used for participant selection. In the 1st step, 9 schools in Beijing were selected and assigned randomly to nutrition intervention ( 3 schools), PA intervention ( 3 schools) or control condition ( 3 schools). In other 5 cities, 6 schools in each city were selected randomly assigned to either combined with nutrition education and PA intervention ( 3 schools) or control condition ( 3 schools). Thus, there are a total of 15 schools in combined intervention and 15 schools in the control group in other 5 cities. In the 2 nd step, 2 classes from each grade in each school were chosen randomly

Geographic region: 6 large cities in China: Beijing, Shanghai, Chongqing, Guangzhou, Jinan and Harbin

Percentage of eligible population enrolled: 96\% participants

Mean age:

- Beijing

- control group: between the ages of 6-9.9 years, $N=314$ (68.3\%) between the ages of $10-13.9$ years, $\mathrm{N}=146(31.7 \%)$.

- nutrition intervention group: between the ages of 6-9.9 years, $N=427(69.4 \%)$, between the ages of $10-13.9$ years, $\mathrm{N}=188(30.6 \%)$.

- PA intervention group: between the ages 6-9.9 years, $N=420$ (71.2\%), between the ages of 10-13.9 years, $\mathrm{N}=170(28.8 \%)$.

- Other 5 centres:

- control group: between the ages of 6-9.9 years, $\mathrm{N}=2357$ ( $71.9 \%)$, between the ages of $10-13.9$ years, $\mathrm{N}=923(28.1 \%)$

- nutrition and PA intervention group: between the ages of 6-9.9 years, $N=2381$ (70.9\%), between the ages of $10-13.9$ years, $\mathrm{N}=975(29.1 \%)$ 
Meng 2013 (Continued)

Sex:

- Beijing

- control group, male $\mathrm{N}=266$ (57.8\%), female $\mathrm{N}=194(42.4 \%)$.

- nutrition intervention group, male $\mathrm{N}=300(48.8 \%)$, female $\mathrm{N}=315(51.2)$

- PA intervention group, male $\mathrm{N}=302(51.2 \%)$, female $\mathrm{N}=288(48.8 \%)$

- Other 5 centres

- control group, male $\mathrm{N}=1644$ (50.1\%), female $\mathrm{N}=1636$ (49.9\%)

- nutrition and PA intervention group: male $\mathrm{N}=1695$ (50.5\%), female $\mathrm{N}=1661$ (49.5\%)

Interventions

To evaluate the effects and the cost effectiveness of a comprehensive intervention programme for childhood obesity that combined nutrition education and PA interventions vs control

Nutrition intervention, PA intervention and their shared common control group were located in Beijing. The combined intervention and its control group were located in other 5 cities. In nutrition education group, 'nutrition and health classes' were given 6 times for the students, 2 times for the parents and 4 times for the teachers and health workers. 'Happy 10' was carried out twice per day in PA group. A classroom-based PA programme for elementary students named 'Happy 10' was used in PA intervention. In each school day, the students conducted 'Happy 10' led by teachers to do a 10-min segment of moderate intensity, age- and space-appropriate exercises. The form of exercises was game, dance or rhythmic gymnastics. Students were also encouraged to develop more forms of exercises they liked. Education about PA was provided to students, parents, health workers and teachers. Each student attended the 'Happy 10'

$10 \mathrm{~min}$ for once, twice a day or $20 \mathrm{~min}$ for each time, once a day

The comprehensive intervention was a combination of nutrition and PA interventions.

"nothing will be done in control schools"

Diet vs PA vs combined diet + PA vs control (2 control groups)

Outcomes

Outcome measures

- Primary outcome: BMI, zBMI, \% overweight/obese, cost-effectiveness

- Secondary outcomes: NR

Process evaluation: NR

Implementation-related Theoretical basis: NR

factors

Resources for intervention implementation: reported

Who delivered the intervention: reported

PROGRESS categories assessed at baseline: child, gender; parent: income

PROGRESS categories analysed at outcome: NR

Outcomes relating to harms/unintended effects: NR

Intervention included strategies to address diversity or disadvantage: NR

Economic evaluation: reported, cost-effectiveness analysis

Notes ChiCTR-PRC-09000402

Funding: this project has been funded by China Ministry of Science \& Technology as "Key Projects in the National Science \& Technology Pillar Program during the Eleventh Five-Year Plan Period", grant number 2008BAI58B05. The funders had no role in study design, data collection and analysis, decision to publish, or preparation of the manuscript.

Inclusion criteria: the schools having pupils with an overweight/obesity rate in excess of $10 \%$ 
There are 5 different groups including 2 control groups:

- Nutrition intervention (Beijing)

- PA intervention (Beijing)

- Control (Beijing)

- Combined nutrition and PA intervention (other 5 cities)

- Control (other 5 cities)

The cost-effectiveness ratio was USD 120.3 for BMI and USD 249.3 for zBMI in combined intervention, respectively.

Pilot study is also included in the review: Li 2010a

\section{Risk of bias}

\begin{tabular}{|c|c|c|}
\hline Bias & Authors' judgement & Support for judgement \\
\hline $\begin{array}{l}\text { Random sequence genera- } \\
\text { tion (selection bias) }\end{array}$ & Low risk & Randomly assigned, random number table, 2-step cluster sampling \\
\hline $\begin{array}{l}\text { Allocation concealment } \\
\text { (selection bias) }\end{array}$ & Unclear risk & NR \\
\hline $\begin{array}{l}\text { Blinding (performance } \\
\text { bias and detection bias) } \\
\text { All outcomes }\end{array}$ & Unclear risk & NR \\
\hline $\begin{array}{l}\text { Incomplete outcome data } \\
\text { (attrition bias) } \\
\text { All outcomes }\end{array}$ & Low risk & $89 \%$ retention \\
\hline $\begin{array}{l}\text { Selective reporting (re- } \\
\text { porting bias) }\end{array}$ & Low risk & Protocol/trial registration document seen. All outcomes reported \\
\hline Other bias & Low risk & No additional threats to validity \\
\hline $\begin{array}{l}\text { Other bias- timing of re- } \\
\text { cruitment of clusters }\end{array}$ & Unclear risk & $\begin{array}{l}\text { NR; no CONSORT figure: text in study design suggests that classes of specific } \\
\text { grades in the school, may have been chosen after schools had already been } \\
\text { randomised. }\end{array}$ \\
\hline & & $\begin{array}{l}\text { Quote: "In the first step, } 9 \text { schools in Beijing were selected and assigned ran- } \\
\text { domly to nutrition intervention ( } 3 \text { schools), PA (PA) intervention ( } 3 \text { schools) or } \\
\text { control condition ( } 3 \text { schools). In other five cities, } 6 \text { schools in each city were } \\
\text { selected randomly assigned to either combined with nutrition education and } \\
\text { PA intervention ( } 3 \text { schools) or control condition ( } 3 \text { schools). In the } 2 \text { nd step, } 2 \\
\text { classes from each grade in each school were chosen randomly" }\end{array}$ \\
\hline
\end{tabular}

Mihas 2010

Methods

Study name: Vyronas youth regarding obesity, nutrition and attitudinal styles

Study design: cluster-RCT

Intervention period: 12 weeks

Follow-up period (post-intervention): 12 months

Differences in baseline characteristics: reported 
Mihas 2010 (Continued)

Reliable outcomes: reported

Protection against contamination: NR

Unit of allocation: individual

Unit of analysis: individual accounting for interschool variation

Participants (control baseline) $=105$
$N$ (control follow-up) $=93$
$N$ (intervention baseline) $=108$
$N$ (intervention follow-up) $=98$
Setting (and number by study group): 5 high schools
Recruitment: the Vyronas area was selected because it represents the socio-economic status of the citi-
zens of Athens

Geographic region: Vyronas district, Athens, Greece

Percentage of eligible population enrolled: $72 \%$

Mean age: intervention: $13.1 \pm 0.8$; control: $13.3 \pm 0.9$

Sex: intervention: $51 \%$ female; control: $50.5 \%$ female

Interventions

To assess the short- and long-term effects of a school-based health and nutrition education intervention on diet, nutrition intake and BMI

$12 \mathrm{~h}$ of classroom material during 12 weeks. The health and nutrition components of the programme were conducted by the class home economics teacher supervised by a health visitor or a family doctor. After teachers were provided with preparatory teaching and classroom materials, they undertook special seminars that were designed and conducted to the intervention classes. In co-operation with the school directors, two 3-h seminars were performed by the study authors.

Multi-component workbooks covering mainly dietary issues, but also dental health hygiene and consumption attitudes, were produced with each student being supplied a workbook. Classroom modules were designed to develop behavioural capability, expectations and self-efficacy for healthful eating and healthy foods selection. Learning activities were designed to influence expectancies that placed an important value on achieving these behaviours. Several motivational methods and strategies were used for increasing skills and self-efficacy (i.e. modelling, guided practice, enactment), achieving better self-monitoring (i.e. problem-solving, goal-setting), changing attitudes and beliefs (i.e. self-reevaluation, environmental re-evaluation, arguments, modelling, direct experience) and changing social influence (i.e. modelling, mobilising social support). Cues and reinforcing messages in the form of posters and displays were provided in the classroom.

After the end of the baseline examinations, 2 meetings were organised whereby parents in the intervention group were given a file containing their child's screening results and presentations on the importance of topics relevant to the dietary habits of children were issued. Parents were also encouraged to modify their dietary habits as well as those of their children.

Control: an envelope with all medical screening results plus some brief comments were mailed to the parents. Did not undertake any health education intervention and no parental educational sessions took place.

Diet intervention vs control

Outcome measures
- Primary outcome: energy and nutrient intake, dietary changes, BMI
- Secondary outcomes: NR


Mihas 2010 (Continued)

Process evaluation: NR

Implementation-related factors
Theoretical basis: Social Learning theory

Resources for intervention implementation: reported

Who delivered the intervention: reported

PROGRESS categories assessed at baseline: gender

PROGRESS categories analysed at outcome: NR

Outcomes relating to harms/unintended effects: NR

Intervention included strategies to address diversity or disadvantage: NR

Economic evaluation: NR
Funding: the raw material for health promotion activities covering the thematic areas of 'Nutrition-dietary habits' and 'PA and health' was funded by the Ministry of Education and the National Foundation for the Youth

\section{Risk of bias}

\begin{tabular}{|c|c|c|}
\hline Bias & Authors' judgement & Support for judgement \\
\hline $\begin{array}{l}\text { Random sequence genera- } \\
\text { tion (selection bias) }\end{array}$ & Low risk & Used a computerised random number generator \\
\hline $\begin{array}{l}\text { Allocation concealment } \\
\text { (selection bias) }\end{array}$ & Unclear risk & NR \\
\hline $\begin{array}{l}\text { Blinding (performance } \\
\text { bias and detection bias) } \\
\text { All outcomes }\end{array}$ & High risk & $\begin{array}{l}\text { No blinding of participants who provided outcomes in self-report question- } \\
\text { naires }\end{array}$ \\
\hline $\begin{array}{l}\text { Incomplete outcome data } \\
\text { (attrition bias) } \\
\text { All outcomes }\end{array}$ & Low risk & $9 \%-11 \%$ attrition \\
\hline $\begin{array}{l}\text { Selective reporting (re- } \\
\text { porting bias) }\end{array}$ & Unclear risk & Protocol mentioned in RCT but we were unable to find it or trial registry report \\
\hline Other bias & High risk & $\begin{array}{l}\text { There is a high risk of contamination between intervention and control partici- } \\
\text { pants within same schools }\end{array}$ \\
\hline $\begin{array}{l}\text { Other bias- timing of re- } \\
\text { cruitment of clusters }\end{array}$ & Low risk & Figure 1 indicates recruitment happened prior to randomisation \\
\hline
\end{tabular}

Mo-suwan 1998

\begin{tabular}{ll}
\hline Methods & Study design: cluster-RCT \\
Intervention period: 29.6 weeks \\
Follow-up (post-intervention): 6 months \\
Differences in baseline characteristics: reported \\
Reliable outcomes: all measures validated in children $>6$ years of age \\
Protection against contamination: not clear \\
Unit of allocation: class
\end{tabular}


Mo-suwan 1998 (Continued)

Unit of analysis: child; unit of analysis errors addressed

\begin{tabular}{|c|c|}
\hline \multirow[t]{4}{*}{ Participants } & $\begin{array}{l}\text { Follow-up at } 6 \text { months: } \mathrm{n} \text { (intervention baseline) }=158 \\
\mathrm{~N} \text { (intervention follow-up) }=147 \\
\mathrm{~N} \text { (control baseline) }=152 \\
\mathrm{~N} \text { (control follow-up) }=145 \\
\mathrm{~N} \text { of classes: } 10\end{array}$ \\
\hline & $\begin{array}{l}\text { Outcome data collected for: } 94 \% \text { of baseline } N \text { followed up; } 75 \% \text { of eligible population enrolled }=310 \\
\text { Geographic setting: Thailand }\end{array}$ \\
\hline & Age: 4.5 (SD 0.4) years \\
\hline & Sex: both sexes included; intervention: $56 \%$ boys; controls: $61 \%$ boys \\
\hline \multirow[t]{2}{*}{ Interventions } & $\begin{array}{l}\text { Kindergarten-based PA programme conducted by specially trained staff and including a 15-min walk } \\
\text { and a } 20 \text {-minute aerobic dance session 3-times a week. Study objective was to evaluate the effect of a } \\
\text { school-based aerobic exercise programme on the obesity indexes of preschool children. } \\
\text { Control programme NR, presumably usual school curriculum }\end{array}$ \\
\hline & PA interventions vs control \\
\hline \multirow[t]{5}{*}{ Outcomes } & - BMI \\
\hline & $\cdot$ TSF \\
\hline & - ratio of weight in kg divided by height cubed in meters (WHCU) \\
\hline & - Computation of BMI, WHCU and TSF slopes \\
\hline & Process evaluation: NR \\
\hline
\end{tabular}

Implementation-related factors

\section{Theoretical basis: Not Reported}

Resources for intervention implementation (e.g. funding needed or staff hours required): Not Reported Who delivered the intervention: Reported

PROGRESS categories assessed at baseline: Reported (Gender, SES)

PROGRESS categories analysed at outcome: Reported (Gender)

Outcomes relating to harms/unintended effects: Not Reported

Intervention included strategies to address diversity or disadvantage: Not Reported

Economic evaluation: Not Reported

Notes

Funding: the project was financially supported by the Research Fund from the Songkhlanagarind Hospital Foundation. Trial supported by a grant from the National Research Council of Thailand

\section{Risk of bias}

\begin{tabular}{lll}
\hline Bias & Authors' judgement & Support for judgement \\
\hline $\begin{array}{l}\text { Random sequence genera- } \\
\text { tion (selection bias) }\end{array}$ & Unclear risk & $\begin{array}{l}\text { Classes were randomly allocated to either the exercise group or control group; } \\
\text { no further information }\end{array}$ \\
\hline $\begin{array}{l}\text { Allocation concealment } \\
\text { (selection bias) }\end{array}$ & Unclear risk & NR \\
\hline $\begin{array}{l}\text { Blinding (performance } \\
\text { bias and detection bias) } \\
\text { All outcomes }\end{array}$ & Unclear risk & NR \\
\hline
\end{tabular}


Mo-suwan 1998 (Continued)

Incomplete outcome data Low risk Loss to follow-up was minimal and reasons given for 2 exclusions from analy(attrition bias)

All outcomes

\begin{tabular}{lll}
\hline $\begin{array}{l}\text { Selective reporting (re- } \\
\text { porting bias) }\end{array}$ & Low risk & $\begin{array}{l}\text { Protocol not sought; all outcomes specified in methods were reported in re- } \\
\text { sults }\end{array}$ \\
\hline Other bias & Low risk & No other threats to validity \\
\hline $\begin{array}{l}\text { Other bias- timing of re- } \\
\text { cruitment of clusters }\end{array}$ & Low risk & $\begin{array}{l}\text { No CONSORT figure; text indicates recruitment happened prior to randomisa- } \\
\text { tion }\end{array}$ \\
\hline
\end{tabular}

\section{Morgan 2011}

Methods

Study name: Healthy dads, healthy kids (HDHK)

Study design: RCT

Intervention period: 3 months

Follow-up period (post-intervention): 3 months

Differences in baseline characteristics: reported

Reliable outcomes: reported

Protection against contamination: NR

Unit of allocation: father-child dyads

Unit of analysis: individual

Participants (control baseline) $=26$ fathers, 32 children
$N$ (control follow-up) $=24$ fathers
$N$ (intervention baseline) $=27$ fathers, 39 children
N (intervention follow-up) $=20$ fathers
Setting (and number by study group): 53 fathers ( 72 children) at the University
recreation centre
Recruitment: from the local community via media releases, school newsletters and paid adverts in lo-
cal newspapers
Geographic region: Newcastle, Callaghan, New South Wales, Australia
Percentage of eligible population enrolled: $90 \%$ fathers
Mean age: intervention + control: $8.2 \pm 2.0$
Sex: intervention + control: $46.5 \%$ female
To evaluate the feasibility and efficacy of the 'Healthy dads, healthy kids' (HDHK) programme, which
was designed to help overweight fathers lose weight and be a role model of positive health behaviours
for their children
There were 8 sessions. 5 with just fathers, and 3 that the children joined.


Session 1: 'Weight loss for men' (fathers)

- Programme rationale

- Importance of fathers and their influence on children

- Energy balance and weight loss

- 9 weight loss tips for men website use for eating and activity diaries

Session 2: 'Raising active children in an inactive world' (fathers)

- Obesogenic environments

- PA levels, trends and benefits PA recommendations

- PA goals for dads

- ideas for fitness/activity at home

Session 3: 'Ready to rumble with dad' (fathers and children)

- Rough and tumble play fun fitness circuits

- Fun and active games

Session 4: 'Healthy eating for families-dads matter' (fathers)

- Healthy eating benefits

- Food based guidelines

- Role of fathers in healthy home eating environments

- Authoritative feeding practices, reading food labels

Session 5: 'Sustaining healthy eating at home' (fathers)

- Planning meals

- Australian guide to healthy eating, recommended daily intakes

- Why we eat food?

- Support and strategies for successful dietary changes and relapse prevention

Session 6: 'Fitness, fun and fundamental movement skills' (fathers and children)

- Fundamental movement skill circuit

- Rough and tumble activities

- Partner fitness challenges

Session 7: 'Playing strong' (fathers and children)

- The benefits of strength training, strength training exercises, rough and tumble activities

- Ball and game skills

Session 8: 'Games show and Healthy BBQ' (fathers)

- Programme revision

- Group-based trivia competition with practical challenges to reinforce PA messages (fitness, fundamental movement skills (FMS) etc.)

Conducted at the University recreation centre and delivered by 2 of the male researchers (PJM and $\mathrm{DRL})$, both qualified teachers with expertise in PE.

The wait-list control group received no information or intervention before attending the 3- and 6month follow-up assessment sessions.

Diet and PA combination intervention vs control 
Morgan 2011 (Continued)

- Secondary outcomes: for fathers and children, zBMI, waist z-score, blood pressure, resting heart rate, PA, dietary heart rate

Process evaluation: reported: recruitment, retention, attendance, satisfaction (fathers)

Implementation-related factors
Theoretical basis: SCT

Resources for intervention implementation: reported

Who delivered the intervention: reported

PROGRESS categories assessed at baseline: child: gender; father: SES

PROGRESS categories analysed at outcome: NR

Outcomes relating to harms/unintended effects: NR

Intervention included strategies to address diversity or disadvantage: NR

Economic evaluation: NR
Funding: this study was funded by the Hunter Medical Research Institute and the Gastronomic Lunch. Children in the control group were more likely to be overweight/obese

\section{Risk of bias}

\begin{tabular}{lll}
\hline Bias & Authors' judgement & Support for judgement \\
\hline $\begin{array}{l}\text { Random sequence genera- } \\
\text { tion (selection bias) }\end{array}$ & Low risk & $\begin{array}{l}\text { The random allocation sequence was generated by a computer-based random } \\
\text { number-producing algorithm in block lengths of } 6 \text { to ensure an equal chance } \\
\text { of allocation to each group }\end{array}$ \\
\hline $\begin{array}{l}\text { Allocation concealment } \\
\text { (selection bias) }\end{array}$ & Low risk & $\begin{array}{l}\text { To ensure concealment, the sequence was generated by a statistician and giv- } \\
\text { en to the project manager. Randomisation was completed by a researcher } \\
\text { who was not involved in the assessment of participants and the allocation se- } \\
\text { quence was concealed when enrolling participants. Children in the control } \\
\text { group were more likely to be overweight/obese }\end{array}$
\end{tabular}

Blinding (performance bias and detection bias) All outcomes

\section{High risk}

Participants were blind to group allocation at baseline assessment. Quote: "Although it was our intention to blind assessors at follow up, it was not possible to keep assessors completely blinded, as there were a few cases of treatment group families (and in particular, children) mentioning aspects of their program involvement or wearing their program T-shirts to follow-up assessment sessions"

Incomplete outcome data Low risk 83\% retention, balanced, ITT conducted

(attrition bias)

All outcomes

Selective reporting (re- Unclear risk Protocol or trial register not found
porting bias)

Other bias Low risk Analyses were performed separately for fathers and children

Muckelbauer 2010

$\begin{array}{ll}\text { Methods } & \text { Study design: cluster-RCT } \\ & \text { Intervention period: } 11 \text { months }\end{array}$


Muckelbauer 2010 (Continued)

Follow-up period (post-intervention): nil

Differences in baseline characteristics: reported

Reliable outcomes: reported

Protection against contamination: reported

Unit of allocation: city

Unit of analysis: individual accounting for clustering by school

Participants
N (control baseline) $=1839$
N (intervention baseline) $=1978$
N (intervention follow-up) $=1641$
Setting (and number by study group): elementary schools in deprived areas (16 control and 17 inter-
vention schools)
Recruitment: NR
Geographic region: 2 neighbouring cities, Dortmund and Essen, Germany
Percentage of eligible population enrolled: random sample of schools, $84 \%$ of 3817 children attending
the participating schools, with a higher rate in the intervention group (88\%) than in the control groups
(80\%; $P=0.004)$

Mean age: intervention: $8.26 \pm 0.73$; control: $8.34 \pm 0.76$

Sex: intervention: $49.8 \%$ female; control: $49.7 \%$ female sumption was effective in preventing overweight among children in elementary school

In each intervention school, 1 water fountain, or 2 for schools with $>150$ participants, was installed. The fountains provided cooled, filtered, plain or optionally carbonated water. In addition, each child received a plastic water bottle $(500 \mathrm{~mL})$, and teachers were encouraged to organise filling of the water bottles each morning for all children in the corresponding classes. The educational intervention consisted of four 45-min classroom lessons dealing with the water needs of the body and the water circuit in nature.

At the beginning of the study, teachers received a booklet with the prepared curriculum and necessary materials to implement the lessons in the formal school curriculum.

The lessons were developed by using the results of empirical teaching research and were intended to improve the constructs of intention, attitudes, and perceived behavioural control, on the basis of the theory of planned behaviour.

3 months after the beginning of the study, teachers introduced a motivation unit (i.e. booster sessions) that used a goal-setting strategy to reach a sustained increase in water consumption by giving quantitative targets and feedback. In month 5 after the baseline assessment, each participant received a new water bottle with an improved handling design.

Control schools did not receive any intervention.

Diet (water consumption only) intervention vs control

$\begin{array}{ll}\text { Outcomes } & \text { Outcome measures } \\ \text { - Primary outcome: overweight prevalence } & \text { - Secondary outcomes: BMI SD scores }\end{array}$


Muckelbauer 2010 (Continued)

Process evaluation: reported (implementation)

Implementation-related factors
Theoretical basis: TPB

Resources for intervention implementation: reported

Who delivered the intervention: reported

PROGRESS categories assessed at baseline: gender

PROGRESS categories analysed at outcome: NR

Outcomes relating to harms/unintended effects: reported

Intervention included strategies to address diversity or disadvantage: NR, intervention only included deprived schools

Economic evaluation: reported: costs

NCT00554294
Funding: this study was supported by grant no. 05HS026 of the German Federal Ministry of Food, Agri-
culture, and Consumer Protection. Intervention material (water fountains, bottles, print of the lesson
booklet) was provided by the Association of the German Gas and Water Industries.
The initial costs per water fountain were EUR 2500 and the long-term costs per enrolled child were
EUR $13 /$ year. The educational intervention was presented by the teachers; therefore, no additive costs
emerged.

\section{Risk of bias}

\begin{tabular}{lll}
\hline Bias & Authors' judgement & Support for judgement \\
\hline $\begin{array}{l}\text { Random sequence genera- } \\
\text { tion (selection bias) }\end{array}$ & Unclear risk & $\begin{array}{l}\text { Randomisation was performed at the city level to minimise contamination be- } \\
\text { tween neighbouring schools in } 1 \text { city, no further details }\end{array}$ \\
\hline $\begin{array}{l}\text { Allocation concealment } \\
\text { (selection bias) }\end{array}$ & High risk & $\begin{array}{l}\text { NR. However the model to test for intervention effects on the primary outcome } \\
\text { prevalence of overweight at the follow-up assessment included significant } \\
\text { confounders, besides the fixed intervention effect, although randomisation } \\
\text { was conducted }\end{array}$ \\
\hline
\end{tabular}

\begin{tabular}{|c|c|c|}
\hline $\begin{array}{l}\text { Blinding (performance } \\
\text { bias and detection bias) } \\
\text { All outcomes }\end{array}$ & High risk & $\begin{array}{l}\text { Blinding of outcome assessment for BMI not reported. Participants not blind } \\
\text { Quote: "participants were aware of the behavioural intervention aim." }\end{array}$ \\
\hline
\end{tabular}

Incomplete outcome data Low risk
(attrition bias)

Of 3190 children screened at baseline, a total of 2950 children (92\%) were also measured at the follow-up assessment and were considered for analysis. All outcomes Dropouts $(N=240)$ were similar to analysed participants with respect to the prevalence of overweight ( $24.6 \%$ vs $24.5 \% ; P=0.741)$, mean BMI SDs ( 0.26 vs $0.26 ; \mathrm{P}=0.807)$, mean age ( 8.27 vs 8.30 years; $P=0.574)$, proportion of boys $(50.4 \%$ vs $50.2 \% ; P=0.772)$, and proportion of children with migrational background $(42.1 \%$ vs $44.3 \%$; $P=0.568)$.

$>30 \%$ loss to follow-up - therefore high risk?

\begin{tabular}{lll}
\hline $\begin{array}{l}\text { Selective reporting (re- } \\
\text { porting bias) }\end{array}$ & Low risk & Protocol/trial registration document seen. All outcomes reported \\
\hline Other bias & Low risk & No additional threats to validity \\
\hline
\end{tabular}


Muckelbauer 2010 (Continued)

Other bias- timing of re- Low risk Figure indicates recruitment happened prior to randomisation cruitment of clusters

Study design: cluster-RCT

Intervention period: 6 months

Follow-up period (post-intervention): 6 months

Differences in baseline characteristics: reported

Reliable outcomes: reported

Protection against contamination: NR

Unit of allocation: childcare centre

Unit of analysis: individual

\section{Participants}

$\mathrm{N}($ intervention + control baseline $)=318$ but baseline characteristics for intervention $=238$, control $=69$

$\mathrm{N}$ (intervention + control follow-up) $=185$

Setting (and number by study group): 8 subsidised childcare centres ( $N=6$ intervention, $N=2$ control)

Recruitment: NR

Geographic region: Miami-Dade County, Florida, USA

Percentage of eligible population enrolled: $98 \%$ participants

Mean age: intervention: 2 years $=34,3$ years $=85,4$ years $=87,5$ years $=32$; control: 2 years $=20,3$ years $=23,4$ years $=22,5$ years $=4$. Average age for boys was 3.82 years, average age for girls was 3.91 years.

Sex: intervention: $49.2 \%$ female; control: $47.8 \%$ female

- Teacher component:

- modelled after a modified version of Hip-hop to health

- included 2 training events per centre

- teachers and staff were trained on the role and rationale of the HI-HO programme, taught implementation strategies, and provided lessons to use with the children.

- An additional component that was not included in Hip Hop was weekly technical assistance visits with the teachers and a HI-HO specialist to ensure the implementation of a low-fat, high-fibre diet that included more fruits and vegetables with an emphasis on cultural barriers.

- Parent component:

- specific components included a monthly educational dinner in which nutrition and PA were discussed, monthly newsletters, and at-home activities

- the content of the parent dinners paralleled the information offered in the monthly newsletters but unlike the Hip Hop curriculum, issues were covered that were often of concern to parents of preschool children (e.g. how to introduce new foods and how to encourage eating more fruits and vegetables). In addition, dietitians addressed perceptions related to childhood weight status, which are often engrained in cultural beliefs such as "He will grow out of it" or the idea of "babyfat." 
Natale 2014 (Continued)

$\circ$ parents were encouraged to reduce TV viewing, increase PA, and model healthy eating behaviours for their child at home.

- for each of the six at-home activities that each family completed, they received a healthy snack bag.

- at the end of the programme, parents who attended three or more dinners received a certificate of completion.

- Center-based modifications component:

- these included the development of policies to increase PA and healthy eating. Furthermore, a nutritionist worked with each child care center to modify menus to make them compliant with the policies and also to ensure that the U.S. Department of Agriculture (USDA) nutritional requirements were met.

- The nutritionist ensured that the modifications made to the centres' daily menus were of equal cost as prior food purchases, while simultaneously lowering the daily consumption of saturated fats and trans fatty acids.

- Each centre agreed on a drink policy that included providing water as the primary beverage, not allowing juice or sweetened beverages more than once a week, and changing from whole milk to $1 \%$ milk.

- The snack policy consisted of healthy snacks, such as fresh fruit and/or vegetables, as a substitute for cookies and other high-lipid snacks.

- The PA policy consisted of urging centres to increase PA to $>1 \mathrm{~h}$ per day and to decrease TV viewing to $<60$ min twice a week

Control centres received an attention control programme. Centres received a visit from an injury prevention education mobile unit. The mobile provided parents and teachers with hands-on safety education and information, as part of an ongoing injury prevention programme at the University of Miami.

Diet and PA combination intervention vs control

$\begin{array}{ll}\text { Outcomes } & \text { Outcome measures } \\ \text { - Primary outcome: zBMI } & \text { - Secondary outcomes: Dietary intake, PA }\end{array}$

Process evaluation: NR

Implementation-related factors
Theoretical basis: SEM

Resources for intervention implementation: reported

Who delivered the intervention: reported

PROGRESS categories assessed at baseline: gender, race/ethnicity

PROGRESS categories analysed at outcome: NR

Outcomes relating to harms/unintended effects: NR

Intervention included strategies to address diversity or disadvantage: intervention targeted low-income minority centres, cultural/ethnic modifications, nutritionist ensured that the modifications made to the centres' daily menus were of equal cost as prior food purchases; designed to address health disparities through an innovative community-based model

Economic evaluation: reported - some costs

Notes

Funding: this research was funded by the Miami-Dade County Children's Trust (grant number 764-287). Also assesses relationship between BMI and parent/home intervention activities. All centre menu changes were 'revenue neutral'

\section{Risk of bias}


Natale 2014 (Continued)

$\begin{array}{ll}\begin{array}{l}\text { Random sequence genera- } \\ \text { tion (selection bias) }\end{array} & \begin{array}{l}\text { Quote: "Eight child care centers were randomly assigned to an intervention or } \\ \text { attention control arm" }\end{array}\end{array}$
tion (selection bias) attention control arm"

\begin{tabular}{lll}
$\begin{array}{l}\text { Allocation concealment } \\
\text { (selection bias) }\end{array}$ & Unclear risk \\
\hline $\begin{array}{l}\text { Blinding (performance } \\
\text { bias and detection bias) }\end{array}$ & Unclear risk & NR \\
All outcomes & &
\end{tabular}

\begin{tabular}{|c|c|c|}
\hline $\begin{array}{l}\text { Incomplete outcome data } \\
\text { (attrition bias) } \\
\text { All outcomes }\end{array}$ & High risk & $\begin{array}{l}\text { Attrition NR by group, reports } 58 \% \text { retention for the outcome BMI at one year. } \\
\text { Quote: "Attrition rates were calculated based on available data for child BMI } \\
\text { as well as parent measures for each of the time points. At baseline, there were } \\
318 \text { child and parent dyads; at } 6 \text { months, there were } 239 \text { child and parent } \\
\text { dyads; and at } 1 \text { year, there were } 185 \text { parent and child dyads." }\end{array}$ \\
\hline Other bias & Low risk & No additional threats to validity \\
\hline $\begin{array}{l}\text { Other bias- timing of re- } \\
\text { cruitment of clusters }\end{array}$ & Unclear risk & NR \\
\hline
\end{tabular}

Nemet 2011

Study design: cluster-RCT
Intervention period: 1 school year
Follow-up period (post-intervention): 12 months (for Arab-Israeli subgroup only)
Differences in baseline characteristics: reported
Reliable outcomes: reported
Protection against contamination: NR
Unit of allocation: kindergarten classes
Unit of analysis: individual accounting for clustering

Participants
$\mathrm{N}$ (control baseline) $=378$ Jewish-Israeli, N = 188 Arab-Israeli
months post-intervention
$\mathrm{N}$ (intervention baseline) = 417 Jewish-Israeli, N = 154 Arab-Israeli
$\mathrm{N}$ (intervention follow-up) = 376 Jewish-Israeli and 134 Arab-Israeli (1 school year), 118 Arab-Israeli at
12 months post-intervention
Setting (and number by study group): 30 kindergartens (only include 1 class each, 15 classes in each
group for Jewish-Israeli, 12 kindergartens for Arab-Israeli, 6 in each group)
Recruitment: NR
Geographic region: low socioeconomic status communities, Sharon area, Israel


Nemet 2011 (Continued)

Percentage of eligible population enrolled: NR

Mean age: intervention: $5.20 \pm 0.02$ Jewish-Israeli, 5.36 (0.03) Arab-Israeli; control: $5.24 \pm 0.03$ Jewish-Israeli, 5.40 (0.02) Arab-Israeli

Sex: intervention: 46\% female Jewish-Israeli, 45\% female Arab-Israeli; control: 44\% female Jewish-Israeli; $45 \%$ female Arab-Israeli

Interventions

To prospectively examine the effects of a randomised school-based intervention on nutrition and PA knowledge and preferences, anthropometric measures, and fitness in low socioeconomic kindergarten children

Intervention has a nutrition and PA element.

- Nutrition

- Intervention was designed mainly to improve nutritional knowledge and was based on the nutritional programme 'It Fits Me' ('Tafur Alay') of the Israeli Ministry of Education (www.tafuralay.co.il/). Briefly, the intervention consisted of teaching topics such as food groups, vitamins, healthy food choices, food preparation and cooking methods, and information on fast-food vs home cooking. The topics were taught through short lectures/talks, games, and story reading. Delivered by kindergarten staff. Topics included the following: what do popular Israeli foods contain, fruits and vegetables, what is calcium and why is it important, special dietary consideration during holidays,

- In addition, monthly flyers detailing nutritional information were sent home via the children. Children were asked to present the nutritional information to their parents, and parents were asked to discuss the information with their children.

- PA

- All intervention children took part in $45 \mathrm{~min}$ (divided into three 15-min sessions) per day of exercise training, 6 days/week. Sessions took place indoors and outdoors.

- Activities varied in duration and intensity and were designed primarily as games.

Who delivered: once a week training was directed by a professional youth coach. Similar activities were delivered the rest of the week by the preschool staff. Endurance type activities accounted for most of the time spent in training (about 20\% team sports (soccer, dodge ball) and $80 \%$ running games (tag, hide-and-seek, relays, etc)), with attention also given to co-ordination and flexibility skills. Preschool teachers also were given a CD collection of children's songs, related to the topic of nutrition and exercise.

Training: preschool teachers attended an all-day seminar in which they were acquainted with the programme and were trained by the study team so that preschool staff (i.e. teachers and assistant teachers) could perform all the nutritional aspects of the intervention and most exercise classes. Teachers were given lectures, hands-on sessions (on nutrition and PA), and written material to familiarise them with the programme and enable them to perform it in their classes. During the intervention, kindergarten teachers were invited to 2 additional training days; the goal of these meetings was to collect feedback on the programme and to introduce new materials to the teachers. Adherence to the programme was followed weekly by the study co-ordinator and by the professional youth coach.

Parents and children of the intervention groups only were invited for 2 'Healthy Day Festivals' that focused on the major themes of the programme (introduction of healthy nutrition, prevention of childhood obesity, and beneficial effects of exercise in children). The first festival was performed during the 2 nd month of the programme, and the 2 nd festival was performed toward the middle of the programme. The festivals included lectures given by the study team and games for both children and parents.

Control: participants in the control group were informed that measurements

are part of a survey on PA and nutrition in kindergarten children, and they continued their regular kindergarten schedule.

Diet and PA combination intervention vs control 
Nemet 2011 (Continued)

Outcomes
- Primary outcome: nutrition knowledge and preference, PA knowledge and preference, physical fitness, weight, height, BMI, BMI percentile

- Secondary outcomes: primary/secondary NR

Process evaluation: NR

Implementation-related

factors

Theoretical basis: NR
Resources for intervention implementation: reported

Who delivered the intervention: reported

PROGRESS categories assessed at baseline: gender

PROGRESS categories analysed at outcome: gender

Outcomes relating to harms/unintended effects: NR

Intervention included strategies to address diversity or disadvantage: targeted to Arab-Israeli and Jewish-Israeli low-SES children

Economic evaluation: NR

Notes Funding: the study was supported by a grant from The Rosalinde and Arthur Gilbert Foundation, and the Israel Heart Fund.

\section{Risk of bias}

\begin{tabular}{lll}
\hline Bias & Authors' judgement & Support for judgement \\
\hline $\begin{array}{l}\text { Random sequence genera- } \\
\text { tion (selection bias) }\end{array}$ & Low risk & Randomly assigned by computerised program \\
\hline $\begin{array}{l}\text { Allocation concealment } \\
\text { (selection bias) }\end{array}$ & Unclear risk & NR \\
\hline
\end{tabular}

Blinding (performance Low risk

bias and detection bias)

All outcomes
Measurements performed by trained technicians who were blinded to the assignment of the kindergarten
Incomplete outcome data Unclear risk (attrition bias)

All outcomes
$8.8 \%$ attrition (Jewish-Israeli subgroup) 13\% Arab-Israeli subgroup at end of intervention, $41 \%$ attrition at 1 year post-intervention for Arab-Israeli subgroup. Unlike original baseline cohort, BMI percentile was higher and fitness was lower in the control group that was available for 1-year post-intervention follow-up - unclear whether this is adjusted for in the analyses.

Quote: "Seventy children did not complete the study (8.8\%), because they were absent on the days of follow-up measurements (29/378 control, 41/417 intervention) and therefore were excluded from the study"

All outcomes appear to have been reported, however the results are NR for the entire sample, two papers reports results both short- (5731) and long-term (1240) for Arab-Israeli children and 1 paper reports short-term results for Jewish-Israeli children (6778). None of the papers report that the data are from one larger study. Protocol and trial register report were sought but not found.

\begin{tabular}{lll}
\hline Other bias & Low risk & No additional threats to validity \\
\hline $\begin{array}{l}\text { Other bias- timing of re- } \\
\text { cruitment of clusters }\end{array}$ & Unclear risk & NR; no CONSORT figure \\
\hline
\end{tabular}




Study design: cluster-RCT
Intervention period: 16 weeks +8 weeks maintenance
Follow-up: 8 months
Differences in baseline characteristics: reported
Reliable outcomes: yes for weight, height, TSF (but method of measurement NR)
Protection against contamination: not done
Unit of allocation: school
Unit of analysis: child. Not known if unit of analysis errors addressed

\begin{tabular}{ll}
\hline Participants & N (intervention baseline) $=89$ \\
& N (intervention follow-up) $=84$ \\
& (3 high schools) \\
N (control baseline) $=112$ \\
N (control follow-up) $=106$ \\
(3 high schools) \\
Outcome data collected for all those enrolled i.e. 100\% follow-up \\
\% of eligible population enrolled $=86.8 \%$ of intervention school, $83.6 \%$ of control school \\
Geographical setting: Minnesota, USA
\end{tabular}

Mean age: intervention, 14.9 (SD0.9) years; controls: 15.8 (SD1.1)

Sex: girls only

Interventions $\quad$ High-school based girls only, intervention with priority given to girls with BMI at or above 75 th percentile and who did < 30 min per day 3 times/week PA (eating disorders excluded). Delivery was by school staff and research team, with local guest instructors. Intervention addressed socio-environmental, personal and behavioural factors, with PA 4 times/week, nutrition and social support session every other week for total of 16 weeks with an 8-week maintenance component of lunch time meetings. Control programme NR, presumably usual school curriculum

Combined effects of dietary interventions and PA interventions vs control

- BMI
- PA stages of change (based on the Stages of Change Model)
- Participation in PA based on Godin and Sheppard
- Dietary intake adapted from Youth and Adolescent Food Frequency Questionnaire
- Binge eating adapted from the Minnesota Adolescent Health Survey
- Personal Factors
$\circ$ Harter's Self Perception Profile for Children
$\circ$ Media internalisation
○Self-efficacy to be active
$\circ$ Socio-environmental support

Process evaluation: reported

Implementation-related factors
Theoretical basis: SCT

Resources for intervention implementation (e.g. funding needed or staff hours required): reported

Who delivered the intervention: reported

PROGRESS categories assessed at baseline: reported (race, gender)

PROGRESS categories analysed at outcome: NR

Outcomes relating to harms/unintended effects: reported

Intervention included strategies to address diversity or disadvantage: reported 
Neumark-Sztainer 2003 (Continued)

$$
\text { Economic evaluation: NR }
$$

Notes

Funding: this study was supported by Grant AHA NATL/ 9970064N from the American Heart Association

(D. Neumark-Sztainer, principal investigator)

\section{Risk of bias}

\begin{tabular}{|c|c|c|}
\hline Bias & Authors' judgement & Support for judgement \\
\hline $\begin{array}{l}\text { Random sequence genera- } \\
\text { tion (selection bias) }\end{array}$ & Unclear risk & Only state that schools were randomly assigned to conditions \\
\hline $\begin{array}{l}\text { Allocation concealment } \\
\text { (selection bias) }\end{array}$ & High risk & $\begin{array}{l}\text { NR. Girls in the intervention group had higher BMI values than girls in control } \\
\text { group, more younger children in the intervention than control group, more } \\
\text { older children in control group, also different balance of ethnicities in groups, } \\
\text { all indicating baseline imbalance }\end{array}$ \\
\hline $\begin{array}{l}\text { Blinding (performance } \\
\text { bias and detection bias) } \\
\text { All outcomes }\end{array}$ & Unclear risk & $\begin{array}{l}\text { Girls were aware of intervention assignment. It is reported that trained re- } \\
\text { search staff assessed height and weight and calculated BMI but not reported } \\
\text { whether they were aware of intervention. }\end{array}$ \\
\hline $\begin{array}{l}\text { Incomplete outcome data } \\
\text { (attrition bias) } \\
\text { All outcomes }\end{array}$ & Low risk & $\begin{array}{l}\text { Reasons for missing data given and missing data balanced across groups and } \\
\text { with similar baseline characteristics to completers. }\end{array}$ \\
\hline $\begin{array}{l}\text { Selective reporting (re- } \\
\text { porting bias) }\end{array}$ & Low risk & $\begin{array}{l}\text { Protocol not sought; all outcomes specified in methods were reported in re- } \\
\text { sults }\end{array}$ \\
\hline Other bias & Low risk & \\
\hline $\begin{array}{l}\text { Other bias- timing of re- } \\
\text { cruitment of clusters }\end{array}$ & High risk & $\begin{array}{l}\text { Quote: "Although schools were randomly assigned to conditions, because of } \\
\text { logistical and scheduling issues, girls were recruited after the schools were } \\
\text { randomised. Girls in intervention schools knew they were enrolling in an al- } \\
\text { ternative PE class. Girls in control schools were recruited to participate in a re- } \\
\text { search study about eating and exercise patterns of teens. }\end{array}$ \\
\hline
\end{tabular}

Neumark-Sztainer 2010

\begin{tabular}{ll}
\hline Methods & Study design: cluster-RCT \\
Intervention period: 16 weeks \\
Follow-up period (post-intervention): 5 months \\
Differences in baseline characteristics: reported \\
Reliable outcomes: reported \\
Protection against contamination: NR \\
Unit of allocation: school \\
Unit of analysis: individual accounting for clustering \\
N (control baseline) $=174$ \\
N (control follow-up) $=159$ \\
N (intervention baseline) $=182$ \\
\hline
\end{tabular}


Neumark-Sztainer 2010 (Continued)

$$
\mathrm{N} \text { (intervention follow-up) }=177
$$

Setting (and number by study group): 6 intervention and 6 control high schools in urban and first-ring suburban areas

Recruitment: schools were selected because of their diverse student bodies, girls in intervention and control schools were invited to register for an all-girls PE class as an alternative to the regular coeducational class. Recruitment materials were designed to appeal to inactive girls interested in healthy weight management. Care was used to avoid stigmatising the class in any way. A class description was included in the school catalogue used for class registration. Additionally, posters and flyers about the programme were displayed at schools

Geographic region: Minneapolis/St. Paul metropolitan area of Minnesota, USA

Percentage of eligible population enrolled: $82 \%$ participants

Mean age: intervention + control: $15.8 \pm 1.17$

Sex: intervention + control: $100 \%$ female adolescent girls: 'New moves'

New moves is implemented within schools, as an all-girls PE class, with supplementary group and individual activities. The programme strives to provide a supportive environment in which all girls feel comfortable being physically active and discussing weight-related issues, regardless of their size, shape, or level of PA. Girls in both intervention and control schools participated in an all-girls PE class during the first semester of the school year. Additionally, intervention girls received the New moves curriculum during their PE class and participated in New moves activities throughout the rest of the school year.

New moves programme components included:

- the New moves PE class, which incorporated nutrition and social support/self-empowerment sessions

- individual counselling sessions using MI techniques

- lunch get-togethers (lunch bunches) once a week during the maintenance period (post-16 weeks of step 1 and 2), and a one-off parent-child day retreat to reinforce messages

- minimal parent outreach activities (6 postcards were sent home to reinforce New moves messages)

The New moves PE class was approximately 16 weeks long. Girls participated in PA (Be Fit) 4 days/week and nutrition (Be Fueled) or social support/self-empowerment (Be Fab) classes 1 day/week. Be Fit sessions were taught 3 days/week by school PE teachers and 1 day/week by different community guest instructors who exposed the girls to fun activities (e.g. dance, hip hop, kickboxing) available in the community. New moves intervention staff ran all other programme components. PE teachers participated in a full-day training prior to the start of the intervention and a half-day training in the middle of the programme. Additionally, teachers received regular, ongoing support from New moves staff throughout the programme.

Teachers within control schools did not receive training on New moves until after the study period and were free to conduct their PE classes as they desired during the study period. it is important to note that the control group also received an intervention (i.e. an all-girls class composed of girls with sedentary lifestyles).

Diet and PA combination intervention vs control

Outcomes Outcome measures

- Primary outcome: $\%$ body fat, BMI, PA, sedentary activity, dietary intake, eating patterns, unhealthy weight-control behaviours, and body/self-image

- Secondary outcomes: NR in this paper

Process evaluation: reported: programme satisfaction 
Neumark-Sztainer 2010 (Continued)

\author{
Implementation-related Theoretical basis: SCT, Stages of Change \\ factors \\ Resources for intervention implementation: reported \\ Who delivered the intervention: reported \\ PROGRESS categories assessed at baseline: race/ethnicity \\ PROGRESS categories analysed at outcome: NR \\ Outcomes relating to harms/unintended effects: reported (unhealthy weight control behaviours) \\ Intervention included strategies to address diversity or disadvantage: NR \\ Economic evaluation: NR
}

Notes NCT00250497

Funding: supported by Grant R01 DK063107 (D. Neumark-Sztainer, principal investigator) from the National Institute of Diabetes and Digestive and Kidney Diseases, NIH. Research was supported in part by grant M01-RR00400 from the National Center for Research Resources, the NIH.

The pilot study Neumark-Sztainer 2003 is also included in this Cochrane Review

No girls were excluded due to eating disorder behaviours

Over $75 \%$ of the girls were racial/ethnic minorities and $46 \%$ were overweight or obese.

At follow-up, the percentage of intervention girls engaging in unhealthy weight control behaviours decreased by $13.7 \%(P=0.021)$ as compared to control girls. Additionally, intervention girls showed significant improvements in body satisfaction $(P=0.045)$, perceived athletic competence $(P=0.044)$, and self-worth $(P=0.031)$ as compared to control girls.

A secondary reference to Neumark-Sztainer 2010, Friend et al 2014 (Sch Health. 2014;84: 326-333) evaluates sustainability of the programme in 10 of the schools.

Results: all schools continued all-girls PE classes using New moves components following the study period. Fewer schools continued the nutrition and social support classroom modules and individual coaching sessions while no schools continued

lunch get-togethers. Programme components were sustained in both New moves intervention schools and control schools.

Conclusions: programmes are most likely to be sustained if they (1) fit into the current school structure, (2) receive buy-in by teachers, and (3) require minimal additional funds or staff time. Providing control schools with minimal training and

intervention resources was sufficient to continue programme components if staff perceived the programme was important for students' health and compatible within the school's existing infrastructure.

\title{
Risk of bias
}

Bias Authors' judgement Support for judgement

Random sequence genera- Unclear risk Randomised, no further details

tion (selection bias)

Allocation concealment Unclear risk NR

(selection bias) 
Neumark-Sztainer 2010 (Continued)

\begin{tabular}{|c|c|c|}
\hline $\begin{array}{l}\text { Blinding (performance } \\
\text { bias and detection bias) } \\
\text { All outcomes }\end{array}$ & Unclear risk & $\begin{array}{l}\text { Outcomes measured during intervention (post-class) were done in schools so } \\
\text { unlikely to have been blinded, outcomes measured at } 9 \text { months were done in } \\
\text { the university laboratory so could have been blinded; details NR }\end{array}$ \\
\hline
\end{tabular}

\begin{tabular}{lll}
\hline $\begin{array}{l}\text { Incomplete outcome data } \\
\text { (attrition bias) } \\
\text { All outcomes }\end{array}$ & Low risk & Retention was high at 94\% (intervention: 97\%; control: 91\%). \\
\hline $\begin{array}{l}\text { Selective reporting (re- } \\
\text { porting bias) }\end{array}$ & High risk & $\begin{array}{l}\text { Trial registration found. BMI was not listed in the trial registration report, but is } \\
\text { listed in the outcome data of the trial report. Therefore this outcome is at high } \\
\text { risk of bias. }\end{array}$
\end{tabular}

\begin{tabular}{lll}
\hline Other bias & Low risk & No additional threats to validity \\
\hline $\begin{array}{l}\text { Other bias- timing of re- } \\
\text { cruitment of clusters }\end{array}$ & Low risk & Figure shows recruitment happened prior to randomisation \\
\hline
\end{tabular}

Nollen 2014

\begin{tabular}{ll}
\hline Methods & Study design: RCT \\
Intervention period: 12 weeks \\
Follow-up period (post-intervention): nil \\
Differences in baseline characteristics: reported \\
Reliable outcomes: reported \\
Protection against contamination: NR \\
Unit of allocation: individual \\
Unit of analysis: individual
\end{tabular}

Participants
$\mathrm{N}($ control baseline $)=25$
$\mathrm{N}$ (control follow-up) $=23$
$\mathrm{N}$ (intervention baseline $)=26$
$\mathrm{N}$ (intervention follow-up) $=21$

Setting (and number by study group): after school, no further details

Recruitment: recruited through after-school programmes located in economically disadvantaged neighbourhoods, girls had to request enrolment packet

Geographic region: Kansas City, Missouri, USA

Percentage of eligible population enrolled: $46 \%$

Mean age: intervention: $11.3 \pm 1.5$ (9-14); control: $11.3 \pm 1.7$ (9-14)

Sex: intervention + control: $100 \%$ female

Test the feasibility and potential efficacy of a 12-week stand-alone mobile technology intervention
Intervention was delivered on a MyPal A626 handheld computer (ASUS Computer International,
www.asus.com/us/). The device was comparable in size, weight, and appearance to a smart phone and
used a Microsoft Windows Mobile 6 operating system.


Nollen 2014 (Continued)

It included goal-setting and planning that required girls to set 2 daily goals and an accompanying plan for improving the behaviour addressed in each module, cues to action, and self-monitoring that prompted girls to self-monitor progress toward their goals at 5 preselected times throughout the day and feedback and reinforcement on goal attainment. Use was reinforced through a song-based reward system that provided girls one song/day if they responded to $80 \%$ of daily prompts. In an attempt to discourage use of the programme beyond the required goal-setting and self-monitoring components, the intervention was intentionally designed without gaming, social media, or text messaging formats that could promote rather than diminish screen time.

Both conditions lasted 12 weeks and targeted fruits/vegetables (weeks 1-4); SSBs (weeks 5-8), and screen time (weeks 9-12). The mobile intervention prompted real-time goal setting and self-monitoring and provided tips, feedback, and positive reinforcement related to the target behaviours.

Controls received the same content in a written manual (identical screen shots) but no prompting

Diet intervention vs control

\begin{tabular}{ll}
\hline Outcomes & Outcome measures \\
- Primary outcome: mobile app use, dietary intake (fruit and vegetables, SSBs), screen time, and BMI \\
- Secondary outcomes: NR \\
Process evaluation: reported: programme enjoyment
\end{tabular}

Implementation-related factors
Theoretical basis: 'behavioural weight control principles'

Resources for intervention implementation: reported

Who delivered the intervention: reported

PROGRESS categories assessed at baseline: gender, race/ethnicity, SES (neighbourhood economic disadvantage)

PROGRESS categories analysed at outcome: NR

Outcomes relating to harms/unintended effects: NR

Intervention included strategies to address diversity or disadvantage: NR, but intervention targeted disadvantaged neighbourhoods, and racial and ethnic minority girls

Economic evaluation: NR

Notes

Funding: Dr. Nollen was supported by an award that was co-funded by the Office of Research on Women's Health (ORWH), the Eunice Kennedy Shriver National Institute of Child Health and Human Development (NICHD), National Institute of Allergy and Infectious Diseases (NIAID), and National Institutes of Mental Health (NIMH) (K12 HD052027) and the National Heart Lung and Blood Institute at the NIH (K23 HL090496).

The average rating of programme enjoyment was 4.5 (SD 0.9). Favorite parts of the programme were obtaining songs $(68.2 \%)$ and setting goals $(36.4 \%)$. The least favourite part of the programme was the reminder prompts (31.8\%). Girls used the programme on $63 \%$ of days, responded to $42 \%$ of prompts, and earned an average of 23.9 songs. Study reports that weight loss was not addressed

\section{Risk of bias}

\begin{tabular}{lll}
\hline Bias & Authors' judgement & Support for judgement \\
\hline $\begin{array}{l}\text { Random sequence genera- } \\
\text { tion (selection bias) }\end{array}$ & Unclear risk & Randomisation, no other details \\
\hline $\begin{array}{l}\text { Allocation concealment } \\
\text { (selection bias) }\end{array}$ & Unclear risk & NR \\
\hline \hline
\end{tabular}


Nollen 2014 (Continued)
Blinding (performance
Unclear risk
NR
bias and detection bias)
All outcomes

Incomplete outcome data Low risk $\quad 86.2 \%$ retention, equally balanced
(attrition bias)

All outcomes

Selective reporting (re- Unclear risk Protocol or trial register not found
porting bias)

Other bias Low risk No additional threats to validity

Nyberg 2015

$\begin{array}{ll}\text { Methods } & \text { Study design: cluster-RCT } \\ \text { Intervention period: } 6 \text { months } \\ \text { Follow-up period (post-intervention): } 6 \text { months } \\ \text { Differences in baseline characteristics: reported } \\ \text { Reliable outcomes: reported } \\ \text { Protection against contamination: NR } \\ \text { Unit of allocation: class } \\ \text { Unit of analysis: individual accounting for cluster }\end{array}$

\begin{tabular}{ll}
\hline Participants & $N($ control baseline $)=114$ \\
& $N($ control follow-up $)=112$ \\
& $N($ intervention baseline $)=129$ \\
& $N($ intervention follow-up $)=127$
\end{tabular}

Setting (and number by study group): 8 schools with 14 preschool classes (14 classes in each group)

Recruitment: the schools included were within the school physician's administrational area; parents were informed verbally about the project at regular school meetings and were also informed through a letter written by the research team and the school physician

Geographic region: a municipality in Stockholm County, Sweden

Percentage of eligible population enrolled: $53 \%$ schools, $40 \%$ participants

Mean age: intervention: $6.2 \pm 0.3$; control: $6.2 \pm 0.3$

Sex: intervention: $47 \%$ female; control: $51 \%$ female

Interventions

To evaluate the effectiveness of the 6-month 'Healthy school start' programme on children's PA and healthy eating habits and on the prevention of overweight and obesity in 6-year-old children attending preschool class.

3 components to the intervention: 
Nyberg 2015 (Continued)

- health information for parents (a brochure was developed and sent home to parents with the aim to increase parental knowledge on how to promote children's dietary and PA habits, containing facts and advice for parents within 7 areas:

- parental feeding practices;

- healthy food and family meal times;

- PA

- sweets, snacks, ice-cream and sodas;

- fruit and vegetables;

- physical inactivity, screen time, and commercials;

- sleep

- MI with parents (parents in the intervention group were offered 2 sessions during the intervention period with a trained external provider)

- Teacher-led classroom activities with children with ten 30-min teacher-led sessions (a teacher's manual and a workbook for children were developed to facilitate the classroom activities. The activities were related to the different areas in the brochure, for example discussing the importance of eating fruit and vegetables and thereafter trying a new fruit or vegetable). Teachers were trained for the classroom activities by the research team for $2 \mathrm{~h}$

Control classes were offered the whole programme directly after the 6-month follow-up measurements

Diet and PA combination intervention vs control

\begin{tabular}{ll}
\hline Outcomes & Outcome measures \\
& $\begin{array}{l}\text { - Primary outcome: PA } \\
\text { of underweight, normal weight, overweight and obesity }\end{array}$ \\
& Process evaluation: reported: fidelity, compliance \\
\hline Implementation-related & Theoretical basis: SCT \\
& Resources for intervention implementation: reported \\
& Who delivered the intervention: reported \\
& PROGRESS categories assessed at baseline: child: gender; parent: education; race/ethnicity \\
& PROGRESS categories analysed at outcome: NR (for anthropometric) \\
& Outcomes relating to harms/unintended effects: reported (change prevalence of underweight) \\
Intervention included strategies to address diversity or disadvantage: NR \\
Economic evaluation: NR
\end{tabular}

Notes

ISRCTN32750699

Funding: ES and LSE received funding for this study from the Public Health Fund, Stockholm County Council. GN received funding from the Signhild Engkvist Foundation, the Martin Rind Foundation and the Lars Hierta Memorial Foundation.

\section{Risk of bias}

\begin{tabular}{lll}
\hline Bias & Authors' judgement & Support for judgement \\
\hline $\begin{array}{l}\text { Random sequence genera- } \\
\text { tion (selection bias) }\end{array}$ & Unclear risk & Randomisation, no further details \\
\hline
\end{tabular}


Nyberg 2015 (Continued)

Allocation concealment Unclear risk
(selection bias)

Blinding (performance Unclear risk NR

bias and detection bias)

NR

All outcomes

Incomplete outcome data Low risk $\quad$ Very few dropouts (3\%); ITT done
(attrition bias)
All outcomes

Selective reporting (re- High risk Protocol seen; only reports effect by gender for main outcome (PA); only reporting bias) ports BMI in text (whereas other outcomes reported in tables) and only reports data immediately post-intervention not follow-up. Does not report waist circumference although measured.

Economic variables NR (even though mentioned in the protocol that costs of the intervention will be calculated by an economist)

\begin{tabular}{lll}
\hline Other bias & Low risk & No additional threats to validity \\
\hline $\begin{array}{l}\text { Other bias- timing of re- } \\
\text { cruitment of clusters }\end{array}$ & Low risk & Figure shows recruitment happened prior to randomisation \\
\hline
\end{tabular}

Ostbye 2012

Study name: KAN-DO (Kids and adults now - defeat obesity!)
Study design: RCT
Intervention period: 8 months
Follow-up period (post-intervention): 12 months
Differences in baseline characteristics: reported
Reliable outcomes: reported
Protection against contamination: NR
Unit of allocation: mother and child dyads
Unit of analysis: individual

Participants
N (control baseline) $=200$
N (intervention baseline) $=200$
N (intervention follow-up) $=150$ (child's weight)
Setting (and number by study group): home setting with one group session
Recruitment: postpartum women who were overweight/obese prior to pregnancy and their children
aged 2-5 years, women were primarily identified from state birth certificates and screened for eligibility
at 2-6 months postpartum
Geographic region: Triangle and Triad regions of North Carolina, USA


Ostbye 2012 (Continued)

$$
\text { Percentage of eligible population enrolled: } 28 \% \text { (496/1769) }
$$

Mean age: intervention + control: $3.06 \pm 1.0$

Sex: intervention: $43.5 \%$ female; control: $45.0 \%$ female
To enhance healthy lifestyle behaviours in mother-preschooler (2-5 years old) dyads in North Carolina

Participants in the intervention arm received 8 monthly mailed interactive kits, followed each month by a 20-30-min telephone coaching session using MI techniques. Kits included child activities and incentives reinforcing the month's topic (e.g. a rewards chart, yoga mat, pedometer, portion plate).

The intervention targeted the dyad's healthy weight via instruction in parenting styles and skills, techniques for stress management (including emotion regulation), and education about healthy behaviours. Parenting skill instruction emphasised

- an authoritative parenting style

- routines for sleep and mealtimes

- a supportive home environment,

- role-modeling of healthy eating and PA

- improvement of feeding style

Education about healthy behaviour changes in the dyad targeted:

- decreased intake of sugary drinks and fast food,

- increased fruit and vegetable consumption,

- meals prepared at home,

- MVPA

- decreased sedentary behaviour

Coaching calls reviewed information in the module and addressed motivation, self-efficacy, and barriers to change. The intervention also included one semi-structured group session, where the study coaches and nutritionist reinforced content from the family kits and set aside time for role play and group discussion. A healthy meal and free child care were provided.

Control arm participants received monthly newsletters emphasizing pre-reading skills

PA combination intervention vs control

\section{Outcome measures}

- Primary outcome: diet, PA, sedentary behaviour, all at 8 months post-baseline and child zBMI at 22 months post-baseline

- Secondary outcomes: parenting behaviours, mother's dietary intake and PA, and mother and child weight, all at 8 months post-baseline

Process evaluation: NR

Implementation-related factors
Theoretical basis: SCT

Resources for intervention implementation: reported

Who delivered the intervention: reported

PROGRESS categories assessed at baseline: gender; parent: race/ethnicity, SES (household income), education, marital status

PROGRESS categories analysed at outcome: NR

Outcomes relating to harms/unintended effects: NR 
Intervention included strategies to address diversity or disadvantage: NR (except that intervention targeted overweight mothers)

Economic evaluation: NR

Funding: this study was funded by a grant from the NIH, National Institute of Diabetes, Digestive and Kidney Diseases (R01-DK-07549). Dr. Zucker was supported by grant 1-K23-MH-070-418-01.

Run-in period prior to randomisation

All participants received monetary incentives (totaling USD 100) to complete assessments.

Study is ongoing - this paper only reports 8-month outcomes (22-month outcomes to follow)

\section{Risk of bias}

\begin{tabular}{lll}
\hline Bias & Authors' judgement & Support for judgement \\
\hline $\begin{array}{l}\text { Random sequence genera- } \\
\text { tion (selection bias) }\end{array}$ & Low risk & Computer-generated, with permuted 8-block randomisation \\
\hline $\begin{array}{l}\text { Allocation concealment } \\
\text { (selection bias) }\end{array}$ & Unclear risk & NR \\
\hline $\begin{array}{l}\text { Blinding (performance } \\
\text { bias and detection bias) } \\
\text { All outcomes }\end{array}$ & Unclear risk & NR \\
\hline $\begin{array}{l}\text { Incomplete outcome data } \\
\text { (attrition bias) } \\
\text { All outcomes }\end{array}$ & Low risk & 23\% attrition, balanced across groups \\
\hline $\begin{array}{l}\text { Selective reporting (re- } \\
\text { porting bias) }\end{array}$ & Low risk & $\begin{array}{l}\text { All outcomes specified were reported; protocol paper reports that outcomes } \\
\text { will be reported at 10 months post-baseline but the available outcome paper } \\
\text { reports at 8 months }\end{array}$ \\
\hline \begin{tabular}{l} 
Other bias \\
\hline
\end{tabular} & Low risk & No additional threats to validity \\
\hline
\end{tabular}

Paineau 2008

$\begin{array}{ll}\text { Methods } & \text { Study design: cluster-RCT } \\ \text { Intervention period: } 8 \text { months } \\ \text { Follow-up period (post-intervention): nil } \\ \text { Differences in baseline characteristics: reported } \\ \text { Reliable outcomes: reported } \\ \text { Protection against contamination: NR } \\ \text { Unit of allocation: school } \\ \text { Unit of analysis: family/individual }\end{array}$


Paineau 2008 (Continued)

All analyses were performed according to ITT principles. Missing data for BMI were imputed using the mean value in the whole cohort.

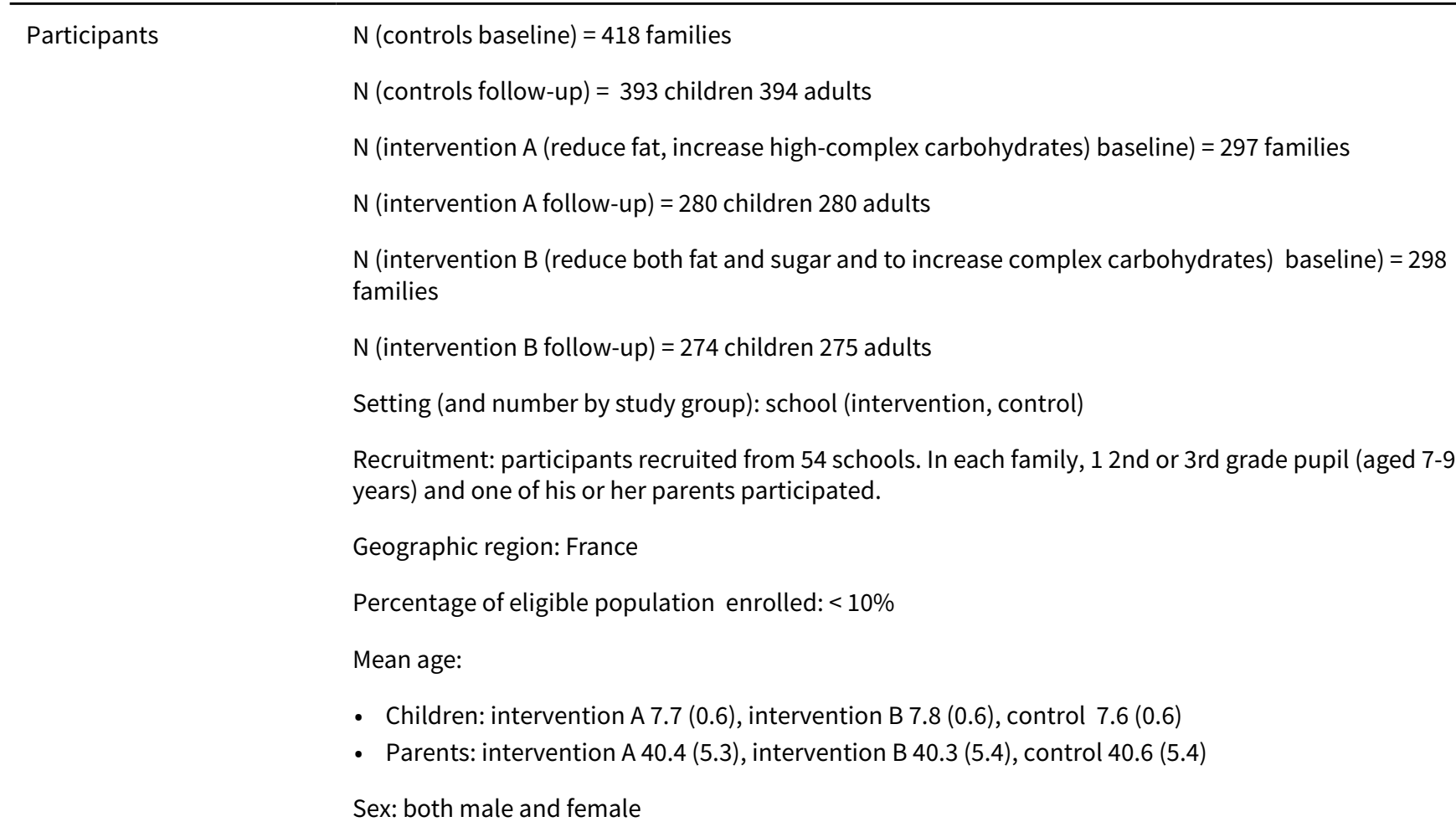

Interventions Intervention group A received advice on how to reduce dietary fats ( $<35 \%$ of total energy intake) and how to increase complex carbohydrates ( $>50 \%$ of total energy intake)

Intervention group B received advice on how to reduce both dietary fats ( $<35 \%$ of total energy intake) and sugars ( $-25 \%$ of initial crude intake) and how to increase complex carbohydrates $(>50 \%$ of total energy intake)

- Computer-based interventions: through the "Etude Longitudinale Prospective Alimentation et Sante" (ELPAS) website, participant families could access self-administered questionnaires (diet, PA, meal preparation, and quality of life) along with updated information, an individual and interactive agenda, an email address, and various other functions. They also performed 3-day dietary records

- Monthly telephone counselling and internet-based monitoring to families ( $30 \mathrm{~min} / \mathrm{month}$ ) by a trained dietician for 8 months. The telephone calls were dedicated to analysing food habits and providing advice on reaching their specific dietary targets

- Monthly newsletters, to both children and parents

- Series of events (e.g. conferences, museum visits), and 3 school-based lessons on nutritional education were programmed in participating schools

Dietary interventions vs control

Outcomes

- Dietary intake: total energy intake, fats, sugars, complex carbohydrates

- Anthropometric measures: height, weight, BMI, z BMI, chest, waist, hip and knee circumferences, blood pressure, heart rate, fat mass, fat-free mass,

- Overall PA: daily screen viewing and activities for clubs

Process evaluation: NR

Implementation-related factors
Theoretical basis: NR

Resources for intervention implementation (e.g. funding needed or staff hours required): reported 
Paineau 2008 (Continued)

Who delivered the intervention: reported

PROGRESS categories assessed at baseline: NR (occupation, gender, race, education, $\mathrm{S}$ for SES)

PROGRESS categories analysed at outcome: NR

Outcomes relating to harms/unintended effects: NR

Intervention included strategies to address diversity or disadvantage: NR

Economic evaluation: NR

\section{Notes}

\section{NCT00456911}

Funding: funding was provided by the French Ministry of Research (2002 Réseau Alimentation Référence Europe 31), and by the ELPAS study's private partners (Avenance Enseignement, the Centre d'Etudes et de Documentation du Sucre, and the Louis Bonduelle Foundation).

The private partners did not participate in conduct of the study; collection, management, analysis, or interpretation of the data; or preparation, review, or approval of the manuscript. The Centre d'Etudes et de Documentation du Sucre participated in the study design.

\section{Risk of bias}

\begin{tabular}{lll}
\hline Bias & Authors' judgement & Support for judgement \\
\hline $\begin{array}{l}\text { Random sequence genera- } \\
\text { tion (selection bias) }\end{array}$ & Low risk & $\begin{array}{l}\text { Randomisation performed according to a computer-generated randomisation } \\
\text { list }\end{array}$ \\
\hline $\begin{array}{l}\text { Allocation concealment } \\
\text { (selection bias) }\end{array}$ & Low risk & $\begin{array}{l}\text { Randomisation occurred at the school level and performed on all units at start } \\
\text { of study }\end{array}$ \\
\hline $\begin{array}{l}\text { Blinding (performance } \\
\text { bias and detection bias) } \\
\text { All outcomes }\end{array}$ & Low risk & $\begin{array}{l}\text { All anthropometric measurements were performed by trained staff, blinded to } \\
\text { the experimental design, at baseline (September-October 2005) and at the end } \\
\text { of the intervention (May-June 2006) }\end{array}$ \\
\hline
\end{tabular}

Incomplete outcome data Low risk (attrition bias)

All outcomes

\begin{abstract}
Quote: "Of the baseline sample, $84.8 \%$ (859 families) completed the study, indicating a dropout rate of $15.2 \%$, with no significant difference in the percentage of dropout between groups $(P=0.46)$. The main reason for dropout was lack of time to complete the dietary records and lack of motivation. Most dropouts occurred in the first 4 months of the study. Those who did and did not complete the study did not differ for sex or initial BMI......Missing data for BMI were imputed using the mean value in the whole cohort."
\end{abstract}

Protocol/trial registration document seen. All outcomes reported

Selective reporting (re- Low risk porting bias)

\begin{tabular}{lll}
\hline Other bias & Low risk & No additional threats to validity \\
\hline $\begin{array}{l}\text { Other bias- timing of re- } \\
\text { cruitment of clusters }\end{array}$ & Low risk & Figure shows recruitment happened prior to randomisation \\
\hline
\end{tabular}

Papadaki 2010

$\begin{array}{ll}\text { Methods } & \text { Study name: DiOgenes } \\ & \text { Study design: RCT; } 5 \text { arms } \\ \text { Intervention period: } 6 \text { months }\end{array}$


Papadaki 2010 (Continued)

Follow-up period (post-intervention): zero

Differences in baseline characteristics: reported

Reliable outcomes: reported

Protection against contamination: NR in this paper

Unit of allocation: family

Unit of analysis: child

The analyses were performed in 2 ways, by completers and ITT principles

\section{Participants}

Eligible families were generally healthy, with at least 1 parent overweight $(\mathrm{BMI}<27 \mathrm{~kg} / \mathrm{m} 2)$ and younger than 65 years, and at least 1 child aged 5-18 years

\section{0 children screened}

827 children ( 381 boys and 446 girls), aged 5-18 years, completed baseline examinations. Families with parents who lost $<8 \%$ of their weight during an 8 -week run-in low-calorie diet period $(N=800)$ were randomly assigned.

658 children examined after 4 weeks, and 492 after 6 months. 465 children completed all assessments and were analysed.

Setting: academic research centre

Recruitment: reported in detail in another paper

Geographic region: volunteer families from 8 countries; Netherlands, Denmark, UK, Greece, Germany, Spain, Bulgaria, and Czech Republic

Percentage of eligible population enrolled: $72.5 \%$ (827/1140)

Mean age: (range 5-18)

Boys mean 11.9 SD $3.4(\mathrm{~N}=201)$

Girls mean 12.4 SD $3.5(\mathrm{~N}=264)$

Sex: $57.9 \%$ female No advice on weight loss was provided because the focus of the study was the ability of the diets to affect outcomes through appetite regulation.

Randomisation was followed by

- in Maastricht and Copenhagen, a 6-month supermarket period (free food provided to families by laboratory shops, in addition to dietary instructions

- in remaining centres, a 6-month dietary-instruction-only period

Implementation of the intervention was NR in this paper

Outcome measures
- Primary outcome measures were changes in anthropometric measurements, zBMI, and body compo-
sition during the intervention
- Secondary outcomes were changes in the proportion of overweight and obese children and changes
in waist-to-hip circumference ratio.

Implementation-related

Theoretical basis: none reported in this paper

factors 
Papadaki 2010 (Continued)

Resources for intervention implementation (e.g. funding needed or staff hours required): NR in this paper

Who delivered the intervention: dietitians

PROGRESS categories assessed at baseline: gender

PROGRESS categories analysed at outcome: NR in this paper

Outcomes relating to harms/unintended effects: NR in this paper

Intervention included strategies to address diversity or disadvantage: NR

Economic evaluation: NR

NCT00390637
Funding: the DiOGenes study was partially funded by the European Community (contract FOOD-
CT-2005-513946). Financial contributions from local sponsors were provided to the supermarket cen-
tres, which also received a number of foods free of charge from food manufacturers. A full list of these
sponsors is available at www.diogenes-eu.org/sponsors/.

\section{Risk of bias}

\begin{tabular}{lll}
\hline Bias & Authors' judgement & Support for judgement \\
\hline $\begin{array}{l}\text { Random sequence genera- } \\
\text { tion (selection bias) }\end{array}$ & Low risk & The randomisation was performed with a web-based randomisation program \\
\hline $\begin{array}{l}\text { Allocation concealment } \\
\text { (selection bias) }\end{array}$ & Unclear risk & No mention of allocation concealment in this paper \\
\hline $\begin{array}{l}\text { Blinding (performance } \\
\text { bias and detection bias) } \\
\text { All outcomes }\end{array}$ & High risk & $\begin{array}{l}\text { No mention that outcome assessors were blind to allocation in this paper; trial } \\
\text { registry entry states open-label }\end{array}$ \\
\hline $\begin{array}{l}\text { Incomplete outcome data } \\
\text { (attrition bias) } \\
\text { All outcomes }\end{array}$ & High risk & $\begin{array}{l}\text { Flow chart and other details reported, but 40\% dropped out in each arm. ITT } \\
\text { done }\end{array}$ \\
\hline $\begin{array}{l}\text { Selective reporting (re- } \\
\text { porting bias) }\end{array}$ & Low risk & $\begin{array}{l}\text { Registered on trial registry and protocol available; all outcomes that were pre- } \\
\text { specified were reported }\end{array}$ \\
\hline \begin{tabular}{l} 
Other bias \\
\hline
\end{tabular} & Low risk & No additional threats to validity \\
\hline
\end{tabular}

\section{Pate 2005}

\begin{tabular}{ll}
\hline Methods & Study design: cluster-RCT \\
Intervention period: 12 months \\
Follow-up period (post-intervention): nil \\
Differences in baseline characteristics: reported \\
Reliable outcomes: reported \\
Protection against contamination: NR \\
Unit of allocation: school
\end{tabular}


Pate 2005 (Continued)

Unit of analysis: school

Missing data at follow-up were imputed by applying a regression method.

\begin{tabular}{|c|c|}
\hline \multirow[t]{10}{*}{ Participants } & $\mathrm{N}$ (controls baseline) $=741$ \\
\hline & $N($ controls follow-up) $=712-741$ \\
\hline & $\mathrm{N}$ (interventions baseline) $=863$ \\
\hline & $\mathrm{N}$ (interventions follow-up) $=827-863$ \\
\hline & Setting (and number by study group): school (intervention $N=12$; control $N=12$ ) \\
\hline & $\begin{array}{l}\text { Recruitment: all 8th-grade girls who attended } 1 / 31 \text { middle schools that fed students to the } 24 \text { partici- } \\
\text { pating high schools were invited to complete the measures. }\end{array}$ \\
\hline & Geographic region: 14 South Carolina counties \\
\hline & Percentage of eligible population enrolled: $34 \%$ \\
\hline & Mean age: intervention: $13.6 \pm 0.6$ years; control: $13.6 \pm 0.6$ years \\
\hline & Sex: $100 \%$ female \\
\hline \multirow[t]{12}{*}{ Interventions } & LEAP (Lifestyle education for activity programme) \\
\hline & $\begin{array}{l}\text { Designed to change both instructional practices and school environment to increase support for PA } \\
\text { among girls }\end{array}$ \\
\hline & Instructional: \\
\hline & - Changes in content and delivery of PE and health education \\
\hline & $\begin{array}{l}\text { - Included a gender-specific, girl-friendly, choice-based instructional programme designed to build ac- } \\
\text { tivity skills and reinforce participation in PA, both inside and outside of class }\end{array}$ \\
\hline & $\begin{array}{l}\text { - Health education lessons to teach skills necessary for adopting and maintaining a physically active } \\
\text { lifestyle }\end{array}$ \\
\hline & Environmental: \\
\hline & - Role modelling by faculty and staff \\
\hline & - Increased communication about PA \\
\hline & - Promotion of PA by the school nurse \\
\hline & - Family- and community-based activities \\
\hline & PA interventions vs control \\
\hline
\end{tabular}

Outcomes

Primary outcome: \% of girls in who reported participating in vigorous PA

Secondary outcomes: prevalence of overweight and at-risk for overweight

Process evaluation: NR

Implementation-related factors
Theoretical basis: reported (SEM drawn from SCT)

Resources for intervention implementation (e.g. funding needed or staff hours required): NR

Who delivered the intervention: reported

PROGRESS categories assessed at baseline: reported (race)

PROGRESS categories analysed at outcome: reported (race)

Outcomes relating to harms/unintended effects: NR 
Pate 2005 (Continued)

Intervention included strategies to address diversity or disadvantage: NR

Economic evaluation: NR

Notes

Funding: this study was funded by a grant from the National Heart, Lung and Blood Institute (R01HL057775).

\section{Risk of bias}

\begin{tabular}{|c|c|c|}
\hline Bias & Authors' judgement & Support for judgement \\
\hline $\begin{array}{l}\text { Random sequence genera- } \\
\text { tion (selection bias) }\end{array}$ & Unclear risk & $\begin{array}{l}\text { Quote: "Schools were paired by school size, percentage of girls who were } \\
\text { African American, urban/suburban or rural location, and class structure (60- or } \\
90 \text {-minute classes). Schools from each pair were randomly assigned to control } \\
\text { or intervention groups }\end{array}$ \\
\hline $\begin{array}{l}\text { Allocation concealment } \\
\text { (selection bias) }\end{array}$ & Unclear risk & $\begin{array}{l}\text { Quote: "Schools from each pair were randomly assigned to control or interven- } \\
\text { tion groups" }\end{array}$ \\
\hline $\begin{array}{l}\text { Blinding (performance } \\
\text { bias and detection bias) } \\
\text { All outcomes }\end{array}$ & Unclear risk & NR \\
\hline $\begin{array}{l}\text { Incomplete outcome data } \\
\text { (attrition bias) } \\
\text { All outcomes }\end{array}$ & Unclear risk & $\begin{array}{l}76 \% \text { individual retention overall stated in text; this is not corroborated by the } \\
\text { figures in table } 1 \text { that indicate }>50 \% \text { loss. Text suggests that } 76 \% \text { refers to } \\
\text { those that received intervention and those that had follow-up, where Interven- } \\
\text { tion started several months after randomisation }\end{array}$ \\
\hline $\begin{array}{l}\text { Selective reporting (re- } \\
\text { porting bias) }\end{array}$ & Unclear risk & Protocol or trial register not found \\
\hline Other bias & Low risk & No additional threats to validity \\
\hline $\begin{array}{l}\text { Other bias- timing of re- } \\
\text { cruitment of clusters }\end{array}$ & Low risk & $\begin{array}{l}\text { No CONSORT figure; text suggests recruitment happened prior to randomisa- } \\
\text { tion }\end{array}$ \\
\hline
\end{tabular}

\section{Patrick 2006}

Study design: RCT
Intervention period: 12 months
Follow-up period (post-intervention): nil
Differences in baseline characteristics: reported
Reliable outcomes: reported
Protection against contamination: NR
Unit of allocation: individual
Unit of analysis: individual
Analyses were conducted under the ITT assumption by replacing missing values at the 12 -month end
point with the most recent available data from either the 6-month or baseline assessment.

Participants N(controls baseline $)=395$ 
Patrick 2006 (Continued)

$$
\begin{aligned}
& N(\text { controls follow-up })=334 \\
& N(\text { interventions baseline })=424 \\
& N(\text { interventions follow-up })=356
\end{aligned}
$$

Setting (and number by study group): community (intervention $N=424$; control $N=395$ )

Recruitment: healthy adolescents scheduled for a 'well-child' visit were recruited through their primary care providers ( $\mathrm{N}=45$ primary care providers) from 6 private clinic sites

Geographic region: San Diego County, California, USA

Percentage of eligible population enrolled: $59 \%$

Mean age: intervention: $12.8 \pm 1.3$ years (girls), $12.6 \pm 1.4$ years (boys); control: $12.6 \pm 1.4$ years (girls), $12.8 \pm 1.3$ years (boys)

Sex: $53 \%$ female

'PACE+' intervention: designed to promote adoption and maintenance of improved eating and PA behaviours

- computer-supported intervention initiated in primary health care settings

- printed manual to take home

- 12 months of stage-matched telephone calls and mail contact

- parent intervention to help parents encourage behaviour change

Control

- adaptation of SunSmart sun protection behaviour programme developed at the University of Rhode Island, Kingston

Combined effects of dietary interventions and PA interventions vs control

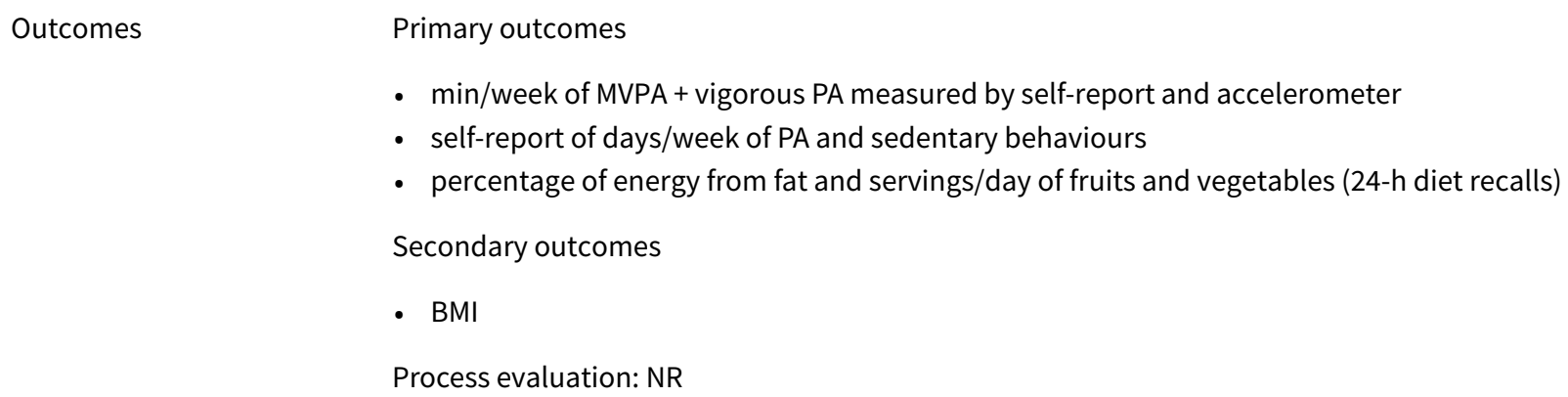


Patrick 2006 (Continued)

Notes
Funding: this project was supported by grants R01CA081495 and R01CA098861-03S1 from the NIH National Cancer Institute, Bethesda, Md. Drs Patrick, Calfas, and Sallis are co-owners of, and receive income from, the Center for Health Interventions, LLC (San Diego, Ca), which is developing products related to the research described in this paper. The terms of this arrangement have been reviewed and approved by San Diego State University and the University of California, San Diego, in accordance with their respective conflict-of-interest policies.

\section{Risk of bias}

\section{Bias}

\section{Authors' judgement Support for judgement}

Random sequence genera- Unclear risk Method for sequence generation NR tion (selection bias)

Allocation concealment $\quad$ Unclear risk $\quad$ Method for allocation concealment NR
(selection bias)

\begin{tabular}{lll}
\hline Blinding (performance & Unclear risk & $\begin{array}{l}\text { Participants were not blinded. NR whether or not outcome assessors were } \\
\text { blinded }\end{array}$
\end{tabular}

All outcomes

\begin{tabular}{|c|c|c|}
\hline $\begin{array}{l}\text { Incomplete outcome data } \\
\text { (attrition bias) }\end{array}$ & Low risk & $\begin{array}{l}\text { Participant flow through study reported and similar rates of attrition across } \\
\text { groups }\end{array}$ \\
\hline
\end{tabular}

All outcomes

Selective reporting (re- Unclear risk Protocol or trial register not found

porting bias)

Other bias Low risk No additional threats to validity

Paul 2011

$\begin{array}{ll}\text { Methods } & \text { Study design: RCT } \\ \text { Intervention period: } 2 \text { nurse home visits (2-3 weeks post birth and at 4-6 months post birth) } \\ \text { Follow-up period (post-intervention): } 1 \text { year } \\ \text { Differences in baseline characteristics: reported } \\ \text { Reliable outcomes: reported } \\ \text { Protection against contamination: NR } \\ \text { Unit of allocation: mother-newborn dyads } \\ \text { Unit of analysis: individual } \\ \text { N (control baseline) }=41 \\ \text { N (control follow-up) }=30 \\ \text { N (intervention baseline) }=39 \text { (soothe/sleep) } \\ \text { N (intervention follow-up) }=29 \\ \text { P (intervention baseline) }=38 \text { (introduction of solids) } \\ \mathrm{N} \text { (intervention follow-up) }=29\end{array}$


Paul 2011 (Continued)

$\mathrm{N}$ (intervention baseline) $=42$ (soothe/sleep and introduction of solids)

$\mathrm{N}$ (intervention follow-up) $=22$

Setting (and number by study group): home-based

Recruitment: mother-newborn dyads intending to breastfeed were recruited from a maternity ward

of a single, academic medical centre

Percentage of eligible population enrolled: NR

Mean age: intervention + control: 39 weeks gestation

Sex: intervention + control: $51 \%$ female (completers)

- Soothe/Sleep"

- One home visit 2-3 weeks post birth by nurse

- Designed to increase sleep duration in early infancy:

- At the first visit parents were taught "alternate strategies to feeding as an indiscriminate first response to infant distress"

- One-on-one instruction and demonstration was provided to teach 5 soothing techniques:

- swaddling

- side or stomach position while awake

- shushing

- swinging

- sucking

- In addition to training participants were given instructional handout and commercially produced video "The Happiest Baby on the block"

- Other instructions to parents:

- emphasize day/night environment difference

- respond to nocturnal awakenings with other soothing and care-taking responses rather than feeding

- "Introduction of solids" T

- to teach parents about hunger and satiety cues and the appropriate time to start solids

- and how to use repeated exposure to overcome infant rejection:

- At the first visit parents were:

- instructed to delay the introduction of complementary foods until infant at least 4 months old and to avoid putting infant cereal into a bottle of breast or formula milk

- given instructional handout to recognise hunger and fullness cues

- asked to inform the research nurse when the child was ready to for solid food

- At the 2nd visit (4-6 months post birth by nurse) parents:

- were taught the importance of repeated exposure to solid foods to improve acceptance of unfamiliar foods and the developmental signs for solid food readiness (such as good head control and sitting with support)

- received hands-on demonstration on feeding their child pureed food and handouts on infant feeding including how to recognise hunger and fullness

- were instructed to begin pureed food when infant calm and alert, not crying or fussing.

- After 2 nd home visit parents asked to feed infants $1 / 4$ pureed vegetables at a similar time each day, for 6 consecutive days over 4 consecutive weeks

- Mothers were provided with the infant foods

All participants (control and other intervention):

- 2 home visits, the first 2-3 weeks after birth, the 2 nd within 2 weeks of the 1st introduction of solid foods (between 4-6 months of age of the infant)

- Content of the visits depended on which group randomised to 
Paul 2011 (Continued)

- Interventions delivered by research nurses

- Received standard infant parenting book with traditional advice on handling infant night awakenings

- Questions about general infant breast feeding and care answered

Infants weighed and measured

Diet intervention vs control

\begin{tabular}{|c|c|}
\hline \multirow[t]{2}{*}{ Outcomes } & $\begin{array}{l}\text { Outcome measures } \\
\text { - Primary outcome: weight for length percentile } \\
\text { - Secondary outcomes: sleep and feeding behaviour, conditional weight gain scores, adverse effects } \\
\text { (growth) }\end{array}$ \\
\hline & Process evaluation: NR \\
\hline
\end{tabular}

Implementation-related factors

\section{Theoretical basis: NR}

Resources for intervention implementation: reported

Who delivered the intervention: reported

PROGRESS categories assessed at baseline: gender; mother: race/ethnicity; SES (household income), education, marital status

PROGRESS categories analysed at outcome: NR

Outcomes relating to harms/unintended effects: reported (gaining insufficient weight)

Intervention included strategies to address diversity or disadvantage: NR

Economic evaluation: NR

Notes

NCT00359242

Funding: this work was supported by grant DK72996 from the National Institute of Diabetes and Digestive and Kidney Diseases (NIDDK) and in part by a General Clinical Research Center grant from NIH (M01RR10732) and GCRC Construction Grant (C06RR016499) awarded to the Pennsylvania State University College of Medicine. Infant food jars were generously donated by Gerber. Additional support was received from the Penn State Children, Youth and Families Consortium and The Children's Miracle Network.

The mean birth weight for these participants was $3.33 \mathrm{~kg}$, equivalent to the 45 th percentile for birth weight for gestational age.

"... We do not have adequate data to assess the extent to which parents' implementation of the

"Soothe/Sleep" intervention may have affected its impact."

\section{Risk of bias}

\begin{tabular}{lll}
\hline Bias & Authors' judgement & Support for judgement \\
\hline $\begin{array}{l}\text { Random sequence genera- } \\
\text { tion (selection bias) }\end{array}$ & Unclear risk & $\begin{array}{l}\text { Randomisation included stratification for maternal prepregnancy BMI with } 2 \\
\text { groups, } \mathrm{BMI}<25 \text { and } \mathrm{BMI} \geq 25 .\end{array}$ \\
& $\begin{array}{l}\text { Mother-newborn dyads were randomised into } 1 / 4 \text { cells using a } 2 \times 2 \text { design to } \\
\text { receive both, } 1 \text {, or no interventions delivered at } 2 \text { nurse home visits }\end{array}$ \\
$\begin{array}{l}\text { Allocation concealment } \\
\text { (selection bias) }\end{array}$ & Unclear risk & $\mathrm{NR}$ \\
\hline
\end{tabular}


Paul 2011 (Continued)

Blinding (performance bias and detection bias)

All outcomes

High risk Assessors (research nurses) were unblinded

\begin{tabular}{lll}
\hline $\begin{array}{l}\text { Incomplete outcome data } \\
\text { (attrition bias) } \\
\text { All outcomes }\end{array}$ & High risk & $\begin{array}{l}31 \% \text { attrition in a relatively small trial, no significant difference between } \\
\text { groups. Non-completers significantly younger and less educated at baseline, } \\
\text { and were more likely to be single, non-white, and Medicaid-insured. }\end{array}$ \\
\hline $\begin{array}{l}\text { Selective reporting (re- } \\
\text { porting bias) }\end{array}$ & High risk & $\begin{array}{l}\text { Trial registration found. BMI was not listed in the trial registration report, but is } \\
\text { listed in the outcome data of the trial report. Therefore this outcome is at high } \\
\text { risk of bias. }\end{array}$
\end{tabular}

Other bias Low risk

\section{Peralta 2009}

\begin{tabular}{|c|c|}
\hline \multirow[t]{9}{*}{ Methods } & Study design: RCT \\
\hline & Intervention period: 6 months \\
\hline & Follow-up period (post-intervention): nil \\
\hline & Differences in baseline characteristics: NR \\
\hline & Reliable outcomes: reported \\
\hline & Protection against contamination: NR \\
\hline & Unit of allocation: child \\
\hline & Unit of analysis: child \\
\hline & All analyses were performed according to ITT principles \\
\hline \multirow[t]{10}{*}{ Participants } & $\mathrm{N}($ controls baseline $)=17$ \\
\hline & $N$ (controls follow-up) $=16$ \\
\hline & $\mathrm{N}$ (interventions baseline) $=16$ \\
\hline & $\mathrm{N}$ (interventions follow-up) = 16 \\
\hline & Setting (and number by study group): secondary school ( $N=1)$ \\
\hline & Recruitment: 7 th graders completing $<49$ laps using Multistage Fitness Test \\
\hline & Geographic region: Australia \\
\hline & Percentage of eligible population enrolled: $58 \%$ \\
\hline & Mean age: $12.5 \pm 0.4$ years \\
\hline & Sex: male only \\
\hline \multirow[t]{3}{*}{ Interventions } & $\begin{array}{l}\text { - Curriculum component: } 1 \times 60 \text {-min curriculum session and } 2 \times 20 \text {-min lunchtime PA sessions per week, } \\
\text { and for } 16 \text { programme weeks; each } 60 \text {-min curriculum session included practical and/or theoretical } \\
\text { components }\end{array}$ \\
\hline & - Practical component: comprised modified games and activities \\
\hline & $\begin{array}{l}\text { - Theoretical components: focused on promoting PA through increasing physical self-esteem and self- } \\
\text { efficacy, reducing time spent in small-screen recreation at weekends, decreasing sweetened beverage }\end{array}$ \\
\hline
\end{tabular}


Peralta 2009 (Continued)

consumption, and increasing fruit consumption and the acquisition and practice of self-regulatory behaviours such as goal-setting, time-management, and identifying and overcoming barriers

- Behaviour modification techniques (e.g. group goals converting time spent in PA to $\mathrm{km}$ to reach a specified destination, and the use of incentives such as small footballs) were used throughout the programme behaviours

- Practical components: modified games and activities

- School staff, PE teacher, facilitated by researcher but included programme champion who also chose peer facilitators (11th graders), one 20-min training session) and $6 \times$ newsletters sent to parents were also involved except for researchers.

(Combined effects of dietary interventions and PA interventions vs control)

\begin{tabular}{ll}
\hline - Height and weight \\
- Waist circumference \\
- \% \%ody fat \\
- Cardiorespiratory fitness \\
- PA using accelerometry \\
- Time spent using small-screen recreation \\
- Sweetened beverage and fruit consumption \\
Process evaluation: reported
\end{tabular}

Implementation-related factors
Theoretical basis: reported (SCT)

Resources for intervention implementation (e.g. funding needed or staff hours required): NR

Who delivered the intervention: reported

PROGRESS categories assessed at baseline: reported (gender)

PROGRESS categories analysed at outcome: NR

Outcomes relating to harms/unintended effects: NR

Intervention included strategies to address diversity or disadvantage: NR

Economic evaluation: NR

Notes

Funding: the study authors thanked participating students, staff and the broader intervention school community for partly funding the study.

All analyses performed according to ITT principles

\section{Risk of bias}

\begin{tabular}{lll}
\hline Bias & Authors' judgement & Support for judgement \\
\hline $\begin{array}{l}\text { Random sequence genera- } \\
\text { tion (selection bias) }\end{array}$ & Low risk & Randomised "using a computer-based number producing algorithm..." \\
\hline $\begin{array}{l}\text { Allocation concealment } \\
\text { (selection bias) }\end{array}$ & Unclear risk & NR \\
\hline $\begin{array}{l}\text { Blinding (performance } \\
\text { bias and detection bias) } \\
\text { All outcomes }\end{array}$ & Low risk & Assessors blinded \\
\hline
\end{tabular}

\begin{tabular}{l}
\hline $\begin{array}{l}\text { Incomplete outcome data } \\
\text { (attrition bias) }\end{array}$ Low risk Only one participant lost at follow-up and ITT done \\
(n)
\end{tabular}


Peralta 2009 (Continued)

All outcomes

Selective reporting (re- Unclear risk $\quad$ Protocol or trial register not found
porting bias)

\begin{tabular}{ll}
\hline Other bias & Low risk \\
& Intervention conducted in 1 school with an absence of a 'true' control group \\
since it was compulsory for all boys to participate in PA
\end{tabular}

\section{Puder 2011}

\section{Methods}

Study name: Ballabeina study

Study design: cluster-RCT

Intervention period: 10 months (school year)

Follow-up period (post-intervention): nil

Differences in baseline characteristics: reported

Reliable outcomes: reported

Protection against contamination: reported

Unit of allocation: class

Unit of analysis: individual accounting for clustering

$$
\begin{aligned}
& N(\text { controls baseline })=310 \\
& N(\text { controls follow-up })=292 \\
& N(\text { interventions baseline })=342 \\
& N(\text { interventions follow-up })=333
\end{aligned}
$$

Setting (and number by study group): 40 public preschool classes ( $N=20$ intervention classes, 10 classes in each of German-speaking and French-speaking, $\mathrm{N}=20$ control classes, 10 classes in each of German speaking and French speaking)

Recruitment: classes from the 2 areas were separately selected after agreement of the school directors and the school health services - all children in Switzerland attend preschool

Geographic region: German- (city of St Gallen; 70,000 inhabitants) and the French- (urban surroundings of Lausanne, Canton Vaud; 50,000 inhabitants) speaking regions of Switzerland - represent 2 culturally distinct regions with different school and preschool systems, at least $40 \%$ of children of migrant background.

Percentage of eligible population enrolled: $90 \%$

Mean age: intervention:5.2 \pm 0.6 ; control:5.2 \pm 0.6

Sex: intervention: $49 \%$ female; control: $51 \%$ female

Interventions
To test the effect of a multidimensional lifestyle intervention on aerobic fitness and adiposity in predominantly migrant preschool children.

The regular teachers performed the majority of the intervention and were supported by a local health promoter. The intervention included PA lessons, adaptation of the built infrastructure; promotion of regional extracurricular PA; playful lessons about nutrition, media use and sleep, funny homework cards and information materials for teachers and parents. 
Puder 2011 (Continued)

- $4 \times 45$-min sessions of PA a week

- 22 sessions on healthy nutrition, media use, and sleep

Control continued their regular school curriculum ( $1 \times 45$-min PA lesson/week in the gym. In the Frenchspeaking region there was 1 additional 45-min rhythmic lesson/week, corresponding to their regular curriculum).

Diet and PA combined intervention vs control

Outcomes

Outcome measures

- Primary outcome: BMI, aerobic fitness

- Secondary outcomes: motor agility, balance, \% body fat, waist circumference, PA, eating habits, media use, sleep, psychological health (quality of life), cognitive abilities

Process evaluation: reported (implementation)

Implementation-related factors
Theoretical basis: SEM

Resources for intervention implementation: reported

Who delivered the intervention: reported

PROGRESS categories assessed at baseline: child, gender; parent: education, race/ethnicity (migrant status)

PROGRESS categories analysed at outcome: parent: education, race/ethnicity (migrant status)

Outcomes relating to harms/unintended effects: reported

Intervention included strategies to address diversity or disadvantage: culturally tailored

Economic evaluation: NR

Notes NCT00674544

Funding: the study was mainly supported by the Swiss National Science Foundation (grant No 3200B0-116837) and Health Promotion Switzerland (project No 2104). Additional funding was obtained from a research award for interdisciplinary research from the University of Lausanne, a Takeda research award, the Wyeth Foundation for the Health of Children and Adolescents, the Freie Akademische Gesellschaft, and an unrestricted educational grant from Nestlé. The funding sources had no role in the study design, data collection, analysis, interpretation of data, in the writing of the report, and in the decision to submit the article for publication.

Main paper (Puder 2011) reports main results; see secondary references: Burgi 2012 for outcome effects by (parental) migrant status and educational level; Niederer 2013 for outcome effects by child weight status and fitness level

\section{Risk of bias}

\begin{tabular}{lll}
\hline Bias & Authors' judgement & Support for judgement \\
\hline $\begin{array}{l}\text { Random sequence genera- } \\
\text { tion (selection bias) }\end{array}$ & Low risk & $\begin{array}{l}\text { Selection and randomisation performed by person not involved in the study, } \\
\text { randomly assigned after stratification for sociocultural and linguistic region }\end{array}$ \\
\hline $\begin{array}{l}\text { Allocation concealment } \\
\text { (selection bias) }\end{array}$ & Low risk & Classes were randomised with the use of opaque envelopes \\
\hline $\begin{array}{l}\text { Blinding (performance } \\
\text { bias and detection bias) } \\
\text { All outcomes }\end{array}$ & Low risk & $\begin{array}{l}\text { Specially trained researchers measured outcomes and were blinded to group } \\
\text { allocation }\end{array}$ \\
\hline
\end{tabular}


Puder 2011 (Continued)

Incomplete outcome data Low risk Low attrition and ITT done.
(attrition bias)

All outcomes

Quote: "None of the 40 preschool classes left the study, and eight children in the intervention group and 18 in the control group had moved away by the end of the year"

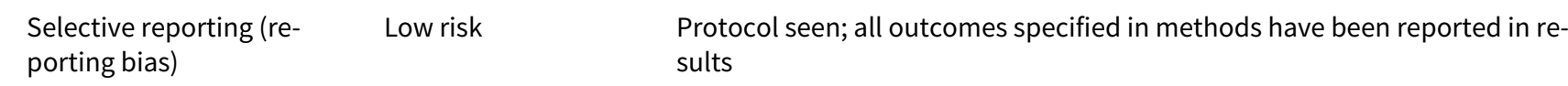

\begin{tabular}{lll}
\hline Other bias & Low risk & Contamination was minimised \\
\hline $\begin{array}{l}\text { Other bias- timing of re- } \\
\text { cruitment of clusters }\end{array}$ & Low risk & Figure shows recruitment happened prior to randomisation \\
\hline
\end{tabular}

Reed 2008

$\begin{array}{ll}\text { Methods } & \text { Study design: cluster-RCT } \\ \text { Intervention period: } 1 \text { school year } \\ \text { Follow-up period (post-intervention): nil } \\ \text { Differences in baseline characteristics: NR } \\ \text { Reliable outcomes: reported } \\ \text { Protection against contamination: NR } \\ \text { Unit of allocation: school } \\ \text { Unit of analysis: individual }\end{array}$

\begin{tabular}{|c|c|}
\hline Participants & $\begin{array}{l}N \text { (controls baseline) }=90 \\
N \text { (controls follow-up) }=81 \\
N \text { (interventions baseline) }=178 \\
N \text { (interventions follow-up) = } 156 \\
\text { Setting: } 10 \text { participating schools randomised, } 3 \text { assigned to usual practice and } 7 \text { assigned to interven- } \\
\text { tion. Of the } 10 \text { schools, } 2 \text { from the usual practice group and } 6 \text { from the intervention group took part in } \\
\text { CV assessment. } \\
\text { Recruitment: elementary schools in Vancouver and Richmond school districts, British Colombia, Cana- } \\
\text { da; 4th and 5th grade children } \\
\text { Geographic region: Canada } \\
\text { Percentage of eligible population enrolled: } 52 \% \\
\text { Mean age: } 9 \text { - } 11 \text { years } \\
\text { Sex: both male and female }\end{array}$ \\
\hline Interventions & $\begin{array}{l}\text { - The goal of the programme (Action schools! BC) was to provide } 150 \mathrm{~min} \text { of PA per week ( } 2 \times 40 \mathrm{~min} \text { PE } \\
\text { classes and } 15 \times 5 \mathrm{~min} / \text { day of extra PA in class throughout the day) }\end{array}$ \\
\hline
\end{tabular}


Reed 2008 (Continued)

- The model emphasised a whole-school approach that targeted 6 action zones:

- school environment

- scheduled PE

- extra-curricular activities

- school spirit

- family and community

- classroom action

- Classroom action was the only prescriptive component and required teachers in the intervention group to deliver 15 min of moderate to intensive PA daily to achieve the 75 min of extra PA per week in addition to the PE classes.

- An intervention facilitator worked with the school action team (comprised of the school principal and/ or teachers) to design a programme that included activities across all 6 action zones

- A support team conducted a 1-day training workshop for teachers in the intervention group to support their action plan. Intervention teachers were also provided a classroom action bin with resources to support their action plan.

- Teachers in both intervention and usual practice (control) groups were asked to record the min of PA per day in activity logs.

PA interventions vs control

Outcomes

- Outcome measures: CV fitness (measured by 20-m shuttle run test), blood pressure (systolic and diastolic), BMI, total cholesterol, HDL, LDL, Apo B, C-reactive protein and fibrinogen at the end of the intervention period.

Process evaluation: NR

Implementation-related factors
Theoretical basis: reported (SEM)

Resources for intervention implementation (e.g. funding needed or staff hours required): reported

Who delivered the intervention: reported

PROGRESS categories assessed at baseline: place, race, gender

PROGRESS categories analysed at outcome: NR

Outcomes relating to harms/unintended effects: NR

Intervention included strategies to address diversity or disadvantage: NR

Economic evaluation: NR
Funding: BC Ministry of Health, 2010 LegaciesNow. Dr. McKay is a Michael Smith Foundation for Health Research Senior Scholar and Dr. Warburton is a Michael Smith Foundation for Health Research Scholar and a CIHR New Investigator

\section{Risk of bias}

Bias Authors' judgement Support for judgement

Random sequence genera- Unclear risk tion (selection bias)

Quote: "(We) stratified schools by size and geographic location (to account for ethnic distribution). Schools were then remotely randomized to either Usual Practice (UP, $\mathrm{n}=3$ ) or Intervention (INT, $\mathrm{n}=7$ ) by an epidemiologist not involved in the trial."

\begin{tabular}{ll}
\hline $\begin{array}{l}\text { Allocation concealment } \\
\text { (selection bias) }\end{array}$ & Quw risk \\
& $\mathrm{n}=3$ ) or Intervention (INT, $\mathrm{n}=7$ ) by an epidemiologist not involved in the trial."
\end{tabular}

Blinding (performance Unclear risk NR for outcome assessment but participants were not blind.


Reed 2008 (Continued)

All outcomes
Quote: "It was not possible for schools to be blinded to random assignment."

\begin{tabular}{|c|c|c|}
\hline $\begin{array}{l}\text { Incomplete outcome data } \\
\text { (attrition bias) } \\
\text { All outcomes }\end{array}$ & Low risk & Overall $11 \%$ loss, $12 \%$ in intervention and $10 \%$ in control \\
\hline $\begin{array}{l}\text { Selective reporting (re- } \\
\text { porting bias) }\end{array}$ & Low risk & $\begin{array}{l}\text { Protocol seen; all outcomes from the protocol are in papers and some addi- } \\
\text { tional outcomes are in papers as well }\end{array}$ \\
\hline Other bias & Low risk & No additional threats to validity \\
\hline $\begin{array}{l}\text { Other bias- timing of re- } \\
\text { cruitment of clusters }\end{array}$ & Low risk & Clusters were recruited before randomisation \\
\hline
\end{tabular}

Reilly 2006

\begin{tabular}{|c|c|}
\hline \multirow[t]{8}{*}{ Methods } & Study design: cluster-RCT \\
\hline & Intervention period: 24 weeks \\
\hline & Follow-up period (post-intervention): 6 months \\
\hline & Differences in baseline characteristics: reported \\
\hline & Reliable outcomes: reported \\
\hline & Protection against contamination: reported \\
\hline & Unit of allocation: nursery \\
\hline & Unit of analysis: individual \\
\hline \multirow[t]{10}{*}{ Participants } & $\mathrm{N}($ controls baseline $)=277$ \\
\hline & $\mathrm{N}$ (controls follow-up) = 259 (at 12 months) \\
\hline & $\mathrm{N}$ (interventions baseline) $=268$ \\
\hline & $\mathrm{N}$ (interventions follow-up) = 245 (at 12 months) \\
\hline & Setting (and number by study group): nurseries (intervention $N=18$; control $N=18$ ) \\
\hline & $\begin{array}{l}\text { Recruitment: } 36 \text { nurseries were randomly selected from a total of } 104 \text { nurseries that were willing to par- } \\
\text { ticipate ( } 124 \text { nurseries in total were initially invited). All families with children in their preschool year at- } \\
\text { tending the } 36 \text { nurseries were eligible to participate. }\end{array}$ \\
\hline & Geographic region: Glasgow, Scotland, UK \\
\hline & Percentage of eligible population enrolled: $47 \%$ (from original 124 invited nurseries) \\
\hline & Mean age: intervention: $4.2 \pm 0.3$ years; control: $4.1 \pm 0.3$ years \\
\hline & Sex: intervention: $52 \%$ female; control: $48 \%$ female \\
\hline Interventions & $\begin{array}{l}\text { Nursery element: } \\
\text { - Enhanced PA programme consisting of } 3 \times 30 \text {-min sessions of PA each week over } 24 \text { weeks. } \\
\text { - } 2 \text { members of staff from each intervention nursery attended } 3 \text { training sessions to deliver the inter- } \\
\text { vention }\end{array}$ \\
\hline
\end{tabular}


Reilly 2006 (Continued)

- For 6 weeks during the intervention, each intervention nursery displayed posters focusing on increasing PA through walking and play

- Capital cost < GBP 200

Home element:

- Each participating family received a resource pack of materials (cost GBP 16) with guidance on linking physical play at nursery and at home, and 2 health education leaflets

Control:

- Usual curriculum and head-teachers agreed not to enhance their physical development and movement curriculum

PA interventions vs control

- Primary outcome: BMI, expressed as a SD score relative to UK 1990 reference data
- Secondary outcomes: PA; sedentary behaviour; fundamental movement skills; process evaluation
Process evaluation: reported

Implementation-related Theoretical basis: NR

factors

Resources for intervention implementation (e.g. funding needed or staff hours required): reported

Who delivered the intervention: reported

PROGRESS categories assessed at baseline: reported (gender)

PROGRESS categories analysed at outcome: reported (gender)

Outcomes relating to harms/unintended effects: NR

Intervention included strategies to address diversity or disadvantage: NR

Economic evaluation: no formal evaluation, however costs of materials provided

Notes ISRCTN36363490

Funding: British Heart Foundation, Glasgow City Council, and the Caledonian Research Foundation. The pilot study was funded by Sport Aiding Medical Research for Kids (SPARKS)

\section{Risk of bias}

Bias Authors' judgement Support for judgement

Random sequence genera- Low risk tion (selection bias)

Quote: "All 36 participating nurseries were allocated to group in advance in one operation with stratified random sampling. Allocations were concealed by carrying out randomisation of the 36 nurseries at the same time and informing the liaison researcher and nurseries together."

\begin{tabular}{lll}
\hline $\begin{array}{l}\text { Allocation concealment } \\
\text { (selection bias) }\end{array}$ & Low risk & $\begin{array}{l}\text { Allocation was by nursery and "allocations were concealed by carrying out } \\
\text { randomisation of the } 36 \text { nurseries at the same time..." }\end{array}$ \\
\hline $\begin{array}{l}\text { Blinding (performance } \\
\text { bias and detection bias) }\end{array}$ & Low risk & $\begin{array}{l}\text { Researchers who made the outcome measures were blinded to nursery al- } \\
\text { location with the exception of the statistician who carried out the allocation } \\
\text { and the contact between the research team and the nurseries. Nurseries were } \\
\text { made aware of their allocation status. }\end{array}$ \\
\hline
\end{tabular}

\begin{tabular}{|c|c|c|}
\hline $\begin{array}{l}\text { Incomplete outcome data } \\
\text { (attrition bias) }\end{array}$ & Low risk & $\begin{array}{l}\text { Participant flow provided and similar proportion of missing data from both } \\
\text { groups }\end{array}$ \\
\hline
\end{tabular}


Reilly 2006 (Continued)

Selective reporting (re- Low risk Trial registration document seen. All outcomes reported porting bias)

\begin{tabular}{lll}
\hline Other bias & Low risk & No additional threats to validity \\
\hline $\begin{array}{l}\text { Other bias- timing of re- } \\
\text { cruitment of clusters }\end{array}$ & Low risk & Figure shows recruitment happened prior to randomisation \\
\hline
\end{tabular}

\section{Robbins 2006}

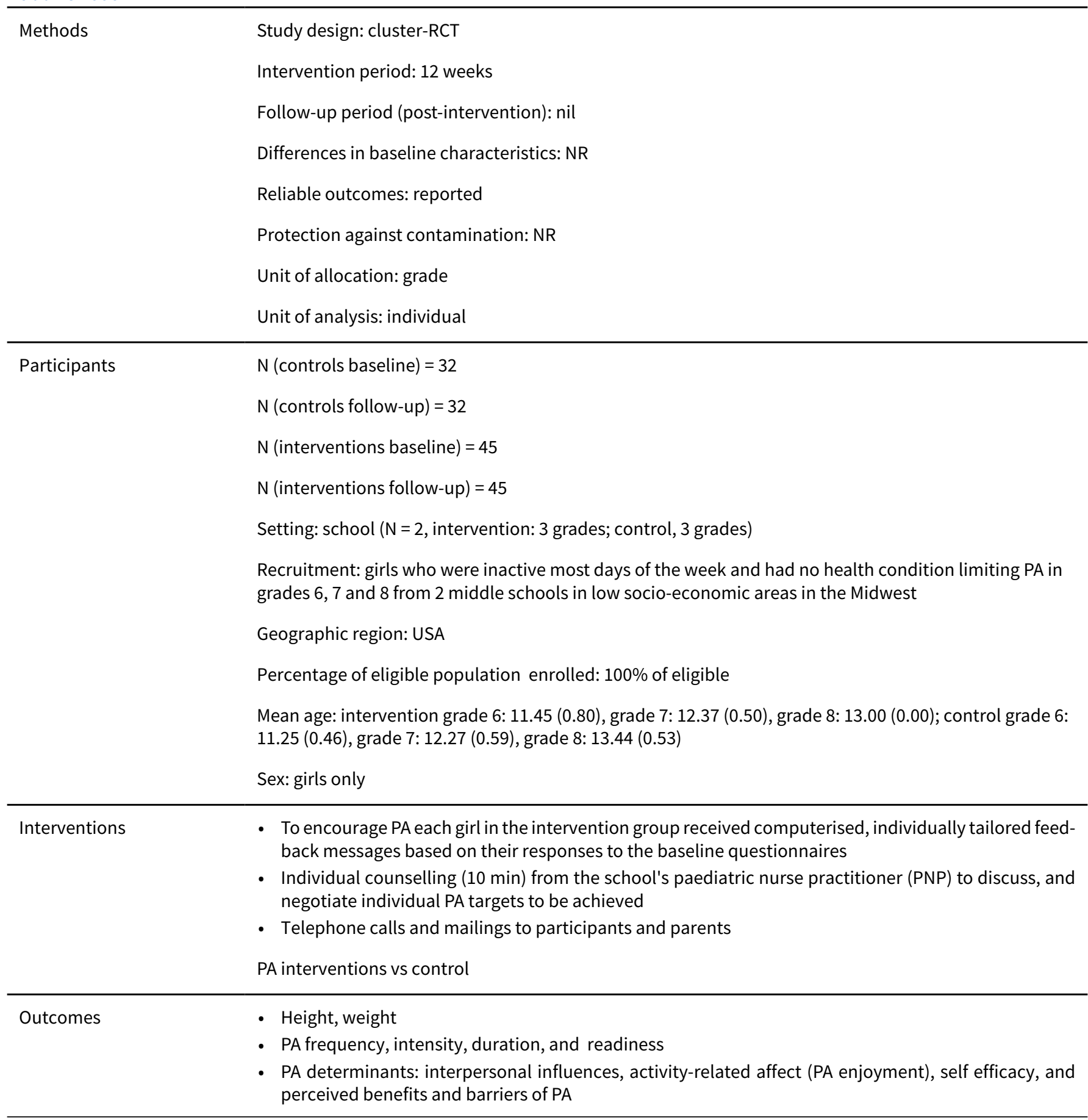


Robbins 2006 (Continued)

Process evaluation: reported

Implementation-related factors
Theoretical basis: Health Promotion Model and TTM

Resources for intervention implementation (e.g. funding needed or staff hours required): reported Who delivered the intervention: reported

PROGRESS categories assessed at baseline: NR

PROGRESS categories analysed at outcome: NR

Outcomes relating to harms/unintended effects: NR

Intervention included strategies to address diversity or disadvantage: reported

Economic evaluation: NR
Notes

Funding: funding to conduct the study was received from The Robert Wood Johnson Foundation.

\section{Risk of bias}

\begin{tabular}{|c|c|c|}
\hline Bias & Authors' judgement & Support for judgement \\
\hline $\begin{array}{l}\text { Random sequence genera- } \\
\text { tion (selection bias) }\end{array}$ & Low risk & $\begin{array}{l}\text { Quote: "Computer assignment to either an intervention or control group was } \\
\text { based upon a numerical code that included school group and grade. Flip-of- } \\
\text { a-coin randomisation identified the grade and school assigned to each condi- } \\
\text { tion" }\end{array}$ \\
\hline $\begin{array}{l}\text { Allocation concealment } \\
\text { (selection bias) }\end{array}$ & Low risk & $\begin{array}{l}\text { Randomisation was at school level and was performed on all units at the start } \\
\text { of the study }\end{array}$ \\
\hline $\begin{array}{l}\text { Blinding (performance } \\
\text { bias and detection bias) } \\
\text { All outcomes }\end{array}$ & Unclear risk & NR \\
\hline $\begin{array}{l}\text { Incomplete outcome data } \\
\text { (attrition bias) } \\
\text { All outcomes }\end{array}$ & Low risk & No loss occurred \\
\hline $\begin{array}{l}\text { Selective reporting (re- } \\
\text { porting bias) }\end{array}$ & Unclear risk & Protocol or trial register not found \\
\hline Other bias & Low risk & No additional threats to validity \\
\hline $\begin{array}{l}\text { Other bias- timing of re- } \\
\text { cruitment of clusters }\end{array}$ & Low risk & Figure indicates recruitment happened prior to randomisation \\
\hline
\end{tabular}

\section{Robinson 2003}

$\begin{array}{ll}\text { Methods } & \text { Study design: RCT } \\ & \text { Intervention period: } 12 \text { weeks } \\ & \text { Follow-up (post-intervention): nil } \\ & \text { Differences in baseline characteristics: reported } \\ & \text { Reliable outcomes: reported } \\ & \text { Protection against contamination: NR } \\ & \text { Unit of allocation: child } \\ & \text { Unit of analysis: child }\end{array}$


Robinson 2003 (Continued)

All analyses were performed according to ITT principles

\begin{tabular}{|c|c|}
\hline \multirow[t]{4}{*}{ Participants } & $\begin{array}{l}N(\text { controls- baseline })=33 \\
N(\text { controls- follow-up })=33 \\
N \text { (interventions- baseline })=28 \\
N \text { (interventions-follow-up })=26\end{array}$ \\
\hline & $\begin{array}{l}\text { Recruitment: all consenting 8-10-year-old, African American girls with } \mathrm{BMI} \geq 50 \text { th percentile for age and } \\
\text { gender, and a parent with a BMI }=25 \text {. Set in Oakland and Palo Alto, California, USA. }\end{array}$ \\
\hline & $\begin{array}{l}\text { Proportion of eligibles participating: not stated, but criteria kept broad. Intended to recruit } 50 \text { and } 61 \\
\text { were enrolled. }\end{array}$ \\
\hline & $\begin{array}{l}\text { Mean age: intervention, } 9.5 \text { (SD 0.8) years; controls: } 9.5 \text { (SD 0.9) } \\
\text { Sex: girls only }\end{array}$ \\
\hline \multirow[t]{2}{*}{ Interventions } & $\begin{array}{l}\text { GEMS study (Girls' health enrichment multi-site studies). After-school dance classes set in communi- } \\
\text { ty centres designed to improve PA, reduce sedentary behaviours and enhance diet. The intervention } \\
\text { called 'START' (Sisters taking action to reduce TV) was delivered by trained university-based dance in- } \\
\text { structors and a female African American intervention specialist. The programme consisted of daily } \\
\text { dance classes during school weeks and reducing TV was covered in } 5 \text { home-based lessons. } 4 \text { communi- } \\
\text { ty lectures were also provided. } \\
\text { Controls received newsletters and health education lectures }\end{array}$ \\
\hline & Combined effects of dietary interventions and PA interventions vs control \\
\hline
\end{tabular}

\begin{tabular}{ll}
\hline Outcomes & BMI \\
- Waist circumference \\
- Physical maturation \\
- DEXA for \% body fat \\
PA: \\
$\circ$ CSA accelerometer, \\
$\circ$ a modification of the Self-Administered PA Checklist (SAPAC), \\
- Dietary intake measured by $2 \times 24-h$ recalls using NDS-R
\end{tabular}

Process evaluation: reported

Implementation-related factors
Theoretical basis: SCT

Resources for intervention implementation (e.g. funding needed or staff hours required): reported Who delivered the intervention: reported

PROGRESS categories assessed at baseline: reported (education, SES)

PROGRESS categories analysed at outcome: NR

Outcomes relating to harms/unintended effects: NR

Intervention included strategies to address diversity or disadvantage: reported

Economic evaluation: NR

Notes

\section{Risk of bias}


Robinson 2003 (Continued)

Random sequence genera- Low risk tion (selection bias)
Quote: "...urn randomization procedure was used to generate the treatment allocation sequences. The different sequences were stored on a computer at the CC (coordinating centre), and accessed using an interactive voice-response telephone system." (Rochon 2003)

\begin{tabular}{|c|c|c|}
\hline $\begin{array}{l}\text { Allocation concealment } \\
\text { (selection bias) }\end{array}$ & Low risk & $\begin{array}{l}\text { The central administration of the study by a co-ordinating centre would sug- } \\
\text { gest that allocation was concealed }\end{array}$ \\
\hline
\end{tabular}

(selection bias)

Outcome assessors were blinded

Blinding (performance Low risk

bias and detection bias)

All outcomes

\begin{tabular}{lll}
\hline $\begin{array}{l}\text { Incomplete outcome data } \\
\text { (attrition bias) } \\
\text { All outcomes }\end{array}$ & Low risk & Missing data minimal and reasons given \\
\hline $\begin{array}{l}\text { Selective reporting (re- } \\
\text { porting bias) }\end{array}$ & Low risk & Protocol seen. All outcomes reported \\
\hline Other bias & Low risk & No additional threats to validity \\
\hline
\end{tabular}

\section{Robinson 2010}

\begin{tabular}{|c|c|}
\hline \multirow[t]{9}{*}{ Methods } & Study name: Stanford GEMS (Girls' health enrichment multi-site studies) \\
\hline & Study design: cluster-RCT \\
\hline & Intervention period: 2 years \\
\hline & Follow-up period (post-intervention): nil \\
\hline & Differences in baseline characteristics: reported \\
\hline & Reliable outcomes: reported \\
\hline & Protection against contamination: NR \\
\hline & Unit of allocation: families/households (1 girl/household) \\
\hline & Unit of analysis: individual \\
\hline \multirow[t]{6}{*}{ Participants } & $\mathrm{N}$ (control baseline $)=134$ \\
\hline & $\mathrm{N}$ (control follow-up) $=118$ \\
\hline & $\mathrm{N}$ (intervention baseline) $=127$ \\
\hline & $\mathrm{N}$ (intervention follow-up) = 107 \\
\hline & Setting (and number by study group): community centres \\
\hline & $\begin{array}{l}\text { Recruitment: recruited from schools, community centres, churches and community events in low- } \\
\text { income, predominantly African-American neighbourhoods. Recruitment strategies were based on } \\
\text { making presentations and distributing fliers to girls and parents at existing after-school programmes, } \\
\text { schools, churches, and neighbourhood and community events (e.g. street fairs, Juneteenth celebra- } \\
\text { tions, African-American cultural events), and making individual presentations to parents and girls in } \\
\text { commercial locations (e.g. food stores, new store openings). They also presented the project to school } \\
\text { parent groups, church groups, and Parks and Recreation Department staff, to enhance the visibility of } \\
\text { Stanford GEMS, especially among community opinion leaders. }\end{array}$ \\
\hline
\end{tabular}


Robinson 2010 (Continued)

Geographic region: Oakland, California, USA

Percentage of eligible population enrolled: $83 \%$

Mean age: intervention: $9.5 \pm 0.9$; control: $9.4 \pm 0.8$

Sex: intervention: $100 \%$ female; control: $100 \%$ female

To test the efficacy of a culturally-tailored after-school dance programme and a family-based intervention to reduce TV, videotape and video game use to reduce BMI gain among lower-SES African-American pre-adolescent girls

Families were randomised to 2-year, culturally-tailored interventions:

- after-school hip-hop, African and step dance classes and a home/family-based intervention to reduce screen media use or

- information-based health education

The GEMS Jewels after-school dance intervention was offered 5 days/week, 12 months/year (excluding school holidays), at community centres in selected neighbourhoods. Daily sessions lasted up to $2.5 \mathrm{~h}$ and started with a 1-h homework period and small snack followed by $45-60 \mathrm{~min}$ of learning and practicing dance routines. 3 styles of dance were taught: traditional African dance, hip-hop, and step. Additional activities to maintain motivation included: 'GEMS Jamboree' dance performances approximately every 8 weeks for families and friends, including awards for each girl based on Kwanzaa principles; videotaped feedback; allowing girls to teach each other and choreograph routines; opportunities for participant choice and control; and performances at public events. Dance classes were led by female African-American college students and/or recent graduates from the local community where possible, to serve as role models for dance, maintaining cultural identity, and educational achievement.

Sisters taking action to reduce television (START) is a home-based, screen-time reduction intervention designed to incorporate African or African-American history and culture, including up to 24 lessons over 2 years. Young adult, African-American female 'START mentors' met with families in their homes to deliver each lesson, following the screen-time reduction model developed over several prior studies.

Control: the 'Health education comparison intervention' was selected to address the possibility of resentful demoralisation and/or compensatory rivalry. It consisted of state-of-the-art, culturally-tailored, authoritative, information-based health education on nutrition, PA, and reducing CV and cancer risk. It included 24 monthly newsletters for the girls (Felicia's healthy news flash) and their parents/guardians (Stanford GEMS health report), and quarterly community centre health lectures (Family fun nights). The same monitoring and incentive schedules employed for our experimental treatment condition were used.

PA intervention vs control (health education)

Outcome measures

- Primary outcome: BMI, zBMI

- Secondary outcomes: waist circumference, TSF thickness, resting blood pressure, resting heart rate, fasting serum insulin, glucose, lipids, PA, screen time, dietary intake, psychosocial (weight concerns and depressive symptoms)

Process evaluation: reported (attendance)

Implementation-related Theoretical basis: Social Cognitive Model

factors

Resources for intervention implementation: reported

Who delivered the intervention: reported

PROGRESS categories assessed at baseline: parent: SES (household income), household education, marital status 

BMI

Outcomes relating to harms/unintended effects: weight concerns, depressive symptoms, injuries/illness, height-growth velocity, BMI loss

Intervention included strategies to address diversity or disadvantage: targeted African-American families with low SES; intervention culturally tailored

Economic evaluation: NR

\section{Notes}

\section{NCT00000615}

Funding: this research was funded by a co-operative agreement UO1 HL62663 from the National Heart, Lung, and Blood Institute, NIH. An NHLBI Program Officer (EO) was a member of the co-operative agreement Steering Committee and as a co-author on the manuscript, participated in interpretation of the data and preparation of the manuscript. The NHLBI Program Officer and other NHLBI scientific staff provided input on design and conduct of the study, but were not involved in collection, management or analysis of the data.

Pilot study is included in this Cochrane Review (Robinson 2003); girls were required to have a BMI $\geq$ 25 th percentile for age and/or at least 1 overweight parent/guardian (BMI $\geq 25 \mathrm{~kg} / \mathrm{m} 2$ ). Girls were excluded with $\mathrm{BMI}>35 \mathrm{~kg} / \mathrm{m} 2$.

Median attendance rates at dance classes were only $12 \%, 1 / 5$ of the goal rate.

Systematic monitoring of all injuries and other medical problems requiring a visit to a medical care provider, height-growth velocity, and BMI loss suggested no increased risk associated with participation in the study as a whole or between intervention groups (all $\mathrm{P}$ $\geq 0.20$ ). No injuries or illnesses were judged to be "probably" or "definitely" related to study participation

"Treatment group girls attended only mean $\pm \mathrm{SD}=0.21 \pm 0.22$ (median $=0.12$, Interquartile (IQ) range $=$ 0.02-0.34, minimum 0, maximum 0.81 ) of possible dance classes, from randomisation to their last assessment. Attendance rates fell over the course of the study (Figure 2). Two main challenges impacted dance class attendance. First, changes in community centre leadership or episodes of violent crime at or near the community centres where dance classes were held necessitated changing intervention sites six times. Second, the local transportation vendor ended service abruptly early in the study. They eventually provided their own vans and drivers but attendance rates never fully recovered.

At FU4 girls reported practicing dance outside of class a mean $\pm \mathrm{SD}=2.7 \pm 2.6$ days per week ( $45 \%$ on 3 or more days per week) for a mean $\pm \mathrm{SD}=.83 \pm .50$ hours ( $37 \%$ for 1 hour or more) confirming the motivating aspect of the intervention. We were able to deliver mean $\pm S D=12.4 \pm 6.3$ (median $=13$, IQ range $=7-18$ ) out of 25 possible START lessons. $70 \%$ of families received at least the first seven lessons, defined as the basic skills portion of the intervention, $29 \%$ received $7-14$ lessons, $34 \%$ received $15-20$ lessons, and $7 \%$ received 21 or more. $77 \%$ hooked up at least one TV Allowance electronic TV time manager $(12 \%$ two or more) and the mean \pm SD reported weekly screen time budget goal was $10.0 \pm 2.4$ hours ( median $=10$, IQ range $=7.5-12$ ).

All 24 educational newsletters were able to be sent to valid addresses for $94 \%$ of active placebo health education girls and parents/guardians. $87 \%$ of girls reported reading at least half of the Felicia's Healthy News Flash newsletters ( $66 \%$ almost all or all). Families attended $1.1 \pm 1.4$ (median $=1$, IQ range $=0-2$ ) of eight possible evening health education events. Additional Saturday summer Health Education Fairs were attended by $31 \%$ of 94 families enrolled by the summer of the first year and $14 \%$ of 127 families in the 2 nd summer of the study. $80 \%$ of parents/guardians reported reading at least half of the Stanford GEMS Health Report newsletters (54\% almost all or all). All elements of the Treatment and Comparison interventions were rated highly for fun and helpfulness by girls and parents/guardians." 
Robinson 2010 (Continued)

\begin{tabular}{|c|c|c|}
\hline Bias & Authors' judgement & Support for judgement \\
\hline $\begin{array}{l}\text { Random sequence genera- } \\
\text { tion (selection bias) }\end{array}$ & Low risk & $\begin{array}{l}\text { Quote: "Families/households were randomized by computer using Efron's bi- } \\
\text { ased coin randomization toto produce similar sample sizes in each group" }\end{array}$ \\
\hline $\begin{array}{l}\text { Allocation concealment } \\
\text { (selection bias) }\end{array}$ & Unclear risk & NR \\
\hline $\begin{array}{l}\text { Blinding (performance } \\
\text { bias and detection bias) } \\
\text { All outcomes }\end{array}$ & Low risk & $\begin{array}{l}\text { Data collection was scheduled every } 6 \text { months in participants' homes by } \\
\text { trained, female African-American research assistants, blinded to experimental } \\
\text { assignment }\end{array}$ \\
\hline $\begin{array}{l}\text { Incomplete outcome data } \\
\text { (attrition bias) } \\
\text { All outcomes }\end{array}$ & Low risk & Low attrition (12\%-16\%) and balanced between groups \\
\hline $\begin{array}{l}\text { Selective reporting (re- } \\
\text { porting bias) }\end{array}$ & High risk & $\begin{array}{l}\text { Trial registration found. No outcomes listed in trial registration document. BMI } \\
\text { was not listed in the trial registration report }\end{array}$ \\
\hline Other bias & Low risk & No additional threats to validity \\
\hline $\begin{array}{l}\text { Other bias- timing of re- } \\
\text { cruitment of clusters }\end{array}$ & Low risk & Figure shows recruitment happened prior to randomisation \\
\hline
\end{tabular}

\section{Rodearmel 2006}

\begin{tabular}{|c|c|}
\hline \multirow[t]{8}{*}{ Methods } & Study design: RCT (see Notes, below) \\
\hline & Intervention period: 13 weeks \\
\hline & Follow-up period (post-intervention): nil \\
\hline & Differences in baseline characteristics: reported \\
\hline & Reliable outcomes: reported \\
\hline & Protection against contamination: reported \\
\hline & Unit of allocation: family \\
\hline & Unit of analysis: individual \\
\hline \multirow[t]{6}{*}{ Participants } & $\begin{array}{l}N \text { (controls baseline): families } N=23 \text {; target girls } N=14 \text {; target boys } N=11 \text {; other girls } N=9 \text {; other boys } \\
N=10\end{array}$ \\
\hline & $\begin{array}{l}N \text { (controls follow-up): families } N=19 \text {; target girls } N=12 \text {; target boys } N=8 \text {; other girls } N=6 \text {; other boys } \\
N=6\end{array}$ \\
\hline & $\begin{array}{l}N \text { (interventions baseline): families } N=82 \text {; target girls } N=40 \text {; target boys } N=53 \text {; other girls } N=30 \text {; other } \\
\text { boys } N=22\end{array}$ \\
\hline & $\begin{array}{l}N \text { (interventions follow-up): families } N=62 \text {; target girls } N=29 \text {; target boys } N=39 \text {; other girls } N=16 \text {; oth- } \\
\text { er boys } N=18\end{array}$ \\
\hline & Setting (and number by study group): families (intervention $N=82$; control $N=23$ ) \\
\hline & $\begin{array}{l}\text { Recruitment: families from Fort Collins area with at least one } 8 \text { - to } 12 \text {-year-old child who was at-risk-for- } \\
\text { overweight or overweight (?85th percentile BMI-for-age) (target child) who would participate with at } \\
\text { least } 1 \text { parent or guardian were recruited. Recruitment by printed flyers and email advertising }\end{array}$ \\
\hline
\end{tabular}


Rodearmel 2006 (Continued)

Geographic region: Fort Collins, Colorado, USA

Percentage of eligible population enrolled: NR

Mean age:

Intervention:

- Target girls $10.1 \pm 0.2$

- Target boys $9.8 \pm 0.2$

- Other girls $12.8 \pm 0.7$

- Other boys $11.8 \pm 0.4$

Control:

- Target girls $9.9 \pm 0.4$

- Target boys $9.9 \pm 0.2$

- Other girls $11.8 \pm 0.8$

- Other boys $12.0 \pm 0.7$

Sex: intervention 55\% female; control 56\% female

Interventions Intervention group:

- families asked to maintain their usual eating and step patterns for the first week of the study to establish baseline, then asked to make 2 small lifestyle changes consisting of:

o increasing their daily walking by 2000 steps/day above baseline levels and

- consuming 2 servings/day of ready-to-eat cereal, 1 at breakfast and 1 for a snack.

- Provided with a step counter and a group-specific step and cereal log and free cereal

Control group:

- asked to maintain their usual eating and step patterns throughout the study.

- provided with a step counter and a group-specific step and cereal log

Both groups:

- all family members asked to record their daily steps and cereal servings consumed

- attended 3 group meetings throughout study period for measurement and data collection

- given magnets and stickers with written reminders to record daily data. Also provided with calculators

Combined effects of dietary interventions and PA interventions vs control

\begin{tabular}{ll}
\hline Outcomes & - Steps \\
- Cereal servings consumed \\
- Bood intake \\
- Fody weight/adiposity \\
$\circ$ bodylts: \\
$\circ$ BMI \\
$\circ \%$ boight \\
- For children: \\
$\circ \%$ BMI-for-age \\
$\circ \%$ body fat
\end{tabular}

Process evaluation: reported

Implementation-related factors
Theoretical basis: NR

Resources for intervention implementation (e.g. funding needed or staff hours required): reported 
Rodearmel 2006 (Continued)

Who delivered the intervention: reported

PROGRESS categories assessed at baseline: reported (gender)

PROGRESS categories analysed at outcome: reported (gender)

Outcomes relating to harms/unintended effects: NR

Intervention included strategies to address diversity or disadvantage: NR

Economic evaluation: NR

Notes
Funding: this work was supported by NIH Grants DK042549 and DK048520 and by the W.K. Kellogg Institute.

Deciding if this RCT is cluster-randomised or not depends upon which outcome data are looked at. The unit of allocation is the family. So technically a cluster-RCT. However the study authors specified a single 'target child' per family. Therefore for data for the target child it is an RCT. However if data from 'other children' in the family are assessed it is a cluster-RCT with the family as the cluster. However, we did not extract any numerical data from this study as they do not present change in BMI or zBMI.

\section{Risk of bias}

\begin{tabular}{lll}
\hline Bias & Authors' judgement & Support for judgement \\
\hline $\begin{array}{l}\text { Random sequence genera- } \\
\text { tion (selection bias) }\end{array}$ & Unclear risk & $\begin{array}{l}\text { Quote: "We randomly assigned 105 families to the experimental (EXP; } \mathrm{n} \text { 82) or } \\
\text { control (CON; } \mathrm{n} 23 \text { ) groups." }\end{array}$ \\
\hline $\begin{array}{l}\text { Allocation concealment } \\
\text { (selection bias) }\end{array}$ & Unclear risk & NR \\
\hline $\begin{array}{l}\text { Blinding (performance } \\
\text { bias and detection bias) } \\
\begin{array}{l}\text { All outcomes } \\
\hline\end{array}\end{array}$ & Unclear risk & NR \\
\hline
\end{tabular}

Incomplete outcome data High risk

Clusters lost (23\% families), $25 \%$ in intervention and $18 \%$ in control

(attrition bias)

All outcomes

Selective reporting (re- Unclear risk Protocol or trial register not found
porting bias)

Other bias Low risk

Other bias- timing of re- Low risk

cruitment of clusters

Rosario 2012

$\begin{array}{ll}\text { Methods } & \text { Study design: cluster-RCT } \\ \text { Intervention period: } 6 \text { months } \\ \text { Follow-up period (post-intervention): nil } \\ \text { Differences in baseline characteristics: reported } \\ \text { Reliable outcomes: reported }\end{array}$


Rosario 2012 (Continued)

Protection against contamination: reported

Unit of allocation: school

Unit of analysis: individual accounting for clustering

\begin{tabular}{|c|c|}
\hline \multirow[t]{10}{*}{ Participants } & $\mathrm{N}($ control baseline $)=231$ \\
\hline & $N($ control follow-up) $=143$ \\
\hline & $\mathrm{N}$ (intervention baseline) $=233$ \\
\hline & $\mathrm{N}$ (intervention follow-up) = 151 \\
\hline & Setting (and number by study group): 7 public elementary scl \\
\hline & $\begin{array}{l}\text { Recruitment: } 7 / 80 \text { public elementary schools were randomly } \\
\text { study. The number of schools involved was according to cons } \\
\text { and implementation of the programme. }\end{array}$ \\
\hline & Geographic region: urban, Portugal \\
\hline & Percentage of eligible population enrolled: $81 \%$ participants \\
\hline & Mean age: intervention + control: $8.3 \pm 1.2(6-12)$ \\
\hline & Sex: intervention + Control: $51.5 \%$ female \\
\hline
\end{tabular}

Interventions

To assess the impact of a 6-month nutrition programme

Teachers of the intervention group had 12 sessions of $3 \mathrm{~h}$ each with the researchers during 6 months, which included the following contents:

- session 1, how to promote health and prevent disease, lifestyle determinants of health, obesity - definitions and descriptions of the problem, risk factors and health problems;

- session 2, key concepts in food and nutrition;

- sessions 3 and 4, dietary guidelines (the Portuguese food wheel), healthy eating advice for children, covering the 5 main food groups, and interventions to help children and their families to consume healthy foods and plan well-balanced meals and snacks;

- session 5, teach children about the importance of water, and teaching strategies to replace consumption of SSBs with water;

- sessions 6 and 7, appropriate PA levels and healthy-eating behaviours such increasing fruit and vegetable intake and decreasing energy-dense micronutrient-poor foods;

- session 8, teaching strategies and learning theory in the classroom;

- session 9, strategies to reduce screen exposure time;

- session 10, global assessment of the training programme;

- sessions 11 and 12, healthy cooking and strategies to get children and their families involved in healthy cooking

72-h duration, distributed between active learning strategies ( $36 \mathrm{~h}$ with the researchers) and the delivery of the learnt contents to the children ( $36 \mathrm{~h}$ ). 15 teachers

Diet intervention vs control

Outcomes

Outcome measures

- Primary outcome: zBMI

- Secondary outcomes: energy intake, PA, prevalence, incidence or remission of overweight/obesity, consumption of low-nutrient, energy-dense (LNED) foods: SSBs and solids (see secondary reference for Rosario 2012: Rosario et al. 2013)

Process evaluation: NR 
Rosario 2012 (Continued)

Implementation-related factors
Theoretical basis: Health Promotion Model and SCT

Resources for intervention implementation: reported

Who delivered the intervention: reported

PROGRESS categories assessed at baseline: gender; parent: education

PROGRESS categories analysed at outcome: NR

Outcomes relating to harms/unintended effects: NR

Intervention included strategies to address diversity or disadvantage: NR

Economic evaluation: NR

Notes NCT01397123

Funding: this work was supported by the Fundação para a Ciência e Tecnologia (FCT), Projeto PEst-OE/ SAU/UI0617/2011

Included the programme in the progression of teaching career

\section{Risk of bias}

\begin{tabular}{lll}
\hline Bias & Authors' judgement & Support for judgement \\
\hline $\begin{array}{l}\text { Random sequence genera- } \\
\text { tion (selection bias) }\end{array}$ & Low risk & $\begin{array}{l}\text { Schools were randomised according to a random number generator, with } \\
\text { blinding to schools }\end{array}$ \\
\hline $\begin{array}{l}\text { Allocation concealment } \\
\text { (selection bias) }\end{array}$ & Low risk & $\begin{array}{l}\text { Schools were randomised according to a random number generator, with } \\
\text { blinding to schools }\end{array}$ \\
\hline $\begin{array}{l}\text { Blinding (performance } \\
\text { bias and detection bias) } \\
\text { All outcomes }\end{array}$ & Low risk & Children and outcomes assessors were blinded to group assignment \\
\hline $\begin{array}{l}\text { Incomplete outcome data } \\
\text { (attrition bias) } \\
\text { All outcomes }\end{array}$ & High risk & $\begin{array}{l}\text { High attrition: 35\% and 38\% (intervention and control respectively), equally } \\
\text { balanced }\end{array}$ \\
\hline $\begin{array}{l}\text { Selective reporting (re- } \\
\text { porting bias) }\end{array}$ & Low risk & \begin{tabular}{l} 
Protocol/trial registration document seen. All outcomes reported \\
\hline $\begin{array}{l}\text { Other bias } \\
\text { Other bias- timing of re- }\end{array}$
\end{tabular} \\
\begin{tabular}{ll} 
cruitment of clusters \\
\hline
\end{tabular} & Low risk & $\begin{array}{l}\text { Significant difference between groups at baseline for parental education so } \\
\text { this was adjusted for in subsequent analyses }\end{array}$ \\
\hline
\end{tabular}

Rosenkranz 2010

$\begin{array}{ll}\text { Methods } & \text { Study name: SNAP (Scouting nutrition \& activity program) } \\ \text { Study design: cluster-RCT } \\ \text { Intervention period: } 4 \text { months } \\ \text { Follow-up period (post-intervention): nil }\end{array}$


Rosenkranz 2010 (Continued)

Differences in baseline characteristics: reported

Reliable outcomes: reported

Protection against contamination: NR

Unit of allocation: troops

Unit of analysis: individual accounting for clustering

\begin{tabular}{|c|c|}
\hline \multirow[t]{10}{*}{ Participants } & $\mathrm{N}($ control baseline $)=42$ \\
\hline & $N($ control follow-up $)=39$ \\
\hline & $\mathrm{N}$ (intervention baseline) $=34$ \\
\hline & $\mathrm{N}$ (intervention follow-up) = 33 \\
\hline & $\begin{array}{l}\text { Setting (and number by study group): } 7 \text { Girl Scout Junior troo } \\
\text { tervention troops, } 4 \text { control troops) }\end{array}$ \\
\hline & $\begin{array}{l}\text { Recruitment: } 7 \text { troops agreeing to participate completed a pre } \\
\text { period }\end{array}$ \\
\hline & Geographic region: 3 Midwestern towns, Kansas, USA \\
\hline & Percentage of eligible population enrolled: $75 \%$ participants \\
\hline & Mean age: intervention: $10.6 \pm 1.1$; control: $10.5 \pm 1.3$ \\
\hline & Sex: intervention: $100 \%$ female; control: $100 \%$ female \\
\hline
\end{tabular}

Interventions

To evaluate an intervention designed to prevent obesity by modifying Girl Scout troop meeting environments, and by empowering girls to improve the quantity and/or quality of family meals in their home environments

The intervention consisted of 3 main components:

- an interactive educational curriculum delivered by troop leaders

- troop meeting policies implemented by troop leaders

- badge assignments completed at home by Girl Scouts with parental assistance

A trained research assistant observed each troop during 7 full meetings between time 1 (October 2007) and time 2 (April 2008) assessments. The educational curriculum consisted of 8 modules, delivered over the course of about 4 months. Modules were designed to require 60-90 min to deliver. Meetings were held at the Girl Scouts organisation's property (4 troops), at a troop leader's home ( 2 troops), or at a community centre ( 1 troop). Troop leaders underwent $2 \mathrm{~h}$ of training by the 1 st author prior to intervention commencement

Target behaviours of the intervention included:

- frequent family meals

- parent-child shared PA

- elimination of TV during mealtimes

- drinking water instead of SSBs at mealtimes

- including fruit/vegetables in family meals

- practicing good manners during family meals

- helping parents prepare family meals and cleaning up afterwards

Each module consisted of a discussion of intervention target behaviours, worksheet for goal setting and self-monitoring, physically active recreation session (e.g. walking, dancing, yoga, and active games), fruit/vegetable snack recipe preparation, family meal role-playing, clean-up period, and description of the take-home assignment. 
Rosenkranz 2010 (Continued)

Troop meeting policies included:

- providing 15 min per meeting for physically active recreation

- troop leaders participating in physically active recreation with girls

- provision of a fruit/vegetable snack prepared by girls

- troop leaders eating fruit/vegetable snack with girls

- troop leaders verbally promoting PA, fruit/vegetable consumption in troop meetings and for home, and verbally promoting family meals for home

- prohibition of SSBs, candy, and TV watching during meetings.

Control: NR

Diet and PA intervention vs control

\begin{tabular}{ll}
\hline Outcomes & Outcome measures \\
- Primary outcome: BMI, BMI percentile, zBMI \\
- Secondary outcomes: PA, meeting snacks, dietary intake \\
Process evaluation: reported (implementation)
\end{tabular}

Implementation-related factors
Theoretical basis: SCT

Resources for intervention implementation: reported

Who delivered the intervention: reported

PROGRESS categories assessed at baseline: child, race/ethnicity; parent: education, SES (free/reduced or not)

PROGRESS categories analysed at outcome: NR

Outcomes relating to harms/unintended effects: NR

Intervention included strategies to address diversity or disadvantage: NR

Economic evaluation: NR

Notes NCT00949637

Funding: Funding for this project was provided, in part, by the Sunflower Foundation: Health Care for Kansans, a Topeka-based philanthropic organisation with the mission to serve as a catalyst for improving the health of Kansans.

3 troop leader self-rating averages over the 8 modules ranged from 1.52-1.86 (zero $=$ no implementation to $2.0=$ full implementation). Troops differed $(F 2,18=21.5, P<.001)$ in overall implementation with averages of $1.43,1.86$, and 1.84 (mean $=1.71$ ).

\section{Risk of bias}

\section{Bias Authors' judgement Support for judgement}

Random sequence genera- Low risk Random number generator, stratified by troop size tion (selection bias)

\begin{tabular}{lll}
$\begin{array}{l}\text { Allocation concealment } \\
\text { (selection bias) }\end{array}$ & Unclear risk & $\begin{array}{l}\text { Quote: "Troops were stratified into large }(\mathrm{N}=4) \text { and small size troops ( } \mathrm{n}=3 \text { ) and } \\
\text { then according to a random number generator were } \\
\text { randomized (by first author) within strata to the control or intervention condi- } \\
\text { tions" }\end{array}$ \\
\hline $\begin{array}{l}\text { Blinding (performance } \\
\text { bias and detection bias) }\end{array}$ & Low risk & $\begin{array}{l}\text { At study commencement, research assistants were blind to condition of each } \\
\text { troop }\end{array}$ \\
\hline
\end{tabular}


Rosenkranz 2010 (Continued)

All outcomes

Incomplete outcome data Low risk Low attrition overall (6\%); ITT done
(attrition bias)

(attrition bias)

All outcomes

Selective reporting (re- Low risk Protocol/trial registration document seen. All outcomes reported
porting bias)

\begin{tabular}{ll}
\hline Other bias $\quad$ Low risk $\quad$ No additional threats to validity \\
\hline
\end{tabular}

Other bias- timing of re- Low risk $\quad$ Figure shows recruitment happened prior to randomisation

cruitment of clusters

Roth 2015

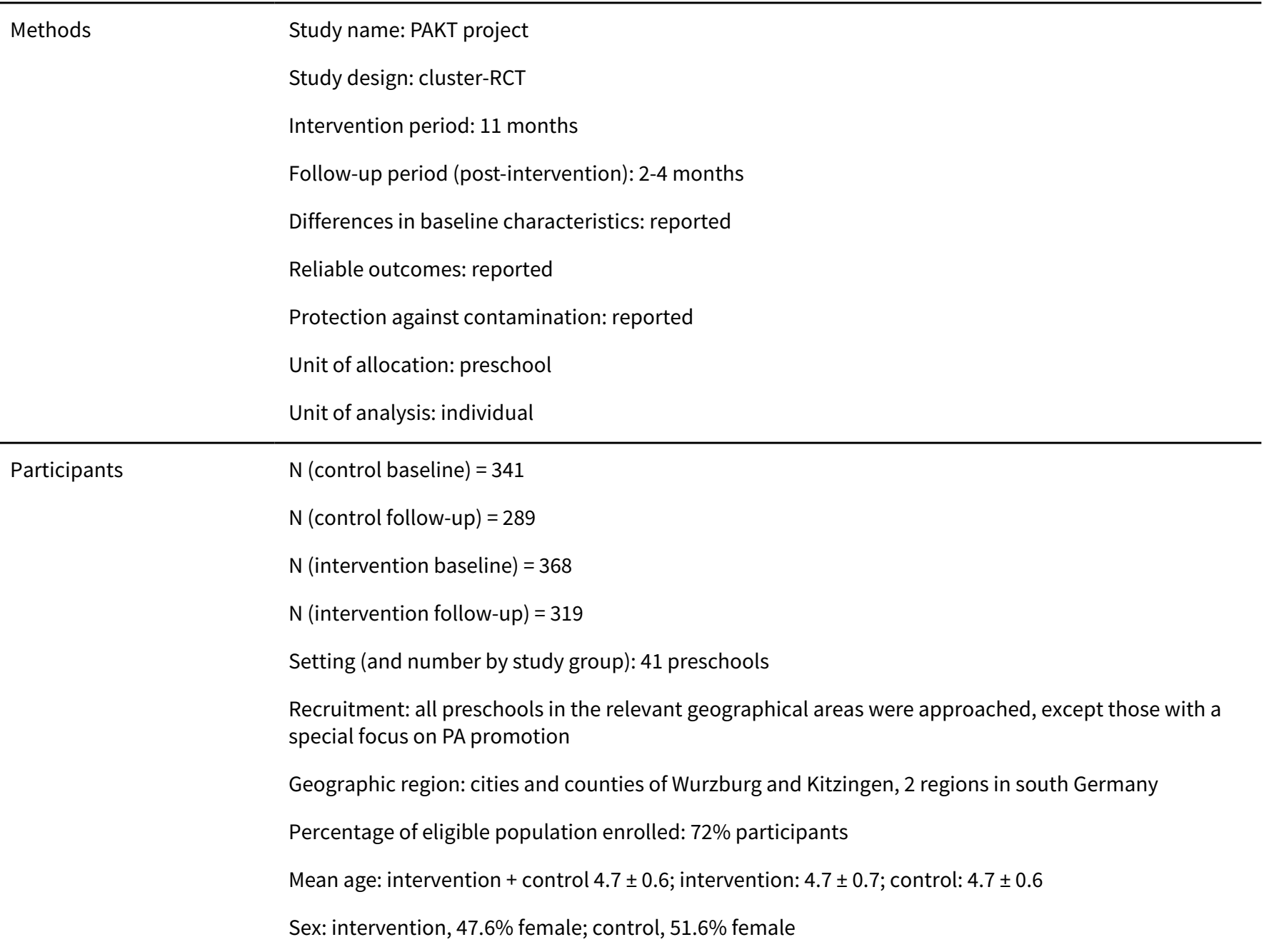

Interventions Aimed to evaluate a multicomponent, child-appropriate preschool intervention programme led by preschool teachers to enhance PA and motor skill performance in 4- and 5-year-old children.

Daily PA session lasting 30 min and PA homework over 1 academic year. Intervention was designed by professional. Intervention included educational components for parents and teachers. 
Roth 2015 (Continued)

Implementation of the intervention was monitored at least once per 8 weeks.

PA intervention vs control

Outcome measures
- $\begin{aligned} & \text { Outcomes } \\ & \text { - Secondary outcomes: BMI centile, sum of } 4 \text { skinfolds (triceps, biceps, subscapular, and suprailiac), } \\ & \text { blood pressure, accidents and infections, MVPA and composite motor skills at final follow-up, single } \\ & \text { motor performance tasks including the obstacle course, one-foot stand, balancing backward, stand- } \\ & \text { ing long jump, jumping to-and-fro sidewise, and target throw at all time points. }\end{aligned}$

Process evaluation: NR

Implementation-related factors
Theoretical basis: NR

Resources for intervention implementation: reported

Who delivered the intervention: reported

PROGRESS categories assessed at baseline: gender, SES, race/ethnicity (migrant status)

PROGRESS categories analysed at outcome: NR

Outcomes relating to harms/unintended effects: reported (accidents and infections)

Intervention included strategies to address diversity or disadvantage: NR

Economic evaluation: NR

Notes

Funding: the authors declare that the institution of household, KCR and KR had financial support from the German Federal Ministry of Education and Research (BMBF) (Grant Nr. 01EL0606, BMBF) and from the BARMER GEK (formerly Gmuender Ersatz-Kasse GEK) for the submitted project.

The funders had no role in study design, data collection and analysis, decision to publish, or preparation of the manuscript.

Implemented by preschool teachers without further costs

\section{Risk of bias}

\begin{tabular}{lll}
\hline Bias & Authors' judgement & Support for judgement \\
\hline $\begin{array}{l}\text { Random sequence genera- } \\
\text { tion (selection bias) }\end{array}$ & Low risk & $\begin{array}{l}\text { Computer-generated random number table stratified for urban or rural loca- } \\
\text { tion }\end{array}$ \\
\hline $\begin{array}{l}\text { Allocation concealment } \\
\text { (selection bias) }\end{array}$ & Low risk & $\begin{array}{l}\text { Randomisation was performed by a person blinded to the identity of the } \\
\text { preschool }\end{array}$ \\
\hline $\begin{array}{l}\text { Blinding (performance } \\
\text { bias and detection bias) } \\
\text { All outcomes }\end{array}$ & Low risk & Outcome assessors blinded \\
\hline $\begin{array}{l}\text { Incomplete outcome data } \\
\text { (attrition bias) } \\
\text { All outcomes }\end{array}$ & Low risk & 13\%-15\% attrition \\
\hline $\begin{array}{l}\text { Selective reporting (re- } \\
\text { porting bias) }\end{array}$ & Unclear risk & Protocol or trial register not found \\
\hline \begin{tabular}{l} 
Other bias \\
\hline
\end{tabular} & Low risk & No additional threats to validity \\
\hline
\end{tabular}


Roth 2015 (Continued)

Other bias- timing of re- Low risk Figures shows recruitment happened prior to randomisation cruitment of clusters

Rush 2012

\begin{tabular}{|c|c|}
\hline \multirow[t]{9}{*}{ Methods } & Study name: Project Energize \\
\hline & Study design: cluster-RCT \\
\hline & Intervention period: 2 years \\
\hline & Follow-up period (post-intervention): nil \\
\hline & Differences in baseline characteristics: reported \\
\hline & Reliable outcomes: reported \\
\hline & Protection against contamination: NR \\
\hline & Unit of allocation: schools \\
\hline & Unit of analysis: individual accounting for clustering \\
\hline \multirow[t]{10}{*}{ Participants } & $\mathrm{N}$ (control baseline) $=\mathrm{NR}$ \\
\hline & $N$ (control follow-up) $=660$ \\
\hline & $\mathrm{N}$ (intervention baseline) $=\mathrm{NR}$ \\
\hline & $N$ (intervention follow-up) $=692$ \\
\hline & Setting (and number by study group): 124 schools (62 intervention, 62 control) \\
\hline & Recruitment: NR \\
\hline & Geographic region: Waikato Region of New Zealand \\
\hline & Percentage of eligible population enrolled: $50 \%$ participants \\
\hline & Mean age: intervention + control: 5 years and 10 years \\
\hline & Sex: intervention: $49 \%$ female; control: $50 \%-51 \%$ female \\
\hline
\end{tabular}

To compare changes in blood pressure and body composition in children who attended Energize schools with children in control schools. The trial also aimed to identify predictors of increase in body fat and blood pressure over 2 years in relation to age, sex, ethnicity, rurality and social deprivation.

Children

- Each of the 11 team Energize staff ('energizers') was allocated between 6 and 8 schools each, by the team manager.

- Classes modelled included fundamental movement skill training, ideas for 'huff and puff' fitness activities, modified games, and ball activities and sport-related games, where keeping children moving as much as possible throughout each session was the focus.

- Also, energizers promoted active transport, lunchtime games, bike days and leadership training for students to be leaders of PAs before and after school.

- Assist each school with a range of healthy-eating initiatives. These included canteen makeovers. Healthy fund raising was promoted.

- Nutrition 'nuggets' were also provided every week in the school newsletter.

- A home-school link programme that provided opportunities for parents to attend 3 information-based sessions, which included a 45-min practical nutrition class. 
Rush 2012 (Continued)

Teachers and local community:

- Project offered assistance to teachers, parents and the local community. This was implemented through a range of activities, such as professional development and evenings with a dietitian

Each control school involved in the project worked with their energizer to develop an individualised action plan based on the individual needs of the school.

Given no additional resourcing or information

Diet and PA combination intervention vs control

$\begin{array}{ll}\text { Outcomes } & \text { Outcome measures } \\ \text { - Primary outcome: BMI, body fat, resting blood pressure (all SD scores) } \\ \text { - Secondary outcomes: NR } \\ \text { Process evaluation: NR }\end{array}$

Implementation-related factors
Theoretical basis: NR

Resources for intervention implementation: reported

Who delivered the intervention: reported

PROGRESS categories assessed at baseline: gender, SES, place (rural/urban)

PROGRESS categories analysed at outcome: gender, race/ethnicity

Outcomes relating to harms/unintended effects: NR

Intervention included strategies to address diversity or disadvantage: NR

Economic evaluation: reported (direct costs)

\section{ACTRN12610000132044}

Funding: the Waikato District Health Board funds the Project Energize programme and its evaluation. The Ministry of Health, New Zealand has contributed to evaluation funding.

Implementation: while the evaluation measurements were undertaken 2 years from the commencement of the intervention, the nature of the intervention process meant that it was able to be implemented only in a graduated way, reflecting the characteristics and capacities of individual schools. This led to a shorter duration of intervention implementation before endpoint measurements for lower-decile schools, where a higher proportion of Maori children attend. Sport Waikato was contracted by the Waikato District Health Board to deliver Project Energize. 'Team Energize' are either teachers or graduates in the fields of exercise and nutrition, or PE, employed by Sport Waikato to support the delivery and development of the programme in each intervention school.

Following this RCT the intervention was rolled out as a region-wide whole-school nutrition and PA programme.

Costs: the programme is cost-effective, the main costs are the salaries of the Energizers and team leader and the travel required to move between schools. We calculate that the average cost of the intervention for each child, each year, is < NZD 40 and this could be improved by further efficiencies.

\section{Risk of bias}

\section{Bias \\ Authors' judgement Support for judgement}

Random sequence genera- Unclear risk

Stratified by rurality and social deprivation and randomised, no further details tion (selection bias) 
Rush 2012 (Continued)

\begin{tabular}{lll}
$\begin{array}{l}\text { Allocation concealment } \\
\text { (selection bias) }\end{array}$ & Unclear risk & NR \\
\hline $\begin{array}{l}\text { Blinding (performance } \\
\text { bias and detection bias) } \\
\text { All outcomes }\end{array}$ & Low risk & $\begin{array}{l}\text { The measurement teams were trained in all measurements and blind to the al- } \\
\text { location of the school at baseline and follow-up. }\end{array}$ \\
\hline
\end{tabular}

\begin{tabular}{|c|c|c|}
\hline $\begin{array}{l}\text { Incomplete outcome data } \\
\text { (attrition bias) }\end{array}$ & High risk & $\begin{array}{l}20 \% \text { of the younger children and } 43 \% \text { of the older children were lost to fol- } \\
\text { low-up; NR by group }\end{array}$ \\
\hline
\end{tabular}

All outcomes

Selective reporting (re- Low risk Protocol seen; all outcomes specified in methods were reported in results porting bias)

\begin{tabular}{lll}
\hline Other bias & Low risk & No additional threats to validity \\
\hline $\begin{array}{l}\text { Other bias- timing of re- } \\
\text { cruitment of clusters }\end{array}$ & Unclear risk & NR \\
\hline
\end{tabular}

Safdie 2013

Study design: cluster-RCT
Intervention period: 18 months
Follow-up period (post-intervention): nil
Differences in baseline characteristics: reported
Reliable outcomes: reported
Protection against contamination: NR
Unit of allocation: school
Unit of analysis: individual (overweight/obesity, food intake, number of steps taken) and school-level
(food availability and MVPA in PE classes and recess)

(food availability and MVPA in PE classes and recess)

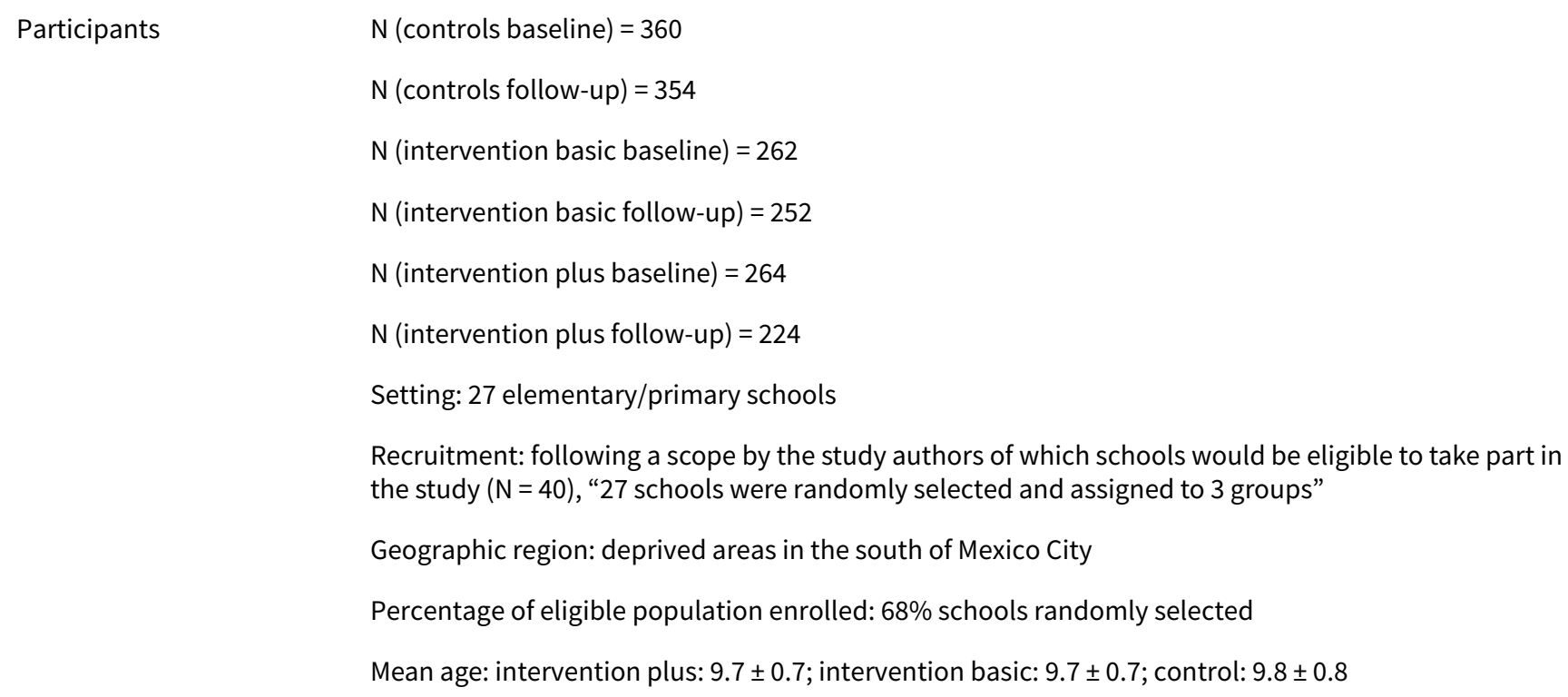


Safdie 2013 (Continued)

Sex: intervention plus: $54.0 \%$ female; intervention basic: $48.4 \%$ female; control, $48.6 \%$ female

Interventions
Basic: support to improve general environment (obesogenic environment) of the school, including the types of offering of foods and drinks (provided by external vendors) as snacks for the children at recess/break time, and quality of PE lessons (in terms of amount of MVPA promoted) and during recess sessions. For the basic intervention, this came in the form of educational leaflets for the school and the external vendors. Also there was mass communications and marketing to children to encourage them to eat healthy snacks, drink water instead of sugary drinks, and be more physically active. The schools in the basic arm were limited to using existing school infrastructure and resources.

Plus: the schools in the 'plus' arm received, in addition to the basic intervention, specialist PE/PE teachers who taught 1 extra PE class/week, and provided 15-min activity (callisthenics) sessions 4 times/week during morning recess. They also received additional financial investment to support the school's efforts in implementing the intervention.

The basic programme focused on improving norms related to nutrition and PA at the schools and was limited to using existing school infrastructure and resources. The plus programme implemented all the components incorporated in the basic programme and included additional financial investment and human resources. No changes were made to existing nutrition or PA practices in control schools.

Diet and PA intervention (basic and plus) vs control

- Primary outcome: BMI, weight, height, overweight and obesity, food and beverage availability at school, food intake at recess, PA opportunities during PE classes and recess, children's PA (steps taken) at school

- Secondary outcomes: primary/secondary not specified

Process evaluation: reported (implementation)

Implementation-related factors
Theoretical basis: ecological principles, TPB, HBM, SCT

Resources for intervention implementation: reported

Who delivered the intervention: reported

PROGRESS categories assessed at baseline: gender

PROGRESS categories analysed at outcome: NR

Outcomes relating to harms/unintended effects: NR

Intervention included strategies to address diversity or disadvantage: NR but intervention targeted schools classified by the Ministry of Education as having students of low SES and receiving benefits from the Federal School Breakfast Program

Economic evaluation: NR
Funding: the project was supported by the Pan American Health Organization (PAHO), the HLHP program of the International Life Science Institute (ILSI), the Mexican Council for Science and Technology (Conacyt), and the Mexican Ministry of Health (SSa). This work was carried out with support from the Global Health Research Initiative (GHRI), a collaborative research funding partnership of the Canadian Institute of Health Research, the Canadian International Development Agency, Health Canada, the International Development Research Centre, and the Public Health Agency of Canada.

One of the plus schools changed during year 2 to become a full-time school, and data from this school was therefore excluded from the analysis.

\section{Risk of bias}

Bias Authors' judgement Support for judgement


Safdie 2013 (Continued)

$\begin{array}{ll}\begin{array}{l}\text { Random sequence genera- } \\ \text { tion (selection bias) }\end{array} & \begin{array}{l}27 \text { schools were randomly selected and assigned, no further details to one of } \\ \text { three conditions }\end{array}\end{array}$

$\begin{array}{ll}\begin{array}{l}\text { Allocation concealment } \\ \text { (selection bias) }\end{array} & \text { Unclear risk NR }\end{array}$

Blinding (performance $\quad$ Unclear risk
bias and detection bias)
All outcomes

\begin{tabular}{|c|c|c|}
\hline $\begin{array}{l}\text { Incomplete outcome data } \\
\text { (attrition bias) } \\
\text { All outcomes }\end{array}$ & High risk & $\begin{array}{l}1 \text { cluster lost from one of the intervention arms. The study authors state that } \\
886 \text { students ( } 52 \%) \text { were selected for outcome evaluation, from the } 1712 \text { stu- } \\
\text { dents who participated in the study. It is unclear how these } 886 \text { students were } \\
\text { selected from the total } 1712 \text { (not in flow chart). }\end{array}$ \\
\hline
\end{tabular}

\begin{tabular}{lll}
\hline $\begin{array}{l}\text { Selective reporting (re- } \\
\text { porting bias) }\end{array}$ & Unclear risk & Protocol or trial register not found \\
\hline Other bias & Low risk & No additional threats to validity \\
\hline $\begin{array}{l}\text { Other bias- timing of re- } \\
\text { cruitment of clusters }\end{array}$ & High risk & $\begin{array}{l}\text { Recruitment of clusters happened before randomisation of clusters, however } \\
\text { participants appear to have been recruited after randomisation. }\end{array}$ \\
\hline
\end{tabular}

Sahota 2001

$\begin{array}{ll}\text { Methods } & \text { Study design: cluster-RCT } \\ \text { Intervention period: } 1 \text { year } \\ \text { Follow-up (Post-intervention): nil } \\ \text { Differences in baseline characteristics: reported } \\ \text { Reliable outcomes: reported } \\ \text { Protection against contamination: not done. (Schools that were controls } 1 \text { year received the interven- } \\ \text { tion the following year) } \\ \text { Unit of allocation: school } \\ \text { Unit of analysis: child } \\ \text { Unit of analysis errors addressed }\end{array}$

$\begin{array}{ll}\text { Participants } & \text { For weight and height: } \mathrm{n} \text { (controls baseline) }=312 \\ \mathrm{~N} \text { (controls follow-up) }=303 \\ \mathrm{~N} \text { (intervention baseline) }=301 \\ \mathrm{~N} \text { (intervention follow-up) }=292 \\ \mathrm{~N} \text { of schools: } 10 \\ \text { Recruitment: not clear } \\ \text { Geographical setting: northern UK } \\ \text { Proportion of eligibles participating: for weight and height: control 97\%, intervention 96\% } \\ \text { Mean age: intervention: } 8.36 \text { (0.63) years; control: } 8.42 \text { (0.63) years } \\ \text { Sex: both sexes included. Intervention: } 51 \% \text { boys; control: } 59 \% \text { boys } \\ \text { School-based intervention. 'Active programme promoting lifestyle in schools' (APPLES). The pro- } \\ \text { gramme was designed to influence diet and PA and not simply knowledge. Targeted at the whole } \\ \text { school community including parents, teachers and catering staff. The programme consisted of teacher } \\ \text { training, modifications of school meals and the development and implementation of school action } \\ \text { plans designed to promote healthy eating and PA. } \\ \text { Control schools received usual curriculum } \\ \text { Combined effects of dietary interventions and PA interventions vs control }\end{array}$


Sahota 2001 (Continued)

\begin{tabular}{|c|c|}
\hline Outcomes & $\begin{array}{l}\text { - BMI } \\
\text { - Dietary intake - 24-h recall and 3-day food diaries } \\
\text { - PA - frequency of PA and sedentary behaviour was measured by questionnaire. } \\
\text { - Psychological measures - } 3 \text { validated measures including a Self-Perception Profile for Children, a } \\
\text { questionnaire to distinguish global self-worth from competence and a measure of dietary restraint } \\
\text { Process evaluation: reported }\end{array}$ \\
\hline $\begin{array}{l}\text { Implementation-related } \\
\text { factors }\end{array}$ & $\begin{array}{l}\text { Theoretical basis: multi-component health promotion programme, based on the Health Promoting } \\
\text { Schools concept } \\
\text { Resources for intervention implementation (e.g. funding needed or staff hours required): reported } \\
\text { Who delivered the intervention: reported } \\
\text { PROGRESS categories assessed at baseline: reported (race, gender) } \\
\text { PROGRESS categories analysed at outcome: NR } \\
\text { Outcomes relating to harms/unintended effects: reported } \\
\text { Intervention included strategies to address diversity or disadvantage: NR } \\
\text { Economic evaluation: NR }\end{array}$ \\
\hline Notes & $\begin{array}{l}\text { Funding: the research was funded by a grant from the Northern and Yorkshire Region Research and De- } \\
\text { velopment Unit }\end{array}$ \\
\hline
\end{tabular}

\section{Risk of bias}

Bias Authors' judgement Support for judgement

\begin{tabular}{|c|c|c|}
\hline $\begin{array}{l}\text { Random sequence genera- } \\
\text { tion (selection bias) }\end{array}$ & Low risk & $\begin{array}{l}\text { Quote: "We randomised them to receive the intervention or to serve as the } \\
\text { comparison school using the toss of a coin." }\end{array}$ \\
\hline
\end{tabular}

\begin{tabular}{|c|c|c|}
\hline $\begin{array}{l}\text { Allocation concealment } \\
\text { (selection bias) }\end{array}$ & Low risk & $\begin{array}{l}\text { Schools were recruited, then all were randomised at the same time at the start } \\
\text { of the study and interventions were implemented throughout participating } \\
\text { schools. }\end{array}$ \\
\hline $\begin{array}{l}\text { Blinding (performance } \\
\text { bias and detection bias) } \\
\text { All outcomes }\end{array}$ & High risk & Outcome assessment was not blinded \\
\hline $\begin{array}{l}\text { Incomplete outcome data } \\
\text { (attrition bias) } \\
\text { All outcomes }\end{array}$ & Low risk & No cluster loss; 93\% individual retention for BMI data \\
\hline $\begin{array}{l}\text { Selective reporting (re- } \\
\text { porting bias) }\end{array}$ & Unclear risk & Protocol or trial register not found \\
\hline Other bias & Low risk & No additional threats to validity \\
\hline $\begin{array}{l}\text { Other bias- timing of re- } \\
\text { cruitment of clusters }\end{array}$ & Low risk & Figure indicates recruitment happened prior to randomisation \\
\hline
\end{tabular}




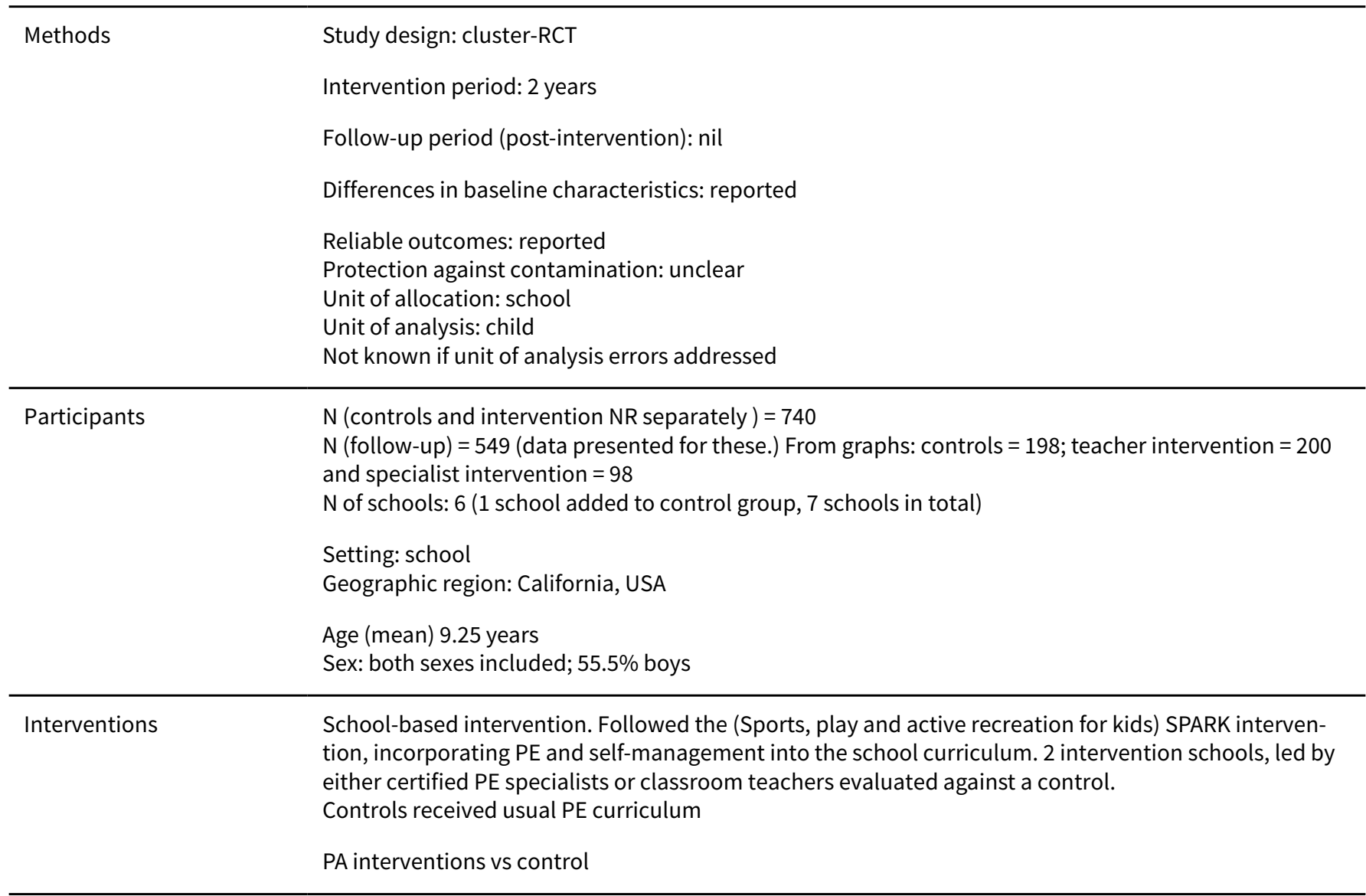

\section{Outcomes}

- Weight status: BMI presented at fall 1990, spring 1991, fall 1991 and spring 1992

Process evaluation: NR

Implementation-related factors
Theoretical basis: Behaviour Change and self-management

Resources for intervention implementation (e.g. funding needed or staff hours required): reported

Who delivered the intervention: reported

PROGRESS categories assessed at baseline: reported (race, gender)

PROGRESS categories analysed at outcome: reported (gender)

Outcomes relating to harms/unintended effects: NR

Intervention included strategies to address diversity or disadvantage: NR

Economic evaluation: NR

Notes Funding: NIH Grant HL 44467

\section{Risk of bias}

\begin{tabular}{lll}
\hline Bias & Authors' judgement & Support for judgement \\
\hline $\begin{array}{l}\text { Random sequence genera- } \\
\text { tion (selection bias) }\end{array}$ & High risk & $\begin{array}{l}12 \text { schools were "randomly assigned" to the } 3 \text { experimental conditions, how- } \\
\text { ever an additional school was recruited and added to the control group after } \\
\text { this process was conducted. }\end{array}$ \\
\hline
\end{tabular}


Sallis 1993 (Continued)

\begin{tabular}{lll}
$\begin{array}{l}\text { Allocation concealment } \\
\text { (selection bias) }\end{array}$ & Unclear risk & NR \\
\hline $\begin{array}{l}\text { Blinding (performance } \\
\text { bias and detection bias) }\end{array}$ & Unclear risk & NR \\
All outcomes & &
\end{tabular}

\begin{tabular}{|c|c|c|}
\hline $\begin{array}{l}\text { Incomplete outcome data } \\
\text { (attrition bias) } \\
\text { All outcomes }\end{array}$ & High risk & $\begin{array}{l}\text { Missing data ( } 26 \%) \text { not provided by study group and reasons for attrition not } \\
\text { given }\end{array}$ \\
\hline
\end{tabular}

\begin{tabular}{lll}
\hline $\begin{array}{l}\text { Selective reporting (re- } \\
\text { porting bias) }\end{array}$ & Unclear risk & Protocol or trial register not found \\
\hline Other bias & Low risk & No additional threats to validity \\
\hline $\begin{array}{l}\text { Other bias- timing of re- } \\
\text { cruitment of clusters }\end{array}$ & Unclear risk & NR \\
\hline
\end{tabular}

Salmon 2008

Study design: cluster-RCT
Intervention period: 6 months
Follow-up period (post-intervention): 1 year (assessments at 6, 12 months post-intervention)
Differences in baseline characteristics: reported
Reliable outcomes: reported
Protection against contamination: reported
Unit of allocation: class
Unit of analysis: individual

\footnotetext{
Participants N (controls baseline $)=62$

$\mathrm{N}$ (controls 12 -month follow-up) $=55$

$\mathrm{N}$ (behavioural modification $(\mathrm{BM})$ intervention baseline $)=66$

$\mathrm{N}($ BM 12-month follow-up $)=60$

$\mathrm{N}$ (fundamental motor skills (FMS) intervention baseline) $=74$

$\mathrm{N}($ FMS 12-month follow-up) $=69$

$\mathrm{N}(\mathrm{BM} / \mathrm{FMS}$ baseline $)=93$

$\mathrm{N}(\mathrm{BM} / \mathrm{FMS} 12-$ month follow-up $)=84$

Setting (and number by study group): 17 classes across 3 schools. Number of classes in each trial group NR

Recruitment: all grade 5 students within 3 selected government schools located across 4 campuses in low-SES areas

Geographic region: Melbourne, Australia
} 
Salmon 2008 (Continued)

Percentage of eligible population enrolled: $78 \%$

Mean age: male 10 years 8 months \pm 5 months; female 10 years 8 months \pm 4 months

Sex: $51 \%$ female

- Behaviour modification (BM) group: in addition to the usual PE and sports classes, 19 lessons (40-50 min each) were delivered in classroom by 1 qualified $P E$ teacher for 1 school year. Lessons incorporated self-monitoring time spent in PA and screen behaviours, health benefits of PA, sedentary behaviour environments, decision-making and identifying alternatives to screen behaviours, intelligent TV viewing and reducing viewing time, advocacy of reduced screen time, use of pedometers and group games

- Fundamental Motor Skills (FMS) group: in addition to the usual PE and sports classes, 19 lessons (40-50 min each) were delivered either in the indoor or outdoor PA facilities at each school for 1 school year. Lessons focused on mastery of 6 fundamental movement skills (run, throw, dodge, strike, vertical jump, and kick). The interventionist taught the skills with an emphasis on enjoyment and fun through games and maximum involvement for all the children.

- BM/FMS group: children in this group received both BM and FMS lessons.

PA interventions vs control

- BMI
- Outcomes
- Objectively assessed PA (accelerometer) - PA measured for 8 days during waking hours, except when
bathing or swimming
- Self-reported screen behaviours
- Self-reported enjoyment of PA (5-point Likert scale)
- Bastery of fundamental movement skills
- Food intake: children were asked to complete a 22-item food-frequency questionnaire to determine
the energy density of their diet
Process evaluation: reported

Implementation-related factors

\section{Theoretical basis: SCT and Behavioural Choice theory}

Resources for intervention implementation (e.g. funding needed or staff hours required): reported Who delivered the intervention: reported

PROGRESS categories assessed at baseline: reported (place, gender, education, SES)

PROGRESS categories analysed at outcome: reported (gender)

Outcomes relating to harms/unintended effects: reported

Intervention included strategies to address diversity or disadvantage: reported

Economic evaluation: NR
Funding: this study was funded by the Victorian Health Promotion Foundation. Jo Salmon is supported by a National Heart Foundation of Australia and Sanofi-Aventis Career Development Award. Kylie Ball is supported by a National Health and Medical Research Council/National Heart Foundation of Australia Career Development Award. David Crawford is supported by a Victorian Health Promotion Foundation Senior Research Fellowship.

\section{Risk of bias}


Salmon 2008 (Continued)

Random sequence genera- Low risk Randomised by withdrawing a ticket from a container tion (selection bias)

\begin{tabular}{|c|c|c|}
\hline $\begin{array}{l}\text { Allocation concealment } \\
\text { (selection bias) }\end{array}$ & Low risk & $\begin{array}{l}\text { Allocation was by class and all classes were randomised at the start of the } \\
\text { study }\end{array}$ \\
\hline
\end{tabular}

\begin{tabular}{|c|c|c|}
\hline $\begin{array}{l}\text { Blinding (performance } \\
\text { bias and detection bias) } \\
\text { All outcomes }\end{array}$ & Low risk & $\begin{array}{l}\text { The } 5 \text { specialist evaluators who examined video tapes of children performing } \\
\text { the fundamental movement skills to assess the children's mastery of these } \\
\text { skills were blind to the group assignment. }\end{array}$ \\
\hline
\end{tabular}

\begin{tabular}{lll}
\hline Incomplete outcome data & Unclear risk & No cluster lost; individual loss 25\% overall and similar across groups; gener- \\
(attrition bias) & $\begin{array}{l}\text { alised estimating equation models were used to account for data missing at } \\
\text { random }\end{array}$ &
\end{tabular}

\begin{tabular}{lll}
\hline $\begin{array}{l}\text { Selective reporting (re- } \\
\text { porting bias) }\end{array}$ & Unclear risk & Protocol or trial register not found \\
\hline Other bias & Low risk & No additional threats to validity \\
\hline $\begin{array}{l}\text { Other bias- timing of re- } \\
\text { cruitment of clusters }\end{array}$ & Low risk & Figure indicates recruitment happened prior to randomisation \\
\hline
\end{tabular}

Santos 2014

\begin{tabular}{|c|c|}
\hline \multirow[t]{9}{*}{ Methods } & Study name: Healthy buddies \\
\hline & Study design: cluster-RCT \\
\hline & Intervention period: 10 months \\
\hline & Follow-up period (post-intervention): nil \\
\hline & Differences in baseline characteristics: NR \\
\hline & Reliable outcomes: reported \\
\hline & Protection against contamination: NR \\
\hline & Unit of allocation: school \\
\hline & Unit of analysis: individual accounting for clustering \\
\hline \multirow[t]{9}{*}{ Participants } & $\mathrm{N}($ control baseline $)=347$ \\
\hline & $N$ (control follow-up) $=273$ \\
\hline & $\mathrm{N}$ (intervention baseline) $=340$ \\
\hline & $\mathrm{N}$ (intervention follow-up) $=310$ \\
\hline & Setting (and number by study group): 20 elementary schools ( 10 in each group, 5 urban and 5 rural) \\
\hline & Recruitment: schools randomly selected \\
\hline & Geographic region: Manitoba Province in Canada \\
\hline & Percentage of eligible population enrolled: $7 \%$ schools enrolled then 20 randomly selected \\
\hline & Mean age: intervention: $9.3 \pm 9.1-9.5$; control: $8.8 \pm 8.6-9.0$ \\
\hline
\end{tabular}


Santos 2014 (Continued)

Sex: intervention: $54 \%$ female; control: $48 \%$ female

Interventions To test the hypothesis that a school-based, peer-led healthy living programme would reduce adiposity and increase PA among children.

- Teachers delivering the 'Healthy buddies' lesson plans attended a 2-day training seminar at the beginning of the 2009-2010 academic school year

- -21 lesson plans were provided to teachers to be delivered during the school year to older students (programme content focused on PA, promoting healthy foods, and having a healthy body image using the slogans: "Go Move!" (activity), "Go Fuel!" (nutrition), and "Go Feel Good!" (body image))

- At each intervention school, older class was paired with a younger class. Each week, the older students received a 45-min healthy living lesson from their classroom teacher. Later that week, the older students acted as peer mentors, teaching a 30-minute lesson to their younger 'buddies'.

- As part of the 30-min lessons, The "GoFuel!" component included lessons about distinguishing nutritious from unhealthy foods and beverages. As part of the "Go Feel Good!" component, students were taught to value themselves and classmates based on individual traits rather than peer influence

- For the "Go Move" aspect, two 30-min structured aerobic fitness sessions/week, called fitness loops, with the student pairs

Waiting list control group to receive the intervention after a 1-year delay

Diet and PA combination intervention vs control

Outcome measures
Outcomes
- Primary outcome: waist circumference, zBMI
take
Process evaluation: NR

Implementation-related factors
Theoretical basis: NR

Resources for intervention implementation: reported

Who delivered the intervention: reported

PROGRESS categories assessed at baseline: gender, race/ethnicity, rural/urban

PROGRESS categories analysed at outcome: NR

Outcomes relating to harms/unintended effects: NR

Intervention included strategies to address diversity or disadvantage: NR

Economic evaluation: NR
Funding: role of the sponsor: the funding agency, the Province of Manitoba, helped in the design of the study, enrolling schools to participate and training teachers, but it had no role in the collection of data, statistical analyses, or interpretation of findings or in the preparation, review, or approval of the manuscript.

Teachers delivering the 'Healthy buddies' lesson plans attended a 2-day training seminar; older students providing peer-led lessons were trained by the teachers in their weekly lesson.

\section{Risk of bias}

\begin{tabular}{lll}
\hline Bias & Authors' judgement & Support for judgement \\
\hline $\begin{array}{l}\text { Random sequence genera- } \\
\text { tion (selection bias) }\end{array}$ & Low risk & $\begin{array}{l}\text { Use of computer-generated random sequence, and blocked to ensure equal } \\
\text { representation }\end{array}$
\end{tabular}


Santos 2014 (Continued)

From rural and First Nations (ie, indigenous) schools in both intervention and control arms

\begin{tabular}{|c|c|c|}
\hline $\begin{array}{l}\text { Allocation concealment } \\
\text { (selection bias) }\end{array}$ & Low risk & $\begin{array}{l}\text { Randomisation was performed by an investigator who was not involved in da- } \\
\text { ta collection }\end{array}$ \\
\hline $\begin{array}{l}\text { Blinding (performance } \\
\text { bias and detection bias) } \\
\text { All outcomes }\end{array}$ & Low risk & $\begin{array}{l}\text { Unable to blind control group as on waiting list and parents consented; re- } \\
\text { search assistants who did the measurements were blinded to study assign- } \\
\text { ment }\end{array}$ \\
\hline $\begin{array}{l}\text { Incomplete outcome data } \\
\text { (attrition bias) } \\
\text { All outcomes }\end{array}$ & Low risk & $\begin{array}{l}\text { Low attrition rate and well balanced across groups (if exclude the } 1 \text { school of } \\
40 \text { participants that dropped out immediately post-randomisation) } 89 \% \text { vs } \\
91 \% \text { attrition, control vs intervention }\end{array}$ \\
\hline $\begin{array}{l}\text { Selective reporting (re- } \\
\text { porting bias) }\end{array}$ & Low risk & Protocol/trial registration document seen. All outcomes reported \\
\hline Other bias & Low risk & $\begin{array}{l}\text { Presents baseline characteristics but doesn't report whether there were any } \\
\text { significant differences between groups - however all outcomes were adjusted } \\
\text { for baseline measures }\end{array}$ \\
\hline $\begin{array}{l}\text { Other bias- timing of re- } \\
\text { cruitment of clusters }\end{array}$ & Low risk & $\begin{array}{l}\text { Randomisation occurred after eligibility of schools was determined. } \\
\text { Quote: "See figure 1." }\end{array}$ \\
\hline
\end{tabular}

Sevinc 2011

\begin{tabular}{|c|c|}
\hline \multirow[t]{8}{*}{ Methods } & Study design: cluster-RCT \\
\hline & Intervention period: 8 months \\
\hline & Follow-up period (post-intervention): nil \\
\hline & Differences in baseline characteristics: reported \\
\hline & Reliable outcomes: reported \\
\hline & Protection against contamination: NR \\
\hline & Unit of allocation: group ( 2 schools in each group) \\
\hline & Unit of analysis: individual \\
\hline \multirow[t]{9}{*}{ Participants } & $N($ controls baseline $)=2926$ \\
\hline & $N($ controls follow-up) $=2654$ \\
\hline & $\mathrm{N}$ (intervention 1 baseline) = 1932 \\
\hline & $\mathrm{N}$ (intervention 1 follow-up) = 1897 \\
\hline & $\mathrm{N}$ (intervention 2 baseline) $=1989$ \\
\hline & $\mathrm{N}$ (intervention 2 follow-up) $=1815$ \\
\hline & $\begin{array}{l}\text { Setting (and number by study group): schools, intervention } 1(N=2) \text {, intervention } 2(N=2) \text {, control }(N= \\
\text { 2). Each group comprised of one low-SES and one high-SES school }\end{array}$ \\
\hline & Recruitment: all schools involved in a half-day education system; randomly sampled \\
\hline & Geographic region: Denizli, Turkey \\
\hline
\end{tabular}


Sevinc 2011 (Continued)

Percentage of eligible population enrolled: $98.9 \%$ participants

Mean age: (intervention + control): $7-13$ years

Sex: intervention 1: 50.3\% female; intervention 2: $49.8 \%$ female; control: $49.1 \%$ female

Interventions

Aim: to determine the efficiency of applying both PA and healthy nutrition programmes and only a healthy nutrition programme for preventing obesity in primary school students (aged 7-13) in Denizli, to determine the relationship of this efficiency with the possible variables, and to construct an obesity control programme aimed at the students.

Associated study name: Get into motion for health

Intervention description

Intervention group 1: PA combined with healthy nutrition education programme. Initial weekly PE classes were $2 \mathrm{~h}$ in total and increased to $3 \mathrm{~h}$ on different days of the week. During these sessions standard PA and sport programmes, specific to the age range of the children were applied. Does not state who delivered the PA programme.

Intervention group 2: healthy nutrition education programme only. Education on the importance of healthy nutrition and the methods of preventing obesity were given to the students, their parents, and the teachers. Boxed milk was also distributed to the students for them to drink during meal time. Moreover, to supply healthy eating options for the students in the school canteens, water, freshly squeezed fruit juice, buttermilk, milk and seasonal fruits were sold.

Who delivered/training: personnel of the Health Training Division of the City Health Administration/teachers

Diet and PA combination intervention vs dietary intervention only vs control

PA intervention vs control (health education)

$\begin{array}{ll}\text { Outcomes } & \text { Outcome measures } \\ \text { - Primary outcome: BMI } & \text { Secondary outcomes: NR } \\ & \text { Process evaluation: NR }\end{array}$

Implementation-related Theoretical basis: NR

factors

Resources for intervention implementation: reported

Who delivered the intervention: reported

PROGRESS categories assessed at baseline: gender; parent: SES (income)

PROGRESS categories analysed at outcome: gender; parent: SES (income)

Outcomes relating to harms/unintended effects: NR

Intervention included strategies to address diversity or disadvantage: targeted both low- and high-SES schools

Economic evaluation: NR

Notes $\quad$ Funding: NR

In one of the schools in intervention group 1, some of the equipment required for the PA programme could not be obtained, and an insufficiency in directing the school canteens to supply healthy food instead of fast food and carbonated drinks might be counted among the limitations of the study. 
Sevinc 2011 (Continued)

Risk of bias

\begin{tabular}{|c|c|c|}
\hline Bias & Authors' judgement & Support for judgement \\
\hline $\begin{array}{l}\text { Random sequence genera- } \\
\text { tion (selection bias) }\end{array}$ & Unclear risk & $\begin{array}{l}\text { From the low- and high-value SES regions, } 3 \text { schools each (a total of } 6 \text { schools) } \\
\text { were selected by using a simple random sampling method. These schools } \\
\text { were randomly divided into } 3 \text { groups consisting of } 1 \text { school from the low- and } 1 \\
\text { school from the high-SES level. Of these groups, } 2 \text { were again randomly select- } \\
\text { ed as intervention groups and the remaining } 1 \text { as the control group }\end{array}$ \\
\hline $\begin{array}{l}\text { Allocation concealment } \\
\text { (selection bias) }\end{array}$ & Unclear risk & NR \\
\hline $\begin{array}{l}\text { Blinding (performance } \\
\text { bias and detection bias) } \\
\text { All outcomes }\end{array}$ & Unclear risk & NR \\
\hline $\begin{array}{l}\text { Incomplete outcome data } \\
\text { (attrition bias) } \\
\text { All outcomes }\end{array}$ & Low risk & $94 \%$ retention of those 'reached' \\
\hline $\begin{array}{l}\text { Selective reporting (re- } \\
\text { porting bias) }\end{array}$ & Unclear risk & Protocol or trial register not found \\
\hline Other bias & Low risk & No additional threats to validity \\
\hline $\begin{array}{l}\text { Other bias- timing of re- } \\
\text { cruitment of clusters }\end{array}$ & Low risk & Figures suggest recruitment happened prior to randomisation \\
\hline
\end{tabular}

Shin 2015

Methods
Study design: RCT. Dyads were recruited. 1 child and 1 caregiver
Intervention period: 8 months
Follow-up period (post-intervention): nil
Differences in baseline characteristics: reported
Reliable outcomes: reported
Protection against contamination: NR
Unit of allocation: youth-caregiver dyads
Unit of analysis: individual
N (control baseline) $=242$ intervention and control
N (control follow-up) $=63$
N (intervention follow-up) 89
Setting (and number by study group): community: 7 recreation centres and 21 corner shops (interven-
tion) and 7 recreation centres (control)
Recruitment: recreation centres randomly selected
Geographic region: East and West Baltimore, USA


Shin 2015 (Continued)

Percentage of eligible population enrolled: $63 \%$

Mean age: intervention: 13.0 (1.6); control: 13.0 (1.4

Sex: intervention: $59.6 \%$ female; control: $57.1 \%$ female

Interventions

To increase availability and selection of healthful foods through nutrition promotion and education.

During the intervention, materials and activities, such as taste tests, cooking demonstrations, giveaways, shelf labels, and point-of-purchase health communication materials such as posters and flyers, were introduced in intervention recreation centres, local corner stores, and carryout restaurants. Interventions in each venue were interconnected and reinforced each other.

Each of the intervention's 5 phases focused on a single aspect of healthful eating: healthful beverages, healthful breakfast, cooking at home/healthful lunch, healthful snacks, and selecting more healthful options at carryout restaurants.

Youth peer educators were recruited from each intervention recreation centre and trained by interventionists to assist in health promotions.

Diet intervention vs control

Outcome measures
- Primary outcomes: behavioural intentions, self-efficacy, knowledge, and outcome expectancies, food
purchasing and preparation patterns, BMI for age percentiles
- Secondary outcomes: NR
Process evaluation: NR

Implementation-related factors
Theoretical basis: SCT

Resources for intervention implementation: reported

Who delivered the intervention: reported

PROGRESS categories assessed at baseline: gender, education

PROGRESS categories analysed at outcome: NR

Outcomes relating to harms/unintended effects: NR

Intervention included strategies to address diversity or disadvantage: intervention targeted already overweight low-income African American youth living in an environment where healthful foods are less available

Economic evaluation: NR

Notes

Funding: the study authors received no financial support for the research, authorship, and/or publication of this article. Resources NR in great detail; no information about control

\section{Risk of bias}

\begin{tabular}{lll}
\hline Bias & Authors' judgement & Support for judgement \\
\hline $\begin{array}{l}\text { Random sequence genera- } \\
\text { tion (selection bias) }\end{array}$ & Unclear risk & Randomisation, no further details \\
\hline $\begin{array}{l}\text { Allocation concealment } \\
\text { (selection bias) }\end{array}$ & Unclear risk & NR \\
\hline $\begin{array}{l}\text { Blinding (performance } \\
\text { bias and detection bias) }\end{array}$ & Unclear risk & NR \\
\hline
\end{tabular}


Shin 2015 (Continued)

All outcomes

Incomplete outcome data High risk $\quad 63 \%$ retention of dyads (152/242). 38\% clusters lost
(attrition bias)

(attrition bias)

All outcomes

Selective reporting (re- Unclear risk $\quad$ Protocol or trial register not found
porting bias)
porting bias)

Other bias Low risk No additional threats to validity

\section{Sichieri 2009}

\begin{tabular}{ll}
\hline Methods & Study design: cluster-RCT \\
Intervention period: 7 months of 1 school year \\
Follow-up period (post-intervention): nil \\
Differences in baseline characteristics: reported \\
Reliable outcomes: reported \\
Protection against contamination: reported \\
Unit of allocation: school \\
Unit of analysis: individual with clustering by class \\
All analyses were performed according to ITT principles
\end{tabular}

\section{Participants}

$$
\begin{aligned}
& N(\text { controls baseline })=608 \\
& N(\text { controls follow-up })=493 \\
& N(\text { interventions baseline })=526 \\
& N(\text { interventions follow-up })=434
\end{aligned}
$$

Setting (and number by study group): 47 classes ( $N=23$ intervention; $N=24$ control) in 22 schools

Recruitment: all 4th graders from 22 public schools in metropolitan city of Niteroi were invited to participate.

Geographic region: Niteroi, Rio de Janeiro, Brazil

Percentage of eligible population enrolled: $98 \%$

Mean age: intervention: $10.9 \pm 0.81$; control: $10.9 \pm 0.75$

Sex: intervention, $53.1 \%$ female; control, $52.6 \%$ female

- Healthy lifestyle education programme, social marketing

- Simple messages encouraging water instead of SSB

- Formative and developmental work performed prior

- Classroom quizzes, games, activities to promote water over SSB

- Children make drawings and songs

- $10 \times 1$-h sessions of activities facilitated by 4 trained researchers who were assigned for each class 
Sichieri 2009 (Continued)

- Activities required 20-30 min and teachers encouraged to reinforce the messages during their lessons

- Printed materials provided to RAs and music teachers to facilitate sessions

Dietary interventions vs control

Outcome measures
- Primary outcome: change in BMI, carbonated SSB and juice intake
- Secondary outcomes: overweight and obesity
Process evaluation: NR

Implementation-related factors
Theoretical basis: NR

Resources for intervention implementation (e.g. funding needed or staff hours required): reported Who delivered the intervention: reported

PROGRESS categories assessed at baseline: reported (gender, race)

PROGRESS categories analysed at outcome: reported (gender)

Outcomes relating to harms/unintended effects: NR

Intervention included strategies to address diversity or disadvantage: NR

Economic evaluation: NR

$\begin{array}{ll}\text { Notes } & \text { Funding: the study was supported by the Brazilian National Research Council - CNPq. Grant number: } \\ 500404 / 2003-8-\text { CNPq }\end{array}$

\section{Risk of bias}

\begin{tabular}{lll}
\hline Bias & Authors' judgement & Support for judgement \\
\hline $\begin{array}{l}\text { Random sequence genera- } \\
\text { tion (selection bias) }\end{array}$ & Unclear risk & NR \\
& $\begin{array}{l}\text { Quote: "We began the study by ranking schools based on the prevalence of } \\
\text { overweight and of obesity, and randomisation was generated by blocking of } \\
\text { four schools. The last two in the list were randomly assigned to intervention or } \\
\text { control groups, balancing the groups by BMI." }\end{array}$ \\
\hline $\begin{array}{l}\text { Allocation concealment } \\
\text { (selection bias) }\end{array}$ & Low risk & Randomisation at school level and all schools randomised at start of study \\
\hline $\begin{array}{l}\text { Blinding (performance } \\
\text { bias and detection bias) } \\
\text { All outcomes }\end{array}$ & Unclear risk & NR \\
\hline
\end{tabular}

Incomplete outcome data Low risk

19\% overall individual loss and balanced across groups; ITT done

(attrition bias)

All outcomes

Selective reporting (re- High risk
porting bias)

Trial registration found. BMI was not listed in the trial registration report, but is listed in the outcome data of the trial report. Therefore this outcome is at high risk of bias.

\begin{tabular}{lll}
\hline Other bias & Low risk & No additional threats to validity \\
\hline $\begin{array}{l}\text { Other bias- timing of re- } \\
\text { cruitment of clusters }\end{array}$ & Low risk & Figure shows recruitment happened prior to randomisation \\
\hline \hline
\end{tabular}




Methods
Study name: JuvenTUM Project
Intervention period: 12 months
Follow-up period (post-intervention): nil
Differences in baseline characteristics: reported
Reliable outcomes: reported
Protection against contamination: reported
Unit of allocation: schools
Unit of analysis: individual

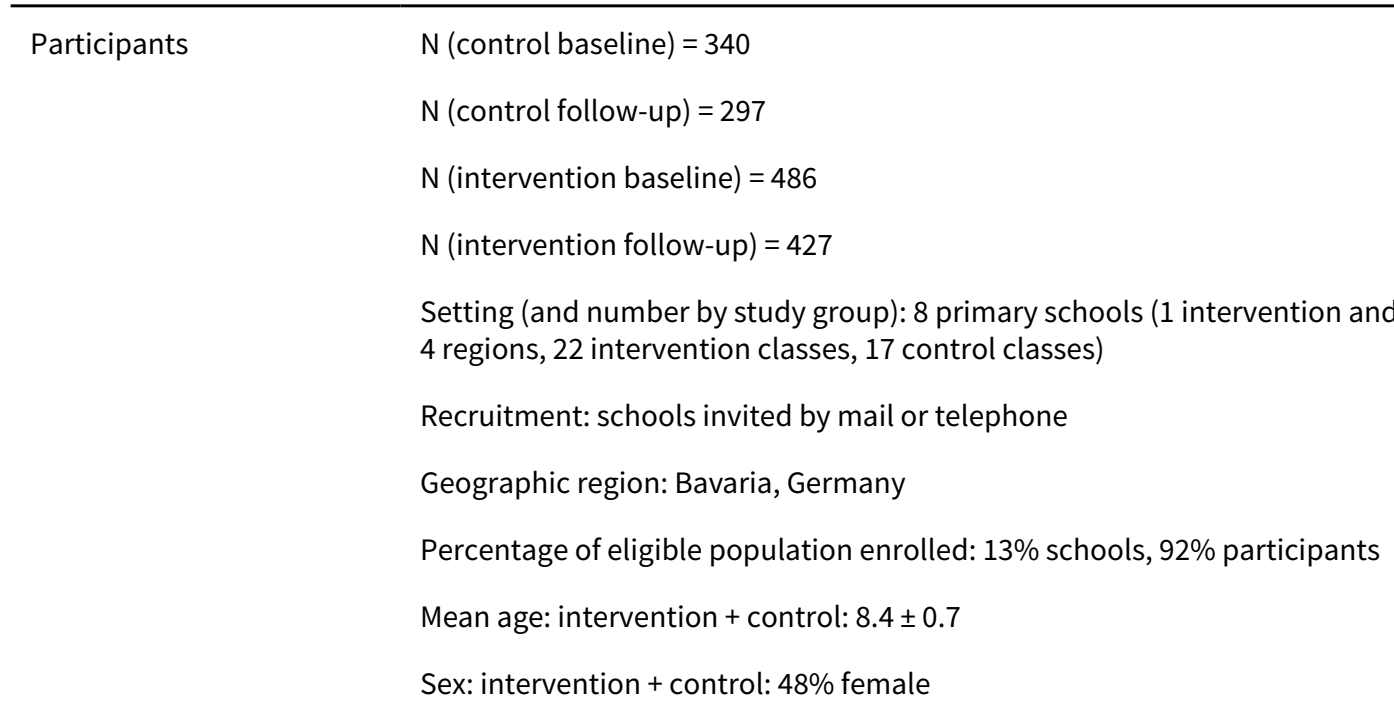

Interventions

To evaluate a simple and ubiquitously applicable school-based educational programme to increase PA, fitness, and life-style awareness and to improve health obesity measures.

- PE lessons: $45 \mathrm{~min} / \mathrm{month}$ given by trained PE teachers (in addition to usual 2-3 45-min lessons given by usual teachers)

- Training sessions for the teachers with info about health-related topics, games and examples for active breaks and organisation of school-specific improvements (playground, healthy break, teacher health)

- Training sessions for the parents with info about health-related topics, games and example for active breaks and sports activities for families.

- Journals with info for families about PA, sports possibilities and little homework tasks for the families

- School environmental settings (e.g. the physical environment, organisation of school breaks, playing during school time, and sports facilities) were altered to promote more PA. These changes were designed to increase physical movement, promote healthier food availability and choices (more vegetables and fruits and less energy-dense food), and reduce media consumption.

Diet + PA combination intervention vs control

Outcomes

Outcome measures

- Primary outcome: PA

- Secondary outcomes: BMI, BMI SD score, waist circumference, physical fitness, media consumption 
Siegrist 2013 (Continued)

Process evaluation: NR

Implementation-related factors

Theoretical basis: NR
Resources for intervention implementation: reported

Who delivered the intervention: reported

PROGRESS categories assessed at baseline: NR

PROGRESS categories analysed at outcome: NR

Outcomes relating to harms/unintended effects: reported (underweight)

Intervention included strategies to address diversity or disadvantage: NR

Economic evaluation: NR
NCT00988754

Funding: this work has been funded by a grant from the Bavarian State Ministry of the Environment and Public Health (Gesund.Leben.Bayern) (LP 00001-FA 08).

Baseline waist circumference was less in the control group $(P=0.035)$, adjusted for in analyses; no significant change was observed for children below the 10th percentile

\section{Risk of bias}

\begin{tabular}{|c|c|c|}
\hline Bias & Authors' judgement & Support for judgement \\
\hline $\begin{array}{l}\text { Random sequence genera- } \\
\text { tion (selection bias) }\end{array}$ & Unclear risk & Randomisation, no further details \\
\hline $\begin{array}{l}\text { Allocation concealment } \\
\text { (selection bias) }\end{array}$ & Unclear risk & NR \\
\hline $\begin{array}{l}\text { Blinding (performance } \\
\text { bias and detection bias) } \\
\text { All outcomes }\end{array}$ & Low risk & $\begin{array}{l}\text { Quote: "all children, parents and teachers needed to be informed about the } \\
\text { group allocation. The main co-ordinator of the study is also not blinded to the } \\
\text { group assignments of the schools. However, the medical examiners are not } \\
\text { aware of the group allocation of the participating children. The medical exam- } \\
\text { iners were responsible for measuring anthropometry." }\end{array}$ \\
\hline $\begin{array}{l}\text { Incomplete outcome data } \\
\text { (attrition bias) } \\
\text { All outcomes }\end{array}$ & Low risk & $12 \%$ attrition equally balanced \\
\hline $\begin{array}{l}\text { Selective reporting (re- } \\
\text { porting bias) }\end{array}$ & Low risk & $\begin{array}{l}\text { Protocol seen; all outcomes reported to some extent - but not all data in ta- } \\
\text { bles i.e. media consumption, sports club participation were reported. However } \\
\text { these outcomes are not analysed within this review. }\end{array}$ \\
\hline Other bias & Low risk & No additional threats to validity. \\
\hline $\begin{array}{l}\text { Other bias- timing of re- } \\
\text { cruitment of clusters }\end{array}$ & Low risk & See figure 1 . Recruitment happened before randomisation \\
\hline
\end{tabular}

\section{Simon 2008}

Methods Study design: cluster-RCT


Simon 2008 (Continued)

Intervention period: 4 years

Follow-up period (post-intervention): nil

Differences in baseline characteristics: reported

Reliable outcomes: reported

Protection against contamination: reported

Unit of allocation: school

Unit of analysis: school, Individual

Sensitivity analysis conducted using ITT population to compare this with analysis using data from only those participants who completed the study.

\begin{tabular}{|c|c|}
\hline \multirow[t]{10}{*}{ Participants } & $\mathrm{N}($ controls baseline $)=479($ blood samples $N=326)$ \\
\hline & $\mathrm{N}$ (controls follow-up) $=358$ \\
\hline & $\mathrm{N}$ (interventions baseline) $=475$ (blood samples $\mathrm{N}=304)$ \\
\hline & $\mathrm{N}$ (interventions follow-up) $=374$ \\
\hline & Setting (and number by study group): 8 schools ( 4 in each group) \\
\hline & $\begin{array}{l}\text { Recruitment: } 4 \text { pairs of matched schools randomly selected out of } 77 \text { public middle schools of the De- } \\
\text { partment of Bas-Rhin. All 6th graders in randomised schools were eligible. }\end{array}$ \\
\hline & Geographic region: Eastern France \\
\hline & Percentage of eligible population enrolled: $91 \%$ (surveys); $73 \%$ (blood samples) \\
\hline & Mean age: intervention: $11.7 \pm 0.7$; control: $11.6 \pm 0.6$ \\
\hline & Sex: intervention: $52.6 \%$ female; control: $47.4 \%$ female \\
\hline \multirow[t]{8}{*}{ Interventions } & Programme began during 1st school year and ran until end of 4 th school year \\
\hline & - Educational component focusing on PA and sedentary behaviours \\
\hline & $\begin{array}{l}\text { - New opportunities for PA offered in lunchtime, breaks and after-school hours taking account of bar- } \\
\text { riers to PA }\end{array}$ \\
\hline & - Activities organised by formal physical educators, no competitive aspect \\
\hline & - Enjoyment highlighted to help less confident children \\
\hline & - Sporting events and cycling to school days \\
\hline & - Parents and educators encouraged to support PA through regular meetings \\
\hline & PA interventions vs control \\
\hline
\end{tabular}

Outcomes

- Primary outcome: BMI

- Secondary outcomes:

- self-reported leisure PA? assessed with the Modifiable Activity Questionnaire for adolescents.

- time spent in front of TV/video and in active commuting between home and school

- self-efficacy and intention toward PA (lower scores indicating better outcomes) were assessed with the Stanford Adolescent Heart Health Programmes questionnaire

- CV risk factors

Process evaluation: reported

Implementation-related factors
Theoretical basis: Behaviour Change and SEM

Resources for intervention implementation (e.g. funding needed or staff hours required): NR 
Simon 2008 (Continued)

Who delivered the intervention: reported

PROGRESS categories assessed at baseline: reported (gender, SES)

PROGRESS categories analysed at outcome: reported (gender, SES)

Outcomes relating to harms/unintended effects: NR

Intervention included strategies to address diversity or disadvantage: NR

Economic evaluation: NR

NCT00498459
Funding: this study was supported by grants from The Regional Health Insurance of Alsace-Moselle; Na-
tional Program of Research in Human Nutrition (INSERM and INRA); French Public Authorities within
the National Nutritional Health Program and through the Youth and Sports Department; Conseil Gener-
al du Bas-Rhin; Municipalities of Drusenheim, Illkirch-Graffenstaden, Obernai and Schiltigheim and The
International Longevity Centre. The funding sponsors had no role in the design and protocol develop-
ment of the study, in data collection, analysis and interpretation or in manuscript preparation.

\section{Risk of bias}

\begin{tabular}{|c|c|c|}
\hline Bias & Authors' judgement & Support for judgement \\
\hline $\begin{array}{l}\text { Random sequence genera- } \\
\text { tion (selection bias) }\end{array}$ & Low risk & $\begin{array}{l}\text { Randomisation of } 77 \text { schools included stratification, it would be therefore be } \\
\text { reasonable to assume this process was mediated with a computer }\end{array}$ \\
\hline $\begin{array}{l}\text { Allocation concealment } \\
\text { (selection bias) }\end{array}$ & Low risk & Randomised at the school level and all schools randomised at start of study \\
\hline $\begin{array}{l}\text { Blinding (performance } \\
\text { bias and detection bias) } \\
\text { All outcomes }\end{array}$ & Unclear risk & NR \\
\hline $\begin{array}{l}\text { Incomplete outcome data } \\
\text { (attrition bias) } \\
\text { All outcomes }\end{array}$ & Low risk & $24 \%$ individual loss, balanced. ITT done \\
\hline $\begin{array}{l}\text { Selective reporting (re- } \\
\text { porting bias) }\end{array}$ & Low risk & Protocol/trial registration document seen. All outcomes reported \\
\hline Other bias & Low risk & No additional threats to validity \\
\hline $\begin{array}{l}\text { Other bias- timing of re- } \\
\text { cruitment of clusters }\end{array}$ & Low risk & Randomisation happened before enrolment \\
\hline
\end{tabular}

Singh 2009

Study design: cluster-RCT
Intervention period: 8 months
Follow-up period (post-intervention): 4 months and 12 months post-intervention (12-and 20-month
observations respectively)
Differences in baseline characteristics: reported
Reliable outcomes: reported


Singh 2009 (Continued)

Protection against contamination: reported

Unit of allocation: school

Unit of analysis: individual with multilevel analysis that included student, class, school

All analyses were performed according to ITT principles

\begin{tabular}{ll}
\hline Participants & $N$ (controls baseline) $=476$ \\
& $N$ (controls follow-up $)=$ NR by group \\
& $N$ (interventions baseline $)=632$ \\
& $N$ (interventions follow-up) $=$ NR by group
\end{tabular}

Setting (and number by study group): schools ( $N=10$ intervention; $N=8$ control). Targeted adolescents with lower socio-economic and educational level. 3 classes in each school were included.

Recruitment: participating schools were asked to select 3 classes of 1st-year students. Selection of classes was based on practical reasons.

Geographic region: The Netherlands

Percentage of eligible population enrolled: $84 \%$

Mean age: intervention: boys $=12.8 \pm 0.5$, girls $=12.6 \pm 0.5$; control: boys $=12.9 \pm 0.5$, girls $=12.7 \pm 0.5$

Sex: intervention: $53 \%$ female; control: $47 \%$ female

- Reduction in consumption of SSBs

- Reduction in consumption of high-sugar, high-fat-content snacks

- Reduction in sedentary behaviour

- Increase in active transport behaviour

- Maintenance of level of sports participation

- Individual component: - educational programme covering 11 biology and PE lessons

- Environmental component:

- School-specific advice on selection of school canteen and possible change options

- Financial encouragement of schools to offer additional PA options

- Utilised the Intervention Mapping Protocol, which facilitates a systematic process of designing health promotion interventions and based on theory and empirical evidence

- Behaviour change methods used:

- self-monitoring, self-evaluation

- reward

○ increasing skills

- goal setting

- environmental changes

- social encouragement

- social support

- information regarding behaviour

- personalised messages

Combined effects of dietary interventions and PA interventions vs control 
Singh 2009 (Continued)

- Primary outcome

- Changes in body composition (i.e. waist circumference, skinfold thickness and BMI)

- Secondary outcomes

- Changes in dietary and PA behaviour (EBRBs)

- Consumption of SSBs (i.e. consumption of soft drinks and fruit juices)

- Consumption of high-energy snacks (i.e. consumption of savoury snacks and sweet snacks)

- Screen-viewing behaviour (i.e. time spent on TV viewing and computer use)

- Active commuting to school

Process evaluation: reported

Implementation-related factors
Theoretical basis: reported (Intervention mapping protocol, Behaviour Change and Environmental frameworks)

Resources for intervention implementation (e.g. funding needed or staff hours required): reported

Who delivered the intervention: reported

PROGRESS categories assessed at baseline: reported (gender, race)

PROGRESS categories analysed at outcome: reported (gender, race)

Outcomes relating to harms/unintended effects: NR

Intervention included strategies to address diversity or disadvantage: reported

Economic evaluation: NR
Funding: this study is part of the Netherlands Research Programme for Weight Gain Prevention and is funded by grant $2000 Z 002$ from the Netherlands Heart Foundation, the Dutch Ministry of Health, Welfare, and Sports, and the Royal Association of Teachers of PE (KVLO). None of the funders had input into protocol development, data collection, or analyses or interpretation.

Protocol published separately. Refer to secondary references for Singh 2009: Singh et al. BMC Public Health 2006, 6:304 doi:10.1186/1471-2458-6-304 and Singh et al. Arch Pediatr Adolesc Med 2007;161:565-571 for 8-month outcome data.

\section{Risk of bias}

\begin{tabular}{lll}
\hline Bias & Authors' judgement & Support for judgement \\
\hline $\begin{array}{l}\text { Random sequence genera- } \\
\text { tion (selection bias) }\end{array}$ & Low risk & $\begin{array}{l}\text { Quote: "the schools were randomly assigned to either the intervention or con- } \\
\text { trol group, using SPSS statistical software (SPSS Inc, Chicago, Ill) for random } \\
\text { selection of a sample" }\end{array}$ \\
\hline $\begin{array}{l}\text { Allocation concealment } \\
\text { (selection bias) }\end{array}$ & Low risk & $\begin{array}{l}\text { Randomisation occurred at the school level and was performed on all units at } \\
\text { the start of the study }\end{array}$ \\
\hline $\begin{array}{l}\text { Blinding (performance } \\
\text { bias and detection bias) }\end{array}$ & High risk & $\begin{array}{l}\text { Research assistants involved in conducting measurements and delivering } \\
\text { intervention materials were not blinded. Other members of the research } \\
\text { team who helped with the measurements were blinded. After randomisation, } \\
\text { schools were informed about the group allocation }\end{array}$ \\
\hline $\begin{array}{l}\text { Incomplete outcome data } \\
\begin{array}{l}\text { (attrition bias) } \\
\text { All outcomes }\end{array}\end{array}$ & Low risk & $\begin{array}{l}17 \% \text { and } 21 \% \text { loss of individuals in the control and intervention schools re- } \\
\text { spectively; ITT done. }\end{array}$ \\
& & $\begin{array}{l}\text { Quote: "All analyses were performed according to the intention-to-treat princi- } \\
\text { ple. Missing values were not imputed" }\end{array}$ \\
\hline
\end{tabular}


Singh 2009 (Continued)

Selective reporting (re- Low risk Study protocol seen; all outcomes specified were reported porting bias)

\begin{tabular}{lll}
\hline Other bias & Low risk & No additional threats to validity \\
\hline $\begin{array}{l}\text { Other bias- timing of re- } \\
\text { cruitment of clusters }\end{array}$ & Low risk & Figure shows recruitment happened prior to randomisation \\
\hline
\end{tabular}

\section{Skouteris 2016}

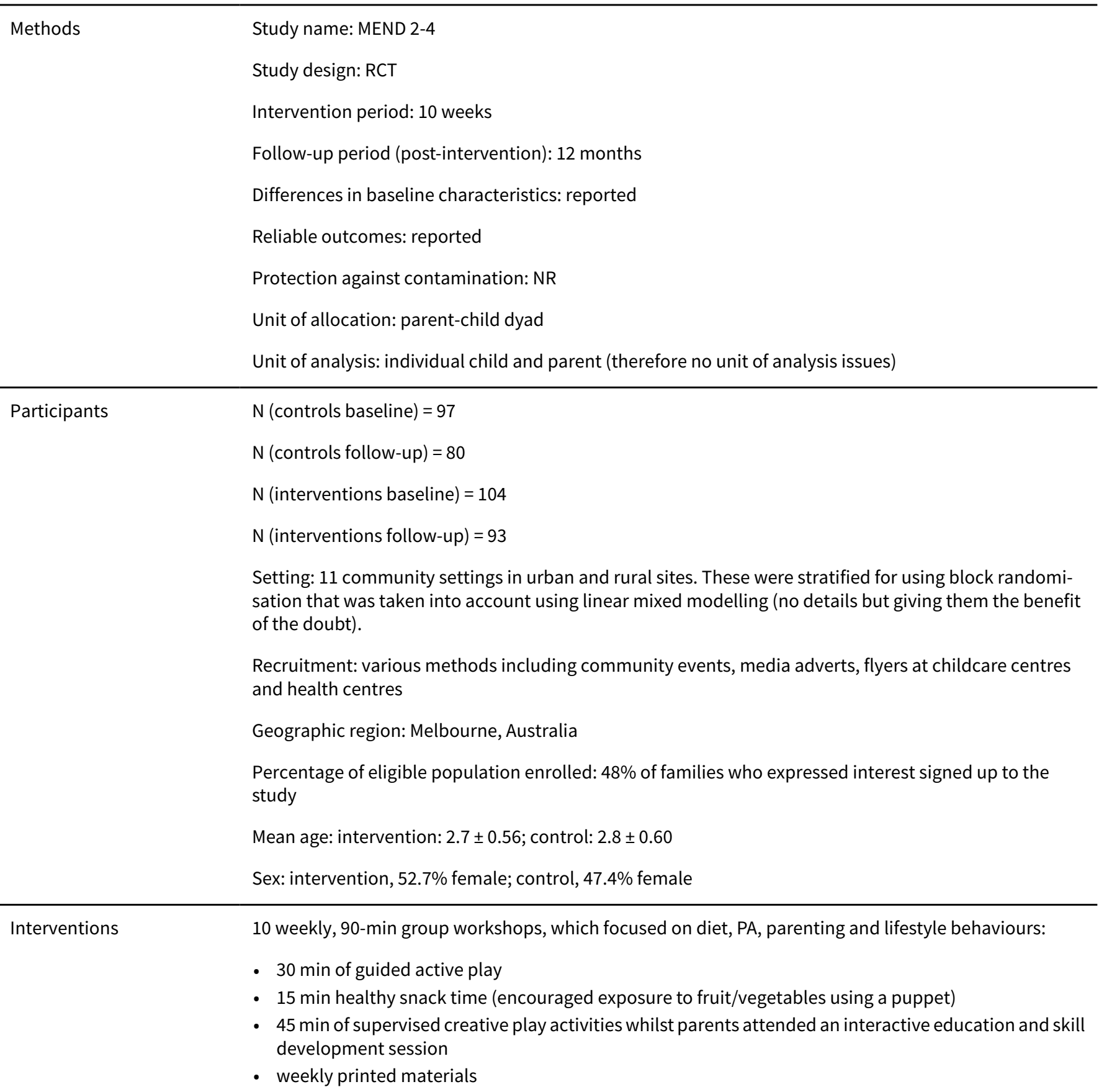


Skouteris 2016 (Continued)

- workshops delivered by trained MEND leaders (nurse or childcare worker, trained via a 2-day MEND training course). MEND leaders were monitored regularly

The waiting list control group did not receive any intervention, but were offered the programme at study completion.

Diet and PA combination intervention vs control

Outcome measures
- Primary outcome: dietary intake, vegetable intake, fruit intake, high-energy snack foods, sweet drinks,
water, plain milk, eating habits, fussiness, satiety responsiveness, and neophobia
- Secondary outcomes: PA, sedentary behaviour, zBMI
Process evaluation: "Programmes were implemented as intended"

Implementation-related

Theoretical basis: learning and social cognitive theories

factors

Resources for intervention implementation: reported

Who delivered the intervention: reported

PROGRESS categories assessed at baseline: gender; parent: SES (family income), education, occupation, marital status

PROGRESS categories analysed at outcome: NR

Outcomes relating to harms/unintended effects: NR

Intervention included strategies to address diversity or disadvantage: NR but does state that, "despite targeting recruitment strategies at families who were at high risk of being in need of an obesity prevention intervention, children in our study sample fell mainly in the healthy weight range"

Economic evaluation: NR

Notes

ACTRN12610000200088

Funding: this study was funded by an Australian Research Council Linkage Grant (ARC LP100100049)

A voucher draw (supermarket vouchers worth AUD 50-250) encouraged participant retention.

\section{Risk of bias}

\begin{tabular}{lll}
\hline Bias & Authors' judgement & Support for judgement \\
\hline $\begin{array}{l}\text { Random sequence genera- } \\
\text { tion (selection bias) }\end{array}$ & Low risk & Computer-generated, randomised in blocks pertaining to their community site \\
\hline $\begin{array}{l}\text { Allocation concealment } \\
\text { (selection bias) }\end{array}$ & Low risk & Concealed in opaque envelopes \\
\hline $\begin{array}{l}\text { Blinding (performance } \\
\text { bias and detection bias) } \\
\text { All outcomes }\end{array}$ & Low risk & $\begin{array}{l}\text { Outcome assessors blind to allocation, programme facilitators and partici- } \\
\text { pants not blinded }\end{array}$ \\
\hline $\begin{array}{l}\text { Incomplete outcome data } \\
\text { (attrition bias) }\end{array}$ & Unclear risk & $\begin{array}{l}\text { Differential dropout: 18\% attrition in intervention and 14\% attrition in control } \\
\text { All outcomes }\end{array}$ \\
\hline $\begin{array}{l}\text { Selective reporting (re- } \\
\text { porting bias) }\end{array}$ & Low risk & Protocol/trial registration document seen. All outcomes reported \\
\hline
\end{tabular}


Skouteris 2016 (Continued)
Other bias
Low risk
No additional threats to validity

Slusser 2012

$\begin{array}{ll}\text { Methods } & \text { Study design: RCT } \\ \text { Intervention period: } 17 \text {-weeks (10 cohorts over } 17 \text { months) } \\ \text { Follow-up period (post-intervention): } 35 \text { weeks } \\ \text { Differences in baseline characteristics: reported } \\ \text { Reliable outcomes: reported } \\ \text { Protection against contamination: NR } \\ \text { Unit of allocation: individual } \\ \text { Unit of analysis: individual }\end{array}$

Participants
$\begin{aligned} & \mathrm{N} \text { (control baseline) }=80 \\ & \mathrm{~N}(\text { control follow-up) }=37 \\ & \mathrm{N} \text { (intervention baseline) }=80 \\ & \mathrm{~N} \text { (intervention follow-up) }=44 \\ & \text { Setting (and number by study group): healthcare clinic preschools including Head start, family centre } \\ & \text { and Children's Bureau serving low-income predominantly Latino families } \\ & \text { Recruitment: at clinic visits or in classrooms of community sites (Latino with at least } 1 \text { child 2-4 years) } \\ & \text { Geographic region: Los Angeles, USA } \\ & \text { Percentage of eligible population enrolled: } 100 \% \\ & \text { Mean age: intervention + control: } 2-4 \\ & \text { Sex: intervention: } 55.7 \% \text { female; control: } 56.7 \% \text { female }\end{aligned}$

Interventions To examine the effectiveness of a multicomponent parent training programme on the prevention of overweight and obesity among Latino children aged 2-4

Parent training intervention to promote optimal nutrition and PA. Used a bilingual social worker as a facilitator for the classes. $7 \times 90$-min weekly modules and 2 booster sessions, 1 /month after the end of the 7 weeks and final booster session a month later. Included parent homework.

Wait list control group

Diet and PA combination intervention vs control

\begin{tabular}{ll}
\hline Outcomes & Outcome measures \\
& - Primary outcome: zBMI \\
& Process evaluation: NR
\end{tabular}

$\begin{array}{ll}\begin{array}{l}\text { Implementation-related } \\ \text { factors }\end{array} & \text { Theoretical basis: Social Learning theory } \\ & \text { Resources for intervention implementation: reported }\end{array}$


Who delivered the intervention: reported

PROGRESS categories assessed at baseline: child, race/ethnicity; parent: race/ethnicity, marital status, SES (health insurance)

PROGRESS categories analysed at outcome: NR

Outcomes relating to harms/unintended effects: NR

Intervention included strategies to address diversity or disadvantage: culturally modified

Economic evaluation: NR

Notes

Funding: study was funded by the generous gifts of: Joseph Drown Foundation, Simms/Mann Family Foundation, and Venice Family Clinic.

Study analyses focuses on subset of children with a BMI > 50th percentile at baseline

\section{Risk of bias}

\begin{tabular}{|c|c|c|}
\hline Bias & Authors' judgement & Support for judgement \\
\hline $\begin{array}{l}\text { Random sequence genera- } \\
\text { tion (selection bias) }\end{array}$ & Low risk & $\begin{array}{l}\text { Stratified by gender and BMI percentile and randomly assigned using comput- } \\
\text { er program }\end{array}$ \\
\hline $\begin{array}{l}\text { Allocation concealment } \\
\text { (selection bias) }\end{array}$ & Low risk & $\begin{array}{l}\text { Stratified by gender and BMI percentile and randomly assigned using comput- } \\
\text { er program }\end{array}$ \\
\hline $\begin{array}{l}\text { Blinding (performance } \\
\text { bias and detection bias) } \\
\text { All outcomes }\end{array}$ & Low risk & $\begin{array}{l}\text { The current paper reports the results of anthropometric assessments compar- } \\
\text { ing } \mathrm{t} 1 \text { and } \mathrm{t} 3 \text { with } \mathrm{t} 3 \text { measurements administered by an } \\
\text { assessor who was not aware of group assignment. }\end{array}$ \\
\hline $\begin{array}{l}\text { Incomplete outcome data } \\
\text { (attrition bias) } \\
\text { All outcomes }\end{array}$ & High risk & $\begin{array}{l}21 \% \text { and } 29 \% \text { attrition (intervention and control respectively), also excluded } \\
\text { from analysis all children with baseline BMIs }<50 \text { th percentile ( } 24 \% \text { interven- } \\
\text { tion and } 25 \% \text { control). There was differential dropout in this subset that was } \\
\text { accounted for in the analyses. }\end{array}$ \\
\hline $\begin{array}{l}\text { Selective reporting (re- } \\
\text { porting bias) }\end{array}$ & Unclear risk & Protocol or trial register not found \\
\hline Other bias & Low risk & No additional threats to validity \\
\hline
\end{tabular}

Smith 2014

\section{Methods}

Study name: Active teen leaders avoiding screen-time (ATLAS)

Study design: cluster-RCT

Intervention period: 8 months

Follow-up period (post-intervention): 10 months

Differences in baseline characteristics: reported

Reliable outcomes: reported

Protection against contamination: NR

Unit of allocation: school 
Smith 2014 (Continued)

Unit of analysis: individual accounting for clustering

\begin{tabular}{|c|c|}
\hline \multirow[t]{10}{*}{ Participants } & $\mathrm{N}($ control baseline $)=284$ \\
\hline & $\mathrm{N}$ (control follow-up) = 195 \\
\hline & $\mathrm{N}$ (intervention baseline) $=283$ \\
\hline & $\mathrm{N}$ (intervention follow-up) = 191 \\
\hline & $\begin{array}{l}\text { Setting (and number by study group): } 14 \text { co-educational public secondary schools in areas with } \\
\text { cioeconomic index (SEI) value of } \leq 5 \text { (lowest } 50 \% \text { ): } 7 \text { schools in each group }\end{array}$ \\
\hline & Recruitment: NR \\
\hline & Geographic region: Newcastle, Hunter, and Central Coast regions of New South Wales, Australia \\
\hline & Percentage of eligible population enrolled: $70 \%$ schools, $42 \%$ participants \\
\hline & Mean age: intervention: $12.7 \pm 0.5$; control: $12.7 \pm 0.5$ \\
\hline & Sex: intervention: $0 \%$ female; control: $0 \%$ female \\
\hline
\end{tabular}

Interventions

To evaluate the effects of a multicomponent, school-based obesity prevention intervention incorporating smartphone technology on weight and health behaviours of male adolescents,

teacher professional development, provision of fitness equipment to schools, face-to-face PA sessions, lunchtime student mentoring sessions, researcher-led seminars, a smartphone application and website, and parental strategies for reducing screen-time

'ATLAS' is a multicomponent intervention designed to prevent unhealthy weight gain by increasing PA, reducing screen-time, and lowering SSB consumption among adolescent boys attending schools in low-income areas:

- For teachers

- Teacher professional development: two 6-h workshops (pre-programme and mid-programme to provide a rationale for the programme and outline the intervention strategies (ie, programme components, behavioural messages))

- 1 fitness instructor session (each school received 1 visit during their regularly scheduled sport session from a practicing fitness instructor (i.e. personal trainer) while the teacher observed)

- For parents

- 4 parent newsletters

- For students

- 3 × 20-min researcher-led seminars (seminars provide key information surrounding the programme's components and behavioural messages, including current recommendations regarding youth PA, screen-time, and RT)

- $20 \times 90$-min enhanced school sport sessions (sport sessions delivered by teachers at the study schools, behavioural messages reinforced during cool down times)

- Lunchtime PA-mentoring sessions $(6 \times 20$ min sessions, recruiting and instructing grade 7 boys in elastic tubing RT)

- Constant pedometer and ATLAS smartphone app access (15-17 weeks, smartphone app and website are used for PA monitoring, recording of fitness challenge results, tailored motivational messaging, peer assessment of RT skills, and goal-setting for PA and screen time)

The control group participated in usual practice (i.e. regularly scheduled school sports and PE lessons) for the duration of the intervention but received an equipment pack and a condensed version of the programme after the 18-month follow-up assessments.

PA intervention vs control 
Smith 2014 (Continued)

- Primary outcome: BMI, waist circumference

- Secondary outcomes: $\%$ body fat, PA, screen time, SSB intake, muscular fitness, RT skill competency

Process evaluation: reported (implementation)

Implementation-related factors
Theoretical basis: Self-determination theory and SCT

Resources for intervention implementation: reported

Who delivered the intervention: reported

PROGRESS categories assessed at baseline: race/ethnicity, SES

PROGRESS categories analysed at outcome: NR

Outcomes relating to harms/unintended effects: reported

Intervention included strategies to address diversity or disadvantage: targeted boys at risk of obesity:

failing to meet international PA or screen-time guidelines

Economic evaluation: NR

\section{ACTRN 12612000978864}

Funding: this study was funded by an Australian Research Council Discovery Project grant (DP120100611). The sponsor had no involvement in the design or implementation of the study, in analyses of data, or in the drafting of the manuscript.

An equipment pack valued at approximately AUD 1000 (including pedometers, elastic tubing devices, boxing gloves, focus pads and hanging gym handles) was provided to each school if needed.

On average, schools conducted $79 \%$ of intended school sports sessions and $64 \%$ of intended lunchtime sessions. Sixty-five percent of boys attended $\$ 70 \%$ of the sport sessions but only $44 \%$ of boys attended at least two-3rds of lunchtime sessions.

Participant satisfaction with the ATLAS intervention was high, but satisfaction with the lunchtime sessions was somewhat lower. Smartphone (or similar device) ownership was reported by $70 \%$ of boys, and $63 \%$ reported using either the iPhone or Android version of the ATLAS app. Almost one-half of the group agreed or strongly agreed that the "push prompt" messages reminded them to be more active, reduce their screen-time, and drink fewer sugary drinks, and $44 \%$ of participants agreed or strongly agreed that the ATLAS app was enjoyable to use. Teacher satisfaction with the intervention was high.

\section{Risk of bias}

\begin{tabular}{lll}
\hline Bias & Authors' judgement & Support for judgement \\
\hline $\begin{array}{l}\text { Random sequence genera- } \\
\text { tion (selection bias) }\end{array}$ & Low risk & $\begin{array}{l}\text { Randomisation was performed by an independent researcher with the use of } \\
\text { a computer-based random number-producing algorithm. }\end{array}$ \\
\hline $\begin{array}{l}\text { Allocation concealment } \\
\text { (selection bias) }\end{array}$ & Low risk & Randomisation was performed by an independent researcher with the use of \\
& $\begin{array}{l}\text { a computer-based random number-producing algorithm. Also assessors were } \\
\text { blinded to treatment allocation at baseline but not at follow-up. }\end{array}$
\end{tabular}

$\begin{array}{ll}\begin{array}{l}\text { Blinding (performance } \\ \text { bias and detection bias) }\end{array} & \text { High risk } \\ \text { All outcomes } & \begin{array}{l}\text { Assessors were blinded to treatment allocation at baseline but not at fol- } \\ \text { low-up. }\end{array}\end{array}$

Incomplete outcome data Low risk

Retention $85.6 \%$ at 8 months and $76.8 \%$ at 18 months; ITT done

(attrition bias)

All outcomes 
Smith 2014 (Continued)

Selective reporting (re- Low risk Protocol not sought; all outcomes specified in methods have been reported
porting bias)

\begin{tabular}{lll}
\hline Other bias & Low risk & No additional threats to validity \\
\hline $\begin{array}{l}\text { Other bias- timing of re- } \\
\text { cruitment of clusters }\end{array}$ & Low risk & Figure shows recruitment happened prior to randomisation \\
\hline
\end{tabular}

\section{Spiegel 2006}

\begin{tabular}{|c|c|}
\hline \multirow[t]{8}{*}{ Methods } & Study design: cluster-RCT \\
\hline & Intervention period: 5-6 months \\
\hline & Follow-up period (post-intervention): nil \\
\hline & Differences in baseline characteristics: NR \\
\hline & Reliable outcomes: reported \\
\hline & Protection against contamination: reported \\
\hline & Unit of allocation: classroom \\
\hline & Unit of analysis: individual (not adjusted for clustering by classroom) \\
\hline \multirow[t]{10}{*}{ Participants } & $\mathrm{N}$ (controls baseline) $=572$ \\
\hline & $\mathrm{N}$ (controls follow-up) $=479$ \\
\hline & $\mathrm{N}$ (interventions baseline) $=619$ \\
\hline & $\mathrm{N}$ (interventions follow-up) $=534$ \\
\hline & Setting: classrooms in schools \\
\hline & $\begin{array}{l}\text { Recruitment: 4th and 5th graders from } 16 \text { schools (69 classes) in four states (Delaware, Florida, Kansas, } \\
\text { and North Carolina) }\end{array}$ \\
\hline & Geographic region: USA \\
\hline & Percentage of eligible population enrolled: NR \\
\hline & Mean age: NR (4th and 5th graders; ages 9-10) \\
\hline & Sex: both male and female \\
\hline \multirow[t]{7}{*}{ Interventions } & - The WAY intervention programme was teacher-led \\
\hline & - Intervention teachers participated in workshops and received programme materials. \\
\hline & $\begin{array}{l}\text { - The programme was integrated throughout the school year with activities ranging in engagement } \\
\text { time from } 20 \mathrm{~min} \text { to more extensive activities that require } \geq 1 \mathrm{~h}\end{array}$ \\
\hline & $\begin{array}{l}\text { - Students were engaged in multidisciplinary activities in language arts, mathematics, science, and } \\
\text { health content, building their academic skills while developing their health attitudes, behavioural in- } \\
\text { tent, and, ultimately, behaviour }\end{array}$ \\
\hline & - Used directed-reflective journaling and class discussions with students that were reinforced over time \\
\hline & $\begin{array}{l}\text { - Students were provided with an orientation to the programme and activities through video and print } \\
\text { resources }\end{array}$ \\
\hline & $\begin{array}{l}\text { - Intervention classes followed a 10-min aerobic exercise routine each day during class time. The video } \\
\text { provided a common baseline exercise routine for all intervention classes }\end{array}$ \\
\hline
\end{tabular}


Spiegel 2006 (Continued)

- The programme activities were organised into 7 discrete modules.

- Module 1 orients students to the programme and the concept of wellness and has them record a baseline description of their understanding, interpretations, and attitudes of themselves and wellness.

- Module 2 is where the students learn how to collect, report, and analyse data about themselves and their health and reflect on their attitudes and beliefs related to the data

- and their health behaviours.

- Module 3 focuses on PA and fitness. Students learn about the F.I.T.T. (Frequency, Intensity, Time, and Technique) principles, how to design a basic workout routine, and how to incorporate PA into their daily routine

- Module 4 addresses nutrition and diet

- Module 5 is where students learn more about their bodies (how they move, the parts and systems of their bodies); how their behaviours influence their bodies; how researchers learn about their bodies (medical technology and research); how to be a good consumer of health information; and basic information and attitudes about disease transmission.

- Module 6 provides an orientation to genetics and family health history as a resource to examining personal health.

- Module 7 is where students practice the information and skills they learned in class. They conclude the year with a review of their personal goals and a personal assessment of their progress toward the goal

Combined effects of dietary interventions and PA interventions vs control

\begin{tabular}{ll}
\hline Outcomes & Height, weight \\
& - Diet (survey) \\
& PA levels (survey) \\
Process evaluation: reported
\end{tabular}

Implementation-related factors

Theoretical basis: reported (Theory of Reasoned Action, Constructivism)

Resources for intervention implementation (e.g. funding needed or staff hours required): reported

Who delivered the intervention: reported

PROGRESS categories assessed at baseline: reported (place, SES)

PROGRESS categories analysed at outcome: NR

Outcomes relating to harms/unintended effects: NR

Intervention included strategies to address diversity or disadvantage: reported

Economic evaluation: NR

Notes

Funding: this study was commissioned by the Institute for America's Health, a not-for-profit 501 (c)3 organisation striving to enhance the health of all Americans through research and education (www.healthy-america.org).

\section{Risk of bias}

\begin{tabular}{lll}
\hline Bias & Authors' judgement & Support for judgement \\
\hline $\begin{array}{l}\text { Random sequence genera- } \\
\text { tion (selection bias) }\end{array}$ & Unclear risk & $\begin{array}{l}\text { Quote: "To reduce sample bias, participants in the intervention and compar- } \\
\text { ison groups at each school were selected through random sampling tech- } \\
\text { niques." }\end{array}$ \\
\hline $\begin{array}{l}\text { Allocation concealment } \\
\text { (selection bias) }\end{array}$ & Unclear risk & $\begin{array}{l}\text { Quote: "Intervention and comparison classes were randomly selected at each } \\
\text { school" }\end{array}$ \\
\hline
\end{tabular}


Spiegel 2006 (Continued)

Blinding (performance $\quad$ Unclear risk $\quad$ NR
bias and detection bias)

All outcomes

\begin{tabular}{|c|c|c|}
\hline $\begin{array}{l}\text { Incomplete outcome data } \\
\text { (attrition bias) } \\
\text { All outcomes }\end{array}$ & Unclear risk & $\begin{array}{l}\text { Numbers randomised or analysed NR, only that total was } 1013 \text { and groups } \\
\text { were } 479 \text { (control) and } 534 \text { (intervention) but they say low attrition. } \\
\text { Quote: "There was a } 16.2 \% \text { attrition rate in the comparison group (N } 479 \\
\text { matched measures between baseline to post-data) and a } 13.7 \% \text { attrition rate } \\
\text { in the intervention group (N } 534 \text { matched)." }\end{array}$ \\
\hline
\end{tabular}

\begin{tabular}{lll}
\hline $\begin{array}{l}\text { Selective reporting (re- } \\
\text { porting bias) }\end{array}$ & Unclear risk & Protocol or trial register not found \\
\hline Other bias & High risk & Risk of contamination \\
\hline $\begin{array}{l}\text { Other bias- timing of re- } \\
\text { cruitment of clusters }\end{array}$ & Unclear risk & NR \\
\hline
\end{tabular}

\section{Stolley 1997}

$\begin{array}{ll}\text { Methods } & \begin{array}{l}\text { Study design: RCT } \\ \text { Intervention period: } 12 \text { weeks }\end{array} \\ & \text { Follow-up (post-intervention): nil } \\ \text { Differences in baseline characteristics: reported } \\ \text { Reliable outcomes: reported } \\ \text { Protection against contamination: not possible } \\ \text { Unit of allocation: child } \\ \text { Unit of analysis: child }\end{array}$

\begin{tabular}{|c|c|}
\hline \multirow[t]{3}{*}{ Participants } & $\begin{array}{l}N \text { (intervention baseline })=32 \text { mothers and } 32 \text { daughters } \\
N \text { (control baseline })=30 \text { mothers and } 33 \text { daughters } \\
N \text { (intervention follow-up) }=20 \text { mothers and } 23 \text { daughters have dietary data reported however, stated } \\
\text { that in all } 51 \text { mothers }(78 \%) \text { and } 54 \text { daughters }(83 \%) \text { had data collected }\end{array}$ \\
\hline & $\begin{array}{l}\text { Unable to separate intervention from control figures with data provided } \\
\text { Geographical setting: Chicago, USA }\end{array}$ \\
\hline & $\begin{array}{l}\text { Age: } 7-12 \text { years; mean age intervention } 9.9 \text { (SD 1.3); controls } 10.0 \text { (SD 1.5) years } \\
\text { Sex: girls only }\end{array}$ \\
\hline \multirow[t]{2}{*}{ Interventions } & $\begin{array}{l}\text { Set up within a community-based tutoring programme this intervention examined the effectiveness of } \\
\text { a culturally specific obesity-prevention programme for low-income, inner-city African American, pread- } \\
\text { olescent girls and their mothers. } \\
\text { Programme focused on adopting a low-fat, low-calorie diet and increased activity. } \\
\text { Controls were offered a general health programme. }\end{array}$ \\
\hline & Combined effects of dietary interventions and PA interventions vs control \\
\hline \multirow[t]{2}{*}{ Outcomes } & $\begin{array}{l}\text { Mother and daughters: } \\
\circ \text { body weight and height } \\
\circ \% \text { overweight } \\
\circ \text { daily caloric intake, total fat gram intake, \% calories from fat, saturated fat, dietary cholesterol } \\
\text { assessed by Quick Check for Fat (QCF) and analysed with Quick Check Diet (QCD). }\end{array}$ \\
\hline & - Parental completion of a self-report measure of parental support and role modelling around food. \\
\hline
\end{tabular}


Stolley 1997 (Continued)

Process evaluation: reported

Implementation-related factors

\section{Theoretical basis: NR}

Resources for intervention implementation (e.g. funding needed or staff hours required): NR

Who delivered the intervention: reported

PROGRESS categories assessed at baseline: reported (occupation, gender, education, SES)

PROGRESS categories analysed at outcome: NR

Outcomes relating to harms/unintended effects: NR

Intervention included strategies to address diversity or disadvantage: reported

Economic evaluation: NR

Notes $\quad$ Funding: non-industry. This project was supported by grants from the American Heart Association of Metropolitan Chicago

\section{Risk of bias}

\begin{tabular}{lll}
\hline Bias & Authors' judgement & Support for judgement \\
\hline $\begin{array}{l}\text { Random sequence genera- } \\
\text { tion (selection bias) }\end{array}$ & Unclear risk & NR \\
\hline $\begin{array}{l}\text { Allocation concealment } \\
\text { (selection bias) }\end{array}$ & Unclear risk & NR but there was no baseline imbalance \\
\hline $\begin{array}{l}\text { Blinding (performance } \\
\text { bias and detection bias) } \\
\text { All outcomes }\end{array}$ & Unclear risk & NR \\
\hline $\begin{array}{l}\text { Incomplete outcome data } \\
\text { (attrition bias) } \\
\text { All outcomes }\end{array}$ & High risk & $\begin{array}{l}78 \% \text { of mothers completed the study with a difference in weight between } \\
\text { completers and dropouts. Thinner mothers were more likely to drop out (P< } \\
0.05)\end{array}$ \\
\hline $\begin{array}{l}\text { Selective reporting (re- } \\
\text { porting bias) }\end{array}$ & Unclear risk & Protocol or trial register not found \\
\hline \begin{tabular}{l} 
Other bias \\
\hline
\end{tabular} & Low risk & No additional threats to validity \\
\hline
\end{tabular}

Story 2003

$\begin{array}{ll}\text { Methods } & \text { Study design: RCT } \\ & \text { Follow-up: } 12 \text { weeks } \\ & \text { Differences in baseline characteristics: reported } \\ & \text { Reliable outcomes: reported } \\ \text { Protection against contamination: NR } & \text { Unit of allocation: child } \\ \text { Unit of analysis: child }\end{array}$

\begin{tabular}{ll}
\hline Participants & N (controls baseline) $=27$ \\
& N (controls follow-up) $=27$ \\
& N (intervention baseline) $=26$ \\
& N (intervention follow-up) $=26$
\end{tabular}


Story 2003 (Continued)

Proportion of eligibles participating: not stated, but criteria kept broad. Intended to recruit 50 and 61 were enrolled

Geographical setting: Minnesota, USA

Mean age: intervention 9.4 (SD 0.9); controls 9.1 (SD 0.8) years

Sex: girls only

\begin{abstract}
Interventions
- After-school classes set in schools designed to improve skill building and practice in support of health behaviour messages in the programme.

- Included drinking water, eating more fruit, vegetables and low fat foods, increasing PA reducing TV watching and enhancing self-esteem.

- The intervention was delivered by African American GEMS staff. Family contact and activities supported the intervention.
\end{abstract}

Controls received a 12-week programme unrelated to nutrition and PA (enhancing self-esteem and cultural enrichment)

Combined effects of dietary interventions and PA interventions vs control

- BMI
- Waist circumference
- Physical maturation
- DEXA for \% body fat
- PA: CSA accelerometer, a modification of the Self-Administered PA Checklist (SAPAC), GEMS Activity
Questionnaire(GAQ) computerised
- Dietary intake measured by two 24-h recalls using NDS-R
- Psychological variables:
$\circ$ body silhouettes McKnight Risk Factor Survey, and Stunkard 1983
$\circ$ Healthy choice behavioural intentions (diet)
$\circ$ Self-efficacy for healthy eating
$\circ$ PA outcomes expectations, and a self-efficacy measure.
Process evaluation: reported

Implementation-related factors

Theoretical basis: SCT, youth development, and resiliency based approach

Resources for intervention implementation (e.g. funding needed or staff hours required): reported Who delivered the intervention: reported

PROGRESS categories assessed at baseline: reported (race, education, SES)

PROGRESS categories analysed at outcome: NR

Outcomes relating to harms/unintended effects: reported

Intervention included strategies to address diversity or disadvantage: reported

Economic evaluation: NR

Notes

\title{
Risk of bias
}

\begin{tabular}{lll}
\hline Bias & Authors' judgement & Support for judgement \\
\hline $\begin{array}{ll}\text { Random sequence genera- } \\
\text { tion (selection bias) }\end{array}$ & Low risk & $\begin{array}{l}\text { Quote: "...urn randomization procedure was used to generate the treatment al- } \\
\text { location sequences. The different sequences were stored on a computer at the }\end{array}$
\end{tabular}


Story 2003 (Continued)

CC, and accessed using an interactive voice-response telephone system." (See secondary reference for Story 2003 ,

\begin{tabular}{lll}
\hline $\begin{array}{l}\text { Allocation concealment } \\
\text { (selection bias) }\end{array}$ & Unclear risk & NR \\
\hline $\begin{array}{l}\text { Blinding (performance } \\
\text { bias and detection bias) }\end{array}$ & Unclear risk & NR \\
All outcomes & \\
\hline
\end{tabular}

Incomplete outcome data Low risk

(attrition bias)

Missing data minimal (1 participant)

All outcomes

\begin{tabular}{lll}
\hline $\begin{array}{l}\text { Selective reporting (re- } \\
\text { porting bias) }\end{array}$ & Low risk & $\begin{array}{l}\text { Protocol seen; all outcomes from the protocol are in papers and some addi- } \\
\text { tional outcomes are in papers as well }\end{array}$ \\
\hline Other bias & Low risk & No additional threats to validity \\
\hline
\end{tabular}

Story 2012

\begin{tabular}{|c|c|}
\hline \multirow[t]{9}{*}{ Methods } & Study name: Bright start \\
\hline & Study design: cluster-RCT \\
\hline & Intervention period: 2 school years (14 weeks in kindergarten and 31 weeks during 1st grade) \\
\hline & Follow-up period (post-intervention): nil \\
\hline & Differences in baseline characteristics: reported \\
\hline & Reliable outcomes: reported \\
\hline & Protection against contamination: NR \\
\hline & Unit of allocation: school \\
\hline & Unit of analysis: individual accounting for clustering \\
\hline \multirow[t]{10}{*}{ Participants } & $\mathrm{N}$ (control baseline $)=187$ \\
\hline & $\mathrm{N}$ (control follow-up) = 187 \\
\hline & $\mathrm{N}$ (intervention baseline) $=267$ \\
\hline & $\mathrm{N}$ (intervention follow-up) = 267 \\
\hline & Setting (and number by study group): 14 kindergarten schools on a Native American Indian reservation \\
\hline & $\begin{array}{l}\text { Recruitment: all } 14 \text { schools on the reservation were recruited into the study in } 1 / 2 \text { cohorts of } 6 \text { and } 8 \\
\text { schools, respectively }\end{array}$ \\
\hline & Geographic region: Pine Ridge Reservation in South Dakota, USA \\
\hline & Percentage of eligible population enrolled: $96 \%$ participants \\
\hline & Mean age: intervention: 5.84 ; control: 5.76 \\
\hline & Sex: intervention: $48 \%$ female; control: $50 \%$ female \\
\hline
\end{tabular}


Story 2012 (Continued)

Interventions
To reduce excessive weight gain by increasing PA and healthy eating practices through changes in school and household environments

The goals of the intervention were to: increase PA at school to at least $60 \mathrm{~min} /$ day; modify school meals and snacks; and involve families in making behavioural and environmental changes at home.

- PA at school

- Aim was $60 \mathrm{~min} /$ day (during school days). Led by class teachers and PE teachers "Kindergarten and first grade teachers were trained in all approaches through a two-day structured training." "PE teachers were trained by a CATCH PE expert to incorporate CATCH PE. "Teachers were provided with an "Action Toolbox" of various easy and developmentally age-appropriate ways to implement exercise throughout the school day."

- Playground equipment such as balls and jump ropes

- Healthy eating at school

- Daily during school hours throughout intervention as was based on improving school based diet.

- Intervention delivered by teachers and food-service staff.

- "Food-service staff at the intervention schools were trained during each of the two years on specific goals, including to: offer $1 \%$ white milk instead of $2 \%$ or whole milk, eliminate chocolate or other flavored milks, serve recommended portion sizes, purchase and use lower-calorie/fat foods, offer low-fat salad dressing in a portion-controlled container, provide more fruits and vegetables, and offer second helpings only on fruits and vegetables."

- "Teachers were trained to limit daily snacks in the classroom, and if used, to be only low-fat and low-sugar foods." Teachers were given a large supply of items to be used as rewards instead of food e.g. stickers, stamps, pencils

- Family environment

- 4 family based events ( 3 x family fun nights, 1 x summer event) throughout the intervention period. Unclear how long these were for.

- Motivational phone calls (stopped after 2nd family fun night).

- Quarterly newsletter. Take home incentives provided "e.g. magnets with behavioural messages, refrigerator water dispenser, vegetable steamer, basketball, jump rope, and fresh fruits/vegetables)"

- Bright start research staff set behavioural goals with parents

- Lakota research staff provided motivational telephone calls to parents.

Diet and PA combination intervention vs control

- Primary outcome: BMI, \% body fat, prevalence of overweight and obesity

- Secondary outcomes: \% of calories from fat and nutrient content in school meals, duration of PA at school, and food intake at home

Process evaluation: reported

Implementation-related factors
Theoretical basis: NR

Resources for intervention implementation: reported

Who delivered the intervention: reported

PROGRESS categories assessed at baseline: gender

PROGRESS categories analysed at outcome: gender

Outcomes relating to harms/unintended effects: NR

Intervention included strategies to address diversity or disadvantage: targeted Native American Indian children, intervention was tailored to the Lakota language and Native American culture

Economic evaluation: NR 
Story 2012 (Continued)

Notes
Funding: this research was supported by Grant \# 1 R01 HL078846 from the NIH, Bethesda, MD, USA.

Based on parent report and school records, 99.3\% of children were of Native American Indian heritage, with almost all children from what is commonly known as the Oglala Sioux Tribe, but more correctly the Lakota people.

Motivational phone calls had to be stopped due to logistics of using cell phones as means of communication. There are drop spot areas on the reservation with no phone signal and many phones had no voice mail.

\section{Risk of bias}

\begin{tabular}{|c|c|c|}
\hline Bias & Authors' judgement & Support for judgement \\
\hline $\begin{array}{l}\text { Random sequence genera- } \\
\text { tion (selection bias) }\end{array}$ & Unclear risk & Randomisation, no further details \\
\hline $\begin{array}{l}\text { Allocation concealment } \\
\text { (selection bias) }\end{array}$ & Unclear risk & Randomisation, no further details \\
\hline $\begin{array}{l}\text { Blinding (performance } \\
\text { bias and detection bias) } \\
\text { All outcomes }\end{array}$ & Unclear risk & NR \\
\hline $\begin{array}{l}\text { Incomplete outcome data } \\
\text { (attrition bias) } \\
\text { All outcomes }\end{array}$ & Low risk & $\begin{array}{l}\text { Very low attrition - there were only } 3 \text { children whose families moved from in- } \\
\text { tervention to control schools; data for children were analysed according to the } \\
\text { original assignments of study condition. }\end{array}$ \\
\hline $\begin{array}{l}\text { Selective reporting (re- } \\
\text { porting bias) }\end{array}$ & Low risk & Protocol/trial registration document seen. All outcomes reported \\
\hline Other bias & Low risk & No additional threats to validity \\
\hline $\begin{array}{l}\text { Other bias- timing of re- } \\
\text { cruitment of clusters }\end{array}$ & Unclear risk & $\begin{array}{l}\text { No CONSORT figure but text shows recruitment happened prior to randomisa- } \\
\text { tion. }\end{array}$ \\
\hline
\end{tabular}

Telford 2012

\begin{tabular}{ll} 
Methods & Study design: cluster-RCT \\
Intervention period: 2 years \\
Follow-up period (post-intervention): nil \\
Differences in baseline characteristics: reported \\
Reliable outcomes: reported \\
Protection against contamination: reported \\
Unit of allocation: schools \\
Unit of analysis: individual accounting for clustering \\
N (control baseline) $=$ NR \\
N (control follow-up) $=308$ \\
N (intervention baseline) $=$ NR \\
\hline Participants
\end{tabular}


Telford 2012 (Continued)

$\mathrm{N}$ (intervention follow-up) $=312$

Setting (and number by study group): 13 elementary schools ( 32 classes) to the specialist-taught PE group and 16 schools ( 36 classes) to control PE group

Recruitment: schools recruited from an Australian education jurisdiction through invitations to the principals in 2005 (as part of the 'Lifestyle of our kids' study)

Geographic region: Canberra, Australia

Percentage of eligible population enrolled: $97 \%$ schools

Mean age: grade 3, no further details (age 8/9?)

Sex: intervention, $49 \%$ female; control, $48 \%$ female

Interventions

Aim: to investigate whether PE delivered by visiting specialist PE teachers in elementary schools influenced the academic performance and body composition of mid-elementary school children.

2 classes of 45-50 min of PE per week for 75/80 weeks of school over the 2-year period. The general classroom teachers associated with the specialist-taught group conducted the remaining 50-60 min of $\mathrm{PE}$ in 2 or 3 extra sessions per week.

The content of the specialist PE differed from the common practice PE in various ways:

Median \% of class time devoted to vigorous PA was $14.6 \%$ ( $21.5 \%$ in common practice). Devoted significantly more lesson time to activities related to fitness, including strength, flexibility and static and dynamic postural activities ( $17.6 \%$ specialist and $2.1 \%$ common practice). More emphasis placed on strength, balance, and postural control. Teachers spent more time demonstrating and participating in activities.

PA intervention vs control

\begin{tabular}{|c|c|}
\hline Outcomes & $\begin{array}{l}\text { Outcome measures } \\
\text { - Primary outcome: height, weight, BMI, \% body fat, PA, cardiorespiratory fitness, numeracy, reading } \\
\text { and writing scores } \\
\text { - Secondary outcomes: primary/secondary not specified } \\
\text { Process evaluation: reported (dose) }\end{array}$ \\
\hline
\end{tabular}

Implementation-related

Theoretical basis: NR

factors

Resources for intervention implementation: reported

Who delivered the intervention: reported

PROGRESS categories assessed at baseline: gender, race/ethnicity

PROGRESS categories analysed at outcome: gender

Outcomes relating to harms/unintended effects: NR

Intervention included strategies to address diversity or disadvantage: NR

Economic evaluation: NR

Notes

Funding: this research received financial support from the Commonwealth Education Trust (London, UK)

Sustainability and economic viability of the intervention programme was enhanced by an ongoing course of professional development for the classroom teachers provided by the visiting specialists.

\section{Risk of bias}


Telford 2012 (Continued)

\begin{tabular}{|c|c|c|}
\hline Bias & Authors' judgement & Support for judgement \\
\hline $\begin{array}{l}\text { Random sequence genera- } \\
\text { tion (selection bias) }\end{array}$ & Unclear risk & Randomly assigned, no further details \\
\hline $\begin{array}{l}\text { Allocation concealment } \\
\text { (selection bias) }\end{array}$ & Unclear risk & NR \\
\hline $\begin{array}{l}\text { Blinding (performance } \\
\text { bias and detection bias) } \\
\text { All outcomes }\end{array}$ & Unclear risk & NR \\
\hline $\begin{array}{l}\text { Incomplete outcome data } \\
\text { (attrition bias) } \\
\text { All outcomes }\end{array}$ & Unclear risk & $\begin{array}{l}620 \text { had measurements at baseline and 2-year follow-up. } 130 \text { additional chil- } \\
\text { dren had insufficient data from baseline/follow-up, potentially } 17 \% \text { attrition, } \\
\text { groups NR }\end{array}$ \\
\hline $\begin{array}{l}\text { Selective reporting (re- } \\
\text { porting bias) }\end{array}$ & Low risk & Protocol/trial registration document seen. All outcomes reported \\
\hline Other bias & Low risk & No additional threats to validity \\
\hline $\begin{array}{l}\text { Other bias- timing of re- } \\
\text { cruitment of clusters }\end{array}$ & Unclear risk & Text suggests recruitment happened prior to randomisation \\
\hline
\end{tabular}

\section{Thivel 2011}

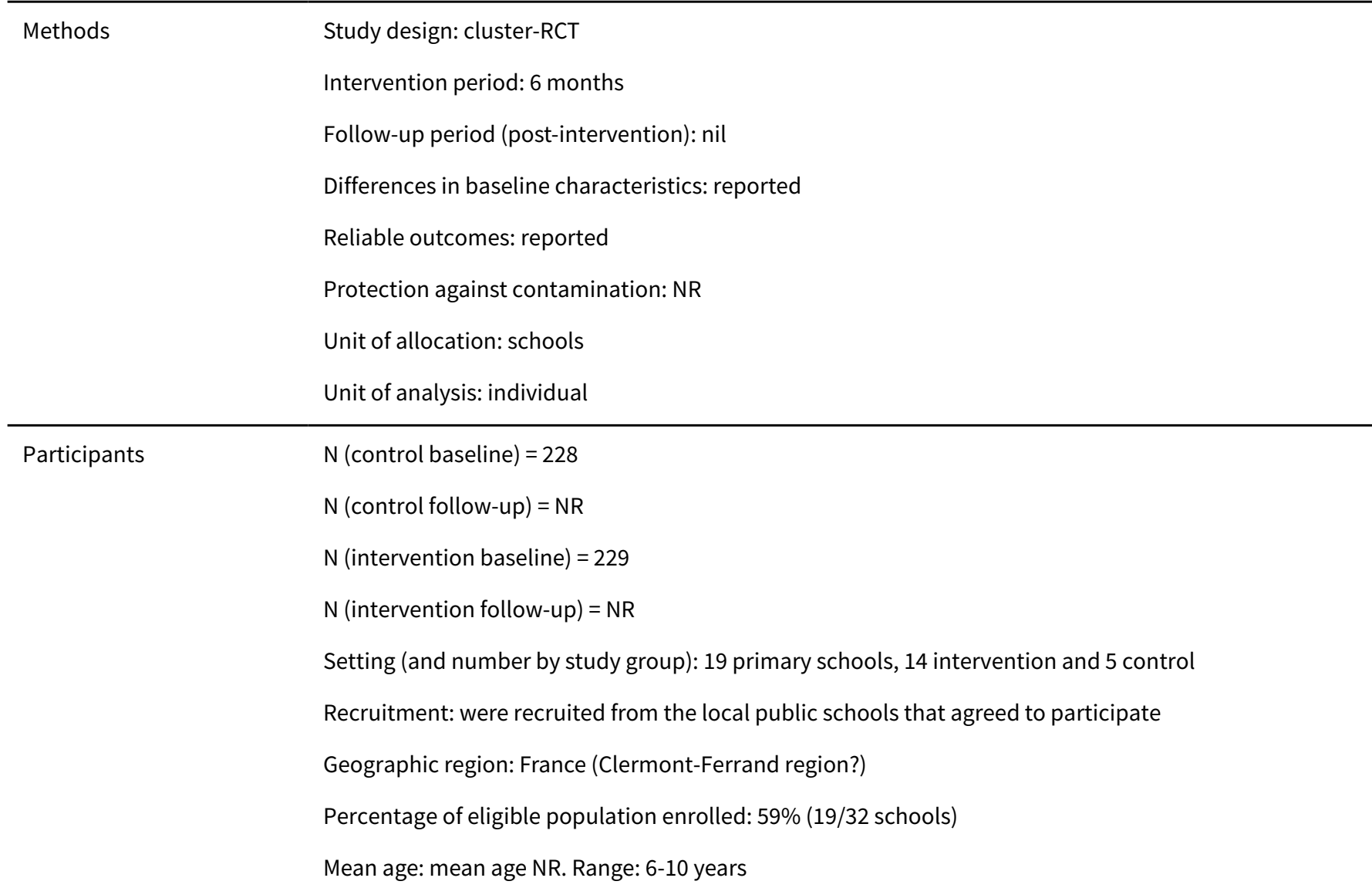


Thivel 2011 (Continued)

Sex: intervention, $52 \%$ female; control, $49 \%$ female
Aim: to assess the effect of a 6-month PA programme on body composition and physical fitness among primary schoolchildren

Primary objective: to increase time spent in PA and minimise inactivity. $120 \mathrm{~min}$ (2 times for $60 \mathrm{~min}$ ) of supervised physical exercise in addition to $2 \mathrm{~h}$ of PE classes/week. The additional sessions consisted of a 10-min warm up followed by psychometric activities and exercises to improve co-ordination, flexibility, strength, speed and endurance. The content of the programme was designed to enhance pleasure and enjoyment during exercise, in order to encourage children's participation in PA during the intervention but also to motivate them to maintain an active lifestyle on a long-term basis.

Who delivered: sports science students. The additional hours per week were managed and taught by sports science students as part of their training; they were themselves supervised by a member of the investigation staff.

Control: children in the control group did not have any intervention and followed their habitual $2 \mathrm{~h}$ of PA education per week

PA intervention vs control

Notes

Outcome measures

- Primary outcome: BMI, waist circumference, skinfolds, fat-free mass, physical fitness

- Secondary outcomes: primary/secondary not specified

Process evaluation: NR

Implementation-related factors
Theoretical basis: NR

Resources for intervention implementation: reported

Who delivered the intervention: reported

PROGRESS categories assessed at baseline: gender

PROGRESS categories analysed at outcome: NR

Outcomes relating to harms/unintended effects: NR

Intervention included strategies to address diversity or disadvantage: NR

Economic evaluation: NR

Funding: this study was funded by grants from the French National Plan for Nutrition and Health (PNNS), the Comite Regional Executif des Actions de Sante d'Auvergne (CREAS), the Caisse Régionale d'Assurance Maladie d'Auvergne (CRAMA), the Appert Institutes, the town of Clermont-Ferrand, and the governing bodies of the Clermont-Ferrand school system.

\section{Risk of bias}

\begin{tabular}{lll}
\hline Bias & Authors' judgement & Support for judgement \\
\hline $\begin{array}{l}\text { Random sequence genera- } \\
\text { tion (selection bias) }\end{array}$ & Unclear risk & Randomly assigned, no further details. \\
& $\begin{array}{l}\text { Quote: "Schools that agreed to take part in the study (19 schools) were ran- } \\
\text { domly assigned as interventional (229 children/14 schools) and observational } \\
\text { schools (228 children/five schools)" }\end{array}$ \\
\hline
\end{tabular}

Allocation concealment $\quad$ Unclear risk $\quad$ Randomly assigned, no further details.
(selection bias)

(selection bias) 
Thivel 2011 (Continued)

Quote: "Schools that agreed to take part in the study (19 schools) were randomly assigned as interventional ( 229 children/14 schools) and observational schools (228 children/five schools)"

\begin{tabular}{|c|c|c|}
\hline $\begin{array}{l}\text { Blinding (performance } \\
\text { bias and detection bias) } \\
\text { All outcomes }\end{array}$ & Unclear risk & $\begin{array}{l}\text { No information about people recording the interventions. The children as- } \\
\text { signed in the control group were not aware that an intervention was taking } \\
\text { place in other schools }\end{array}$ \\
\hline
\end{tabular}

\begin{tabular}{lll}
\hline $\begin{array}{l}\text { Incomplete outcome data } \\
\text { (attrition bias) } \\
\text { All outcomes }\end{array}$ & Unclear risk & No reporting of attrition \\
\hline $\begin{array}{l}\text { Selective reporting (re- } \\
\text { porting bias) }\end{array}$ & Unclear risk & Protocol or trial register not found \\
\hline Other bias & Low risk & No additional threats to validity \\
\hline $\begin{array}{l}\text { Other bias- timing of re- } \\
\text { cruitment of clusters }\end{array}$ & Low risk & Schools were recruited after randomisation. See Figure 1 \\
\hline
\end{tabular}

Velez 2010

$\begin{array}{ll}\text { Methods } & \text { Study design: RCT } \\ \text { Intervention period: } 12 \text { weeks } \\ \text { Follow-up period (post-intervention): nil } \\ \text { Differences in baseline characteristics: reported } \\ \text { Reliable outcomes: reported } \\ \text { Protection against contamination: NR } \\ \text { Unit of allocation: individual } \\ \text { Unit of analysis: individual }\end{array}$

$\begin{array}{ll}\text { Participants } & \text { (control baseline) }=\text { NR (31 in intervention and control at baseline) } \\ N \text { (control follow-up) }=13 \\ \text { N (intervention baseline) }=\text { NR } \\ \text { N (intervention follow-up) }=15 \\ \text { Setting (and number by study group): predominantly Hispanic high school } \\ \text { Recruitment: recruited from PE classes } \\ \text { Geographic region: New Jersey, USA } \\ \text { Percentage of eligible population enrolled: NR } \\ \text { Mean age: intervention + control: } 16.14 \pm 0.19 \\ \text { Sex: intervention: } 33 \% \text { female; control: } 54 \% \text { female } \\ \text { To assess the effects of a RT programme on the muscular strength, body composition, and self-concept } \\ \text { of normal Hispanic adolescents and those who are overweight/obese. }\end{array}$


The 12-week RT programme, which consisted of 35-40-min sessions, 3 nonconsecutive days/week, in lieu of PE class.

RT workouts were divided into upper body and lower body days. Because of several school-related schedule conflicts, participants were required to complete $30 / 36$ sessions possible to be included in final analyses.

For each session, the researchers met the participants at the high school weight room and led them through each planned workout in a 1:3 or 1:4 trainer to participant ratio. Participants were exposed to a familiarisation session that included instruction on warming up, equipment use, exercise performance, and rating of perceived exertion (RPE).

Each session began with a 5-min systemic warm-up to increase body temperature and reduce the chance of injury.

Workouts were divided into upper body and lower body days.

The participants performed 2-3 sets of 10-15 repetitions on a subset of upper-body exercises including bench press, seated row, shoulder press, lat pulldowns, flies, bicep curls, and tricep pushdowns or lower-body exercises including squats, Romanian dead lift, leg extensions, leg curls, lunges, and calf raises. Between each of the sets they were allowed to rest for 60-90 seconds permitting an adequate amount of time for recovery.

These exercises were done at a moderate intensity, defined as approximately $80 \%$ of the adolescents' 10RM (repetition maximum) determined earlier. RPE was used to assess the participants' perception of the intensity of the workout and was administered after each exercise was completed. If the participants were able to complete 15 repetitions, the load was increased by $5 \%$ by the researchers

The participants in the control group underwent 12 weeks of the typical PE/health class. Activities included such things as soccer, volleyball, basketball, floor hockey, and other various individual and team sport games performed each day of the week.

Participants to continue eating their normal diet.

PA intervention vs control

Outcomes Outcome measures

Primary outcome: muscular strength, BMI, physical self-perception and self-concept, lean body mass, $\%$ body fat, squat weight, shoulder press weight, seated row weight, bench press weight, fat mass

Secondary outcomes: primary/secondary NR

Process evaluation: NR

Implementation-related factors

\section{Theoretical basis: NR}

Resources for intervention implementation: reported

Who delivered the intervention: reported

PROGRESS categories assessed at baseline: gender

PROGRESS categories analysed at outcome: NR

Outcomes relating to harms/unintended effects: NR

Intervention included strategies to address diversity or disadvantage: NR

Economic evaluation: NR Sports Research Council 
Velez 2010 (Continued)

Risk of bias

\begin{tabular}{|c|c|c|}
\hline Bias & Authors' judgement & Support for judgement \\
\hline $\begin{array}{l}\text { Random sequence genera- } \\
\text { tion (selection bias) }\end{array}$ & Unclear risk & $\begin{array}{l}\text { Matched on \% body fat before being randomly assigned. } \\
\text { Quote: "Subjects were randomly assigned to a control group } \\
\text { (CON; } N=15) \text { or to a resistance training group (RT; } N=13)\end{array}$ \\
\hline $\begin{array}{l}\text { Allocation concealment } \\
\text { (selection bias) }\end{array}$ & Unclear risk & NR \\
\hline $\begin{array}{l}\text { Blinding (performance } \\
\text { bias and detection bias) } \\
\text { All outcomes }\end{array}$ & Unclear risk & NR \\
\hline $\begin{array}{l}\text { Incomplete outcome data } \\
\text { (attrition bias) } \\
\text { All outcomes }\end{array}$ & High risk & $\begin{array}{l}\text { Low attrition ( } 3 / 31 \text { ) but dropouts affected the equivalence achieved for body } \\
\text { composition and weight through the matching technique used, therefore } \\
\text { change scores were calculated to account for baseline values. }\end{array}$ \\
\hline $\begin{array}{l}\text { Selective reporting (re- } \\
\text { porting bias) }\end{array}$ & Unclear risk & Protocol and trial registry report sought but not found \\
\hline Other bias & Low risk & No additional threats to validity \\
\hline
\end{tabular}

Verbestel 2014

Study design: cluster-RCT
Intervention period: 12 months
Follow-up period (post-intervention): nil
Differences in baseline characteristics: reported
Reliable outcomes: reported
Protection against contamination: NR
Unit of allocation: town/municipality
Unit of analysis: individual accounting for clustering

\begin{tabular}{|c|c|}
\hline \multirow[t]{7}{*}{ Participants } & $N($ control baseline $)=65$ \\
\hline & $N$ (control follow-up) $=54$ \\
\hline & $\mathrm{N}$ (intervention baseline) $=126$ \\
\hline & $\mathrm{N}$ (intervention follow-up) = 99 \\
\hline & $\begin{array}{l}\text { Setting (and number by study group): daycare centres in } 6 \text { towns/municipalities ( } 22 \text { in control and } 35 \\
\text { intervention daycare centres) }\end{array}$ \\
\hline & $\begin{array}{l}\text { Recruitment: selection of the } 6 \text { towns/municipalities was based on } 5 \text { socio-economic characteristics } \\
\text { and } 2 \text { communities with a low, } 2 \text { communities with a medium and } 2 \text { communities with a high SES were } \\
\text { selected. In each community, all daycare centres were invited for participation. Within each daycare } \\
\text { centre, parents of all children aged 9-24 months were invited }\end{array}$ \\
\hline & Geographic region: Flanders, Belgium \\
\hline
\end{tabular}


Verbestel 2014 (Continued)

Percentage of eligible population enrolled: $51 \%$ daycare centres

Mean age: intervention: $15.84 \pm 2.75$ months

Control: $14.90 \pm 2.43$ months

Sex: intervention: $46.8 \%$ female; control: $43.8 \%$ female

Interventions

To evaluate the effects of a 1-year family-based healthy lifestyle intervention implemented through daycare centres on toddlers' zBMI and reported activity- and dietary-related behaviours

A family-based healthy lifestyle intervention was developed and implemented through daycare centres, with 2 main components:

- Guidelines and tips presented on an A3 take-home poster

- a colourful and animated A3 sheet with 5 stickers. Each sticker dealt with a targeted behaviour (see below) and provided parents with practical information and/or strategies. The stickers were distributed to the parents every 2 months and were gradually stuck on the poster by the parents. The stickers were always accompanied by a letter with information about the target behaviour.

- A tailored information sheet for parents about their children's activity and dietary related behaviours, arriving every 2 months with the above stickers and corresponding to the targeted behaviour. Two types of information were included:

- fixed part including a short overview of the current recommendations

- variable part included normative feedback in which the child's baseline behaviour was related to the current recommendations

Target behaviours:

- consumption of water

- consumption of milk

- consumption of fruit and vegetables

- increasing PA and decreasing screen-time behaviour

- consumption of sweets and savoury snacks.

No intervention provided to control

Diet and PA combination intervention vs control

Outcomes

Outcome measures

- Primary outcome: zBMI

- Secondary outcomes: PA, screen time, dietary intake

Process evaluation: NR

Implementation-related factors

Theoretical basis: (i) theories of information processing; (ii) the Elaboration Likelihood Model; and

(iii) the Precaution-Adoption Process Model

Resources for intervention implementation: reported

Who delivered the intervention: reported

PROGRESS categories assessed at baseline: gender, SES (mother's education)

PROGRESS categories analysed at outcome: NR

Outcomes relating to harms/unintended effects: NR

Intervention included strategies to address diversity or disadvantage: NR

Economic evaluation: NR 
Verbestel 2014 (Continued)

Notes
Funding: the work was supported by the Ministry of the Flemish Community (Department of Economics, Science and Innovation; Department of Welfare, Public Health and Family). The work was performed by the Centre of Expertise for Welfare, Public Health and Family, which is a consortium of researchers from the Catholic University of Leuven, Ghent University, Vrije Universiteit Brussel and KH Kempen.

\section{Risk of bias}

\begin{tabular}{|c|c|c|}
\hline Bias & Authors' judgement & Support for judgement \\
\hline $\begin{array}{l}\text { Random sequence genera- } \\
\text { tion (selection bias) }\end{array}$ & Unclear risk & Matched pairs, randomly allocated, no further details \\
\hline $\begin{array}{l}\text { Allocation concealment } \\
\text { (selection bias) }\end{array}$ & High risk & $\begin{array}{l}\text { Local professionals and daycare centres in the communities were already } \\
\text { aware of their group allocation at the start of the study }\end{array}$ \\
\hline $\begin{array}{l}\text { Blinding (performance } \\
\text { bias and detection bias) } \\
\text { All outcomes }\end{array}$ & High risk & $\begin{array}{l}\text { After randomisation, parents received a letter in which they were informed } \\
\text { about the study but their group assignment was not revealed at that time. } \\
\text { However, the daycare centres distributed the letters to the parents and they } \\
\text { may have incorporated this information in their communication to the par- } \\
\text { ents. Further, blinding of the parents in the intervention communities could } \\
\text { not be maintained throughout the study as parents received specific materials } \\
\text { as part of the intervention. Also, the researchers who conducted the measure- } \\
\text { ments were not blinded to group allocation. }\end{array}$ \\
\hline $\begin{array}{l}\text { Incomplete outcome data } \\
\text { (attrition bias) } \\
\text { All outcomes }\end{array}$ & High risk & Unbalanced: $21 \%$ attrition in intervention and $14 \%$ in control \\
\hline $\begin{array}{l}\text { Selective reporting (re- } \\
\text { porting bias) }\end{array}$ & Unclear risk & $\begin{array}{l}\text { Protocol mentioned in the RCT. But we were unable to locate a copy. Nor of the } \\
\text { trial registration. }\end{array}$ \\
\hline Other bias & Low risk & None identified \\
\hline $\begin{array}{l}\text { Other bias- timing of re- } \\
\text { cruitment of clusters }\end{array}$ & Low risk & $\begin{array}{l}\text { No participant flow chart but text indicates recruitment happened prior to ran- } \\
\text { domisation. }\end{array}$ \\
\hline
\end{tabular}

\section{Viggiano 2015}

$\begin{array}{ll}\text { Methods } & \text { Study design: cluster-RCT } \\ \text { Intervention period: } 20 \text { weeks } \\ \text { Follow-up period (post-intervention): } 1 \text { month and } 13 \text { months } \\ \text { Differences in baseline characteristics: reported } \\ \text { Reliable outcomes: reported } \\ \text { Protection against contamination: NR } \\ \text { Unit of allocation: schools } \\ \text { Unit of analysis: individual accounting for clustering } \\ \text { N (control baseline) }=1447 \\ \mathrm{~N} \text { (control follow-up) }=421\end{array}$


Viggiano 2015 (Continued)

\author{
$\mathrm{N}$ (intervention baseline $)=1663$ \\ $N$ (intervention follow-up) $=624$ \\ Setting (and number by study group): 20 middle/high schools; 10 intervention and 10 control \\ Recruitment: 12 public middle schools and 8 public high schools were invited \\ Geographic region: Campania, Italy
}

Percentage of eligible population enrolled: 95\% participants

Mean age: intervention: 13.3 (13.2-13.4); control: 13.0 (12.9-13.04)

Sex: intervention, $45 \%$ female; control, $49 \%$ female

Interventions

Aim: to confirm the effectiveness of Kaledo in improving nutritional knowledge and promoting longterm healthy dietary behaviour in a large cohort study

Kaledo is an educational board game. A typical game session requires 2-4 players and lasts about 15$30 \mathrm{~min}$. A game session represents a journey through daily meals of the Mediterranean diet. At the start, each player receives four chips and sets the energy expenditure of his/her kaleidoscope on the value corresponding to his/her Basal Metabolic Rate (BMR is obtained by consulting a simple table on the kaleidoscope which is based on age and weight).

During a game session, the players move their pawns on the 59 boxes on the board and, consequently, they receive nutrition cards (common food items of Mediterranean diet) or activity cards (common daily activity) as indicated in the destination boxes. A player can refuse to take a card by leaving one chip. In this way, he can try to balance the total energy intake (EI) given by the nutrition cards with the total energy expenditure (EE) given by the activity cards and the BMR. At the end of the game, the winner is the person with maximum points calculated on the bases of energy balance (maximum 5 points), best food items (maximum 4 points), and food variety (maximum 1 point). 7 special boxes on the board act as a punishment or a reward during the game and they are associated with specific dietary behaviour in real life (e.g. a fast food lunch).

Who delivered: children played themselves, aided by the classroom teacher.

Training: before the beginning of the trial, teachers were trained to use the game and they were instructed to select different students to play the game together at each game session.

Control group: the control group did not participate in any game session with Kaledo.

Diet and PA intervention (board game) vs control

Outcomes Outcome measures

- Primary outcome: score on the Adolescent Food Habits Checklist, scores on 6 sections of a dietary questionnaire (food habits, eating behaviour, nutrition knowledge), zBMI.

- Secondary outcomes: NR

Process evaluation: NR

Implementation-related factors
Theoretical basis: NR

Resources for intervention implementation: reported

Who delivered the intervention: reported

PROGRESS categories assessed at baseline: gender

PROGRESS categories analysed at outcome: gender

Outcomes relating to harms/unintended effects: NR

Intervention included strategies to address diversity or disadvantage: NR 
Viggiano 2015 (Continued)

Economic evaluation: NR

Notes The pilot study is already included in this Cochrane Review: Amaro 2006

\section{Risk of bias}

\begin{tabular}{|c|c|c|}
\hline Bias & Authors' judgement & Support for judgement \\
\hline $\begin{array}{l}\text { Random sequence genera- } \\
\text { tion (selection bias) }\end{array}$ & Low risk & $\begin{array}{l}\text { Quote: "Computer generated list of random numbers with the restriction of } \\
\text { balancing the number of middle and high schools between the two groups" }\end{array}$ \\
\hline $\begin{array}{l}\text { Allocation concealment } \\
\text { (selection bias) }\end{array}$ & Unclear risk & NR \\
\hline $\begin{array}{l}\text { Blinding (performance } \\
\text { bias and detection bias) } \\
\text { All outcomes }\end{array}$ & Unclear risk & NR \\
\hline $\begin{array}{l}\text { Incomplete outcome data } \\
\text { (attrition bias) } \\
\text { All outcomes }\end{array}$ & High risk & $\begin{array}{l}\text { High attrition; } 35 \% \text { in intervention and } 25 \% \text { in control at } 6 \text { months and } 62 \% \text { in } \\
\text { intervention and } 71 \% \text { in control at } 18 \text { months }\end{array}$ \\
\hline $\begin{array}{l}\text { Selective reporting (re- } \\
\text { porting bias) }\end{array}$ & Unclear risk & Protocol and trial registry report sought but not found \\
\hline Other bias & Low risk & No additional threats to validity \\
\hline $\begin{array}{l}\text { Other bias- timing of re- } \\
\text { cruitment of clusters }\end{array}$ & Low risk & $\begin{array}{l}\text { Schools were recruited before randomisation. } \\
\text { Quote: "The } 20 \text { schools participating in the trial were randomly allocated } \\
\text { to two independent groups by a computer-generated list of random numbers } \\
\text { with the restriction of balancing the number of middle and high schools be- } \\
\text { tween the two groups. Each group was then randomly assigned to a treatment } \\
\text { condition." }\end{array}$ \\
\hline
\end{tabular}

Vizcaino 2008

$\begin{array}{ll}\text { Methods } & \text { Study design: cluster-RCT } \\ \text { Intervention period: } 24 \text { weeks (during the 2004-2005 academic year) } \\ \text { Follow-up period (post-intervention): nil } \\ \text { Differences in baseline characteristics: reported } \\ \text { Reliable outcomes: reported } \\ \text { Protection against contamination: reported } \\ \text { Unit of allocation: school } \\ \text { Unit of analysis: individual } \\ \text { All analyses were performed according to ITT principles } \\ \text { N (controls baseline) }=606 \\ \text { N (controls follow-up) }=579\end{array}$


Vizcaino 2008 (Continued)

$$
\begin{aligned}
& N \text { (interventions baseline) }=513 \\
& N \text { (interventions follow-up) }=465 \\
& \text { Setting (and number by study group): schools ( }=10 \text { intervention; } N=10 \text { control) }
\end{aligned}
$$

Recruitment: selected 20 schools in 20 towns in the Province of Cuenca, Spain. 4th and 5th-grade children in participating schools were invited to participate.

Geographic region: Cuenca, Spain

Percentage of eligible population enrolled: $79 \%$

Mean age: intervention: boys $=9.4 \pm 0.7$ years, girls $=9.4 \pm 0.7$ years; control: boys $=9.5 \pm 0.7$ years, girls $=9.4 \pm 0.6$ years

Sex: intervention: $48.9 \%$ female; control: $49.6 \%$ female

- Implemented during the 2004/2005 academic year, consisted of 3 x 90-min sessions/ week for 24 weeks. These were held after school.

- 90-min session included 15 min of stretching, 60 min of aerobic resistance and 15 min of muscular strength/resistance exercise

- Non-competitive recreational PA programme (Movi) adapted to children's age and held at the schools athletic facilities? usually children went home after class then returned to school for programme.

- PA sessions planned by 2 qualified PE teachers and supervised by sports instructors

- Sessions included sports with alternative equipment (pogo sticks, Frisbees, jumping balls, parachutes, etc, co-operative games, dance and recreational athletics)

- Sports instructors had 2-day training programme and written plan of activities for each session was developed for standardisation

- Standard PE curriculum continued in both intervention and control schools.

- Further details at www.movidavida.org

PA intervention vs control

- BMI
- TSF thickness
- \% body fat
- Blood pressure
- 12 -h fasting blood samples to measure: total cholesterol, triglycerides, apo A and apo B
Process evaluation: reported

Implementation-related factors
Theoretical basis: NR

Resources for intervention implementation (e.g. funding needed or staff hours required): reported Who delivered the intervention: reported

PROGRESS categories assessed at baseline: reported (gender)

PROGRESS categories analysed at outcome: reported (gender)

Outcomes relating to harms/unintended effects: NR

Intervention included strategies to address diversity or disadvantage: reported

Economic evaluation: no formal evaluation, however average cost per child was provided (EUR 28 / child/month) 
Vizcaino 2008 (Continued)

de Salud Carlos III, Red de Investigacion en Actividades Preventivas y de Promocion de Salud (grant RD06/0018/ 0038)

\section{Risk of bias}

\begin{tabular}{|c|c|c|}
\hline Bias & Authors' judgement & Support for judgement \\
\hline \multirow{2}{*}{$\begin{array}{l}\text { Random sequence genera- } \\
\text { tion (selection bias) }\end{array}$} & Low risk & Used a computer-generated procedure. \\
\hline & & $\begin{array}{l}\text { Quote: "10 schools were randomized to the intervention group, and } 10 \text { to the } \\
\text { control group" }\end{array}$ \\
\hline $\begin{array}{l}\text { Allocation concealment } \\
\text { (selection bias) }\end{array}$ & Low risk & $\begin{array}{l}\text { Randomisation occurred at the school level and "Schools were informed of the } \\
\text { result of randomisation after they agreed to participate in the study" }\end{array}$ \\
\hline $\begin{array}{l}\text { Blinding (performance } \\
\text { bias and detection bias) } \\
\text { All outcomes }\end{array}$ & High risk & $\begin{array}{l}\text { Nurses who made the anthropometric and blood pressure measurements } \\
\text { were not blinded to intervention allocation. Laboratory analysts who deter- } \\
\text { mined blood lipids were blinded to school allocation }\end{array}$ \\
\hline $\begin{array}{l}\text { Incomplete outcome data } \\
\text { (attrition bias) } \\
\text { All outcomes }\end{array}$ & Low risk & Low rates of attrition between groups; ITT done \\
\hline $\begin{array}{l}\text { Selective reporting (re- } \\
\text { porting bias) }\end{array}$ & Unclear risk & Protocol and trial registry report sought but not found \\
\hline Other bias & Low risk & None identified \\
\hline $\begin{array}{l}\text { Other bias- timing of re- } \\
\text { cruitment of clusters }\end{array}$ & Low risk & Figure indicates recruitment happened prior to randomisation \\
\hline
\end{tabular}

\section{Wang 2012}

\begin{tabular}{ll}
\hline Methods & Study design: cluster-RCT \\
Intervention period: 12 months \\
Follow-up period (post-intervention): nil \\
Differences in baseline characteristics: reported \\
Reliable outcomes: reported \\
Protection against contamination: NR \\
Unit of allocation: schools \\
Unit of analysis: individual \\
N (control baseline) $=527$ \\
N (control follow-up) $=482$ \\
N (intervention baseline) $=476$ \\
N (intervention follow-up) $=449$ \\
Setting (and number by study group): 6 primary schools
\end{tabular}


Wang 2012 (Continued)

Recruitment: stratified random sampling: each 2 schools were selected from schools with large $>1000$ students), middle (500-1000 students), and small scales ( $<5002$ classes were randomly chosen within each school

Geographic region: Jinan City, Shandong Province, China

Percentage of eligible population enrolled: random sampling

Mean age: Grades 2-5: 7-11 years

Sex: NR

To explore the effective intervention mode to control child obesity
$\begin{aligned} & \text { Interventions } \\ & \text { effects and prevention methods of child obesity, and ways to build up a healthy diet. } \\ & \text { - Happy ten minutes (Happy } 10 \mathrm{~min} \text { ): school teachers organised students to do exercise in } 2 \text { sessions } \\ & \text { of 'happy ten minutes' every day. The exercise reached the moderate PA level and was either indoors } \\ & \text { or outdoors. } \\ & \text { Diet and PA intervention vs control }\end{aligned}$
$\begin{aligned} & \text { Outcome measures } \\ & \text { Outcomes }\end{aligned}$
Primary outcome obesity-related knowledge, attitudes, behaviour, blood lipids, blood glucose, preva-
lence overweight/obesity
Secondary outcomes: primary/secondary not specified
Process evaluation: NR

Implementation-related factors
Theoretical basis: NR

Resources for intervention implementation: reported

Who delivered the intervention: reported

PROGRESS categories assessed at baseline: NR

PROGRESS categories analysed at outcome: NR

Outcomes relating to harms/unintended effects: NR

Intervention included strategies to address diversity or disadvantage: NR

Economic evaluation: NR

Notes

Funding: Ministry of Science and Technology's "Eleventh Five-Year" National Science and Technology Support Plan Key Project (Project Number: 2008BAl58B05)

Review author (G Yang) data extracted this study as it is published in Chinese (English abstract)

\section{Risk of bias}

\begin{tabular}{lll}
\hline Bias & Authors' judgement & Support for judgement \\
\hline $\begin{array}{l}\text { Random sequence genera- } \\
\text { tion (selection bias) }\end{array}$ & Unclear risk & Randomly chosen \\
\hline $\begin{array}{l}\text { Allocation concealment } \\
\text { (selection bias) }\end{array}$ & Unclear risk & NR \\
\hline $\begin{array}{l}\text { Blinding (performance } \\
\text { bias and detection bias) }\end{array}$ & Unclear risk & NR \\
\hline
\end{tabular}


Wang 2012 (Continued)

All outcomes

\begin{tabular}{|c|c|c|}
\hline $\begin{array}{l}\text { Incomplete outcome data } \\
\text { (attrition bias) } \\
\text { All outcomes }\end{array}$ & Low risk & $\begin{array}{l}\text { Low attrition; } 27 / 476(5.6 \%) \text { in intervention group and } 45 / 527(8.5 \%) \text { in control } \\
\text { group withdrawals }\end{array}$ \\
\hline $\begin{array}{l}\text { Selective reporting (re- } \\
\text { porting bias) }\end{array}$ & Unclear risk & Protocol and trial registry report sought but not found \\
\hline $\begin{array}{l}\text { Other bias- timing of re- } \\
\text { cruitment of clusters }\end{array}$ & Unclear risk & NR \\
\hline
\end{tabular}

Warren 2003

\begin{tabular}{ll}
\hline Methods & Study design: RCT \\
& Intervention period: 14 months \\
& Follow-up (post-intervention): nil \\
& Differences in baseline characteristics: reported \\
& Reliable outcomes: reported \\
& Protection against contamination: NR \\
& Unit of allocation: child \\
Unit of analysis: child
\end{tabular}

\begin{tabular}{|c|c|}
\hline \multirow[t]{5}{*}{ Participants } & $\begin{array}{l}N \text { (controls and interventions - baseline })=218 \\
N(\text { controls follow-up })=54 \\
N(3 \text { interventions follow-up })=164\end{array}$ \\
\hline & Recruitment: all consenting 5-7 year-olds from 3 primary schools. Set in central UK \\
\hline & Proportion of eligibles participating: not stated \\
\hline & Mean age: all groups 6.1 (SD 0.6) years \\
\hline & Sex: both sexes; $51 \%$ boys \\
\hline
\end{tabular}

Interventions

- School and family-based interventions focusing on nutrition, PA, or both, upon the prevalence of overweight/obesity

- The setting was lunchtime clubs where an interactive and age-appropriate nutrition and/or PA curriculum was delivered by the project team.

- Controls received an education programme covering the non-nutritional aspects of food and human biology.

Combined effects of dietary interventions and PA interventions vs control

\begin{tabular}{ll}
\hline Outcomes & BMI \\
- Skinfolds measured at 5 sites (biceps, triceps, subscapular, supra-iliac, calf) \\
- Nutrition knowledge: validated questionnaire \\
- PA: children and parents completed basic questions about habitual activity (not validated) \\
- Diet: parents reported on behalf of children a 24-h recall and a food frequency questionnaire \\
Process evaluation: reported
\end{tabular}

Implementation-related factors
Theoretical basis: Social Learning theory

Resources for intervention implementation (e.g. funding needed or staff hours required): reported 
Warren 2003 (Continued)

Who delivered the intervention: reported

PROGRESS categories assessed at baseline: reported (gender, education)

PROGRESS categories analysed at outcome: NR

Outcomes relating to harms/unintended effects: NR

Intervention included strategies to address diversity or disadvantage: reported

Economic evaluation: NR

Notes Funding: non-industry. The project was funded by the UK Food Standards Agency

\section{Risk of bias}

\begin{tabular}{|c|c|c|}
\hline Bias & Authors' judgement & Support for judgement \\
\hline $\begin{array}{l}\text { Random sequence genera- } \\
\text { tion (selection bias) }\end{array}$ & Unclear risk & $\begin{array}{l}\text { NR. } \\
\text { Quote: "Children were randomly allocated to one of four groups" }\end{array}$ \\
\hline $\begin{array}{l}\text { Allocation concealment } \\
\text { (selection bias) }\end{array}$ & Unclear risk & $\begin{array}{l}\text { NR. } \\
\text { Quote: "Children were randomly allocated to one of four groups" }\end{array}$ \\
\hline $\begin{array}{l}\text { Blinding (performance } \\
\text { bias and detection bias) } \\
\text { All outcomes }\end{array}$ & High risk & $\begin{array}{l}\text { The assessors were involved with delivering the intervention. Parents also pro- } \\
\text { vided information. Therefore assessment was not blinded. } \\
\text { Quote: "Baseline assessments were made before randomisation." } \\
\text { Quote: "Assessors were involved in the delivery of the intervention" } \\
\text { Quote: "Assessments were made by teachers and parents" }\end{array}$ \\
\hline $\begin{array}{l}\text { Incomplete outcome data } \\
\text { (attrition bias) } \\
\text { All outcomes }\end{array}$ & Low risk & $\begin{array}{l}\text { Attrition was not }>30 \% \text {. Similar numbers missing from each group. Reasons } \\
\text { for withdrawal given and characteristics of withdrawals investigated }\end{array}$ \\
\hline $\begin{array}{l}\text { Selective reporting (re- } \\
\text { porting bias) }\end{array}$ & Unclear risk & Protocol and trial registry report sought but not found \\
\hline Other bias & Low risk & None identified \\
\hline
\end{tabular}

\section{Weeks 2012}

$\begin{array}{ll}\text { Methods } & \text { Study design: RCT } \\ \text { Intervention period: } 8 \text { months } \\ \text { Follow-up period (post-intervention): nil } \\ \text { Differences in baseline characteristics: reported } \\ \text { Reliable outcomes: reported } \\ \text { Protection against contamination: reported } \\ \text { Unit of allocation: individual }\end{array}$


Weeks 2012 (Continued)

Unit of analysis: individual

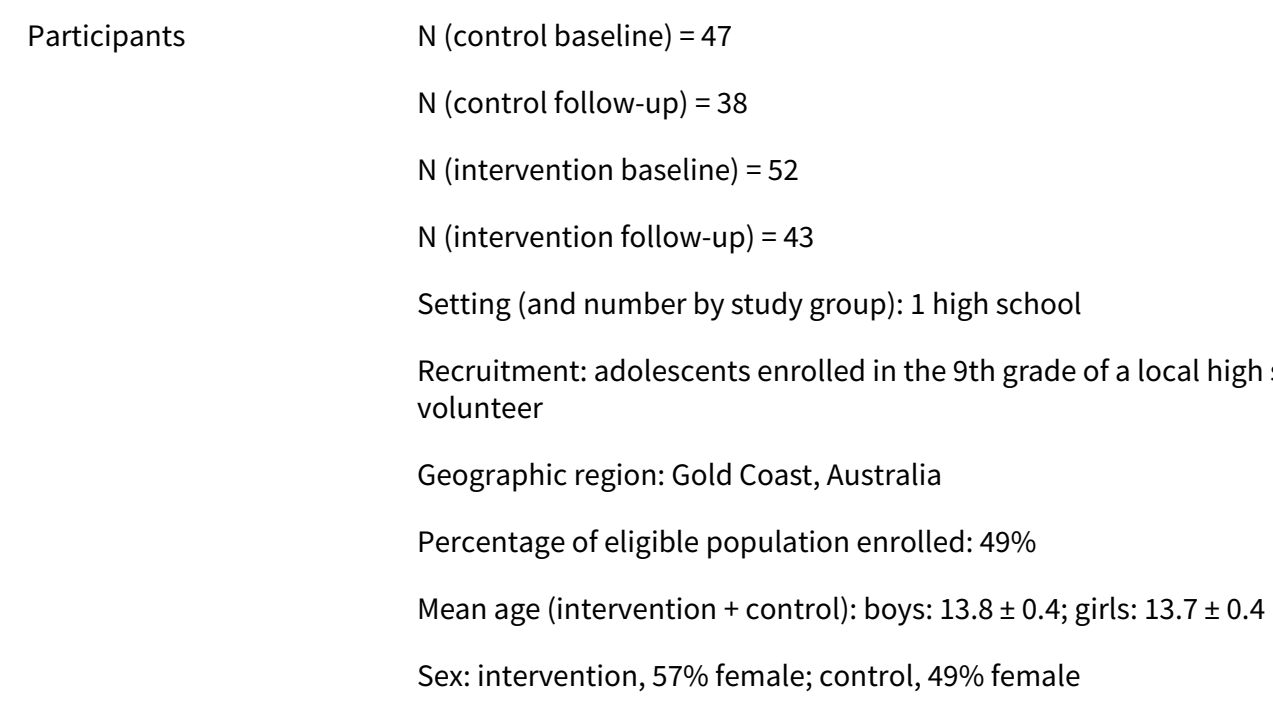

Interventions

Aim: to determine the effect of a regular, brief, in-school jumping regime on muscle and fat mass in healthy adolescent boys and girls, in comparison with controls.

The intervention group participated in 10 min of supervised jumping activity at the start of each PE class, i.e. 2 x per week for 8 months, excluding holidays. Each bout of jumping comprised at least some of the following manoeuvres: jumps, hops, tuck-jumps, jump-squats, stride jumps, star jumps, lunges, side lunges and skipping. A typical 10-min session included approximately 300 jumps performed at approximately $1-3 \mathrm{~Hz}$ and at a height of $0.2-0.4 \mathrm{~m}$.

Who delivered: the instructor demonstrated all jumping activities and co-ordinated the routine at each session. Jumping sessions were occasionally supplemented with upper limb strengthening activities, such as push-ups and exercises with resistive bands.

Control group: participants undertook regular PE warm ups and stretching directed by their usual PE teacher at a time that corresponded with intervention group activities, i.e. at the beginning of every PE class, twice per week for a period of 8 months, excluding holidays. Control activities were focused on improving flexibility and general preparedness for PA.

PA intervention vs control

Outcome measures
- Primary outcome: whole body bone-free lean tissue and fat mass
- Secondary outcomes: BMI, physical maturity, muscle power, PA
Process evaluation: reported (compliance)

Implementation-related factors
Theoretical basis: NR

Resources for intervention implementation: reported

Who delivered the intervention: reported

PROGRESS categories assessed at baseline: gender

PROGRESS categories analysed at outcome: gender

Outcomes relating to harms/unintended effects: NR

Intervention included strategies to address diversity or disadvantage: NR 
Weeks 2012 (Continued)

$$
\text { Economic evaluation: NR }
$$

Notes

Funding: there were no external funding sources. Intervention did not need additional staffing or equipment and with minimal disruption to daily school activities. Mean compliance for the intervention was $80 \%$.

\section{Risk of bias}

\begin{tabular}{|c|c|c|}
\hline Bias & Authors' judgement & Support for judgement \\
\hline $\begin{array}{l}\text { Random sequence genera- } \\
\text { tion (selection bias) }\end{array}$ & Unclear risk & Randomised, no further details \\
\hline $\begin{array}{l}\text { Allocation concealment } \\
\text { (selection bias) }\end{array}$ & Unclear risk & Randomised, no further details \\
\hline $\begin{array}{l}\text { Blinding (performance } \\
\text { bias and detection bias) } \\
\text { All outcomes }\end{array}$ & Unclear risk & NR \\
\hline $\begin{array}{l}\text { Incomplete outcome data } \\
\text { (attrition bias) } \\
\text { All outcomes }\end{array}$ & Low risk & $18 \%$ attrition; ITT done \\
\hline $\begin{array}{l}\text { Selective reporting (re- } \\
\text { porting bias) }\end{array}$ & Unclear risk & Protocol and trial registry report sought but not found \\
\hline Other bias & Low risk & None identified \\
\hline
\end{tabular}

\section{Wen 2012}

\begin{tabular}{ll}
\hline Methods & Study name: The health beginnings trial (HBT) \\
Study design: $\mathrm{RCT}$ \\
Intervention period: 24 months \\
Follow-up period (post-intervention): 24 months \\
Differences in baseline characteristics: reported \\
Reliable outcomes: reported \\
Protection against contamination: NR \\
Unit of allocation: mother and child dyad? \\
Unit of analysis: individual \\
N (control baseline) $=330$ \\
N (control follow-up) $=234$ \\
N (intervention baseline) $=337$ \\
N (intervention follow-up) $=249$ \\
Setting (and number by study group): home-based
\end{tabular}


Wen 2012 (Continued)

Recruitment: research assistants gave pregnant women attending antenatal clinics a letter of invitation and information about the study

Geographic region: socially and economically disadvantaged areas of Sydney, Australia

Percentage of eligible population enrolled: $86 \%$ participants

Mean age: intervention + control: 2 years

Sex: NR

Interventions

To assess the effectiveness of a home-based early intervention on children's BMI at age 2 .

8 home visits (1-2 h per visit) from specially trained community nurses delivering a staged, home-based intervention, one in the antenatal period, and seven at 1, 3, 5, 9, 12, 18 and 24 months after birth. Timing of the visits was designed to coincide with early childhood developmental milestones. 4 community nurses were recruited and trained to ensure consistency of delivering the intervention.

The key intervention messages included:

- Breast is best

- No solids for me until 6 months

- I eat a variety of fruit and vegetables every day

- Only water in my cup

- I am part of an active family

Families in both the control and intervention group received the usual childhood nursing service from community health service nurses. All new mothers in the state of New South Wales received at least 1 nurse visit for general support at home. Some vulnerable families are offered multiple home visits. To maximise the retention rate in this study, they posted home safety promotion materials to women in the control group at six and 12 months.

Diet and PA combination intervention vs control

Outcomes Outcome measures

- Primary outcome: exclusive breast feeding, timing of introduction of solids, and practice of "tummy time" at 6 months, breastfeeding, cup use, bottle at bedtime, food as reward at 12 months, BMI at 24 months (Wen 2012)

- Secondary outcomes: dietary behaviours, PA and TV viewing all at 24 months (Wen 2012), costs (Hayes 2014 (Wen 2012 secondary reference))

Process evaluation: NR

Implementation-related factors
Theoretical basis: NR

Resources for intervention implementation: reported

Who delivered the intervention: reported

PROGRESS categories assessed at baseline: mothers: race/ethnicity, education, SES (income), employment status, marital status

PROGRESS categories analysed at outcome: NR

Outcomes relating to harms/unintended effects: NR

Intervention included strategies to address diversity or disadvantage: intervention targeted most socially and economically disadvantaged areas of Sydney

Economic evaluation: reported (Hayes 2014 (Wen 2012 secondary reference)) 
Methods: economic evaluation of a RCT, the 'Healthy beginnings' (HB) trial, from the perspective of the health funder. Intervention resources were determined from local health district records in 2012 AUD. Health-care resource utilisation was determined through patient-level data

linkage.

Results: the cost of HB intervention in the clinical trial over 2 years was AUD 1309 per child (2012 AUD).

The incremental cost-effectiveness ratio was AUD 4230 per unit BMI avoided and AUD 631 per 0.1 reduction in zBMI. It was estimated that the programme could be delivered in practice for AUD 709 per child; with incremental cost-effectiveness ratios of AUD 2697 per unit BMI avoided and AUD 376 per 0.1 reduction in BMI z-score.

Conclusions: "We present the first economic evaluation of an effective obesity prevention initiative in early childhood. HB is a moderately priced intervention with demonstrated effectiveness that offers similar or better value for money than existing obesity prevention or treatment interventions targeted at older children."

\section{Risk of bias}

\begin{tabular}{lll}
\hline Bias & Authors' judgement & Support for judgement \\
\hline $\begin{array}{l}\text { Random sequence genera- } \\
\text { tion (selection bias) }\end{array}$ & Low risk & $\begin{array}{l}\text { Random allocation was concealed by sequentially numbered, sealed opaque } \\
\text { envelopes containing the group allocation, which was determined by a com- } \\
\text { puter-generated random number with a block size of } 50 \text { with a 1:1 allocation } \\
\text { ratio }\end{array}$ \\
& &
\end{tabular}

Allocation concealment Low risk Random allocation was concealed by sequentially numbered, sealed, opaque
(selection bias) envelopes containing the group allocation, which was determined by a computer-generated random number with a block size of 50 with a 1:1 allocation ratio

\begin{tabular}{ll}
\hline $\begin{array}{l}\text { Blinding (performance } \\
\text { bias and detection bias) }\end{array}$ & Low risk \\
$\begin{array}{ll}\text { All outcomes } & \text { Immediately after baseline data collection, the nurse opened the sealed enve- } \\
& \text { lope and informed the mother of her group. We applied blinding to treatment } \\
& \text { allocation for data collection, data entry, and analysis; } 2 \text { research assistants } \\
\text { not involved in the implementation of the intervention collected outcome da- } \\
\text { ta in the woman's home. }\end{array}$
\end{tabular}

\begin{tabular}{|c|c|c|}
\hline $\begin{array}{l}\text { Incomplete outcome data } \\
\text { (attrition bias) } \\
\text { All outcomes }\end{array}$ & Low risk & $\begin{array}{l}24 \% \text { attrition in intervention and } 27 \% \text { attrition in control, imputed missing da- } \\
\text { ta and balanced across groups }\end{array}$ \\
\hline
\end{tabular}

\begin{tabular}{lll}
\hline $\begin{array}{l}\text { Selective reporting (re- } \\
\text { porting bias) }\end{array}$ & Low risk & Protocol/trial registration document seen. All outcomes reported \\
\hline Other bias & Low risk & No additional threats to validity \\
\hline
\end{tabular}

Whittemore 2013

$\begin{array}{ll}\text { Methods } & \text { Study name: Health(e)teen } \\ & \text { Study design: cluster-RCT } \\ \text { Intervention period: } 3 \text { months? } \\ \text { Follow-up period (post-intervention): } 3 \text { months? }\end{array}$


Whittemore 2013 (Continued)

Differences in baseline characteristics: reported

Reliable outcomes: reported

Protection against contamination: NR

Unit of allocation: class

Unit of analysis: individual accounting for clustering

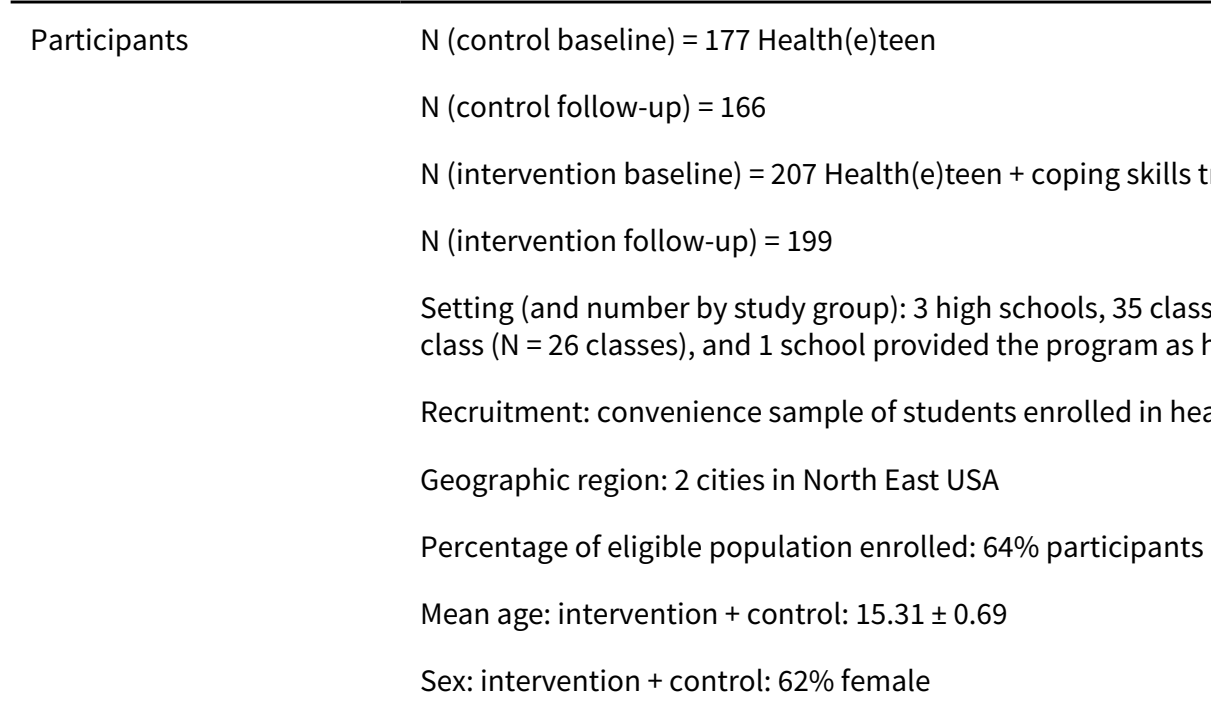

Interventions

To compare the effectiveness of 2 school-based internet obesity prevention programs, Health(e)teen and Health(e)teen + CST in diverse adolescents on BMI, health behaviours, and self-efficacy at 3 and 6 months

Components of the Health(e)teen and Health(e)teen + CST program were

- lessons

- including self-assessment, simulations, problem-solving, repetition, and individualised feedback

- there were 8 lessons on the topics of nutrition, PA, metabolism, and portion control

- lessons provided content, goal-setting and self-monitoring

- A reality TV concept of the program included diverse relatable characters who demonstrated typical situations (social modelling) in videos, text, and lesson commentary

- Lessons were highly interactive, and students received individualised feedback via self-assessments and questions on content.

- goal setting

- self-monitoring

- health coaching: providing individualised feedback, encouragement and social persuasion

- social networking: providing individualised feedback, encouragement and social persuasion

Students were encouraged to record their food intake and PA each time they logged on, and the program provided a visual display of their progress. Students also set goals and monitored progress with completing goals. A blog by a "coach" the opportunity to interact with a health coach (graduate nursing student) and other students, and a personal journal section were other components of the program.

Health(e)teen + CST included all the aforementioned components and the addition of 4 lessons on coping skills training (total of 12 lessons).

CST lessons included

- social problem solving

- stress reduction

- assertive communication 
Whittemore 2013 (Continued)

- conflict resolution

Lessons provided content on stress reduction, assertive communication, conflict resolution, and social problem solving as it relates to healthy eating and PA

Teachers were provided access to the websites and guidelines to promote student participation. The program was developed to be self-standing, with teacher involvement required only to help students log onto the program and monitor student activity to assure that students were participating in the program (rather than exploring other websites). Teachers were also instructed to prompt students to complete lessons and self-monitoring as well as explore all components of the program

Diet and PA combination intervention vs diet and PA combination (internet)

Outcome measures
- Primary outcome: BMI
- Secondary outcomes: sedentary behaviour, nutrition behaviour, self-efficacy
Process evaluation: reported (satisfaction at 3 months, data usage)

Implementation-related factors
Theoretical basis: theory of interactive technology, Social Learning theory

Resources for intervention implementation: reported

Who delivered the intervention: reported

PROGRESS categories assessed at baseline: child, gender, race/ethnicity; parent: SES (income), education

PROGRESS categories analysed at outcome: NR

Outcomes relating to harms/unintended effects: NR

Intervention included strategies to address diversity or disadvantage: NR

Economic evaluation: NR

\section{Notes}

Funding: NIH/NINR: RC1NR011594

Study participants received a gift card for completion of data collection (USD 25.00 at time 1; USD 30.00 at times 2 and 3 ).

Because program implementation was different in some classes (homework vs classroom), a mixed model analyses was done exploring the effect of implementation by program.

Satisfaction with the programs was high. The mean satisfaction score was $3.58(+.68)$. There was no significant difference between groups with respect to satisfaction $(P=0.26)$. Participation was also high, with adolescents completing $83 \%$ of lessons (median 100\%). In each group, more than half of participants completed all lessons (53\% of participants in Health(e)teen + CST and 70\% in Health(e)teen). Adolescents completed self-monitoring assessments 5.26 times (+ 2.75; median 5) over the 8-12 lessons. Adolescents of the Health $(e)$ teen + CST completed fewer lessons $(P=0.001)$ yet had higher participation in self-monitoring $(P<0.001)$

\section{Risk of bias}

\begin{tabular}{lll}
\hline Bias & Authors' judgement & Support for judgement \\
\hline $\begin{array}{l}\text { Random sequence genera- } \\
\text { tion (selection bias) }\end{array}$ & Unclear risk & Randomisation no further details \\
\hline
\end{tabular}


Whittemore 2013 (Continued)

\begin{tabular}{l}
$\begin{array}{l}\text { Allocation concealment } \\
\text { (selection bias) }\end{array} \quad$ Unclear risk Randomisation no further details \\
\hline
\end{tabular}

\begin{tabular}{|c|c|}
\hline $\begin{array}{l}\text { Blinding (performance } \\
\text { bias and detection bias) } \\
\text { All outcomes }\end{array}$ & Unclear risk \\
\hline
\end{tabular}

\begin{tabular}{lll}
\hline $\begin{array}{l}\text { Incomplete outcome data } \\
\text { (attrition bias) } \\
\text { All outcomes }\end{array}$ & Low risk & Low attrition: 5\% \\
\hline $\begin{array}{l}\text { Selective reporting (re- } \\
\text { porting bias) }\end{array}$ & Low risk & Protocol/trial registration document seen. All outcomes reported \\
\hline Other bias & Low risk & No additional threats to validity \\
\hline $\begin{array}{l}\text { Other bias- timing of re- } \\
\text { cruitment of clusters }\end{array}$ & Low risk & Figure shows recruitment happened prior to randomisation \\
\hline
\end{tabular}

Wilksch 2015

Study name: Media smart; life smart; the helping, encouraging, listening and protecting peers (HELPP)
Study design: cluster-RCT
Intervention period: 5 weeks
Follow-up period (post-intervention): 11 months
Differences in baseline characteristics: reported
Reliable outcomes: reported
Protection against contamination: reported
Unit of allocation: class
Unit of analysis: individual accounting for clustering

\begin{tabular}{|c|c|}
\hline \multirow[t]{10}{*}{ Participants } & $N($ control baseline $)=473$ \\
\hline & $N$ (control follow-up) = 346 \\
\hline & $\mathrm{N}$ (intervention baseline) = $255 \mathrm{HELPP}$ \\
\hline & $\mathrm{N}$ (intervention follow-up) $=170$ \\
\hline & $\mathrm{N}$ (intervention baseline) $=347$ life smart \\
\hline & N (intervention follow-up) = 279 \\
\hline & $\mathrm{N}$ (intervention baseline) = 269 media smart \\
\hline & $N$ (intervention follow-up) $=219$ \\
\hline & $\begin{array}{l}\text { Setting (and number by study group): } 12 \text { schools across } 3 \text { Australian states ( } 10 \text { co-educational and } 2 \\
\text { girls only) }\end{array}$ \\
\hline & $\begin{array}{l}\text { Recruitment: schools were invited to participate based on a staff member previously expressing an in- } \\
\text { terest in body image programmes }(\mathrm{N}=4) \text { or where schools were geographically located within } 1 \mathrm{~h} \text { of } \\
\text { the participating university in that state }(\mathrm{N}=8)\end{array}$ \\
\hline
\end{tabular}


Wilksch 2015 (Continued)

Geographic region: South Australia, Victoria, Western Australia

Percentage of eligible population enrolled: $27 \%$ schools; $1316 / 1441$ participants 'correctly matched across waves for inclusion in anlayses'

Mean age: intervention $\times 3+$ control: $13.21 \pm 0.68$

Sex: intervention $\times 3+$ control: $64 \%$ female

Interventions

The aim of this research was to investigate the efficacy of an obesity-prevention programme (Life smart) and two eating disorder-prevention programmes (Media smart and HELPP) against each other and a no-intervention control condition with young adolescent girls and boys from pre- to post-intervention and over a 12-month follow-up

All 3 programmes were developed around the evidence-based principles of being interactive; avoiding psychoeducation about eating disorders and obesity; and having multiple sessions with 8 lessons of 50-min duration delivered at the rate of 2 lessons/week. The programmes were presented by postgraduate psychology students who had attended a training session run by the programme developers covering principles of effective programme delivery followed by three 2-h workshops for each intervention. Presenters received training in all 3 programmes and were required to deliver each programme in order to reduce the likelihood of presenter effects contaminating programme outcomes

Diet and PA combination intervention ( $x 3$ ) vs control

\begin{tabular}{ll}
\hline Outcomes & Outcome measures \\
& - Primary outcome: weight concerns, BMI \\
& Process evaluation: NR \\
\hline $\begin{array}{l}\text { Implementation-related } \\
\text { factors }\end{array}$ & Theoretical basis: NR \\
& Resources for intervention implementation: reported \\
& Who delivered the intervention: reported \\
& PROGRESS categories assessed at baseline: gender, SES (Index of Community Socio- Educational Ad- \\
& vantage (ICSEA)) \\
& PROGRESS categories analysed at outcome: gender \\
& Outcomes relating to harms/unintended effects: reported (risk of eating disorders) \\
Intervention included strategies to address diversity or disadvantage: NR \\
Economic evaluation: NR
\end{tabular}

Notes

Funding: this research was funded by a Butterfly Research Institute Grant. S.M.W. held a research fellowship funded by the South Australian Centre for Intergenerational Health and now holds a research fellowship funded by the National Health and Medical Research Council. S.B.A. is supported by the Ellen Feldberg Gordon Fund for Eating Disorders Research and the Programs to prevent eating disorders and obesity 1821 www.cambridge.org/core/terms. doi.org/10.1017/S003329171400289X Downloaded from www.cambridge.org/core. University of Bristol Library, on 14 Mar 2018 at 15:47:04, subject to the Cambridge Core terms of use, available at US Maternal and Child Health Bureau, Health Resources and Services Administration, training grants MC00001 and the Leadership Education in Adolescent Health Project 6T71-MC00009.

(Index of Community Socio- Educational Advantage (ICSEA)) The mean ICSEA rating was 1104 (range $=972-1183$ ), indicating above average socio-economic advantage, consistent with anecdotal reports from program presenters suggesting a predominantly white sample as reflecting Australian society. 
Wilksch 2015 (Continued)

Media smart girls (mean $=19.78, \mathrm{SD}=3.42$ ) had a significantly lower BMI than HELPP girls (mean = $21.01, \mathrm{SD}=3.76$, Effect size $=0.33$ )

\section{Risk of bias}

\begin{tabular}{|c|c|c|}
\hline Bias & Authors' judgement & Support for judgement \\
\hline \multirow{2}{*}{$\begin{array}{l}\text { Random sequence genera- } \\
\text { tion (selection bias) }\end{array}$} & Unclear risk & Randomisation, no further details. \\
\hline & & $\begin{array}{l}\text { Quote: "Classes in the intervention grade were randomly allocated to one of } \\
\text { the three programs. Where the intervention grade had at least three classes, } \\
\text { each class would receive a different program." }\end{array}$ \\
\hline $\begin{array}{l}\text { Allocation concealment } \\
\text { (selection bias) }\end{array}$ & Unclear risk & $\begin{array}{l}\text { NR. Significant differences between groups for girls on regular eating and BMI, } \\
\text { NR whether this was adjusted for in the analysis }\end{array}$ \\
\hline $\begin{array}{l}\text { Blinding (performance } \\
\text { bias and detection bias) } \\
\text { All outcomes }\end{array}$ & Unclear risk & NR \\
\hline $\begin{array}{l}\text { Incomplete outcome data } \\
\text { (attrition bias) } \\
\text { All outcomes }\end{array}$ & Unclear risk & Retention ranged from $74 \%$ to $81 \%$; ITT done \\
\hline $\begin{array}{l}\text { Selective reporting (re- } \\
\text { porting bias) }\end{array}$ & Unclear risk & Protocol and trial registry report sought but not found \\
\hline Other bias & Low risk & No additional threats to validity \\
\hline $\begin{array}{l}\text { Other bias- timing of re- } \\
\text { cruitment of clusters }\end{array}$ & Low risk & Figure shows recruitment happened prior to randomisation \\
\hline
\end{tabular}

Williamson 2012

$\begin{array}{ll}\text { Methods } & \text { Study name: LA health study } \\ \text { Study design: cluster-RCT } \\ \text { Intervention period: } 28 \text { months } \\ \text { Follow-up period (post-intervention): nil } \\ \text { Differences in baseline characteristics: reported } \\ \text { Reliable outcomes: reported } \\ \text { Protection against contamination: NR } \\ \text { Unit of allocation: school clusters } \\ \text { Unit of analysis: individual accounting for clustering } \\ \text { N (controls baseline) }=587 \\ \text { N (controls follow-up) }=18 \text { months: } 421,28 \text { months: } 447 \\ \text { N (primary prevention (PP) baseline) }=713 \\ \text { N (PP follow-up) }=18 \text { months: } 584,28 \text { months: } 489\end{array}$


Williamson 2012 (Continued)

$$
\begin{aligned}
& \text { N (combination PP + secondary prevention (SP) baseline })=760 \\
& N \text { (combination PP + SP follow-up) }=18 \text { months: 614, } 28 \text { months: } 553
\end{aligned}
$$

Setting (and number by study group): 17 school clusters (each cluster described as an exclusive set of elementary schools and the middle or junior high school into which they feed): primary prevention (5 clusters), combination of primary prevention and secondary prevention (6 clusters) and control ( 6 clusters)

Recruitment: top-down approach, i.e. first sought the support of the highest levels of school administration and progressively sought support at lower levels. Students were recruited in the school environment by a variety of methods, including presentations to students and parents, fliers, and word of mouth.

Geographic region: Louisiana, USA

Percentage of eligible population enrolled: $74 \%$ school clusters; $42 \%$ participants

Mean age: PP: $10.5 \pm 1.2$; SP: $10.5 \pm 1.2$; control: $10.6 \pm 1.2$

Sex: PP: $58.8 \%$ female; SP: $57.2 \%$; control: $60 \%$ female to a control group for prevention of weight/fat gain in the entire sample and overweight children

Name: LA health study

- Intervention: PP

- Modification of the school environment to promote healthy nutrition and PA with 3 primary objectives:

- modify environmental cues related to healthy eating and activity

- modify the cafeteria food service programme

- modify the PE programmes as described in the SPARK study (reference 24) and to reduce sedentary behaviour.

- The programme used an environmental approach that was developed and tested in the 'Wise mind' study.

- Recommendations from the American Academy of Pediatrics were followed.

- The contents of vending machines were modified to meet dietary guideline criteria and activity goals set

- 60 min of moderate to vigorous activity per day and

- $<2 \mathrm{~h}$ /day of TV viewing and video gaming

- Intervention: PP + SP

- Combination of PP (identical to above) and SP.

- SP employed a classroom instruction component combined with an internet-based approach. The internet intervention of this study was delivered as part of regular classroom instruction, combined with synchronous (online) internet counselling and asynchronous (email) communications for children and their parents.

- Tailoring: the website was programmed to recognise whether a participant was overweight or obese at baseline and slightly different programs were presented to overweight and non-overweight children, which was effective for minimising the potential for stigmatising overweight children.

- Control

- The control group for the RCT received none of the prevention components that are hypothesised to yield weight gain prevention.

- Teachers received information about the LA health project, instruction on modelling and implementing content standards as they relate to LA health, and technology training critical to project implementation. Treatment-specific training began after random assignment of schools, and was held at locations convenient for the teachers

Diet and PA with and without added classroom and internet education component vs control 
Williamson 2012 (Continued)

\begin{tabular}{|c|c|}
\hline Outcomes & $\begin{array}{l}\text { Outcome measures } \\
\text { - Primary outcome: zBMI, \% body fat } \\
\text { - Secondary outcomes: PA, energy intake } \\
\text { Process evaluation: reported (integrity) }\end{array}$ \\
\hline $\begin{array}{l}\text { Implementation-related } \\
\text { factors }\end{array}$ & $\begin{array}{l}\text { Theoretical basis: Social Learning theory } \\
\text { Resources for intervention implementation: reported } \\
\text { Who delivered the intervention: reported } \\
\text { PROGRESS categories assessed at baseline: gender, race/ethnicity, SES (enrolment in the free or re- } \\
\text { duced-cost lunch programme) } \\
\text { PROGRESS categories analysed at outcome: gender, race/ethnicity } \\
\text { Outcomes relating to harms/unintended effects: NR } \\
\text { Intervention included strategies to address diversity or disadvantage: NR however } 81.7 \% \text { of partici- } \\
\text { pants described as being low SES at baseline } \\
\text { Economic evaluation: NR }\end{array}$ \\
\hline Notes & $\begin{array}{l}\text { NCT00289315 } \\
\text { Funding: this project was supported by the National Institute for Child Health and Human Development } \\
\text { of the NIH (R01 HD048483) and the U.S. Department of Agriculture (58-6435-4-90). In addition, this work } \\
\text { was partially supported by the NORC Center Grant \#1P30 DK072476 entitled "Nutritional Programming: } \\
\text { Environmental and Molecular Interactions" sponsored by NIDDK, and C. Martin was supported by NIH } \\
\text { grant K23 DK068052 (PI: C. Martin) }\end{array}$ \\
\hline
\end{tabular}

\section{Risk of bias}

Bias Authors' judgement Support for judgement

Random sequence genera- Unclear risk 17 school clusters were randomly assigned to $1 / 3$ study arms tion (selection bias)

\begin{tabular}{lll}
$\begin{array}{l}\text { Allocation concealment } \\
\text { (selection bias) }\end{array}$ & Unclear risk & NR \\
\hline $\begin{array}{l}\text { Blinding (performance } \\
\text { bias and detection bias) } \\
\text { All outcomes }\end{array}$ & Low risk & $\begin{array}{l}\text { Quote: "measurements were conducted by two independent assessment } \\
\text { teams who travelled together" }\end{array}$ \\
\hline
\end{tabular}

\begin{tabular}{|c|c|c|}
\hline \multirow{2}{*}{$\begin{array}{l}\text { Incomplete outcome data } \\
\text { (attrition bias) } \\
\text { All outcomes }\end{array}$} & Low risk & $\begin{array}{l}\text { Attrition rate was } 14 \%, 16 \% \text { and } 24 \% \text { in PP, SP and control respectively at end } \\
\text { of study }\end{array}$ \\
\hline & & $\begin{array}{l}\text { Quote: "The results were compared with results from a last observation car- } \\
\text { ried forward (LOCF) intent-to-treat approach to evaluate the reliability of the } \\
\text { findings and the same results were found" }\end{array}$ \\
\hline $\begin{array}{l}\text { Selective reporting (re- } \\
\text { porting bias) }\end{array}$ & Unclear risk & $\begin{array}{l}\text { Protocol/trial registration document seen. All outcomes reported. However } \\
\text { both intervention arms were combined and compared with control as no sig- } \\
\text { nificant difference between groups at follow-up for primary outcomes }\end{array}$ \\
\hline Other bias & Low risk & None identified \\
\hline
\end{tabular}


Williamson 2012 (Continued)

Other bias- timing of re- Low risk $\quad$ Figure and text both indicate recruitment happened prior to randomisation cruitment of clusters

Yilmaz 2015

$\begin{array}{ll}\text { Methods } & \text { Study design: RCT } \\ \text { Intervention period: } 8 \text { weeks } \\ \text { Follow-up period (post-intervention): } 7 \text { months } \\ \text { Differences in baseline characteristics: reported } \\ \text { Reliable outcomes: reported } \\ \text { Protection against contamination: NR } \\ \text { Unit of allocation: families } \\ \text { Unit of analysis: individual }\end{array}$

Participants

$\mathrm{N}$ (control baseline $)=201$

$\mathrm{N}$ (control follow-up) $=176$

$\mathrm{N}$ (intervention baseline $)=211$

$\mathrm{N}$ (intervention follow-up) $=187$

Setting (and number by study group): primary care setting and home

Recruitment: fliers were introduced to parents who brought their children to hospital for well-child care visit

Geographic region: Ankara, Turkey

Percentage of eligible population enrolled: $59 \%$ families

Mean age: intervention: $3.52 \pm 1.28$; control: $3.49 \pm 1.22$

Sex: intervention: $51 \%$ female; control: $54 \%$ female

To determine if a simple intervention aimed at preschool-aged children, applied at the health maintenance visits, in the primary care setting, would be effective in reducing screen time. Home visits to collect data at 2, 6 and 9 months.

The participants in the study group were exposed to the 4 intervention components at 2-week intervals. The intervention consisted of 3 printed materials and interactive CDs and 1 counselling call, intending to decrease screen time.

The 1st set of printed materials was given after the baseline questionnaire, followed by a counselling phone call 2 weeks later. The 2 nd and 3 rd printed materials were distributed at the 4 th and 6 th week. A follow-up questionnaire was done 8 weeks after the start of the study.

The printed materials and CDs were aimed to decrease screen time at home. CD recordings included harmful effects of TV, video and computer games and a list of alternative activities to watching TV and other screens. In order to reduce screen time, parents were asked to read age-appropriate books to their children daily; a family mealtime with TV turned off was advised; children were encouraged to offer alternative activities to watching TV, such as reading books; the parents were supported to place 'no TV or screen' signs on each TV or screen at home. Alternative ways of spending time was offered when 
Yilmaz 2015 (Continued)

not sitting in front of a screen. Then the parents were supported to remove their child's TV or computer from his or her bedroom.

The 2 nd component of the intervention was a counselling call. This call encouraged families to make their home screen-free; it provided the benefits of a screen-free home, and difficulties to establish and keep a screen-free home.

The 3rd component included a picture book showing a family while making their home screen-free. It gave data about increasing conversation among family members, decreasing children's screen time and consequences of increased screen time, such as violence.

The 4th component included information about stories of families that were able to decrease their screen time.

PA (screen time) intervention vs control

\begin{tabular}{ll}
\hline Outcomes & Outcome measures \\
- Primary outcome: screen time & Secondary outcomes: eating meals in front of TV, zBMI, aggressive behaviour \\
& Process evaluation: NR \\
\hline $\begin{array}{l}\text { Implementation-related } \\
\text { factors }\end{array}$ & Theoretical basis: SCT \\
& Resources for intervention implementation: reported \\
& Who delivered the intervention: NR \\
& PROGRESS categories assessed at baseline: gender; parent: employment, income, home owner- \\
ship/type of housing & PROGRESS categories analysed at outcome: NR \\
& Outcomes relating to harms/unintended effects: NR \\
Intervention included strategies to address diversity or disadvantage: NR \\
Economic evaluation: NR
\end{tabular}

Notes Funding: NR

\section{Risk of bias}

Bias Authors' judgement Support for judgement

Random sequence genera- Low risk Quote: "The list of random numbers was used to assign families to study or tion (selection bias) control group"

\begin{tabular}{ll}
\hline $\begin{array}{l}\text { Allocation concealment } \\
\text { (selection bias) }\end{array}$ & Unclear risk \\
& $\begin{array}{l}\text { Quote: "The list of random numbers was used to assign families to study or } \\
\text { control group" }\end{array}$ \\
\hline
\end{tabular}

\begin{tabular}{|c|c|c|}
\hline $\begin{array}{l}\text { Blinding (performance } \\
\text { bias and detection bias) } \\
\text { All outcomes }\end{array}$ & Low risk & $\begin{array}{l}\text { Data collecting residents were uninformed about group assignment. The fami- } \\
\text { lies in the control group were not aware of counselling interventions. Until the } \\
\text { end of data collection the investigators were blind to results. }\end{array}$ \\
\hline
\end{tabular}

Incomplete outcome data Low risk Attrition 13.3\%, completer analysis (attrition bias)

All outcomes 
Yilmaz 2015 (Continued)

Selective reporting (re- Unclear risk $\quad$ Protocol and trial registry report sought but not found
porting bias)

Other bias Low risk None identified

Zask 2012

\begin{tabular}{|c|c|}
\hline \multirow[t]{9}{*}{ Methods } & Study name: Tooty fruity vegie \\
\hline & Study design: cluster-RCT \\
\hline & Intervention period: 10 months \\
\hline & Follow-up period (post-intervention): nil \\
\hline & Differences in baseline characteristics: NR \\
\hline & Reliable outcomes: reported \\
\hline & Protection against contamination: NR \\
\hline & Unit of allocation: pre-school \\
\hline & Unit of analysis: individual accounting for clustering \\
\hline \multirow[t]{10}{*}{ Participants } & $\mathrm{N}$ (control baseline) = 163 (data were collected from 80.7\% at baseline) \\
\hline & $\mathrm{N}$ (control follow-up) $=152$ \\
\hline & $\mathrm{N}$ (intervention baseline) = 335 (data were collected from $80.7 \%$ at baseline) \\
\hline & $\mathrm{N}$ (intervention follow-up) = 286 \\
\hline & Setting (and number by study group): 31 preschools ( 18 intervention and 13 control) \\
\hline & $\begin{array}{l}\text { Recruitment: preschools in the New South Wales North Coast area }(\mathrm{N}=40) \text { were asked to submit an ex- } \\
\text { pression of interest to participate in the programme. } 30 \text { preschools volunteered and the team deter- } \\
\text { mined that it would have the capacity and resources to provide the intervention to } 18 \text { of them }\end{array}$ \\
\hline & Geographic region: north coast of New South Wales, Australia \\
\hline & Percentage of eligible population enrolled: $75 \%$ preschools volunteered and 18 were chosen \\
\hline & Mean age: intervention + control: $50.5 \pm 6.7$ months girls; $58.8 \pm 6.8$ months boys \\
\hline & Sex: intervention + control: $48.3 \%$ female \\
\hline \multirow[t]{6}{*}{ Interventions } & $\begin{array}{l}\text { Aimed to decrease overweight and obesity prevalence among children by improving fundamental } \\
\text { movement skills, increasing fruit and vegetable intake and decreasing unhealthy food consumption }\end{array}$ \\
\hline & $\begin{array}{l}\text { - PA interventions } \\
\text { - Structured twice-weekly fundamental movement skill development through prescribed games } \\
\text { suitable for a wide age range }\end{array}$ \\
\hline & $\begin{array}{l}\text { - Playground environment review and alterations to encourage more active movement and better } \\
\text { access to sports equipment during free play times. }\end{array}$ \\
\hline & ○ Small grants for sports equipment. \\
\hline & - Workshop for parents on limiting sedentary time, promoting PA and fundamental movement skills \\
\hline & $\begin{array}{l}\text { - A monthly 4-page newsletter containing tips of healthy eating and active playing ideas was provid- } \\
\text { ed to each parent. }\end{array}$ \\
\hline
\end{tabular}


- Healthy eating interventions

- Review and adjustment of food and nutrition policies to explicitly identify appropriate and inappropriate foods in lunch boxes.

- Communication of new policy to parents along with lunchbox displays

- Colourful posters on 'better foods' and 'foods better left out' on display all year

- Distribution of the 'Family feud/food' DVD, which models practical ways to improve children's eating habits, for their parent library

- Parents' workshops on positive parenting in relation to healthy eating and feeding 'fussy' eaters

- Simple consistent messages for children about 'sometimes' and 'everyday' foods; puppets, staff in fruit and vegetable costumes, stories, role-play, growing, cooking, and taste-testing fruit and vegetables were all used to reinforce this message

- Staff acting as role models and giving positive reinforcement to children about eating healthy food and drinking water

- Drinking water made more accessible.

- Healthy eating interventions

- Review and adjustment of food and nutrition policies to explicitly identify appropriate and inappropriate foods in lunch boxes.

- Communication of new policy to parents along with lunchbox displays

- Colourful posters on 'better foods' and 'foods better left out' on display all year

- Distribution of the 'Family feud/food' DVD, which models practical ways to improve children's eating habits, for their parent library

- Parents' workshops on positive parenting in relation to healthy eating and feeding 'fussy' eaters

- Simple consistent messages for children about 'sometimes' and 'everyday' foods; puppets, staff in fruit and vegetable costumes, stories, role-play, growing, cooking, and taste-testing fruit and vegetables were all used to reinforce this message

- Staff acting as role models and giving positive reinforcement to children about eating healthy food and drinking water

- Drinking water made more accessible.

Preschools that acted as control schools in 1 year, were on a waiting list for an intervention and were offered the full programme in subsequent years (the programme continued beyond 2007).

Diet and PA combination intervention vs control

\section{Outcomes}

Outcome measures

- Primary outcome: fundamental movement skills, fruit and vegetable served in lunchbox, unhealthy items in lunchbox, zBMIs, waist circumference, food intake, PA and sedentary behaviours

- Secondary outcomes: not clear which outcomes were primary/secondary

Process evaluation: NR

Implementation-related factors
Theoretical basis: NR

Resources for intervention implementation: reported (not much detail)

Who delivered the intervention: NR

PROGRESS categories assessed at baseline: gender

PROGRESS categories analysed at outcome: NR

Outcomes relating to harms/unintended effects: NR

Intervention included strategies to address diversity or disadvantage: NR

Economic evaluation: NR 
Zask 2012 (Continued)

Of the 1005 records collected, there were 966 complete records of lunch box audits ( $96.1 \%), 952$ complete records for anthropometric measures (94.7\%), 789 complete records of fundamental movement skills testing (78.5\%), and 699 returned parent surveys (69.6\%). Waist circumference data were only available in 498 cases in 18 preschools (10 intervention and 8 control) as records in other preschools were deemed unreliable.

Small grants for sports equipment, no further details

\section{Risk of bias}

Bias Authors' judgement Support for judgement

Random sequence genera- High risk

tion (selection bias)

Quote: "A random allocation in a ratio of approximately 1.4:1. Six intervention
and one control preschool participated in the pilot stage in 2006 to test the in-
tervention's feasibility. The 2006 control preschool became an intervention
preschool in 2007 with additional 11 intervention and 12 control preschools.
Overall, there were 18 intervention and 13 control preschools. Data from both
2006 and 2007 preschools were used in the final analyses."
Comment: it is not clear whether this study is fully randomised.

\begin{tabular}{|c|c|c|}
\hline $\begin{array}{l}\text { Allocation concealment } \\
\text { (selection bias) }\end{array}$ & High risk & As above \\
\hline $\begin{array}{l}\text { Blinding (performance } \\
\text { bias and detection bias) } \\
\text { All outcomes }\end{array}$ & High risk & $\begin{array}{l}\text { Unlikely to be blinded, especially when } 1 \text { control school became an interven- } \\
\text { tion school }\end{array}$ \\
\hline $\begin{array}{l}\text { Incomplete outcome data } \\
\text { (attrition bias) } \\
\text { All outcomes }\end{array}$ & High risk & $\begin{array}{l}\text { Data were collected from } 80.7 \% \text { and } 67.2 \% \text { of all children enrolled pre- and } \\
\text { post-intervention respectively. In addition, there were reliability issues with } \\
\text { waist circumference measurement so only data in which the same tester mea- } \\
\text { sured waist circumference pre- and post-intervention were included }\end{array}$ \\
\hline $\begin{array}{l}\text { Selective reporting (re- } \\
\text { porting bias) }\end{array}$ & Unclear risk & Protocol and trial registry report sought but not found \\
\hline Other bias & Low risk & No additional threats to validity \\
\hline $\begin{array}{l}\text { Other bias- timing of re- } \\
\text { cruitment of clusters }\end{array}$ & Low risk & Recruitment happened before randomisation. \\
\hline
\end{tabular}

ANCOVA: analysis of covariance; ASE: Attitude, social influence and self-efficacy model; BEM: Behavioral Ecological Model; BMI: bodymass index; CCHC: childcare health consultants; CDC: Centers for Disease Control and Prevention; CI: confidence interval; clusterRCT: cluster-randomised controlled trial; CSA accelerometer: computer sciences applications accelerometer; CV: cardiovascular; DEXA: dual X-ray absorptiometry; FAS: full analysis sample; FV: fruit and vegetables; GEMS: Girls' health enrichment multisite studies; HBM: Health Belief Model; HDL-C: high-density lipoprotein cholesterol; HOMA-IR: homeostatic model assessment of insulin resistance; IMB model: information-motivation-behavioural skills model; IMD: Index of Multiple Deprivation; ITT: intention to treat; LDL-C: low-density lipoprotein cholesterol; LGA: local government area; LOCF: last observation carried forward; MD: mean difference; MET: metabolic equivalent; MI: Motivational Interviewing; MVPA: moderate to vigorous physical activity; N: number; NDS-R: Nutrition Data System computer program; N/A: not applicable; NIH: National Institutes of Health; NR: not reported; PA: physical activity; PE: physical education; PROGRESS: Place, race, occupation, gender, religion, education, socio-economic status, social status) checklist; PSHE: personal, social and health education; RCT: randomised controlled trial; RT: resistance training; SAPAC: self-administered physical activity checklist; SCT: Social Cognitive Theory; SD: standard deviation; SE: standard error; SEM: Social Ecological Model; SES: socioeconomic status; SSB: sugarsweetened beverages; TPB: Theory of Planned Behaviour; TSF: triceps skinfold; TTM: Transtheoretical Model (Stages of Change); TV: television; WHCU: weight/height cubed; WIC: women's, infants' and children's centre; zBMI: body-mass index z score

\section{Characteristics of excluded studies [ordered by study ID]}




\begin{tabular}{|c|c|}
\hline Study & Reason for exclusion \\
\hline Adab 2014 & Not an RCT \\
\hline Al-Nakeeb 2007 & Longitudinal cohort study - no intervention \\
\hline Alexander 2014 & Not an RCT \\
\hline Almas 2013 & Not an RCT \\
\hline Alves 2008 & Intervention designed for the treatment of childhood obesity \\
\hline Annesi 2015 & Age/aim/duration/outcome \\
\hline Anzman-Frasca 2013 & Age/aim/duration/outcome \\
\hline Ara 2006 & Longitudinal cohort study - no intervention \\
\hline Arbeit 1992 & Aim of the trial was to prevent CVD \\
\hline Ask 2006 & Cluster allocation with $<6$ groups \\
\hline Ask 2010 & Not an RCT \\
\hline Bacardi-Gascon 2012 & Not an RCT \\
\hline Balas-Nakash 2010 & Not an RCT \\
\hline Baranowski 2012 & Age/aim/duration/outcome \\
\hline Beard 2012 & Age/aim/duration/outcome \\
\hline Bellows 2013b & Not an RCT \\
\hline Bergh 2012a & Age/aim/duration/outcome \\
\hline Bergh 2012b & Age/aim/duration/outcome \\
\hline Berry 2007 & Parent-targeted intervention designed specifically for the treatment of obesity \\
\hline Berry 2011 & Not an RCT \\
\hline Berry 2014 & Age/aim/duration/outcome \\
\hline Bilic-Kirin 2013 & Not an RCT \\
\hline Birch 2012 & Age/aim/duration/outcome \\
\hline Bjelland 2015 & Age/aim/duration/outcome \\
\hline Bollela 1999a & Aim of the trial was to improve nutritional intake \\
\hline Bollela 1999b & Aim of the trial was to improve nutritional intake \\
\hline Borys 2000 & Aim was to improve dietary habits of families \\
\hline Briganti 2014 & Not an RCT \\
\hline
\end{tabular}




\begin{tabular}{|c|c|}
\hline Study & Reason for exclusion \\
\hline Bruss 2010 & Not an RCT \\
\hline Buchan 2010 & Age/aim/duration/outcome \\
\hline Burguera 2011 & Age/aim/duration/outcome \\
\hline Burke 1998 & Aim was to improve PA \\
\hline Buscemi 2015 & Age/aim/duration/outcome \\
\hline Cairella 1998 & Aim was to improve nutritional intake \\
\hline Cameron 2014 & Age/aim/duration/outcome \\
\hline Campbell 2014 & Age/aim/duration/outcome \\
\hline Carrel 2005 & $\begin{array}{l}\text { Intervention recruited only overweight or obese participants so considered treatment for } \\
\text { the purposes of this review }\end{array}$ \\
\hline Casazza 2006 & Intervention was $<12$ weeks \\
\hline Centis 2012 & Not an RCT \\
\hline Chomitz 2003 & Aim was to increase parent awareness of child weight status \\
\hline Copeland 2010 & Age/aim/duration/outcome \\
\hline Cordova 2012 & Not an RCT \\
\hline Cruz 2014 & Age/aim/duration/outcome \\
\hline Cullen 1996 & Aim of the trial was to prevent children's behaviour disorders \\
\hline D'Agostino 1999 & Aim of the trial was to improve nutritional intake \\
\hline da Silva 2013 & Age/aim/duration/outcome \\
\hline Daley 2006 & Intervention designed specifically for the treatment of obesity \\
\hline Daniels 2014 & Age/aim/duration/outcome \\
\hline Danielzik 2005 & Intervention was $<12$ weeks \\
\hline Davis 2011 & Not an RCT \\
\hline De Bock 2010 & Age/aim/duration/outcome \\
\hline De Ruyter 2013 & Age/aim/duration/outcome \\
\hline de Silva-Sanigorski 2010a & Not an RCT - tier 2 \\
\hline de Silva-Sanigorski 2010b & Not an RCT - tier 2 \\
\hline de Silva-Sanigorski 2010c & Not an RCT - tier 2 \\
\hline
\end{tabular}




\begin{tabular}{|c|c|}
\hline Study & Reason for exclusion \\
\hline de Silva-Sanigorski 2012 & Not an RCT - tier 2 \\
\hline DeBar 2011 & Age/aim/duration/outcome \\
\hline Della 2013 & Not an RCT \\
\hline Dixon 2000 & Aim of the trial was to improve nutritional intake \\
\hline Donnelly 1996 & Cluster allocation with $<6$ groups \\
\hline Donnelly 2011 & Age/aim/duration/outcome \\
\hline Donnelly 2013 & Age/aim/duration/outcome \\
\hline Dzewaltowski 2010 & Age/aim/duration/outcome \\
\hline Economos 2007 & Cluster allocation with $<6$ groups \\
\hline Economos 2013 & Not an RCT - tier 2 \\
\hline Ehlert 2010 & Age/aim/duration/outcome \\
\hline Elinder 2012 & Not an RCT \\
\hline Eskicioglu 2014 & Not an RCT \\
\hline Evans 2012 & Age/aim/duration/outcome \\
\hline Fitzgibbon 2013 & Not an RCT \\
\hline Flattum 2011 & Age/aim/duration/outcome \\
\hline Flodmark 1993 & Intervention designed specifically for the treatment of obesity \\
\hline Florea 2005 & Intervention designed specifically for the treatment of obesity \\
\hline Flores 1995 & Cluster allocation with $<6$ groups \\
\hline Fonseca 2007 & Comparative study-No intervention \\
\hline Fotu 2011 & Not an RCT - tier 2 \\
\hline Francis 2010 & Age/aim/duration/outcome \\
\hline French 2012 & Not an RCT \\
\hline Frenn 2013 & Not an RCT \\
\hline Gabriele 2010 & Age/aim/duration/outcome \\
\hline Gao 2013 & Age/aim/duration/outcome \\
\hline Gately 2005 & Intervention duration $<12$ weeks \\
\hline Gatti 2015 & Not an RCT \\
\hline
\end{tabular}




\begin{tabular}{|c|c|}
\hline Study & Reason for exclusion \\
\hline Gatto 2015 & Not an RCT \\
\hline Gesell 2012 & Age/aim/duration/outcome \\
\hline Goldfield 2006 & Intervention duration $<12$ weeks \\
\hline Goldfield 2007 & Intervention duration was $<12$ weeks \\
\hline Graf 2011 & Not an RCT \\
\hline Greening 2011 & Not an RCT \\
\hline Hakanen 2010 & Age/aim/duration/outcome \\
\hline Hardy 2010 & Age/aim/duration/outcome \\
\hline Harrell 1998 & Intervention $<12$ weeks duration \\
\hline Harrell 1999 & Intervention $<12$ weeks duration \\
\hline Hartmann 2010 & Age/aim/duration/outcome \\
\hline Hatzis 2010 & Not an RCT - tier 2 \\
\hline Hauner 2012 & Age/aim/duration/outcome \\
\hline Hawthorne 2011 & Age/aim/duration/outcome \\
\hline He 2004 & Intervention designed specifically for the treatment of obesity \\
\hline Hernandez 2014 & Age/aim/duration/outcome \\
\hline Herrick 2012 & Not an RCT \\
\hline Hoelscher 2010 & Not an RCT \\
\hline Hoffman 2011 & Not an RCT \\
\hline Hollar 2010 & Not an RCT \\
\hline Hollar 2010a & Not an RCT \\
\hline Hollar 2010b & Not an RCT \\
\hline Hollywood 2013 & Not an RCT \\
\hline Hopper 1996 & Aim of the trial was to prevent CVD \\
\hline Horodynski 2004 & Aim of the trial was to improve nutritional intake \\
\hline Howard 1996 & Aim of the trial was to prevent CVD \\
\hline Huang 2012 & Age/aim/duration/outcome \\
\hline Huberty 2011 & Age/aim/duration/outcome \\
\hline
\end{tabular}




\begin{tabular}{|c|c|}
\hline Study & Reason for exclusion \\
\hline Ildiko 2007 & Intervention designed specifically for the treatment of obesity \\
\hline Jago 2006 & Intervention duration $<12$ weeks \\
\hline Jago 2011 & Age/aim/duration/outcome \\
\hline Jensen 2013a & Age/aim/duration/outcome \\
\hline Jensen 2013b & Age/aim/duration/outcome \\
\hline Jiang 2007 & Cluster allocation with $<6$ groups \\
\hline Johnson 2012 & Age/aim/duration/outcome \\
\hline Johnston 2012 & Age/aim/duration/outcome \\
\hline Jurg 2006 & Study did not report to be measuring any of the primary outcomes of the review \\
\hline Karanja 2010 & Not an RCT \\
\hline Karanja 2012 & Not an RCT - tier 2 \\
\hline Karp 2014 & Age/aim/duration/outcome \\
\hline Katz 2010 & Not an RCT \\
\hline Katz 2011 & Not an RCT \\
\hline Kesztyus 2013 & Age/aim/duration/outcome \\
\hline Kilanowski 2010 & Age/aim/duration/outcome \\
\hline Kim 2014 & Age/aim/duration/outcome \\
\hline King 2014 & Age/aim/duration/outcome \\
\hline Klakk 2013 & Not an RCT \\
\hline Klish 2012 & Not an RCT \\
\hline Koblinsky 1992 & Aim of the trial was to improve nutritional intake \\
\hline Kremer 2011 & Not an RCT - tier 2 \\
\hline Krombholz 2012 & Not an RCT \\
\hline LaBresh 2014 & Age/aim/duration/outcome \\
\hline Lachausse 2012 & Age/aim/duration/outcome \\
\hline Lagstrom 1997 & Aim of the trial was to improve nutritional intake \\
\hline Lakes 2013 & Not an RCT \\
\hline Lambourne 2013 & Age/aim/duration/outcome \\
\hline
\end{tabular}




\begin{tabular}{|c|c|}
\hline Study & Reason for exclusion \\
\hline Lazorick 2014 & Age/aim/duration/outcome \\
\hline LeMaster 2011 & Age/aim/duration/outcome \\
\hline Li 2010b & Age/aim/duration/outcome \\
\hline Lin 2012 & Not an RCT \\
\hline Lionis 1991 & $\begin{array}{l}\text { Aim of the trial was to assess the effects of a health education intervention aimed at reduc- } \\
\text { ing risk for CVD and cancer }\end{array}$ \\
\hline Llaurado 2014 & Not an RCT - tier 2 \\
\hline Lloyd 2012 & Not an RCT \\
\hline Louzada 2012 & Age/aim/duration/outcome \\
\hline Lubans $2012 b$ & Age/aim/duration/outcome \\
\hline Luepker 1996 & Aim of the trial was to prevent CVD \\
\hline Lumeng 2015 & Age/aim/duration/outcome \\
\hline Lynch 2012 & Not an RCT - tier 2 \\
\hline Lytle 2006 & Aim of the trial was to improve nutritional intake \\
\hline Maggiulli 2015 & Not an RCT \\
\hline Mailey 2012 & Age/aim/duration/outcome \\
\hline Manger 2012 & Not an RCT \\
\hline Manios 1998 & Aim of the trial was to improve PA and fitness \\
\hline Manios 1999 & Aim of the trial was to improve nutritional intake \\
\hline Martinez-Andrade 2014 & Age/aim/duration/outcome \\
\hline Matvienko 2010 & Not an RCT \\
\hline McAuley 2010 & Not an RCT - tier 2 \\
\hline McCallum 2007 & Intervention designed specifically for the treatment of obesity \\
\hline McGarvey 2004 & Intervention duration $<12$ weeks \\
\hline McMurray 2002 & Intervention $<12$ weeks duration \\
\hline Melnyk 2007 & Intervention was $<12$ weeks \\
\hline Millar 2011 & Not an RCT - tier 2 \\
\hline Mistry 2011 & Not an RCT \\
\hline
\end{tabular}




\begin{tabular}{|c|c|}
\hline Study & Reason for exclusion \\
\hline Moodie 2010a & Age/aim/duration/outcome \\
\hline Moodie 2010b & Age/aim/duration/outcome \\
\hline Moodie 2013 & Not an RCT - tier 2 \\
\hline Morgan 2012 & Age/aim/duration/outcome \\
\hline Muckelbauer 2011 & Age/aim/duration/outcome \\
\hline Mustila 2012a & Not an RCT \\
\hline Mustila 2012b & Not an RCT \\
\hline Mustila 2012c & Not an RCT \\
\hline Mustila 2013 & Not an RCT \\
\hline Navarro 2013 & Not an RCT - tier 2 \\
\hline Nichols 2014 & Age/aim/duration/outcome \\
\hline Niinikoski 1997 & Aim was to improve nutritional intake \\
\hline Nogueira 2014 & Age/aim/duration/outcome \\
\hline Obarzanek 1997 & Aim of the trial was to improve nutritional intake \\
\hline Oehrig 2001 & Aim of trial was to improve cardiovascular risk factors \\
\hline Padilla 2012 & Not an RCT \\
\hline Pettman 2014 & Not an RCT - tier 2 \\
\hline Plachta-Danielzik 2011 & Age/aim/duration/outcome \\
\hline Pope 2013 & Age/aim/duration/outcome \\
\hline Pratt 2013 & Age/aim/duration/outcome \\
\hline Prins 2012 & Age/aim/duration/outcome \\
\hline Puma 2013 & Age/aim/duration/outcome \\
\hline Quarles 2011 & Age/aim/duration/outcome \\
\hline Rask-Nissila 2000 & Aim of trial was to examine neurological development \\
\hline Rawlins 2013 & Age/aim/duration/outcome \\
\hline Reed 2013 & Age/aim/duration/outcome \\
\hline Reinehr 2007 & Intervention designed specifically for the treatment of obesity \\
\hline Resaland 2014 & Not an RCT \\
\hline
\end{tabular}




\begin{tabular}{|c|c|}
\hline Study & Reason for exclusion \\
\hline Resnicow 2005 & Intervention designed specifically for the treatment of obesity \\
\hline Richmond 2013 & Not an RCT \\
\hline Robinson 1999 & Cluster allocation with $<6$ groups \\
\hline Ronsley 2013 & Age/aim/duration/outcome \\
\hline Roofe 2010 & Not an RCT \\
\hline Roofe 2011 & Age/aim/duration/outcome \\
\hline Rueda 2013 & Age/aim/duration/outcome \\
\hline Rush 2014 & Age/aim/duration/outcome \\
\hline Rush 2014a & Age/aim/duration/outcome \\
\hline Sadowsky 1999 & Intervention duration $<12$ weeks \\
\hline Sanders 2014 & Not an RCT - tier 2 \\
\hline Schuna 2013 & Age/aim/duration/outcome \\
\hline Sherwood 2013a & Age/aim/duration/outcome \\
\hline Shofan 2011 & Not an RCT \\
\hline Sigmund 2012 & Not an RCT \\
\hline Sigmund 2013 & Not an RCT \\
\hline Simonetti 1986 & This trial was conducted before 1990 and so was excluded from this review \\
\hline Singhal 2010 & Not an RCT \\
\hline Singhal 2011 & Age/aim/duration/outcome \\
\hline Slusser 2013 & Not an RCT \\
\hline Smith 2012 & Not an RCT - tier 2 \\
\hline Spark 1998 & Aim of the trial was to improve nutritional intake \\
\hline Spencer 2012 & Not an RCT \\
\hline Spieker 2015 & Age/aim/duration/outcome \\
\hline Springer 2013 & Age/aim/duration/outcome \\
\hline Stenevi-Lundgren 2009 & Aim of the trial was to improve bone health outcomes \\
\hline Stephens 1998 & Aim of the trial was to improve fitness levels \\
\hline Stewart 1995 & Aim was to improve nutritional intake \\
\hline
\end{tabular}




\begin{tabular}{|c|c|}
\hline Study & Reason for exclusion \\
\hline Stice 2015 & Age/aim/duration/outcome \\
\hline Stock 2007 & Cluster allocation with $<6$ groups \\
\hline Strauss 2011 & Age/aim/duration/outcome \\
\hline Swinburn 2011 & Age/aim/duration/outcome \\
\hline Talvia 2004 & Aim of trial was to improve nutritional intake. \\
\hline Tamir 1990 & Aim of the trial was to prevent CVD \\
\hline Tarro 2014a & Not an RCT - tier 2 \\
\hline Tarro 2014b & Not an RCT - tier 2 \\
\hline Taveras 2011 & Not an RCT \\
\hline Taylor 2005 & Intervention duration $<12$ weeks \\
\hline Telford 2013a & Age/aim/duration/outcome \\
\hline Telford 2013b & Age/aim/duration/outcome \\
\hline Tershakovec 1998 & Trial conducted in hypercholesterolaemic children \\
\hline Toftager 2014 & Age/aim/duration/outcome \\
\hline Toruner 2010 & Age/aim/duration/outcome \\
\hline Toruner 2015 & Not an RCT \\
\hline Treuth 2007 & Cross-sectional study design. Not evaluating the intervention \\
\hline Trudeau 2000 & This was not an intervention study \\
\hline Tucker 2011 & Not an RCT \\
\hline Utter 2011 & Not an RCT - tier 2 \\
\hline Van Ryzin 2013 & Age/aim/duration/outcome \\
\hline Vandongen 1995 & Aim of the trial was to prevent CVD \\
\hline Wake 2011 & Age/aim/duration/outcome \\
\hline Wallen 2010 & Age/aim/duration/outcome \\
\hline Wang 2011 & Age/aim/duration/outcome \\
\hline Weber 2014 & Age/aim/duration/outcome \\
\hline Willi 2012 & Age/aim/duration/outcome \\
\hline Williams 1998 & Aim of the trial was to prevent CVD \\
\hline
\end{tabular}




\begin{tabular}{|c|c|}
\hline Study & Reason for exclusion \\
\hline Williams 2011 & Age/aim/duration/outcome \\
\hline Williamson 2006 & $\begin{array}{l}\text { Intervention recruited only overweight or obese participants so considered treatment for } \\
\text { the purposes of this review }\end{array}$ \\
\hline Williamson 2007 & Cluster allocation with $<6$ groups \\
\hline Wilson 2010 & Age/aim/duration/outcome \\
\hline Winter 2011 & Not an RCT \\
\hline Wright 2012 & Age/aim/duration/outcome \\
\hline Wright 2014a & Age/aim/duration/outcome \\
\hline Wright 2014b & Age/aim/duration/outcome \\
\hline Yang 2014 & Age/aim/duration/outcome \\
\hline Yildirim 2013 & Age/aim/duration/outcome \\
\hline Yin 2012 & Not an RCT \\
\hline Zanirati 2011 & Not an RCT \\
\hline Zhou 2014 & Not an RCT \\
\hline
\end{tabular}

CVD: cardiovascular disease; PA: physical activity; RCT: randomised controlled trial

Characteristics of studies awaiting assessment [ordered by study ID]

Adamo 2013

Methods

Participants

Interventions

Outcomes

\title{
Notes
}

\section{Adamo 2014}

\section{Methods}

\section{Participants}

\author{
Interventions
}

Outcomes 
Adamo 2014 (Continued)

Notes

Adamo 2017

Methods

\section{Participants}

Interventions

Outcomes

\section{Notes}

Allender 2016

\section{Methods}

Participants

\section{Interventions}

Outcomes

Notes

\section{Alvarez-Bueno 2017}

Methods

\section{Participants}

Interventions

\section{Outcomes}

Notes

Anderson 2014

Methods

\section{Participants}

Interventions

\section{Outcomes}


Anderson 2014 (Continued)

Notes

Anderson 2016

Methods

\section{Participants}

Interventions

\section{Outcomes}

\section{Notes}

\section{Andrade 2016}

\section{Methods}

\section{Participants}

\section{Interventions}

\section{Outcomes}

\section{Notes}

\section{Annesi 2017}

Methods

\section{Participants}

Interventions

\section{Outcomes}

\section{Notes}

Arlinghaus 2017

\section{Methods}

\section{Participants}

Interventions

\section{Outcomes}


Arlinghaus 2017 (Continued)

Notes

Armstrong 2015

Methods

\section{Participants}

Interventions

\section{Outcomes}

\section{Notes}

Atkinson 2015

Methods

Participants

Interventions

\section{Outcomes}

\section{Notes}

\section{Bacopoulou 2015}

Methods

\section{Participants}

Interventions

\section{Outcomes}

\section{Notes}

Barbosa 2015

\section{Methods}

\section{Participants}

\section{Interventions}

\section{Outcomes}


Barbosa 2015 (Continued)

Notes

Barbosa 2017

Methods

\section{Participants}

Interventions

\section{Outcomes}

\section{Notes}

\section{Barnes 2015}

Methods

\section{Participants}

\section{Interventions}

\section{Outcomes}

\section{Notes}

\section{Beck 2017}

Methods

\section{Participants}

Interventions

\section{Outcomes}

Notes

Beets 2014

Methods

\section{Participants}

Interventions

\section{Outcomes}


Beets 2014 (Continued)

Notes

Belanger 2016

Methods

\section{Participants}

Interventions

\section{Outcomes}

Notes

Benden 2014

Methods

Participants

\section{Interventions}

Outcomes

Notes

Bergh 2014

Methods

\section{Participants}

Interventions

\section{Outcomes}

Notes

Bips

Methods

\section{Participants}

Interventions

\section{Outcomes}


Bips (Continued)

Notes

Birnbaum 2017

Methods

\section{Participants}

Interventions

Outcomes

\section{Notes}

\section{Bogart 2016}

\section{Methods}

Participants

\section{Interventions}

Outcomes

Notes

Bonuck 2016

Methods

\section{Participants}

Interventions

\section{Outcomes}

Notes

Braun 2016

Methods

\section{Participants}

Interventions

\section{Outcomes}


Braun 2016 (Continued)

Notes

Brophy-Herb 2017

Methods

\section{Participants}

Interventions

\section{Outcomes}

\section{Notes}

Bryant 2017

\section{Methods}

Participants

Interventions

\section{Outcomes}

\section{Notes}

Buhler 2015

Methods

\section{Participants}

Interventions

\section{Outcomes}

\section{Notes}

\section{Burgermaster 2017}

\section{Methods}

\section{Participants}

\section{Interventions}

\section{Outcomes}


Burgermaster 2017 (Continued)

Notes

Burke 2017

Methods

\section{Participants}

Interventions

Outcomes

\section{Notes}

Buscemi 2014

Methods

Participants

\section{Interventions}

Outcomes

Notes

Bustos 2016

Methods

\section{Participants}

Interventions

\section{Outcomes}

Notes

Byrd-Bredbenner 2015

\section{Methods}

\section{Participants}

\section{Interventions}

\section{Outcomes}


Byrd-Bredbenner 2015 (Continued)

Notes

Byrd-Bredbenner 2017

\section{Methods}

\section{Participants}

Interventions

Outcomes

\section{Notes}

Byrd-Bredbenner 2017a

\section{Methods}

Participants

\section{Interventions}

Outcomes

\section{Notes}

\section{Byrd-Bredbenner 2018}

Methods

\section{Participants}

Interventions

\section{Outcomes}

\section{Notes}

Byrne 2016

\section{Methods}

\section{Participants}

Interventions

\section{Outcomes}


Byrne 2016 (Continued)

Notes

Caballero 1998

Methods

\section{Participants}

Interventions

\section{Outcomes}

\section{Notes}

\section{Camacho-Cardenosa 2016}

\section{Methods}

Participants

\section{Interventions}

\section{Outcomes}

\section{Notes}

\section{Campbell 2016}

\begin{tabular}{ll}
\hline Methods & RCT \\
\hline Participants & Children \\
\hline Interventions & Diet and or physical activity \\
\hline Outcomes & Adiposity and or anthropometric outcomes \\
\hline Notes &
\end{tabular}

\section{Cao 2014}

Methods

\section{Participants}

\section{Interventions}

\section{Outcomes}


Cao 2014 (Continued)

Notes

Carlin 2015

Methods

\section{Participants}

Interventions

\section{Outcomes}

\section{Notes}

\section{Carraway 2015}

\section{Methods}

Participants

\section{Interventions}

\section{Outcomes}

\section{Notes}

\section{Cavero-Redondo 2017}

Methods

\section{Participants}

Interventions

\section{Outcomes}

Notes

\section{Cecchetto 2017}

\section{Methods}

\section{Participants}

\section{Interventions}

\section{Outcomes}


Cecchetto 2017 (Continued)

Notes

Chamberland 2017

\section{Methods}

\section{Participants}

Interventions

\section{Outcomes}

\section{Notes}

Chen 2015

\section{Methods}

Participants

\section{Interventions}

\section{Outcomes}

\section{Notes}

\section{Chen 2017}

Methods

\section{Participants}

Interventions

\section{Outcomes}

\section{Notes}

\section{Cloutier 2015}

\begin{tabular}{ll} 
Methods & RCT \\
\hline Participants & Children \\
\hline Interventions & Diet and or physical activity \\
\hline
\end{tabular}


Cloutier 2015 (Continued)

Notes

Cocca 2016

Methods

\section{Participants}

Interventions

\section{Outcomes}

\section{Notes}

\section{Costa 2017}

\section{Methods}

Participants

\section{Interventions}

\section{Outcomes}

\section{Notes}

\section{Creanor 2016}

Methods

\section{Participants}

Interventions

\section{Outcomes}

Notes

Cunningham-Sabo 2016

\section{Methods}

\section{Participants}

\section{Interventions}

\section{Outcomes}


Cunningham-Sabo 2016 (Continued)

Notes

Daniels 2014a

Methods

\section{Participants}

Interventions

Outcomes

\section{Notes}

Daniels 2015

Methods

Participants

\section{Interventions}

\section{Outcomes}

\section{Notes}

\section{Daniels 2015a}

Methods

\section{Participants}

Interventions

\section{Outcomes}

Notes

Davis 2016

Methods

\section{Participants}

Interventions

\section{Outcomes}


Davis 2016 (Continued)

Notes

Davis 2016a

Methods

\section{Participants}

Interventions

\section{Outcomes}

\section{Notes}

de Greeff 2016

Methods

Participants

Interventions

\section{Outcomes}

\section{Notes}

de Moraes 2017

Methods

\section{Participants}

Interventions

\section{Outcomes}

\section{Notes}

de Villiers 2016

\section{Methods}

\section{Participants}

\section{Interventions}

\section{Outcomes}


de Villiers 2016 (Continued)

Notes

Delisle 2015

Methods

\section{Participants}

Interventions

\section{Outcomes}

\section{Notes}

Dordevic 2015

\section{Methods}

\section{Participants}

\section{Interventions}

\section{Outcomes}

\section{Notes}

Doring 2016

Methods

\section{Participants}

Interventions

\section{Outcomes}

Notes

Doring 2016a

Methods

\section{Participants}

Interventions

\section{Outcomes}


Doring 2016a (Continued)

Notes

Drummy 2016

Methods

\section{Participants}

Interventions

\section{Outcomes}

\section{Notes}

Dundee Family Health Study

\section{Methods}

\section{Participants}

\section{Interventions}

\section{Outcomes}

\section{Notes}

\section{Dunker 2017}

Methods

\section{Participants}

Interventions

\section{Outcomes}

\section{Notes}

Dunton 2015

\section{Methods}

\section{Participants}

\section{Interventions}

\section{Outcomes}


Dunton 2015 (Continued)

Notes

Eat Healthy for Families

Methods

\section{Participants}

Interventions

Outcomes

\section{Notes}

\section{Edwardson 2015}

Methods

Participants

\section{Interventions}

\section{Outcomes}

\section{Notes}

Effectiveness of the run-a-mile intervention

Methods

\section{Participants}

Interventions

\section{Outcomes}

Notes

Eldridge 2016

Methods

\section{Participants}

Interventions

\section{Outcomes}


Eldridge 2016 (Continued)

Notes

Eline 2016

Methods

\section{Participants}

Interventions

Outcomes

\section{Notes}

\section{Eneli 2015}

Methods

\section{Participants}

\section{Interventions}

\section{Outcomes}

\section{Notes}

\section{Eno 2017}

Methods

\section{Participants}

Interventions

\section{Outcomes}

\section{Notes}

\section{Esquivel 2015}

\section{Methods}

\section{Participants}

\section{Interventions}

\section{Outcomes}


Esquivel 2015 (Continued)

Notes

Evans 2016

Methods

\section{Participants}

Interventions

\section{Outcomes}

\section{Notes}

Families Preventing Diabetes Together

\section{Methods}

\section{Participants}

\section{Interventions}

\section{Outcomes}

\section{Notes}

\section{Fangupo 2015}

Methods

\section{Participants}

Interventions

\section{Outcomes}

\section{Notes}

\section{Farmer 2017}

\section{Methods}

\section{Participants}

\section{Interventions}

\section{Outcomes}


Farmer 2017 (Continued)

Notes

Folkvord 2016

Methods

\section{Participants}

Interventions

\section{Outcomes}

\section{Notes}

Friedrich 2015

Methods

Participants

Interventions

\section{Outcomes}

\section{Notes}

Fulkerson 2014

Methods

\section{Participants}

Interventions

\section{Outcomes}

\section{Notes}

Fulkerson 2015

\section{Methods}

\section{Participants}

\section{Interventions}

\section{Outcomes}


Fulkerson 2015 (Continued)

Notes

Fulkerson 2017

Methods

\section{Participants}

Interventions

\section{Outcomes}

\section{Notes}

Galland 2016

\begin{tabular}{ll}
\hline Methods & RCT \\
\hline Participants & Children \\
\hline Interventions & Diet and or physical activity \\
\hline Outcomes & Adiposity and or anthropometric outcomes \\
\hline
\end{tabular}

Notes

Gallotta 2015

Methods

\section{Participants}

Interventions

\section{Outcomes}

Notes

Gallotta 2016

Methods

\section{Participants}

\section{Interventions}

\section{Outcomes}


Gallotta 2016 (Continued)

Notes

Gao 2017

Methods

\section{Participants}

Interventions

\section{Outcomes}

\section{Notes}

Gholami 2015

\section{Methods}

Participants

\section{Interventions}

\section{Outcomes}

\section{Notes}

Goldfield 2016

Methods

\section{Participants}

Interventions

\section{Outcomes}

\section{Notes}

Gorin 2014

Methods

\section{Participants}

\section{Interventions}

\section{Outcomes}


Gorin 2014 (Continued)

Notes

Gortmaker 1999b

Methods

Participants

Interventions

\section{Outcomes}

\section{Notes}

Greve 2015

Methods

Participants

Interventions

\section{Outcomes}

\section{Notes}

Grillich 2016

Methods

\section{Participants}

Interventions

\section{Outcomes}

Notes

\section{Gross 2016}

Methods

\section{Participants}

\section{Interventions}

\section{Outcomes}


Gross 2016 (Continued)

Notes

Gruber 2015

Methods

\section{Participants}

Interventions

Outcomes

\section{Notes}

Grydeland 2013

Methods

Participants

Interventions

\section{Outcomes}

\section{Notes}

Gunawardena 2016

Methods

\section{Participants}

Interventions

\section{Outcomes}

Notes

Ha 2014

Methods

\section{Participants}

\section{Interventions}

\section{Outcomes}


Ha 2014 (Continued)

Notes

Habib-Mourad 2014a

Methods

\section{Participants}

Interventions

\section{Outcomes}

\section{Notes}

Haines 2016

\begin{tabular}{ll}
\hline Methods & RCT \\
\hline Participants & Children \\
\hline Interventions & Diet and or physical activity \\
\hline Outcomes & Adiposity and or anthropometric outcomes \\
\hline
\end{tabular}

Notes

Haire-Joshu 2015

Methods

\section{Participants}

Interventions

\section{Outcomes}

Notes

Hammersley 2017

\section{Methods}

\section{Participants}

\section{Interventions}

\section{Outcomes}


Hammersley 2017 (Continued)

Notes

Handel 2017

Methods

\section{Participants}

Interventions

Outcomes

\section{Notes}

Hankonen 2016

\section{Methods}

\section{Participants}

\section{Interventions}

\section{Outcomes}

\section{Notes}

\section{Hannon 2015}

Methods

\section{Participants}

Interventions

\section{Outcomes}

\section{Notes}

Harder-Lauridsen 2014

\section{Methods}

\section{Participants}

Interventions

\section{Outcomes}


Harder-Lauridsen 2014 (Continued)

Notes

Healthy Caregivers-Healthy Children (HC2) Phase II

Methods

\section{Participants}

Interventions

Outcomes

\section{Notes}

Hejazi 2017

\section{Methods}

\section{Participants}

\section{Interventions}

\section{Outcomes}

\section{Notes}

Helle 2017

Methods

\section{Participants}

Interventions

\section{Outcomes}

Notes

Helping pre-school children to avoid obesity

\section{Methods}

\section{Participants}

\section{Interventions}

\section{Outcomes}


Helping pre-school children to avoid obesity (Continued)

Notes

Hingle 2015

Methods

\section{Participants}

Interventions

Outcomes

\section{Notes}

Hjorth 2016

\begin{tabular}{ll}
\hline Methods & RCT \\
\hline
\end{tabular}

\begin{tabular}{ll}
\hline Participants & Children \\
\hline Interventions & Diet and or physical activity
\end{tabular}

Outcomes

Adiposity and or anthropometric outcomes

Notes

Hohman 2016

Methods

\section{Participants}

Interventions

\section{Outcomes}

Notes

Hohman 2017

Methods

\section{Participants}

\section{Interventions}

\section{Outcomes}


Hohman 2017 (Continued)

Notes

Hohman 2018

Methods

\section{Participants}

Interventions

\section{Outcomes}

Notes

Hollar 2015

Methods

Participants

Interventions

\section{Outcomes}

Notes

Hollis 2015

Methods

\section{Participants}

Interventions

\section{Outcomes}

Notes

Hollis 2016

Methods

\section{Participants}

\section{Interventions}

\section{Outcomes}


Hollis 2016 (Continued)

Notes

Horodynski 2015

Methods

Participants

Interventions

\section{Outcomes}

\section{Notes}

\section{Horton 2013}

\section{Methods}

Participants

\section{Interventions}

\section{Outcomes}

\section{Notes}

\section{Hrafnkelsson 2014}

Methods

\section{Participants}

Interventions

\section{Outcomes}

Notes

Huang 2015

Methods

\section{Participants}

\section{Interventions}

\section{Outcomes}


Huang 2015 (Continued)

Notes

Huang 2015a

Methods

\section{Participants}

Interventions

Outcomes

\section{Notes}

Hughes 2016

Methods

Participants

\section{Interventions}

\section{Outcomes}

\section{Notes}

Hull 2016

Methods

\section{Participants}

Interventions

\section{Outcomes}

Notes

Iaia 2017

\begin{tabular}{ll} 
Methods & RCT \\
\hline Participants & Children \\
\hline Interventions & Diet and or physical activity \\
\hline
\end{tabular}


Iaia 2017 (Continued)

Notes

Jaakkola 2015

Methods

\section{Participants}

Interventions

\section{Outcomes}

\section{Notes}

Janeiro

Methods

Participants

Interventions

\section{Outcomes}

\section{Notes}

Jones 2015

Methods

\section{Participants}

Interventions

\section{Outcomes}

Notes

\section{Katan 2016}

\section{Methods}

\section{Participants}

\section{Interventions}

\section{Outcomes}


Katan 2016 (Continued)

Notes

Kaufman-Shriqui 2016

Methods

\section{Participants}

Interventions

Outcomes

\section{Notes}

Kennedy 2018

Methods

Participants

Interventions

\section{Outcomes}

Notes

\section{Kesztyus 2017}

Methods

\section{Participants}

Interventions

\section{Outcomes}

Notes

Kharofa 2015

Methods

\section{Participants}

\section{Interventions}

\section{Outcomes}


Kharofa 2015 (Continued)

Notes

Kipping 2016

\begin{tabular}{ll} 
Methods & RCT \\
\hline Participants & Children \\
\hline Interventions & Diet and or physical activity
\end{tabular}

Outcomes

Adiposity and or anthropometric outcomes

Notes

Knowlden 2015

Methods

Participants

Interventions

Outcomes

Notes

Knowlden 2016

Methods

Participants

Interventions

\section{Outcomes}

Notes

Knowlden 2017

Methods

Participants

Interventions

\section{Outcomes}


Knowlden 2017 (Continued)

Notes

Kobel 2014

Methods

\section{Participants}

Interventions

\section{Outcomes}

\section{Notes}

Kobel 2017

\section{Methods}

Participants

\section{Interventions}

\section{Outcomes}

\section{Notes}

\section{Kobel 2017a}

Methods

\section{Participants}

Interventions

\section{Outcomes}

\section{Notes}

Kocken 2016

\section{Methods}

\section{Participants}

\section{Interventions}

\section{Outcomes}


Kocken 2016 (Continued)

Notes

Kong 2016

Methods

\section{Participants}

Interventions

Outcomes

\section{Notes}

Kovalskys 2016

Methods

Participants

\section{Interventions}

Outcomes

Notes

Kovalskys 2017

Methods

\section{Participants}

Interventions

\section{Outcomes}

Notes

Kovalskys 2017a

Methods

\section{Participants}

Interventions

\section{Outcomes}


Kovalskys 2017a (Continued)

Notes

Kuni 2015

Methods

\section{Participants}

Interventions

Outcomes

\section{Notes}

La 2016

Methods

Participants

\section{Interventions}

\section{Outcomes}

\section{Notes}

\section{LaChausse 2017}

Methods

\section{Participants}

Interventions

\section{Outcomes}

\section{Notes}

Lakshman 2015

\section{Methods}

\section{Participants}

\section{Interventions}

\section{Outcomes}


Lakshman 2015 (Continued)

Notes

Lappe 2017

Methods

\section{Participants}

Interventions

\section{Outcomes}

\section{Notes}

\section{Larsen 2015}

\section{Methods}

\section{Participants}

\section{Interventions}

\section{Outcomes}

\section{Notes}

\section{Lau 2015}

Methods

\section{Participants}

Interventions

\section{Outcomes}

Notes

Leme 2015

Methods

\section{Participants}

\section{Interventions}

\section{Outcomes}


Leme 2015 (Continued)

Notes

Leme 2016

Methods

\section{Participants}

Interventions

Outcomes

\section{Notes}

Lent 2014

Methods

Participants

Interventions

\section{Outcomes}

\section{Notes}

Lerner-Geva 2015

Methods

\section{Participants}

Interventions

\section{Outcomes}

\section{Notes}

Li 2017

Methods

\section{Participants}

\section{Interventions}

\section{Outcomes}


Li 2017 (Continued)

Notes

Liberato 2016

Methods

\section{Participants}

Interventions

\section{Outcomes}

\section{Notes}

Lichtenstein 2011

\begin{tabular}{ll}
\hline Methods & Cluster RCT \\
\hline Participants & Primary school children \\
\hline Interventions & Physical activity \\
\hline Outcomes & BMI, waist circumference, blood pressure \\
\hline Notes & This paper is in German and requires translation \\
\hline
\end{tabular}

\section{Llargués 2017}

Methods

\section{Participants}

Interventions

\section{Outcomes}

Notes

Lloyd 2014

Methods

\section{Participants}

\section{Interventions}

\section{Outcomes}


Lloyd 2014 (Continued)

Notes

Lloyd 2015

Methods

\section{Participants}

Interventions

\section{Outcomes}

\section{Notes}

\section{Lloyd 2017}

\section{Methods}

\section{Participants}

\section{Interventions}

\section{Outcomes}

\section{Notes}

Lloyd 2017a

Methods

\section{Participants}

Interventions

\section{Outcomes}

Notes

Lloyd 2018

Methods

\section{Participants}

Interventions

\section{Outcomes}


Lloyd 2018 (Continued)

Notes

Lopes 2016

Methods

\section{Participants}

Interventions

\section{Outcomes}

\section{Notes}

Lubans 2010

\section{Methods}

Participants

\section{Interventions}

\section{Outcomes}

\section{Notes}

Lubans 2012a

Methods

\section{Participants}

Interventions

\section{Outcomes}

\section{Notes}

Lubans 2016

\section{Methods}

\section{Participants}

\section{Interventions}

\section{Outcomes}


Lubans 2016 (Continued)

Notes

Lubans 2016a

Methods

\section{Participants}

Interventions

\section{Outcomes}

\section{Notes}

Lubans 2016b

\section{Methods}

\section{Participants}

\section{Interventions}

\section{Outcomes}

\section{Notes}

\section{Lumeng 2017}

Methods

\section{Participants}

Interventions

\section{Outcomes}

Notes

Luszczynska 2016

\section{Methods}

\section{Participants}

\section{Interventions}

\section{Outcomes}


Luszczynska 2016 (Continued)

Notes

Lynch 2016

Methods

\section{Participants}

Interventions

\section{Outcomes}

\section{Notes}

Madsen 2015

Methods

Participants

\section{Interventions}

Outcomes

Notes

Madsen 2017

Methods

\section{Participants}

Interventions

\section{Outcomes}

Notes

Magarey 2016

Methods

\section{Participants}

\section{Interventions}

\section{Outcomes}


Magarey 2016 (Continued)

Notes

Maha 2016

Methods

\section{Participants}

Interventions

Outcomes

\section{Notes}

Martinez-Vizcaino 2015

Methods

\section{Participants}

\section{Interventions}

\section{Outcomes}

\section{Notes}

\section{McEachan 2016}

Methods

\section{Participants}

Interventions

\section{Outcomes}

\section{Notes}

McManus 2015

\section{Methods}

\section{Participants}

Interventions

\section{Outcomes}


McManus 2015 (Continued)

Notes

Melnyk 2015

Methods

\section{Participants}

Interventions

Outcomes

\section{Notes}

Messiah 2017

Methods

Participants

\section{Interventions}

Outcomes

Notes

Minossi 2015

Methods

\section{Participants}

Interventions

\section{Outcomes}

Notes

Mohammed 2015

\section{Methods}

\section{Participants}

\section{Interventions}

\section{Outcomes}


Mohammed 2015 (Continued)

Notes

Moir 2016

Methods

\section{Participants}

Interventions

Outcomes

\section{Notes}

Moore 2016

Methods

\section{Participants}

\section{Interventions}

Outcomes

\section{Notes}

\section{Mora 2015a}

Methods

\section{Participants}

Interventions

\section{Outcomes}

\section{Notes}

More jumping and better eating at home and school

\section{Methods}

\section{Participants}

Interventions

\section{Outcomes}


More jumping and better eating at home and school (Continued)

Notes

Morgan 2014

Methods

\section{Participants}

Interventions

Outcomes

\section{Notes}

Muller 2016

Methods

Participants

\section{Interventions}

\section{Outcomes}

Notes

\section{Muzaffar 2014}

Methods

\section{Participants}

Interventions

\section{Outcomes}

Notes

Muzaffar 2016

Methods

\section{Participants}

Interventions

\section{Outcomes}


Muzaffar 2016 (Continued)

Notes

Nanney 2016

Methods

\section{Participants}

Interventions

Outcomes

\section{Notes}

Natale 2016

Methods

Participants

\section{Interventions}

\section{Outcomes}

Notes

Natale 2017

Methods

\section{Participants}

Interventions

\section{Outcomes}

Notes

Natale 2017a

Methods

\section{Participants}

Interventions

\section{Outcomes}


Natale 2017a (Continued)

Notes

Nezami 2016

Methods

\section{Participants}

Interventions

\section{Outcomes}

\section{Notes}

Nezami 2017

\section{Methods}

\section{Participants}

\section{Interventions}

\section{Outcomes}

\section{Notes}

Nezami 2017a

Methods

\section{Participants}

Interventions

\section{Outcomes}

\section{Notes}

\section{Nogueira 2014a}

\section{Methods}

\section{Participants}

\section{Interventions}

\section{Outcomes}


Nogueira 2014a (Continued)

Notes

Nogueira 2015

Methods

\section{Participants}

Interventions

\section{Outcomes}

\section{Notes}

Nogueira 2017

Methods

Participants

Interventions

\section{Outcomes}

\section{Notes}

Novotny 2015

Methods

\section{Participants}

Interventions

\section{Outcomes}

\section{Notes}

Novotny 2017

Methods

\section{Participants}

\section{Interventions}

\section{Outcomes}


Novotny 2017 (Continued)

Notes

Nudging, Healthy Diet and Physical Activity

Methods

Participants

Interventions

Outcomes

Notes

NUTRICIA

Methods

Participants

Interventions

Outcomes

Notes

Nyberg 2016

Methods

\section{Participants}

Interventions

\section{Outcomes}

Notes

Nystrom 2017

Methods

\section{Participants}

Interventions

\section{Outcomes}


Nystrom 2017 (Continued)

Notes

Ochoa 2017

Methods

\section{Participants}

Interventions

\section{Outcomes}

Notes

\section{Ochoa-Aviles 2017}

\section{Methods}

Participants

\section{Interventions}

\section{Outcomes}

\section{Notes}

\section{Olsen 2017}

Methods

\section{Participants}

Interventions

\section{Outcomes}

Notes

\section{Omorou 2015}

\section{Methods}

\section{Participants}

\section{Interventions}

\section{Outcomes}


Omorou 2015 (Continued)

Notes

Omorou 2015a

Methods

\section{Participants}

Interventions

\section{Outcomes}

\section{Notes}

Pamplona 2015

\section{Methods}

\section{Participants}

\section{Interventions}

\section{Outcomes}

\section{Notes}

Parkinson 2015

Methods

\section{Participants}

Interventions

\section{Outcomes}

\section{Notes}

\section{Paul 2016}

\section{Methods}

\section{Participants}

\section{Interventions}

\section{Outcomes}


Paul 2016 (Continued)

Notes

Pellanda 2015

Methods

\section{Participants}

Interventions

\section{Outcomes}

\section{Notes}

Penalvo 2013

Methods

Participants

Interventions

\section{Outcomes}

\section{Notes}

\section{Penalvo 2015}

Methods

\section{Participants}

Interventions

\section{Outcomes}

\section{Notes}

Pinket 2016

\section{Methods}

\section{Participants}

\section{Interventions}

\section{Outcomes}


Pinket 2016 (Continued)

Notes

Price 2015

Methods

\section{Participants}

Interventions

\section{Outcomes}

\section{Notes}

\section{Prina 2014}

\section{Methods}

\section{Participants}

\section{Interventions}

\section{Outcomes}

\section{Notes}

Quintiliani 2014

Methods

\section{Participants}

Interventions

\section{Outcomes}

Notes

Raat 2013

Methods

\section{Participants}

\section{Interventions}

\section{Outcomes}


Raat 2013 (Continued)

Notes

Raine 2017

Methods

\section{Participants}

Interventions

\section{Outcomes}

\section{Notes}

Rangan 2017

Methods

Participants

Interventions

\section{Outcomes}

\section{Notes}

Razani 2016

Methods

\section{Participants}

Interventions

\section{Outcomes}

\section{Notes}

\section{Redfern 2016}

\section{Methods}

\section{Participants}

\section{Interventions}

\section{Outcomes}


Redfern 2016 (Continued)

Notes

Rerksuppaphol 2017

Methods

Participants

Interventions

\section{Outcomes}

\section{Notes}

\section{Reyes-Morales 2016}

\section{Methods}

Participants

Interventions

\section{Outcomes}

\section{Notes}

\section{Richmond 2016}

Methods

\section{Participants}

Interventions

\section{Outcomes}

\section{Notes}

\section{Rosario 2015}

\section{Methods}

\section{Participants}

\section{Interventions}

\section{Outcomes}


Rosario 2015 (Continued)

Notes

Rosario 2017

Methods

\section{Participants}

Interventions

\section{Outcomes}

\section{Notes}

Roth 2010

Methods

Participants

Interventions

\section{Outcomes}

\section{Notes}

\section{Ruiter 2015}

Methods

\section{Participants}

Interventions

\section{Outcomes}

Notes

Rush 2016

\section{Methods}

\section{Participants}

\section{Interventions}

\section{Outcomes}


Rush 2016 (Continued)

Notes

Salazar 2014

Methods

\section{Participants}

Interventions

\section{Outcomes}

\section{Notes}

\section{Sanchez-Lopez 2015}

\section{Methods}

\section{Participants}

\section{Interventions}

\section{Outcomes}

\section{Notes}

Santos 2012

Methods

\section{Participants}

Interventions

\section{Outcomes}

\section{Notes}

\section{Santos 2015}

\section{Methods}

\section{Participants}

\section{Interventions}

\section{Outcomes}


Santos 2015 (Continued)

Notes

Santos 2016

Methods

\section{Participants}

Interventions

Outcomes

\section{Notes}

Santos-Beneit 2015

\section{Methods}

\section{Participants}

\section{Interventions}

\section{Outcomes}

\section{Notes}

\section{Savage 2016}

Methods

\section{Participants}

Interventions

\section{Outcomes}

Notes

Sayers 2017

Methods

\section{Participants}

Interventions

\section{Outcomes}


Sayers 2017 (Continued)

Notes

Schoffman 2017

Methods

\section{Participants}

Interventions

\section{Outcomes}

Notes

Schuh 2017

Methods

Participants

Interventions

\section{Outcomes}

\section{Notes}

\section{Schwartz 2015}

Methods

\section{Participants}

Interventions

\section{Outcomes}

Notes

Seguin 2017

Methods

\section{Participants}

\section{Interventions}

\section{Outcomes}


Seguin 2017 (Continued)

Notes

Seward 2016

Methods

\section{Participants}

Interventions

Outcomes

\section{Notes}

Sgambato 2016

\section{Methods}

Participants

\section{Interventions}

\section{Outcomes}

\section{Notes}

\section{Shah 2016}

Methods

\section{Participants}

Interventions

\section{Outcomes}

\section{Notes}

Sherwood 2015

\section{Methods}

\section{Participants}

\section{Interventions}

\section{Outcomes}


Sherwood 2015 (Continued)

Notes

Simons 2014

Methods

\section{Participants}

Interventions

\section{Outcomes}

Notes

Simons 2015

Methods

Participants

Interventions

\section{Outcomes}

\section{Notes}

\section{Skouteris 2010}

Methods

\section{Participants}

Interventions

\section{Outcomes}

\section{Notes}

\section{Skouteris 2014}

\section{Methods}

\section{Participants}

\section{Interventions}

\section{Outcomes}


Skouteris 2014 (Continued)

Notes

Smedegaard 2016

Methods

\section{Participants}

Interventions

\section{Outcomes}

Notes

Smith 2015

\section{Methods}

\section{Participants}

\section{Interventions}

\section{Outcomes}

\section{Notes}

Smith 2017

Methods

\section{Participants}

Interventions

\section{Outcomes}

\section{Notes}

Smith 2018

Methods

\section{Participants}

Interventions

\section{Outcomes}


Smith 2018 (Continued)

Notes

Sobko 2016

Methods

\section{Participants}

Interventions

\section{Outcomes}

\section{Notes}

\section{Spieker 2015a}

\section{Methods}

Participants

\section{Interventions}

\section{Outcomes}

\section{Notes}

\section{Steenbock 2017}

Methods

\section{Participants}

Interventions

\section{Outcomes}

\section{Notes}

\section{Stettler 2015}

\section{Methods}

\section{Participants}

Interventions

\section{Outcomes}


Stettler 2015 (Continued)

Notes

Suchert 2015

Methods

\section{Participants}

Interventions

\section{Outcomes}

\section{Notes}

Sun 2017

Methods

Participants

Interventions

\section{Outcomes}

\section{Notes}

\section{Sutherland 2016}

Methods

\section{Participants}

Interventions

\section{Outcomes}

Notes

Tang 2016

Methods

\section{Participants}

\section{Interventions}

\section{Outcomes}


Tang 2016 (Continued)

Notes

Tarro 2017

Methods

Participants

Interventions

\section{Outcomes}

\section{Notes}

Taveras 2012

\begin{tabular}{ll}
\hline Methods & RCT \\
\hline Participants & Low-income children aged 2-5 years from health centres in Boston \\
\hline Interventions & diet, sleeping, screen time (mainly home-based) \\
\hline Outcomes & BMl is secondary outcome \\
\hline Notes & NCT01565161 \\
\hline
\end{tabular}

Taylor 2013

\begin{tabular}{ll}
\hline Methods & RCT \\
\hline Participants & Overweight and obese pregnant women BMI $>25$ \\
\hline Interventions & Mother diet and PA, breast or bottle feeding, infant diet and parental feeding practice, infant PA \\
\hline Outcomes & Weight \\
\hline Notes & ISRCTN56735429 \\
& Now published: McEachan 2016 \\
\hline
\end{tabular}

Taylor 2016

\section{Methods}

\section{Participants}

\section{Interventions}


Taylor 2016 (Continued)

Outcomes

\section{Notes}

Taylor 2017

Methods

\section{Participants}

Interventions

Outcomes

\section{Notes}

Taylor 2017a

Methods

\section{Participants}

Interventions

\section{Outcomes}

Notes

Thayer 2017

\section{Methods}

Participants

\section{Interventions}

\section{Outcomes}

\section{Notes}

\section{The Healthy School Start Plus Intervention Study}

\section{Methods}

\section{Participants}

Interventions 
The Healthy School Start Plus Intervention Study (Continued)

Outcomes

\section{Notes}

Thompson 2013

Methods

\section{Participants}

Interventions

Outcomes

Notes

Tirlea 2016

Methods

\section{Participants}

Interventions

\section{Outcomes}

Notes

Tomayko 2016

\section{Methods}

Participants

\section{Interventions}

Outcomes

\section{Notes}

Tomayko 2017

\section{Methods}

\section{Participants}

Interventions 
Tomayko 2017 (Continued)

Outcomes

\section{Notes}

\section{Toscano 2017}

\section{Methods}

\section{Participants}

Interventions

\section{Outcomes}

\section{Notes}

\section{van Grieken 2017}

Methods

\section{Participants}

Interventions

\section{Outcomes}

Notes

van Nassau 2014

\section{Methods}

\section{Participants}

\section{Interventions}

\section{Outcomes}

\section{Notes}

\section{Vilchis-Gil 2016}

\section{Methods}

\section{Participants}

\section{Interventions}


Vilchis-Gil 2016 (Continued)

Outcomes

\title{
Notes
}

Wade 2017

Methods

\section{Participants}

Interventions

\section{Outcomes}

\section{Notes}

\section{Wagner 2017}

Methods

\section{Participants}

Interventions

\section{Outcomes}

Notes

Walther 2011

\begin{tabular}{ll}
\hline Methods & Cluster RCT \\
\hline Participants & School children \\
\hline Interventions & Physical activity (sport) \\
\hline Outcomes & Maximal oxygren uptake, motor coordination, blood pressure, prevalences of overweight/obesity \\
\hline Notes & This paper is in German and requires translation \\
\hline
\end{tabular}

\section{Walton 2015}

\section{Methods}

\section{Participants}

\author{
Interventions
}


Walton 2015 (Continued)

Outcomes

\section{Notes}

\section{Walton 2016}

Methods

\section{Participants}

Interventions

\section{Outcomes}

\section{Notes}

\section{Wang 2017}

Methods

\section{Participants}

Interventions

\section{Outcomes}

\section{Notes}

Wasser 2015

\section{Methods}

\section{Participants}

\section{Interventions}

\section{Outcomes}

\section{Notes}

\section{Waters 2017}

\section{Methods}

\section{Participants}

\section{Interventions}


Waters 2017 (Continued)

Outcomes

\title{
Notes
}

Welk 2015

Methods

\section{Participants}

Interventions

\section{Outcomes}

\section{Notes}

Wen 2015

Methods

\section{Participants}

Interventions

\section{Outcomes}

Notes

Wendel 2016

\section{Methods}

Participants

\section{Interventions}

\section{Outcomes}

\section{Notes}

\section{Wessel 2015}

\section{Methods}

\section{Participants}

\author{
Interventions
}


Wessel 2015 (Continued)

Outcomes

\title{
Notes
}

Wessel 2015a

Methods

\section{Participants}

Interventions

Outcomes

\section{Notes}

Wieland 2016

Methods

\section{Participants}

Interventions

\section{Outcomes}

Notes

Wilken 2013

\section{Methods}

Participants

\section{Interventions}

\section{Outcomes}

\section{Notes}

\section{Wilksch 2017}

\section{Methods}

\section{Participants}

\author{
Interventions
}


Wilksch 2017 (Continued)

Outcomes

\section{Notes}

\section{Wolfenden 2016b}

Methods

\section{Participants}

Interventions

\section{Outcomes}

\section{Notes}

Wright 2016

Methods

\section{Participants}

Interventions

\section{Outcomes}

Notes

Xin 2016

Methods

Participants

Interventions

\section{Outcomes}

\section{Notes}

\section{Xu 2015}

\section{Methods}

\section{Participants}

Interventions 
Xu 2015 (Continued)

\title{
Outcomes
}

\section{Notes}

\section{Xu 2016}

Methods

\section{Participants}

Interventions

\section{Outcomes}

\section{Notes}

Xu 2017

Methods

\section{Participants}

Interventions

\section{Outcomes}

\section{Notes}

Yoshinaga 2016

Methods

Participants

\section{Interventions}

\section{Outcomes}

\section{Notes}

\section{Zafiropulos 2015}

\author{
Methods
}

\section{Participants}

Interventions 
Zafiropulos 2015 (Continued)

Outcomes

\section{Notes}

\section{Zota 2016}

Methods

\section{Participants}

Interventions

Outcomes

Notes

Characteristics of ongoing studies [ordered by study ID]

Adab 2015b

\begin{tabular}{ll}
\hline Trial name or title & WAVES (West Midlands active lifestyle and healthy eating in school children \\
\hline Methods & Cluster-RCT \\
\hline Participants & School children aged 6-7 years \\
\hline Interventions & Diet and PA \\
\hline Outcomes & zBMI, cost per QALY \\
\hline Starting date & Sept 2010-August 2015 \\
\hline Contact information & p.adab@bham.ac.uk \\
\hline Notes & ISRCTN97000586 \\
\hline
\end{tabular}

Adams 2012

\begin{tabular}{ll}
\hline Trial name or title & HCSF (Healthy children, strong families \\
\hline Methods & RCT \\
\hline Participants & $2-5$ years Native American Indian children \\
\hline Interventions & Diet and PA via home visits \\
\hline Outcomes & zBMI, primary caregiver BMI \\
\hline Starting date & NR \\
\hline
\end{tabular}


Adams 2012 (Continued)

Contact information

alex.adams@fammed.wisc.edu

\section{Notes}

\section{Barlow 2008}

Trial name or title

Empowering mothers to prevent obesity at weaning

\section{Methods}

\begin{tabular}{ll}
\hline Participants & Women with pre-pregnancy obesity $(\mathrm{BMI}>35)$ \\
\hline Interventions & $\begin{array}{l}\text { Feasibility RCT of the effectiveness of an intervention aimed at empowering mothers to prevent } \\
\text { obesity at weaning }\end{array}$
\end{tabular}

\section{Outcomes}

\begin{tabular}{ll}
\hline Starting date & 01 April 2007. Project end date: 31 August 2009 \\
\hline Contact information & $\begin{array}{l}\text { Jane Barlow, Professor of Public Health in the Early Years, University of Warwick, Coventry } \\
\text { Jane.barlow@warwick.ac.uk }\end{array}$ \\
\hline Notes & \\
\hline
\end{tabular}

Bundy 2011

\begin{tabular}{ll}
\hline Trial name or title & Sydney playground project \\
\hline Methods & Cluster-RCT \\
\hline Participants & School child red aged 5-7 years \\
\hline Interventions & PA \\
\hline Outcomes & Primary: PA (accelerometer); secondary outcomes include anthrompometrics \\
\hline Starting date & 01 May 2009 \\
\hline Contact information & anita.bundy@sydney.edu.au \\
\hline Notes & ACTRN12611000089932 \\
\hline
\end{tabular}

\section{Draper 2010}

\begin{tabular}{ll}
\hline Trial name or title & HealthKick \\
\hline Methods & Cluster-RCT \\
\hline Participants & Low income schools in South Africa \\
\hline
\end{tabular}


Draper 2010 (Continued)

\begin{tabular}{lc} 
Interventions & Diet and PA NR \\
\hline Outcomes & NR \\
\hline Starting date catherine.draper@uct.ac.za \\
\hline Notes
\end{tabular}

Dreyhaupt 2012

\begin{tabular}{ll}
\hline Trial name or title & Komm mit in das gesunde boot - grundschule \\
\hline Methods & Cluster-RCT \\
\hline Participants & Primary school children aged 5-8 years \\
\hline Interventions & Diet and PA \\
\hline Outcomes & Waist circumference, skinfold thickness, 6-min run \\
\hline Starting date & 2010 \\
\hline Contact information & juergen.steinacker@uniklinik-ulm.de \\
\hline Notes & DRKS00000494 \\
\hline
\end{tabular}

\section{Flattum 2015}

\begin{tabular}{ll}
\hline Trial name or title & HOME plus (Healthy home offerings via the mealtime environment) \\
\hline Methods & RCT \\
\hline Participants & Children aged 8-12 years and their parents \\
\hline Interventions & Diet and reducing sedentary behaviours \\
\hline Outcomes & BMI \\
\hline Starting date & July 2010-June 2016 \\
\hline Contact information & flatt018@umn.edu \\
\hline Notes & NCT01538615 \\
\hline
\end{tabular}

\section{Glazebrook 2011}

Trial name or title STAK (Steps to active kids)


Glazebrook 2011 (Continued)

\begin{tabular}{ll} 
Methods & Cluster-RCT \\
\hline Participants & $\begin{array}{l}\text { School children aged 9-11 years with low level of exercise self-efficacy, asthma and over- } \\
\text { weight }\end{array}$ \\
\hline Interventions & PA \\
\hline Outcomes & BMI, exercise self-efficacy \\
\hline Starting date & 01 April 2010-31 December 2012 \\
\hline Contact information & cris.glazebrook@nottingham.ac.uk \\
\hline Notes & ISRCTN12650001 \\
\hline
\end{tabular}

\begin{tabular}{ll}
\hline Hesketh 2013 & Melbourne infant feeding, activity and nutrition trial (InFANT) program follow-up \\
\hline Trial name or title & Cluster-RCT \\
\hline Participants & First-time parents of infants aged 4-20 months \\
\hline Interventions & Diet and PA \\
\hline Outcomes & BMI, waist circumference, diet, PA, sedentary time \\
\hline Starting date & Mid 2001-end 2013 \\
\hline Contact information & kylie.hesketh@deakin.edu.au \\
\hline Notes & ISRCTN81847050 \\
\hline
\end{tabular}

Horodynski 2011a

\begin{tabular}{ll}
\hline Trial name or title & Healthy toddlers trial \\
\hline Methods & RCT \\
\hline Participants & $\begin{array}{l}\text { Economically and educationally disadvantaged mother-toddler dyads, toddlers aged 12-36 } \\
\text { months }\end{array}$ \\
\hline Interventions & Diet (home-based) \\
\hline Outcomes & Diet intake and eating skills \\
\hline Starting date & 01 October 2010 \\
\hline Contact information & millie@msu.edu \\
\hline Notes & ACTRN12610000981022 \\
\hline
\end{tabular}


Horodynski 2011b

\begin{tabular}{ll}
\hline Trial name or title & Healthy babies trial \\
\hline Methods & RCT \\
\hline Participants & Economically and educationally disadvantaged mother-infant dyads (0-6 months) \\
\hline Interventions & Healthy transition to solids (home-based) \\
\hline Outcomes & Infant growth pattern \\
\hline Starting date & February 2010 \\
\hline Contact information & millie@msu.edu \\
\hline Notes & ACTRN12610000415000 \\
\hline
\end{tabular}

Miller 2012

\begin{tabular}{ll}
\hline Trial name or title & Growing healthy study \\
\hline Methods & RCT \\
\hline Participants & Low-income preschoolers starting Head Start \\
\hline Interventions & Diet (3 arms) \\
\hline Outcomes & BMI, skinfold thickness \\
\hline Starting date & March 2011-May 2015 \\
\hline Contact information & alimill@umich.edu \\
\hline Notes & NCT01398358 \\
\hline
\end{tabular}

Murphy 2013

\begin{tabular}{ll}
\hline Trial name or title & SWITCH (Smart weight in teenagers choosing health) \\
\hline Methods & RCT \\
\hline Participants & Adolescents aged 11-16 years attending dental surgeries BMI $\geq 85$ th centile \\
\hline Interventions & Reduce soft drink consumption (brief intervention using MI) \\
\hline Outcomes & BMI, waist circumference \\
\hline Starting date & July 2011 (Recruitment 01/04/2012 to 01/06/2012) \\
\hline Contact information & jessie.porter@ucl.ac.uk; r.watt@ucl.ac.uk \\
\hline
\end{tabular}


Murphy 2013 (Continued)

Notes

ISRCTN04152711

Myers 2014

\begin{tabular}{ll}
\hline Trial name or title & EB4K with play (Energy balance for kids with play) \\
\hline Methods & Cluster-RCT \\
\hline Participants & Low-income urban schools \\
\hline Interventions & Diet and PA \\
\hline Outcomes & Nutrition knowledge, dietary intake, MVPA, fitness, zBMI score \\
\hline Starting date & Autumn 2011 \\
\hline Contact information & danaeg@berkley.edu \\
\hline Notes & Funded by Healthy Weight Commitment Foundation (food and beverage organisations) \\
\hline
\end{tabular}

Olsen 2012

\begin{tabular}{ll}
\hline Trial name or title & Healthy start project \\
\hline Methods & RCT \\
\hline Participants & $\begin{array}{l}\text { Children aged 2-6 years at risk (high birth weight, mother overweight prior to pregnancy or familial } \\
\text { low SES); excluded overweight children }\end{array}$ \\
\hline Interventions & Diet and PA, also focus on stress and sleep (1 intervention and 2 control arms) \\
\hline Outcomes & Anthropometric \\
\hline Starting date & May 2009-December 2020 \\
\hline Contact information & njo@ipm.regionh.dk \\
\hline Notes & NCT01583335 \\
\hline
\end{tabular}

Ostbye 2015

\begin{tabular}{ll}
\hline Trial name or title & Keys (Keys to healthy family child care homes) \\
\hline Methods & Cluster-RCT \\
\hline Participants & Children in family care homes \\
\hline Interventions & Diet and PA (home-based) \\
\hline Outcomes & PA and dietary intake (BMI is secondary) \\
\hline \hline
\end{tabular}


Ostbye 2015 (Continued)

\begin{tabular}{ll} 
Starting date & NR \\
\hline Contact information & courtney.mann@dm.duke.edu \\
\hline
\end{tabular}

Notes

Paul 2014

\begin{tabular}{ll}
\hline Trial name or title & INSIGHT (Intervention nurses start infants growing on healthy trajectories) \\
\hline Methods & RCT \\
\hline Participants & First-time mothers and their newborns \\
\hline Interventions & Parenting vs safety group \\
\hline Outcomes & zBMI at 3 years \\
\hline Starting date & January 2012-June 2019 \\
\hline Contact information & ipaul@psu.edu \\
\hline Notes & NCT01167270; 1-year outcomes published in Savage 2016 \\
\hline
\end{tabular}

\section{Piek 2010}

\begin{tabular}{ll}
\hline Trial name or title & Animal fun for pre-primary children \\
\hline Methods & RCT and nested cohort \\
\hline Participants & Preschool children aged 4.5-6 years attending government school in low-SES area \\
\hline Interventions & Whole-of-class PA programme \\
\hline Outcomes & Motor and psychosocial skills \\
\hline Starting date & 26 April 2009 \\
\hline Contact information & j.piek@curtin.edu.au \\
\hline Notes & ACTRN12609000869279; unclear if anthropometrics will be measured for intervention vs control \\
\hline
\end{tabular}

\section{Po'e 2013}

\begin{tabular}{ll}
\hline Trial name or title & GRWO (Growing right onto wellness) \\
\hline Methods & RCT \\
\hline Participants & Children aged 3-5 years from minority communities who are not obese \\
\hline
\end{tabular}


Po'e 2013 (Continued)

\begin{tabular}{ll} 
Interventions & Healthy lifestyle vs school readiness programmes set in local recreation centres and libraries \\
\hline Outcomes & BMI \\
\hline Starting date & NR, protocol published 2013 \\
\hline Contact information & shari.barkin@vanderbilt.edu \\
\hline Notes &
\end{tabular}

Reifsnider 2013

\begin{tabular}{ll}
\hline Trial name or title & $\begin{array}{l}\text { A randomized controlled trial to prevent childhood obesity through early childhood feeding and } \\
\text { parenting guidance }\end{array}$ \\
\hline Methods & RCT \\
\hline Participants & $\begin{array}{l}\text { Women 30-36 weeks pregnant whose babies are at high risk for obesity due to ethnicity, income } \\
\text { and maternal BMI }\end{array}$ \\
\hline Interventions & $\begin{array}{l}\text { Structured Community Health Worker (CHW)--provided home visits, using an intervention created } \\
\text { through community-based participatory research, to standard care received through the Special } \\
\text { Supplemental Nutrition Program for Women, Infants, and Children (WIC) }\end{array}$ \\
\hline Outcomes & Anthropometric \\
\hline Starting date & NR, protocol published 2013 \\
\hline Contact information & elizabeth.reifsnider@asu.edu \\
\hline Notes & NCT01905072
\end{tabular}

Sanchez-Gomez 2012

\begin{tabular}{ll}
\hline Trial name or title & $\begin{array}{l}\text { SAVINHEARTS (A clinical trial of two educational strategies in cardiovascular health in child popula- } \\
\text { tion) }\end{array}$ \\
\hline Methods & Cluster-RCT \\
\hline Participants & Children aged 7-8 years in public primary schools in Madrid \\
\hline Interventions & $\begin{array}{l}\text { Music concert about obesity prevention and cardiovascular health vs participatory class with same } \\
\text { messages and makes healthy breakfast }\end{array}$ \\
\hline Outcomes & Knowledge questionnaire and attitude test (BMI is secondary) \\
\hline Starting date & $\begin{array}{l}\text { January 2012- January 2015 } \\
\text { Contact information }\end{array}$ \\
$\begin{array}{l}\text { Sanchez-Gomez LM: Agencia de Evaluación de Tecnología Sanitarias (AETS), ISCIII. Instituto de In- } \\
\text { vestigación Sanitaria del Hospital Universitario de La Princesa (IP).C/ Monforte de Lemos 5, Madrid } \\
28029, \text { Spain }\end{array}$
\end{tabular}


Sanchez-Gomez 2012 (Continued)

Notes

NCT01418872

Sherwood 2013

\begin{tabular}{ll}
\hline Trial name or title & NET-Works (Now everybody together for amazing and healthful kids) \\
\hline Methods & Low-income racially/ethnically diverse preschool age children \\
\hline Participants & Diet and PA involving home, community, primary care and community \\
\hline Interventions & BMI \\
\hline Outcomes & June 2012-April 2017 \\
\hline Starting date & nancy.sherwood@healthpartners.com \\
\hline Contact information & NCT01606891 \\
\hline Notes &
\end{tabular}

\section{Siegrist 2011}

\begin{tabular}{ll}
\hline Trial name or title & JuvenTUM 3 \\
\hline Methods & Cluster-RCT \\
\hline Participants & School children aged 10-14 years in Germany \\
\hline Interventions & Diet and PA involving home and school \\
\hline Outcomes & BMl, waist and arm circumferences, skinfold thickness, also micro- and macrovascular function \\
\hline Starting date & July 2008-December 2012 \\
\hline Contact information & siegrist@sport.med.tum.de \\
\hline Notes & NCT00988754 \\
\hline
\end{tabular}

\section{Slawson 2015}

\begin{tabular}{ll}
\hline Trial name or title & Team up for healthy living \\
\hline Methods & Cluster-RCT \\
\hline Participants & Adolescents in Southern Appalachia \\
\hline Interventions & $\begin{array}{l}\text { Diet and PA involving undergraduate students as peer facilitators and Theory of Planned Behav- } \\
\text { iour }\end{array}$ \\
\hline Outcomes & BMI, diet, PA and sedentary behaviours \\
\hline \hline
\end{tabular}


Slawson 2015 (Continued)
Starting date
NR

Contact information

Slawson DL, East Tennessee State University College of Public Health, USA

Notes

Sobko 2011

\begin{tabular}{ll}
\hline Trial name or title & EarlY STOPP (Stockholm obesity prevention program) \\
\hline Methods & RCT \\
\hline Participants & Overweight and/or obese parents with infants starting at 1 year in Stockholm \\
\hline Interventions & Diet and PA and sleep (dietitian, physiotherapist or nurse delivers) \\
\hline Outcomes & BMI \\
\hline Starting date & January 2010-March 2016 \\
\hline Contact information & tanja.sobko@ki.si \\
\hline Notes & ES-2010 \\
\hline
\end{tabular}

\section{Taylor 2011}

\begin{tabular}{ll}
\hline Trial name or title & POI.nz (Prevention of overweight in infancy) New Zealand \\
\hline Methods & RCT (4 arm) \\
\hline Participants & Mothers recruited at booking of maternity services \\
\hline Interventions & Education+support vs food, activity and breastfeeding group vs sleep group vs usual care \\
\hline Outcomes & Weight velocity and zBMI \\
\hline Starting date & May 2009-April 2017 \\
\hline Contact information & barry.taylor@otago.ac.nz \\
\hline Notes & NCT00892983 \\
\hline
\end{tabular}

\section{Tovar 2013}

\begin{tabular}{ll}
\hline Trial name or title & Live well \\
\hline Methods & RCT \\
\hline Participants & Immigrant mothers and children \\
\hline
\end{tabular}


Tovar 2013 (Continued)

\begin{tabular}{ll} 
Interventions & Family meals \\
\hline Outcomes & NR, baseline data published 2013 \\
\hline Starting date & alison_tovar@mail.uri.edu \\
\hline Contact information & \\
\hline Notes
\end{tabular}

Walters 2012

\begin{tabular}{ll}
\hline Trial name or title & Healthy hearts across generations \\
\hline Methods & RCT \\
\hline Participants & Native American Indian families (at risk) \\
\hline Interventions & MI \\
\hline Outcomes & BMI - in adults (unclear if child) \\
\hline Starting date & NR \\
\hline Contact information & KW5@uw.edu \\
\hline Notes & \\
\hline
\end{tabular}

Ward 2011

\begin{tabular}{ll}
\hline Trial name or title & My parenting SOS \\
\hline Methods & RCT \\
\hline Participants & Families with young children \\
\hline Interventions & Parenting skills on healthy eating and PA \\
\hline Outcomes & \% body fat \\
\hline Starting date & Jluy 2009- June 2012 \\
\hline Contact information & dsward@email.unc.edu \\
\hline Notes & NCT00998348 \\
\hline
\end{tabular}

\section{Waters 2007}

Trial name or title Fun ' $n$ ' healthy in Moreland


Waters 2007 (Continued)

Methods

\begin{tabular}{ll}
\hline Participants & Primary school children in 24 schools in Moreland, an inner city suburb of Melbourne, Australia \\
\hline Interventions & $\begin{array}{l}\text { Intervention is a facilitated approach to supporting school to implement an evidence-based ap- } \\
\text { proach with interventions based on priorities within the school, ensuring focus on diet, PA and } \\
\text { child health and well-being }\end{array}$ \\
\hline Outcomes & BMI, child health and well-being \\
\hline Starting date & 2004-2010 \\
\hline Contact information & Www.mchs.org.au/ \\
\hline Notes & ACTRN12607000385448 \\
\hline
\end{tabular}

\section{Wen 2012a}

\begin{tabular}{ll}
\hline Trial name or title & Healthy beginnings trial phase 2 \\
\hline Methods & RCT \\
\hline Participants & Children aged up to 2 years \\
\hline Interventions & Infant feeding practices \\
\hline Outcomes & BMI, dietary, PA and screen-time behaviours \\
\hline Starting date & NR \\
\hline Contact information & Imwen@email.cs.nsw.gov.au \\
\hline Notes & $\begin{array}{l}\text { ACTR12607000 168459 phase 2 is a longer, no-treatment follow-up and cost-effectiveness } \\
\text { analysis }\end{array}$ \\
\hline
\end{tabular}

Wyatt 2013

\begin{tabular}{ll}
\hline Trial name or title & HeLP (Healthy lifestyles programme) \\
\hline Methods & Cluster-RCT (32 UK schools) \\
\hline Participants & 9 -10 years \\
\hline Interventions & Healthy lifestyles \\
\hline Outcomes & BMl, cost-effectiveness analysis \\
\hline Starting date & 01 March 2012-31 October 2016 \\
\hline Contact information & katrina.wyatt@pms.ac.uk \\
\hline
\end{tabular}


Wyatt 2013 (Continued)
Notes
ISRCTN15811706

Xu 2012

\begin{tabular}{ll}
\hline Trial name or title & CLICK-Obesity \\
\hline Methods & Cluster-RCT (8 urban schools in China) \\
\hline Participants & 4th graders \\
\hline Interventions & Diet and PA including environment \\
\hline Outcomes & Body composition \\
\hline Starting date & NR \\
\hline Contact information & f.xufei@gmail.com \\
\hline Notes & ChiCTR-ERC-11001819 \\
\hline
\end{tabular}

\section{Zoorob 2013}

\begin{tabular}{ll}
\hline Trial name or title & Healthy families study \\
\hline Methods & RCT \\
\hline Participants & Hispanic families with children aged 5-7 years \\
\hline Interventions & $\begin{array}{l}\text { A 12-month intervention promotes healthy eating behaviours, increased physical activity, and de- } \\
\text { creased sedentary behaviour, with an emphasis on parental modeling and experiential learning for } \\
\text { children. vs oral health control }\end{array}$ \\
\hline Outcomes & BMI \\
\hline Starting date & June 2010 to May 2016 \\
\hline Contact information & rzoorob@mmc.edu \\
\hline Notes & NCT01156402 \\
\hline
\end{tabular}

BMI: body-mass index; FMS: Fundamental Movement Skills; MI: motivational interviewing; MVPA: moderate to vigorous physical activity; NR: not reported; PA: physical activity; QALY: quality-adjusted life year; RCT: randomised controlled trial; TPB: Theory of Planned Behaviour; WIC: Women's, infants' and children's centre; zBMI: body-mass index z score

\section{DATA AND ANALYSES}


Comparison 1. Dietary interventions versus control: age 0-5 years

\begin{tabular}{lllll}
\hline $\begin{array}{l}\text { Outcome or subgroup } \\
\text { title }\end{array}$ & No. of studies & $\begin{array}{l}\text { No. of partici- } \\
\text { pants }\end{array}$ & Statistical method & Effect size \\
\hline $1 \mathrm{zBMI}$ & 1 & 520 & Mean Difference (Random, 95\% Cl) & $-0.14[-0.32,0.04]$ \\
\hline
\end{tabular}

Analysis 1.1. Comparison 1 Dietary interventions versus control: age 0-5 years, Outcome 1 zBMI.

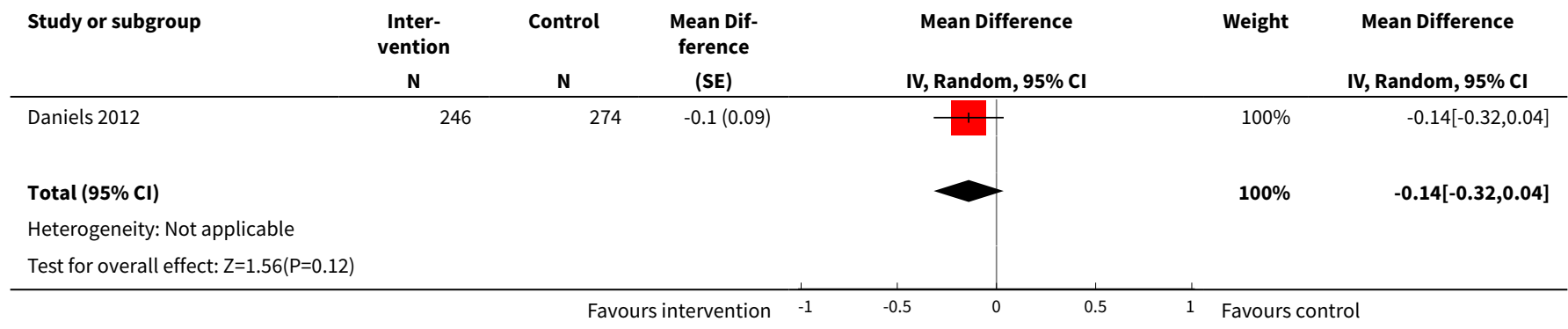

\section{Comparison 2. Physical activity interventions versus control: age 0-5 years}

\begin{tabular}{|c|c|c|c|c|}
\hline Outcome or subgroup title & No. of studies & $\begin{array}{l}\text { No. of partici- } \\
\text { pants }\end{array}$ & Statistical method & Effect size \\
\hline $\begin{array}{l}1 \text { zBMI. Physical activity vs } \\
\text { control - setting }\end{array}$ & 4 & 1053 & $\begin{array}{l}\text { Mean Difference (Random, 95\% } \\
\mathrm{Cl} \text { ) }\end{array}$ & $0.01[-0.10,0.13]$ \\
\hline 1.1 Health system & 2 & 495 & $\begin{array}{l}\text { Mean Difference (Random, 95\% } \\
\mathrm{Cl} \text { ) }\end{array}$ & $0.02[-0.14,0.17]$ \\
\hline 1.2 Childcare/preschool & 2 & 558 & $\begin{array}{l}\text { Mean Difference (Random, 95\% } \\
\mathrm{Cl} \text { ) }\end{array}$ & $0.01[-0.17,0.19]$ \\
\hline $\begin{array}{l}2 \text { BMI. Physical activity vs con- } \\
\text { trol - setting }\end{array}$ & 5 & 2233 & $\begin{array}{l}\text { Mean Difference (Random, 95\% } \\
\mathrm{Cl} \text { ) }\end{array}$ & $-0.22[-0.44,0.01]$ \\
\hline 2.1 Health system & 1 & 143 & $\begin{array}{l}\text { Mean Difference (Random, 95\% } \\
\mathrm{Cl} \text { ) }\end{array}$ & $-0.2[-0.59,0.19]$ \\
\hline 2.2 Childcare/preschool & 4 & 2090 & $\begin{array}{l}\text { Mean Difference (Random, 95\% } \\
\mathrm{Cl} \text { ) }\end{array}$ & $-0.23[-0.50,0.05]$ \\
\hline
\end{tabular}

Analysis 2.1. Comparison 2 Physical activity interventions versus control: age 0-5 years, Outcome 1 zBMI. Physical activity vs control - setting.

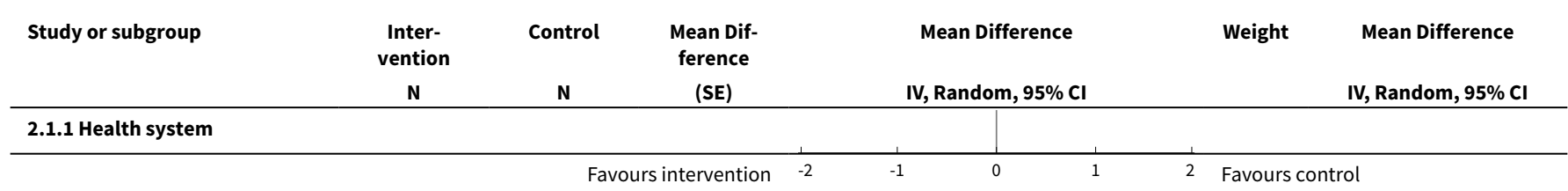




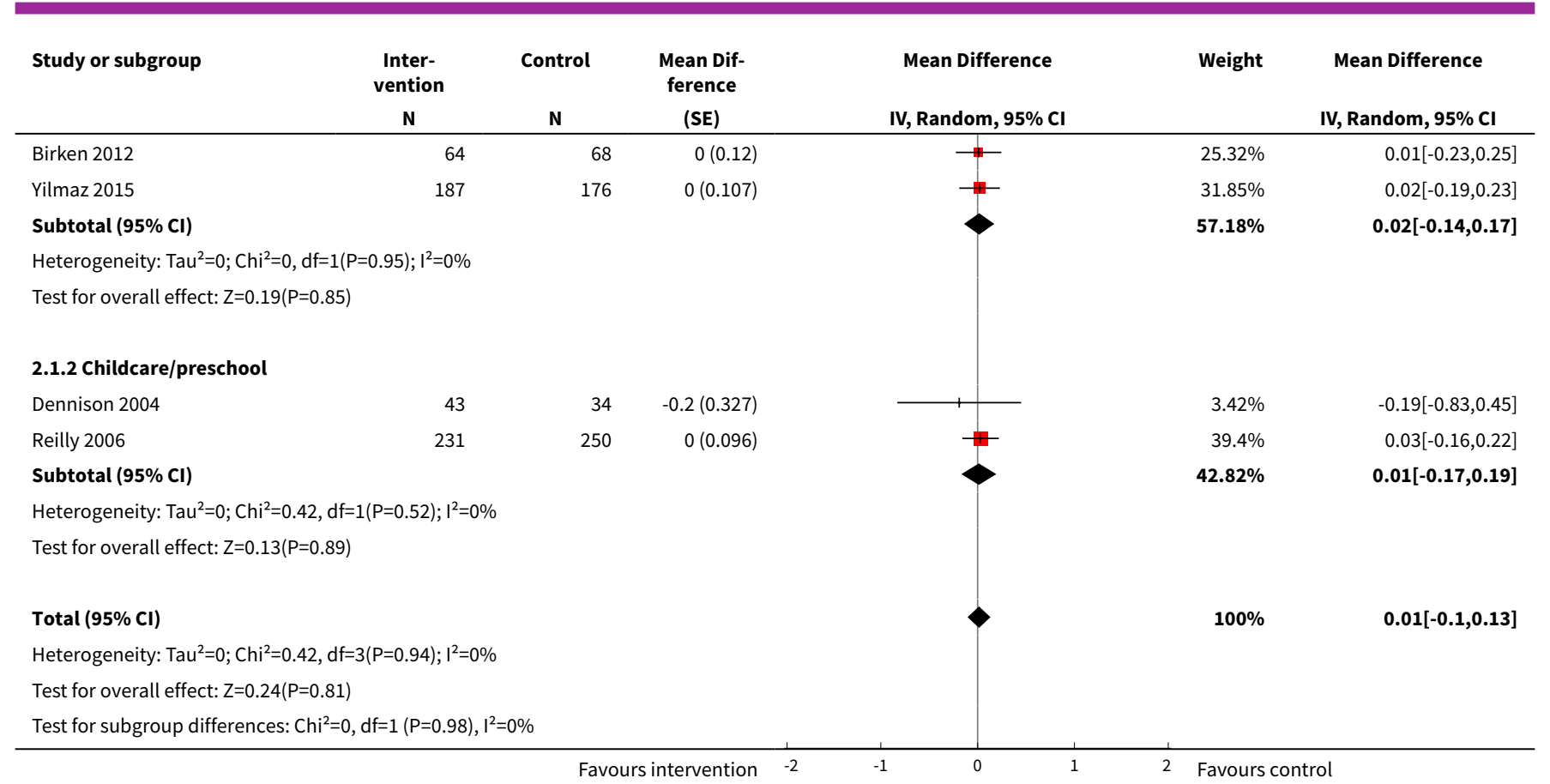

Analysis 2.2. Comparison 2 Physical activity interventions versus control: age 0-5 years, Outcome 2 BMI. Physical activity vs control - setting.

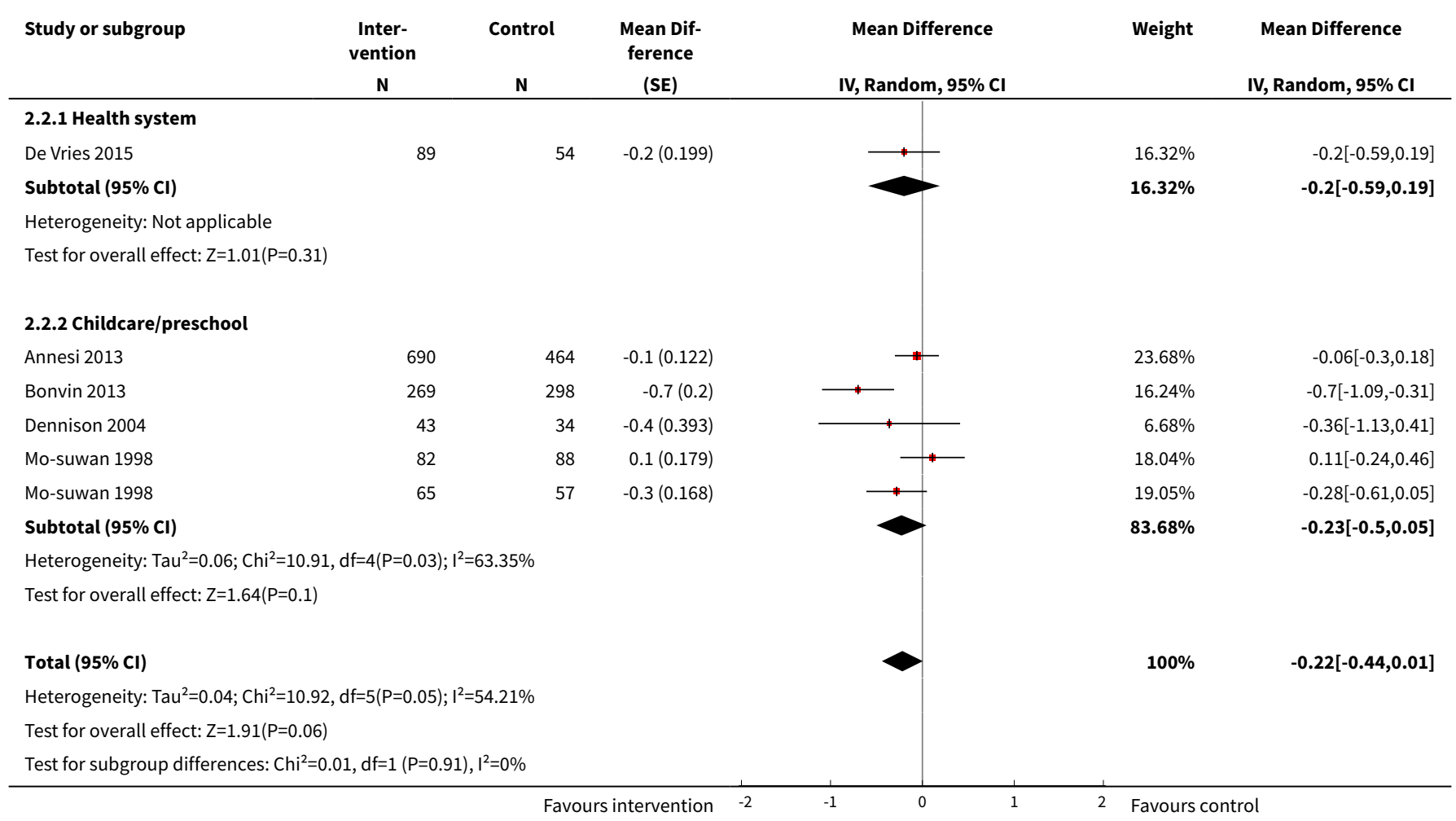


Comparison 3. Diet and physical activity interventions versus control: age $0-5$ years

\begin{tabular}{|c|c|c|c|c|}
\hline Outcome or subgroup title & No. of studies & $\begin{array}{l}\text { No. of partici- } \\
\text { pants }\end{array}$ & Statistical method & Effect size \\
\hline $\begin{array}{l}1 \text { zBMI. Diet and physical activity } \\
\text { vs control - setting }\end{array}$ & 16 & 6261 & $\begin{array}{l}\text { Mean Difference (Random, } \\
95 \% \mathrm{Cl} \text { ) }\end{array}$ & $-0.07[-0.14,-0.01]$ \\
\hline 1.1 Childcare/preschool & 10 & 4913 & $\begin{array}{l}\text { Mean Difference (Random, } \\
95 \% \mathrm{Cl} \text { ) }\end{array}$ & $-0.04[-0.09,0.01]$ \\
\hline 1.2 Health system & 1 & 121 & $\begin{array}{l}\text { Mean Difference (Random, } \\
95 \% \mathrm{Cl} \text { ) }\end{array}$ & $-0.24[-0.46,-0.02]$ \\
\hline 1.3 Wider community & 2 & 632 & $\begin{array}{l}\text { Mean Difference (Random, } \\
95 \% \mathrm{Cl} \text { ) }\end{array}$ & $-0.02[-0.13,0.09]$ \\
\hline 1.4 Home & 3 & 595 & $\begin{array}{l}\text { Mean Difference (Random, } \\
95 \% \mathrm{Cl} \text { ) }\end{array}$ & $-0.13[-0.35,0.09]$ \\
\hline $\begin{array}{l}2 \text { zBMI. Diet and physical activity } \\
\text { vs control - duration }\end{array}$ & 16 & 6261 & $\begin{array}{l}\text { Mean Difference (Random, } \\
95 \% \mathrm{Cl} \text { ) }\end{array}$ & $-0.07[-0.14,-0.01]$ \\
\hline $\begin{array}{l}\text { 2.1 Duration of intervention } \leq 12 \\
\text { months }\end{array}$ & 13 & 4235 & $\begin{array}{l}\text { Mean Difference (Random, } \\
95 \% \mathrm{Cl} \text { ) }\end{array}$ & $-0.09[-0.17,-0.01]$ \\
\hline $\begin{array}{l}\text { 2.2 Duration of intervention }>12 \\
\text { months }\end{array}$ & 3 & 2026 & $\begin{array}{l}\text { Mean Difference (Random, } \\
95 \% \mathrm{Cl} \text { ) }\end{array}$ & $-0.02[-0.09,0.06]$ \\
\hline $\begin{array}{l}3 \text { BMI. Diet and physical activity } \\
\text { vs control - setting }\end{array}$ & 11 & 5536 & $\begin{array}{l}\text { Mean Difference (Random, } \\
95 \% \mathrm{CI} \text { ) }\end{array}$ & $-0.11[-0.21,-0.00]$ \\
\hline 3.1 Home & 2 & 778 & $\begin{array}{l}\text { Mean Difference (Random, } \\
95 \% \mathrm{Cl} \text { ) }\end{array}$ & $-0.33[-0.55,-0.10]$ \\
\hline 3.2 Wider community & 1 & 75 & $\begin{array}{l}\text { Mean Difference (Random, } \\
95 \% \mathrm{Cl} \text { ) }\end{array}$ & $-0.59[-0.94,-0.24]$ \\
\hline 3.3 Childcare/preschool & 8 & 4683 & $\begin{array}{l}\text { Mean Difference (Random, } \\
95 \% \mathrm{Cl} \text { ) }\end{array}$ & $-0.05[-0.14,0.05]$ \\
\hline $\begin{array}{l}4 \text { BMI. Diet and physical activity } \\
\text { vs control - duration }\end{array}$ & 11 & 5536 & $\begin{array}{l}\text { Mean Difference (Random, } \\
95 \% \mathrm{Cl} \text { ) }\end{array}$ & $-0.11[-0.21,-0.00]$ \\
\hline $\begin{array}{l}\text { 4.1 Duration of intervention }>12 \\
\text { months }\end{array}$ & 1 & 667 & $\begin{array}{l}\text { Mean Difference (Random, } \\
95 \% \mathrm{Cl} \text { ) }\end{array}$ & $-0.29[-0.56,-0.02]$ \\
\hline $\begin{array}{l}\text { 4.2 Duration of intervention } \leq 12 \\
\text { months }\end{array}$ & 10 & 4869 & $\begin{array}{l}\text { Mean Difference (Random, } \\
95 \% \mathrm{Cl} \text { ) }\end{array}$ & $-0.09[-0.20,0.01]$ \\
\hline
\end{tabular}


Analysis 3.1. Comparison 3 Diet and physical activity interventions versus control: age 0-5 years, Outcome 1 zBMI. Diet and physical activity vs control - setting.

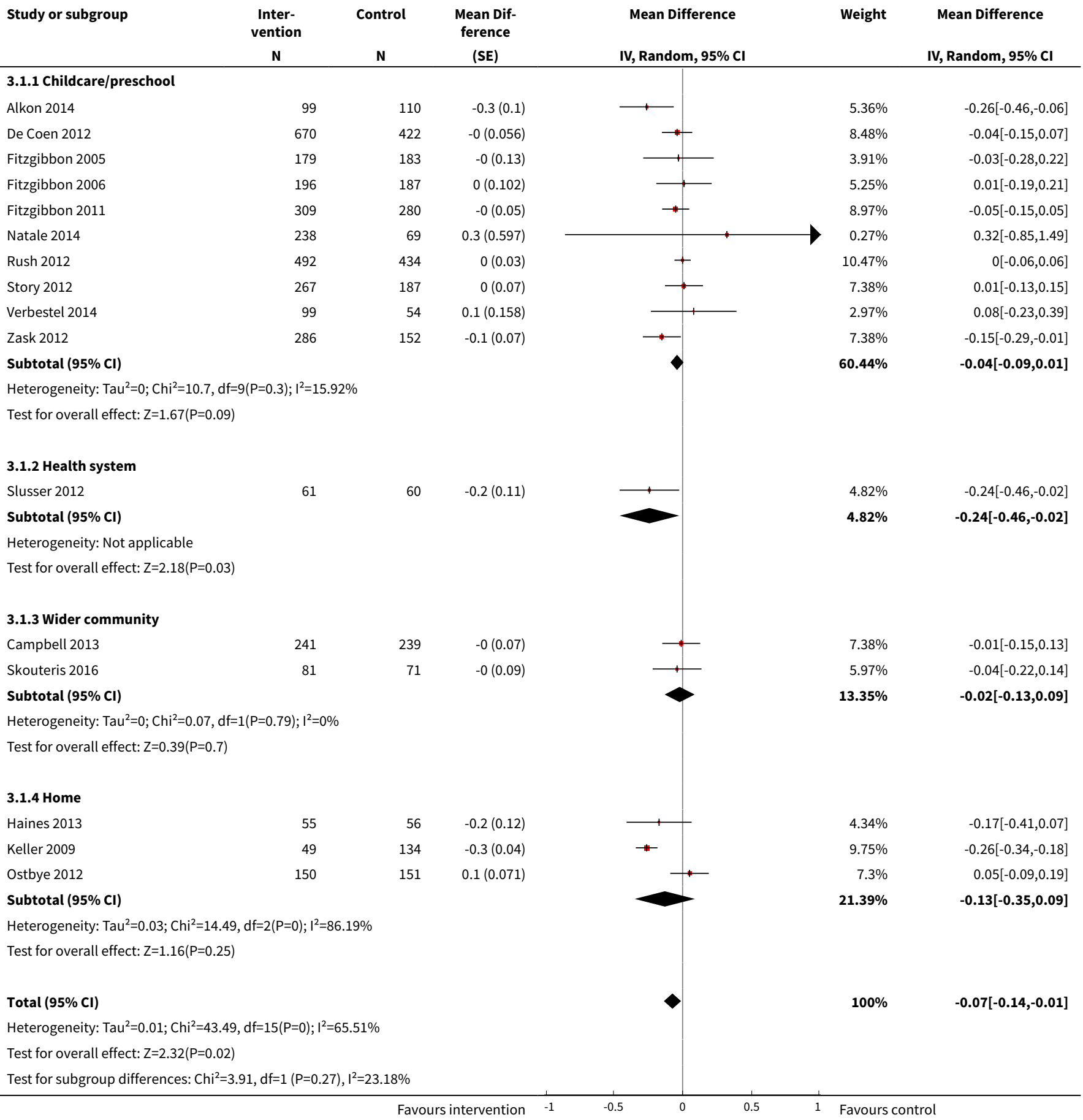


Analysis 3.2. Comparison 3 Diet and physical activity interventions versus control: age 0-5 years, Outcome 2 zBMI. Diet and physical activity vs control - duration.

\begin{tabular}{|c|c|c|c|c|c|c|}
\hline Study or subgroup & $\begin{array}{c}\text { Inter- } \\
\text { vention } \\
\mathbf{N} \\
\end{array}$ & Control & $\begin{array}{c}\text { Mean Dif- } \\
\text { ference } \\
\text { (SE) } \\
\end{array}$ & IV, Random, 95\% CI & Weight & $\begin{array}{c}\text { Mean Difference } \\
\text { IV, Random, } 95 \% \text { Cl }\end{array}$ \\
\hline \multicolumn{7}{|c|}{ 3.2.1 Duration of intervention $\leq 12$ months } \\
\hline Alkon 2014 & 99 & 110 & $-0.3(0.1)$ & $\longrightarrow$ & $5.36 \%$ & $-0.26[-0.46,-0.06]$ \\
\hline Fitzgibbon 2005 & 179 & 183 & $-0(0.13)$ & & $3.91 \%$ & $-0.03[-0.28,0.22]$ \\
\hline Fitzgibbon 2006 & 196 & 187 & $0(0.102)$ & + & $5.25 \%$ & $0.01[-0.19,0.21]$ \\
\hline Haines 2013 & 55 & 56 & $-0.2(0.12)$ & & $4.34 \%$ & $-0.17[-0.41,0.07]$ \\
\hline Keller 2009 & 49 & 134 & $-0.3(0.04)$ & $\rightarrow$ & $9.75 \%$ & $-0.26[-0.34,-0.18]$ \\
\hline Natale 2014 & 238 & 69 & $0.3(0.597)$ & & $0.27 \%$ & $0.32[-0.85,1.49]$ \\
\hline Ostbye 2012 & 150 & 151 & $0.1(0.071)$ & + & $7.3 \%$ & $0.05[-0.09,0.19]$ \\
\hline Rush 2012 & 492 & 434 & $0(0.03)$ & - & $10.47 \%$ & $0[-0.06,0.06]$ \\
\hline Skouteris 2016 & 81 & 71 & $-0(0.09)$ & - & $5.97 \%$ & $-0.04[-0.22,0.14]$ \\
\hline Verbestel 2014 & 99 & 54 & $0.1(0.158)$ & 1 & $2.97 \%$ & $0.08[-0.23,0.39]$ \\
\hline Zask 2012 & 286 & 152 & $-0.1(0.07)$ & $\longrightarrow$ & $7.38 \%$ & $-0.15[-0.29,-0.01]$ \\
\hline Subtotal $(95 \% \mathrm{CI})$ & & & & & $76.76 \%$ & $-0.09[-0.17,-0.01]$ \\
\hline \multicolumn{7}{|c|}{ Heterogeneity: Tau $^{2}=0.01 ; \mathrm{Chi}^{2}=40.53, \mathrm{df}=12(\mathrm{P}<0.0001) ; \mathrm{I}^{2}=70.39 \%$} \\
\hline \multicolumn{7}{|c|}{ Test for overall effect: $Z=2.3(P=0.02)$} \\
\hline \multicolumn{7}{|c|}{ 3.2.2 Duration of intervention $>12$ months } \\
\hline Campbell 2013 & 241 & 239 & $-0(0.07)$ & - & $7.38 \%$ & $-0.01[-0.15,0.13]$ \\
\hline De Coen 2012 & 670 & 422 & $-0(0.056)$ & $\rightarrow$ & $8.48 \%$ & $-0.04[-0.15,0.07]$ \\
\hline Story 2012 & 267 & 187 & $0(0.07)$ & + & $7.38 \%$ & $0.01[-0.13,0.15]$ \\
\hline Subtotal $(95 \% \mathrm{Cl})$ & & & & & $23.24 \%$ & $-0.02[-0.09,0.06]$ \\
\hline \multicolumn{7}{|c|}{ Heterogeneity: $\operatorname{Tau}^{2}=0 ; \mathrm{Chi}^{2}=0.33, \mathrm{df}=2(\mathrm{P}=0.85) ;\left.\right|^{2}=0 \%$} \\
\hline \multicolumn{7}{|c|}{ Test for overall effect: $\mathrm{Z}=0.47(\mathrm{P}=0.64)$} \\
\hline \multicolumn{7}{|c|}{ Heterogeneity: $\operatorname{Tau}^{2}=0.01 ; \mathrm{Chi}^{2}=43.49, \mathrm{df}=15(\mathrm{P}=0) ; \mathrm{I}^{2}=65.51 \%$} \\
\hline \multicolumn{7}{|c|}{ Test for overall effect: $Z=2.32(P=0.02)$} \\
\hline Test for subgroup dif & $2, d f=1(P=0$. & ${ }^{2}=45.1 \%$ & & & & \\
\hline
\end{tabular}

\section{Analysis 3.3. Comparison 3 Diet and physical activity interventions versus control: age 0-5 years, Outcome 3 BMI. Diet and physical activity vs control - setting.}

\begin{tabular}{|c|c|c|c|c|c|c|}
\hline Study or subgroup & $\begin{array}{c}\text { Inter- } \\
\text { vention } \\
\mathbf{N}\end{array}$ & Control & $\begin{array}{c}\text { Mean Dif- } \\
\text { ference } \\
\text { (SE) }\end{array}$ & IV, Random, $95 \% \mathrm{CI}$ & Weight & $\begin{array}{c}\text { Mean Difference } \\
\text { IV, Random, 95\% CI }\end{array}$ \\
\hline \multicolumn{7}{|l|}{ 3.3.1 Home } \\
\hline Haines 2013 & 55 & 56 & $-0.4(0.2)$ & $\longrightarrow$ & $4.78 \%$ & $-0.4[-0.79,-0.01]$ \\
\hline Wen 2012 & 337 & 330 & $-0.3(0.14)$ & $\longrightarrow$ & $7.28 \%$ & $-0.29[-0.56,-0.02]$ \\
\hline Subtotal $(95 \% \mathrm{Cl})$ & & & & & $12.06 \%$ & $-0.33[-0.55,-0.1]$ \\
\hline \multicolumn{7}{|c|}{ Heterogeneity: $\mathrm{Tau}^{2}=0 ; \mathrm{Chi}^{2}=0.2, \mathrm{df}=1(\mathrm{P}=0.65) ; \mathrm{I}^{2}=0 \%$} \\
\hline \multicolumn{7}{|c|}{ Test for overall effect: $Z=2.84(P=0)$} \\
\hline 3.3.2 Wider commu & & & & & & \\
\hline
\end{tabular}




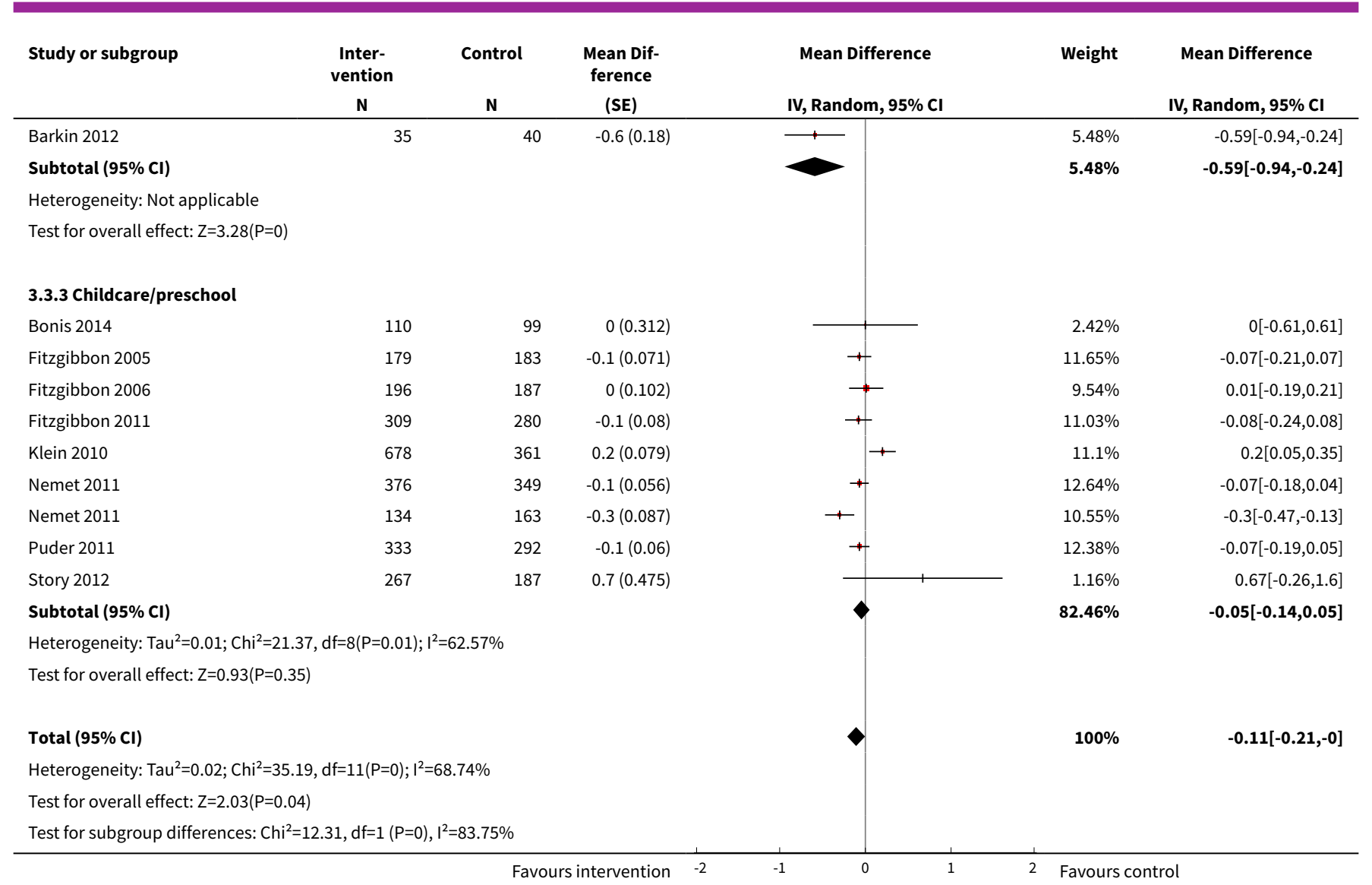

Analysis 3.4. Comparison 3 Diet and physical activity interventions versus control: age 0-5 years, Outcome 4 BMI. Diet and physical activity vs control - duration.

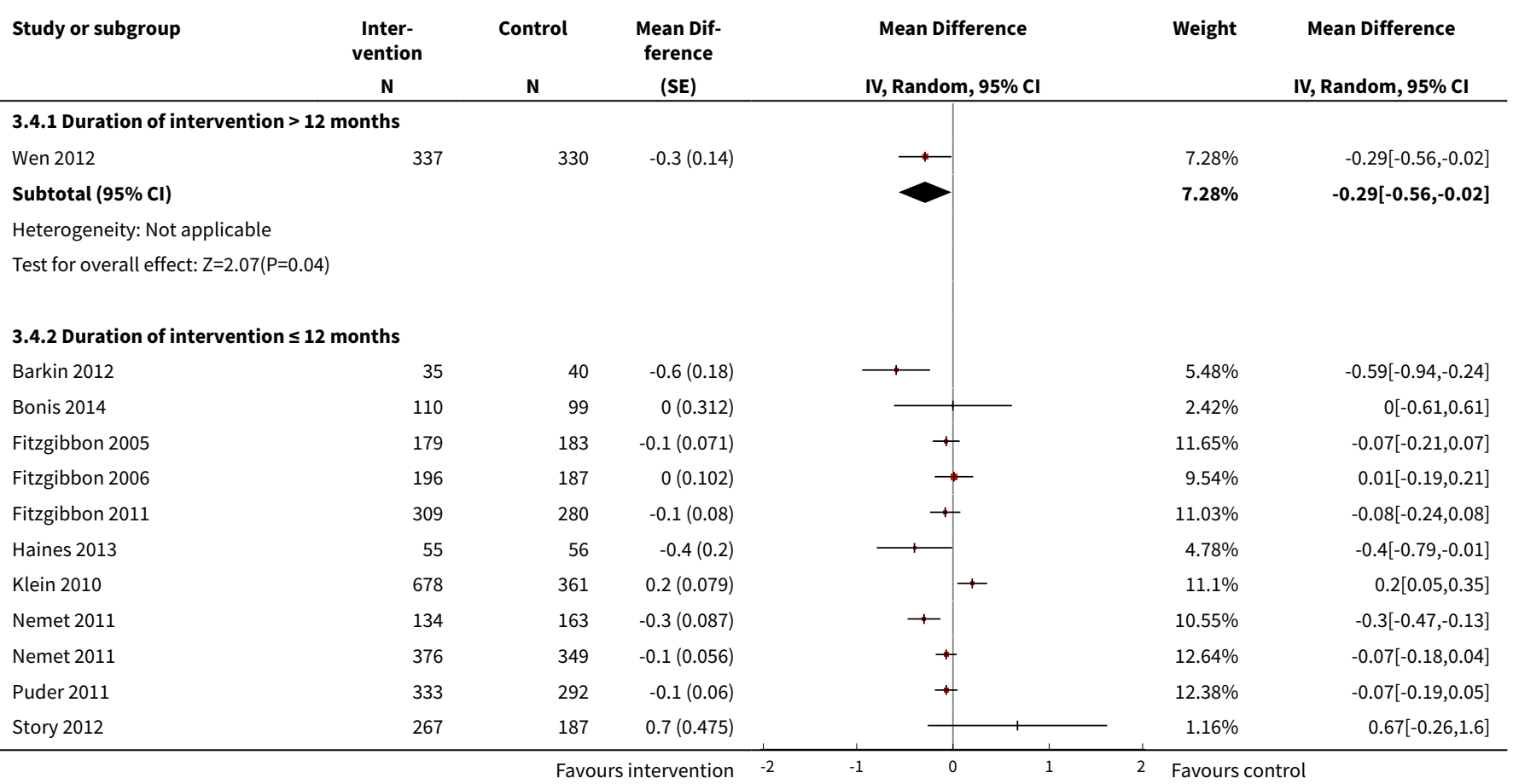




\begin{tabular}{|c|c|c|c|c|c|c|}
\hline Study or subgroup & $\begin{array}{c}\text { Inter- } \\
\text { vention } \\
\mathbf{N} \\
\end{array}$ & N & $\begin{array}{c}\text { Mean Dif- } \\
\text { ference } \\
\text { (SE) }\end{array}$ & IV, Random, 95\% CI & Weight & IV, Random, 95\% CI \\
\hline Subtotal $(95 \% \mathrm{Cl})$ & & & & $\gamma$ & $92.72 \%$ & $-0.09[-0.2,0.01]$ \\
\hline \multicolumn{7}{|c|}{ Heterogeneity: $\mathrm{Tau}^{2}=0.02 ; \mathrm{Chi}^{2}=32.83, \mathrm{df}=10(\mathrm{P}=0) ; \mathrm{I}^{2}=69.54 \%$} \\
\hline \multicolumn{7}{|c|}{ Test for overall effect: $Z=1.7(P=0.09)$} \\
\hline Total $(95 \% \mathrm{Cl})$ & & & & $\gamma$ & $100 \%$ & $-0.11[-0.21,-0]$ \\
\hline \multicolumn{7}{|c|}{ Heterogeneity: $\mathrm{Tau}^{2}=0.02 ; \mathrm{Chi}^{2}=35.19, \mathrm{df}=11(\mathrm{P}=0) ; \mathrm{I}^{2}=68.74 \%$} \\
\hline \multicolumn{7}{|c|}{ Test for overall effect: $Z=2.03(P=0.04)$} \\
\hline \multicolumn{7}{|c|}{ Test for subgroup differences: $\mathrm{Chi}^{2}=1.71, \mathrm{df}=1(\mathrm{P}=0.19), \mathrm{I}^{2}=41.48 \%$} \\
\hline
\end{tabular}

Comparison 4. Dietary interventions versus control: age 6-12 years

\begin{tabular}{lllll}
\hline $\begin{array}{l}\text { Outcome or sub- } \\
\text { group title }\end{array}$ & No. of studies & $\begin{array}{l}\text { No. of partici- } \\
\text { pants }\end{array}$ & Statistical method & Effect size \\
\hline 1 zBMI - setting & 9 & 7231 & Mean Difference (Random, 95\% Cl) & $-0.03[-0.06,0.01]$ \\
\hline 1.1 School & 8 & 6771 & Mean Difference (Random, 95\% Cl) & $-0.02[-0.06,0.01]$ \\
\hline 1.2 Wider community & 1 & 460 & Mean Difference (Random, 95\% Cl) & $-0.16[-0.35,0.04]$ \\
\hline 2 BMI - setting & 6 & 5061 & Mean Difference (Random, 95\% Cl) & $-0.02[-0.11,0.06]$ \\
\hline 2.1 School & 5 & 4601 & Mean Difference (Random, 95\% Cl) & $-0.02[-0.10,0.07]$ \\
\hline 2.2 Wider community & 1 & 460 & Mean Difference (Random, 95\% Cl) & $-0.74[-1.68,0.19]$ \\
\hline
\end{tabular}

Analysis 4.1. Comparison 4 Dietary interventions versus control: age 6-12 years, Outcome 1 zBMI - setting.

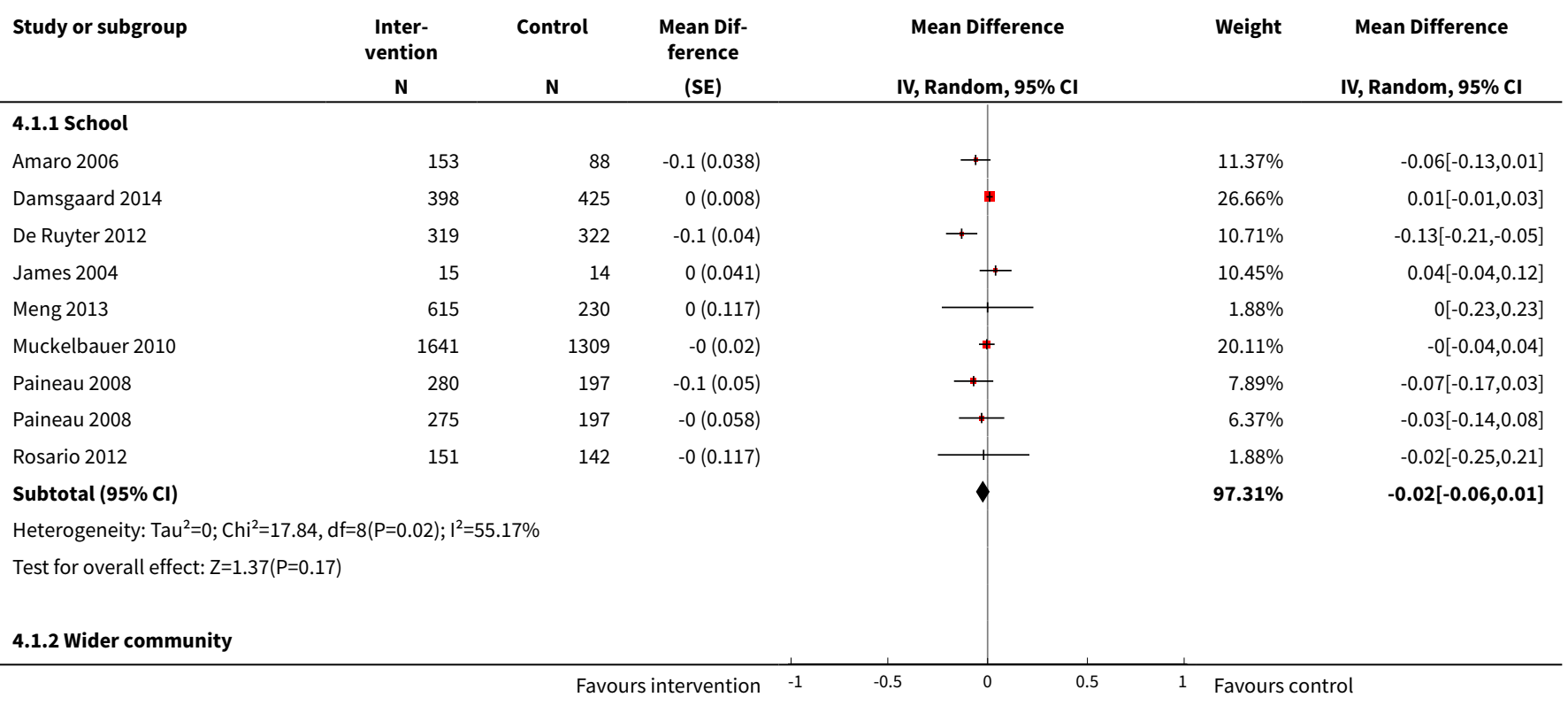




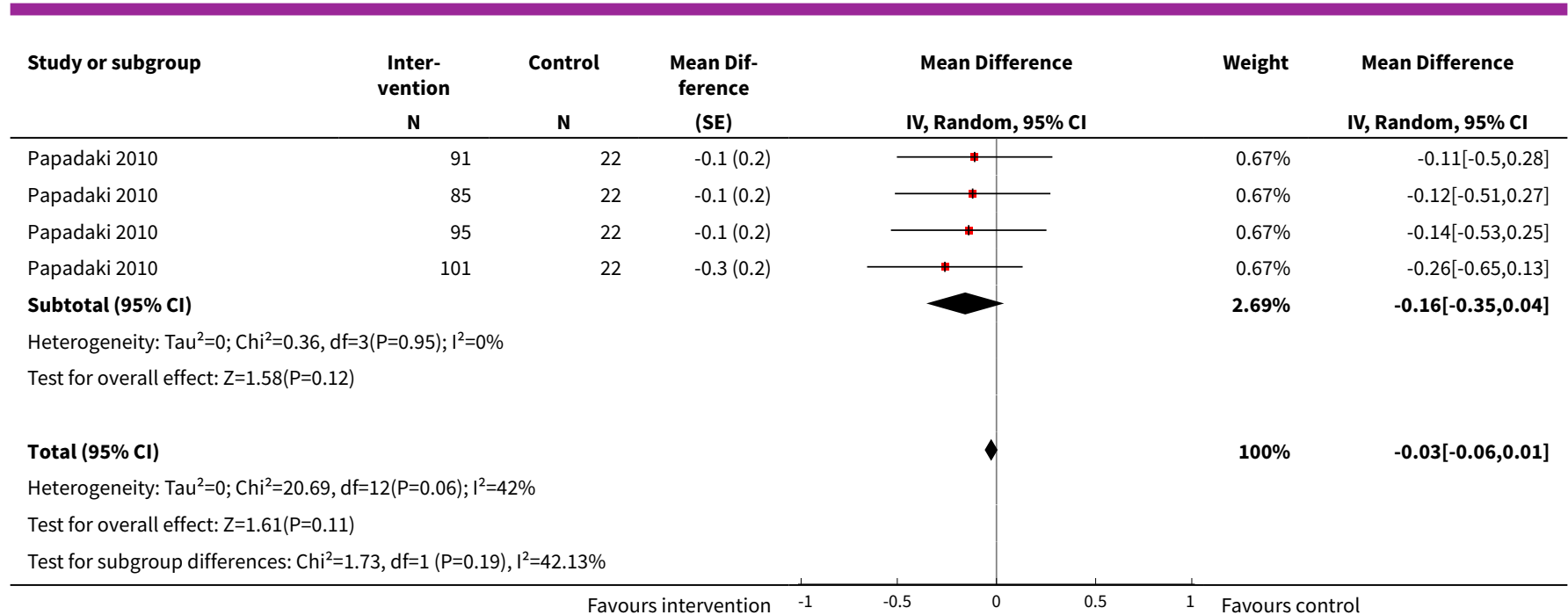

Analysis 4.2. Comparison 4 Dietary interventions versus control: age 6-12 years, Outcome 2 BMI - setting.

\begin{tabular}{|c|c|c|c|c|c|c|}
\hline Study or subgroup & $\begin{array}{c}\text { Inter- } \\
\text { vention } \\
\mathbf{N}\end{array}$ & Control & $\begin{array}{l}\text { Mean Dif- } \\
\text { ference } \\
\text { (SE) }\end{array}$ & $\begin{array}{c}\text { Mean Difference } \\
\text { IV, Random, } 95 \% \mathrm{CI}\end{array}$ & Weight & $\begin{array}{c}\text { Mean Difference } \\
\text { IV, Random, 95\% CI }\end{array}$ \\
\hline \multicolumn{7}{|l|}{ 4.2.1 School } \\
\hline James 2004 & 15 & 14 & $-0.1(0.092)$ & + & $22.03 \%$ & $-0.1[-0.28,0.08]$ \\
\hline Meng 2013 & 615 & 230 & $0(0.26)$ & 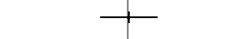 & $2.75 \%$ & $0.02[-0.49,0.53]$ \\
\hline Paineau 2008 & 274 & 197 & $-0(0.093)$ & $\#$ & $21.56 \%$ & $-0.02[-0.2,0.16]$ \\
\hline Paineau 2008 & 280 & 197 & $-0.1(0.086)$ & + & $25.22 \%$ & $-0.07[-0.24,0.1]$ \\
\hline Sevinc 2011 & 1815 & 37 & $\begin{array}{r}-0.1 \\
(481.031)\end{array}$ & & $0 \%$ & $-0.07[-942.87,942.73]$ \\
\hline Sichieri 2009 & 434 & 493 & $0.1(0.082)$ & $\#$ & $27.62 \%$ & $0.1[-0.06,0.26]$ \\
\hline Subtotal $(95 \% \mathrm{CI})$ & & & & 1 & $99.19 \%$ & $-0.02[-0.1,0.07]$ \\
\hline \multicolumn{7}{|c|}{ Heterogeneity: $\mathrm{Tau}^{2}=0 ; \mathrm{Chi}^{2}=3.26, \mathrm{df}=5(\mathrm{P}=0.66) ; \mathrm{I}^{2}=0 \%$} \\
\hline \multicolumn{7}{|c|}{ Test for overall effect: $Z=0.37(P=0.71)$} \\
\hline Papadaki 2010 & 101 & 22 & $-1.2(0.95)$ & & $0.21 \%$ & $-1.23[-3.09,0.63]$ \\
\hline Papadaki 2010 & 91 & 22 & $-0.5(0.96)$ & & $0.2 \%$ & $-0.54[-2.42,1.34]$ \\
\hline Papadaki 2010 & 85 & 22 & $-0.5(0.96)$ & 1 & $0.2 \%$ & $-0.55[-2.43,1.33]$ \\
\hline Papadaki 2010 & 95 & 22 & $-0.6(0.95)$ & & $0.21 \%$ & $-0.65[-2.51,1.21]$ \\
\hline Subtotal $(95 \% \mathrm{Cl})$ & & & & & $0.81 \%$ & $-0.74[-1.68,0.19]$ \\
\hline \multicolumn{7}{|c|}{ Heterogeneity: $\mathrm{Tau}^{2}=0 ; \mathrm{Chi}^{2}=0.36, \mathrm{df}=3(\mathrm{P}=0.95) ; \mathrm{I}^{2}=0 \%$} \\
\hline \multicolumn{7}{|c|}{ Test for overall effect: $Z=1.56(P=0.12)$} \\
\hline Total $(95 \% \mathrm{Cl})$ & & & & 1 & $100 \%$ & $-0.02[-0.11,0.06]$ \\
\hline \multicolumn{7}{|c|}{ Heterogeneity: $\operatorname{Tau}^{2}=0 ; \mathrm{Chi}^{2}=5.92, \mathrm{df}=9(\mathrm{P}=0.75) ; \mathrm{I}^{2}=0 \%$} \\
\hline \multicolumn{7}{|c|}{ Test for overall effect: $Z=0.51(P=0.61)$} \\
\hline Test for subgroup dif & $1, \mathrm{df}=1(\mathrm{P}=0$. & ${ }^{2}=56.7 \%$ & & & & \\
\hline
\end{tabular}


Comparison 5. Physical activity interventions versus control: age 6-12

\begin{tabular}{|c|c|c|c|c|}
\hline Outcome or subgroup title & No. of studies & $\begin{array}{l}\text { No. of partici- } \\
\text { pants }\end{array}$ & Statistical method & Effect size \\
\hline $\begin{array}{l}1 \text { zBMI. Physical activity vs con- } \\
\text { trol - setting }\end{array}$ & 7 & 6841 & $\begin{array}{l}\text { Mean Difference (Random, } \\
95 \% \mathrm{Cl} \text { ) }\end{array}$ & $-0.02[-0.06,0.02]$ \\
\hline 1.1 Wider community & 2 & 481 & $\begin{array}{l}\text { Mean Difference (Random, } \\
95 \% \mathrm{Cl} \text { ) }\end{array}$ & $-0.07[-0.19,0.05]$ \\
\hline 1.2 School & 5 & 6360 & $\begin{array}{l}\text { Mean Difference (Random, } \\
95 \% \mathrm{Cl} \text { ) }\end{array}$ & $-0.03[-0.07,0.00]$ \\
\hline $\begin{array}{l}2 \text { zBMI. Physical activity vs con- } \\
\text { trol - duration }\end{array}$ & 7 & 6841 & $\begin{array}{l}\text { Mean Difference (Random, } \\
95 \% \mathrm{Cl} \text { ) }\end{array}$ & $-0.02[-0.06,0.02]$ \\
\hline $\begin{array}{l}2.1 \text { Duration of intervention } \leq 12 \\
\text { months }\end{array}$ & 2 & 995 & $\begin{array}{l}\text { Mean Difference (Random, } \\
95 \% \mathrm{Cl} \text { ) }\end{array}$ & $0.00[-0.09,0.09]$ \\
\hline $\begin{array}{l}\text { 2.2 Duration of intervention }>12 \\
\text { months }\end{array}$ & 5 & 5846 & $\begin{array}{l}\text { Mean Difference (Random, } \\
95 \% \mathrm{Cl} \text { ) }\end{array}$ & $-0.03[-0.08,0.02]$ \\
\hline $\begin{array}{l}3 \text { BMI. Physical activity vs control } \\
\text { - setting }\end{array}$ & 14 & 16410 & $\begin{array}{l}\text { Mean Difference (Random, } \\
95 \% \mathrm{Cl} \text { ) }\end{array}$ & $-0.10[-0.14,-0.05]$ \\
\hline 3.1 Wider community & 2 & 481 & $\begin{array}{l}\text { Mean Difference (Random, } \\
95 \% \mathrm{Cl} \text { ) }\end{array}$ & $-0.19[-0.50,0.12]$ \\
\hline 3.2 School & 12 & 15929 & $\begin{array}{l}\text { Mean Difference (Random, } \\
95 \% \mathrm{Cl} \text { ) }\end{array}$ & $-0.10[-0.14,-0.06]$ \\
\hline $\begin{array}{l}4 \text { BMI. Physical activity vs control } \\
\text { - duration }\end{array}$ & 14 & 16410 & $\begin{array}{l}\text { Mean Difference (Random, } \\
95 \% \mathrm{Cl} \text { ) }\end{array}$ & $-0.10[-0.14,-0.05]$ \\
\hline $\begin{array}{l}\text { 4.1 Duration of intervention } \leq 12 \\
\text { months }\end{array}$ & 11 & 13705 & $\begin{array}{l}\text { Mean Difference (Random, } \\
95 \% \mathrm{Cl} \text { ) }\end{array}$ & $-0.11[-0.15,-0.06]$ \\
\hline $\begin{array}{l}4.2 \text { Duration of intervention }>12 \\
\text { months }\end{array}$ & 3 & 2705 & $\begin{array}{l}\text { Mean Difference (Random, } \\
95 \% \mathrm{Cl} \text { ) }\end{array}$ & $0.00[-0.14,0.14]$ \\
\hline
\end{tabular}

Analysis 5.1. Comparison 5 Physical activity interventions versus control: age 6-12, Outcome 1 zBMI. Physical activity vs control - setting.

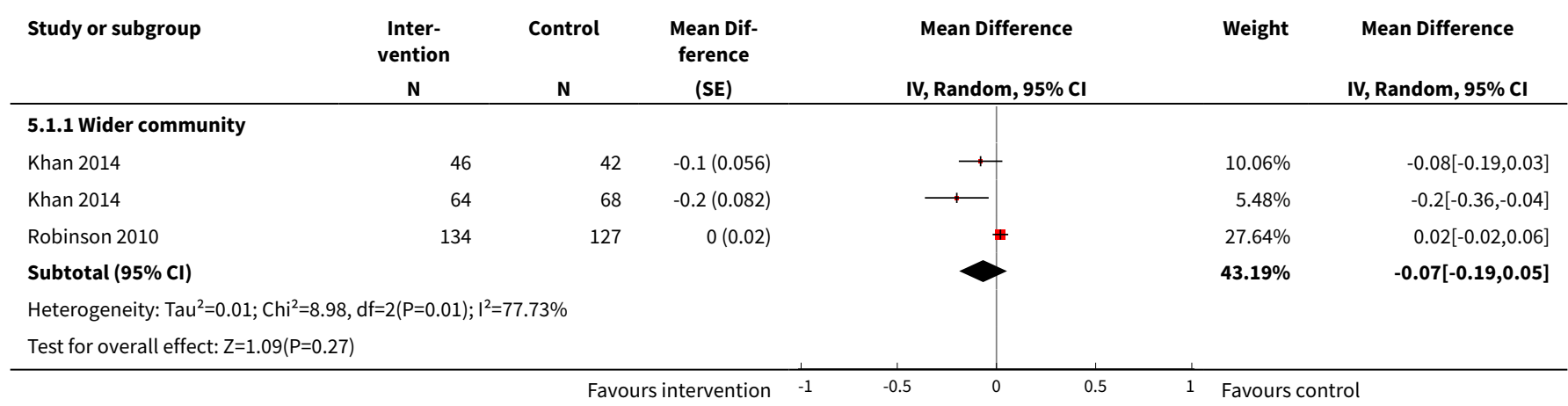




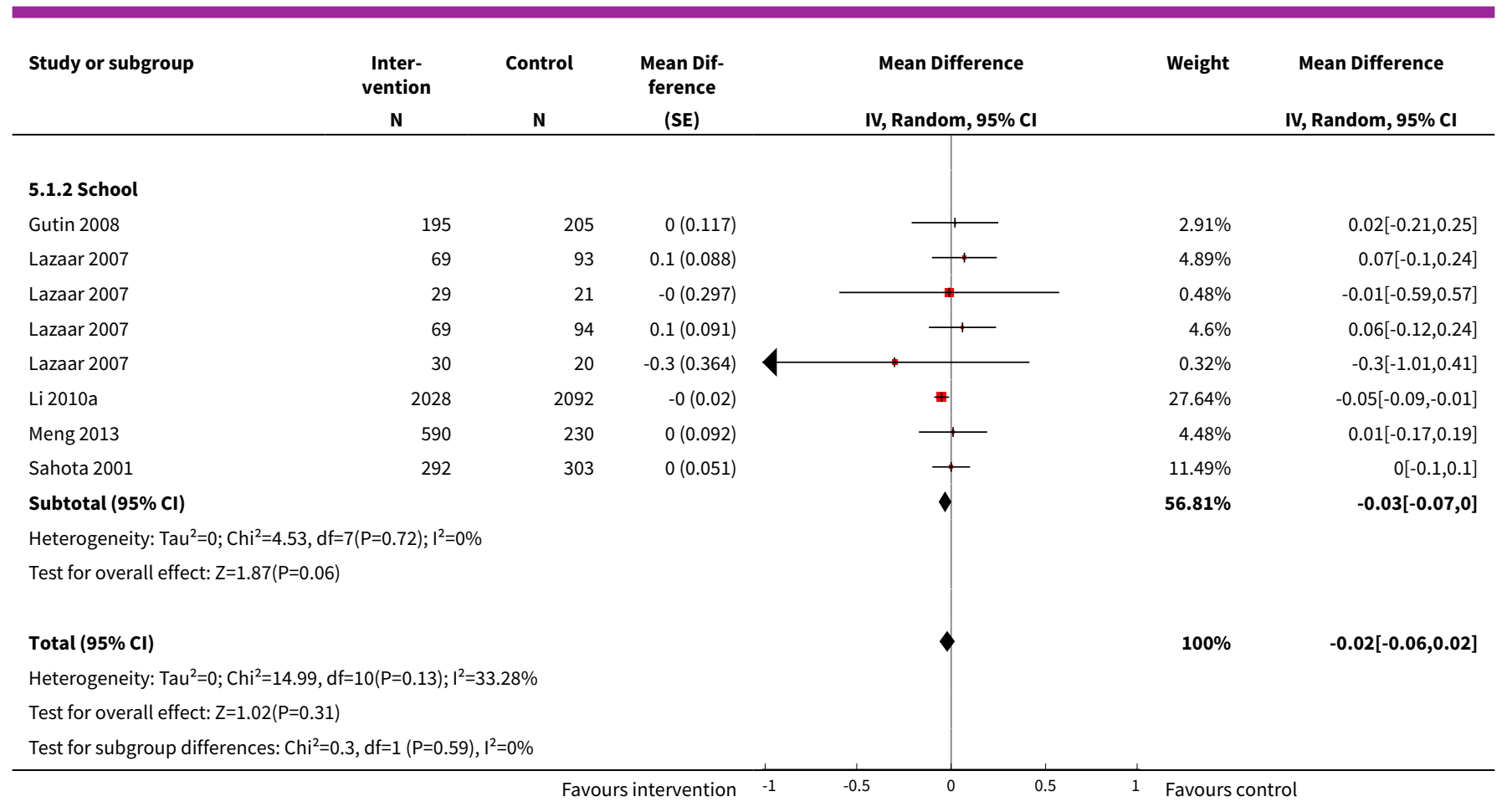

Analysis 5.2. Comparison 5 Physical activity interventions versus control: age 6-12, Outcome 2 zBMI. Physical activity vs control - duration.

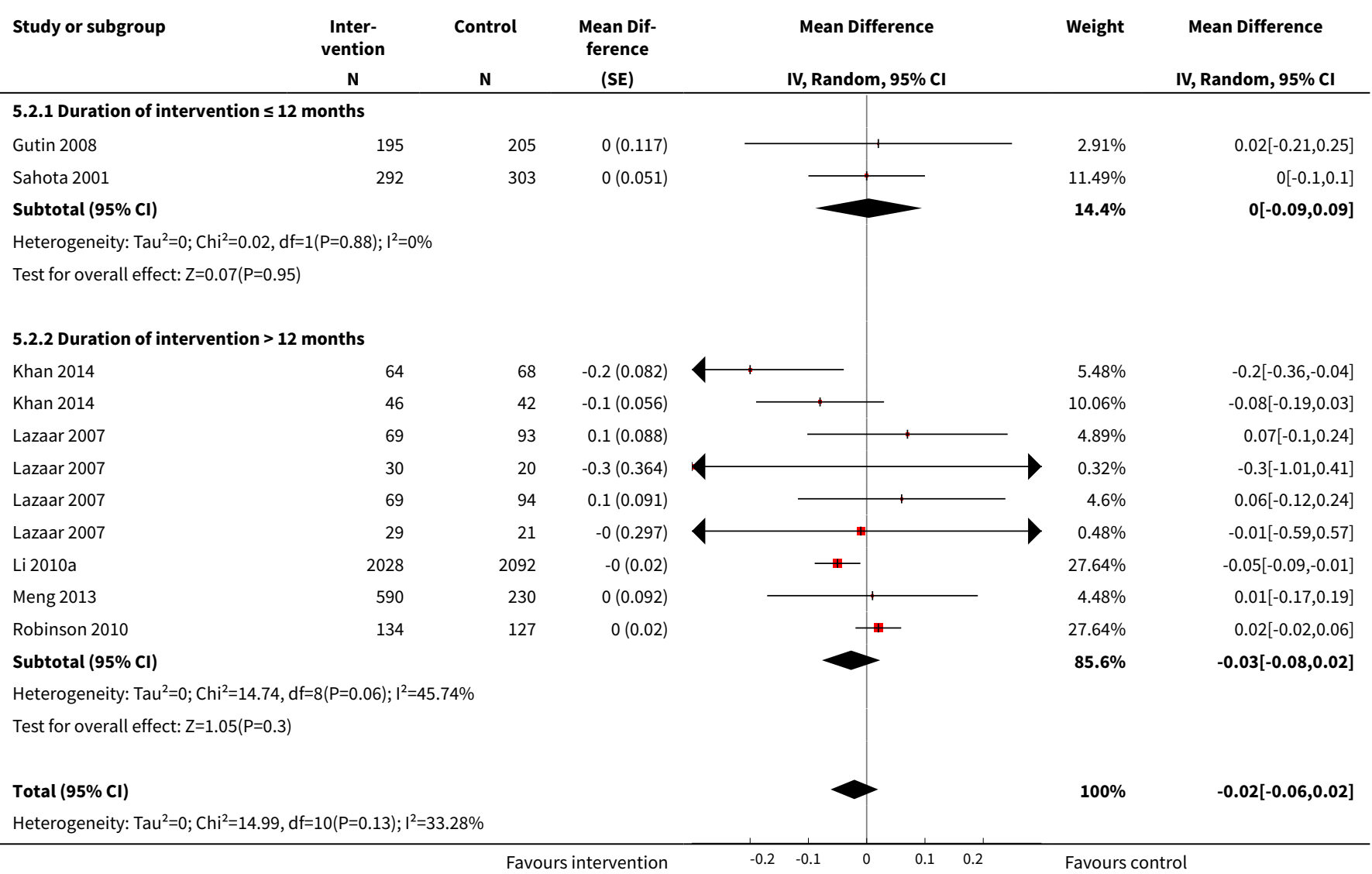




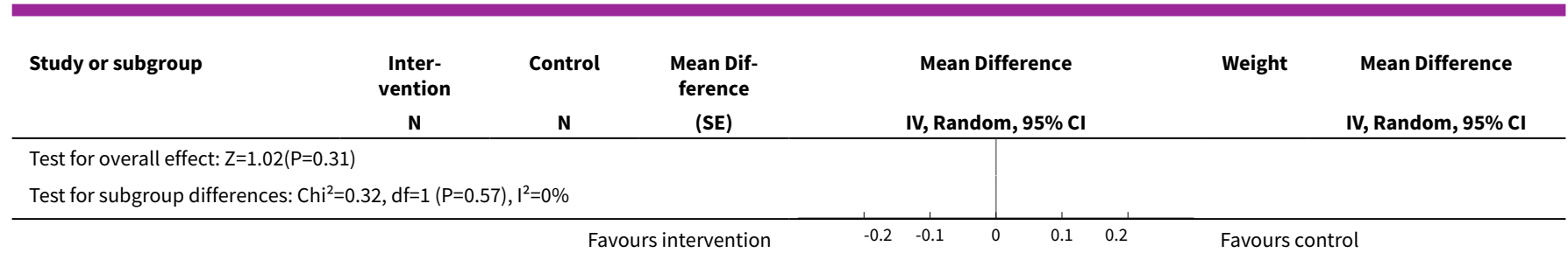

Analysis 5.3. Comparison 5 Physical activity interventions versus control: age 6-12, Outcome 3 BMI. Physical activity vs control - setting.

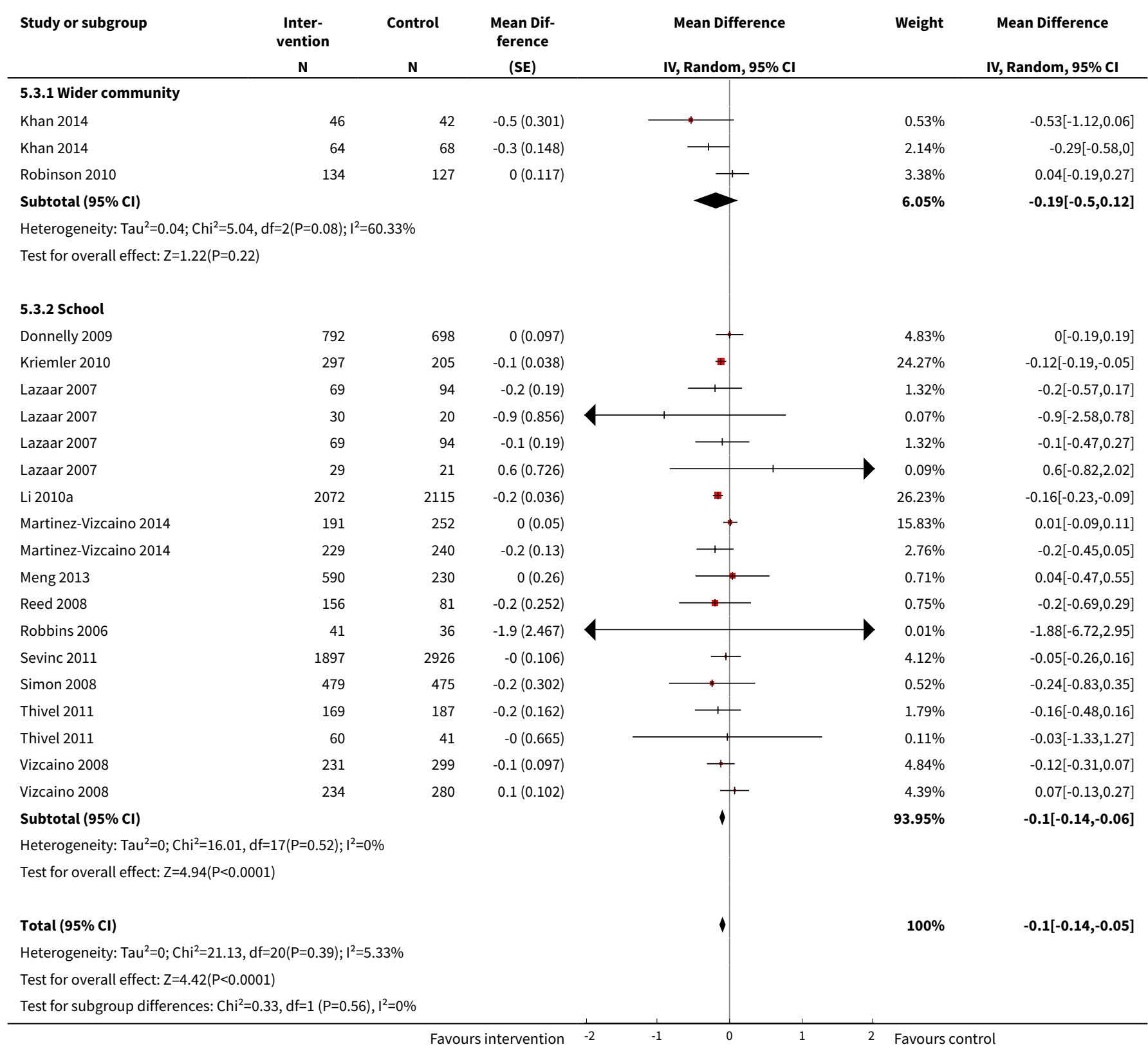


Analysis 5.4. Comparison 5 Physical activity interventions versus control: age 6-12, Outcome 4 BMI. Physical activity vs control - duration.

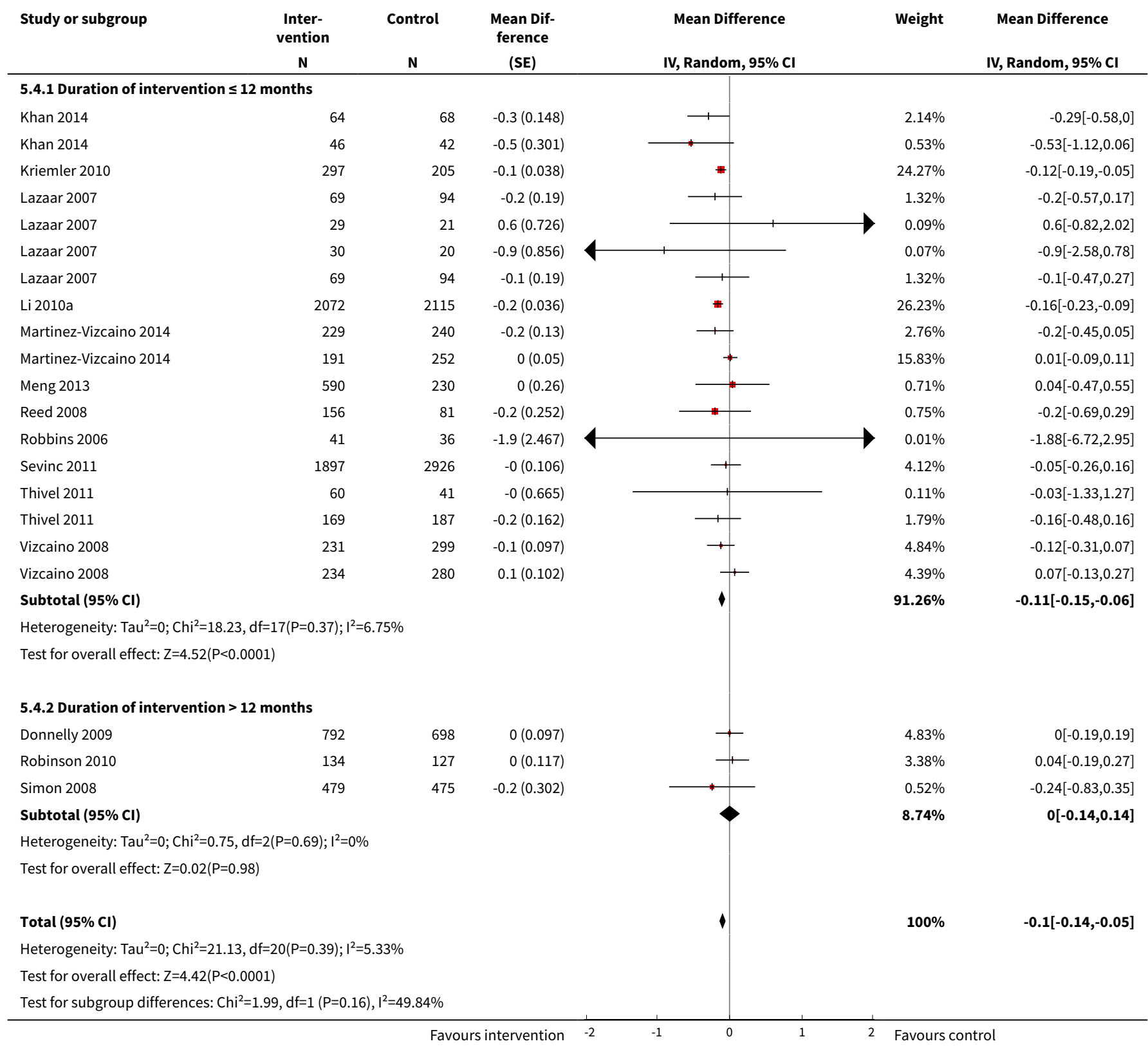

Comparison 6. Diet and physical activity interventions vs control: age 6-12 years

\begin{tabular}{lllll}
\hline Outcome or subgroup title & No. of studies & $\begin{array}{l}\text { No. of partici- } \\
\text { pants }\end{array}$ & Statistical method & Effect size \\
\hline $\begin{array}{l}1 \text { zBMI. Diet and physical activity } \\
\text { vs control - setting }\end{array}$ & 20 & 24043 & $\begin{array}{l}\text { Mean Difference (Random, } \\
95 \% \mathrm{Cl} \text { ) }\end{array}$ & $-0.05[-0.10,-0.01]$ \\
\hline 1.1 Home & 1 & 134 & $\begin{array}{l}\text { Mean Difference (Random, } \\
95 \% \mathrm{Cl})\end{array}$ & $0.03[-0.04,0.10]$ \\
\hline
\end{tabular}




\begin{tabular}{|c|c|c|c|c|}
\hline Outcome or subgroup title & No. of studies & $\begin{array}{l}\text { No. of partici- } \\
\text { pants }\end{array}$ & Statistical method & Effect size \\
\hline 1.2 Wider community & 4 & 657 & $\begin{array}{l}\text { Mean Difference (Random, } \\
95 \% \mathrm{Cl} \text { ) }\end{array}$ & $-0.04[-0.39,0.31]$ \\
\hline 1.3 School & 15 & 23252 & $\begin{array}{l}\text { Mean Difference (Random, } \\
95 \% \mathrm{Cl} \text { ) }\end{array}$ & $-0.04[-0.08,-0.01]$ \\
\hline $\begin{array}{l}2 \text { zBMI. Diet and physical activity } \\
\text { vs control - duration }\end{array}$ & 20 & 24043 & $\begin{array}{l}\text { Mean Difference (Random, } \\
95 \% \mathrm{Cl} \text { ) }\end{array}$ & $-0.05[-0.10,-0.01]$ \\
\hline $\begin{array}{l}2.1 \text { Duration of intervention }>12 \\
\text { months }\end{array}$ & 8 & 11779 & $\begin{array}{l}\text { Mean Difference (Random, } \\
95 \% \mathrm{Cl} \text { ) }\end{array}$ & $-0.05[-0.10,0.00]$ \\
\hline $\begin{array}{l}2.2 \text { Duration of intervention } \leq 12 \\
\text { months }\end{array}$ & 12 & 12264 & $\begin{array}{l}\text { Mean Difference (Random, } \\
95 \% \mathrm{Cl} \text { ) }\end{array}$ & $-0.06[-0.12,0.01]$ \\
\hline $\begin{array}{l}3 \text { BMI. Diet and physical activity vs } \\
\text { control - setting }\end{array}$ & 25 & 19498 & $\begin{array}{l}\text { Mean Difference (Random, } \\
95 \% \mathrm{Cl} \text { ) }\end{array}$ & $-0.05[-0.11,0.01]$ \\
\hline 3.1 School & 16 & 18747 & $\begin{array}{l}\text { Mean Difference (Random, } \\
95 \% \mathrm{Cl} \text { ) }\end{array}$ & $-0.04[-0.10,0.02]$ \\
\hline 3.2 Wider community & 9 & 751 & $\begin{array}{l}\text { Mean Difference (Random, } \\
95 \% \mathrm{Cl} \text { ) }\end{array}$ & $-0.08[-0.29,0.13]$ \\
\hline $\begin{array}{l}4 \text { BMI. Diet and physical activity vs } \\
\text { control - duration }\end{array}$ & 25 & 19498 & $\begin{array}{l}\text { Mean Difference (Random, } \\
95 \% \mathrm{Cl} \text { ) }\end{array}$ & $-0.05[-0.11,0.01]$ \\
\hline $\begin{array}{l}4.1 \text { Duration of intervention }>12 \\
\text { months }\end{array}$ & 8 & 5704 & $\begin{array}{l}\text { Mean Difference (Random, } \\
95 \% \mathrm{Cl} \text { ) }\end{array}$ & $-0.08[-0.18,0.03]$ \\
\hline $\begin{array}{l}\text { 4.2 Duration of intervention } \leq 12 \\
\text { months }\end{array}$ & 17 & 13794 & $\begin{array}{l}\text { Mean Difference (Random, } \\
95 \% \mathrm{Cl} \text { ) }\end{array}$ & $-0.04[-0.11,0.04]$ \\
\hline
\end{tabular}

Analysis 6.1. Comparison 6 Diet and physical activity interventions vs control: age 6-12 years, Outcome 1 zBMI. Diet and physical activity vs control - setting.

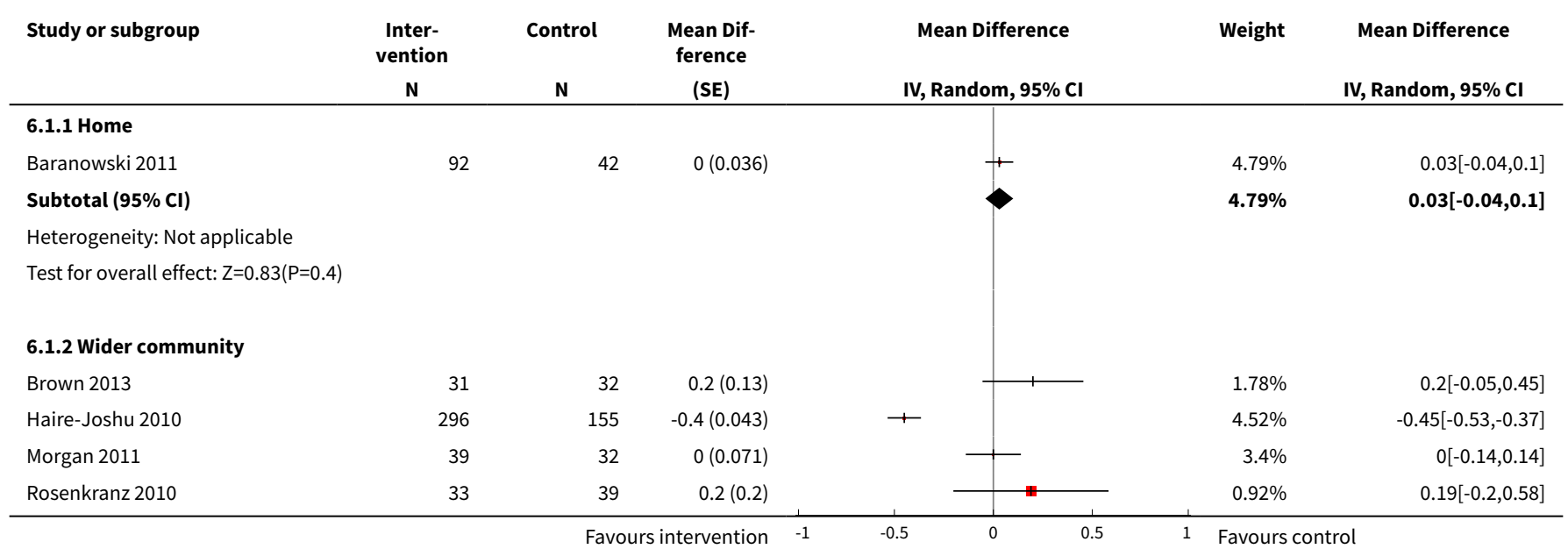




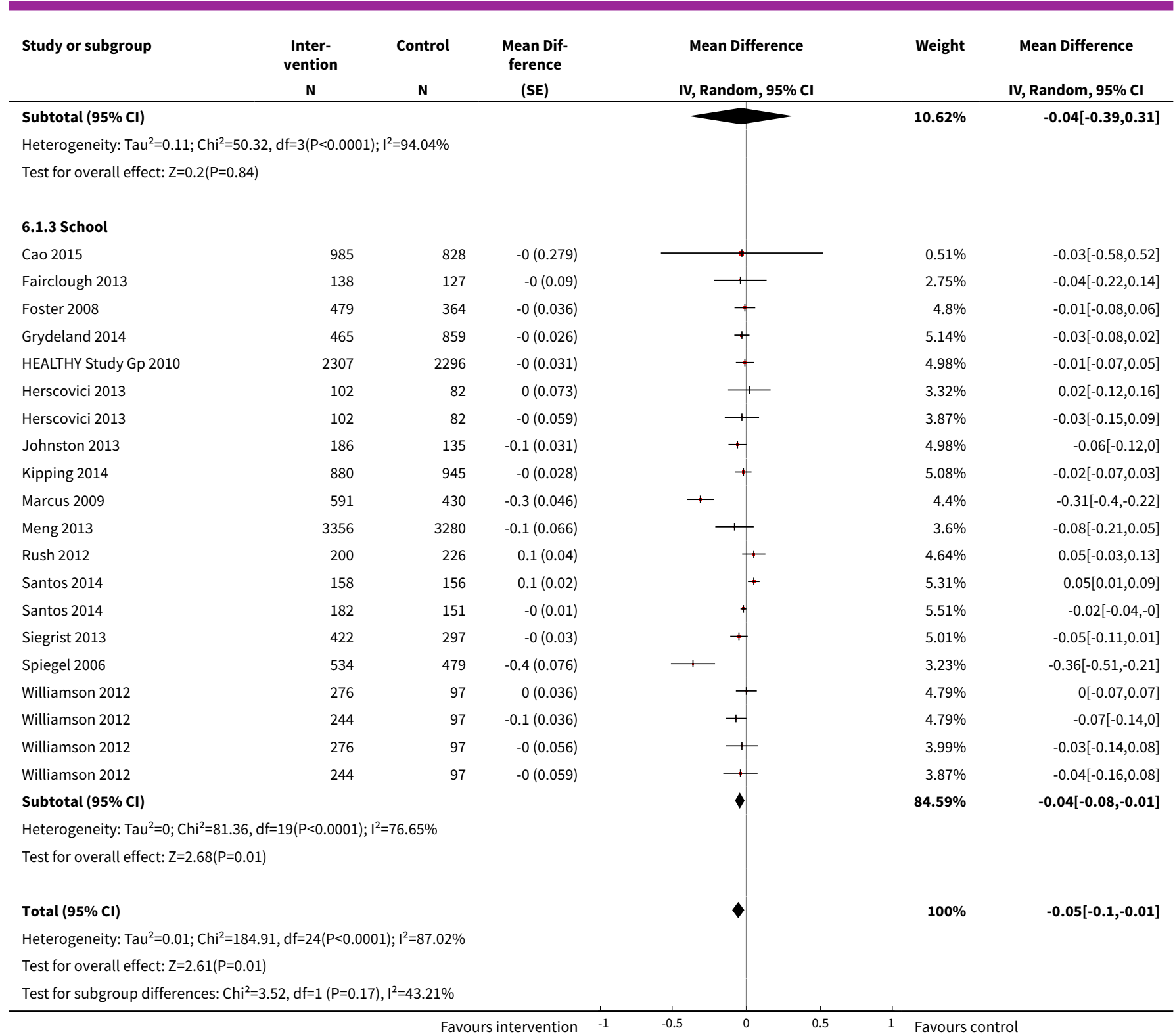

Analysis 6.2. Comparison 6 Diet and physical activity interventions vs control: age 6-12 years, Outcome 2 zBMI. Diet and physical activity vs control - duration.

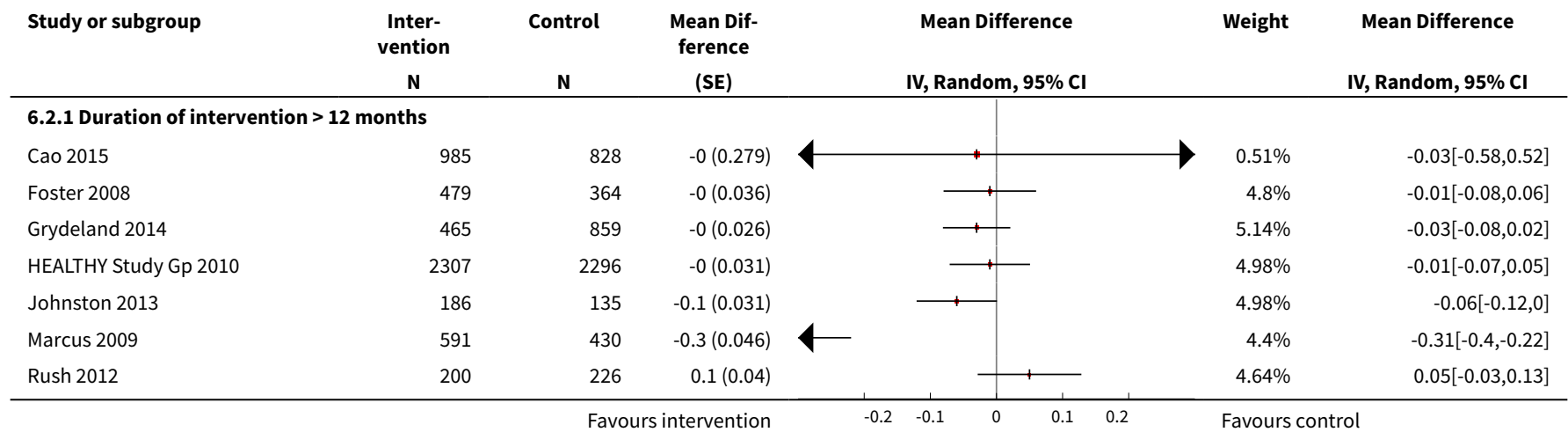




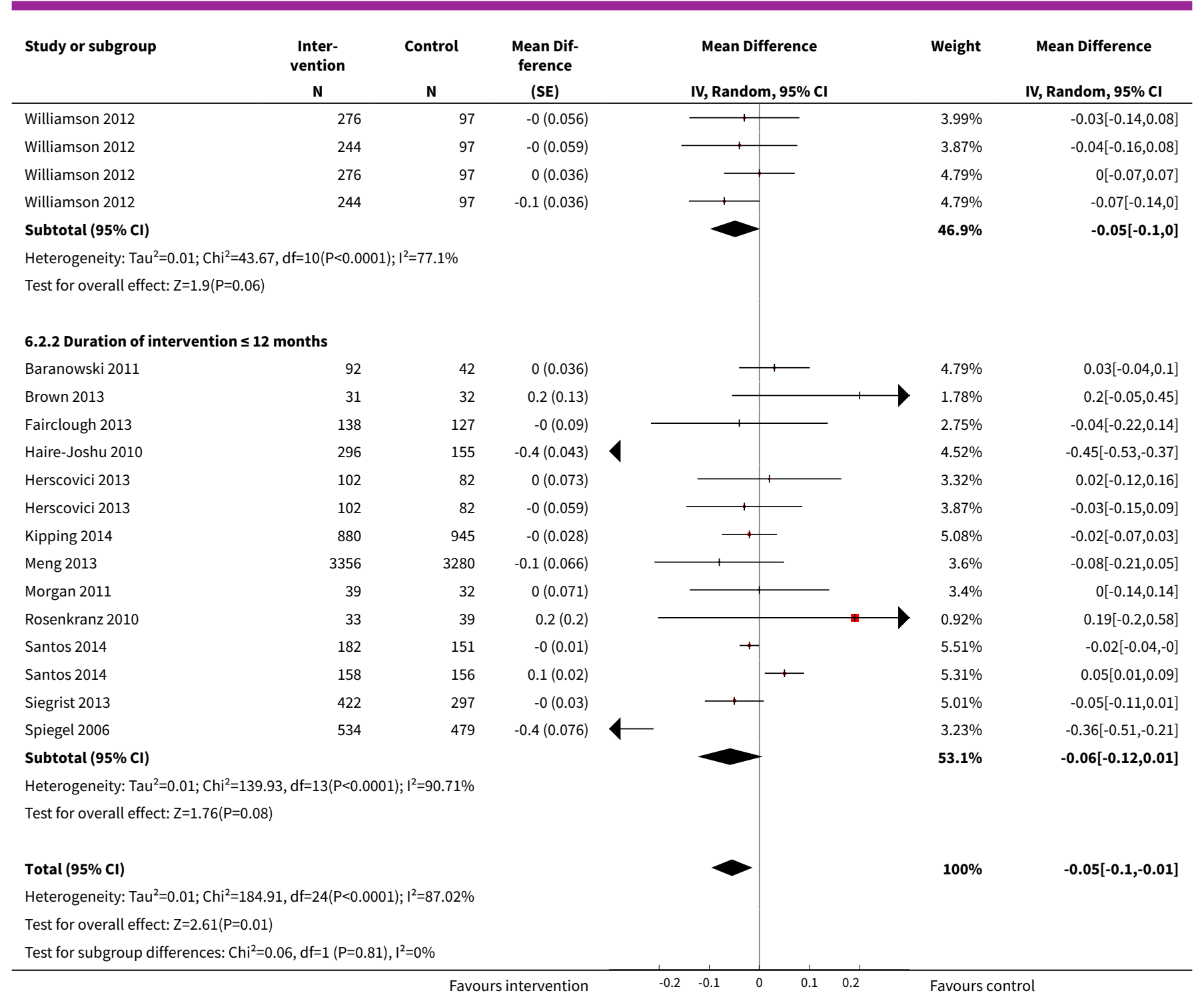

Analysis 6.3. Comparison 6 Diet and physical activity interventions vs control: age 6-12 years, Outcome 3 BMI. Diet and physical activity vs control - setting.

\begin{tabular}{|c|c|c|c|c|c|c|}
\hline Study or subgroup & $\begin{array}{c}\text { Inter- } \\
\text { vention } \\
\mathbf{N}\end{array}$ & $\begin{array}{l}\text { Control } \\
\text { N }\end{array}$ & $\begin{array}{l}\text { Mean Dif- } \\
\text { ference } \\
\text { (SE) }\end{array}$ & $\begin{array}{c}\text { Mean Difference } \\
\text { IV, Random, } 95 \% \mathrm{CI}\end{array}$ & Weight & $\begin{array}{c}\text { Mean Difference } \\
\text { IV, Random, 95\% CI }\end{array}$ \\
\hline \multicolumn{7}{|l|}{ 6.3.1 School } \\
\hline Caballero 2003 & 727 & 682 & $-0.1(0.107)$ & $\rightarrow$ & $5.92 \%$ & $-0.1[-0.31,0.11]$ \\
\hline Foster 2008 & 479 & 364 & $-0(0.117)$ & $\longrightarrow$ & $5.13 \%$ & $-0.04[-0.27,0.19]$ \\
\hline Gentile 2009 & 529 & 587 & $0(0.036)$ & + & $18.7 \%$ & $0[-0.07,0.07]$ \\
\hline Habib-Mourad 2014 & 188 & 175 & $0.2(0.158)$ & - & $3.1 \%$ & $0.18[-0.13,0.49]$ \\
\hline Herscovici 2013 & 102 & 82 & $-0.1(0.162)$ & 1 & $2.96 \%$ & $-0.15[-0.47,0.17]$ \\
\hline Herscovici 2013 & 102 & 82 & $0(0.219)$ & 1 & $1.71 \%$ & $0.02[-0.41,0.45]$ \\
\hline Jansen 2011 & 583 & 653 & $0(0.07)$ & + & $10.6 \%$ & $0.03[-0.11,0.17]$ \\
\hline Jansen 2011 & 657 & 729 & $-0.1(0.06)$ & + & $12.57 \%$ & $-0.1[-0.22,0.02]$ \\
\hline
\end{tabular}




\begin{tabular}{|c|c|c|c|c|c|c|}
\hline Study or subgroup & $\begin{array}{c}\text { Inter- } \\
\text { vention } \\
\mathbf{N}\end{array}$ & Control & $\begin{array}{l}\text { Mean Dif- } \\
\text { ference } \\
\text { (SE) }\end{array}$ & $\begin{array}{c}\text { Mean Difference } \\
\text { IV, Random, } 95 \% \mathrm{CI}\end{array}$ & Weight & $\begin{array}{c}\text { Mean Difference } \\
\text { IV, Random, 95\% CI }\end{array}$ \\
\hline Johnston 2013 & 186 & 135 & $-0.2(0.204)$ & \begin{tabular}{l|l}
1 \\
\end{tabular} & $1.95 \%$ & $-0.25[-0.65,0.15]$ \\
\hline Kipping 2008 & 249 & 223 & $0.1(0.186)$ & 1 & $2.31 \%$ & $0.1[-0.26,0.46]$ \\
\hline Levy 2012 & 259 & 247 & $-0.7(0.5)$ & \begin{tabular}{l|l} 
& \\
\end{tabular} & $0.35 \%$ & $-0.7[-1.68,0.28]$ \\
\hline Levy 2012 & 239 & 252 & $-0.5(0.327)$ & t & $0.8 \%$ & $-0.5[-1.14,0.14]$ \\
\hline Llargues 2012 & 272 & 236 & $-0.4(0.465)$ & + & $0.4 \%$ & $-0.4[-1.31,0.51]$ \\
\hline Magnusson 2012 & 90 & 76 & $0.3(0.24)$ & 7 & $1.44 \%$ & $0.31[-0.16,0.78]$ \\
\hline Meng 2013 & 3356 & 3280 & $-0.2(0.128)$ & 1 & $4.45 \%$ & $-0.19[-0.44,0.06]$ \\
\hline Safdie 2013 & 252 & 177 & $1.3(0.633)$ & & $0.22 \%$ & $1.3[0.06,2.54]$ \\
\hline Safdie 2013 & 224 & 177 & $-0.1(0.663)$ & & $0.2 \%$ & $-0.1[-1.4,1.2]$ \\
\hline Siegrist 2013 & 422 & 297 & $0(0.26)$ & & $1.24 \%$ & $0[-0.51,0.51]$ \\
\hline Story 2003 & 26 & 27 & $0.2(0.2)$ & $\begin{array}{l}1 \\
\end{array}$ & $2.03 \%$ & $0.2[-0.19,0.59]$ \\
\hline Subtotal $(95 \% \mathrm{Cl})$ & & & & $\checkmark$ & $89.52 \%$ & $-0.04[-0.1,0.02]$ \\
\hline \multicolumn{7}{|c|}{ Heterogeneity: $\mathrm{Tau}^{2}=0 ; \mathrm{Chi}^{2}=22.54, \mathrm{df}=19(\mathrm{P}=0.26) ; \mathrm{I}^{2}=15.7 \%$} \\
\hline \multicolumn{7}{|c|}{ Test for overall effect: $Z=1.43(P=0.15)$} \\
\hline \multicolumn{7}{|c|}{ 6.3.2 Wider community } \\
\hline Baranowski 2003 & 17 & 14 & $0.6(0.381)$ & & $0.59 \%$ & $0.6[-0.15,1.35]$ \\
\hline Beech 2003 & 21 & 9 & $-0.4(0.25)$ & - & $1.33 \%$ & $-0.38[-0.87,0.11]$ \\
\hline Beech 2003 & 21 & 9 & $-0.4(0.25)$ & - & $1.33 \%$ & $-0.4[-0.89,0.09]$ \\
\hline Brown 2013 & 31 & 32 & $0(0.245)$ & & $1.39 \%$ & $0[-0.48,0.48]$ \\
\hline Chen 2010 & 35 & 32 & $1.1(0.76)$ & & $0.15 \%$ & $1.08[-0.41,2.57]$ \\
\hline Chen 2011 & 26 & 24 & $0(0.18)$ & - & $2.46 \%$ & $0.01[-0.34,0.36]$ \\
\hline Klesges 2010 & 153 & 150 & $-0.1(0.26)$ & & $1.24 \%$ & $-0.1[-0.61,0.41]$ \\
\hline Nollen 2014 & 23 & 21 & $0.1(0.526)$ & & $0.31 \%$ & $0.06[-0.97,1.09]$ \\
\hline Robinson 2003 & 28 & 33 & $-0.3(0.23)$ & - & $1.57 \%$ & $-0.32[-0.77,0.13]$ \\
\hline Rosenkranz 2010 & 33 & 39 & $1.2(0.918)$ & & $0.1 \%$ & $1.2[-0.6,3]$ \\
\hline Subtotal $(95 \% \mathrm{Cl})$ & & & & & $10.48 \%$ & $-0.08[-0.29,0.13]$ \\
\hline \multicolumn{7}{|c|}{ Heterogeneity: $\operatorname{Tau}^{2}=0.03 ; \mathrm{Chi}^{2}=12.03, \mathrm{df}=9(\mathrm{P}=0.21) ; \mathrm{I}^{2}=25.2 \%$} \\
\hline \multicolumn{7}{|c|}{ Test for overall effect: $Z=0.72(P=0.47)$} \\
\hline Total $(95 \% \mathrm{Cl})$ & & & & $\checkmark$ & $100 \%$ & $-0.05[-0.11,0.01]$ \\
\hline \multicolumn{7}{|c|}{ Heterogeneity: $\mathrm{Tau}^{2}=0 ; \mathrm{Chi}^{2}=34.98, \mathrm{df}=29(\mathrm{P}=0.21) ; \mathrm{I}^{2}=17.1 \%$} \\
\hline \multicolumn{7}{|c|}{ Test for overall effect: $Z=1.59(P=0.11)$} \\
\hline Test for subgroup dif & $1, \mathrm{df}=1(\mathrm{P}=0$. & ${ }^{2}=0 \%$ & & & & \\
\hline
\end{tabular}

Analysis 6.4. Comparison 6 Diet and physical activity interventions vs control: age 6-12 years, Outcome 4 BMI. Diet and physical activity vs control - duration.

\begin{tabular}{|c|c|c|c|c|c|c|}
\hline Study or subgroup & $\begin{array}{c}\text { Inter- } \\
\text { vention } \\
\mathbf{N} \\
\end{array}$ & Control & $\begin{array}{l}\text { Mean Dif- } \\
\text { ference } \\
\text { (SE) } \\
\end{array}$ & $\begin{array}{c}\text { Mean Difference } \\
\text { IV, Random, 95\% CI }\end{array}$ & Weight & $\begin{array}{c}\text { Mean Difference } \\
\text { IV, Random, 95\% CI }\end{array}$ \\
\hline \multicolumn{7}{|c|}{ 6.4.1 Duration of intervention > 12 months } \\
\hline Caballero 2003 & 727 & 682 & $-0.1(0.107)$ & + & $5.92 \%$ & $-0.1[-0.31,0.11]$ \\
\hline Foster 2008 & 479 & 364 & $-0(0.117)$ & + & $5.13 \%$ & $-0.04[-0.27,0.19]$ \\
\hline Grydeland 2014 & 465 & 859 & $-0.1(0.056)$ & + & $13.46 \%$ & $-0.1[-0.21,0.01]$ \\
\hline Klesges 2010 & 153 & 150 & $-0.1(0.26)$ & $\longrightarrow$ & $1.24 \%$ & $-0.1[-0.61,0.41]$ \\
\hline Llargues 2012 & 272 & 236 & $-0.4(0.465)$ & $\longrightarrow+$ & $0.4 \%$ & $-0.4[-1.31,0.51]$ \\
\hline
\end{tabular}




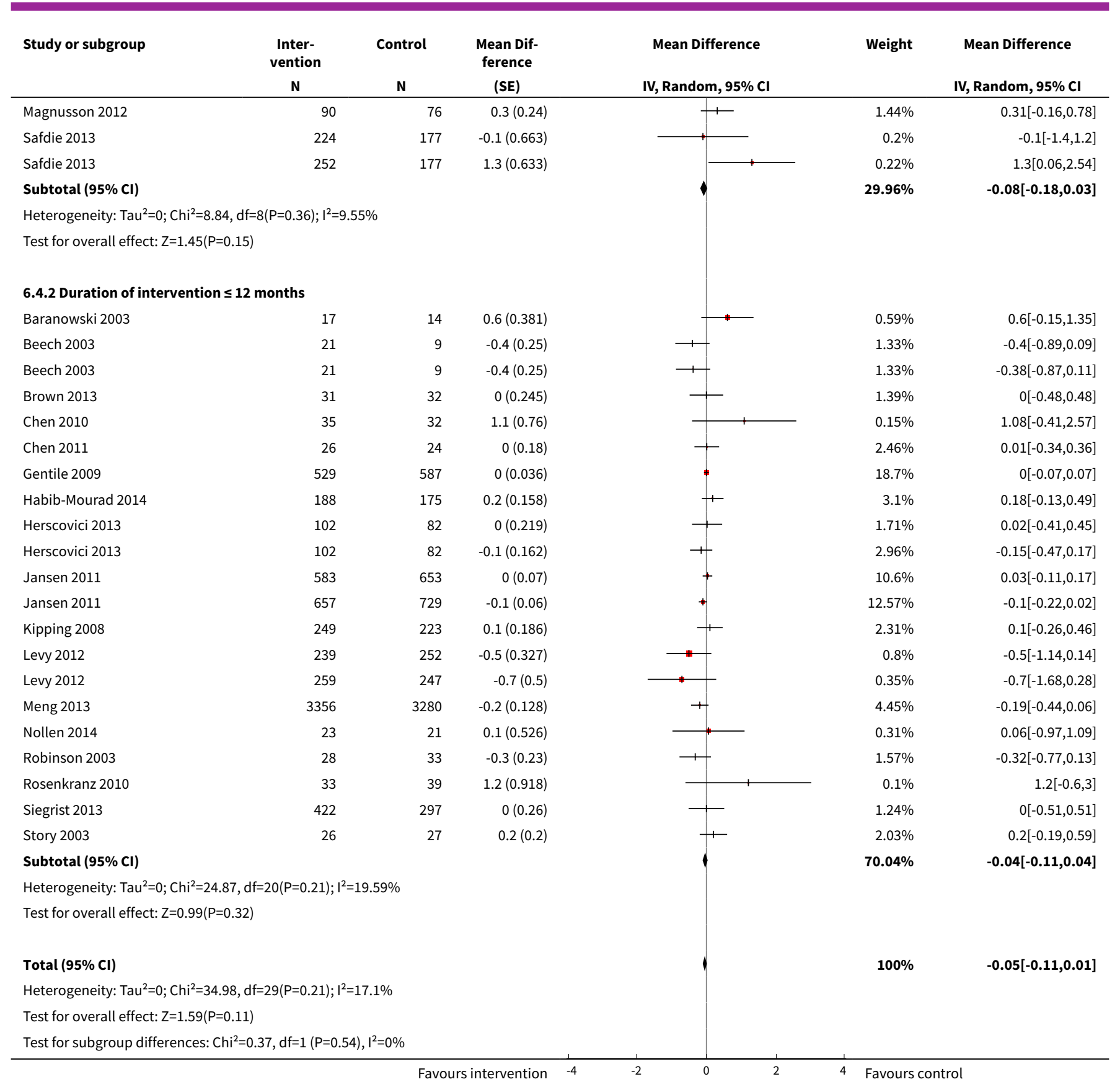

\section{Comparison 7. Diet interventions versus control: age 13-18 years}

\begin{tabular}{lllll}
\hline $\begin{array}{l}\text { Outcome or sub- } \\
\text { group title }\end{array}$ & No. of studies & $\begin{array}{l}\text { No. of partici- } \\
\text { pants }\end{array}$ & Statistical method & Effect size \\
\hline 1 BMI - setting & 2 & 294 & Mean Difference (Random, 95\% Cl) & $-0.13[-0.50,0.23]$ \\
\hline 1.1 Home & 1 & 103 & Mean Difference (Random, 95\% Cl) & $-0.14[-0.54,0.26]$ \\
\hline 1.2 School & 1 & 191 & Mean Difference (Random, 95\% Cl) & $-0.1[-0.99,0.79]$ \\
\hline
\end{tabular}


Analysis 7.1. Comparison 7 Diet interventions versus control: age 13-18 years, Outcome 1 BMI - setting.

\begin{tabular}{|c|c|c|c|c|c|c|}
\hline Study or subgroup & $\begin{array}{l}\text { Inter- } \\
\text { vention } \\
\quad \mathrm{N}\end{array}$ & $\begin{array}{c}\text { Control } \\
\text { N }\end{array}$ & $\begin{array}{l}\text { Mean Dif- } \\
\text { ference } \\
\text { (SE) }\end{array}$ & $\begin{array}{l}\text { Mean Difference } \\
\text { IV, Random, } 95 \% \mathrm{CI}\end{array}$ & Weight & $\begin{array}{l}\text { Mean Difference } \\
\text { IV, Random, } 95 \% \mathrm{CI}\end{array}$ \\
\hline \multicolumn{7}{|l|}{ 7.1.1 Home } \\
\hline Ebbeling 2006 & 53 & 50 & $-0.1(0.204)$ & - & $83.2 \%$ & $-0.14[-0.54,0.26]$ \\
\hline Subtotal $(95 \% \mathrm{Cl})$ & & & & & $83.2 \%$ & $-0.14[-0.54,0.26]$ \\
\hline \multicolumn{7}{|c|}{ Heterogeneity: Not applicable } \\
\hline \multicolumn{7}{|c|}{ Test for overall effect: $Z=0.69(P=0.49)$} \\
\hline \multicolumn{7}{|l|}{ 7.1.2 School } \\
\hline Mihas 2010 & 98 & 93 & $-0.1(0.454)$ & + & $16.8 \%$ & $-0.1[-0.99,0.79]$ \\
\hline Subtotal $(95 \% \mathrm{Cl})$ & & & & & $16.8 \%$ & $-0.1[-0.99,0.79]$ \\
\hline \multicolumn{7}{|c|}{ Heterogeneity: Not applicable } \\
\hline \multicolumn{7}{|c|}{ Test for overall effect: $\mathrm{Z}=0.22(\mathrm{P}=0.83)$} \\
\hline Total $(95 \% \mathrm{Cl})$ & & & & & $100 \%$ & $-0.13[-0.5,0.23]$ \\
\hline \multicolumn{7}{|c|}{ Heterogeneity: Tau $^{2}=0 ; \mathrm{Chi}^{2}=0.01, \mathrm{df}=1(\mathrm{P}=0.94) ; \mathrm{I}^{2}=0 \%$} \\
\hline \multicolumn{7}{|c|}{ Test for overall effect: $Z=0.72(P=0.47)$} \\
\hline Test for subgroup dif & $1, d f=1(P=0$ & $1^{2}=0 \%$ & & & & \\
\hline
\end{tabular}

\section{Comparison 8. Physical activity interventions versus control: age 13-18 years}

\begin{tabular}{|c|c|c|c|c|}
\hline $\begin{array}{l}\text { Outcome or subgroup } \\
\text { title }\end{array}$ & No. of studies & $\begin{array}{l}\text { No. of partici- } \\
\text { pants }\end{array}$ & Statistical method & Effect size \\
\hline 1 zBMI - setting & 1 & 100 & Mean Difference (Random, 95\% Cl) & $-0.20[-0.30,-0.10]$ \\
\hline 1.1 School & 1 & 100 & Mean Difference (Random, 95\% Cl) & $-0.20[-0.30,-0.10]$ \\
\hline 2 zBMI - duration & 1 & 100 & Mean Difference (Random, 95\% Cl) & $-0.20[-0.30,-0.10]$ \\
\hline $\begin{array}{l}\text { 2.1 Duration of interven- } \\
\text { tion } \leq 12 \text { months }\end{array}$ & 1 & 100 & Mean Difference (Random, 95\% Cl) & $-0.20[-0.30,-0.10]$ \\
\hline 3 BMI - setting & 4 & 720 & Mean Difference (Random, 95\% Cl) & $-1.53[-2.67,-0.39]$ \\
\hline 3.1 School & 4 & 720 & Mean Difference (Random, 95\% Cl) & $-1.53[-2.67,-0.39]$ \\
\hline $4 \mathrm{BMI}$ - duration & 4 & 720 & Mean Difference (Random, 95\% Cl) & $-1.53[-2.67,-0.39]$ \\
\hline $\begin{array}{l}\text { 4.1 Duration of interven- } \\
\text { tion } \leq 12 \text { months }\end{array}$ & 4 & 720 & Mean Difference (Random, 95\% Cl) & $-1.53[-2.67,-0.39]$ \\
\hline
\end{tabular}


Analysis 8.1. Comparison 8 Physical activity interventions versus control: age 13-18 years, Outcome 1 zBMI - setting.

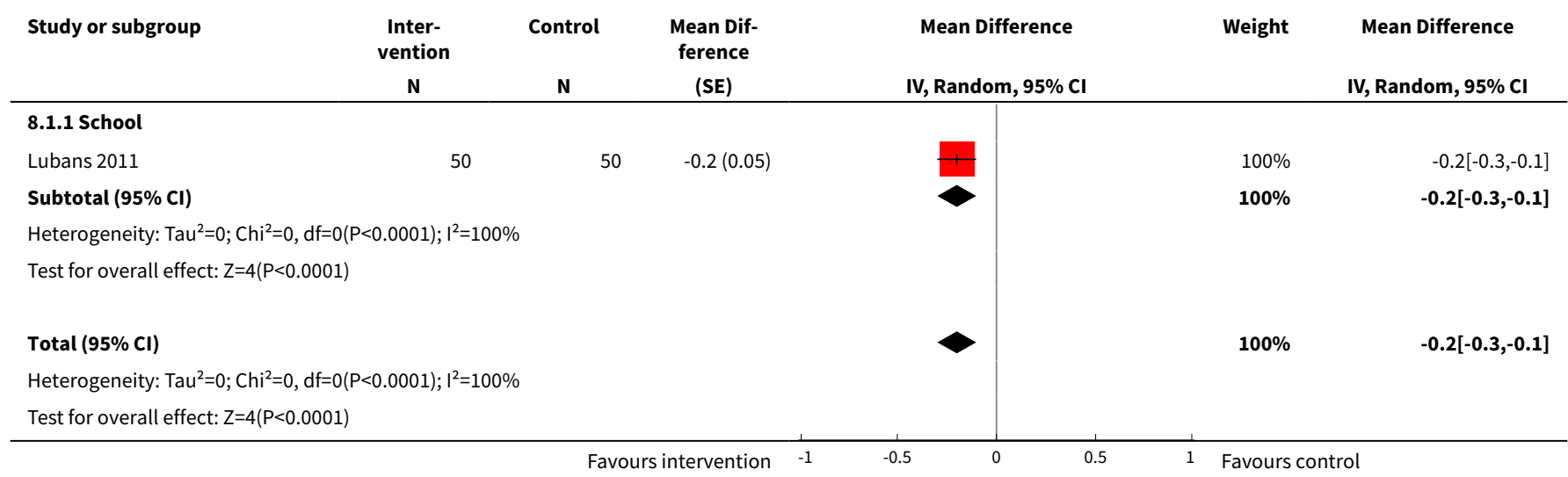

Analysis 8.2. Comparison 8 Physical activity interventions versus control: age 13-18 years, Outcome 2 zBMI - duration.

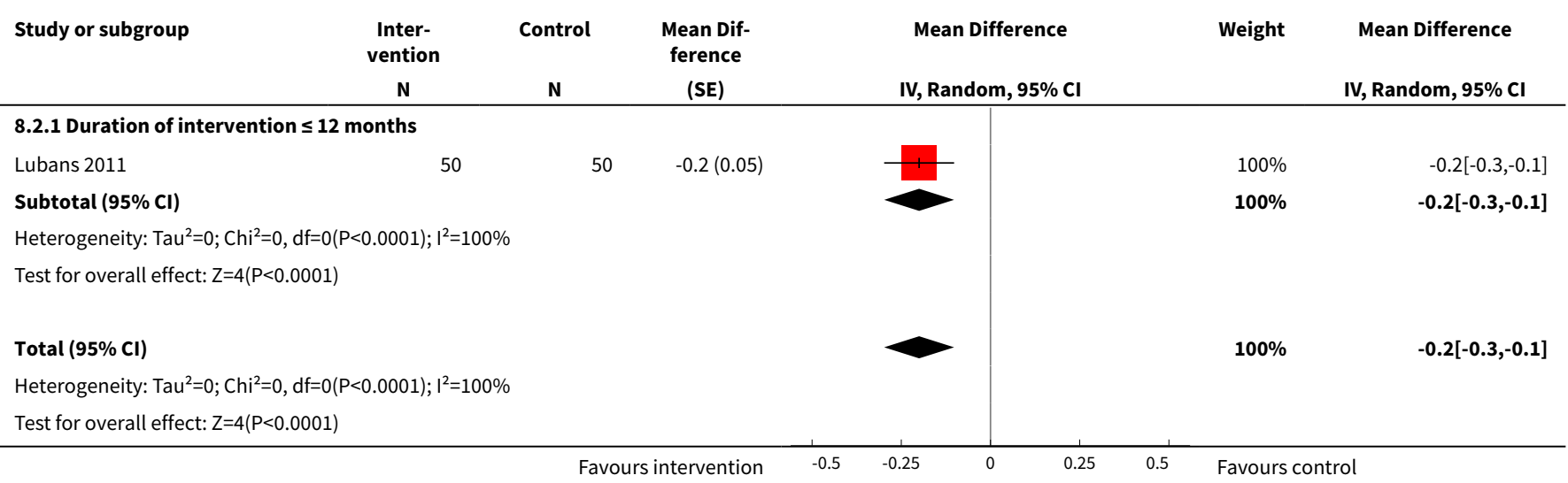

Analysis 8.3. Comparison 8 Physical activity interventions versus control: age 13-18 years, Outcome 3 BMI - setting.

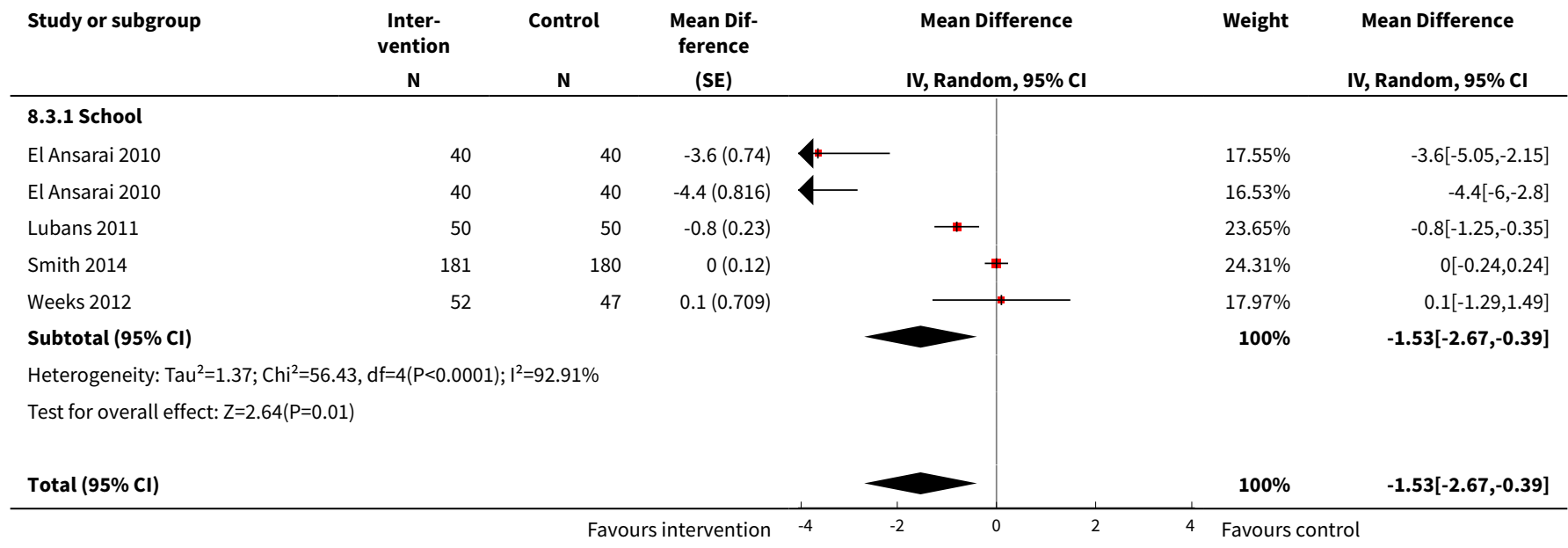




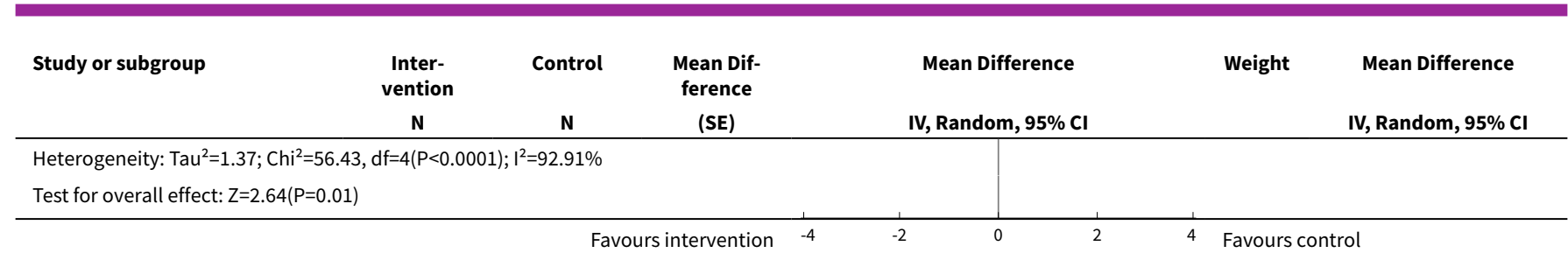

\section{Analysis 8.4. Comparison 8 Physical activity interventions} versus control: age 13-18 years, Outcome 4 BMI - duration.

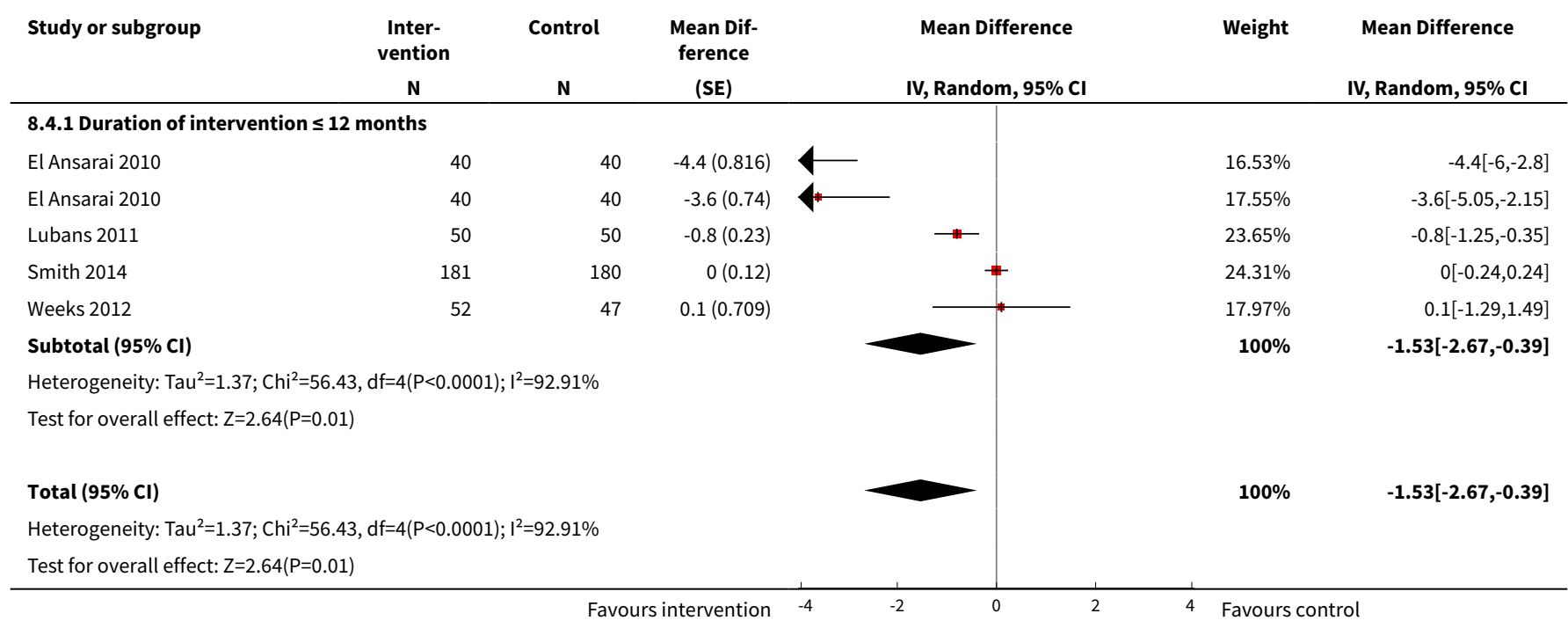

\section{Comparison 9. Diet and physical activity interventions versus control: age 13-18 years}

\begin{tabular}{lllll}
\hline $\begin{array}{l}\text { Outcome or subgroup ti- } \\
\text { tle }\end{array}$ & No. of studies & $\begin{array}{l}\text { No. of partici- } \\
\text { pants }\end{array}$ & Statistical method & Effect size \\
\hline 1 zBMI - setting & 6 & 16543 & Mean Difference (Random, 95\% Cl) & $0.01[-0.05,0.07]$ \\
\hline 1.1 Home & 1 & 75 & Mean Difference (Random, 95\% Cl) & $0.06[-0.13,0.26]$ \\
\hline $\begin{array}{lllll}1.2 \text { School } \\
2 \text { zBMI - duration }\end{array}$ & 5 & 16468 & Mean Difference (Random, 95\% Cl) & $0.00[-0.06,0.06]$ \\
\hline $\begin{array}{l}2.1 \text { Duration of interven- } \\
\text { tion } \leq 12 \text { months }\end{array}$ & 3 & 16543 & Mean Difference (Random, 95\% Cl) & $0.01[-0.05,0.07]$ \\
\hline $\begin{array}{l}2.2 \text { Duration of interven- } \\
\text { tion > 12 months }\end{array}$ & 3 & 2525 & Mean Difference (Random, 95\% Cl) & $-0.09[-0.18,0.00]$ \\
\hline $\begin{array}{l}3 \text { BMI - setting } \\
\text { 3.1 School }\end{array}$ & 8 & 14018 & Mean Difference (Random, 95\% Cl) & $0.01[-0.02,0.04]$ \\
\hline
\end{tabular}




\begin{tabular}{lllll}
\hline $\begin{array}{l}\text { Outcome or subgroup ti- } \\
\text { tle }\end{array}$ & No. of studies & $\begin{array}{l}\text { No. of partici- } \\
\text { pants }\end{array}$ & Statistical method & Effect size \\
\hline 4 BMI - duration & 8 & 16583 & Mean Difference (Random, 95\% Cl) & $-0.02[-0.10,0.05]$ \\
\hline $\begin{array}{l}4.1 \text { Duration of interven- } \\
\text { tion }>12 \text { months }\end{array}$ & 2 & 12904 & Mean Difference (Random, 95\% Cl) & $-0.04[-0.17,0.09]$ \\
\hline $\begin{array}{l}\text { 4.2 Duration of interven- } \\
\text { tion } \leq 12 \text { months }\end{array}$ & 6 & 3679 & Mean Difference (Random, 95\% Cl) & $-0.03[-0.11,0.05]$ \\
\hline
\end{tabular}

\section{Analysis 9.1. Comparison 9 Diet and physical activity interventions} versus control: age 13-18 years, Outcome 1 zBMI - setting.

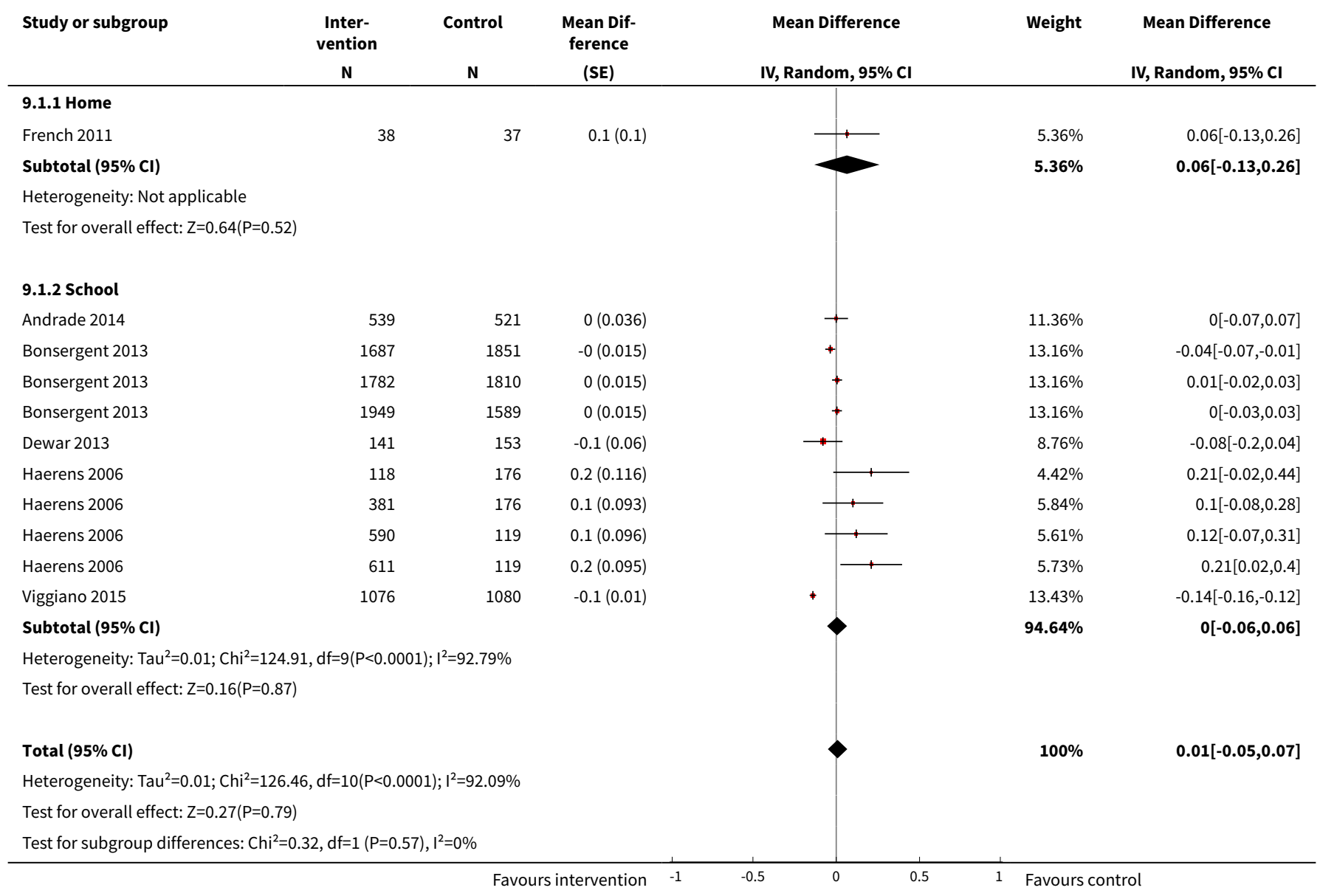


Analysis 9.2. Comparison 9 Diet and physical activity interventions versus control: age 13-18 years, Outcome 2 zBMI - duration.

\begin{tabular}{|c|c|c|c|c|c|c|}
\hline Study or subgroup & $\begin{array}{c}\text { Inter- } \\
\text { vention } \\
\mathbf{N} \\
\end{array}$ & $\begin{array}{l}\text { Control } \\
\text { N }\end{array}$ & $\begin{array}{c}\text { Mean Dif- } \\
\text { ference } \\
\text { (SE) } \\
\end{array}$ & $\begin{array}{c}\text { Mean Difference } \\
\text { IV, Random, } 95 \% \mathrm{CI}\end{array}$ & Weight & $\begin{array}{c}\text { Mean Difference } \\
\text { IV, Random, 95\% CI }\end{array}$ \\
\hline \multicolumn{7}{|c|}{ 9.2.1 Duration of intervention $\leq 12$ months } \\
\hline Dewar 2013 & 141 & 153 & $-0.1(0.06)$ & $\longrightarrow$ & $8.76 \%$ & $-0.08[-0.2,0.04]$ \\
\hline French 2011 & 38 & 37 & $0.1(0.1)$ & $1+$ & $5.36 \%$ & $0.06[-0.13,0.26]$ \\
\hline Viggiano 2015 & 1076 & 1080 & $-0.1(0.01)$ & $*$ & $13.43 \%$ & $-0.14[-0.16,-0.12]$ \\
\hline \multicolumn{7}{|c|}{ Heterogeneity: $\mathrm{Tau}^{2}=0 ; \mathrm{Chi}^{2}=5.03, \mathrm{df}=2(\mathrm{P}=0.08) ; \mathrm{I}^{2}=60.23 \%$} \\
\hline \multicolumn{7}{|c|}{ Test for overall effect: $Z=1.86(P=0.06)$} \\
\hline \multicolumn{7}{|c|}{ 9.2.2 Duration of intervention $>12$ months } \\
\hline Andrade 2014 & 539 & 521 & $0(0.036)$ & + & $11.36 \%$ & $0[-0.07,0.07]$ \\
\hline Bonsergent 2013 & 1782 & 1810 & $0(0.015)$ & + & $13.16 \%$ & $0.01[-0.02,0.03]$ \\
\hline Bonsergent 2013 & 1949 & 1589 & $0(0.015)$ & + & $13.16 \%$ & $0[-0.03,0.03]$ \\
\hline Haerens 2006 & 611 & 119 & $0.2(0.095)$ & 1 & $5.73 \%$ & $0.21[0.02,0.4]$ \\
\hline Haerens 2006 & 590 & 119 & $0.1(0.096)$ & 1 & $5.61 \%$ & $0.12[-0.07,0.31]$ \\
\hline Haerens 2006 & 381 & 176 & $0.1(0.093)$ & $\begin{array}{l}1 \\
1\end{array}$ & $5.84 \%$ & $0.1[-0.08,0.28]$ \\
\hline Haerens 2006 & 118 & 176 & $0.2(0.116)$ & 1 & $4.42 \%$ & $0.21[-0.02,0.44]$ \\
\hline Subtotal $(95 \% \mathrm{Cl})$ & & & & 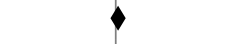 & $72.45 \%$ & $0.01[-0.02,0.04]$ \\
\hline \multicolumn{7}{|c|}{ Heterogeneity: $\mathrm{Tau}^{2}=0 ; \mathrm{Chi}^{2}=16.44, \mathrm{df}=7(\mathrm{P}=0.02) ; \mathrm{I}^{2}=57.42 \%$} \\
\hline \multicolumn{7}{|c|}{ Test for overall effect: $Z=0.6(P=0.55)$} \\
\hline Total $(95 \% \mathrm{Cl})$ & & & & & $100 \%$ & $0.01[-0.05,0.07]$ \\
\hline \multicolumn{7}{|c|}{ Heterogeneity: $\operatorname{Tau}^{2}=0.01 ; \mathrm{Chi}^{2}=126.46, \mathrm{df}=10(\mathrm{P}<0.0001) ; \mathrm{I}^{2}=92.09 \%$} \\
\hline \multicolumn{7}{|c|}{ Test for overall effect: $Z=0.27(P=0.79)$} \\
\hline Test for subgroup dif & $3, \mathrm{df}=1(\mathrm{P}=0.0$ & $\left.\right|^{2}=73.88 \%$ & & & & \\
\hline
\end{tabular}

Analysis 9.3. Comparison 9 Diet and physical activity interventions versus control: age 13-18 years, Outcome 3 BMI - setting.

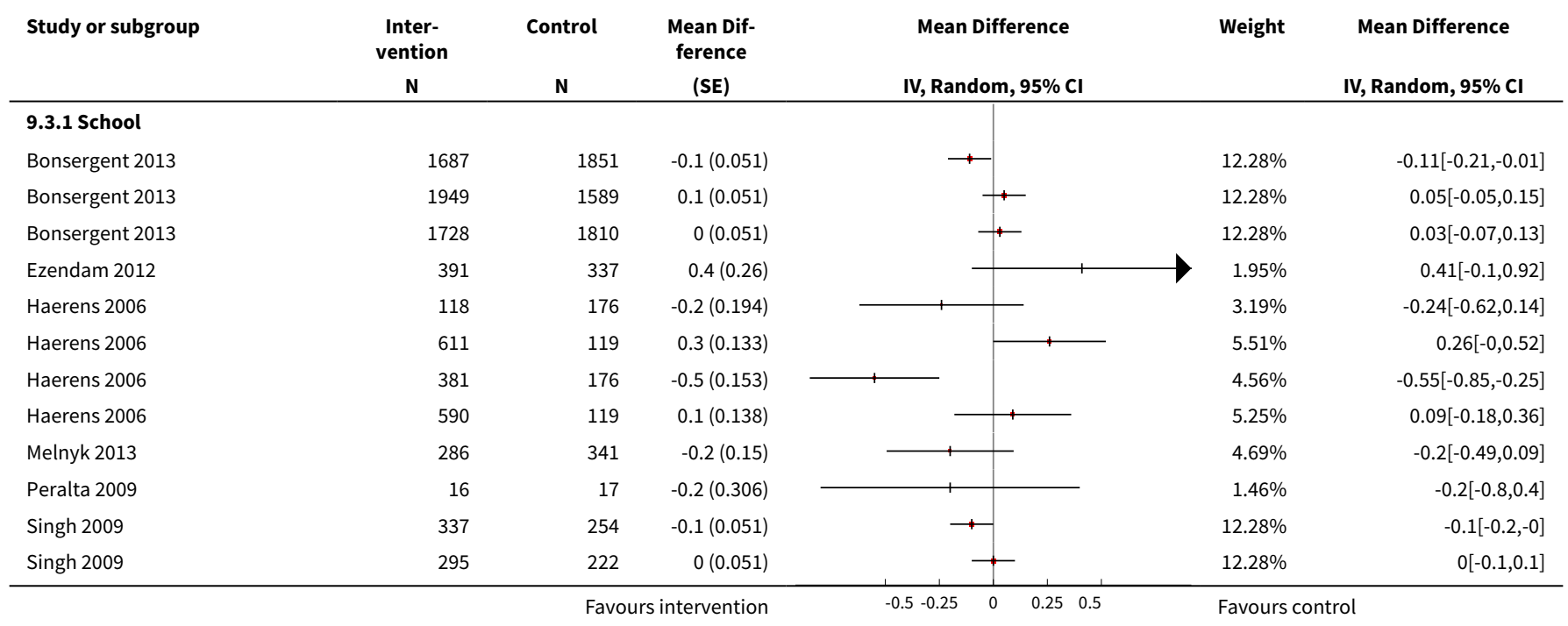




\begin{tabular}{|c|c|c|c|c|c|c|}
\hline Study or subgroup & $\begin{array}{c}\text { Inter- } \\
\text { vention } \\
\mathbf{N}\end{array}$ & Control & $\begin{array}{l}\text { Mean Dif- } \\
\text { ference } \\
\text { (SE) }\end{array}$ & $\begin{array}{c}\text { Mean Difference } \\
\text { IV, Random, 95\% CI }\end{array}$ & Weight & IV, Random, 95\% CI \\
\hline Whittemore 2013 & 199 & 166 & $0.5(0.582)$ & * & $0.43 \%$ & $0.5[-0.64,1.64]$ \\
\hline Wilksch 2015 & 173 & 236 & $0.1(0.112)$ & + & $6.79 \%$ & $0.07[-0.15,0.29]$ \\
\hline Wilksch 2015 & 173 & 236 & $0.1(0.148)$ & & $4.78 \%$ & $0.05[-0.24,0.34]$ \\
\hline Subtotal $(95 \% \mathrm{CI})$ & & & & & $100 \%$ & $-0.02[-0.1,0.05]$ \\
\hline \multicolumn{7}{|c|}{ Heterogeneity: $\mathrm{Tau}^{2}=0.01 ; \mathrm{Chi}^{2}=33.07, \mathrm{df}=14(\mathrm{P}=0) ; \mathrm{I}^{2}=57.67 \%$} \\
\hline \multicolumn{7}{|c|}{ Test for overall effect: $Z=0.63(P=0.53)$} \\
\hline Total $(95 \% \mathrm{Cl})$ & & & & & $100 \%$ & $-0.02[-0.1,0.05]$ \\
\hline \multicolumn{7}{|c|}{ Heterogeneity: $\mathrm{Tau}^{2}=0.01 ; \mathrm{Chi}^{2}=33.07, \mathrm{df}=14(\mathrm{P}=0) ; \mathrm{I}^{2}=57.67 \%$} \\
\hline \multicolumn{7}{|c|}{ Test for overall effect: $\mathrm{Z}=0.63(\mathrm{P}=0.53)$} \\
\hline
\end{tabular}

Analysis 9.4. Comparison 9 Diet and physical activity interventions versus control: age 13-18 years, Outcome 4 BMI - duration.

\begin{tabular}{|c|c|c|c|c|c|c|}
\hline Study or subgroup & $\begin{array}{c}\text { Inter- } \\
\text { vention } \\
\mathbf{N}\end{array}$ & $\begin{array}{l}\text { Control } \\
\text { N }\end{array}$ & $\begin{array}{c}\text { Mean Dif- } \\
\text { ference } \\
\text { (SE) }\end{array}$ & IV, Random, $95 \% \mathrm{CI}$ & Weight & $\begin{array}{c}\text { Mean Difference } \\
\text { IV, Random, 95\% CI }\end{array}$ \\
\hline \multicolumn{7}{|c|}{ 9.4.1 Duration of intervention $>12$ months } \\
\hline Bonsergent 2013 & 1728 & 1810 & $0(0.051)$ & + & $12.28 \%$ & $0.03[-0.07,0.13]$ \\
\hline Bonsergent 2013 & 1949 & 1589 & $0.1(0.051)$ & + & $12.28 \%$ & $0.05[-0.05,0.15]$ \\
\hline Bonsergent 2013 & 1687 & 1851 & $-0.1(0.051)$ & + & $12.28 \%$ & $-0.11[-0.21,-0.01]$ \\
\hline Haerens 2006 & 590 & 119 & $0.1(0.138)$ & + & $5.25 \%$ & $0.09[-0.18,0.36]$ \\
\hline Haerens 2006 & 381 & 176 & $-0.5(0.153)$ & + & $4.56 \%$ & $-0.55[-0.85,-0.25]$ \\
\hline Haerens 2006 & 118 & 176 & $-0.2(0.194)$ & + & $3.19 \%$ & $-0.24[-0.62,0.14]$ \\
\hline Subtotal $(95 \% \mathrm{CI})$ & & & & 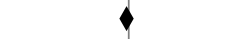 & $55.35 \%$ & $-0.04[-0.17,0.09]$ \\
\hline \multicolumn{7}{|c|}{ Heterogeneity: $\mathrm{Tau}^{2}=0.02 ; \mathrm{Chi}^{2}=24.3, \mathrm{df}=6(\mathrm{P}=0) ; \mathrm{I}^{2}=75.3 \%$} \\
\hline \multicolumn{7}{|c|}{ Test for overall effect: $Z=0.6(P=0.55)$} \\
\hline \multicolumn{7}{|c|}{ 9.4.2 Duration of intervention $\leq 12$ months } \\
\hline Ezendam 2012 & 391 & 337 & $0.4(0.26)$ & 1 & $1.95 \%$ & $0.41[-0.1,0.92]$ \\
\hline Melnyk 2013 & 286 & 341 & $-0.2(0.15)$ & + & $4.69 \%$ & $-0.2[-0.49,0.09]$ \\
\hline Peralta 2009 & 16 & 17 & $-0.2(0.306)$ & 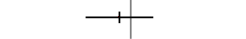 & $1.46 \%$ & $-0.2[-0.8,0.4]$ \\
\hline Singh 2009 & 337 & 254 & $-0.1(0.051)$ & * & $12.28 \%$ & $-0.1[-0.2,-0]$ \\
\hline Singh 2009 & 295 & 222 & $0(0.051)$ & + & $12.28 \%$ & $0[-0.1,0.1]$ \\
\hline Whittemore 2013 & 199 & 166 & $0.5(0.582)$ & *- & $0.43 \%$ & $0.5[-0.64,1.64]$ \\
\hline Wilksch 2015 & 173 & 236 & $0.1(0.148)$ & $\rightarrow$ & $4.78 \%$ & $0.05[-0.24,0.34]$ \\
\hline Wilksch 2015 & 173 & 236 & $0.1(0.112)$ & + & $6.79 \%$ & $0.07[-0.15,0.29]$ \\
\hline Subtotal $(95 \% \mathrm{Cl})$ & & & & 1 & $44.65 \%$ & $-0.03[-0.11,0.05]$ \\
\hline \multicolumn{7}{|c|}{ Heterogeneity: $\operatorname{Tau}^{2}=0 ; \mathrm{Chi}^{2}=8.58, \mathrm{df}=7(\mathrm{P}=0.28) ; \mathrm{I}^{2}=18.41 \%$} \\
\hline \multicolumn{7}{|c|}{ Test for overall effect: $Z=0.67(P=0.51)$} \\
\hline \multicolumn{7}{|c|}{ Test for overall effect: $Z=0.63(P=0.53)$} \\
\hline \multicolumn{7}{|c|}{ Test for subgroup differences: $\mathrm{Chi}^{2}=0.02, \mathrm{df}=1(\mathrm{P}=0.89), \mathrm{I}^{2}=0 \%$} \\
\hline & & Favo & ntervention & -2 & Favours & \\
\hline
\end{tabular}


Comparison 10. Dietary interventions versus physical activity interventions: age 6-12 years

\begin{tabular}{llll}
\hline $\begin{array}{l}\text { Outcome or sub- } \\
\text { group title }\end{array}$ & No. of studies & $\begin{array}{l}\text { No. of partici- } \\
\text { pants }\end{array}$ & Statistical method \\
\hline $1 \mathrm{zBMI}$ & 1 & 1205 & Mean Difference (Random, 95\% Cl) \\
\hline $2 \mathrm{BMI}$ & 2 & 4917 & Mean Difference (Random, $95 \% \mathrm{Cl})$ \\
\hline
\end{tabular}

Analysis 10.1. Comparison 10 Dietary interventions versus

physical activity interventions: age 6-12 years, Outcome 1 zBMI.

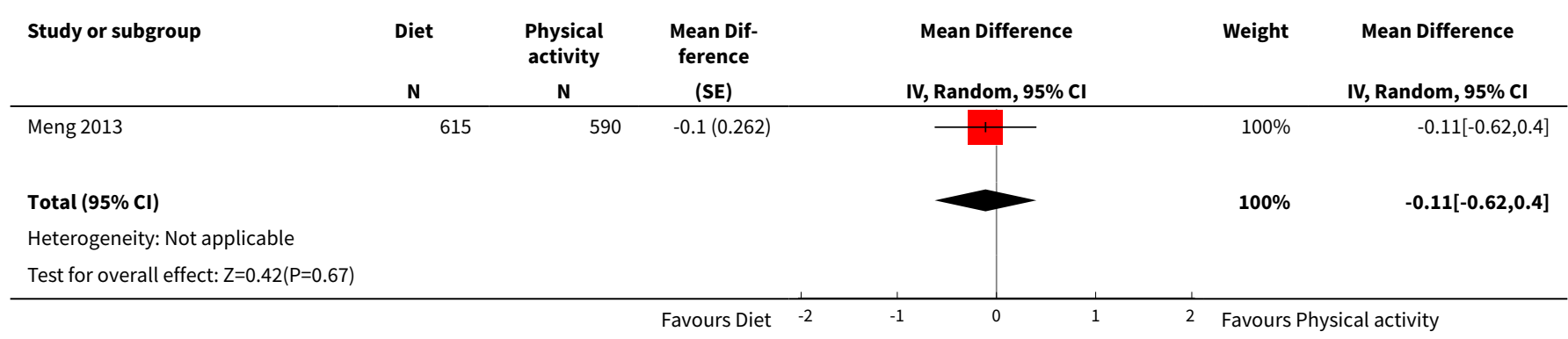

Analysis 10.2. Comparison 10 Dietary interventions versus physical activity interventions: age 6-12 years, Outcome 2 BMI.

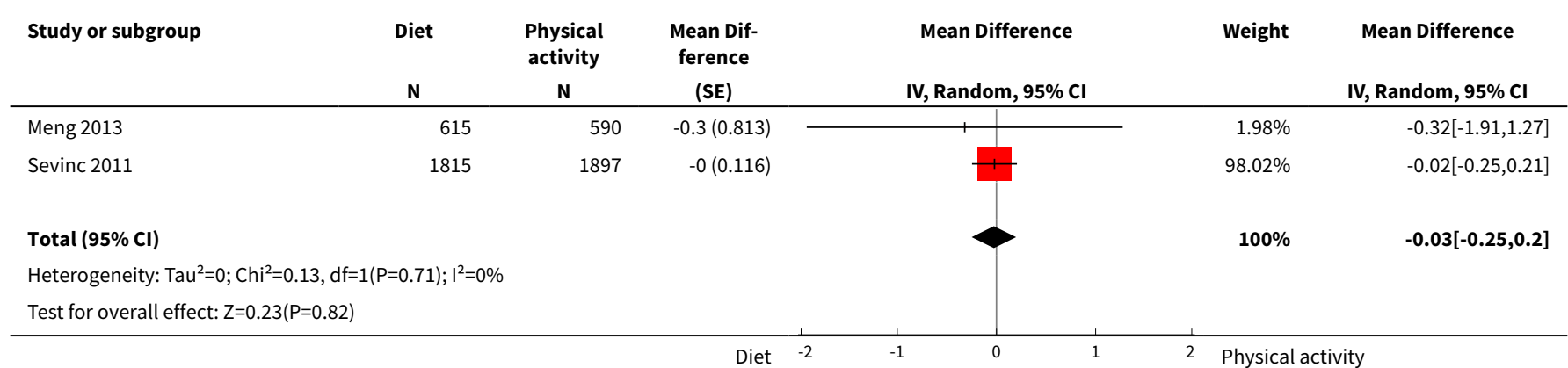

Comparison 11. Diet and physical activity versus physical activity interventions: age 6-12 years

\begin{tabular}{lllll}
\hline $\begin{array}{l}\text { Outcome or sub- } \\
\text { group title }\end{array}$ & No. of studies & $\begin{array}{l}\text { No. of partici- } \\
\text { pants }\end{array}$ & Statistical method & Effect size \\
\hline $1 \mathrm{zBMI}$ & 1 & 3946 & Mean Difference (Random, 95\% Cl) & $-0.16[-0.57,0.25]$ \\
\hline $2 \mathrm{BMI}$ & 1 & 3946 & Mean Difference (Random, 95\% Cl) & $-0.04[-1.05,0.97]$ \\
\hline
\end{tabular}


Analysis 11.1. Comparison 11 Diet and physical activity versus physical activity interventions: age 6-12 years, Outcome 1 zBMI.

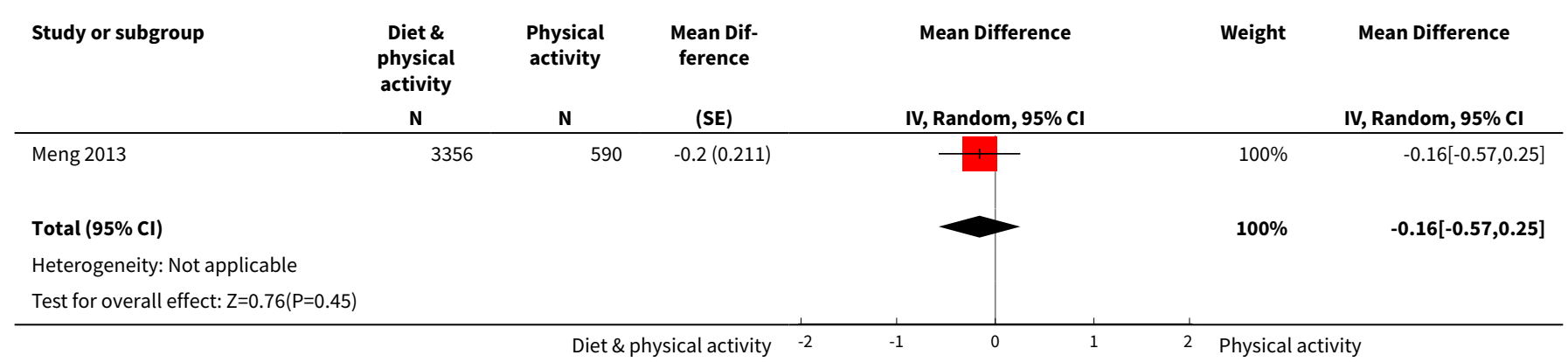

Analysis 11.2. Comparison 11 Diet and physical activity versus physical activity interventions: age 6-12 years, Outcome 2 BMI.

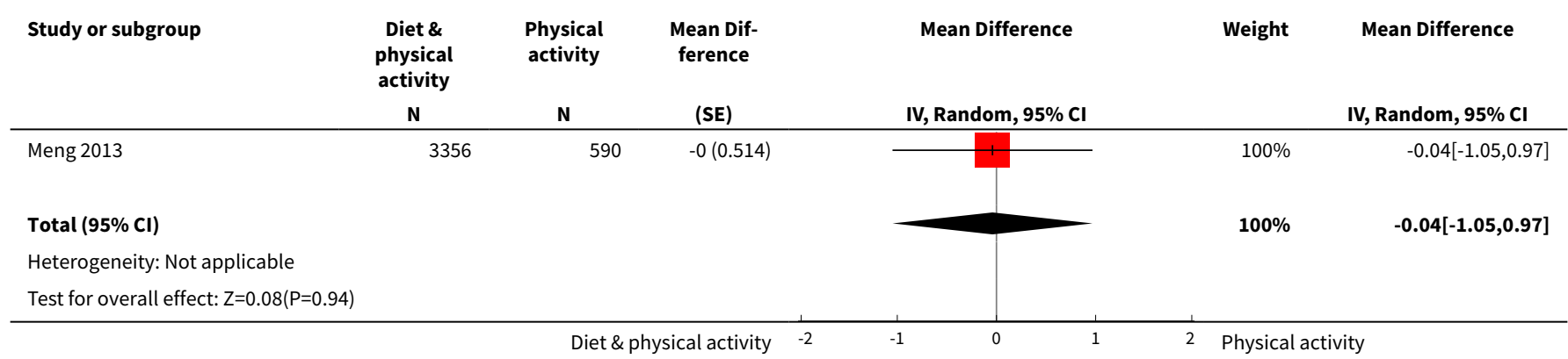

Comparison 12. Dietary interventions versus diet and physical activity interventions: age 6-12 years

\begin{tabular}{lllll}
\hline $\begin{array}{l}\text { Outcome or sub- } \\
\text { group title }\end{array}$ & No. of studies & $\begin{array}{l}\text { No. of partici- } \\
\text { pants }\end{array}$ & Statistical method & Effect size \\
\hline $1 \mathrm{zBMl}$ & 1 & 3971 & Mean Difference (Random, 95\% Cl) & $0.05[-0.38,0.48]$ \\
\hline $2 \mathrm{BMI}$ & 1 & 3971 & Mean Difference (Random, 95\% Cl) & $-0.28[-1.67,1.11]$ \\
\hline
\end{tabular}

Analysis 12.1. Comparison 12 Dietary interventions versus diet and physical activity interventions: age 6-12 years, Outcome 1 zBMI.

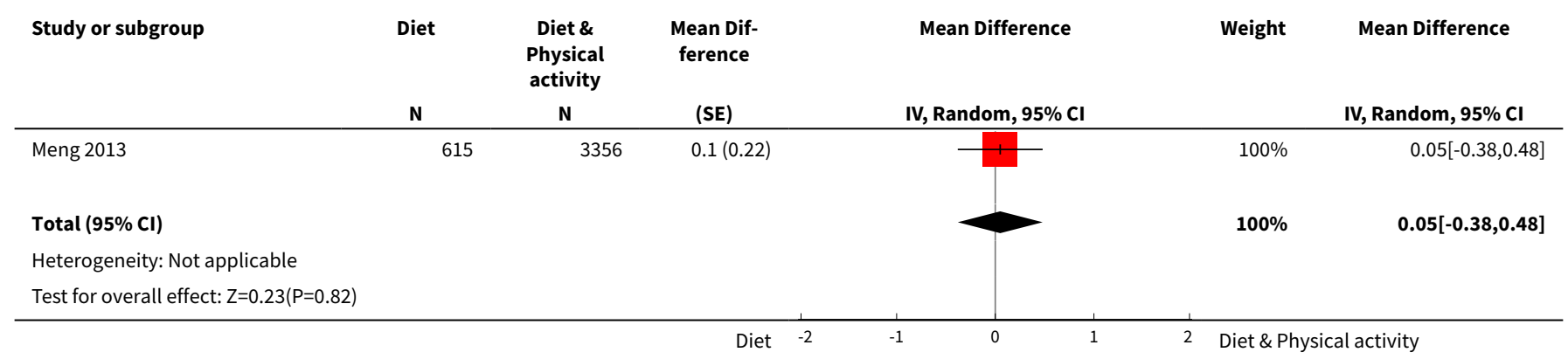


Analysis 12.2. Comparison 12 Dietary interventions versus diet and physical activity interventions: age 6-12 years, Outcome 2 BMI.

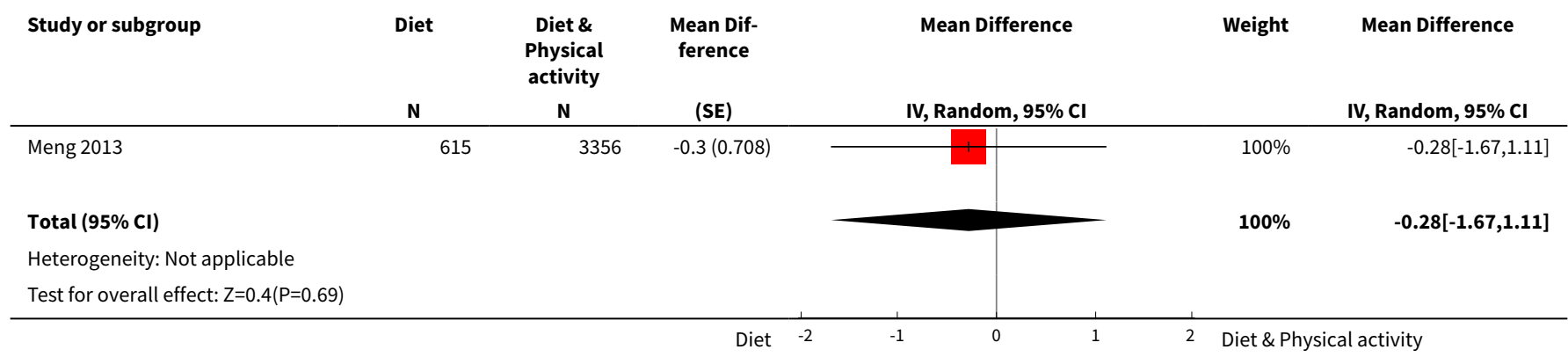




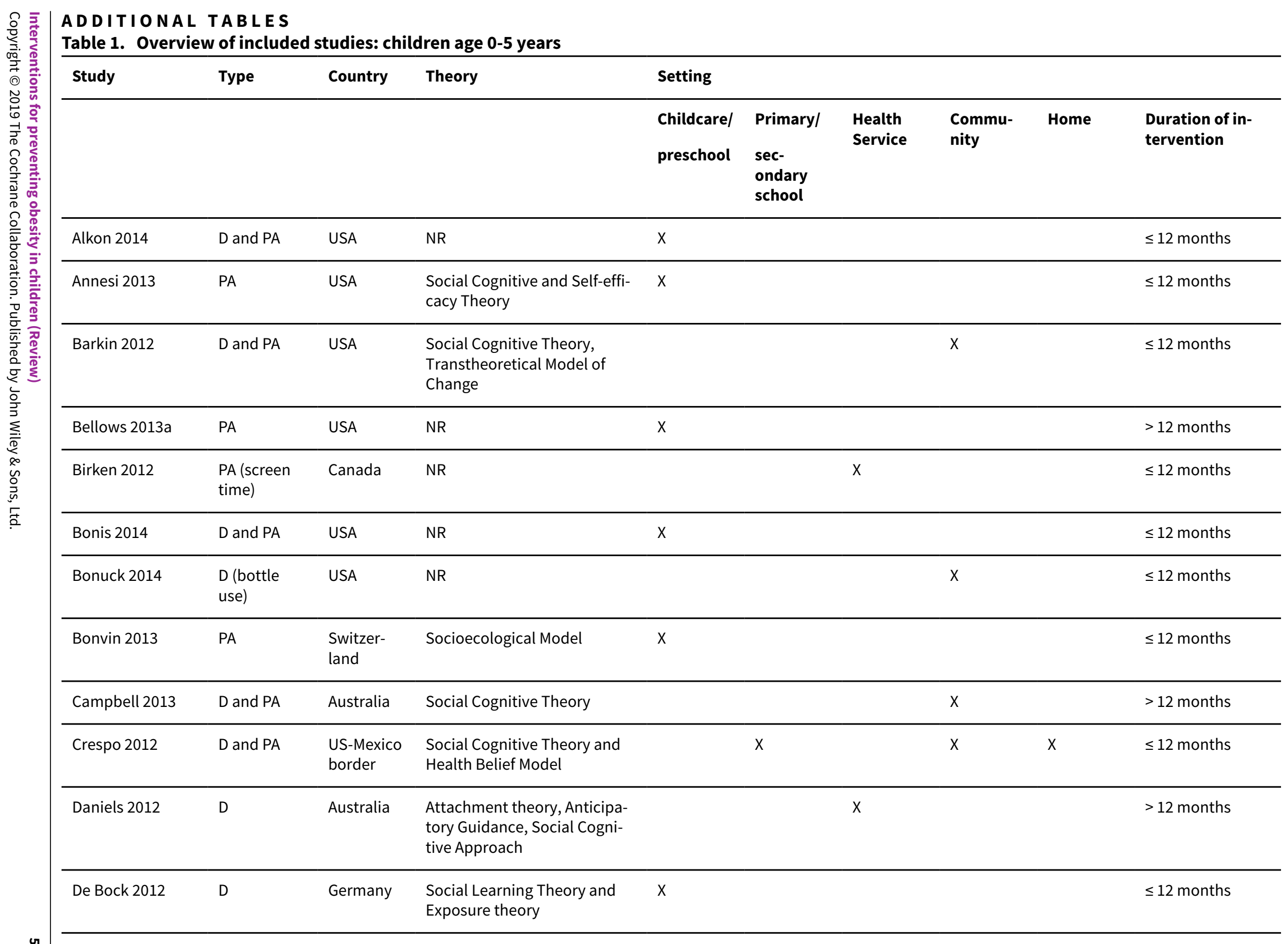




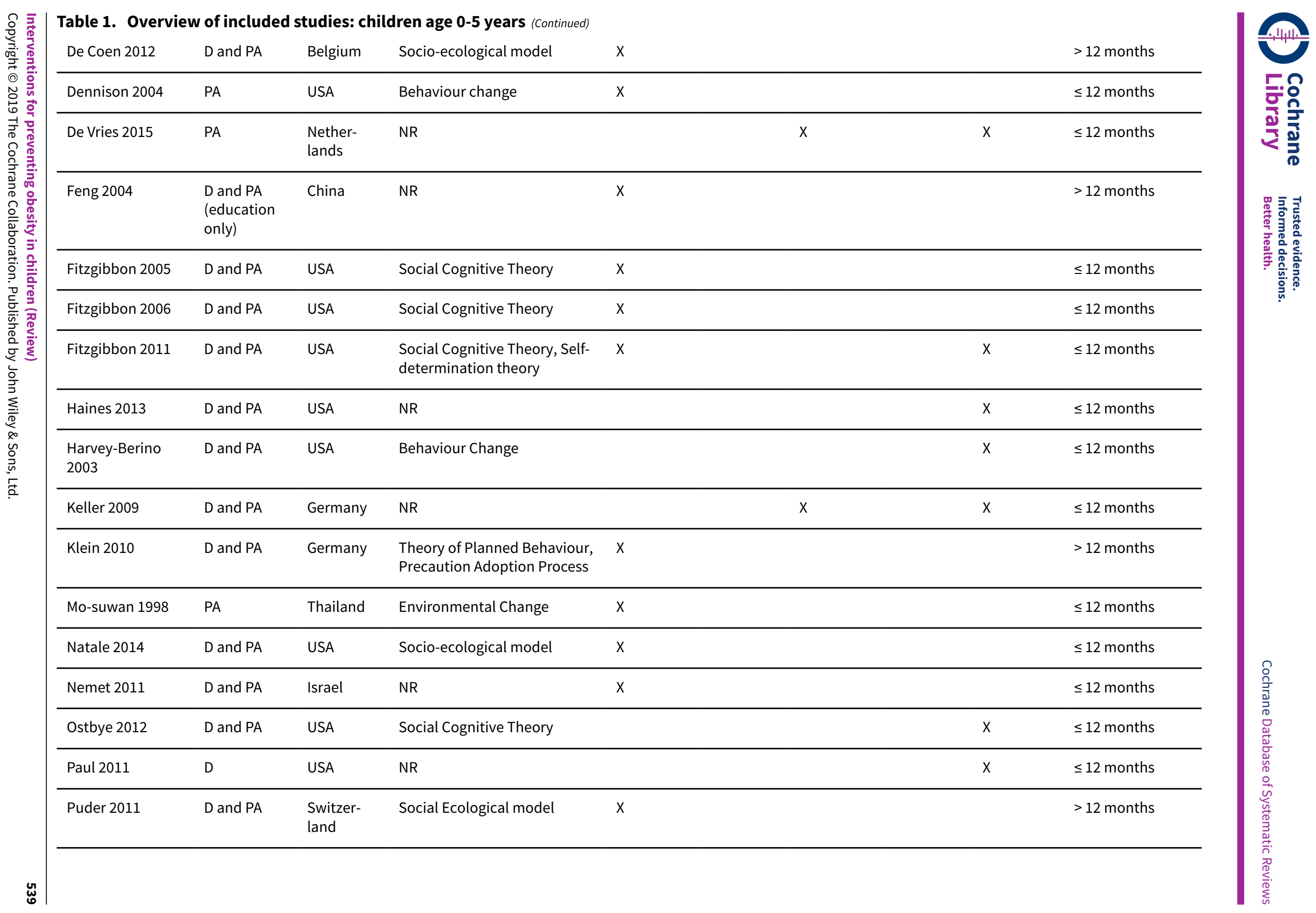




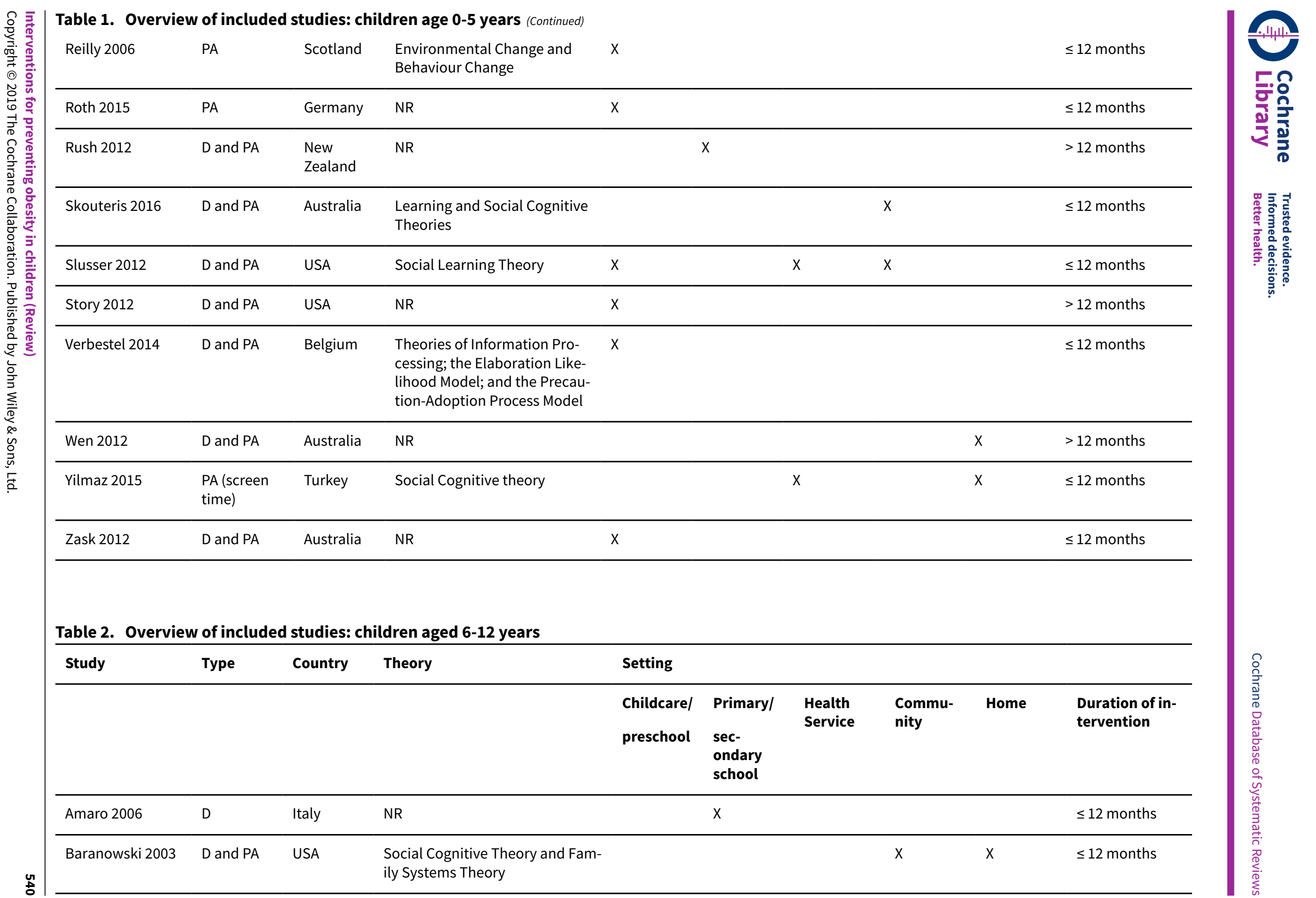




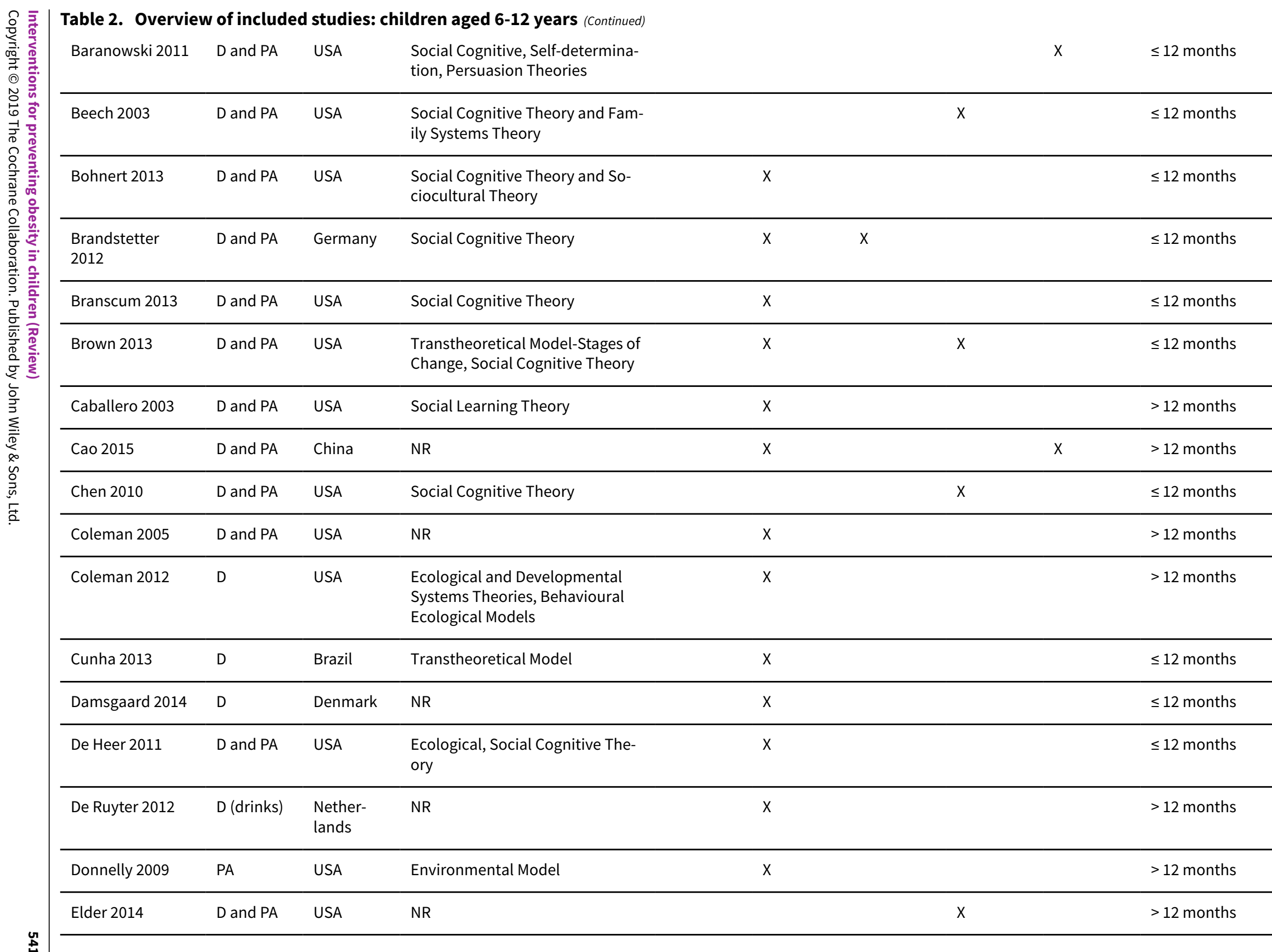




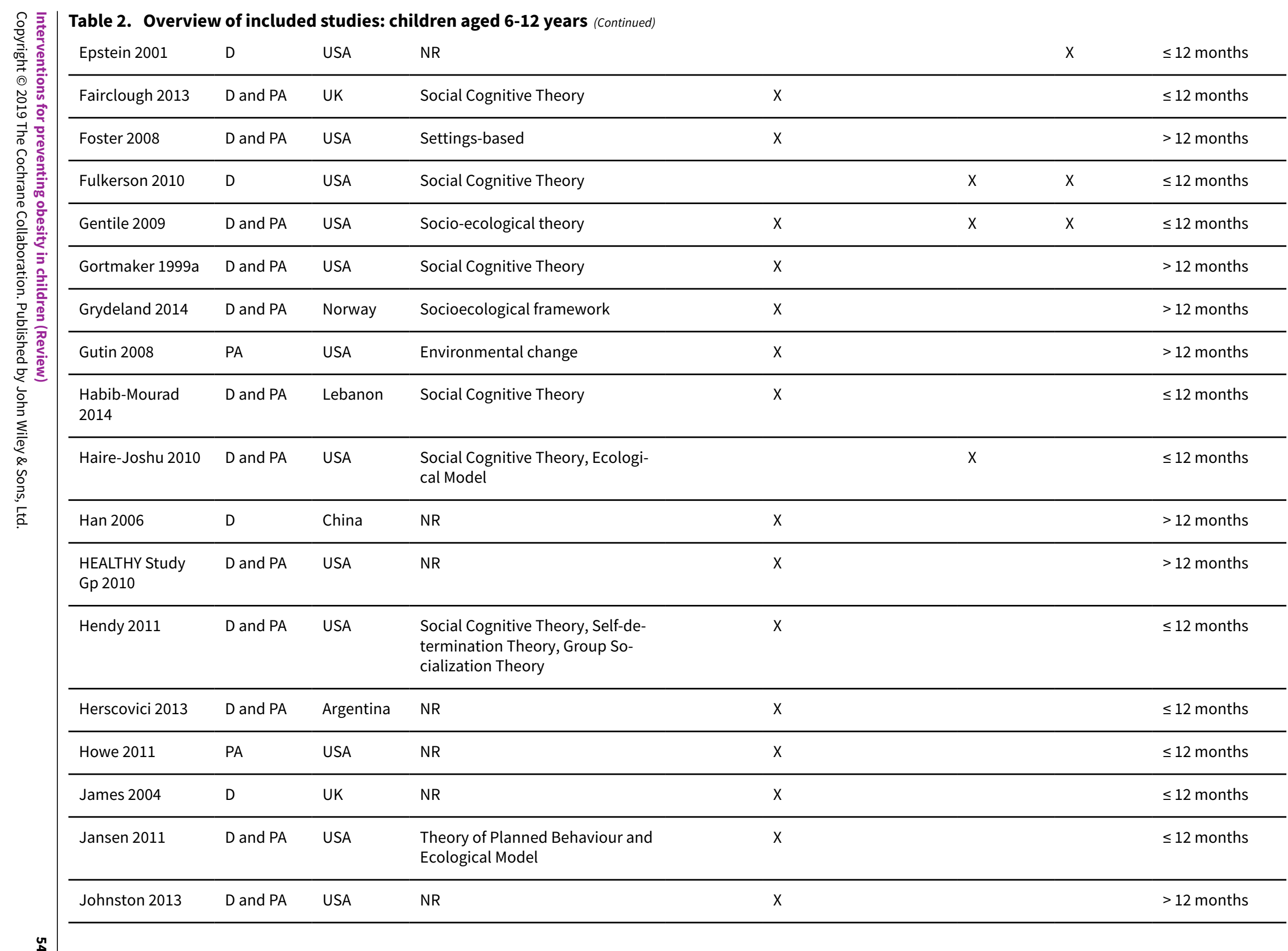




\begin{tabular}{|c|c|c|c|c|c|c|}
\hline Kain 2014 & $\mathrm{D}$ and $\mathrm{PA}$ & Chile & NR & $x$ & & $\leq 12$ months \\
\hline Khan 2014 & PA & USA & NR & & $x$ & $\leq 12$ months \\
\hline Kipping 2008 & $D$ and $P A$ & UK & $\begin{array}{l}\text { Social Cognitive Theory and Be- } \\
\text { havioural C }\end{array}$ & $x$ & & $\leq 12$ months \\
\hline Kipping 2014 & $D$ and $P A$ & UK & Social Cognitive Theory & $x$ & & $\leq 12$ months \\
\hline Klesges 2010 & $D$ and $P A$ & USA & NR & & $x$ & $>12$ months \\
\hline Kriemler 2010 & PA & $\begin{array}{l}\text { Switzer- } \\
\text { land }\end{array}$ & Socio-ecological Model & $x$ & & $\leq 12$ months \\
\hline Lazaar 2007 & PA & France & NR & $x$ & & $\leq 12$ months \\
\hline Levy 2012 & $\mathrm{D}$ and $\mathrm{PA}$ & Mexico & NR & $x$ & & $\leq 12$ months \\
\hline Li 2010a & PA & China & NR & $x$ & $x$ & $\leq 12$ months \\
\hline Llargues 2012 & $D$ and $P A$ & Spain & $\begin{array}{l}\text { Investigation, Vision, Action and } \\
\text { Change Methodology }\end{array}$ & $x$ & & $>12$ months \\
\hline $\begin{array}{l}\text { Macias-Cervantes } \\
2009\end{array}$ & PA & Mexico & NR & & $x$ & $\leq 12$ months \\
\hline Madsen 2013 & PA & USA & NR & $x$ & & $\leq 12$ months \\
\hline Magnusson 2012 & $D$ and $P A$ & Iceland & NR & $x$ & & $>12$ months \\
\hline Marcus 2009 & $\mathrm{D}$ and $\mathrm{PA}$ & Sweden & NR & $x$ & & $>12$ months \\
\hline $\begin{array}{l}\text { Martinez-Vizcaino } \\
2014\end{array}$ & PA & Spain & Socio-ecological model & $x$ & & $\leq 12$ months \\
\hline Meng 2013 & $\begin{array}{l}\text { D, D and } \\
\text { PA, PA }\end{array}$ & China & NR & $x$ & & $\leq 12$ months \\
\hline Morgan 2011 & $\mathrm{D}$ and $\mathrm{PA}$ & Australia & Social Cognitive Theory & & $x$ & $\leq 12$ months \\
\hline $\begin{array}{l}\text { Muckelbauer } \\
2010\end{array}$ & D (water) & Germany & Theory of Planned Behaviour & $x$ & & $\leq 12$ months \\
\hline
\end{tabular}




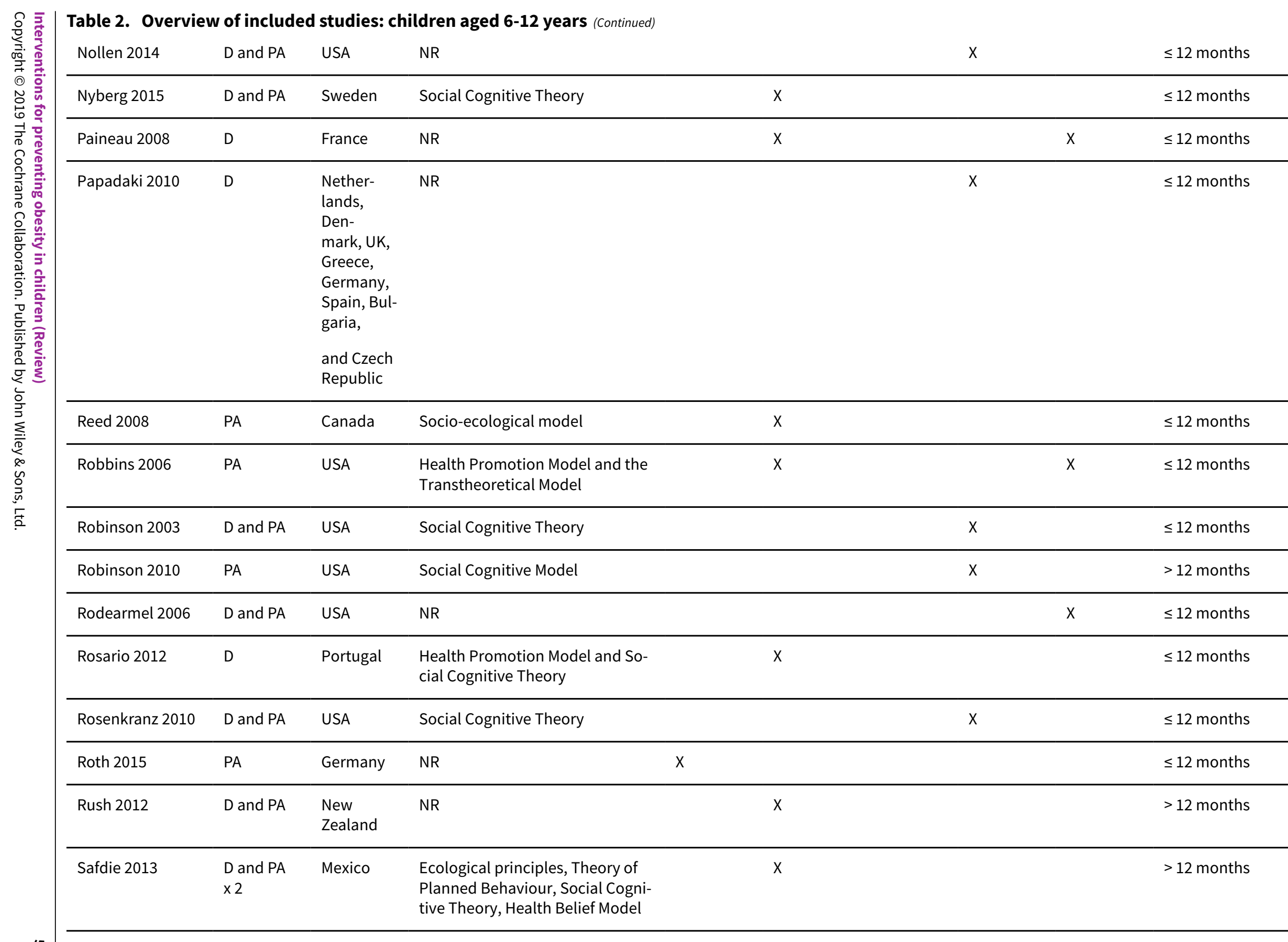




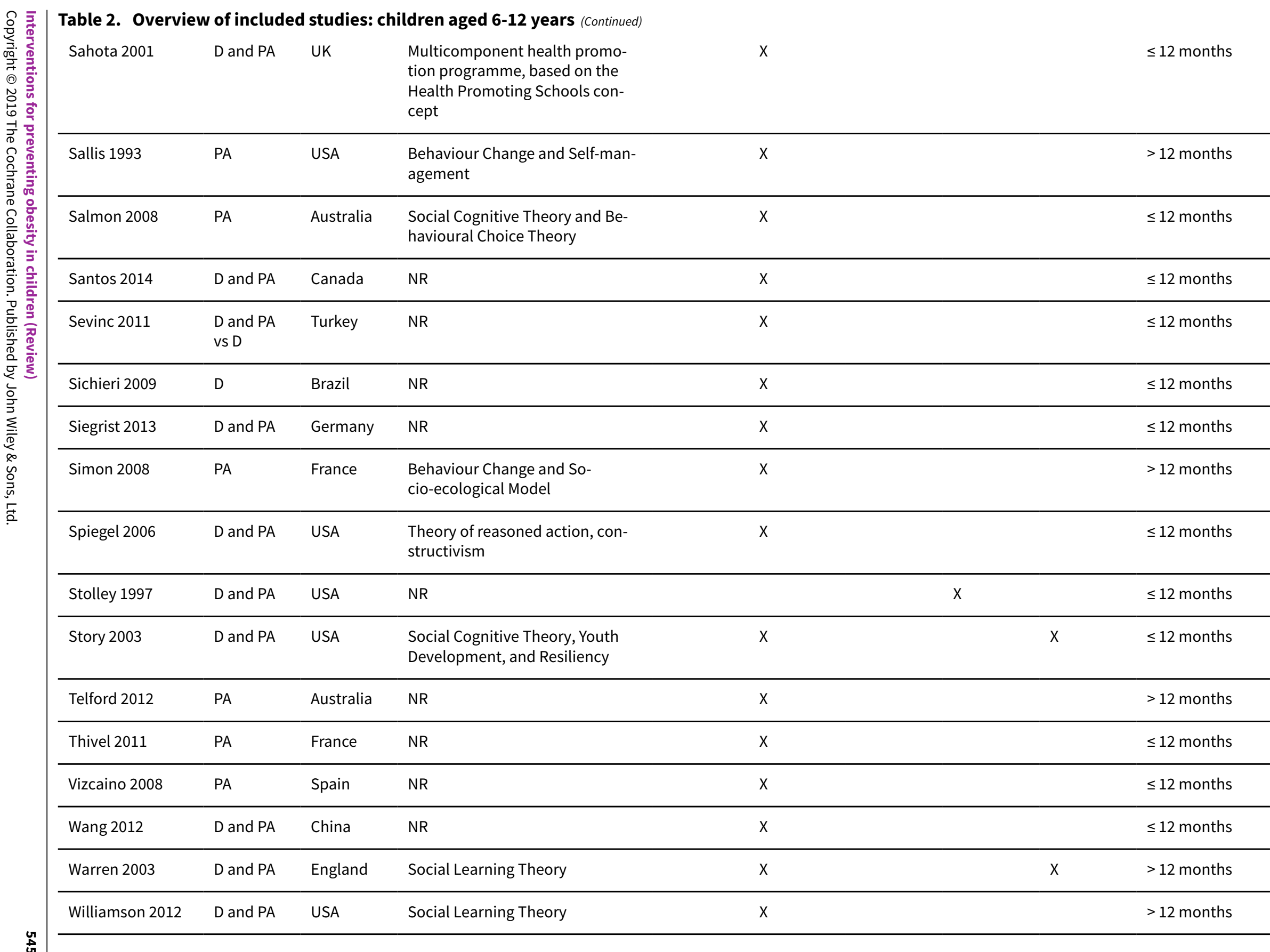


Table 3. Overview of included studies: children age 13-18 years

\begin{tabular}{|c|c|c|c|c|c|c|c|c|c|}
\hline \multirow[t]{2}{*}{ Study } & \multirow[t]{2}{*}{ Type } & \multirow[t]{2}{*}{ Country } & \multirow[t]{2}{*}{ Theory } & \multicolumn{5}{|l|}{ Setting } & \multirow{2}{*}{$\begin{array}{l}\text { Duration of in- } \\
\text { tervention }\end{array}$} \\
\hline & & & & $\begin{array}{l}\text { Childcare/ } \\
\text { preschool }\end{array}$ & $\begin{array}{l}\text { Prima- } \\
\text { ry/ sec- } \\
\text { ondary } \\
\text { school }\end{array}$ & $\begin{array}{l}\text { Health } \\
\text { Service }\end{array}$ & $\begin{array}{l}\text { Commu- } \\
\text { nity }\end{array}$ & Home & \\
\hline Andrade 2014 & $\mathrm{D}$ and $\mathrm{PA}$ & Ecuador & $\begin{array}{l}\text { Social Cognitive Theory, Informa- } \\
\text { tion-Motivation Behavioral Skills } \\
\text { Model, Control Theory, Trans- the- } \\
\text { oretical Model, Theory of Planned } \\
\text { Behavior }\end{array}$ & & $\mathrm{x}$ & & & & $>12$ months \\
\hline Black 2010 & $\mathrm{D}$ and $\mathrm{PA}$ & USA & $\begin{array}{l}\text { Social Cognitive Theory and Moti- } \\
\text { vational Interviewing }\end{array}$ & & & & $x$ & $x$ & $\leq 12$ months \\
\hline $\begin{array}{l}\text { Bonsergent } \\
2013\end{array}$ & $\mathrm{D}$ and $\mathrm{PA}$ & France & NR & & $x$ & $x$ & $x$ & & $>12$ months \\
\hline $\begin{array}{l}\text { Christiansen } \\
2013\end{array}$ & PA & Denmark & Social Ecological framework & & $x$ & & & & $>12$ months \\
\hline Dewar 2013 & $\mathrm{D}$ and $\mathrm{PA}$ & Australia & Social Cognitive Theory & & $x$ & & & & $\leq 12$ months \\
\hline Ebbeling 2006 & $\mathrm{D}$ & USA & NR & & & & & $x$ & $\leq 12$ months \\
\hline El Ansarai 2010 & PA & Egypt & NR & & $x$ & & & & $\leq 12$ months \\
\hline Ezendam 2012 & $\mathrm{D}$ and $\mathrm{PA}$ & $\begin{array}{l}\text { Nether- } \\
\text { lands }\end{array}$ & $\begin{array}{l}\text { Theory of Planned Behavior, Pre- } \\
\text { caution Adoption Process Model, } \\
\text { Implementation Intentions }\end{array}$ & & $\mathrm{x}$ & & & & $\leq 12$ months \\
\hline Farias 2015 & PA & Brazil & NR & & $x$ & & & & $\leq 12$ months \\
\hline French 2011 & $\mathrm{D}$ and $\mathrm{PA}$ & USA & NR & & & & $x$ & $x$ & $\leq 12$ months \\
\hline Haerens 2006 & $D$ and $P A$ & Belgium & $\begin{array}{l}\text { Theory of Planned Behaviours and } \\
\text { Transtheoretical Model }\end{array}$ & & $\mathrm{x}$ & & & & $>12$ months \\
\hline
\end{tabular}




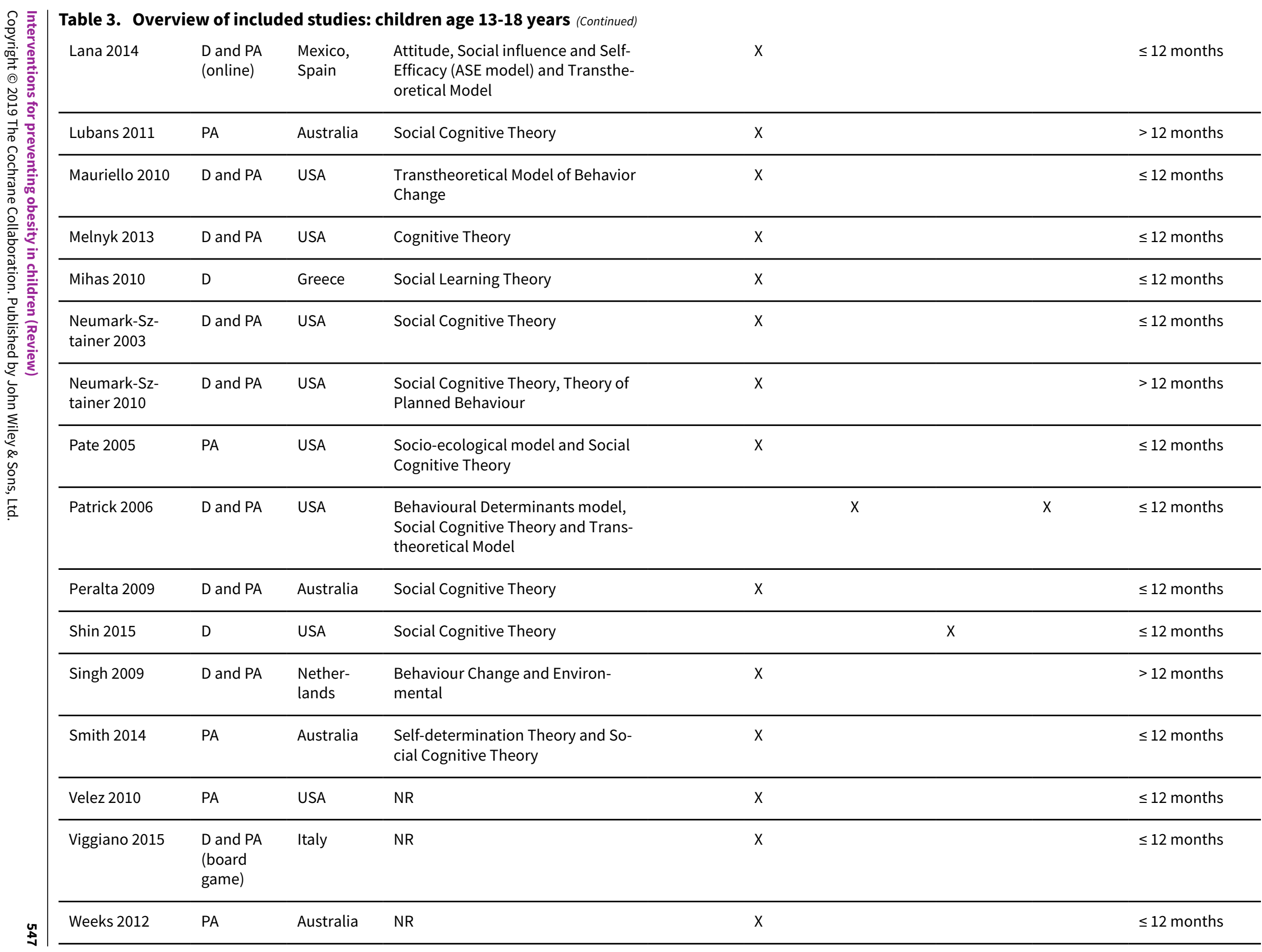




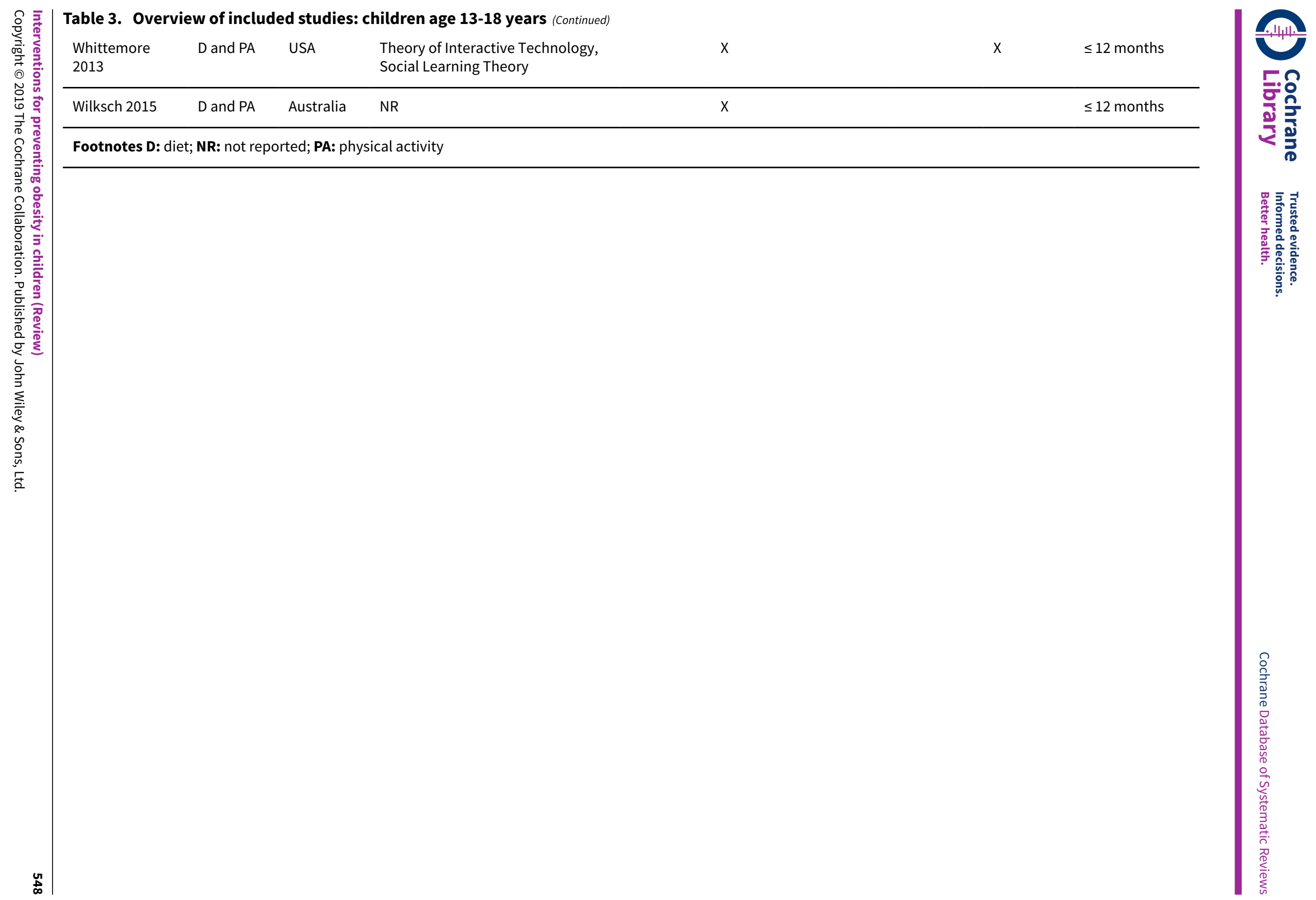


Table 4. Type of comparisons

\begin{tabular}{|c|c|c|}
\hline Study & Type & Control \\
\hline Alkon 2014 & $\mathrm{D}$ and $\mathrm{PA}$ & Waitlist \\
\hline Amaro 2006 & $\mathrm{D}$ & No intervention \\
\hline Andrade 2014 & $\mathrm{D}$ and $\mathrm{PA}$ & Usual care \\
\hline Annesi 2013 & PA & Usual care \\
\hline Baranowski 2003 & $\mathrm{D}$ and $\mathrm{PA}$ & Day camp \\
\hline Baranowski 2011 & $D$ and $P A$ & Health-related video games \\
\hline Barkin 2012 & $\mathrm{D}$ and $\mathrm{PA}$ & School-readiness programme \\
\hline \multirow[t]{2}{*}{ Beech 2003} & 1. D and PA child-targeted & Self-esteem \\
\hline & 2. D and PA parent-targeted & \\
\hline Bellows 2013a & PA (plus diet) & Diet intervention only \\
\hline Birken 2012 & PA (screen time) & Safe media use \\
\hline Black 2010 & $\mathrm{D}$ and $\mathrm{PA}$ & No intervention \\
\hline Bohnert 2013 & $\mathrm{D}$ and $\mathrm{PA}$ & No intervention \\
\hline Bonis 2014 & $\mathrm{D}$ and $\mathrm{PA}$ & Waitlist \\
\hline \multirow[t]{7}{*}{ Bonsergent 2013} & $\begin{array}{l}\text { 1. D and PA education + environ- } \\
\text { ment + screening strategies }\end{array}$ & No intervention \\
\hline & $\begin{array}{l}\text { 2. D and PA education + environ- } \\
\text { ment strategies }\end{array}$ & \\
\hline & $\begin{array}{l}\text { 3. D and PA education + screen- } \\
\text { ing strategies }\end{array}$ & \\
\hline & 4. D and PA education strategy & \\
\hline & $\begin{array}{l}\text { 5. D and PA environment }+ \\
\text { screening strategies }\end{array}$ & \\
\hline & $\begin{array}{l}\text { 6. D and PA environment strate- } \\
\text { gy }\end{array}$ & \\
\hline & 7. D and PA screening strategy & \\
\hline Bonuck 2014 & D (bottle use) & No intervention \\
\hline Bonvin 2013 & PA & Waitlist \\
\hline Brandstetter 2012 & $\mathrm{D}$ and $\mathrm{PA}$ & $\begin{array}{l}\text { Usual care presumed as intervention integrated into school curricu- } \\
\text { lum }\end{array}$ \\
\hline Branscum 2013 & D and PA (theory-based) & Knowledge-based D and PA \\
\hline Brown 2013 & $\mathrm{D}$ and $\mathrm{PA}$ & Alcohol and drug comparison \\
\hline Caballero 2003 & $\mathrm{D}$ and $\mathrm{PA}$ & Usual care presumed as no details but school-based intervention \\
\hline
\end{tabular}


Table 4. Type of comparisons (Continued)

\begin{tabular}{|c|c|c|}
\hline Campbell 2013 & $\mathrm{D}$ and $\mathrm{PA}$ & Newsletters on non-obesity-focused themes \\
\hline Cao 2015 & $\mathrm{D}$ and $\mathrm{PA}$ & No intervention \\
\hline Chen 2010 & $\mathrm{D}$ and $\mathrm{PA}$ & Waitlist \\
\hline Chen 2011 & $\mathrm{D}$ and $\mathrm{PA}$ & $\begin{array}{l}\text { General health information related to nutrition, dental care, safety, } \\
\text { skin care, and risk-taking behaviours }\end{array}$ \\
\hline Christiansen 2013 & PA & Usual care \\
\hline Coleman 2005 & $\mathrm{D}$ and $\mathrm{PA}$ & No intervention (financial incentive to participate) \\
\hline Coleman 2012 & $\mathrm{D}$ & Usual care presumed as no details but school-based intervention \\
\hline \multirow[t]{3}{*}{ Crespo 2012} & 1. D + PA family-only & No intervention \\
\hline & 2. D + PA community-only & \\
\hline & 3. D + PA family + community & \\
\hline Cunha 2013 & $\mathrm{D}$ & No intervention \\
\hline Damsgaard 2014 & $\mathrm{D}$ & Packed lunch from home \\
\hline Daniels 2012 & $\mathrm{D}$ & Usual care \\
\hline De Bock 2012 & $\mathrm{D}$ & Waitlist \\
\hline De Coen 2012 & $\mathrm{D}$ and $\mathrm{PA}$ & $\begin{array}{l}\text { Usual care presumed as no details but primarily school-based inter- } \\
\text { vention }\end{array}$ \\
\hline De Heer 2011 & $\mathrm{D}$ and $\mathrm{PA}$ & Health workbooks and incentives \\
\hline De Ruyter 2012 & $\mathrm{D}$ (drink) & $\begin{array}{l}\text { Similar sugar-containing drink in participants who commonly drank } \\
\text { them }\end{array}$ \\
\hline De Vries 2015 & PA & Standard care without PA recommendations \\
\hline Dennison 2004 & PA & Health and safety programme \\
\hline Dewar 2013 & $\mathrm{D}$ and $\mathrm{PA}$ & Usual care? presumed as no details but school-based intervention \\
\hline Donnelly 2009 & PA & $\begin{array}{l}\text { Usual care - regular classroom instruction without physically active } \\
\text { lessons }\end{array}$ \\
\hline Ebbeling 2006 & $\mathrm{D}$ (drink) & Usual drink consumption \\
\hline El Ansarai 2010 & $\begin{array}{l}\text { PA (plus 'normal' exercise } \\
\text { schedule provided by the } \\
\text { school) }\end{array}$ & Usual care 'normal' exercise schedule provided by the school \\
\hline Elder 2014 & $D$ and $P A$ & No intervention - measurement only \\
\hline Epstein 2001 & D (fruit + veg) & D (fat + sugar) \\
\hline Ezendam 2012 & $\mathrm{D}$ and $\mathrm{PA}$ & No intervention \\
\hline
\end{tabular}


Table 4. Type of comparisons (Continued)

\begin{tabular}{lll} 
Fairclough 2013 & D and PA & Did not teach a specific unit focused on healthy eating and PA \\
\hline Farias 2015 & PA & Usual care physical activity at school \\
\hline Feng 2004 & D and PA (education only) & No intervention - translated \\
\hline Fitzgibbon 2005 & D and PA & General health intervention \\
\hline Fitzgibbon 2006 & D and PA & General health intervention \\
\hline Fitzgibbon 2011 & D and PA & General health intervention \\
\hline Foster 2008 & D and PA & No intervention \\
\hline French 2011 & D and PA & No intervention \\
\hline Fulkerson 2010 & D & No intervention \\
\hline
\end{tabular}

\begin{tabular}{ll}
\hline Dentile 2009 & D and PA (plus community com- Community component only \\
ponent)
\end{tabular}

\begin{tabular}{lll}
\hline Gortmaker 1999a & D and PA & Usual care health curricula and PE classes \\
\hline Grydeland 2014 & D and PA & Usual care presumed as no details but school-based intervention \\
\hline Gutin 2008 & PA & No intervention presumed as no details (after-school intervention) \\
\hline Habib-Mourad 2014 & D and PA & Usual curriculum \\
\hline Haerens 2006 & 1. D+PA parent & Usual care presumed as no details but school-based intervention \\
& 2. D+PA child alone & \\
\hline
\end{tabular}

\begin{tabular}{|c|c|c|}
\hline Haines 2013 & $\mathrm{D}$ and $\mathrm{PA}$ & Mailed materials focused on child development \\
\hline Haire-Joshu 2010 & $\mathrm{D}$ and $\mathrm{PA}$ & Usual care \\
\hline Han 2006 & $\mathrm{D}$ & $\begin{array}{l}\text { Usual care presumed as no details but school-based intervention - } \\
\text { translated }\end{array}$ \\
\hline Harvey-Berino 2003 & $\begin{array}{l}D \text { and PA (plus parenting sup- } \\
\text { port) }\end{array}$ & $\begin{array}{l}\text { Parenting support but refrained from discussing child or parent eating } \\
\text { and exercise behaviour }\end{array}$ \\
\hline HEALTHY Study Gp 2010 & $\mathrm{D}$ and $\mathrm{PA}$ & No intervention - assessment only \\
\hline Hendy 2011 & D and PA (token rewards) & Token rewards for three "Good Citizenship Behaviors." \\
\hline Herscovici 2013 & $\mathrm{D}$ and $\mathrm{PA}$ & Usual care presumed as no details but school-based intervention \\
\hline Howe 2011 & PA & $\begin{array}{l}\text { No intervention and were not allowed to stay for the after-school in- } \\
\text { tervention but rather instructed not to change their daily after-school } \\
\text { routine }\end{array}$ \\
\hline James 2004 & $\mathrm{D}$ (drinks) & Usual care presumed as no details but school-based intervention \\
\hline Jansen 2011 & $D$ and $P A$ & Usual care curriculum \\
\hline
\end{tabular}


Table 4. Type of comparisons (Continued)

\begin{tabular}{lll} 
Johnston 2013 & D and PA & Self-help \\
\hline Kain 2014 & D and PA & Usual care presumed as no details but school-based intervention \\
\hline Keller 2009 & D and PA & No intervention - study translated in previous version of review \\
\hline Khan 2014 & PA & $\begin{array}{l}\text { Maintain regular after-school routine, financial incentive for measure- } \\
\text { ments }\end{array}$ \\
\hline Kipping 2008 & D and PA & Waitlist \\
\hline Kipping 2014 & D and PA & Standard teaching \\
\hline Klein 2010 & D and PA & No intervention \\
\hline Klesges 2010 & D and PA & Self-esteem and social efficacy \\
\hline Kriemler 2010 & PA & Not informed of an intervention group \\
\hline Lana 2014 & 1. D and PA online only & No intervention presumed as no details \\
\hline
\end{tabular}

\begin{tabular}{|c|c|c|}
\hline Lazaar 2007 & PA & Usual care presumed as no details but school-based intervention \\
\hline Levy 2012 & $\mathrm{D}$ and $\mathrm{PA}$ & Usual care presumed as no details but school-based intervention \\
\hline Li 2010a & PA & No intervention \\
\hline Llargues 2012 & $\mathrm{D}$ and $\mathrm{PA}$ & Usual care presumed as no details but school-based intervention \\
\hline Lubans 2011 & PA & Waitlist \\
\hline Macias-Cervantes 2009 & PA & Maintain the same level of physical activity \\
\hline Madsen 2013 & PA & No intervention presumed as no details \\
\hline Magnusson 2012 & $\begin{array}{l}\text { D and PA (plus } 2 \text { x 40-min PA + } \\
\text { incentives) }\end{array}$ & $2 \times 40-$ min PA + incentives \\
\hline Marcus 2009 & $\mathrm{D}$ and $\mathrm{PA}$ & Normal curriculum \\
\hline Martinez-Vizcaino 2014 & $\begin{array}{l}\text { PA (plus } 2 \mathrm{~h} / \text { week of physical ac- } \\
\text { tivity at low to moderate inten- } \\
\text { sity) }\end{array}$ & $\begin{array}{l}\text { Standard physical education curriculum ( } 2 \mathrm{~h} / \text { week of physical activity } \\
\text { at low to moderate intensity) }\end{array}$ \\
\hline Mauriello 2010 & D and PA (multimedia) & No intervention \\
\hline Melnyk 2013 & $\mathrm{D}$ and $\mathrm{PA}$ & Attention control programme - common health topics \\
\hline \multirow[t]{3}{*}{ Meng 2013} & 1. D & No intervention \\
\hline & 2. $P A$ & \\
\hline & 3. $D$ and $P A$ & \\
\hline
\end{tabular}


Table 4. Type of comparisons (Continued)

\begin{tabular}{|c|c|c|}
\hline Mo-suwan 1998 & PA & Usual care presumed as no details but school-based intervention \\
\hline Morgan 2011 & $\mathrm{D}$ and $\mathrm{PA}$ & Waitlist \\
\hline Muckelbauer 2010 & $\mathrm{D}$ (water) & No intervention \\
\hline Natale 2014 & $\mathrm{D}$ and $\mathrm{PA}$ & Attention control - safety education curriculum \\
\hline Nemet 2011 & $\mathrm{D}$ and $\mathrm{PA}$ & Regular kindergarten schedule \\
\hline Neumark-Sztainer 2003 & $D$ and $P A$ & $\begin{array}{l}\text { Regular physical education class and minimal intervention (written } \\
\text { materials on healthy eating and physical activity at baseline) }\end{array}$ \\
\hline Neumark-Sztainer 2010 & $\begin{array}{l}\text { D and PA (plus all-girls PE class } \\
\text { during the first semester) }\end{array}$ & All-girls PE class during the first semester then usual PE \\
\hline Nollen 2014 & $\begin{array}{l}\text { D and PA (screen time only, via } \\
\text { mobile technology) }\end{array}$ & Same content in a written manual but no prompting \\
\hline Nyberg 2015 & $\mathrm{D}$ and $\mathrm{PA}$ & Waitlist \\
\hline Ostbye 2012 & $\begin{array}{l}\text { D and PA (plus financial incen- } \\
\text { tives) }\end{array}$ & $\begin{array}{l}\text { Monthly newsletters emphasising pre-reading skills plus financial in- } \\
\text { centives }\end{array}$ \\
\hline \multirow[t]{2}{*}{ Paineau 2008} & $\begin{array}{l}\text { 1. reduce fat }+ \text { increase complex } \\
\text { carbohydrate }\end{array}$ & No advice \\
\hline & $\begin{array}{l}\text { 2. reduce both fat+sugar+in- } \\
\text { crease complex carbohydrate }\end{array}$ & \\
\hline \multirow[t]{4}{*}{ Papadaki 2010} & $\begin{array}{l}\text { 1. low protein /low glycaemic } \\
\text { index }\end{array}$ & $\begin{array}{l}\text { National dietary guidelines, with medium protein content and no spe- } \\
\text { cific instructions on glycaemic index }\end{array}$ \\
\hline & $\begin{array}{l}\text { 2. low protein/high glycaemic } \\
\text { index }\end{array}$ & \\
\hline & $\begin{array}{l}\text { 3. high protein/low glycaemic } \\
\text { index }\end{array}$ & \\
\hline & $\begin{array}{l}\text { 4. high protein/high glycaemic } \\
\text { index }\end{array}$ & \\
\hline Pate 2005 & PA (plus enrolled in PE) & Enrolled in PE classes \\
\hline Patrick 2006 & $\begin{array}{l}\text { D and PA (plus lottery tickets for } \\
\text { small cash prizes) }\end{array}$ & Sun protection plus lottery tickets for small cash prizes \\
\hline \multirow[t]{3}{*}{ Paul 2011} & 1. soothe/sleep & No intervention \\
\hline & 2. introduction to solids & \\
\hline & 3. combination & \\
\hline Peralta 2009 & PA & Physical activity curriculum sessions \\
\hline Puder 2011 & $\mathrm{D}$ and $\mathrm{PA}$ & Regular school curriculum \\
\hline Reed 2008 & PA & Usual care \\
\hline
\end{tabular}


Table 4. Type of comparisons (Continued)

\begin{tabular}{|c|c|c|}
\hline Reilly 2006 & PA & Usual care curriculum \\
\hline Robbins 2006 & PA & Handout listing the PA recommendations \\
\hline Robinson 2003 & $\mathrm{D}$ and $\mathrm{PA}$ & $\begin{array}{l}\text { Active comparison - health education programme to promote health- } \\
\text { ful diet and activity patterns via newsletters and delivering health ed- } \\
\text { ucation lectures }\end{array}$ \\
\hline Robinson 2010 & PA & Information-based health education \\
\hline Rodearmel 2006 & $\mathrm{D}$ and $\mathrm{PA}$ & $\begin{array}{l}\text { Maintain usual eating and step patterns (given step counter and logs } \\
\text { same as intervention group) }\end{array}$ \\
\hline Rosario 2012 & $\mathrm{D}$ & Usual care presumed as no details but school-based intervention \\
\hline Rosenkranz 2010 & $\mathrm{D}$ and $\mathrm{PA}$ & No intervention presumed (Girl Scouts USA) \\
\hline Roth 2015 & PA & Usual care presumed, pre-school setting \\
\hline Rush 2012 & $D$ and $P A$ & No additional resourcing or information \\
\hline Safdie 2013 & $\begin{array}{l}\text { 1. Basic D and PA } \\
\text { 2. Basic D and PA plus financial } \\
\text { investment and resources }\end{array}$ & $\begin{array}{l}\text { No changes were made to existing nutrition or physical activity prac- } \\
\text { tices }\end{array}$ \\
\hline Sahota 2001 & $\mathrm{D}$ and $\mathrm{PA}$ & Usual care presumed as no details but school-based intervention \\
\hline Sallis 1993 & PA & Usual care PE \\
\hline Salmon 2008 & $\begin{array}{l}\text { 1. Behaviour modification of PA } \\
\text { 2. Fundamental movement } \\
\text { skills } \\
\text { 3. Combination }\end{array}$ & Usual care curriculum \\
\hline
\end{tabular}

\begin{tabular}{lll}
\hline Santos 2014 & D and PA & Usual care regular curriculum \\
\hline Sevinc 2011 & 1. D & Usual care presumed as no details but school-based intervention \\
& 2.D and PA & \\
\hline
\end{tabular}

\begin{tabular}{lll}
\hline Shin 2015 & D & No intervention \\
\hline Sichieri 2009 & D (drinks) & $\begin{array}{l}2 \times \text { 1-h general sessions on health issues and printed general advices } \\
\text { regarding healthy diets }\end{array}$ \\
\hline Siegrist 2013 & D and PA & Usual care \\
\hline Simon 2008 & PA & Usual care school curriculum \\
\hline Singh 2009 & D and PA & Usual care regular curriculum \\
\hline Skouteris 2016 & D and PA & Waitlist \\
\hline Slusser 2012 & D and PA & Waitlist \\
\hline
\end{tabular}


Table 4. Type of comparisons (Continued)

\begin{tabular}{|c|c|c|}
\hline Smith 2014 & PA & $\begin{array}{l}\text { Waitlist and usual practice (i.e. regularly scheduled school sports and } \\
\text { PE) }\end{array}$ \\
\hline Spiegel 2006 & $\mathrm{D}$ and $\mathrm{PA}$ & Data collection only \\
\hline Stolley 1997 & $\mathrm{D}$ and $\mathrm{PA}$ & Attention placebo group \\
\hline Story 2003 & $\mathrm{D}$ and $\mathrm{PA}$ & $\begin{array}{l}\text { "active placebo," non-nutrition/PA condition, promoting self-esteem } \\
\text { and cultural enrichment }\end{array}$ \\
\hline Story 2012 & $\mathrm{D}$ and $\mathrm{PA}$ & Usual care presumed as no details but school-based intervention \\
\hline Telford 2012 & PA & Usual care, common practice PE \\
\hline Thivel 2011 & PA & Not aware of the intervention in other schools \\
\hline Velez 2010 & PA & No intervention \\
\hline Verbestel 2014 & $\mathrm{D}$ and $\mathrm{PA}$ & No intervention presumed as no details \\
\hline Viggiano 2015 & D and PA (board game) & No intervention \\
\hline Vizcaino 2008 & $\begin{array}{l}\text { PA (plus standard PE curriculum } \\
\text { ( } 3 \text { h/week of PA at low to mod- } \\
\text { erate intensity) }\end{array}$ & Standard PE curriculum ( $3 \mathrm{~h} /$ week of PA at low to moderate intensity) \\
\hline Wang 2012 & $\mathrm{D}$ and $\mathrm{PA}$ & $\begin{array}{l}\text { Usual care presumed as no details but school-based intervention - } \\
\text { translated }\end{array}$ \\
\hline \multirow[t]{3}{*}{ Warren 2003} & 1. D & Educational programme about food in a 'non-nutrition' sense \\
\hline & 2. PA & \\
\hline & 3. D and PA & \\
\hline
\end{tabular}

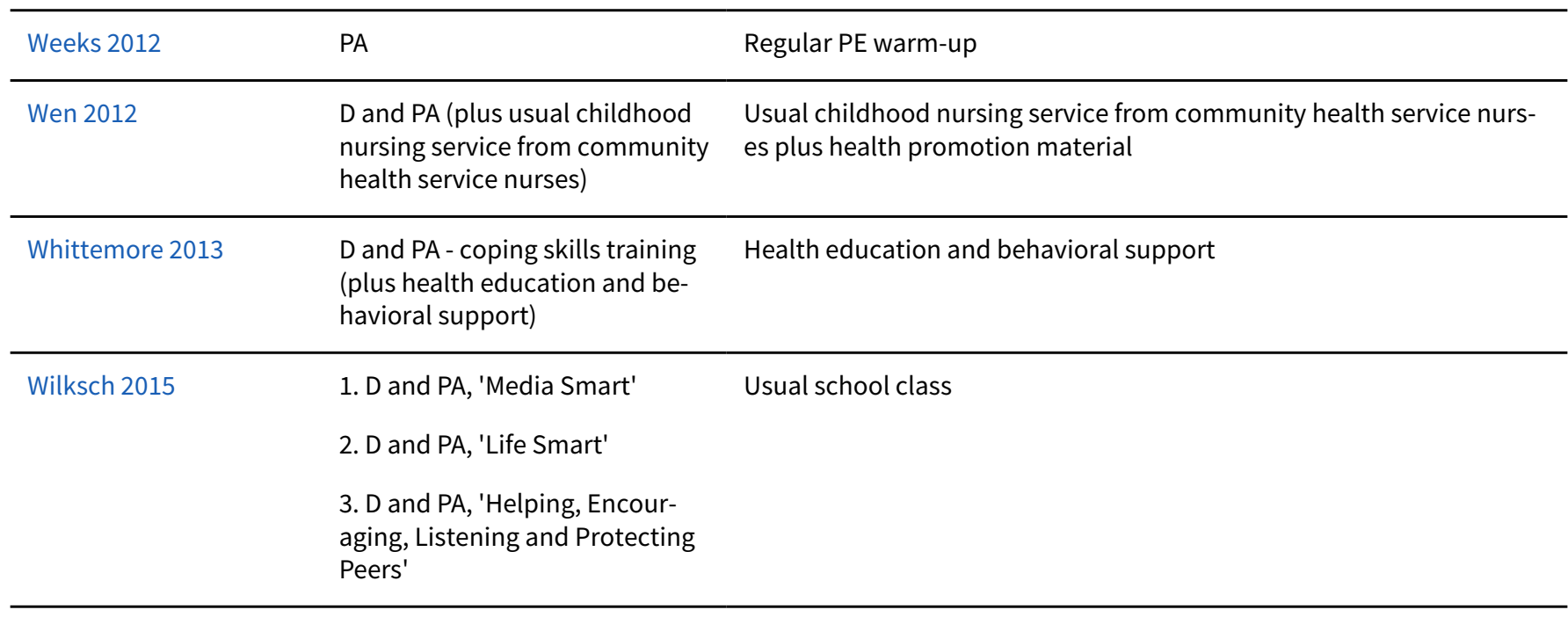

Williamson $2012 \quad$ 1. D and PA, primary prevention No intervention

+ environmental modification

2. D and PA, primary + sec-

ondary prevention with an 
Table 4. Type of comparisons (Continued)

added classroom and internet education component

\begin{tabular}{lll}
\hline Yilmaz 2015 & PA (screen time) & Not aware of the intervention \\
\hline Zask 2012 & D and PA & Waitlist \\
\hline
\end{tabular}

D: diet; PA: physical activity; PE: physical education 
Table 5. Source of funding in the studies

\begin{tabular}{|c|c|c|c|c|c|c|c|}
\hline \multirow[t]{2}{*}{ Age group } & \multicolumn{5}{|c|}{ Source of funding } & \multirow{2}{*}{$\begin{array}{l}\text { Was the writing of re- } \\
\text { ports and research inde- } \\
\text { pendent from industry }\end{array}$} & \multirow{2}{*}{$\begin{array}{l}\text { Source of funding was } \\
\text { from food/nutrition or } \\
\text { intervention industry }\end{array}$} \\
\hline & $\begin{array}{l}\text { Non-industrya: } \\
\text { number }(\%)\end{array}$ & $\begin{array}{l}\text { Not reported: } \\
\text { number (\%) }\end{array}$ & $\begin{array}{l}\text { Not funded: } \\
\text { number }(\%)\end{array}$ & $\begin{array}{l}\text { Industryb: } \\
\text { number (\%) }\end{array}$ & $\begin{array}{l}\text { Industry and non- } \\
\text { industry: number } \\
(\%)\end{array}$ & & \\
\hline $0-5$ & $28(71.8)$ & $6(15.4)$ & $0(0)$ & $0(0)$ & $5(12.8)$ & $2 / 5$ & $3 / 5^{c}$ \\
\hline $6-12$ & $69(81.2)$ & $7(8.2)$ & $0(0)$ & $1(2.4)$ & $7(8.2)$ & $3 / 8$ & $6 / 8^{d}$ \\
\hline $13-18$ & $26(89.7)$ & $1(3.4)$ & $2(6.9)$ & $0(0)$ & $2(6.9)$ & $1 / 2$ & $2 / 2^{e}$ \\
\hline
\end{tabular}

aFunding from government organisations, not-for-profit organisations, charities etc.

bAny source that was from commercial or profit-making organisations including trusts and foundation organisations originating from commercial sources.

cDaniels 2012 (Heinz), Paul 2011 (Gerber food - Nestlé), Puder 2011 (Wyeth foundation, Nestlé).

dDamsgaard 2014 (Danæg A/S, Naturmælk, Lantmännen A/S, Skærtoft Mølle A/S, Kartoffelpartnerskabet, AkzoNobel Danmark, Gloria Mundi and Rose Poultry A/S); Grydeland 2014 (Thorne-Holst related to Chocolate manufacturer Marabou); Kain 2014 (Corporea Tesmontes A food processing company); Paineau 2008 (CEDUS Association for sugar beet producers France); Papadaki 2010 (Numerous food suppliers including Coca-Cola, Unilever and Kellogs); Rodearmel 2006 (WK Kellogs Institute for Food and Nutrition Research). eBonsergent 2013, Wyeth Foundation (Nestlé); and Patrick 2006 (the PACE trial) indicated that three authors received income from an organisation that developed the intervention used in the trial.

Table 6. Adverse event data as reported in studies in children aged 0 to 5 years

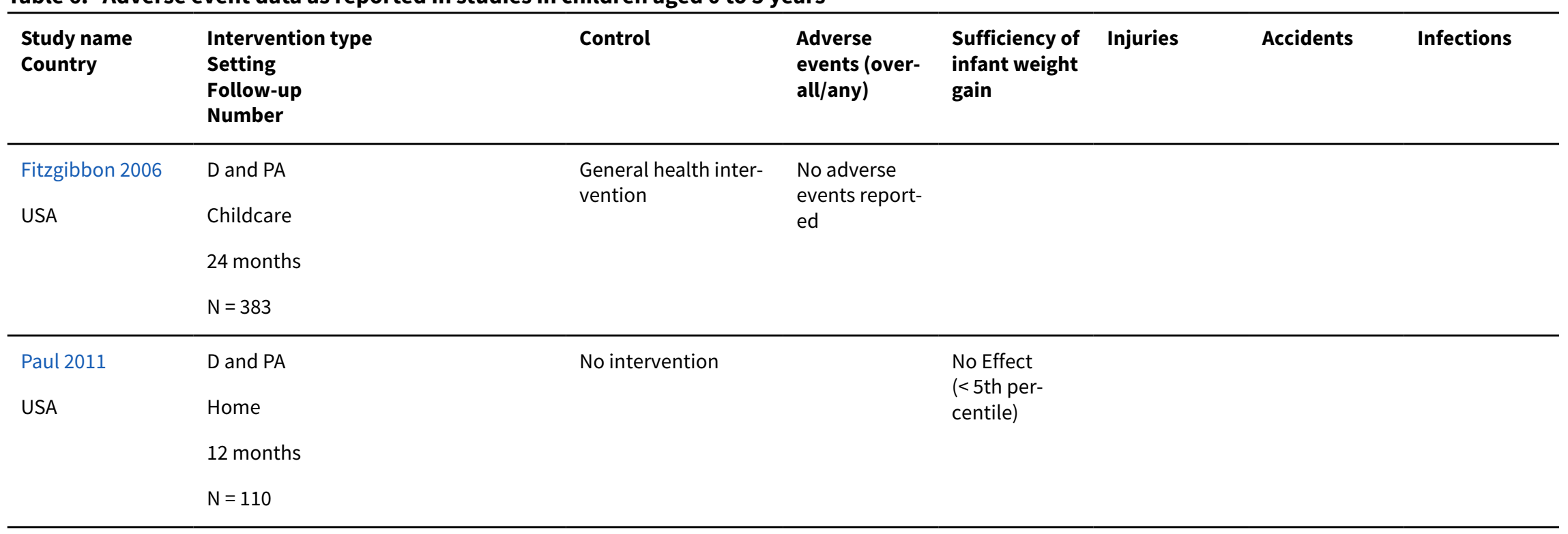




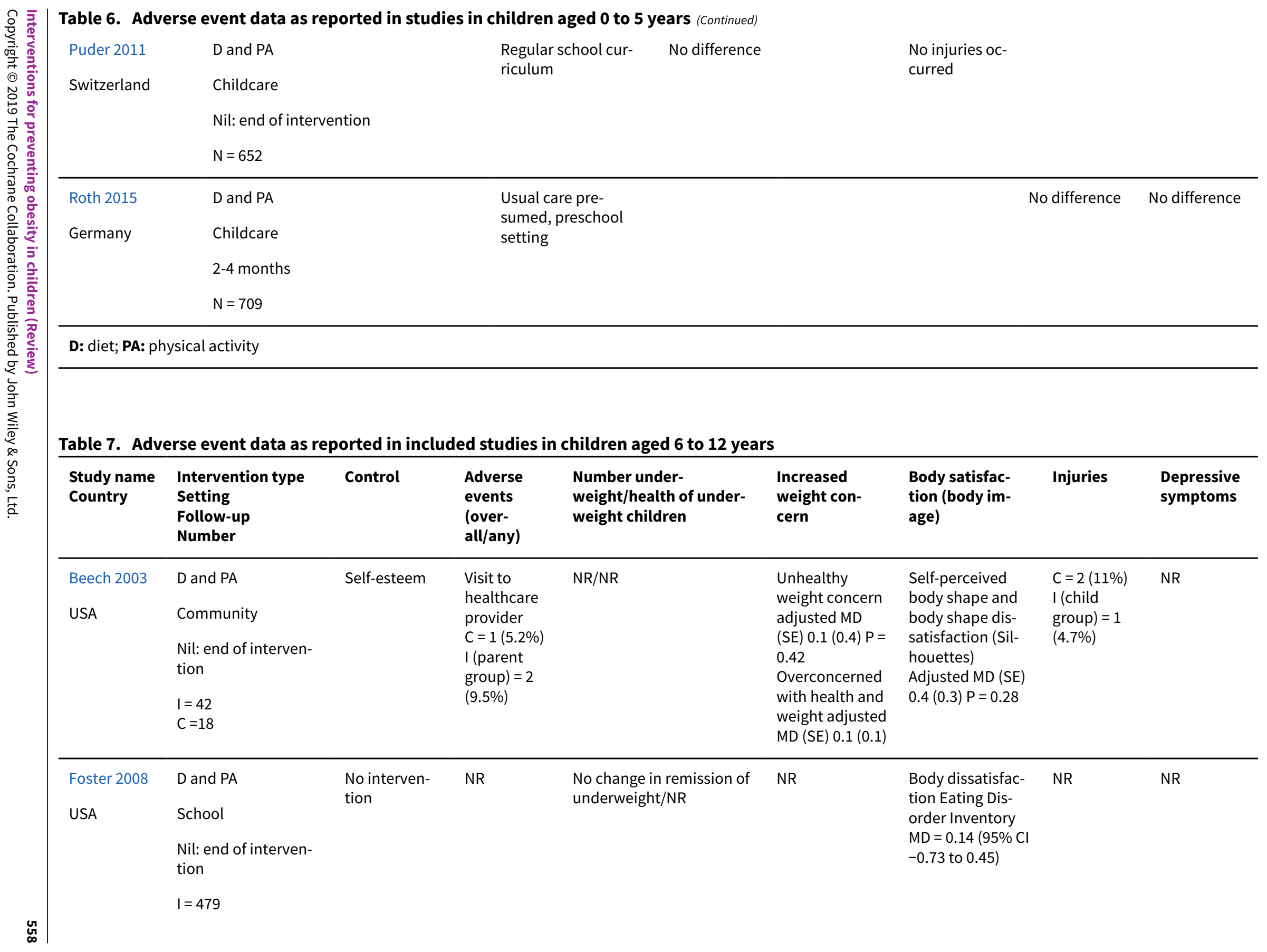




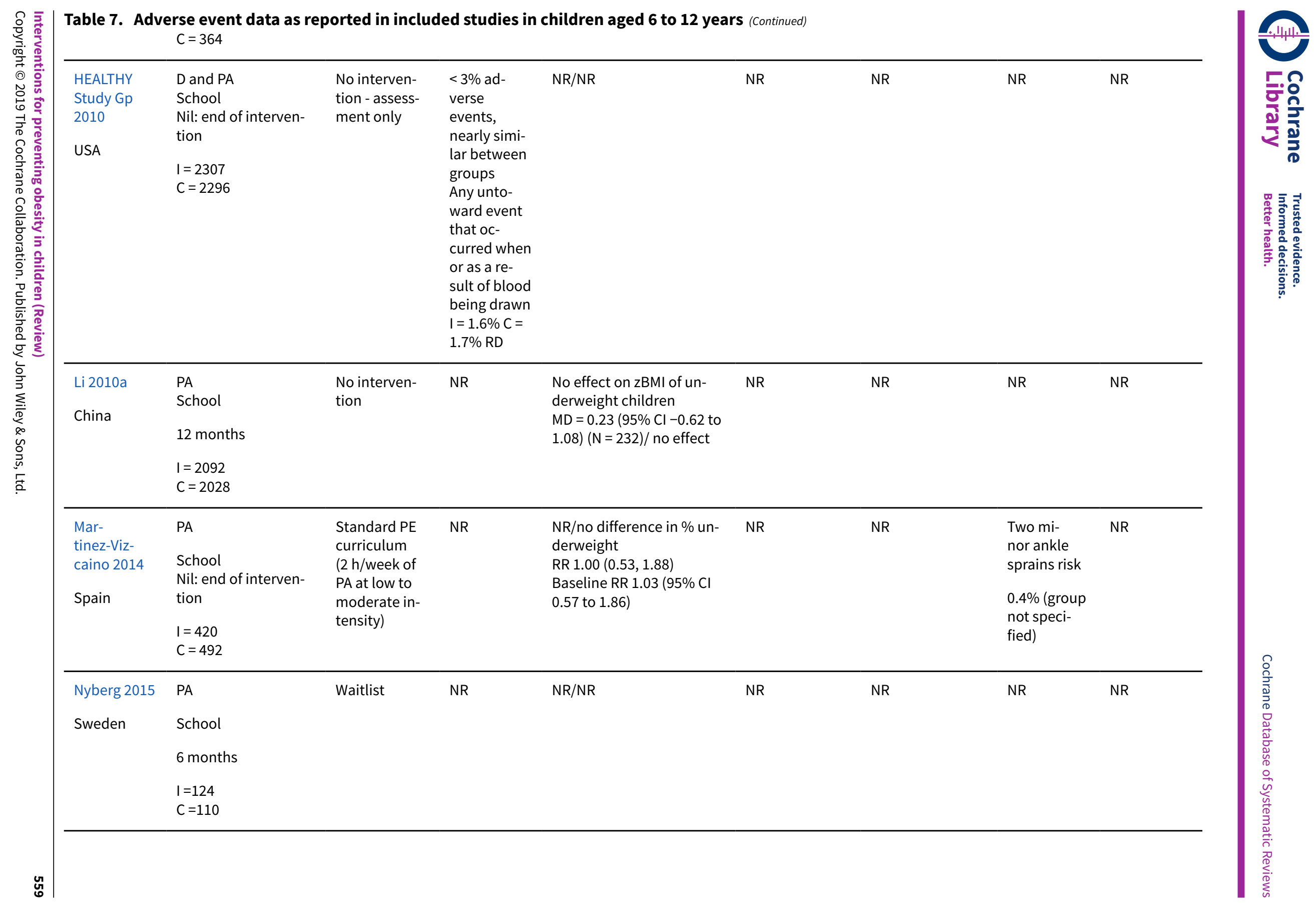


Table 7. Adverse event data as reported in included studies in children aged 6 to 12 years (Continued)

\begin{tabular}{|c|c|c|c|c|c|c|c|c|}
\hline $\begin{array}{l}\text { Robinson } \\
2010 \\
\text { USA }\end{array}$ & $\begin{array}{l}\text { PA } \\
\text { Community } \\
\text { Nil: end of interven- } \\
\text { tion } \\
\text { I }=107 \\
C=118\end{array}$ & $\begin{array}{l}\text { Active com- } \\
\text { parison- } \\
\text { health edu- } \\
\text { cation pro- } \\
\text { gramme to } \\
\text { promote } \\
\text { healthful di- } \\
\text { et and activi- } \\
\text { ty patterns via } \\
\text { newsletters } \\
\text { and delivering } \\
\text { health educa- } \\
\text { tion lectures }\end{array}$ & NR & $\begin{array}{l}\text { NR/no change } \\
\text { No difference in percent of } \\
\text { underweight RR } 1.11 \text { ( } 95 \% \\
\text { Cl } 0.3 \text { to } 4.0 \text { ) }\end{array}$ & $\begin{array}{l}\text { No effect } \\
\text { Overconcern } \\
\text { with } \\
\text { Weight and } \\
\text { Shape (Scale } \\
0-100 \text { ), using } \\
\text { the McKnight } \\
\text { Risk Factor Sur- } \\
\text { vey } \\
\text { Difference } \\
\text { in means of } \\
\text { change/year } \\
0.26(95 \% \text { Cl } \\
-2.18 \text { to } 2.71 \text { ) } \\
\text { Baseline = } \\
29.21 ; C=27.85\end{array}$ & $\begin{array}{l}\text { Self-perceived } \\
\text { body shape } \\
\text { and body shape } \\
\text { dissatisfaction } \\
\text { (Silhouettes) } \\
\text { Difference in } \\
\text { means of change/ } \\
\text { year }-0.04 \text { (95\% } \\
\text { Cl }-0.15,0.08 \text { ) } \\
\text { Baseline }=1.11 \text {; C } \\
=1.78\end{array}$ & NR & $\begin{array}{l}\text { Children's } \\
\text { Depression } \\
\text { Inventory } \\
\text { Scale (0-20) } \\
\text { Reduced for } \\
\text { intervention } \\
\text { group MD } \\
\text { change/year } \\
-0.21 \text { (95\% } \\
\text { Cl-0.42, } \\
-0.001) \\
\text { Baseline } \\
=2.09 ; C= \\
2.74\end{array}$ \\
\hline $\begin{array}{l}\text { Siegrist } 2013 \\
\text { Germany }\end{array}$ & $\begin{array}{l}\text { D and PA } \\
\text { School } \\
\text { Nil: end of interven- } \\
\text { tion } \\
I=427 \\
C=297\end{array}$ & Usual care & NR & $\begin{array}{l}\text { Waist circumference of } \\
\text { children }<10 \text { th centile for } \\
\text { weight did not differ be- } \\
\text { tween the intervention } \\
\text { and control group }(\mathrm{P}= \\
0.373) / \mathrm{NR}\end{array}$ & NR & NR & NR & NR \\
\hline
\end{tabular}

C: control; D: diet; I: intervention; MD: mean difference; NR: not reported; PA: physical activity; PE: physical education; RD: risk difference; RR: risk ratio; SE: standard error; zBMI: body-mass index z score

Table 8. Adverse event data as reported in included studies in children aged 13 to 18 years

\begin{tabular}{|c|c|c|c|c|c|c|c|c|c|}
\hline $\begin{array}{l}\text { Study } \\
\text { name } \\
\text { Country }\end{array}$ & $\begin{array}{l}\text { Interven- } \\
\text { tion type } \\
\text { Setting } \\
\text { Follow-up } \\
\text { N }\end{array}$ & Control & $\begin{array}{l}\text { Unhealthy } \\
\text { weight con- } \\
\text { trol }\end{array}$ & Binge eating & $\begin{array}{l}\text { Clinical } \\
\text { levels of } \\
\text { shape or } \\
\text { weight } \\
\text { concern }\end{array}$ & $\begin{array}{l}\text { Body sat- } \\
\text { isfaction } \\
\text { (body im- } \\
\text { age) }\end{array}$ & Self-acceptance/self-worth & $\begin{array}{l}\text { Depres- } \\
\text { sive } \\
\text { symp- } \\
\text { toms }\end{array}$ & Anxiety \\
\hline $\begin{array}{l}\text { Melnyk } \\
2013 \\
\text { USA }\end{array}$ & $\begin{array}{l}\text { D and PA } \\
\text { School } \\
6 \text { months }\end{array}$ & $\begin{array}{l}\text { Attention control } \\
\text { programme cov- } \\
\text { ering common } \\
\text { health topics }\end{array}$ & NR & NR & NR & NR & NR & $\begin{array}{l}\text { No effect: } \\
\text { I= } 47.03 \\
\text { (46.21 to } \\
47.85) ; C= \\
46.55 \text { ( } 45.8\end{array}$ & $\begin{array}{l}\text { No effect: } \\
\text { I = 47.40 } \\
(46.5 \text { to } \\
48.31) ; C \\
=46.95\end{array}$ \\
\hline
\end{tabular}




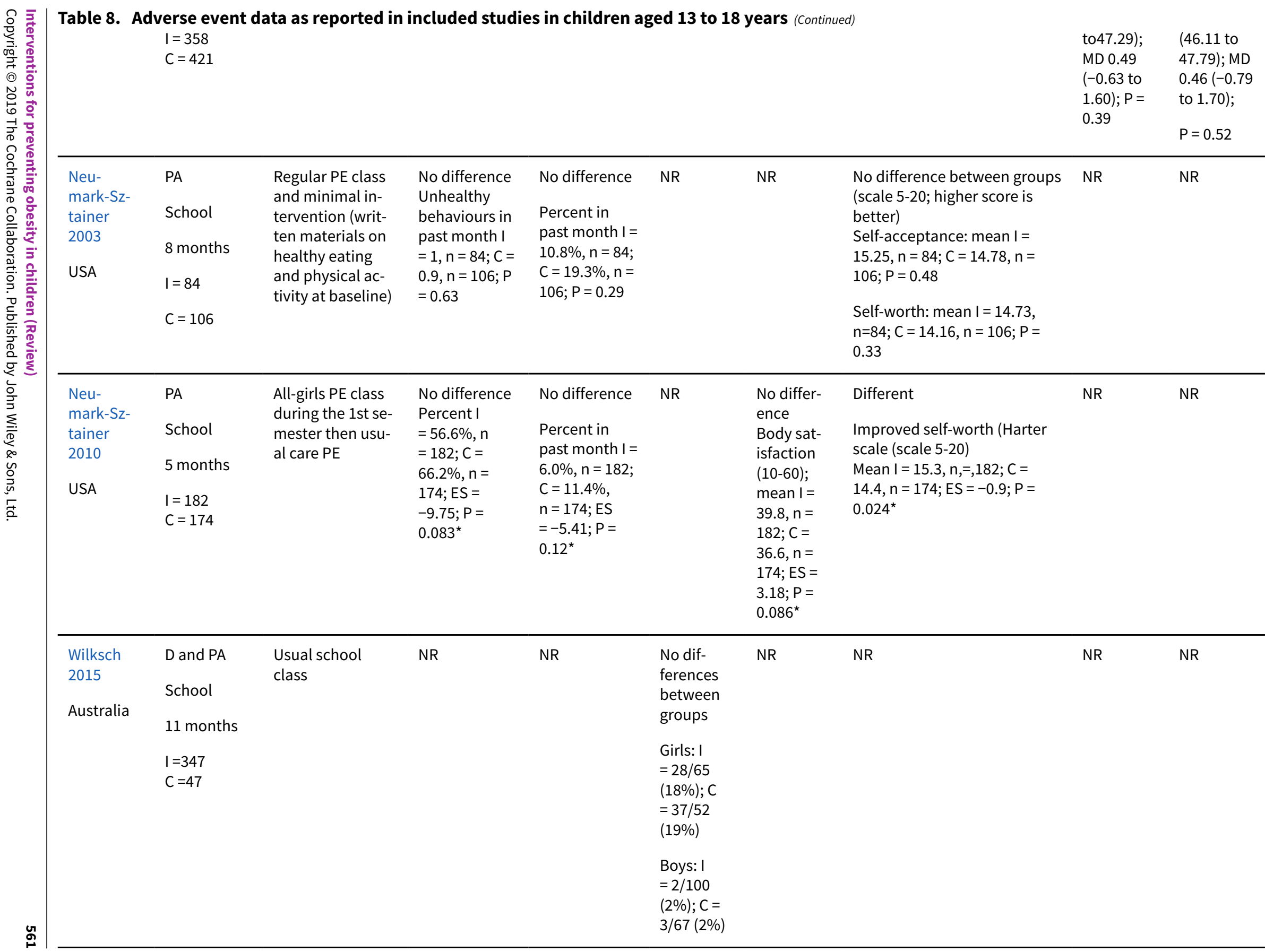




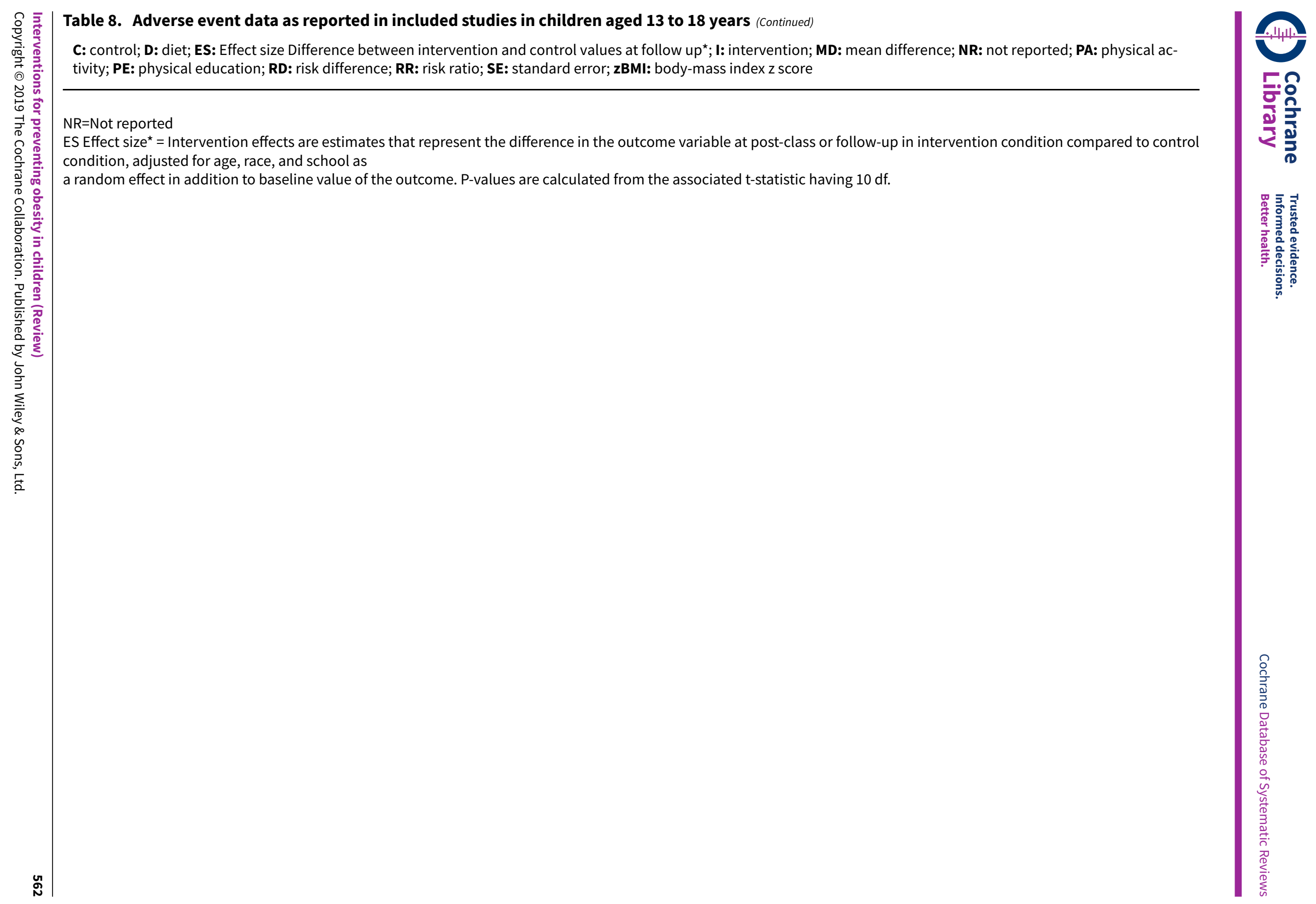


Table 9. Number of study intervention arms addressing the primary outcomes of BMI and zBMI

\begin{tabular}{|c|c|c|c|c|c|c|}
\hline \multirow[t]{2}{*}{ Age group } & \multirow[t]{2}{*}{ Outcome } & \multicolumn{5}{|c|}{ Intervention type } \\
\hline & & Dietary & $\begin{array}{l}\text { Physical activ- } \\
\text { ity }\end{array}$ & $\begin{array}{l}\text { Diet and physical activ- } \\
\text { ity }\end{array}$ & $\begin{array}{l}\text { Total } \\
\text { BMI }\end{array}$ & $\begin{array}{l}\text { Total } \\
\text { zBMI }\end{array}$ \\
\hline \multirow[t]{2}{*}{$0-5$ years } & BMI & 1 & 4 & 11 & 16 & \\
\hline & zBMI & 1 & 4 & 15 & & 20 \\
\hline \multirow[t]{2}{*}{$6-12$ years } & BMI & 5 & 13 & 25 & 43 & \\
\hline & zBMI & 7 & 6 & 18 & & 31 \\
\hline \multirow[t]{2}{*}{$13-18$ years } & BMI & 2 & 5 & 6 & 13 & \\
\hline & zBMI & 0 & 1 & 6 & & 7 \\
\hline Total & & & & & 72 & 58 \\
\hline
\end{tabular}

BMI: body-mass index; zBMI: body-mass index z score

Table 10. List of studies in meta-analyses: children aged 0 to 5 years, outcome BMI, intervention and setting Children aged 0-5 years

\begin{tabular}{|c|c|c|c|}
\hline \multirow[t]{2}{*}{ Setting } & \multicolumn{3}{|c|}{ Intervention type } \\
\hline & Diet & Physical activity & Diet and physical activity \\
\hline \multirow[t]{2}{*}{ Home } & & & Wen 2012 \\
\hline & & & Haines 2013 \\
\hline \multirow[t]{8}{*}{ Childcare } & & Annesi 2013 & Bonis 2014 \\
\hline & & Bonvin 2013 & Fitzgibbon 2005 \\
\hline & & Dennison 2004 & Fitzgibbon 2006 \\
\hline & & Mo-suwan 1998 & Fitzgibbon 2011 \\
\hline & & & Klein 2010 \\
\hline & & & Nemet 2011 \\
\hline & & & Puder 2011 \\
\hline & & & Story 2012 \\
\hline Healthcare & De Vries 2015 & & \\
\hline Wider community & & & Barkin 2012 \\
\hline
\end{tabular}


Table 10. List of studies in meta-analyses: children aged 0 to 5 years, outcome BMI, intervention and setting (Continued) School

\begin{tabular}{llll} 
Count & 1 & 4 & 11 \\
\hline
\end{tabular}

BMI: body-mass index

Table 11. List of studies in meta-analyses: children aged 6 to 12 years, outcome BMI, intervention and setting Children aged 6-12

\begin{tabular}{|c|c|c|c|}
\hline \multirow[t]{2}{*}{ Setting } & \multicolumn{3}{|c|}{ Intervention type } \\
\hline & Diet & Physical activity & Diet and physical activity \\
\hline Home & - & - & - \\
\hline Childcare & - & - & - \\
\hline Healthcare & - & - & - \\
\hline \multirow[t]{9}{*}{ Wider community } & Papadaki 2010 & Khan 2014 & Baranowski 2003 \\
\hline & & Robinson 2010 & Beech 2003 \\
\hline & & & Brown 2013 \\
\hline & & & Chen 2010 \\
\hline & & & Chen 2011 \\
\hline & & & Klesges 2010 \\
\hline & & & Nollen 2014 \\
\hline & & & Robinson 2003 \\
\hline & & & Rosenkranz 2010 \\
\hline \multirow[t]{8}{*}{ School } & Sichieri 2009 & Donnelly 2009 & Caballero 2003 \\
\hline & James 2004 & James 2004 & Foster 2008 \\
\hline & Meng 2013 & Kriemler 2010 & Gentile 2009 \\
\hline & Paineau 2008 & Lazaar 2007 & Grydeland 2014 \\
\hline & & Li 2010a & Habib-Mourad 2014 \\
\hline & & Martinez-Vizcaino 2014 & Herscovici 2013 \\
\hline & & Reed 2008 & James 2004 \\
\hline & & Robbins 2006 & Jansen 2011 \\
\hline
\end{tabular}


Table 11. List of studies in meta-analyses: children aged 6 to 12 years, outcome BMI, intervention and

setting (Continued)

\begin{tabular}{lcc} 
& Simon 2008 & Johnston 2013 \\
\hline & Thivel 2011 & Kipping 2008 \\
\hline & Vizcaino 2008 & Levy 2012 \\
\hline & Llargues 2012 \\
\hline & & Magnusson 2012 \\
\hline Count & & Siegrist 2013 2013 \\
\hline BMI: body-mass index & $\mathbf{1 3}$ & Story 2003 \\
\hline
\end{tabular}

Table 12. List of studies in meta-analyses: children aged 13 to 18 years, outcome BMI, intervention and setting

\section{Children aged 13-18 years}

\begin{tabular}{llll}
\hline Setting & \multicolumn{2}{l}{ Intervention type } & \\
\hline & Diet & Physical activity & Diet and physical activity \\
\hline Home & Ebbeling 2006 & &
\end{tabular}

\section{Childcare}

\section{Healthcare}

Wider community

\begin{tabular}{|c|c|c|c|}
\hline School & Mihas 2010 & El Ansarai 2010 & Bonsergent 2013 \\
\hline & & Lubans 2011 & Ezendam 2012 \\
\hline & & Neumark-Sztainer 2003 & Haerens 2006 \\
\hline & & Smith 2014 & Melnyk 2013 \\
\hline & & Weeks 2012 & Peralta 2009 \\
\hline & & & Singh 2009 \\
\hline & & & Whittemore 2013 \\
\hline & & & Wilksch 2015 \\
\hline Count & 2 & 5 & 6 \\
\hline
\end{tabular}


Table 12. List of studies in meta-analyses: children aged 13 to 18 years, outcome BMI, intervention and settining (Gontinued) index

Table 13. List of studies in meta-analyses: children aged 0 to 5 years, outcome zBMI, intervention and setting Children aged 0-5 years

\begin{tabular}{lll}
\hline Setting & \multicolumn{2}{l}{ Intervention type } \\
\cline { 2 - 3 } Diet $\quad$ Physical activity Diet and physical activity
\end{tabular}

\begin{tabular}{ll}
\hline Home Haines 2013 \\
\hline
\end{tabular}

Keller 2009

Ostbye 2012

\begin{tabular}{lll}
\hline Childcare & Dennison 2004 & Alkon 2014 \\
\hline & Reilly 2006 & De Coen 2012 \\
\hline
\end{tabular}

Fitzgibbon 2005

Fitzgibbon 2006

Fitzgibbon 2011

Natale 2014

Story 2012

Verbestel 2014

Zask 2012

\begin{tabular}{|c|c|c|c|}
\hline Healthcare & Daniels 2012 & Birken 2012 & Slusser 2012 \\
\hline
\end{tabular}

\section{Wider community \\ (1)}

(10)

\section{School}

Count

1

4

Campbell 2013

Skouteris 2016

zBMI: body-mass index z score

Table 14. List of studies in meta-analyses: children aged 6 to 12 years, outcome zBMI, intervention and setting

\section{Children aged 6-12 years}


Table 14. List of studies in meta-analyses: children aged 6 to 12 years, outcome zBMI, intervention and Sestting (Continued) Intervention type

\begin{tabular}{|c|c|c|c|}
\hline & Diet & Physical activity & Diet and physical activity \\
\hline Home & & & Baranowski 2011 \\
\hline \multicolumn{4}{|l|}{ Childcare } \\
\hline \multicolumn{4}{|l|}{ Healthcare } \\
\hline \multirow[t]{4}{*}{ Wider community } & Papadaki 2010 & Khan 2014 & Brown 2013 \\
\hline & & Robinson 2010 & Haire-Joshu 2010 \\
\hline & & & Morgan 2011 \\
\hline & & & Rosenkranz 2010 \\
\hline \multirow[t]{13}{*}{ School } & Amaro 2006 & De Ruyter 2012 & Cao 2015 \\
\hline & Damsgaard 2014 & Gutin 2008 & Fairclough 2013 \\
\hline & James 2004 & Lazaar 2007 & Foster 2008 \\
\hline & Muckelbauer 2010 & Li 2010a & Grydeland 2014 \\
\hline & Paineau 2008 & & HEALTHY Study Gp 2010 \\
\hline & Rosario 2012 & & Herscovici 2013 \\
\hline & & & Johnston 2013 \\
\hline & & & Kipping 2014 \\
\hline & & & Marcus 2009 \\
\hline & & & Santos 2014 \\
\hline & & & Siegrist 2013 \\
\hline & & & Spiegel 2006 \\
\hline & & & Williamson 2012 \\
\hline Count & 7 & 6 & 18 \\
\hline
\end{tabular}

zBMI: body-mass index z score

Table 15. List of studies in meta-analyses: children aged 0 to 5 years, outcome zBMI, intervention and setting

\section{Children aged 13-18 years}

Setting Intervention type


Table 15. List of studies in meta-analyses: children aged 0 to 5 years, outcome zBMI, intervention and

setting (continued) Diet Physical activity Diet and physical activity

\begin{tabular}{llll}
\hline Home & - & - & French 2011 \\
\hline School & - & Lubans 2011 & Andrade 2014 \\
\hline
\end{tabular}

Bonsergent 2013

Dewar 2013

Haerens 2006

Viggiano 2015

$\begin{array}{llll}\text { Count } & 0 & 1 & 6\end{array}$

zBMI: body-mass index z score

APPENDICES

Appendix 1. Search strategies 2018

\begin{tabular}{ll}
\hline Component & Interventions for preventing obesity in children \\
\hline Review area & Obesity prevention strategies for children \\
\hline Populations/aspect & Children/adolescents in any setting \\
\hline Interventions & $\begin{array}{l}\text { Any interventions aimed at preventing obesity in children (including diet/psychosocial/exercise } \\
\text { etc.) }\end{array}$ \\
\hline Study designs & RCTs \\
\hline Exclusions & Animal studies \\
\hline $\begin{array}{l}\text { How the information was } \\
\text { searched }\end{array}$ & Databases: MEDLINe, Embase, Cochrane (CENTRAL), CINAHL, PsycINFO \\
& Language: English \\
& Date parameters: from dates of last searches of draft Cochrane Review June 2105 \\
\hline
\end{tabular}

Search terms and date searched

See MEDLINE strategy (below). This is the strategy used in the 2015 update with the addition of RCT filter (Cochrane sensitive best balance (Lefebvre 2011)).

Searched 5 January 2018

Search results
MEDLINE/Premedline $=3287$

Embase $=4057$

CINAHL $=458$

Cochrane CENTRAL $=2046($ includes all 2015) 
(Continued)

$$
\begin{aligned}
& \text { Psycinfo }=658 \\
& \text { Total }=10506
\end{aligned}
$$

Cochrane systematic reviews $(\mathrm{SR})=411$

Total including the SRs $=10,917$

Totals deduplicated $=5847$ (5485 plus 362 SRs from the Cochrane Library not picked up elsewhere)

Database: Ovid MEDLINE(R) Epub Ahead of Print, In-Process and Other Non-Indexed Citations, Ovid MEDLINE(R) Daily and Ovid MEDLINE(R) $<1946$ to Present $>5$ January 2018

Search Strategy:

1 exp Obesity/ (202741)

2 Weight Gain/ (30968)

3 exp Weight Loss/ (40755)

4 obes $^{\star}$.af. (345017)

5 (weight gain or weight loss).af. (158726)

6 (overweight or over weight or overeat ${ }^{\star}$ or over eat ${ }^{\star}$ ).af. (76258)

7 weight change ${ }^{\star}$.af. (10816)

8 ((bmi or body mass index) adj2 (gain or loss or change)).af. (4318)

9 or/1-8 (480272)

10 exp Behavior Therapy/ (72104)

11 social support/ (68041)

12 exp Psychotherapy, Group/ (26861)

13 ((psychological or behavio? $\left.r^{\star}\right)$ adj (therapy or modif* or strateg* or intervention $\left.{ }^{\star}\right)$ ).af. (64921)

14 (group therapy or family therapy or cognitive therapy).af. (38550)

15 ((lifestyle or life style) adj (chang* or intervention*)).af. (13662)

16 counsel?ing.af. (125997)

17 social support.af. (83956)

18 (peer adj2 support).af. (3747)

19 (children adj3 parent ${ }^{\star}$ adj3 therapy).af. (101)

20 or/10-19 (330899)

21 exp Obesity/dh (Diet Therapy] (7585)

22 exp Diet Therapy/ (53110)

23 Fasting/ (35664)

24 (diets or diet or dieting).af. (455218)

25 (diet $^{\star}$ adj (modif* or therapy or intervention* or strateg*)).af. (30218)

26 (low calorie or calorie control* or healthy eating).af. (8754)

27 (fasting or modified fast*).af. (118905)

28 exp Dietary Fats/ (91576)

29 (fruit or vegetable*).af. (125846)

30 (high fat $^{\star}$ or low fat* or fatty food $\left.{ }^{\star}\right)$.af. (44662)

31 formula diet*.af. (726)

32 or/21-31 (708647)

33 exp Exercise/ (179352)

34 exp Exercise Therapy/ (46020)

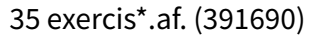

36 (aerobics or physical therapy or physical activity or physical inactivity).af. (182908)

37 (fitness adj (class* or regime* or program $\left.{ }^{\star}\right)$ ).af. (912)

38 (aerobics or physical therapy or physical training or physical education).af. (118570)

39 dance therapy.af. (350)

40 sedentary behavio?r.af. (4204)

41 or/33-40 (565681)

42 exp Complementary Therapies/ (220825)

43 (alternative medicine or complementary therap* or complementary medicine).af. (39726)

44 (hypnotism or hypnosis or hypnotherapy).af. (12438)

45 (acupuncture or homeopathy or homoeopathy).af. (33648) 
46 (chinese medicine or indian medicine or herbal medicine or ayurvedic).af. (73724)

47 or/42-46 (294165)

48 ((diet or dieting or slim*) adj (club* or organi?ation)).af. (35)

49 (weightwatcher ${ }^{\star}$ or weight watcher ${ }^{\star}$ ).af. (167)

50 (correspondence adj (course* or program*)).af. (91)

51 (fat camp* or diet ${ }^{\star}$ camp $^{\star}$ ).af. (26)

52 or/48-51 (319)

53 exp Health Promotion/ (72719)

54 exp Health Education/ (162980)

55 (health promotion or health education).af. (191843)

56 (media intervention* or community intervention*).af. (2223)

57 health promoting school ${ }^{*}$.af. (302)

58 ((school or community) adj2 program*).af. (23166)

59 ((school or community) adj2 intervention*).af. (9538)

60 (family intervention* or parent* intervention).af. (2115)

61 (parent $^{\star}$ adj2 (behavio?r or involve* or control ${ }^{\star}$ or attitude ${ }^{\star}$ or educat $\left.^{\star}\right)$ ). af. (36435)

62 or/53-61 (342124)

63 exp Health Policy/ (101913)

64 (health polic $^{\star}$ or school polic* or food polic ${ }^{\star}$ or nutrition polic $\left.{ }^{\star}\right)$.af. (133063)

6563 or $64(161488)$

66 exp Obesity/pc (Prevention and Control] (18369)

67 exp Primary Prevention/ (146391)

68 (primary prevention or secondary prevention).af. (64098)

69 (preventive measure ${ }^{\star}$ or preventative measure ${ }^{\star}$ ).af. (23224)

70 (preventive care or preventative care).af. (5195)

71 (obesity adj2 (prevent* ${ }^{\star}$ or treat*)).af. (26373)

72 or/66-71 (257782)

739 and ( 20 or 32 or 41 or 47 or 52 or 62 or 65 or 72$)(192518)$

74 exp child/ or exp infant/ or adolescent/ (3549829)

75 (child* or adolescen* or infant* or pediatr* or paediatr* or boys or girls or youth or youths or teenage* or young people or young person or young adult* or schoolchildren or school children).af. (4751805)

7674 or 75 (4751805)

7773 and 76 (59570)

78 (exp animals/ not humans.sh.) or (rat or rats or mouse or mice or rodent ${ }^{\star}$ ).ti. (5017766)

7977 not 78 (57565)

80 controlled clinical trial.pt. (101735)

81 randomi\#ed.ab. (542771)

82 placebo.ab. (210412)

83 randomly.ab. (311814)

84 (clinical trials as topic or controlled clinical trials as topic).sh. (208213)

85 trial.ti. (203294)

86 exp randomized controlled trial/ or exp randomized controlled trials as topic/ (638598)

87 or/80-86 (1349493)

8879 and 87 (9638)

89 limit 88 to $y r=" 2016$-Current" (1872)

$90\left(201506^{\star}\right.$ or $201507^{\star}$ or $201508^{\star}$ or $201509^{\star}$ or $20151^{\star}$ or $2016^{\star}$ or $2017^{\star}$ or $2018^{\star}$ ).ed,dc,dp,ep. (4310717)

9188 and 90 (3287)

9289 or $91(3287)$

\section{Appendix 2. Search strategies 2015}

\section{CENTRAL}

\section{5, Issue 5 (via Cochrane Library)}

\section{Searched 10 June 2015}

\section{Limits: CENTRAL 2005, Issue 1 to 2015, Issue 5}

1. MeSH descriptor Obesity explode all trees

2. MeSH descriptor Body Weight Changes explode all trees

3. $\left(\right.$ obes $\left.^{\star}\right)$ 
4. ("weight gain" or "weight loss")

5. (overweight or "over weight" or overeat* or (over next eat $\left.{ }^{\star}\right)$ )

6. (weight next change ${ }^{\star}$ )

7. ((bmi or "body mass index") near (gain or loss or change $\left.{ }^{\star}\right)$ )

8. (1 OR 2 OR 3 OR 4 OR 5 OR 6 OR 7 )

9. MeSH descriptor Behavior Therapy explode all trees

10. MeSH descriptor Social Support explode all trees

11. MeSH descriptor Psychotherapy, Group explode all trees

12. ((psychological or behavio? $\left.{ }^{\star}\right)$ near (therapy or modif* or strateg $^{\star}$ or intervention $\left.{ }^{\star}\right)$ )

13. ("group therapy" or "family therapy" or "cognitive therapy")

14. (lifestyle or "life style") near (chang* or intervention*)

15. counsel?ing

16. "social support"

17. (peer near2 support)

18. (children near3 parent* near3 therapy)

19. (9 OR 10 OR 11 OR 12 OR 13 OR 14 OR 15 OR 16 OR 17 OR 18)

20. MeSH descriptor Obesity explode all trees with qualifier: $\mathrm{DH}$

21. MeSH descriptor Diet Therapy explode all trees

22. MeSH descriptor Fasting, this term only

23. (diets or diet or dieting)

24. diet $^{\star}$ near (modif ${ }^{\star}$ or therapy or intervention ${ }^{\star}$ or strateg ${ }^{\star}$ )

25. "low calorie" or (calorie next control*) or "healthy eating"

26. (fasting or (modified next fast $\left.{ }^{\star}\right)$ )

27. MeSH descriptor Dietary Fats explode all trees

28. (fruit or vegetable ${ }^{\star}$ )

29. (high next fat ${ }^{\star}$ ) or (low next fat ${ }^{\star}$ ) or (fatty next food ${ }^{\star}$ )

30. formula next diet ${ }^{\star}$

31. (20 OR 21 OR 22 OR 23 OR 24 OR 25 OR 26 OR 27 OR 28 OR 29 OR 30)

32. MeSH descriptor Exercise explode all trees

33. MeSH descriptor Exercise Therapy explode all trees

34. exercis*

35. (aerobics or "physical therapy" or "physical activity" or "physical inactivity")

36. fitness near (class ${ }^{\star}$ or regime* or program ${ }^{\star}$ )

37. ("physical training" or "physical education")

38. "dance therapy" 
39. sedentary next behavio? $r^{\star}$

\section{0. (32 OR 33 OR 34 OR 35 OR 36 OR 37 OR 38 OR 39)}

41. MeSH descriptor Complementary Therapies explode all trees

42. "alternative medicine" or (complementary next therap*) or "complementary medicine"

43. (hypnotism or hypnosis or hypnotherapy)

44. (acupuncture or homeopathy or homoeopathy)

45. ("chinese medicine" or "indian medicine" or "herbal medicine" or ayurvedic)

46. (41 OR 42 OR 43 OR 44 OR 45)

47. (diet* or slim $\left.{ }^{\star}\right)$ near (club* or organi?ation)

48. (weightwatcher ${ }^{\star}$ or (weight next watcher $\left.{ }^{\star}\right)$ )

49. correspondence near (course* or program*)

50. (fat or diet $\left.{ }^{\star}\right)$ next camp*

51. (47 OR 48 OR 49 OR 50)

52. MeSH descriptor Health Promotion explode all trees

53. MeSH descriptor Health Education explode all trees

54. ("health promotion" or "health education")

55. ("media intervention"” or "community intervention")

56. (health next promoting next school ${ }^{\star}$ )

57. ((school or community) near2 program $\left.{ }^{\star}\right)$

58. ((school or community) near2 intervention*)

59. (family next intervention $\left.{ }^{\star}\right)$ or (parent ${ }^{\star}$ next intervention ${ }^{\star}$ )

60. (parent ${ }^{\star}$ near2 (behavio? $r^{\star}$ or involve* or control ${ }^{\star}$ or attitude* or educat $\left.^{\star}\right)$ )

61. (52 OR 53 OR 54 OR 55 OR 56 OR 57 OR 58 OR 59 OR 60)

62. MeSH descriptor Health Policy explode all trees

63. (health next polic ${ }^{\star}$ ) or (school next polic ${ }^{\star}$ ) or (food next polic ${ }^{\star}$ ) or (nutrition next polic ${ }^{\star}$ )

64. (62 OR 63)

65. MeSH descriptor Obesity explode all trees with qualifier: PC

66. MeSH descriptor Primary Prevention explode all trees

67. ("primary prevention" or "secondary prevention")

68. (preventive next measure ${ }^{\star}$ ) or (preventative next measure ${ }^{\star}$ )

69. ("preventive care" or "preventative care")

70. (obesity near2 (prevent ${ }^{\star}$ or treat $\left.{ }^{\star}\right)$ )

71. (65 OR 66 OR 67 OR 68 OR 69 OR 70)

72. (19 OR 31 OR 40 OR 46 OR 51 OR 61 OR 64 OR 71)

73. (8 AND 72) 
74. MeSH descriptor Child explode all trees

75. MeSH descriptor Infant explode all trees

76. (child* or adolescen* or infant $\left.{ }^{\star}\right)$

77. (teenage* or "young people" or "young person" or (young next adult*))

78. (schoolchildren or "school children")

79. pediatr $^{\star}$ or paediatr ${ }^{\star}$ )

80. (boys or girls or youth or youths)

81. MeSH descriptor Adolescent, this term only

82. (74 OR 75 OR 76 OR 77 OR 78 OR 79 OR 80 OR 81)

83. (73 AND 82)

\section{Ovid MEDLINER}

(1946 to May Week 5 2015)

\section{Searched 10 June 2015}

\section{Limits: publication year 2010 to search date}

1. exp Obesity/

2. Weight Gain/

3. exp Weight Loss/

4. obes\$.af.

5. (weight gain or weight loss).af.

6. (overweight or over weight or overeat\$ or over eat\$).af.

7. weight change\$.af.

8. ((bmi or body mass index) adj2 (gain or loss or change)).af.

9. or/1-8

10. exp Behavior Therapy/

11. social support/

12. exp Psychotherapy, Group/

13. ((psychological or behavio?r\$) adj (therapy or modif\$ or strateg\$ or intervention\$)).af.

14. (group therapy or family therapy or cognitive therapy).af.

15. ((lifestyle or life style) adj (chang\$ or intervention\$)).af.

16. counsel?ing.af.

17. social support.af.

18. (peer adj2 support).af.

19. (children adj3 parent\$ adj3 therapy).af.

20. or $/ 10-19$

21. exp OBESITY/dh (Diet Therapy] 
22. exp Diet Therapy/

23. Fasting/

24. (diets or diet or dieting).af.

25. (diet\$ adj (modif\$ or therapy or intervention\$ or strateg\$)).af.

26. (low calorie or calorie control\$ or healthy eating).af.

27. (fasting or modified fast\$).af.

28. exp Dietary Fats/

29. (fruit or vegetable\$).af.

30. (high fat\$ or low fat\$ or fatty food\$).af.

31. formula diet\$.af.

32. or/21-31

33. exp Exercise/

34. exp Exercise Therapy/

35. exercis\$.af.

36. (aerobics or physical therapy or physical activity or physical inactivity).af.

37. (fitness adj (class\$ or regime\$ or program\$)).af.

38. (aerobics or physical therapy or physical training or physical education).af.

39. dance therapy.af.

40. sedentary behavio?r.af.

41. or $/ 33-40$

42. exp Complementary Therapies/

43. (alternative medicine or complementary therap\$ or complementary medicine).af.

44. (hypnotism or hypnosis or hypnotherapy).af.

45. (acupuncture or homeopathy or homoeopathy).af.

46. (chinese medicine or indian medicine or herbal medicine or ayurvedic).af.

47. or/42-46

48. ((diet or dieting or slim\$) adj (club\$ or organi?ation)).af.

49. (weightwatcher\$ or weight watcher\$).af.

50. (correspondence adj (course\$ or program\$)).af.

51. (fat camp\$ or diet\$ camp\$).af.

52. or/48-51

53. exp Health Promotion/

54. exp Health Education/

55. (health promotion or health education).af.

56. (media intervention\$ or community intervention\$).af. 
57. health promoting school\$.af.

58. ((school or community) adj2 program\$).af.

59. ((school or community) adj2 intervention\$).af.

60. (family intervention\$ or parent\$ intervention).af.

61. (parent\$ adj2 (behavio?r or involve\$ or control\$ or attitude\$ or educat\$)).af.

62. or/53-61

63. exp Health Policy/

64. (health polic\$ or school polic\$ or food polic\$ or nutrition polic\$).af.

65.63 or 64

66. $\exp \mathrm{OBESITY/pc} \mathrm{(Prevention} \mathrm{and} \mathrm{Control]}$

67. exp Primary Prevention/

68. (primary prevention or secondary prevention).af.

69. (preventive measure\$ or preventative measure\$).af.

70. (preventive care or preventative care).af.

71. (obesity adj2 (prevent\$ or treat\$)).af.

72. or/66-71

73. randomized controlled trial.pt.

74. controlled clinical trial.pt.

75. Random Allocation/

76. Double-Blind Method/

77. single-blind method/

78. Placebos/

79. *Research Design/

80. intervention studies/

81. evaluation studies/

82. Comparative Study/

83. exp Longitudinal Studies/

84. cross-over studies/

85. clinical trial.tw.

86. clinical trial.pt.

87. latin square.tw.

88. (time adj series).tw.

89. (before adj2 after adj3 (stud\$ or trial\$ or design\$)).tw.

90. ((singl\$ or doubl\$ or trebl\$ or tripl\$) adj5 (blind\$ or mask)).tw.

91. placebo\$.tw. 
92. random\$.tw.

93. (matched communities or matched schools or matched populations).tw.

94. control\$.tw.

95. (comparison group\$ or control group\$).tw.

96. matched pairs.tw.

97. (outcome study or outcome studies).tw.

98. (quasiexperimental or quasi experimental or pseudo experimental).tw.

99. (nonrandomi?ed or non randomi?ed or pseudo randomi?ed or quasi randomi?ed).tw.

100. prospectiv\$.tw.

101. volunteer\$.tw.

102. or/73-101

103. 20 or 32 or 41 or 47 or 52 or 62 or 65 or 72

104. 9 and 102 and 103

105. Animals/

106. exp Child/

107. Adolescent/

108. exp Infant/

109. (child\$ or adolescen\$ or infant\$).af.

110. (teenage\$ or young people or young person or young adult\$).af.

111. (schoolchildren or school children).af.

112. (pediatr\$ or paediatr\$).af.

113. (boys or girls or youth or youths).af.

114. or/106-113

115. 104 not 105

116. 114 and 115

117. limit 116 to $y r=$ "2010-Current"

\title{
Embase OVID
}

(1996 to 2015 Week 23)

\section{Searched 11 June 2015}

\section{Limits: publication years 2010 to search date}

\author{
1. exp obesity/ \\ 2. weight gain/ \\ 3. weight reduction/ \\ 4. obes\$.af. \\ 5. (weight gain or weight loss).af.
}


6. (overweight or over weight or overeat\$ or over eat\$).af.

7. weight change\$.af.

8. ((bmi or body mass index) adj2 (gain or loss or change)).af.

9. or/1-8

10. behavior therapy/

11. social support/

12. family therapy/

13. group therapy/

14. ((psychological or behavio?r\$) adj (therapy or modif\$ or strateg\$ or intervention\$)).af.

15. (group therapy or family therapy or cognitive therapy).af.

16. ((lifestyle or life style) adj (chang\$ or intervention\$)).af.

17. counsel?ing.af.

18. social support.af.

19. (peer adj2 support).af.

20. (children adj3 parent\$ adj3 therapy).af.

21. or $/ 10-20$

22. exp diet therapy/

23. (diets or diet or dieting).af.

24. (diet\$ adj (modif\$ or therapy or intervention\$ or strateg\$)).af.

25. (low calorie or calorie control\$ or healthy eating).af.

26. (fasting or modified fast\$).af.

27. exp fat intake/

28. (fruit or vegetable\$).af.

29. (high fat\$ or low fat\$ or fatty food\$).af.

30. formula diet\$..af.

31. $\mathrm{or} / 22-30$

32. exp exercise/

33. exp kinesiotherapy/

34. exercis\$.af.

35. (aerobics or physical therapy or physical activity or physical inactivity).af.

36. (fitness adj (class\$ or regime\$ or program\$)).af.

37. (aerobics or physical therapy or physical training or physical education).af.

38. dance therapy.af.

39. sedentary behavio?r.af.

40. or/32-39 
41. exp alternative medicine/

42. (alternative medicine or complementary therap\$ or complementary medicine).af.

43. (hypnotism or hypnosis or hypnotherapy).af.

44. (acupuncture or homeopathy or homoeopathy).af.

45. (chinese medicine or indian medicine or herbal medicine or ayurvedic).af.

46. or/41-45

47. ((diet or dieting or slim\$) adj (club\$ or organi?ation)).af.

48. (weightwatcher\$ or weight watcher\$).af.

49. (correspondence adj (course\$ or program\$)).af.

50. (fat camp\$ or diet\$ camp\$).af.

51. or/47-50

52. exp health education/

53. (health promotion or health education).af.

54. (media intervention\$ or community intervention\$).af.

55. health promoting school\$.af.

56. ((school or community) adj2 program\$).af.

57. ((school or community) adj2 intervention\$).af.

58. (family intervention\$ or parent\$ intervention).af.

59. (parent\$ adj2 (behavio?r or involve\$ or control\$ or attitude\$ or educat\$)).af.

60. or/52-59

61. health care policy/

62. (health polic\$ or school polic\$ or food polic\$ or nutrition polic\$).af.

63.61 or 62

64. exp obesity/pc (Prevention]

65. primary prevention/

66. (primary prevention or secondary prevention).af.

67. (preventive measure\$ or preventative measure\$).af.

68. (preventive care or preventative care).af.

69. (obesity adj2 (prevent\$ or treat\$)).af.

70. or/64-69

71. exp clinical trial/

72. exp Randomized Controlled Trial/

73. randomization/

74. exp Double-Blind procedure/

75. exp Single-Blind procedure/ 
76. exp Crossover procedure/

77. clinical trial.tw.

78. ((singl\$ or doubl\$ or trebl\$ or tripl\$) and (mask\$ or blind\$)).tw.

79. latin square.tw.

80. placebo/

81. placebo\$.tw.

82. random\$.tw.

83. Comparative Study/

84. evaluation/

85. clinical trial.tw.

86. latin square.tw.

87. (before adj2 after adj3 (stud\$ or trial\$ or design\$)).tw.

88. ((singl\$ or doubl\$ or trebl\$ or tripl\$) adj5 (blind\$ or mask)).tw.

89. (matched communities or matched schools or matched populations).tw.

90. control\$.tw.

91. (comparison group\$ or control group\$).tw.

92. matched pairs.tw.

93. (outcome study or outcome studies).tw.

94. (quasiexperimental or quasi experimental or pseudo experimental).tw.

95. (nonrandomi?ed or non randomi?ed or pseudo randomi?ed or quasi randomi?ed).tw.

96. prospectiv\$.tw.

97. volunteer\$.tw.

98. or/71-97

99. 21 or 31 or 40 or 46 or 51 or 60 or 63 or 70

100. 9 and 98 and 99

101. animal/

102. exp child/

103. $\exp$ ADOLESCENT/

104. exp preschool child/

105. exp infant/

106. (child\$ or adolescen\$ or infant\$).af.

107. (teenage\$ or young people or young person or young adult\$).af.

108. (schoolchildren or school children).af.

109. (pediatr\$ or paediatr\$).af.

110. (boys or girls or youth or youths).af. 
111. or/102-110

112. 100 not 101

113. 111 and 112

114. limit 113 to $y r=$ "2010 $-2015 "$

\section{PsyciNfo}

\section{2 to June Week 22015}

\section{Searched 15 June 2015}

\section{Limits: date range: 2005 to 2010}

1. exp overweight/

2. weight control/

3. obes ${ }^{\star}$.tw.

4. weight gain*.tw.

5. weight loss ${ }^{\star}$.tw.

6. (overweight or over weight).tw.

7. weight loss/

8. weight gain/

9. (overeat ${ }^{\star}$ or over eat $\left.{ }^{\star}\right)$.tw.

10. weight change ${ }^{\star}$. tw.

11. ((bmi or body mass) adj3 (gain ${ }^{\star}$ or loss ${ }^{\star}$ or change $\left.{ }^{\star}\right)$ ).tw.

12. or/1-11

13. (adolescence 1317 yrs or childhood birth 12 yrs or infancy 223 mo or neonatal birth 1 mo or preschool age 25 yrs or school age 6 $12 \mathrm{yrs})$.ag.

14. (child* or adolescen*).tw.

15. (child* or adolescen $^{\star}$ or infant $\left.{ }^{\star}\right)$.tw.

16. pediatr $^{\star}$ or paediatr $\left.{ }^{\star}\right) . t w$.

17. (boys or girls or youth or youths).tw.

18. or/13-17

19. 12 and 18

20. exp experimental design/

21. exp clinical trials/

22. (clinical ${ }^{*}$ stud $^{\star}$ or single-blind or single blind or triple-blind or triple blind).tw.

23. (random* or clinical trial ${ }^{\star}$ or controlled study or double-blind or double blind).tw.

24. (matched communit* or matched school* or matched population*).tw.

25. ((control or comparison) adj group).tw.

26. (outcome study or outcome studies).tw. 
27. matched pair ${ }^{*}$.tw.

28. (quasiexperimental or quasi experimental or pseudo experimental).tw.

29. prospectiv $^{\star}$.tw.

30. volunteer ${ }^{\star}$. tw.

31. ((before and after) adj3 (trial* or study or studies or design*)).tw.

32. time series.tw.

33. latin square.tw.

34. or/20-33

35. 19 and 34

36. limit 35 to $y r=" 2010-2015 "$

\section{CINAHL}

\section{Searched 11 June 2015}

\section{Limits: publication date March 2010 to search date}

1. (MH "Obesity+")

2. (MH "Weight Gain")

3. (MH "Weight Loss")

4. (TI obese or obesity) OR (AB obese or obesity)

5. ( $\mathrm{TI}$ weight gain or weight loss) $\mathrm{OR}$ ( $\mathrm{AB}$ weight gain or weight loss)

6. (TI weight change*) OR ( $A B$ weight change $\left.{ }^{\star}\right)$

7. (TI bmi N2 loss) OR (AB bmi N2 loss)

8. (TI bmi N2 gain) OR (AB bmi N2 gain)

9. (TI bmi N2 change) OR (AB bmi N2 change)

10. (TI body mass index N2 change) OR (AB body mass index N2 change)

11. (TI body mass index N2 gain) OR (AB body mass index N2 gain)

12. (TI body mass index N2 loss) OR (AB body mass index N2 loss)

13. (1 or 2 or 3 or 4 or 5 or 6 or 7 or 8 or 9 or 10 or 11 or 12$)$

14. (MH “Child+")

15. (MH "Child")

16. (MH “Infant+")

17. (MH "Adolescence")

18. (( TI child $^{\star}$ or adolescen ${ }^{\star}$ or infant $\left.^{\star}\right)$ OR (AB child* or adolescen* or infant $\left.^{\star}\right)$ )

19. ((TI teenage\$ or young people or young person or young adult $\left.t^{\star}\right)$ OR (AB teenage or young people or young person or young adult $\left.\left.{ }^{\star}\right)\right)$

20. (TI schoolchildren) OR (AB schoolchildren)

21. (14 or 15 or 16 or 17 or 18 or 19 or 20$)$

22. 13 and 21 
23. (MH "Study Design+")

24. (MH "Evaluation Research+")

25. (MH "Comparative Studies")

26. (MH "Random Assignment")

27. (MH "Random Sample+")

28. (MH "Placebos")

29. (MH “Clinical Trials")

30. (PT “CLINICAL TRIAL”)

31. clin* N25 trial*

32. clin* N25 stud*

33. latin square

34. time series

35. TX random*

36. TX matched communities or matched schools or matched populations

\section{TX comparison group ${ }^{\star}$}

38. TX matched pair*

39. TX outcome study or outcome studies

40. TX quasiexperimental or quasi experimental or pseudo experimental

41. TX nonrandomi* or pseudorandomi* or quasirandomi*

42. $T X$ prospectiv*

43. TX volunteer

44. $(23$ or 24 or 25 or 26 or 27 or 28 or 29 or 30 or 31 or 32 or 33 or 34 or 35 or 36 or 37 or 38 or 39 or 40 or 41 or 42 or 43 )

45. 22 and 44, Limiters - Published Date: 20100101-20151231

\section{Appendix 3. Search strategies 2010}

\section{CENTRAL 2010, Issue 1}

\section{Searched 26 March 2010}

Limits: CENTRAL; 2005, Issue 1 to 2010, Issue 1

1. MeSH descriptor Obesity explode all trees

2. MeSH descriptor Body Weight Changes explode all trees

3. $\left(\right.$ obes $\left.^{\star}\right)$

4. ("weight gain" or "weight loss")

5. (overweight or "over weight" or overeat ${ }^{\star}$ or (over next eat*))

6. (weight next change*)

7. ((bmi or "body mass index") near (gain or loss or change $\left.{ }^{\star}\right)$ )

8. (1 OR 2 OR 3 OR 4 OR 5 OR 6 OR 7 )

9. MeSH descriptor Behavior Therapy explode all trees

10. MeSH descriptor Social Support explode all trees

11. MeSH descriptor Psychotherapy, Group explode all trees

12. ((psychological or behavio? $\left.r^{\star}\right)$ near (therapy or modif* or strateg* or intervention $\left.{ }^{\star}\right)$ )

13. ("group therapy" or "family therapy" or "cognitive therapy")

14. (lifestyle or "life style") near (chang* or intervention*)

15. counsel?ing 
16. "social support"

17. (peer near2 support)

18. (children near3 parent ${ }^{\star}$ near3 therapy)

19. (9 OR 10 OR 11 OR 12 OR 13 OR 14 OR 15 OR 16 OR 17 OR 18)

20. MeSH descriptor Obesity explode all trees with qualifier: $\mathrm{DH}$

21. MeSH descriptor Diet Therapy explode all trees

22. MeSH descriptor Fasting, this term only

23. (diets or diet or dieting)

24. diet $^{\star}$ near (modif* or therapy or intervention* or strateg*)

25. "low calorie" or (calorie next control ${ }^{\star}$ ) or "healthy eating"

26. (fasting or (modified next fast $\left.{ }^{\star}\right)$ )

27. MeSH descriptor Dietary Fats explode all trees

28. (fruit or vegetable*)

29. (high next fat ${ }^{\star}$ ) or (low next fat $\left.{ }^{\star}\right)$ or (fatty next food ${ }^{\star}$ )

30. formula next diet ${ }^{*}$

31. (20 OR 21 OR 22 OR 23 OR 24 OR 25 OR 26 OR 27 OR 28 OR 29 OR 30)

32. MeSH descriptor Exercise explode all trees

33. MeSH descriptor Exercise Therapy explode all trees

34. exercis ${ }^{\star}$

35. (aerobics or "physical therapy" or "physical activity" or "physical inactivity")

36. fitness near (class* or regime* or program ${ }^{\star}$ )

37. ("physical training" or "physical education")

38. "dance therapy"

39. sedentary next behavio? ${ }^{\star}$

40. (32 OR 33 OR 34 OR 35 OR 36 OR 37 OR 38 OR 39)

41. MeSH descriptor Complementary Therapies explode all trees

42. "alternative medicine" or (complementary next therap*) or "complementary medicine"

43. (hypnotism or hypnosis or hypnotherapy)

44. (acupuncture or homeopathy or homoeopathy)

45. ("chinese medicine" or "indian medicine" or "herbal medicine" or ayurvedic)

46. (41 OR 42 OR 43 OR 44 OR 45)

47. (diet ${ }^{\star}$ or slim*) near (club* or organi?ation)

48. (weightwatcher ${ }^{\star}$ or (weight next watcher $\left.{ }^{\star}\right)$ )

49. correspondence near (course* or program ${ }^{\star}$ )

50. (fat or diet $\left.{ }^{\star}\right)$ next camp*

51. (47 OR 48 OR 49 OR 50)

52. MeSH descriptor Health Promotion explode all trees

53. MeSH descriptor Health Education explode all trees

54. ("health promotion" or "health education")

55. ("media intervention*" or "community intervention*")

56. (health next promoting next school*)

57. ((school or community) near2 program*)

58. ((school or community) near2 intervention*)

59. (family next intervention $\left.{ }^{\star}\right)$ or (parent* next intervention*)

60. (parent ${ }^{\star}$ near2 (behavio? $r^{\star}$ or involve ${ }^{\star}$ or control ${ }^{\star}$ or attitude ${ }^{\star}$ or educat $\left.{ }^{\star}\right)$ )

61. (52 OR 53 OR 54 OR 55 OR 56 OR 57 OR 58 OR 59 OR 60)

62. MeSH descriptor Health Policy explode all trees

63. (health next polic ${ }^{\star}$ ) or (school next polic ${ }^{\star}$ ) or (food next polic ${ }^{\star}$ ) or (nutrition next polic ${ }^{\star}$ )

64. (62 OR 63)

65. MeSH descriptor Obesity explode all trees with qualifier: PC

66. MeSH descriptor Primary Prevention explode all trees

67. ("primary prevention" or "secondary prevention")

68. (preventive next measure ${ }^{\star}$ ) or (preventative next measure ${ }^{\star}$ )

69. ("preventive care" or "preventative care")

70. (obesity near2 (prevent* or treat $\left.{ }^{\star}\right)$ )

71. (65 OR 66 OR 67 OR 68 OR 69 OR 70)

72. (19 OR 31 OR 40 OR 46 OR 51 OR 61 OR 64 OR 71)

73. (8 AND 72)

74. MeSH descriptor Child explode all trees

75. MeSH descriptor Infant explode all trees

76. (child* or adolescen ${ }^{\star}$ or infant ${ }^{\star}$ )

77. (teenage* or "young people" or "young person" or (young next adult*)) 
78. (schoolchildren or "school children")

79. (pediatr ${ }^{\star}$ or paediatr ${ }^{\star}$ )

80. (boys or girls or youth or youths)

81. MeSH descriptor Adolescent, this term only

82. (74 OR 75 OR 76 OR 77 OR 78 OR 79 OR 80 OR 81)

83. (73 AND 82)

\section{Ovid MEDLINE (1950 to March Week 2 2010)}

\section{Searched 24 March 2010}

Limits: entry date Feb 2005-search date

1. exp Obesity/

2. Weight Gain/

3. exp Weight Loss/

4. obes\$.af.

5. (weight gain or weight loss).af.

6. (overweight or over weight or overeat\$ or over eat\$).af.

7. weight change\$.af.

8. ((bmi or body mass index) adj2 (gain or loss or change)).af.

9. or/1-8

10. exp Behavior Therapy/

11. social support/

12. exp Psychotherapy, Group/

13. ((psychological or behavio?r\$) adj (therapy or modif\$ or strateg\$ or intervention\$)).af.

14. (group therapy or family therapy or cognitive therapy).af.

15. ((lifestyle or life style) adj (chang\$ or intervention\$)).af.

16. counsel?ing.af.

17. social support.af.

18. (peer adj2 support).af.

19. (children adj3 parent\$ adj3 therapy).af.

20. or/10-19

21. exp OBESITY/dh (Diet Therapy]

22. exp Diet Therapy/

23. Fasting/

24. (diets or diet or dieting).af.

25. (diet\$ adj (modif\$ or therapy or intervention\$ or strateg\$)).af.

26. (low calorie or calorie control\$ or healthy eating).af.

27. (fasting or modified fast\$).af.

28. exp Dietary Fats/

29. (fruit or vegetable\$).af.

30. (high fat\$ or low fat\$ or fatty food\$).af.

31. formula diet\$.af.

32. or $/ 21-31$

33. exp Exercise/

34. exp Exercise Therapy/

35. exercis\$.af.

36. (aerobics or physical therapy or physical activity or physical inactivity).af.

37. (fitness adj (class $\$$ or regime $\$$ or program $\$$ )).af.

38. (aerobics or physical therapy or physical training or physical education).af.

39. dance therapy.af.

40. sedentary behavio?r.af.

41. or $/ 33-40$

42. exp Complementary Therapies/

43. (alternative medicine or complementary therap\$ or complementary medicine).af.

44. (hypnotism or hypnosis or hypnotherapy).af.

45. (acupuncture or homeopathy or homoeopathy).af.

46. (chinese medicine or indian medicine or herbal medicine or ayurvedic).af.

47. or/42-46

48. ((diet or dieting or slim\$) adj (club\$ or organi?ation)).af.

49. (weightwatcher\$ or weight watcher\$).af.

50. (correspondence adj (course\$ or program\$)).af.

51. (fat camp\$ or diet\$ camp\$).af. 
52. or/48-51

53. exp Health Promotion/

54. exp Health Education/

55. (health promotion or health education).af.

56. (media intervention\$ or community intervention\$).af.

57. health promoting school\$.af.

58. ((school or community) adj2 program\$).af.

59. ((school or community) adj2 intervention\$).af.

60. (family intervention\$ or parent\$ intervention).af.

61. (parent\$ adj2 (behavio?r or involve\$ or control\$ or attitude\$ or educat\$)).af.

62. or/53-61

63. exp Health Policy/

64. (health polic\$ or school polic\$ or food polic\$ or nutrition polic\$).af.

65.63 or 64

66. exp OBESITY/pc (Prevention and Control]

67. exp Primary Prevention/

68. (primary prevention or secondary prevention).af.

69. (preventive measure\$ or preventative measure\$).af.

70. (preventive care or preventative care).af.

71. (obesity adj2 (prevent\$ or treat\$)).af.

72. or/66-71

73. randomized controlled trial.pt.

74. controlled clinical trial.pt.

75. Random Allocation/

76. Double-Blind Method/

77. single-blind method/

78. Placebos/

79. *Research Design/

80. intervention studies/

81. evaluation studies/

82. Comparative Study/

83. exp Longitudinal Studies/

84. cross-over studies/

85. clinical trial.tw.

86. clinical trial.pt.

87. latin square.tw.

88. (time adj series).tw.

89. (before adj2 after adj3 (stud\$ or trial\$ or design\$)).tw.

90. ((singl\$ or doubl\$ or trebl\$ or tripl\$) adj5 (blind\$ or mask)).tw.

91. placebo\$.tw.

92. random\$.tw.

93. (matched communities or matched schools or matched populations).tw.

94. control\$.tw.

95. (comparison group\$ or control group\$).tw.

96. matched pairs.tw.

97. (outcome study or outcome studies).tw.

98. (quasiexperimental or quasi experimental or pseudo experimental).tw.

99. (nonrandomi?ed or non randomi?ed or pseudo randomi?sed or quasi randomi?ed).tw.

100. prospectiv\$.tw.

101. volunteer\$.tw.

102. or $/ 73-101$

103. 20 or 32 or 41 or 47 or 52 or 62 or 65 or 72

104. 9 and 102 and 103

105. Animals/

106. exp Child/

107. Adolescent/

108. exp Infant/

109. (child\$ or adolescen\$ or infant\$).af.

110. (teenage\$ or young people or young person or young adult\$).af.

111. (schoolchildren or school children).af.

112. (pediatr\$ or paediatr\$).af.

113. (boys or girls or youth or youths).af. 
114. or/106-113

115. 104 not 105

116. 114 and 115

117. limit 116 to Date of Publication from 20050201-

\section{Embase OVID (1980 to 2010 Week 11)}

\section{Searched 24 March 2010}

Limits: entry 2005-2010

1. exp obesity/

2. weight gain/

3. weight reduction/

4. obes\$.af.

5. (weight gain or weight loss).af.

6. (overweight or over weight or overeat\$ or over eat\$).af.

7. weight change $\$$.af.

8. ((bmi or body mass index) adj2 (gain or loss or change)).af.

9. or/1-8

10. behavior therapy/

11. social support/

12. family therapy/

13. group therapy/

14. ((psychological or behavio?r\$) adj (therapy or modif\$ or strateg\$ or intervention\$)).af.

15. (group therapy or family therapy or cognitive therapy).af.

16. ((lifestyle or life style) adj (chang\$ or intervention\$)).af.

17. counsel?ing.af.

18. social support.af.

19. (peer adj2 support).af.

20. (children adj3 parent\$ adj3 therapy).af.

21. or $/ 10-20$

22. exp diet therapy/

23. (diets or diet or dieting).af.

24. (diet\$ adj (modif\$ or therapy or intervention\$ or strateg\$)).af.

25. (low calorie or calorie control\$ or healthy eating).af.

26. (fasting or modified fast\$).af.

27. exp fat intake/

28. (fruit or vegetable\$).af.

29. (high fat\$ or low fat\$ or fatty food\$).af.

30. formula diet\$.af.

31. or/22-30

32. exp exercise/

33. exp kinesiotherapy/

34. exercis\$.af.

35. (aerobics or physical therapy or physical activity or physical inactivity).af.

36. (fitness adj (class\$ or regime\$ or program\$)).af.

37. (aerobics or physical therapy or physical training or physical education).af.

38. dance therapy.af.

39. sedentary behavio?r.af.

40. or/32-39

41. exp alternative medicine/

42. (alternative medicine or complementary therap\$ or complementary medicine).af.

43. (hypnotism or hypnosis or hypnotherapy).af.

44. (acupuncture or homeopathy or homoeopathy).af.

45. (chinese medicine or indian medicine or herbal medicine or ayurvedic).af.

46. or/41-45

47. ((diet or dieting or slim\$) adj (club\$ or organi?ation)).af.

48. (weightwatcher\$ or weight watcher\$).af.

49. (correspondence adj (course $\$$ or program $\$)$ ). af.

50. (fat camp\$ or diet\$ camp\$).af.

51. or/47-50

52. exp health education/

53. (health promotion or health education).af.

Interventions for preventing obesity in children (Review) 
54. (media intervention\$ or community intervention\$).af.

55. health promoting school\$.af.

56. ((school or community) adj2 program\$).af.

57. ((school or community) adj2 intervention\$).af.

58. (family intervention\$ or parent intervention).af.

59. (parent\$ adj2 (behavio?r or involve $\$$ or control\$ or attitude $\$$ or educat\$)).af.

60. or/52-59

61. health care policy/

62. (health polic\$ or school polic\$ or food polic\$ or nutrition polic\$).af.

63.61 or 62

64. exp obesity/pc (Prevention]

65. primary prevention/

66. (primary prevention or secondary prevention).af.

67. (preventive measure $\$$ or preventative measure\$).af.

68. (preventive care or preventative care).af.

69. (obesity adj2 (prevent\$ or treat\$)).af.

70. or/64-69

71. exp clinical trial/

72. exp Randomized Controlled Trial/

73. randomization/

74. exp Double-Blind procedure/

75. exp Single-Blind procedure/

76. exp Crossover procedure/

77. clinical trial.tw.

78. ((singl\$ or doubl\$ or treble\$ or tripl\$) and (mask\$ or blind\$)).tw.

79. latin square.tw.

80. placebo/

81. placebo\$.tw.

82. random\$.tw.

83. Comparative Study/

84. evaluation/

85. clinical trial.tw.

86. latin square.tw.

87. (before adj2 after adj3 (stud\$ or trial\$ or design\$)).tw.

88. ((singl\$ or doubl\$ or trebl\$ or tripl\$) adj5 (blind\$ or mask)).tw.

89. (matched communities or matched schools or matched populations).tw.

90. control\$.tw.

91. (comparison group\$ or control group\$).tw.

92. matched pairs.tw.

93. (outcome study or outcome studies).tw.

94. (quasiexperimental or quasi experimental or pseudo experimental).tw.

95. (nonrandomi?ed or non randomi?ed or pseudo randomi?sed or quasi randomi?ed).tw.

96. prospectiv\$.tw.

97. volunteer\$.tw.

98. or/71-97

99. 21 or 31 or 40 or 46 or 51 or 60 or 63 or 70

100. 9 and 98 and 99

101. animal/

102. exp child/

103. $\exp$ ADOLESCENT/

104. exp preschool child/

105. exp infant/

106. (child\$ or adolescen\$ or infant\$).af.

107. (teenage\$ or young people or young person or young adult\$).af.

108. (schoolchildren or school children).af.

109. (pediatr\$ or paediatr\$).af.

110. (boys or girls or youth or youths).af.

111. or/102-110

112. 100 not 101

113. 111 and 112

114. 113 and (2005-2010]/py 


\section{PsycINFO 1806 to March Week 32010}

\section{Searched 24 March 2010}

Limits: Date Range: 2005-2010

1. exp overweight/

2. weight control/

3. obes*.tw.

4. weight gain ${ }^{\star}$. tw.

5 . weight loss ${ }^{\star}$.tw.

6. (overweight or over weight).tw.

7. weight loss/

8. weight gain/

9. (overeat ${ }^{\star}$ or over eat $\left.{ }^{\star}\right)$.tw.

10. weight change*.tw.

11. ((bmi or body mass) adj3 (gain ${ }^{\star}$ or loss ${ }^{\star}$ or change $\left.e^{\star}\right)$ ).tw.

12. or/1-11

13. (adolescence 1317 yrs or childhood birth 12 yrs or infancy 223 mo or neonatal birth 1 mo or preschool age 25 yrs or school age 6

$12 \mathrm{yrs})$.ag.

14. $\left(\right.$ child $^{\star}$ or adolescen $\left.{ }^{\star}\right)$.tw.

15. (child* or adololescen ${ }^{\star}$ or infant $\left.{ }^{\star}\right)$.tw.

16. (pediatr* or paediatr $\left.{ }^{\star}\right)$.tw.

17. (boys or girls or youth or youths).tw.

18. or/13-17

19. 12 and 18

20. exp experimental design/

21. exp clinical trials/

22. (clinical ${ }^{\star}$ stud $^{\star}$ or single-blind or single blind or triple-blind or triple blind).tw.

23. (random* or clinical trial ${ }^{\star}$ or controlled study or double-blind or double blind).tw.

24. (matched communit* or matched school ${ }^{\star}$ or matched population $\left.{ }^{\star}\right)$.tw.

25. ((control or comparison) adj group).tw.

26. (outcome study or outcome studies).tw.

27. matched pair*.tw.

28. (quasiexperimental or quasi experimental or pseudo experimental).tw.

29. prospectiv $^{\star}$. tw.

30. volunteer*.tw.

31. ("before and after" adj3 (trial* or study or studies or design $\left.{ }^{\star}\right)$ ).tw.

32. time series.tw.

33. latin square.tw.

34. or $/ 20-33$

35. 19 and 34

36. limit 35 to Date Range: 2005 to 2010

\section{CINAHL Plus with full text}

\section{Searched 25 March 2010}

Limits: entry date Feb 2005 -

\section{1. (MH "Obesity+")}

2. (MH "Weight Gain")

3. (MH "Weight Loss")

4. (TI obese or obesity) OR (AB obese or obesity)

5. (TI weight gain or weight loss) OR ( $A B$ weight gain or weight loss)

6. (TI weight change $\left.{ }^{\star}\right)$ OR (AB weight change $\left.{ }^{\star}\right)$

7. (TI bmi N2 loss) OR (AB bmi N2 loss)

8. (TI bmi N2 gain) OR (AB bmi N2 gain)

9. (TI bmi N2 change) OR (AB bmi N2 change)

10. (TI body mass index N2 change) OR (AB body mass index N2 change)

11. (TI body mass index N2 gain) OR (AB body mass index N2 gain)

12. ( $\mathrm{TI}$ body mass index N2 loss) OR (AB body mass index N2 loss)

13. (1 or 2 or 3 or 4 or 5 or 6 or 7 or 8 or 9 or 10 or 11 or 12)

14. (MH "Child+")

15. (MH "Child") 
16. (MH "Infant+")

17. (MH "Adolescence")

18. (TI child* or adolescen ${ }^{\star}$ or infant $\left.{ }^{\star}\right)$ OR (AB child* or adolescen* or infant $\left.{ }^{\star}\right)$

19. (TI teenage $\$$ or young people or young person or young adult $\left.t^{\star}\right)$ OR (AB teenage\$ or young people $r$ young person or young adult $t^{\star}$ )

20. ( $\mathrm{TI}$ schoolchildren) OR (AB schoolchildren)

21. (14 or 15 or 16 or 17 or 18 or 19 or 20 )

22. 13 and 21

23. (MH "Study Design+")

24. (MH "Evaluation Research+")

25. (MH "Comparative Studies")

26. (MH "Random Assignment")

27. (MH "Random Sample+")

28. (MH "Placebos")

29. (MH "Clinical Trials")

30. (PT "CLINICAL TRIAL")

31. clin* N25 trial*

32. clin* N25 stud*

33. latin square

34. time series

35. TX random ${ }^{\star}$

36. TX matched communities or matched schools or matched populations

37. TX comparison group*

38. TX matched pair ${ }^{\star}$

39. TX outcome study or outcome studies

40. TX quasiexperimental or quasi experimental or pseudo experimental

41. TX nonrandomi* or pseudorandomi* or quasirandomi*

42. TX prospectiv*

43. TX volunteer

44. (23 or 24 or 25 or 26 or 27 or 28 or 29 or 30 or 31 or 32 or 33 or 34 or 35 or 36 or 37 or 38 or 39 or 40 or 41 or 42 or 43 )

45. 22 and 44

46. 45 and em 200502-

\section{Appendix 4. Search strategies 2005}

\section{CENTRAL (in The Cochrane Library) (2005 update)}

\section{5, Issue 1}

1. $\exp$ OBESITY/

2. exp Weight Gain/

3. exp Weight Loss/

4. obes\$.af.

5. (weight gain or weight loss).af.

6. (overweight or over weight or overeat\$ or over eat\$).af.

7. weight change\$.af.

8. ((bmi or body mass index) adj2 (gain or loss or change)).af.

9. or/1-8

10. exp Behavior Therapy/

11. exp Social Support/

12. exp Family Therapy/

13. exp Psychotherapy, Group/

14. ((psychological or behavio?r\$) adj (therapy or modif\$ or strateg\$ or intervention\$)).af.

15. (group therapy or family therapy or cognitive therapy).af.

16. ((lifestyle or life style) adj (chang\$ or intervention\$)).af.

17. counsel?ing.af.

18. social support.af.

19. (peer adj2 support).af.

20. (children adj3 parent\$ adj therapy).af.

21. or $/ 10-20$

22. exp OBESITY/dh (Diet Therapy]

23. exp Diet, Fat-Restricted/

24. exp Diet, Reducing/

25. exp Diet Therapy/ 
26. exp FASTING/

27. (diets or diet or dieting).af.

28. (diet\$ adj (modif\$ or therapy or intervention\$ or strateg\$)).af.

29. (low calorie or calorie control\$ or healthy eating).af.

30. (fasting or modified fast\$).af.

31. exp Dietary Fats/

32. (fruit or vegetable\$).af.

33. (high fat\$ or low fat\$ or fatty food\$).af.

34. formula diet\$.af.

35. or/22-34

36. exp EXERCISE/

37. exp Exercise Therapy/

38. exercis\$.af.

39. (aerobics or physical therapy or physical activity or physical inactivity).af.

40. (fitness adj (class\$ or regime\$ or program\$)).af.

41. (aerobics or physical therapy or physical training or physical education).af.

42. dance therapy.af.

43. sedentary behavio?r.af.

44. or/36-43

45. exp Complementary Therapies/

46. (alternative medicine or complementary therap\$ or complementary medicine).af.

47. (hypnotism or hypnosis or hypnotherapy).af.

48. (acupuncture or homeopathy or homoeopathy).af.

49. (chinese medicine or indian medicine or herbal medicine or ayurvedic).af.

50. or/45-49

51. ((diet or dieting or slim\$) adj (club\$ or organi?ation)).af.

52. (weightwatcher\$ or weight watcher\$).af.

53. (correspondence adj (course $\$$ or program $\$)$ ).af.

54. (fat camp\$ or diet\$ camp\$).af.

55. or/51-54

56. exp Health Promotion/

57. exp Health Education/

58. (health promotion or health education).af.

59. (media intervention\$ or community intervention\$).af.

60 . health promoting school\$.af.

61. ((school or community) adj2 program\$).af.

62. ((school or community) adj2 intervention\$).af.

63. (family intervention $\$$ or parent $\$$ intervention).af.

64. (parent $\$$ adj2 (behavio?r or involve $\$$ or control\$ or attitude $\$$ or educat $\$)$ ).af.

65. or/56-64

66. exp Health Policy/

67. exp Nutrition Policy/

68. (health polic\$ or school polic\$ or food polic\$ or nutrition polic\$).af.

69. or/66-68

70. $\exp$ OBESITY/pc (Prevention and Control]

71. exp Primary Prevention/

72. (primary prevention or secondary prevention).af.

73. (preventive measure or preventative measure\$).af.

74. (preventive care or preventative care).af.

75. (obesity adj2 (prevent\$ or treat\$)).af.

76. or/70-75

77. randomized controlled trial.pt.

78. controlled clinical trial.pt.

79. exp Controlled Clinical Trials/

80. exp Random Allocation/

81. exp Double-Blind Method/

82. exp Single-Blind Method/

83. exp Placebos/

84. *Research Design/

85. exp Intervention studies/

86. exp Evaluation studies/

87. exp Comparative Study/ 
88. exp Follow-Up Studies/

89. exp Prospective Studies/

90. exp Cross-over Studies/

91. clinical trial.tw.

92. clinical trial.pt.

93. latin square.tw.

94. (time adj series).tw.

95. (before adj2 after adj3 (stud\$ or trial\$ or design\$)).tw.

96. ((singl\$ or doubl\$ or trebl\$ or tripl\$) adj5 (blind\$ or mask)).tw.

97. placebo\$.tw.

98. random\$.tw.

99. (matched communities or matched schools or matched populations).tw.

100. control\$.tw.

101. (comparison group $\$$ or control group\$).tw.

102. matched pairs.tw.

103. (outcome study or outcome studies).tw.

104. (quasiexperimental or quasi experimental or pseudo experimental).tw.

105. (nonrandomi?ed or non randomi?ed or pseudo randomi?sed or quasi randomi?ed).tw.

106. prospectiv\$.tw.

107. volunteer\$.tw.

108. or/77-107

109. 21 or 35 or 44 or 50 or 55 or 65 or 69 or 76

110. 9 and 109 and 108

111. Animals/

112. $\exp$ CHILD/

113. $\exp$ CHILD, PRESCHOOL/ or CHILD/

114. exp INFANT/

115. (child\$ or adolescen\$ or infant\$).af.

116. (teenage $\$$ or young people or young person or young adult\$).af.

117. (schoolchildren or school children).af.

118. (pediatr\$ or paediatr\$).af.

119. (boys or girls or youth or youths).af.

120. or/112-119

121. 110 not 111

122. 121 and 120

\section{MEDLINE (through Ovid) (2005 update)}

\section{Searched 12 February 2005/16 February 2005}

\section{1. exp OBESITY/}

2. exp Weight Gain/

3. exp Weight Loss/

4. obes\$.af.

5. (weight gain or weight loss).af.

6 . (overweight or over weight or overeat\$ or over eat\$).af.

7. weight change\$.af.

8. ((bmi or body mass index) adj2 (gain or loss or change)).af.

9. or/1-8

10. exp Behavior Therapy/

11. exp Social Support/

12. exp Family Therapy/

13. exp Psychotherapy, Group/

14. ((psychological or behavio?r\$) adj (therapy or modif\$ or strateg\$ or intervention\$)).af.

15. (group therapy or family therapy or cognitive therapy).af.

16. ((lifestyle or life style) adj (chang\$ or intervention\$)).af.

17. counsel?ing.af.

18. social support.af.

19. (peer adj2 support).af.

20. (children adj3 parent\$ adj therapy).af.

21. or/10-20

22. exp OBESITY/dh (Diet Therapy]

23. exp Diet, Fat-Restricted/ 
24. exp Diet, Reducing/

25. exp Diet Therapy/

26. $\exp$ FASTING/

27. (diets or diet or dieting).af.

28. (diet\$ adj (modif\$ or therapy or intervention\$ or strateg\$)).af.

29. (low calorie or calorie control\$ or healthy eating).af.

30. (fasting or modified fast\$).af.

31. exp Dietary Fats/

32. (fruit or vegetable\$).af.

33. (high fat\$ or low fat\$ or fatty food\$).af.

34. formula diet\$.af.

35. or/22-34

36. $\exp$ EXERCISE/

37. exp Exercise Therapy/

38. exercis\$.af.

39. (aerobics or physical therapy or physical activity or physical inactivity).af.

40. (fitness adj (class\$ or regime\$ or program\$)).af.

41. (aerobics or physical therapy or physical training or physical education).af.

42. dance therapy.af.

43. sedentary behavio?r.af.

44. or/36-43

45. exp Complementary Therapies/

46. (alternative medicine or complementary therap\$ or complementary medicine).af.

47. (hypnotism or hypnosis or hypnotherapy).af.

48. (acupuncture or homeopathy or homoeopathy).af.

49. (chinese medicine or indian medicine or herbal medicine or ayurvedic).af.

50. or/45-49

51. ((diet or dieting or slim\$) adj (club\$ or organi?ation)).af.

52. (weightwatcher\$ or weight watcher\$).af.

53. (correspondence adj (course $\$$ or program $\$$ )).af.

54. (fat camp\$ or diet\$ camp\$).af.

55. or/51-54

56. exp Health Promotion/

57. exp Health Education/

58. (health promotion or health education).af.

59. (media intervention\$ or community intervention\$).af.

60 . health promoting school\$.af.

61. ((school or community) adj2 program\$).af.

62. ((school or community) adj2 intervention\$).af.

63. (family intervention $\$$ or parent $\$$ intervention).af.

64. (parent\$ adj2 (behavio?r or involve\$ or control\$ or attitude\$ or educat\$)).af.

65. or/56-64

66. exp Health Policy/

67. exp Nutrition Policy/

68. (health polic\$ or school polic\$ or food polic\$ or nutrition polic\$).af.

69. or/66-68

70. exp OBESITY/pc (Prevention and Control]

71. exp Primary Prevention/

72. (primary prevention or secondary prevention).af.

73. (preventive measure\$ or preventative measure\$).af.

74. (preventive care or preventative care).af.

75. (obesity adj2 (prevent\$ or treat\$)).af.

76. or/70-75

77. randomized controlled trial.pt.

78. controlled clinical trial.pt.

79. $\exp$ Controlled Clinical Trials/

80. exp Random Allocation/

81. exp Double-Blind Method/

82. exp Single-Blind Method/

83. exp Placebos/

84. *Research Design/

85. exp Intervention studies/

Interventions for preventing obesity in children (Review) 
86. exp Evaluation studies/

87. exp Comparative Study/

88. exp Follow-Up Studies/

89. exp Prospective Studies/

90. exp Cross-over Studies/

91. clinical trial.tw.

92. clinical trial.pt.

93. latin square.tw.

94. (time adj series).tw.

95. (before adj2 after adj3 (stud\$ or trial\$ or design\$)).tw.

96. ((singl\$ or doubl\$ or trebl\$ or tripl\$) adj5 (blind\$ or mask)).tw.

97. placebo\$.tw.

98. random\$.tw.

99. (matched communities or matched schools or matched populations).tw.

100. control\$.tw.

101. (comparison group\$ or control group\$).tw.

102. matched pairs.tw.

103. (outcome study or outcome studies).tw.

104. (quasiexperimental or quasi experimental or pseudo experimental).tw.

105. (nonrandomi?ed or non randomi?ed or pseudo randomi?sed or quasi randomi?ed).tw.

106. prospectiv\$.tw.

107. volunteer\$.tw.

108. or/77-107

109. 21 or 35 or 44 or 50 or 55 or 65 or 69 or 76

110.9 and 109 and 108

111. Animals/

112. $\exp$ CHILD/

113. $\exp$ ADOLESCENT/

114. $\exp$ CHILD, PRESCHOOL/ or CHILD/

115. exp INFANT/

116. (child\$ or adolescen\$ or infant\$).af.

117. (teenage\$ or young people or young person or young adult\$).af.

118. (schoolchildren or school children).af.

119. (pediatr\$ or paediatr\$).af.

120. (boys or girls or youth or youths).af.

121. or/112-120

122. 110 not 111

123. 122 and 121

124. limit 123 to $y r=1990-2005$

\section{EMBASE (2005 update)}

\section{Dates 1990 to 2005}

1. $\exp$ OBESITY/

2. exp Weight Gain/

3. exp Weight Loss/

4. obes\$.af.

5. (weight gain or weight loss).af.

6. (overweight or over weight or overeat\$ or over eat\$).af.

7. weight change\$.af.

8. ((bmi or body mass index) adj2 (gain or loss or change)).af.

9. or/1-8

10. exp Behavior Therapy/

11. exp Social Support/

12. exp Family Therapy/

13. exp Psychotherapy, Group/

14. ((psychological or behavio?r\$) adj (therapy or modif\$ or strateg\$ or intervention\$)).af.

15. (group therapy or family therapy or cognitive therapy).af.

16. ((lifestyle or life style) adj (chang\$ or intervention\$)).af.

17. counsel?ing.af.

18. social support.af.

19. (peer adj2 support).af.

Interventions for preventing obesity in children (Review)

Copyright $\odot 2019$ The Cochrane Collaboration. Published by John Wiley \& Sons, Ltd. 
20. (children adj3 parent $\$$ adj therapy).af.

21. or/10-20

22. exp OBESITY/dh (Diet Therapy]

23. exp Diet, Fat-Restricted/

24. exp Diet, Reducing/

25. exp Diet Therapy/

26. $\exp$ FASTING/

27. (diets or diet or dieting).af.

28. (diet\$ adj (modif\$ or therapy or intervention\$ or strateg\$)).af.

29. (low calorie or calorie control\$ or healthy eating).af.

30. (fasting or modified fast\$).af.

31. exp Dietary Fats/

32. (fruit or vegetable\$).af.

33. (high fat\$ or low fat\$ or fatty food\$).af.

34. formula diet $\$$.af.

35. or/22-34

36. $\exp$ EXERCISE/

37. exp Exercise Therapy/

38. exercis\$.af.

39. (aerobics or physical therapy or physical activity or physical inactivity).af.

40. (fitness adj (class\$ or regime\$ or program\$)).af.

41. (aerobics or physical therapy or physical training or physical education).af.

42. dance therapy.af.

43. sedentary behavio?r.af.

44. or/36-43

45. exp Complementary Therapies/

46. (alternative medicine or complementary therap\$ or complementary medicine).af.

47. (hypnotism or hypnosis or hypnotherapy).af.

48. (acupuncture or homeopathy or homoeopathy).af.

49. (chinese medicine or indian medicine or herbal medicine or ayurvedic).af.

50. or/45-49

51. ((diet or dieting or slim\$) adj (club\$ or organi?ation)).af.

52. (weightwatcher\$ or weight watcher\$).af.

53. (correspondence adj (course $\$$ or program $\$)$ ).af.

54. (fat camp\$ or diet\$ camp\$).af.

55. or/51-54

56. exp Health Promotion/

57. exp Health Education/

58. (health promotion or health education).af.

59. (media intervention\$ or community intervention\$).af.

60. health promoting school\$.af.

61. ((school or community) adj2 program\$).af.

62. ((school or community) adj2 intervention\$).af.

63. (family intervention $\$$ or parent $\$$ intervention).af.

64. (parent\$ adj2 (behavio?r or involve\$ or control\$ or attitude\$ or educat\$)).af.

65. or/56-64

66. exp Health Policy/

67. exp Nutrition Policy/

68. (health polic\$ or school polic\$ or food polic\$ or nutrition polic\$).af.

69. or/66-68

70. $\exp$ OBESITY/pc (Prevention and Control]

71. exp Primary Prevention/

72. (primary prevention or secondary prevention).af.

73. (preventive measure\$ or preventative measure\$).af.

74. (preventive care or preventative care).af.

75. (obesity adj2 (prevent\$ or treat\$)).af.

76. or/70-75

77. exp Clinical Trial/

78. exp Randomized Controlled Trial/

79. exp Randomization/

80. exp Double-Blind procedure/

81. exp Single-Blind procedure/

Interventions for preventing obesity in children (Review) 
82. exp Crossover procedure/

83. clinical trial.tw.

84. ((singl\$ or doubl\$ or treble\$ or tripl\$) and (mask\$ or blind\$)).tw.

85. latin square.tw.

86. exp PLACEBO/

87. placebo\$.tw.

88. random\$.tw.

89. Comparative Study/

90. exp Evaluation/

91. clinical trial.tw.

92. clinical trial.pt.

93. latin square.tw.

94. (before adj2 after adj3 (stud\$ or trial\$ or design\$)).tw.

95. ((singl\$ or doubl\$ or trebl\$ or tripl\$) adj5 (blind\$ or mask)).tw.

96. placebo\$.tw.

97. random\$.tw.

98. (matched communities or matched schools or matched populations).tw.

99. control\$.tw.

100. (comparison group\$ or control group\$).tw.

101. matched pairs.tw.

102. (outcome study or outcome studies).tw.

103. (quasiexperimental or quasi experimental or pseudo experimental).tw.

104. (nonrandomi?ed or non randomi?ed or pseudo randomi?sed or quasi randomi?ed).tw.

105. prospectiv\$.tw.

106. volunteer\$.tw.

107. or/77-107

108. 21 or 35 or 44 or 50 or 55 or 65 or 69 or 76

109. 9 and 108 and 107

110. Animals/

111. $\exp \mathrm{CHILD/}$

112. $\exp A D O L E S C E N T /$

113. $\exp$ CHILD, PRESCHOOL/ or CHILD/

114. exp INFANT/

115. (child\$ or adolescen\$ or infant\$).af.

116. (teenage\$ or young people or young person or young adult\$).af.

117. (schoolchildren or school children).af.

118. (pediatr\$ or paediatr\$).af.

119. (boys or girls or youth or youths).af.

120. or/111-119

121. 109 not 110

122. 121 and 120

123. limit 122 to $\mathrm{yr}=1990-2005$

\section{PsycINFO (2005 update)}

\section{Date 1990 to 2005}

1. exp OBESITY/

2. exp Weight Gain/

3. exp Weight Loss/

4. obes\$.af.

5. (weight gain or weight loss).af.

6. (overweight or over weight or overeat\$ or over eat\$).af.

7. weight change\$.af.

8. ((bmi or body mass index) adj2 (gain or loss or change)).af.

9. or/1-8

10. exp Behavior Therapy/

11. exp Social Support/

12. exp Family Therapy/

13. exp Psychotherapy, Group/

14. ((psychological or behavio?r\$) adj (therapy or modif\$ or strateg\$ or intervention\$)).af.

15. (group therapy or family therapy or cognitive therapy).af.

16. ((lifestyle or life style) adj (chang\$ or intervention\$)).af. 
17. counsel?ing.af.

18. social support.af.

19. (peer adj2 support).af.

20. (children adj3 parent\$ adj therapy).af.

21. or/10-20

22. exp OBESITY/dh (Diet Therapy]

23. exp Diet, Fat-Restricted/

24. exp Diet, Reducing/

25. exp Diet Therapy/

26. exp FASTING/

27. (diets or diet or dieting).af.

28. (diet\$ adj (modif\$ or therapy or intervention\$ or strateg\$)). af.

29. (low calorie or calorie control\$ or healthy eating).af.

30. (fasting or modified fast\$).af.

31. exp Dietary Fats/

32. (fruit or vegetable\$).af.

33. (high fat\$ or low fat\$ or fatty food\$).af.

34. formula diet\$.af.

35. or/22-34

36. exp EXERCISE/

37. exp Exercise Therapy/

38. exercis\$.af.

39. (aerobics or physical therapy or physical activity or physical inactivity).af.

40. (fitness adj (class\$ or regime\$ or program\$)).af.

41. (aerobics or physical therapy or physical training or physical education).af.

42. dance therapy.af.

43. sedentary behavio?r.af.

44. or/36-43

45. exp Complementary Therapies/

46. (alternative medicine or complementary therap\$ or complementary medicine).af.

47. (hypnotism or hypnosis or hypnotherapy).af.

48. (acupuncture or homeopathy or homoeopathy).af.

49. (chinese medicine or indian medicine or herbal medicine or ayurvedic).af.

50. or/45-49

51. ((diet or dieting or slim\$) adj (club\$ or organi?ation)).af.

52. (weightwatcher\$ or weight watcher\$).af.

53. (correspondence adj (course $\$$ or program\$)).af.

54. (fat camp\$ or diet\$ camp\$).af.

55. or/51-54

56. exp Health Promotion/

57. exp Health Education/

58. (health promotion or health education).af.

59. (media intervention\$ or community intervention\$).af.

60 . health promoting school\$.af.

61. ((school or community) adj2 program\$).af.

62. ((school or community) adj2 intervention\$).af.

63. (family intervention $\$$ or parent $\$$ intervention).af.

64. (parent\$ adj2 (behavio?r or involve\$ or control\$ or attitude\$ or educat\$)).af.

65. or/56-64

66. exp Health Policy/

67. exp Nutrition Policy/

68. (health polic\$ or school polic\$ or food polic\$ or nutrition polic\$).af.

69. or/66-68

70. $\exp$ OBESITY/pc (Prevention and Control]

71. exp Primary Prevention/

72. (primary prevention or secondary prevention).af.

73. (preventive measure\$ or preventative measure\$).af.

74. (preventive care or preventative care).af.

75. (obesity adj2 (prevent\$ or treat\$)).af.

76. or/70-75

77.21 or 35 or 44 or 50 or 55 or 65 or 69 or 76

78. Animals/

Interventions for preventing obesity in children (Review)

Copyright @ 2019 The Cochrane Collaboration. Published by John Wiley \& Sons, Ltd. 
79. (child\$ or adolescen\$ or infant\$).af.

80. (teenage\$ or young people or young person or young adult\$).af.

81. (schoolchildren or school children).af.

82. (pediatr\$ or paediatr\$).af.

83. (boys or girls or youth or youths).af.

84. or/79-82

85.9 and 77 and 84

86.85 not 78

\section{CINAHL (2005 update)}

\section{Date 1990 to 2005}

1. exp OBESITY/

2. exp Weight Gain/

3. exp Weight Loss/

4. obes\$.af.

5. (weight gain or weight loss).af.

6. (overweight or over weight or overeat\$ or over eat\$).af.

7. weight change\$.af.

8. ((bmi or body mass index) adj2 (gain or loss or change)).af.

9. or/1-8

10. exp Behavior Therapy/

11. exp Social Support/

12. exp Family Therapy/

13. exp Psychotherapy, Group/

14. ((psychological or behavio?r\$) adj (therapy or modif\$ or strateg\$ or intervention\$)).af.

15. (group therapy or family therapy or cognitive therapy).af.

16. ((lifestyle or life style) adj (chang\$ or intervention\$)).af.

17. counsel?ing.af.

18. social support.af.

19. (peer adj2 support).af.

20. (children adj3 parent\$ adj therapy).af.

21. or $/ 10-20$

22. $\exp$ OBESITY/dh (Diet Therapy]

23. exp Diet, Fat-Restricted/

24. exp Diet, Reducing/

25. exp Diet Therapy/

26. exp FASTING/

27. (diets or diet or dieting).af.

28. (diet\$ adj (modif\$ or therapy or intervention\$ or strateg\$)).af.

29. (low calorie or calorie control\$ or healthy eating).af.

30. (fasting or modified fast\$).af.

31. exp Dietary Fats/

32. (fruit or vegetable\$).af.

33. (high fat\$ or low fat\$ or fatty food\$).af.

34. formula diet\$.af.

35. or/22-34

36. $\exp$ EXERCISE/

37. exp Exercise Therapy/

38. exercis\$.af.

39. (aerobics or physical therapy or physical activity or physical inactivity).af.

40. (fitness adj (class\$ or regime\$ or program\$)).af.

41. (aerobics or physical therapy or physical training or physical education).af.

42. dance therapy.af.

43. sedentary behavio?r.af.

44. or/36-43

45. exp Complementary Therapies/

46. (alternative medicine or complementary therap\$ or complementary medicine).af.

47. (hypnotism or hypnosis or hypnotherapy).af.

48. (acupuncture or homeopathy or homoeopathy).af.

49. (chinese medicine or indian medicine or herbal medicine or ayurvedic).af.

50. or/45-49 
51. ((diet or dieting or slim\$) adj (club\$ or organi?ation)).af.

52. (weightwatcher\$ or weight watcher\$).af.

53. (correspondence adj (course $\$$ or program\$)).af.

54. (fat camp\$ or diet\$ camp\$).af.

55. or/51-54

56. exp Health Promotion/

57. exp Health Education/

58. (health promotion or health education).af.

59. (media intervention\$ or community intervention\$).af.

60. health promoting school\$.af.

61. ((school or community) adj2 program\$).af.

62. ((school or community) adj2 intervention\$).af.

63. (family intervention\$ or parent\$ intervention).af.

64. (parent\$ adj2 (behavio?r or involve $\$$ or control\$ or attitude $\$$ or educat $\$$ )).af.

65. or/56-64

66. exp Health Policy/

67. exp Nutrition Policy/

68. (health polic\$ or school polic\$ or food polic\$ or nutrition polic\$).af.

69. or/66-68

70. $\exp \mathrm{OBESITY/pc} \mathrm{(Prevention} \mathrm{and} \mathrm{Control]}$

71. exp Primary Prevention/

72. (primary prevention or secondary prevention).af.

73. (preventive measure\$ or preventative measure\$).af.

74. (preventive care or preventative care).af.

75. (obesity adj2 (prevent\$ or treat\$)).af.

76. or/70-75

77. exp study design/

78. exp evaluation research/

79. exp comparative studies/

80. exp Random Assignment/

81. exp Random sample/

82. exp Placebos/

83. exp Prospective Studies/

84. clinical trial.tw.

85. clinical trial.pt.

86. (clin\$ adj25 (trial\$ or stud\$)).mp. (mp=title, cinahl subject headings, abstract, instrumentation]

87. latin square.tw.

88. (time adj series).tw.

89. (before adj2 after adj3 (stud\$ or trial\$ or design\$)).tw.

90. ((singl\$ or doubl\$ or trebl\$ or tripl\$) adj5 (blind\$ or mask)).tw.

91. placebo\$.tw.

92. random\$.tw.

93. (matched communities or matched schools or matched populations).tw.

94. control\$.tw.

95. (comparison group\$ or control group\$).tw.

96. matched pairs.tw.

97. (outcome study or outcome studies).tw.

98. (quasiexperimental or quasi experimental or pseudo experimental).tw.

99. (nonrandomi?ed or non randomi?ed or pseudo randomi?sed or quasi randomi?ed).tw.

100. prospectiv\$.tw.

101. volunteer\$.tw.

102. or/77-101

103. 21 or 35 or 44 or 50 or 55 or 65 or 69 or 76

104. Animals/

105. $\exp$ CHILD/

106. $\exp$ ADOLESCENT/

107. $\exp$ CHILD, PRESCHOOL/ or CHILD/

108. exp INFANT/

109. (child\$ or adolescen\$ or infant\$).af.

110. (teenage $\$$ or young people or young person or young adult $\$$ ).af.

111. (schoolchildren or school children).af.

112. (pediatr\$ or paediatr\$).af. 
113. (boys or girls or youth or youths).af.

114. or/105-113

115.9 and 103

116. 115 and 102 and 114

117. 116 not 104

\section{Appendix 5. Adjusting analyses for the effects of clustering}

Fourteen randomised controlled trials (RCTs) had not adjusted for clustering in their analyses. Two of these studies did not have data that could be used in a meta-analysis (Farias 2015; Sallis 1993). We adjusted data from the remaining 12 studies (5 with zBMI data; 9 with BMI data) and for clustering using the methods described in the Cochrane Handbook for Systematic Reviews of Interventions (Higgins 2011a).

The tables below list:

- the calculation of design effect, and the adjustment to the standard error (SE) of the effect size for the 12 studies;

- effect sizes, both unadjusted and adjusted for clustering, using intracluster correlation coefficient 0.04 for the outcome zBMI;

- effect sizes, both unadjusted and adjusted for clustering, using intracluster correlation coefficient 0.04 for the outcome BMI. 


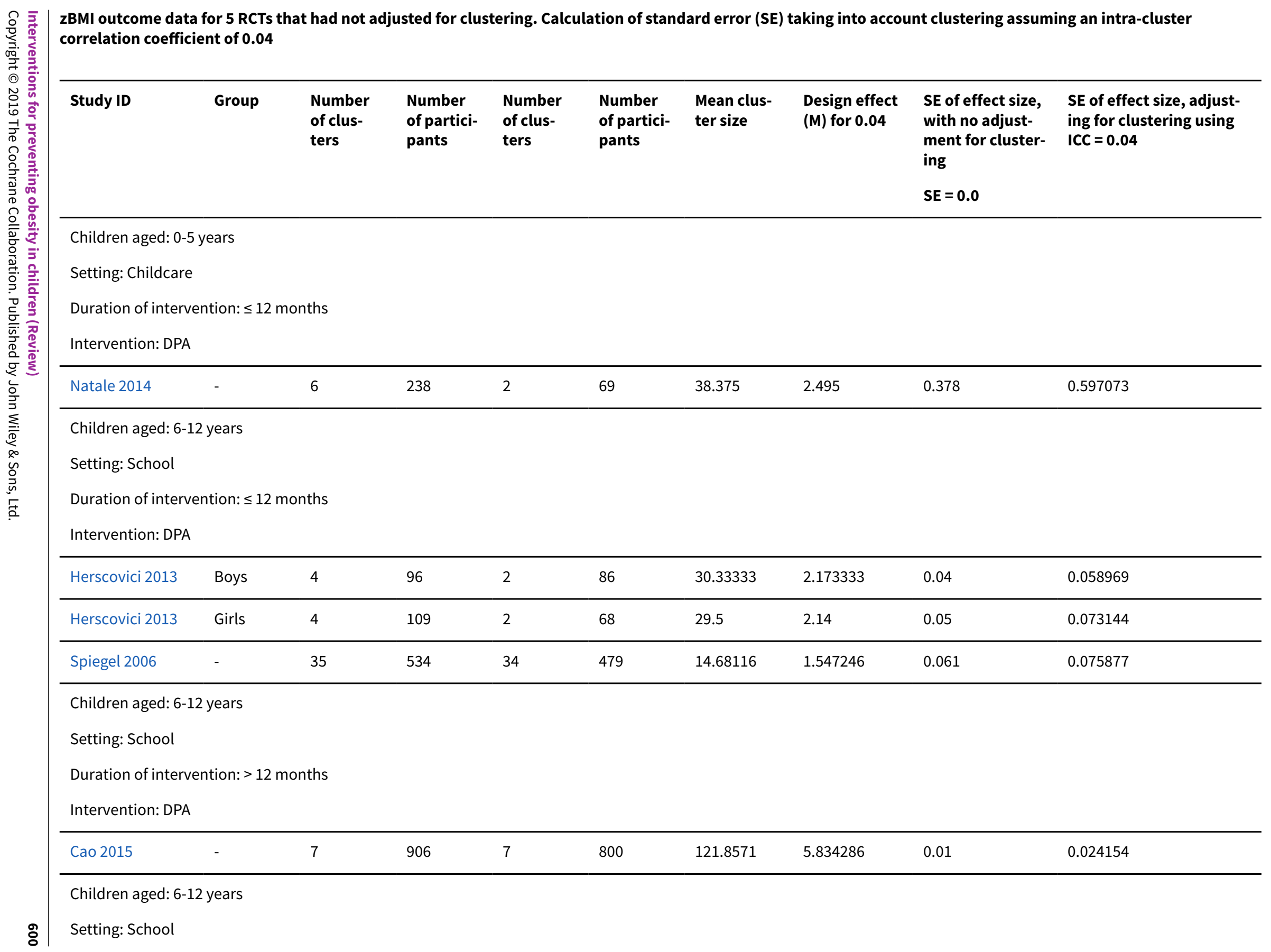




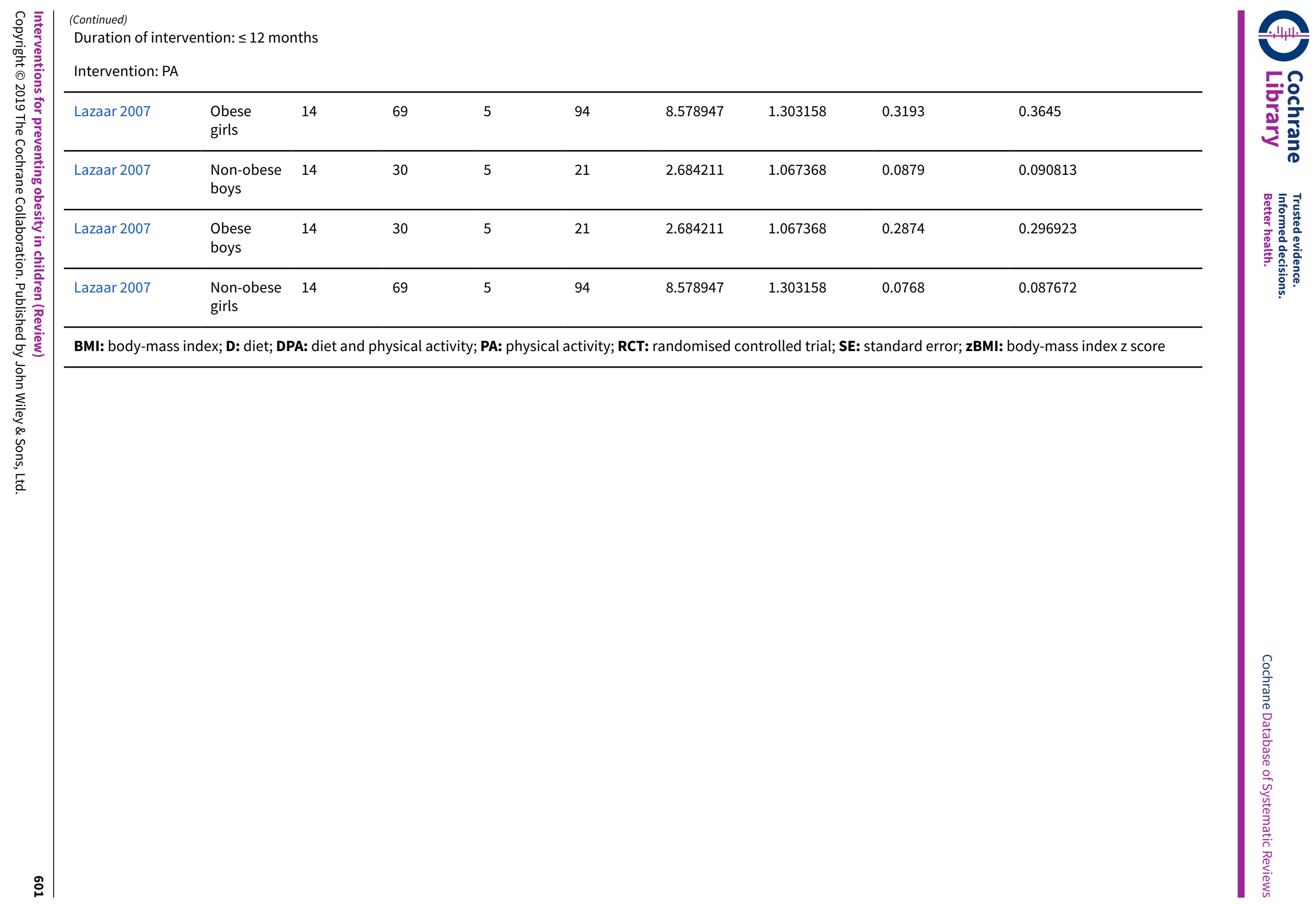




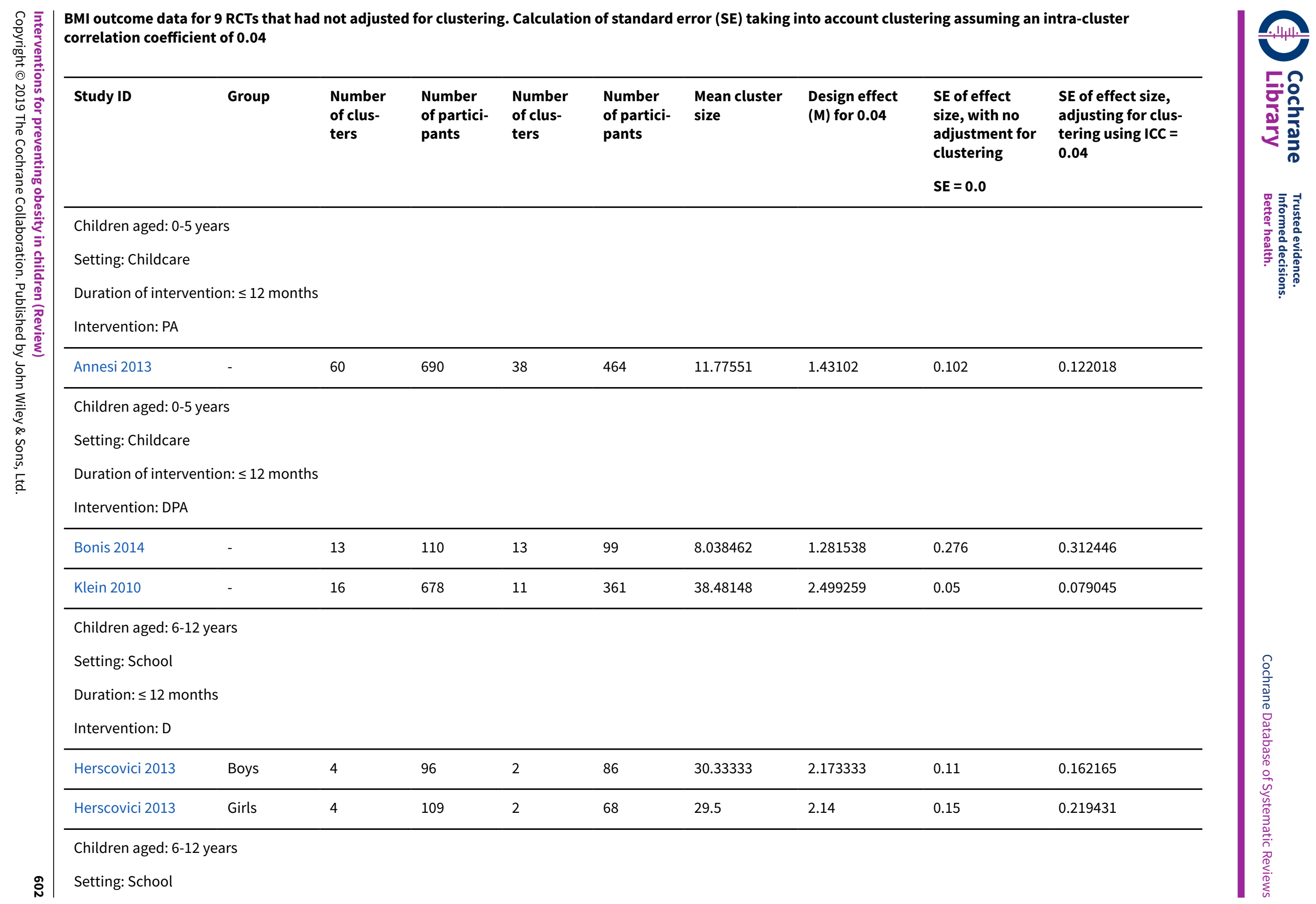




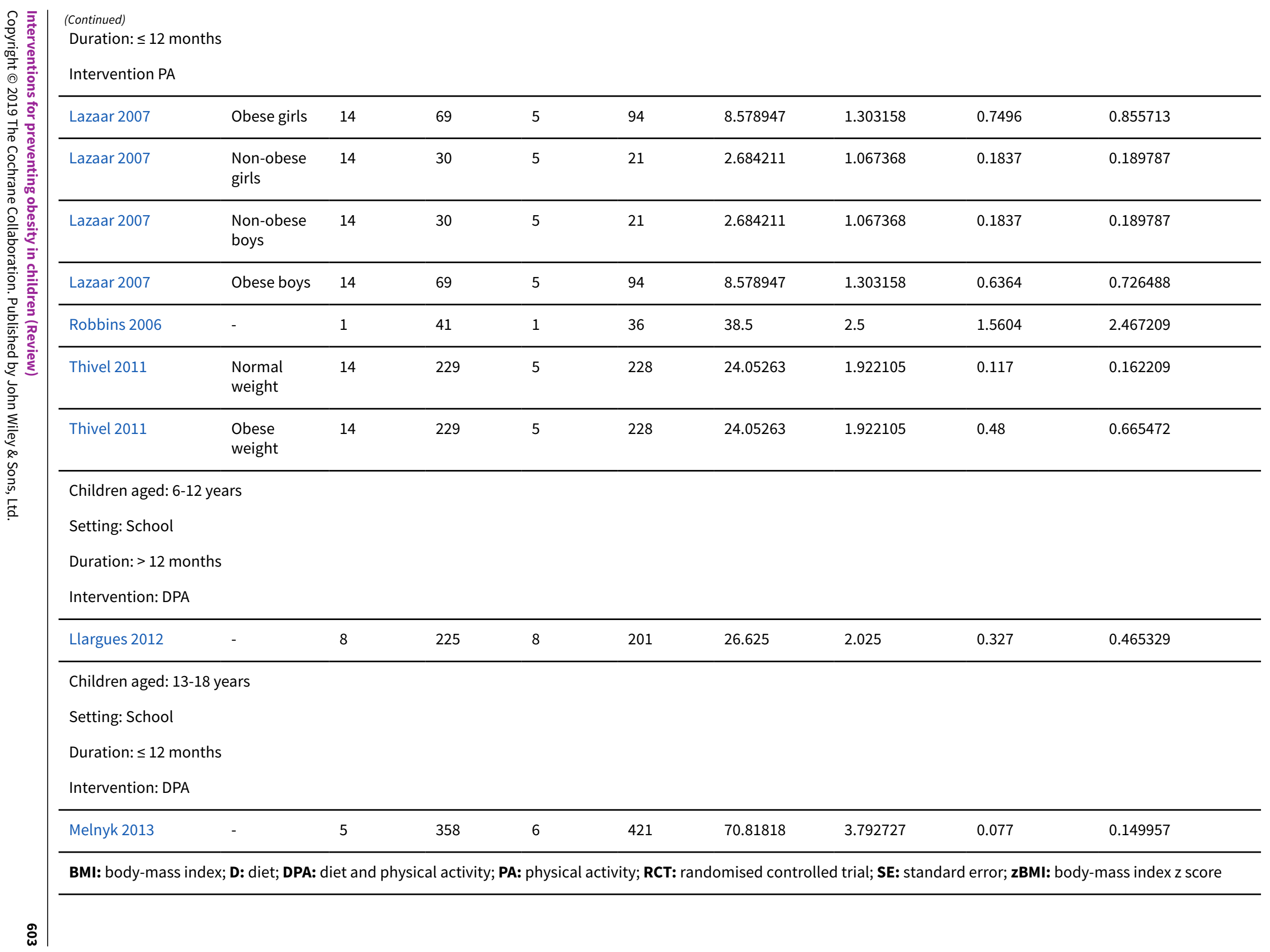


Effect sizes, both unadjusted and adjusted for clustering, using intracluster correlation coefficient 0.04 for the outcome zBMI All interventions. Subgroup: intervention types. Age group: 0 to 5 years. Outcome zBMI

\begin{tabular}{llll}
\hline Comparison/subgroup & Number of RCTs & $\begin{array}{l}\text { Pooled effect sizes not adjust- } \\
\text { ed }\end{array}$ & $\begin{array}{l}\text { Pooled effect sizes adjusted with ICC } \\
\mathbf{0 . 0 4 a}\end{array}$ \\
\hline All interventions vs control & 21 & MD (IV, random, 95\% CI) & MD (IV, random, 95\% CI) \\
\hline Dietary intervention & 1 & $-0.07(-0.12$ to -0.01$)$ & $-0.07(-0.12$ to -0.01$)$ \\
\hline$\cdot$ Physical activity intervention & 4 & $-0.14(-0.32$ to 0.04$)$ & $-0.14(-0.32$ to 0.04$)$ \\
\hline $\begin{array}{l}\text { Diet and physical activity inter- } \\
\text { vention }\end{array}$ & 16 & $0.01(-0.10$ to 0.13$)$ & $0.01(-0.10$ to 0.13$)$ \\
\hline
\end{tabular}

CI: confidence interval; ICC: intracluster correlation coefficient; IV: generic inverse variance; MD: mean difference; Random: random-effects model; RCT: randomised controlled trial; zBMI: body-mass index z score

aFigures in bold indicate a difference from the unadjusted value.

Intervention types. Subgroup: setting/duration of intervention. Age group: 0 to 5 years. Outcome: zBMI

\begin{tabular}{|c|c|c|c|}
\hline Comparison/subgroup & Number of RCTs & $\begin{array}{l}\text { Pooled effect sizes not adjust- } \\
\text { ed }\end{array}$ & $\begin{array}{l}\text { Pooled effect sizes adjusted } \\
\text { with ICC } 0.04^{a}\end{array}$ \\
\hline & & MD (IV, random, 95\% Cl) & MD (IV, random, 95\% CI) \\
\hline Diet vs physical activity control & 1 & $-0.14(-0.32$ to 0.04$)$ & No change to datab \\
\hline Physical activity vs control - setting & 4 & $0.01(-0.10$ to 0.13$)$ & No change to datab \\
\hline - Health system & 2 & $0.02(-0.14$ to 0.17$)$ & No change to datab \\
\hline - Childcare/preschool & 2 & $0.01(-0.17$ to 0.19$)$ & No change to datab \\
\hline $\begin{array}{l}\text { Diet and physical activity vs control - } \\
\text { setting }\end{array}$ & 16 & $-0.07(-0.13$ to -0.01$)$ & $-0.07(-0.14$ to -0.01$)$ \\
\hline - Childcare/preschool & 10 & $-0.04(-0.09$ to 0.01$)$ & $-0.04(-0.09$ to 0.01$)$ \\
\hline - Health system & 1 & $-0.24(-0.46$ to -0.02$)$ & $-0.24(-0.46$ to -0.02$)$ \\
\hline - Wider community & 2 & $-0.02(-0.13$ to 0.09$)$ & $-0.02(-0.13$ to 0.09$)$ \\
\hline - Home & 3 & $-0.13(-0.35$ to 0.09$)$ & $-0.13(-0.35$ to 0.09$)$ \\
\hline $\begin{array}{l}\text { Diet and physical activity vs control - } \\
\text { duration }\end{array}$ & 16 & $-0.07(-0.13$ to -0.01$)$ & $-0.07(-0.14$ to -0.01$)$ \\
\hline - Duration of intervention $\leq 12$ months & 13 & $-0.09(-0.17$ to -0.01$)$ & $-0.09(-0.17$ to -0.01$)$ \\
\hline
\end{tabular}


(Continued)
- Duration of intervention $>12$ months
3
$-0.02(-0.09$ to 0.06$)$
$-0.02(-0.09$ to 0.06$)$

CI: confidence interval; ICC: intracluster correlation coefficient; IV: generic inverse variance; MD: mean difference; Random: random-effects model; RCT: randomised controlled trial; zBMI: body-mass index z score

aFigures in bold indicate a difference from the unadjusted value.

bNo change to data means - there were no RCTs in this subgroup analysis for which the analysis had not adjusted for clustering

All interventions. Subgroup: intervention types. Age group: 6 to 12 years. Outcome: zBMI

\begin{tabular}{llll}
\hline Comparison/subgroup & Number of RCTs & Pooled effect sizes not adjusted & $\begin{array}{l}\text { Pooled effect sizes adjusted with } \\
\text { ICC 0.04a }\end{array}$ \\
\hline All interventions & 34 & MD (IV, random, 95\% CI) & MD (IV, random, 95\% CI) \\
\hline Dietary intervention & 9 & $-0.04(-0.07$ to -0.02$)$ & $-0.04(-0.07$ to -0.02$)$ \\
\hline Physical activity intervention & 7 & $-0.03(-0.06$ to 0.01$)$ & $-0.03(-0.06$ to 0.01$)$ \\
\hline $\begin{array}{l}\text { Diet and physical activity inter- } \\
\text { vention }\end{array}$ & 20 & $-0.02(-0.06$ to 0.02$)$ & $-0.02(-0.06$ to 0.02$)$ \\
\hline
\end{tabular}

Cl: confidence interval; ICC: intracluster correlation coefficient; IV: generic inverse variance; MD: mean difference; Random: random-effects model; RCT: randomised controlled trial; zBMI: body-mass index z score

aFigures in bold indicate a difference from the unadjusted value.

Intervention types. Subgroup: setting/duration of intervention. Age group: 6 to 12 years. Outcome: zBMI

\begin{tabular}{llll}
\hline Comparison/subgroup & Number of RCTs & $\begin{array}{l}\text { Pooled effect sizes not adjust- } \\
\text { ed }\end{array}$ & $\begin{array}{l}\text { Pooled effect sizes adjusted } \\
\text { with ICC } \mathbf{0 . 0 4 a}\end{array}$ \\
\hline Diet vs control - setting & 9 & MD (IV, random, 95\% CI) & MD (IV, random, 95\% CI) \\
\hline$\cdot$ School & 8 & $-0.03(-0.06$ to 0.01$)$ & No change to datab \\
\hline$\cdot$ Wider community & 1 & $-0.02(-0.06$ to 0.01$)$ & No change to datab \\
\hline Diet vs control - duration & 9 & $-0.16(-0.35$ to 0.04$)$ & No change to datab \\
\hline$\cdot$ Duration of intervention $\leq 12$ months & 8 & $-0.03(-0.06$ to 0.01$)$ & No change to datab \\
\hline$\cdot$ Duration of intervention $>12$ months & 1 & $0.00(-0.01$ to 0.02$)$ & No change to datab \\
\hline Physical activity vs control - setting & 7 & $-0.13(-0.21$ to -0.05$)$ & $-0.02(-0.06$ to 0.02$)$ \\
\hline
\end{tabular}




\begin{tabular}{|c|c|c|c|}
\hline - Wider community & 2 & $-0.07(-0.19$ to 0.05$)$ & $-0.07(-0.19$ to 0.05$)$ \\
\hline - School & 5 & $-0.03(-0.06$ to 0.00$)$ & $-0.03(-0.07$ to 0.00$)$ \\
\hline Physical activity vs control - duration & 7 & $-0.02(-0.06$ to 0.02$)$ & $-0.02(-0.06$ to 0.02$)$ \\
\hline - Duration of intervention $\leq 12$ months & 3 & $0.02(-0.02$ to 0.05$)$ & $0.02(-0.02$ to 0.05$)$ \\
\hline - Duration of intervention $>12$ months & 4 & $-0.04(-0.10$ to 0.01$)$ & $-0.05(-0.09$ to -0.00$)$ \\
\hline $\begin{array}{l}\text { Diet and physical activity vs control - } \\
\text { setting }\end{array}$ & 20 & $-0.05(-0.09$ to -0.02$)$ & $-0.05(-0.10$ to -0.01$)$ \\
\hline - Community & 4 & $-0.04(-0.39$ to 0.31$)$ & $-0.04(-0.39$ to 0.31$)$ \\
\hline - School & 15 & $-0.04(-0.07$ to -0.02$)$ & $-0.04(-0.08$ to -0.01$)$ \\
\hline $\begin{array}{l}\text { Diet and physical activity vs control - } \\
\text { duration }\end{array}$ & 20 & $-0.05(-0.09$ to -0.02$)$ & $-0.05(-0.10$ to -0.01$)$ \\
\hline - Duration of intervention $>12$ months & 8 & $-0.05(-0.08$ to -0.01$)$ & $-0.05(-0.10$ to 0.00$)$ \\
\hline - Duration of intervention $\leq 12$ months & 12 & $-0.06(-0.12$ to 0.00$)$ & $-0.06(-0.12$ to 0.01$)$ \\
\hline
\end{tabular}

CI: confidence interval; ICC: intracluster correlation coefficient; IV: generic inverse variance; MD: mean difference; Random: random-effects model; $\mathbf{R C T}$ : randomised controlled trial; zBMI: body-mass index z score

aFigures in bold indicate a difference from the unadjusted value.

bNo change to data means - there were no RCTs in this subgroup analysis for which the analysis had not adjusted for clustering

All interventions. Subgroup: intervention types. Age group: 13 to 18 years. Outcome: zBMI

\begin{tabular}{llll}
\hline Comparison/subgroup & Number of RCTs & Pooled effect sizes not adjusted & $\begin{array}{l}\text { Pooled effect sizes adjusted with } \\
\text { ICC 0.04a }\end{array}$ \\
\hline All interventions & MD (IV, random, 95\% CI) & MD (IV, random, 95\% CI) \\
\hline Physical activity intervention & 1 & $-0.01(-0.07$ to 0.05$)$ & $-0.01(-0.07$ to 0.05$)$ \\
\hline $\begin{array}{l}\text { Diet and physical activity in- } \\
\text { tervention }\end{array}$ & 6 & $-0.20(-0.30$ to -0.10$)$ & $-0.20(-0.30$ to -0.10$)$ \\
\hline
\end{tabular}

CI: confidence interval; ICC: intracluster correlation coefficient; IV: generic inverse variance; MD: mean difference; Random: random-effects model; RCT: randomised controlled trial; zBMI: body-mass index z score

aFigures in bold indicate a difference from the unadjusted value.

Intervention types. Subgroup: setting/duration of intervention. Age group: 13 to 18 years. Outcome: zBMI 


\begin{tabular}{|c|c|c|c|}
\hline Comparison/subgroup & Number of RCTs & Pooled effect sizes not adjusted & $\begin{array}{l}\text { Pooled effect sizes adjusted } \\
\text { with ICC } 0.04^{a}\end{array}$ \\
\hline & & MD (IV, random, 95\% CI) & MD (IV, random, 95\% CI) \\
\hline $\begin{array}{l}\text { Physical activity interventions vs } \\
\text { control - setting }\end{array}$ & 1 & $-0.20(-0.30$ to -0.10$)$ & No change to datab \\
\hline - School & 1 & $-0.20(-0.30$ to -0.10$)$ & No change to datab \\
\hline Duration & 1 & $-0.20(-0.30$ to -0.10$)$ & No change to datab \\
\hline $\begin{array}{l}\text { Duration of intervention } \leq 12 \\
\text { months }\end{array}$ & 1 & $-0.20(-0.30$ to -0.10$)$ & No change to datab \\
\hline DPA vs control - setting & 6 & $0.01(-0.05$ to 0.07$)$ & No change to datab \\
\hline · Home & 1 & $0.06(-0.13$ to 0.26$)$ & No change to datab \\
\hline - School & 5 & $0.00(-0.06$ to 0.06$)$ & No change to datab \\
\hline Duration & 6 & $0.01(-0.05$ to 0.07$)$ & No change to datab \\
\hline $\begin{array}{l}\text { - Duration of intervention } \leq 12 \\
\text { months }\end{array}$ & 3 & $-0.09(-0.18$ to 0.00$)$ & No change to datab \\
\hline $\begin{array}{l}\text { - Duration of intervention }>12 \\
\text { months }\end{array}$ & 3 & $0.01(-0.02$ to 0.04$)$ & No change to datab \\
\hline
\end{tabular}

CI: confidence interval; ICC: intracluster correlation coefficient; IV: generic inverse variance; MD: mean difference; Random: random-effects model; RCT: randomised controlled trial; zBMI: body-mass index z score

aFigures in bold indicate a difference from the unadjusted value.

bNo change to data means - there were no RCTs in this subgroup analysis for which the analysis had not adjusted for clustering.

Effect sizes both unadjusted and adjusted for clustering using intracluster correlation coefficient 0.04 for the outcome BMI All interventions. Subgroup intervention types. Age group: 0 to 5 years. Outcome: BMI

\begin{tabular}{llll}
\hline Comparison/subgroup & Number of RCTs & Pooled effect sizes not adjusted & $\begin{array}{l}\text { Pooled effect sizes adjusted with } \\
\text { ICC } \mathbf{0 . 0 4}\end{array}$ \\
\hline & 16 & MD (IV, random, 95\% CI) & MD (IV, random, 95\% CI) \\
\hline Intervention type & 1 & $-0.12(-0.22$ to -0.02$)$ & $-\mathbf{0 . 1 2}(-\mathbf{0 . 2 2}$ to $-\mathbf{0 . 0 3})$ \\
\hline Dietary intervention & 4 & $-0.20(-0.59$ to 0.19$)$ & $-0.20(-0.59$ to 0.19$)$ \\
\hline Physical activity intervention & 4 & $-0.22(-0.49$ to 0.04$)$ & $-\mathbf{0 . 2 3}(-\mathbf{0 . 5 0}$ to 0.05$)$ \\
\hline $\begin{array}{l}\text { Diet and physical activity inter- } \\
\text { vention }\end{array}$ & 11 & $-0.09(-0.21$ to 0.03$)$ & $-\mathbf{0 . 0 9}(-\mathbf{0 . 2 0}$ to 0.01$)$ \\
\hline
\end{tabular}


(Continued)

BMI: body-mass index; Cl: confidence interval; ICC: intracluster correlation coefficient; IV: generic inverse variance; MD: mean difference; Random: random-effects model; RCT: randomised controlled trial

aFigures in bold indicate a difference from the unadjusted value.

Intervention types. Subgroup setting/duration of intervention. Age group: 0 to 5 years. Outcome: BMI

\begin{tabular}{|c|c|c|c|}
\hline Comparison/subgroup & Number of RCTs & $\begin{array}{l}\text { Pooled effect sizes not adjust- } \\
\text { ed }\end{array}$ & $\begin{array}{l}\text { Pooled effect sizes adjusted } \\
\text { with ICC } 0.04^{a}\end{array}$ \\
\hline & & MD (IV, random, 95\% CI) & MD (IV, random, 95\% CI) \\
\hline Diet vs physical activity control & 16 & $-0.12(-0.22$ to -0.02$)$ & $-0.12(-0.22$ to -0.03$)$ \\
\hline Physical activity vs control - setting & 1 & $-0.20(-0.59$ to 0.19$)$ & $-0.20(-0.59$ to 0.19$)$ \\
\hline - Health system & 4 & $-0.22(-0.49$ to 0.04$)$ & $-0.23(-0.50$ to 0.05$)$ \\
\hline - Childcare/preschool & 11 & $-0.09(-0.21$ to 0.03$)$ & $-0.09(-0.20$ to 0.01$)$ \\
\hline $\begin{array}{l}\text { Diet and physical activity vs control - } \\
\text { setting }\end{array}$ & . & No data for analysis & \\
\hline · Home & 5 & $-0.21(-0.43$ to 0.01$)$ & $-0.22(-0.44$ to 0.01$)$ \\
\hline - Wider community & 1 & $-0.20(-0.59$ to 0.19$)$ & $-0.20(-0.59$ to 0.19$)$ \\
\hline - Childcare/preschool & 4 & $-0.22(-0.49$ to 0.04$)$ & $-0.23(-0.50$ to 0.05$)$ \\
\hline $\begin{array}{l}\text { Diet and physical activity vs control - } \\
\text { duration }\end{array}$ & 11 & $-0.09(-0.21$ to 0.03$)$ & $-0.09(-0.20$ to 0.01$)$ \\
\hline - Duration of intervention $\leq 12$ months & 2 & $-0.33(-0.55$ to -0.10$)$ & $-0.33(-0.55$ to -0.10$)$ \\
\hline - Duration of intervention $>12$ months & 1 & $-0.59(-0.94$ to -0.24$)$ & $-0.59(-0.94$ to -0.24$)$ \\
\hline
\end{tabular}

BMI: body-mass index; Cl: confidence interval; ICC: intracluster correlation coefficient; IV: generic inverse variance; MD: mean difference; Random: random-effects model; RCT: randomised controlled trial

aFigures in bold indicate a difference from the unadjusted value.

All interventions. Subgroup intervention types. Age group: 6 to 12 years

\begin{tabular}{llll}
\hline Comparison/subgroup & Number of RCTs & Pooled effect sizes not adjusted & $\begin{array}{l}\text { Pooled effect sizes adjusted with ICC } \\
\mathbf{0 . 0 4 a}\end{array}$ \\
\hline & & MD (IV, random, $\mathbf{9 5 \%}$ Cl) & MD (IV, random, 95\% CI) \\
\hline Intervention type & 41 & $-0.07(-0.10$ to -0.03$)$ & $-\mathbf{0 . 0 6}(-\mathbf{0 . 1 0}$ to $-\mathbf{0 . 0 3})$ \\
\hline
\end{tabular}


(Continued)

\begin{tabular}{llll}
$\cdot$ Dietary intervention & 5 & $-0.02(-0.11$ to 0.06$)$ & $-0.02(-0.11$ to 0.06$)$ \\
\hline $\begin{array}{l}\text { Physical activity intervention } \\
\text { Diet and physical activity in- } \\
\text { tervention }\end{array}$ & 25 & $-0.10(-0.15$ to -0.05$)$ & $-\mathbf{0 . 1 0}(-\mathbf{0 . 1 4}$ to $-\mathbf{0 . 0 5})$ \\
\hline
\end{tabular}

BMI: body-mass index; CI: confidence interval; ICC: intracluster correlation coefficient; IV: generic inverse variance; MD: mean difference; Random: random-effects model; RCT: randomised controlled trial

aFigures in bold indicate a difference from the unadjusted value.

Intervention types. Subgroup setting/duration of intervention. Age group: 6 to 12 years. Outcome: BMI

\begin{tabular}{llll}
\hline Comparison/subgroup & Number of RCTs & $\begin{array}{l}\text { Pooled effect sizes not ad- } \\
\text { justed }\end{array}$ & $\begin{array}{l}\text { Pooled effect sizes adjusted } \\
\text { with ICC 0.04a }\end{array}$ \\
\hline Diet vs control - setting & MD (IV, random, 95\% CI) & MD (IV, random, 95\% CI) \\
\hline School & 4 & $-0.07(-0.17$ to 0.03$)$ & No change to datab \\
\hline Wider community & 3 & $-0.06(-0.16$ to 0.04$)$ & No change to datab \\
\hline
\end{tabular}

Diet vs control - duration

\begin{tabular}{|c|c|c|c|}
\hline \multicolumn{4}{|l|}{ - Duration of intervention $\leq 12$ months } \\
\hline \multicolumn{4}{|l|}{ - Duration of intervention $>12$ months } \\
\hline Physical activity vs control - setting & 13 & $-0.10(-0.15$ to -0.05$)$ & $-0.10(-0.15$ to -0.05$)$ \\
\hline - Wider community & 2 & $-0.19(-0.50$ to 0.12$)$ & $-0.19(-0.50$ to 0.12$)$ \\
\hline - School & 11 & $-0.10(-0.14$ to -0.05$)$ & $-0.10(-0.14$ to -0.06$)$ \\
\hline Physical activity vs control - duration & 13 & $-0.10(-0.15$ to -0.05$)$ & $-0.10(-0.14$ to -0.05$)$ \\
\hline - Duration of intervention $\leq 12$ months & 10 & $-0.11(-0.16$ to -0.05$)$ & $-0.11(-0.16$ to -0.06$)$ \\
\hline - Duration of intervention $>12$ months & 3 & $0.00(-0.14$ to 0.14$)$ & $0.00(-0.14$ to 0.14$)$ \\
\hline Diet and physical activity vs control - setting & 13 & $-0.10(-0.15$ to -0.05$)$ & $-0.10(-0.14$ to -0.05$)$ \\
\hline - School & 10 & $-0.11(-0.16$ to -0.05$)$ & $-0.11(-0.16$ to -0.06$)$ \\
\hline - Wider community & 3 & $0.00(-0.14$ to 0.14$)$ & $0.00(-0.14$ to 0.14$)$ \\
\hline $\begin{array}{l}\text { Diet and physical activity vs control - dura- } \\
\text { tion }\end{array}$ & 25 & $-0.05(-0.11$ to 0.01$)$ & $-0.05(-0.11$ to 0.01$)$ \\
\hline - Duration of intervention $>12$ months & 8 & $-0.08(-0.19$ to 0.03$)$ & $-0.08(-0.18$ to 0.03$)$ \\
\hline
\end{tabular}


(Continued)
- Duration of intervention $\leq 12$ months
17
$-0.04(-0.11$ to 0.03$)$
$-0.04(-0.11$ to 0.04$)$

BMI: body-mass index; Cl: confidence interval; ICC: intracluster correlation coefficient; IV: generic inverse variance; MD: mean difference; Random: random-effects model; RCT: randomised controlled trial

aFigures in bold indicate a difference from the unadjusted value.

bNo change to data means - there were no RCTs in this subgroup analysis for which the analysis had not adjusted for clustering.

All interventions. Subgroup intervention types. Age group: 13 to 18 years. Outcome: BMI

\begin{tabular}{llll}
\hline Comparison/subgroup & Number of RCTs & Pooled effect sizes not adjusted & $\begin{array}{l}\text { Pooled effect sizes adjusted with } \\
\text { ICC 0.04a }\end{array}$ \\
\hline & 14 & MD (IV, random, 95\% CI) & MD (IV, random, 95\% CI) \\
\hline Intervention type & 2 & $-0.09(-0.20$ to 0.01$)$ & $-\mathbf{0 . 0 9}(-\mathbf{0 . 2 0}$ to 0.02$)$ \\
\hline Dietary interventions & $-0.13(-0.50$ to 0.23$)$ & $-0.13(-0.50$ to 0.23$)$ \\
\hline Physical activity interventions & 4 & $-1.53(-2.67$ to -0.39$)$ & $-1.53(-2.67$ to -0.39$)$ \\
\hline $\begin{array}{l}\text { Diet and physical activity inter- } \\
\text { ventions }\end{array}$ & 8 & $-0.03(-0.11$ to 0.04$)$ & $-\mathbf{0 . 0 2}(-\mathbf{0 . 1 0}$ to 0.05$)$ \\
\hline
\end{tabular}

BMI: body-mass index; CI: confidence interval; ICC: intracluster correlation coefficient; IV: generic inverse variance; MD: mean difference; Random: random-effects model; RCT: randomised controlled trial

aFigures in bold indicate a difference from the unadjusted value.

Intervention types. Subgroup setting/duration of intervention. Age group: 13 to 18 years. Outcome: BMI

\begin{tabular}{llll}
\hline Comparison/subgroup & Number of RCTs & Pooled effect sizes not adjusted & $\begin{array}{l}\text { Pooled effect sizes adjusted } \\
\text { with ICC } \mathbf{0 . 0 4 a}\end{array}$ \\
\hline & 2 & MD (IV, random, 95\% CI) & MD (IV, random, 95\% CI) \\
\hline Dietary interventions - setting & 1 & $-0.13(-0.50$ to 0.23$)$ & No change to datab \\
\hline$\cdot$ Home & 1 & $-0.14(-0.54$ to 0.26$)$ & No change to datab \\
\hline $\begin{array}{l}\text { School } \\
\text { Dietary interventions - duration }\end{array}$ & 4 & $-0.10(-0.99$ to 0.79$)$ & No change to datab \\
\hline $\begin{array}{l}\text { Duration of intervention } \leq 12 \\
\text { months }\end{array}$ & 4 & $-1.53(-2.67$ to -0.39$)$ & No change to datab \\
\hline $\begin{array}{l}\text { Physical activity interventions - set- } \\
\text { ting }\end{array}$ & 4 & $-1.53(-2.67$ to -0.39$)$ & No change to datab \\
\hline
\end{tabular}


(Continued)

\begin{tabular}{|c|c|c|c|}
\hline - School & 4 & $-1.53(-2.67$ to -0.39$)$ & No change to datab \\
\hline - Duration < 12 months & 4 & $-1.53(-2.67$ to -0.39$)$ & No change to datab \\
\hline $\begin{array}{l}\text { Diet and physical activity interven- } \\
\text { tions - setting }\end{array}$ & 8 & $-0.03(-0.11$ to 0.04$)$ & $-0.02(-0.10$ to 0.05$)$ \\
\hline Duration & 8 & $-0.03(-0.11$ to 0.04$)$ & $-0.02(-0.10$ to 0.05$)$ \\
\hline $\begin{array}{l}\text { Duration of intervention } \leq 12 \\
\text { months }\end{array}$ & 2 & $-0.04(-0.17$ to 0.09$)$ & $-0.04(-0.17$ to 0.09$)$ \\
\hline $\begin{array}{l}\text { Duration of intervention }>12 \\
\text { months }\end{array}$ & 6 & $-0.04(-0.13$ to 0.05$)$ & $-0.03(-0.11$ to 0.05$)$ \\
\hline
\end{tabular}

BMI: body-mass index; CI: confidence interval; ICC: intracluster correlation coefficient; IV: generic inverse variance; MD: mean difference; Random: random-effects model; RCT: randomised controlled trial

aFigures in bold indicate a difference from the unadjusted value.

bNo change to data means - there were no RCTs in this subgroup analysis for which the analysis had not adjusted for clustering.

\section{Appendix 6. Studies listed by continent, income level and country of origin}

Asia

\begin{tabular}{lll}
\hline Study ID & Incomea & Country \\
\hline Cao 2015 & High-income & China \\
\hline Feng 2004 & High-income & China \\
\hline Han 2006 & High-income & China \\
\hline Li 2010a & High-income & China \\
\hline Meng 2013 & High-income & China \\
\hline Wang 2012 & High-income & China \\
\hline Mo-suwan 1998 & Upper middle-income & Thailand \\
\hline
\end{tabular}

alncome based on World Bank classification of countries.

\section{Australasia}

\begin{tabular}{lll}
\hline Study ID & Income & Country \\
\hline Campbell 2013 & High-income & Australia \\
\hline \hline
\end{tabular}




\begin{tabular}{|c|c|c|}
\hline Daniels 2012 & High-income & Australia \\
\hline Dewar 2013 & High-income & Australia \\
\hline Lubans 2011 & High-income & Australia \\
\hline Morgan 2011 & High-income & Australia \\
\hline Peralta 2009 & High-income & Australia \\
\hline Rush 2012 & High-income & New Zealand \\
\hline Salmon 2008 & High-income & Australia \\
\hline Skouteris 2016 & High-income & Australia \\
\hline Smith 2014 & High-income & Australia \\
\hline Telford 2012 & High-income & Australia \\
\hline Weeks 2012 & High-income & Australia \\
\hline Wen 2012 & High-income & Australia \\
\hline Wilksch 2015 & High-income & Australia \\
\hline Zask 2012 & High-income & Australia \\
\hline
\end{tabular}

alncome based on World Bank classification of countries.

\section{Europe}

\begin{tabular}{lll}
\hline Study ID & Incomea & Country \\
\hline Amaro 2006 & High-income & Italy \\
\hline Bonsergent 2013 & High-income & France \\
\hline Bonvin 2013 & High-income & Switzerland \\
\hline Brandstetter 2012 & High-income & Germany \\
\hline Christiansen 2013 & High-income & Denmark \\
\hline Damsgaard 2014 & High-income & Denmark \\
\hline De Bock 2012 & High-income & Germany \\
\hline De Coen 2012 & High-income & Belgium \\
\hline De Ruyter 2012 & High-income & Netherlands \\
\hline De Vries 2015 & High-income & Netherlands \\
\hline \hline
\end{tabular}

Interventions for preventing obesity in children (Review) 
(Continued)

\begin{tabular}{|c|c|c|}
\hline Ezendam 2012 & High-income & Netherlands \\
\hline Fairclough 2013 & High-income & UK \\
\hline Grydeland 2014 & High-income & Norway \\
\hline Haerens 2006 & High-income & Belgium \\
\hline James 2004 & High-income & UK \\
\hline Keller 2009 & High-income & Germany \\
\hline Kipping 2008 & High-income & UK \\
\hline Kipping 2014 & High-income & UK \\
\hline Klein 2010 & High-income & Germany \\
\hline Kriemler 2010 & High-income & Switzerland \\
\hline Lazaar 2007 & High-income & France \\
\hline Llargues 2012 & High-income & Spain \\
\hline Magnusson 2012 & High-income & Iceland \\
\hline Marcus 2009 & High-income & Sweden \\
\hline Martinez-Vizcaino 2014 & High-income & Spain \\
\hline Muckelbauer 2010 & High-income & Germany \\
\hline Nyberg 2015 & High-income & Sweden \\
\hline Paineau 2008 & High-income & France \\
\hline Papadaki 2010 & High-income & $\begin{array}{l}\text { Netherlands, Denmark, UK, } \\
\text { Greece, Germany, Spain, Bulgar- } \\
\text { ia, and Czech Republic }\end{array}$ \\
\hline Puder 2011 & High-income & Switzerland \\
\hline Reilly 2006 & High-income & Scotland \\
\hline Rosario 2012 & High-income & Portugal \\
\hline Roth 2015 & High-income & Germany \\
\hline Sahota 2001 & High-income & UK \\
\hline Siegrist 2013 & High-income & Germany \\
\hline Simon 2008 & High-income & France \\
\hline
\end{tabular}




\begin{tabular}{lll}
$\begin{array}{lll}\text { (Continued) } \\
\text { Singh } 2009\end{array}$ & High-income & France \\
\hline Thivel 2011 & High-income & Belgium \\
\hline Verbestel 2014 & High-income & Italy \\
\hline Viggiano 2015 & High-income & Spain \\
\hline Vizcaino 2008 & High-income & England \\
\hline Warren 2003 & High-income & \\
\hline
\end{tabular}

alncome based on World Bank classification of countries.

\section{Europe and Central Asia}

\begin{tabular}{lll}
\hline Study ID & Incomea $^{\text {Country }}$ \\
\hline Sevinc 2011 & Upper middle-income & Turkey \\
\hline Yilmaz 2015 & Upper middle-income & Turkey \\
\hline
\end{tabular}

alncome based on World Bank classification of countries.

\section{Middle East and North Africa}

\begin{tabular}{lll}
\hline Study ID & Incomea $^{\mathbf{a}}$ & Country \\
\hline Nemet 2011 & High-income & Israel \\
\hline El Ansarai 2010 & Lower middle-income & Egypt \\
\hline Habib-Mourad 2014 & Upper middle-income & Lebanon \\
\hline
\end{tabular}

alncome based on World Bank classification of countries.

\section{North America}

\begin{tabular}{lll}
\hline Study ID & Incomea & Country \\
\hline Alkon 2014 & High-income & USA \\
\hline Annesi 2013 & High-income & USA \\
\hline Baranowski 2003 & High-income & USA \\
\hline
\end{tabular}


(Continued)

\begin{tabular}{|c|c|c|}
\hline Baranowski 2011 & High-income & USA \\
\hline Barkin 2012 & High-income & USA \\
\hline Beech 2003 & High-income & USA \\
\hline Bellows 2013a & High-income & USA \\
\hline Birken 2012 & High-income & Canada \\
\hline Black 2010 & High-income & USA \\
\hline Bohnert 2013 & High-income & USA \\
\hline Bonis 2014 & High-income & USA \\
\hline Bonuck 2014 & High-income & USA \\
\hline Branscum 2013 & High-income & USA \\
\hline Brown 2013 & High-income & USA \\
\hline Caballero 2003 & High-income & USA \\
\hline Chen 2010 & High-income & USA \\
\hline Chen 2011 & High-income & USA \\
\hline Coleman 2005 & High-income & USA \\
\hline Coleman 2012 & High-income & USA \\
\hline De Heer 2011 & High-income & USA \\
\hline Dennison 2004 & High-income & USA \\
\hline Donnelly 2009 & High-income & USA \\
\hline Ebbeling 2006 & High-income & USA \\
\hline Elder 2014 & High-income & USA \\
\hline Epstein 2001 & High-income & USA \\
\hline Fitzgibbon 2005 & High-income & USA \\
\hline Fitzgibbon 2006 & High-income & USA \\
\hline Fitzgibbon 2011 & High-income & USA \\
\hline Foster 2008 & High-income & USA \\
\hline French 2011 & High-income & USA \\
\hline Fulkerson 2010 & High-income & USA \\
\hline
\end{tabular}


(Continued)

\begin{tabular}{|c|c|c|}
\hline Gentile 2009 & High-income & USA \\
\hline Gortmaker 1999a & High-income & USA \\
\hline Gutin 2008 & High-income & USA \\
\hline Haines 2013 & High-income & USA \\
\hline Haire-Joshu 2010 & High-income & USA \\
\hline Harvey-Berino 2003 & High-income & USA \\
\hline HEALTHY Study Gp 2010 & High-income & USA \\
\hline Hendy 2011 & High-income & USA \\
\hline Howe 2011 & High-income & USA \\
\hline Jansen 2011 & High-income & USA \\
\hline Johnston 2013 & High-income & USA \\
\hline Khan 2014 & High-income & USA \\
\hline Klesges 2010 & High-income & USA \\
\hline Madsen 2013 & High-income & USA \\
\hline Mauriello 2010 & High-income & USA \\
\hline Natale 2014 & High-income & USA \\
\hline Neumark-Sztainer 2003 & High-income & USA \\
\hline Neumark-Sztainer 2010 & High-income & USA \\
\hline Nollen 2014 & High-income & USA \\
\hline Ostbye 2012 & High-income & USA \\
\hline Pate 2005 & High-income & USA \\
\hline Patrick 2006 & High-income & USA \\
\hline Paul 2011 & High-income & USA \\
\hline Reed 2008 & High-income & Canada \\
\hline Robbins 2006 & High-income & USA \\
\hline Robinson 2003 & High-income & USA \\
\hline Robinson 2010 & High-income & USA \\
\hline
\end{tabular}




\begin{tabular}{|c|c|c|}
\hline Rodearmel 2006 & High-income & USA \\
\hline Rosenkranz 2010 & High-income & USA \\
\hline Sallis 1993 & High-income & USA \\
\hline Santos 2014 & High-income & Canada \\
\hline Shin 2015 & High-income & USA \\
\hline Slusser 2012 & High-income & USA \\
\hline Spiegel 2006 & High-income & USA \\
\hline Stolley 1997 & High-income & USA \\
\hline Story 2003 & High-income & USA \\
\hline Story 2012 & High-income & USA \\
\hline Velez 2010 & High-income & USA \\
\hline Whittemore 2013 & High-income & USA \\
\hline Williamson 2012 & High-income & USA \\
\hline Crespo 2012 & Upper middle-income & US-Mexico border \\
\hline Levy 2012 & Upper middle income & Mexico \\
\hline Macias-Cervantes 2009 & Upper middle income & Mexico \\
\hline Lana 2014 & Upper middle-income & Mexico, Spain \\
\hline Safdie 2013 & Upper middle income & Mexico \\
\hline
\end{tabular}

alncome based on World Bank classification of countries.

\section{South America}

\begin{tabular}{lll}
\hline Study ID & Incomea $^{\text {Country }}$ \\
\hline Herscovici 2013 & High-income & Argentina \\
\hline Kain 2014 & High-income & Chile \\
\hline Andrade 2014 & Upper middle-income & Ecuador \\
\hline Cunha 2013 & Upper middle-income & Brazil \\
\hline Farias 2015 & Upper middle-income & Brazil \\
\hline Sichieri 2009 & Upper middle-income & Brazil \\
\hline \hline
\end{tabular}


alncome based on World Bank classification of countries.

\section{Appendix 7. Theories underpinning the interventions}

\begin{tabular}{|c|c|c|c|}
\hline Theory & $\begin{array}{l}\text { RCTs of children } \\
\text { aged } 0-5\end{array}$ & $\begin{array}{l}\text { RCTs of children } \\
\text { aged 6-12 }\end{array}$ & $\begin{array}{l}\text { RCTs of children } \\
\text { aged 13-18 }\end{array}$ \\
\hline Anticipatory guidance & $\checkmark$ & & \\
\hline Attachment theory & $\checkmark$ & & \\
\hline Attitude, social influence and self-Efficacy (ACE model) & & & $\checkmark$ \\
\hline Behavioural choice theory & & $\checkmark$ & \\
\hline Control theory & & & $\checkmark$ \\
\hline Ecological and developmental systems theories & & $\checkmark$ & \\
\hline Environmental change theory & & $\checkmark$ & \\
\hline Exposure theory & $\checkmark$ & & \\
\hline Family systems theory & & $\checkmark$ & \\
\hline Group socialization theory & & $\checkmark$ & \\
\hline Health belief model & $\checkmark$ & $\checkmark$ & \\
\hline Health promotion model & & $\checkmark$ & \\
\hline Implementation intentions & & & $\checkmark$ \\
\hline Information-motivation behavioral skills model & & & $\checkmark$ \\
\hline Investigation, vision, action and change methodology & & $\checkmark$ & \\
\hline Positive youth development & & $\checkmark$ & \\
\hline Precaution adoption process model & $\checkmark$ & & $\checkmark$ \\
\hline Self-determination theory & $\checkmark$ & $\checkmark$ & $\checkmark$ \\
\hline Social cognitive theory & $\checkmark$ & $\checkmark$ & $\checkmark$ \\
\hline Social learning theory & & $\checkmark$ & $\checkmark$ \\
\hline Sociocultural theory & & $\checkmark$ & \\
\hline Socioecological model & $\checkmark$ & & $\checkmark$ \\
\hline Theories of information processing & $\checkmark$ & & \\
\hline Theory of interactive technology & & & $\checkmark$ \\
\hline
\end{tabular}


(Continued)

Theory of planned behaviour

Theory of reasoned action, constructivism

Transtheoretical model-stages of change

Number of theories $\checkmark$

$\checkmark$

\section{WHAT'S NEW}

\begin{tabular}{lll}
\hline Date & Event & Description \\
\hline 18 July 2019 & $\begin{array}{l}\text { New citation required and conclusions } \\
\text { have changed }\end{array}$ & $\begin{array}{l}\text { The conclusions of this review have changed in that there is } \\
\text { more detail about the effects of the three intervention types on } \\
\text { preventing obesity for children in the three different age groups, } \\
\text { specifying where we found evidence of an effect and where we } \\
\text { found no evidence. We assessed the certainty of evidence for this } \\
\text { review using the GRADE approach. In this review we have identi- } \\
\text { fied and included evidence from low- and middle-income coun- } \\
\text { tries. We have made changes to the methods and meta-analy- } \\
\text { sis, including updating the assessment of risk of bias to account } \\
\text { for biases specific to cluster-RCTs. There have been changes to } \\
\text { the composition of the authorship team since the last review was } \\
\text { published. }\end{array}$ \\
\hline
\end{tabular}

21 May $2019 \quad$ New search has been performed

In this update, we reran the search up to June 2015 and added 108 new randomised controlled trials (RCTs), bringing the total to 153 . We changed the inclusion criteria restricting our search to RCTs and, consequently, have excluded 10 non-randomised studies present in the version published in 2011. We re-ran searches again to January 2018 and provide a list of all potentially relevant studies we identified published between 2015 and 2018. RCT publications are accruing on this topic at the rate of approximately 100 per year. This volume of evidence requires changes to the presentation and preparation of the data for this systematic review. Therefore, in the future this review will be split into three separate Cochrane systematic reviews, each with a new protocol, based on age/developmental stage of the children. Data from the 2018 search will be synthesised into those separate reviews.

\section{HISTORY}

Protocol first published: Issue 4, 1999

Review first published: Issue 1, 2001

\begin{tabular}{lll}
\hline Date & Event & Description \\
\hline 1 August 2013 & Amended & $\begin{array}{l}\text { Republished under new editorial group (from Heart to Public } \\
\text { Health Group), with no changes to the text of the review. }\end{array}$ \\
\hline 27 May 2011 & $\begin{array}{l}\text { New citation required but conclusions } \\
\text { have not changed }\end{array}$ & $\begin{array}{l}\text { In this update, we reran the search for studies up to March 2010 } \\
\text { and 36 additional new studies have now been included (the pre- }\end{array}$ \\
\hline
\end{tabular}




$\begin{array}{lll}\text { Date Event Description } & \text { Den }\end{array}$

vious version of this review included 22 studies, however three of the original 22 studies have now been moved to excluded studies). A meta-analysis has been conducted and demonstrates marked heterogeneity, but with estimates of effects that are unlikely to be due to chance. Data extraction has been expanded in this review update to include a variety of "implementation factors" to aid contextualisation and utilisation of findings.

\begin{tabular}{|c|c|c|}
\hline 3 July 2008 & Amended & Converted to new review format. \\
\hline 1 July 2005 & New search has been performed & $\begin{array}{l}\text { Search strategies run in February 2005. The inclusion criteria } \\
\text { were changed to exclude studies published before } 1990 . \\
\text { Twelve new studies were included. Three long-term studies of } \\
1 \text { year or more (Caballero 2003; James 2004; Warren 2003) and } \\
\text { nine short-term studies of } 3 \text { months to } 1 \text { year (Baranowski 2003; } \\
\text { Beech 2003; Dennison 2004; Harvey-Berino 2003; Kain 2004; Neu- } \\
\text { mark-Sztainer 2003; Pangrazi 2003; Robinson 2003; Story 2003). } \\
\text { One study (Simonetti 1986) was excluded because it was pub- } \\
\text { lished before 1990. This study had been included in earlier ver- } \\
\text { sion of this review. } \\
\text { The conclusions were amended slightly, but the main direction } \\
\text { and intent of the conclusions did not change. The background } \\
\text { section was updated. The methodology used for this update was } \\
\text { changed to include additional search terms and information } \\
\text { from study evaluations in keeping with the broader approach of } \\
\text { health promotion and public health reviews. }\end{array}$ \\
\hline
\end{tabular}

1 April $2002 \quad$ New search has been performed

Search strategies were rerun and review content updated accordingly.

\section{CONTRIBUTIONS OF AUTHORS}

Tamara Brown led the review process up to June 2015, worked on the amended protocol, conducted the searching, developed the extraction template, extracted data, provided advice with data extraction, meta analysis and data synthesis decisions, performed data synthesis, and wrote the review text and contributed to previous versions of this review. She also screened records for the 2018 update search.

Theresa Moore led the process of responding to reviewers' and editors' comments for the 2015 update, including meta-analysis, review structure, interpretation of data, synthesis of evidence, implementation of GRADE, drafting and editing of review text and screening titles and abstracts. She also led the process for the 2018 update search.

Lee Hooper checked data syntheses, interpreted the results, assisted with the draft and helped to revise the manuscript.

Yang Gao helped with data extraction, translation of studies, contributed to previous versions of this review, assisted with the draft and helped to revise the manuscript.

Sharea ljaz assessed risk of bias, helped with data extraction, assisted with the draft and helped to revise the manuscript.

Martha Elwenspoek screened titles and abstracts and commented on the final review.

Amir Zayegh helped with data extraction and commented on the final review.

Sophie Foxen helped with data extraction and commented on the final review.

Lucia Magee helped with data extraction and commented on the final review.

Claire O'Malley helped with searching, data extraction and commented on the final review.

Liz Waters (deceased) initially provided the overall structure and process and contributed to previous versions of this review. 
Carolyn Summerbell: provided the overall structure and process; contributed to previous versions of this review;

amended the protocol; developed the extraction template; extracted data; interpreted the results; revised the manuscript; and commented on the final review. As corresponding author, Carolyn has had full access to the data in the review and takes final responsibility for the decision to submit for publication.

\section{DECLARATIONS OF INTEREST}

Tamara Brown: no conflicts of interest to report Theresa Moore: no conflicts of interest to report Lee Hooper: no conflicts of interest to report Yang Gao: no conflicts of interest to report Amir Zayegh: no conflicts of interest to report Sharea ljaz: no conflicts of interest to report Sophie Foxen: no conflicts of interest to report Lucia Magee: no conflicts of interest to report Claire O'Malley: no conflicts of interest to report Carolyn Summerbell: no conflicts of interest to report Martha Elwenspoek: no conflicts of interest to report

\section{SOURCES OF SUPPORT}

\section{Internal sources}

- Fuse, NIHR (National Institute for Health Research Collaboration) Centre for Translational Research in Public Health, UK.

- Faculty of Health and Social Sciences, Durham University, UK.

- School of Medicine, Deakin University, Australia.

- School of Population and Global Health, University of Melbourne, Australia.

- National Institute for Health Research (NIHR) Collaboration for Leadership in Applied Health Research and Care West (CLAHRC West), UK. - supported the time of Theresa Moore, Sharea Ijaz, Jelena Savovic, Alison Richards and Martha Elvenspoek.

- Population Health Sciences, Bristol Medical School, University of Bristol, UK.

\section{External sources}

- World Health Organization, Switzerland.

- Cochrane Review Support Funding, Cochrane Central Executive, UK.

\section{DIFFERENCES BETWEEN PROTOCOLANDREVIEW}

\section{Objectives}

We have reduced the objectives of this review to an analysis of zBMI scores, BMI and adverse events. Earlier versions of this review included several additional primary and secondary outcomes and we have not attempted to assess the effect of interventions on changes in prevalence of obesity, and rate of weight gain among children under 18 years (see primary outcomes section below for details).

\section{Search}

We have updated the search to 2018 , however we have not yet synthesised evidence from identified potential studies into the review. The rationale for this is that the evidence on this topic is accruing at the rate of 2000 to 4000 records per year, or approximately 200 potentially relevant, full-text papers to assess per year. Added to this, the current scope of this review is too broad to identify nuanced differences in what works for whom in which setting. By publishing the synthesis of the 2015 search we present the most up-to-date, synthesised evidence. The list of potentially relevant studies makes the next tranche of evidence available to researchers. We will now divide this review into three smaller reviews by age group of child. We will prepare new protocols for these reviews in which all methods can be revised and from which we will be able to carry out a more detailed analysis of the effects of interventions.

\section{Searching other resources}

For the 2018 update we searched Clinicaltrials.gov (clinicaltrials.gov/), with the filter 'Applied Filters: Child (birth-17)'. We also searched the WHO International Clinical Trials Registry Platform, search portal (apps.who.int/trialsearch/), using the filter for studies in children.

\section{Types of studies}

Controlled trials without randomisation (CCTs) had been included in this review up to and including the 2011 update. From 2015 onwards we excluded CCTs as there are were sufficient numbers of RCTs available to contribute to this research question. As a result we excluded 10 
CCTs from this review. In the 2011 version we excluded cluster-RCTs with fewer than six clusters, resulting in the exclusion of three studies. In the 2001 and 2002 version, we included studies regardless of publication date. In the 2005 version (and onwards), studies published before 1990 were excluded, resulting in the exclusion of one study. Our rationale for this is that global evidence suggests that the prevalence of overweight and obesity in children, including preschool children, started to rise at the end of the 1980s (de Onis 2010; GBD Obesity Collaboration 2014). Given the lag time between the conception, funding, and the completion of RCTs, we considered a 1990 publication date as a pragmatic and reasonable starting point for the literature in the area.

\section{Data collection}

\section{Indicators of theory and process}

We collected data on indicators of intervention process and evaluation, health promotion theory underpinning intervention design, modes of strategies and attrition rates. We compared where possible, whether the effect of the intervention varied according to these factors. We included this information in descriptive analyses and used it to guide the interpretation of findings and recommendations.

\section{Primary outcomes}

We have reduced the number of primary outcomes to

- zBMI

- BMI

- Adverse events

We are no longer presenting data on the outcomes listed below, although we have recorded which studies reported these outcomes.

- Prevalence of overweight and obesity

- Weight and height

- Ponderal index

- Per cent fat content

- Skin-fold thickness

\section{Selection of studies}

For the 2015 update, one reviewer (TB) screened titles and abstracts, with a random subsample (10\%) checked by another review author (CS). For the 2018 update two review authors (TB and ME) independently assessed, in duplicate, all titles and abstracts, using RAYYAN software (Rayyan-QCRI 2016).

\section{Assessment of risk of bias in included studies}

\section{Selective outcome reporting}

In the 2011 review, studies were at low risk of reporting bias when a published protocol was available, and all specified outcomes were included in the study report; we assessed studies without a published protocol as unclear risk of reporting bias. For this current version, we have followed methods as described in the Cochrane Handbook for Systematic Reviews of Interventions (Higgins 2017) and have sought protocols or trials register reports for all studies, and compared reported outcomes, with those specified a priori. Full details are in the methods.

\section{Measures of treatment effect}

\section{Unit of analysis issues}

For cluster-randomised studies, we assessed whether the study had analysed the data using methods that accounted for clustering. For those studies that had used analyses that were not able to account for clustering, for example using t-tests, we approximated clustering effects using methods as stated in the Cochrane Handbook for Systematic Reviews of Interventions (Higgins 2011a). We ran sensitivity analyses comparing the meta-analyses with and without approximate adjustment for clusters. There were very slight differences in the pooled treatment effects. We then elected to use the outcomes with approximation of adjustment for clustering. Full details are in the methods section.

\section{Data synthesis}

We pooled zBMI data and BMI data separately in the meta-analyses for this update. Previous versions aggregated data from these outcomes using standardised mean differences. Also, we have not presented a pooled analysis for all studies. Instead we have presented distinct comparisons for each age group. We have subgrouped these by setting and duration. We believe the populations, children aged 0 to 5 years, children aged 6 to 12 years and young people aged 13 to 18 years, to be too different, developmentally, to be considered to be a single sample. Interventions that are likely to work on a four-year-old, are unlikely to work in adolescents, and vice versa. We have presented the effects of BMI and zBMI for each of the three age groups as the main analyses in this review. In future this review will be split into three new reviews by the age group of the children, to allow a more detailed analysis of the data. 
This update of the review pooled data using generic inverse variance for zBMI and BMI. Previous versions of the review reported several outcomes including adiposity, physical activity-related behaviours or diet-related behaviours, however, in this version we have reported only results for the anthropometric outcomes ZBMI and BMI. This was because of the volume of outcome data from 153 included studies. We will re-evaluate decisions on the outcome measures of interest and analysis of outcomes in the next update of this review.

Our 2018 update search identified several potentially relevant studies after title and abstract screening. We have not yet extracted data and information about these studies but have classified them as 'Studies awaiting classification' (see Characteristics of studies awaiting classification). This allowed the review authors to publish this systematic review with the synthesis of data from the 2015 search and also to list studies potentially relevant to the review at the next update and make them available to users of this review. With the exceptionally rapid accrual of literature and studies on this topic, updating this review becomes increasingly difficult (See Figure 2). In addition, systematic review and analysis methods have also changed since 2001 when this review was first published. The review team plan to split the review into three new reviews based upon the age of the children, and this will provide an opportunity to update the objectives and analysis methods of the review.

\section{Subgroup analysis and investigation of heterogeneity}

In the 2001, 2002 and 2005 versions, studies we categorised studies into long-term (at least one year) and short-term (at least 12 weeks), referring to the length of the intervention itself or to a combination of the intervention with a follow-up phase. For the 2011 version and this current version, we categorised studies based on target age group ( 0 to 5 years, 6 to 12 years, and 13 to 18 years) rather than study duration, to enhance utility of this review for decision makers as these age groups correspond to stages of developmental and childhood settings.

In earlier versions of this review we evaluated effectiveness by subgrouping according to risk of bias based on one domain only, randomisation. For this review we have used the GRADE process to assess the effects of risk of bias on the outcomes by downgrading evidence if risk of bias affected the treatment effect. See Assessment of risk of bias in included studies.

\section{GRADE and 'Summary of findings' table}

We created 'Summary of findings' tables to summarise the size and certainty of effects of the interventions. This was based on the five GRADE considerations (risk of bias, consistency of effect, imprecision, indirectness and publication bias). We used GRADEpro software (GRADEpro GDT 2015), and followed methods described in the Cochrane Handbook for Systematic Reviews of Interventions (Section 8.5 (Higgins 2017), and Chapter 12, (Schünemann 2017)). In determining consistency of effects for each comparison we looked at the 12 statistic value. For comparisons where the meta-analysis had an 12 statistic value above $60 \%$ we determined these to be at 'serious' inconsistency, if the $1^{2}$ was above $85 \%$ we considered this to be 'very serious' inconsistency. For risk of bias, we examined if the treatment changed markedly upon removal of studies at high risk of bias. If the effect change was small we did not downgrade. However, if the effect size was large then we downgraded the evidence.

\section{NDEX TERMS}

\section{Medical Subject Headings (MeSH)}

*Diet; Behavior Therapy; Body Mass Index; Combined Modality Therapy; Exercise [ ${ }^{*}$ physiology]; Overweight [prevention \& control] [therapy]; Pediatric Obesity [ ${ }^{\star}$ prevention \& control] [therapy]; Quality of Life; Randomized Controlled Trials as Topic; Treatment Outcome

\section{MeSH check words}

Adolescent; Child; Child, Preschool; Female; Humans; Infant; Male 